
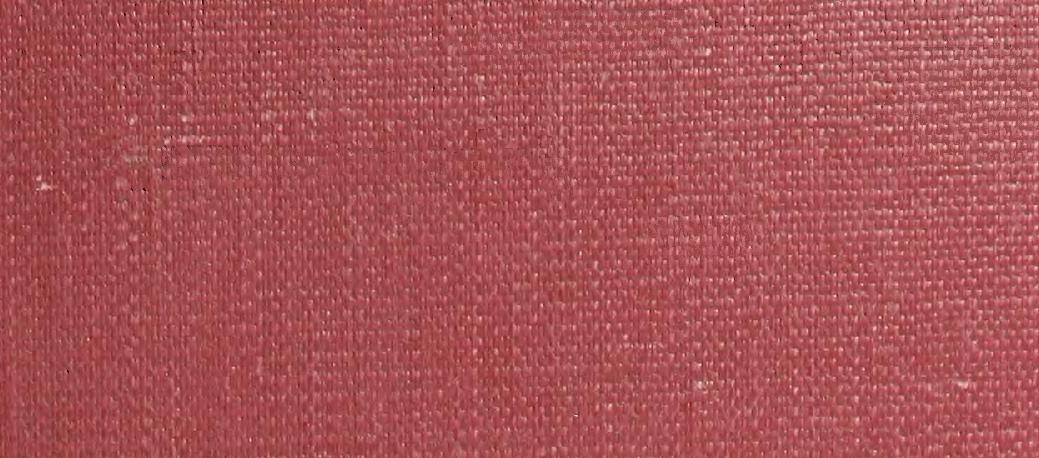


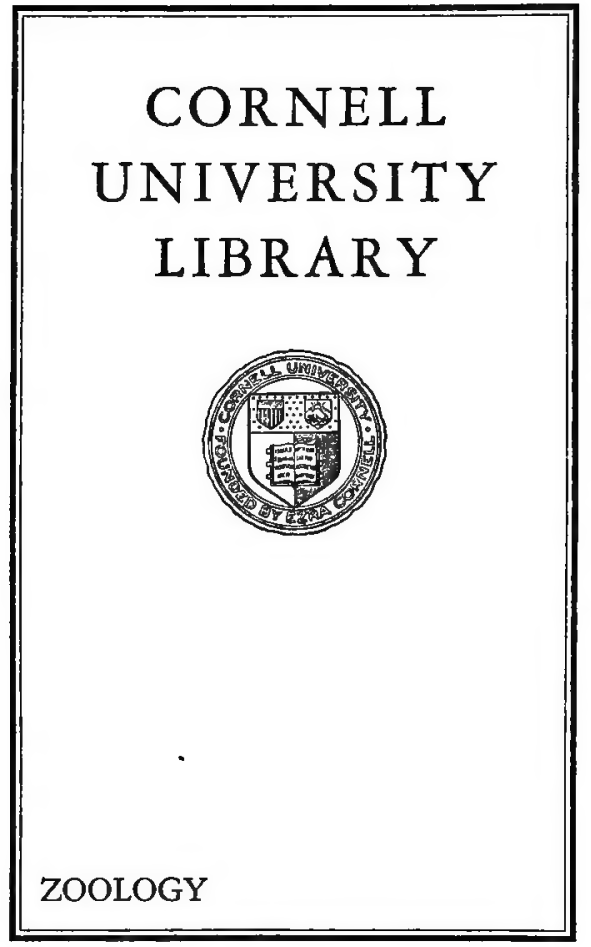


Anatomical

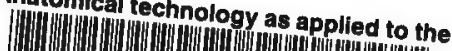

31924003015421 


\section{Cornell University Library}

The original of this book is in the Cornell University Library.

There are no known copyright restrictions in the United States on the use of the text.

http://www.archive.org/details/cu31924003015421 






\title{
ANATOMICAL TECHNOLOGY
} AS APPLIED T'O

\section{THE DOMESTIC CAT:}

\author{
AN INTRODUCTION
}

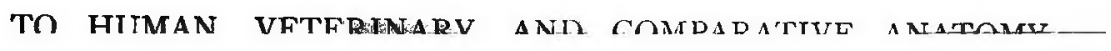

In preparation by the same authors :

"Microscopical Technology, as applied to the domestic cat, the frog and the Meno-branchus."

"Physiological Technology, the methods of painless experimentation (callisection) upon the frog and the domestic cat."

NEW YORK AND CHICAGO:

A. S. BARNES AND COMPANY. 



\title{
ANATOMICAL TECHNOLOGY
}

AS APPLIED TO

\section{THE DOMESTIC CYAT:}

\author{
AN INTRODUCTION
}

TO HUMAN, VETERINARY, AND COMPARATIVE ANATOMY.

\section{WITH ILLUSTRATIONS.}

BY

\section{BURT G. WILDER, B.S., M.D.,}

PRCFESSOR OF PHYSIOLOGY, COMPARATIVE ANATOMY, AND ZOÖLOGY IN CORNELL UNIVERSITY, AND OF HHYSIOLOGY IN THE MEDICAL SCHOOL OF MAINE, MEMBER OF THE AMERICAN NEUROLOGICAL ASSOCIATION, FELLOW OF THE AMERICAN ASSOCIATION FOR THE ADVANCEMENT OF SCIENCE, ETC.

\section{AND}

\section{SIMON H. GAGE, B.S.}

ASSISTANT PROFESSOR OF PHYSIOLOGY AND LECTURER ON MICROSCOPICAL TECHNOLOGY IN CORNELL UNIVERSITY, FELLOW OF THE AMERICAN ASSOCLATION FOR THE ADVANCEMENT OE SCIENCE, ETC.

NEW YORK AND CHICAGO:

A. S. BARNES AND COMPANY. 
Med. 1093

Copyright, 1882, by A. S. Barnes \& Co. ALL RIGHTS RESERVED. 


\section{P R E F A C E.}

THIs work has grown out of our needs as instructors of students preparing for practical work in Human, Veterinary, or Comparative Anatomy. Most of it has been employed in the anatomical laboratory of Cornell University for from one to four years, and we have been led to believe that it may prove useful elsewhere.

Some of our laboratory students aim to be professional naturalists, agriculturists, or veterinarians, but most of them intend to study Medicine or to teach Physiology with other branches in schools and colleges. The latter desire to gain a personal acquaintance with the organs whose functions they are to discuss, and the former require, in addition, a familiarity with anatomical methods and literature; few of them have had any practical training in Biology.

The guides to vertebrate dissection by Straus-Durckheim, Morrell, Rolleston, Krause, Huxley and Martin, Foster and Langley, Bernard, Martin and Moale, and Mojsisovics, present many admirable feutures, but four of them are in French or German, and none have fully answered our requirements.

Of the works above named, several imply that either the frog or the human body has been previously dissected; hence, presumably, the brevity of the directions, the lack of descriptions of instruments and methods, and the fewness or absence of illustrations. They are based upon the frog, turtle, dog, rat or rabbit, or on animals in general, and the ordinary anthropotomical terms of description, upper, lower, etc., are almost uniformly employed. Some dwell only upon points of physiological importance, and in nearly all the references to other publications are few and general. 
So far as we are aware, this work differs from the manuals above mentioned in one or more of the following particulars:-

It assumes no previous anatomical knowledge or experience, yet is rapidly progressive, introducing in succession bones, muscles, viscera, vessels, nerves, brain and organs of sense.

It is based upon the domestic cat (see $\$ 124)$. The terms of description and designation apply to all vertebrates (\$38); they are technical (\$29), and precision and brevity have been especially regarded in their selection.

The purely descriptive portions are subordinated to the practical and directive. There is a General List of the instruments and materials required for ordinary anatomical work ( $\$ 130)$, and directions are given for their care. All directions for dissection and nanipulation begin with special lists of the instruments and materials required (\$ 235). Explicit instructions are given for coarse injections, for the preparation of bones, and for the use of alcohol as a preservative.

Certain regions and organs are quite fully discussed, while others are briefly mentioned or omitted altogether ( $\S 128)$. Unusual space is given to the viscera $(\$ 129)$. The study of the brain includes a consideration of the typical vertebrate brain, descriptions and dissections of the brains of the frog and the Menobranchus, and an approximately complete Descriptive List of the encephalic parts, with References and Synonyms.

There are illustrations, and the methods of making the preparations figured are fully described. The abbreviations are of the technical names only, and they are nearly uniform throughout. As far as possible, the technical names are written in full upon the figures. In the explanations of the figures, the technical names and the abbreviations are alphabetically arranged (\$33).

There are numerous Alphabetical Lists, Tables of Synonyms and Tabular Arrangements of names according to the relations of parts (p. xxvi).

Attention is called to the incompleteness of our information upon certain points.

There are frequent cross references and numerous definite references to other publications $(\S 2)$; the titles of the works and papers cited (three hun- 
dred and thirty in number) are given in an Alphabetical List of Authors at the end.

While, therefore, the book is designed primarily as a guide for beginners, certain features-the references and the suggestions as to lines of inquirymay prove useful to teachers and others who may undertake to add to existing knowledge.

Histological facts and methods do not come within the scope of the work, but at the close of the discussion of most of the organs is given a summary of (A) the abrious or macroscopic structure-that which may be determined with the unaided eye, and (B) the fine or microscopic structure. The latter is in no sense complete. It embraces only the points upon which most standard authors are agreed and which may be demonstrated without a great expenditure of time. Only the structure of the given tissue is considered; bence the presence of vessels and nerres is not mentioned. If it be desired to carry the histological inquiry fartber, the works of Quain, Stricker, Ranvier, Beale, Frey, and the special papers therein referred to, are recommended.

Among the many friends who have aided or encouraged us, our thanks are especially due to Professor Oliver Wendell Holmes for helpful criticism of the terminology and for suggesting the preparation of a manual in which it should be incorporated; to our colleague, Professor J. H. Comstock, and to Professors E. C. Spitzka and T. B. Stowell for valuable suggestions and for the adoption in their writings of the descriptive terms herein employed; also to the last named for a critical revision of the early manuscript of the muscles, and for the important additions to knowledge contained in his recent paper on the vagus nerve of the cat. To all of our laboratory students we are indebted for aid, suggestions and criticisms, and especially to those (see end of Bibliography) who have selected parts of the cat as subjects of their graduation theses.

Our acknowledgmerts are here made to the American Philosophical Society for the use of the four lithographic plates, and to the firms named. in the List of Illustrations for the courteous: loan of electrotypes of instruments manufactured by them.

The original figures were drawn by the persons named in the Note preceding the List of Illustrations. The three ladies have also been our stu- 
dents, and have shown more than ordinary interest in their work. Most of the original drawings were made by our colleague, Professor E. C. Cleaves, whose skill, patience, and accuracy only artists and anatomists can fully appreciate.

There has been constant coöperation throughout, but, it may be proper to state, the senior author holds himself particularly responsible for the Introduction, the Preservation of Soft Parts, the Bones of the Iimbs, the Muscles, the Heart, the Central Nerrous System and the Cranial Nerves; and the junior author for the Preparation of Bones, Coarse Injections, the Skeleton excepting the limbs, the Viscera, the Peripheral Vascular and Nervous Systems and the Organs of Sense.

Notwithstanding our efforts for accuracy, there are donbtless errors of observation and interpretation. Corrections or suggestions will be gladly received. 


\section{O N T E N T S.}

\section{INTRODUCTION.}

References to Publications................................... 1-8

Method of reference to authors................................ $\quad 2$

Catalogue of scientific papers published by the Royal Society of London .. 3

Decimal Systems.

Centigrade Thermometer-Fallrenheit's Thermometer....................

Comparison and reduction of the two thermometric scales.............. 3

Table of equivalent temperatures according to the Fahrenheit and Centi-

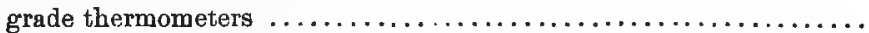

Formulæ for the reduction of the Fahrenheit and Centigrade scales and vice versa.............................................

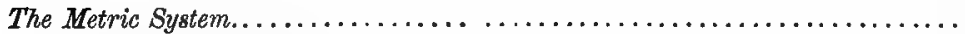

Meter, liter and gram-definitions and common equivalents............

Comparison of the metric and English systems of weights and measures... 5

Table of the metric measures............................... 5

How to learn the metric system............................ 6

Table of equivalents of metric and English measures............... 7

The metric system in medicine.............................. 8

Zoological Classification .................................... 8

Table illustrating classification $\ldots \ldots \ldots \ldots \ldots \ldots \ldots \ldots \ldots \ldots \ldots, \quad 9$

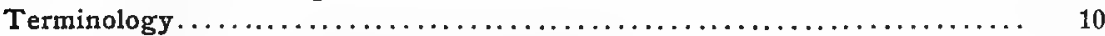

Character of the terminology here used........................ 10

General considerations upon anatomical terminology ................ 11

Aphorisms upon terminology from various sources ................. 12

Brief atatement of the objects and methods of the terminological changes here made......................................... 13

Designation of Orguns-Organonomy........................... 14

General considerations by various authors....................... 14

Importance of brevity...................................... 15

Comparison of technical and vermacular terms in science $\ldots \ldots \ldots \ldots \ldots \ldots$ 15-17

Ease in acquiring technical terms............................ 17

Names indicative of relative position. ........................ 17

The limits of terminological change.......................... 18

Priority and etymological appropriateness of technical terms........... 18

Some inconsistencies in the nomenclature here adopted.............. 19

Names and abbreviations on the figures $\ldots \ldots \ldots \ldots \ldots \ldots \ldots \ldots, 20$ 


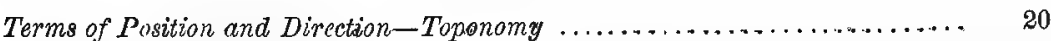

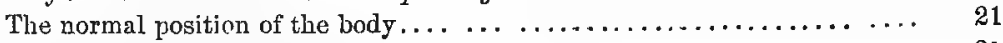

Designation of the aspects of animals. . . . . . . . . . . . . . .

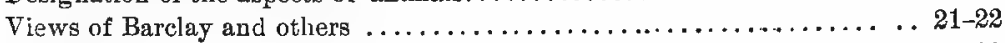

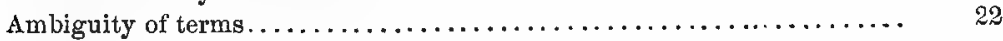

Intrinsic Toponomy ................................... 28

Cephalic and caudal, dorsal, veutral, dextral, sinistral, lateral and mesalsignification and use by various authors . . . . . . . . . . . . $23-24$

Ventrimeson, dorsimeson, intermediate and medial. . . . . . . . . . . .

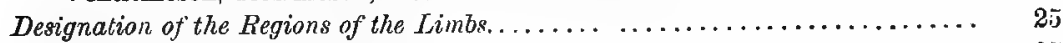

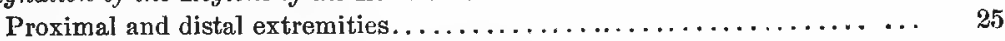

Cephalic and caudal, dorsal and ventral aspects.............. 25-26

Other names for the extremities and aspects................. $20^{2}$

Terms of General Application to the Whote Body.................. 26

Central and peripheral, ectal and ental.................. 26-27

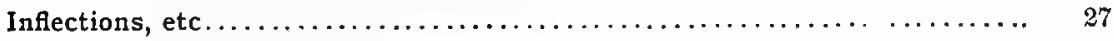

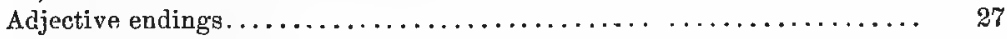

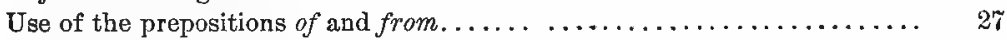

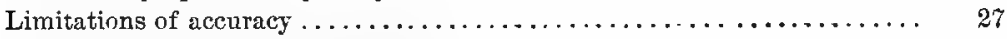

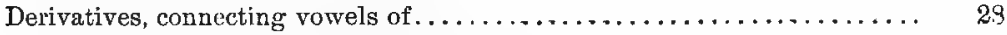

Compound words-Hybrid words....................... 28

Primary Divisions of the Body-Soma and Membra............... 29

Axial portion, soma-A ppendicular portion, membra-Truncus or trunk.. 29

Comparison of right and left (dextral and sinistral) iu the object and the

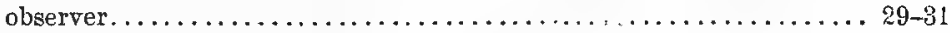

Position and direction on the soma........................ 31

Coltumna vertebralis, its constitution and location $\ldots \ldots \ldots \ldots \ldots \ldots \ldots \ldots 2$

Canalis neuralis and cclum or general body cavity ............. 83

Essential characteristic of all vertebrates. ..................

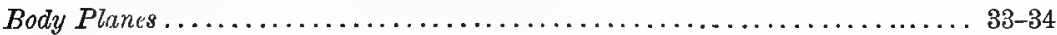

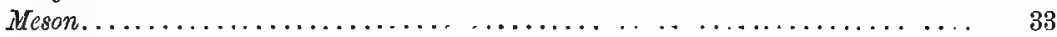

Paired or Lateral Organs-Azygous or Mesnl Organs.... . . . . . . . 33, 43

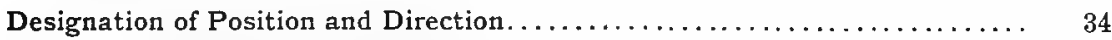

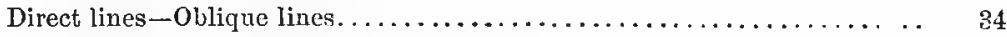

Designation of direct and oblique lines.................. 34-35

Designation of position on oblique and direct lines $\ldots \ldots \ldots \ldots \ldots \ldots \ldots . \ldots \ldots$

Designation of diagonal liues. . . . . . . . . 35

Designation of the relative position of points on diagonal lines. . . . . . . . 86

Designation of Direction and Rulative Position on the Limbs. . . . . . . . . . 36

Ental and ectal and their derivatives...................... 36

The soma and its divisions-Hend, neck, trunk and tail............ 36

The trunk and its divisions-Thorax, abdomen and pelvis ......... 36-37

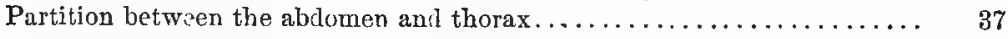

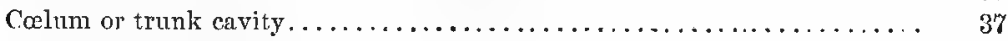

Table of the divisions of the body $\ldots \ldots \ldots \ldots \ldots \ldots \ldots \ldots \ldots \ldots \ldots \ldots \ldots$

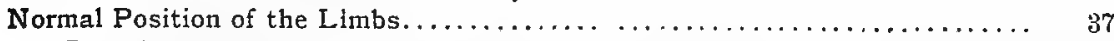

Limb Segments and the Arthra or Joints.................... 40

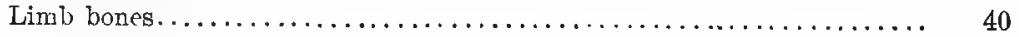

Carpus and tarsus. . . . . . . . . . . . . . . . . 41 
PAGH

Syntropy and antitropy $\ldots \ldots \ldots \ldots \ldots \ldots \ldots \ldots \ldots \ldots \ldots \ldots, 42$

Enumeration of Parts in a Series........................... 42

Use of Terms of Relative Position in a Physiological Sense............. 44

Slip System of Notes..................................... $4 \overline{5}-52$

Slips-Size, etc.-Making notes on........................ 45

Library catalogues and catalogue data for specimens............... 46

References, extracts, clippings and notes proper................. 47

Accumulation and elimination of slips $\ldots \ldots \ldots \ldots \ldots \ldots \ldots \ldots \ldots, 47$

Arrangement and storage of notes............................. 48

Subdivision of notes, with Table.......................... 48-49

Distribution of glips . .................................... 50

Method of using slips .................................. 50

Portfolios for slips and sheets. . . . . . . . .

Miscellaneous suggestions as to the use of slips.................. 51

Origin of the slip system................................. 52

Rules and Aphorisms of General Application in Biology.............. 52-53

Anatomical Technology-Introductory.......................... 55

Reasons for Selection of the Cat for Anatomical Study.................. 55

Importance of methods................................. 55

Comparison of the cat as an anatomical subject with adult human beings, still-born children, horse, rabbit, dog, rat, opossum............ 55-57

Needs of a standard of comparison for comparative anatomists.......... 56 Reasons for treating of only a Part of the Body...................... 57 Reusons for giving unusual Prominence to the Viscerr.................... 58

\section{CHAPTER I.}

\section{INSTRUMENTS AND MATERIAL FOR AN.ITOMICAL TECHNOLOGY.}

Alphabetical list of iustruments and materials needed for anatomical technology, with prices......................................... $59-62$

Dealers' catalogues of instruments and materials ..................... 59

General Character of Instruments.............................. 62

Description of Instruments and Material. . . . . . . . . . . . . . . . . . . .

Arthrotome and beaded bristles.............................6 62-63

Supporting blocks, flexible blor-pipe... ..................... 64

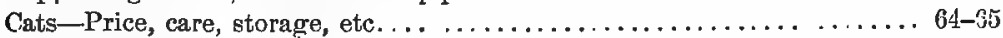

Cbain-hooks, small compressor and dissecting gowa................ 65

Drawing materials, foot lathe and drills, force; $\mathrm{s}$ (coarse and fine), nippers

for cutting bune................................... 67

Dropping-buttle oiler, parchment labels, rubber gloves and tubing, saw... 68

Scales and special weighing pan, scalpels...................... 69

Scissors and sponges ................................ 70

Syringotome, tags for labels, tools, towels, tracer (seeker or finder), sharp

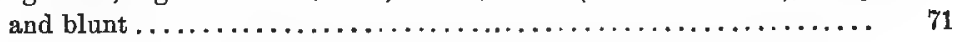

Trays for specimens, tripod magnifier and tunnels or funnels........... 72 
Wetting bottle for 15 per cent. glycerin, waste paper for particles made

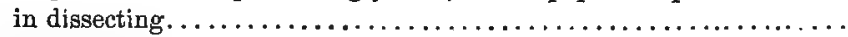

Care of Instruments.

Special directions for blow-pipe, tray, forceps, nippers, saw, scissors, scal-

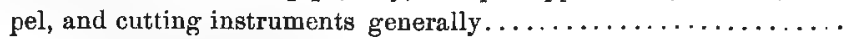

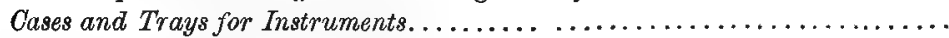

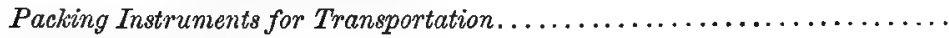

Polishing Instruments.

Sharpening Instruments.

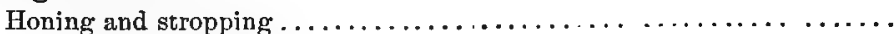

Determination of sharpness. . . . . . . . . . . . . . . . . . . . . .

Killing Animals for Dissection. . . . . . . . . . . . . . . . . . . .

Drowning, killing with chloroform or ether in a box.............

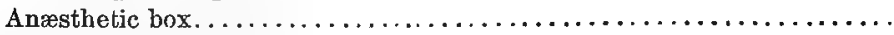

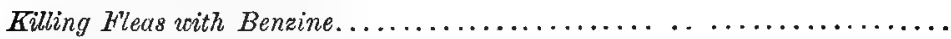

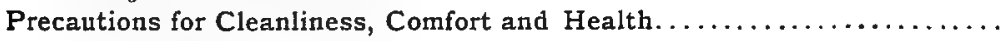

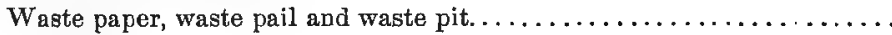

Washing sink. . . . . . . . . . . . . . . . . . . . . . . . .

Deodorizers - Animal charcoal, alcohol, potassium permanganas, sul-

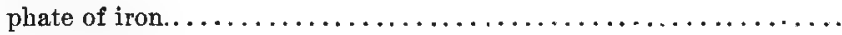

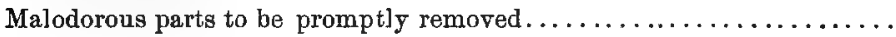

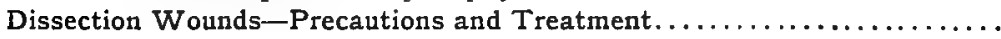

\section{CHAPTER II.}

GENERAL DESCRIPTION OF THE SKELETON, ANATOMICAL LANDMARKS AND ARDOMINAL TRANSECTION.

General Description of the Skeleton.

Components of the skeleton

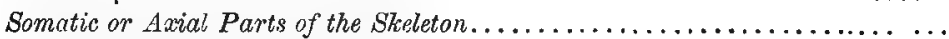

Skull and vertebræ.

Costæ or ribs, sternum, scapula and clavicle.................

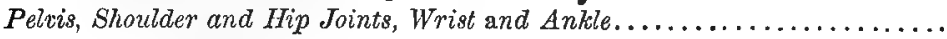

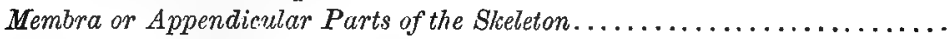

Bones of the limbs . . . . . . . . . . . . . . . . . . . . . . . . .

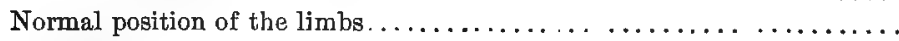

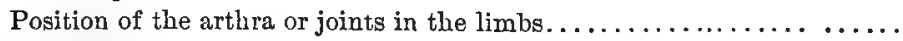

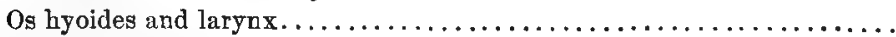

Anatomical landmarks.

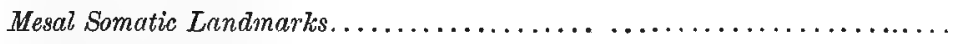

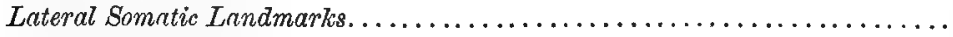

Membral Landmarks

Abdominal Transection. . . . . . . . . . . . . . . . . . . . . . . . .

Parts involved.

Preservation of the Cephalic Half of the Transected Body.............. 


\section{CHAPTER III.}

\section{PREPARATION AND PRESERVATION OF ANATOMICAL SPECIMENS.}

\section{Preparation of Bones}

PAGE

General directions................................. 108

Removing soft parts from alcoholic specimens................ 105

Removal of soft parts by ants or Delmestes................. 105

Putrefactive maceration in water......................... 105

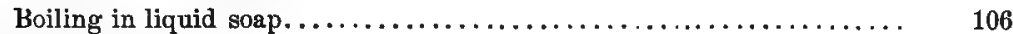

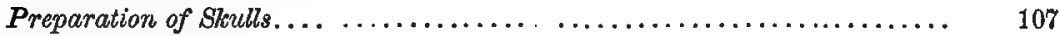

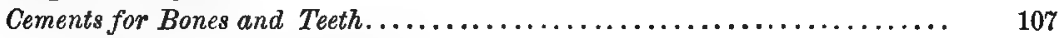

Preparation of Natural Skeletons. . ....................... 108-109

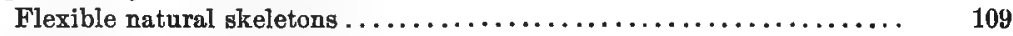

Preparation of the Bones of Young Animals..................... 109

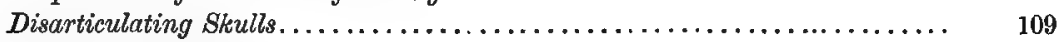

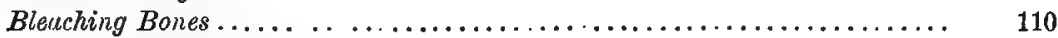

Preservation of Soft Parts. . . . . . . . . . . . . . . . . . . . . . 111-131

Agents preventing decomposition....................... 111

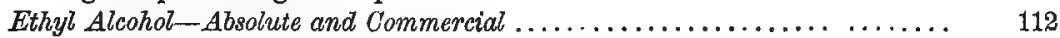

Table of percentages of alcohol. . . . . . . . . . . . . . . . . . . . 112

Leading characteristics of alcohol. ......................... 113

Inflammahility of alcohol and precautions against fire.............. 114

Determination of percentages of alcohol with the alcoömeter......... 114

Determination of and changing the percentage of alcohol. . . . . . . . 115

Table of the amount of alcohol and water to mix in order to obtain a

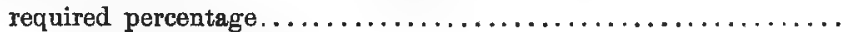

Kind of water to mix with alcohol ...................... 116

Use of Alcohol in Preserving Animals. . . . . . . . . . . . . . . . 117-131

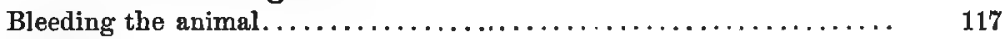

Opening the abdomen and expelling the fæces................. 118

Injection of alcohol into the thorax and abdomen.............. 118

Injection of alcohol into the Jungrs, stomach and intestines . . . . . . . . 119

Injection of the arteries.............................. 119

Treatment of Special Animals, Organs and 7issues ................ 120

Amount of Alcohol required for a Specimen...................... 121

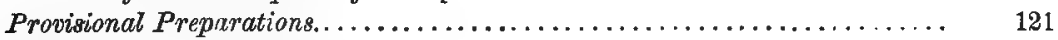

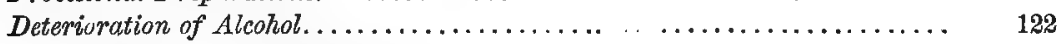

Purification of alcohol by settling and filtration.............. 122

Alcohal Vapor as a Preservative............................... 123

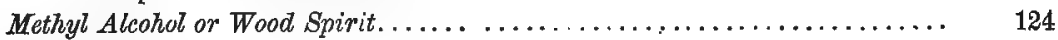

Wickersheimer's Preservative Fluid........................... 124

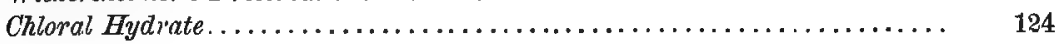

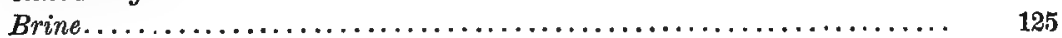

Temporary Storage of Alcoholic Specimen...................... 125

Lakage and evaporation............................. 125

Glass dishes and boxes for specimens..................... $\quad 126$

Exhibition or Permanent Storage of Alcoholic Specimens.............. 126

Metal cans, screw-top cans........................... 126 
Glass jars......................................... 127

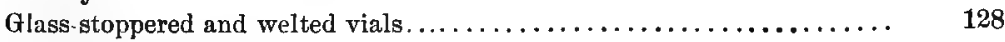

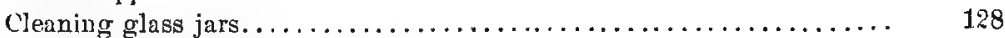

Display of alcoholis specimens. ......................... 129

Packing Alcoholic Specimens for Transportation. ................. 129-130

Frozen Sections and Dissections......................... 131-132

Preparation of the animal for freezing-Making and preserving the

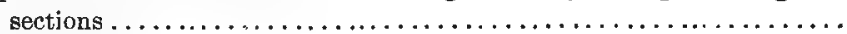

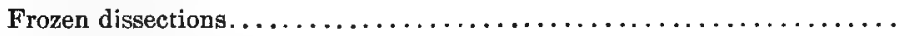

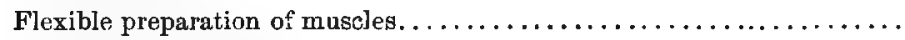

Preparation of Hollow Viscera ... . . . . . . . . . . . . . . . . . 132-135

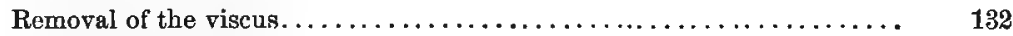

Cleaning, trimming and inflation with alcohol $\ldots \ldots \ldots \ldots \ldots \ldots \ldots . \ldots \ldots$

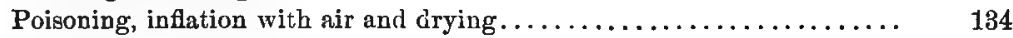

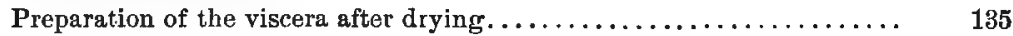

Measuring the Capacity or Volume of Organs.................... 135

\section{CHA P TER IV.}

\section{COARSE INJECTIONS.}

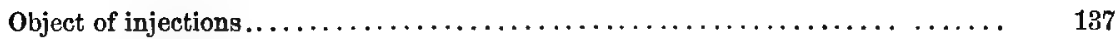

Syringe-Care of syringe, white metal syringe.................. 137

Canulæe and preparation of glass canulæ.................... 138

Brass anatomical syringes. . . . . . . . . . . . . . . . . . . . . 139

Injection Masses. . . . . . . . . . . . . . . . . . . . . . . . . . 139-141

Plaster of Paris Mass................................ 139

Carmine, Berlin blue and various colors for staining plaster......... 139

Preparation of plaster injection masses ... . . . . . . . . . . . . 140

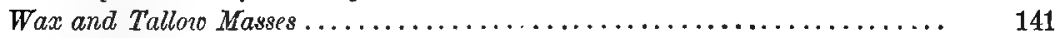

Practical working of wax and tallow injections............... 141

Choice of Specimen and Time for Injection. .................. 141

Arteries and veins to inject.......................... 142

Injection of Femoral Vessels............................. 142-146

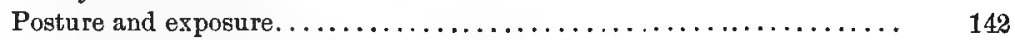

Parting the hair and isolating the vessels ... .............. 143

Incision in the vessel-Ligatures and introduction of the canula. . . . . . 143-145

Making the injection-Tying the vessel. . . . . . . . . . . . . .

Cleaning the syringe and canula....................... 146

Injection of the Abdominal Aorta and of the Postcava............... 147

Injection of the Jugular Vein and the Carotid Artery............... 147-148

\section{CHAPTER V .}

\section{OSTEOLOGY, THE STUDY OF THE BONES.}

Determination of Right and Left with Certain Bones............. 149-151

Position of the bones............................ 149

Determination of Right and Left with Entire Limbs ............... 151-152 
PAGE

Special Mnemonics of the Humerus. . ...................... 152

Description of Bones. ................................. 152-189

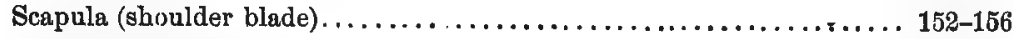

Humerus (bone of brachium or " upper arm "). . . . . . . . . . . 157-161

Carpalia (wrist bones).............................. 161

Clavicula (clavicle or collar bone)........................ 162

Sternum (breast bone).............................. 162

Costæ (ribs) and costal cartilages......................... 163-167

Ligamentum interarticulare and Comparison with that of Man.......... 165, 166

Pelvis.............................................. 167-169

Ilium, ischium, Os cotyloideum, Os innominatum, Os pubis......... 168

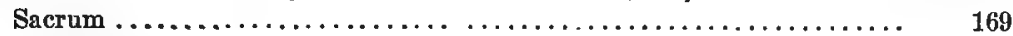

Columna vertebralis .................................. 169-173

Regions of the vertebral column........................ 170

Distinguishing the groups of vertebre.................... 170

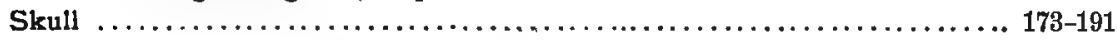

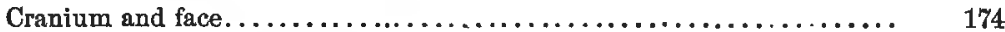

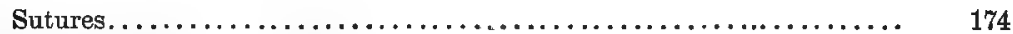

Table of the Bones of the Slcull.......................... 174-176

T'able of Articulations of the Bones of the Bkull ................ 176-179

Description of the dorsum of the skull .................. 179-182

Description of the base of the skull. . . . . . . . . . . . . . . 183-185

Description of the tympanic bulla..................... 185

Description of the mesal aspect of the skull ..... . . . . . . . 185-186

Description of the lamina cribrosa......................... 187

Description of the mandible (lower jaw)...................... 188-189

Table of the Foramina and Canals of the Skull, with Traversing Structures, etc. 190

Structure of Bone................................. 191

\section{CHAPTER VI.}

\section{MYOLOGY, STUDY OF THE MUSCLES.}

\section{General Considerations}

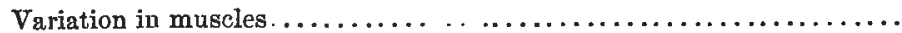

Errors of manipulation and interpretation................. 193-194

Technical Terms of Myology........................... 194

Muscular groups-Subdivisions......................... 194-195

Parts of a muscle.................................... 195

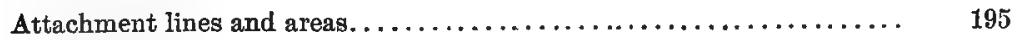

Origin and insertion-Choice of origin and insertion............. 195

Determination of muscular homologies. . . . . . . . . . . . . . 196

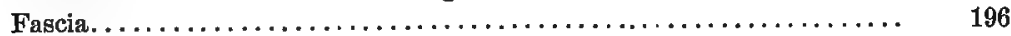

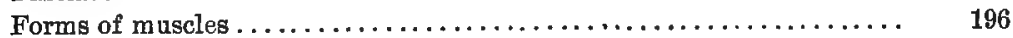

Designation of the borders of muscles.................... 197

Aphorisms for Dissectors............................. 197-198

Horo to use Dissecting Instruments. . . . . . . . . . . . . . . . . . . 199-201

Practical Suggestions to Dissectors .......................... 201

Choice of specimen and removal of superfluous parts............ 201 


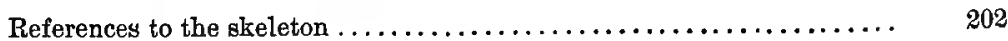

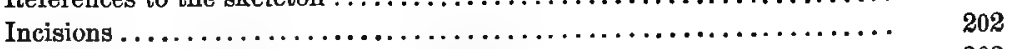

Vessels and nerves and action of muscles................... 202

Transection of muscles................................ 203

Covering and moistening parts under dissection $\ldots \ldots \ldots \ldots \ldots \ldots \ldots \ldots, 203$

Common faults of dissectors.......................... 203

Clipping the hair, cutting and removing the skin............. 204-205

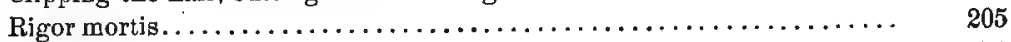

Description of Certain Muscles......................... 206-271

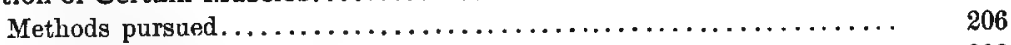

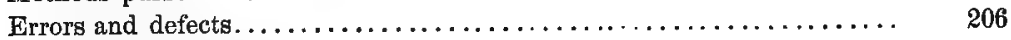

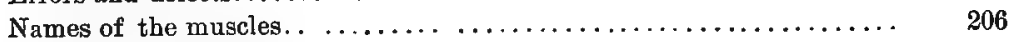

Table of the muscles treated, with synonyms ............... 207

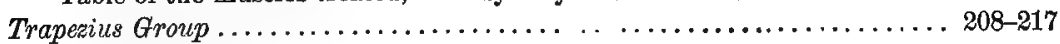

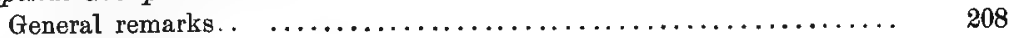

Exposure of the trapezius muscles................... 210

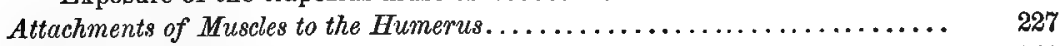

Pectoralis Group .................................. 233-241

General remark on the pectoral group.................... 285

Crossing of the pectoral elements....................... 235

Complexity of the pectoral muscles...................... $\quad 235$

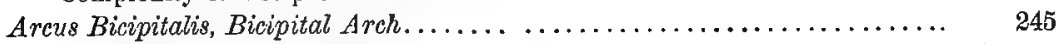

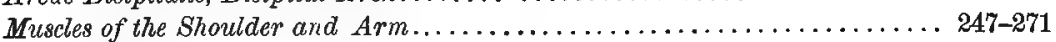

Structure of Muscular Tissue ......................... 272

Muscular fibers, length and connection with tendons............ 272

\section{CHAPTER VII.}

ABDOMINAL AND THORACIC VISCFRA, MOUTH, SALIVARY GLANDS AND NECK.

Abdominal Viscera. ................................ 273-297

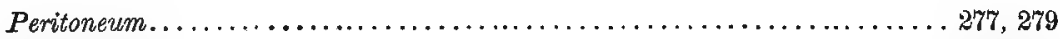

Diaphragma..................................... 277, 311

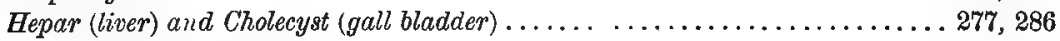

Lobes of the liver and its ducts........................ 286

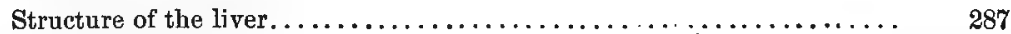

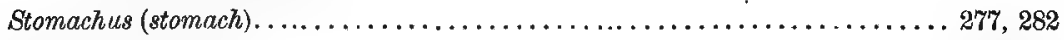

Small Intestine, Duodenum, Jejunum and Tleum, and their Structure... . . 283-284

Large Intestine, Cacum, Colon and Rectum, and their Structure.......... 285

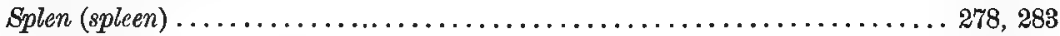

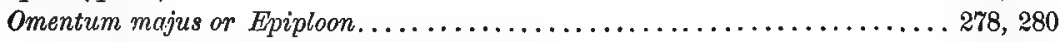

Foramen of Winslow, mesenteric glands................. 280

Pancreas and its Ducts, with the Ampullu of Vater . . . . . . . . . . 287, 290-293

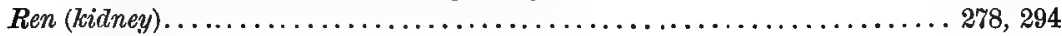

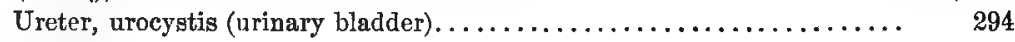

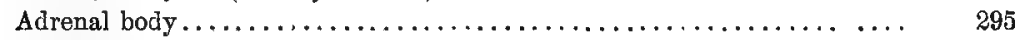

Uterus, Fallopian Tube and Ovary...................... 295-296

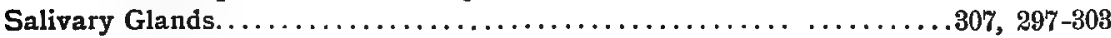

Preparation of the ducts................................... 298 
Exposure of the salivary glands and their ducts.

Nerves associated with the glands and ducts.

Parotid Gland.

Stenon's duct.

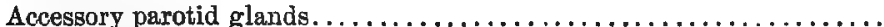

Wharton's duct.

Structure of salivary glands..................

Cavum Oris (Mouth Cavity) and Pharynx.................... 303

Structure of bnccal walls and tongue..................... 304

Neck (Collum) and Trachea.............................. 307

Structure of the trachea.............................. 308

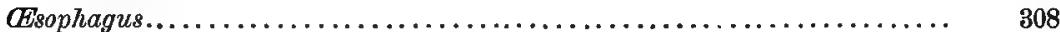

Strncture of the cesophagus........................ 308

Thorax. . . . . . . . . . . . . . . . . . . . . . . . . . . . . . . . . . . . . . . . 308-314

Pleura and septum mediastinale. . . . . . . . . . . . . 309

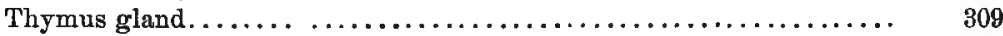

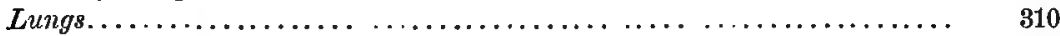

Lobes and air cells of the lungs. . ....................... 310

Bronchi and bronchioli................................ 311

Structure of the lungs.............................. 311

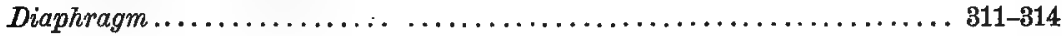

Central tendon $. . \ldots \ldots \ldots \ldots \ldots \ldots \ldots \ldots \ldots \ldots \ldots \ldots \ldots \ldots \ldots \ldots, \quad 311$

\section{CHAPTER VIII.}

\section{VASCULAR SYSTEM.}

General Considerations................................

Blood Vuscular System............................... $\mathbf{3 1 5}$

Arteries, veins and capillaries.......................... 315

Lymph Vascular System........................... 315-316

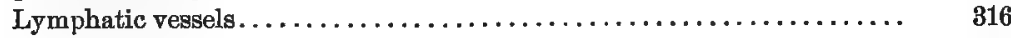

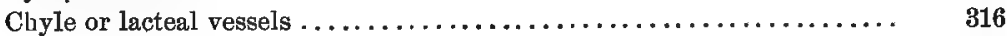

Comparison of the Lymph and Blood Vascular Systems............... 316

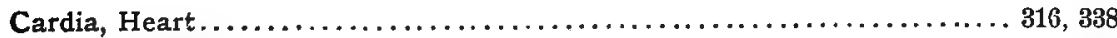

References to the heart and remarks.................... 316

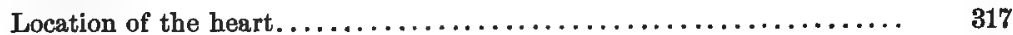

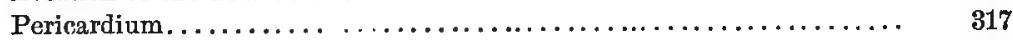

Form and normal position of the heart..................... 318

Designation and recognition of the regions $\ldots \ldots \ldots \ldots \ldots \ldots \ldots \ldots \ldots, \quad 318$

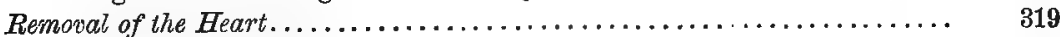

Separation of the heart from the lungs $\ldots \ldots \ldots \ldots \ldots \ldots \ldots \ldots \ldots \ldots . \quad 320$

Preservation of the Heart in Alcohol......................... 321 
Removal of the pericardium and blood................... 321

Tying the vessels, injection of alcohol and hardening........... 321-322

Table of the Principal Parts and Features of the Heart............. 322

List of abbreviations of cardiac names................. $\quad 325$

Descriptive list of the parts of the heart, arranged alphabetically...... 325-333

Dissection of the Heart. ............................ $337-338$

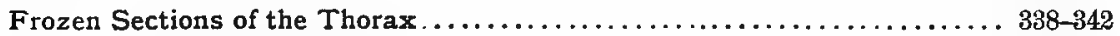

Blood Vessels of the Trunk. . . . . . . . . . . . . . . . . . . . . $342-362$

Table of the systemic and portal veins.................. $342-343$

Table of the systemic vessels of the trunk and arm. . . . . . . . . . 343-344

Veins of the Thorax. ................................ $349-850$

Arteries of the Thorax and Arm . . . . . . . . . . . . . . . . . . $350-355$

Abdominal Blood Vessels . . . . . . . . . . . . . . . . . . . . . . . $355-362$

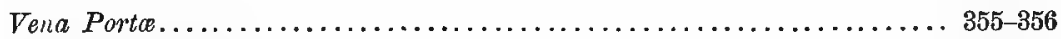

Systemic Abdominal Veins ............................. 356-358

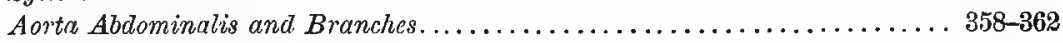

Dilisions of the Aorta Abdominalis......................... 361

Structure of the Heart and Blood Vessels. . . . . . . . . . . . . . . 362-363

Thoracic Ducts and Lymphatic Vessels..................... 363-368

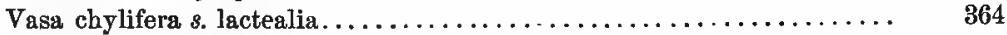

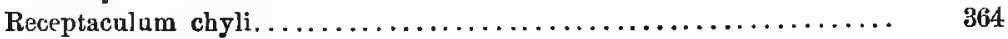

Ductus thoracicus sinister et dexter................... 364-365

Lymphatics of the arms, legs and face................... 367

Injection of the Lymphatic Vessels and Glands.................. 367

\section{CHA P TER IX.}

\section{NEUROLOGY, THE NERVOUS SYSTEM.}

General Considerations..

Nerves, ganglia, alba and cincrea.................... 369

Primary Divisions of the Nervous System. . . . . . . . . . . . . . . 369

Central and Peripheral Portions of the Myelencephalic System......... 369

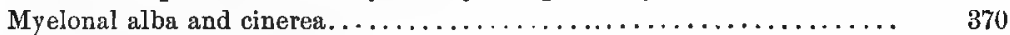

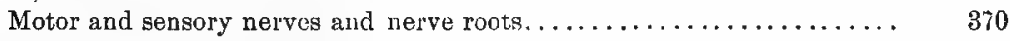

Functions of the Alba and Cinerea....................... 871

Analogies and Differences of the Nervons and Vassular $\$$; stems . . . . . . . 371-372

Myelencephalic or Cerebro-spinal Nervous System. . . . . . . . . . . . . . 372-894

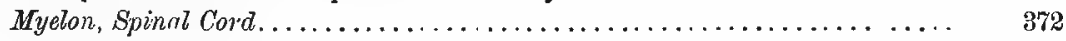

Myelonal or Spinal Nerves........................... 373

Dorsal and ventral (posterior and anterior) primary divisions of the spinal

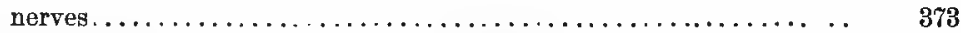

General Directions for Dissecting the Nervous System ............. $\quad \mathbf{3 7 5}$

Demonstration of the ventral primary division of the myelonal nerves of

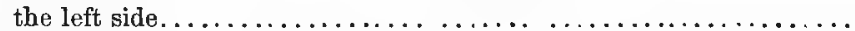


Brachial Plexus and the Principal Nerves of the Right Arm and Scapular

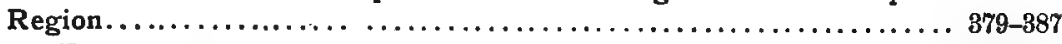

Nervus Vagus......................................... $387-394$

Nervus Sympathicus (sympathetic nerve) . . . . . . . . . . . . . . . . 394

Superior cervical ganglion......................... 395

Thyroid or middle cervical ganglion .................... 395

Vertebral or inferior cervical ganglion.................... 395

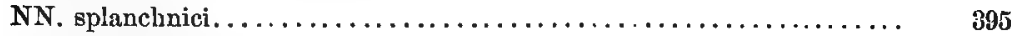

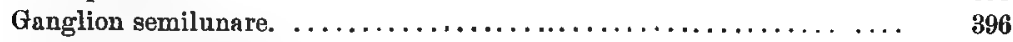

Abdominal sympathic............................... 396

Relations of the Myelencephalic and Sympathic Nerves............... 396-398

Structure of Nervous Matter................................ 398-399

\section{CHAPTER X.}

\section{THE ENCEPHALON OR BRAIN.}

General Considerations.

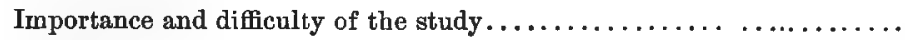

Methods of studying the brain......................... 400

Consideration of the Brain of the Frog and Menobranclus as Simple Brains... 402-403

Partial vocabulary of encephalic names.................. 403-404

Encephalic Segments................................ 404

Names and synonyms, tabular arrangement................. 405

Unequal morphical value of the segnents.................. 406

Advantages in using segmental names................... 406

Names of the brain cavities. . ......................... 406

Objections to the names commonly used.................. 406

Amphibian Brain-Tabular Arrangement of its Principal Parts.......... 409

Ideal, Simple Brain. ................................ 400

General Constitution of the Encephatic Segments................ 412

Metencephalon, epencephalon, mesencephalon, diencephalon, prosen-

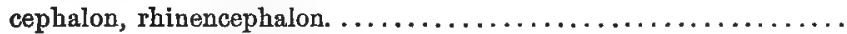

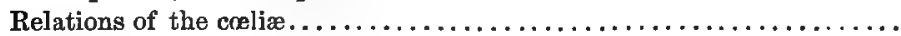

Comparison of the brain with a house................... 413

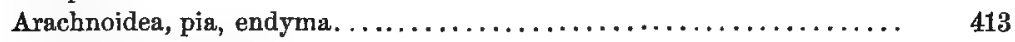

Telæe and plexuses................................. 414

Foramen of Magendie............................ 414, 482

Complete circumscription of the brain cavities............... 414

Conarial tube.................................. 415

Study of the Amphibian Brain......................... 415

Obtaining and killing the animals ... . . . . . . . . . . . $\ldots \ldots \ldots$

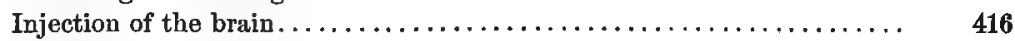

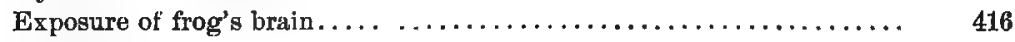

Exposure of the brain of Menobranchus.................. 417

Preservation of amphibian brain....................... 418

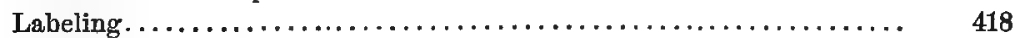

General Inspection of the Amphibian Brain................. 418-420

Dissection of the amphibian brain....................... 420-423 
Removal of the dura................................ 428

Transferring the brain to alcohol, weighing and removing the pia .....

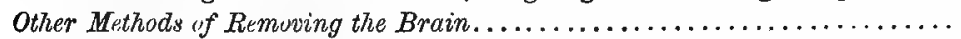

By nipping away the calva, and application to the removal of the brain of infants.

Hemisection of the head with a saw

$431-432$

Mesal and lateral bemisection.

432

Hardening a bemisected brain.

433

435

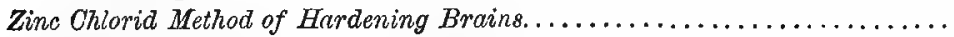

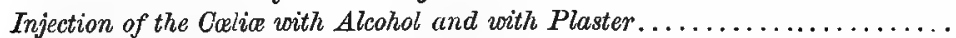

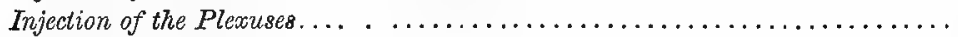

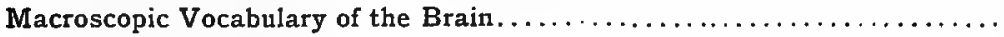

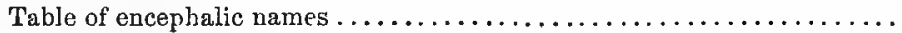

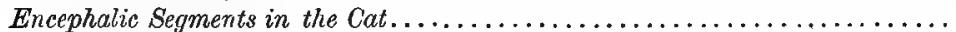

Differences between the brains of cat and frog. . . . . . . . . . . . . .

Hemisection of the brain . . . . . . . . . . . . . . . . . . . . .

Inspection of the mesal aspect. . . . . . . . . . . . . . . . . . . .

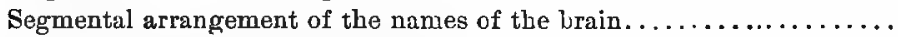

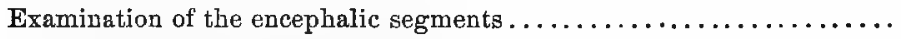

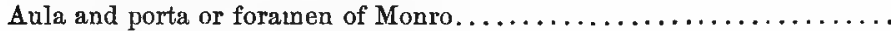

Demonstration of the Procclia, Rhinocclia and Porta (lateral and olfactory ventricles and foramen of Monro) $\ldots \ldots \ldots \ldots \ldots \ldots \ldots \ldots \ldots \ldots$

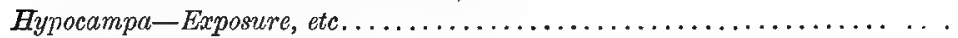

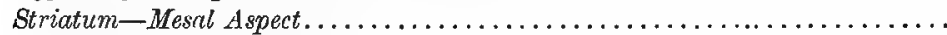

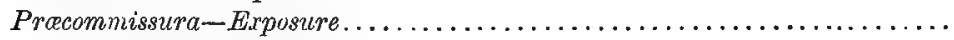

Relations of the plexuses to the membranous walls of the porta, diacœlia

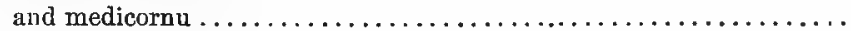

Explanation of the Plates of the Brain...

List of Encephalic Names with Synonyms and References, alphabetically arranged.

Comments upon Figures of Feline Brains

Form of the cerebrum $\ldots \ldots \ldots \ldots \ldots \ldots \ldots \ldots \ldots \ldots \ldots \ldots \ldots$

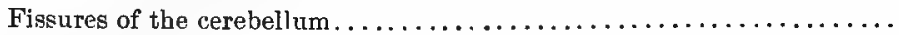

Table of Abridged Synonymy of the Cerebral Fissures..................

The three problems connected with the study of the fissures..........

Formation of the fissures. . . . . . . . . . . . . . . . . . . . . . . .

Structural Relations of Certnin Fissures, with a List...............

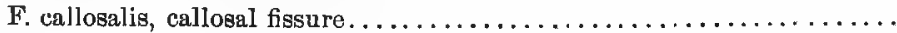

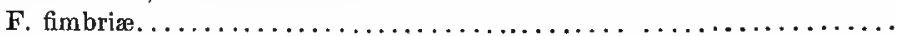

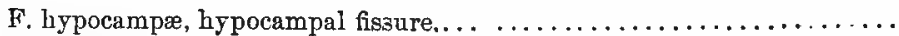

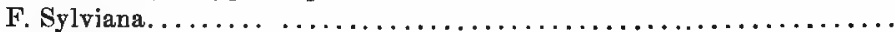

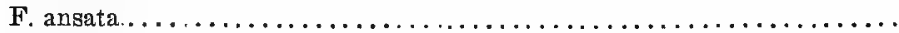

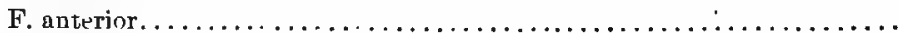

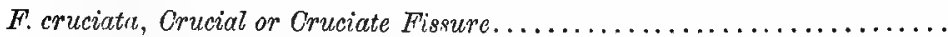

Constant and peculiar characters.

Homology, with views of various authors . 
Designation of the Gyri.

Table showing three ways of enumeruting the arched gyri..........

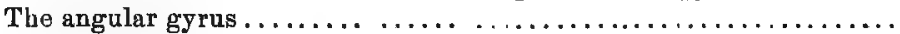

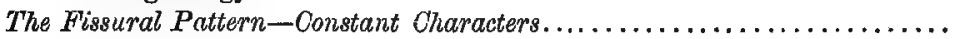

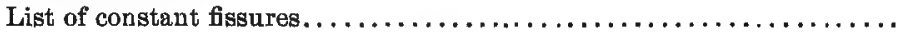

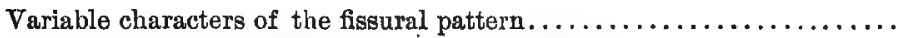

List of inconstant fissures............................. 502

Homology of Human and Feline Fissures, with Diffleulties. . . . . . . . . 502

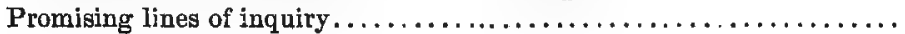

\section{- CHAPTER XI.}

\section{THE CRANIAL NERVES AND THE ORGANS OF SENSE.}

Cranial Nerves-General Considerations.

Comparison of cranial and myelonal nerves. . . . . . . . . . . . . .

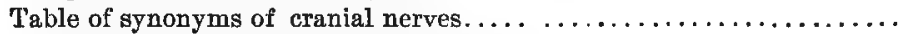

Numerical designation of the cranial nerves by Willis and Sömmering. .

Physiological arrangement of the cranial nerves................

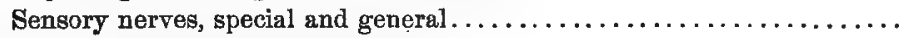

Special Consideration of the Twelve Cranial Nerves... . . . . . . . . . . . 507-510

Table of the Cranial Nerves, giving Ectal Origins, Foramina of Exit, Distribution and Frunction.

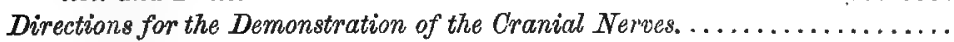

General Considerations.

Skin, tongue, nose, eye, ear, general sensibility $\ldots \ldots \ldots \ldots \ldots \ldots \ldots$

Cutis, the Skin.

Structure and function of its various parts... ................ 512

Appendages of the skin-Hair, claws, sweat and sebaceous glands..... 512

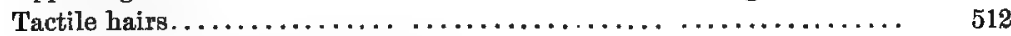

Lingua, the Tongue-Uses, papillæ, nerves .................. 512-513

Nasus, the Nose-Lining membrane, sensibility of its various parts....... 513

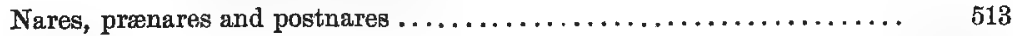

Oculus, the Eye..................................... $514-526$

Appendages of the Eye............................. 514-516

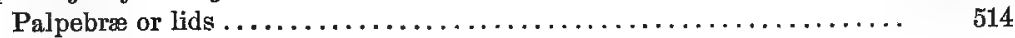

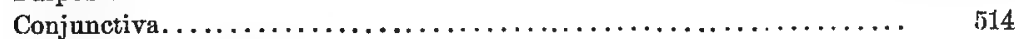

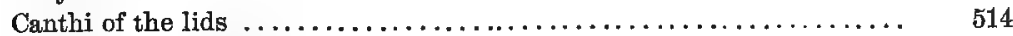

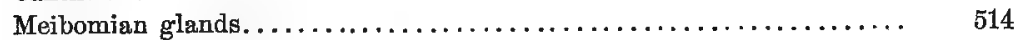

Membrana nictitans-Attachment and office................ 515

Lachrymal apparatus. . . . . . . . . . . . . . . . . . . . . . 515-520

Muscles of the Eye............................... 516-520

Origins of the muscles of the eye...................... 519

Action of the muscles of the eye....................... 519

Nervous supply of the muscles. . . . . . . . . . . . . .

Globus Oculi, Eyeball.............................. 520-526

How to obtain the eyeball of a cat, ox or sheep............... 520

Form and parts of the eyeball. . . . . . . . . . . . . . . . 520 
PAGE

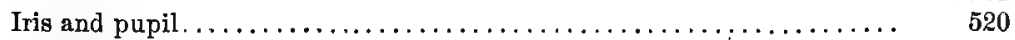

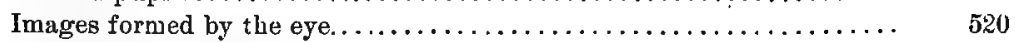

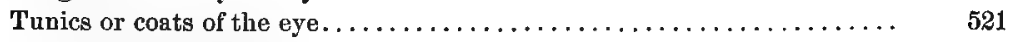

Preservation of an eye in alcohol for the coats.............. 521

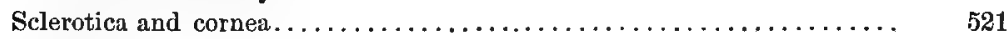

Choroidea, plicæ ciliares. ........................ 521-522

Retina, ora serrata and pars ciliaris retinæ................. 522

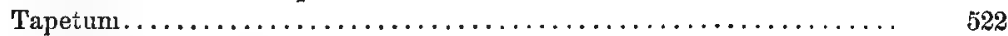

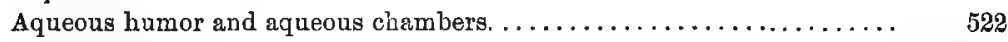

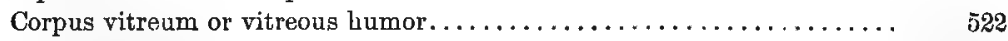

Lens, ligament and capsule . . . . . . . . . . . . . . . . . . 522-523

Organum Auditus, Auris, Ear........................ 526-533

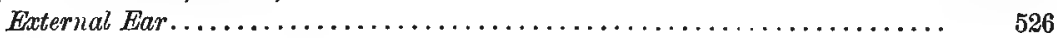

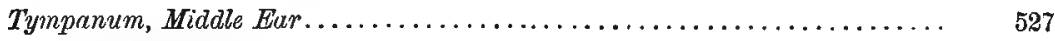

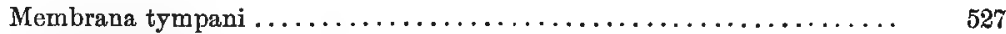

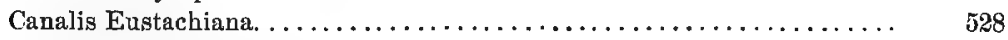

Malleus and tensor tympani muscle....... . . . . . . . . . 528

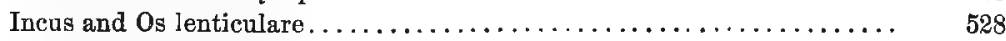

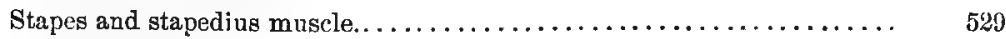

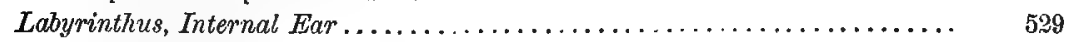

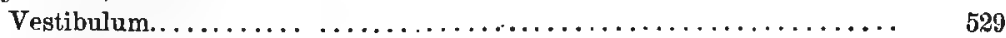

Fenestra ovalis and fenestra rotunda................... 529

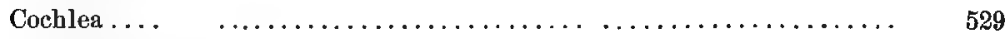

Scala vestibuli and scala tympani...................... 529

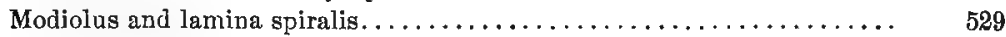

Canales semicirculares.......................... 530

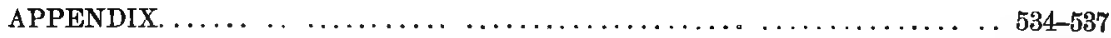

BIBLIOGRAPHY $\ldots \ldots \ldots \ldots \ldots \ldots \ldots \ldots \ldots \ldots \ldots \ldots \ldots \ldots \ldots \ldots \ldots \ldots \ldots \ldots \ldots$

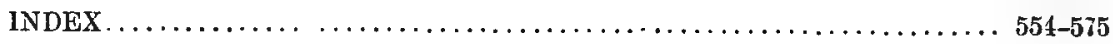




\section{LIST OF ILLUSTRATIONS.}

In respect to twenty-six figures we are indebted to the persons named in parenthesis, either for the idea of a figure or for the use of an electrotype. Of the original figures, the larger number were drawn by E. C. C'leaves, Professor of Free-hand Drawing in Cornell University. The four plates of the brain were drawn and lithographed by Miss G. D. Clements, who also drew Fig. 81-82. Fig. 11-12, 14-19, were drawn by Miss I. M. Curtis; Fig. 124-127, by Mrs. S. S. Phe]ps Gage; and Fig. 92, 110-112, 121-128, by the senior author.

In preparing the drawings for the original figures, the specimens-now preserved in the museum of Cornell University-were photographed with a vertical camera, as suggested by the junior anthor $(6)$. The outlines of the drawings were traced directly from these photographs; hence the relative size and position of parts are accurately represented. Finally the drawings were photo-engraved.

1.-Paper Meter Yard (American Metric Bureau) ................... 6

2-4.-Diagrams of Three Aspects of a Vertebrate, exhibiting the most general

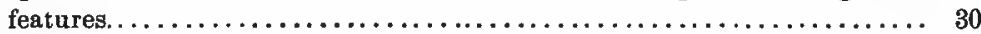

5.-Diagrams of the Three Body Planes, with the various lines of direction.... . 34

6.-Diagram of the Dorsal Aspect of the Cat, with the limbs in the normal position. 38

7.-Diagram of an Ideal Transection of the Thorax of the Cat, with the arms in an approximately normal position, and showing the location of the principal

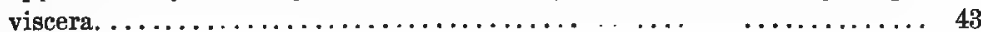

8.-Japanned Tray for Catalogue Slips (Readers' and Writers' Economy Co.) ... 50

9.-Pigeon-hole Case (Readers' and Writers' Economy Co.)............ 51

10.-Stubs's Nippers (Codman and Shurtleff) $\ldots \ldots \ldots \ldots \ldots \ldots \ldots \ldots \ldots \ldots \ldots \ldots$

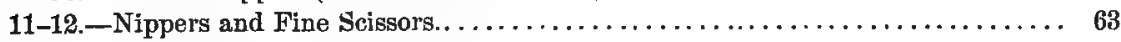

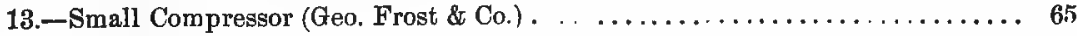

14.-Blunt-pointed Scalpel.............................. 66

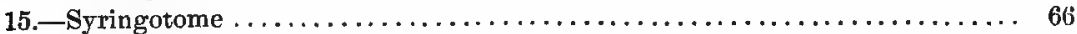

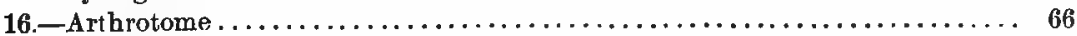

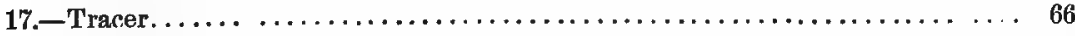

18.-Coarse Forceps................................... 66

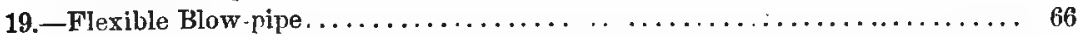

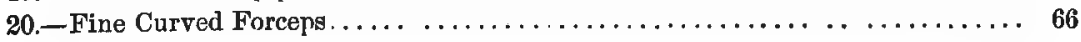


21.-Back-saw (Goodnow and Wightman) $\ldots \ldots \ldots \ldots \ldots \ldots \ldots \ldots \ldots \ldots \ldots \ldots$

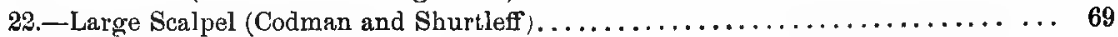

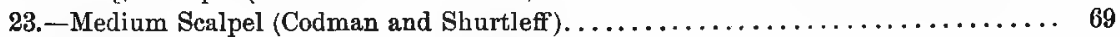

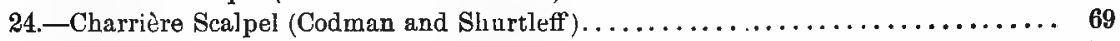

25.-Coarse Scissors, curved flatwise (Codman and Shurtleff) ............. 70

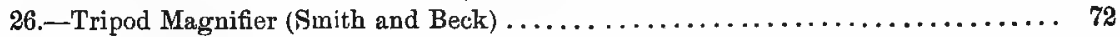

27. -Wetting Bottle for 15 per cent. Glycerin $\ldots \ldots \ldots \ldots \ldots \ldots \ldots \ldots \ldots \ldots \ldots \ldots$

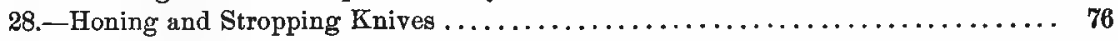

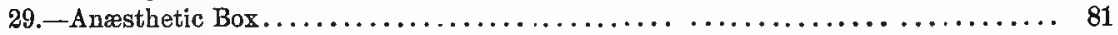

30.-The Skeleton seen from the Left (Strans.Durckheim, A) . . . . . . . . . 88

31.-Covered Glass Box (Whitall, Tatum \& Co.) . . . . . . . . . . . . . . 126

32.-Wide-mouthed Specimen Jars with Covers (Whitall, Tatum \& Co.)......... 127

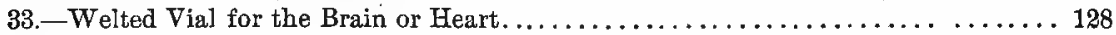

34.-Bottle Brush. .................................... 129

35.-White Metal Syringe with top unscrewed and piston removed $\ldots \ldots \ldots \ldots \ldots 138$

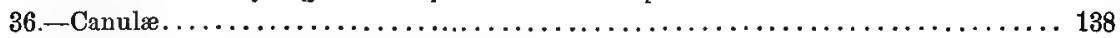

37.-Brass Syringe (Codman and Shurtleff) . . . . . . . . . . . . . . . . . 139

38.-Small Brass Syringe with removable Stop.cock and Canulæ for Fjne Injec-

tions (Codman and Shurtleff) $\ldots \ldots \ldots \ldots \ldots \ldots \ldots \ldots \ldots \ldots \ldots \ldots \ldots \ldots \ldots$

39. - Femoral Vessels exposed for Injection....................... 142

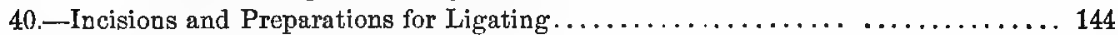

41.-Double Ligature, Surgeon's and Square Knot. . . . . . . . . . . . . . 144

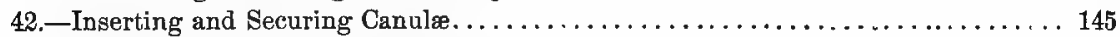

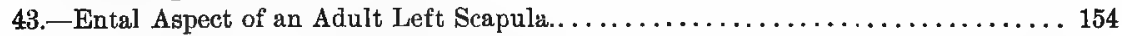

44.-Ectal Aspect of an Adult Left Scapula...................... 155

45.-The Glenoid End of an Adult Right Scapula and the Proximal End of an Adult

Rigbt Humerus. . . . . . . . . . . . . . .

46. - Ventral Aspect of an Adult Right Humerus. . . . . . . . . . . . . . .. 158

47.-Dorsal Aspect of the Carpal Region of a Young Lion and of two Young Dogs

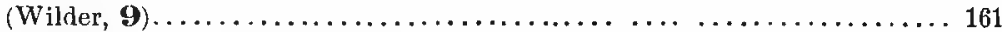

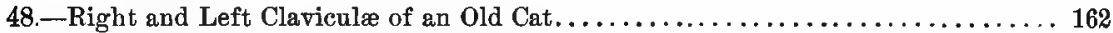

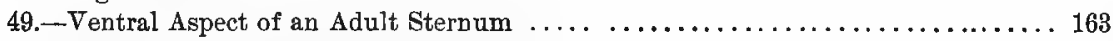

50.-Caudal View of the Seventh Pair of Ribs....................... 166

51.-Ventral View of the Cat's Pelvis with adjacent Vertebræ (modified from S.raus-

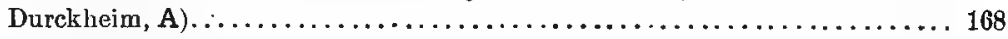

52.-Ventral Aspect of the Cervical and two Thoracic Vertebræ (modified from Straus-

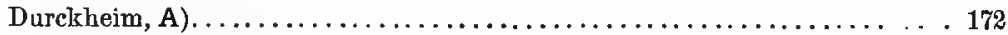

53.-Caudal View of the Fourth Cervical Vertebra ................... 173

54.-Caudal View of the Seventh Thoracic Vertebra................... 173

55. - Caudal View of the Fourth Lumbar Vertebra. . . . . . . . . . . . . . . . 173

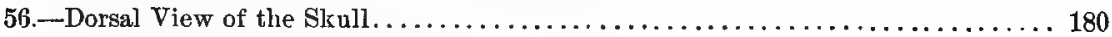

57.-Ventral Aspect or Base of the Skull (modified from Straus-Durckheim, A) . ... 182

58.-Ventro-lateral View of the Left Bulla tympanica and Adjacent Parts ........ 185

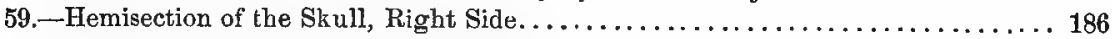

60.-Dorso-caudal View of the Lamina Cribrosa and the Sinus Frontalis. . . . . . . . 188

61. Lateral View of the Left Mandibular Ramus..................... 188

62.-Dorsal View of the Mandible (modified from Straus-Durckheim, A) . . . . . . 189

63. - The Scalpel Held as a Pen (Bernard, A) . . . . . . . . . . . . . . . . . . . 199

64.-The Scalpel Held as a Carving-knife (Bernard, A) . . . . . . . . . . . . . 200

65. - The Scalpel Held as a Violin-Bow (Bernard, A) . . . . . . . . . . . . . . 200 
66.-The Ectal Skeletal Muscles of the Neck and Shoulder............... 211

67.-The Second Layer of Skeletal Muscles of the Neck and Shoulder.......... 218

63-71.-Views of the Four Aspects of the Humerus with Areas of Muscular Attach-

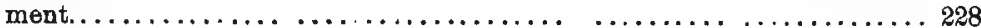

72.-The Pectoralis Group of Muscles. . . . . . . . . . . . . . . . . . . . . 234

73.-Ental Aspect of Left Shoulder Muscles and Ectal Aspect of the Serratus Mag-

nus and Levator Anguli Scapulæ Muscles ................... 246

74.-The Cephalic Aspect of the Left Arm with the Ectal Muscles of the Scapula. 254

75. - The Muscles upon the Ental Aspect of the Scapula and the Caudal Aspect of the Brachium and $A$ tebrachium...................... 262

76. -Lines of Incision for exposing the Thoracic and Abdominal Viscera.........274

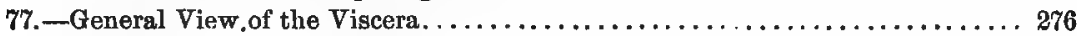

78.-Diagram showing the Relations of the Abdominal Organs and the Perito-

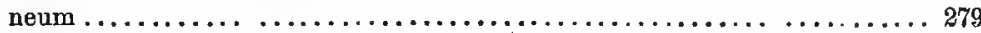

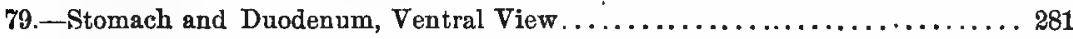

80.-Cæcum and Ileo-cæcal Valve, Ventral View...................... 284

81.-Ventral View of the Pancreas and its Parts (Gage, 3)............... 288

82.-Cholecystis and Pancreatic Reservoir and their Relation with the Duodenum

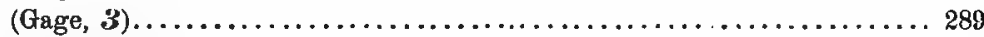

83. - Ventral View of the Human Pancreatic Ducts (Bernard) . . . . . . . . . . . 290

84.-Longitudinal Section of the Ampulia of Vater, showing the Entrance of the Ductus Choledochus and the Duct of Wirsung (Gage, 3); the same in man

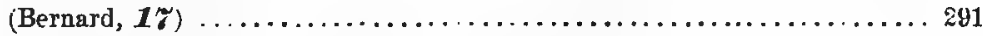

85.-Dorso-ventral Section of the Right Kidney, Caudal View... . . . . . . . . . 298

86.-Longitudinal Dextro-sinistral Section of the Right Kidney, Ventral View . . . 293

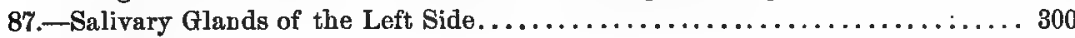

88.-Hemisection of the Head. ................................ 305

89.-Lungs and Trachea. . . . . . . . . . . . . . . . . . . . . . . . 310

90.-Caudal View of the Diaphragm with the Structures that traverse it........ 313

91.-The Dorsal Aspect of the Heart with the Central Portions of the Larger Ves-

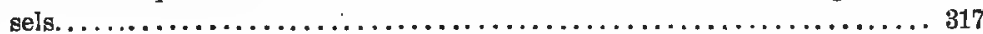

92.-Diagram of the Cardiac Cavities, Dorsal Aspect.................. 323

93.-Dorsal Part of the Heart seen from the Ventral Aspect............... Esiz

94-Longitudinal Segment of the Heart, showing the Right Sinus of Valsalva and

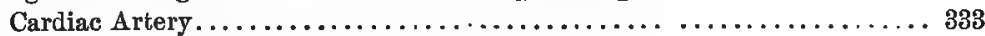

95.-Transection of the Auricles, Caudal Aspect.................... 334

96. -Transection of the Auricles, Cephalic Aspect. . . . . . . . . . . . . . . 335

97. - Transection of the Ventricles, Caudal Aspect................... 336

98.-Transection of the Ventricles, Cephalic Aspect. . . . . . . . . . . . . . 336

99. -Frozen Transection of the Thorax, Caudal Aspect................. 340

100.-Frozen Transection of the Thorax, Cephalic Aspect. . . . . . . . . . . . 341

101.-General View of the Blood Vessels, and Diagrams of the Beginnings of the Postcava and Termination of the Aorta... ................. 345

102.-The Aorta with its Main Branches in the Thorax and Right Arm, also Origin of the Carotid Arteries: Veins with Valves (Quain, A) . . . . . . . . . 347 103.-The Left Thoracic Duct, Sinistral View, and Termination of the Thoracic

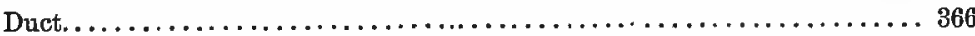

104.-Dorsal Aspect of the Brain and of Part of the Myelon with its Nerves...... 374 105. - Ventral Aspect of the Right Brachial Plexus and its Nerves........... 378 106. - Diagram of the Right Brachial Plexus, Ventral View................ 381 
107.-The Vagus and Sympathic Nerves and a Diagram of the Sacral Part of the

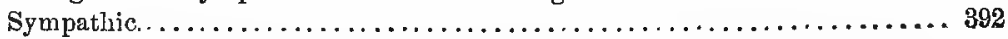

108._The Recurrent Laryngeal Nerves, Ventral View (Stowell, 1)............ 394 109.-Transection of the Thorax, showing the Origin of a Pair of Spinal Nerves and the Relations of the Sympathic and Cerebro-Spinal Systems, Cephalic View. 397 110.-Longitudinal Dextro-Sinistral Section of an Ideal, Simple Brain, showing the Relations of the Cavities, the Sequence of the Encephalic Segments, and the Relations of the Coliæ................................. 408 111.-Mesal Aspect of the Right Half of an Ideal, Simple Brain, showing the Contour and Constitution of the Coelian Floors and Roofs . . . . . . . . . . . 408 112.-Transection of Several Segments of the Ideal Brain, showing the Coliæ and their Parietes . ................................... 408

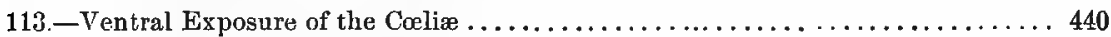

114.-Dorsal Aspect of the Mesencephalon, with Adjacent Parts.............441

115.-Dorsal Aspect of the Callosum after Partial Removal of the Hemispheres....... 442

116. - Diagram of the Base of the Brain. ........................ 443

117.-Diagram of the Mesal Aspect of the Left Hemiencephalon. . . . . . . . . 446-447

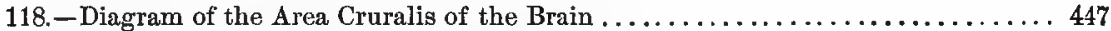

119.-Plaster Casts of the Medicornua Inverted .................... 458

120.-Plaster Casts of the Diacolia (Dorsal Portion), Aula, and Right Porta and Præcornu of the Sheep, inverted $\ldots \ldots \ldots \ldots \ldots \ldots \ldots \ldots \ldots \ldots \ldots \ldots \ldots \ldots$

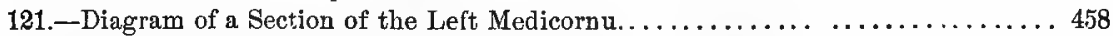

122 -Diagram of a Transection of the Brain through the Diacclia . . . . . . . . 458

123.-Diagram of a Transection of the Porta (Foramen of Mon ro) . . . . . . . . . 458

124.-Cerebral Fissures, Lateral Aspect........................... 494

125.-Cerebral Fissures, Mesal Aspect. . . . . . . . . . . . . . . . . . . . . . . . . 494

126.-Section of the Eye, in situ $\ldots \ldots \ldots \ldots \ldots \ldots \ldots \ldots \ldots \ldots \ldots \ldots \ldots \ldots \ldots$

127.-Diagram of the Ear. . . . . . . . . . . . . . . . . . . . . . . . . . 532

128.-Transection of a Coil of the Cochlea (Quain, A.) ................. 533

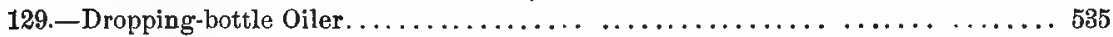

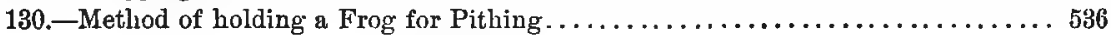

\section{FOUR PLATES OF THE BRAIN. (Wilder, 14.)}

For the use of these plates we are indebted to the American Philosophical Society of Philadelphia.

1.-Dorsal Aspect of the Brain.

2.-Sinistral Aspect of the Brain.

PLATE I.

\section{PLATE II.}

3.-Basis Encephali, or Ventral Aspect of the Brain.

4.-Mesal Surface of the Right Hemiencephalon.

PLATE III.

5.-Cephalic Aspect of the Prosencephalon.

6.-Caudal Aspect of the Prosencephalon, with part of the Diencephalon.

7.-Dorsal Aspect of the Diencephalon and Mesencephalon. 


\section{FIGURE}

8.-Caudal Aspect of the Mesencephalon, with parts of the adjoining regions.

9.-Sinistral Aspect of the Mesencephalon and Diencephalon.

10.-Dorsal Aspect of the Diencephalon, including the Diatela.

11.-Area Cruralis, with part of the Pons and of the Area Præchiasmatica.

12.-Dorsal Aspect of the Metencephalon.

13.-Part of an Oblique Transection of the Prosencepbalon and Diencephalon to show the Form and Position of the Crista Fornicis.

\section{PLATE IV.}

14.-Ventro-caudal View of the Fornix, with the adjacent parts.

15.-Dorsal Aspect of the Proccelia.

16.-Rhinocelia and Procœlia.

17.-Mesal Aspect of the Right Hemisphere, with the Lobus Olfactorius.

18.-Right Procœlia seen from the Right or Ectal Side.

19.-Left Præcornu and Porta exposed from the Left or Ectal Side.

20.-Transection of the Fornix with the Crista. 


\section{LIST OF TABLES AND ALPHABETICAL LISTS.}

Table of some equivalent Temperatures, according to the Centigrade and Fahrenheit scales. . . . . . . . . . . . . . . .

Table of the Metric Measures. . . . . . . . . . . . . . . . . . .

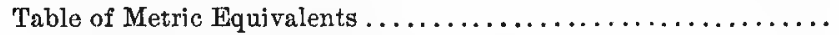

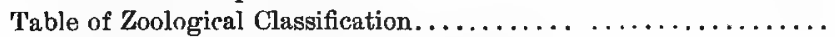

Table of the Principal Divisions of the body..............

Table illustrating the Subdivision of Notes. . . . . . . . . . . .

List of Anatomical Instruments and Materials .............

Table of the Specific Gravities of different Percentages of Alcohol..

Table of the Ratios of Alcohol and Water to produce a given

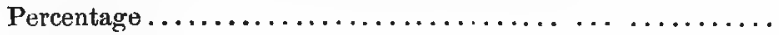

Composition of Wickersheimer's Liquid ................

Table of the Bones of the Skull, slıowing the Synonyms and An-

thropotomical Equivalents . . ................. 174-176

Table of Articulations of the Bones of the Skul]............ 176-179

PAGE

4

$59-62$

Table of the Cranial Foramina and of the Structures which traverse them

Table of the Synonyms of forty Muscles.

Table of the Principal Parts and Features of the Heart. ........

Table of the Systemic veins of the Trunk and of the Portal Vins..

Table of the Systemic Arteries of the Trunk and Arm ..... .....

List of the Principal Parts of the Amphibian Brain, with their

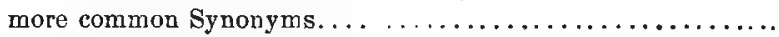

Table of the Names and Synonyms of the Encephalic Segments...

Tabular arrangement of the Principal Parts of the Amplibian

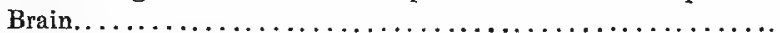

List of Abbreviations of general Enceplualic names.............

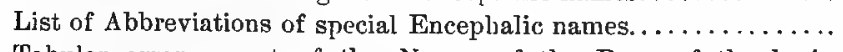

Tabular arrangement of the Names of the Parts of the brain according to the Segments...................448,449

List of Synonyms and References for the Encephalic parts. . . . . . 471-491

List of writers who have figured the Feline Brain. . . . . . . . . 491-493

Abridged Synonymy of the Cerebral Fissures............... 496

List of writers who have mentioned the Cruciate Fissure... . . . 499, 500

Table showing four Methods of enumerating the "arched gyri"...

Tabje of the Synonyms of the Cranial Nerves................

Provisional Physiological Arrangement of the Cranial Nerves.....

Table of the Origin, Distribution, etc., of the Cranial Nerves......
5

7

9

39

40

112

116

124

BECTION

6
9
14
16
80
108
130
261

273

300

494

495

562

604

844

914,915

916, 917

403

1058

1061

409

436

1069

1327

1128

1138

1180-1333

1334

1342

1361

501

505

1363

1374

506

1377

510

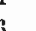

\section{0}




\section{INTRODUCTION.}

$\S 1$. There are five matters of general application :-

1. The reference to other Publications.

2. The designation of Weights, Measures and Temperatures.

3. The names of Animals, and of the Groups to which they belong.

4. The designation of the Parts of animals, and the indication of their Position and Direction.

5. The making and arrangement of Notes.

The treatment of these matters in the present work may be characterized. briefly as follows:-

1. The citations are numerous and explicit.

2. Only decimal systems are employed,- - the Centigrade scale and the Metric system.

3. The classification adopted is in accordance with generally accepted views.

4. The terminology is intended to have the following features: general application to all Vertebrates; intelligibility to all nations; accuracy; brevity ; simplicity ; consistency ; uniformity of abbreviation.

5. What may be called the slip-system of notes is recommended.

\section{I.-REFERENCES TO PUBLICATIONS.}

§ 2. We have thought it best to make somewhat full references to other Manuals and Compendiums, and to the Works and Papers of original observers. Our reasons are :-

1. This work is designed to be used not only by the general student, but also; as an elementary introduction, by those who are themselves to become investigators. In our opinion, these latter cannot too soon become familiar with the sources of original information, and with the views of the present and past leaders in scientific progress. 
2. Upon some points, especially that of Terminology, we feel that the acceptance of our ideas will be more ready and complete if it be shown that they are shared by other and more widely known writers.

3. While we are responsible for whatever may prove to be erroneous, we are very loth to run the risk of receiving, even from beginners, credit for having first made an observation or an experiment, or first devised an instrument or a mode of manipulation, the honor of which belongs properly to others.

4. On the other hand, since our statements as to the structure of the cat do not always accord with those of other writers, our own papers are frequently referred to in evidence that those statements have already been submitted to competent scientific tribunals.

§3. References.-In the text, the capital letter or Arabic numeral directly following an author's name indicates the place of the work or paper upon the List. This letter or numeral is in black letter.

The second Arabic numeral designates the number of the page. When the introductory portions of a book are separately paged the Roman numeral designating the page is preceded by the letter $p$.

When a work consists of two or more volumes, the number of the volume in question is indicated by a Roman numeral placed between the two Arabic numerals.

When two or more works or papers are referred to after the name of the same author, their letters or numbers are separated by a semicolon.

The numbers of two or more pages or volumes are separated by commas, or by short dashes when the passages in question extend orer sereral pages.

For example: Rolleston, A, 10, refers to the tenth page in the body of the work of the "Forms of Animal Life." Rolleston, A, p. x, refers to the tenth page of the Introductory portion of the same work. Agassiz, A, iv, 10, refers to the tenth page of the fourth volume of the "Contributions to the Nat. Bist. of the U.S." Wyman, 34, 10, refers to the tenth page of the "Anatomy of the Nervons System of Rana pipiens," which was published among the "Smithsonian Contributions to Knowledge," and is hence regarded as a paper.

On account of the large number of citations, we have usually omitted the words volume and page and their abbreviations. This is regarded as permissible by Bigelow; A, 49 .

The following is the mode of reference :-

Near the end of the book is a "List of Publications referred to." In that list, the names of Authors occur in alphabetical order.

Under each name, the titles are in two groups, including respectively Separate Books, and Papers published in Journals or by Scientific Societies. 
The works are designated by letters, the papers by Arabic numerals. The former have no definite order, and no significance is to be attached to their sequence.

The papers are numbered, so far as possible, as in the "Catalogue of Scientific Papers" published by the Royal Society of London (A), where the order is intended to be chronological. The eight volumes of that Catalogue already published include the papers which have appeared between the years 1800 and 1873 . On our list, the papers issued since the latter date are assigned provisional numbers in italics.

In the case of papers, as in the Royal Society Catalogue "when possible, both the rolume and the year have been given. With Transactions of Societies the year to which the volume belongs, and not the year of publication, has been given. A date enclosed in brackets marks the time when a paper was read, which occasionally precedes by some years the date of the volume in which it is printed."

We shall be thankful for corrections or suggestions which may make the Bibliography more extensive and accurate.

\section{II.-THE DECIMAL SYSTEMS.}

§ 4. The two decimal systems used in scientific work are:-

1. The measurement of Temperature upon the centigrade scale by the thermometer of Celsius.

2. The metric system of Weights and Measures.

\section{THE CENTIGRADE THERMOMETER.}

\$5. Upon this, the Thermometer of Celsius, 0 (zero) represents the temperature of melting ice. The point attained by the column of Mereury at the temperature of boiling water is marked 100 (one hundred).

Between these two points, the scale is divided into 100 degrees in groups of 10 each.

According to this scale, the average temperature of the human body is between 37 and 38 , and that of the comfortable atmosphere of a sittingroom in winter about 20 .

Fahrenheit's Thermometer.-Upon this, the melting point of ice is marked 32 , and the boiling point of water 212 .

\$6. Comparison and Reduction of the two Scales.-Since the Fahrenheit thermometer is largely used in English-speaking countries, the following Table and Formulæ may be useful. The former is taken from -Littré et Robin, (A, 1594, Article "Thermometre"); the latter from Dunglison, (A, 488, Article "Heat"). 


\section{TABLE}

OF SOME EQUIVALENT TEMPERATURES ACCORDING TO THE THERMOMETRIC SCALES OF CELSIUS (CENTIGRADE) AND FAHRENHEIT.

\begin{tabular}{c|c}
\hline \hline. & F. \\
\hline 0 & 32 \\
10 & 50 \\
20 & 68 \\
30 & 86 \\
35 & 95 \\
{$[37.2+]$} & {$[99]$} \\
40 & 104 \\
50 & 122 \\
60 & 140 \\
70 & 158 \\
80 & 176 \\
90 & 194 \\
100 & 212 \\
\hline
\end{tabular}

Formulæ for the reduction of the Fahrenheit to the centigrade scale, and vice versa.

To reduce F. to $\mathrm{C}$., subtract 32 , multiply by 5 , and divide by 9 . To reduce C. to F., multiply by 9 , divide by 5 , and add 32 .

$1 \mathrm{C}$. equals $1.8 \mathrm{~F}$. $1 \mathrm{~F}$. equals $.555 \mathrm{C}$.

\section{THE METRIC SYSTEM.}

§ \%. Definition.-The Metric System of Weights and Measures is based upon the meter as a standard of length.

The Meter.-This, the unit of length, equals, approximately, one tenmillionth part of the quadrant of the meridian circle which passes through Dunkirk and Barcelona; it is thus about one forty-millionth part of the earth's circumference as measured upon that line.

In common English measure, the meter is $39.3 \% 0 \% 9$ inches, or about 3 feet 3 inches and a third, or about three and one third inches more than a yard.

The Liter.-This is the unit of capacity. It represents the space occupied by a cube whose edge is one tenth of a meter.

The liter corresponds nearly to our quart; more accurately, it is $\mathbf{1 . 0 5 6}$ common quart; 0.880 imperial quart; 0.907 dry quart.

The Gram - This is the unit of weight. It represents the weight of a cube of distilled water whose edge is one hundredth of a meter, and at a temperature of $4^{\circ} \mathrm{C}$. 
The gram is 15.432 Troy grains; or 0.564 aroirdupois drams; or 0.035 avoirdupois ounces. The U.S. nickel five-cent piece weighs five grams, and is, moreover, one fiftieth of a meter $(2 \mathrm{~cm}$.) in diameter.

The other measures of length, capacity, and weight are decimal divisions or multiples of the meter, the liter, and the gram, and their names are so formed as to indicate their value in each case.

§ 8. As compared with the English or any other system of weights and measures, the Metric System has the following desirable features:-

1. It has a single basis, and involves fewer separate terms; hence it is more easily learned.

2. It is decimal; hence it is more easily used.

3. It is already practically international, and largely, if not chiefly, employed in the best kinds of scientific work.

We do not feel called upon for a general discussion of the merits of the Metric System. Its origin, nature, and advantages have been admirably set forth in the works of F. A. P. Barnard, J. Pickering Putnam, Persifor Frazer, etc, and are periodically urged by able writers in various Journals, medical, scientific, and sociological. Philosophical treatises upon the general subject are published by "The American Metrological Society," and "The American Metric Bureau" prints a "Bulletin"(A). The final issue of "The Harvard Register" contains a compact and at the same time comprehensive plea "In favor of the Metric System," the force of which is rather increased than diminished by the article just following it upon the opposite side.

Practically, out of the twenty-three or four names for measures of length, capacity, and weight which may be employed, only about one-third are in common use by scientific men. These are, the meter, liter, and gram; the centimeter and millimeter, being respectively the hundredth and the thousandth of the meter; the milligram, the thousandth of the gram; the kilogram or thousand grams; and the cubic centimeter, which is the same as milliliter, the thousandth part of the liter.

$\$ 9$. The following Table includes all the regular metric denominations. But only the eight or nine whose names are printed in capitals are in general use.

\section{TABLE OF THE METRIC MEASURES.}

\begin{tabular}{|c|c|c|c|}
\hline & LENGTH. & WEIGHT. & CAPACITY. \\
\hline & MILLIMETER. & MILLIGRAM. & $\begin{array}{l}\text { Milliliter. Thousandths. } \\
\text { (CUBIC CENTIMETER.) }\end{array}$ \\
\hline DIVISIONS. & $\begin{array}{l}\text { CENTIMETER. } \\
\text { Decimeter. }\end{array}$ & $\begin{array}{l}\text { Centigram. } \\
\text { Decigram. }\end{array}$ & $\begin{array}{l}\text { Centiliter. Hundredths. } \\
\text { Deciliter. Tenths. }\end{array}$ \\
\hline UNITS. & METER. & GRAM. & LITER. \\
\hline MULTIPLES. & $\begin{array}{l}\text { Dekameter. } \\
\text { Hek tometer. } \\
\text { KILOMETER. } \\
\text { Myriometer. }\end{array}$ & $\begin{array}{l}\text { Dekagram. } \\
\text { Hektogram. } \\
\text { KILOGRAM. } \\
\text { Myriogram. }\end{array}$ & $\begin{array}{l}\text { Dekaliter. Tens. } \\
\text { Hektoliter. Hundreds. } \\
\text { Kiloliter. Thousands. } \\
\text { Myrioliter. T'en thous. }\end{array}$ \\
\hline
\end{tabular}


\$10. How to Learn the Metric System.-According to our experience, there are three chief requisites :-

$\$ 11$. 1. Absolute certainty of the significance of gram, liter and meter, as the units of measure of weight, capacity and length, respectively.

With the child or unlearned person, this may be purely an effort of memory. But most persons can save something by connecting gram with gravity, liter with liquid, and meter with measure, or with the word itself as it occurs in thermometer, barometer, etc.

\section{\$12. 2. Certainty of the force of the prefixes.}

With the child, this too is a matter of memory. But the prefixes of the names of the divisions are from the Latin, while those of the multiples are from the Greek. Since Greent increases while Latin diminishes, it has been proposed to combine the initials of the four words in a single mnemonic word gild. We suggest, instead, that the $g r$. of Greek be associated with the same letters in greater, and that Latin and less have the same initial.

Deci, centi, and mille are familiar to most persons in the words decimate, centipede and millipede, while deka, hekto and kilv, are known to some in decade, decalogue, hekatomb, and chiliad. Of these six prefixes, however, centi, milli and kilo, are much more often used than the others.

\section{\$13. 3. Personal familiarity with some metric measure.}

The shortest and surest way to a knowledge of the metric system is to carry some metric measure, or have one always at hand. The five-cent nickel piece is 2 centimeters in diameter. and weighs 5 grams. The student is advised to carry a metric rule in the pocket, and to keep another always upon the table.

The prices of Rules and Tapes vary from 3 cents to as many dollars. A list of the styles and prices may be obtained from the American Metric Bureau ( $A$ ), and from dealers in such articles.

Fig. 1.

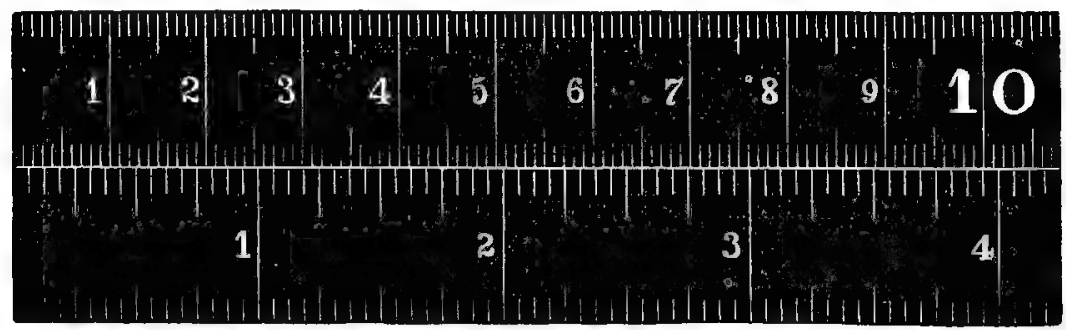

Fig. 1. A section, about one tenth, of the paper meter-yard, fumished for 20 cents by the American Metric Bureau (A, No. 26, 410). The face has the meter and the yard side by side, and the back bears a complete Table of metric and English equivalents, embracing 198 separate entries. The whole folds into the length of a decimeter, so as to be easily carried in the pocket, and the Table may be consulted without unfolding the paper, "Moisture affects the absolute length, but not the accuracy of comparison." 
Teachers may aid the introduction of the system by obtaining metric Models and Apparatus, by hanging metric Charts upon the walls, by making their own diagrams on cloth a yard wide but a meter long, and by pointing to them with the graduated Sticks of one or two meters in length. But the best of all agencies is the placing of some metric measure, if only the 5-cent rule, in the hands of each pupil.

An efficacious yet inexpensive way of diffusing elementary information and arousing curiosity respecting the system is to employ, in business correspondence, envelopes or postal cards bearing a metric ruling along one edge. This ruling is done free of charge by the Metric Bureau.

\$14. Reduction to and from the Present System.-Pending the universal adoption of the metric system, it is often necessary to effect a reduction to and from the older measures.

The following Table of Equivalents is selected from the Tables on the back of the "meter-yard," (Am. Met. Bureau, A, 444-448), in Foster and Langley, (A, 263), and in Egleston, (A).

\begin{tabular}{|c|c|c|c|}
\hline Abbreviations. & Measures. & Accurate. & Approximate. \\
\hline $\begin{array}{l}\text { crn. } \\
\text { c. c., or cu. cm. } \\
\text { fl. oz. } \\
\text { ft. } \\
\text { ft. } \\
\text { U. S. gall. } \\
\text { U. S. gill. } \\
\text { gr. Troy. } \\
\text { g. } \\
\text { “. } \\
\text { in. } \\
\text { kilo., or kg. } \\
\text { "“ } \\
\text { “. } \\
\text { km. } \\
\text { l. } \\
\text { “" } \\
\text { m. } \\
\text { “ } \\
\text { ، } \\
\text { mile. } \\
\text { mm. } \\
\mu .\end{array}$ & $\begin{array}{l}\text { centimeter. } \\
\text { cubic centimeter. } \\
\text { fluid ounce. } \\
\text { foot. } \\
\text { “" } \\
\text { U. S. wine gallon. } \\
\text { U. S. wine gill. } \\
\text { Troy grain. } \\
\text { gram. } \\
\text { “" } \\
\text { inch. } \\
\text { kilogrum. } \\
\text { “" } \\
\text { “" } \\
\text { kilometer. } \\
\text { liter. } \\
\text { “" } \\
\text { meter. } \\
\text { “" } \\
\text { ، } \\
\text { ‘ } \\
\text { mile. } \\
\text { millimeter. } \\
\text { " micromillimeter." }\end{array}$ & $\begin{array}{c}0.393 \text { in. } \\
0.033 \text { fl. oz. } \\
29.578 \text { c. c. } \\
0.304 \text { meter. } \\
30.479 \text { cm. } \\
3.785 \text { literg. } \\
118.31 \text { c. c. } \\
.064 \text { gram. } \\
15.432 \text { gr. Troy. } \\
0.564 \text { dram avoir. } \\
0.035 \text { o. avoir. } \\
2.539 \text { cm. } \\
2.204 \text { Ib. avoir. } \\
2.679 \text { lb. Troy. } \\
32.15 \text { oz. Troy. } \\
3527 \text { oz. avoir. } \\
0.621 \text { mile. } \\
0.264 \text { U. S. gall. } \\
1.056 \text { U. S. quarts. } \\
2.113 \text { U. S. pints. } \\
0.546 \text { fathom. } \\
1.093 \text { yard. } \\
9.842 \text { hands. } \\
3.280 \text { feet. } \\
39.370 \text { inches. } \\
1.609 \text { kilometers. } \\
0.039 \text { inch. } \\
0.001 \text { mm. } \\
\\
28.349 \text { grams. } \\
31.103 \text { g. } \\
0.473 \text { liter. } \\
0.453 \text { kilo. } \\
0.373 \text { kilo. } \\
0.946 \text { liter. } \\
0.914 \text { meter. }\end{array}$ & 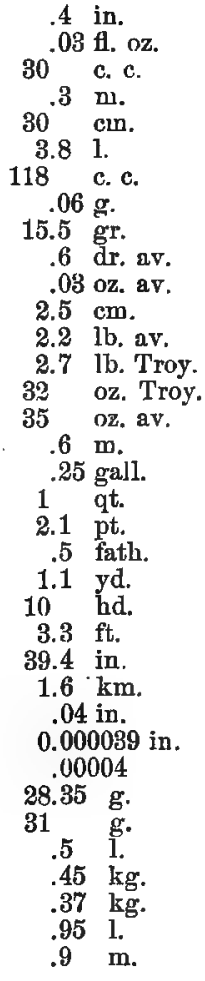 \\
\hline
\end{tabular}


The "micromillimeter" is not an original member of the Metric System, and the desirability of using the word is strongly questioned. In either case, the symbol for the thousandth of the millimeter should be the Greek $\mu$ rather than $r m m$.

\$15. The Metric System in Medicine.-Notwithstanding the obvious and considerable hindrances to a change involving commercial interests, the new system is surely though slowly making its way among Physicians and Pharmacists.

The matter is constantly discussed in the Medical Journals, and "Reduction Tables" have been publisbed in very compact form. Dr. Kreider has one, in the Medical Record for Oct. 23, 1880; Dr. F. H. Brown has another in " The Medical Register for New England," which has been reprinted as a pocket leaflet by the Metric Bureau ; while another leaflet, first printed by the Metric Bureau (A, 345-348), by Dr. E. Wigglesworth, presents "The Metric System in a Nut-shell," with especial reference to the needs of the medical profession.

\section{ZOOLOGICAL CLASSIFICATION.}

\$16. As this is in no sense a treatise upon either Zoology or Comparative Anatomy, but simply a guide to certain practical methods of work, we give only such an outline of the Classification of Animals as may serve to indicate the generally accepted taxonomic relations of the forms here considered.

Fuller information and discussion of this matter may be found in the works and papers of Gegenbaur, Hæckel, Huxley, Owen, etc., and in the condensed Summary of Pascoe (A) ; see also Balfour, A, ii, 1.

It did not seem worth while, in the accompanying Table, to indicate the view entertained by many Zoologists, that the primary division of both the Animal Kingdom and the Vertebrate Branch is dichotomous: the one into the Protozoa and the Metazoa; the other into the Acrania and the Craniota.

The names of the eight animals more or less fully treated of are given in the column at the right of the page. One, the amoba, is a member of the lowest division of the Animal Kingdom, the Protozoa, and consists of but a single cell, a mass of nearly homogeneous but nucleated protoplasm.

The other seven belong to the highest division, the branch Vertebrata, the essential character of which, as will be more fully indicated farther on, is that there is a dorsal and a ventral cavity, between which is a sub-cylindrical axis of membrane, cartilage, or bone.

Man, the Cat, Dog, and Rabbit are members of the class Mammalia, including the Vertebrates which are warm-blooded, and are brought forth alive. The Frog and Menobranchus are Amphibia or Batrachians, which differ from the Reptiles in having gills or water-breathing organs at some period of their lives. 

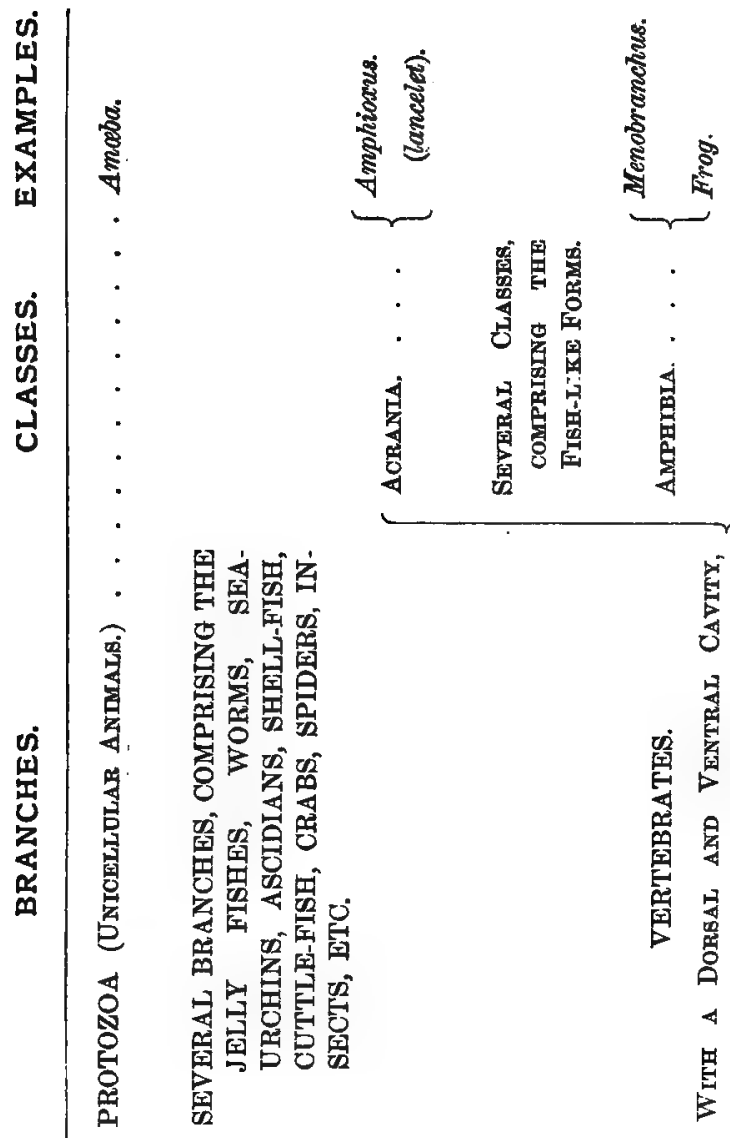

स्ड़

- 鄫专

- 20

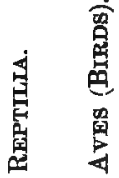

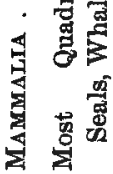
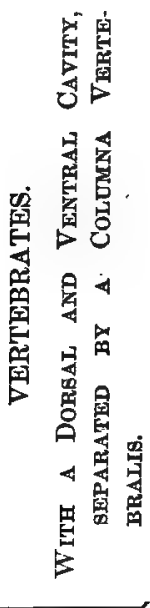

5
0
0
0
$Z$
1
4
4
4
4
4 
The lowest vertebrate is the little Amphioxus lanceolatus, usually assigned to a distinct class, the Acrania. Between the Amphioxus and the Amphibia are all the "fishes," common and uncommon, including the Sharks and Rays, and many for which there are no popular names. Likewise, between the Amphibia and the Mammals are the classes Reptiles and Birds, no examples of which are herein considered.

\section{IV.-TERMINOLOGY.}

\$22. Whoever does any systematic practical work in anatomy, whether by investigation or teaching, is impelled, sooner or later, to suggest some modifications of the language previously employed in recording or communicating observations and ideas.

Such modifications are often put forth in publications without explanation or apology, and their subsequent adoption by others may then depend less upon their intrinsic value, than upon the reputation and authority of the proposers.

In the following pages, especially in the account of the Brain, there will be found a few new words, for the introduction of which reasons will be duly offered.

Many other words and phrases will appear more or less unusual, some to one reader and some to another. Those, however, who are most familiar with the best original anatomical publications in not only their own language, but also those of other nations, will, upon careful serutiny, perceive that the present work really contains very few terms for which there is not sound precedent in the writings of recognized authorities, in the use of either the same words, or of words analogous in character and formation.

From the productions of working anatomists in all parts of the world we have endeavored to select, without bias or partiality, the terms which seem to us best suited to the object, and most easily used.

As a whole, therefore, the Terminology here employed is that of no one nation or writer. Much less should it be regarded as our own, excepting in so far as we have succeeded in our efforts to combine the elements from various sources into a consistent and Lomogeneous whole.

But while the prestige of authoritative precedent might lead us to make a personal trial of any assemblage of terms, it would not, by itself, warrant an introduction into a purely elementary work like the present. It is proper, therefore, to say that, with very few exceptions, all of the names and descriptive terms here employed have withstood the severe practical test of use by a large number of students for from one to seven years.

This test is called severe because these students have been comparatively mature, and have done practical work in anatomy with the view of becoming either naturalists, teachers or physicians; and because they have not simply listened to lectures in which the terms were used, but have been required to employ them in writing their own descriptions of parts; because, finally, they have been urged to make suggestions and criticisms with entire freedom.

On what, therefore, may be called experimental grounds, we feel justified in presenting this Revised Terminology of Anatomy to students other than our own; but since each year has brouglut about some change therein, we bave no reason to think it perfect or complete, and we ask the co-operation of all who may undertake to follow out our directions with the view of rendering it better adapted to the needs of Practical Workers in Anatomy. 
§23. Some General Considerations upon Anatomical Terminology.-These remarks are based largely upon an article (Wilder, 9), which although published under the name of the senior author, was prepared with the advice and co-uperation of the junior.

That article referred more particularly to the brain, but covered most of the questions connected with the naming and description of other parts. Hence the following expressions, the only published comments which have come under our notice, may fairly be considered applicuble to the whole subject :-

"The Nation" for April 21, 1881, contains a brief notice of the article, evidently by an experienced teacher of Anatomy, containing the admission that "there is certainly ample room for a Reform of Anatomical Nomenclature."

The following are extracts from a Letter (Spitzka, 7 ) to the editor of "Science," published in that journal for April 9,1881, by Dr. E. C. Spitzka, the author of many papers upon the Anatomy of the Brain :-

" It is with mingled pleasure and profit that I have read the very suggestive paper on cerebral nomenclature contributed to your latest issues by Professor Wilder. Some of the suggestions which he has made have been latent in my own mind for years, but I have lacked the courage to bring them before my colleagues. Now that he has broken ground, those who prefer a rational nomenclature to one which, like the present reigning one, is based upon erroneous principles, or rather on no principles at all, will be rejoiced at the precedent thus set for innovations. * * * He who has himself been compelled to labor under the curse of the old system, the bexeath, below, under, in front of, inside, external, between, etc., will l'ook upon the simple ventral, dorsal, lateral, mesal, cephatic, proximal, caudal, distal, etc., as so many boons. I have no hesitation in saying that the labor of the anatomical student will be diminished fully one-half when this nomenclature shall have been definitely adopted. * * *

In proceeding to comment on some of the terms proposed by Professor Wilder, I wish it to be distinctly understood that I do so merely tentatively and to promote discussion ; in so doing I feel certain that I am carrying out that writer's wish. It is but just to state that the majority of the terms cannot be discussed; they are perfection and simplicity combined."

With the permission of the writer, "Science" for May 28, 1881, printed the following Letter (Holmes, 1), from one who has been the Professor of Anatomy in the Harvard Medical School for more than the third of century :-

“Boston, May 3, 1881.

“Dear DR. WILDER:-I have read carefully your paper on Nomenclature. I entirely approve of it as an attempt, an attempt which I hope will be partially successful, for no such sweeping change is, I think, ever adopted as a whole. But I am struck with the reasonableness of the system of changes you propose, and the fitness of wany of the special terms you have suggested.

"The last thing an old teacher wants is, as you know full well, a new set of terms for a familiar set of objects. It is hard instructing ancient canine individuals in new devices. It is hard teaching old professors new tricks. So my approbation of your attempt is a sic vos non vobis case so far as I am concerned. * * *

"What you have to do is to keep agitating the subject, to go on training your students to the new terms-some of which you or others will doubtless see reasons for changing-to improve as far as possible, fill up blanks, perhaps get up a small manual in which the new terms shall be practically applied, and have faith that sooner or later the best part of your innovations will find their way into scientific use. The plan is an ex- 
cellent one; it is a new garment which will fit Science well, if that capricious and fantastic and old-fashioned dressing lady can only be induced to try it on.

"Always very truly yours,

"Oliver Wendell Holmes."

See also Appendix, § 1443.

That Terminology is worthy of attention, is indicated also by the care bestowed upon the language of modern Chemistry and Mathematics, and by the following Aphorisms:-

\$24. "Questious of Definition are of the very highest importance in Philosophy, and they need to be watched accordingly."-Duke of Argyll, 1 .

"In all sciences, Nomenclature is an object of importance; and each term should convey to the student a definite meaning."-Dunglison, A, Preface.

"Every art is full of conceptions, which are peculiar to itself; and, as the use of language is to convey our conceptions to one another, language must supply signs for those conceptions."-Huxley, C, 14.

"Everything in Science ought to be real, ingenuous and open; every expression that indicates duplicity, or equivocation, reservation, wavering or inconsistency, is a reproach to it."-Barclay, A., 89.

"There is a necessity for perfect definiteness of language in all truly Scientific work."-P. G. Tait, 1.

"Technical terms are the tools of thought." *

"Only an inferior hand persists in toiling with a clumsy instrument, when a better one lies within his reach. *** A single substantive term is a better instrument of thonght than a paraphrase."-Owen, A, I, pp. xii, xiv.

"As morphology deals with forms and relations of position, it demands a careful selection of terms and a methodical nomenclature."-Goodsir, A, II, 83.

"To designate the locations of organs by the relation of animals to the surface of the earth is as far from philosophical as it would be to define the position of a house or of a tree by reference to the planet Jupiter."-Wilder, 9, 122.

The progress of Comparative Anatomy has been hindered by the use of anthropotomical terms and methods.

"There is not one person in a hundred who can describe the commonest occurrence with even an approach to accuracy."-Huxley, C.

"The test of the accuracy and completeness of a description is, not that it may assist, but that it caunot mislead."-Wilder, $9,123$.

Errors of personal equation are diminished by the use of exact terms. 
"A name is a short substitute for a definition, and where no definition exists, there can be no name."-Packard and Cope, $\boldsymbol{1}$.

"The one essential of naming is that distinct objects shall have distinct names; and the second essential is, that each object or concept shall have but one name."-Idem.

"Life is too short to spend in digging for Truth with a long-handled shovel when a trowel will serve the purpose; nor is it becoming that any nation, however wise and great, should ask all the rest to take their intellectual food with chop-sticks of its peculiar pattern."-Wilder, $9,124$.

"The personal convenience and preferences of all existing anatomists should be held as of little moment as compared with the advantages which reform may ensure to the vastly more numerous anatomical workers of the future."-Idem, $13 \%$.

The two following may serve to show that we have not been unmindful of the dangers and disadvantages of terminological change.

"Nothing is more pernicious than to attempt to tamper with wellunderstood and universally accepted symbols."-Anonymous Reviewer ("The Athenæum," June 4, 1881).

"He who, affected by the cacoethes reformandi, insists upon reform for the sake of an ideal perfection; is apt to appear as nothing better than a troublesome and useless pedant."-Wilder, 9,124 .

\$25. Brief Statement of the Objects and Methods of the Terminological Changes here made.-To render the Vocabulary of Anatomy equally applicable to all Vertebrates, and equally intelligible to all Nations.

To facilitate the Recognition of parts by students, and lessen the labor of Memorizing.

To abridge the length of Descriptions, and at the same time increase their Accuracy.

To include in this Vocabulary, so far as practicable, only such terms as are brief, simple, exact, significant, of classical origin, and capable of inflection.

To propose as few changes as possible, and to introduce new names only for parts apparently unknown or unnamed before (e.g., crista fornicis), or in the place of semi-descriptive appellations undesirably long or incapable of inflection, as e.g., cimbia for tractus transversus pedunculi, porta for foramen Monroi.

To consider brevity as an especially desirable characteristic of such names as are most frequently employed.

When a part is known by a descriptive phrase, to select therefrom some characteristic word as the technical designation; e. g., iter (a tertio ad ventriculum quartum). 
When two or more parts are similar, or have similar relations, to distinguish them by joining to some common title already in use, prefixes indicative of their relative positions; e.g., postgeniculatum, progeniculatum.

To shorten the names of several parts by omitting the word corpus, and using the neuter adjective as a substantive.

To keep modern usage and the rules of classical etymology constantly in mind, but not to be hindered thereby from the employment or even the formation of terms which are eminently desirable from the practical standpoint.

To discard terms which indicate size, those which refer to the natural attitude of man or animals, most vernacular names, and all names of the reproductive organs which have been applied needlessly to other parts.

The terms employed by anatomists form two divisions: those which indicate the position or direction of organs, and those by which the organs themselves are designated. Since, also, writers have often treated of them separately, it will be convenient here to consider anatomical toponomy and organonomy under distinct headings.

\& 26. Designation of Organs-Organonymy.-There are probably few investigators or teachers of comparative anatomy who have not been impressed, in some degree, with the desirability of some modification of the prevailing nomenclature of organs, - the "bizarre nomenclature of anthropotomy," (Owen, A, II, 143)-based as it is upon the peculiar features of the human body, which has been fitly characterized, from a morphological point of view, as " not a model, but a monstrosity."

This impression may give rise to special papers, like those of Owen, (166), Maclise (1), and Pye-Smith (1), or simply to more or less extended remarks upon the subject, with or without the use or presentation of new terms.

More thian one hundred pages of Vicq d'Azyr's great Anatomy (A) are deroted to a "Vocabulaire anatomique, angmente d'un grand nombre de termes nouveaux."

In the Preface to his "Anatomie du Chat" (A, pp. xiv-xvii), StrausDurckbeim devotes several pages to a discussion of anatomical nomenclature, and the body of the work contains many original names. Professor H. S. Williams calls attention (A, Preface), to the "crying need of a standard and uniform nomenclature of comparative anatomy."

In the Preface to their recent account of the morphology of the skull (A), Parker and Bettany say: "It has been attempted to narrate the facts by means of a consistent terminology, amplifying what Prof. Huxley has so admirably developed." Several of Huxley's papers (as \%0), contain new terms, most of which have been generally accepted, and in a greater or less degree the same is true of the elder Agassiz (A), Gegenbaur (59), Hæckel (A), Marsh (I), and others. 
\$2\%. Nomina Impudica.-Several parts, especially of the brain, have received names originally applied to portions of the reproductive apparatus which they were fancied to resemble. While it may perhaps be urged in extenuation that the patres anatomici entertained a notion as to the representation of the entire organism in the brain, some of their terms certainly indicate an entire freedom from apprehension that the mysteries of encephalic anatomy ever would be discussed by ordinary mortals, much less by women, or under circumstances requiring propriety of speech.

On the other hand, many names of general or special application have been, at times or by certain writers, needlessly applied to the male and female organs. Among the 50-60 medical synonyms for vulva are sinus, folliculus, annulus, hiatus, ostium, sulcus, trema, delta, cava, fovea, mesa, porta, and fundus. Among the 70-80 synonyms of penis are vomer, vas, clavus, cauda, vena, gladius, radix, ramus, columna, trabs, pyramis, and spina. These terms should not be lost to clean anatomical uses because heedless or filthy-minded writers have so misapplied them.

$\S 28$. Importance of Brevity.-As has been stated, and as will be exemplified in the vocabulary, we place great stress upon brevity as a desirable characteristic of anatomical terms.

So long as the study of anatomy was nearly confined to members of the medical profession, they being comparatively few in number, and, by ancient tradition at least, not wholly averse to clothing their discourse in a sesquipedalian garb impenetrable to the vulgar eye, it mattered little whether the statement of a given fact or idea required one minute or tive.

But now, thanks to the popular writings of Agassiz, Dana, Gray, Darwin, Hæckel, Huxley, Owen and others, in so far especially as they have aroused a personal interest in the problems of Evolution, natural history instruction is given systematically in all schools and colleges, and the time seems to have come when, in the words of the naturalist first-named, "Scientific truth must cease to be the property of the few ; it must be woven into the common life of the world." It is probable, indeed, that those who employ anatomical language to a greater or less extent at the present day are at least one hundred times as numerous as when Dr. Barclay's praiseworthy effort at reform was received with indifference or opposition.

In our opinion, therefore, the single names trochiter, trochin, epicondylus, and epitroch lea are worthy of aloption in place of the compound terms greater and lesser tuberosity, external and internal condyle of the humerus, notwithstanding their proposer, Chanssier, has burdened the myological division of anatomy with the most unwieldly set of terms that could have been devised.

\$29. Technical Terms.-It may be asked: In the face of this rapid popularization of anatomical knowledge, is it worth while to introduce, or even to retain, any purely technical terms? 
Apparently some German scientists have determined upon a negative reply to this inquiry, and their papers, even those of strictly scientific nature, teem with vernacular words, and with wonderful compounds thereof.

For abundant examples, the reader may consult any "Dictionary of German Medical Terms," as that of Cutter (A).

If this kind of verbifaction be tolerable under any circumstances, it certainly would be justified by the extent and importance of the contributions to knowledge which appear first in the German scientific periodicals.

Upon this point, however, we can do no better than to quote the very recent judgment of one who is at the same time an investigator, a promoter of "the diffusion of knowledge," and an admirer of the methods and results of German science :-

"Every art is full of conceptions which are peculiar to itself ; and, as the use of language is to convey our conceptions to one another, language must supply signs for those conceptions. Either existing signs may be combined in loose and cumbrous paraphrases, or ncw signs, having a well-understood and definite signification, may be invented. Science is cosmopolitan, and the difficulties of the study of zoology would be prodigiously increased if zoologists of different nationalities used different technical terms for the same thing. They need a universal language; and it has been found convenient that the language shall be Latin in form, and Latin or Greek in derivation."-Huxley, C, 14.

Unless it can be shown that there is a distinction between the methods of designating entire organisms, and the parts thereof, the foregoing passages should silence the objections of those who would have us retain a vocabulary as vague as was that of Chemistry in the days of quicksilver, vitriol and copperas-a vocabulary which combines the ponderous stiffness of the cloister with the puerile vagueness of the nursery. Tuberculum bigeminum anterius must give way to lobi optici, or some even shorter term; while trachea must take the place of windpipe, weasand, luftröre and conduit arien.

Is it not worth considering, too, that any avoidance of the use of technical terms is, after all, only partial and delusive? The principal human organs have, of course, received popular names which may, in great measure, be applied to the other Vertebrates, especially the Mammals. But there are many other organs of which the butcher takes little or no account. Shall we construct vernacular phrases, and write of the liver-vein, the kidneyartery, the gullet-nerves, and the sweetbread-tube? Unless we are prepared to give up such convenient and elastic terms as hepatic, renal, pancreatic, pulmonary, cardiac, etc., why should not the student be informed, at the outset of his anatomical and physiological enquiry, that nearly all the divisions and appendages of the principal organs have received titles derived from the classical names of the organs themselves? These derivatives and com- 
pounds would then have some significance, instead of appearing like troublesome verbal complications.

So too with the names of the various groups of animals, nearly all of which are based upon the technical names for some of the organs. The determined "vernacularist" may delude himself with the belief that he is defying the classics in calling Amphioxus by the name Lancelet; but he cannot appreciate the progress or the present condition of systematic zoology without learning that to the same lowest vertebrate have been applied the terms acrania, leptocardia, cirrostomi, cephalochorda, and pharyngobranchii. Why then should he not bave been informed already that cardia, cirrus, stoma, pharynx and branchia are technical names for heart, gill, etc.?

In short, while the small beginnings of Physiology and Zoology may be acquired by the use of vernacular words alone, any considerable progress in exact knowledge would be excessively inconvenient if not impossible, at least with the French or English student, without the aid of a certain number of technical terms.

Nor are these terms so numerous as to constitute anything more than a purely sentimental burden. As has been well-said by one who is in the position to recognize to the full the value of purely classical training, "A doctor, lawyer, or popular exhorter who cannot learn by heart, in a week, all the technical terms and phrases of Latin origin which he encounters in his common professional occupation, has not wits enough for his calling." Eliot, 1, 359.

That there is no inherent obstacle to the employment of technical terms of classical derivation is shown by the readiness with which such words as petroleum and phylloxera have become domesticated along with the objects which they represent. There are scores of animals, like the Rhinoceros, Hippopotamus, and Ichneumon, for which there are no English vernacular names; while the youngest student of Botany accepts Hepatica, Anemone, and even Rhododendron without difficulty or hesitation. Homely as it sounds, stomach is of classical origin, and the use of caul for omentum, sweet-bread for pancreas, or blind-gut for cacum, would surprise a class in Elementary Physiology.

Even the late Jeffries Wyman, who saw no objection to forearm, and used near rather than proximal for the first row of carpalia, accepted intermembral as "good," and freely employed, if indeed he did not originate, the adjective pretibial, which probably would have come into general use had. not the bone in question proved to be the homologue of the intermedium.(Morse, 18, 13.)

$\$ 30$. Names Indicative of Relative Position.-Where four or more similal parts form a series, they are usually numbered $1,2,3$, 4, etc., beginning with the one nearest the head, or the middle line, as the case may be. Thus, of the ribs, the first is next to the neck; among the several groups of 
vertebræ, cervical, thoracic, and lumbar, the most cephalic is the first; so too, in the normal position of the limbs (to be explained farther on), the pollex and primus (great toe) are on the borders nearer the head, and may sometimes be designated as the first digit and dactyl.

In designating the fractional portions of the length of a bone, the proximal half, third, fourth, fifth, etc., is the first; the rest following in order toward the distal end.

When, however, the series embraces only two or three similar parts, the general name for them all has been ustually followed (in Latin) or preceded (in English) by some word indicative of relative position; as, e. g., processus superior, and middle commissure.

This plan effects a saving in the number of different words without the risk of ambiguity, just as when we say John Smith senior, junior, and third. But all such terms are open to the objection of being compound, and therefore incapable of inflection.

In some cases, therefore, the more general terms have been combined with the distinctive prefixes to form single words, like supraspinatus and mesoglutcus. Owen has also employed (A, III, 519) postcava and proecava and the senior author has proposed $(\mathbf{2 2}, 306)$ entopectoralis and ectopectoralis, and, more recently $(\boldsymbol{9}$ and $\boldsymbol{1} \boldsymbol{1})$, a series of similar names for parts of the brain; e. g., pracommissura, medicommissura, postcommissura, ctc.

\$31. The Limits of Terminological Change.-As has been stated already, the modifications here proposed are intended to provide for what seem to be actual necessities, irrespective of purely theoretical considerations, and of any desire for a perfectly uniform and consistent terminology. It may be well, however, to specify certain general limitations to cluanges of anatomical nomenclature.

Priority is practically of little moment in respect to the names of organs, since it is usually difficult to ascertain when and by whom they were first applied. An example of this is afforded by the phrase foramen of Monro, (Wilder, 3). Nor, indeed, has priority always been held sacred in systematic zoology. Owen's "Deinosaurians" was proposed nine years later than von Meycr's "Pachypoda;" yet, as stated by Huxley $(\mathbf{1 0 8}, 33)$, it has becn retained, notwithstanding the small size of some members of the group.

Etymological appropriateness is sometimes disregarded, as in the caso just mentioned, and in the more familiar names Reptiles, Vertebrates, Edentates, etc. Prof. Huxley has recently expressed the common sense vicw of the matter as follows:-

"If well understood terms which have acquired a definite scientific connotation are to be changed whenever advancing knowledge renders them etymologically inappropriate, the nomenclature of taxonomy will before long become hopelessly burdened." (C, 16.)

So, too, the names of organs have sometimes been given in reference to 
some variahle or unessential character, or have even conveyed an erroneous idea; yet no one now thinks of discarding either rectum, arteria, or carotid.

Sometimes even brevity and etymological accuracy yield to established usage. The word cubitum, proposed by the senior author in $18 \% 2(\mathbf{1 0}, 21)$ as the technical equivalent of fore-arm, is both shorter than antebrachium, and more in accordance with its classical employment; but the latter word seems to be more generally preferred, and we are ready to accept it.

In another case, even though a new term has not yet come into general use, a special vitality may be imparter to it by the authority of those who may have adopted it. No marked or persistent disfavor is likely to be shown to terms which, like myelon, can clain Prof. Owen as father, and find a godfather in Prof. Huxley.

Even Milne-Edwards, while intimating (A, XI, 234) that anatomical nomenclature has been created in sufficient perfection, frankly admits the superiority of myelon over moelle épiniere.

§32. Some Inconsistencies.-It will be noted (Fig. 6) that we have refrained from giving technical names to the membral arthra (joints) or have merely added them in parentheses.

This is partly because the need of names for parts so familiar seems less urgent than in the case of some other organs. Still, it is certainly undesirable that the carpo-metacarpal arthron should be called wrist in man, and knee in the horse, and the chief cause of our inconsistency in this respect has been our inability to decide upon the relative merits of the various names which might be applied. The names suggested are those which were proposed by the senior author in $18 \% 1(\mathbf{1 0}, 21-24)$; but they do not appear to have been adopted to any considerable extent.

Strict logical consistency, also, would impel us to substitute entoscapular for subscapular in the designation of a fossa and muscle of the scapula; so too, supraspinous, supraspinatus, infraspinous, and infraspinatus, should be praspinatus, etc.; notwithstanding the demands of consistency and logic, and the example of Owen (A, III, 44), we decline to interfere with these brief and old-established titles of well-known parts.

Further information concerning the changes in the names of organs will be given in the Index and in the Lists and Tables of names in the several chapters.

It will be noted, also, that of the two Latin names for heart we have adopted the longer cardia, rather than the shorter cor. This is because derivatives and compounds of the former are by far the more numerous and familiar. Hence the selection is really in accordance with the more general principle that all measures of reform should have regard to the practicability as well as the abstract suitability of a change.

The case with stomach is less simple; although the word is directly derived from the Greek $\sigma \tau o ́ \mu \tilde{a} \chi o s$, it is practically a vernacular term, while 
most of the derivatives and compounds are formed from the Latin gaster. Perhaps we should have adopted the latter as the technical designation for the organ.

8 33. Names and Abbreviations on the Figures.-Whatever may be the practice of different writers, probably all will agree that figures for the information of students, and especially such as are to be used in dissection, are more helpful if the following conditions are observed:-

1. So far as possible, the technical names of the parts should be written upon the parts themselves.

2. Where there is not room for the names upon the figure itself, they should generally be written at the side, and connected with the parts by distinct lines.

3. When abbreviations are employed, they should be of the technical rather than of the vernacular terms, and should be uniform, at least for all figures of the same organ or region.

Whoever examines the figures in the present work will see that an attempt has been made to conform to the above named conditions. That success has been only partial, will be at once anticipated and excused by all who have undertaken the same task. The need of abbreviation was greatest with the brain, where many distinct parts are crowded within a small space. Here, as a rule, only names of more general application, such as Fissura, Gyrus, Sulcus, etc., and their abbreviations, have been commenced with capitals; but this distinction has not been observed in all other cases.

In the explanations of the figures, the abbreviations are given in alphabetical order.

\$ 34. Terms of Position and Direction-Toponymy.-Like other solids, the body of an animal has six general aspects.

As with other elongated solids, these aspects are two endi and four sides.

Were the body simply a mass of homogeneous material, like, for example, an oral of wood, the supporting side would be called the bottom, and described as the lower side; the opposite side would then be upper, and be called top; of the two other sides, either might bo called right, and the opposite would then be left; finally, either of the two ends night be named front, and the other end would then be known as back. To indicate the two ends as anterior and posterior, and the top and bottom as superior. and inferior, would render only more evident the fact that the various aspects of the mass are determined and named according to their relations to the observer, or to the surface of the earth.

But with the animal body, at least the body of the adnlt vertebrate, there are constant and more or less marked distinctions between the opposite ends and sides, so that these various aspects have fixed and definito relations to one another and to certain other objects or influences. 
Food and drink are received at one end of the body, and the organs of special sense are there located. Between one of the sides and the nervous cord known as the myelon or spinal cord, there always intervenes a subcylindrical rod, usually of bone or cartilage, the Columna vertebralis, or spinal column, while the opposite side is not so separated from the myelon. The two remaining sides differ less from one another, but distinctions have been observed, some of which have been commented upon by the senior author in papers $13,12,13$, and 18 .

It is obvious that the comparison of the corresponding aspects in different animals will be more easily made and more instructive, if the animals can be placed, actually or ideally, in some common position, and if the aspects can be called always by the same names.

$\S 35$. The Normal Position of the Body.-Taking as the natural attitude of an animal that which it assumes in ordinary locomotion, there are wide differences among Vertebrates. The head of man points directly away from the eurth, and the longitudinal axis of the body forms a right angle with its surface; with the gorilla and some other apes the axis is slightly inclined; with birds it forms a smaller angle with the supporting surface; but with the larger number of vertebrates the body is nearly or quite horizontal.

As the question is entirely one of bodily organization, and has no reference to mental or spiritual preeminence, there nerer has been made any serious objection to regarding the normal position of vertebrate animals as that of the majority of them, in which the body axis is horizontal, and the aspect nearer the earth is that which is separated from the myelon by the Columna vertebralis.

\$ 36. Designation of the Aspects.-Instead, however, of applying to the various aspects names naturally suggested by the parts themselves, irrespective of the particnlar attitude assumed by the animal, anatomists, probably influenced by the greater practical importance of the human body, have almost universally employed terms which are strictly applicable only to man in the erect attitude. In order, therefore, that a comparison might be instituted between corresponding parts of man, a cat and a fish, it was necessary, at least constructively, to erect the two latter upon their tails.

Notwithstanding the logical inconsistency of such a course, and the risk of misunderstandings, no effort at reformation seems to have been made until early in the present century, when Dr. Barclay, the anatomical preceptor of Professor Owen, published a little volume (A) entitled: "A New Anatomical Nomenclature, relating to the Terms which are expressive of Position and Aspect in the Animal System."

\$3\%. The key note of Barclay's view of the subject is struck in the following paragraph :- 
"The vague ambiguity of such terms as superior, inferior, anterior, posterior, etc., must Lave been felt and acknowledged by every person the least versant with anatomical description."

Some of Barclay's new terms have been occasionally used by $\mathrm{Owen}$, but most of his contemporaries and immediate successors seem to have been quite indifferent to his suggestions, and only within a comparatively few years has the subject again received attention.

Dunglison admits $(A, 61)$ that "Great confusion has prevailed with anatomists in the use of the terms before, behind, etc." Spitzka forcibly states $(\mathbf{1}, 75$, note 1$)$ the objections to the use of anterior, etc., and refers $(\gamma, 165)$ to the gradual disuse of the equivalent German terms by Henle, Gudden and others; more exact terms, also, are occasionally employed by several writers who do not explicitly condemn the current toponomy; Cones, 1, 150; Cleland, 1, 170 ; Gegenbaur, A, 491; Rolleston, B, 33, note; Huxley, 2 .

In previous publications (A, 69, and 1, fere) Mivart more or less consistently discards anterior and posterior, and his recent work (B, 258, note,) characterizes them as "Unfortunate as applied to a quadruped like the cat."

Finally, the need of a rudical "change of base" has been proclaimed in one of the very strongholds of anthropotomy:-

"Now that the more extended study of comparative anatomy and embryonic development is largely applied to the elucidation of the human structure, it is very desirable that descriptive terms should be sought which may, without ambiguity, indicate position and relation in the organism at once in man and animals. Such terms as cephalic and caudal, dorsal and ventral, etc., are of this kind, and ought, whenever this may be done consistently with sufficient clearness of description, to take the place of those which are only applicable to the peculiar attitude of the human body."-Quain, A, i, 6.

This is certainly explicit as to the principle involved, and it is to be hoped that later editions of this standard Human Anatomy may display its practical application to the body of the work.

The ambiguity here alluded to is not merely hypothetical. In a recent work (Mivart, B) the $\boldsymbol{M}$. sterno-mastoideus is described (p. 134) as arising "beneath the anterior part of the pectoralis major," but on $p$. 145 a part of the $M$. ectopectoralis is said to arise "beneath the munubrium [præsternum]." In the former case beneath means entad of or dorsad of, while in the latter the same word signifies ectad of or ventrad of. The experienced human or comparative anatomist may know what is intended, and the context would enable any one, with a little study, to determine the matter by exclusion; but there are so many instances in which, by reason of the $a b$ sence of planes and straight lines, the context must be depended upon in a greater or less degree, that needless ambiguities should be avoided.

The foregoing illustration, however, by no means exhausts the list of possible ambiguities. In the normal position of a vertebrate, the heart is 
beneath the Columna vertebralis; in the natural attitude of man, it is beneath the bifurcation of the trachea; in the position in which both man and quadrupeds are commonly dissected, the heart is beneath the sternum; finally, it may be said to be beneath the ribs or the $M M$. intercostales in the sense of being covered by them. The single English word here means successively ventrad of, caudad of, dorsad of, and entad of the organs on four different sides of it. Whatever may be thought best by the writers of descriptions for advanced students, we hold that the use of such terms in a work expressly designed for beginners would be little else than a self-stultification of its authors and a mockery of its readers.

$\S 38$. The Intrinsic Toponymy.-Following the suggestion of Barclay, and the more or less consistent example of the other writers above named, we shall wholly discard all terms which "contain an allusion to the situation of different objects, as they stand with respect to the heavens and the earth ;" and shall designate the aspects and regions of the body by terms derived from names which have been applied to the parts themselves. Hence we shall speak of the cephalic and the caudal ends or aspects or regions; of the dorsal and ventral aspects or regions; and of the dextral and sinistral aspects and regions.

Such terms constitute a Toponrmical Vocabulary which is based upon intrinsic instead of purely extrinsic and accidental relations.

\$39. Cephalic and Cauda1.-Barclay proposed the words attantal and sacral for the designation of the position of parts lying toward the head or the tail in reference to an imaginary plane dividing the body at about the middle of its length.

In many of the lower vertebrates, however, there is no distinct atlas or sacrum, and in any case these terms would not apply strictly to parts beyond the bones in question; hence Barclay devised for the head an entirely new set of terms, inial, glabellar, etc. So far as we know, atlantal and sacral occur only in the writings of Owen (A, III, 519) and Turner $(1,819)$.

Thacher has employed $(\mathbf{1}, 282$ and 292) orad as both adjective and adverb, but the correlative aborad, which might have been expected, has not been observed by us in his papers.

Cephalic and caudal are employed by Cleland (1), and are recommended by Quain as stated above. Their signification is obvious, and practically there seems to be no serious objection to their use, although it is possible to imagine cases where some ambiguity might arise from the fact that each is employed in two senses, the one relative, and the other absolute. For example, in the absolute sense, only the vertebræ of the tail are caudal; but relatively,caudal may be used to designate one or more vertebræ in any part of the series, which are situated nearer than others to the caudal end of the body. So too, we may speak of the caudal aspect of the skull, or of the cephalic members of the series of caudal vertebræ. 
\$ 40. Dorsal and Ventral.-By their derivation, and by their application to distinctly differentiated and universally recognized regions, these two terms are perfectly acceptable so far as the vertebrates are concerned.

Should the alleged correspondence of the ventral region of the Vertebrate with the tergal region of the Arthropod prove to be one of true homology, it may be desirable in time to discard dorsal and ventral for more suitable terms, but for the present, if on practical grounds alone, it seems well to retain them.

As stated above, these are among the terms recommended in Quain, and they have been somewhat generally employed by anatomists; Huxley, A, 1 et seq.; Owen, A, III, 3 ; Sanders, 1 ; Hadlich 1, 97, note ; Mivart, A, 69 , $\mathrm{B}, 263$ and $\mathbf{1}$, fere.

'To avoid ambiguity, it will be better to employ clorsal only in the sense bere indicated, and to characterize the costiferous vertebræ as thoracic.

$\$ 41$. Dextral and Sinistral. - These are simply more technical equivalents of the English right and left. No objection has been made to them, or substitutes offered for them.

842. Lateral.-Usually the two sides are so similar that what is true of the one is, approximately at least, true also of the other. Hence it is often desirable to employ a general term applicable to either or both the right or the left. Such a term is lateral, the derivation of which properly indicates the fact that, as compared with the two ends or the top and bottom, the right and the left are the sides. Lateral is therefore a more general term, while dextral and sinistral are specific.

\$43. Mesal.-In the restricted literal sense of entering into the composition of the head or the tail, certain parts may be said to be absolutely cephalic or caudal; but in most cases these terms, like dorsal and ventral, merely indicate a greater proximity of the part so characterized to one or the other of the four aspects in comparison with some other part, named or implied. Eren when used in an apparently absolute sense, they are really relative terms.

This is because there has not been detected any structural line or plane of demarcation between the two ends of the body, or between the back and the belly.

But with right and left, the case is different. The right eye and the right kidney are not simply nearer than the left to the right side or aspect of the body; they also form parts of the right half of the body, and this half, notwithstanding the numerous instances of continuity, is so readily distinguishable from the left, that one may almost describe the body as if it were formed by the union of two pieces, similar, but reversed in form and position.

The plane of junction of the right and left sides is at or near the middle of the body, and has been known as the middle or median plane, a longi- 
tudinal line therein being called a middle or median line. But since certain parts lie upon or cross this plane, and since it is sometimes desirable to speak of lateral parts as more or less near it, Barclay proposed $(A, 121)$ for it the single word mesion.

We have not met with this word in other writings, although mesial and mesiad are not infrequent.

Nevertheless, it seems desirable to designate this middle plane by a single word which is at once significant, short and capable of inflection.

Such a word is meson, from the Greek ro $\mu \dot{\varepsilon} \sigma o v$, the middle, equivalent to the more ponderons Latin meditullium. This word and its deriratives were proposed $(9)$ by the senior author.

For convenience, any point or line therein may be called meson, but the lines most frequently referred to in description constitute the dorsal and ventral borders of the plane, and may be known as dorsimeson and ventrimeson respectively.

To avoid ambiguity, it would be well to employ mesal and its derivatives only in reference to the meson; intermediate (middle) may then be applied to the second of any series of three similar parts; while medial could be used in reference to the digitus medius.

$\S 44$. Convenient additions to the vocabulary of toponymy would be terms of single words, corresponding with meson, but indicating respectively the dorsal, ventral and other aspects of the body. Wo refrain, however, from making any specific suggestion.

$\S 45$. Designation of the Regions of the Limbs.-The body as a whole, with the cat as with most vertebrates, consists of two general divisions, axial and appendicular; the former is the body proper or soma; the latter are the limbs or membra.

On account of the approximately vertical position of the arms and legs in the natural attitude of man, their attached and free ends had been called superior and inferior, or upper and lower.

For these terms, as inapplicable to the limbs of many animals (fishes and turtles, etc.), as are the terms anterior and posterior (in the anthropic sense) to the rest of their bodies, Barclay wisely substituted proximal and distal, which have been very generally adopted.

We speak, therefore, of the attached end of any appendage, as limb, ear, barbel, tentacle, horn, spine, as the proximal end, its free or unattached extremity being in like manner called distal. The same terms apply to the corresponding ends of the segments, bones and muscles of these appendages.

As has been well remarked by Mivart $(2,509)$, "The tail, to a certain extent, partakes of the natures of both the trunk and the limbs. It is like the limbs in that it is solid, that it contains no body-cavity, and is not traversed by the alimentary canal." Still, the tail is really a division of the soma, and its two ends should be designated as cephalic and caudal. 
Barclay seems not to have concerned himself for other than English uisers of his new terms, and we can only conjecture what he would have made the classical forms of proximal and distal. Following analogy, they may be rendered proximalis and distalis, though no such Latin words exist.

For the four other aspects (borders or sides) of each limb, Barclay. proposed the terms ulnar, radial, anconal and thenar, tibial, fibular, rotular and popliteal.

These have been employed to some extent by later writers, but they are open to at least three objections: they are specific instead of general; the bones from which they are derived are not recognizable in "fishes ;" with many mammals, the ulna and radius are crossed instead of parallel, and with some the ulna and tibia are but slightly developed.

Huxley and some other English anatomists have employed the general terms preaxial, postaxial, epaxial, and hypaxial. But these words are liable to misconception because uxial has been used already in reference to not only the axis vertebra, but also the entire skeleton of the trunk as contradistinguished from that of the limbs.

Perhaps the chief objection to all these terms is that they are not really necessary, and introduce undesirable verbal complications.

The limbs are certainly part of the body, and whether or not, as held by Thacher ( $\boldsymbol{1}$ ) and Mivart (1), they are essentially and primarily only isolated and differentiated portions of continuons lateral fold, there seems to be a general assent to Huxley's proposition (4) that, for comparison, the limbs should be regarded as extended laterad at right angles with the soma.

Hence it seems to us most natural to apply to the aspects of the limbs the same terms which are applied to the corresponding regions of the soma. Thus each limb presents a dorsal and a ventral aspect, a cephalic and caudal aspect, and a proximal and distal end.

$\S 46$. Terms of General Application to the Whole Body.-Central and peripheral were proposed by Barclay, and have been very generally used by anatomists. They are especially applicable to the parts of the nervous and vascular systems, since the vessels and the nerves may be said to radiate from or converge to the heart and the myelencephalon (cerebro-spinal axis) as anatomical and physiological centers.

Barclay also recognized the need of terms denoting nearness or remoteness with respect to the surface of any part of the body, and proposed dermal in the one case, central doing duty also in the other.

Most anatomists, however, have contented themselves with the older words, outer and inner, superficial and deep, sublime and profound.

Of these terms, three are incapable of inflection; all are rery commonly employed in a metaphysical sense, and are therefore more or less ambiguous; while the last two are quite inappropriate to the insignificant structures with which they are often associated. 
The need of other terms than those in use was so generally and so strongly felt among the students in the Anatomical Laboratory of Cornell University that the suggestion to employ entul and ectal was welcomed, and they were published in the article $(9)$ alroady mentioned. Derived respectively from $\dot{\varepsilon} \nu$ tós and $\dot{k} k$ ós their significance is obvious, while their brevity and capacity for inflection will probably commend them to accurate working anatomists.

Both words are already familiar in the words ectozoa, entozoa, entoptic, entoglutceus, ectoglutceus, ctc. $\Delta$ s a rule, it will probably be well to employ them in reference to lamince or surfaces.

$\S 4 \%$ Inflections. - Barclay proposed that the various adjective forms should be converted into adrerbs by substituting for the ending al the letters $a d$, the Latin equivalent of the English ward. Thus dorsal, ventral, dextral, sinistral and lateral became dorsud, ventrad, dextrad, sinistrad, and laterad. Substituting mesal for mesial, and cephalic and caudal for atlantal and sacral, we have in addition the terms mesad, cephalad and caudad.

Proxinal, distal, ental and ectal are readily converted into the adverbs proximad, distad, entad and ectad.

For example, the dura (mater) may be described as ectad of the brain, but entad of the cranium. A part may be divided by cutting either ecto-entad, from without inward, or ento-ectad, from within outward.

The adverbial forms occur less frequently than the adjectives, but dorsad is used by Huxley, as reported in Nature, Jan. 6, 1881, p. 281, and this together with ventrad and mesiad are systematically employed by Mivart in a recent paper, $(\mathbf{1}$,$) although not in his latest work (B).$

\$48. Use of the Prepositions of and From.-Of is used with terms of relative position, when the rerbs to be or to lie are expressed or understood. Thus we say, the elbow is distad of the shoulder.

From is used with active verbs implying extension or passage from one point to another; thus, the myelon extends caudad from the brain; the humerus extends distad from the shoulder, etc.

\$49. Limitations to Accuracy.-The use of the terms above enumerated certainly renders it possible to be more definite in description. Yet absolute accuracy and exactness are often unattainable, with an animal like the cat, where there are few plane surfaces or straight lines. It often becomes necessary to designate the relative positions of two parts, or the direction of a line upon a more or less curved or undulating surface. For example, on Fig. 7 representing approximately the outline of a transection of the body, the point $a$ is obviously laterad of the point $b$. So too, the point $c$ is dorsad of the point $d$. But the point $c$ may be describerd as latero-ventrad of $b$, or dorso-mesad of $d$; it would seldom happen, however, that the curvature should be equal on both sides, and usually the needed qualification would be supplied by the context. 
\$50. Derivatives.-With derivative words the connecting vowel is commonly $i$; e. g., alipes, claviger, fatifer, fidicen, fluctigena, decimanus, neuvilemma, and xiphisternum. But classical exceptions are mulomedicus, quadrupedus, noctuvigilus, and decumanus. In common English and scientific terms of Latin or Greek origin the $o$ is common; $e . g$, ambodexter, burgomaster, gastrotomy, termonology, ventroinguinal, lateroflexion, mucopurolent, vasomotor, curvograph, neuroglia, oculospinar, pleuroperitoneal, xiphosura, septopyra, hemoglobin, cephalotribe, etc. Rarely is it $e$ as in venesection.

Both analogy and euphony lead one to use the $i$ when the first part of the word is of Latin origin, and the $o$ with the Greek.

Hence we have dorsimeson, ventrimeson, dorsicumbent, latericumbent, dextriflexion, sinistriversion, cephaloduction, caudiduction, etc. ?

$\S 51$. Compound Words. - The two Latin compounds known to us are veneri-vagus and vesti-contubernium. 'The following common or technical English compound words are selected from Webster's English Dictionary, or the Medical Dictionaries of Dunglison, or Littré et Rubin, or from the writings of Barclay, Humphrey (E), and Straus-Durckheim: Anglo-Saxon, concavo-convex, dextro-gyrate, ventro-appendicular, costo-vertebral, custoalaris, cnudo-pedal, isseo-cutaneous, occipito-scapularis, dorso-lateral, meso-dorsal, sternoclavicular, clavo-cucullaire, clavi-sternal, clavio-humeralis. By analogy with the foregoing, compound terms of direction should read dorso-ventral, caudo-cephalic, meso-lateral, sinistro-cephalic, etc.

§52. Combination of Words.-The names of two or more organs or tissues may be conjoined like the words for regions; thus we say musculo-tendinous, or gastro-hepatic. But the names of organs are never combined with the names of regions; hence such a term as dorso-gastric does not occur.

§53. Hybrid Words.-Some of the terms already mentioned are formed by the union of Latin with Greek words; e.g., dorsimeson, meso-luteral, and caudo-cephalic; several others are likely to be employed; e. g., claco-mastoideus, and felitomy.

Beyond the occasional intimation, in the dictionaries, that a term is hybrid, the subject seems to be ignored, and it might fairly be inferred that literary authorities entertain one or the other of two opposite convictions: either mongrel words are verbal monstrosities which will be shunned instinctively by all well-regulated minds, or there is no more serious objection to their use, or even their creation, than to the employment, or even the production, of mules, or the mixed varieties of grapes and roses.

However this may be, the fact is that the Latin and the Greek tongues have united to form the following nine hybrids which may be found in Latin writings: anticalo, biclinium, cryptoporticus, dentarpagr, epitogium, monosolis, monoloris, pseudoflavus, and pseudourbanus. Of these, the third only occurs with any degree of frequency.

Whoever will spend the time to lnok through an Unabridged Dictionary of the English language-and the interest as well as the instructiveness of such a search can hardly be realized by those who use the volume only for occasional reference-will find that, after excluding the twenty-five or more words ending with meter, which may perhaps be derived directly from the Latin form metrum, there are more than one hundred hybrid words, many of them in good standing. Many more are to be gleaned from the Dictionaries of Medicine and the other Arts and Sciences.

Nevertheless, it is probable that a due regard for the feelings of the classical purists in whose eyes language was not made for man, but rather man for language, will lead scientists to refrain from the introduction of mongrel terms when others will serve the purpose, and we shall be pleased to receive suggestions leading to the substitution of wholly unobjectionable words for any of the hybrids which may occur in the present work. 


\section{Practical Application of the Foregoing Considerations in the Designation of Some Parts, and in the Indication of their Relative Position and Direction.}

$\S 54$. The Primary Divisions of the Body.-Soma and Membra.-The entire body of a normal and complete Vertebrate presents a principal axial portion, and an appendicular portion, the arms and legs, which may be spoken of, collectively, as the limbs or membra.

Neither the classical nor the vernacular terms for these divisions have been used with desirable exactitude. With the ancients, corpus might signify either the entire body, or the trunk as distinguished from the head, while the English body may refer to either the whole body in distinction from the mind, the axial part in distinction from the appendicular part, or the principal portion of the former in distinction from the lead and the tail. Truncus usually meant the body apart from the limbs, but the head and the trunk are sometimes spoken of as distinct regions.

In view of this lack of discrimination we venture to suggest that the entire physical part of an animal be called the corpus or body; that truncus and trunk be applied only to that part of the axial portion which intervenes between the neck and the tail ; and that the entire axial portion, including head, neck, trunk and tail be denominated the soma.

It is true that the Greek $\sigma \omega \mu a$ was generally equivalent to the Latin corpus, and that many of its deriratives and compounds refer to the entiro organism; but the term somatome was proposed by Goodsir to indicate a vertebral segment, of which the limbs are merely occasional components. Somite and somalopleure are used by Balfour, A, II, 3, 141.

The undesirability of the introduction of a somewhat unfamiliar term is fully admitted. It is probable, however, that it need not be emploved very often.

$\$ 55$. Figure 2 is a diagram of the dorsal aspect of the cat, intended to illustrate certain very general features of it or of any other vertebrate provided with two pairs of limbs.

The outline of the soma is elliptical, the larger end corresponding to the head, and the smaller with the tail.

The arms and legs are represented as lateral appendages projecting at right angles with the longitudinal somatic axis, or meson.

Each limb has an attached or proximal end, and a free or distal end.

The right and left sides of the entire body are antitropic or symmetrical with each other; that is, they are reversed repetitions of one another in opposite directions.

§56. Beginners in Anatomy are sometimes confused by the fact that, with some figures, the right is at their own right, while with others the right of the figure is upon their left.

This confusion may be aroided by a preliminary exercise with a familiar object :- 


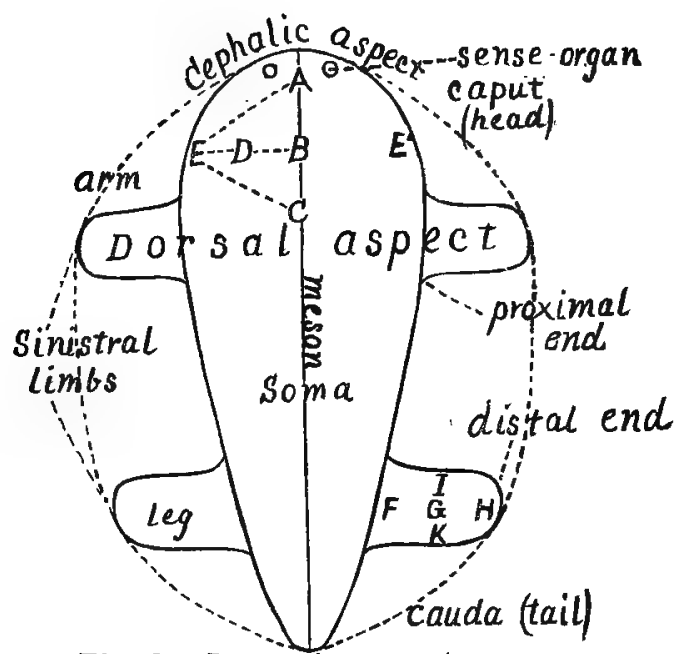

Fig. 2 Dorsal aspect

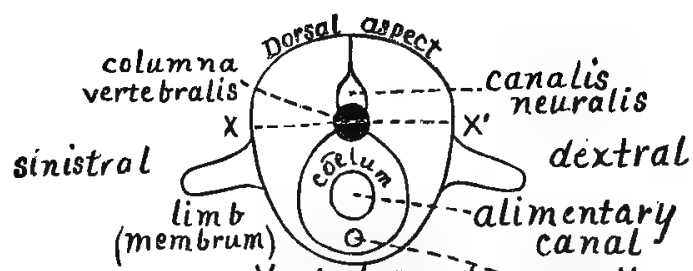

Ventral aspect---cardia

Fig. 3. Transection

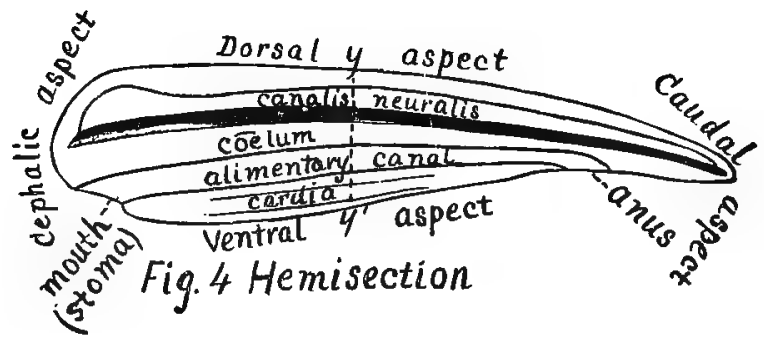

DLagrams of THrete Aspects of a Vertebrate, Exhibiting the most General Features. 
Hold a book and note its several aspects-the top and bottom, the back and front, the right and left sides. For the purpose of comparison, these aspects may be considered to correspond with the head and caudal aspect, the back and tho belly, the right and left sides of a man or a cat.

When the book is held so as to permit the reading of a title printed across the back, the various aspects coincide in position and direction with the body-aspects. The right is at the right of the observer, and the left at his left; the back faces in the same direction with his own, while the top of the book is upward, and its bottom down.

But when the book is held just ready to be opened, the top and bottom bave the same directions as before, but the back and front, the right and left sides are reversed. The right of the book is opposite the left of the observer, and vice versa.

In the first case, the observer and the book are related as are two persons one behind the other; in the second case, the relation is that of two persons facing each other, and as when one views his own image in a mirror.

Again, if the book is held so that the lower end is in view, the right and left still correspond with those of the observer; but if it is turned so as to expose the top, then the right and left are reversed. The same difference exists in the case of transections of objects. If a book were cut across, there would be exposed two cut surfaces, the bottom surface of the upper part, and the top surface of the lower part. With an animal, these would be called the caudal surface of the cephalic part, and the cephalic surface of the caudal part. If the former is ricwed, the right and left of the surface coincide with those of the observer; if the latter, then the right and left are reversed.

As with objects, so with their representations in pictures and diagrams. The right and the left, the dorsal and the ventral aspects, are to be so designated, whatever may be their position on the page or with respect to the observer.

The foregoing remarks concern symmetrical figures, which represent either the dorsal, the ventral, the caudal or the cephalic aspects of an animal, or its parts. As a rule, in the present work, such figures are so placed that the meson coincides in direction with that of the observer, and with the longer diameter of the page, as, e. g., Fig. 2 and 3.

Figures which, like Fig. 4, are unsymmetrical, and represent the lateral aspects of animals or their parts, are usually so placed that the meson lies across the page, and at a right angle with that of the observer. Usually, also, in accordance with distinguished precedent, as remarked in a paper (17) by the senior anthor, the cephalic end of such figures is turned toward the left of the page and of the observer.

\$ 5\%. Position and Direction on the Soma.-The letters A, B, C, D, E, F, G, H, I, K, with the dotted lines between them are introduced for 
the sake of illustrating some of the more common cases of designation of relative position and lines of direction.

The points A, B and C lie at or upon the meson, and are therefore mesal. So also, the dotted lines between the points are mesal lines.

$\mathrm{D}$ is not at the meson, and is therefore laterall (in this case sinistrad) of $\mathrm{B}$; but it lies nearer the meson than $\mathrm{E}$, and is therefore mesad of it.

$\mathrm{B}$ lies cephalad of $\mathrm{C}$, but caudail of $\mathrm{A}$.

$\mathrm{E}$ lies latero-cephalad of $\mathrm{C}$, and latero-cauclad of $\mathrm{A}$.

$\mathrm{A}$ lies meso-cephalad of $\mathrm{E}$, and $\mathrm{C}$ meso-caudad. The line $\mathrm{A}, \mathrm{B}, \mathrm{C}$ is a cephalo-caudal, or caudo-cephatic line, or it may be described as extending caudad from $\mathrm{A}$, or cephalad from $\mathrm{C}$.

The line E, D, B, is a meso-sinistral, or sinistro-mesal line, and may be said to extend either mesad from $\mathrm{E}$, or laterad (sinistrad) from B.

The line A, $\mathrm{E}$ extends meso-cephalad from $\mathrm{E}$, or sinistro-caudad from A.

The line A, B, C coincides with the meson, and a cut upon it would be a hemisection.

The line E, D, B, E' is a transrerse line, or at right angles with the meson, and a cut therein would be a transection.

Finally, an organ on the moson, and represented, for instance, by B, is not only mesal in position, but also called azygous or unpaired; while two sinilar organs, one upon each side, and represented for instance by $\mathrm{E}^{\prime}$, are lateral in position, and called paired organs. Each such paired organ may be called the platetrope of the other, or its lateral homologue, or the fellow of the opposite side.

The letters npon the right leg have similar relations, excepting that proximal and distal take the place of mesal and lateral or dextral and sinistral.

I and $\mathrm{K}$, for example, lie respectively cephalad and caudad of $\mathrm{G}$; while $G$ lies caudad of $I$ and cephalad of $K$. F lies proximad of $G$, and $G$ of $H$; $\mathrm{H}$ is distad of $\mathrm{G}$, and the relative locations of the three may be designated as proximal, clistal and intermediate.

A. The reasons for repressnting the limbs in the position here given them will be more advantageously presented in $\S 80$.

B. The dotted lines connecting the two extremities of the soma with the distal ends of the limbs illustrate the idea referred to on $p .26$ that the limbs are essentially remnants of two contisuous lateral folds; see also Balfour, A, III, 501.

\$58. Figure 3 represents an ideal transection of the body of a cat, or any other vertebrate possessing limbs.

Only some of the more constant and essential features are here shown.

Near the middle of the figure is the section of the Columna vertebralis.

'The Cotumna vertebralis or spinal colimn may be of bone, cartilage, or a semi-solid material with membraneous walls, according to the animal ex- 
amined, the degree of development, or the part which is divided. It lies upon the meson, and serves therefore as a boundary between the right and the left sides of the animal.

In most animals it is located, in nearly its whole length, nearer that side of the body which is commonly upward in ordinary locomotion; that is, it is nearer the dorsal aspect. There is no definite plane of contact of the dorsal with the rentral region as of the right with the left side; but, for convenience, the two regions may be regarded as meeting at a line $\mathrm{X}, \mathrm{X}^{\prime}$, passing from side to side through the middle of the Columna vertebralis.

Dorsad of the Columna is a canal, the Canalis neuralis, containing the myelencephalon or cerebro-spinal axis, (brain and spinal cord). Ventrad of the Columna is a cavity, usually more capacious, the coelum, or general body cavity, in which are the heart, alimentary canal, and other viscera.

This arrangement of principal organs, in two cavities, on opposite sides (but not on the right and left) of a mesal axis is a constant character of all Vertebrates, and, with perhaps a few exceptions, is peculiar to the group.

\$59. Figure 4 represents an ideal hemisection (section on the meson) of such a body as is represented in figures in 2 and 3 . The cephalic aspect or head looks toward the left.

$\$ 60$. The Body Planes.-For the sake of precision in the use of toponomical terms, the planes already referred to will be more distinctly defined; see Fig. 5.

\$61. 1. The Meson.-This is a plane passing lengthwise or the body, and dividing the whole into approximately equal and similar right and left halves.

For convenience, the dorsal and ventral borders of this plane may be called the dorsimeson and the ventrimeson respectively.

Organs like the nose, the stomach, and the urocyst (bladder) appearing upon both sides of the meson are said to be mesal or azygous. They are, at least primarily and approximately, symmetrical in themselves ; that is, they consist of similar right and left halves.

Organs like the eyes, the lungs, and the kidneys, which form pairs, the one upon the right and the otber upon the left of the meson, are called lateral or paired antitropous organs. Either of them is symmetrical with its fellow (platetrope), but not in itself.

§ 62. 2. An imaginary transverse, dorso-ventral plane, at right angles with the meson, and dividing the body into a cephalic and a caudal region.

§63. 3. An imaginary longitudinal, dextro-sinistral plane, extending the whole length of the body, and dividing it into a dorsal and a ventral region.

These three body-planes correspond in direction with the three dimensions of a solid, length, height, and width. Each is at a right angle with both the others. The longitudinal sematic axis lies in the meson. 
As already indicated, the position of the meson is fixed; that of the other two planes is purely conventional and for convenience in a given case.

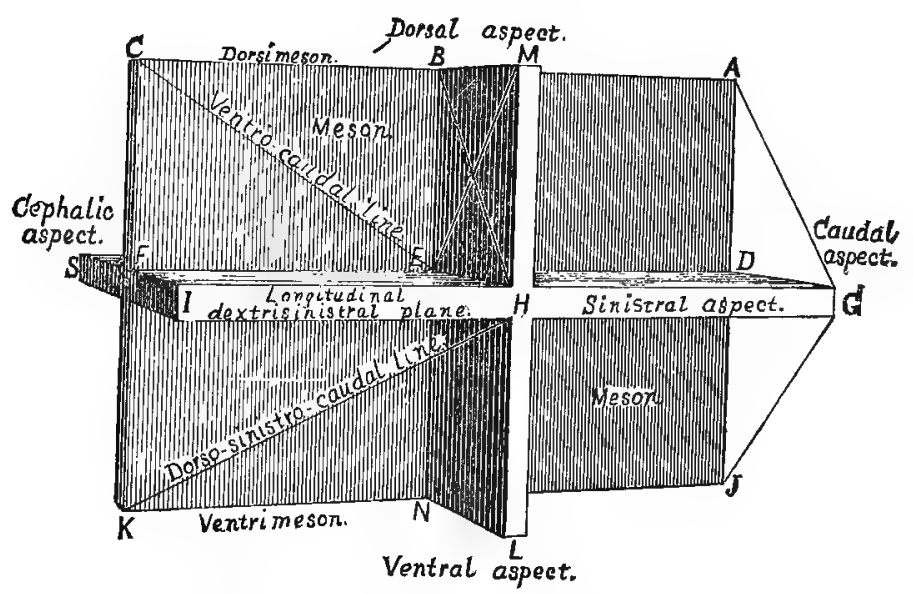

Fig. 5.-Diagram of the three Body-ptanes, with the VARIOUS LINES OF DIRECTION.

\$64. Designation of Position and Direction.-By the use of terms derived from meson, and from the names of the general regions or aspects of the body, it is possible to designate positions and lines of direction with considerable precision.

There are two general cases :-

1. Where the points to be located are on a line which itself lies in one of the three body planes, or in a plane parallel to one of them.

2. Where the points to be located are on a line which is not in either of these planes, but connects some two of them like the diagonal of a solid; e. g., the points $\mathrm{H}, \mathrm{K}$, at the ends of the line HK.

Under case 1, the lines may be either direct or oblique.

\&65. Direct lines may be perpendicular to the meson, as the line SFI, or any other line passing directly from one side of the body to the other. Such a line would be the line connecting the eyes, or the heads of two ribs.

Direct lines may lie either in the meson itself, or in an imaginary plane parallel thereto and either parallel or perpendicular to the longitudinal dorso-sinistral plane; e.g., ABC or MHL. Such lines pass directly from the dorsal to the ventral aspect of the body, or from the caudal to the cephalic aspect.

All direct lines may be also characterized as coinciding with the intersection of two of the three body-planes, or of planes parallel thereto. 
$\S 66$. Oblique lines lie in one plane, but are not perpendicular to either of the other planes; neither do they coincide with the intersection of two of the body planes, or of planes parallel thereto. They are comparable with the diagonal of a parallelogram. Such lines are EC; $\mathrm{ME} ; \mathrm{BH}$.

$\S 6 \%$. Designation of Direct Lines.-This requires a term of two words, of which one indicates the point of departure and the other the point of approach.

On the figure of the model, the line $\mathrm{ABC}$ is a cephalo-caudal line, or extends cephalo-caudad; or the order may be reversed by saying that it is a caudo-cephalic line, or extends caudo-cephalad. The line CFK is a dorsoventral or ventro-dorsal line, and the line IFS is dextro-sinistral or sinistrodextral.

\$68. Designation of the Relative Positions of Points upon Direct Lines.-This requires a term of but one word, adverbial in form, and indicating a point of approach.

In Fig. 5, C is cephalad of $\mathrm{B}$, and dorsad of $\mathrm{F}$; $\mathrm{A}$ is candad of $\mathrm{B} ; \mathrm{H}$ is ventrad of M, but dorsad of $\mathrm{L}$.

In the body, the sternum is ventrad of the heart, the Columna vertebralis is dorsad, and the diaphragm is caudad of the same organ.

In man, the sternum would be said to be before, or in front of, or anterior to the heart; but in animals it might be described as below, under or inferior to the same organ.

It should be kept in mind that these terms are relative, not absolute. The diaphragm, for example, lies cephalad of the stomach, but caudad of the heart.

\&69. Designation of Oblique Lines.-This requires a term of two words, both of which indicate points of approach. The points of departure are usually apparent from the context. Thus, the line E, C, extends both toward the head and the back; hence it is called a cephalo-dorsal line, or described as passing cephalo-dorsad. The same line could be described as caudo-ventral, or as extending caudo-ventrad.

In man, such a line would be described as extending forward (ventrad) and downward (caudad). In comparative anatomy, it might be said to pass downward (ventrad) and backward (caudad).

$\$$ 70. Designation of the Relative Position of Points upon Oblique Lines.- This requires a term of two words, in the adverbial form.

$\mathrm{On}$ the line $\mathrm{CE}, \mathrm{O}$ is cephalo-dorsad of $\mathrm{E}$, while $\mathrm{E}$ lies caudo-ventrad of $\mathrm{C}$.

In man, $\mathrm{C}$ would be said to be above and behind; in animals, in front and above.

§ 71. Designation of Diagonal Lines not in Either of the Three Planes.-This requires a term of three words, all of which indicate points of approach; the order of the words is immaterial. 
In Fig. 5 the line HK may be described as having a dorso-sinistro-caudal direction. In man, it would be said to extend backward (dorsad), to the left (sinistrad), and downward (caudad). But with an animal it would be described as passing upward, to the left, and backward.

\section{$\$ 72$. Designation of the Relative Position of Points upon} Diagonal Lines not in Either of the Planes.-A term of three words is needed, with the adverbial termination. Thus $\mathrm{H}$ is dorso-sinistro-caudad of $\mathrm{K}$; or $\mathrm{K}$ is ventro-meso-cephalad of $\mathrm{H}$.

$\$ 73$. In all these cases, it is sometimes more convenient, and equally intelligible, to substitute for the more specific terms dextral and sinistral, the more general terms mesal and lateral. The line ME, for example, might be called dorso-lateral instead of dorso-sinistral; or it might be called ventromesal, and the context would show which side was referred to. If, while dissecting upon the left side, the student were directed to cut mesad for $2 \mathrm{~cm}$., he would cut towards the right, that is dextrad.

\$74. Designation of Direction and Relative Position upon the Limbs. - The rarious terms are employed and combined as for the soma, excepting that in place of mesal and lateral, or dextral and sinistral, there are used the terms proximal and distal.

\$\%. Ectal and Ental, and their Derivatives.-The general significance and uses of these terms have been indicated in $\S 46$. Other examples will be given in connection with Fig. $\%$.

A special employment of the two words in combination is for the sake of designating the direction of an incision. Ordinarily incisions are carried from the surface inward, that is ecto-entad; sometimes, however, it is desirable to divide parts, especially the skin and abdominal parietes, by an incision in the opposite direction-ento-ectad.

$\S \% 6$. Figure 6 is intended to show certain features of the cat, regarded as a Mammal, and not simply as a Vertebrate, as in Figures 2, 3, and 4.

As in Fig. 2, the body is ventricumbent, so as to expose the dorsal aspect.

The limbs (arms and legs) are extended at each side at right angles with the axis of the soma, in what is commonly regarded as their normal position.

The principal divisions of the body are named in the Table on p. 39 .

\& $\% \%$. The Soma and its Divisions.-The largest division of the soma is the trunk. The tail forms an extension in one direction, while the other end is continued as the neck and head. The neck is narrower than the head or trunk, and the head itself consists of the cranium and the face.

$\S$ \%8. The Trunk and its Divisions.-There are readily distinguished three regions of the trunk. The intermediate region or abdomen has only fleshy sides, while the more caudal-pelvis-has the Os innominatum on 
each side, and the more cephalic-thorax-is enclosed by the ribs (costce) and their cartilages (costicartilagines).

The ventral and larger cavities of these three trunk regions are, strictly speaking, cavum abdominis, cavum pelvis, and cavum thoracis; but they are usually called by the names of the regions themselves.

The abdominal and pelvic cavities are continuous; but between the abdomen and thorax, in the cat as in all other Mammals, there intervenes a musculo-tendinous partition-the diaphragma.

With all the lower Vertebrates, the diaphragm is absent or incomplete, so that the three cavities are continuous, and constitute the colum or trunk cavity. Sometimes it is convenient to apply this general name with Mammals.

A part of the alimentary canal is introduced for the sake of illustrating a somewhat peculiar case in the designation of relative positions which will be referred to in connection with the explanation of Fig. $7 ; \S 91$.

$\S$ 79. The Vertebræ.-As shown in Fig. 30, in the adult cat, the vertebræ form a continuous series from the caudal aspect of the cranium to the tip of the tail. In this diagram, however, only the thoracic vertebræ are shown by a series of squares representing their centra (bodies). The lines extended dextrad from the centra indicate the right costce (ribs). The centra and costre are included in this diagram merely to illustrate the method of notation of parts which form a series. The most cephalic is numbered 1 , etc.

$\S 80$. Normal Position of the Limbs.-For accurate comparison of the limbs with each other, or with those of other animals, it is desirable that they should be placed, or regarded as placed, in some uniform normal position.

One of the many possible natural attitudes of the cat's limbs is shown in Fig. 30, and there is great variety among other Mammals.

Huxley has proposed (4) that all limbs should be compared as if in the position shown in Figures 2 and 6 . In this condition they stand out at right angles with the body (soma), the extensor surfaces (with the convexities of the elbow and knee) being placed dorsally, and the flexor surfaces ventrally, with both pair of limbs. This is the position which they have in the embryo Mammal, according to Kölliker, and it continues throughout life in some Amphibia and Reptiles.

There is some difference of opinion as to whether the ventral aspects of the limbs should not be regarded as facing more or less directly mesad; but in other respects Huxley's view has been adopted by several anatomists, including Mivart, Pagenstecher, Rolleston, Flower, Coues, and the senior author, in whose paper $(10,9-17)$ will be found fuller references. See also Balfour, A, II, כ08.

Owing to changes which occur after the first formation of the limbs, it 


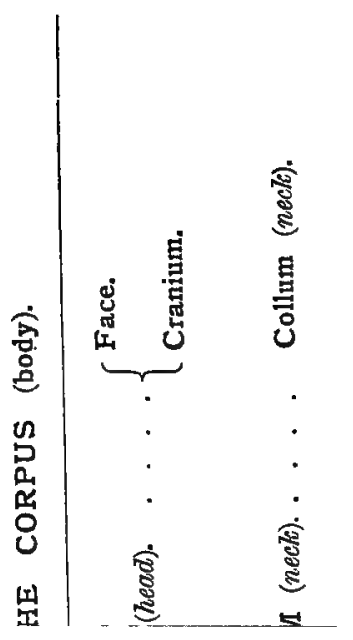

ह

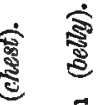

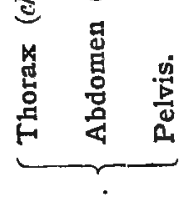

产
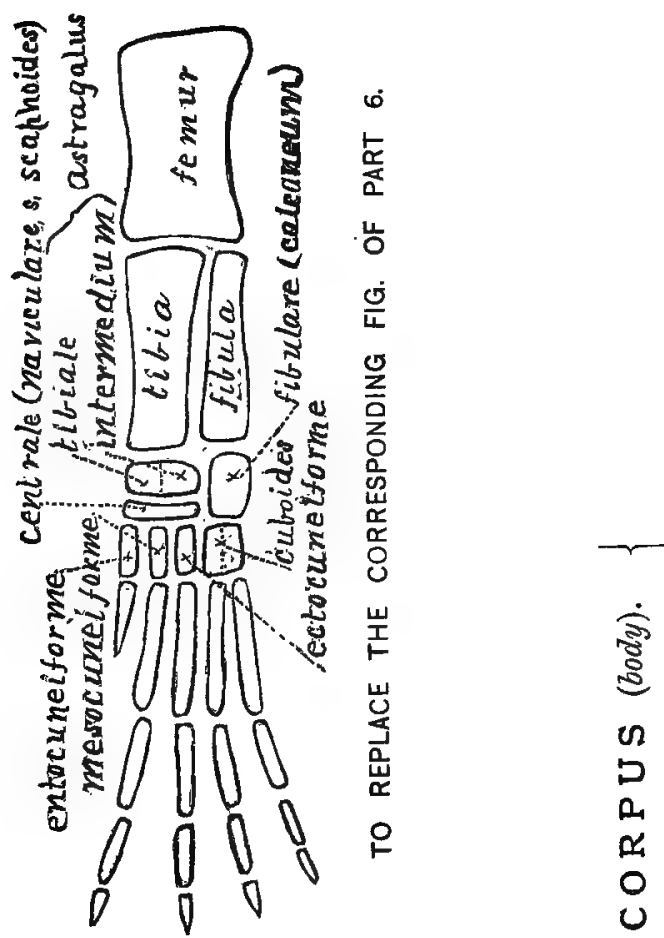

है है

5
5
3
3
3
3
3
3

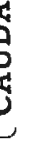

है

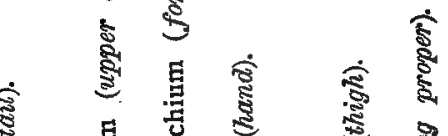

क्षे

总

क्ष

垫

हิ

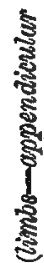

突 



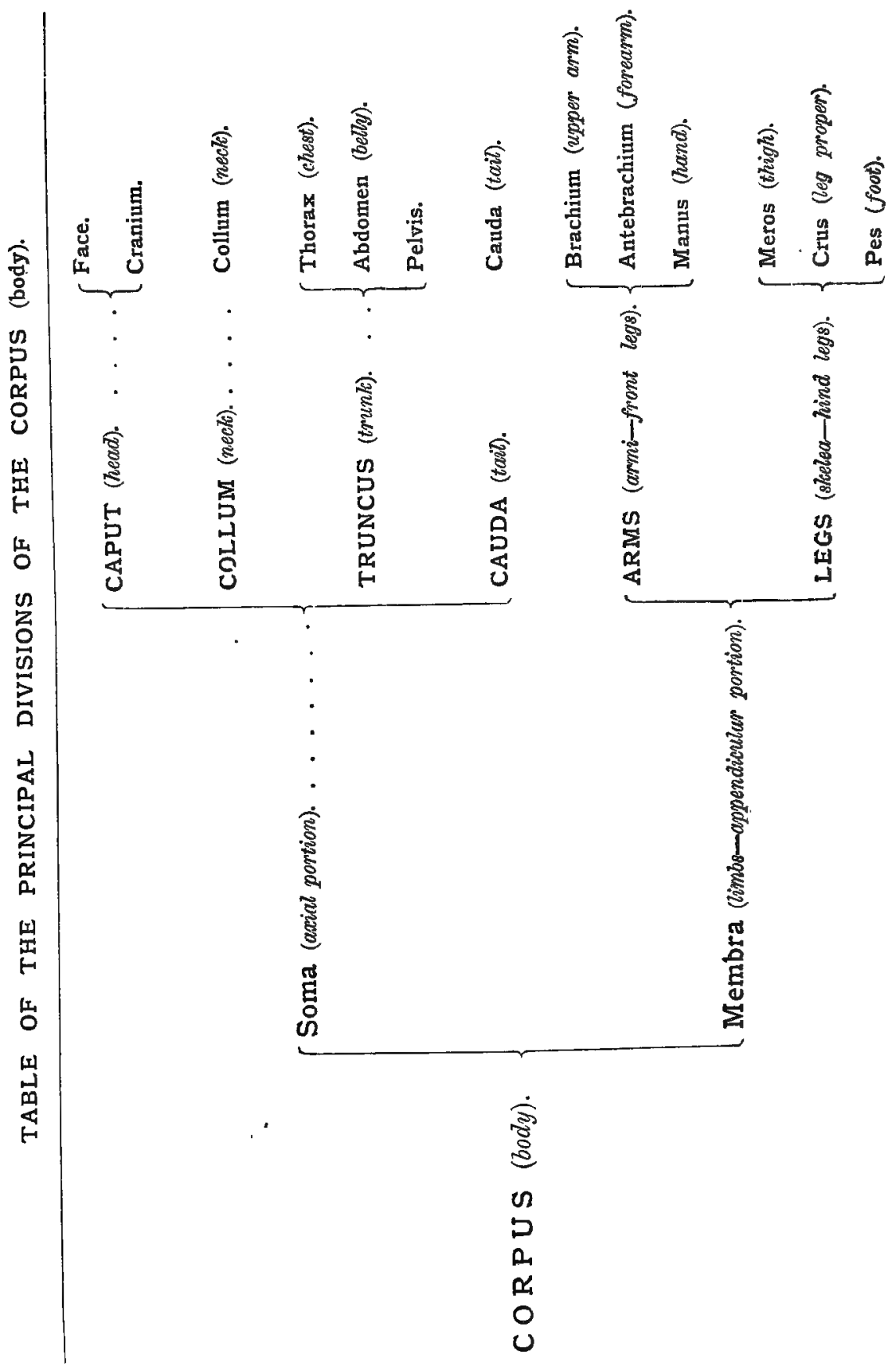


is not easy, with most Mammals, to replace them in the normal position. It may, however, be readily accomplished with an orang, and without much difficulty with the arms of a cat or a child.

The student may exemplify the normal position of the arms approximately by getting upon all-fours, placing the palms upon the floor at either side, with the fingers pointed laterad; the convexities of the elbows should then be made to look dorsad and laterad, upward and outward. If now the arms be extended laterad to their utmost length, still keeping the palms against the floor, and the elbows away from it, the limbs will have nearly the position shown in figures 6 and $\%$.

§ 81. Limb Segments. -With the cat, as with most air-breathing Vertebrates, each limb presents at least three divisions or segmenta, connected with each other and with the soma by three arthra (joints or articulations).

These segments and arthra are thus proximal, intermediate, and distal. As shown upon the right of the diagram, the proximal segments are brachium (upper arm) and meros (thigh). The intermediate segments are antebrachium (forearm) and crus (leg proper). 'The distal segments are the manus (hand) and pes (foot).

The entire limbs are joined with the trunk by the shoulder and hip joints, while the elbow and knee intervene between the brachium and antebrachium, the meros and crus, and the wrist and ankle between the antebrachium and manus, the crus and pes respectively.

The technical names for the arthra placed in parenthesis are those which were proposed in 1873 by the senior author $(\mathbf{1 0}, 18)$; but as they have not been generally adopted they are not insisted upon here.

Each manus and pes also presents a threefold division, carpus, metacarpus, and digits (thumb and fingers); tarsus, metatarsus, and dactyls (toes).

The distinctive names for the digits and dactyls are placed under them. The primus (great toe) is represented by a dotted line, in consideration of its absence from the cat.

\$ 82. Limb Bones.-On the left side of the diagram are shown the bones corresponding to the segments already enumerated.

The proximal segments have each a single bone, the fumerus and femur, which are represented as subcylindrical, with enlarged extremities.

The intermediate segments have each two bones, ulna and radius, tibia and fibula, which, in the normal positjon of the limbs, lie side by side, the radius and tibia on the cephalic aspect of the limbs, and the ulna and fibula on the caudal. These bones are likerwise subcylindrical, but the ulna and tibia are larger at their proximal ends, while the radius and fibula increase more or less distad. The patella (knee-pan) and the olecranon and other special features are not shown. 
\$ 83. Marsh has proposed (1) to apply general names to the corresponding bones of the arm and leg. Thus, the bones of the proximal segments are the Ossa propodialia; the radius and ulna, the tibia and fibula, constitute the epipodialia; the bones of the carpus and tarsus are mesopudialia; the metacarpalin and metatarsalia are-as indeed they have previously been called-the metapodialia, and the old term phalanges is retained for the bones of the digits and dactyls.

$\S 84$. Carpus and Tarsus.-The carpatia (bones of the carpus), and the tarsalia (bones of the tarsus), are variously interpreted by different writers; see Balfour, A, II, 508.

'The following general description, based chiefly upon the researches of Gegenbaur, is given by Huxley, A, 31 :-

"There is reason to believe that, when least modified, the carpus and the tarsus are composed of skeletal elements which are alike in number and arrangement.

"One of these, primitively situated in the centre of the carpus or tarsus, is termed the centrale; on the distal side of this are fre carpalia, or tarsalia, which articulate with the several metacarpal or metatarsal bones; while, on its proximal side are three bones-one radiale or tibiale, articulating with the radius or tibia; one ulnare or fibulare, with the ulna or fibula; and one intermedium, situated between the foregoing.

"Carpal and tarsal bones or cartilages, thus disposed, are to be met with in some Amphibia and Cholonia, but, commonly, the typical arrangement is disturbed by the suppression of some of these elements, or their coalescence with one another.

"Thus, in the carpus of man, the radiale, intermedium, and ulnare are represented by the scaphoides, lunare, and cuneiforme respectively. The pisiforme is a sesamoid bone, developed in the tendon of the $M$. flexor carpi ulnaris, which has nothing to do with the primitive carpus. The centrale is not represented in a distinct shape, having probably coalesced with one of the other elements of the carpus. The fourth and fifth carpalia have coalesced, and form the single urciforme. See $\$ 421$.

"In the tarsus of man the astragalus represents the coalesced tibiale and intermedium; the calcaneum the fibulare. The naviculare (scaphoides of Anthropotomy) is the centrale. Like the corresponding bones in the carpus, the fourth and fifth tarsalia have coalesced to form the cuboides."

$\$ 85$. In the cat, so far as we can judge from the figures and statements of Flower (36, 138), and Mivart (B, 96, Fig. 60), and from our own observations, the condition of things is as follows :-

In the tarsus, aside from peculiarities of shape, the tarsalia and their connections are as in man.

In the carpus, the same is the case, excepting that the scapho-lunare represents not only the scaphoides and the lunare-the radiale and intermedium of the primitive carpus-but also the centrale. Flower found 
this element distinct in a dog; the senior author has observed (19, 301, Fig. 1,) it as a separate center of ossification in a young lion, shown in Figure 47; and it is apparently shown, though not alluded to, by Mivart, (B, Fig. 60.)

There are many interesting and important questions connected with the composition of the carpus and tarsus of different Vertebrates, and those interested in the subject may consult Gegenbaur (Lankester), A, 4r9-481, 487, 488, and the papers of that anatomist, Morse (18 and $\boldsymbol{1}$ ) Marsh (1), and Wilder (16).

$\S 86$. The metapodials are comparatively simple elements. In the diagram, the pollical (first) metacarpal is made shorter than the rest, as is the case in the cat, and the primal (first) metatarsal is represented as a slight rudiment, the renainder of it and the whole dactyl being absent, and hence shown by dotted lines.

The ordinary digits and dactyls have each three phalangeal segments, proximal, internediate, and distal. The pollex has but two, and in those Mammals which have a primus, this usually consists of but two phalanges.

$\S 87$. This peculiarity of the pollex and primus has always constituted a main argument in favor of the prevailing view as to the correspondence of the limbs with each other-intermembral homology.

According to this view, the cephalic borders of the arm and leg correspond, morphically as well as telically; the radius is the homologue of the tibia, and the ulna of the fibula; and the pollex is both the homologue and the analogue of the primus.

This, the syntropical idea of intermembral homologies, has been adopted, in one form or another, by nearly all anatomists.

The other idea, that of untitropy, has been advocated or accepted by a much smaller number, including the elder Agassiz, Coues, Dana, Foltz, Wyman, and the senior author.

According to this view, the two ends of the soma, and thus the cephalic and caudal aspects of the limbs, are reversed or symmetrical repetitions of one another, as are the cor. responding organs upon the right and left sides. The radius thus becomes the homologue of the fibula, and the tibia of the ulna, while the pollex represents the quintus, and the minimus the primus.

Fully recognizing the apparent objections to this view, we nevertheless believe it to be correct, and would refer those interested in the sulject to the paper (10) by the senior author, where these objections are discussed and the opinions of other writers stated, with a List of Works and Papers treating of the general question.

§8. Enumeration of Parts in a Series.-As has been stated ( $\$ 30$, 79), two or more similar parts which form a series are enumerated in order, beginning with the one rearest the head, or the meson, or the proximal end of the more comprehensive part of which they are subdivisions.

On the diagram (Fig. 6) are given some illustrations of this method. The rertebræ have been referred to already. Of the digits and dactyls, the most cephalic in each series may be designated as the first; but it is evident that this might cause confusion in the case of animals having only four or a less number of fingers or toes. 
It is often desirable to describe the place of origin or insertion of muscles as from or upon a given fraction of the entire length of a long bone. When the area extends over a third of the whole length the thirds would be designated usually as proximal, middle or distal;' but when fourths or smaller subdivisions are employed, they may be designated as first, second, etc., as shown upon the femur in Fig. 6.

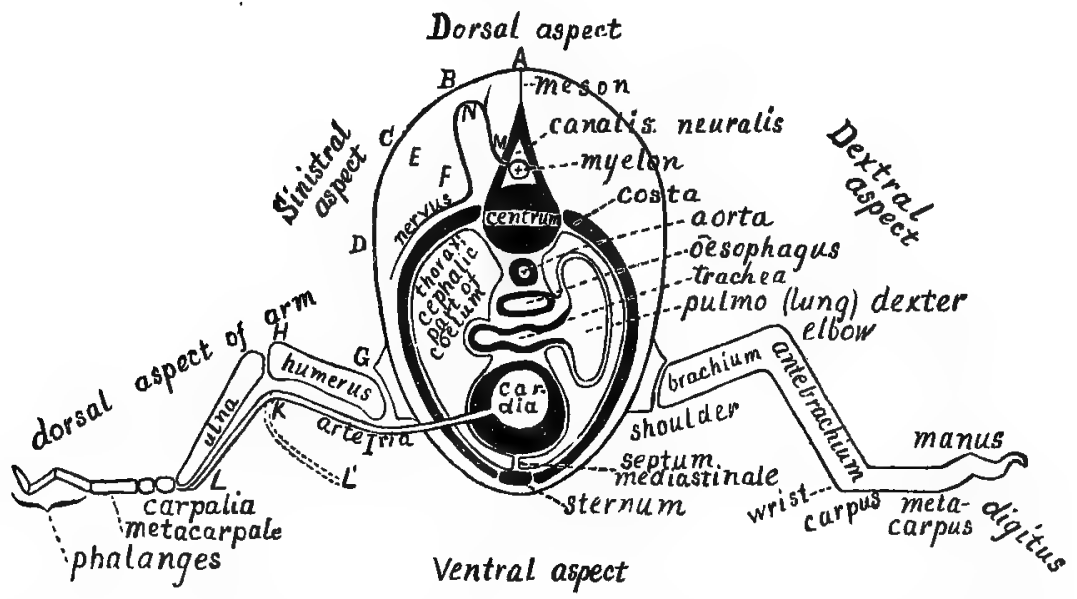

Fig. 7.-Diagram of an Ideal Tranghotion of the Thorax of the Cat, with the Arms in an Approximately Normal Position, and showing the Location of the Principal Viscera.

\$ 89. In Fig. 7, the cut surface is viewed from the caudal aspect, so that the right and left parts are as in the other diagram (Fig. 6).

As a whole, the body is symmetrical, the two halves being reversed repetitions of one another on opposite sides of the meson.

No definite separation exists between the dorsal and the ventral regions. We may, however, speak of the dorsal and the ventral aspects, and the vertebral column, or main axis of the soma, intervenes between the dorsal cavity, or Canatis neuralis, and the ventral cavity, the calum, whose more cephalic division or thorax is bere transected.

The Canalis neuralis contains the myelon, and the colum the organs of organic life, the thoracic, abdominal and pelvic viscera.

The following parts and organs are mesal or approximately so, at least in the embryo: nose, tongue, myelon, centra vertebrarum, aorta, osophagus, trachea, heart, sternum.

The following are in pairs: eyes, ears, kidneys, costce, costicartilagines (costal cartilages), pulmones (lungs), pleurce, limbs.

The right Iung is shown as a single and simple sack, communicating 
with the trachea. The left lung is reduced to give place for the names of the carity.

Each pleura is seen to be a closed sack, which lines the corresponding side of the thorax to form the ectal or parietal lamina, and is reflected upon the viscera to form the ental or visceral lamina. Between the heart and the sternum, on the meson, the two pleuræ are in contact, and form the septum mediastinale.

For the sake of simplicity, the pericardium is omitted, and the heart shown as a single cavity with regular walls. The single vessel emerging from the heart will be referred to in $\$ 91$.

The spaces on either side, between the skin and the osseous walls of the coelum and Canalis neuralis are occnpied by the muscles, etc.

The arms have their elbows directed dorsad, and the palms ventrad. The caudal aspect is seen, the bones including the ulna and the ulnare (unciforme), and the minimal metacarpal and phalanges.

\$ 90. Relative Positions. - The letters A-L are introduced into the diagram for the sake of illustrating certain common cases of designation of relative position.

The point $A$ is at the meson on the dorsal aspect of the body; its position may be described as dorsimesal. B, C, and D are lateral in position, or, to be more specific, sinistral.

Relatively, $\mathrm{B}$ is laterad of $\mathrm{A}$, while $\mathrm{C}$ is dorsad of $\mathrm{D}$. Since they are upon a curved surface, B may be described as either dorsad or mesad of $\mathrm{C}$; while $\mathrm{C}$ may be said to lie either ventrad or laterad of $\mathrm{B}$. Which expression should be employed would depend upon the context; in either case, practically, there is no room for ambiguity.

Ectal and Ental._Of the three planes represented by C, E, F, the former is relatively ectal, the last is ental, and the third intermediate.

Proximal and Distal. $-\mathrm{G}$ is at the proximal end of the humerus, and $\mathrm{H}$ at the distal end. $\mathrm{G}$ is thus proximad of $\mathrm{H}$, and $\mathrm{H}$ distad of $\mathrm{G}$.

Central and Peripheral.-On the artery, I is centrad of $\mathrm{K}$, and $\mathrm{K}$ is peripheral in comparison with $\mathrm{I}$, but centrad of $\mathrm{L}$.

On the nerve which leaves the myelon, $\mathrm{M}$ and $\mathrm{N}$ are relatively central and peripheral.

§ 91. Use of some Terms of Relative Position in a Physiological Sense.-Central and peripheral, and sometimes other terms are employed in a physiological rather than in a strictly topographical sense.

For example, on the norve the ventral curve is really, that is topographically, nearer the myelon and the meson than $\mathrm{N}$; but $\mathrm{N}$ would nevertheless commonly be described as centrad of the curve.

A striking case of this sort is furnished by the recurrent laryngeal branch of the $N$. vagus. In a part of its course, it curves around one of the great arteries near the heart, and then passes cephalad to the larynx. Now the larynx is much nearer the brain, where 
the $N$. vagus arises, than is the heart; topographically, therefore, the termination of the nerve is nearer the brain than part of its trunk; yet the termination would be described as the peripheral portion.

On the artery shown in Fig. \%, ventrad of the bones of the left arm, K is peripherad of $\mathrm{I}$, and $\mathrm{L}$ of $\mathrm{K}$. If the arm should be so flexed as to bring the artery into the position of the dotted lines, $\mathrm{L}^{\prime}$ would be topographically nearer the heart than $\mathbf{K}$, but would nevertheless be a peripheral point on the vessel.

A somewhat similar case is offered by the abdominal portion of the alimentary canal as shown on Fig. 6 . In the strict topographical sense, the ccecum and contiguous portion of the small intestine lie caudad of the colon. In some cases, a description would refer to this fact, but it would be nevertheless understood that, physiologically and in respect to the course of the contents of the canal, the crecum is intermediate between the small intestine and the colon, and is therefore caudad of the one and cephalad of the other. The relation in the one case is merely of contiguity; in the other, of structural and functional continuity.

\section{V.-THE SLIP-SYSTEM OF NOTES.}

\$ 92. The following suggestions as to "notes" are here introduced not because, like the foregoing matters, they render what follows more intelligible or available, but because, taking for granted that none will have got so far in the volume without the intention to do some serious scientific or literary work, we desire to put them in possession of a method which has proved most useful to us, and which we shall be glad to have adopted by our readers even if their only employment of it is in recording criticisms of our statements and ideas.

\$93. The essential feature of a "slip-system" is the use of separate slips of uniform and convenient size.

Accessory features concern the precise size and form of the slips, the way of writing upon them, and the manner of their filing and distribution for reference.

\$ 94. After constant use of the slips for fifteen and seven years respectively, we make the following specific recommendations :-

\$ 95. The Slips. - Excluding those used in the Catalogues of Libraries and Museums (which will be referred to fartber on) the note-slips should be of unruled paper; white, blue, light brown or yellow in color ; slightly sized, so as to take either the pencil or the pen ; moderately stiff, but not. thick; and of the size of the U.S. Postal Card, at present $13 \times 7.7 \mathrm{~cm}$.

\$96. Making Notes.-Some of these slips should be carried in the pocket at all times, preferably in the pocket-book, which is usually large enough for folded lettcrs. 
Temporary memoranda may be made across the slip, but all other notes should be written lengthwise, and preferably on one side only.

$\$ 97$. Slip notes are of the following kinds: museum catalogues; library catalogues ; references ; extracts ; clippings ; statements of observations or ideas, original or otherwise, with or without drawings.

§98. Library Catalogues.-The titles of books and papers should comprise at least the following data: the author's name and surname or initials ; the title and subtitle of the book or paper. With a book, the edition, size, number of pages and illustrations ; the place and date of publication. With a paper, the Periodical in which it was first published, dates of presentation and publication, the volume, part, pages and illustrations. With botll, references to Translations and Abstracts.

The foregoing data are entered in various orders, and with different kinds of abbreviation. Those who desire to adopt some standard method of writing titles should consult the "Catalogue of Scientific Papers" published by the Royal Society of London, the publications of "The American Library Association," and the "List of Periodicals taken by the Public Libraries near Boston."

A. One of the valuable suggestions of the Library Association is that the more com. mon given names should always be indicated by the initial, this initial being distinguished from the same letters used elsewhere by a colon following: For example, E. might stand for Edwin, Eghert, Edmund, etc., but E: would always signify Edward.

B. Another suggestion, that the sizes of books should be indicated not by numbers but by the initials of the words signified by the numbers, as 0 . for octavo, etc., is carried out in the "List" just mentioned, and is made more available by the use of the "Book Size Rule," provided by the Readers and Writers Economy Company, being a metric rule, $30 \mathrm{~cm}$. (1 ft.) long; this is also a convenient desk rule.

\$ 99. Catalogue Data.-On the face of the Museum Catalogue Card should be noted the following: Muscum number, original number, class (of animals), series (of specimens or preparations), genus, species, common name, locality, sex, age, dates of collection, death, and reception (which do not always coincide). This should leare the lower half of the face for a brief description of the preparation; purpose for which it was made; reference to any original list, to publications, etc.

On the reverse of the slip should be, after a repetition of the museum number: the original weight of the brain, and of the body, and the ratio in decimals; the present (usually alcoholic) weight of the brain and body; the capacity (of an inflated preparation); the length from vertex to anus (of an entire example); by whom collected; when and by whom prepared; donor or seller; his address; original cost, expressage, etc., hours' work; estimated present value; museum numbers of other specimens from the same indrvidual; numbers of other preparations from members of the same litter; numbers of other specimens with which this may be instructively compared. 
With care in the arrangement and abbreviation of these points, all may be entered, together with others not here specified. We have not yet fully determined upon the best form for the Catalogue slip, and hope others will offer the results of their experience.

For library and museum catalogues, the slips should be of heavier and stiffer paper than for ordinary notes, and the surface should be well-sized, since ink is commonly employed.

$\S 100$. References.-The slips may be used for brief references to works, papers, persons, addresses, localities, museums, etc., constituting, in fact, permanent memoranda, which may be filed with more elaborate or extended materials upon the same subjects.

$\S 101$. Extracts. - Upon one side of the slip may be written from 40 to 50 words, either by hand, or with the type-writer when the lines are at medium distance, $1 \mathrm{~cm}$. apart. At short distance, the number is just doubled, and, if necessary, both sides may be written on, or two or more slips may be used, or a sheet may be used, and then folded to the slip size.

\$102. Clippings.-Clippings from periodicals and newspapers may often be accommodated upon the slip. When no longer than the slip is wide, they may be pasted at either end, with the lines always lengthwise of the slip. When the length is greater than the width of the slip, the columns, if narrow, may be pasted side by side; but usually so much as exceeds the width must be pasted on the remaining space so that the lines run across the slip. By thus covering both sides, a single slip may receive an entire column of "The Medical Record," or "Science;" more than half a column of "The Nation," and nearly half a column of the "New York Daily Tribune."

Even when unaccompanied by the signature of some well-established authority, such olippings serve as memoranda which may aid in looking up the matter farther, and as more or less valuable confirmatory evidence.

$\S 103$. Notes Proper.-The most common and most important use of the slips is as a ready and convenient vehicle for the many kinds of information which the scientific or literary worker desires to record and to use.

The Slip is Always at Hand.-It may be written upon without parade, and under almost any circumstances, whether sitting at the table, or riding in the cars, or even on horseback; whether engaged in regular work, or conversing with friends, or even at night and in the darkness. Under all these conditions, more or less favorable, the essence or "pith" of a fact or idea may be recorded, though sometimes in a shape quite unintelligible to others and well-nigh so to the writer, unless an early opportunity is taken for putting it in better form.

$\S 104$. Accumulation and Elimination of Slips.-The beginner's object is to accumulate the brief records of what he has learned, but is not sure of remembering when wanted.

After the first year, however, many of the things thus recorded become familiar as the alphabet, and much also that is supposed true to-day may be 
disproved to-morrow, or superseded by more accurate descriptions, more perfect drawings, and more logical discussions.

With the ordinary note-book, or Index Rerum, all these untruths, half-truths, or truisms must forever remain where they were placed. From being merely superfluous, they soon become burdensome, then confusing, and finally a source of loss and exasperation.

Not so the slip; the instant its usefulness is at an end, into the wastebasket it goes, making room for more desirable successors.

§ 105. Arrangement and Storage of Notes.-After trying many different plans, we make the following practical suggestions :-

- \$106. Slip-Portfolios. *-The slip-portfolio is for bolding the notes upon a given subject.

It is made from a piece of stiff Manilla paper, $16 \times 13 \mathrm{~cm}$., and folded over a pencil so that the folded edge is left rounded. The slips are introduced, the title of the subject is written along the top, and the whole is bound together by a rubber band $7 \mathrm{~cm}$. Iong by $1 \mathrm{~mm}$. thick, like No. 372 of the Price-list of the R. and W. Ec. Co.

Such a portfolio will hold 50 slips, but usually when there are more than 25 they should be subdivided.

$\$ 10 \%$ Subdivision of Notes.-Let us suppose that the student is collecting information respecting the heart of the cat. While his notes are few, all may be contained in a single portfolio. As they multiply, they naturally fall into four categories relating respectively to the structure of the organ, its functions, its development, and the titles of works and papers treating of it. As the notes increase under each of these heads, anatomical notes may be divided naturally into sets relating respectively to the appearances which are visible to the eye, those which require the aid of the microscope, and those which involve special kinds of manipulntion, as boiling, etc., in order to display the arrangement of the fibers. The macroscopic anatomy may refer to the auricles, the vontricles or the valves, and each of these headings may be still further subdivided.

We see, therefore, that without the subdivision of the notes upon five quite extensive topics, the heart might require the use of a dozen separate portfolios. Since all of these concern the heart, they may all be bound together by a stronger band, say No. $385,8 \mathrm{~cm}$. long and $3 \mathrm{~mm}$. wide; or, they might be bound in two sets, the one including all the divisions of the gross anatomy, and the other the rest.

Such a plan of subdivision is not only convenient for reference, but furnishes an exercise in Natural Classification; see p. $49, \S 108$.

$\S 109$. Distribution.- Since it is not always convenient to assign the

* The emplogment of the portfolios was suggested to us by Wm. Nichols, M.D., of Boston, Mass. 


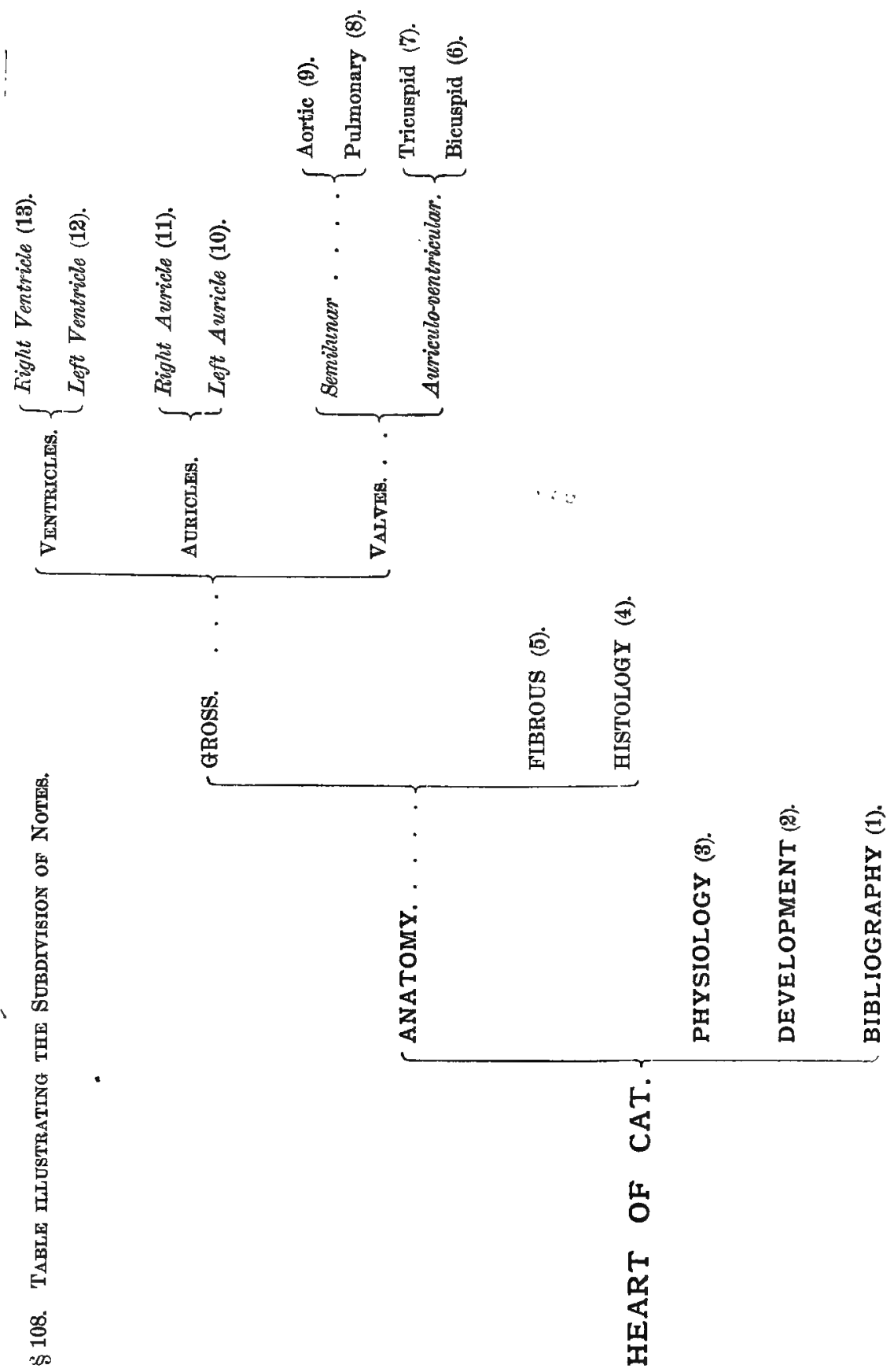


notes to their proper places at the time they are made, it is well to have an extra portfolio, which, in this case, would be marked, "Heart of Cat; Distribution."

When its contents are to be distributed, the other portfolios are arranged upon the table in some natural order; the removal of their bands will permit the upspringing of the side so as to expose the heading, and facilitate the introduction of slips.

\$110. Slip-box.-This should be of stout tin, preferably Japanned. The inside measures of length and width should be $22 \mathrm{~cm} . \times 14 \mathrm{~cm}$. (about $9 \times 5.5$ in.), which is nearly the size of the ordinary sheet of note paper.

The depth is less simply stated. If the box has a folding lid, like the "Japanned tray, postal size," here figured (Fig. 8), a depth of $8 \mathrm{~cm}$. will be needed. But the scientific student has such abundant need for shallow trays, that he will usually find it more conrenient to have, in place of the folding lid, a single cover, $2 \mathrm{~cm}$. deep. This should overlap the edge of the box for $1 \mathrm{~cm}$., resting on a welt. The depth of the box itself will then be only $7 \mathrm{~cm}$.

If the slips are kept in envelopes instead of portfolios, the box and corer together should have a depth of $9 \mathrm{~cm}$. The Japanned tray, "standard size," is only $5 \mathrm{~cm}$. deep, and if the "postal size" is ordered, care should be taken that it is of the actual width required for the postal card, since some the of so-called "postal slips" are only $12.5 \mathrm{~cm}$. long.

The free edges of the box and cover should. be turned, and the latter should be "wired."

§111. Cost of Materials. - The tin box may be had for from 50 cents to $\$ 1.00$. A tin cash-box, with lock, costs $\$ 1.50$. The Japanned tray, postal size, with slips, blocks and guides, is $\$ 2.65$.

"Roll Manilla," 40 inches wide, is $8 \mathrm{cts}$. per yard; including the cutting, the slipfolios cost at the rate of 10 cts. for 25-35.

The bands are rated at $\$ 2.20$ per M. for No. 372 , and $\$ 5.85$ for No. 385 . By the hun. dred they cost proportionally a little more.

The slips may be cut by hand, but it is usually cheaper to have the cutting done by the stationer. They cost from $50 \mathrm{cts}$. to $\$ 1.00$ per $M$.

\$112. Other Methods.--The slips may be placed in envelopes, sealed at the side, and with the right end cut off. If this plan is adopted, it is true economy to nse stout envelopes which will not readily fray and tear. Cloth-lined envelopes of the proper width, $9 \mathrm{~cm}$. (three and one-half inches), may be had for about $\$ 2.50$ per hundred, and are practically indestructible.

Another way is to write the heading upon a card the size of the slips, and put

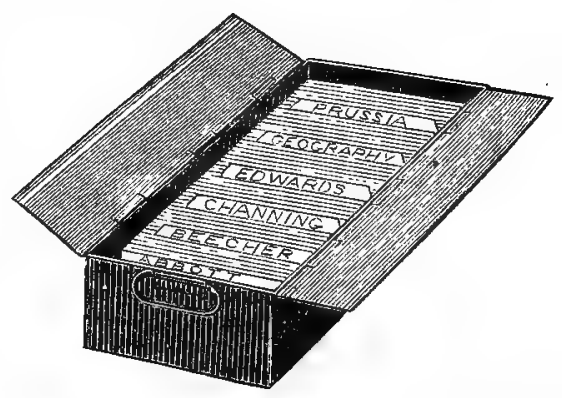

Fig. 8. -The Japanned Tray, for Cata. LOGUe SLIPS. READERS AND WRITERS Economy Company. 
the band over all. Finally, there is the method exemplified by the accompanying figure (Fig. 8), which seems, however, better adapted for museum and library catalogues.

\$113. Using the Slips.-For ordinary reference, the portfolio is held in the left hand, the band removed, and the slips turned until the desired one is found.

As a basis for manuscript or lecture-notes, they may be arranged in the proper order by spreading them out upon the table; more or less condensation and elimination will theu occur. The selected or condensed notes may be used as lecture-notes, or their substance transcribed to sheets.

$\$ 114$. For carefully prepared manuscript, the following stages of composition are recommended :-

1. Slips, hand-written. 2. Slips, selected, condensed, and, if possible, type-written. 3. Sheets, hand or type-written, into which the type-written slips may sometimes be incorporated by pasting. 4. Sheets, carefully typewritten, a corrected copy of the first set.

Manuscript so prepared, especially if in addition an interval of at least one week elapses between the first and the second copies, will usually need few changes in proof.

\$ 115. Sheet-Portfolios.-The sheets referred to are of the standard note size, about $21 \times 12.5 \mathrm{~cm}$. Such sheets and drawings of similar size may be conveniently kept in sheet-portfolios of the Manilla paper, double the size of the sheet, say about $21 \times 27 \mathrm{~cm}$.

Portfolios of sheets, or the sheets themselves, may be conveniently kept in the "Pigeon Hole Case," "pamphlet size," supplied at $\$ 1.50$ by the R. \& W. Ec. Co. The case shown in the figure is known as the "Billet size." For " note sheets," there are only two tiers of holes.

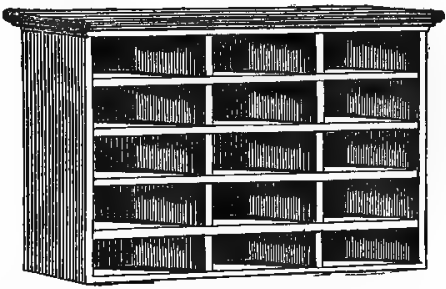

Fig. 9.-UNIFORM, INTERCHANGEable Pigeon Hole Case, Billet SIZE. READERS \& WRITERs ECoNoMY Co., (A.)

\$116; Miscellaneous Suggestions.-A. By writing the portfolio headings with pencil, they may be changed as desired, and to any extent. Even if written with ink or pfinted, however, each portfolio presents four surfaces which may be used in turn.

\$117. B. The type-nriter or calligraph can be adjusted to any width of paper. Of course, the longer the line the fewer the shiftings; but the narrower sheet is more easily manipulated, especially when changes or divisions are required, and it is often a real advantage to be able, without delay, to write alternately upon the sheets and the slips.

§118. C. While assorting a large number of slips for which portfolios have not been prepared, it is sometimes desirable to remove them all without mixing the separated piles. This may be accomplished, either by interposing a blank slip between the successive piles, or by placing them crosswise, and carrying a band over them diagonally. 
$\S 119$. Origin of the Slip-system.-It is probable that the slip-system has been devised independently by many individuals, and we are informed that Mr. Folsom, the Librarian of the Boston Athenæum, proposed the use of slips in Card Catalogues at the Librarian's Convention in New York in 185:. As a means of recording and arranging scientific information, however, the first published notice known to us is the following by the senior author (1\%), communicated to the Boston Society of Natural History, May 15, 1867:-

\$120. "It consists in the brief statement of facts, ideas, or references to books, written upon one side of a slip of paper equal to the sixtb part of a sheet of note paper.

"A few of these blanks are carried in the pocket, and advantage is thus taken of opportunities for recording and preserving information which the time, place, or state of mind would not permit to be written out in full, or which might be forgotten before a fitting opportunity should occur.

“These slips are distributed at leisure into envelopes bearing the title of the subject.

"By keeping the slips separate, it is evident that an indefinite subdivision of each general subject can be made by simply increasing the number of envelopes and redistributing the slips.

"The slips may be used either for simple reference, or, if in preparation of a lecture or communication, by arranging them on the table in any desired order, and transcribing parts of them in the form of notes; while for a written paper they serve to indicate the general order of discussing a subject.

"The method proposed makes sure of the essential fact or idea in a brief form, and the slips, being kept separate and of uniform size, may easily be arranged, carried and arranged, or rearranged in any order at any time."

This plan was presented in a lecture at the session of the Anderson School of Natural History on Penikese Island, in July 1873, and, according to a report in the N. Y. Tribune, "was almost universally and immediately adopted."

\section{\$121. Rules and Aphorisms of General Application.}

"In the order of Nature, doing comes before thinking; Art before Science"-Joseph Henry (Mayer, 1, 95).

"Personal familiarity alone makes knowledge alive."-Philip Gilbert Hamerton.

"Practice the utmost rigidity and thoroughness in research, without regard to time consumed, or value of results." - Henry James Clark (Pack$\operatorname{ard}, \boldsymbol{1})$.

“Lenteur dans la marche; aridité dans l'étude; solidité dans les principes; sûreté dans les résultats; ce sont là les attributs des sciences d’observation."-Bichat, A, i, p. v.

Correct methods are the keys of knowledge.

Whoever has learned how to work has taken a long step toward independence of teachers and books. 
"The method may determine the result."-Louis Agassiz.

"If researches take at the first step a wrong direction they diverge the farther from the truth the farther they are followed."-Gaboriau.

"It is often as if the truth were rather whispered than spoken by Nature."-Owen.

Accuracy is more to be desired than speed.

Books may be consulted in haste, but Nature demands deliberation.

Non-discrimination is no proof of identity

Ignorance of a specimen's locality may cause delay; an error respecting it may create confusion.

As is the locality to an individual, so is the individual to any of its parts.

"There is so close a solidarity between ourselves and the animal world that our inaccessible inward parts may be supplemented by theirs. ***A sheep's heart or lungs or eye must not be confounded with those of man; but so far as the comprehension of the elementary facts of the physiology of circulation and of respiration and of vision goes, the one furnishes the needed anatomical data as well as the other."-Huxley, 3.

"Carpenters and tailors do not learn their trades upon rosewood and cloth of gold."-Wilder, 2,8 -

"Felitomy should be the stepping-stone to anthropotomy."-Idem, $\mathbb{2}, 6$.

"No medical student should be allowed to dissect the human cadaver until he has familiarized himself with the anatomy of the cat."-Clevenger, $1,1$.

He who can skillfully dissect a cat will find little difficulty in dissecting a man.

"Fiat experimentum in corpore vili."

The softer the parts, the harder their study.

Soft parts are perishable.

Hurried dissection is rarely trustworthy.

The thorough examination of soft parts can be made only under one or more of the following conditions: A. Limitation of the inquiry; B. Abundance of material; C. Continuity of dissection; D. Co-operation of several dissectors ; E. Preservation of the parts.

Filth and bad odors cannot always be aroided, but their continuance is rarely necessary.

Accurate knowledge of a few things is better than vague ideas concerning many.

First expressions are rarely correct or perfect.

A description is perfect only when it enables one who has never seen the object to form a correct image of it. 


$$
\text { • }
$$




\section{ANATOMICAL TECHNOLOGY.}

\section{INTRODUCTORY.}

$\S 123$. This work treats mainly of the Gross Anatomy of certain portions of the Domestic Cat, and of the Methods of their Examination and Preservation.

Reasons for Selection and Preference.-There shall be given here a condensed statement of the reasons for selecting the Cat, for giving almost exclusive attention to the Cephalic region of the body, and for devoting to the Brain and other Viscera a relatively large amount of space.

$\S 124$. For Selecting the Cat.-Three things are to be learned by the student of Anatomy and Physiology, whether Human, Veterinary or Comparative : methods of manipulation; fundamental facts of structure and function; and terms of expression.

Most of the methods might be learned upon any mammal, but convenience and economy are consulted by the use of one which is at the same time widely distributed, common, easily kept in confinement, and of moderate size so as to be readily manipulated and cheaply preserved.

Methods cannot be practised without some knowledge of the nature, location, and uses of the parts concerned; and the record or communication of results involves the use of terms; hence there is effected a substantial saving of time, mental effort and expense by employing, in the acquisition of methods, a form anatomically and physiologically comparable with those which the student is preparing himself to investigate.

The adult human subject is inconveniently large, not always easy to obtain, and often expensive when all things are considered. The immature jndividuals (still-births) which 
may sometimes be had, lack the firmness of texture which is desirable for the examination of certain parts.

The veterinarian is especially interested in the horae and the cow; but these are too large and costly for elementary work; in less degree, the sheep and the pig are open to the same objection.

The needs of the comparative anatomist were well expressed in the course of a "Laboratory talk," by the late Prof. Jeffries Wyman, as recorded by the senior author $(2,5):-$

"In commenting upon the unsatisfactory vature of some published notes of dissections, he said : 'Much of this is due to the lack of suitable standards for comparison. The human body is not a suitable standard for the lower vertebrates. The best thing any anatomist can do is to prepare complete accounts of the structure of a few forms, each typical of some large group. The fowl could represent the birds, and the cat the mammals. The cat's anatomy should be done first, because it would also serve as an introduction to Human Anatomy, and thus become an important aid to Medical Education." "

Theoretically, among known forms, the wants of the comparative anatomist might be more fully met by the more generalized opossum or raccoon. The tiny musk-deer of Java could be more easily dissected and preserved than most of our hoofed quadrupeds; while a medium sized monkey, especially after some confinement, is the best possible substitute for the human subject.

Unfortunately, however, none of these animals is sufficiently abundant in all parts of the civilized world, and we must seleet such as are to be had. The rat is certainly too small for most purposes, and not easy to obtain unmutilated. The rabbit, like the rat, belongs to a group of peculiar mammals, the Rodents, with no special advantages in respect to either human or veterinary anatomy. Dogs vary greatly in size and mode of life, so that no one of the many breeds can be fairly spoken of as the dog, and although, as stated by the senior author $(\mathbf{2 1}, 308)$, the pectoral muscles present less variation than might be expected, few comparisons have been made of the other soft parts excepting the brain, where, as regards the disposition of the cerebral fissures, the variation is considerable and perplexing (Wilder, 12, 242).

So far as we can judge from published records and our own observations, cats are much less subject to variation than dogs. They are both walkers and climbers, and therefore comparable with the domestic quadrupeds on the one hand, and with the monkeys, and through them with man, on the other. They are common in most civilized lands, fertile, easily reared, and may be kept in confinement, even in considerable numbers, without difficulty. They quickly succumb to angsthetics, and their size is such as to facilitate both dissection and preservation in alcohol. They are quiet, while dogs are noisy.

$\S 125$. Of a less practical nature, yet well worth mention, is the remarkable combination in the cat's organization, of delicacy, agility and strength; a combination which seems almost perfectly adapted to the prolongation of individual life and the perpetuation of the species. Indeed, as well remarked by Mivart (B, 493,) "Something may be said in favor of cats being the highest of mammals, if man is considered merely in his animal capacity, in which alone he can be brought into comparison with other organisms." See also Minot, $\mathbf{1}$, and Dana, $1 \mathbf{2 3}, 160$.

This idea will be acceptable to those who prefer the purely teleological aspect of Nature. Others, however, may find ample ground for discussion respecting the universal operation of the doctrine of Final Causes, at least in the present state of our knowledge. Teleology has not yet explained the existence of the insignificant clavicle, the rudimentary primal metacarpal, the little pocket at the border of the ear, or the cusps on the deciduous mandibular canine teeth.

$\$ 126$. In short, while freely conceding the advantages which might be presented by 
other forms, especially if it were practicable to supply a separate standard for the anthropotomist, the zoologist and the veterinarian, we nevertheless believe that even then these three might be advantageously compared with the cat as a fourth and intermediate form, and that, when all points are considered-size, habit, distribution, physical endowment and zoolugical position-the cat should be chosen over all others, both as a single standard for comparison, and as a subject of elementary and preliminary anatomical and physiological work.

$\S 127$. Nor is this choice wholly without precedent. It is true that descriptive and practical works upon Mammals have been more often hased upon others than the cat. For obvious reasons, the horse has been the subject of many publications ; the rabbit is selected by Krause (A), the rabbit and the dog by Foster and Langley (A), the rat by Rolleston (A) and Morrell (A), while Coues has described (47) the skeleton and muscles of the opossum.

Yet Straus-Durckheim devoted a magnificent monograph to the bones, ligaments and muscles of the cat ; and reduced copies of his outline plates, with a translation of the "Explanations," have been published by our colleagne, Prof. Henry S. Williams. The skeleton is delineated and named, in connection with those of the Duck and the Codfisb, by E. Tulley Newton (A), and as the work was "prepared under the supervision of Prof. Huxley," the usefulness of the cat has probably been appreciated by that zoologist. Finally, the recent volume by Mivart (B) purports to describe the entire structure of the cat, although no practical directions are given, and, according to notices in The Nation for Jnne 2, 1881, and in Science, and The Athenceum for June 4, the author seems to have made somewhat frequent and wholly unspecified substitutions of human anatomy for that of the cat.

The junior author las published two papers $(1,3)$ upon the anatomy of the cat ; and the desire for a complete account of its brain, expressed by the senior author in 1873 $(11,229)$, has been recently, in part, fulfilled in the papers numbered $2,3,4,5,6$, $\%, 8,9,12,13$ and 14 .

\section{$\S 128$. Reasons for Treating of only Part of the Body.-This} work is primarily an explanation of methods, and the descriptions of organs are mainly in illustration thereof.

The account of only forty muscles covers an equal number of pages. To devote a proportionate space to the 150 or more other muscles, and to all the arteries, veins and nerves would swell the volume to undesirable dimensions. Some selection was therefore necessary.

Of the two general regions of the body, the cephalic is certainly more familiar to most l,ersons, more interesting, more employed in art, more often used in experiment, and more subject to injury and disease. To obtain and prepare the heart and the brain involves some manipulation of the thorax and head.

The arm of the cat is more complete than the leg, since it has all five of the digits, and presents the interesting and important provision for the rotation of one of the epipodial bones about the other.

Notwithstanding our doubts respecting the homologies of the $M$. clavo-trapezius and some of the antebrachial muscles, the myology of the arm is in a more satisfactory state than that of the leg, where the great "adductors" are likely to puzzle anatomists for some time to come.

In short, the same practical considerations which have led most anatomists to describe the muscles of the antebrachium with more fullness than those of the back, have induced us to select the arm rather than the leg for the more detailed descriptions. 
8129. Reasons for Giving Unusual Prominence to the Viscera.-It is stated by Macalister (British Association Report, 1877, p. 94), that "at least 600 bodies are annually examined in the dissecting rooms of Great Britain and Ireland;" yet how few are the published observations respecting the characters, constant or variable, of any parts other than the bones and the muscles. In this country the case is still worse, and we have no journal especially devoted to Anatomy and Physiology.

This comparative neglect of the internal organs is probably due, in great part, to the fact that, as remarked by the enior author, $(2,9)$, the average " human subject is rarely available for the study of viscera. Usually some of them are diseased. The beart is apt to be full of injection-mass. The brain and abdominal viscera decay so rapidly that some of their important features are soon obliterated; and when, as is customary, their removal is postponed until after the examination of the overlying muscles, their condition is often such as to render them unfit for preservation. How many students have gained a good view of the thoracic duct, or the sympathetic ganglia? How often has it been ascertained whether a subject has two pancreatic ducts or only one? Is a satisfictory examination of the brain made by the majority of dissectors?" In short, do not the larger number of medical students regard the viscera, even the heart, as simply a mass of ill smelling material, difficult to examine, not very instructive, and worthy only of the waste pail?

Whatever be the cause of this inattention to the viscera, the fact leads us to give ample space and illustration to these organs as they exist in an animal peculiarly adapted for their study and preservation. Moreover, although a predilection for surgery prevails among medical students, most of the diseases they will have to treat affect the viscera rather than the skeleton and its muscles; physiology, too, is largely splanchnological, and a correct knowledge of the brain is searly more desirable in connection with the progress of rational Psychology.

Lastly, notwithstanding the general preference of systematic zoologists for skeletal characters-a preference certainly based upon convenience, and, in respect to fossil forms, upon absolute necessity-we are disposed to think, as suggested by Gill ( $\boldsymbol{1}$, p. xxvii) and the senior author $(\mathbf{2 2}, \mathbf{1 8 9})$, that the more comprehensive vertebrate divisions should be founded upon cerebral and cardiac characters. 


\section{H A P T E R I.}

LIST OF INSTRUMENTS AND MATERIAL FOR ANATOMICAL TECHNOLOGY-DESCRIPTION OF INSTRUMENTS AND APPARATUS-CARE, POLISHING AND SHARPENING OF INSTRUMENTS-METHODS OF KILLING ANIMALS-PRECAUTIONS FOR CLEANLINESS-DEODORIZERS.

\section{$\S 130$. Anatomical Instruments and Material.-The follow-} ing is a General List of the Supplies required for anatomical work. A Special List will be given in connection with each kind of manipulation.

The names are arranged in alphabetical order. Common letters are used for the names of instruments and materials which are desirable, especially in a large laboratory. The names in black letter are of articles which are regarded by us as indispensable to the performance of the best kind of work.

Several articles are not marked indispensable because for them may be substituted others which, although less perfect and satisfactory, may be cheaper or more easily obtained. For example, ordinary cotton may be used instead of the absorbent, a pail or box in place of the anæsthetic-box, crockery instead of glass, etc. Of the two syringes, the cheaper is marked indispensable, but the more expensive will answer the purpose more conveniently.

In the first column are given the numbers of the figures of the instruments and apparatus in this work. In the second column, the sections are named when possible. Usually these sections occur within the present chapter, but in some cases, as with Alcohol, Jars, etc., the articles are treated of elsewhere, as may be ascertained from the Index.

In the last column are given the maximum prices of the less familiar articles. They are usually taken from dealers' lists, and are therefore based upon the ordinary weights and measures. It will be understood that prices vary according to the quality of the goods, the state of the market, and the distance of the dealer from the place of manufacture.

Illustrated Catalogues of Anatomical and Surgical Instruments, of Tools, of Glass-ware, and of Chemical Apparatus and Supplies, may be obtained of dealers, as, for instance, .Codman \& Shurtleff, of Boston, Shepard \& Dudley, of New York, and Snowden, of Philadelphia; A. J. Wilkinson, and Goodnow \& Wightman, of Boston; Whitall, Tatum \& Co., of New York ; J. \& H. Berge, of New York, and others.

\section{ARTICLE.}

Absorbent cotton. . . . . . . . . . . . . . . . .

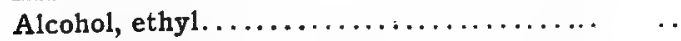

Alcoömeter (alcoholometer), or hydrometer....

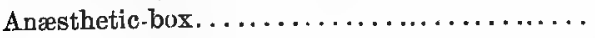

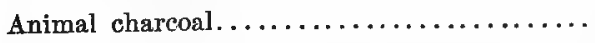

Arseniate of soda. . . . . . . . . . . . . . . .

Arthrotome.

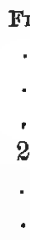

16
SEc.

134

ch. III

"6

194

198

ch. III

135
PER

$\mathrm{lb}$.

gall.

..

.

lb.

$1 \mathrm{b.}$

.
Price $\$ 1.00$

2.50

2.00

1.50

10

20

1.25 
ArTicle.

Atomizer or spraying apparatus

Bags, coarse, for cats.

Basins, graniteware.

Beaded bristles.

Benzine

Bistoury, concave, blunt point.

"، 66

small, straight, blunt point

$"$ sharp point

Blocks, $15 \times 7 \times 5 \mathrm{~cm}$

Blow pipe, flexible.

Bone chisel

Bottle brushes

Butcher knife, small

Cans and boxes, metal.

Canulæ, giass.

Carbolic acid, strong

Cats

Chain hooks.

Chamois leather

Chloroform.

Clove oil.

Compressor, small

Corks, assorted.

Cork presser.

Cosmoline or vaseline.

Cotton, common.

Deodorizers

Dishes, glass, covered

Dissecting-gown

Drawing materials.

Drills and stock

Emery, finest.

Enterotome

Ether, sulphuric.

rilter

Foot lathe.

Forceps, coarse.

" fine, curved.

Glycerin, common.

Graduate, glass

Hone, finest Arkansas oil-stone.

Hone, medium.

Hydrometer jar, $12 \times 2$ inches.

Injecting materials, colors, etc............

Instrument cases. . . . . . . . . . . . . . .

Jars, glass, wide-mouthed

Knitting needle, smallest.

Lamp, Bunsen or spirit

Metric rule, weights and measures.
Fie.

.

Sec.

Per

Price

1.25

136

..

1.25

14

.

19

.

34

..

.

36

..

․

13

141

ch. III

139

140

lb.

2.00

33

ch. III doz.

3.80

142

143

144

159

2.00

5.00

25.00

145

146

1.00

1.25

$1 b$.

50

184

184

ch. III

ch. IV

171

ch. III 
ARTiole.

Muslin, cheap

Nail brush.

Needles, post mortem.

Nippers, large and medium.

Nippers, small, Stubs's.

Nitric acid.

Oil, olive or sewing-machine

Oiler, glass or metal

Parchment for labels.

Parchment numbers.

Permanganate of potassa.

Pins, assorted, and ribbon.

Plaster, adhesive.

Plaster of Paris, finest dental

Ṕliers, round nose, 5-inch.

Probe, silver, with eyelet.

Rouge, jeweller's

Rubber bands, assorted.

Rubber gloves.

Rubber sheeting

Rubber tubing

Saw, back, small.

Scales, large and small.

Scalpels, large, medium and Charriere.....

Scissors, coarse, curved flatwise.........

Scissors,

edgewise.

" medium, curved flatwise.

" fine, curved edgewise.

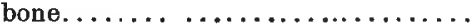

hair.

Silicate of soda.

Sodium chloride (common salt)

Sponges, small.

Strop, razor.

Syringe, brass, with canula.............

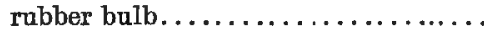

white metal

Syringotome.

Tags for labels.

Tenaculum.

Tools, carpenter's

Towels, fine crash, $45 \times 70 \mathrm{~cm} . \ldots \ldots \ldots \ldots$.

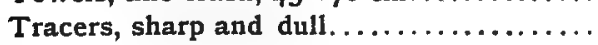

Trays, tin, copper, or wood.............

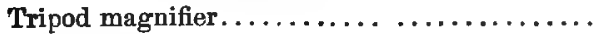

Tubing, g]ass, small.

Tunnels, fluted tube.

Turpentine, spirits of

Twine and thread, linen.
Fra.

SEO.

Pens

Prior

11

146

146

147

149

148

198

202

ch. IV

$\begin{array}{lll}\cdots & \cdots & \cdots \\ \ldots & \ldots & 50\end{array}$

183

106

150

151

152

153

155

$22-24$

25

156

"

"

$2 J$

$\begin{array}{ll}\cdots & 157 \\ \cdots & 158\end{array}$

158

160

185

38

ch. IV

pt. III

ch. IV

161

15

.

163

164

165

166

167

pt. III

26

.

.

.

. 


\begin{tabular}{|c|c|c|c|c|}
\hline ARTICLE. & Fire. & Sre. & Per & Prict \\
\hline Vials, large-mouthed... . . . . . . . . . . . . . . & . & ch. III & .. & .. \\
\hline Waste pail, covered $\ldots \ldots \ldots \ldots \ldots \ldots \ldots \ldots$ & .. & 196 & .. & .. \\
\hline Waste papera $\ldots \ldots \ldots \ldots \ldots \ldots \ldots \ldots \ldots \ldots$ & .. & 195 & .. & . \\
\hline Watch glasses. . . . . . . . . . . . . . . & .. & pt. II & doz. & 2.60 \\
\hline Wetting bottle $\ldots \ldots \ldots \ldots \ldots \ldots \ldots \ldots \ldots \ldots$ & 27 & 170 & .. & .. \\
\hline Wire, brass, copper and iron. . . . . . . . . . . . . & .. & . & .. & .. \\
\hline
\end{tabular}

$\S 133$. It will be seen from the foregoing list that the absolutely necessary instruments for ordinary anatomical work are comparatively few and inexpensive.

In purchasing instruments, the student should remember that their value depends not upon their handles, their finish, or their cost, but upon the adaptation of their size, form and temper to the work in view.

On the other hand, while perfect instruments alone will not insure a good dissection, they are generally more easy to use, and more durable. Hence it is cheaper, in the end, to purchase the best.

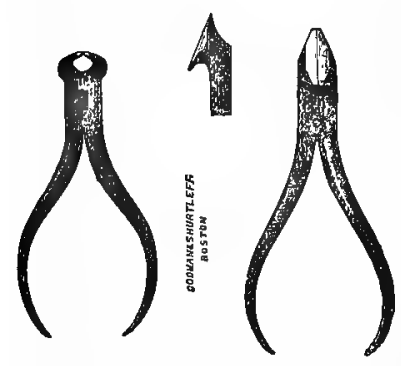

Left figure, ordinary "cutting nippers." Right figure, "diagonal side cutting nippers," called nippers in this work. Middle figure, blades of nippers seen from the side.

$\$$ 134. Absorbent Cotton.--This is cotton freed from all impurities, and especially from the oil which ordinary cotton retains in small amount. It is therefore not only cleaner, but capable of absorbing water or alcohol instantly. On this account Fig. 10.-Stubs's Nippers, $\times .33 ; 8146$. it is to be preferred for the cushions which are made for freshly prepared brains, embryos, and other soft and delicate specimens to rest upon while hardening. It should also be used for packing small or delicate alcoholic specimens for transportation; the ordinary cotton occupies much nore room at first than after it is thoroughly soaked, and a space is thus left in which the specimen can be shaken to and fro. When ordinary cotton must be used for either of these purposes, it should be first thoroughly soaked. For dry packing, the ordinary cotton will answer.

\& 135. Arthrotome-Fig. 16.--This is a strong scalpel, two edged for about $1 \mathrm{~cm}$. from the point. The handle is steel, continuous with the blade, and roughened like that of the anthropotomical, "cartilage knife." The arthrotome should be used for the rougher 


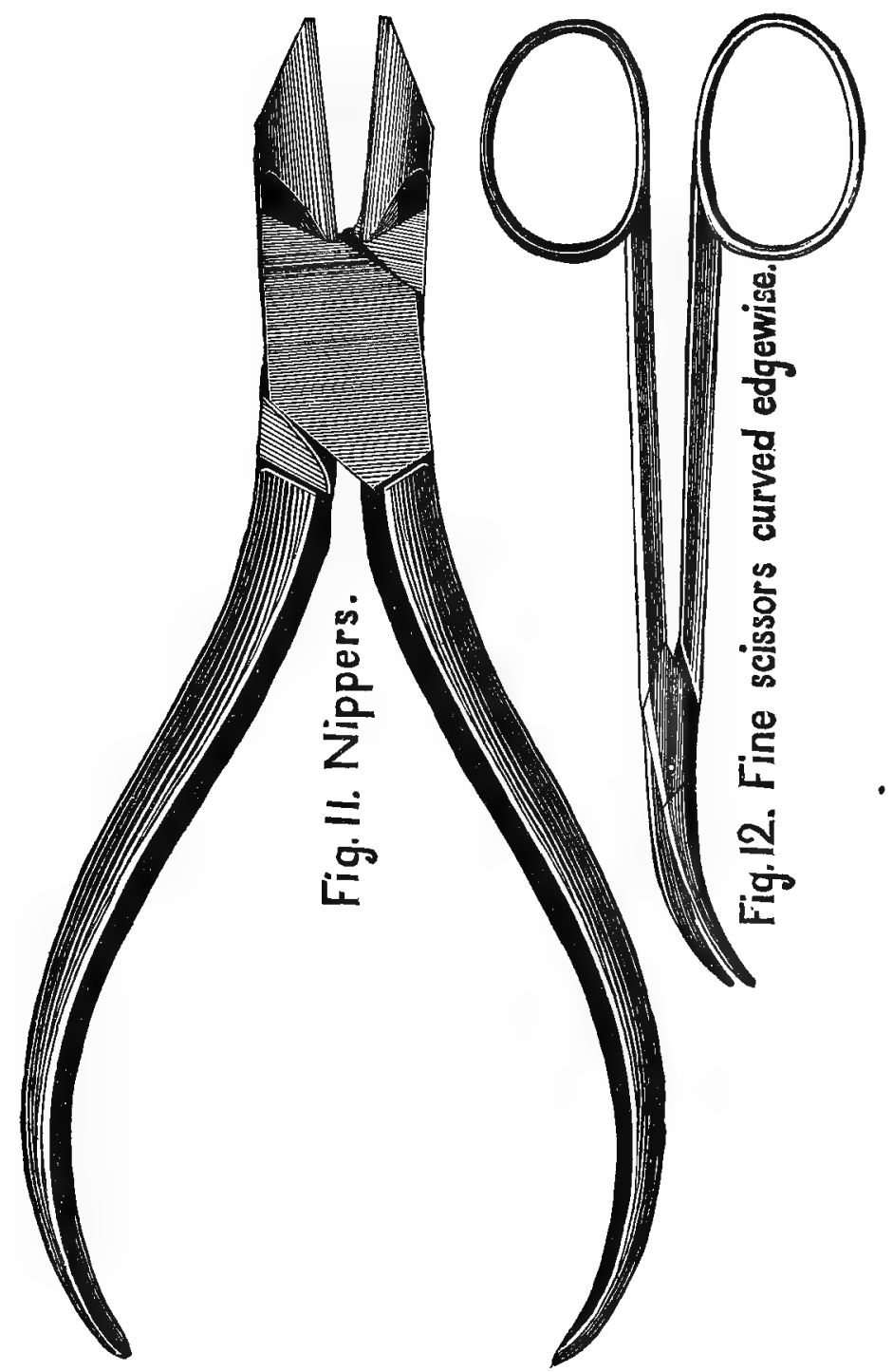

FIG. 11.-NrPPERs ; $\times 1$; $\$ 146$. German "Side cutting Nippers." Fia. 12.-.FInE ScIssors, curved edgewise ; $\times 1 ; \$ 156$.

work, as cutting cartilages and separating arthra, so as to avoid dulling and nicking the more delicate blades of the scalpels. Any thick bladed scalpel may be ground into a tolerable arthrotome.

\& 136. Beaded Bristles.-These are easily made. Cut from a 
hair broom a bunch of the bristles, and select the longer and more perfect ones. Cut off the split end so as to leave each bristle 5-7 mm. long. Melt red sealing wax in the flame of a lamp, and dip into it the larger ends of several bristles at once; lay them down separately and dip more until each has a bit of the wax. Then take them one by one, hold them near the flame, and turn them between the fingers so that the wax assumes the form of a small tapering bead. These bristles are often useful in probing for slender holes and canals, especially in the brain and other soft parts.

$\S 137$. Blocks. - These are of wood, well oiled, and with the edges rounded. They are used for supporting the parts under dissection, but a folded wet towel may sometimes serve the same purpose.

$\S 138$. Flexible Blow Pipe-Fig. 19.-This is the whole or part of the ordinary metallic blow pipe, with the addition of a piece of rubber tubing $30-40 \mathrm{~cm}$. long. The blow pipe may be filed in two, and the pieces used for finer and coarser work respectively.

Unlike the short, straight and stiff blow pipe, this may be bent in any direction, and the object inflated may be held at a convenient distance from the eye. Since inflation is temporary injection, the advantage of witnessing the effects during the operation are obvious.

A. The idea of attaching a flexible tube to the metal blow pipe was first suggested to us by Mr. C. F. Clark, a student, in 1874 .

B. A blow pipe of any size may be made by drawing a bit of glass tube to a point, as in making a canula, and attaching the rubber tube.

$\S 139$. Cats. - The price of cats varies from five to twenty-five cents. The owners of superfluous animals, especially of such as are too old for usefulness or comfort, are sometimes glad to have them painlessly killed.

The isolated student can usually obtain a cat when it is needed, but a laboratory must keep siveral on hand. Contrary to general expectation, cats rarely quarrel in captivity, and the fiercest of them generally become approachable within a few days. But the fleas which probably infest all to some extent, seem to multiply more rapidly when several cats are confined together, and some persons-though comparatively few-are annoyed by them; hence certain precautions should be observed.

If possible, the cats should be kept in a separate building. If they are confined in part of a building otherwise occupied, the room should be isolated by double doors, etc. The walls should be thoroughly plastered, or made of closely matched boards.

At least one window should be reached by the sun, and a raised platform should be so placed that the cats can sun themselves on it. The windows should be covered with 
strong wire netting, and always open a little at the top. In summer the ventilation cannot be too free; in winter the room should be kept at a moderate temperature (10 to $15^{\circ} \mathrm{C}$.).

At least once a year, the room should be thoroughly cleaned; and then washed with a solution of sulphate of iron. Benzine should be poured or sprayed into all the corners and cracks to kill the fleas. If possible, the room should be vacant during the hottest months.

S'allow boxes of dry earth should be placed upon the floor, and the earth changed frequently. Should soiling of the floor occur, the feces shonld be removed at once, the spot well washed and saturated with some dendorizer, and then covered with a box. An uncleanly cat should be promptly removed. The male cat is retromingent; if one side of the earth boxes reaches to the height of ajout $30 \mathrm{~cm}$. (about 1 foot) above the earth, the walls of the room may be protected from their strong-smelling urine.

Cats like separate beds, which may be provided by placing boxes containing a little hay or "excelsior" along the sides of the room, preferably at a little distance from the floor. T'ue cats are better pleased if half the top of the box is left upon it.

Fresì catnip should be strewn about the room occasionally when obtainable; the dried lerb is a good substitute in the winter.

Graham crackers and water should always be accessible, milk should be supplied daily, and miat once or twice a week. The milk vessels should be kept clean.

\$ 140. Chain Hoolss-(See Codman \& Shurtleff, A, 43, Fig. 14). - These are used for fixing or suspending parts under dissection. In most cases, with so small an animal as the cat, these purposes can be accomplished by the use of the "small compressor," with strings or straps of appropriate length.

\$141. Compressor, Small-Fig. 13. -This name is given to what is known among dealers in wearing apparel

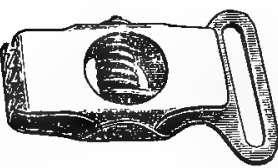

Frg. 13.-SMall CoMPRESSOR ; $\times 1 ; \S 141$. as the "Royal Garment Clasp, No. 1." The spring which closes it is quite strong, and the sharp teeth enable it to retain its hold under considerable tension. By means of the eyelet, the compressor may be attached by a string or a strap to the loop at the side of the tray. For some purposes the teeth may be removed.

\$ 142. Dissecting Gown. - In some cases, the clothes are sufficiently protected by an apron and pair of sleeves, or even by a towel upon the lap. But generally, especially while injecting, removing viscera, preparing bones, or performing experiments, one should wear a gown of some smooth black stuff, like silesia. The gown should nearly reach the ankles, and the sleeves should be held at the wrists by elastic bands. It may open in front or behind, but the buttons should be concealed lest they catch upon the edge of a dish or jar. Soiling of the wristbands by the dye of the gown may be prevented by facing the sleeves with white linen. 


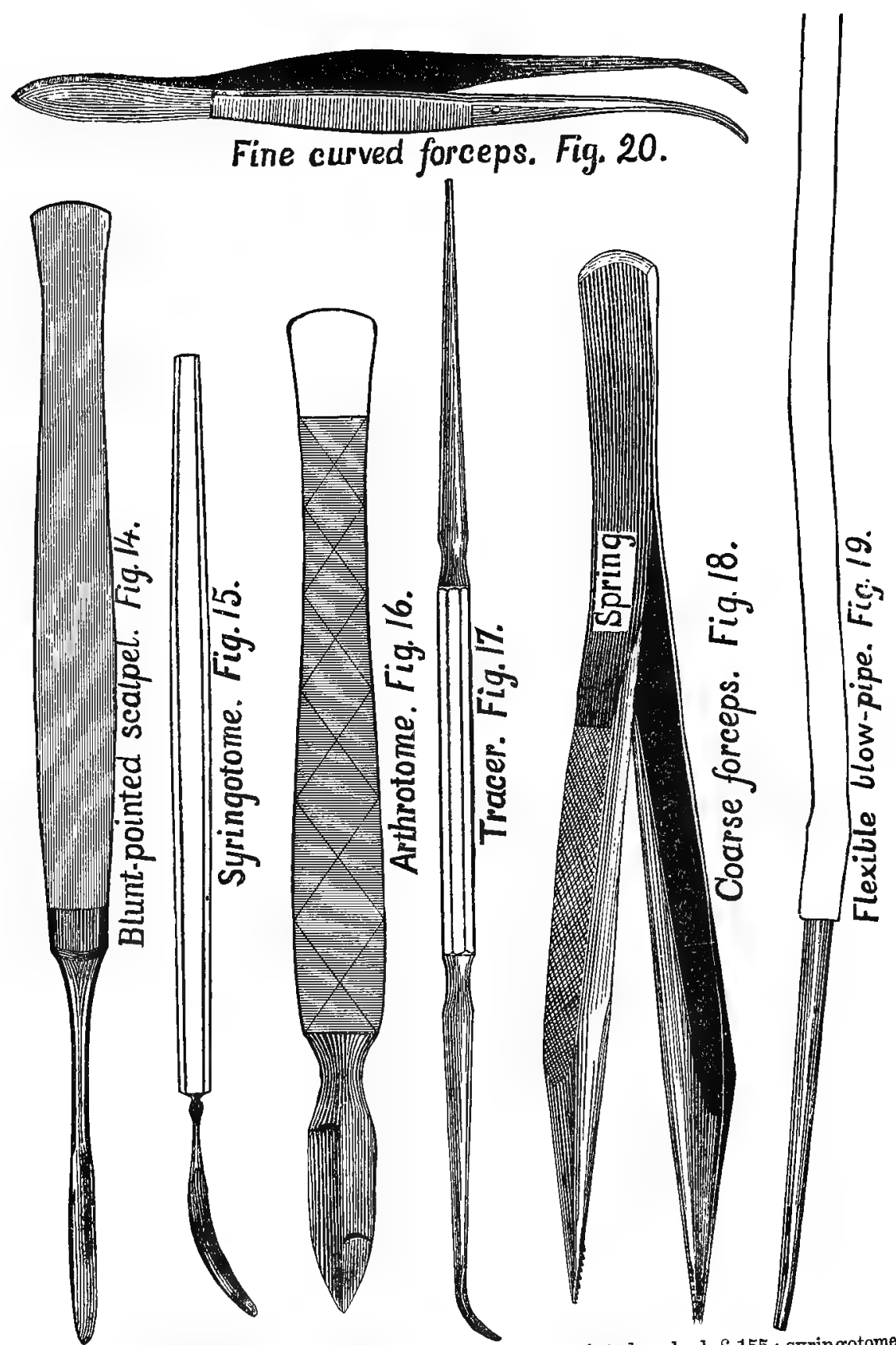

Figures 14-20.-Various instruments; $\times 1$. Blunt-pointed scalpel, $\$ 155$; syringotome $\S 161$; arthrotome, $\S 135$; tracer, $\S 166$; coarse forceps, $\S 145$; flexible blow-pipe, $\S 130$ fine curved forceps, 145 . 
\$ 143. Drawing Materials. - These are required in all kinds of Natural History work, and may be conveniently mentioned here. In addition to the writing pencil, there should be at least two for drawing, the one of medium, and the other of considerable hardness. All pencils should be "hexagonal" to prevent rolling, or else provided with a hexagonal rubber eraser. For the neatest work, however, it is well to use a separate eraser with a brush for removing the crumbs from the paper. (See Readers and Writers Economy Co., A.) It is so often desirable to double the size of objects that the "duplicating dividers" are more useful in place of or in addition to the ordinary dividers. India ink is used for some drawings and for writing upon parchment (see $\$ 149$ ).

\$ 144. Drills.-A laboratory should have a foot lathe like, for example, the one figured by Goodnow \& Wightman, A, 73. But most of the holes required in bone, wood or metal, may be made by a small "Hand drill," like, for example, that figured on p. 14 of the same Catalogue.

$\S 145$. Forceps, Coarse and Fine-Figs. 18, 20.-Both pairs are absolutely necessary, and the fine ones should be curved. With nearly all forceps, the spring is too strong; it should be only sufficient to separate the blades when the pressure of the fingers is relaxed. If the dealers will not supply forceps with the proper spring, the desired change may be effected with a file or grindstone. The "Coxeter" style of coarse forceps is to be preferred. Those represented in Fig. 18, have the blades excavated so as to be lighter. than those formerly made.

\$ 146. Nippers - Fig. 10, 11.-These are the "diagonal side cutting nippers or pliers" of the dealers in hardware. The obliquity of the blades to the handles gives them great advantages over either the "side cutting" pliers, or the "cross cutting," which are shown in Fig. 10, left figure. Seven sizes are made, ranging from 10-20 cm. (4-8 in.) in length. Those of 10 and $15 \mathrm{~cm}$. are best adapted to anatomical work upon small animals. The larger of these will cut any of the bones of cats less than two years old, but the larger bones of older individuals may require the saw. For some purposes the points should be quite sharp, and may be made so with a file or upon a grindstone.

A. "Pointed nippers with oblique jaws" are mentioned by Newton (B, 22, 174), but they do not appear to be in general use by anatomists. The nippers have been used in anatomical work, especially for the removal of the brain, by the senior author since 1871, and are mentioned in his paper, $11,158$. 
B. The German instruments answer very well for most purposcs, and are much less expensive thun the others: They are imported by Messrs. H. Boker \& Co., of New York, and may be had of A. J. Wilkinson in Boston, and of Messrs. Treman, King \& Co. in Ithaca, and of larger dealers generally. The "Stubs's" nippers are more finely tempered, and better finished. The smallest size (Fig. 10) are sold by Messrs. Codman \& Shurtleff for $\$ 1$. The "bone forceps" of the surgical price lists are still more expensive.

C. The "cross cutting" nippers are employed chiefly for cutting wire and for other mechanical purposes.

$\$ 147$. Oiler.-A neat substitute for the ordinary metal oiler may be made by suspending a dropping-tube in the mouth of a vial of oil. One may then graduate the amount more exactly, and apply it more accurately; see Appendix.

$\S 148$. Parchment Numbers.-Sheets of numbers of any size may be had at a reasonable rate from "Collins' Printing House," Philadelphia. If they are to be used with alcoholic specimens, that fact should be mentioned so that proper drying ink may be employed. The numbers may be pasted upon dry specimens, or placed in the alcohol with wet ones, or attached to muscles during dissection by means of the small "ribbon pins."

\$ 149. Parchment for Labels.-Numbers and memoranda to be attached to alcoholic specimens should be written upon parchment with a saturated solution of India ink in either glacial acetic acid, or acetic acid No. 8. The writing should be allowed to dry before exposure to the alcohol. Parchment is written upon more easily if the surface is first rubbed with a rubber eraser.

$\S 150$. Rubber Gloves.-These are an efficient protection against contact with poisonous or malodorous substances. Of course, fine dissection cannot be done in gloves, but it is sometimes desirable to protect the hands, especially in the manipulation of the intestines, and while macerating bones. Like other rubber articles, the gloves should be wiped dry after using, and laid in a cool, dark place.

$\S 151$. Rubber Tubing.-For the blow pipe, and for canulæ, the lumen should be $3 \mathrm{~mm}$. (1-8th inch); the size next most useful in the laboratory is $6 \mathrm{~mm}$.

\& 152. Saw-Fig. 21.-This should have a thin blade, and the teeth should be but slightly "set." It is used chiefly for bisecting the head for the removal of the brain, and should not be employed for mechanical purposes.

\& 153. Scales.-Very few cats

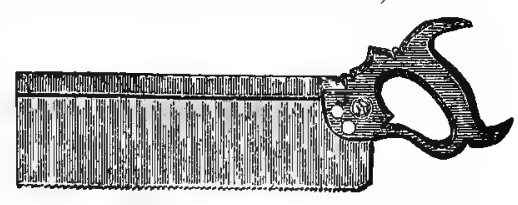

FIG. 21.-BACK SAw ; $x .14$. weigh as much as 5 kilos., and heavier ones can be weighed entire 
upon ordinary scales. For most anatomical purposes, therefore, the scales need not be arranged for more than 4-5 kilos., or about 10 lbs. avoir. Weights of less than 1 gram should be determined by the smaller or "prescription" scales.

Of the larger scales there are two styles, the "open " and the "box." An example of the former is the "Druggist's trip scales," figured by J. \& H. Berge, A, 63; of the latter, is the "Ebony box scale," figured by Whitall, Tatum \& Co., A, 74. With a capacity of 10 lbs., the former costs $\$ 7$, and the latter $\$ 14$. In selecting scales, it would be well to obtain the advice of some chemist or physicist.

$\S 154$. Weighing Pan.-The scale pans accompanying the scales above mentioned are about $20 \mathrm{~cm}$. in diameter, and will contain any separate organ of the cat, or the head or limbs. For weighing an adult cat entire, a larger pan is needed. For this purpose, one of the ordinary trays, $30 \times 40 \mathrm{~cm}$. may be used, but it is better to provide a special pan. It should be oval or oblong, about $25 \times 35 \mathrm{~cm}$., made of stout tin, and with a rim about $3 \mathrm{~cm}$. high. Its weight may be diminished by punching out disks not more than $1 \mathrm{~cm}$. in diameter, until it exactly balances some weight, as 500 grams, or a piece of lead, which, of course, must be used always with the pan. If the cat to be weighed is stiff, it may sometimes be made to rest upon the ordinary scale pan without touching anything; otherwise this special pan should be used, the arms and legs and tail being kept within it.

§ 155. Scalpels-Figs. 22, 23, 24.-These are single edged, and thin bladed knives, with ebony or ivory handles. The medium size

FIG. 22.

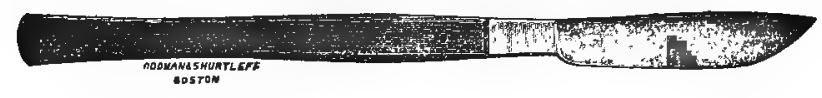

FIG. 23.

FIG, 24,
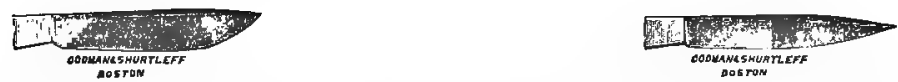

Fig. 22-Large Scalpel, for sections of brain, etc.; $\S 155 ; \times .5$. Fig. 23.-Mrdiuxstzed SCALPEL, for ordinary dissecting; $\times .5 ; \S 155$. Fig. 24.-Charriere SCALPEL, for finer dissecting; $x .5 ; \S 155$.

(Fig. 23) answers for most purposes, and will last a long time if carefully used. The "Charriere" is employed for finer-but not the finest-dissecting, and the large scalpel should be kept-perfectly smooth and keen for making macroscopic sections of the brain, heart, etc. 
\$ 156. Scissors-Figures 12, 25.-All dissecting scissors should be curved. The curvature gives three advantages: better adap-

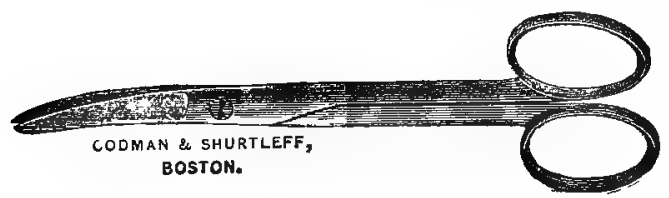

Fig. 25.-Coarse Scissors Curved Elatwise; $\times .5 ; \S 156$. tation to the surfaces of animals and their parts, which are rarely plane; the power to change the direction of the cut without corresponding movement of the band or of the subject; the effect of the cutting is more easily observed. For most purposes, the blades should be curved flatwise, as in Fig. 25, but those curved edgewise, as in Fig. 12, are sometimes very convenient. All coarse scissors should have lock joints, so that the blades may be separated for cleaning and sharpening. Fine scissors should have sharp and well matched points. The medium coarse scissors are well adapted to work upon the brain and heart, and other organs for which the coarse scissors are too large, and the fine ones unnecessarily delicate.

$\S 15 \%$. The bone scissors are simply a strong, blunt-pointed pair of coarse scissors, with which the cartilages, ribs, and other hard or tough parts may be cut, so as to save the edges. of the proper dissecting scissors. A straight pair will answer the purpose quite well, and usually cost somewhat less.

$\S 158$. The hair may be removed with a pair of common cheap scissors; but it is better, especially in a large laboratory, to provide a special pair of coarse scissors, curved flatwise, and with the points quite blunt.

$\$ 159$. The enterotome supplied in post-mortem cases is a pair of long scissors, one blade of which is enlarged and rounded, and projects beyond the other so as to precede it in opening an intestine. Similar instruments might be made for the cat, but the same end may be accomplished by guarding one of the blades of a pair of coarse scissors, especially such as are curved edgewise. Cleland suggests (A, 155) sticking a bit of costicartilage upon one blade, but a small rubber stopper will serve the purpose.

$\$ 160$. Sponges.--Sponges may be conveniently classed as carriage, bathing, anatomical and surgical. The last are fine grained, and specially prepared, so as to be somewhat expensive. A few such, of conical shape, are desirable, especially for experimentation. For ordinary anatomical purposes, sponges should be small enough 
to be easily grasped, and should be freed from sand and grit before using. The larger and coarser kinds are useful in various ways.

All sponges should be washed after using, and boiled occasionally for a few moments. It is said that they may be freshened by soaking in brine to which a little iodine has been added.

$\S 161$. Syringotome-Fig. 15.-This is sometimes called "canaliculus knife." It is a small, concave, blunt pointed bistoury, which is very convenient for delicate work upon the brain and heart, and for following and slitting up narrow canals.

The syringotome was found very serviceable by the senior author in tracing out the tortuous canals upon the heads of sharks and skates at the Museum of Comparative Zoology in 1866-7. At his suggestion it was included in the set of dissecting instruments supplied to the students of "The Anderson School of Natural History at Penikese Island " in 1873. Excepting with the brain, however, most of the uses of the syringotome may be subserved by the less expensive tracer.

§162. Tags.-Two sizes of tags are needed : the smaller are for numbers or brief memoranda sufficient to identify the specimen; they are used by stationers and dry goods dealers. The larger are the smallest size of "Dennison's Shipping tag," and are 3.5 cm. wide by 7.2 long; they should be provided with strings, and the eyelet should be guarded by a metal ring.

$\S 163$. Tenaculum-See Codman \& Shurtleff, A, 42, Fig. 8.This is seldom needed in felitomy.

\$164. Tools, Carpenter's.-A laboratory should contain the ordinary tools, as saw, hammer, screw drivers, awls, bits and stock, rat tail and three cornered files, screw hooks and eyes, etc.

$\S 165$. Towels.-Excepting the roller towels, these should be short. The finer crash is more expensive, but wears longer than the cheaper stuffs, and there is less lint; it may be had in rolls, which may be cut into the desired lengths.

§ 166. Tracer-Fig. 17.-This is prepared from a piece of hexagonal or octagonal steel rod, about $15 \mathrm{~cm}$. long, and $4 \mathrm{~mm}$. in diameter. The middle third is left as a handle; one of the terminal thirds tapers to a blunt point, and serves as a probe for some purposes; the other end tapers in like manner, and is bent at the tip so as to form about the fourth of the periphery of a circle $1 \mathrm{~cm}$. in diameter; the concavity is then sharpened.

The value of the tracer in isolating vessels and nerves can hardly be overestimated. A dull tracer may be used also in lifting vessels and nerves that have been isolated already, as in experiments. 
A. A tracer may be made by any one from a piece of steel rod, or from a dental " excavator," but the saving so effected is hardly worth the trouble. The instrument is made by Codman \& Shurtleff, and perhaps by others.

B. The tracer is apparently similar to the "seeker" of the English anatomists, and the "finder" of the Germans. The instrument was introduced into the laboratory of Cornell University with a microscope presented by the late Hon. John Stanton Gould. The con. version of the opposite end into a tapering probe was suggested by $\mathrm{Mr}$. Willis $\mathrm{N}$. Rudd, a student, and the sharpening of the concavity was first proposed by the junior author.

\$ 167. Trays-Fig. 78. - These are usually made of stout tin, but copper is more durable. If wood is used, it should be thoroughly oiled. A wire loop should be soldered at the middle of each end, and on each side at about one-sixth of the distance from each end.

Four sizes of trays are convenient in the dissection of cats : $15 \times 20,20 \times 30,30 \times 40$, and $40 \times 60 \mathrm{~cm}$. The depth of the two smaller sizes should be about $1 \mathrm{~cm}$., and that of the two larger about $2 \mathrm{~cm}$. The largest size should be stiffened by diagonals upon the bottom.

$\S 168$. Tripod Magnifier-Fig. 26.-This is a simple magnifier with a large field, and mounted upon a tripod which may be placed

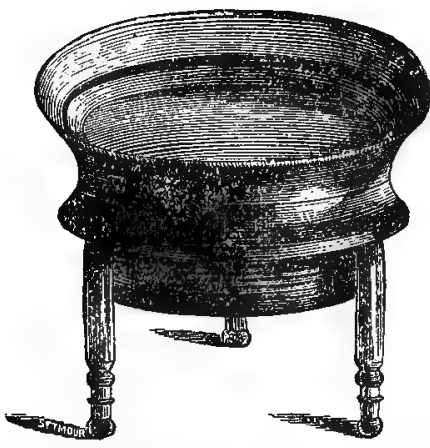

Fig. 26.-TRipod MagNiflen; $\times 1 ; \S 168$.

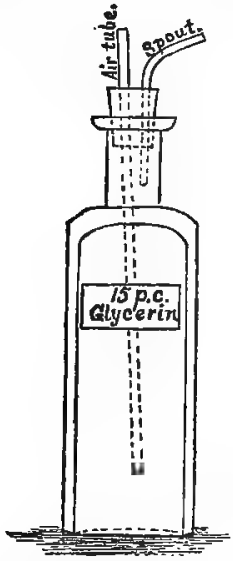

Fig. 27.-Wetting Bottle; $\$ 170$. in liquid. While examining delicate objects out of liquid, it is safer to turn the legs toward the eye, or remove them altogether. The object should be held so as to receive the best light.

$\S 169$. Tunnels.-Several sizes are desirable, of both glass and tin. The latter should have the slender part fluted. When the larger part of a tin tunnel, or either part of a glass one, is set into a bottle or jar, a thick string should be interposed between it and the mouth of the vessel to permit the escape of air as the liquid enters. 
$\S 170$. Wetting Bottle-Fig. 27. -The bottle is about $16 \times 5 \mathrm{~cm}$., so as not to be easily overturned. The mouth is closed by a cork through which are passed two slender glass tubes. One, the airtube, is straight, and reaches nearly to the bottom of the bottle; the other, the spoul, extends but little below the cork, and is curved so as to permit the ready application of the contents.

$\S 171$. Wetting Mixture.-The cheaper commercial glycerin is to be mixed with water in the proportion of 15 per cent. by measure, or 12 per cent. by weight, and about 25 drops of clove oil is to be added to each liter of the mixture.

$\S 172$. Waste Papers.-These are pieces of paper of several sizes, corresponding respectively to the slips, the sheets, and the trays.

The quality of paper is not material, so long as it is firm enough to hold together when slightly wet. The "roll Manilla" paper of medium thickness is strong and cheap, but the ordinary slips and sheets which have been used by writing on one or both sides may be kept for this purpose.

With fine dissections, or when only connective tissue or small muscles are to be removed, the waste slip may be placed in a corner of the tray.

With coarser work, and when skin, fat, and the larger muscles or viscera are to be removed, use the waste sheet.

When the cat is to be transected or eviscerated, or when some larger animal is under dissection, have at hand extra trays containing tile corresponding waste papers.

The waste should be disposed of as soon as possible, but in case the opportunity for re-examination is desired, the tray containing it may be set aside, and the paper will obviate the objectionable adhesion of the waste to the tray itself which might otherwise occur.

\section{CARE OF INSTRUMENTS.}

$\S 173$. In general, all instruments should be washed, thoroughly dried, and slightly oiled as soon as possible after using.

The washing may be done with a cloth or sponge, but the nail brush should be used for the joints of nippers, and the teeth of the saw. The wiping may be done with a towel, and then with a bit of clotid or chamois slightly oiled.

All joints should be kept well oiled. 
The oiling should be especially thorough when instruments are to be packed away or disused for some time, and particularly at the sea shore.

Scalpels and other instruments with wooden or ivory handles should not be allowed to soak in water, lest the rivets become loose after drying. Wooden handles should be occasionally oiled.

$\$ 174$. Blow Pipe.-Usually this needs only to be wiped, first with a moist, and then with a dry cloth. After using, be sure that the lumen is free; if clogged, open it with the fine knitting needle, or a wire.

$\S$ 175. Forceps.-Clean the serrated parts with the nail brush, draw a cloth or towel between the blades, and then wipe.

$\S$ 176. Nippers.-Clean the joint and blades well with the nail brush, wipe dry, and oil the joint.

$\$ 17 \%$. Saw.-Use the nail brush, moving it from the back toward the teeth. Wipe, carrying the towel in the same direction. Then wipe dry, and oil.

$\S 178$. Scalpels and Cutting Instruments Generally.-These instruments, and especially such as have keen edges and delicate points, should be protected from contact with each other and with other objects. If not kept in a case, they may be laid in a small tray, like the cover of a note box lined with chamois or velveteen. When several are to be carried at once, each handle should be held between two fingers so that the-blades may not touch.

In wiping a scalpel, hold it firmly in the left hand, and let the cloth cover the right thumb and index, as in wiping a table knife; do not let the cloth come upon the edge. The scalpel should be wiped four times: first with a moist cloth to remove all blood and fragments; then with a dry cloth; then with an oiled cloth or chamois, and finally with a clean dry cloth or chamois. The fingers should not touch the blade after the final wiping.

\$ 179. Scissors.-If the blades are lock jointed, they should be separated. The blades are to be treated like the scalpels. Clean the joint thoroughly, and keep it oiled.

$\S 180$. Trays.-If waste papers are used, the trays will usually require only rinsing, after which they should be set up on edge to dry. Where many trays are used, there should be a suitable rack for them.

$\S 181$. Instrument Cases.-The experienced anatomist rarely uses the "case" in which, probably, his first instruments were 
purchased. The ordinary case never has room for all the necessary instruments, and is a less convenient receptacle for them while in use than a shallow tray like the cover of a note box; see $\$ 110$.

If the student is unable to resist the temptation to possess a regular dissecting case, he is advised to obtain one of the more costly, as likely to contain better instruments. The case commonly selected by the special students at Cornell University is sold by Messrs. Codman \& Shurtleff at $\$ 9$, and contains the following instruments : three assorted scalpels, coarse and tine forceps, coarse and fine curved scissors ; arthrotome, and blow pipe. Similar cases may probably be had elsewhere. The additional instruments must be purchased separately.

$\S$ 182. Packing Instruments for Transportation.-All scalpels and delicate pointed instruments generally should be packed as follows: thrust the point into a bit of cork, then wrap well in a piece of thin paper, such as is supplied in packages under the name of "star mills," "diamond," etc. The paper should project well beyond the blade, and be twisted or bent over so as to keep the cork in place. Thus wrapped, the most delicate instruments may be sent by mail in pasteboard or light wooden boxes, or otherwise transported. Since no written communication is permitted upon such a package without payment of letter rates, the consignee should be notified at the same time. Whether for mailing or any. other kind of transportation, the heavier instruments, or such as need no special protection, should be packed separately from the more delicate. Small cigar boxes answer a good purpose, but the most suitable boxes for instruments are made by the Swift Manufacturing Co. of New York, and others; they are long and narrow, and provided with lids.

\section{POLISHING INSTRUMENTS.}

\$ 183. A. Instruments that have become tarnished may be repolished by rubbing with a piece of chamois or cloth on which has been put oil and rouge. Perhaps the best way to apply the polishing material is to wrap the cloth around the end of the index for small instruments, or roll the cloth or chamois into a bundle for large instruments. In both cases the surface to be polished is rubbed as in scouring household knives, taking care to avoid contact with the edge of the instrument. 
B. Rust should be removed with a dull knife and then the polishing may be done as above. When an instrument is badly tarnished, the polishing with rouge is a tedious process. In that case, fine emery may be used to remove the tarnish, and then rouge for the final polish. The emery should be applied as directed for rouge.

C. If one has access to a polishing wheel the process is shortened greatly. The same materials are used as in polishing by hand and in the same order.

D. Fmery of exceeding fineness may be easily prepared as follows: Fill a high narrow vessel like a preserving jar, nearly full of water, and put into it about 200 grammes of ordinary flour of emery. Agitate thoroughly, and after the vessel has stood half a minute pour off the liquid into another dish. Add more water to the first vessel and agitate again, and pour off the liquid as before. The larger particles sink first, and hence the emery in the water poured off is much finer than that left. Allow the emery to settle from the first and second washings and pour off the water and dry the emery. Several grades may be obtained in this simple way.

E. Instruments may be very nicely polished by using, in place of rouge, the fine whitish ashes that may be found in the upper parts of stoves in which anthracite coal is burned.

F. If rouge is added to the oiled leather used for wiping the instruments after they are washed $(\S 178)$, the polish maty be retained indefinitely.

\section{SHARPENING INSTRUMENTS.}

$\S 184$. Honing.-For honing, it is desirable to have two oil stones, one very fine for finishing, and one somewhat coarser for

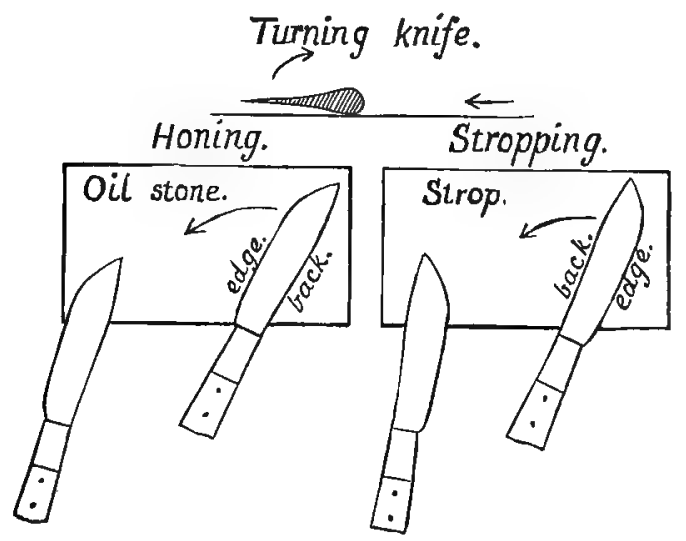

Fig 28.-Honing and Stropping Finves. The upper figure shows how the knife is to be turned upon its back in reversing the movement. The left figure shows the edgeward movement of the blade in honing; the right, the backward movement in stropping. $\& \& 184,185$. 
commencing the sharpening, and for sharpening the coarser instruments.

A. Place several drops of fine olive or sewing-machine oil on the stone and, with a cloth devoted to the purpose, rub the surface to remove all dirt and expose the cutting particles of the stone. After the stone is well wiped, put two or three more drops of oil upon it, and spread it around with a scalpel blade.

B. Look at the edge of the instrument to be sharpened with the tripod magnifier, holding the edge of the blade up and between the eye and the light. This is to see if there are any nicks in the edge. If there are nicks, they should be removed by rubbing the edge on the fine stone. After making two or three sweeps across the stone, look at the edge again to see if all the nicks are removed. If they are not, continue to grind the edge on the stone till they are. If the nicks are slight the edge need be ground off only in their immediate vicinity. If they are deep, however, the entire edge should be removed or it will become wavy.

When the edge is smooth and free from nicks it should be honed; if quite dull, first on the coarse and then on the fine stone.

C. In case the instrument is a scalpel, (1) grasp the handle in such a way that the index and medius shall oppose the pollex, and the end of the handle shall touch the palm. (2) Place the blade flat on the stone as shown in Fig. 28, and then lift the back very slightly. (3) Move the knife with a curving sweep toward the left, as shown by the arrow, so that the point of the blade shall be at the lower left corner at the end of the sweep. (4) Then turn the blade over, always turning the edge away from the stone. Do this by rolling the handle in the fingers. (5) After the knife is turned, it should be moved across the stone from left to right exactly as described for the motion from right to left. The handle, of course, points in the opposite direction.

In this method of honing, which is that employed by the best cutlers, the edge precedes the back; the blade is so placed on the stone that it follows the handle, and the sharpening is from heel to point. If the blade were pushed across the stone instead of being drawn as above, the sharpening would be from point to heel.

D. In the beginning of the honing, one may press quite firmly and draw the same side of the blade over the stone three or four times without turning it; but when the edge becomes thin, the blade should be turned at every sweep. 
E. In case the edge should turn over, producing the so-called wire edge, it must be removed by drawing the edge along some finegrained substance like horn or ebony. One should be careful not to get any of the detached wire edge on the stone, as it would be liable to produce nicks in the edge of the knife.

F. (1) Use the coarse stone until the knife will cut a thin shaving from the convex surface of smooth writing paper. (2) Wrap the paper around a lead pencil, remove the pencil and rest the blade flat upon the paper. Press down slightly, and push the blade, edge forward, along the top of the curve. If the knife is sharp, it will cut a thin shaving from the paper. (3) Another very excellent way to judge of moderate sharpness is to rest the tang of the blade on the end of the medius, and to feel of the edge by moving the ball of the index along it in such a way that if a cut were made it would be a mere shaving from the cuticle like that from the paper. If the knife is sharp, it will take hold, as it is called, that is, one can feel that it is cutting. The ball of the index is very sensitive, and one can judge quite correctly of the smoothness and sharpness of the edge. Those who object to trying the edge on the skin can employ a bit of smooth grained cork.

G. (1) When the edge is sufficiently sharp throughout its entire extent to cut a shaving from the cylindrical paper, or to take hold of the finger or the edge of the cork, the fine stone may be used.

(2) In using the fine stone the blade should be turned at erery sweep. Use the fine stone until the knife will cut a hair near its base or near the point where it is held.

(3) It often happens that some parts of an edge are sharp and others not. In such a case the dull parts alone can be applied to the stone by using the edge of the stone.

When the scalpel will cut a hair close to a fixed point, it is sufficiently sharp for ordinary dissecting.

$\S 185$. Stropping. - A good razor strop is required, as, e. g., "Emersons'."

The strop is to give the final keenness and smoothness to the edge of a cutting instrument. It is a waste of time to employ it before the degree of sharpness indicated for the fine stone is attained, as the strop sharpens very slowly.

A. (1) Grasp the knife exactly as for honing. (2) The blade is cxrried across the strop with a long curving sweep just as described for honing except that the back of the blade precedes the 
edge. (Fig. 28.) (3) The blade should be turned at the ond of every sweep across the strop, thus drawing it from right to left as . often as it is drawn from left to right.

B. (1) Use first the red and then the black side of the strop. (2) Press only moderately. The nearer a perfect edge is attained the more lightly should one press.

(3) Continue the stropping on the red side until the knife will cut a hair of the head $1 \mathrm{~cm}$. from the point where it is grasped by the fingers; then employ the black side.

(4) Continue to use this side until the knife will cut a hair from the head $2-3 \mathrm{~cm}$. from the point where it is held, or, what is a better test, until it will cut the fine hairs on the dorsum of the hand and wrist half a centimeter from their base when the knife is moved distad-toward the ends of the fingers. If the knife has a perfect edge it will cut these fine hairs so easily that one can hardly tell by the feeling when a hair is divided.

\$186. Scissors.-These are nuuch more difficult to sharpen than scalpels, and the fine ones should be sent to the makers unless one is very skillful. Place the blade so that the oblique face formed by grinding shall rest flat on the stone. Draw the blade, edge foremost, across the stone with a curving sweep as for scalpels $(\S 184, \mathbf{C}[3])$. Test for sharpness with the finger or by attempting to cut moistened tissue paper $(\S 184, \mathbf{F}[3])$.

\section{$\S 187$. Tracer, Syringotome and Concave Edges Generally.}

In sharpening instruments of this kind one should use the edge of the stone instead of its face. The edge of the stone should be somewhat rounded. In sharpening, draw the blade along the stone so that the edge precedes the back as for scalpels $(\$ 184, \mathbf{C}[3])$. Test the sharpness with the finger $(\$ 184, \mathbf{F}[3])$.

$\S 188$. The care and sharpening of instruments are considered by Mnjsisovics, $A, 13$; IIoltzapffel, A, III, 1026-1156 ; Hyrtl, A, 23-27 ; Straus-Durckheim, B, 1, 158-160.

\section{KILLING ANIMALS FOR DISSECTION.}

$\S 189$. There is usually no difficulty in taking a cat when it is wanted. Such as will not come when called may be secured by means of a strong net, or by using a bag attached like a net to a hoop and pole.

The bag referred to is of strong coarse material, and commonly used for oats. In such a bag the cat may be left for several hours; 
but the closer-woven bags which are used for flour do not admit - sufficient air.

$\S 160$. IMethods of Killing-(Bernard, A, 149-182).-Two things are to be considered in killing animals for dissection :-

(1.) The death should be as nearly painless as possible.

(2.) None of the organs or tissues to be examined should be injured by the method employed.

\$191. Drowning fulfils the above requirements fairly well. Judging from the experience of human beings, death by drowning is attended with very brief physical discomfort.

Place the cat in a wire cage, or loose meshed bag, and immerse it completely in water for four or five minutes. Usually a cat cannot be resuscitated after it has been completely immersed for ninety seconds; after four or five minutes, spontaneous resuscitation is altogether improbable.

\$192. Chloroforming is preferable to drowning, since no liquid is drawn into the lungs, and the hair is not filled with water. The death, too, with cats, seems to be quietly going to sleep. There is usually no struggling, showing that the period of intoxication by the chloroform is very short.

Place the cat in the anæsthetic box (see Fig. 29). This is easily accomplished if the cat is in a bag by placing the mouth of the bag in the box, whereupon the cat will usually walk in of its own accord. After the cat is in the box and the door closed and fastened, remove the cork from the hole in the edge and pour 5-10 cc. of chloroform upon the furled curtain. Then unfurl the curtain by means of the string; this will expose a greater surface from which the chloroform can evaporate. Usually the cat will be asleep in three minutes, and dead in twenty minutes. Do not remove it from the box till all signs of respiration have ceased.

If ether is used, $15-20 \mathrm{cc}$. is required for a cat.

A. If one does not possess the anæsthetic box, cats may be chloroformed as follows: Place a newspaper on the floor, and invert over it a large wash-bowl or a small tight box or pai]. Put the cat under the receptacle, and pour $10 \mathrm{cc}$. of chloroform on a sponge or a bit of cotton, and put it under the receptacle with the cat. The box, or whatever is used, must be held down, or a weight must be placed upon it, while the animal is coming under the influence of the anæsthetic.

B. Dogs moan while becoming anæsthetized, but presumably they and all other animals may be killed painlessly with chloroform or ether. The amount required to kill an animal varies with its size, and with the size and closeness of the box.

$\S 193$. Killing Fleas.-If the cat has fleas, as is usually the case, 
open the box after fifteen minutes, and throw over it $20 \mathrm{cc}$. of benzine. Reclose the door of the box and leave it for ten or fifteen minutes longer. Fleas revive from the chloroform used in killing the cat, but they do not revive if benzine is used.

Do not use the benzine until the cat is completely anæsthetized, for it causes great discomfort.

$\S$ 194. The Anæsthetic Box-Fig. 29.-This is a close box, the base, frame and door being of wood, and the rest of "double-thick"

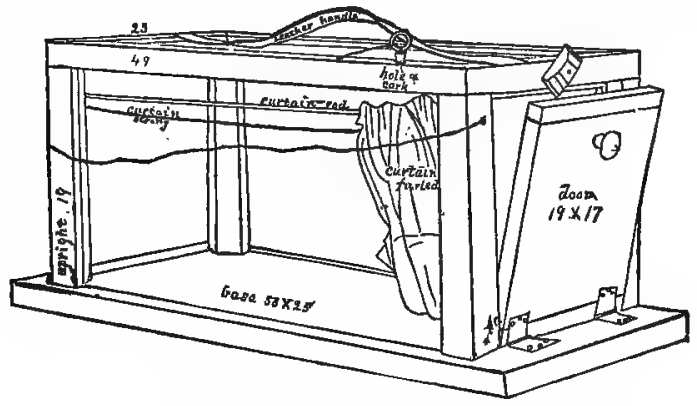

FIG. 29. -THE ANसSTHETIC-BOX; $\times$.1.

glass. The dimensions are given in the figure. The base and frame should be grooved for the reception of the glass, and the whole should be put together with screws to permit the renewal of the glass in case of breakage.

Along one side, near the top, runs a thick brass wire, on which slides a curtain, moved by a string. Just above the curtain, when furled, is a hole, which is closed by a cork secured from loss by a string. The leather handle is convenient in lifting and moving the box.

The cost of the cat-box, including the glass, is about $\$ 1.50$.

Almost any close vessel or box will answer for the administration of a fatal dose of chloroform, but for experimental purposes the progress of anæsthesia must be observed.

\section{PRECAUTIONS FOR CLEANLINESS, COMFORT AND HEALTH.}

$\S 195$. Waste Papers.-These have been referred to in $\S 172$, and are mentioned here again because of the tendency of beginners to neglect a very essential element of neatness in anatomical work.

$\S 196$. Waste Pail.-This should bə of galvanized iron, copper 
or zinc, never of wood or tin ware. The cover should be of the same material, and fit closely. The pail should be emptied at night, rinsed, and a few cc. of permanganate of potash solution poured into it. Once a week it should be thoroughly scoured and dried.

\$ 196. Waste Pit.-The single student may readily dispose of the refuse of his work, but some special provision must be made for a laboratory.

In cities, communication may sometimes be had with the sewer. The opening into the sewer should have at least two lids, closely fitting, and should be within a closet or cupboard provided with a special flue so that the sewer gas may not be drawn into the laboratory.

In smaller towns, and at many universities, the readiest mode of disposal of refuse is to have a pit dug in dry soil at a convenient distance. The mouth of the pit should be kept from falling in by a cask or box, and should be closely covered. The earth removed in digging the pit should be kept at hand under cover, and some of it sprinkled over the offal at night. When filled within half a meter of the surface, the pit should be filled up compactly with earth.

$\S 197$. Sink.-This should be of iron, galvanized iron, zinc or copper, or lined with one of these materials. It should be slightly inclined, the outlet should be at the lower end, and guarded with a movable grating. The escape-pipe should be of ample size, and trapped at some point which can be reached.

Excepting just over the escape, the sink should be covered by one or more wooden lids on hinges which may be raised from the front and rested against the wall behind the sink. These lids should be of hard wood, and well oiled or painted. In each lid should be cut an oval or elliptical hole for a basin. The longer diameter of the hole should correspond with that of the sink, so that the basin may be emptied without lifting it from its place.

If there is no general water supply, a water cask or lead lined box may be placed above the sink. In any case, the faucets should be nearly on a level with the face of one standing at the sink, and the water should be conducted therefrom through flexible rubber tubes ending a little above the basin. The tube must be firmly secured to the faucet, and must not be sharply bent if the pressure of water is considerable. 
Hair, plaster of Paris, sand, and fine particles generally must not be thrown in the sink.

The sink should be thoroughly emptied and washed at night. A convenient instrument for scraping out the sink, or cleaning a table or tray, is a piece of heavy rubber moulding, provided with a handle.

$\S 198$. Deodorizers.-Most of the unpleasant smells which would otherwise attend work in practical anatomy may be avoided by preserving the material in alcohol, by removing the intestines within twenty-four hours after death, and by the observance of other due precautions for cleanliness. But maceration is necessarily offensive, and sometimes valuable specimens are more or less decomposed before their reception; it is therefore necessary in some cases to employ deodorizers.

Animal Charcoal.-This effective deodorizer may be sprinkled over the surface of offensive specimens, and is especially serviceable when such have to be transported. Its use in the improvement of old alcohol is described in Ch. III.

Alcohol. - If the specimen is of moderate size, and is to be preserved as a whole or in great part, the putrefaction may be checked by immersion in strong alcohol, from 75 to 95 per cent. The alcohol may be poured over the specimen, or the latter may be immersed in it, or covered by cloths saturated with it. In either case, the alcohol will become offensive, and must be deodorized by filtration before mixing with other alcohol or use upon other specimens. The stronger the alcohol, the more decided is its action, but its clearness is unessential.

Potassium Permanganas.-This is an excellent deodorizer. A saturated solution should be kept at hand, and a few cc. poured into the waste pail at night, and into any other malodorous jar or vessel. It stains the skin temporarily.

Sulphate of Iron-Copperas.-This cheap deodorizer may be used in place of the more efficacious but more expensive permanganate of potash. The coarsely powdered. crystals, or a saturated solution, may be placed in the sink, pail, or pit.

A solution of Chloride of Lead is recommended in The Medical Record, August 20,1881, p. 222.

\& 199. Discharges from the Cat.-The following precautions are always desirable, and should never be neglected in demonstrations or experiments upon cats before a class :- 
When the cat is dead, or, in experiment, quite asleep, place it upon a tray. Roll some common cotton between the fingers into a somewhat firm conical plug or suppository about $5 \times 1 \mathrm{~cm}$.

Dorsiduct the tail of the cat so as to expose the anus and open it slightly. With the large forceps grasp the plug obliquely near the tip and force it into the rectum. Then push it completely beyond the constricted orifice with the forceps or a smooth, rounded stick. If the cat has been affected by diarrhea, it may be necessary to insert a second plug.

Let the buttocks of the cat project slightly beyond the edge of the tray, over the sink or some other receptacle; then press firmly and steadily upon the abdomen just cephalad of the pubes. If the cat is a female, the urine will usually flow out readily; if it does not appear, as is often the case with males, it is not likely to be forced out during the subsequent operations.

The urine of cats has a very offensive odor, and should not be allowed to flow into the tray, or to reach the hair of the animal.

Remove any escaped urine or feces with a bit of cotton, followed by washing if necessary.

If the left hypochondrium is prominent, or if there is other evidence that the stomach is distended with food, let the mouth project beyond the tray over a receptacle, and compress the whole abdomen. If any matters escape from the stomach, the mouth should be washed afterward with a stream of water.

$\S 200$. Malodorous Parts.-On account of their contents, the stomach and intestine become offensive very soon after death, especially if exposed to the air.

In some cases it may not be necessary to open the abdomen during the dissection of a fresh specimen; decomposition will then proceed less rapidly, and the effects will be less obvious.

If the abdomen is opened, these hollow viscera should be either removed soon, or so treated as to lessen or prevent the production of offensive odors. The large intestine especially may usually be examined during the first or second day, so as to be removed.

When any part of the alimentary canal is divided, the site of the intended incision should be freed from its contents for at least $2 \mathrm{~cm}$. ; then two ligatures should be applied at least $1 \mathrm{~cm}$. apart, and the cut made between them ; see Fig. 41 .

When it is undesirable to remove the intestine, most of the offensiveness may be avoided by expelling the contents. The con- 
tents of the large intestine are readily forced out by manipulation, the cotton plug of course having been removed. Those of the small intestine may be made to flow out with a stream of water injected into it near the stomach by means of a syringe, or from a faucet.

The water should be pressed out of the intestine, and alcohol then thrown into it. The alcohol may be retained if the plug is returned to the anus; or a ligature may be placed about the rectum.

\$201. Dissection Wounds.-Slight dissection wounds have occasionally been received in the anatomical laboratory of Cornell University, but the results have been nowise different from similar cuts inflicted under ordinary circumstances.

So far as our experience goes, it is probable that no danger need be apprehended from a wound received during the dissection of any well preserved alcoholic specimen, or of any healthy cat, whether fresh or otherwise.

In proportion to the number of human bodies annually dissected or examined in necropsies, serious dissection wounds are very few. Indeed, the actual number of such cases is so small that statistics are wanting to enable us to determine with accuracy the conditions under which the consequences are likely to be injurious. A few writers believe these results to be due to "the absorption and irritation of a putrescent fluid ; but this explanation will hardly account for the frequency of the disease after contact with recent bodies before putrefaction has set in, and especially of persons who have died of acute disease, such as puerperal fever, peritonitis, etc., and for the affection of several persons at the same time, from the same body and with the same symptoms."

In view of the insufficiency of our knowledge, and of the fact that dissection wounds are most apt to occur with beginners, one of the advantages of the cat as a subject of preliminary anatomical work is, that the subject may be obtained healthy and fresh, and bo preserved in alcohol at slight expense.

- 8 202. Precautions.-Dissection wounds should be avoided by care in the use of cutting and pointed instruments, and by guarding against contact with the sharp points and edges of bones which have been broken or cut.

Before commencing work upon a suspected animal, or upon decomposing flesh, or upon macerated bones, the hands may be anointed with some kind of fat, as cosmoline, vaseline, olive oil or "cold cream."

If the skin is already broken, rubber gloves may be worn, as in macerating or in handling offensive viscera, etc., where no delicacy of manipulation is required. In ordinary dissection upon a suspected subject, the cuts or abrasions may be cauterized with strong carbolic or nitric acid, or covered by several thicknesses of adhesive plaster. 
If the hands can be kept dry, the common court plaster or isinglass plaster will suffice; but if they are to be wet, it is safer to use the diachylon or lead-plaster, which does not easily wash off, but must be warmed before application or removal.

In cauterizing, use a slender, pointed stick; dip it first into the acid, and then into the open place of the skin.

\$ 203. Treatment._" Local applications, if used at all, must be employed immediately, decisively, and effectually. Should a person be in bad health at the time of receiving a prick or wound during dissection, no matter what condition the subject may be in, it behoves him to pay immediate attention to the injury, and anticipate any further consequences."

"Should the wound be received while engaged on a recent subjcct, and one which had died from acute disease, much more caution and attention are required, even if he be in the best of health. The part should be well cleansed, and a temporary ligature applied immediately above (centrad of) the wound; then the bleeding, if any, should be encouraged to flow freely; where there is no bleeding, suction must be employed; [this may be by the mouth if the skin and mucous membrane are intact, otherwise by means of a tube, or, better, a cupping glass the exhaustion of which is produced by means of a rubber bulb]." Then cauterize as directed above.

The foregoing directions for immediate treatment are taken from Holmes (T.) A, 621, and Clarke, A, 54.

Whenever ill effects are anticipated, proper medical advice should be sought without delay. 


\section{H A P T E R I I.}

GENERAL DESCRIPTION OF THE SKELETON-ANATOMICAL LANDMARKS-ABDOMINAL TRANSECTION.

\section{GENERAI DESCRIPTION OF THE SKELETON.}

$\S 204$. The softer parts of the body are protected, supported, or enabled to exert themselves to greater mechanical advantage by a framework-the skeleton.

The skeleton consists of bones (Ossa), and cartilages (Cartilagines), which are more or less closely united at sutures (Sutura), or movable upon each other at Arthra (joints or articulations). At the arthra and at some of the sutures the undesirable displacement of the parts is checked by bands of inelastic fibrous tissue-the ligaments (Ligamenta).

Firmer in texture, more definite in outline, and more constant in form, number and position than most of the soft parts, the bones and cartilages serve as convenient guides to the identification and description of the latter, and therefore naturally precede them as subjects of anatomical study.

The following general account of the skeleton as a whole is given in advance of the detailed description of the individual bones in order that the directions for certain preliminary operations may be more easily understood and followed.

$\S 205$. Fig. 30.-The skeleton seen from the left.

This figure has been reduced by photography from that of Straus-Durckheim (A, P1. 11), and has been further modified as follows: the dextral costæ (ribs) have been omitted for the sake of clearness; the last costicartilage has been shortened so as to show its actual condition; the principal bones have been named, and the costre and vertebræ have been numbered; the shading is likewise altered somewhat.

$\S 206$. Like the body as a whole, the skeleton comprises a somatic ("axial") portion including the bones of the head, neck, thorax, abdomen, pelvis and tail, and a membral ("appendicular") portion, including the bones of the arms and legs. 


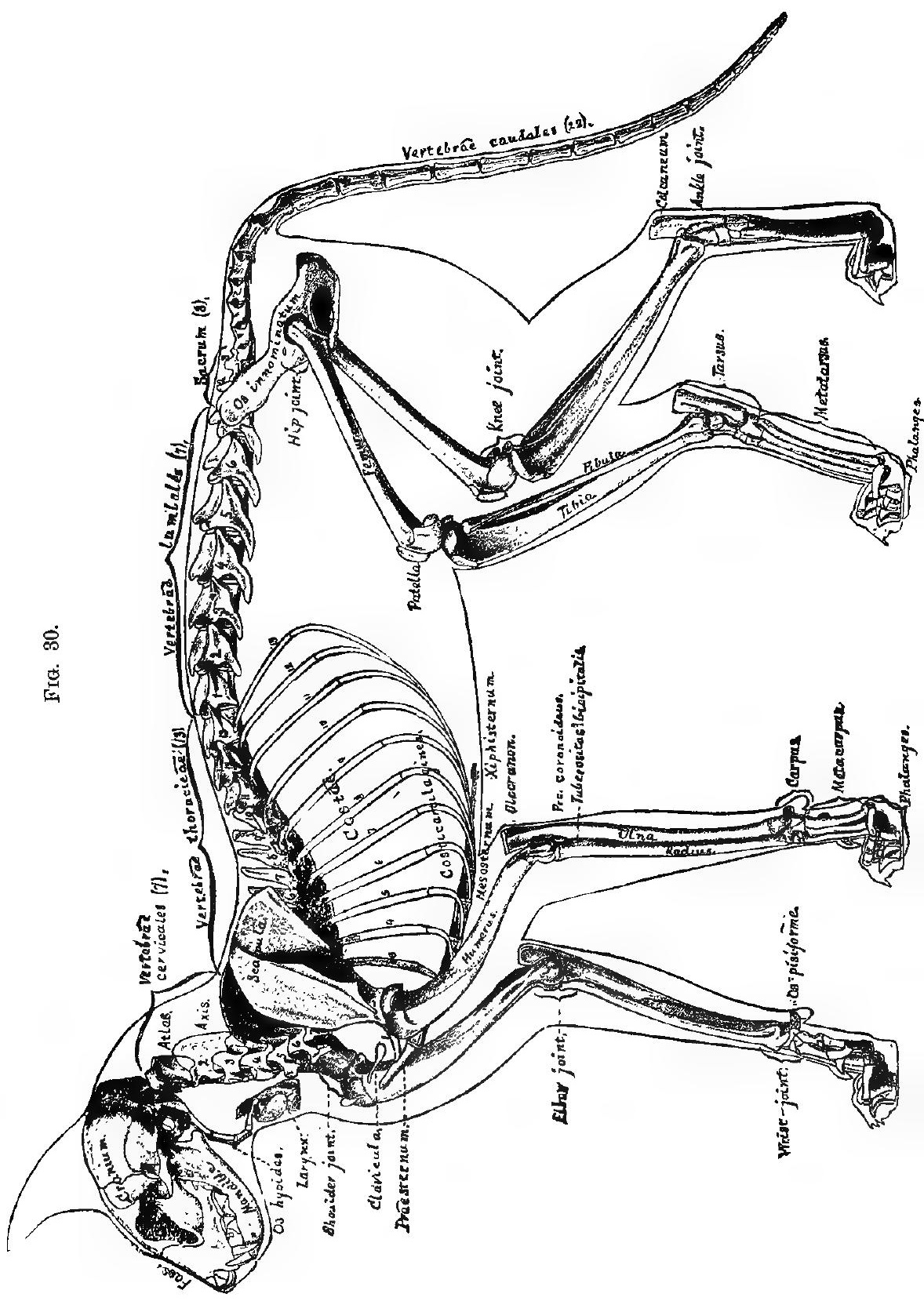


The membral bones have been shown from the dorsal aspect in the normal position of the parts in Fig. 6, but in Fig. 30 the animal is represented from the left side, in one of its natural attitudes while standing or walking.

In this attitude the limbs are directed ventrad instead of laterad, and are thus nearly parallel to each other; while their segments and the general divisions of the somatic skeleton, the head, neck, trunk and tail, form angles with each other, imparting to the whole skeleton a graceful and spirited appearance.

\$207. Skull-(Fig. 56-62).-The cephalic division of the somatic skeleton is the skull, consisting of the cranium or braincase, and the face, to which appertains the mandible or lower jaw. The transverse ridge at the junction of the dorsal and caudal aspects of the skull is the Crista lamdoidalis (Fig. 56, Cst. Imd.); the darkly shaded area between the cranium and the face represents the lefit orbit, and the longitudinal irregular bar just ventrad of it is the zygoma, or arcus zygomaticus (Fig. 56).

The Os hyoides will be described in $\$ 224$.

\$ 208. Vertebræ-(Fig. 51-55).-Caudad from the skull extends a series of bony segments-the Vertebro-constituting the Columna vertebralis or spine or spinal column. As seen from the side, the Columna presents two curvatures in opposite directions, like an elongated letter $s$.

For convenience and more or less naturally, the vertebræ may be grouped in five divisions, cervical, thoracic, lumbar, pelvic or sacral and caudal. The numbers following the names of these groups upon Fig. 30 are those which are most commonly observed, but variations sometimes occur, as will be mentioned in the description of the Columna vertebralis.

The vertebræ of each group have certain features in common, and also individual peculiarities by which, excepting most of the caudales, they may be distinguished when completely isolated. On the entire skeleton, the groups are most readily recognized on account of the connections of three of them with other parts, as follows :-

The thirteen thoracic vertebræ are costiferous, that is, connected with the ribs; the cervical series begins at the skull, and the three sacral vertebræ are united so as to form a single bone-the $s a$ crum-to which is attached the $O s$ innominatum on either side. The lumbar vertebræ intervene between the thoracic and the sa- 
crum, while the caudal vertebræ extend caudad of the last-named bone.

The first and second cervical vertebræ (Fig. 52), are also called, respectively, atlas and axis. The former is expanded laterally in wide transverse processes. The latter presents a Spina neuralis, which is both long and high.

The seventh cervical spine and the first five thoracic are hidden in Fig. 30. The first nine or ten thoracic spines are directed more or less decidedly caudad, but the last two or three, like the lumbar, are directed cephalad, while the tenth or eleventh has an intermediate direction. The various processes become less and less distinct among the caudal vertebræ, and the caudal members of the series are little more than subcylindrical segments of bone.

Unlike most anatomists, Straus-Durckheim includes (B, I., 480, Pl. xi,, B, C), the eleventh, twelfth and thirteenth thoracic vertebræ with the lumbar series.

\$209. Costæ-(Fig. 50). - Each costa (rib) is seen to be connected by its dorsal end with the thoracic region of the Columna vertebralis, and at its ventral with a costicartilago. Part of the first costicartilage appears in Fig. 30 just cephalad of the left shoulder joint, but the second rib and its cartilage are wholly concealed by the scapula and humerus.

\$210. Sternum-(Fig. 49). - The sternum, or breast-bone, consists of a series of eight or nine mesal segments called sternebro. The caudal piece is the xiplisternum or ctl. ensiformis, and is partly cartilaginous. The cephalic segment is the prosternum or manubrium, and the intervening six or seven sternebræ constitute the mesosternum.

\$ 211. Scapula - shoulder blade-(Fig. 43-45). - The ectal aspect of this bone, as shown in Fig. 30 and 44, presents a ridge, the mesoscapula, which has three prominences-acromion, metacromion, and tuberositas.

\$ 212. Clavicula-The clavicle or collar bone-(Fig. 48).-In the cat these bones are small and imbedded in the muscles, without direct attachment to other bones. They are shown in Fig. 30, one on each side, between the shoulder joints.

A. In the cat the scapula appears as a part of the arm. In man, however, and in many other vertebrates, the clavicles are larger and articulated with the scapula and the sternum. In most reptiles and birds, and in two Mammals (Echidna and Oruithorhynchus) the scapula and the sternum are connected by a stout osseous bar-the coracoid-which is represented in the cat as in man by merely a process-the Prc. coracoideus; Fig. $43,45$. 
B. The two scapulæ with the clavicles and the coracoid bones are commonly regarded as constituting a sort of belt-the scapular arch or shoulder girdle.

\$ 213. Pelvis-Pelvic Girdle-(Fig. 51).-This limb girdle is complete in the cat. Each lateral piece-Os innominatum-is attached to the sacrum by its dorsal end, and ventrad joins its platetrope (fellow of the opposite side) at the symphysis pubis. The rounded vertebral end of each os innominatum is the Crista $i l i i$, and the caudal end is the ischiatic tuberosity.

$\S 214$. Shoulder and Hip Joints.-These are the proximal arthra of the arm and the leg respectively, forming their points of attachment with the shoulder girdle and the pelvis. Both are ball-andsocket joints, but the former is the more free, and the position of the entire joint may be changed on account of the suspension of the scapula in the muscles.

$\S 215$. Elbow and Knee.-These are both hinge joints, the latter being less encompassed by bone, and hence somewhat the freer of the two. At the knee the femur articulates with the tibia only, while the elbow is between the humerus and both the ulna and radius.

§ 216. Wrist and Ankle.-The latter is a trie hinge joint, but the former combines features of the hinge and the ball-and-socket varieties.

\& 217. The Bones of the Limbs.-All of these have been named in the Introduction in connection with the description of Fig. 6 ( $\$ 82-85)$, and some will be described hereafter with more detail. Reference will be made here only to certain general features of the larger bones, and to the attitudes of the entire limbs.

Antagonism of the Membral Segments.-It will be noted that, excepting the distal segments, the corresponding segments of the limbs point in opposite directions, and that the same antagonism exists between the principal elements of the scapular arch and the pelvic girdle. As a necessary concomitant, any two successive segments, excepting in the case of the manus and antebrachium, point in opposite directions.

From this relation of the segments there are two results : First, that the weight of the body rests upon columns which are not only near its opposite ends, but also tend to counteract each other for the most part, so that stability is more easily maintained. Nevertheless, by exception, the distal segments coincide in direction, so that both limbs may strike the ground in one direction, and thus propel 
the body in the other. Second, these limbs are more elastic than if the several segments were in the same line, and the muscles act upon the bones to better mechanical advantage.

$\S 218$. By some writers (Wyman, 75, 253; Coues, 1, 15), and formerly by the senior author $(5,45)$, this opposed or symmetrical or antitropic relation of the scapula and ilium, and of the propodial and epipodial bones of the arm and leg has been regarded as evidence in favor of a general symmetrical homology between the two limbs. The senior author, however, has admitted $(\mathbf{1 0}, 15)$ that this antagonistic relation is secondary and telical rather than primary and morphical, and has fully assented to the view that in their normal position both pairs of limbs extend laterad from the trunk, and their flexures are in the dorso-ventral rather than in the cephalo-caudal direction.

$\S 219$. To replace the limbs in their normal and primitive position (see $\$ 45$ ), it is necessary to rotate the elbow cephalad and the knee candad, and then-if the commonly accepted view be correctto lateriduct both limbs until they are at right angles with the meson, as in Fig. 6.

This rotation will leave the convexities of the elbow and the knee facing dorsad (as in Fig. 7), and that of the ankle, with the plantar aspect of the pes, facing ventrad. In the arm, however, the corresponding aspect of the manus-the palm-will be left facing dorsad, and the ulna and radius will be crossed instead of parallel like the corresponding tibia and fibula. But if the manus be supinated, the ulna and radius will be parallel, and the palm will face ventrad like the sole.

The restoration just described is assumed to have taken place in the following brief account of some of the bones and their prominences:-

$\S 220$. The proximal end of the humerus presents an elevation, the trochiter or Tuberositas major (Fig. 45, 46), which is cephalic in the normal position of the parts, but lateral in their natural attitude. At the distal end of the same bone (Fig. 46) are two elevations, the epicondylus and epitrochlea, commonly called external and internal condyles. In the natural attitude, as seen in Fig. 30, the epicondyle shows on the left arm, and the epitrochlea on the right.

The ulna projects dorsad and proximad of the elbow as a thick process, the olecranon. Just distad of the joint, on the ventral side of the bone, is an elevation-the Processus coronoideus-for the attachment of the $M$. brachialis.

The proximal end of the radins is the capitellum, while the distal end of each antebrachial bone presents a short Prc. styloideus. 
The individual carpatia are not easily distinguished, but the $O$. pisiforme is seen on the right side; see Fig. 47.

$\S 221$. At the proximal end of the femur is a marked process, the trochanter, which is naturally visible in the lateral view of the bone, but normally has a caudal position. At the distal end of the same bone are the cephalic and caudal ("inner and outer") condyles.

At the convexity of the knee, and thus normally dorsad of the joint, is the patella, which answers in some respects to the olecranon, but is really only a very large $O$. sesamoideum.

The tibia is seen to be both larger and longer than the fibula, which indeed does not enter into the composition of the knee. The malleoli, cephalic and caudal (internal and external), are slight elevations of the distal ends of the tibia and fibula respectively. Of the tarsalia, the prominent calcaneum is easily recognized.

$\S 222$. In ordinary locomotion neither the palm nor the sole are in contact with the ground excepting at the junction of the metacarpal and metatarsal regions with the digits and dactyls; the body is supported upon the ball of the foot and the dactyls and upon the corresponding parts of the manus, and the cat is thus a typical digitigrade. We can imitate its condition so far as concerns the elevation of the heel and the support of the body mainly upon the ball of the foot; but the human digits and dactyls cannot be brought into the state of the cat's, where the proximal phalanges are flexed dorsad, the intermediate ventrad, and the distal ones again dorsad so as to keep the sharp claw points off the ground.

\$ 223. The Positions of the Arthra.-It will be noted that the arthra of the arm are ventrad of the corresponding arthra of the leg, although the manus and pes are upon the same plane, and the vertebral ends of the scapula and ilium are at nearly the same level.

Since there is little difference in either length or inclination between the humerus and femur, and the radius and tibia, this difference in the levels of the arthra must be associated with the greater length of the pes as compared with the manus, and with both the length and more nearly vertical direction of the scapula as compared with the ilium.

Notwithstanding the popular designation of the wrist of a horse as its "knee," it should be kept in mind that the true knee of the mammalian quadruped is a joint 
of the leg, and, like its counterpurt the elbow, is but slightly removed from the level of the ventral border of the trunk. The ankle of the quadruped is often called hough or hock.

\section{\$ 224. Os Hyoides and Larynx-(Fig. 30).-Dependent from} the caudal region of the basis cranii is an inverted segmented bony and cartilaginous arch-the Os hyoides. Connected with the summit or ventral end of this arch is the larynx, a cartilaginous case forming the principal organ of the voice. (In Fig. 30 the larynx is placed too nearly in contact with the fifth Vertebra cervicalis).

The keystone of the arch appears in Fig. 30 only by its sinistral end, shown as a small subquadrate area close to the angle formed by the junction of the ventral outlines of the head and the neck. The lateral bar extending from this point caudad and slightly ventrad is one of a pair of pieces called thyro-hyals, which are additions to the arch itself, and are most directly connected with the larynx.

Each lateral half or pier of the arch consists of four segments, as follows :-

Next to the basihyal is the cerato-hyal. Then comes the. epinyal, and then the stylo-hyal. In the figure the dotted line from the words os hyoides is drawn to the arthron between the cerato-hyal and epihyal. The three pieces mentioned are bony; the fourth piece, which appears in the figure along the side of the bulla, is cartilaginous, and has been called Ctl. stylo-hyoidea. It is attached at the bottom of a deep pit just laterad of the bulla; (see Fig. 57, Fs. tyh.). The sequence of the osseous segments may be connected with the alphabetical order of the initials of their names, $B, C, E$, and $S$.

A. For fuller accounts of the Os hyoides, and for the origin of the names of the seg. ments, consult Owen, A, II., 506 ; Flower, A, 123. -Straus-Durckheim's account (A, I, 450$453)$ is not very satisfactory. The figures of Mivart ( $B$, 78) are good, but he ascribes to the cat a small osseous segment-the tympano-hyal-which exists in the $\operatorname{dog}$ (Flower, A, 123), but which we have failed to find in even old cats, and which is not mentioned by Straus-Darckheim.

B. In man the thyro-hyals are disproportionately large, and are called the greater cornua, while the cerato-hyals are called lesser cornua. Quain, A, I, 50 ; Gray, A, 206 ; Parker and Bettany, A, 305.

The following parts of the larynx may be recognized in the figure :-

The largest and ventrally placed piece, and that which is reached 
by the dotted line, is the Ctl. thyroidea. In man it forms the mesal projection known as "Adam's apple." Just ventrad of it in the figure, but caudad in the normal position of the parts, is the ring shaped Ctl. cricoidea. Each thyro-hyal segment of the Os hyoides is directly connected with the larynx by a small $C t t$. arytenoidea, one of which is indistinctly shown in the figure at the dorsal (really cephalic) end of the prolongation of the Ctl. cricoidea. The epiglottis is seen projecting just dorsad of the thyro-hyals.

\section{ANATOMICAL LANDMARKS.}

\$225. During dissection and experimentation it is often desirable to determine the lines and limits of incisions, or to ascertain the location and outline of parts which are obscured by the skin or other soft parts. The elevations and depressions which may serve as guides are called anatomical landmarks.

The landmarks here described should be carefully studied, first upon the prepared slreleton by the aid of figures and descriptions, and then upon the entire cat by the aid of preparations of the soft parts, and frozen sections and dissections.

Most of the landmarks are more easily recognized upon the living or freshly killed animal, but they should be sought also during the continuance of rigor mortis, and upon specimens hardened by alcohol. Finaily, their recognition should be practised also with the eyes closed.

Whoever intends to perform experiments should become sufficiently intimate with some cat to be permitted to manipulate all the accessible parts. The late Prof. Jeffries Wyman once told the sonior author that in Paris he lived in the same house with StrausDurckheim; and that the indefatigable felitomist wonld sometimes sit by the bour holding a cat, and passing his fingers from point to point over the muscular elevations, the joints and other bony prominences with which his mind was occupied at that time.

The landmarks form three groups, mesal somatic, lateral somatic, and membral.

§ 226. IMesal Somatic Landmarks - Crista lambdoidalis(Fig. 56).-Prominent as is this crest upon the prepared skull, it is by no means easy to find upon the entire animal. The cervical muscles just caudad of the occiput are very firm and compact, so that the change of substance is not very marked to the touch. The crista, however, is nearly in a line with the most caudal convexity of the ears, and may be more distinctly felt during alternate ventriduction and dorsiduction of the head.

\$ 227. Spina Neuralis Axialis-The axial spine-(Fig. 30).-The somewhat sharp caudal projection of this spine may be felt at the dorsimeson between the cervical muscles $3-4 \mathrm{~cm}$. from the Crista lambdoidalis, and not far from the scapulæ. 
Spina Neuralis Thoracica Prima-The first thoracic spinous process.-This is about half as long again as that of the seventh cervical vertebra, and larger at the tip tlian the succeeding thoracic spines. In the living animal, it is at the bottom of the interscapular depression, but one or both of the scapulæ may be ventriducted so as to leave it more prominent.

In man, it is the seventh cervical spine which is longer than the rest, whence the name Vertebra prominens sometimes applied to the vertebra.

Sp. Nrl. Thr. 13-(Fig. 30).-This is a little cephalad of the vertebral ends of the last ribs, and may sometimes be distinguished from the other thoracic spines by its greater cephalo-caudal extent, wherein it resembles the lumbar series. The tenth or eleventh spine is usually quite short, so as to leave a slight hiatus about $2 \mathrm{~cm}$. cephalad of the thirteenth. In counting the spines beginning with the first, the thirteenth will usually appear to be the twelfth on account of the short one just mentioned. If the lumbar series be counted, attention must be paid to the point next mentioned.

Spina Lumbalis \%-The last lumbar spine.-This projects just cephalad of a line between the Cristæ iliorum, while the first sacral spine projects between the Cristæ, so as to be hidden by them in the figure of the entire skeleton (Fig. 30).

\$228. Pubes-(Fig. 51).-The cephalic border of the pubic bone is easily felt both at and laterad of the ventrimeson.

Epigastrium and Xiphisternum-(Fig. 72). - The epigastrium is a subtriangular area at about the junction of the cephalic and middle third of the trunk. Its latero-cephalic borders are formed by the ninth and tenth costicartilagines. The xiphisternum (Fig. $30,49,72$,) may be felt on the meson in a lean cat, but it is sometimes obscured by fat.

Prosternum-(Fig. 30, 49).-This is easily distinguished either on passing the finger cephalad along the sternum to the neck, or caudad along the neck until it reaches the somewhat sharply projecting point.

Larynx - (Fig. 30). - This forms a compressible ventrimesal prominence about midway between the præsternum and the chin when the head is dorsiducted so as to bring the ventral surface of the neck and head into the same plane.

Lateral Somatic Landmarks.-These, of course, are in pairs, but they will be spoken of in the singular number. 
§ 229. Zygoma-The Arcus zygomaticus-(Fig. 30, 56).-Both the dorsal and ventral borders of this may usually be felt distinctly, although in old males it is more or less obscured by the thickness of the skin and connective tissue upon the cheeks. The dorsal border is nearly in line with the lateral angle of the eye.

Diapophysis atlantalis-Transverse process of the atlas vertebra-(Fig. 30, 52).-This may be felt as a ridge just caudad of the base of the ear. The caudal angle is more distinct, and the soft parts on its ventral side are less prominent and firm than those upon the dorsal.

Scapula-(Fig. 30, 44).-The middle of the convex vertebral margin of the scapula projects dorsad of the intervening cervical neural spines, so as to give rise to the Depressio interscapularis which is so marked while the living cat is on its feet. Upon a lean animal, the following scapular prominences may be easily determined: mesoscapula, with its tuberosity (Fig. 44); gleno-vertebral angle (Fig. 43); (the coraco-vertebral angle is so heavily overlaid with muscle as to be less easily felt); acromion (Fig. 44, 45); metacromion.

\$230. Costa 13-Last rib.-The abdominal parietes just caudad of the last rib are easily indented by the finger almost to the apex of the triangular area which intervenes between it and the lateral border of the vertebral muscles (Fig. 30).

Crista ilii.-When the finger is carried along the border of the vertebral muscles at about the same distance from the meson as the apex of the angle between them and the last rib, the Crista ili $i$ is felt as a rounded ridge nearly dorsad of the knee (Fig. 51).

Tuberositas ischii-Ischiatic tuberosity. - This is felt as a blunt prominence ventro-laterad of the anus.

Clavicula -'The clavicle or collar bone-(Fig. 30, 48, 67, 72).-In young or lean animals this may be felt by pinching up the skin and subjacent muscles between the shoulder and the præsternum.

Membral Landmarks.-These, likewise, are in pairs, but are spoken of in the singular number.

$\S 231$. Arthra.-After what has been said in the general description of the skeleton ( $\$ 214-223$ ), there will be no difficulty in ascertaining the position of any of the membral arthra. Most of the bony prominences also may be recognized readily from their relations to the arthra.

Trochiter-(Fig. 30, 46)-The greater tuberosity of the humerus.- 
This forms a marked projection at the convexity of the shoulder, a little ventro-cephalad of the acromion.

Olecranon, Epicondylus and Epitrochlea-(Fig. 30, 46).--These prominences about the elbow joint are recognized without difficulty even in fat animals if the parts are manipulated between the thumb and fingers.

M. biceps-(Fig. 74, 75).-The fusiform body of this muscle may be felt on the ventral aspect of the antebrachium by rolling the soft parts gently between the fingers upon the humerus. It is less distinct than in man, on account of the more distal extension of the insertion lines of the pectoralis group of muscles.

$\S 232$. Capitellum radii-Head of the radius.-By alternately pronating and supinating the manus while a finger is pressed upon the elbow a little ventro-distad of the epicondylus, the capitellum may be felt during its rotation.

Processus styloides ulnce et radii-The styloid processes of the ulna and radius.-These are to be felt at the caudal and cephalic sides of the wrist joint.

Os pisiforme-(Fig. 30).-This forms a marked and somewhat movable projection just proximad of the wrist, and on the caudoventral border of the antebrachium. Just distad of it is a prominent callosity - the hypothenar eminence (Fig. 105).

§ 233. Trochanter-Trochanter major femoris-(Fig. 30).-This projects considerably from the hip, a little ventrad of a line between the Crista ilii and the Tuberositas ischii, and a little nearer the latter. Its movement is distinct when the leg is moved.

Patella-The knee pan.-When the crus is extended so as to relax the muscles connected with the patella, this bone is easily moved from side to side.

Calcaneum.-This forms the marked projection on the ventral aspect of the leg just proximad of the ankle; it is sometimes called the hock.

Malleoli.-These processes of the distal ends of the tibia and fibula are readily distinguished at the cephalic and caudal sides of the ankle.

\section{ABDOMINAL TRANSECTION.}

$\S 234$. Since most of the dissections herein described involve only the thorax, neck, head and arms, it is usually more convenient and economical to divide the body into caudal and cephalic parts. 
The former may then be thrown away, and the latter preserved, or dissected while fresh. The kidneys are important visceral landmarks, and are easily preserved, hence they may be retained with the cephalic part.

Abdominal transection may be performed alone, but it is more conveniently done when another person holds the cat in the desired positions. The assistant, also, may read the directions to the operator.

§ 235. Instruments and Materials. - Arthrotome; tracer ; medium scalpel; coarse curved scissors; hair scissors; block; twine, about half a meter; skeleton; wide mouthed jar (about $6 \times 12,15$ or 18 in.) ; alcohol, 52-67 per cent., to half fill the jar; coarse syringe ; large tray.

$\S 236$. Parts Involved.-The following parts are more or less directly involved in Abdominal Transection. From the figures and sections referred to, enough should be learned to enable the operator to recognize them during the operation :-

- Aorta.-Fig. 101.

Columna Vertebralis.-The spinal column.

Costo-Ribs.-Fig. 30, 50, 73.

Costicartilagines-The costal cartilages.-Fig. 30, 50.

Crista ilii-The crest of the ileum.-Fig. 30, 51.

Diaphragma-The diaphragm.-Fig. 90, 101.

Epigastrium-The "pit of the stomach."-Fig. 30, 72.

Fibro-cartilago intervertebralis-The (sixth lumbar) intervertebral disk of fibro-cartilage.-Fig. 51 .

Hepar-The liver.-Fig. 77.

Intestinum Tenue-The small intestine.-Fig. 77.

Ligamentum Suspensorium Hepatis-The suspensory ligament of the liver.

Mesenterium.-Fig. 78.

OEsophagus-The gullet.-Fig. 107.

Parietes Abdominales-The abdominal parietes, the muscular and membranous lateral and ventral walls of the abdomen.-Fig. 77, 101.

Pelvis-The pelvic girdle.-Fig. 30, 51.

Postcava-Vena cava inferior s. ascendens.-Fig. 101.

Rectum.-Fig. 77.

Renes-The kidneys.-Fig. 79, 101.

Stomachus-The stomach.-Fig. 79, 81. 
Thorax-The chest.-Fig. 30, 77.

Vertebrce lumbales-The lumbar vertebræ, with their diapophyses and zygapophyes.-Fig. 30, 51, 55.

Xiphisternum-The ensiform cartilage of the sternum.-Fig. $30,49$.

\$237. Operation.-Place the cat dorsicumbent, the head to the left. Pinch up the right abdominal parietes about midway between the crista ilii and the last rib.

With the arthrotome, transfix the fold so pinched up, and cut ento-ectad to the surface. If only the skin is divided at the first attempt, pinch up the muscles and repeat, the transfixion, but be careful not to include any of the intestines within the fold.

Into the incision so made, insert the left index and medius, the hand being supinated, and lift the parietes away from the viscera. Introduce the scalpel, with the edge directed ectad, and divide the parietes on each side until the thicker vertebral muscles are reached a few centimeters from the dorsimeson." The dorsal ends of this transverse incision should be just caudad of the kidneys.

About $2 \mathrm{~cm}$. sinistrad of the ventrimeson, divide the parietes longitudinally to the pelvis and the epigastrium, making sure not to cut the xiphisternum or costicartilages.

Turn aside the four rectangular flaps so formed, and draw the intestines caudad. Introduce the pronated left hand between the liver and the parietes until the fingers are met by the diaphragm. Partly flex the fingers, and push them somewhat dorsad; then draw the liver and stomach caudad; this will expose the Tendo centralis of the diaphragm, which should be punctured with the tracer. The admission of air into the thorax will permit the farther caudiduction of the liver and stomach.

\$238. If the cephalic part is to be preserved, ligate the rectum and then the stomach and liver, and inject 84-95 per cent. alcohol into the aorta, as directed in $\$ 285$. After two hours a plaster injection may be made if desired. Then proceed as in $\S 239$.

If the parts are to be dissected fresh, or if the muscles are to be exposed and the thorax opened so soon as to render the alcohol injection unnecessary, proceed as in $\$ 239$.

$\S 239$. Insert the curved scissors, keeping the convexity toward the diaphragm, and divide the Ligamentum suspensorium of the liver, the postcava and the abdominal asophagus; while so doing, the viscera should be drawn ventrad as well as caudad, so as to put the parts named upon the stretch. 
When the liver and stomach are free, draw them and the intestines caudad, dividing the mesal peritoneal attachments (mesenterium) to a point $5-6 \mathrm{~cm}$. caudad of the kidneys, but do not remove the latter.

Compress the rectum between the fingers so as to force its contents in both directions ; ligate it in two places about $2 \mathrm{~cm}$. apart, and cut between the ligatures (Fig. 41). The viscera thus freed may be thrown away unless wanted for some purpose.

$\S 240$. Place the block under the cat opposite the kidneys. Press upon the thick muscles just caudad of the kidneys, and about $2 \mathrm{~cm}$. laterad of the meson. The sixth lumbar diapophyses (see Fig. 30) will be felt. With the arthrotome, divide all the soft parts on the ventral aspect of the lumbar vertebra between the tips of the sixth diapophyses, and scrape them caudad for about $2 \mathrm{~cm}$. This will expose a transverse whitish swelling, the intervertebral fibro-cartilage between the sixth and seventh lumbar vertebræ.

If the kidneys are not to be kept, or if it is desirable to make the whole preparation shorter, the columna vertebralis may be divided between the second and third or third and fourth lumbar vertebræ.

Push the arthrotome into this fibro-cartilage, and cut dextrad and sinistrad as far as possible. Then cut latero-cephalad on each side to the tip of the diapophysis; then directly laterad so as to divide the thick vertebral muscles.

Grasp the cephalic part of the cat just cephalad of the incision, and lift it from the tray. If this does not disjoint the zygapophyses, bend the caudal part downward until the ligaments give way, and the zygapophyses are separated. Cut the remaining soft parts and the skin, and thus complete the transection. The caudal part may be thrown away.

Knead the thorax so as to expel the blood, place the cephalic part-which may be spoken of now as the cat-upon a clean dry tray, or upon paper laid in a tray, and remove the hair, as directed in Chap. VI. ; note the precautions as to the disposal of hair, $\S 197$.

$\S 241$. Preservation.-Tie the twine firmly about the last lumbar vertebra (which is the sixth if the directions have been followed), and make a loop through which may be passed a second piece of twine already attached to the ring of the jar cover, or, better, an S-hook connected with the cover by a string of suitable length. To the vertebra should also be tied a tag bearing the brief record of the sex of the cat, its age or apparent period of growth, 
its color, the date of killing, and the name or initials of the dissector; a similar tag should be attached to the jar. The cat may then be suspended in the jar head downward; all parts should be immersed in the alcohol. If any parts press closely against the sides, some cotton or "excelsior" should be interposed.

If the cat can be left in the alcohol for at least a week, the parts are more completely and uniformly hardened.

$\S 242$. Thoracic Transection. - This is sometimes desirable when only the abdominal and pelvic regions are to be examined or preserved.

The primary incision should be transverse, about $2 \mathrm{~cm}$. cephalad of the epigastrium. The mesosternum may be divided with the nippers, and the incision then carried on either side in the 7 th intercostal space. The thoracic viscera are readily separated from the diaphragm, the cesophagus, aorta, and postcava may be cut 1-2 $\mathrm{cm}$. from the diaphragm, and the dislocation of the columna vertebralis effected between the seventh and eighth thoracic vertebræ.

If the abdominal viscera are to be preserved, strong alcohol should be injected into the aorta, and into the abdomen by a slit just large enough to admit the canula ; the contents of the stomach and rectum should be, if possible, washed out with a stream of water, aided by kneading; alcohol should then be injected into both cavities, the esophagus tied, and the rectum plugged with cotton.

The abdominal viscera are easily displaced, and it is well to let the caudal region of the body, with or without the legs, rest dorsicumbent in alcohol for several days, the jar being securely closed, and placed on its side in a tray. The alcohol should be renewed at the end of a week.

\$ 243. Removal of the Tail.-In some cases it may be desirable to remore the tail.

Girdle it with the arthrotome near the base, and divide it with the nippers and bone scissors. With a young animal, the bone scissors may be used without the nippers.

With a freshly killed animal, when bleeding is nndesirable, a twine ligature may be tied firmly just cephalad of the point of intended removal.

If only the muscles, vessels, and nerves are to be studied, the claw points may be removed with nippers. 


\section{CHAPTER III.}

PREPARATION OF BONES-PERMANENT PREPARATION OF SOFT PARTS-THE CSE OF ALCOHOL, ETC.-JARS, ETC.-FROZEN SECTIONS AND DISSECTIONS-INFLATED PREPARATIONS-MEASUREMENT OF VOLUME AND CAPACITY.

\section{PREPARATION OF BONES.}

Special Instruments and Material :-Arseniate of Soda-Beans or Peas-Benzine - Bone Drill - Cement - Ether - Glycerin - Labels ( $\$ 162$ )-Liquid Soup - Macerating Dishes - Nail or Tooth Brushes - Salt Solution - Syringe - Turpentine Oil - Wicker. sheimer's Liquid.

$\S 244$. There are four principal methods of preparing bones for scientific purposes :-

(1) Cutting and scraping the soft parts from alcoholic specimens.

(2) Allowing Ants or Dermestes to remove the soft parts of fresh specimens.

(3) Maceration (putrefactive) in water.

(4) Boiling with liquid soap.

General Directions.-A. If one can choose his specimen, a young adult should be selected. In too young animals the epiphyses are apt to separate easily from the diaphyses, and the symphyses open too easily. On the other hand, old animals sometimes have exostoses, or abnormal growths on their bones, and the symphyses and sutures are liable to be entirely obliterated.

B. If possible, have at hand for reference a perfect skeleton of the part to be prepared, so that the exact position of delicate bones and processes may be seen, and hence not be lost or broken through inadvertence.

C. Whatever method is employed, it is better to divide the animal into several parts by cutting some of the principal arthra, as the humero-scapular, the femoro-innominate, the occipito-atlantal, and the lumbo-thoracic.

D. Labeling.-The whole animal should bear a label giving the name, date, sex, and, if possible, the age. Each separated part 
should bear a label giving the above general data, and also the name of the part. This is imperatively necessary with the vertebræ, the ribs, and the phalanges; hence each must be carefully labeled as it is separated from the rest of the body.

E. If the methods of maceration or heating with liquid soap are employed, parts like the pelvis and scapulæ, and limb bones, exclusive of the manus and pes, which cannot be mistaken, may be put together and labeled as for the whole animal. Parts that might be difficult to distinguish should be kept in separate dishes, and each properly labeled. The vertebræ might be divided into the four sets-cervical, thoracic, lumbar, and sacral; each set should then be connected by attaching a Manilla hemp string to a wire and passing it through the neural canal and tying the ends. The order of the bones cannot then be changed as they separate in the macerating process.

The caudal vertebræ that have no neural canal must be numbered or put in separate dishes or vials and properly labeled.

The ribs of one side at least should be removed one by one, and tied in order on a string, or put into separate dishes.

Never put bones of different animals in the same dish, unless they differ so greatly in size or conformation that confusion would be impossible.

F. The humerus, femur, tibia, and radius should be drilled at or near their ends, so that the oily matter in the medullary canal may be removed. The tibia and radius may be drilled in the articular surfaces, care being taken to reach the center of the thickness of each bone. The distal end of the femur may be drilled, but the proximal hole should be made on the ventral side, between the trochanter and the articular head. With the humerus, the proxintal hole may be made in the articular surface, about midway between the base of the trochiter and the trochin, but cephalad of the slight extension of the Canalis bicipitalis. The distal hole may be drilled half way through the diaphysis, from a point on the cephalic aspect, at the junction of the third and fourth quarters.

After the holes are made, the medullary matter may be broken up with a wire, and most of it expelled by syringing, first with warm water and afterward with liquid soap, or spirits of turpentine, or ether. The syringing should be repeated at the close of whatever process is adopted for removing the flesh.

With most langer animals, and with some smaller ones, it may be desirable to drill the ulna and fibula also. 
\$ 245. Removing Soft Parts from Alcoholic Specimens.Animals that have been preserved in alcohol may have their bones cleaned by simply cutting and scraping away the soft parts. It will be necessary to take great care, however, or the delicate processes, especially of the skull, will be broken in getting off the tough connective tissue. After the bones are cleaned as well as possible, simply allow them to dry, or finish the cleaning as directed hereafter $(\$ 249)$.

\section{\$ 246. Allowing Ants or Dermestes to remove the Soft} Parts of Fresh Specimens.-The skin and most of the flesh should be dissected away, and the partially cleaned bones left in a damp room. Dermestes will find them, and the larvæ of the beetles will remove the soft parts.

If the aid of ants is to be sought, the bones prepared as just directed should be sprinkled with sugar or smeared with molasses or honey. Then they should be placed in a box pierced with small holes. The box should be put by an ant's nest, and some sugar sprinkled around the holes leading into it. The ants will clean the bones more satisfactorily than the Dermestes. It usually takes about a week for them to clean a cat's arm.

$\S 247$. Maceration in Water.-Employ stone-ware, porcelain or glass dishes, if possible, to avoid discoloration of the bones. The bones are freed from skin and most of the soft parts, separated, placed in separate dishes as directed above, and covered completely with clean soft water. The dishes should then be placed in a room where the temperature does not fall below 18-20 C. If the room is still warmer, the maceration will proceed all the more rapidly. The water should be changed on the third day, and again on the tenth, to avoid discoloration.

It requires from a fortnight to two months for complete maceration. The bones of very large animals may require even a longer time.

If possible, maceration should be done in a separate building, and during warm weather. If done during cold weather, the fire should not be allowed to go out, or adipocere, a waxy substance, may form, which is difficult to remove. If the maceration is done in a room or closet, there should be a special ventilating flue ( $\& 196)$. During maceration the bones should be occasionally examined. When the soft parts separate readily, the water covering them should be carefully poured off, and a gentle stream of fresh water 
allowed to play upon them. Use a nail or tooth brush to remove the flesh, and if it is necessary employ the scissors, forceps, and a dull but smooth-edged knife to clean some parts; the nippers aid in tearing off ligaments and tendons. After all the soft parts are removed, rinse the bones well with clean water, and place them on white paper in a dry room. Do not fail to accompany each bone by its proper label $(\$ 244 \mathbf{D})$.

'The vertebræ, ribs, etc., should be kept on a string after cleaning, or when dry they may be numbered. The skeleton of each animal should be kept in a separate box and properly labeled.

$\S 248$. Liquid Soap Process.-The cleaning is done in this process by heating the bones in a dilution of the following mixture: Rain (or distilled) water, $2000 \mathrm{cc}$. ; strong ammonia, $150 \mathrm{cc}$. ; nitrate of potash (saltpetre), 12 grams ; hard soap, 75 grams.

Prepare the bones by removing the slin and most of the soft parts, as directed for macerating ( $\$ 247$ ).

Place the bone, or-if several are to be prepared from the same animal--those that cannot be mistaken for one another, in a vessel containing water, 4 parts, and liquid soap, 1 part.

Prepare enough of this mixture to completely immerse the bones. Boil in this forty minutes; then pour off the liquid and add a similar amount prepared in the same way. Boil again for half an hour; and usually the muscles may be removed by the hands, a smooth stick, or a scalpel handle.

As it would take too long for the bones to cool spontaneously before working upon them, they may be placed directly in cold water.

After removing all the muscles that come off readily, replace the bones in the dish and continue the boiling until the soft parts may be readily removed with a nail brush.

When removing the soft parts, it is necessary to be careful not to lose small bones.

After all the soft parts are removed, it is usually best to boil the bones for half an hour in a mixture of equal parts of the liquid soap and water to remove the last remnants of grease.

Finally rinse the bones well with clean water and lay them upon white paper to dry.

This is by far the best method of preparing bones: (1) The liquid soap saponifies the fat and aids in softening connective tissue. (2) The bones come out white and free from grease. (3) It requires 
but a very short time to prepare a skeleton or a part of a skeleton. (4) It is especially adapted for skulls, as the teeth are much less liable to fall out, and the gelatinized dental periosteum serves as a cement. (5) There is no danger of blood poisoning (septicæmia) if fresh, healthy animals are used. There is no danger in any case after the bones have been well boiled.

$\S 249$. Bones are not satisfactorily cleaned by ants or Dermestes. The cleaning may be very satisfactorily completed, however, by boiling in the mixture of liquid soap (\$248).

The final cleaning of alcoholic specimens is best done in the same way.

\$250. Preparation of Skulls-A. By Maceration.-Separate the skull, remove the skin, the eyes and the tongue. Then separate the mandible, but let the os hyoides remain. With a tracer or a piece of wire, inserted through the Froramen magnum (Fig. 57), break up the brain, taking care, however, not to injure the projecting bony tentorium (Fig. 59). After the brain is broken up it should be washed out with a syringe.

Place the skull and mandible in the macerating dish so that the teeth shall be uppermost; then, if the water is changed carefully, the teeth are less apt to fall out and be lost.

The cleaning should be done as described above ( $\$ 247)$, except that greater care is usually necessary.

B. By the Liquid Soap Process.-The skall should be prepared as described for maceration, except that the mandible need not be separated. Proceed as directed above $(\$ 248)$.

Skulls can be much more quickly and safely prepared by the liquid eoap process than by any other.

$\S 251$. Cements for Bones and Teeth.-The pelvis and mandible often separate at their symphyses, and the teeth may become loose and be in danger of falling out. This latter is especially liable to occur with macerated skulls. To unite bones, one should use :-

A. Liquid gelatin (see Formula, \& 1446). "Attach the bones firmly by a rubber band or string' while the gelatin is drying. 'The method is the same as for gluing wood.

Teeth may be fastened in their sockets by the same substance, only it is unnecessary to bind them in while the gelatin is drying. The skull should rest on its dorsal side, and the mandible on its ventral side, so that the teeth may remain in place during the drying. 
B. An excellent cement, for teeth especially, is the following :--

Silicate of soda or potassa (liquid glass) mixed to a paste with powdered chalk. This hardens quickly, therefore it must be used soon after it is prepared. Neither this nor the gelatin will resist water.

As soon as a macerated skull is rinsed, the teeth that are loose should be taken out one at a time, the socket partly filled with one of the cements and the tooth replanted. In this way the teeth may be kept in their proper places, and as the skull dries the teeth will be firmly fixed. Although the teeth of skulls prepared by the liquid soap process may be loose before the skull is dry, the gelatinized dental periosteum will usually fasten them very firmly as the skull dries. If any of the teeth come out they should be replanted as directed above.

Need of Accuracy,-So far as concerns the general aspect of the skull, the incisors may be interchanged, and missing teeth may be replaced by teeth from other skulls. It is to be feared that such substitutions are sometimes made with skulls prepared for sale. Unless the changes are carefully specified, such skulls have no real scientific value as regards the teeth.

When two or more of the teeth drop out at the same time, unless direct observation or comparison with a fresh or alcoholic specimen warrants absolute certainty, it is better to keep those teeth in a box separate from the rest.

\section{\$252. Preparation of Natural Skeletons.-By a natural} skeleton is meant one in which the bones are held together by their natural ligaments. Such a skeleton may be prepared according to either of the processes described above. One must, however, watch that the ants may remove only the muscles, or that, in the process of maceration, only the muscles decay. In all the procosses, the muscles are softened or removed first. If the liquid soap process is employed, proceed as follows: Boil the bones in the mixture as directed $(\$ 248)$ until the muscles may be removed without much trouble. It will be found necessary, however, to allow the boiling to proceed only to the point where the muscles will separate from the bones by using the hands, a smooth stick like a scalpel handle, or a dull knife blade. The ligaments will be found considerably swollen and somewhat softened. The softened ectal surface may be cautiously scraped off; then the preparation should be soaked for 3-6 hours in a saturated solution of arseniate of soda (about 25 per cent.), to poison the ligaments and protect them from Dermestes. Then the part should be arranged as nearly as possible in a natural 
position and fastened with pins or strings and allowed to dry. The swollen ligaments will shrink very greatly, so that what might have seemed a very imperfect preparation when moist will be excellent when dry. When the specimen is dry, rough projections of ligament may be removed with a sharp knife.

Flexible Natural skeletons.-If it is desired to have the ligaments flexible, the preparation may be soaked after it is partly dry for from 16 to 12 hours in either Wickerscheimer's fluid ( $\$ 299$ ), or a mixture of a saturated aqueous solution of arseniate of soda, 4 parts, and glycerine 1 part.

$\S 253$. Preparation of the Bones of Young Animals.-The liquid soap process is to be preferred. But during the boiling the bones must be carefully watched, and the boiling should be carried little farther than for making natural skeletons. The epiphyses must not be allowed to separate from the diaphyses.

The skulls of new born kittens may be nicely prepared in this way, and the sutures show with great distinctness.

§ 254. Disarticulating Skulls.-Choose a young or barely mature animal for this preparation, since the cranial sutures are liable to be obliterated in adults.

Prepare the skull by the liquid soap process (\$ 248). Continue the final boiling for half an hour longer than for a skull that is not to be disarticulated. While still moist, the bones may be separated by steady traction. This may be done with the greatest ease if half grown animals are used.

Skulls that have become dry may be boiled for half an hour in the liquid soap to soften the gelatinized connective tissue binding them together.

Macerated young skulls may be disarticulated by carefully pulling and prying the bones apart.

An excellent plan is to fully disarticulate one side of a skúll, and to leave the other with the bones in situ.

Disarticulation of Large Skulls.-If a large skull is prepared by the liquid soap process it should be thoroughly softened by soaking in water two or three days or by boiling an hour. Then fill the cranial cavity with dry beans or peas, force a cork tightly into the foramen magnum (Fig. 57) and place the skull in water. The swelling of the peas will force the bones apart. Macerated 
skulls should be treated as just described, but they need not be boiled.

$\S 255$. Bleaching Bones.-In order that bones should be white and clean, it is necessary that they be relieved of all their grease and blood. Neither of these ends is accomplished when the flesh is removed by insects-ants or Dermestes. Both are fairly well accomplished by proper maceration; and both still better by the liquid soap process.

The old method, and the one still largely employed in whitening bones, is to place them where they are constantly exposed to the action of the sun, rain and dew. The following methods are, however, more speedy and satisfactory :-

The blood is usually got rid of in the process of boiling or maceration. If, however, one wishes to prepare bones in the best possible manner, the entire vascular system may be washed out with normal salt solution. To do this, insert a canula into the arch of the aorta (Fig. 101); open the præcava (Fig. 101) just peripherad of the heart, and then, with a syringe, inject salt solution into the aorta until it runs uncolored from the præcava.

\$256. Freeing Bones from Grease.-There are four ways of removing grease from bones:-

A. By suspending them in spirits of turpentine-Oleum terebinthinæ rectificatum-(ordinary commercial oil of turpentine will do), for three or four weeks. An indefinite soaking in turpentine will do no harm if the bones are suispended, and more than four weeks may be necessary for large bones.

The turpentine should be rery fluid.

The bones must be suspended so that the thick, oily substance that settles to the bottom of the vessel cannot touch them.

After the bones have soaked for a sufficient time in the turpentine, they should be exposed to sunlight but not to rain.

B. Suspension in benzine (common commercial benzine will do very well). The bones should be treated as directed for turpentine.

C. Soaking the bones in sulphuric ether. The bones need not be suspended, simply placing them in the vessel with the ether will be sufficient. The ether dissolves the grease very quickly, so that small bones like those of the cat are entirely freed from grease in a fortnight or even a less time. The bones should be exposed to light as directed above. 
Ether is expensive, but the use of it for removing grease from bones does not injure it for anasthetizing animals.

As turpentine, beuzine, and ether are very volatile, they must be kept in tight vessels. The preserving jars (Fig. 32) answer very well for the bones and either of these agents.

Either of the three preceding methods may be employed for removing grease from natural skeletons. The following method is also good for that purpose, but not quite so safe, as there is some danger of loosening the ligaments.

D. Soaking the bones in liquid soap. Place the bones in a dish of liquid soap ( $\$ 248$ ) and let them remain for three or four days, in some cases longer, then wash them very thoroughly with clean water. Dry them and expose them to the sun ( 256). It is not usually. necessary to treat bones that have been prepared by the liquid soap process, but bones that have been macerated or prepared by insects may be relieved of their grease in this way. It may be desirable to mix the liquid soap with an equal amount of water and boil for half an hour or more.

\section{GENERAL REFERENCES TO THE PREPARATION OF BONES.}

Baker, E, A, Appendix.

Heath, A, Appendix.

Straus-Durckheim, B, I, 285.

Brown, A, 94.

Witt, A, Appendix.

Treasury of Natural History, A, 790.

\section{THE PRESERVATION OF SOFT PARTS.}

\$257. Practically, a bone once properly cleaned is imperishable, and needs only to be protected from dust or injury. But all the soft parts of the body are more or less prone to decompose at common temperatures and under ordinary conditions; even when treated with preservatives, they are liable to deteriorate unless constantly cared for.

Many agents have been employed for the delay or prevention of decomposition, and for the permanent preparation of soft parts. Personal experience enables us to speak confidently of only three-cold, arseniate of soda, and alcohol.

A few other agents-brine, methyl alcohol, chloral and Wickersheimer's liquid-will be briefly mentioned.

$\S 258$. Cold.-Decomposicion is prevented by a temperature of 0 C. (32 F.), and is more or less retarded at temperatures between 0 and 10 .

Cold may be employed alone for freezing specimens so as to keep indefinitely; more 
often, decomposition is simply retarded by it, while the specimen is temporarily protected by arseniate of soda or permanently preserved by means of alcohol.

Frozen sections and dissections will be discussed later in this chapter.

\$259. Arseniate of Soda-Sodinn Arsenias. $-\mathrm{A}$ saturated solution of this poisonous salt may be used for the injection of specimens which are to be dissected fresh, or for the poisoning of inflated preparations.

Water dissolves about one fourth of its weight of arseniate; hence the saturated solution is 20-25 per cent. It should not be allowed to remain upon the dissector's skin longer than necessary.

Here and elsewhere, when a solution or mixture is mentioned, the menstruum is vater unless the contrary is stated.

\section{ALCOHOL.}

\$260. Alcohol is almost essential in practical anatomy; its qualities are active, and its management requires constant care.

General Description.-The alcohol commonly employed for Natural History purposes is, strictly speaking, ethylic alcohol, and a member of a group including several others; the amylic, butylic, propylic, methylic, etc. The last named variety will be mentioned again in \$298.

Ethyl alcohol is colorless, volatile, and inflammable; it has a vinous odor and pungent taste; it coagulates albuminous substances and extracts more or less water from organisms immersed in it. Its composition is $\mathrm{C}_{2} \mathrm{H}_{6} \mathrm{O}$. Its specific gravity is 796 .

Absolute Alcohol.-The foregoing statements as to composition and specific gravity really apply only to pure or absolute alcohol, that is, alcohol which has been freed from water and all impurities. This, however, is quite expensive (about $\$ 8.00$ per gal.), and is required only for some histological purposes.

Ordinary or Commercial Alcohol contains from 5-60 per cent. of water. Most of these mixtures are commonly called alcohols, but some of the grades have also special names, as indicated upon the following Table :-

§ 261. Table of Specific Gratities of different Percentages of alcohols AT $155_{5}^{\circ} \mathrm{C}, 60^{\circ} \mathrm{F}$.

100, .796, Absolute alcohol.

95, .809, [Commercial alcohol.]

9 2, .817, Alcohol fortius, U.S.

89,825 , Lightest spirit obtained by ordinary distillation.

$85, .885$, Alcohol, U. S., spiritus rectificatus, rectified spirit.

\author{
$84, .838$, Spiritus rectificatus, $\mathrm{Br}$. \\ $75, .860$. \\ $60, .896$. \\ $50, .917$, [Strong liquors, whisky, etc.] \\ $49, .920$, Spiritus tenuior, proof spirit, $\mathrm{Br}$. \\ 39, .941, Alcolol dilutum, U. S.
}

United States Dispensatory, 13th edition, 1880. See also Watts, A, and Baley, A.

Alcohol as received from the warehouse usually contains about 95 per cent. of absolute alcohol; but that which has been kept for some time, especially if frequently exposed to the air, is apt to range from 90-94 per cent., on account of evaporation and the alsorption of water from the air. 
The strong liquors-brandy, gin, rum, and whisky-contain from 45-55 per cent. of absolute alcohol. A mixture containing less than 20 per cent. is not directly useful in zoology.

\section{\$262. The Leading Characteristios of Alcohol.-Ethyl alcohol} has twelve prominent characteristics, of which one half are desirable and the other half undesirable from the zoological point of view :It is simple, cleanly, colorless, and fragrant, generally obtainable, and-as a preservative-absolutely efficient.

On the other hand, it is costly, volatile, inflammable, and decolorant, quick to absorb water from the air, and-under certain conditions-corrosive of some metals.

§ 263. Alcohol should be used with discretion, always closely covered, secured from fire, and kept in vessels of glass, hard wood, copper, zinc, or galvanized iron.

\$264. The Cost of Alcohol.-This, of course, is an extrinsic feature, and varies much in different parts of the world. In most civilized countries, to the actual cost of its production is added a heavy government tax, and the retail price in the United States is about $\$ 2.50$ per gallon, or $50-75$ cents per liter.

This bigh price of the liquid best adapted for the preservation of specimens has directly and seriously retarded all anatomical and zoological progress. At various times between 1863 and 1879, at the request of the late Prof. Louis Agassiz and others, with the co-operation of the Hon. S. Hooper and others, Congress wisely made provisions (U. S. Revised Statutes, §3297), by which, under very stringent conditions and with heavy pecuniary liabilities in case of the slightest misapplication, museums and other educational institutions " may withdraw alcohol from bonded warehouses without payment of tax, for the sole and exclusive purposes of use in the chemical laboratory, or for the preservation of Natural History specimens belonging to such institutions." The cost of alcohol so obtained is about one sixth of the retail price. See Appendix.

The instructions for so obtaining alcohol for scientific purposes are printed in No. 7 , Series 7 of the U. S. Revenue Depurtment, p. 43, which may be had from the Collectors.

Since no variation from the prescribed forms is permitted, great care must bo taken in making out the application and bond ; those who obtain alcohol annually will save troublo and sometimes serious delay by liaving the forms printed.

After making out the form of the application and bond, and inserting the names of the two sureties, some proprietor of a bonded warehouse should be asked to set aside the number of barrels (40-45 gals. each) of alcohol desired, and to send a memorandum of the marks and other items required in the papers. If the forms are printed, a copy of the application form may be sent, so that the various numbers may be filled in, together with the number of the collection district in which the warehouse is located. This copy should be retained for reference in making the assurance afterward required.

The papers should then be promptly filled out, care being taken that the signatures of the sureties coincide as to initials and abbreviations with the names as entered in the body of the bond. The papers are to be transmitted to the Commissioner of Internal Revenue through the Collector of the district in which the institution is located. If the papers have been properly made out, the permit may be received within ten days.

No form is prescribed for the assurance of the Revenue Department that the alcohol 
has been used for the prescribed purposes within the spe ified time. It is necessary to make oath before the proper officer to this effect, giving the marks of the alcohol and the date of the original application. This affidavit is to be transmitted to the Commissioner of Internal Revenue throngh the Collector of the district in which the institution is located.

Since it is not always easy to ascertain the location of warehouses, it may be proper to mention that alcohol may be obtained promptly under the foregoing conditions from Messrs. Geo. \& Thos. Farthing, of Buffalo, in the 30th collection district of the State of New York.

\& 265. Inflammability.-At ordinary temperatures-15-20 C.-a mixture of alcohol and water containing 30 per cent. or less of absolute alcohol cannot we ignited, and promptly extinguishes a lighted match dipped into it; 35 per cent. ignites with difficulty, and the flame is extinguished by the lightest current of air ; 40 and 45 per cent. ignite more readily, but burn gently and slowly. Even 65 per cent. does not buru fiercely, and the flame is easily extinguished. Specimens saturated with alcohol are more combustible in proportion to the strength of the alcohol.

Other Inflammable Substances. - Still more volatile and inflammable are the ether, benzine and spirits of turpentine which are used in anatomical work.

§ 266. Precautions against Fire.-Alcohol, benzine, ether, and spirits of turpentine in bulk should be stored in a fire-proof vault, or in some small building apart from valuable collections and apparatus. In the laboratory there should be not more than 20 liters of $9 \tilde{0}$ per cent. alcohol, and of the other liquids only enough for current uses. All of them should be kept in glass or copper vessels, well stoppered and at a safe distance from all lights and beating apparatus. They should never be opened within one meter of a light, and if there is a current of air toward the light, the distance should be at least doubled.

When removed from alcohol for examination or dissection, specimens should be washed off with water, and kept wet with the 1is per cent. glycerin solution, as directed elsewhere. This precaution is the more essential when anatomical work is done by artificial light.

Cotton and cloths which have been saturated with alcohol or other inflammable liquids should be dried on trays in the sun or wind rather than near a fire. Common cotton should be thrown away after use in alcohol, but absorbent cotton may be saved if thoroughly dried.

Safety matches are to be preferred. All matches should be kept in metal or glass boxes. After using, matches should be put into a glass or metal receptacle, and never thrown on the floor.

Smoking in a laboratory where alcohol is used should be absolutely forbidden.

$\S 267$. Determination of the Percentage of Alcohol in an Alcoholic Liquid.-This may be done by means of either a Specific Gravity Hydrometer or an Alcoömeter (alcoholometer). For zoological purposes the latter instrument is more convenient and sufficiently exact.

\$ 268. Alcoömeter (alcoholometer).-This is a form of hydrometer or areometer especially adapted to determining the volume or weight percentage of alcohol in a mixture of alcohol and water.

It is a graduated tube, loaded so as to rest vertically in any liquid capable of floating it. The alcoömeter of Tralles is commonly employed in this country; it indicates the volume per cent.

In pure water the instrument sinks only to zero, the lower end 
of the scale. In absolute alcohol it sinks to 100, the upper end. Mixtures of the two liquids permit it to sink to various depths, and the number corresponding with the surface of the liquid indicates the percentage of alcohol by volume.

A. Alcohol that has been used for the preservation of specimens usually holds in solution or suspension substances of greater specific gravity than alcohol or even water. Their presence increases the specific gravity of the liquid, and causes the per cent. of alcohol to appear less than it really is. Old alcohol should therefore be cleared (\$ 294), if necessary, before testing with the alcoömeter; but filtration will not, of course, remove materials which are in real solution.

B. As stated by Günther ( $8 \mathrm{~A}, 697$ ), the Britisl hydrometer is so arranged that the zero corresponds with proof spirit, about 49 per cent. The other grades are designated as so many degrees above or below proof, and two degrees equal but one per cent. For example, our 95 per cent. alcohol would be 92 above proof, while 20 below proof would indicate the presence of 39 per cent. of absolute alcohol.

§ 269. Hydrometer Jar.-This is a tall and narrow glass jar, mounted on a foot. The alcohol to be tested is poured into it, and the scale may be read through the glass.

Any glass jar of sufficient height (e.g. the $3 \times 10 \mathrm{in}$. jar of Whitall, Tatum \& Co., A), will answer in using the alcoömeter, but the narrowness of the proper hydrometer jar requires a less quantity of the liquid, and the scale is read more easily. A cylindrical graduate on a foot, and holding about 500 cc., makes an excellent hydrometer jar, but is more expensive. Finally, a cheap one may be made by corking one end of a tall lamp chimney or piece of large glass tubing, and fixing it into a hole in a wooden disk.

Unless care is sure to be observed in introducing the alcoömeter, a piece of soft sponge or some cotton should be pushed to the bottom of the jar.

$\$ 270$. Changing the Percentage of Absolute Alcohol in a Mixture.-This may be done by mixing two grades of alcohol, or by adding water to one of them. The due proportions may be ascertained either by experiment or by the arithmetical method known as alligation alternate.

§ 271. Rule of Alligation Alternate.-Find the difference between the required per cent. and the per cent. of each of the liquids to be combined. Write the reciprocal of each of these different numbers, and reduce the fractions to a common denominator. The numerators will then represent the proportionate volumes of the two liquids.

Examples,-Water and 95 per cent. alcohol are to be so combined that the per cent. of the mixture will be $50 ; 95-50=45 ; 50-00$ (the water) $=50$. The reciprocals of the two numbers are $\frac{1}{45}$ and $\frac{1}{50}$; reduced to a common denominator, these fractions become $\frac{9}{450}$ and $\frac{10}{450}$ respectively; hence 9 volumes of water are to be added to 10 volumes of alcohol. On the alcoömeter the per cent. of the mixture will be indicated as about 55 .

Again, two grades of alcohol, respectively 75 and 30 per cent, are to be combined so 
that the per cent. of the mixture shall be $50 ; 50-30=20 ; 75-50=25$. The reciprocals are $\frac{1}{2} \delta$ and $\frac{1}{25}$; reduced to a common denominator, these fractions become $\frac{5}{10} \sigma$ and $\frac{4}{100}$, consequently 5 volumes of 30 per cent. alcohol are to be added to 4 volumes of $7 \tilde{0}$ per cent. in order that the per cent. of the mixture may be 50 . Upon the alcoömeter the per cent. is about 52 .

Owing to the unequal specific gravities of alcohol and water, and the contraction which occurs when they are mixed, the results obtained by this method are only approximate, as indicated by the alcoömeter. They are, however, sufficiently close for most purposes.

When liquids of different specific gravities are mixed, they should be thoroughly shaken together before testing with the alcoömeter.

\$ 272. Determining the Ratio by Experiment.-When a given volume of alcohol is to be made either stronger or weaker, the end may be reached experimentally in either of two ways: The entire volume of the alcohol to be changed may be placed in a jar, and the modifying liquid - whether water or weaker or stronger alcoholmay then be introduced gradually until the desired per cent. is attained; or a given volume of the alcohol to be changed may be placed in the hydrometer jar, and given volumes of the other liquid. induced. Having ascertained the ratios, larger volumes may be mixed accordingly.

In both these cases, the two liquids must be thoroughly shaken or stirred together before testing.

\$273. Reduction of 95 per cent. Alcohol by the Addition of Water.-Since this is the change most frequently made, we have ascertained by the alcoömeter the results of the mixture of water and alcohol in various simple ratios. These ratios are given in the following Table :-

\begin{tabular}{|c|c|c|c|c|c|}
\hline \multirow{2}{*}{$\begin{array}{l}\text { REQUIRED PER- } \\
\text { CENTAGE. }\end{array}$} & \multicolumn{2}{|c|}{ VOLUMES, } & \multirow{2}{*}{$\begin{array}{l}\text { REQUIRTD PER- } \\
\text { CENTAGE. }\end{array}$} & \multicolumn{2}{|c|}{ VOLUMES. } \\
\hline & Alcohol. & Water. & & Alcohol. & Water. \\
\hline $\begin{array}{l}84 \\
82 \\
78 \\
75 \\
67 \\
62 \\
60 \\
59\end{array}$ & $\begin{array}{l}6 \\
5 \\
4 \\
3 \\
2 \\
1.5 \\
1.25 \\
1.2\end{array}$ & $\begin{array}{l}1 \\
1 \\
1 \\
1 \\
1 \\
1 \\
1 \\
1\end{array}$ & $\begin{array}{l}55 \\
48 \\
45 \\
42 \\
35 \\
30 \\
22\end{array}$ & $\begin{array}{l}1.1 \\
1 \\
1 \\
1 \\
1 \\
1 \\
1\end{array}$ & $\begin{array}{l}1 \\
1 \\
1.25 \\
1.5 \\
2 \\
3 \\
4\end{array}$ \\
\hline
\end{tabular}

§ 274. Water to be Mixed with Alcohol.-If the mixture is for the hardening or storage of specimens, any clean water will answer. But if for the exhibition of finished preparations, the water should be either distilled, or rain water filtered; otherwise the mixture is liable to be clouded. 
\$ 275. The Economios of Alcohol.-In the economical employment of alcohol, four matters are to be considered: (1) The use of different grades for appropriate purposes; (2) The prevention of evaporation; (3) The avoidance of leakage; (4) The improvement of "old" (deteriorated) alcohol.

$\S 276$. The $U_{s e}$ of Appropriate Grades.-Upon zoological specimens alcohol is employed for three distinct purposes: preparation; storage; exhibitior. Different degrees of clearness and strength are needed for these different purposes, and the safety of specimens, economy, and the appearance of collections depend upon the use of each grade for its appropriate purpose. For example, the commercial (95 per cent.) alcohol is just strong enough for some purposes, much too strong for others, and needlessly clear and pure for others.

The following remarks apply to all vertebrates, and the more general ones to most invertebrates also. Among the invertebrates the jelly-fishes and some other soft forms cannot be satisfactorily preserved by means of alcohol, and most of the rest should be treated like the brains and embryos of vertebrates.

$\S 277$. Strong alcohol coagulates the tissues so as to form a firm ectal layer, through which it afterward passes with difficulty. With a small specimen, the only objection may be the corrugation and distortion of the specimen. But with a large and fleshy one, the outside may harden while the interior is actually decomposing.

There is no difficulty in preserving the skeletal muscles and the limbs, but the brain and abdominal viscera, especially the liver, are not only more prone to decomposition than the muscles, but naturally excluded by them from the alcohol. Unless, therefore, definite measures are taken prior to immersion (\$286), some of the viscera will surely fail to be preserved. These measures will vary according to the size of the specimen and the use to be made of it, and, under some circumstances, the opportunity for using the required instruments.

$\S 278$. The four accessory measures are named in the order of their simplicity: (1) Freely opening the abdomen; (2) Injecting alcohol into the thorax and abdomen; (3) Injection of alcohol into the large hollow viscera ; (4) Injection of alcohol into the arteries.

\& 279. As a preliminary to any of them, the animal should be bled, if practicable, while under the influence of an anæsthetic. As soon as the cat is quite asleep, the femoral vessels may be exposed as directed for coarse injections (Fig. 39). and the V. femoralis divided. When the venous flow slackens, the artery may be opened. In this way most of the blood in the larger vessels is removed, and preservation is facilitated. 
\$ 280. (1) Freely Opening the Abdomen.-This is sometimes, as when collecting in the field, the only practicable method. The abdomen is opened by a longitudinal incision a little dextrad of the meson, never-as commonly directed-on the meson itself. A second incision is to be made at a right angle with the first, extending to the dextral margin of the abdominal cavity; these two incisions are shown in Fig. 76 , but the transverse one should not cross the meson.

The specimen is to be placed in 52-67 per cent. alcohol, the hand introduced, and the viscera lifted and moved slightly, so as to permit the penetration of the liquid to all parts; cotton may be interposed. The liver especially should be displaced, and-unless its preservation is especially desirable-most of it should be removed, the cholecyst (gall bladder) being left.

$\$ 281$. This method is much more efficacious if combined with the third, as follows:-

The rectum is compressed so as to expel its contents per anum, and the small intestine treated in like manner, if its contents seem to be considerable. The stomach is manipulated and compressed so as to expel its contents through the mouth. A slit about $1 \mathrm{~cm}$. long is then made in the free surface of the duodenum near the stomach, and alcohol injected, first through the pylorus into the stomach, and then into the small intestine, so as to fill both it and the large. The escape of the alcohol from the anus may be prevented by a plug of cotton. For all injections of alcohol, a rubber bulb syringe is more convenient than one with a piston. After injecting, it should be thoroughly rinsed out with water.

The lungs may be filled with alcohol by passing a curved canula attached to a rubber tube from the mouth through the glottis, or by opening the trachea.

\$ 282. (2) Injection of 58-67 per cent. Alcohol into the Thorax. and Abdomen. - This is less efficacious than the other methods, but may be adopted when it is desirable to mutilate a specimen as little as possible, as in making a permanent preparation of an entire animal.

The incisions for the introduction of the canula should be made obliquely, so as to leave a valvular orifice which is less apt to permit the escape of the injected liquid. Both sides of the thorax should be injected. Of course this method is more efficacious if combined with the third. 
\$ 283. (3) Injection of 58-67 per cent. Alcohol into the Stomach, Intestine, and Lungs.-If the left hypochondrium is prominent, or if it is known that there is much food in the stomach, the entire abdomen should be compressed, so as, if possible, to expel the contents through the mouth. Pressure of the abdomen just cephalad of the pubes will probably expel part of the contents of the large intestine, and most of the rest may be washed out with water introduced from a syringe or from a faucet. Alcohol should then be injected into the abdomen, as directed in $\$ 282$, or the cavity may be opened, as directed in $\S 280$. In the latter case, both the stomach and small intestine may be filled from the duodenum. In the former, alcohol may be injected into the large intestine through the anus, and into the stomach from the mouth. The anus should be plugged with cotton to retain the alcohol; if the stomach is filled only moderately, the alcohol will probably be retained by it. The lungs may be filled in either of the two ways mentioned in $\$ 281$.

of course, neither of the three measures above described is of direct service in preserving the membral muscles or the brain. These parts are immediately reached by the alcohol only according to the fourth method, which is efficacious for all parts, but somewhat more complicated than the other methods.

§ 284. (4) Injection of Alcohol into the Arteries.-This should be done in all cases when practicable, or unless there are special objections. With entire animals, or the cephalic or caudal halves, there is no practical difficulty; with smaller portions, as the head or a single limb, it may be necessary to tie or secure with compressors other vessels than the one injected, so as to prevent the escape of alcohol ; but in some cases even this may be omitted, the injection being done in a dish, so that the escaping alcohol is saved.

The following directions apply particularly to the cat:-

If the entire animal is to be preserved, provide at least 2 liters of 84-95 per cent. alcohol and a syringe and canula of appropriate size. After bleeding ( $\$ 279$ ), close the vein by a ligature both centrad and peripherad of the point of division, and have ready a small compressor for application to the artery. Inject the alcohol into the $A$. femoralis, as directed for plaster (see Chap. III.).

Inject the alcohol slowly, so as to give it time for penetrating. the smaller vessels, and stop as soon as the resistance is decidedly increased, lest the vessels be ruptured. This last precaution is indispensable if a plaster or gelatin injection is to be made afterward. 
Place a small compressor on the artery just centrad of the point of injection, and withdraw the canula. At least two hours should elapse before dissection is begun or an injection made with plaster or gelatin. In the interval the hair may be clipped (see Chap. V1.). After clipping, if no other injection is to be made, the animal may be placed in alcohol, 42-55 per cent.

$\S 285$. If only the cephalic half of the body is to be preserved, not more than one liter of alcohol may be needed, and the operation of abdominal transection should be carried as far as to open the abdomen and puncture the diaphragm (\$238).

Then, in place of removing the viscera at once, proceed as follows:-

Grasp the rectum as far caudad as possible, and force its contents cephalad. Place two ligatures on it (Fig. 41), about one $\mathrm{cm}$. apart, and cut between them.

With the scissors and tracer divide the mesentery of the large intestine and that of the small intestine, to a point opposite the cephalic end of the left kidney (Fig. 101, ren).

Pass a strong thread from this point around the stomach and liver, 1-2 cm. from the diaphragm, and tie it very firmly. The ligature should cut deeply into the substance of the liver.

With the tracer expose the aorta opposite the caudal end of the left kidney. Open the aorta as directed (Chap. IV.) for the A.femoralis. Inject alcohol as directed $(\S 284)$; then inject alcohol into the lungs as directed in $\$ 281$. Remove the abdominal viscera by an incision $1-2 \mathrm{~cm}$. caudad of the ligature, taking care not to displace the latter.

$\S 286$. Treatment of Special Organs and Tissues.-The blood should be washed off with water or weak brine. Very vascular parts, like the liver or spleen, should be gently manipulated so as to expel most of the blood.

In respect to the strength of alcohol required, the soft parts form three groups, as follows:-

Brains, embryos, the liver, spleen, the glands, and most invertebrates should be laid upon cotton in natural attitudes, first in alcohol of 52-67 per cent.; after two days in 95 per cent., for a week ; lastly, for exhibition or storage, in 75-95 per cent.

Muscular organs, including the heart and alimentary canal, may be hardened and permanently preserved in 42-55 per cent. The same strength is adapted to most entire animals. 
Ligaments, bones, and cartilages should be placed in 42-55 per cent. for two days, and then kept in 22-30 per cent.

When the same specimen contains two or more of these kinds of organs, the strength of the alcohol should be adapted to the more perishable, provided these latter are to be fully preserved.

For injection into the viscera or abdomen, 52-67 per cent. ; for arterial injection, 84-95 per cent., is to be preferred, but any strength above 30 per cent. would be of some service.

While hardening, specimens should be kept in a cool place.

$\S 287$. Fresh Specimens should not be in contact with the sides of the vessel. In order that all parts may be reached by the alcohol, the specimen should be suspended so as to hang freely, or cotton or "excelsior" interposed between the jar and parts which would be in contact with it, or the specimen jar laid upon one side, and shifted daily for a week, so that no part of the specimen is in contact for more than 24 hours. With well-preserved specimens, this precaution need not be observed.

\$288. Flexible Specimens. - If it is desirable that flexible specimens should harden without contortion, they should be suspended from the cover of the jar, or the jar itself laid upon the side, as suggested in $\S 287$; it is safer to place the jar upon a tray. The mouth end should be slightly raised and very securely closed.

\$ 289. Amount of Alcohol Required.-With fresh specimens the alcohol should not be less than twice the volume of the specimen. The results are more satisfactory if the ratio is as $4: 1$. The smaller amount may be more safely used with specimens when alcohol has been injected into the arteries $(\$ 286)$.

A. Specimens which have been once saturated with alcohol may be stored or placed on exhibition in the minimum quantity required to cover them; in this way a single jar or can may hold a large number of specimens. But fresh or partly preserved specimens, especially of viscera, should have plenty of space and abundance of alcohol. Neverthe. less, experienced collectors are sometimes led to fill a jar with fresb specimens, in the hope, apparently, that the inevitable laws of decay will be overruled in tleeir favor. In most cases, a single well-preserved specimen is of more value to science than ten which have been insufficiently cared for. What is worth preserving at all is worth preserving well.

B. With museum specimens, and for exhibition, the odor of the alcohol is less important; but the clearness and strength are essential.

C. Old alcohol, whether filtered or not, may be used for storage, and for the primary treatment of some fresh specimens.

§ 290. Provisional Preparations.-It is not always possible or even desirable to make the final preparation of a part upon a fresh specimen, or immediately upon the reception of one in alcohol. In 
these cases, however, if the scope of the final preparation is determined, most of the parts not involved may be removed at once, thus saving alcohol or storage room, and-if the specimen is fresh-insuring the more complete access of the alcohol. This measure is particularly important in the case of viscera or massive muscular organs, and especially the brain of large animals and man. Even with the brain of the cat, if the aulic region, for instance, is to be examined, it is well to remove the dorsal and lateral parts of the hemispheres.

$\$ 291$. Deterioration of Alcohol.-During its use upon specimens, alcohol is subject to four kinds of deterioration :-

It may become colored by solution of coloring matters; turbid from the suspension of small particles; offensive from the solution of malodorous matters; and weak from the evaporation of the pure alcohol, the impartation of alcohol to the specimen, the abstraction of water from the specimen, and the absorption of moisture from the atmosphere.

\$292. Purification.-Aside from distillation for the sake of strengthening, alcohol may be purified and improved in three ways: settling; filtration for clearing; filtration for deodorizing.

\$293. Settling.-Let the alcohol stand for a few hours undisturbed. Place a second jar close to it, so that the first need not be carried, and pour the alcohol into it very carefully, and without reversing the tilt until the dregs begin to approach the mouth. If the alcohol is less than 20 per cent., or if the dregs are very foul, they should be thrown away. But usually the dregs should be poured into a more slender jar, and allowed to settle a second time.

\$294. Filtration, for Clearing. - When alcohol is simply somewhat turbid, as usually is the case after the settling, or if its intended use does not require deodorizing, the following process is sufficient: Push some cotton into the narrow part of a large tunnel, set the tunnel into a jar, and cover it closely. According to the compactness of the cotton will the alcohol filter through more or less rapidly, and with greater or less change of color and clearness. The same cotton should not be used a second time.

A. Clearing may be effected at the same time with deodorizing, if the perforated lid of the filter to be described is covered with muslin, and if one or more layers of muslin are so placed that the alcohol must pass through them before reaching the animal charcoal.

B. During the filtration of alcohol, unless it is conducted into a receiver with a mouth just large enough to receive the discharging end of the funnel, or the tube connected therewith, let these pass throngh a lole in a metal, wooden, or pasteboard cover of the jar. 
\$ 295. Filtration for Deodorization and Decoloration.-This requires the use of animal charcoal, and should follow the settling and clearing already described.

Any kind of water filter containing animal charcoal may be used, but the simpler and cheaper forms are sufficient, and the charcoal should be capable of renewal. Such is the earthenware filter made for water under the direction of our colleague, Prof. A. A. Breneman, and for sale by Messrs. Rankin \& Son of Ithaca. Unless the alcohol is clear, the perforated lid should be wrapped in a cloth, and two or more layers of cloth placed over the top of the filter, resting on the lid, so that the alcohol may be cleared of suspended impurities before it reaches the charcoal. In the absence of a proper filter, a bag of the charcoal may be pushed firmly into a large tunnel ; the tunnel for this purpose should have the smaller end larger than usual.

§ 296. Crystalline Deposits.-From some specimens, especially brains, strong alcohol extracts substances which are precipitated as white crystals when the percentage of alcohol is diminished or the temperature lowered. The resulting turbidity and clouding of the glass require a thorough washing of the specimen and the jar, and the renewal of the alcohol. The old alcohol should settle in a cool place, and then be filtered through cotton. Even after filtering, it should be used only for hardening and storage.

When two grades of "old alcohol," whether filtered or not, are mixed, the deposits above mentioned are liable to occur; hence, if a clear mixture is desired, a trial should be made first with small quantities of the liquids.

\$ 297. Strengthening.-So far from increasing, the strength of alcohol is likely to be reduced by the processes of filtration and settling, especially unless precautions are taken against evaporation. A slight improvement may be effected by treatment with quicklime, and distillation is, of course, an efficient means. The readier method, however, is simply to add to the weaker spirit enough 95 per cent. alcohol to impart to the mixture the required strength (\$\$ 271,272$)$.

When alcohol is purchased at retail, none should be wasted. But if it is obtained free of tax, it is sometimes cheaper to throw away the weaker grades, especially the dregs after settling, rather than to spend much time in their improvement.

§ 298. Alcohol Vapor..-The antiseptic and preservative properties of the vapor of alcohol may be utilized in two ways :-

(1) Upon Fresh Specimens. - When a fresh specimen is to be dissected somewhat rap. idly, say within one week in warm weather, or two or three in cold, it will keep with little change if placed in a close vessel at the bottom of which is enough 95 per cent. alcohol to keep the atmosphere completely saturated with the vapor. In these cases the skin should be removed only as needed, and the exposed parts covered, when not under examination, with cloths wrung out in alcohol.

(2) Alcohotic Specimens.-Any specimen which has been acted upon throughout by alcohol will keep almost indefinitely in the vapor. Hence, in some cases, especially where a specimen is under daily dissection, after it has been fully saturated with alcohol, the 
latter may be poured off, and a small amount of strong alcohol left at the bottom of the jar.

In this case, however, if water or the wetting liquid has been applied to the specimen, a little alcohol should be poured over it whenever it is returned to the jar, or that which is already at the bottom may be shaken up so as to moisten the surface.

\& 299. Methyl Alcohol.-This is more often called wood spirit or wood naphtha. Its odor is unpleasant, and it is not used as beverage. In England it is said that no tax is imposed upon a mixture containing 11 per cent. of methyl alcohol. In this country it is not subject to tax, but costs about $\$ 1.50$ per gallon. According to Günther $(A, 697)$, it is less efficient than ethyl alcohol as a preservative, at least for fishes. Hence, whep ethyl alcohol can be had free of tax, there is no object in using the methylic variety; even when the former costs its full retail price, unless large quantities were wanted, the saving in cost would hardly compensate for the unpleasant smell of the wood spirit.

$\S 300$. Wickersheimer's Liquid.-Several formulæ for making this preservative have been published in this country, as, e.g., in "The Popular Science Monthly," March, 1880, p. 717 ; "The Medical Record," April 17, 1880, and April 30, 1881, p. 501.

In the last named, it is stated that the original formula has been altered, and that Messrs. Poerz \& Flohr of Berlin prepare two kinds, one intended for injections, and the other for immersing bodies. Their composition is as follows :-

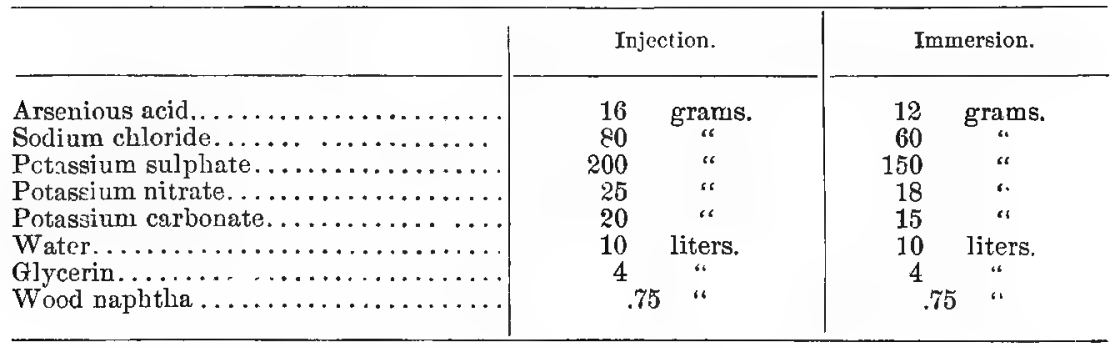

According to the previous article in the "Record," the various salts are to be dissolved in the boiling water, and the solution is to be cooled and filtered before the other two liquids are added. It is probable that the cheaper glycerin will answer, and that ordinary (ethyl) alcohol is to be preferred to the methylic variety which is specified.

Application.- Preparations that are to be preserved dry are immersed in the liquid for 6-12 days according to size, and then dried in the open air.

Hollow organs, like the lungs, must be filled witl the liquid, then laid in a vessel of the same, and afterward distended with air and dried.

Our own experience with this liquid does not enable us to give a decided opinion as to its merits. We are disposed to think that it will answer a good purpose with preparations of the ligaments, and of hollow viscera where flexibility is desired. With.dry inflated preparations, the cheaper and simpler arseniate of soda is equally efficacious, while all ordinary "wet preparations" are, in our opinion, more surely and conveniently preserved by alcohol.

\$ 301. Chloral Hydrate.-A solution of this substance has been used by Dr. W. W. Keen and some other anatomists. The results seem to be satisfactory in most respects 
so far as concerns man and other Vertebrates, but less so with the Invertebrates, at least as reported by Prof. A. E. Verrill in a letter to Dr. Keen.

Dr. Keen has published two papers upon the subject ( $\boldsymbol{l}$ and $\%$ ), and kindly permits us to print the following brief statemeni of his experience up to November 1, 1881 :-

"As to the strength of the solution, I should use 20-30 grains to the ounce of water. [This is 4-6 per cent., or in the proportion of 1 gram of chloral to 24-16 cc. of water.] For the Invertebrates and for vegetable tissues, my own experience, though limited, is favorable. It preserves vertebrate organs, excepting the brain, for a few years at least. I have had no failure, and some of my specimens aro now nine years old. The colors are rather less affected than by alcobol. The jars do not need to be hermetically sealed, hence the specimens are always accessible. The specimens are also less apt to dry when exposed. The cost of the solution is much less than that of alcohol on which the tax is paid. For the preservation of human subjects for dissection, it is most excellent, excepting that it will not keep them very long in hot weather, and is not well adapted for keeping them over the summer. For use in cool and cold weather, and especially for the dissection of nerves, it holds its own."

The foregoing statements from so distinguished an anatomist certainly warrant a careful trial of chloral; our own experience is as yet too limited for the publication of the results.

§ 302. Brine.-A saturated solution of rock or dairy salt is sometimes used alone for the temporary preservation of anatomical material; it is aaid to be more efficacious when the specimen is previously injected with a saturated solution of arseniate of suda. In one of the leading medical schools of this country this plan is successfully and economically followed for keeping human subjects even through the summer months.

$\S 303$. Exhibition and Storage of Alcoholic Specimens.-As was stated in $\$ 262$, while alcohol is a perfectly efficient preservative so far as concerns all vertebrates, its cost, volatility, and corrosive action necessitate certain measures which may be conveniently discussed in connection with the general subject of the storage and exhibition of specimens.

$\S 304$. Temporary Storage.-For this purpose, and for brief transportation, alcoholic specimens may be placed in vessels made of wood or earthenware or any kind of metal; but vessels so employed should be carefully examined as often as once a month, so as to guard against evaporation, leakage, or rusting.

Leakage or evaporation are readily detected from the diminution of alcohol or the dampness of the bottom of the vessel or of the floor, but rusting may not be apparent unti] loss or damage has occurred. When alcoholic specinens are kept in tin ware, the metal is certain, sooner or later, to be corroded at some point of contact with a specimen. A mass of rust is formed which may prevent the eseape of the alcohol so long as the specimens are undisturbed. But this rust is liable to be dislodged by any movement, or even by a blow upon the side of the vessel, and thus permit the escape of the alcohol.

\$ 305. Leakage from Imperfect Glass Vessels.-Small jars and vials sometimes have small holes or cracks which escape notice at the time of filling, but which permit the loss of alcohol and the damage of the contents. In some cases, part of the bottom of a small jar may be so thin that it is pulled off by simply adhering to the paint of a slelf. 
$\S 306$. Corks should be used only for brief transportation or temporary storage. The best of them are insufficient barriers against either evaporation or leakage, and the poorer ones siould not be used at all with specimens. Corks may be rendered more secure by soaking in oil, or covering with Brunswick black, or with a solution of paraffine in benzine, benzole, or turpentine. Rubber stoppers are sometimes used.

$\S 307$. Glass Dishes and Boxes.-The anatomist has use for glass dishes of almost every size. A few sizes are kept in stock by Messrs. Berge (A, No. 257), and Whitall, Tatum \& Co. (A, No. 2265). Covers for them may be cut from window glass, or these or other

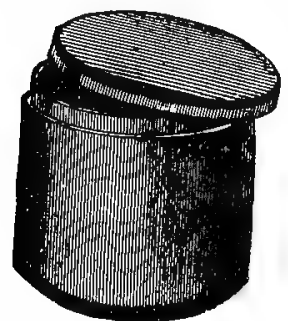

FIG. 31. - COVERED Grass Box; x.25; $\S 306$. sizes may be ordered to have the edges ground for the reception of covers ground near the edge.

The covered box shown in Fig. 31 is very useful for the hardening of brains and other small specimens. The largest size advertised by the firms above mentioned (Nos. 202 and 2590) has a capacity of nearly $500 \mathrm{cc}$, and costs $\$ 3.80$ per dozen.

The covers of these boxes and dishes do not fit accurately, and their contents should be under constant supervision.

$\S 308$. Metal Boxes.-As the name implies, the box usually differs from the jar or the can in its relatively greater horizontal extent and in having a cover which is coextensive with the box, and is readily remored and replaced. The accessibility of the contents thus compensates partly for the evaporation of the alcohol which must occur in some degree. The cover should fit closely and have a wide flange, but such a box should be under constant supervision. Large boxes should be supported by an outer box of wood.

For the preservation of cats while under occasional examination, a box of galvanized iron may be $60 \mathrm{~cm}$. long, 40 wide, and 25 deep. An entire cat may rest in it lengthwise, and either the cephalic or caudal half crosswise. Around the inside, half way from the bottom, extends a ledge on which may be laid a metal plate perforated with holes $2-3$ $\mathrm{cm}$. in diameter. On this plate may be placed a second tier of specimens, which are thus kept from pressing upon those at the bottom. If preferred, the bottom may contain only alcohol, the vapor of which will suffice for the keeping of the specimens upon the plate, provided they have been already thoroughly saturated with alcohol. The box may be supported upou bosses or upon short movable legs. The cost of such a box is $\$ 4-\$ 5$.

\section{\$ 309. Exhibition or Permanent Storage_-For these purposes,} and for transportation occupying more than a month, alcoholic specimens can be safely entrusted only to tightly closing vessels of glass, copper, zinc or galvanized iron. Of course, glass must be used for exhibition ; it is also cheaper than metal for tightly closing vessels, provided the risk of breakage is not regarded.

$\S 310$. Metal Cans.-Three kinds of metal cans are useful in connection with alcoholic specimens. For bringing alcohol from the barrel, and for containing the supply for current use, the ordinary five gallon oil can is well adapted, especially if provided with a faucet from which the alcohol may be drawn into jars without, lifting or tipping the can. The small gallon or half.gallon can is desirable for pouring the alcohol into small vials. These cans may be made of tin, but copper is more secure.

Screvo-top Cans.-For the permanent storage of alcoholic specimens, especially if occasional moving is necessary, copper cans with screw-tops are desirable. They are more 
expensive than jars of the same capacity, but are less subject to injury, especially during transportation.

- A convenient size has the following dimensions: Height, $30 \mathrm{~cm}$.; diameter, $40 \mathrm{~cm}$; diameter of the orifice, $26 \mathrm{~cm}$; cenpacity, 30-35 liters. The rings of the screw-top are made of brass, and should not weigh over 1300 grams. The bottom should be protected from abrasion by bosses. (At the Museum of Comp. Zoology in Cambridge each can is enclosed in a wooden case which facilitates handling and may be used in transportation.) The edge of the cover-ring is notched for the admission of the iron bar with which it is screwed or unscrewed. Such a can costs s:10-\$12; this is more than the cost of a glass jar of equal capacity, but the latter is liable to break, especially during transportation, while the can is practically indestructible.

\& 311. Glass Jars.-With the exceptions indicated in the foregoing sections, glass is to be preferred for all alcoholic specimens. Glass jars may be considered in five groups: fruit jars; specimen jars with covers; specimen jars with neck and ground glass stoppers ; welted jars ; compressed jars.

$\S$ 312. Fruit Jars.-There are many styles of these, but most of them are made of more or less opaque glass. The orifice is seldom more than; $5.5 \mathrm{~cm}$. in diameter. They are thus unsuited for exhibition or for holding wide specimens; but their cheapness and strength adapt them well for the storage and transportation of such specimens as they will contain.
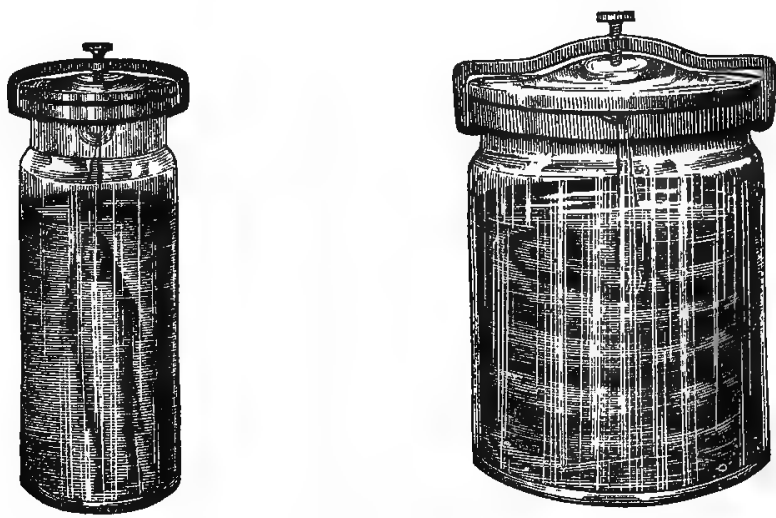

Fig. 32.-Wide Modthed Spqctmen Jars with Cover; $\S 312$.

§ 313. Wide Mouthed Jars with Covers-(Fig. 32).-These are made on the general pattern of the "Millville" fruit jar, but are of clear glass, and have mouths nearly as wide as the jars; the cover has a ring from which specimens may be suspended. Messrs. Whitall, Tatum \& Co., in addition to what is said in their catalogue $(A, 2600)$, will send a price list of 13 sizes, ranging from $4 \times 3$ in. to $23 \times 9$. The following special lengths of standard widths have been made by the same firm at the following prices for each jar: $18 \times 3$ in., $\$ 1.15 ; 23 \times 4, \$ 2.05 ; 32 \times 4, \$ 3.30$; tuese tall jars are provided with a " foot."

These jars are of course less handsome than the glass stoppered kind, but their strength and cheapness well adapt them for museum and laboratory use. 
§ 314. Glass Stoppered Jars.-These are made to order by any extensive glass manufactory. The Dorflinger Glass Company, White Mills, Wayne Co., Pa., issue a price list of 51 different sizes, ranging from $2 \times 1$ in. to $22 \times \%$.

\$ 315. Welted Jars-(Fig. 33).-With the specimen jars above described, the neck is strengthened by a constriction. This involves one of two alternatives: either the speci-

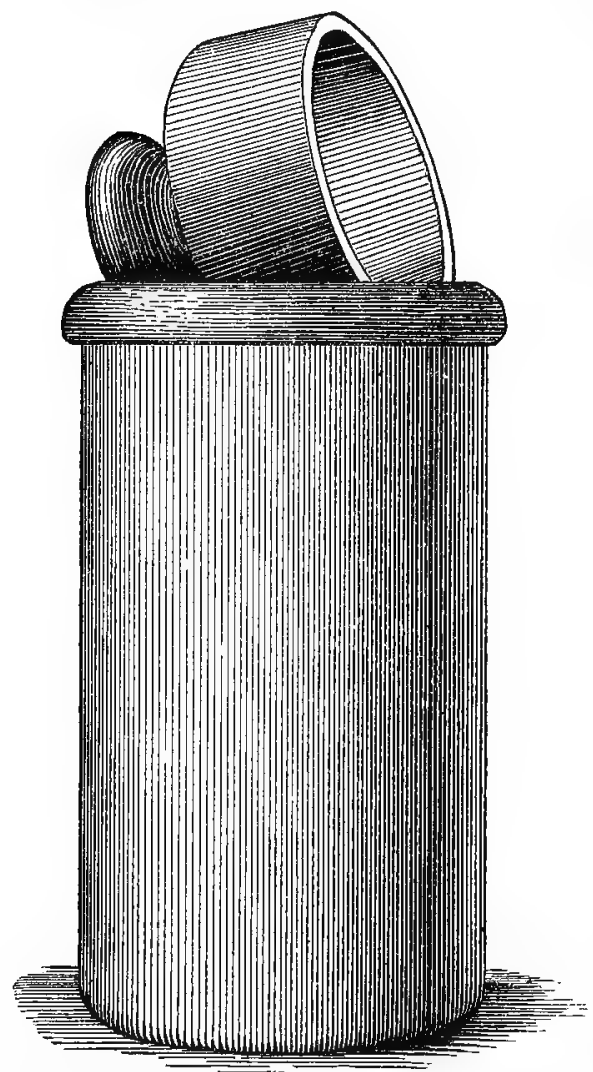

Fig. 33.-WeLted VIal for the Bratn or HEART OF THE CAT ; slightly reduced; $\$ 314$ men must be more or less compressed in entering or leaving the jar, or the body of the jar is needlessly large. With some kinds of specimens these are not very serious objections. But with brains and embryos it is desirable that all pressure should be avoided, and also that they should be close to the side of the jar. For such specimens the jars should be of uniform cliameter throughout, and the requisite thickening at the mouth may bu gained by a welt.

The size represented in Fig. 33 is adapted to the brain or the heart of the cat. Its inside measures are $4 \mathrm{~cm}$. wide, and $6 \mathrm{~cm}$. to the lower border of the part which is grouud for the stopper. A smaller size of the same height, but only $3 \mathrm{~cm}$. in diameter, is adapted for either half of the brain. Both sizes are made to order Ly Messrs. Whitall, Tatum \& Co. for $\$ 2.50$ per dozen.

$\S 316$. Compressed Jars.-The ordinary circular jar is poorly adapted for the display of flat specimens like frozen sections and some fishes. Messrs. Whitall, Tatum \& Co. have molds for two sizes of compressed jars similar to the photographers" "bath." They are $15 \mathrm{~cm}$. high and 21 or $12 \mathrm{~cm}$. wide. The larger cost $\$ 12$ per dozen, and the smaller $\$ 6$. The tors are level and ground off for thin glass covers, which may be cemented on. The covers cost a small sum in addition to the price of the jars.

\& 31\%. Cleaning Jars.-When large enough, jars should be washed on the inside with a sponge. If they will not admit the hand, the washing may be done with a swab made by tying a sponge upon the end of a stick, or with the bottle brushes, one of which is shown in Fig. 34. For long jars and large tubes there are bottle brushes with long handles; or a wire may be attached to the handle of a short brush ; or the ring of the handle may be cut off, 
and the end of the wire let into a hole in a stick of the desired length. If the jar is greasy, it may be cleaned perfectly by means of the liquid soap (\$248).

After washing, glass jars should be well rinsed with clean water, and dried with a soft but not linty towel, and finally-if for the exhibition of specimens-polished inside and out with a bit of old silk or chamois leather.

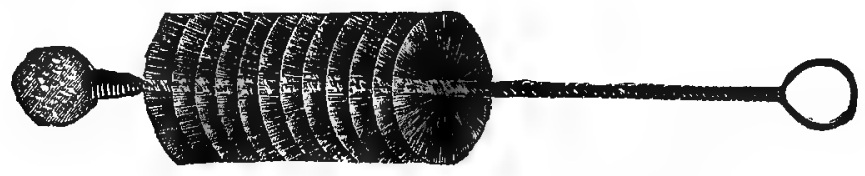

Fig. 34.-Bottle BRdsh ; reduced; § 316.

$\S$ 318. Display of Alcoholic Specimens.-Of course the alcohol should be clear and the jar clean; but as the jars are commonly cylindrical while many specimens are more or less compressed, the surface of the specimen is sometimes undesirably distant from the side of the jar, and the image is distorted by the intervening liquid. When the compressed jars mentioned in \$315 are not available, a smaller bottle or jar of clear glass may be introduced so as to crowd the specimen against one side of the jar. The inner jar must be loaded with lead or filled with alcohol or water to such an extent as to just sink it in the alcohol. If water is used, the smaller jar must be very tightly closed. See Appendix, § 1456 .

As a rule, an exhibition jar should contain only one specimen.

§ 319. Packing Alcoholic Specimens for Transportation.There are certain precautions of general application, but the details vary according to the nature of the specimens and the conditions of transportation.

In most cases the specimens should be packed before the alcohol is poured in, and the vessel should always be filled.

Unless a pail is used, or a box or basket with an unmistakable handle, and "This side up" prominently inscribed, the package should be prepared for any position.

In respect to transportation, specimens may be grouped as coarse, delicate, and scaly.

Coarse Specimens.-Under this head come most entire examples of the larger number of vertebrates. These, or their firmer parts, may be packed with no precaution other than to fill the vacancies with hay or "excelsior" before introducing the alcohol. 
Scaly Specimens.-Most "fishes" and some Reptiles should be wrapped in gauze or netting, or any porous cloth or soft paper. The wrapper should be secured with a string.

Delicate Specimens. - The delicacy may be due to smallness or softness, or to the presence of projecting parts which are liable to injury.

The safest way is to put each specimen in a separate vial or jar quite full of alcohol, or in a small box with saturated cotton which may be immersed in a larger jar or can.

When several delicate specimens are to be packed in the same vessel, they should be separated by layers of cotton thoroughly saturated with alcohol. After the alcohol is poured in, the jar should stand for an hour at least to allow time for settling. If any occur, more cotton should be put in.

Packing without Alcohol.-When the time of transmission by mail or express is short, specimens may be safely sent if covered with a layer of alcoholic cotton, then wrapped in rubber sheeting, and packed in a wooden or metal box, or in a vial properly protected.

Secure Closure of Vessels.-Always important with alcoholic specimens, this is essential before transportation. In all cases the vessel should be laid on its side or stood upon the upper end after closing, so as to determine the possibility of leakage.

The glass stoppers should be anointed with glycerin or fine oil, or a solution of paraffine in benzine or turpentine, and pushed in with a slight screwing motion. The jar should then be rolled in cloth, and the cloth turned tightly over the upper end of the jar and tied down so as to secure the stopper.

The covers of fruit jars and specimen jars should be screwed down more firmly than usual. If possible, all jars should stand upright during transportation, and be well packed in hay or other soft material.

Welted Vials may be let through holes in a board so as to be supported by the welt. The board may rest on cleets in a box.

Packing boxes of tin are usually closed by soldering.

When earthen jars are used, the corks should be pushed below the level of the rim of the mouth, and the space filled up with a thick paste of plaster of Paris.

\$ 320. Miscellaneous Suggestions. - Specimens having prominent appendages, whether arms, legs, wings, fins or spines, especially if they are stiff or angular, should be put into jars tail first, so that their removal may be unobstructed. 
This rule may sometimes require modification, as with some " fishes" with delicate caudal fins, which might be broken by the pressure. The specimen may be suspended from the cover of the jar, or the tail may rest upon cotton, or, finally, the jar may be of such size as to permit the withdrawal of the specimen tail first.

\& 321. In some cases, when a delicate or valuable specimen is in a jar or vial with a mouth too small for its easy or safe extraction, the jar should be sacrificed. Place its mouth just over the edge of a waste pail or box, and rap it smartly with a hammer so as to break off the top. In removing the specimen, avoid the bits of glass which may have fallen into the jar.

When the glass stopper of a jar cannot be removed in the ordinary way, tap the handle on both sides, alternately, with a piece of wood or the edge of a razor-strop. If this fails to loosen the stopper, or if the handle breaks off, the jar may be broken open as directed above.

$\$ 322$. In removing large specimens from alcohol, especially if the hair or feathers remain, squeeze the alcohol out with the hands, then suspend the specimen for a short time from a hook over the jar or some other receptacle, and finally rinse it off with a stream of water. In this way some alcohol is saved, drying is retarded, and the dissector avoids the odor and stimulating effect of the rapor of the alcohol.

\section{FROZEN SECTIONS AND DISSECTIONS.}

\$323. Frozen Sections.-These are sections of desired thickness made of a specimen which has been frozen hard so that the parts retain their natural relative positions.

Such preparations have been made in Europe since 1883, and in some cases they have been preserved as permanent preparations. A series of frozen sections of the head, in the museum of the Medical School of Maine, were made by Dr. Thomas Dwight, and formed the basis of his work on the head (A). See also his more recent work (B).

Frozen sections of a eat were made by us on the 21st of Jan., 1879, and were briefly described by the senior author in $\mathbf{2 1}$. The method was as follows :-

$\S 324$. The cat was killed with chloroform, the arteries injected with red plaster and the veins with blue. It was then carefully supported in a natural attitude in a box of hay, which was placed out of doors for three days. The cat was then perfectly rigid, and was rapidly cut with a sharp wide-bladed saw into transections about $1 \mathrm{~cm}$. thick. The cutting was done in a cool room, and each section when made was placed upon a glass plate, held under a gentle stream of water, and both surfaces freed from hair and fat particles with a soft nail brush. It was then laid on one side in a dish of 95 per cent. alcohol, and put in a cool place.

As the sections thawed, they were hardened by the alcohol so as to retain their form. Each was then mounted in a compressed jar $(\$ 316)$. In some cases the viscera were secured from falling out by means of insect pins. The cavities of the heart were better seen after the removal of the plaster. 
$\S 325$. The foregoing is the method followed in making the transections shown in Fig. 99 and 100 . The following modifications may be desirable :-

1. The animal should be bled, when anæsthetized, as directed in $\S 279$.

2. Probably sawdust would be better than hay for support during the freezing.

3. 'The freezing was done in winter, but artificial cold might be produced at any time.

4. More satisfactory results might be reached by making sections of only part of a single individual. For example, the thoracic transections would be more true to nature if the lungs had been filled with water from the trachea ; this, however, would have spoiled the neck. In like manner the stomach and intestine or other hollow organs could be distended to their normal size.

5. For the middle region of the abdomen, including the intestines and part of the uterus and urocyst, a more satisfactory result might be reached by filling those organs with water, and keeping up the pressure during the freezing. A sharp broad-bladed knife could be used to transect the abdomen ventro-dorsad, and a narrow-bladed saw used for dividing the vertebræ.

$\S 326$. Frozen Dissections. - While frozen sections display the actual relative position of organs which lie in a given plane, they are unsatisfactory for the same reason that microscopic sections often are : it is rare that a complete view is afforded of any aspect of an organ. Hence we believe that for many purposes there should be a combination of freezing with ordinary dissection. For example, with a frozen cat, most of the thorax could be removed with the saw. Then, before thawing took place, all the rest of the thorax could be cut or nipped away so as to expose the entire cephalic surface of the diaphragm, with the csophagus, great vessels and nerves projecting for a short distance. The pelvic region could then be removed, and the preparation mounted in strong alcohol so as to retain its form.

\$ 327. Flexible Preparations of Muscles.-Dr. Thomas Dwight has published (1) the results of experiments by himself and others. We are unable to contribute anything to the subject.

\$328. Preparation of Hollow Viscera.-Aside from preservation like other soft parts, the stomach and cæcum, the lungs, the urocyst (urinary bladder), and cholecyst (gall bladder) may be prepared by permanent inflation with air or alcohol so as to display their size, form and subdivisions with more or less of their texture.

Since the stomach is most frequently prepared in this way, the following directions apply more directly to it, but they may be readily modified for the other organs named. The parts of the stomach are shown in Fig. 81.

\$ 329. Removal.--The viscus should be removed from the body as soon as possible after death. If, however, the stomach or cæcum has been freed from its contents as described in $\$ 283$, the removal may be longer delayed.

There should be left attached to the viscus a portion of each canal continuous therewith, about $5 \mathrm{~cm}$. long if possible. With large specimens this length may be greater, and with small ones it must sometimes be less. To secure so much of the œsophagns of 
the cat, the diaphragm must be cut through; after division of the tube, a circlet of the diaphragm may be left attached thereto.

\$330. Cleansing.-The organ should be manipulated in water so as to expel the contents and dislodge the mucus from the ental surface. When possible, as with the cæcum and with the stomachs of some of the lower vertebrates, the organ should be everted. In any case the ental surface should be thoroughly cleansed with a stream of water from a faucet or syringe.

If desired, the capacity of the viscus may be measured as directed in $\S 334$.

Normal salt solution should then be thrown into the organ, and it should be allowed to soak in the same for a few hours, or until the blood is removed. If any part projects above the surface, it should be covered with a layer of absorbent cotton.

§331. Trimming.-After sufficient soaking, expel the n. s. s., and place the organ upon a clean tray or dish. Provide two compressors or some linen thread, blowpipe, coarse forceps and coarse scissors curved flatwise.

Close the free end of the œsophagus with a compressor or ligature. Into the duodenum introduce the blowpipe, inflate the organ to a moderate degree, and close the orifice with a compressor or ligature. If the latter is employed, the orifice may be controlled by the fingers of the operator or of an assistant while the thread is applied.

In trimming, the fat and vessels and connective tissue are to be grasped with the forceps, and cut off close to the viscus. The convexity of the scissors must be kept toward the organ, and care taken not to wound it with the point of the forceps.

The subsequent steps in the operation vary according to the agent-air or alcohol - to be used for the permanent inflation.

\$332. Inflation with Alcohol.-This is better adapted to the lungs, and to delicate specimens which might not withstand the pressure and manipulation involved when air is employed.

Place the organ in 42-55 per cent. alcohol, in a dish, or better in the jar in which it is to be preserved. Cut off the ligatured smaller end, and secure in the orifice the canula of a syringe, or a canula connected by a rubber tube with the canula of a syringe; place a ligature at the orifice; then distend the organ with the same per cent. of alcohol. If a bulb syringe is used, or if the injecting syringe is provided with a cock, it is only necessary to tighten the lig- 
ature at the time of desired distension; otherwise, the reflux of the alcohol may be prevented by a small compressor.

The jar selected should be so large that the inflated organ may swim without pressure against the sides. It may be kept from the bottom either by suspending it by a thread from the loop on the jar cover, or by attaching a piece of cork or a bulb of thin glass.

$\$ 333$. Inflation with Air, and Drying.-This method is less expensive, but more complicated:-

After trimming, cleansing, soaking, and trimming as above directed ( $\$ 330,331$ ), the organ is to be partly filled with a saturated solution of arseniate of soda (\$259), and placed in the same. The projecting part should be covered with absorbent cotton, and the whole should be turned at least once. According to size, it should remain in the solution for from 3-12 hours. If left too long, the tissues are gelatinized and rendered incapable of withstanding pressure and manipulation.

Expel any liquid contents as completely as possible, and let the organ drain from a clean smooth surface.

Select two fine-grained corks adapted respectively to the two orifices. With a rat-tailed file girdle each with a furrow at about the middle of the length. Perforate the larger, and fit very closely into it a piece of glass tube projecting about $3 \mathrm{~cm}$. from the larger end. Fill the grooves with mucilage, and secure each cork by winding about it at the level of the groove a string or rubber band.

To the glass tube attach a rubber tube. Inflate the organ, and apply a compressor near the glass tube. Put a tack or a small screw-eye into each cork, and suspend the organ from screw-hooks in a natural position, in a warm but not dusty place. If the organ is large, or drags too heavily upon the slender œsophagus and intestine, support it by bands of parchment or oiled paper.

Connect the rubber tube with a gas jet, or with the outlet of a gasometer, or other apparatus by which continuous pressure may be made. Remove the compressor, and turn the gas on cautiously, so as not to distend the organ unduly.

The escape of gas will be slight, but its accumulation should be aroided, and the place well ventilated.

If no artificial means of inflation are available, air may be blown in from the mouth, or injected with a syringe, but in these cases constant attention is required to prevent collapse. 
When the organ is perfectly dry, the ends containing the corks may be cut off, and fresh corks secured with mucilage only. In at least one of the corks should be a screw-eye to which the label may be attached, and by which the specimen may be suspended from a screw-hook.

Finally, by excising one or more pieces of the wall with a keen scalpel, the position and shape of the orifices may be displayed.

A. Dried inflated specimens should be kept free from dust and moisture, in close cases, or boxes or jars. They should not be varnished.

B. Inflated preparations which have been dried without poisoning, or have been insufficiently poisoned, may be sprayed with the solution of arseniate of soda by means of an atomizer. This should be done cautiously and over a small area at a time, lest the entire organ collapse.

C. According to a paragraph in the "American Naturalist" for March, 1881, page 282, " Dry specimens may be freed from parasites by spraying with anhydrous sulphurous acid."

\$ 334. To measure the Capacity of an Organ.-This may be done in either of two ways:-

1. The organ may be filled with water from a vessel of known capacity, and the amount measured.

2. The organ may be filled, and its contents allowed to escape into a vessel and then measured.

In employing either method, certain precautions should be observed :-

1. The organ should be completely emptied of its contents.

2. It should not be measured until after the cessation of any contraction which may exist at or soon after death.

3. If possible, it should be held by an assistant.

4. It should rest in a dish of water so that the water used in measuring may not exert undue pressure.

5. Since only the capacity of the organ itself is desired, care must be taken to let the water go no farther than the outlets. With the stomach, for example, the pylorus should be closed, and the introduced water should not rise into the cesophagus.

6. If the organ is not to be preserved, the pylorus may be tied. But as tying injures the parts, a specimen which is to be preserved may have the outlet held by an assistant or closed by a compressor or by other mechanical means.

7. If the cardiac orifice is large, the water may be poured in. If small, it may be introduced through a tunnel or through a canula connected with a syringe. In the one case hydrostatic pressure 
must be avoided, and in the other no more force should be used than will suffice to propel the water very gently.

8. If the contained water is to be measured, the organ should be brought to the edge of the vessel, and the latter filled so that the outlet of the organ may be carried over its edge. Then the contents are to be carefully expressed into another vessel. In no case must the organ be made to support the weight of the contained water.

9. While full, the organ should be measured as to length, width and height, and as to its girth at one or more points.

$\S 335$. To measure the Volume of an Organ.-The following method is proposed by Dr. H. P. Bowditch $(\mathbf{1}, 149)$ : "Plunge the organ into a vessel already full, and measure the overflow."

Of course the full vessel must stand in a dish from which the overflow may be collected, and the ordinary anatomical tray will not answer. In some cases, therefore, the following method, though less simple, may be more easily adopted, and the result will be almost equally exact :-

Attach a cord securely to some part of the organ. Place it in a vessel, and fill the latter with water. Then remove the organ by means of the cord, and the amount required to fill the vessel will represent the volume of the organ. In most cases, the space occupied by the cord may be disregarded.

General References to the Preservation of Soft Parts.-The following are in addition to the references given on p. 111 :-U. S. Dispensatory, 13th ed., Art. Alcohol ; Watts, A ; Baley, A ; Günther, A, Appendix ; Kecn, 1, 2; Dwight, A, B, and I ; Reeves, A; Highley, 1 ; Hyrtl, A ; Mojsisovies, A, 26-37. 


\title{
CHAPTER IV.
}

\author{
COARSE IN JECTION S.
}

§ 336. Special Apparatus and Material, :-Acid, Acetic, No. 8, mixed with an equal volume of water-Aniline Red (Magenta)-Aniline Blue-Beeswax-Berlin Blue (see \$ 1449)-Carmine No. 40-Chrome Yellow-Chrome Green-Cobalt Blue-Dishes (2) of 100 cc. capacity-Magenta or Aniline Red-Mixing Dish, 400 cc. capacity-Pestle of Wood or Porcelain-Pins-Plaster of Paris, Finest Dental-Red Lead-Sponge-Syringe with assorted Canulæ-Thread, Linen No. 25-35, Cotton No. 20-Turpentine, Oil ofVarnish, Copal-Vermilion, American or Chinese.

$\S 337$. The object of injections is to render the blood vessels more apparent, and thus to facilitate their detection. The importance of a perfect familiarity with their position and relations cannot be overestimated from the surgical and experimental standpoints.

$\S 338$. Syringe.-A syringe is usually employed to force the injecting mass into the blood vessels. It should have the following features :-(A) Ample capacity, so that one syringe full will fill the entire arterial or venous system of the animal to be injected. Of course this does not apply to large animals like horses. One of $200 \mathrm{cc}$. is adapted to cats. (B) The piston of the syringe should fit well and be leather packed (Fig. 35). (C) There should be canuly of various sizes corresponding to the different vessels to be injected. The brass anatomical syringes (Fig. 37, 38) are best, but quite expensive.

Care of the Syringe.-As soon as an injection is finished, expel any remaining plaster into the waste pail. Then fill the syringe several times with clean water and expel it. Force a part of a syringe full through the canula, so it may be entirely emptied. Finally, it is best to unscrew the top of the barrel and remove the piston, so that the barrel may be entirely emptied. Wipe the piston with an old towel and oil the leather packing before returning the piston to the barrel.

If glue or wax mass is employed, the syringe should be cleaned with hot voater.

If the piston becomes so loose that the mass passes it instead of being forced out through the canula, remove the piston, and make the leather packing flare slightly (Fig. 35).

If the canula becomes clogged, it may be easily opened by using a small knitting needle.

§ 339. White-Metal Syringe (Fig. 35).-Syringes of this kind are cheap and answer very well for both coarse and fine injections. The metal canula is much too large to insert in an ordinary vessel, but that difficulty may be easily overcome by the use of glass can. ula (Fig. 36) and a rubber tube for connection (Fig. 42). 
Barrel.-The barrel of the syringe is the large cylindrical part in which the piston fits. The injecting mass is drawn into it by pulling the piston in the direction of the handle.
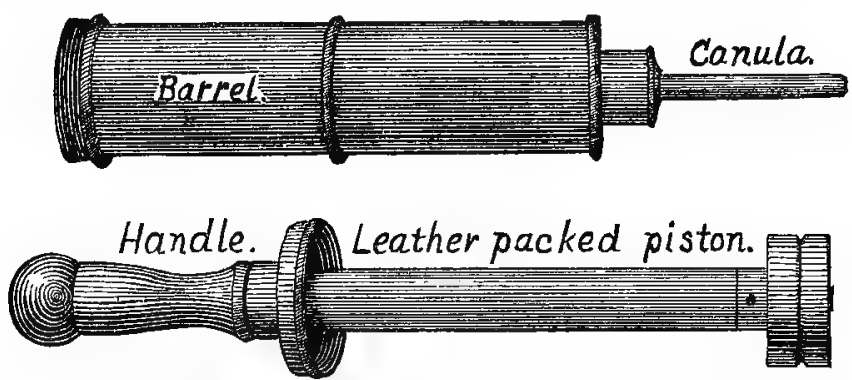

Fig. 35.-White-Metal Syringe with Top Unscrewed and Pigton REMOVED ; $\times .3$.

Canula.-The canula is the smaller part which serves to connect the syringe with the object to be injected.

Handle.--The handle is a continuation of the piston. It projects from the barrel, and by it the piston is moved.

Piston.-The piston of a syringe is within the barrel. Its end should be packed with leather, as shown in the figure, so that it will fit very closely.

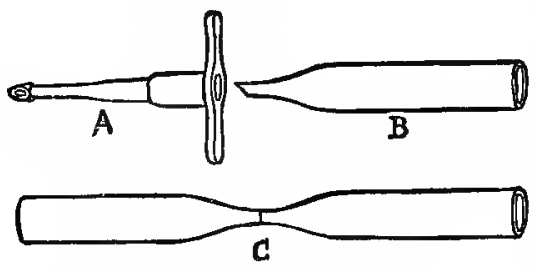

Fig. 36.-CANUL $\mathbb{R} ; \times .6$.

Canula or Nozzle.-The canula serves to connect the syringe with the object to be in. jected. The end should be cut off obliquely. It is then like a wedge, and may be inserted into the vessel much more easily than one cut aquarely off.

The caliber of the canula should be as great as can be put into the vessel to be injected. For plaster injections ( $\S 341$ ) the canula should not be less than $1 \mathrm{~mm}$. at its narrowest point.

A.-Metal canula with lateral arms at the end next the syringe, and a slight shoulder at the small end.

B.-A glass canula cut off obliquely at its small end.

C. - A glass tube drawn out for two canulæ.

$\S 340$. To prepare glass canulæ, take a glass tube about $6 \mathrm{~mm}$. in diameter and from $8-10 \mathrm{~cm}$. in length, and heat its middle evenly in a Bunsen flame. When the glass is softened, draw the two ends apart until the tube is sufficiently reduced in caliber in the middle; then after cooling make a fine scratch with a file as shown in $\mathbf{C}$, and the two may be broken apart. When separated, grind the end tirst on a fine file with water to make it oblique as in $\mathbf{A}$ and $\mathbf{B}$, and finally on the fine oil stone to make it smooth. Heat the larger end in the flame to round the sharp edges. 
In this figure (Fig. . 37) of the regular brass anatomical syringe, are shown four canula of various sizes and a separable stop-cock. The stop-cock fits upon the end of the short

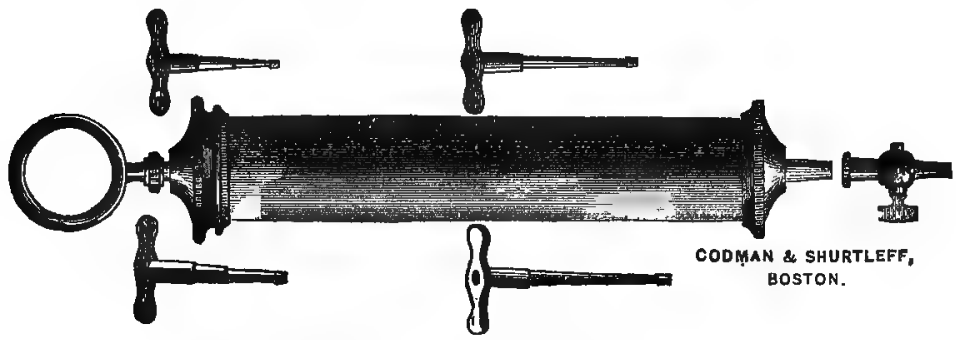

FIG. 37.-Brass SyrINGE; $\times .5$.

canula of the syringe, and the canulw for insertion in the vessels fit upon the end of the . stop-cock. In most syringes of this kind the stop-cock may be dispensed with and the canulæ fitted directly to the syringe if desired.

\section{INJECTION MASSES.}

§ 341. Plaster of Paris Mass.-The most convenient mass is composed of the finest plaster of Paris stained with carmine sollttion for arteries and with Berlin blue for veins. To facilitate the preparation of the mass, a quantity of both colors should be kept in stock.

§ 342. Carmine Solution.This is prepared by grinding to a paste 4-5 grams of carmine No.

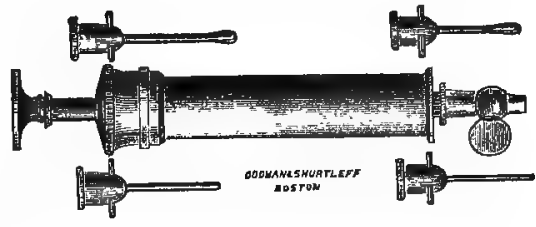

Frg. 38.-Small Brass Sxringet with ReMovabLe Stop-Cock aND CANUL, For Fine Injections ; $x .3$. 40 in 20 cc. of water and then dissolving it in $50 \mathrm{cc}$. of strong ammonia. To this solution is then added $75 \mathrm{cc}$. of glycerin and 500 cc. of water. After shaking well, filter through fine flannel or absorbent cotton.

$\S 343$. Blue.-Berlin blue ( $\$ 1449$ ), a saturated aqueous solution, 500 cc., glycerin, 75 cc. Mix the glycerin and the blue, and filter as for red. The glycerin preserves the solutions and retards the setting of the plaster (Gage, 1, 717).

- \& 344. Various Colors-A. Red.-Plaster mass may be well colored by American or Chinese vermilion, red lead or a solution of aniline red (magenta): Magenta, 2.5 grams ; 50 per cent. alcohol, $100 \mathrm{cc}$. 
B. Blue.-The plaster mass may be colored blue by cobalt or ultramarine blue or a solution of aniline blue: Aniline blue, 2.5 grams ; 50 per cent. alcohol, 100 cc.

C. Yellow or Green.-Employ chrome yellow or green.

Permanent preparations should not be made of an animal injected with a mass colored by one of the aniline dyes, for they are soluble in alcohol and fade in the light. The vermilion and cobalt blue are the most permanent, but the carmine and Berlin blue last many years.

$\$ 345$. Preparation of Plaster Injection Masses.-The masses should be used immediately after preparation, and before the plaster has time to set.

Approximately the same volume of plaster and liquid should be employed for ordinary injections. If, however, one wishes the mass to fill the smallest vessels, the liquid should be increased so that the ratio is as $1-2$ or even $1-3$.

A. Mass Colored with Carmine.-Measure out 100 cc. of the finest plaster of Paris and put it into a mixing dish, a tea or coffee cup, that will hold about $400 \mathrm{cc}$. Add to this plaster about $100 \mathrm{cc}$. of the carmine solution ( $\$ 343$ ), and mix thoroughly with a wooden or porcelain pestle. Finally, add slowly and with constant stirring the 50 per cent. acetic acid. Add the acid till the color changes to bright red and the odor of the acid in the mass is quite perceptible. An excess of acid is less injurious than a deficiency.

B. Mass Colored with Berlin Blue.-Plaster same as for carmine. Add $100 \mathrm{cc}$. of the Berlin blue solution ( $\$ 343$ ), and stir well: No acid is necessary.

C. Masses Stained with Aniline.-Measure out $100 \mathrm{cc}$. of plaster and put it into the mixing dish as directed above; then add, for red, $20 \mathrm{cc}$. of the magenta solution and $100 \mathrm{cc}$. of the 15 per cent. glycerin solntion $(\$ 171)$. Stir thoroughly. For blue, add $50 \mathrm{cc}$. of the blue aniline solution and 75 cc. of the 15 per cent. glycerin.

D. Mass Colored with Vermilion or Red Lead.-Put 25 grams of the dry color into the mixing dish and add 25 cc. of 15 per cent. glycerin. Grind the color thoroughly to crush all the lumps. Finally, add $100 \mathrm{cc}$. of plaster and $100 \mathrm{cc}$. of 15 per cent. glycerin, and mix very thoroughly.

E. Masses Colored with Cobalt or Ultramarine Blue, Chrome Yellow or Green.-Employ 15 cc. of the color, and prepare as directed for the vermilion ( $\$ 344$ ). 


\section{A STARCH INJECTION MASS.}

(To replace pp. 139-140, Wilder and Gage's Anatomical Technology.)

[From Thr New York Medical Journal, June 7, 1884.]

A coarse injection mass which is cold-flowing, may be forced nearly to the capillaries, rapidly hardens after injection, leaves the vessels flexible, does not dull dissecting instruments, is suitable for permanent dry or alcoholic preparations, is simple in its manipulation, cleanly and economical, seems to be fully realized in the starch mass introduced by Ad. Pansch, of Kiel, and since recommended, with various modifications, by Wikszemski, Dalla Rosa, Meyer, and Browning.*

As starch is insoluble in alcohol and cold water, it becomes hard when injected into the blood-vessels simply by the exudation of the liquid with which it is mixed. (That the starch grains forming the mass remain entirely unchanged may be easily demonstrated by making a microscopic examination of the contents of an injected vessel. )

The mass originally recommended by Pansch consisted of wheat-flour and cold water, to which was added a sufficient quantity of the desired coloring matter. Later experiments have shown that pure starch is better than flour.

\section{Mass for Ordinary Injections :}

Dry starch ("laundry" is good) $\ldots \ldots \ldots \ldots \ldots \ldots \ldots$ I vol. $21 / 2$ per cent. aqueous solution of chloral hydrate, .... I " " 95 per cent. alcohol, $\uparrow \ldots \ldots \ldots \ldots \ldots \ldots \ldots \ldots$. I/4 "

Since almost any animal injected may afford some organ worth preserving, it seems better to employ permanent colors for tingeing the mass. Among those which are available, probably vermilion, red lead, ultramarine, chrome orange, yellow, or green, are preferable. ,

* See Ad. Pansch, "Archiv für Anatomie und Entwickl.," 1877, pp. 480-482, and 1881. pp, 76-78; Wikszemski, same journal, 1880, pp. 232-234; Dalla Rossa, same, pp. 371-377 : Herm. von Meyer, same, 1882, pp. 60, 61, and 1883, pp. 265, 266 ; Prowning, "Annals of Anatomy and Surgery," 1884. pp. 24, 25.

$\dagger$ The chloral and alcohol prevent fermentation in the mass when it is kept in stock; the alcohol also increases the fluidity and likewise the more rapid hardening in the vessels; both, of course, act as a preservative upon the animal injected. 


\section{Preparation of the Color.}

Dry color, .................... I vol.

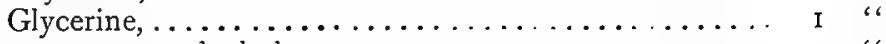

95 per cent. alcohol, ................. I "

To avoid lumps, which would clog the cannulæ, or small vessels, the color is thoroughly ground with the liquid in a mortar. It is stored in a well-stoppered bottle, and is prepared for use simply by shaking.

\section{Special Mass.}

For the injection of brains, and, perhaps, for other rapidly perishing specimens, it seems best, as suggested by Prof. Wilder, to use strong preservatives in preparing the mass:

Corn starch (that used for food). ........... I vol

5 per cent. aqueous solution of chloral hydrate, .... $1 / 2$ "

95 per cent. alcohol. $\ldots \ldots \ldots \ldots \ldots \ldots \ldots \ldots \ldots, 3 / 4$ “

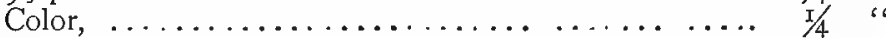

For convenience and economy, a considerable quantity of either of the masses described above may be prepared at once, and kept in a widemouthed specimen or fruit jar. A smooth stick in each jar is convenient for stirring the mass, which should always be done just before using. The syringe may be filled directly from the jar, and any mass remaining in the syringe after the injection is finished may be returned to the jar.

If it is desired to have the mass enter very fine vessels, some of the stock mass, as given above, diluted with an equal volume of water or chloral solution, may be injected first, and immediately followed by the undiluted mass, or, for large animals, a mass containing twice the usual amount of starch. In whatever form the starch is used, it is necessary to work somewhat expeditiously, because the exudation of the liquid in the smaller vessels takes place so rapidly that the mass hardens very quickly in them. The larger the vessel, the more slowly, of course, do the exudation and, consequently, the hardening take place. It sometimes happens that large vessels, like the aorta, are not fully distended after the exudation of the liquid. In this case some mass containing double the ordinary amount of starch can be advantageously injected in two hours or longer after the first injection.

\section{Dry Preparations.}

Finally, if vessels injected with the starch mass are dissected free, soaked a day or two in Wickersheimer's preservative, and then dried, they retain their form, and, to a great degree, their flexibility.

S. H. GAGE, Cornell University, Ithaca, N. Y., April 25, 1884. 
$\S$ 346. Wax and Tallow Masses.-Wax Mass.-Beeswax, 2 parts ; Canada balsam, 2 parts ; Vermilion, 2 parts.

Mix the wax and Canada balsam and melt over a water bath. Then grind the vermilion thoroughly in a small amount of mastic varnish, and add it to the mixture. Heat over the water bath for half an hour or more. This mass flows very finely. Hyrtl, A, 616.

Tallow Mass.-Tallow, 900 grams; Magnesia usta (calcined magnesia), 15 grams; Vermilion, 30 grams.

Grind the magnesia and vermilion in a small amount of mastic varnish or turpentine, before adding to the melted tallow. Harrison, A, II., 866.

\$ 347. Practical Working of Wax and Tallow Injections.First. The animal must be warmed to $38-40^{\circ} \mathrm{C}$. This is best done in a large galvanized iron dish that may be covered and the water kept hot by means of a Bunsen burner or in some other way. All the heating should be done in a water bath so that no burning may occur.

When the animal is warmed through (the time required depends on the size of the animal), the canula should be put in position (\$358). Warm the syringe thoroughly by filling it with hot water and slowly emptying it. Warm the mass till it is quite fluid, and stir it well. If it is not heated above $50^{\circ} \mathrm{C}$., it will not burn most mammalian tissues. Fill the syringe with the mass and force it out, to make sure the mass is thoroughly mixed. Then fill the syringe, connect it with the canula in the vessel, and force the mass in rather more rapidly than directed for plaster (\$359), but the operation should not be so long continued.

One cannot inject Fishes or Amphibia with wax mass, as Hyrtl has well said, for the heat required to warm the subject and the mass would cook the tissues. For them, plaster or some other cold flowing mass, or glue which remains liquid at a low temperature, must be used.

After the injection is finished, the animal should remain in a cool place for at least three or four hours before the dissection is commenced.

\$ 348. Choice of Specimen for Injection.--A young adult and lean cat is best. It should be fasting except for special purposes.

$\S 349$. Time of Injection.-Inject before the rigor mortis comes on. If that is impossible, it is better to put it into warm water $\left(35^{\circ} \mathrm{C}\right.$.) for an hour to make the muscles flexible. This is not abso- 
lutely necessary, but the smaller vessels will be more likely to be injected if the muscles are flexible.

$\S 350$. Arteries to Inject for a Complete Injection of the Animal, named in order of Desirability : $\mathbf{A}$. femoralis, A. carotidea, Aorta (Fig. 39, 101).

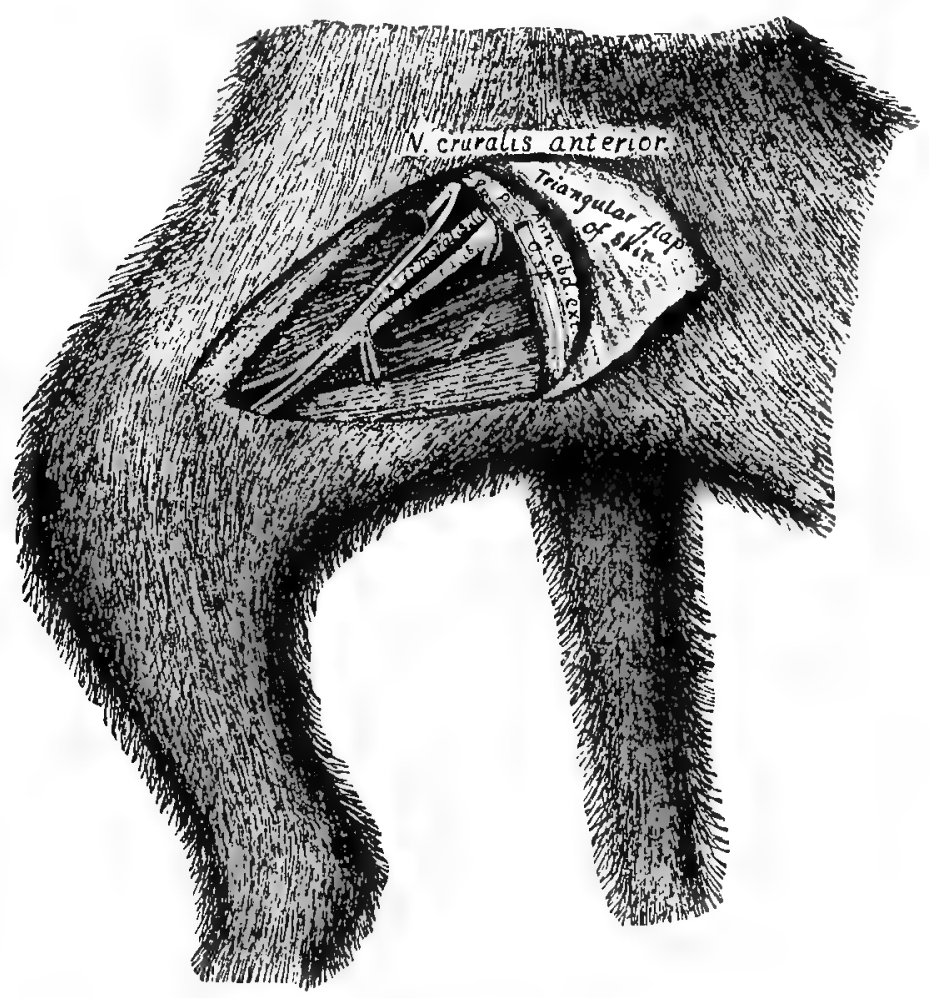

Fia. 39.-Femoral, Vessels; $\times$.5.

$\S 351$. Veins to Inject: $\mathbf{V}$. femoralis, $\mathbf{V}$. jugularis externa, Postcava (Fig. 101). As to Cleanliness, see $§ 199$.

INJECTION OF THE FEMORAL VESSELS.

$\S$ 352. Posture.-Place the cat dorsicumbent, as in Fig. 76.

§ 353. Exposure.-Grasp the meros close to the trunk with the pollex and index, and the femur will be felt in an interval between 
the muscles on the cephalic side (upper side in the present posture). The femoral vessels are just entad of the skin and connective tissue, and parallel with the femur in the proximal third of the meros. To expose the vessels, lift a triangular flap of skin (Fig. 39).

§ 354. Parting the Hair.-Before making the incisions, wet the hair well with a sponge, and with a comb part it along the lines where the incisions are to be made. Incisions can be very much more neatly and easily made after the skin is thus exposed.

FIa. 39.-Preparation.-The arteries were injected caudad from the aorta abdominalis (\$ 101); then the veins were injected from the V. poplitæa in the popliteal space, in the concavity of the knee (Fig 30). After half an hour the triangular flap of skin was dissected free and turned to the left and secured by a pin passing through its tip into the muscles of the left meros. All the fat and connective tissue were then removed with the tracer, fine forceps and scissors.

A. (Arteria) femoralis-Femoral artery.-This is the continuation of the A. iliaca externa (\$101). It is between the vein and nerve.

Ann. abd. ext. Annulus abdominalis exterior s. ectalis-External (ectal) inguinal ring.-This is the ectal opening of the inguinal canal through which the chorda spermatica passes into the abdominal cavity.

C. sp. Chorda spermatica-Spermatic cord. - This is a bundle of structures passing from the testis to the abdominal cavity. It is composed mainly of the spermatic artery and vein and the vas deferens.

Lg. P. Ligamentum Poupartii (Poupart's ligament, Crural arch).-This is a ligamentous or aponeurotic arch dorsad of which pass the femoral vessels.

N. (Nervus) cruralis anterior (Anterior crural nerve).-This is the largest branch of the lumbar plexus of nerves. It is both muscular and cutaneous in distribution. Quain, A, I, 604.

Scarpa's Triangle.-This is the triangular depression on the cephalic side of the meros in which the femoral vessels are found. Quain, A, I, 454; Gray, A, 546.

V. (Vena) femoralis-Femoral vein.-The femoral vein is continued by the $V$. iliaca communis as it passes into the abdomen. It is caudad of the artery as the two pass dorsad of the Ligamentum Poupartii, but ventrad of it in the meros.

\& 355. Dissection.-With the tracer and fine forceps, very carefully dissect the artery free from the vein, nerve and connective tissue for about $2 \mathrm{~cm}$. from the abdominal wall. Be very careful not to stretch the artery. It seems only about half as thick as the vein.

\$356. Incisions in the Vessel.-When the artery is free, put the handle of a scalpel wet in 15 per cent. glycerin entad of it (Fig. 40); with a Charrière scalpel then make a V-shaped incision that will include about one third the width of the vessel as it is flattened on the scalpel handle. Cut entirely through to the scalpel handle on which the vessel rests. The Charrière should be held pen-like (Fig. 63), and so that the apex of the $V$ (Fig. 40) shall point periph- 
erad. A more convenient, but less safe way for beginners, is to

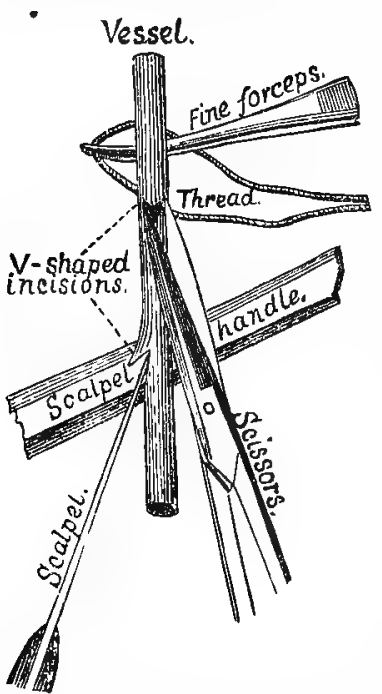

Fici, 10.-Incisions and PREPARATION FOR LIGATURING: $\times .5$. employ scissors as sloown in Fig. 40. It is sometimes desirable also to cut the $V$ from apex to base as shown in Fig. 42. Enlarge the opening in the vessel by inserting the probe wet with 15 per cent. glycerin.

$\$ 35 \%$. Ligatures. $-W$ hile the probe is still in the vessel, with the fine forceps grasp the middle of a thread $30-40 \mathrm{~cm}$. long and push the loop through entad of the vessel, and at a point centrad of the incision (Fig. 40). The loop may then be grasped by the fingers or forceps and drawn through as far as desired.

Instead of pushing the thread through as directed above, one may put the forceps entad of the vessel first and grasp the loop and pull it through. Bernard, A, 263.

When the thread is through as far as desired, cut the loop. The thread nearest the incision is for tying the canula in the vessel ; the other is for ligaturing the vessel when the injection is finished. Both threads should be loosely tied in a surgeon's knot (Fig. 41), so that they may be quickly tightened.

§ 358. Introduction of the Canula.When the threads are prepared, remove the probe from the vessel, select a canula of the proper size, that is, as large as can be put into the vessel, and make sure that it is open by blowing into it or forcing water through it with the syringe. Having wet its small end, put it into the vessel so that the injection will be centrad. To insert the canula, grasp one edge of the $V$-shaped incision with the fine forceps and pull it open. Now introduce

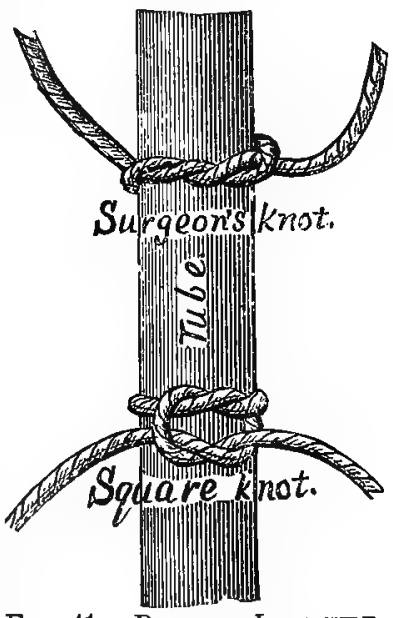

Fya. 41.-Dodbue Ligature; $\times 1$. the canula and push gently with a slight twisting motion. At the same time pull with an equal force in the opposite direction with the 
fine forceps by which the edge of the incision is grasped. As soon as the canula is in the vessel, let go the edge of the slit and grasp the whole vessel where it sheaths the canula. Pinch quite firmly, and pull while the canula is pushed into the vessel for about $1 \mathrm{~cm}$. The canula being smooth slips into the vessel notwithstanding the pressure of the forceps. Rest the elbows on the table to steady the hands.

The canula should be very smooth, and the serrations on the forceps must not be too deep or they will cut the vessel. The canula may be polished as directed for instruments $(\S 183)$, and any roughness may be removed by the oil stone.

As soon as the canula is properly inserted, put the thread nearest the incision (Fig. 40) so that it will press on the canula within the vessel, and then tighten the knot. If the canula has an enlargement near the end (Fig. 36, A), it cannot escape when tied as in Fig. 42. If there is no enlargement or a glass canula is used, the thread must be tied to some part of the canula outside the vessel. If there are transverse projections (Fig. 36, A), the thread should be tied around one of them. If a glass canula is used, tie as shown in Fig. 42. All the threads should be knotted in a hard knot finally, and the ends should be cut within a centimeter of the last knot.

\$ 359. Making the Injection.-As soon as the canula is secured in the vessel, fill the

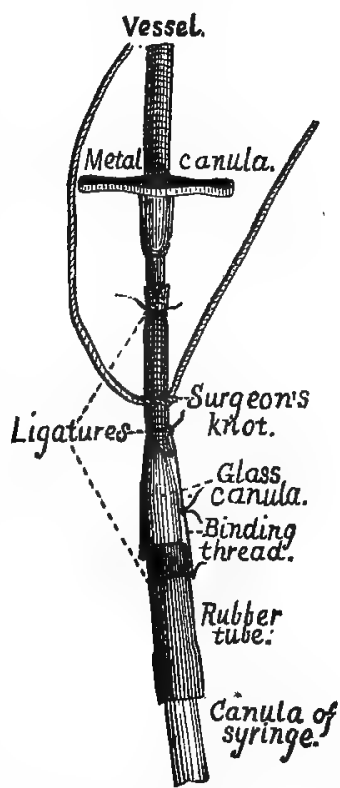

Fig. 42.-Insemtivg ANd Securing Candla; $\times \mathbf{5}$. syringe partly with water or normal salt solution, connect it with the canula in the vessel, and force a little of the liquid in to make sure the canula is open and properly inserted. In connecting the canula and syringe, grasp the canula with one hand and hold it firmly while making the connection. Do the same in separating them.

After forcing a small amount of water into the vessel, separate the canula and syringe, expel the water, and then prepare the mass as directed above ( $\$ 345$ ). Stir the mass thoroughly, and then fill the syringe, being sure to lower the syringe as the mass is drawn 
into it, so that no air may get into the syringeConnect the syringe with the canula which is in the vesse (358). Hold the canula firmly with one hand and pull toward the syringe as the mass is forced into the vessel. Be sure that the vessel is -not looped or twisted in the least, but drawn peripherad just enough to straighten it. Force the piston down steadily and continuously ; do not allow it to stop until the injection is finished. If the canula becomes clogged, the resistance will be complete, and there will be an entire absence of the elastic feeling which comes from the distended arteries. Very often the canula may be opened by pulling the piston back a little and then forcing it down quickly.

One can tell only by experience when the injection is finished. The vessels are usually filled, however, when the piston returns slightly on remitting the pressure. If the injection is carried too far or the pressure is too great, the semilunar valves (Fig. 102) are liable to be ruptured and the heart filled, or some vessel may give way.

$\S 360$. Tying the Vessel.-As soon as the injection is finished, tie the vessel with the thread provided for the purpose (Fig. 42), draw back the piston slightly, and then cut the string holding the canula in the vessel and remove the canula.

$\S 361$. Cleaning the Canula, Syringe and Mixing Dish.-Do this immediately after the injection is finished before the plaster has time to set. Expel the plaster remaining in the syringe into the waste pail ; then fill the syringe with water and empty it. Do this several times, and then force some clean water through the canula. Finally, it is best to unscrew the top of the syringe and pour out any liquid that has passed the piston.

Throw any plaster remaining in the mixing dish into the waste pail and clean out the dish very thoroughly. The plaster must not be thrown into the sink lest it should set and clog the waste pipe. The dissection may commence in half an hour after the injection is tinished.

\$362. Injection of the Femoral Vein-Fig. 39.-Dissect the vein free for 2 or $3 \mathrm{~cm}$. from the abdominal wall as described for the artery ( $\$ 353)$. Compress the vein and force the blood centrad. It will pass very readily and leave the vein nearly empty. Now press on the vein just as it enters the abdomen and try to force the blood peripherad. There will be seen a bulging in the vessel extending about $.5 \mathrm{~cm}$. from the body wall. There are two valves at 
the peripheral end of the enlargement which prevent the flow of blood in this direction. Make a V-shaped incision in the vein centrad of the valves in the same manner as directed for the artery $(\S 356)$. Compress the thorax and hold the cat upright to facilitate the flow of blood.

When as much blood as possible is removed, insert the canula (Fig. 42), and inject plaster prepared as directed (\$ 359).

The injection should be made as directed for the arteries, but it should be remembered that the walls of the veins are thinner than those of the arteries, and hence the pressure must be more moderate.

On account of the valves in the systemic veins (Fig. 102), it is necessary to inject centrad. The injection may be made to pass the valves sometimes, however, by manipulating the part while the mass is forced in.

$\$ 363$. Injection of the Aorta Abdominalis.-Posture and Exposure.-Place the cat dorsicumbent, and then expose the abdominal viscera as shown in Fig. 79, and directed hereafter.

Turn the four flaps aside, and then turn the intestines to the left. The right kidney will be seen on the right side, and in the middle line the postcava (Fig. 101).

\$364. Dissection, and Insertion of Canula.-With the tracer tear away the mesentery (Fig. 80) and connective tissue in the middle line opposite the caudal margin of the right kidney. The aorta is dorsad and sinistrad of the postcava in this region, and between the two great psoas muscles. Free it for $2-3 \mathrm{~cm}$. Make a V-shaped incision with scissors (Fig. 40), insert and fasten the canula, and inject as directed above $(\$ 359)$.

If only the cephalic part of the body is to be studied, it is necessary to inject cephalad only, but if the entire animal is to be studied, one should inject first cephalad and then caudad. The second injection should be made as soon as possible after the first.

As soon as the injection is made, the postcava should be opened to allow the blood to escape.

§ 365. Injection of the Postcava.-The exposure is the same as for the Aorta abdominalis. Inject as directed for the V. femoralis (\$362)。

$\S 366$. Injection of the V. Jugularis Externa.-Posture.-Place the cat dorsicumbent and a block flatwise under the neck and shoulders. Rotate the head so that the nose points away from the side to be injected.

Exposure.-Press upon the throat and find the larynx (Fig. 30, 
$\$ 224)$. Then press upon the side of the neck near the bead and determine the position of the wing of the atlas, diapophysis atlantalis (Fig. 52, § 229). Part the hair along a caudo-cephalic line from about midway between the wing of the atlas and the larynx, and then commencing opposite the cephalic edge of the wing of the atlas, carry an incision directly caudad for $3-5 \mathrm{~cm}$. Divaricate the skin, and the external jugular vein will appear, full of blood.

Injection.-Dissect the vein free as directed for the A. femoralis, make a V-shaped incision, insert the canula, and then remove as much blood as possible, as directed ( $\S 362)$. Finally, inject with blue plaster (\$343).

It is impossible to fill the small vessels with plaster on account of the valves (Fig. 102). If one wishes to inject the venous system of the cat, it may be done successfully in most cases by injecting blue gelatin into the jugular or femoral vein. See Appendix, $\S 1450$.

\$ 367. Injection of the Arteria Carotidea.-Posture.-The posture should be the same as for the V. jugularis externa ( $\$ 366$ ).

Exposure.-Make an incision through the skin as directed for the external jugular vein $(\S 366)$. Dissect up the mesal edge of the skin to the ventrimeson. Determine the position of the larynx, and then make an incision $2-3 \mathrm{~cm}$. long just laterad of the larynx and through the sterno-mastoid muscle. Divaricate the cut edges of the muscle, and there will be seen extending parallel with the trachea the carotid artery, the vagus and sympathic nerves, and the $V$. jugularis interna (Fig. 101).

Dissection.-With the tracer and forceps, dissect the artery free from the nerve and vein. Make a V-shaped incision, insert the canula, and inject centrad as dirested for the $A$. femoralis ( $\$ 359$ ).

All the arteries of the body will be filled excepting those cephalad of the place of injection on the side injected. To fill those, one must inject cephalad after the general injection is made.

References to Coarse Injections.--Bernard, A, 191, 262 ; Gage, 1, 717; Harrison, A, II, 865 ; Heath, A, 547 ; Hyrtl, A, 615 ; Straus-Durckheim, B, I, 90; Mojsisovics, A, $15-24$. 


\section{H A P T E R V.}

\section{OSTEOLOGY-THE STUDY OF THE BONES.}

THE DETERMINATION OF THE RIGHT AND LEFT WITH CER'TAIN BONES-SPECIAL MNEMONICS OF THE HUMERUS-DETALIED DESCRIPTION OF THE SCAPULA, HUMERUS, CARPALIA, Clavicula, sTERNUM, COSTA, PELVIS, VeRTEBRA AND SKULL.

§363. A general description of the whole skeleton has been given oi pp. 87-95. In accordance with the general plan of this work as stated in $\S 128$, certain parts of the skeleton are also described somewhat in detail, while others are mentioned only incidentally or not at all.

Sooner or later, of course, the student will consult some complete treatise upon Human, Veterinary or Comparative Anatomy, and thus acquire the information here omitted. We believe, however, that he will do well to make for himself drawings and descriptions of the bones not fully described here; the drawings should be in outline or but slightly shaded; the descriptions should be in two parts, general and brief, and special and detailed.

\section{DETERMINATION OF RIGHT AND LEFT.}

\$369. The right and left of paired bones may be determined by reference to a mounted skeleton or figure. The longer membral bones and the parts of the shoulder and pelvic girdles may also be distinguished by means of the following special formulæ:-

In all cases, except with the scapula, innominatum and fibula, the long axis of the given bone is placed horizontally from the left to the right of the observer, and the distal end is made to point to the side to which the given bone belongs.

At the end of the formula for determining the side of the body to which a long bone belongs, are placed directions for the recognition of one of the extremities and two of the sides or aspects which are not opposite. The end and the sides not given may then be readily ascertained. Finally, there are directions for determining 
the aspects of the limbs as wholes, together with some special mnemonics for the humerus.

\$ 370. Clavicula-Clavicle, collar bone (Fig. 48).-A. Man.It should be placed with the greater concavity up, the projecting part of the thick, mesal or sternal end toward the observer; then the flattened lateral end will point to the side to which the bone belongs.

B. Cat.-It should be held with the subcylindrical end mesad and curving downward, the great concavity toward the observer; the flattened end will then point to the side to which the bone belongs. In young cats the two ends are so nearly alike that it is difficult to determine right and left.

\$ 371. Scapula-Shoulder blade (Fig. 43-45).-The gleno-vertebral angle ( $\$ 383$ ) should be held toward the observer and the glenoid fossa down; then the mesoscapula will be on the side to which the bone belongs.

§ 372. Humerus-(Fig. 46).-The bone should be held with the olecranon fossa (Fig. 71) up, the epitrochlea toward the observer (the musculo-spiral groove in man away from him). In the cat and many other animals, rarely in man also, there is a foramen $(F m$. epitrochleare, Fig. 46), near the caudal border of the distal end $(\S 417)$.

The olecranon fossa, the deepest of the distal fossæ (Fig. 71) is on the dorsal aspect. The Foramen epitrochleare in the cat and the most prominent apophysis (epitrochlea) of the distal end are on the caudal side in both cat and man.

§ 373. Radius-(Fig. 30). - The bicipital tuberosity should be held down, the styloid process on the side away from the observer. The styloid process is the most distal part of the bone. It is on the cephalic side, while the bicipital tuberosity, which is near the proximal end, is mostly on the ventral side. The distal end is the larger.

\$374. Ulna-(Fig. 30). - The great sigmoid cavity should be held down, the lesser sigmoid cavity from the observer; then the smaller end points to the side.

The great sigmoid cavity is on the ventral aspect at the proximal end; the lesser one is on the cephalic aspect just distad of the greater one and continuous with it.

\$ 375. Innominatum-Pelvic bone (Fig. 30, 51).-It should be held with the ischiatic tuberosity toward the observer, the pubic 
arthral facet down, the cotyloid fossa looking to one side; the fossa will be on the side to which the bone belongs.

\$ 376. Femur-(Fig. 30). -The bone should be placed with the head pointing away from the observer, the intercondylar fossa or notch down.

The nearly spherical arthral head is situated at the proximal extremity ; it faces approximately cephalad. The longitudinal concavity of the whole bone and the intercondylar fossa or notch are on the ventral aspect.

$\$ 377$. Tibia-(Fig. 30).--It should be held with the tuberosity for the patellar ligament up, the malleolus facing away from the observer; the end bearing the malleolus will point to the side to which the bone balongs.

The most distal part (malleolus) is on the cephalic, and the longitudinal concavity on the ventral aspect.

\& 378. Fibula-(Fig. 30).-If the bone be placed horizontally, with its distal extremity toward the observer, and the distal arthral surface up, then the deep rough depression at the latero-distal margin of the arthral surface will be on the side to which the bone belongs; or, in grasping this end with the pollex and index, the pollex of the side to which the bone belongs may be easily put into this depression.

This method, so far as we know, was first devised by G. S. Sheppard, a student in the anatomical laboratory of Cornell University.

The distal arthral surface is on the cephalic aspect, and the depression for the ligament spoken of below is at the ventro-distal edge of this surface.

The arthral surface of the distal extremity is on the side, while that of the proximal extremity is nearly on the end. The depression spoken of at the distal end is for the attachment of the "posterior fasciculus of the external lateral ligament" of the ankle.

\section{$\$ 379$. Determination of the Right and Left with the Entire} Limbs.-The sides of the whole arm may be recognized by remembering that the capitellum, radius and pollex (thumb) are on the cephalic side, while the olecranon process and fossa are on the dorsal side, and the convexity of the elbow points dorsad.

The sides of the whole leg may be determined, since the tibia and primus (great toe) are on the cephalic aspect, and the convexity of the knee faces dorsad. 
$\S 380$. Special Mnemonics of the Humerus.-With a bone having such numerous and important anatomical relations, so variously placed in different animals and in the same animal at different times, so frequently involved in surgery, and so generally represented in painting and statuary, it is very desirable that the names and relative positions of the parts should be promptly remembered. The following mnemonic suggestions may prove useful to some :-

Of the two ends of the bone, proximal and distal, the former is the larger and has the longer name.

'The cephatic side is also called radial, and both these names are longer than the corresponding words caudal and ulnar, which apply to the opposite side.

With one exception, the principal features of the cephalic side have longer uames than the similar parts upon the caudal side. Trochiter, capitellum and Fs. radiatis are longer than trochin, trochlea and Fs. ulnaris. The trochiter itself also is larger than the trochin.

Epicondylus equals epitrochlea in length, but the latter is readily associated with trochlea, and itself suggests the name of the Fm. epitrochleare.

In the normal position of the arm, the deep olecranon fossa (Fig. 71) is uppermost with both man and cat. With the cat also, the longer and more decided dorsal concavity of the bone as a whole may be, though somewhat remotely, associated with the back of a saddle horse.

$\S 381$. The selected portions of the skeleton are here described in the following order, which is mainly that of their simplicity :-

Scapula, humerus, ulna, radius, carpus, clavicula, sternum, costæ (ribs), pelvis, vertebræ, and skull. The other membral bones and the Os hyoides have been briefly described in $\$ \$ 220-224$.

THE SCAPULA (Fig. 30, 43, 44, 45, 67, 74, 75).

Raferences.-Straus-Durckheim, A, I, 507-509 ; Parker, A, 215, Pl. xxx. ; Owen, A, II, 488; Gray, A, 218-223; Quain, A, I, 81; Chauveau, A, 81; Chauveau (Fleming), A, 72 ; Flower, A, 221 and 229 ; Humphrey, A, 363-370 ; Mivart, B, 89-91; Leyh, A, 170, 171.

\$382. General Description.-The scapula (shoulder blade or blade bone) is a flat irregular bone imbedded in the muscles on the lateral aspect of the cephalic region of the thorax (Fig. 30, 67, 74, 75), and articulating with the humerus to form the shoulder joint.

As seen from its ental or ectal aspect (Fig. 43 and 44 ), the out- 
line of the scapula may be characterized as either subtriangular or approximacely semicircular. The former term is more commonly employed, perhaps because it is more applicable to the human scapula. But if the two scapulæ of the cat are placed with their straighter sides in apposition, they will be seen to cover an area which is approximately circular, although the borders are more or less undulating.

\$ 383. The Margins and Angles of the Scapula.-It is at least convenient to regard the scapula as triangular, and as presenting therefore three sides (margines) and three angles (anguli).

The thicker (glenoid or arthral) angle articulates with the humerus, and presents several elevations and depressions which will be described separately. Its larger part is occupied by a concave surface, the Fossa glenoidea, for articulation with the humerus; hence that border of the bone which is separated from the fossa only by its lip is called the Margo glenoideus. Between the fossa and the other border springs a hook-shaped projection, the Prc. coracoideus, and the border is thence named Mrg. coracoideus. The intermediate border is called the Mrg. vertebralis from its proximity to the Columna vertebralis.

Of the borders, the glenoid is the longest and straightest. The coracoid is the shortest and least regular, and its outline varies in different individuals. According to the observations of Parker (A, 215, Pl. xxx., Fig. 1-3), in the cat and in some other Carnivora this margin ossifies from an independent center and remains for some time separate from the rest of the bone. The vertebral border is intermediate in length, and presents a nearly regular curvature. With young individuals this margin is cartilaginous, representing a suprascapula, but later it becomes coössified with the rest.

The angles, respectively more and less obtuse, formed by the junction of the vertebral margin with the other two, are called coracovertebral and gleno-vertebral.

The emargination of the coracoid border near the neck of the bone (at the lower end of the dotted space in Fig. 43) is known as the Incisura coraco-scapularis (Flower, A, 223). In the human scapula it is deeper and commonly called the suprascapular notch.

$\$$ 384. Description of Fig. 43.-The ental aspect of an adult left scapula.

Acromion.-This is more distinctly seen in Fig. 44 and 45 .

$\S 385$. Area Musculares-Areas of the attachment of muscles.-Upon these two figures of the scapula and upon four views of the humerus (Fig. 68-71), the areas of muscular 
attachment are enclosed by interrupted lines. Following the method of Gray (A) and H. S. Williams (1), the origins are indicated by dots and the insertions by short lines. It is easy to associate these two marks with the letters $o$ and $i$, which form respectively the initials of origin and insertion.

The scapula affords origin to the following 10 muscles: Subscapularis, supraspinatus, infraspinatus, meditriceps, spino-deitvideus, acromio-deltoideus, teres (major), micostalis

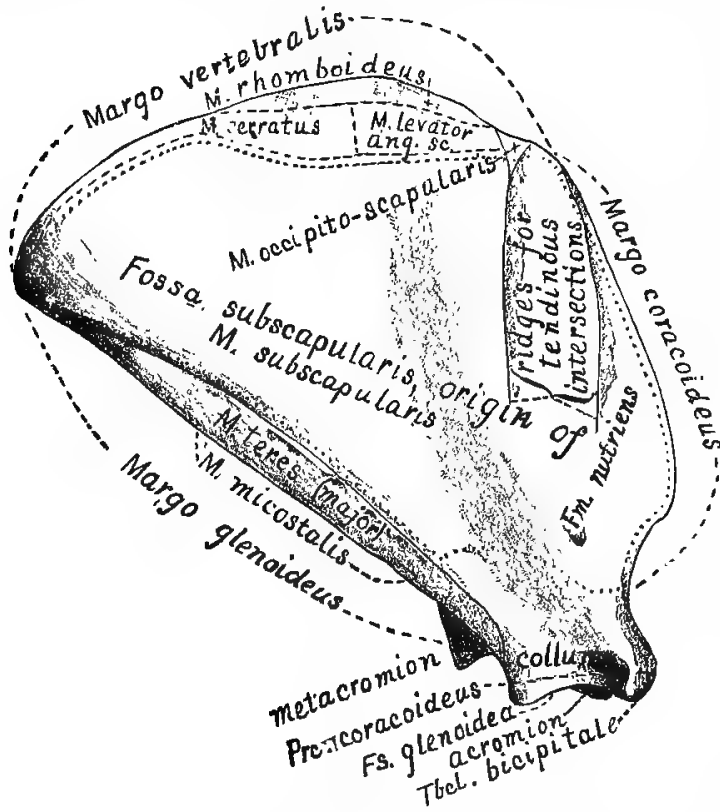

Fia. 43.-The Ental Aspect of an addut Left SCAPULA ; $\times 1$. (teres minor), biceps and coracoideus. Upon it are inserted the following 7 muscles : Acromio-trapezius, spino-trapezinis, rhomboideus, serratus, levator anguli scapula, levator claviculce and occipito-scapularis. On the figure the insertion area of the last named muscle is represented as too near the coraco-vertebral angle.

\$ 386. Collum - Neck. This is the thickened and slightly constricted portion of the scapula connecting the glenoid end or angle with the body of the bone. According to Quain (A, II, 83), its limits are differently assigned by anatomists and surgeons.

\$387. Froramen NutriensThe vascular foramen.-There may be 1,2 or 3 of these foramina upon the ental aspect, and their location is quite variable. They always enter obliquely so as to point toward the glenoid end of the bone, and at least one of them is traceable to the base of the mesoscapiula.

- Frossa Glenoidea.-See description of Fig. 45, § 399.

§ 388. Fossa subscripularis.-This name is applied to the entire ental surface of the bone; as shown upon the figure, however, the muscle of the same name does not arise from the whole area. Most of the margin of the fossa is more or less raised. The shaded area represents a shallow longitudinal furrow which coincides nearly with the mesoscapula (Fig. 44).

Between the furrow and the coracoid margin are two well marked ridges for the attachment of tendinous intersections of the $M$. subscapularis; there are usually other ridges which are most distinct in old individuals. Near the glenoid margin is a prominent ridge which separates the $F_{s}$. subserpularis proper from the slallow furrow which gives origin to the $M$. teres. The $M$. mirostulis arises from the middle two fifths of the Mrg. glenoideus, and its glenoid third gives origin to the $M$. meditriceps.

Metacromion.-This is better shown in Fig. 44 and 45.

§ 389. Processus Coracoideus-The coracoid process.-This, as better seen in Fig. 45, 
projects sharply entad between the Fs. glenoidea and the Mrg. coracoideus. From its extremity arises the M. coracuideus (Fig. 75 ).

Ridges. -See Fs. subscapularis.

Tuberculum Bicipitale.-See Fig. 45.

$\S 390$. Description of Fig. 44.-The ectal aspect of the scapula.

The principal feature of this surface is the subtriangular lamina which projects therefrom; its direction is approximately longitudinal, but it is more nearly parallel with the longer part of the coracoid border, and it inclines in the opposite direction. It begins as a triangular elevation at the vertebral border of the scapula, and rises rapidly to about the middle of the length of the bone, where its edge is thickened and roughened. From this point toward the glenoid end its elevation remains nearly uniform, but there are projections which will be described presently. This

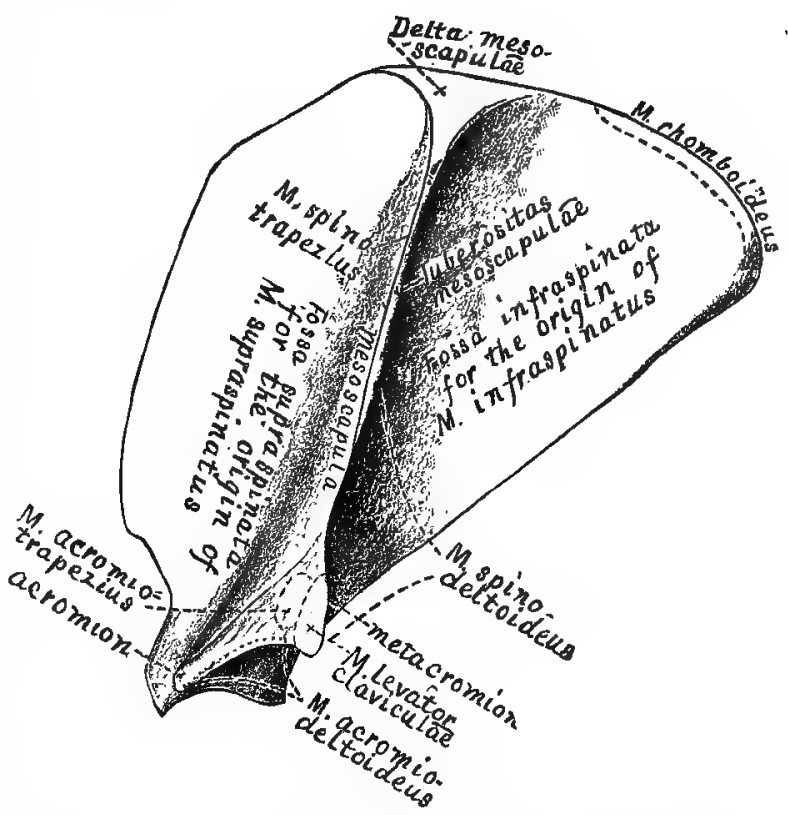

Fig. 44.-The Eotal Aspect of an Adult Left ScapULA; $\times 1$. ectal ridge is the mesoscapula or spine of the scapula.

§ 391. In the light of Embryology and Comparative Anatomy, the entire scapula may be regarded as essentially a subeylindrical bar. With most Mammals the sides are produced in three directions so that a transection is irregularly $T$-shaped; the upright of the $T$ represents the ectal ridge just described, and the two arms of the cross-piece represent the two lamina at right angles therewith which constitute the principal part of the bone, and whose borders are respectively glenoid and coracoid. By Parker (A, 215), these three regions are called respectively mesoscapula, postscapula and prosecapula.

Consistently with these names, the gTenoid border should be called Mrg. postscapularis, and the coracoid Mrg. prascapularis; the fossæ between them and the mesoscapula should also be similarly designated. Since, however, the anthropotomical name for mesoscapula is spine, and in the natural attitude of man its direction is approximately horizontal, these fosss have been named iufraspinous and supraspinous, while the muscles arising therefrom are called infraspinatus and supraspinatus. Until it shall be agreed to change the names of these muscles, it will probably be more convenient to retain the anthropotomical names for the fossæ.

\$ 392. Acromion-Prc. acromialis-Acromion process (Fig. 30, 43, 46, 47).-This forms the free extremity of the mesoscapula. Its ectal border is deflected slightly from the line of the mesoscapula toward the coracoid border. In man it articulates with the clavicle, but in the cat it is connected therewith by only a slender ligament or strip of fascia. 
$\$ 393$. Delta Mesoscapula-The delta of the mesoscapula (Fig. 44).-We suggest this as a suitable designation of the slightly elevated triangular rough surface at the vertebral end of the misoscapula.

§ 391. Fussa Infraspinata-The infraspinous fossa.-This is the larger of the two ectal fosse, and is distinctly triangular in shape. It is overbung by the mesoscapula and metacromion.

\$ 395. Fossa Supraspinata-The supraspinous fossa.-This is less regular in form than the infraspinous fossa, and its area is less extensive; but the musele which occupies it is larger than might be inferred, both on account of the inclination of the mesuscapula and its own projection beyond the coracoid border of the scapula.

Mesoscapula-Spine of the scapula.-See above $(\$ 390)$.

ร 396. Metacromion-(Fig. 43, 67).-At its greatest elevation, near the glenoid end, the mesoscapula is produced over the infraspinoas fossa as a quadrate or subtriangular process, the metacromion. Its ectal surface and free border are rough for the insertion of the

A. Glenoid end of right scapula.
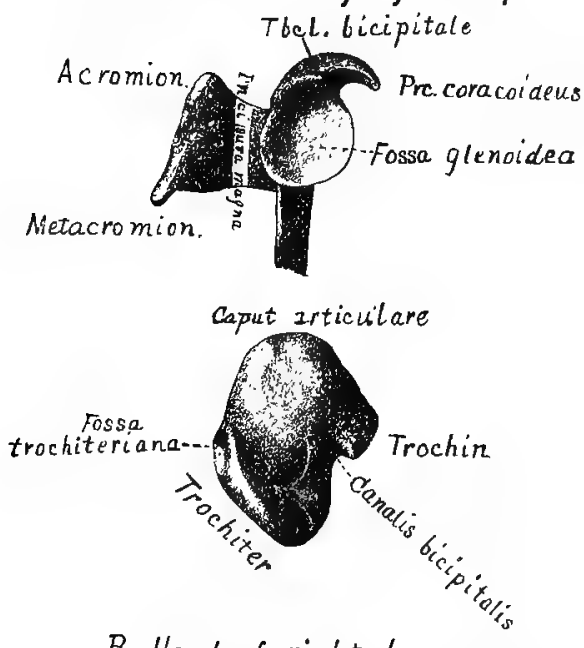

$B$ Head of right himerus,

Frg. 45.-THE GLENOID END OF AN ADUxT Righ' Scapula (A), and the Proximal END OF AN ADULT RIGHT Humerus (B); slightly reduced.

biceps at the root of the Prc. (processus) coracoidens. Near this end, the ental border of the fossa presents a slight emargination.

§ 400. Pro. (processus) Coracoideus-The coracoid process.-This shows here to the best advantage as a hook-like process comparable with the beak of some birds (whence its name), or with a half-bent finger, as suggested by Humphrey (A, 366). At its base, opposite the emargination near the smaller end of the Fs. glenoidea, is a nutrient foramen.

\$ 401. Explanation of Fig. 45, B.-This represents the proximal end of a right humerus, with the dorsal side uppermost. When, therefore, the humerus and the scapula are in contact at the shoulder, the upper and narrower part of the arthral surface of the former is received by the lower and wider part of the Fossi glenoidea. 
The general outline of the proximal end of the bone is approximately that of an irregular lozenge, the two smaller angles blunted, and one of the sides strongly indented. The angles are dorsal and ventral, caudal and cephalic, while the sides face obliquely ventrocephalad, etc.

The following descriptions should be considered in connection with what is sajd of the parts under Fig. 46 :-

§ 402. Canalis Bicipitalis-The bicipital canal or groove (Fig. 30, 46, 69, 70, 75).-This appears as a notch between the troehin and the trochiter, and is overhung more by the former. Through it passes the tendon of the M. biceps on its way to the Tbcl. bicipitale of the scapula (Fig. 45, A).

$\S 403$. Caput Articulare-The arthral head or anatomical head of the humerus (Fig. 30, 46, 68-71). -This presents a smooth convex surface which, as viewed perpendicularly to the proximal end of the bone, is irregularly circular in outline. Really, however, it extends upon the dorsal aspect of the bone, and is, as a whole, approximately triangular. It is much larger than the Frs. glenoidea of the scapula with which it articulates, being twice as long and more than half as wide again.

$\S 404$. Fossa Trochiteriana-The trochiterian fossa (Fig. 30, 68). -This is a depression upon the cephalic aspect of the proximal extremity of the humerus near the tip of the trochiter. Upon it is inserted the M. infrospinatus.

§ 405. Troehin-The caudal, "inner" or " lesser" tuberosity (Fig. 3, 46, 69-73).-This forms the caudal obtuse angle of the lozenge represented by the entire proximal aspect.

Notwithstanding the objections of Hyrt (B, 200), we have employed the names epicendylus, epitrochlea, trochiter and trochin, which were proposed by Chaussier and adopted by Straus-Durckbeim (A, I, 512).

\$ 406. Trochiter-The cephalic, "outer" or "greater" tuberosity (Fig. 30, 46, 68, 69, $71,67,74)$. - The proximal border of this forms the ventro-cephalic side of the lnzenge represented by the entire proximal end of the bone.

THE HUMERUS (Fig. 6, 7, 30, 45, 46, 67-75, 105).

References.-Straus-Durckheim, A, I, 511-514; Owen, A, II, 511; Humphrey, A, 371-377; Chauveau, A, 83, 84; Chauveau (Fleming), A, 73, 74; Flower, A, 239, 246; Leyh, A ; Gray, A, 228-228; Quain, A, I, 85-87.

\$ 407. General Description.-This is the single bone of the brachium, the proximal segment of the arm. According to the membral terminology suggested by Marsh (see $\$ 83$ ), it is the cephalic 0 . propodiale. Its proximal and distal ends form with the scapula and with the ulna and radius the shoulder and elbow joints respectively.

In all Vertebrates excepting the "fishes," that is, in all Amphibia, Reptiles, Birds and Mammals, excepting the armless Amphibia (Cæcilians) and Reptiles (serpents and a few lizards), the humerus is present and gives attachment to numerous and important muscles. In the cat, as partly shown upon Fig. 68-71, it affords origin and insertion to many muscles. 
The humerus of the cat presents a subcylindrical shaft-the diaphysis, and two enlarged and irregular ends-the Extremitates proximales and distales.

Viewed from the ventral or dorsal aspect, the caudal and ce-

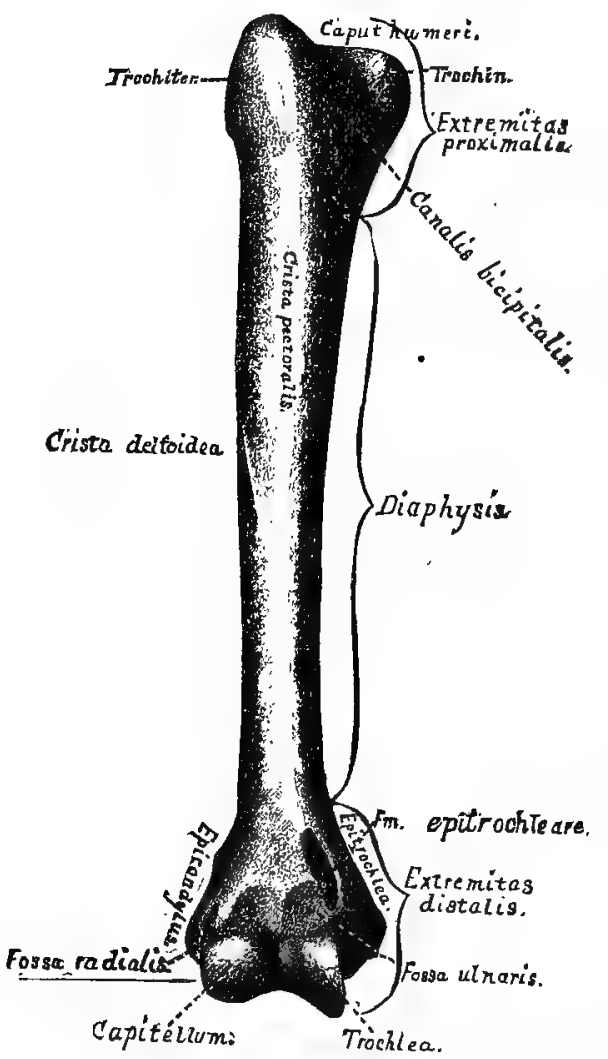

Fig. 46.-Tine Ventral (ANTerior) Aspect of an AdDit Right Humerus; $x 1$. phalic outlines of the entire bone are concave, the concavity being both greater and more regular on the caudal side (on which the three braces are placed). Seen, however, from either the caudal or cephalic side, as in Fig. 68 and 70, the dorsal and ventral outlines are S-shaped. On the dorsal side the sharper curvature is near the distal end, while the reverse is the case with the ventral side. In other words, the proximal extremity is more enlarged dorsad, and the distal one ventrad.

The cat's humerus does not present the appearance of having been twisted which characterizes that of man and some other Mammalsan appearance which is due, at least in part, to the development of ridges with intervening furrows having a more or less distinctly spiral arrangement for the attachment or accommodation of muscles, vessels or nerves.

$\S 408$. Description of Fig. 46.-This represents the ventral aspect of the right humerus, that which is most commonly and fully examined and compared. The same aspect of the left humerus is represented in Fig. 69, and the cephalic, candal and dorsal aspects in Fig. 68,70 and 71 respectively.

§ 409. Canalis Bicipitalis-The bicipital canal or groove (Fig. 30, 45, 46, 69, 70).This is a manlsed depression upon the ventro-caudal aspect of the prosimal extremity. As better shown in Fig. 45, it lies between the eminences called trochiter and trochin. In the 
fresh state it is converted into a foramen or closed canal by tendons which are inserted upon these parts. Through it plays the tendon of the $M$. biceps, the one which represents the "long" or "glenoid" head of the muscle in man. By Straus-Durckheim (A. I, 512), the canal is called " coulisse bicipitale."

\$ 410. Capitellum (humeri)-The radial head, external or outer condyle (Fig, $30,46,6$ ). -The distal end of the bone presents a smooth saddle-shaped surface, the caudal border of which is raised, and the cephalic border rounded. As seen from the ventral aspect, this arthral surface is nearly equally divided by a slight ridge, and the convex caudal portion is the capitellum. This surface narrows as it is continued over the distal end of the bone, so that its shape, if extended in a plane, would be approximately lanceolate or pear-shaped, with a distinct emargination at the cephalic side of the base.

With the capitellum articulates the fossa at the proximal end of the radius; this end, unfortunately, being likewise named capitellum. It would be well if anatomists could agree to call one of these parts capitellum and tho other capitulum.

\$ 411. Caput (humeri) Articulare-The arthral or anatomical head of the humerus.The smooth convex arthral surface of this part hardly appears in this view of the bone, but is shown in Fig. 71. In man it is distinguished from the non-arthral surface of the rest of the Ext. proximaiis by a furrow or constriction, the anatomical neck. In the cat, this neck is not clearly defined.

§ 412. Crista Deltoidea-The deltoid ridge-"Crête deltoïdienne externe," StrausDurckheim (A, I, 513)-(Fig. 46,68,69).-This is a narrow, nearly straight, and-in well marked adult humeri-sharply defined raised line extending from the tubercle for the insertion of the $M$. micostalis (Fig. 69) at the base of the trochiter on the cephalic aspect of the proximal extremity distad and ventrad, to lose itself on the third fourth of the ventral border. The sharpness which characterizes its proximal portion disappears at about the middle of the bone.

\$ 413. Cristr Pectoratis-The pectoral ridge-“"Crêto deltoïdienno antérieure," StrausDurckheim-(Fig. 46, 69).-This name is applied by us to the ill defined rough line upon the proximal two thirds of the ventral aspect of the humerus.

This and the Crs. deltoidea converge distad, but cease to be distinct before meeting. The long triangular interval between them is called by Straus-Durckheim (A, I., 513), "empriente deltoïdienne." Mivart applies (B, 92, Fig. 53), the name deltoid ridge to what seems to correspond to this interval, and inaccurately states that the two parts of the $\boldsymbol{M}$. deltoideus above mentioned are inserted upon it.

§ 414. Diaphysis-The shaft (Fig. 46, 30, 68-71).-Although the shaft of the humerus may be generally described as approximately cylindrical, it is slightly compressed, so that at any point its cephalo-caudal diameter is less than its dorso-ventral. It is slightly curved so as to present a ventral convexity.

For convenience of description, the entire bone may be said to consist of a subcylindrical shaft and enlarged extremities. Strictly speaking, however, the diaphysis includes all but the proximal and distal epiphyses, and these are less extensive than the regions designated as extremitates. In a young animal the proximal epiphysis separates along an undulating line passing distad of the caput articulare and the tubercle for the insertion of the $M$. micostalis. The greatest length of the epiphysis equals only about one eighth of the length of the entire humerus, whereas the Ext.proximalis includes about one fifth. The distal epiphysis also includes merely so much as bears the arthral surfaces with the epitrochlea and epicondylus, whereas the proximal limit of the Ext. distalis embraces also the Fm. epitrochleare.

Upon the caudal aspect of the diaphysis, near the junction of the first and second fourths, and nearer the ventral than the dorsal border, is to be seen, with adult humeri, a 
narrow longitudinal depression ronghened for the attachment of the conjoined tendons of the MM. teres (major) and latissimus (dorsi). It is indicated by an interrupted line on Fig. 70. On Fig. 43 it does not appear, but its proximal end would be opposite the proximal end of the longer brace. Its length equals the dorso-ventral diameter of the bone opposite its distal end.

§ 415. Epicondylus-The epicondyle or external condyle (Fig, 30, 46, 68, 69, 71, 74).This is a rough subconical eminence upon the cephalic aspect of the distal extremity. To it is attached the proximal end of the cephalic (external) lateral ligament of the elbow, and from it arise the MM. Extensor ulnaris and Ext. minimi. Obliquely proximad from the epicondylus extends the Crista epicondylaris, which loses itself on the dorsal aepect of the diaphysis nearly opposite the point of nearest approximation of the deltoid and peetoral crests upon the ventral aspect.

The epicondyle has been called "outer" or "external condyle," and more recently by Markoe (1) and others " external epicondyle."

§ 416. Epitrochlea-The epitrochlea or internal condyle (Fig. 30, 46, 69, 70, 71, 75).This is is a rough subconical eminence upon the caudal aspect of the distal extremity. It is larger than the epicondylus, and springs abruptly from the nearly plane caudal surface formed by the candal prominence of the trechlea. Proximad, however, it is directly continuous with the bar forming the caudal boundary of the Fm. epitrochleare.

§ 417. Fm. (Foramen) Epitrochleare-The epitrochlear or supracondyloid foramen (Fig. 30, 46, 69, 70, 75, 105).-This is a narrow perforation from the ventral to the dorsal aspect of the humerus near the caudal side of the distal extremity. Through it pass the $\mathrm{N}$. medius and the A. brachialis on their way from the dorso-caudal to the ventral aspect of the arm.

In the cat, the Fm. epitrochleare is within the naturally assigned limits of the Ext. distrlix, but it is wholly within the diaphysis; its distal boundary is sometimes very near the diaphysio-epiphysial suture, and sometimes separated therefrom by a space equal to the longer diameter of the orifice.

The Fin. epitrochleare exists in the other Felidæ and in some other Carnivora (Flower, A, 246i, and oceasionally-partly circumseribed by ligament-in man (Humphrey, A, 373; Quain, A, I, 87). Usually, howevir, the human humerus presents merely an emargination of the bone, along which pass the median nerve and the brachial artery.

\$ 418. Fossa Radialis and Fs. Uharis-The radial and ulnar fossæ of the humerus (Fig. 46).-On the ventral (anterior) aspect of the distal extremity of the humerus, just proximad of the arthral surfaces of the trochlea and capitellum, are two slight depressions, against which, in the strongly flexed condition of the elbow, abut the coronoid process of the ulna (Fig. 30, Pre. coronoideus), and the side of the capitellum or proximal arthral end of the radius (Fig. 30); hence their respective names. Between the two fossæ, especially in well marked bones, is a slight ridge. The Fossa ulnaris is described, but not named, by Strans-Durckheim (A, I., 513).

$\S 419$. The Fossa olecranalis, on the dorsal aspect of the distal extremity, will be described in connection with Fig. 71.

\& 420. Trochin and Trochiter-The caudal (lesser or inner) and the cephalic (greater or outer) humeral tuberosities (Fig. 30, 45, 68-71).-These parts of the proximal extremity have been described in connection with Fig. 45, and their muscular attachments will be mentioned in connection with Fig. 68-71. See also above, Canalis bicipitalis. It has been mentioned under Fig. 45 that while the trochin is wholly caudal in position, the trochiter is abont equally ventral and cephalic.

Trochlea-(Fig. 46, 69, 71).-This is the half saddle shaped arthral surface at the caudal side of the distal end of the humerus. Its caudal border is raised and sharply 
defined, but cephalad it is continuous with the capitellum, the limits of the two surfaces being indicated by a slight ridge. Ventrad, the two surfaces are nearly equal in extent, but dorsad the capitellum gradually narrows and disappears as seen in Fig. 71, while the trochlea continues upon the dorsal aspect of the bone and has a decidedly oblique direction.

\section{THE CARPALIA (Fig. 6, 30, 47).}

The bones of the carpus (wrist) have been enumerated in $\$ 84$.

References.-In addition to the references given in $\$ \S 84,85$, see Gegenbaur, $B$; Straus-Durckheim, A, I, 518-524; Mivart, B, 96-98; Gray, A, 235-241; Quain, A, I, 90-93, 99; Chauveau, A, 88, 89; Chauveau (Fleming), A, 78, 80; Leyh, A, 17\%-182; Flower, A, 252-260; Humphrey, A, 387-390.

- \$ 421. Explanation of Fig. 47.-This represents the dorsal aspect of the carpus and of the contiguous parts of the metacarpalia of an Asiatic lion seven months old, and of two young dogs. The lettering is nearly uniform in the three figures, but the present description refers only to the lion.

$\mathbf{P}, \mathbf{I}, \mathbf{M}, \mathbf{A}, \mathbf{M i}$, the metacarpalia of the pollex, index, medius, annularis and minimus; $p$, the $O$. pisiforme, which is really a $888 a-$ moid bone in the tendon of the $M$. flexor ulnaris, and not a true carpal element; $u, m, t d$ and $t m$, the unciforme, magnum, trapezoid and trapezium respectively,
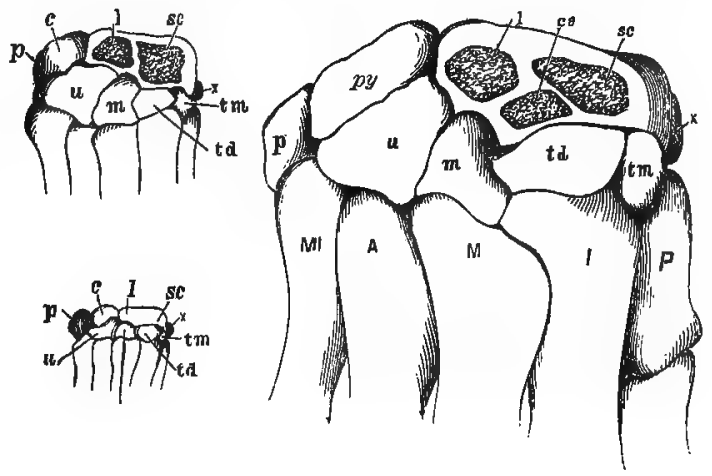

Fig. 47.-The Dorsal Aspect of the Carpal Region OF A YoUNG LION (LARgest FIGURE) AND OF Two Yodnt Dogs. (From Wilder, 19, 301, Fig. 1.) forming the distal row of carpalia; as stated in $\S 84$, the unciforme is supposed to represent two elements of the typical or primitive carpus. .

The proximal row consists of but two cartilaginous pieces, commonly known as the cuneiforme ( $p y)$ and the scapho-lunare. Since cuneiforme has become well established in the names of three of the tarsal bones, we have, upon the suggestion of Prof. 0. C. Marsh and in accordance with the custom of some European anatomists, substituted therefor the term pyramidale, which was employed by Straus-Durckheim (A, I., 520), This element represents the ulnare of the primitive carpus.

The larger proximal piece is a single mass of cartilage, but a section shows that ossification has begun from three separate centers. The two larger correspond to the scaphoides $(8 c)$ and the lunare $(l)$ of man, and to the radiale and intermedium of the primitive carpus. The third and smaller center (ce) probably represents the centrale of the primitive carpus, which is not distinct in man.

So far as appears from the figure of Mivart (B, Fig. 60), the carpus of the cat is essentially similar to that of the lion. 
CLAVICULA (Fig. 30, 48, 66, 67, 72).

The clavicle or collar bone is briefly described in $\$ 212$.

References.-Straus-Durckheim, A, I, 509; Flower, A, 228; Gegenbaur (Lankester), A, 477 ; Gegenbaur, C ; Gray, A, 215-218; Quain, A, I, 84, 96 ; Parker, A, 215; Huщphrey, A, 359-363.

\$ 422. Explanation of Fig. 48.-This represents the unusually large and well marked clavicles or collar bones of an old male cat. Their mesal or sterual ends are apposed, and are seen to be approximately cylindrical.

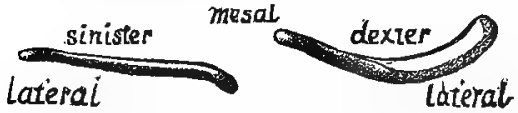

Fig. 48.-The Right and Lefrt ClaVICULA OF AN OLD CAT; $\times 1$.

The left is placed in nearly its natural attitude in the body, showing that its cephalocaudal diameter is nearly uniform, and that each end curves slightly caudad, the mesal end the more decidedly.

The right is so placed as to show the caudal aspect, and display the dorsal concarity. The mesal half is nearly straight, but the lateral is quite regularly curved. The dorso-ventral diameter of the bone increases gradually toward the lateral end, which is about twice the width of the mesal.

Directly or indirectly, the clavicle affords attachment to the Mm. clavo-trcpezius, clnvomastoideus and clavo-deltoideus, but as it is connected with the sternum and the scapula only by ligaments, it is moved with the muscles instead of forming an efficient fulcrum for their action.

THE STERNUM (Fig. 7, 30, 50, 72, 73, 99, 100).

The sternum or breast bone was briefly described in $\S 210$.

References.-Straus-Durckheim, A, I, 496, 497; Mivart, B, 49, 50; Flower, A, 73 : Humphrey, A, 321-329 ; Gray, A, 207-210; Quain, A, I, 25-27 ; Chauveau, A, 75; Chauveau (Fleming), A, 66 ; Loyh, A, 164-166 ; Parker, A, 215.

\& 423. Explanation of Fig. 49.-The ventral aspect of an adult sternum, with the contiguous parts of the costicartilagines. Incomplete views of the sternum are given in Fig. 30, 50, 72, 73, 99 and 100 .

The sternum consists of a mesal series of osseous or partly cartilaginous segments called sternebros, united by cartilages. The figure was drawn from a dried sternum, and the intersternebral cartilages are not shown distinctly, neither is indicated the line of junction of the osseous and cartilaginous portions of the caudal segment.

Of the sternebræ, the most cephalic and the most caudal have received special names, prasternum and xiphisternum. The intervening segments constitute the mesosternum.

8 424. Mesosternum.-As inclicated in \$210, there may be either 6 or 7 mesosternebra, making the total number of sternebræ 8 or 9 . The variation is due to the presence or absence of a short and nearly cubical piece between the xiphisternum and the sixth con. stant mesosterneber. This piece is neither figured nor described by Mivart (B, Fig. 24), or Parker (A, Pl. xxx, Fig. 3), and does not appear distinctly in the figures of StrausDurckheim (A, Pl. vi, Fig. 2, and Pl. vii, Fig. 2), notwithstanding his intimation (A, I, 516) that it is always present. In the sterna examined by ns this seventh piece is sometimes quite large, and in other cases so small as to be unrecognizable, at least from the surface. We have also observed considerable diversity as to the number of costicartilagines which reach the sternum, but are not yet prepared to say whether 8 or 9 is the more frequent 
number. The costicartilages which reach the sternum articulate therewith diarthrodially, but there is considerable diversity as to the extent of the synovial capsules.

Each of the constant mescsternebræ is two or three times as long as wide, and slightly enlarged at the ends so as to be somewhat of a dumb-bell shape.

\$ 425. Prosternum.-This is sometimes called manubrium from its form in man. It is nearly twice as long as the average mesosterneber, and the cephalic half, which is cartilage in the kitten, is compressed and tapers to a blunt point. On each side is an oblique shoulder for the attachment of the first costicartilage, so that the entire præsternum is shaped somewhat like the head of a lance. Its ventral aspect is prominent on the meson, forming the presternal keel.

\$ 426. Xiphisternum.-This is also called the xiphoid or ensiform cartilage. In the adult cat only the caudal third or fourth is cartilage, and its tip is enlarged into a disk. The rest of the xiphisternum tapers caudad from its base.

The sternum affords attachment to the $M M$. ectopectoralis, entopectoralis, sterno-mastoideus, sterno-hyoideus and some others.

$\$ 427$. Relation of the Sternebrce to the Costicartilagines.-While dissecting, it is often desirable to designate the number of a sterneber when the sternum is so covered by muscles as to make the enumeration difficult. In these cases the ribs or their cartilages may usually be counted from the most cephalic of the series, their relations to the sternebræ being as follows:-

With the six constant mesosternebræ, the cartilages are attached at the cephalic end, so that the third mesosterneber, for example, which is the fourth sterneber, would be the segment just caudad of the point of attachment of the fourth cartilage. The first cartilages are connected with the sides of the præsternum, which is really the first sterneber; the relations of the eighth and ninth cartilages are less definite and constant.

\section{COSTAS (RIBS) AND COSTICARTILAGINES (COSTAL CARTI- LAGES). Fig. 30.}

References.-Straus-Durckbeim, A, I, 492, and II, 57; Quain, A, I, 27, 141 ; Gray, A, 210, 295 ; Flower, A, 85 ; Chauvean (Fleming), A, 67, 140 ; Chàveau, A ; Leyh, A, 162, 209 ; Humphrey, A, 329-337; Mivart, B, 50-52.

$\S 428$. The costæ or ribs-13, rarely 14 , on each side-constitute a series of arched, highly elastic

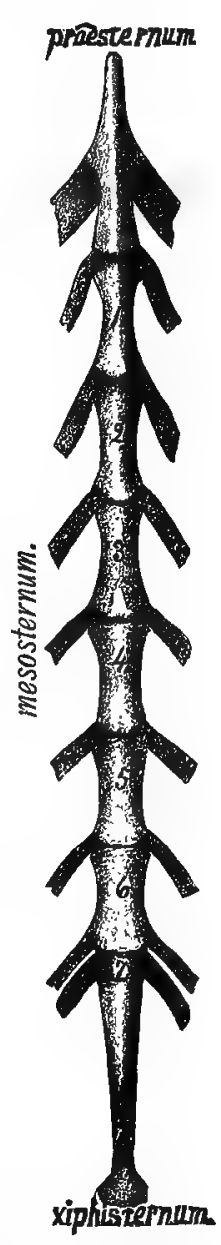

FIG. 49.-T'HE VENTRAL ASPECT OF AN ADULT STERNOM ; $\times 1$. bones which, with their continuations, the costicartilagines, the sternum and the thoracic vertebræ, form the conical skeleton or framework of the thorax (Fig. 30 and 50).

The ribs and their cartilages are slender and subcylindrical in form. They present, in expiration and moderate inspiration, a com. pound curve, the convexities being caudal and lateral (Fig. 30 and 
50). The ribs proper have each a general caudal inclination from the tuberculum to the arthron costicartilaginis, while the cartilage has a cephalic inclination from the arthron to the sternal end (Fig. 30). In full inspiration the ventral or sternal ends of the ribs are nearly ventrad of the vertebral ends, the caudal convexity being partly or entirely obliterated (Fig. 50).

$\S 429$. Special Characters.--The capitellum of the 12th and 13 th ribs--14th if present-articulates with but a single vertebra.

The capitellum of the first articulates to a slight extent with the body of the last cervical, but the diarthrodial part of the articulation is entirely confined to the body of the first thoracic (Flower, A, 23). The first rib and those last named above $(12,13,14)$, possess no ligamentum interarticulare (Fig. 50).

The 12th and 13th ribs--14th if present-possess no arthral tuberculum; it is also sometimes absent from the 11th. The tuberculum of each of the others articulates diarthrodially with the diaphysis of the vertebra corresponding to it in number; thus, the tuberculum of the first articulates with the diapophysis of the first vertebra, and that of the seventh with the diapophysis of the seventh, etc.

430. Sternal, Asternal and Floating Ribs.-The cartilages of the first nine ribs (Fig. 49)-rarely of only the first eight-are articulated with the sternum, and hence are called sternal or true ribs. The remaining three (or four) are called asternal or false ribs, as their cartilages do not reach the sternum. Finally the 13th-14th if present-is not attached to the one just cephalad of it by connective tissue, as are the 10th, 11 th and 12th, but ends independently in the abdominal muscles, and hence is called a floating or vertebral rib. (Humphrey, A, 329, 337 ; Hutchinson, A, 1016.

$\S 431$. Methods of Demonstration.-The form and the various parts of the ribs are best made out in those that have been completely deprived of their soft parts as directed above ( $\$ 248)$. The relations and mobility of the ribs singly and collectively, and their arthra and ligaments, must be studied on fresh or alcoholic specimens.

§ 432. Elasticity and Mobility.-Take as lean a cat as possible, place it dorsicumbent, and, commencing at the ventrimeson, remove the skin and muscles covering the sternum, and the ribs with their cartilages of one side. Press upon the thorax, and the elasticity of the ribs will be felt. Grasp the second mesosterneber 
and pull ventro-cephalad. This will show the way in which the capacity of the thorax is increased.

§ 433. Arthra-Joints.-Remove the muscles covering the tuberculum and cervix of the seventh rib for example, and the ligaments will be seen as white bands holding the tuberculum to the diapophysis and the capitellum in the socket formed by the demifacets of the two vertebræ (Fig. 52, Ar̃thron capitelli). Cut away the ligaments on the caudal side of the tuberculum with the arthrotome and bend the rib cephalad. This will expose the smooth arthral surface of this diarthrodial joint. That of the capitellum may be demonstrated in the same way.

To demonstrate the amphiarthrodial joints at the arthron costicartilaginis, the finger or some solid substance should be placed entad of the arthron, and then the ectal surface of the bone and cartilage should be sliced away with the arthrotome. The cartilage and bone will be found continuous, the ends not being separate and smooth for gliding upon each other as with the capitellum and tuberculum. The end of the rib is but very slightly hollowed out to receive the cartilage, thus differing from the condition in man, where the cartilage is implanted in a deep pit.

The diarthrodial joints of all the sternal ribs ( 8 or 9 ) at the junction of the costal cartilages and sternum ( $\S \S 424,430)$, may be demonstrated by slicing off the ectal surface of the conjoined sternum and cartilage as directed for the Arthron costicartilaginis.

$\S 434$. Ligamentum interarticulare-Interarticular ligament (Fig. 50).-This is most satisfactorily demonstrated in one of the middle ribs, the seventh for example. The muscles should be removed as for demonstrating the tubercular and capitellar joints, and the ligaments binding the tuberculum to the diapophysis should be cut. Nip away the neural arch (Fig. 53) and remove the short segment of exposed myelon. This will expose the floor of the neural canal. Dissect the dorsal (posterior) common ligament from the intervertebral fibro-cartilage, move the rib, and the ligamentum interarticulare will be seen, as a rope in a pulley, passing across the floor of the neural canal in a groove on the dorsal surface of the intervertebral fibro-cartilage, and connecting the heads of the pair of ribs $(\S 444)$.

§ 4.35. This ligament differs so remarkably from its homologue in man that a brief comparison is added:-

In man the ligament is plate-like. In the cat it is thick and band-like. In man it 
completely divides the arthral surface of the capitellum into two parts corresponding to the demifacets of the two vertebræ (Fig. 52, Arthron capitelli). In the cat it but partially divides the surface. In man the ligament joins the intervertebral fibro-cartilage, and does not extend from side to side. In the cat it passes from side to side in a synovial groove lined by an extension of the synovial membrane lining the joints of the capitella of the pair of ribs. Hence, while in man there are four separate synovial cavities for the capitella of each pair of ribs possessing the interarticular ligament, in the cat there is but one, since the arthra of the capitella communicate with each other through the groove for the Ligamentum interarticulare.

References.-Bichat, A, I, 231; Mayer, 47, 273; Chauveau, A, 140 ; Leyh, A, 210; Cleland, 4 and 5 ; Quain, A, I, 141; Gray, A, 296 ; Gage, 5, 421.

\$ 436. Preparation.-Fig. 50 represents two costæ and costicartilagines separated from the body and in the position assumed

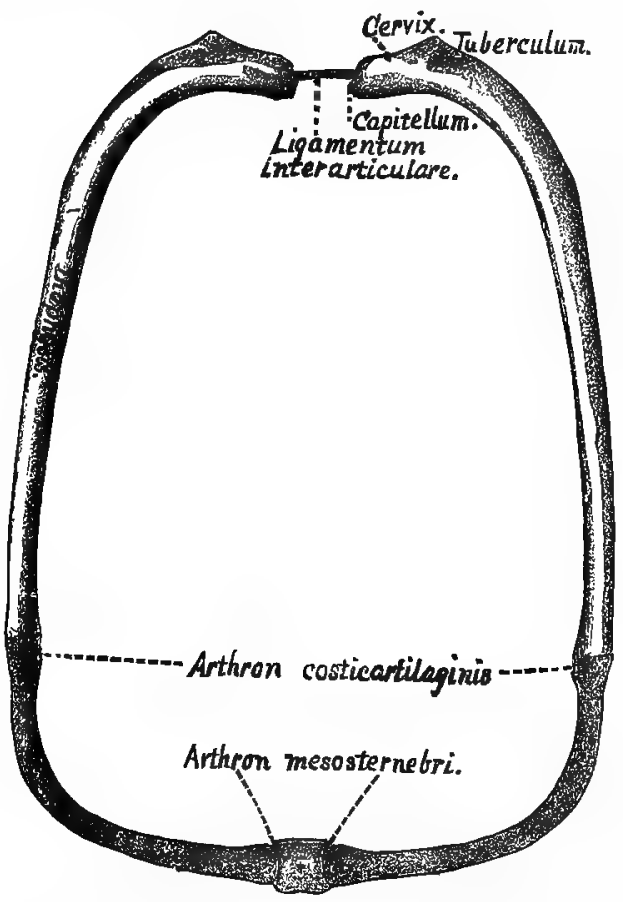

Fig. 50.-Caudal View of the Seventh Pair of COSTA (RIBS); $\times 1$. during the fullest possible inspiration-that is, drawn ventrad, laterad and cephalad so as to obliterate their caudal convexity (Fig. 30), and give the greatest capacity to the thorax. The cleaning process was carried only sufficiently far to remove the soft parts, leaving the interarticular ligament and the connections of the costal cartilages and sternum ( $\$ 252,427)$.

8437 . Arthron costicartilaginis.--The amphiarthrodial articuiation between the sternal end of the diaphysis and its cartilage ( $(433)$.

$\$ 438$. Arthron mesosternebri - Costo-sternal articulation. - The diarthrodial articulation of the costal cartilage with the mesosternum. The first costal cartilage articulates with a single sterneber (præsternum), the other eight with two ( $\$ 430$, Fig. 49).

$\S$ 439. Capitellum-Head.-The vertebral end of the rib ends in a somewhat pear-shaped capitellum or head which artjculates diarthrodially with the demifacets of two contiguous vertebræ (Fig. 52, Arthron capitelli.)

$\$ 440$. Cervix-Neck. - The cervix or neck of the rib is the somewhat constricted part immediately following the capitellum and between it and the tuberculum. 
$\S 441$. Costa-Rib.-The costa or rib proper is the bony part of the arch extending from the capitellum to the Arthron costicartilaginis. It has two extremitiesvertebral or dorsal and sternal or ventral; two surfaces-ectal and ental t two edgescephalic and caudal. The vertebral end bears the capitellum: the ectal surface is next the skin ; the cephalic edge faces toward the head.

\$ 442. Costicartilago-Costal cartilage.-The cartilaginous continuation of the rib which passes from the sternal end of the diaphysis toward the sternum (\$423, Fig. 49). In the figure it is deeply shaded and is between the Arthron costicartilaginis and the mesosterneber.

$\S 443$. Diaphysis (Costæ)-Shaft.-The diaphysis of the rib is the part between the tuberculum and the Artbron costicartilaginis.

$\$ 444$. Ligamentum interarticulare, ck.-Interarticular ligament.-This is a strong, smooth, band-like ligament connecting, through the floor of the neural canal, the heads of opposite ribs (2d to 11th pairs inclusive); (\$ 434).

$\$ 445$. Mesosternebra, az.-The square area between the sternal ends of the costal cartilages represents the caudal end of the 5th mesosterneber (\$ 424 , Fig. 49 ).

$\S 446$. Tuberculum-Tubercle.-The tuberculum is an elevation on the ectal surface of the rib just at the end of the cervix. It bears a smooth arthral facet which articulates (diarthrodially) with the diapophysis of the 7th thoracic vertebra (Fig. 52 and $\S 433$ ).

\section{PELVIS.}

General References to the Pelvis.-Straus-Durckheim, A, I, 499, and II, 63 ; Quain, A, I, 100,122, 159 ; Gray, A, 245 ; Chauveau (Fleming), A, 91,161; Chauveau, A, 75, 152 ; Leyh, A, 166, 212 ; Flower, A, 281 and 33 ; Humphrey, A, 438; Jouliu, 1 ; Mivart, 8 and 19; Wilder, 10. A chronological bibliograply is given at the end of the last.

$\S 447$. Preparation.-The soft parts were fully removed by one of the processes already described (\$ 244-256). Parts of the first and second sacral vertebræ were removed with nippers to expose the Arthron ilio-sacrale. To show the lines of junction between the ilium, ischium, Os pubis and Os cotyloideum, it is necessary to prepare the pelvis of a cat retaining its milk teeth.

$\S$ 448. Arthron ilio-sacrale-Ilio-sacral articulation. -This is the articulation between the sacrum and the ilium. In the figtire the arthral surface on the ilium is brought into view by the removal of part of the sacrum. The caudal third of this surface is diarthrodial, the cephalic two thirds amphiarthrodial; the motion, however, is very limited.

The lateral masses of only the first sacral vertebra articulate directly with the ilium.

$\$ 449$. Crista ilii-Iliac crest.-The iliac crest is the prominent dorso-cephalic projection of the ilium (Fig. 30, 51, $\$ 230$ ).

$\$ 450$. Fm. (Foramen) obturatorium-Obturator or thyroid foramen.-The large oblong space bounded by the os pubis and the ischium. 
\& 451. Fossa cotyloidea-Cotyloid fossa, Acetabulum.-The cotyloid fossa is a deep cup-shaped cavity in the lateral aspect of the innominate bone which articulates diarthrodially with the head of the femur. The third of the circumference of the cavity next the

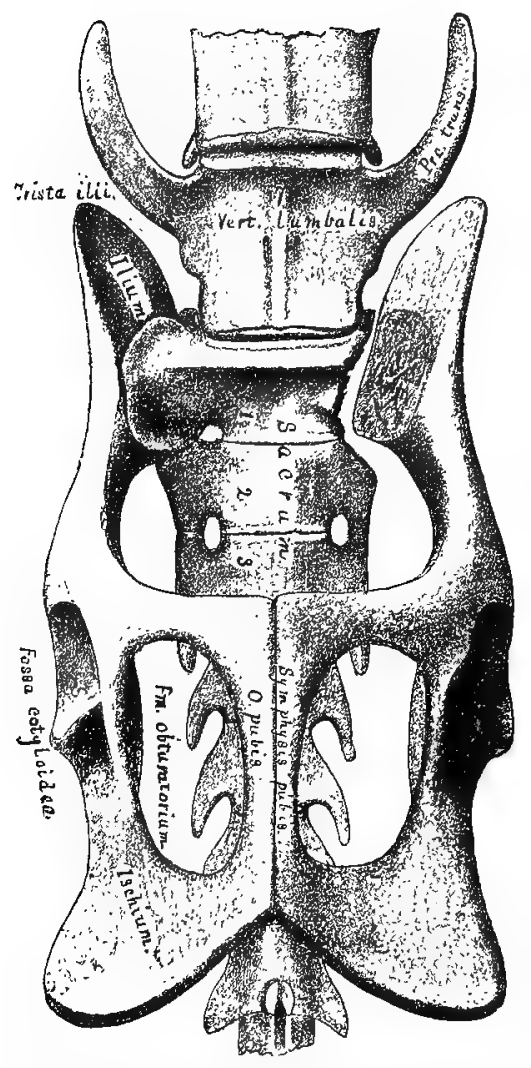

Fig. 51.-Ventral View of the Cat's Pelvis with Adjacent Vertebrat. (Modified from Straus-Durckheim.) $\times 1$. obturator foramen is absent, thus producing the cotyloid notch. A rough depression extends from this notch about half way across the floor of the fossa.

$\S 452$. Ilium.-This is the cephalic part of the os innominatum. It articulates with the sacrum and forms a considerable part of the cotyloid fossa.

$\S 453$. Ischium-The dorsocaudal part of the pelvic bone. It forms a large part of the cotyloid fossa and about one third of the pubic symphysis.

$\S 454$. Os cotyloideum-Cotyloid bone.-A small bone forming the ventral part of the floor of the cotyloid fossa. StrausDurckheim, A, I, 502; MilneEdwards, A, X, 358. It has not been detected in man, and can be seen as a separate bone only in immature cats.

$\S 455$. Os innominatum-Innominate or pelvic bone.-The os innominatum is made up of the ilium, the ischium, the os pubis and the os cotyloideum. These four bones are completely united in adult life. The right and left pelvic bones together form the pelvic girale.

$\$ 456$. Os pubis-Pubic bone.-It is the cephalo-ventral of the bones forming the pelvic girdle. It forms only a small part of the cotyloid fossa and about two thirds of the symphysis pubis.

\& 457. Pelvis, $\alpha z$.- "The two innominate bones, together with 
the sacrum, form the pelvis, a complete circle of bone, or rather a short tube."-Flower, A, 284.

Prc. trans., Processus transversus. - Transverse process or diapophysis of the 7th lumbar vertebra.

\$ 458. Sacrum, $a z$._ "The portion of the vertebral column to which the pelvic girdle is attached." It is composed of three vertebræ, which in adult life are more or less completely consolidated.

In an immature cat which would show well the lines of demarcation between the bones forming the Os innominatum, the sacral vertebræ would be but partly coössified.

$\S 459$. Symphysis pubis, $a z$.-This is the linear articulation (amphiarthrodial) between the ventral aspects of the two innominate bones. Its cephalic three fourths is formed by the Os pubis and the caudal fourth by the ischium. In fully adult animals the symphysis usually becomes anchylosed.

Vert. (Vertebra) lumbalis.-The last or 7th lumbar vertebra (Fig. 55).

Vertebræ caudales, az.-Tail vertebra.-These follow immediately after the sacrum (Fig. 30).

$\S 460$. In addition to the parts named above, the following should be mentioned :-

A. The Ilio-pectineal Line and Eminence--Neither are shown in Straus-Durckheim's figure, from which this was copied. Both should show on the right side, however. The line extends from the ilio-sacral articulation to the most prominent part of the pubis. Opposite the cephalic edge of the acetabulum the line presents an eminence which is at or very near the junction of the ilium and pulbis.

B. The Tuberosity of the Ischium.-This is the most prominent thickened part of the ischium. It forms the extreme dorso-caudal part of the whole pelvis. It is upon this part the cat rests when sitting on her haunches.

COLUMNA VERTEBRALIS.

References.-Straus.Durckheim, A, 458; Quain, A, I, 9, 25 ; Gray, A, 132 ; Hum. phrey, A, 113 ; Milne.Edwards, A, X, 225 ; Leyl, A, 153; Chauveau, A, 19 ; Owen, A, II, 483; Owen, 231 ; Cuvier, A, I, 170 ; Flower, A, 10 ; Mivart, 3 and 24; Maclise, A, 622 ; Cleland, 7 and 13 ; Wilder, 10.

$\S 461$. The Columna vertebralis, spine, vertebral or spinal column, consists of a series of osseous segments called vertebr $a$ arranged in close connection with each other and forming the bony axis of the body. It is nearer the dorsal than the ventral aspect in the cat and in most other Vertebrates. Its position and curves are shown in Fig. 30. It is prolonged caudad beyond the trunk to form the 
axial support of the tail ; and cephalad it is articulated diarthrodially with the occipital region of the skull. "The different vertebræ, with some exceptions ( $\$ 458$ ), remain through life distinct from each other, though closely connected by means of fibrous structures which permit a certain but limited amount of motion between them."

$\$ 462$. "Although the vertebræ of different regions present great diversities of form, there is a certain general resemblance among them showing a common plan of structure. This plan is, however, worked out variously in different regions by change of form and the suppression or superaddition of parts, thus fitting them to fulfill their special purpose."-Flower, A, 10.

In general, each vertebra is composed of a solid subcylindrical centrum or body, and a bony arch (neural arch), with various processes, extending dorsad therefrom (Fig. 52-55).

$\$ 463$. Regions of the Vertebral Column.-For convenience of description, the whole vertebral column has been divided into five regions, named in order from the head :-Cervical (7) ; Thoracic or Dorsal (13); Lumbar (7); Sacral (3); Caudal or Tail (22), (Fig. 30).

The middle Cervical, Thoracic and Lumbar vertebræ are shown in Fig. 53-55, where the special characters of these three groups are well illustrated.

$\S$ 464. Distinguishing the Five Groups of Vertebræ and the Ends of the First Four Groups.-The cervical vertebræ, except the 7th, are distinguished by the presence in the diapophysis of the vertebrarterial foramen $(\$ 473)$.

The 7th cervical is distinguished from the thoracics by the absence from its prominent diapophysis of an Arthron tuberculi, and by the absence of an Arthron capitelli. from the cephalic end of the centrum (Fig. 52). It differs from the other groups by its short wide centrum, large neural foramen and slender neural spine.

The thoracic vertebræ differ from all the others by the presence of an Arthron capitelli on the cephalic end of the centrum or upon both ends.

The Iumbar vertebræ may be distinguished by their long subcylindrical centra, and by the cephalic inclination of the neurapophyses and of all the diapophyses except the first.

The sacral vertebræ are more or less completely anchylosed.

The caudal vertebræ differ from the others by the smallness or 
absence of the neural foramen, the caudal inclination of the diapophyses when present, and by the presence in some (2d to 9 th) of the so-called chevron bones.

$\S 465$. The chevron bones are small ossicles attached to the cephalo-ventral part of the caudal vertebre and forming an open or closed arch through which passes the caudal continuation of the $A$. sacra media.

The ends of the vertebræ may be distinguished very readily by remembering that the arthral surface of the proaygapophysis (Fig. 53), situated at the cephalic end of the vertebra, faces either dorsad or dorso-mesad, while that of the postaygapophysis faces ventrad or ventro-laterad. It follows from this that the postzygapophyses overlie the præzygapophyses like the tiles on a roof. Especially in the thoracic and lumbar regions, the Incisura vertebralis or intervertebral notch is much deeper on the caudal than on the cephalic side. The above characters apply also to man.

\section{$\S 466$. Demonstration.-All of the general as well as special} points relating to the vertebral column may be demonstrated on a flexible natural skeleton ( $\$ 252)$ and one entirely divested of its soft parts. The relation of the myelon and other soft parts must of course be demonstrated on a fresh or alcoholic specimen.

$\S 467$. Preparation-(Fig. 52).-The cleaning ( $\$ 252$ ), was carried sufficiently far to divest this part of the vertebral column of all its soft parts except the intervertebral fibrocartilages.

§ 468. Arthron capitelli-Capitellar articulation.-This is the diarthrodial arthral cavity formed in two adjacent thoracic vertebræ for the reception of the capitellum or head of the rib (Fig. 50). The part of the articulation in each vertebra is called a demifacet. The 12th and 13th vertebræ have each a complete capitellar arthral surface. The caudal part of the last cervical vertebra supports part of the capitellum of the first rib; Flower, A, 23.

\$ 469. Arthron tuberculi.-This is the diarthrodial facet on the diapophysis for articulation with the rib corresponding in number to the vertebra from which the diapophysis arises (Fig. 50, 52). It is not present in the last two thoracic vertebræ.

$\S 470$. Atlas.-The atlas is the first cervical vertebra, and thus the first of the entire series. It articulates diarthrodially with the occipital condyles (Fig. 57). The broad lateral masses are the diapophyses or transverse processes, and are sometimes called "wings of the atlas."

$\$ 471$. Axis.-This is the second of the cervical vertebræ. It articulates diartlirodially with the atlas, but with the third vertebra by the interposition of fibro-cartilage, that is, amphiarthrodially, like the remaining vertebre, except the sacrum ( $\$ 458$ ). Its neural spine is a long sharp ridge (Fig. 30).

Cerv.-Vertebræ cervicales, cervical vertebræ 1-7.

$\S$ 472. Fibro-cartilago intervertebralis, $a z$.-Intervertebral fibro-cartilage.-Between each of the centra, except the first and second and the parts of the sacrum, is this very dense, tough and elastic fibrous material. "The elasticity provides for the vertebræ always returning to their normal relation to each other and the column generally when they have been disturbed therefrom by muscular action." -Flower, A, 12.

$\S 4$ 473. Foramen vert., Fm. vertebrarteriale.-This is the canal through the bases 


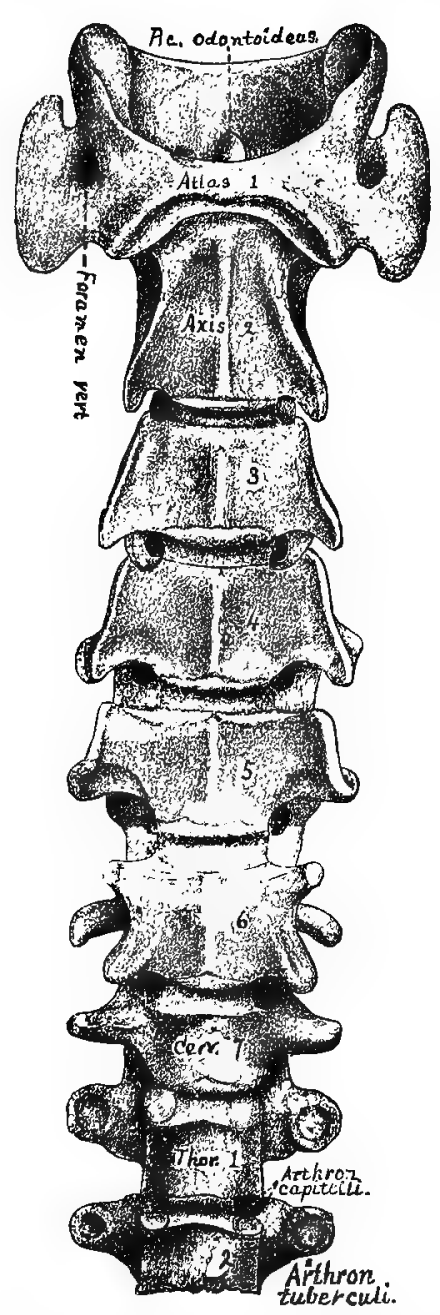

Fig. 52.-Ventral Aspect of the Cervical and of two Thoracio Vertebras. (From Straus. Durckheim.) $\times 1$. of the diapophyses of all but the last (rarely of the last) cervical vertebræ. Through it pass the vertebral artery and vein.

\& 474. Foramen atlantale-Atlantal foramen.This is a passage through the cephalic edge of the atlas just dorsad of the occipito-atlantal arthron. It transmits the first or suboccipital nerve and the vertebral artery; Straus-Durckheim, A, I, 470. It is present in man only as an exception; Quain, A, I, 12.

8 475. Lamella ventralis-Ventral or inferior lamella.-This is the thin plate-like projection extending ventrad from the diapophyses of some of the cervical vertebræ. It is most marked in the 6 th. Flower, A, 22, 23.

$\S$ 476. Prc. (Processus) odontoideus, $a z$.-Odontoid process.-This is a tooth-like projection from the cephalic part of the centrum of the axis. It articulates diartlirodially with the atlas, and serves as a pivot on which the atlas and head rotate. It is kept from encroaching upon the neural canal by a strong ligament.

\$ 477. Explanation of Fig. 53-55.-Anapophysis-Accessory tuluercle. -This is a slender process extending laterad from the caudal part of the neural arch. It is ventrad of the postzygapophysis, and with it clasps the præzygapophysis of the followiug vertebra. It is present in the first six lumbar vertebræ, and markedly in the 9th to the 13th thoracics, where, in man, it is called the inferior tubercle; Quain, A, I, 14.

\$ 478. Arcus neuralis, az.-Neural arch.-This is a bony arch projecting from the dorsal aspect of the centrum. It is called neural arch because it overarches and encloses the myelon or neural axis.

$\S 479$. Canalis neuralis, $\alpha z$. - Neural canal.-The neural canal is formed by the neural foramina, each foramen being a short segment of the canal. It is cnclosed by the neural arches and by ligaments, and contains the myelon.

\$ 480. Centrum, az-Body.-The vertebral centrum is the solid subcylindrical ventral portion of the vertebra. Contiguous vertebral centra articulate amphiarthrodially by means of an interposed disk of fibro-cartilage. For exceptions, see $\S 458$.

\& 481. Diapophysis-Transverse process.-The diapophysis is the lateral projection of the vertebra. Its base, in the cervical vertebre (Fig. 52), except the 7th, contains the vertebrarterial foramen $(\$ 473)$.

§ 492. Fm. (Foramen) neurale, az.-Vertebral ring or foramen.-This is the space 
enclosed by the neural arch and the dorsal aspect of the centrum. The neural foramina of all the vertebræ, together with the ligaments, form the neural canal.

$\S 483$. Fm. (Foramen) vertebrarteriale.-This foramen is found in the base of the diapophyses of all the cervical vertebræ except the last, and rarely in that. In man it is usually present in the last; Humphrey, A, 142. This foramen transmits the Arteria et Vena vertebralis (Fig. 104).

8 484. Inc. (Incisura) vertebralis-Notch.-The pedicles are notched, especially on the caudal side. When the vertebræ are united these notches form the so-called intervertebral foramina, through which pass the vessels and nerves to and from the neural canal.

§ 48j. Lm. (Lamina) neuralis-Lamina. - The two laminæ form the roof of the neural arch. Laterally they join the pedicles, and from their junction at the meson springs the neural spine.

$\S$ 486. Metapophysis-Mammillary process.-This is a dorso-lateral projection of the præzygapophyses of the lumbar vertebre. It corresponds to the internal tubercle of the thoracic transverse processes; Quain, A, I, 15.

$\S 487$. Pedicle.-The pedicle of a vertebra forms the side of the neural arcb. It extends dorsad and joins the lamina.

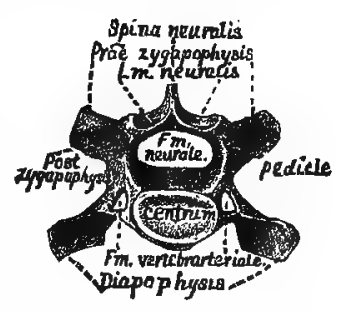

Fig. 53.-Caudal View OF THE FOURTH CERVICAL VERTEBRA; $\times 1$.

$\S 488$. Præzygapophysis-Superior articulating process.-This process articulates

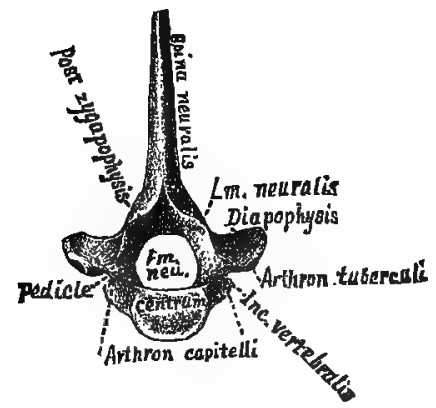

Fig. 54.-Caddal View of The Seventh ThORACIO VERTEBRA; $\times 1$.

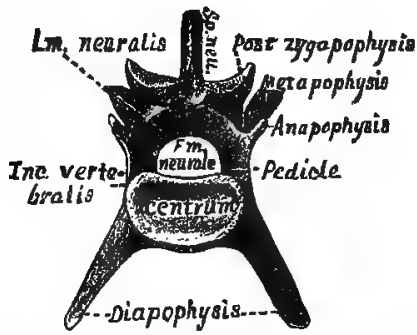

Fig. 55.-CAUdal View OF the Fourth LUMBar Vertebia ; $\times 1$.

diarthrodially with the postzygapophysis of the preceding vertebra, The arthral surface of this process faces nearly dorsad or dorso-mesad.

$\S \cdot 489$. Postzygapophysis-Inferior articulating process.-The postzygapophysis articulates diarthrodially with the præzygapophysis of the vertebra immediately following it. Its arthral surface faces nearly ventrad or ventro-laterad.

§ 490. Sp. neu.-Spina neuralis-Neural spine, Spinous process, az.-This is a mesal process arising from the neural arch and extending approximately dorsad.

\section{THE SKULL.}

General References to the Skull.-Straus-Durckheim, A, I, 380; Quain, A, I, 31; Gray, A, 149 ; Humphrey, A, 175; Darling and Ranney, A, 17 ; Cuvier, A, II, 177 ; Owen, 
A., II, 492 ; Flower, A, 94; Huxley, B, 245 ; Morrell, A; Milne-Edwards, A, X, 306 ; Gegenbaur, A, 463; Leyh, A, 122 ; Chauveau, A, 37 ; Chauveau (Fleming), A, 33 ; Parker and Bettany, A ; Parker, 25; Turner, 4, 848-9 ; Huxley, 3, 238; Wyman, 76 : Cuvier, B, VII.

§ 491. "The skull (Fig. 56-62) is the term commonly applied to the portion of the axial [somatic] skeleton situated within the head." It is a strong bony case or frame enclosing the brain and affording support and protection to the organs of sight, smell, taste and hearing.

It consists of several irregular bones, most of which are immovably united by sutures (synarthroses), so that their relative position, and hence the form of the skull, are constant.

In addition to the skull proper, there are articulated to its base the mandible (Fig. 62) and the hyoid apparatus (Fig. 30, § 224).

$\S$ 492. Cranium and Face.-For convenience, anatomists have divided the skull into these two regions. The cranium is the caudal part of the skull; it encloses and protects the brain. The face is the cephalic part; it surrounds the mouth and nasal passages, and, with the cranium, completes the cavities for the eyes. Quain, A, I, 31, 74; Flower, A, 94, 102.

The number of separable bones entering into the formation of the skull varies with the age of the animal. The teeth are not included. The bones given in the following tables and figures may be easily demonstrated on the skull of a cat with milk teeth.

$\S 493$. Sutures.-The sutures or lines of union between the various bones of the skull have not all received special names, but all may be properly named by forming a compound term of the names of the two bones united, as; Sutura parieto-frontalis instead of S. coronalis; S. maxillo-pramaxillaris, etc. Quain, A, I, $56,58,131$.

\section{\$ 494. Bones of the Skull. Modified from the Tables of Quain, A, 74, and Flower, A, 104. (Fig. 56-62.)}

In the following Tables of the bones, the names in parentluesis beneath a given name are synonyms; and the names behind the small or secondary braces are the anthropotomical equivalents of those in black letter behind the large braces.

The word $O s$ (bone), or its equivalent $O$, is to be understood before all the teclunical names of the bones excepting Vomer and Mandibula. 
C R A I U M .

\begin{tabular}{|c|c|c|}
\hline \multirow{5}{*}{$\begin{array}{l}\text { MESAL (5).. } \\
\text { (Single or Azy- } \\
\text { gous Bones). }\end{array}$} & BASIOCCIPITALE.... & $\begin{array}{l}\text { BASTlaR PROCESS OF THE OCCIPITAL } \\
\text { BONE. }\end{array}$ \\
\hline & BASISPHENOIDEUM & $\begin{array}{l}\text { CACDAI PART OF THE BODY OF THE } \\
\text { SPHENOID BONE, INCLUDING THE } \\
\text { SELLA TURCICA. }\end{array}$ \\
\hline & 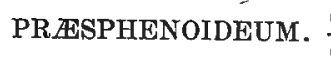 & $\begin{array}{l}\text { CEPHALIC PART OF THE BODY OF THE } \\
\text { SPHENOID BONE. }\end{array}$ \\
\hline & SUPRAOCCIPITALE. & $\begin{array}{c}\text { TABULAR PART OF THE OCCIPITAL } \\
\text { BONE. }\end{array}$ \\
\hline & INTERPARIETALE. & $\begin{array}{l}\text { DORSO-CEPHALIC ANGLE OF THE TABU- } \\
\text { LAR PORTION OF THE OCCIPITAL. }\end{array}$ \\
\hline
\end{tabular}

LATERAL (I0). (Paired Bones).

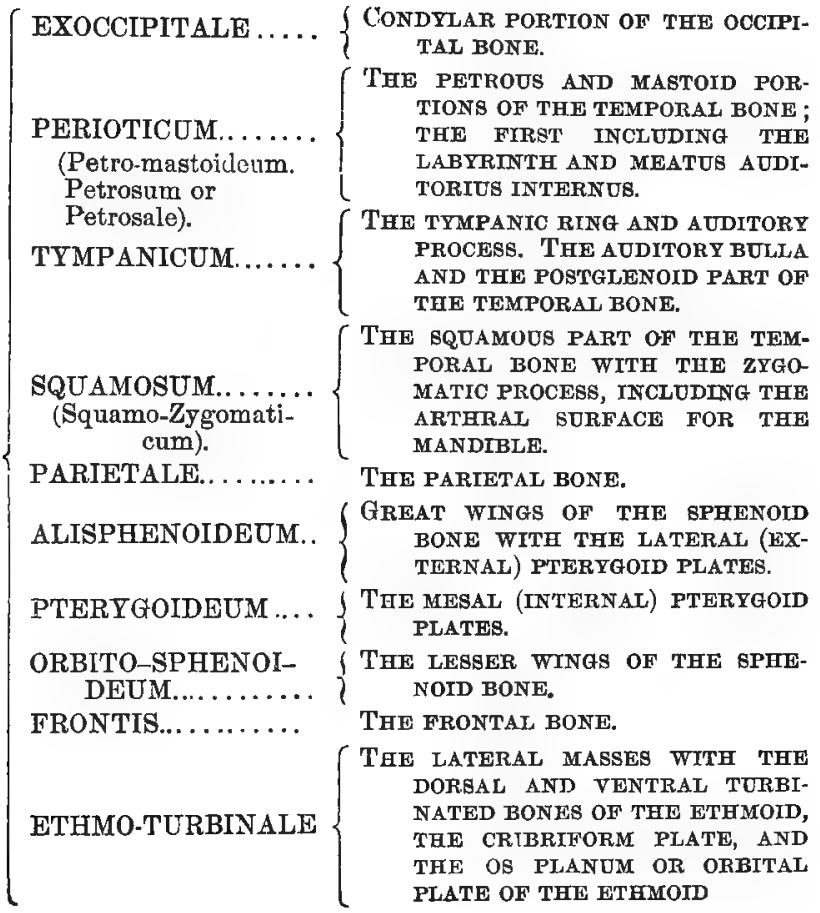

MESAL (2)..... (Single or Azygous Bones).
FACE. 


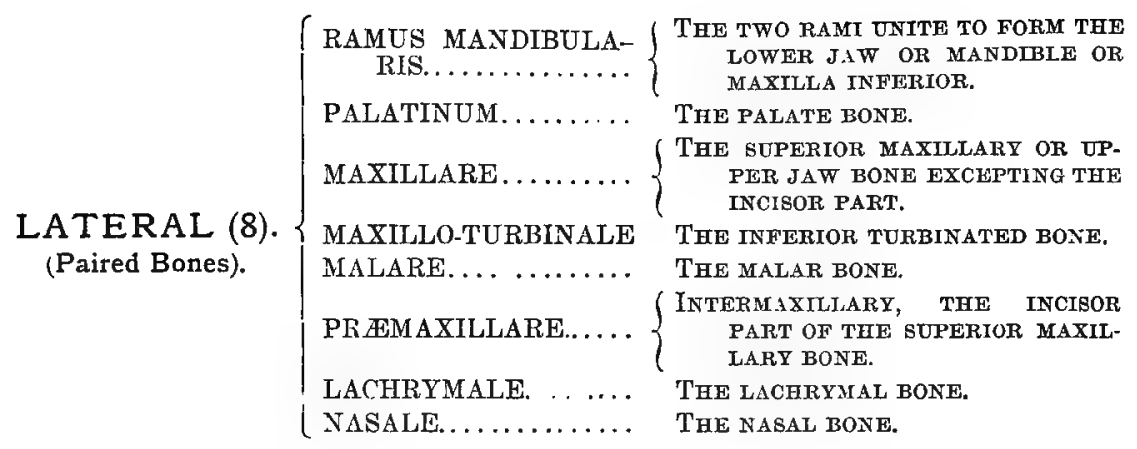

\section{$\S 495$. Articulations of the Bones of the Skull. (Fig. 54-60).}

The following list represents the bones approximately as given in standard works on Human Anatomy. They are readily distinguishable in a nearly adult cat.

The parts forming a compound bone are given in parenthesis immediately below the name of the bone under consideration. See $O_{s}$ occipitale.

The Arabic number in parenthesis, after a bone and just before the brace, indicates the number of bones with which it articulates. The parts of a compound bone are numbered as one. See Os parietale.

In giving the articulations of the mesal bones, the Arabic numeral 2 is placed in parenthesis after all the lateral bones with which it articulates, to indicate that it is connected with both. See Fomer.

If but one of the parts of a compound bone articulates with a given bone, the name of that part is given instead of the name of the whole bone; but if more than one compound enters into the articulation, the name of the whole bone is given, and that is followed by a brace and the names of the components. See Os parietale.

OS OCCIPITALE (6). (Basioccipitale, Exoccipitale, Supraoccipitale, with the Interparietale).
C R A N U M .

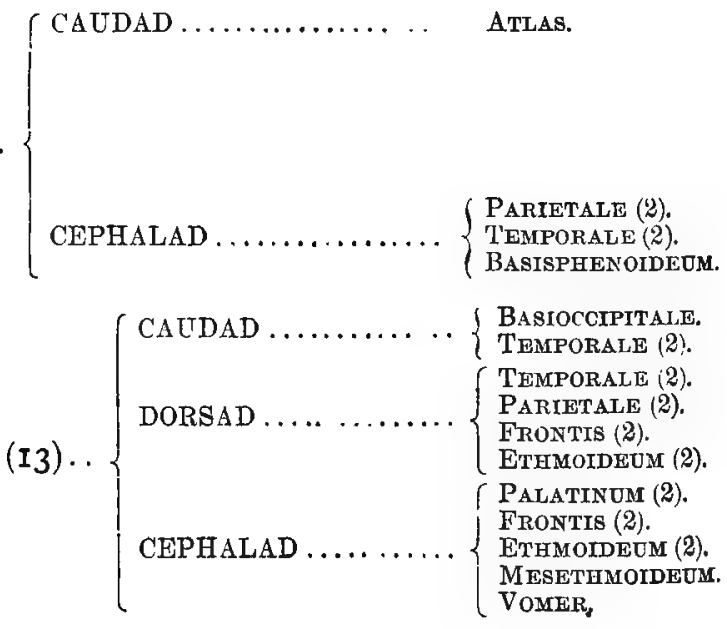

(Basisphenoideum, Alisphenoideum, Pterygoideum, Præsphenoideum, Orbito-sphenoideum). 


\section{OS TEMPORALE (6).. (Perioticum, Tympanicum, Squamosum).

$$
\begin{aligned}
& \text { CAUDAD.... OcctPitale. } \\
& \text { DORSAD.... ParteTALE. } \\
& \text { VENTRAD. }\left\{\begin{array}{l}
\text { RAMUS MANDIBULARIS. } \\
\text { HYOIDES. }
\end{array}\right.
\end{aligned}
$$

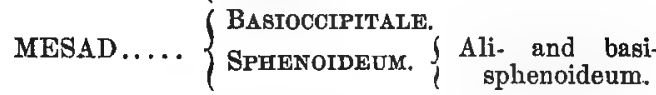

$$
\begin{aligned}
& \text { CEPHALAD. }\left\{\begin{array} { l } 
{ \text { SPHENoIdetr. } } \\
{ \text { PARIETALE. } } \\
{ \text { MaLARE. } }
\end{array} \left\{\begin{array}{l}
\text { Ali- and basi- } \\
\text { sphenoideum. }
\end{array}\right.\right.
\end{aligned}
$$

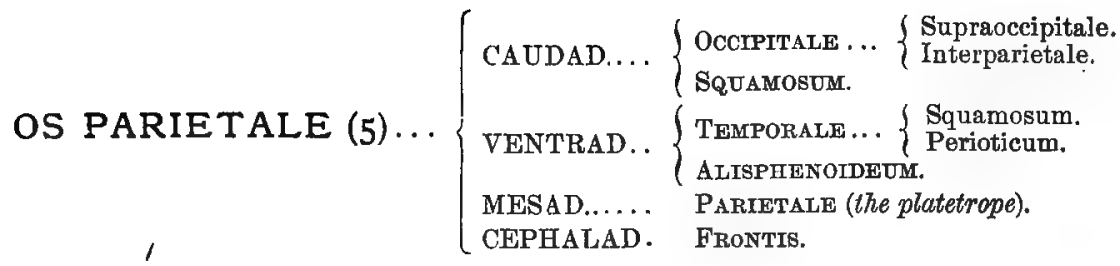

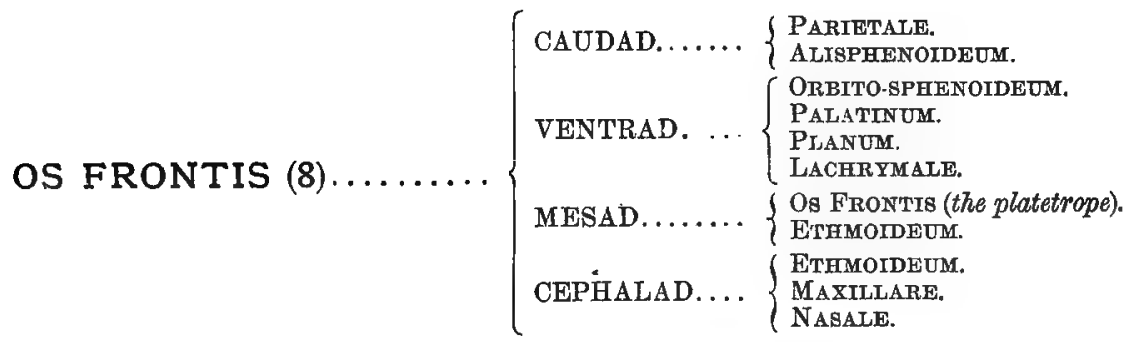

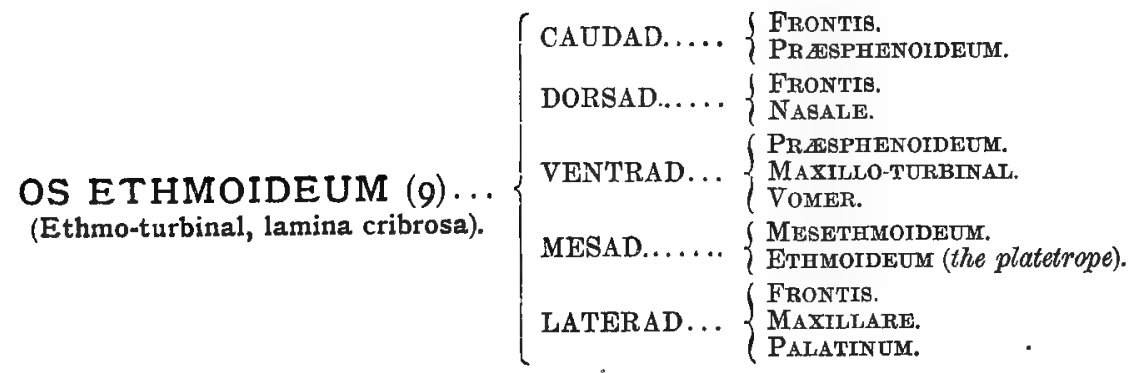

FACE.

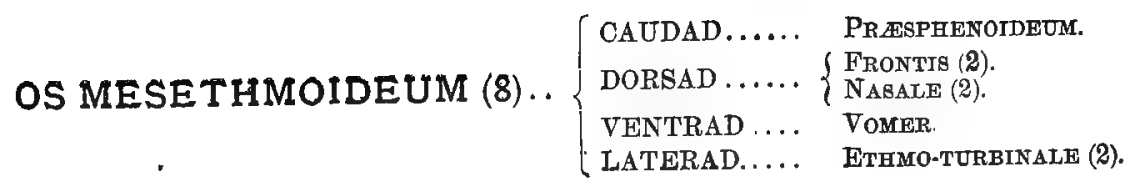


VOMER (I0)..............

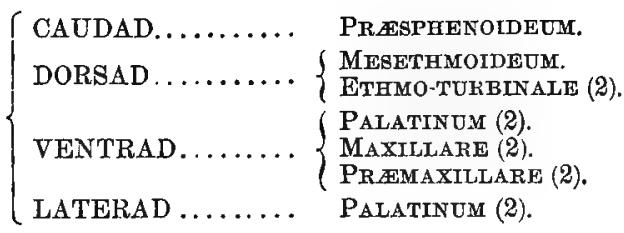

RAMUS MANDIBULA- \{ CAUDO-DORSAD. SqUamosum.

RIS (3)............. $\{$ MESAD......... RM. MNDBL. (the platetrope).

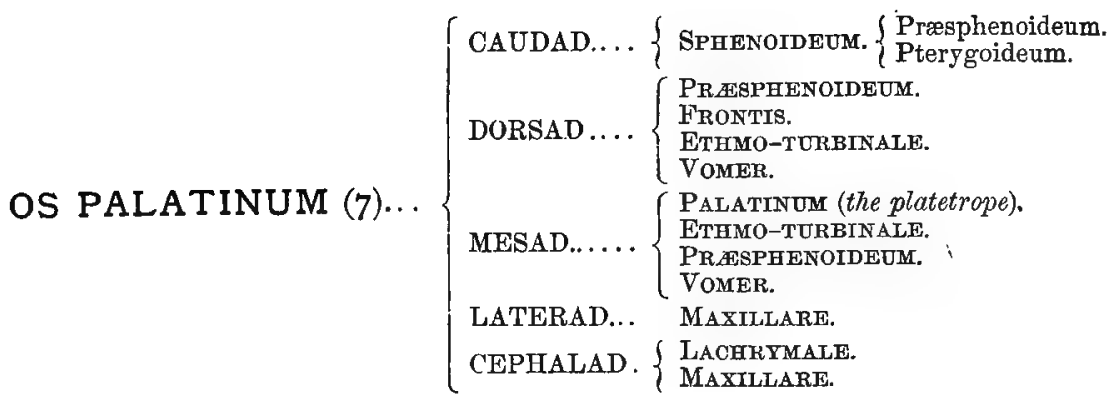

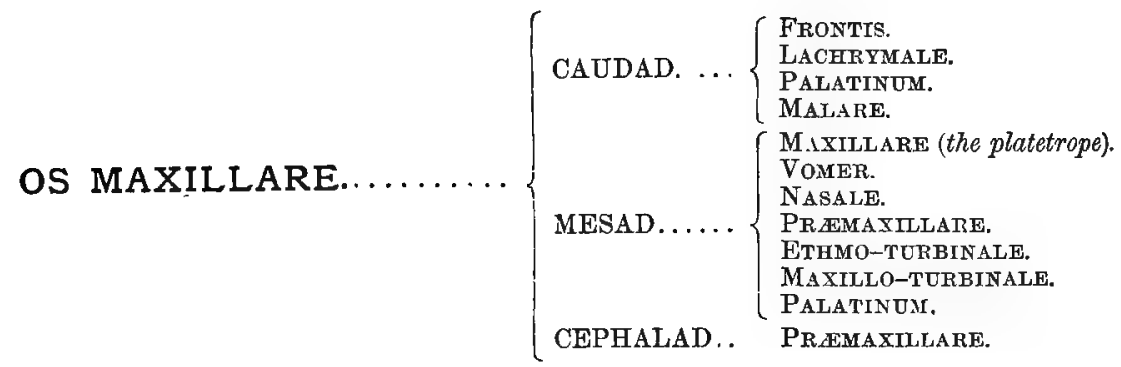

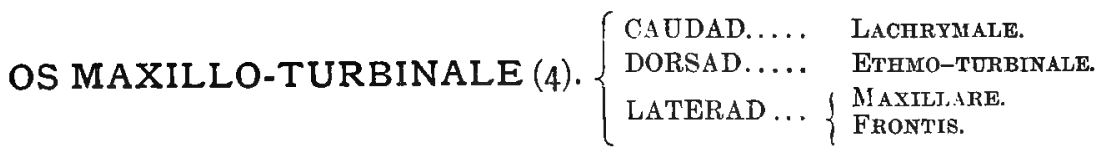

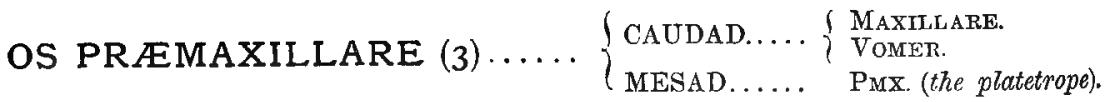

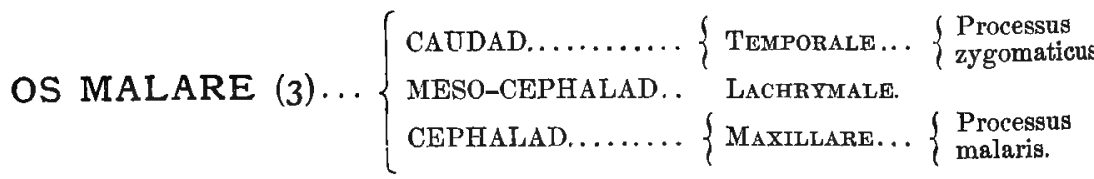


OS LACHRYMALE (5)....

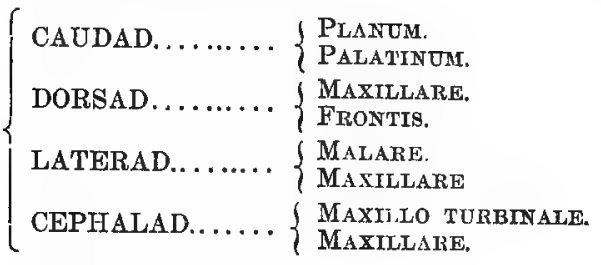

OS NASALE (6).............

All the figures of the skull are of Felis domestica.

In the description of the figures, under the general heads, which are arranged alphabetically, the special parts are named commoncing at the caudal extremity.

For fuller information concerning any of the bones or foramina, refer to the Table of Bones and of Foramina, pp. 175, 190.

$\S 496$. Preparation. - The soft parts were entirely removed $(\$ 250, \mathbf{B})$. The roof of the left side was then removed by sawing carefully with a fine saw to the meson at a level just dorsad of the orbits and about $2 \mathrm{~mm}$. dorsad of the foramen magnum. The frontal and parietal bones were then removed with nippers by separating them at the coronal, the sagittal and the lambdoidal sutures. Finally, the interparietal and supraoccipital were removed by sawing on the meson till the first incision was reached. This exposed the frontal sinus and the ental surface of the base of the skull.

§ 497. Cn. Ich., Canalis lachrymalis-Lachrymal canal.-This canal passes cephalo-ventrad and lies between the maxilla and maxillo-turbinal. It finally opens into the nasal cavity just ventrad of the maxillary attachment of the maxillo-turbinal and nearly opposite the fang of the canine tooth.

\& 498. Cst. Imd., Crista lambdoidalis.-The lambdoidal crest corresponds to the superior curved line of the occipital in Human Anatomy. Flower, A, 112, 132.

§ 499. Cst. temp., Crista temporalis-Temporal crest. -This marks the dorsal limit of the temporal fossa and muscle. In young cats it is considerably separated from the meson, but in adults it approaches it and may be mesal from the lambdoidal crest to the coronal suture. The mesal part is then called sagittal crest ; Flower, A, 112.

Fm. m., Foramen magnum, az.

Fm. j., Foramen jugulare-s. lacerum posterius.-Jugular or posterior lacerated foramen.

Fm. ov., Foramen ovale.

Fm. rt., Foramen rotundum.

Fm. 1. a., Foramen lacerum anterius-8. lacerum orbitale, s. fissura sphenoidalis.-Anterior or orbital lacerated foramen, sphenoidal fissure.

Fm. op., Foramen opticum-Optic foramen. 
$\$ 500$. Fm. plt., Foramen palatinum posterius-Posterior palatine foramen.-The passage between tile opening shown in this figure and Fig. 60 is known as the posterior palatine canal. Quain, A, I, 373.

§ 501. Fm. sphplt., Foramen spheno-palatinum-Spheno-palatine foramen.-It leads from the orbit into the nasal cavity.

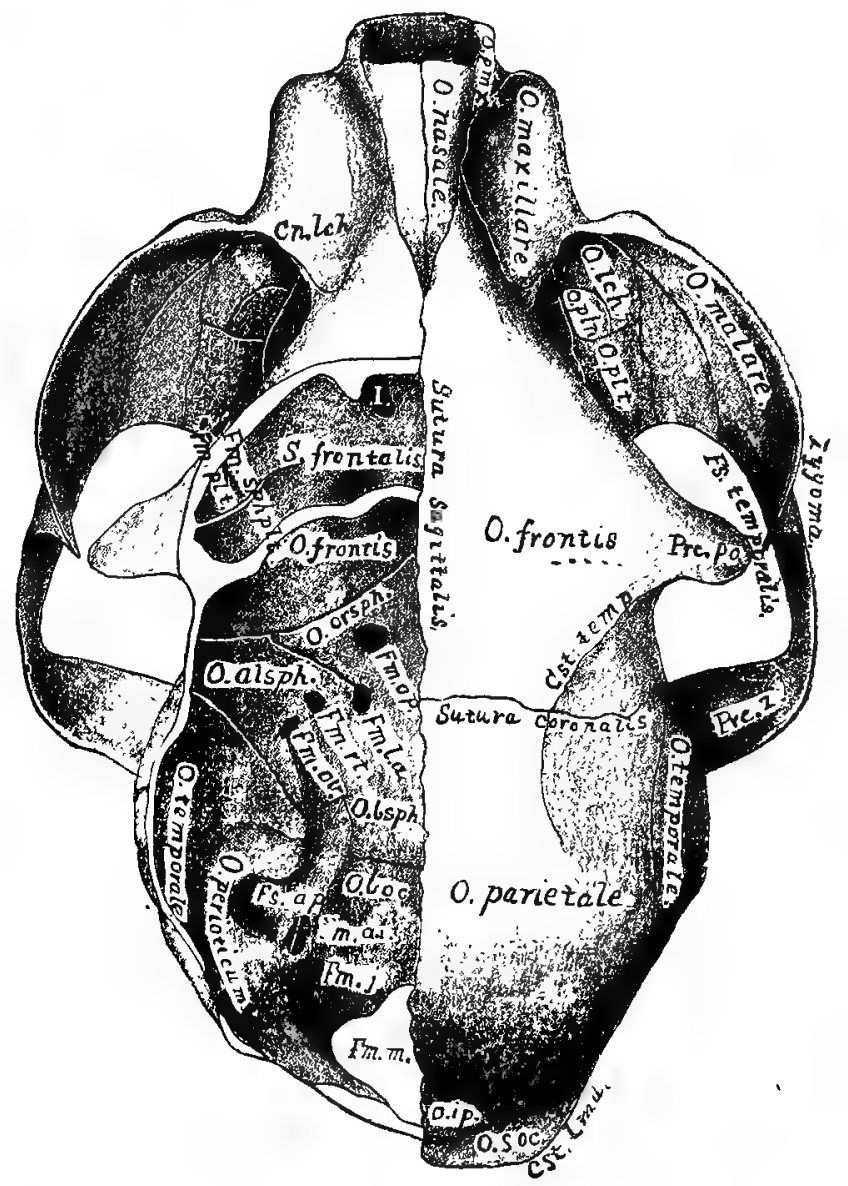

FIG. 56.-Dorsat VIEW OF THE SkULL; $\times 1.2$.

\$02. Fs. ap., Fossa appendicularis.-Fossa for the appendicular lobe of the cerebellum.

503. F. (Fossa) temporalis-Temporal fossa.-This is the space on the side of the skull caudad of the postorbital process of the frontal. By mistake, the name in this figure is written partly in the orbit.

\$ 504. Fossa orbitalis-Orbital fossa, orbit.-In this space the eyes are found in living animals. Unlike the orbital fossa of man, that of the cat is not separated from the temporal by a plate of bone, but opens widely into it. 
$\S 505$. I., Infundibulum.-This is the opening from the frontal sinus to the nasal cavity.

\$506. M. a. i., Meatus auditorius internus-Internal auditory meatus.-This is divided into two parts very near its mouth, as shown by the white line. The ventral part receives the auditory nerve (VIII), the dorsal part, the facial (VII). The facial in its course curves round in the dorso-lateral part of the Pars petrosa and finally emerges at the stylo. mastoid foramen. This passage through the skul from the M. a. i. to the stylo-mastoid foramen is known as the Aquæductus Fallopii. In the Pars petrosa, about $2 \mathrm{~mm}$. from its mouth, there branches cephalad a small canal, the Hiatus Fallopii, which opens opposite the ventral end of the osseous tentorium.

\$ 507. O. soc., Os supraoccipitale, $a z$.-Supraoccipital bone.-This forms the caudal part of the roof of the cranial cavity.

§ 503. O. i. p., Os interparietale, az.-Interparietal bone.-A small bone, separate in young animals. It is cephalad of the supraoccipital and is wedged in between the caudal ends of the parietals.

§ 509. O. parietale-Parietal bone.-The parietal bone forms a large part of the side and roof of the cranial cavity.

\$ 510. O. perioticum-s. petrosum.-This is a part of the temporal bone; it encloses the internal ear, and is divided into two parts, Pars petrosa and Pars mastoidea', the latter appearing on the ectal surface of the skull (Fig. 57).

\$ 511. O. temporale-Temporal bone.-This forms part of the floor and side of the cranium, and by its zygomatic process helps to enclose the temporal fossa.

$\$ 512$. O. boc., Os basioccipitale, $a z$.-Basioccipital bone.-It forms part of the floor of the cranium.

\$ 513. O. bsph., Os basisphenoideum, az.-It forms the caudal part of the body of the sphenoid bone, and helps to make the floor of the cranial cavity.

$\$ 514$. O. alsph., Os alisphenoideum.-This is one of the greater wings of the sphenoid and forms part of the side and floor of the cranium.

\$ 515. O. orsph., Os orbito-sphenoideum.-This is one of the lesser wings of the sphenoid; it forms part of the floor and side of the cranium and part of the mesal wall of the orbit.

§ 516. O. frontis-The frontal bone.-The frontal bone forms a large part of the roof and part of the side of the cephalic region of the cranial cavity and the mesal wall of the orbit. It also covers the caudal part of the nasal cavity.

\$ 517. O. plt., Os palatinum-Palate bone.-This is a very complex boue, forming part of the orbit, of the roof of the mouth, and of the floor and side of the nasal cavity.

$\S 518$. O. malare-8. Os zygomaticum, os jugale-Malar, zygomatic or jugal bone.This bone counects the malar and zygomatic processes of the maxilla and the temporale, and with them encloses laterally the orbit and the temporal fossa, and completes the $Z$ ygoma or zygomatic arch.

\$ 519. O. pln., Os planum. - This is not a separate bone, but merely the orbital part of the ethmo-turbinal. It is often absent.

§ 520. O. Ich., Os lachrymale-Lachrymal bone.-It is a thin quadrilateral bone forming part of the cephalic wall of the orbit and the caudal part of the nasal cavity.

§ 521. O. maxillare-Maxilla, superior maxillary or upper jaw bone.-This is a complex bone which forms a great part of the face. In it are implanted the dorsal (upper) molar, præmolar and canine teeth.

522. O. nasale-Nasal bone. $-A$ thin irregular bone forming part of the roof of the nasal cavity. 


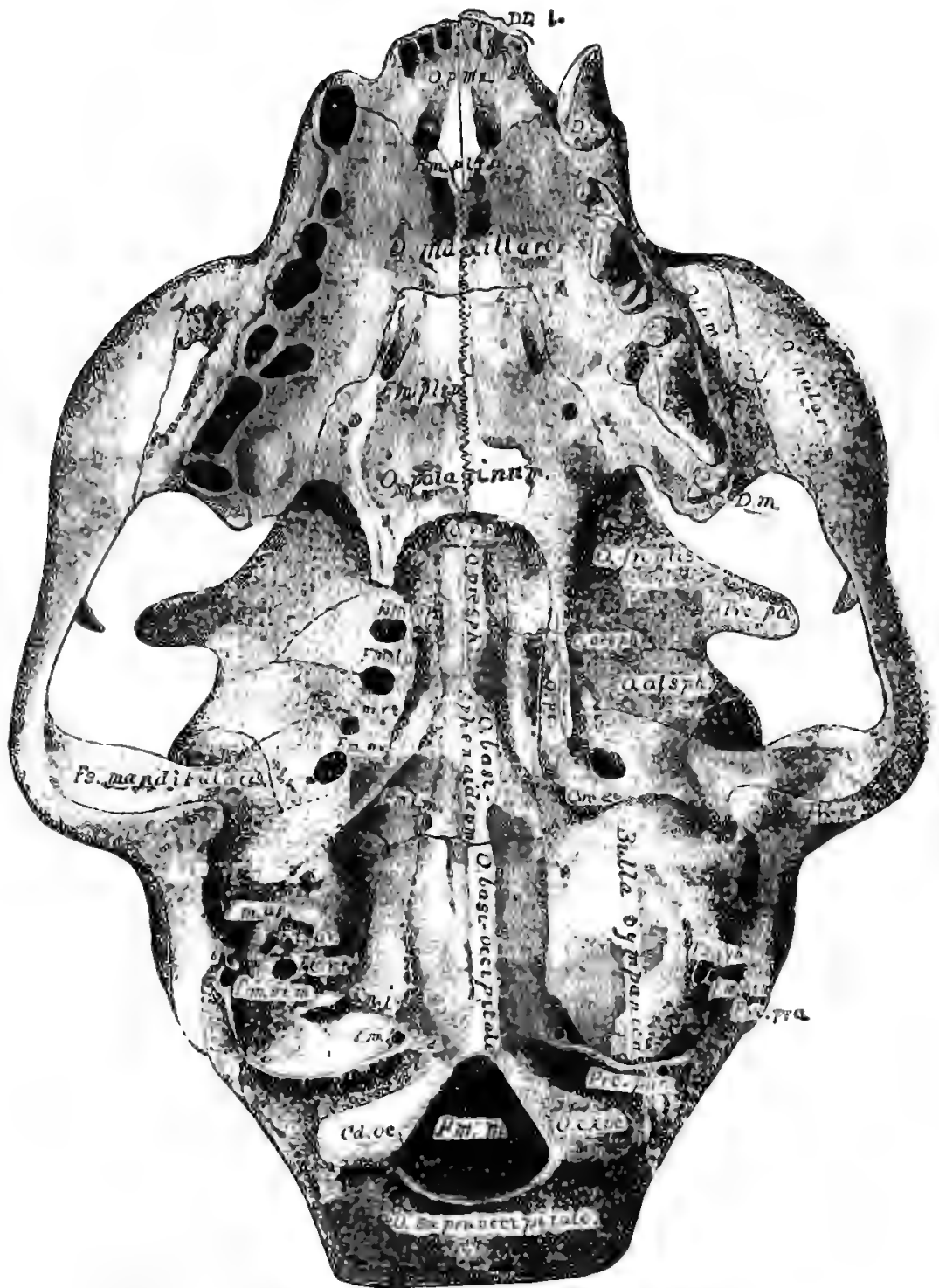

Fra. 57- Ventral. ABPEet on BAse of THE Sigull. (Modified from Straus-Durek. heim) ; $\times 1.75$.

\$ 523. O. pmx., Os præmaxillare-Pruemaxillary or intermaxillary bone. -It forms the most crphalic part of the skull, aud in it are implanted the dorsal (apper) incisor teeth.

Prc. z., Processus zygomaticus.-Zygomatic process of the temporal bone. 
Prc. po., Processus postorbitale.-Post orbital process of the frontal bone.

$\S$ 624. S. (Sinus) frontalis-Frontal sinus.-This cavity in the frontal bone is separated from its platetrope by a bony partition (Fig. 60). It communicates with the nasal cavity through the infundibulum (I.) and is lined by an extension of the nasal mucous membrane.

§ 525. Sutura lambdoidalis, $a z$.-Lambdoidal or occipito-parietal suture.-This is the synarthrodial articulation between the parietal, the interparietal and the supraoccipital bones.

§26. Sutura sagittalis, az.-Sagittal suture.-The synarthrodial articulation between the right and left frontal and the parietal bones. It extends from the nasal boues to the lambdoidal suture.

In Human Anatomy, the sagittal suture is confined to the articulation of the two parietals with each other, the two frontals uniting so early that they are considered as a single bone.

$\S 527$. Sutura coronalis-Coronal or parieto-frontal suture-The synarthrodial articulation between the frontal and parietal bones.

\$528. Fig. 57-Preparation. - The skull was thoroughly cleaned, and while still moist the following structures were removed from the right side :-

(A) The tympanic bulla was removed by inserting an arthrotome between it and the basioccipital and prying steadily, thus exposing the Pars petrosa, the Foramen lacerum jugulare and $F m$. lacerum medium, the Fenestra ovalis and Fenestra rotunda.

(B) The Os pterygoideum and a part of the Os palatinum were removed by the nippers to expose the row of foramina.

(C) The teeth were extracted with the nippers to expose the alveoli (sockets of the teeth).

a, b.-Fractures made in removing the bulla.

Bulla tympanica.-Tympanic or auditory bulla (Fig. 58).

\$ 529. Cd. oc., Condylus occipitalis-Occipital condyle.-The occipital condyle is formed mostly by the exoccipital, but somewhat also by the basioccipital. It articulates diarthrodially with the atlas.

Cn. eu., Canalis Eustachiana.-Eustachian canal (Fig. 58).

D. m., Dens molaris.-Molar tooth.

DD. pm., Dentes præmolares.-Præmolar teeth.

D. c., Dens caninus.-Canine tooth.

DD. i., Dentes incisores.-Incisor teeth.

Fm. m., Foramen magnum, az.

$\S 530$. Fm. cd., Foramen condylare-Condylar foramen.-This foramen is sometimes nearly hidden either by the projection of the bulla or its unusual approach to the Fm. jugulare.

\$51. Fm. j., Foramen jugulare-s. lacerum posterius.-Jugular or posterior lacerated foramen.

\$ 532. Fm. stm., Foramen stylo-mastoideum-Stylo-mastoid foramen.-This is the ectal termination of the Aquaductus Fallopii (Fig. 56, 59, M. a. i.).

§ 533. Fm. 1. m., Foramen lacerum medium-s. Fm. spheno-petrosum-Middle 
lacerated, or spheno-petrosal foramen.-The opening of this foramen on the ectal surface of the skull is just within the mouth of the Eustachian canal. It perforates the lateral edge of the cephalic projection of the bulla, and may be seen on most prepared skulls by looking into the mouth of that canal.

The existence of the Foramen lacerum medium in the cat is not mentioned by any of the authorities, so far as we are aware; but the foramen so named in this figure is between the cephalic extremity of the purs petrosa and the alisphenoideum-the position occupied by the foramen lacerum medium in man, as stated by Quain, A, I, 62, and in the dog, as stated by Flower, A, 101. This foramen in the cat also transmits an artery, which has been called internal carotid in the table of foramina $(\$ 562)$. It is so called since it anastomoses with the cerebral vessels within the cranium, and arises from the carotid at the proper place-slightly centrad of the origin of the lingual-although it passes along the mesal side of the bulla and not in a carotid canal, as stated by Mivart, B, 208, 209. The artery is imbedded in the wall of the Eustachian tube in its passage along the Eustachian canal to the foramen.

Fm. ov., Foramen ovale.

Fm. rt., Foramen rotundum.

\$534. Fm. 1. a., Foramen lacerum anterius-8. fissura sphenoidalis-Anterior lacerated foramen or sphenoidal fissure.

Fm. op., Foramen opticum, transmitting the Nervus opticus.

$\S 535$. Fm. plt. p., Foramen palatinum posterius-Posterior palatine foramen.There are usually two openings of the posterior palatine canal in the roof of the mouth as shown in this figure. (See also Fig. 56.)

Fm. plt. a., Foramen palatinum anterius-8. Fm. incisore.-Anterior palatine or incisor foramen.

$\S 536$. Fs. tyh., Fossa thyrohyalis.-This is the pit into which is inserted the dorsal part of the Os hyoides (\$224).

$\S 537$. Fs. (Fossa) mandibularis-Mandibular or glenoid fossa.-This receives the mandibular condy]e and articulates diarthrodially with it (Fig. 62).

$\S 538$. Ft. rt., Fenestra rotunda.-This opening connects the tympanum with the scala tympani of the cochlea. In the living body it is covered with membrane.

$\S 539$. Ft. ov., Fenestra ovalis.-This connects the tympanum with the vestibula. In living bodies it is closed by the stapes and its connecting soft structures.

$\S 540$. M. a. e., Meatus auditorius externus-External auditory meatus.-This extends from the side of the head to the ectal surface of the Membrana tympani.

§ 541. Margo alveolaris-Alveolar margin or border of the jaw.-In this border of the jaw the teeth are implanted.

O. supraoccipitale, $a z$.-Supraoccipital bone.

O. exoc., Os exoccipitale.-Exoccipital bone.

O. basioccipitale, $a z$.-Basioccipital bone.

O. basisphenoideum, az.-Basisphenoid bone.

O. alsph., Os alisphenoideum (Fig. 56).

O. pt., Os pterygoideum.-Pterygoid bone.

O. præsph., Os præsphenoideum, $a z$.

O. orsph., Os orbito-sphenoideum (Fig. 56).

O. frontis (Fig. 56).

O. vm., Os vomeris.-Vomer (Fig. 59).

O. palatinum.-Palate bone (Fig. 56).

0. malare.-Malar hone (Fig. 56).

O. maxillare.-Maxillary or upper jaw boue (Fig. 56). 
O. pmx., Os præmaxillare,-Præmaxillary or intermaxillary bone.

8 542. Prc. par., Processus paroccipitalis-s. paramastoideus-Paramastoid or paroccipital process.-This is a curved shelf-like projection of the exoccipital which abuts against the caudal end of the bulla.

Prc. pro., Processus mastoideus-8. pars mastoidea.-Mastoid portion of the perioticum.

Prc. z., Processus zygomaticus.-Zygomatic process of the temporal bone.

Prc. po., Processus postorbitale.-Postorbital process of the frontal bone.

§ 543. Fig. 58-Preparation.-The soft parts were removed; then, while the skull was yet moist, the ventral face of the bulla was ground off .on. a fine emery-stone. It might be removed with a watch-spring saw or on an ordinary grindstone. In grinding, the pressure should be only moderate to avoid breaking the delicate septum.

§ 544. Bulla tympanica-The auditory or tympanic bulla.-The bulla is a hollow subspherical part of the o. tympanicum enclosing the tympanum or middle ear. A delicate bony septum divides the cavity into two un. Fig. 58. - Ventro - LATHRAL equal parta. This septum arises from the floor, and extends dorsad, but leaves a space over the fenestra ro-

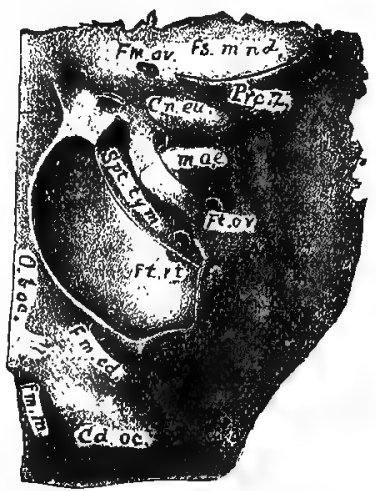

VIEW OF THE LEFT BULLA ANd AdJaCent Parts ; $\times 1.5$. tunda which puts the two chambers in communication.

In the lateral aspect of the bula is the external auditory meatus (M. a. e.); attached to the ectal part of this is the external ear, and to the ental part the Memibrana tympani or ear drum, which completely separates the tympanum or middle ear from the exterior of the body. Flower, A, 110, and 26, 4; Huxley, B, 249 : Straus-Durckheim, A, I, 409.

Cd. oc., condylus occipitalis. -Occipital condyle.

\$ 545. Cn. eu., Canalis Eustachiana-Eustachian canal.-This is a short bony tube leading from the tympanum to the ventral surface of the skull. Flower, A, 111.

Fm. m., Foramen magnum, az.

Fm. cd., Foramen condylare.-Condylar foramen.

Fm. ov., Foramen ovale.

Fs. mnd., Fossa mandibularis.-Mandibular or glenoid fossa (Fig. 57),

Ft. rt., Fenestra rotunda (Fig. 57).

Ft. ov., Fenestra ovalis.

M. a. e., Meatus auditorius externus.-External auditory meatus.

O. boc., Os basioccipitale, az.-Basioccipital bone.

Prc. z., Processus zygomaticus.-Zygomatic process of the temporal bone.

Spt. tym., Septum tympanicum.-The bony partition dividing the interior of the bulla into two unequal chambers.

\& 546. Fig. 59-Preparation.-The soft parts were removed $(\$ 250, \mathbf{B})$, and then the section was made with a watch-spring saw while the skull was 'still moist. The section was made about $2 \mathrm{~mm}$. 
to the left of the meson to avoid injuring the mesethmoideum and other mesal parts. The remaining parts of the left half were afterward removed with the nippers; the septa of the frontal and sphenoidal sinuses were likewise partly removed.

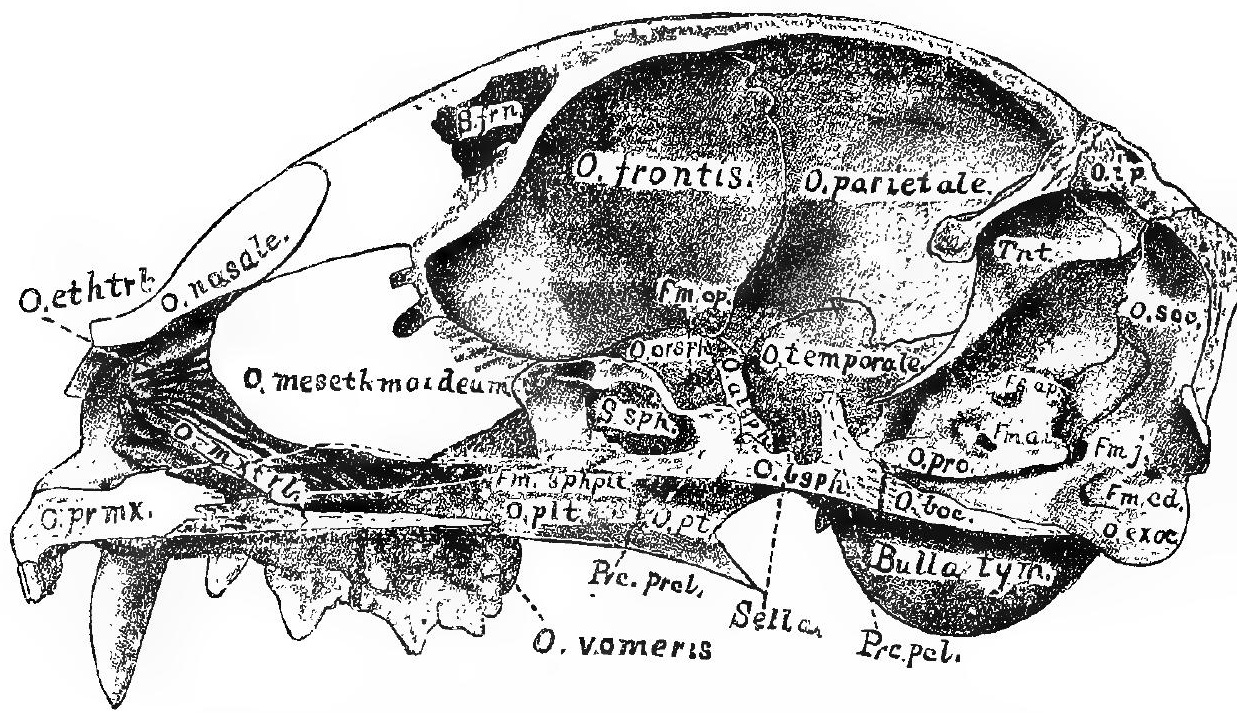

Frg. 59.-Hemisection of the Skuld, Riget Side; $\times 1.5$.

Bulla tym., Bulla tympanica.-Tympanic or auditory bulla (Fig. 58).

Fm. j., Foramen jugulare-s. Fm. lacerum posterius.-Jugular or posterior lacerated foramen.

Opposite the occipital condyle and nearly caudad of the condylar foramen is an unnamed opening for a vein.

Fm. op., Foramen opticum.-Optic foramen.

Fm. sphplt., Foramen spheno-palatinum.-Spheno-palatine foramen.

Fs. ap., Fossa appendicularis (Fig. 56).

8 547. Fossa of the Cranial Cavity.-(A) Fossa cerebellaris, az.-Cerebellar fossa.- This is the part of the cranial cavity caudad of the osseous tentorium. It contains the cerebellum and medulla (Fig. 88).

(B) Fossa cerebralis, az.-Cerebral fossa.- This is the part of the cavity of the skull between the tentorium caudad and the olfactory fossa cephalad. It contains the cerebrum, thalamus, part of the optic lobes and the crura (Fig. 88).

(C) Fossa olfactoria-8. rhinencephalica-Olfactory or rhinencephalic fossa.-This is the smallest of the fossæ. It is limited cephalad by the Lamina cribrosa and extends caudad to the dorso-ventral ridge formed by the frontal and orbito-sphenoid. It lodges the rhinencephalon or olfactory lobes (Fig. 88).

M. a. i., Meatus auditorius internus.--Iuternal auditory meatus (Fig. 57).

O. exoc., Os exoccipitale.-Exoccipital bone (Fig. 56).

O. soc., Os supraoccipitale, $a z$.-Supraoccipital bone. 
O. ip., Os interparietale, az.-Interparietal bone.

O. boc., Os basioccipitale, $a z$-Basioccipital bone.

O. pro., Os perioticum.-Periotic bone (Fig. 59).

O. bsph., Os basisphenoideum, $\alpha z$.-Basisphenoid bone.

o. temporale.-Temporal bone.

O. parietale.-Parietal bune (Fig. 56).

O. pt., Os pierygoideum.-Pterygoid bone.

O. alsph., Os alisphenoideum.-Alisphenoid bone.

O. plt., Os palatinum.-Palate bone (Fig. 57).

O. orsph., Os orbito-sphenoideum.

O. frontis. - Frontal bone (Fig. 56).

$\S 548$. O. vomeris, az.-Vomer.-The vomer is a mesal bone forming part of the septum of the nasal cavities.

$\S 549$. O. mesethmoideum, $\alpha z$.-Vertical plate of the ethmoid.-This is a mesal bone forming most of the partition between the nasal cavities.

o. Nasale.-Nasal bone.

O. mxtrb., Os maxillo-turbinale-Maxillary or inferior turbinated bone.-It is a greatly plicated bone occupying the ventral p.ut of the nasal cavity.

\$ 550. O. ethtrb., Os ethmo-turbinale-Ethmo-turbinal bone-Chis is also a greatly plicated bone; it occupies the dorsal and greater half of the nasal cavity. It projects caudad into both the frontal and the sphenoidal sinuses (Fig. 60).

O. pmx., Os præmaxillare.--Præmaxillary or intermaxillary bone (Fig. $\tilde{5} 7$ ).

O. $\mathrm{mx}$., Os maxillare-The upper jaw bone.-In this figure it is not marked, but it is the deeply shaded part just caudad of the O. Prmx.

Prc. pcl., Processus postclinoideus, az.-Posterior clinoid process.

Prc. prcl., Processus præclinoideus, az.-Præclinoid process.

Sella, Sella turcica, az.-This is a space or pit formed by the two clinsid processes (Fig. 88).

$\$ 551$. S. sph., Sinus sphenoidalis-Sphenoidal sinus.-This is a space in the præspbenoid bone. It is separated from its platetrope by a bony partition. It is lined by a continuation of the nasal mucous membrane, and communicates freely with the nasal cavity.

S. frn., Sinus frontalis.-Frontal sinus (Fig. 56, 60).

\$ 552. Tent., Tentorium. - The osseous tentorium cerebelli is a moderately thick plate of bone projecting from the parjetal bones into the cranial cavity; it separates the cerebrum from the cerebellum (Fig. 88).

Each parietal bone furnishes half of the tentorium, and its halves are conjoined at the meson by a ventral continuation of the sagittal suture ; (see Fig. 88).

§553. Fig. 60-Preparation.-The skull was divested of its soft parts $(\S 250, \mathbf{B})$. It was then sawed obliquely across its cephalic half so as to include the optic foramina, and to fully expose the frontal sinuses. The left lateral wall of the olfactory fossa was nipped away, and likewise the lateral projections of the maxilla.

Cr. Ich., Canalis lachrymalis.-Lachrymal canal (Fig. 56).

Fm. (Foramen) opticum-Optic foramen.-The optic chiasma rests in the groove between the two foramina (Fig. 114). 
Fm. sphplt., Foramen spheno-palatinum.-Spheno-palatine foramen.

Fm. infor., Foramen infraorbitale.-Infraorbital foramen.

$\S 554$. Lm. (Lamina) cribrosa-Cribriform plate of the ethmoid.--The mesethmoid separates the bones of the two sides. The cribsiform plate is the cravial part of the ethmoid, the ethmo-turbinals properly belonging to the face.

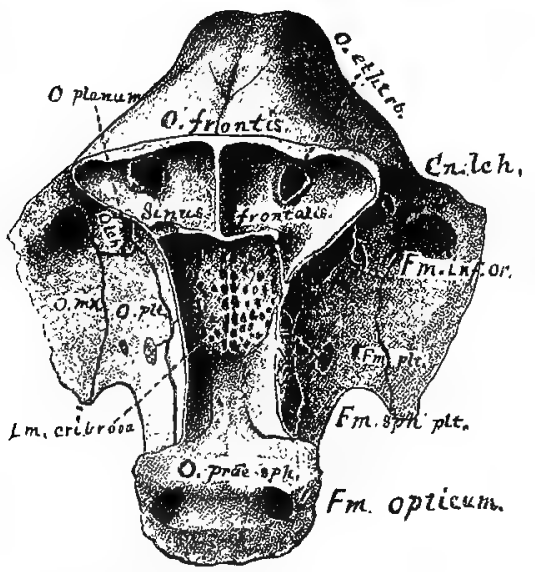

FIG. 60.-DORSO-CADDAL VIEW OF THE

Lamina Cribrosa aNd the Sinus Frontalis ; $\times \mathbf{1 . 5}$.

O. præsph., Os præsphenoideum, az.Præsphenoid bone.

O. plt., Os palatinum.-Palate bone.

O. Ich., Os lachrymale. - Lachrymal one (Fig. 56).

O. planum (Fig. 56).

O. ethtrb., Os ethmo-turbinale-Ethmo-turbinal bone.-In this figure is shown the rounded scroll-like part projecting into the frontal sinus ( $\$ 550)$.

O. frontis.-Frontal bone.

Sinus frontalis.-Frontal sinus (Fig. 56).

§ 555. Fig. 61-Preparation.After the removal of the soft parts $(\$ 250, \mathbf{B})$, the rami were separated at the sympliysis menti (Fig. 62).

\$ 5j6. An. (Angulus) mandibularis.Mandibular angle.

Cd. mndb., Condylus mandibularis.The condyle or arthral head of the mandible (Fig. 62).

D. m., Dens molaris.-Molar tooth.

DD. pm., Dentes præmolares_Præmolar teeth.-The bicuspides of Anthropotomy.

D. c., Dens caninus.-Canine tooth.

DD. i., Dentes incisores.Incisor teeth.

Fm. m, Foramen mentaleMental or labial foramen.-There ase almost invariably two on each side in the cat.

Fs. (Fossa) coronoidea-Coronoid fossa.-From this fossa arises a large part of the masseter muscle.

Prc. cor., Processus coronoi-

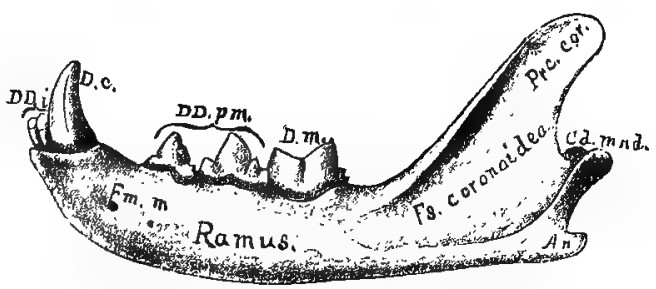

Fig. 61.--Lateral View of the Left MandibU. LAR RAMUS; $\times 1$. deus.-Coronoid process.

$\S 557$. Ramus, Ramus mandibularis-Mandibular ramus.-The mandible is made up of two similar bones joined at the symphysis menti, and these rami form the framework of the floor of the mouth; Flower, A, 120.

In Human Anatomy, the "ramus" is the so called "ascending part," and not the entire half of the mandible as here; Quain, A, I, 54.

§ 558. Fig. 62-Preparation.-All the soft parts were removed 
$(\S 250, \mathbf{B})$; while still moist the two rami were slightly separated at the symphysis menti, and the teeth of the right side were extracted with the nippers to show the alveoli, as in Fig. 57.

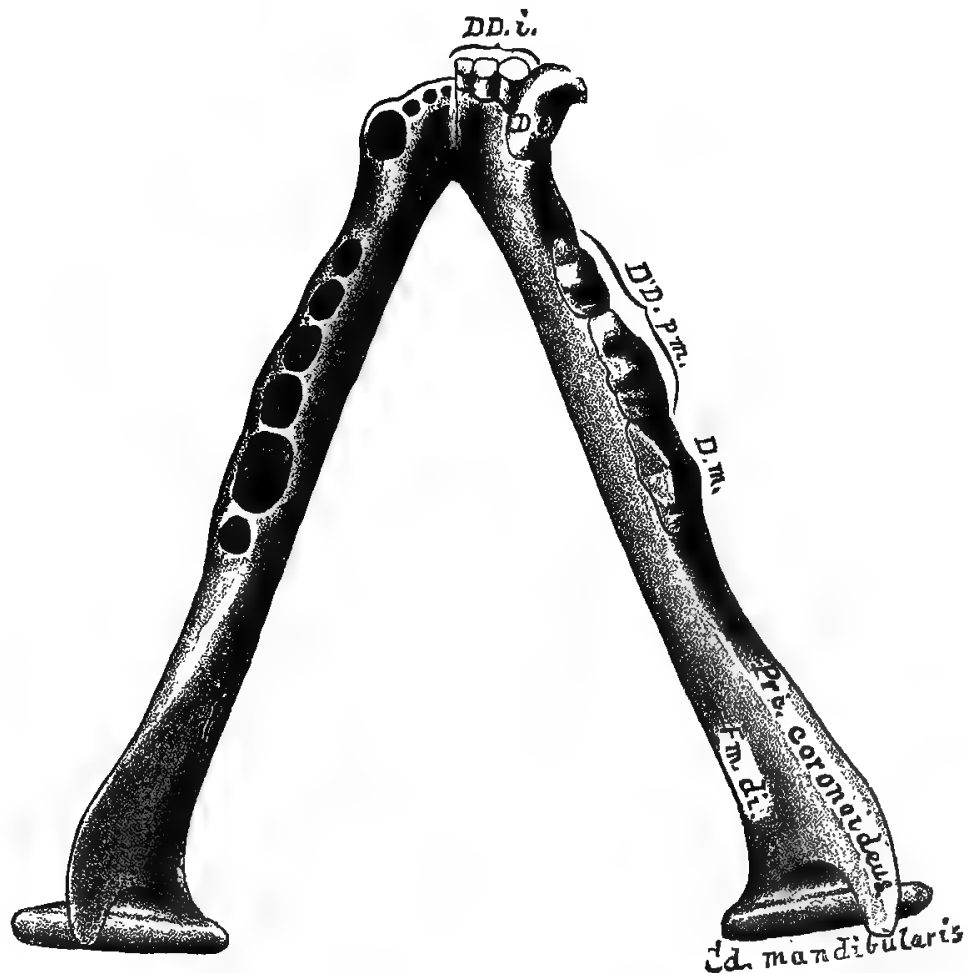

Fig. 62.-Dorsal View of THE MaNdible. (Modified from Straus-Durckheim). $\times 1.75$.

\$ 559. Alveoli.-These are the cavities in which the teeth are implanted.

\$ 560. Cd. (Condylus) mandibularis-Mandibular condyle or arthral bead.-This smooth cylindrical process articulates diarthrodially with the Fossa mandibularis of the temporal (Fig. 5i).

D. m., Dens molaris.-Molar tooth.

DD. pm., Dentes præmolares.-Præmolar teeth.

D. c., Dens caninus.-Canine tooth; Wilder, 15.

DD. i., Dentes incisores.-Incisor teeth.

Fm. di., Foramen dentale inferior.-Inferior dental foramen.

Margo alveolaris-Alveolar margin.-The margin or border of the jaw in which the teeth are implanted.

Prc. (Processus) coronoideus.-Coronoid process.

\$ 561. Symphysis menti.-The symphysia is the amphiarthrodial articulation between the two rami. It is indicated in the figure by a dark line just to the left of the incisor teeth. In old cats this symphysis often becomes anchylosed, but in young individ. uals considerable motion is possible between the two rani. 


\section{§562. Table of the Foramina and Canals of the Skull, the Bones enclosing them, and the Principal Structures to which they give Passage.}

The foramina are enumerated, commencing at the caudal end of the skull, Fig. 56, 62. All are lateral or paired except the foramen magnum, which is mesal.

ForAmINA.
Foramen magnum .........
Foramen condylare........
Foramen jugulare..........
Foramen stylo-mastoideum..
Meatus auditorius internus..
Aquæductus Fallopii........
Hiatus Fallopii.............
Meatus auditorius externus..
Foramen lacerum medium.
(\$ 533$). . . . . . . . . . . . .$.
Canalis Eustachiana........

Foramen ovale.

Foramen rotundum.

Foramen lacerum anterius..

Foramen opticum

Foramina olfactoria.

Foramen palatinum posterius

Foramen spheno-palatinum. .

Foramen palatinum anterius.

Foramen orbitale..........

Canalis lachrymalis.

Foramen infraorbitale.

Foramen dentale inferius....

Foramina mentalia.........
BONES ENCLOSING THE FORAMINA.

$\{$ Basi-ex- and supraoccipitale........ Exoccipitale........

(Basi- and ex-ocoipitale, tympanicum and pars petrosa..

\{ Tympanicum, pars ? mastoidea.......

Pars petrosa......

(Pars petrosa, pars mastoidea, tym panicum.

Pars petrosa........

Tympanicum.... ...

\} Pars petrosa, basi- 1 sphenoideum ..

Alisphenoideum, bul la tympanica....

Alisphenoideum ....

Alisphenoideum ....

fAli-and orbito-sphenoideum..........

Orbito-sphenoideum.

Lamina cribrosa ....

Palatinum.........

Palatinum..........

( Maxillare,præmax-\} illare. .........

Palatinum, maxillare

(Lachrymale, max-

$\{$ illare, maxillo- $\}$ turbinale.......

Maxillare...........

Mandibula.........

Mandibula .........
STRUCTURES THAT TRAVERSE THE FORAMINA.

$\{$ Myelon, N. (spinalis) accessorins $\{(\mathrm{XI}), \mathrm{A}$. basi]aris.

N. hypoglossus (XII).

( $N$. vagus $(X), N$. (spinalis) accessorius (XI), N. g-losso-pharyngeus (IX), Vena jugularis.

N. facialis (VII).

(N. auditorius internus (VIII), N. facialis (VII), $A$. auditoria interma.

N. facialis (VII).

$\{N$. petrosalis superficialis (ec$\{$ talis), (branch of Vidian).

Admits air to the membrana tympani.

Arteria carotidea interna.

(Tuba Eustachiana, A. carotidea interna ( $\$ 533)$.

(N. maxillaris inferior (3d division of $V$ ); small meningeal artery.

\}N. maxillaris superior (2d or

ziddle division of $\mathrm{V}$ ).

$\mathrm{N}$. ophthalmicus (1st division of V), N. oculomotorius (III), N. abducens (VI), N. trochlearis (IV), A.carotidea externa (large branch from the rete mirabile).

$\{\mathrm{N}$. opticus (II) ; a meningeal ar. $\{$ tery.

NN. olfactorii.

NN. palatini, AA. palatinæ.

\{ NN. spheno-palatini, AA. splee. no-palatinæ.

N. naso-palatinus, A. nasalis.

N. oculo-nasalis, A ethmoidalis.

Ductus lachrymalis.

N. infraorbitalis, A infraorbitalis. IN. dentalis inferior, $A$. et $V$. \} dentales inferiores.

N. mentalis, A. mentalis. 
\$ 568. The capacity of a prepared skull may be obtained by filling it through the Fm. magnum with sand or fine shot, and then pouring the material into a graduate glass. If the material used in determining the capacity is fine enough to pass through the foramina, they must be plugged in some way.

The weight of the brain may be obtained approximately by reckoning the cubic centimeters of capacity as grams and adding 4 per cent. (Wyman, $\mathbf{7 6}$ ). Thus, if a cat's skull has a capacity of $25 \mathrm{cc}$, the brain of the same cat would weigh approximately 26 grams. Wyman's statement refers only to the human brain, but presumably the specific gravity of the cat's brain is nearly or quite identical with that of man.

$\S 565$. Obvious Structure of Bone.-In life the surface is covered with a dense layer of connective tissue, the periosteum. Fntad of this periosteum is a layer of compact bone. The intermediate part of all bones is, however, more or less loose in structure, something like a sponge, hence it is called spongy or cancellated bone. This is especially abundant toward the end of long bones and in the vertebral centra.

§ 566. Microscopic Structure.-A solid mass containing: A. Haversian canals, cylindrical channels shown as circles in cross section, as cylinders in longitudinal sections of long bones. These canals contain the blood vessels, as may be demonstrated by examining a finely injected cat's scapula (see Frey, A). They anastomose freely, and open either upon the ectal surface or within the medullary eanal.

B. Lacunce and canaliculi. These are the spaces occupied by the protoplasmic bone cells or corpuscles and their prolongations. They appear in outline like irregularly fusiform connective tissue corpuscles with many fine prolongations or branches. These branches anastomose with the branches of neighboring lacunæ, and sometimes open into an Haversian canal.

In transections of long bones the solid matter and lacunæ are seen to be arranged in more or less concentric lamellæ around the Haversian canals. 


\section{CHAPTER VI.}

\section{MYOLOGY-THE STUDY OF THE MUSCLES.}

GENERAL CONSIDERATIONS-FREQUENCY OF MUSCULAR VARTATIONS-EXPLANATION OF TECHNJCAL TERMS-APHORISMS FOR DISSECTORS-LIST OF INSTRUMENTS AND MATERLALS-FOW TO DBE DISSECTING INSTRUMENTS-PRACTICAL SUGGESTIONSCLIPPING THE H.IR-CUTTING THE SKIN-REMOVAL OF THE SKIN-NAMES AND SYNONYMS OF THE MUSCLES HEREIN DESCRIBED-DESCRIPTIONS OF FORTY MUSCLES OF THE OEPHALIC REGION OF THE BODY, WITH DIRECTIONS FOR THEIR DISEECTION-THE STIRUCTURE OF MUSCLE.

General References to Myotomy.-Bernard, A, 182-206 ; Chauveau (Fleming), 184186; Cleland, A, 1-7 ; Heath (Keen), A, Appendix ; Hodges, A ; Hyrtl, A, 59-67; Mojsisovics, A, 1-15; Straus-Durckheim, B, 130-162 ; Reeves, A.

\$ 567. Muscular Homologies.-The following works and papers deal with the general and special homologies of muscles; the first three embrace all Vertebrates and all regions of the body; the others refer mainly to the limbs of the Mammalia: Humphrey, E, 105-188; Meckel, A, V, VI ; Cuvier, A, I ; Quain, A, I, 185; Mivart, 1 ; Macallister, 9, 12, 19; Rolleston, 13 ; Krause, A ; Coues, 1, 36, 47 ; Wilder, 1, 4, $10,20$.

\$568. General Considerations.-For the reasons stated in $\$ 204$, Practical Anatomy always begins with the skeleton, including the bones and cartilages, the arthra (joints) and ligaments.

Of the soft parts, the muscles are most easily prepared, examined and preserved, and they serve, together with the bones, as landmarks for the recognition of the vessels and nerves; hence Myology naturally succeeds Osteology.

Yet the practical study of the muscles is not without its difficulties.

Upon the limbs, excepting the distal segments, the muscles are much more numerous than the bones. For example, in the arm and shoulder girdle, if we omit tbe manus, there are five bones, scapulı, clavicle, humerus, ulna and radius. Excluding those which arise from the bumerus and are inserted upon the manus, more than thirty distinct muscles are attached to these five bones.

The larger bones also are readily recognized, even when covered by soft parts; but two or more muscles in the same locality may have the same general forn and direction, so that their determination may involve a careful esamination of their attachments.

Hence, whereas most of the bones may be prepared in the sime way, the general rules for dissection must be modified and supplemented with respect to each muscle, in order that it may be exposed, dissected, examined and removed to the best advantage.

\$ 569. Just how explicit the directions stould be has not, apparently, been fully determined by anatomical teachers and writers. Some "Laboratory Directions" are so 
meager as to be, according to our experience, of no value whatsoever. Nowhere, in zootomical works, have we found them so complete as in the "Dissector's Guides" which are used in the Medical Schools. Yet even these, in our opinion, are not altogether suited to the needs of the beginner. They are not sufficiently full; all the parts in a given region are considered at once, a plan better adapted to the advanced student; finally, too little attention is given to typographical and paragraphic details which might facilitate the recognition of statements and reference to other parts of the work.

Without assuming to have decided correctly in this matter, we have acted upon the belief that dissection is a fine art, and by no means easy to acquire; that the beginner is liable to fall into grave errors as to manipulation, fact and interpretation; and that, upon the whole, it is better for him to follow even an imperfect method than none at all.

$\S 570$. Variation.-Another difficulty met with in the study of muscles is the frequency of variations and anomalies not only as to size and shape, but also as to connections, vascular and nervous supply, and even presence.

All standard works upon Human Anatomy record the existence of such variations, and it is probabls that the careful examination of any human subject would disclose one or more departures from the condition regarded as normal.

Notwithstanding the intrinsic probability that any other Mammals, at least the domesticated species, would vary in a similar manner, most dissectors of the lower animals seem to have assumed that what is true of one individual is true of the whole species, and the myological descriptions of Straus-Durckheim (A), Chauveau (A), Coues (47), and Kranse (A) rarely mention departures from rule. Yet no two anthropoid apes have been found to agree in all respects, as may appear from the published dissections of Duvernoy (100), Humphrey (10), Champneys (1), Wyman (47), Macalister (21, 41), Barnard (1), the senior author (1) and others, and Huxley has distinctly expressed $(\boldsymbol{A}, 410)$ the belief that "endless varieties will no doubt be met with by those who carry their inquiries farther" than by the dissection of single individuals of a species; see also the remark of Galton, $\boldsymbol{1}, 175$, note 30 .

The senior author has remarked upon the existence of individual variations among domesti sated dogs $(\mathbf{2 1}, 308)$, and we may add that no one of the scores of cats dissected by us or our students has failed to present some peculiarity of muscular arrangement.

The records of these variations have not as yet been put into shape for publication, and in the present descriptions it has seemed better to give, in most cases, only what seems to be the most usual structure. The student is reminded, however, that his very first dissection may disclose some feature hitherto unobserved.

\$ 571. Errors of Manipulation.-The beginner should bear in mind that nothing is more easy than to commit some error of manipulation-whether by a cut too many or a cut too few-which may greatly affect the appearance of the parts, and lead to very mistaken conclusions. As a rule, therefore, supposed anomalies should not be published until submitted to competent criticism, or carefully checked by the dissection of other individ. uals, or still better of the other half of the same.

In all cases, the student will do well to recall the adrice of Cuvier to a young medical student who ventured to tell him that he had discovered something very new and remarkable in a human body: Cuvier replied : "Go and anatomize an insect, the largest you can find; then reconsider your observation, and if it appear to be correct, I will believe you on your word." After making the dissection, the' student confessed that he bad been in error ; (Lee, A, 56).

As has been suggested by the senior author $(22,307)$, it is doubtful whether any dis* section by beginners should be published at all, excepting upon the approval of an experienced anatomist, after thorough examination. 
\$ 572. Errors of Interpretation.-When an author denies the existence of a part the presence of which is affirmed by other writers, the discrepancy may be due to either of five causes :-

1. Its absence is the rule, and its presence in the other cases was exceptional.

2. It was absent, though normally present.

3. It was present, but accidentally overlooked.

4. It was observed but not recognized, or was mistaken for some other part.

5. It was seen, but purposely ignored.

Of course the last named explanation is also the last to be entertained, but either of the other four contingencies is ljable to occur with even experienced dissectors and learned anatomists.

For example, the M. entopectoralis (Fig. 72), "pectoralis minor," is said by Straus-Durekheim (A, II, 336), and implied by Meckel (A, VI, 249), and Mivart (B, 145), to be wanting with the cat; its existence in any Carnivora is also denied by Cuvier (A, I, 370), and A. H. Young (1, 171). Owen, however, speaks (A, III, 50), of the "pectoralis minor of the dog," and its presence in that animal is admitted by Haughton (115) and Wood $(7,52)$, as it is in the cat by the senior author, who refers $(\mathbf{2 0}, 306)$ to it as found in all the Felidæ and Canidæ examined by him.

The muscle considered by the writers last named to represent the "pectoralis minor" of man is so large in most Carnivora that it was not recognized by the five writers first vamed as the representative of that rather insignificant muscle; in the bear and skunk, however, as stated by the senior author in the paper referred to, it is again smaller than the $M$. ectopectoralis.

In illustration of the second case, the M. supinator longus (Fig. 74) is said by Meckel (A, VI, 303), and Huxley (A, 355) to be wanting with the dog. Chauveau, however, affirms (A, 290) its presence in dogs of all breeds, and it has been repeatedly observed by the senior author. Hence we may conclude, provisionally at least, that it was exceptionally absent in the individuals dissected by Meckel and Huxley.

\section{THE TECHNICAL TERMS OF MYOLOGY.}

\section{§573. Musculus-Muscle.-The name for a mass of muscular} fibers. Such a muscle may or may not be a true muscular integer.

What constitutes a muscular integer has not been determined; as stated in the senior author's brief discussion of the subject $(1 \mathbf{0}, 63)$, the phrase " morphological integer" seems to have been first used with reference to the muscles by Coues $(1,223)$, but the general question is discussed, directly or indirectly, by Owen, Parker, Spencer, and more recently, Humphroy (E). In the present work, it has seemed best to us to recognize as separate muscles, or as distinct divisions of muscles, all the muscular masses whose origins and insertions are fairly constant and capable of definite description.

In applying separate names to the divisions of the human trapezius (Fig. 66, $\$ 607$ ), and deltoideus (Fig. 66, $\$ 674$ ), and yet treating the long and short heads of the coracoideus (Fig. 75, $\S 668$ ), as a single muscle, we are certainly open to the charge of inconsistency. In the present transitional state of opinion respecting muscular integers, entire consistency is hardly to be expected.

\$ 574. Muscular Groups. - It is sometimes convenient to speak of two or more muscles collectively as a group, as, e.g., the pectoralis group, the trapezius group, the triceps group. 
\$ 575. Subdivisions.-As has been well stated by Humphrey $(\mathbf{E}, 110)$, the longitudinal subdivision of a muscle may be either vertical or horizontal. For the sake of distinctness, we shall call the subdivisions of the former kind divisiones, and those of the latter lamince. For example, as has been remarked by the senior author (20, 306), the "M. ectopectoralis (Fig. 72) tends to separate into superimposed laminæ, while the entopectoralis tends to form fasciculi." In the former the "cleavage" is horizontal, in the latter vertical.

$\S 576$. Parts of a Muscle.-The essential and usually largest portion of a muscle is the mass of muscular fibers; this is called its body or belly.

Sometimes one-rarely if ever both-of the ends of a muscle is attached to bone directly or rather to its periosteum. This is the case with the humeral end of the $M$. brachialis (Fig. 74, $\S 692$ ), and the $M$. entotriceps, div. intermedia (Fig. 75, §686).

More often, however, there intervenes between the muscular portion and the bone a cord or sheet of white inelastic fibrous tissue, constituting the tendon. The attachment is then said to be tendinous, while in the former case it was muscular.

Tendous may be so short as to be hardly distinguishable, like the coracoid tendon of the $M$. coracoideus $(\$ 668$ ), or they may be longer than the muscular portion, like the humeral tendon of the same muscle (Fig. 75).

Sometimes, especially with thin flat muscles like the laminæ of the M. ectopectoralis (Fig. 72), the tendinous sheet may be so short as to be practically absent.

\$ 577. Attachment Lines and Areas.-Muscular attachments usually, and tendinous attachments sometimes, cover considerable areas (brachialis, Fig. 68); in other cases the attachment is along lines (entopectoralis, divisio caudalis, Fig. 69).

$\$ 578$. Origin and Insertion.-Of the two attachments of a muscle, one is called origin and the other insertion. Usually, but not always, the origin is from the more fixed part of the body, and the insertion is upon the more movable part.

\$579. Choice of Origin and Insertion.-With the membral muscles, one attachment is generally nearer the soma ( $\$ 54)$ or the proximal end of the limb, and this attachment is always called the origin. Thus the scapular attachments of the MM. biceps (Fig. 75) and subscapularis (Fig. 73) are the origins of those muscles; so 
also, the origins of the MM. entopectoralis (Fig. 72) and clavotrapezius (Fig. 66) are, respectively, their sternal and vertebral attachments.

But the question is less easy with some muscles which connect different parts of the soma with each other, or which extend between the head and the scapula, clavicle or sternum.

Without feeling sure as to what is best, we have adopted the following basis :-

As between the head and the neck or the trunk, the latter are more central and afford origin to the muscles of the head.

The sternum is part of the trunk, and sternal attachments are therefore origins.

The scapula and clavicle appertain to the arm rather than to the trunk, and attachments thereto, as compared with attachments to the head or neck or trunk, are insertions.

It will be seen that the acceptance of these rules entails some apparent contradictions: For example, the clavo-mastoideus (Fig. 67), like the clavo-trapezius (Fig. 66), arises from the skull and is inserted upon the clavicle; but the sterno-mastoideus (Fig. 67), like the splenius (Fig. 67), arises from the trunk and is inserted upon the skull.

Hence the origin of the clavo-mastoideus practically coincides with the insertion of the sterno-mastoideus. In man, where the clavicle joins the sternum, the two muscles are commonly described as one, under the name of sterno-cleido-mastoideus, and one part of the muscle therefore arises where the other is inserted, and vice versa.

\$580. The Determination of Muscular Homologies.-Of course the function of a muscle depends upon its insertion, but there is considerable difference of opinion as to whether the origin or the insertion is the better guide to the determination of its homology. We are disposed to assign greater morphical importance to the origin, according to the views of Barnard $(1,114)$. As to the value of vascular and nervous supply, see Cunningham (1) and Gadow (3).

§581. Fascia.-This is simply a thin sheet of the same kind of fibers as the tendon, but the fibers may present two kinds of arrangement. Sometimes the tendon itself is so thin as to be called a fascia, or it may be continued as a thin sheet upon one or the other face of the muscle; in both these cases, the fibers are nearly or quite parallel. The name fascia, however, is more often given to a sheet of fibers crossing one another in various directions, and forming a sheath or covering for a muscle or a group of muscles. A fascia is also called an aponeurosis, but the name is objectionable on account of both length and etymology.

$\$ 582$. Forms of Muscles.-The body of a muscle may be fusiform or spindle-shaped, like that of the M. biceps (Fig. 73); toniate (strap-shaped or ribbon-like), as with the occipito-scapularis (Fig. 67) ; fan-shaped like the subscapularis (Fig. 73); quadri- 
lateral like the acromio-trapezius, or triangular like the spino-trapezius (Fig. 66). There are other and less usual forms which will be indicated in special cases.

$\$ 583$. Designation of the Borders of Muscles.-Many of the muscles are thin and triangular or tæniate, so as to present sharply defined borders, in place of the more or less rounded aspects or surfaces of a fusiform muscle like the biceps.

Such a flat muscle may become twisted upon its axis in such a way as to change the relations of the borders to the body-planes. In these cases, for the sake of convenience, it will be considered that the muscle has the general direction which it had at its origin, although this may sometimes involve an apparent contradiction of the terms in which the insertion is described.

For example, the $M$. pecto-antebrachialis, dv. cephalica (Fig. 72), arises at the meson, and its borders are called cephatic and caudal throughout its whole length, notwithstanding the fact that the line of insertion upon the ulna has a proximo-distal direction, so that, as based thereon, the borders would be proximal and distal.

$\S 584$. Connect.-For the exposure of the ectal layer of muscles, certain areas of skin must be lifted. The lines of incision which circumscribe such areas are said to "connect" certain parts or points, usually some of the "landmarks" elsewhere (\$\$ 225-233) enumerated.

$\$ 585$. Girdle. -When the skin, especially of a limb, is divided by an incision encircling the part, the latter is said to be "girdled."

$\S 586$. Transect.-In order to examine fully the attachments of a muscle, it is usually desirable to divide it transversely and reflect the two ends in opposite directions. For the sake of brevity, this entire operation will be indicated by the use of the single word transect.

\section{APHORISMS FOR DISSECTORS.}

§587. 1. "Without skilful manipulation we can neither teach by demonstration facts which have been already discovered, nor hope to extend the limits of observation and experimental knowledge."-L. S. Beale, $\mathbf{A}$.

2. "A piece of true dissection ought to turn out an object of wonder and beauty."-Goodsir, A, I, 24.

3. "An anatomist therefore in these curious things had need to have a fine and dainty hand, and at command."-Crooke, A, 460. 
4. "The best workman uses the best tools."-Owen.

5. The value of instruments depends not upon their handles, their finish or their cost, but upon the adaptation of their size, form and temper to the work in hand.

6. Fingers are often the best forceps.

7. Handling and cutting are necessary evils.

8. Neglect of the knife may leave the truth concealed; its misuse may establish an error.

9. "Let the eye go before the hand, and the mind before the eye."-O. W. Holmes.

10. Fat is the anatomist's worst enemy.

11. "Drying is even worse than decomposing." -R. M. Hodges.

12. The skin makes the best wrapper.

13. There are two sides, but only one meson.

14. The bones are the guides to the muscles, the muscles to the vessels, and the vessels to the nerves.

15. The attachments of a muscle determine its homology and function; the thickness and length of its body indicate respectively its power and the distance through which it may contract.

16. The attachments of a muscle are often closely associated with those of others, but its body is usually distinct.

17. In dissecting muscles, the science consists in discriminating between fascia or tendon and mere connective tissue; the art, in removing the latter so as to leave the muscles distinct.

18. Dissection according to direction favors the acquisition of methods and the learning of names and specified relations; but the knowledge of parts is not complete until they have been approached and examined from all sides. See also $§ 122$.

\section{$\$ 588$. List of Instruments and Materials for the Dissection} of Muscles.-Arthrotome, Fig. 16, \& 135; blocks, § 137 ; coarse forceps, Fig. 18, 145 ; fine forceps, Fig. 20, $\$ 146$; scalpels, medium and Charrière, Fig. 23, 24, $\$ 155$; coarse scissors, curved flatwise, Fig. 25, § 156 ; hair scissors, § 158; towels, § 165 ; sharp tracer, Fig. $17, \S 166$; tray, $\S 167$; waste pail, $\S 195$; waste papers, $\S 172$; wetting bottle, Fig. $27, \& 170$.

\$ 589. The Material for Dissection.-A lean animal should be preferred; it should be divided by abdominal transection ( $\$ 234$ ); injected with alcohol ( $\$ 285)$, but not-for the first dissection-with plaster ; kept in 42-55 per cent. alcohol (\$286), and not allowed to dry. 


\section{HOW TO USE DISSECTING INSTRUMENTS.}

§ 590. Hyrtl complains $(\mathbf{A}, 62)$ that " some people hold the forceps like fire-tongs, and the scalpel like a cheese-knife." It is true that most anatomical instruments are for either grasping or cutting, but their proper and successful employment demands much more care and delicacy than is needed in ordinary household operations. The good whittler is not necessarily an expert dissector, and even the coarse scissors are to be handled very differently from shears.

The anatomist, like the surgeon-who is an anatomist and something more,-should have such command over his muscles and nerves that whatever instrument is in his hands becomes for the time being like a part of himself, an extension of his fingers, sharper, firmer and more slender, yet almost equally mobile and sensitive.

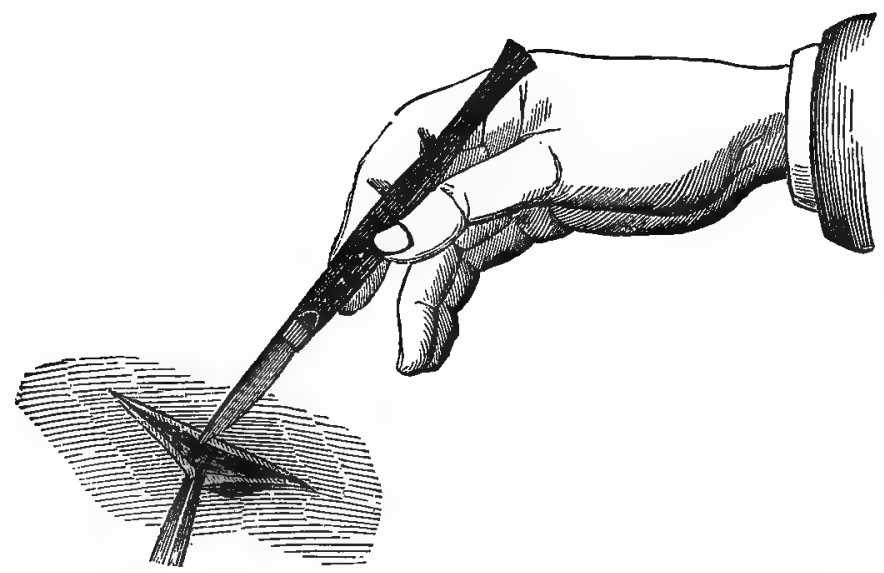

Fig. 63.- The Scalpet Heid as a Pen. (From Bernard).

$\S 591$. Use of the Scalpel ( $\$ 155)$.-The scalpel may be held in either of three general ways :-

A. Like a Pen (Fig. 63). - The edge is directed backward and downward, or forward and upward. This is for ordinary dissection.

B. Like a Carving-knife (Fig. 64).- The edge may be directed upward or downward. This is for the division of more resisting tissues.

C. Like a Violin-bow (Fig. 65).-The scalpel is held between the tip of the pollex on one side and the tips of the other digits upon the 
other. This is for long sweeping strokes, where the greatest freedom is desired.

Minor modifications of these three ways will be readily adopted as the dissector becomes familiar with the instrument.

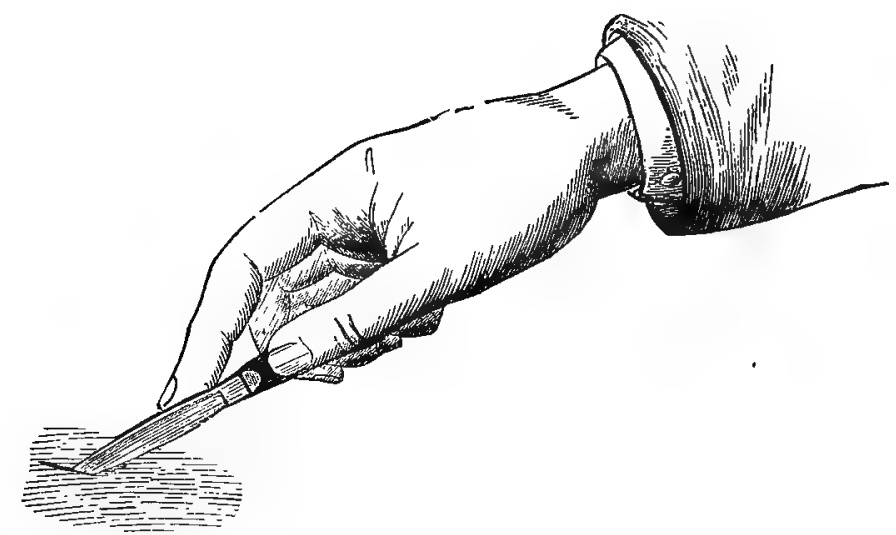

Fig. 64.-The Scaupel Held as a Carting-entfe. (From Bernard).

$\S 592$. Use of the Scissors ( $\$ 156)$.-Contrary to the more usual custom, dissecting scissors should be held with the pollex and medius. The index then serves both to steady the instrument and to

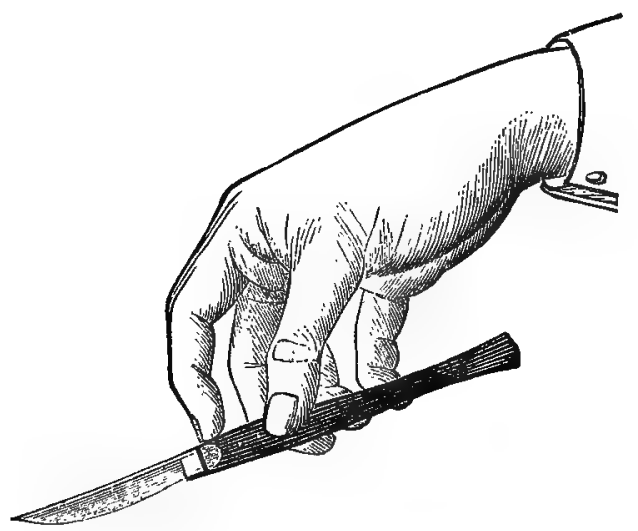

Fig. 65.-The Scatpel Held as a Violin-mow. (From Bernard).

aid the medius in its opposition to the more powerful pollex. In nearly all cases the points of curved scissors should be turned upward and away from the part under dissection. This precaution is es- 
pecially needful in the trimming of vessels and nerves and inflated hollow viscera (\$331).

$\S 593$. Use of the Forceps $(\S 145)$. - The forceps are commonly held between the pollex and the index. In long continued dissections the medius may be substituted for the index at intervals. The digits should be employed in place of the forceps when practicable, both as a relief from fatigue and to avoid crushing the tissues.

The forceps should be used upon muscles as little as possible, and vessels and nerves should be grasped by their sheath of connective tissue. For the separation of slender muscles and of vessels and nerves, the safest way sometimes is to insert the tip of the closed forceps and then allow the blades to separate gently.

$\S 594$. Use of the Tracer $(\$ 166)$. - The tracer is to be held like a pen or pencil. Its form permits a rotation on its axis, so that the point may have any desired direction.

The tracer should be more constantly in the hand than any other instrument. Scalpels and other sharp instruments should only be used when the tracer will not answer the purpose.

The tracer is also very useful in detecting the position of concealed hard parts, as ribs, cartilages and vertebræ. The point may be introduced deeply without impairing the condition of the parts for dissection, and the curvature enables one to lift upon it the ribs or cartilages so as to count them more accurately.

$\S 595$. Use of the Arthrotome ( $\$ 135)$.-As its name implies, the distinctive use of this instrument is for the division of joints and other rough operations which might injure the more delicate edge of the scalpels. Yet the student should accustom himself to accurate and careful manipulation, and endeavor to separate the contiguous bones at an arthron without injuring the cartilages. He should try to feel with the point of the instrument.

\section{PRACTICAL SUGGESTIONS FOR DISSECTORS.}

$\S 596$. 1. Select a lean animal for all anatomical purposes, and especially for the dissection of the muscles, vessels and nerves. The directions for killing are given in $\$ 192$.

2. Take the precautions for cleanliness which are described in $\$ 199$.

3. Remove superfluous parts of the animal, the tail in some cases ( $\$ 243$ ), the caudal or cephalic region of the body, according to the 
directions for abdominal and thoracic transection which are given in $\$ \S 237,242$.

4. See that all the instruments, materials and books for dissection, reference and record are at hand and in order before getting the subject or beginning to work.

5. Refer constantly to a skeleton, or to the bones especially concerned, or to accurate figures.

6. When possible, the head of the subject should be toward the left.

7. When a part from which or toward which an incision is to be carried lies upon the meson, the incision should extend $1-2 \mathrm{~cm}$. beyond the meson. This permits the flap of skin to be reflected across the meson to that extent. If, however, this flap of skin is to be removed, the incision removing it should run parallel with the meson, and upon the side under examination.

Objections to Mesal Incisions.-Both the ectal and ental aspects of the skin present special features at some mesal points, and several muscles besides those of the skin itself arise at the meson, and are liable to injury by a mesal incision. There are two sides, presumably similar, but there is only one meson.

8. Incisions should be as long as may be consistently with the shape and structure of the parts, and the dissector's knowledge of them.

9. When important or delicate parts are liable to be injured, the cuts should be shorter and more carefully made.

10. Vessels and nerves should be dissected from the center toward the periphery, so as to avoid the risk of missing, cutting or tearing the branches.

11. Vessels injected with plaster should be divided with the arthrotome or the bone scissors.

12. In place of the block, especially while the separated arm is under dissection, a folded wet towel may be used; this permits a kind of bed to be made which keeps the arm in place.

13. "To put any group of muscles on the stretch, put the parts concerned in the position into which they would be brought by their antagonistic muscles. For example, to put the flexors of the manus on the stretch, put the manus in a state of extension, and vice versa." - Heath (Keen), $\mathbf{A}, 16$.

14. Study the actions of a muscle by pulling it in the line it naturally occupies. Note the difference between a direct and an indirect action. (Wilder, 4.) 
15. As a rule, muscles must be divided and reflected before the attachments can be fully determined. The attachments are usually more distinct upon the ental aspect.

16. "When several similar muscles of a group-as those upon the antebrachium-are to be transected, cut them at different levels, so as the more easily to match their proximal and distal parts."Heath (Keen), A, 16.

17. The borders of a thin muscle should be grasped and slightly raised, first with the forceps and then with the fingers. If the other border is accessible, it should be treated in the same way, and then the entire width at about the middle raised to permit the passage of a scalpel.

18. In transecting a wide muscle, cut one border, then lift it, keeping the sides of the cut separate, and cut a little deeper, applying the scalpel to the edge of the muscle.

19. Avoid cutting muscles at their attachments. If it is desirable to remove part of a large muscle, leave a small piece of the body attached to each tendon. If necessary-for special reasons-to remove an entire muscle, insert the edge of the arthrotome in the angle formed by the attached ends and the bones.

20. Parts under dissection should be wet occasionally with a mixture of water, glycerin and clove-oil ( $\$ 170)$.

21. Parts which have been exposed, but are no longer under actual dissection, should be covered with skin or rubber-sheeting, or with a bit of cloth wet with the glycerin mixture; and a dry towel should be laijd over all.

22. "Put all fragments on a piece of paper."-Hodges, $\mathbf{A}$.

597. Avoid especially the following: drying, tailing, pecking. The prevention of drying has been already considered.

Tailing is the making of a shallow cut at the beginning or the end of an incision, It is especially apt to occur with beginners, and while dividing the skin. To avoid it, hold the point of the scalpel perpendicularly to the surface at both the beginning and the end of the incision.

Pecking.-We use this homely word to designate one of the most common and most pernicious faults of anatomical beginners, the habit of aimlessly poking and pinching the parts, especially while showing them to the teacher or demonstrator. It reminds the observer of nothing so much as the dabbing and pecking which hens inflict upon a piece of meat. The student should 
bear in mind that a single false cut, and even a pinch in the wrong place, may mar his work beyond repair; he should exercise constant self-control, and never touch the specimen excepting for a definite and sufficient reason.

Pecking is only one of several forms of what may be called anatomical Philistinism; a lack of appreciation of delicacy, whether in structure or in methods of manipulation.

$\S$ 598. Clipping the Hair.-Unless the skin is to be preserved, or there is some other objection, the hair should be removed from a specimen which is to be preserved in alcohol and dissected at intervals.

If the hair is allowed to remain, it interferes with the accuracy of dermal incisions, and with the ease of making them ; it is apt to become detached and disfigure the dissections : finally, unless considerable time is spent in squeezing out the alcohol when the specimen is removed from the jar and the water when it is returned thereto, the hair causes both a waste and a weakening of the alcohol in which the specimen is preserved.

Use the hair scissors ( $\$ 158$ ) or a pair of ordinary scissors with the points blunted. The hair is more easily cut against its inclination, that is, with the scissor points directed cephalad upon the soma and proximad upon the limbs. After abdominal transection, begin at the cut border of the skin.

Clipping is facilitated by wetting the tips of the hair with a sponge only moderately full of water or weak alcohol. Cut close to the skin. The operation usually occupies about an hour. Do not put the removed hair into the sink. Place it in the waste-pail, to be buried.

$\S 599$. Cutting the Skin.-If the hair remains, wet it with 15 per cent. glycerin, or dilute alcohol, or water, along the line of the proposed incision. Then, with a small comb, or the tracer, or the handle of a scalpel, part the hair evenly along the same line so as to expose the skin clearly (\$354). If the hair has been removed, indicate the line of incision by a scratch made with the tracer or the point of the scalpel.

Place the tips of the left index and medius, one upon each side of this line, at the cut border of the skin, and divaricate them so as to stretch the intervening skin.

Grasp the scalpel like a pen, at an angle of $45^{\circ}$, and divide the skin by a single steady stroke as far as the tension exists.

At the beginning and end of the stroke, make the scalpel nearly perpendicular so as to avoid tailing ( $\$ 597$ ). 
Unless one is quite familiar with both the locality and the art of dissecting, this first incision should merely divide the skin proper. The borders may be still farther divaricated, and a similar incision made through the connective tissue and fat, and in some cases the dermal muscle ( $\S 629)$, until the darker red and closer texture shows that the ordinary skeletal muscles have been reached.

With lean animals this second incision will be very shallow, but in some cases the fat forms thick layers between the dermal muscles and the skin and deeper muscles. On the cheeks of old males, and sometimes on other regions, the skin and connective tissue are so thick as to puzzle the beginner.

Shift the tips of the index and medius, and repeat the operation to the end of the line. The separate strokes should join each other accurately, so that the entire incision is straight and smooth-edged.

After the skin is divided, the subcutaneous fat and connective tissue may usually be cut to the proper depth by a single long steady stroke.

$\S 600$. Removing the Skin.-The edge of the area of skin to be removed, preferably at the angle formed by two incisions, should be grasped, first by the forceps and then by the fingers, and lifted so that the scalpel may be applied to the connective tissue by which it and the fat are held loosely to the deeper muscles.

Excepting in the case of some rare form (which should not be dissected by a beginner), the skin should be kept well upon the stretch, and the edge of the scalpel should be applied against the tissues to be exposed, following the direction of the muscular fibers. The object is to remove with the skin all the subcutaneous fat, connective tissue and ectal fascia, so as to expose at once and fully the surface of the muscles, etc., to be examined.

This, the anatomical method of removing the skin, is more diffcult than the "flaying" of the butcher or the "skinning" of the taxidermist. Both of these desire the skin free of fat and connective tissue, and therefore keep the edge of the knife turned toward it. The taxidermist must avoid stretching, but this is easier than to follow strictly the above method. The beginner will usually be tempted to get the skin off in the easiest and quickest way, which is that of the butcher; but he then is obliged practically to repeat the operation for the removal of the tissues which should have been lifted with the skin.

$\S 601$. Rigor Mortis.-The spontaneous stiffening of the mus- 
cles which supervenes soon after death renders dissection difficult. The condition usually passes off within a few hours, and may be speedily overcome by immersing the animal for 40-60 minutes in water at about $35^{\circ} \mathrm{C}$. $\left(95^{\circ} \mathrm{F}\right.$.).

\section{DESCRIPTIONS OF CERTAIN MUSCLES.}

$\S 602$. Limitation.-For the reasons stated in $\S 128$, the myological portion of this work directly relates to only about one fifth of the whole number of muscles which have been enumerated in the cat. As with the bones, however, ( $\$ 368$ ), the student is advised to pursue the subject further, making original drawings and descriptions of at least one muscle in another part of the body. Whether it can be at once identified with some muscle in man is of less importance, so far as the training of the pupil is concerned, than the accurate determination of its connections and other characters.

$\S 603$. The Method here Followed.-With few exceptions, each of the forty muscles here considered is described under the following heads :-

1. Synonymy. - We have given the names for the same muscle employed by StransDurckheim and Mivart, and the names for what appears to us to be the homologous muscle in man and in the horse. The anthropotomical names are taken from "Gray" and "Quain," and the hippotomical ones from the French and the English (Fleming's) editions of "Chauveau." In some cases, we have been unable to satisfy ourselves as to the homology. We should have been glad to include references to the works of Leyh (A) and Gurlt (A) upon the horse, to Krause's Anatomy of the rabbit (A), and to Coues's paper on the opossum (47). The authors' names are indicated by the initial letters only.

2. Figures.-Here are enumerated the figures wherein the muscle appears.

3. General Description. - This is a brief indication of the general form and connections.

4. Posture.-We have indicated the position of the body or limb which seems most farorable to the examination of the muscle.

5. Exposure.-Here are given directions for bringing the muscle into view by the removal of the skin or overlying parts.

6. Dissection.-This includes the operations by which the borders of the muscle are to be raised, its body transected and the ends reflected so as to display the attachments.

7 and 8. Origin and insertion.-Here are given more detailed descriptions of the two attachments.

In addition to the above, a complete account of each muscle should embrace its nervous and vascular supply, its actions, direct, indirect and associated, and its variations.

Errors and Defects.-During the past four years the descriptions and directions here given have been employed by the students in the anatomical laboratory of Cornell University. Inasmuch, however, as annual modifications have been found necessary, we cannot hope that their present form is altogether what it should be, and we shall be very grateful for the correction of errors and the pointing out of defects.

We desire here to repeat the expression of our sense of obligation to Prof. T. B. Stowell, who has kindly followed the descriptions and directions scalpel in hand, and bas given us the benefit of many valuable suggestions and criticisms as to both the facts and the method of stating them, and as to the extent of variation in different individuals.

A former special student, Dr. E. M. Howard, generously placed at our disposal for comparison his manuscript descriptions of the muscles of the cat.

$\$ 604$. The Names of the Muscles.-The number, extent and nature of the changes proposed in the names of the muscles are set forth in the Table on p. 207.

In that Table the names in the left hand column are those adopted in the present work; 
those in the right hand column are Latinized from those employed by Straus-Durckheim ; those in the middle column occur in standard worlss upon Human Anatomy, or in the writings of anthropotomists. In the last column are words or abbreviations indicating the changes which have been made in the names employed by Straus-Durckheim (S.-D.) or anthropotomists (anth.): thus, abbrev. = abbreviated ; tr'l'd = translated ; unif. = unified.

$\S 605$. TABLE.

\begin{tabular}{|c|c|c|c|c|}
\hline Here Adopted. & Fig. & Anthropotomy. & Strains-Durckheim. & Changes. \\
\hline Spino-trapezius... . . & 66 & Trapezius (in part). & Dorso-cucullaris .... & S.-D., unif. \& tr'l'd. \\
\hline Acromio-tra pezius . . & 66 & & Acromio-cúcullarís. & S.-D., translated. \\
\hline Clavo-trapezius.... & 66 & " $\quad$ " $\quad \ldots$ & Clavo-cucullaris.... & S.-D., transiated. \\
\hline Occipito-scapularís... & 67 & Rhomboideus capitis & Occipito-scapularis. & S.-D. \\
\hline Rhomboideus ..... & 67 & Rhomboideus major. & Rhomboideus ..... & S.-D. anth., abbrev. \\
\hline Sterno-mastoideus .. & 67 & $\left\{\begin{array}{c}\text { Sterno mastoide- } \\
\text { us (in part)... }\end{array}\right\}$ & Sterno-mastoideus. . & S. D. \& anth. \\
\hline Clavo-mastoideus... & 67 & $\left\{\begin{array}{r}\text { Sterno-mastoide- } \\
\text { us (in part)... }\end{array}\right\}$ & Cleido mastoideus. . & S. D., translated. \\
\hline Levator clavicu & 67 & Levator claviculæ.. & Transv.-scapularis. & Anth. \\
\hline lis... & 66 & & Dermo-humeralis... & S.-D. \\
\hline Latissimus & 67 & Latissimus dorsi.... & Latissimus dorsi.... & S. D.; anth., abbrev. \\
\hline Clavo-deltoideus.... & 66 & Deltoideus (in part). & Delto-clavicularis... & S.-D, transpo \\
\hline Pecto-antebrachialis. & 72 & & Pecto-antebrachialis & S. D. \\
\hline Ectopectoralis..... & 72 & Pectoralis major... & $\begin{array}{l}\text { Large pectoral..... } \\
\text { Grand pect. (in }\end{array}$ & Generalized. \\
\hline Entopectoralis.. & 72 & Pectoralis minor... & $\left\{\begin{array}{c}\text { part) and sterno } \\
\text { trochiterianus.. }\end{array}\right\}$ & Generalized. \\
\hline Xiphi-humeralis.... & 72 & & Graud pect. (in part). & New. \\
\hline Serratus magnus. $\because$ & 73 & Serratus magnus. & Serr. mag. (in part). & Anth. and S.-D. \\
\hline $\left.\begin{array}{r}\text { Levator anguli } \\
\text { scapulæ....... }\end{array}\right\}$ & 73 & Lev. ang. sca & $\begin{array}{lllll} & 4 & & 4 & \end{array}$ & Anth. \\
\hline Coracoideus ....... & 75 & Coracoideus. & Coraco-brachialis... & Anth., abbrev.; S.-D. \\
\hline Subscapularis ..... & 73 & Subs & Subscapularis...... & Anth. \& S.-D. \\
\hline aspinatus...... & 67 & Supraspinatus..... & Supraspinatus ...... & Anth. \& S.-D. \\
\hline Spino-deltoideus. . & 67 & Deltoideus (in part). & Delto spinalis....... & S.-D., transposed. \\
\hline Acromio-deltoideus.. & 67 & " & $\begin{array}{l}\text { Delto-acromialis... } \\
\text { Infraspinatus ..... }\end{array}$ & $\begin{array}{l}\text { S.-D., transposed. } \\
\text { Anth. \& S.-D. }\end{array}$ \\
\hline $\begin{array}{l}\text { Infraspinatus. . . . } \\
\text { Micostalis......... }\end{array}$ & $\begin{array}{l}67 \\
74\end{array}$ & $\begin{array}{l}\text { Infraspinatus....... } \\
\text { Teres minor....... }\end{array}$ & $\begin{array}{l}\text { Infraspinatus . . } \\
\text { Micostalis....... }\end{array}$ & S.-D. \\
\hline Tere & 75 & Teres major........ & $\begin{array}{l}\text { Micostalis. . . . . . . . . } \\
\text { Teres............. }\end{array}$ & S.-D.; anth., abbrev. \\
\hline Epitrochleari & 75 & Dorso-epitrochlearis & Triceps (in part)... & Anth., abbrev. \\
\hline Meditriceps.... & 74 & Triceps (in part).... & Triceps medius..... & S.-D., abbrev. \\
\hline Ectotriceps..... & 74 & "6 " & Triceps externus... & Generaliz \\
\hline Entotriceps . & 75 & $\left\{\begin{array}{r}\text { Triceps (in part) } \\
\text { and anconeus... }\end{array}\right.$ & $\left\{\begin{array}{c}\text { Tri. int. (In part) } \\
\text { and anconeus. }\end{array}\right\}$ & S.-D., abbrev. \\
\hline Supinator longus. & 74 & Supinator longus... & Supinator longus... & Anth. \& S -D. \\
\hline s......... & 74 & Biceps .......... . & Biceps . . . ....... & Anth. \& S.-D. \\
\hline $\begin{array}{l}\text { Brachialis. . . . . } \\
\text { Extensor }\end{array}$ & 74 & Brachialis anticus... & Brachialis ..... & S.-D.; anth., abbrev. \\
\hline $\left.\begin{array}{l}\text { Extensor (carpi) } \\
\text { radialis longior. }\end{array}\right\}$ & 75 & $\left\{\begin{array}{c}\text { Ex. carpi rad. } \\
\text { longior } \ldots . . .\end{array}\right\}$ & Radialis primus... & Anth., abbrev. \\
\hline $\left.\begin{array}{c}\text { Extensor (carpi) } \\
\text { radialis brevior. }\end{array}\right\}$ & 75 & $\left\{\begin{array}{r}\text { Ex. } \quad \text { carpi rad. } \\
\text { brevior....... }\end{array}\right\}$ & Radialis secundus... & Anth, abbrev. \\
\hline $\begin{array}{l}\text { Extensor communis. } \\
\text { Extensor minimi ... }\end{array}$ & 74 & Ex. digitorum com. . & Ex. dig. communis. & Anth., abbrev. \\
\hline $\begin{array}{l}\text { Extensor minimi ... } \\
\text { Extensor ulnaris ... }\end{array}$ & 74 & Ex. minimi digiti $\cdots$ & Ex proprius minimi & Anth, abbrev. \\
\hline $\begin{array}{l}\text { Extensor ulnaris . . } \\
\text { Indicator ......... }\end{array}$ & 74 & Ex. carpi ulnaris.... & Cubitalis...... & $\begin{array}{l}\text { Anth., abbrev. } \\
\text { S.D. }\end{array}$ \\
\hline Indicator ..... & $\ddot{70}$ & $\begin{array}{l}\text { Extensor indicis.... } \\
\text { Pr. radii teres...... }\end{array}$ & $\begin{array}{l}\text { Indicator .......... } \\
\text { Pronator teres...... }\end{array}$ & S.-D. S. -D.; anth., abbrev. \\
\hline Flexor radialis .... & 75 & Flx. carpi radialis. . & Cercialis.. . . . . . & Anth., abbrev. \\
\hline
\end{tabular}


Where more than one name is used in Human Anatomy, the shortest is here given; the coracoideus, for example, is often called coraco-brachialis, and the sterno-mastoideus, sterno-cleido-mastoideus.

The occipito-scapularis, levator clavioulce and dorso-epitrochlearis occur in man only as anomalies, and have received names in addition to those here selected.

The names employed by Straus-Durckheim have been put into their classical and technical form, excepting in the case of the "large pectoral " and "grand pectoral." It is probable that one cause of the slight use made of the names of this eminent anatomist is the fact that he chose to publish them in the vernacular form.

\$606. The Sources of the Names Here Used.-Of the names of the 40 muscles here described, seven are employed both by Straus-Durckheim and in the standard works upon Human Anatomy; these are: sterno-mastoideus, serratus magnus, subscapularis, supraspinatus, infraspinatus, biceps, and supinator longus.

The following three names have been applied in Human Anatomy, but are not used by Straus-Durckheim : levator clavicula, levator anguli scapulc, and coracoideus.

The following nine names are used by Straus-Durckheim, but not by anthropotomists : occipito-scapularis, rhomboideus, micostalis, teres, brachialis, indicator, pronator teres, dermohumeralis, and pecto antebrachialis. The last two refer to muscles which do not exist in man, and four of the others are but slight modifications of the anthropotomical names.

The following eight names are abbreviations of the anthropotomical names: latissimus, epitrochlearis, extensor radialis longior, ex. rad. brevior, ex. communis, ex. ulnaris, ex. minimi, and flexor radialis.

The following seven names are translations or transpositions of those used by StrausDurckheim : acromio-trapezius, clavo-trapezius, spino-deltoideus, acromio-deltoideus, clavodeltoideus, clavo-mastoideus, and meditriceps. As to the hybrid nature of some of these, terms, see $\$ 53$.

The following four names are the names in common use modified after the analogy of the now almost universally adopted ectoglutaus : ectopectoralis, entopectoralis, ectotriceps, entotriceps.

Two names remain to be accounted for. One of these, spino-trapezius, was substituted for Straus-Durckhein's dorso-cucullaris for the sake of uniformity with respect to the other divisions of the human trapezius, and the correlative division of the deltoideus.

This leaves us responsible for but one entirely new name, xiphi-humeralis. While the muscle so designated seems to us sufficiently distinct to demand a separate appellation, we are not particularly pleased with the name, and stand ready either to accept a shorter one or to regard the muscle as only a division of the ectopectoralis or entopectoralis when the proper evidence is forthcoming.

\section{THE TRAPEZIUS GROUP.}

\& 607. General Remark.-The human $M$. trapezius, a single muscle, seems to be represented in the cat by three nearly distinct muscles, which are here called-beginning with the most caudal-spino-trapezius, acromio-trapezius, and clavo-trapezius. They extend from the cervical and thoracic dorsimeson to the scapula and clavicle.

The names of all the muscles are in italics. To avoid frequent repetitions, the capital $M$, the initial of Musculus, will be prefixed only when otherwise there might be some risk of misunderstanding.

$\S$ 608. Explanation of Fig. 66.-The left ectal skeletal muscles of the neck and shoulder. 
Preparation.-A subtriangular flap of skin was lifted as directed in $\S 610$, together with the dermal muscles connected therewith. The flap was reflected dorsad, but is omitted from the figure; the reflected dorsal end of the dermal muscle called supra-cervicocutaneus, however, is shown. Both cut edges of the skin were lifted and retracted or reflected slightly.

The dermo-humeralis ( $\$ 629$ ) was mostly removed with the skin, but its brachial end appears just dorsad of the elbow. The fat and connective tissue have been removed so as to leave the borders of the muscles more distinct. From the hiatus trapezii, just dorsad of the convexity of the shoulder, the fat has been removed so as to expose the lymphatic gland (Gl. lymphatica), and the hiatus itself was extended dorsad so as to expose the ventral or lateral margin of the occipito-scapularis.

In this and the other descriptions of the myological figures, the parts are enumerated under three heads : bones, etc. ; muscles ; vessels, nerves, glands, etc.

Bones.-Clavicula-Collar bone (\$ 42\%). - In this figure the bone itself does not appear, but the position of its mesal or sternal end is indicated by the dotted line from the word clavicula; see rhaphé.

Mesoscapula-Spine of the scapula $(\$ 390)$. - The position of this ridge of the scapula, intervening between the $M M$. acromio-trapezius and spino-deltoideus, is approximately indicated by the name. It is more distinetly shown in Fig. 67.

Metacromion ( $\$ 396$ ). - This process of the glenoid end of the mesoscapula may be felt through the muscles at a pint corresponding with the begiuning of its name.

Frascia.-This strip of fascia-hardly deserving the name of ligament-passes from the lateral or scapular end of the clavicle to the surface of the $M$. supraspinatus, as better shown in Fig. 67.

Rhaphê (trapezio-deltoidea) ( $\$ 616)$.-This line or seam of connective tissue, between the $M$. clavo-deltoideus and the $M M$. clavo-trapeaius and clavo-mastoideus (Fig. 67), coincides nearly with the position of the clavicle. It is usually more distinct upon the ental aspect, and is somewhat exaggerated in the figure. The word is sometimes spelled raphe.

Spina neurales vertebr. (vertebrarum) thoraci. (thoracicorum),-The third and thirteenth thoracic (" dorsal") neural spines (Fig. 30). -The spines themselves do not appear, but the numbers 9 and 13 indicate the positions of the third and the last of the series.

Muscles.-In the figure the name of each muscle is preceded by $M$, the initial of Musculus.

Acromio-deltoideus - The acromial portion of the deltoid ( $\$ 670)$. - This is the intermediate one of the three muscles which, in the cat, seem to represent the single deltoid musele of man. It and the spino-deltoideus are inserted, like the human deltoid, upon the humerus; but the third portion, the clavo-deltoideus (the "delto-claviculaire" of Straus-Durckheim) is associated with the brachialis and inserted upon the uina.

Acromio-tropezius-The acromial portion of the human trapezius ( $\$ 613)$. - This is the intermediate one of the three muscles which, in the cat, appear to us to represent the human trapezius $(\$ 607)$. In the figure the name is written obliquely across the scapuli $\mathrm{c}$ end of the muscle; the word tendon, near its vertebral end, indicates the imperfectly 
defined tendon of origin ( $\S 614)$. Upon the muscle are also written the names $M$. occipito. scapularis, hiatus trapezii, and Gl. Iymphatica.

Cervico-auricularis $(\$ 615)$.-.This muscle of the ear is shown at the dorsimeson between the ear and the M. supra.cervico-cutaneus; its name is omitted.

Clavo-deltoideus (\$ $63 \%$ ). - See also acronio-deltoideus.

Olavo-trapezius $(\$ 615)$.-See also acromio-trapezius.

Dermo-humeralis ( $\$ 629$ ).-Most of this dermal muscle was removed with the skin ; the name is written across the brachial end which passes between the $M M$. triceps and latissimus to be attached to the latter and the bicipital arch.

Latissimus ( $\$ 635$ ). - This is more commonly called latissimus dorsi. Its caudal portion is covered by the skin; its dorso-cephalic angle is overlapped by the spino-trapezius, but exposed in Fig. $6 r$.

Levator clavicula ( $\$ 627)$.- The scapular end is seen to emerge from entad of the clavotrapezius, to partljo overlap the acromio-trapezius and to be inserted upon the imperfectly defined metacromion rather than upon the clavicle, as might be supposed from the name.

Occipito-scapularis ( $\$ 617$ ). -This is fully shown in Fig. 67. Here, its lateral border appears at tue dorsal end of the hiatus trapezii which has been enlarged so as to show the position of the muscle.

Spino-deltoideus (\$ 674).--See also the acromio-deltoideus.

Spino-trapezius (\$ 611).-See the acromio-trapezius.

Sterno-mastoideus (\$ 622).-Part of this shows darkly between the skin of the neck aud the clavo-trapezius. It is more fully shown in Fig. 67 and 72.

Supra-cervico-cutrneus ( $\$ 615$ ).-Most of this cervical dermal muscle was lifted with the skin, and only its reflected dorsal end is shown.

Teres ( $\$ 680$ ). - This appears in the interval between the spino-trapezius, spino-deltoideus, latissimus and triceps. By inadvertence, the name is written with the addition of the qualifying adjective major; since the muscle more often called teres minor is here designated as micostalis, there seems to be no need of a compound term for the muscle in question.

Triceps.-The name is written across the two largest portions of the muscle commonly known as triceps in mav. The $M$. is upon the scapular head, here called meditriceps $(\$ 682)$, and most of the rest of the name is upon the part here called ectotriceps $(\$ 684)$; the surface of the latter presents a superficial furrow.

Other Parts.-Gl. (glandula lymphatica). - A somewhat large lymphatic gland embedded in the fat which occupies the hiatus trapezii.

Gl. (glandula) parotis-The parotid salivary glaud (Fig. 87).-The candal border of this usually pale gland is exposed by slightly cephaloducting the occipito-presternal edge of sizin.

Gl. (glundula) submaxillaris-The submaxillary salivary gland (Fig. 87).-This appears a little ventrad of the $G l$. parotis, and is usually of a deeper color.

Hiatus trapezii (\$ 613).-This is an elongated lozenge-shaped interval between the cephalic margin of the $M$. acromio-trapezius and the caudal and dorso-caudal margins respectively of the $M M$. clavo-trapezius and lecator clavicula. In reality, tbe very open angle formed by the intersection of these two borders is near the middle of the length of the hiatus, but in the preparation the margins of the $M M$. clavo-trapezius and acromiotrapezius have been artificially separated a little farther dorsad.

$V$. (vena) jugularis-The jugular vein (Fjg. 101).-This is exposed between the $M$. clavo-trapezius and the margin of the skin, where it obliquely crosses the $M$. sterno-mastoideus.

$\S 609$. Exposure.-It is usually more convenient to expose the 
entire trapezius group by lifting a single large flap of skin. This flap, however, may be divided afterward so that one or two of the muscles may be covered while the other is under examination. In so doing, the skin is more easily divided ento-ectad (from within outward).

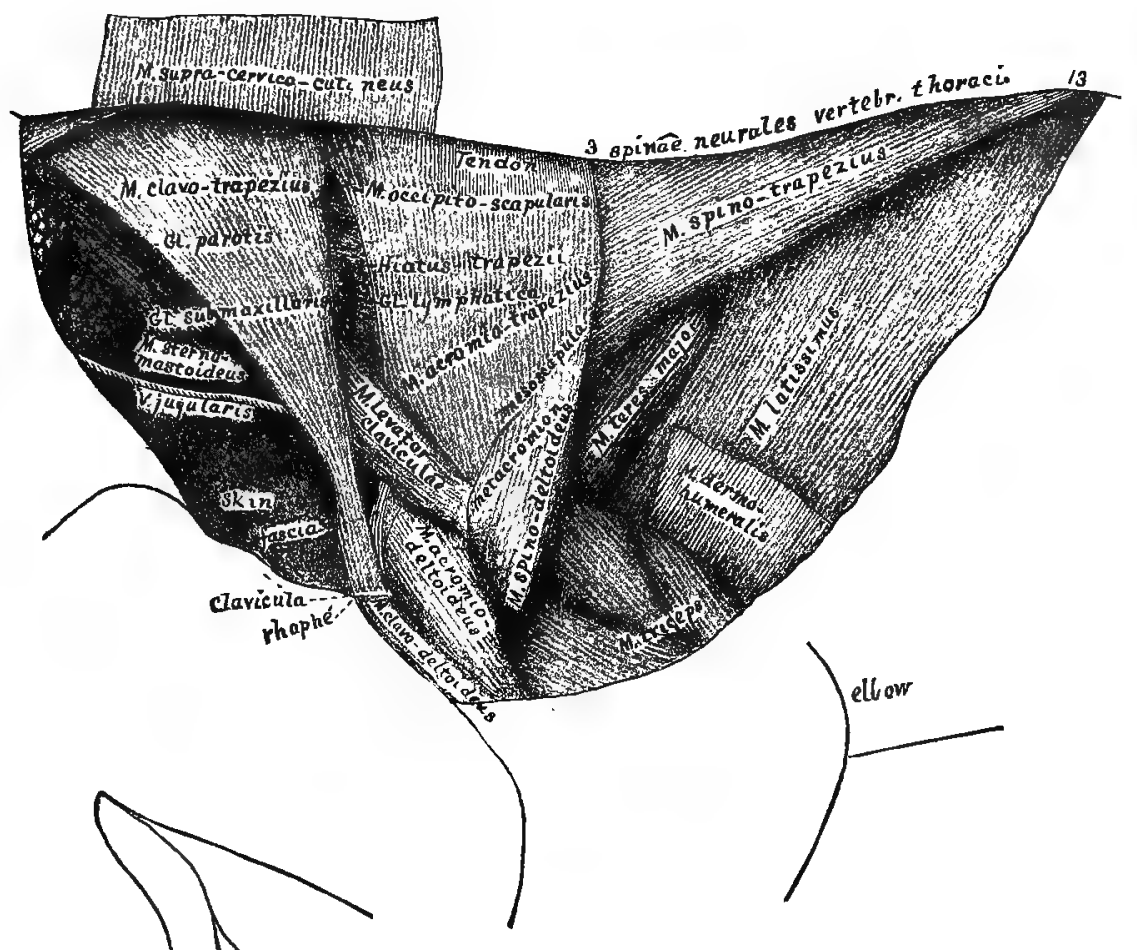

Fig. 66.-The Ectal Skeletal Muscues of the Left Shoulder and Neck, after removal of tee DermaI MUscles. (The lett side of the head is shown in outline below in order to facilitate the student's recognition of the regions in this his first systematic dissection.) 
$\$ 610$. Caution.-In making all incisions through the skin of the cat, it is necessary, in addition to the general directions in $\$ \mathbf{5 9 9}$, to keep in mind the presence of the $M$. dermo-humeralis $(\$ 629)$ and other muscles constituting the panniculus carnosus, a thin muscular layer between the skin and the proper skeletal muscles.

The dermal muscle is usually to be divided and lifted with the skin. With fleshy animals, the risk of cutting at the same time the underlying skeletal muscles is obviated by the intervention of a layer of fat which should also be removed with the skin $(\$ 600)$. With lean animals, the two sets of muscles may usually be distinguished from the thinness and paleness of the dermal layer, and from the fact that it is moved when the skin is pulled in.any direction.

Connect $(\$ 58 x)$ the prosternum (Fig. 30 and $49, \S 228$ ), with the thirteenth thoracic neural spine (Fig. 30 and $66, \S 227$ ), and with a point $1 \mathrm{~cm}$. cephalad of the crista lambdoidalis (Fig. 56, $\$ 226$ ).

The occipito-presternal incision should have a slight caudal convexity so as to skirt the base of the ear. The vertebro-presternal incision should cross the brachium at about the junction of its first and second fourths; this incision may be commenced at the middle of its length, and be carried thence in both directions. Lift the flap at the presternal angle, and remove with the skin the fat, connective tissue and dermal muscles. Reflect it across the dorsimeson.

\section{SPINO-TRAPEZIUS.}

§ 611. Synonymy.-The caudal part of the human trapezius, Q., A, 1, 187; G., A, 373 ; dorso-cucullaire, S. D., A, II, 334 ; portion dorsale du tratize, Ch., A, Fig. 90, " 1 ," 216 ; dorsal trapezius, Ch. (F1.), A, 203 ; hinder portion of the trapezius, Miv., B, 137.

Figures.-Ectal aspect $(66)$; insertion $(67,44)$; transection $(99,100)$.

General Description.-An elongated triangle, from the thoracic dorsimeson to the mesoscapula and the surface of the MM. supraspinatus and infraspinatus.

Dissection.-The ventro-caudal border will appear as a slightly raised line nearly parallel with the presterno-vertebral margin of the skin. In recent specimens the color of the muscle is usually a brighter red than that of the subjacent $M$. Latissimus.

Lift the border near its middle, and trace it mesad, noting that, about $1 \mathrm{~cm}$. from the meson, the muscular fibers are replaced by a thin tendon which is not always easily separated from the subjacent muscle. Then trace the border ventro-cephalad, noting that it 
thickens slightly as it crosses the vertebral border of the scapula, and ends upon the fascia covering the $M$. infraspinatus.

Continue to lift the ventro-caudal border, and dissect up the middle part of the muscle as far cephalad as possible ; then pull it caudad, and at the same time dorsad or ventrad. This will indicate the cephalic border, which is much shorter than the other, and extends latero-ventrad from a point between the 1 st and 4 th thoracic neural spines.

The dorsal half of the cephalic border is muscular, and separable without much difficulty from the adjacent caudal border of the $M$. acromio-trapezius. Opposite, or slightly dorsad of, the vertebral border of the scapula, the border of the $M$. spino-trapezius becomes tendinous, thin and indistinct, so that its true limits are best ascertained by pulling upon the muscular portion. It is also overlapped to some extent by the $M$. acromio-trapezius.

The cephalic border of the muscle may easily be traced, entad of the tendon of the $M$. acromio-trapezius, to a point $8-10 \mathrm{~mm}$. cephalad of the mesoscapula, and about the same distance from the nearest part of the border of the scapula. Here it terminates upon the fascia covering the $M$. supraspinatus.

The muscle should now be transected ( $\$ 586)$, and the ental surface cleared of fat, especially near the attachments.

$\$ 612$. Origin.-From the tips and interspinous ligaments of most or all of the thoracic neural spines. The attachment of the cephalic border may be at any point between the 1st and 4th spine, and that of the ventro-caudal border at any point between the 11th and 13th. The origin of the caudal $2-3 \mathrm{~cm}$. is by a triangular tendon, the lateral angle of which is $1 \mathrm{~cm}$. from the meson. 'The rest of the muscle arises by fleshy fibers: Opposite the cephalic 3 or 4 spines there are sometimes slight intervals filled with loose connective tissue; opposite the others, the corresponding intervals, when they exist, are occupied by a firm fascia which practically renders the attachment continuous across the spines.

Insertion.-Along a curved s-shaped line obliquely crossing the mesoscapula (Fig. 44). The cephalic two thirds of the insertion is by a thin tendon 1-2 cm. long and $1-1.5 \mathrm{~cm}$. wide, which is attached to the fascia upon the ectal aspect of the supraspinatus, and to the mesoscapular tuberosity which it crosses very obliquely. Here it joins the caudal and usually fleshy third of the insertion, which extends upon the fascia covering the infraspinatus at an angle of 
about 45 degrees with the mesoscapula. On the ectal surface of the spino-trapezius, along the line of union of the muscular and tendinous parts, is received the insertion of the acromio-trapezius (Fig. 67).

\section{ACROMIÓ-TRAPEZIUS.}

\$ 613. Synonymy.-The intermediate part of the human trapezius, G., 373; Q., I, 187; acromin-cucullaire, S.-D., A, II, 333 ; portion cervicale du trcppéze, Ch., A, 216, and Fig. 90 ; cervical trapeaius, Ch. (Fl.), A, 303 ; anterior part of the trapezius, Miv., B, 137 .

Figures.--Ectal aspect (66); ental aspect and insertion (67); insertion area (44).

General Description.-Thin ; sub-trapezoidal ; from the dorsimeson, in the caudai part of the cervical region and sometimes also the cephalic part of the thoracic, to the metacromion, and the adjacent surface of the $M$. spino-trapezius.

Dissaction.-The caudal border has been indicated in describing the spino-trapezius, which is often slightly overlapped by it. It nearly coincides with a line drawn between the vertebral ends of the two mesoscapulæ. Lift it at the vertebral border of the scapula, and note that, 8-10 mm. from the meson, it becomes a thin tendon which may be traced across the meson to its platetrope without severing any definite attachments to the subjacent parts. Ventrad it may be traced to a point about $1 \mathrm{~cm}$. caudad of the mesoscapula, and 1.5 to $2 \mathrm{~cm}$. from the nearest part of the vertebral border of the scapula where it terminates upon the ectal surface of the spinotrapezius.

The cephalic border of the muscle may be recognized as forming the caudal margin of a narrow intermuscular interval, the hiatus trapezii, at the side of the neck, about midway between the convexity of the shoulder and the meson. This interval is filled with connective tissue and fat, in which are imbedded a lymphatic gland and an artery.

Follow this border to the meson, noting that, for about $1 \mathrm{~cm}$. therefrom, it is either continuous with the caudal border of the next muscle (clavo-trapezius) or slightly overlapped by it. They may be separated, however, without cutting fibers. Ventrad of the hiatus this border is usually overlapped for $3-5 \mathrm{~mm}$. by the levator claviculae, the border of which must be lifted with care. Transect the acromio-trapezius at the vertebral border of the scapula.

\$ 614. Origin.-In two or three parts: In the caudal tive eighths the thin tendon is continuous across the meson with its platetrope, 
and is connected with the subjacent parts only by small nerves and vessels. It thus spans the interval between the vertebral borders of the scapulæ, which project slightly dorsad of the intervening vertebral region. In the next two or three eighths the median raphé of the tendon is joined by a low median fascia which springs from the supraspinous ligaments. Sometimes, in the cephalic eighth, the muscular fibers reach the meson. The line of origin extends from the caudal end of the axial spine to some point between the 1st and 4th thoracic spines, thus filling the interval between the spinotrapezius and clavo-trapezius.

Insertion.-In three parts: (A) the cephalic fifth is attached to the ectal surface of the metacromion (Fig. 44), and is usually overlapped by the dorsal border of the levator clavicula; (B) the caudal fourth or fifth is attached to the ectal surface of the spinotrapezius, along a dorso-caudal line forming an angle of 30-45 degrees with the mesoscapula; (C) the intervening portion of the muscle is inserted upon the glenoid border of the mesoscapula.

Remark.-The most notable feature of this muscle is the wide tendon of origin, which with its platetrope forms a heart-shaped area with its apex directed cephalad. The office of the muscle seems to be mainly that of a ligament, to prevent the separation of the vertebral borders of the scapulæ.

\section{CLAVO-TRAPEZIUS.}

\$ 615. Synonymy.-The cephalic or clavicular portion of the buman trapezius, G., A, 373 ; Q., A, I, 187 ; clavo-cucullaire, S.-D., A, II, 332 ; part of the "portion antérieure du mastoido-humeral," Ch., A, 209 ; part of the anterior or superior portion of the mastoidohumeralis, Ch. (Fl.), A, 197 ; cephalic part of the "cephalo-humeral," Miv., B, 147, and Wood, 9, 101, Fig. 23, "c." It is thought by some that this muscle does not represent any part of the human tropezius.

Figures.-Ectal aspect (66); ental aspect of reflected ends (67); clavicular end (72).

General Description.-A wide tæniate muscle, from the occiput and the cephalic part of the dorsimeson to the clavicle and the trapezio-deltoid raphé.

Dissection.-The larger part of the cephalic border of the hiatus mentioned under the dissection of the acromio-trapezius is formed by the caudal border of the clavo-trapezius. Trace this border dorsad, bearing in mind its close union with the cephalic border of the acromio-trapezius. Then trace it ventrad, using great care in lifting it from the subjacent levator claviculo, and noting that, ven- 
trad of the point of crossing, the interval between the two muscles is filled with a fascia so dense as to practically unite them.

Lift the skin from $1-2 \mathrm{~cm}$. along the occipito-presternal line. Dissect up the caudal border of the clavo-trapezius for $2-3 \mathrm{~cm}$. at the junction of its dorsal and middle thirds, and pull it caudad. This may serve to indicate the position of the ventro-cephalic border near the cut edge of the skin. Moreover, between the border of the clavo-trapezius and the caudal border of the adjacent sternomastoideus, just caudad of the ear, will be seen emerging one or two nerves.

Follow the border of the clavo-trapezius to the crista lambdoidalis, noting that, for 15-20 mm. therefrom, it is closely united with the caudal border of the adjacent sterno-mastoideus. The two muscles may usually be separated without cutting fibers, but sometimes a slender fasciculus passes from one to the other obliquely at about the midale of their length.

Part of the occipitsl end of the muscle is covered by a small triangular muscle belonging to the ear. This, the $M$. cervico-auricularis (subcervico-pavilien of Straus-Durckheim, A, I, 194), is shown in Fig. 66, but not named; it must be removed with great care to a point just cephalad of the crista lambdoidalis. Trace the ventrocephalic border of the clavo-trapezius to the clavicle, which may be felt in the muscles between the præsternum and the convexity of the shoulder.

Transect the muscle at its middle; in reflecting the ventral end of the muscle, use great eare in separating it from the subjacent clavo-mastoideus, which is also attached to the clavicle. After reflecting the dorsal part, divide it lengthwise along a line corresponding with the angle formed by the meson with the crista lambdoidalis; the wider of the strips so formed may then be reflected across the meson, and the narrower upon the head, as shown in Fig. 67.

$\S 616$. Origin. - In two parts: (A) by a thin tendon $5-10 \mathrm{~mm}$. long, from the mesal $10-15 \mathrm{~mm}$. of the crista lambdoidalis; (B) by fleshy fibers from the supraspinous ligament for $4-5 \mathrm{~cm}$. from the crest to the caudal end of the axial neural spine (Fig. 30, $\$ \$ 208,471$ ).

Insertion.--The details of the insertion differ considerably in individuals, and perhaps in the same individual according to age and the development of the clavicls. Sometimes the sternal end of the clavicle projects about $1 \mathrm{~mm}$. mesad of the $M$. clavo-trapezius, 
while in other cases the muscle extends mesad of the bone so as to join the border of the ectopectoralis.

Pull the muscle dorso-cephalad, and note its apparent continuation across the clavicle with the clavo-deltoideus. The muscles, however, are joined by a raphé, the trapezio-deltoid, which is more apparent upon the ental aspect. Most of the clavo-trapezius is inserted upon this raphé; but the ental fibers of the cephalo-ventral third or fourth are attached directly to the ventral border of the sternal and straighter half of the clavicle. Variations in the mode of insertion should be noted, drawn and reported.

\section{OCCIPITO-SCAPULARIS.}

$\$ 617$. Synonymy.-There seems to be some doubt as to its human homologue; rhomboideus capitis, Miv., B, 145, and Wood, 9, 92, Fig 23, “ $d$ ” ; occipito-scupulaive, S.-D., A, II, 331 ; part of rhomböde, Ch., A, 202 ; part of rhomboideus, Ch. (Fl.), A, 188.

Figures.-Slightly at the dorsal end of the hiatus trapaii (66); ectal aspect (67); lateral border (73); insertion area (45).

Exposure.-by the removal of the MM. acromio- and clavotrapezius.

General Description.-Narrow, tæniate, near-and nearly parallel with-the dorsimeson, from the crista lambdoidalis to the coraco-vertebral angle of the scapula.

Dissection.-About midway between the occiput and the scapula the lateral border of the muscle appears as a slightly raised line 10-15 $\mathrm{mm}$. from the meson. Trace it nearly to the occiput. Lateriduct the middle of the cephalic half of the muscle so as to indicate its mesal border ; then transect.

$\S 618$. Origin.-By fleshy fibers from the crista lambdoidalis, entad of the origin of the clavo-trapezius, beginning $5-10 \mathrm{~mm}$. from the meson, and extending $12-15 \mathrm{~mm}$. laterad to a point nearly in line with the temporo-parietal suture $(\$ 493)$, where it is overlapped by the dorsal border of the sterno-mastoideus. Candad, the muisele gradually approaches the lateral border of the rhomboideus. At the junction of the third with the last fourth, the muscle narrows and thickens, so as to become prismatic rather than tæniate.

Insertion.-The narrowing. muscle is wedged pretty closely between the rhomboideus at its mesal side and the levator anguli scapulo at its lateral side, and is inserted either between these muscles upon the coraco-vertebral angle of the scapula (Fig. 45), 6-16 mm. cephalad of the mesoscapula, or upon the ental surface of the 
second muscle close to its own insertion; in some cases, these two forms of attachment coexist.

$\S 619$. Explanation of Fig. 67. -The second layer of skeletal muscles of the left shoulder and neck.

Preparation.-After the reflection of the skin and dermal muscles as in Fig. 66, the following muscles were transected and reflected: spino-trapezius, acromio-trapezius, clavo-trapezius and

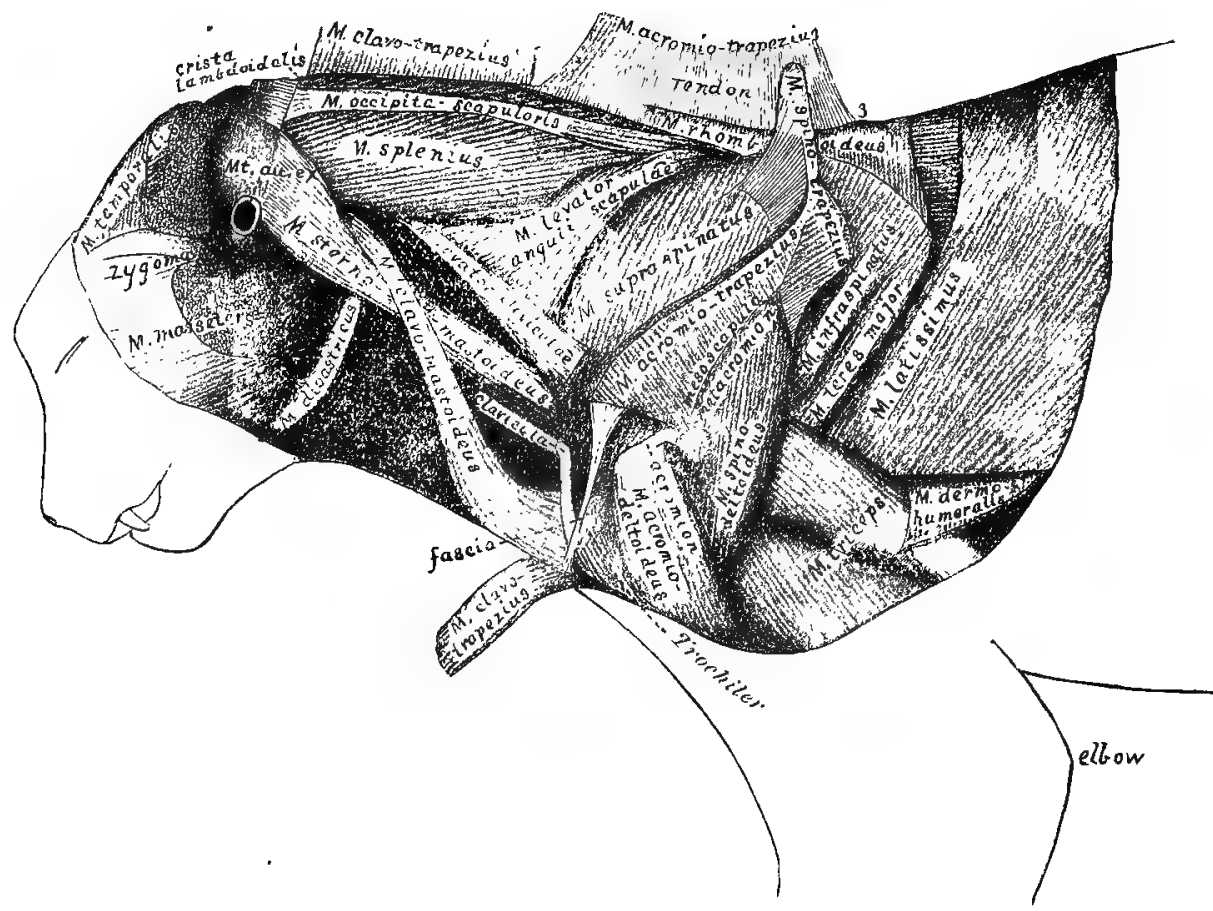

Fig. 67.-The Second Layer of Skeletal Muscles of the Neck and Shodlder.

levator clavicula. The skin was also removed from the left side of the head, together with the external ear, the parotid and submaxillary salivary glands, and parts of the MM. temporalis and masseter.

Bones.-Acromion (\$392).-This process of the mesoscapula is seen to afford origin to the acromio-deltoideus.

Clavicula $(\$ \& 422,608)$.-By the reflection of the clavo-trapezins and the deflection of the clavo-mastoideus, the clavicle is brought into view. Its name is connected with its sternal end. 
Crista lambdoidalis-The lambdoid ridge of the skull (Fig. 56).-The crest itself does not distinctly appear, but its position coincides with the lines of attachment of the occipitoscapularis and sterno-mastoideus and part of the clavo-trapezins.

Mesoscapula and metacromion (Fig. 44 and § 608).

Trochiter-The larger or cephalic tuberosity of the humerus (Fig. 30 and 74, $\S 420$ ).

Zygoma-The zygomatic arch (Fig. 30, 56, $\$ \S 207,229$ ).-The outlines of this prominent bony arch are shown just dorsad of the name.

Muscles.-The acromio-deltoideus ( $(670)$, dermo-humeralis ( $(629$ ), spino-deltoideus $(\$ 674)$, teres ( $\$ 680$ ), and triceps ( $\$ 3882,684$ ), are sufficiently described in the explanation of Fig. 66 ( $\$ 608$ ), and the supraspinatus, infraspinatus and teres (the word major is superfluous) in the explanation of Fig. 74 (\$ 672). The masseter, temporalis, splenius and digastricus are not particularly described in this work.

Acromio-trapezius (Fig. 66, $\$ 613$ ).-This was transected so as to leave the semicordate tendon wholly in the vertebral part, which is reflected dorsad. The scapular part is slightly lifted so as to show the manner of its connection with the ectal surface of the spino-trapezius.

Clavo-trapezius (Fig. 66, § 615).-The clavicular end has been reflected. The other and much wider end was longitudinally divided from the angle between the cephalic and mesal parts of the origin, and the two portions thus formed were reflected respectively cephalad and dorsad. The name is written upon the latter only.

Latissimus (Fig. 66, \& 685).-By the removal of the vertebral, end of the spino-trapezius, the dorso-cephalic angle of this muscle is exposed. In the interval between its cephalic border and the caudal border of the rhomboideus, are seen some of the proper vertebral muscles. By inadvertence, the fibers in the dorso-cephalic corner are not represented as parallel with the border.

Levator anguli scapulce (Fig. 78, $\$ 686$ ). - A part of the ectal aspect is seen between the splenius and the supraspinatus. The ental aspect of the whole muscle appears in Fig. 73.

Levalor ctaviculoe (\$ 627).-This has been transected near its insertion upon the metacromion, and the metacromial end is somewhat indistinctly seen reflected upon the spinodeltoideus.

Occipito-scapularis (\$ 617). - The cephalic attachment is seen to have been covered by that of the clavo-trapezius, and its scapular end is wedged in between the rhomboideus and the levator anguli scapulas.

Rhomboideus ( $\S 620$ ). - The cephalic border is not satisfactorily represented in this figure. The scapular end of the muscle is better shown in Fig. 74.

spino-trapezius (Fig. 66, § 611).-The vertebral end has been removed altogether. The scapular end is lifted a little so as to show its relations with the acromio-trapezius and with the ectal aspects of the supraspinatus, infraspinatus and spino-deltoideus.

Sterno-mastoideus (Fig. 72, $\$ 622$ ).-The removal of the clavo-trapezius, the salivary glands and the external ear has exposed its cranial attachment and its intersection with the clavomastoideus. Its sternal end and its connection with its platetrope are shown in Fig. 72.

Other Parts.-Mt. au. ex.-Mextus auditorius externus.-Its lumen is exposed by the removal of the concha or external ear with the skin.

\section{RHOMBOIDEUS.}

\$ 620. Synonymy.-The human rhomboideus major, with probably the $r$ minor also, G., A, 375 ; Q., A, 191 ; rhomboïde, S.-D., A, II, 334; rhomboüde, Ch., A, 202 ; rhomboideus, Ch. (FI.), A, 188; rhomboideus major, Miv., B, 145.

Figures.-Ectal aspect (67); scapular end, ectal aspect (74); scapular end, ental aspect $(75) ;$ insertion area $(44,45)$. 
General Description.-Trapezoidal in outline; from the caudal part of the cervical dorsimeson and the cephatic part of the thoracic, to the vertebral border of the scapula.

Exposure.-By the removal of the MM. spino- and acromiotrapezius.

Posture.-Ventricumbent, with one or two blocks lengthwise under the thorax so as to permit the ventriduction of the shoulder. Usually the body must be steadied by cords or chain-hooks. Lateriduct the vertebral border of the scapula so as to render the $M$. rhomboideus tense.

Dissection.-The ventro-cephalic border of the muscle has been exposed by the reflection of the occipito-scapularis. The caudal border may be seen along a line running nearly laterad from the 4th or 5th thoracic spine, opposite the gleno-vertebral angle of the scapula. Note that the texture of the muscle is looser than that of the trapezii, and that the caudal border is thinner than the ventrocephalic, excepting the dorso-cephalic third of the latter. This muscle may be safely transected by cutting ecto-entad; in reflecting it, note that the thickest part is opposite the 1st and $2 \mathrm{~d}$ thoracic spines.

$\$ 621$. Origin. - In two parts: (A) from the caudal two or three fifths of the cervical supraspinous ligament; in the latter case it includes the caudal two thirds of the axial spine, and thus extends about $1 \mathrm{~cm}$. cephalad of the acromio-trapezius ; (B) from the sides of the tips of the first four thoracic spines and from the interspinous ligaments caudad of each of them, excepting, sometimes, the 4th.

Insertion.-At least two forms of insertion are found. The simpler is as follows (Fig. 44, 45): the cephalic three fourths is attached by short tendinous fibers along the vertebral border of the scapula, passing gradually from its ental to its ectal margin. The cephalic half or third of this portion is closely united with the insertion of the levator anguli scapulce. The caudal fourth is attached by fleshy fibers upon the gleno-vertebral angle in apposition with the origin of the teres.

The other mode of insertion presents four divisions: (A) the cephalic $8 \mathrm{~mm}$. is attached by fleshy fibers to the ental aspect of the lev. ang. scap. close to its insertion upon the ental margin of the vertebral border of the scapula, and caudo-ventrad of the insertion of the occipito-scapularis; (B) the next 6-8 $\mathrm{mm}$., constituting the thickest part of the muscle, is attached by fleshy fibers to the ental 
margin of the vertebral border, opposite the triangular space at the vertebral end of the mesoscapula; (C) the next $2-2.3 \mathrm{~cm}$. is attached by a thin tendon, $2-3 \mathrm{~mm}$. long, upon the ectal margin of the vertebral border from opposite the middle of the space just mentioned, and thus slightly overlapping part $\mathbf{B}$, to within $8-10 \mathrm{~mm}$. of the gleno-vertebral angle of the scapula; (D) the caudal 5-6 $\mathrm{mm}$. is separated from the rest by an interval 1-5 mm. wide, and is inserted by fleshy or very short tendinous fibers upon the ectal aspect of the gleno-vertebral angle in apposition with the origin of the teres. The scapular end of the muscle may be separated more or less readily into four divisions corresponding with the parts of the insertion just described, and the caudal division is sometimes quite distinct, with a decided extension toward the teres.

\section{STERNO-MASTOIDEUS.}

Remark.-This is strictly a muscle of the neck, but is here described on account of its close relations with the muscles of the shoulder and with blood-vessels.

$\$ 622$. Synonymy.-The sternal part of the human sterno-cleido-mastoid, G., A, 357; Q., A, 1, 292 ; sterno-mastoidien, S.-D., A, II, 248 ; sterno-maxillaire, Ch., A, 210 ; sternomaxillaris, Ch. (Fl.), A, 198 ; sterno-mastoid, Miv., B, 134.

Figures.-Lateral aspect of cephalic half $(66)$; lateral aspect $(67)$; united sternal portions (72); sternal portion (73).

§623. General Description.-Tæniate, along the ventro-cephalic border of the clavo-trapezius, from the prasternum to the mastoid process of the temporal bone and the ventral part of the crista lambdoidalis.

Posture.-Latericumbent; a block transversely under the neck ; the head hanging.

Exposure-Connect the angle of the mouth with the occipitopresternal line $(\$ 610)$ by an incision corresponding with the direction of the margin of the upper lip. Note that the skin of the cheek, especially in old males, is very thick. Dissect up both edges of the skin for about $1 \mathrm{~cm}$.

The zygomx (Fig. 30, 56, 67) may be felt as a firm bony arch between the $M$. temporalis (Fig. 67) dorsad, and the $M$. masseter ventrad.

Just caudad of the zygoma may be felt the cartilaginous meatus auditorius (Fig. 67, Mt. au. ex.), partly embraced by the small, pale and rather loose-textured parotid gland (Fig. 87, 107). Divide the meatus close to the head, and reflect the flap, together with the 
ear and parotid, for $2-3 \mathrm{~cm}$., taking care not to cut caudad of the crista.

Then reflect the ventral flap for about the same distance. In the depression just caudad of the mandibular angle note the firmer textured and darker colored submaxillary gland (Fig. 66, 87). Its dorsal border is often quite firmly attached to the ventral border and ectal surface of the sterno-mastoideus. Remove the gland, together with the dense connective tissue in the groove between the head and the neck ventrad of the origin of the occipito-scapularis.

Along the ventral border of the space occupied by the submaxillary gland is the Vena juguzaris (Fig. 101), which lies upon the ectal aspect of the sterno-mastoideus, crossing very obliquely from its ventral to its dorsal border. Divide the vein at the middle of its length, reflect the ends and free the surface of the muscle from fat and connective tissue.

Dissection.-The dorsal border of the muscle is apparent at about its middle, where it was crossed by the $V$. jugularis, and where it in turn crosses the ventral border of the subjacent clavomastoideus. Lift this border, taking care not to include the fibers of the clavo-mastoideus. A little cephalad of the middle of the length of the muscle dissect from the dorsal to the ventral border.

In reflecting the cephalic part, note that the ventral border is thickened as if folded upon itself, and that, at the occiput, the dorsal border may orerlap the occipito-scapularis for half the width of the latter. In reflecting the candal part, note that, about the middle of the length of the whole muscle, it joins its platetrope, the fibers appearing to interdigitate to some extent (Fig. 72). About $2 \mathrm{~cm}$. cephalar of the presternum the muscle is overlapped by the ectal lamina of the ectopectoralis.

\$ 624. Origin.-On account of the overlapping just mentioned, the farther dissection of the sternal end of the muscle is better deferred until after the examination of the ectopectoralis.

Insertion. - The cephalic attachment of the muscle is by a tendon 2-7 mm. long which is inserted upon the crista lambdoidalis laterad of, and usually overlapping to some extent, the origin of the occipito-scapularis. The line of insertion extends not only along the crista, but also upon the rather sharp ridge of the mastoid process, which ceases suddenly at a slight elevation just dorso-caudad of the stylo-mastoid foramen, $3-4 \mathrm{~mm}$. from the meatus. The tendon is here a little thicker than at other points, and between it and the 
tendon of the subjacent splenius (Fig. 67) there is sometimes a welldefined depression.

\section{CLAVO-MASTOIDEUS.}

$\S 625$. Synonymy.-The clavicular part of the human sterno-cleido-mastoid, G., A, 357 ; Q., A, I, 292 ; “cleido-mastoidien," S.-D., A, II, 333 ; part of the " mastoido-humeral," Ch., A, 209, Fig. 90; part of the mastoido-humeralis, Ch. (Fl.), A, 196; part of the cephalohumeral, Miv., B, 147 ; cleido-mastoid, Wood, 9, 101, Fig. 23, "b."

Figures. - Lateral aspect (67); ventral border of clavicular part (72).

General Description.-Narrow, tæniate, from the clavicle to the mastoid process.

Posture. - Latericumbent, the block transversely under the neck, and the head hanging.

Exposure.-By the reflection of the clavo-trapezius and the sterno-mastoideus.

Dissection. - The dorsal border is apparent at about its middle. Raise it, and draw the muscle dorsad so as to indicate its ventral border. In reflecting the cephalic part, note that, about $18 \mathrm{~mm}$. from the head, the muscle is perforated by a nerve, near which, entad of the muscle, lies the separate lateral half of the thyroid body. In reflecting the caudal part, note the constant increase in width to the clavicle, that the $V$. jugularis lies mesad of it, and usually a lymphatic gland entad of it, while its dorsal border is attached by a firm fascia to the ventral border of the levator claviculat.

§626. Origin.--In two nearly equal parts: (A) the ventral part arises, with the corresponding part of the insertion of the clavo-trapezius, from the ventro-cephalic border of the sternal half or three fifths of the clavicle; (B) the remainder arises from the ental aspect of the trapezio-deltoid raphe, but is connected with the scapular part of the clavicle by a strong fascia which might be regarded as a common tendon of attachment for the corresponding parts of the clavo-trapezius, clavo-mastoideus and clavo-deltoideus.

Insertion.-By fleshy fibers upon the ventral and caudal borders of the mastoid process, and upon its mesal side.

Remark.--Respecting the choice of origin and insertion for this muscle, see $\S 579$.

\section{LEVATOR CLAVICUL $\mathbb{E}$.}

§ 627. Synonymy.-" Transverso-scapulaire," S.-D., A, II, 331 ; "levator clavicula," Wood, 9, 95, Fig. 23, " $e$ " ; trachelo-acromialis, Huxley, A, 418 ; levator scapularis, Miv., $\mathrm{B}, 148$; not found normally in man.

Figures.-Scapular part $(66,67)$; insertion area (45). 
Posture.-Latericumbent, with a block transversely under the neck. The posture must be changed often and greatly in the course of the exposure and dissection.

Exposure.-By the reflection of the clavo-trapezius, sternomastoideus and clavo-deltoideus. Note that, after the reflection of the above named muscles and the occipito-scapularis, the broad transverse process of the atlas is covered, dorsally, by a thick mass of muscle, the most ectal of which, the splenius (Fig. 67), presents a smooth and convex surface. Remove this mass by deep incisions with the arthrotome as follows: Laterad from the cephalic end of the origin of the rhomboideus; then cephalad to the occiput; then laterad close to the occiput, but without severing any of the muscular attachments along the crest; finally, beginning with the meson, dissect up the mass from the vertebræ.

General Description.-Narrow, tæniate, from the basioccipital bone (Fig. 57) and atlantal transverse process or diapophysis (Fig. 52) to the metacromion (Fig. 44).

Dissection.-The dorsal border of the scapular end has been indicated $(\$ 614)$ in connection with the insertion of the cephalic border of the acromio-trapezius. Trace it cephalad, and draw the middle of the muscle dorsad so as to indicate its ventral border.

In reflecting the caudal part, note a lymphatic gland close to the coracoid border of the supraspinatus. In reflecting the cephalic part, note that, a little caudad of the atlantal transverse process, its plane gradually changes from dorso-ventral to dextro-sinistral, and that there are signs of subdivision.

Remove the muscles rentrad and cephalad of the atlantal transverse process, but without cutting the attachment of this muscle to its ventral surface. Feel for the prominent auditory bulla just mesad of the $M$. masseter and the meatus, and carefully dissect off the $M$. digastricus, which covers it. Aroid the external carotid artery which skirts the meso-cephalic border of the bulla, and the hyoid arch (Fig. 30) which lies imbedded in the muscles between it and the artery.

Then use the tracer to clear away the connective tissue, and the arthrotome to scrape the ventral surface of the transverse process of the atlas, excepting where it is occupied by the origin of the levator clavicula. Between the border of the process and the larynx and trachea note and remove the lateral lobe of the thyroid body. 
Note, but do not remove, the carotid artery, and the nervous trunk representing the conjoined $N N$. vagus and sympathicus (Fig. 107).

\$ 628. Origin.-The larger and constant head arises by fleshy fibers from the ventral surface of the atlantal transverse process along an oblique line extending from the junction of the process with the body of the vertebra, $3-4 \mathrm{~mm}$. from the meson, latero-caudad to a point about $3 \mathrm{~mm}$. cephalad of the caudo-lateral angle of the process. This line of origin is $6-8 \mathrm{~mm}$. long, and is nearly parallel with the oblique meso-caudal border of the arthral surface in contact with the axis.

The smaller and less constant head is about $3 \mathrm{~mm}$. wide and is the more direct continuation of the ventral border of the muscle. Opposite the latero-cephalic angle of the transverse process it becomes a thin flat tendon which is closely applied to the ventrolateral aspect of a muscle, the $M$. rectus anticus capitis, which extends along the ventral side of the vertebræ, and is inserted with it into the basioccipital bone nearer the bulla (Fig. 57) than the meson, and about midway between the jugular foramen (Fig. 57, $F m . j$.) and the cephalic angle of the bulla. This, the occipital head of the muscle, is sometimes absent, and other irregularities have been observed in the cephalic end of the muscle.

Insertion.-By a strong tendon 1-2 mm. long and a little narrower than the muscle, upon the ectal surface of the metacromion (Fig. 45, 67), close to its free border. The dorsal border slightly overlaps the ventral border of the acromio-trapezius near its insertion, and the ventral border is firmly joined by a strong fascia with the clavo-trapezius.

\section{DERMO-HUMERALIS.}

§ 629. Synonymy.-“ Dermo-humeral," S.-D., A, II, 251; part of the "pannicule charnu," Ch., A, 200; part of the fleshy pannicle, Ch. (Fl.), A, 180 ; part of the panniculus carnosus, Miv., B, 136 ; not represented in man.

Figures.-Humeral part, ectal aspect $(66,74)$; partly reflected $(67,72,73)$.

Posture.-Latericumbent, the ventral region toward the dissector; a block transversely under the thorax, just caudad of the elbows.

Exposure-C Connect the last (13th) thoracic spinous process with the ventrimeson by a dorso-ventral incision. Be careful to divide only the skin, with the subcutaneous fat and connective tissue, together with a thin sheet of pale muscular fibers, the dermo- 
humeralis, and avoid cutting the ental, thicker and darker colored latissimus (Fig. 66), the cephalic part of which was exposed in the dissection of the spino-trapezius.

Begin to lift the flap at the dorsimeson ; remove the fat and connective tissue from the ental surface of the dermo-humeralis, and note that its fibers have nearly the same direction as those of the latissimus. When the caudo-ventral border of the latissimus is reached, a little ventrad of the middle of the incision, be careful not to lift with the skin and dermo-humeralis, the thin dorso-caudal margin of the $M$. xiphi-humeralis (Fig. 72), a member of the pectoralis group of muscles, which arises at the meson, and sometimes adheres quite closely to the dermo-humeralis.

General Description. - As stated by Straus-Durckheim (A, I, 251), "this muscle covers as a mantle the whole thorax and abdomen, ... . but differs from the skin-muscles proper in the attachment of one end to the skeleton ;" this attachment, however, is only indirect.

Dissection.-The cephalic and shorter border of the muscle may be seen on the ental surface of the skin along a dorso-ventral line from the $2 \mathrm{~d}$ or $3 \mathrm{~d}$ thoracic spine to the axilla. The caudo-ventral border is less distinct, but may be detected 1-2 cm. from the meson. Connect the two borders by an incision $3-4 \mathrm{~cm}$. from the brachium. Leave the caudal portion of the muscle upon the skin, but carefully dissect up the narrower and thicker brachial part, freeing both its ectal and ental surfaces from fat and connective tissue. Note that it not only overlies the corresponding part of the latissimus, but that its ental surface becomes intimately united with the ectal surface of that muscle.

$\$ 630$. Origin.-From the skin, along an oblique line extending ventro-cephalad from about the middle of the length of the pelvis upon the caudal aspect of the meros as far as the knee; also along a line which is just laterad of the dorsimeson opposite the $2 \mathrm{~d}$ or $3 \mathrm{~d}$ thoracic spine, but which gradually leaves the meson as it extends caudad to join the pelvic line already mentioned.

Insertion.-From the broad origin abore described the fasciculi converge ventro-cephalad toward the caudal aspect of the brachium. The muscle becomes narrower and thicker, and less closely attached to the skin. Near the dorsal border of the brachium it joins the ectal surface of the subjacent latissimus. The dorsal border, 1-1.3 cm. wide, is attached directly by muscular fibers, but the remain- 
der ends as a thin tendon ranging from $1-3 \mathrm{~cm}$. long. The details of its indirect connection, through the latissimus, with the bicipital arch (Fig. 73), and thus with the humerus, may be examined more conveniently after the dissection of the pectorales.

Remark.-This muscle does not exist in man, where the group of dermal muscles is represented only by the $M$. platysma myoides upon the sides of the neck, and by certain muscles of the face.

\$ 631. Explanation of Figures 68-7I inclusive.-These represent respectively the cephalic (outer), ventral (anterior), caudal (inner), and dorsal (posterior) aspects of the left humerus.

A shaded representation of the ventral aspect of the right humerus is given in Fig. 46. These four figures are little more than outlines for the sake of indicating the attachment areas and lines of the muscles or muscular divisions described in this work which arise from or are inserted upon this bone.

As in the figures of the scapula (Fig. 43, 44), the lines enclosing origins are composed of dots, and those enclosing insertions of short dashes.

In order to place the figures across the page, and so facilitate reference and comparison, many of the parts are undesirably small, and several are so crowded as to be indistinct.

The attachments are at least approximately correct for the majority of cases, but considerable variation is to be expected.

The identification of the muscles, especially those of the pectoralis group, is much facilitated by the use of a carved wooden model of the humerus, enlarged 4 or 5 diameters so as to increase both the attachment areas and the spaces between them. Such a model, made by Mr. H. W. Turner, a special student, has been in use for several years in the Anatomical Laboratory of Cornell University.

The following features of the humerus itself are shown and sufficiently described in the descriptions of Fig. $45 \mathrm{~B}$ and Fig. 46 :-

Cn. (canalis) bicipitalis $(69,70), \S \S 402,409$; capitellum $(69), \$ 410$; caput articulare (71), $\S \S 403,411$; crista deltoidea $(68,69), \S 412$; crs. (crista) epicondylaris $(68,71), \S 415$; epicondylus $(69,71)$, \$ 415 ; epitrochlea $(69,70,71), \S 416 ;$ Fin. (foramen) epitrochleare $(69,70,71)$, § 417 ; trochin $(69,71)$, $\S \S 405,420$; trochiter $(68,69,71)$, $\S 8406,420$; trochlea (69), $\S 420$.

The following parts are not designated upon these figures, but may be recognized from the other figures and descriptions :-

Cristn pecioralis (Eig. 46, $\S 413$ ); fossa ulnaris and fossa radialis (Fig. 46, § 418): fossn trochiteriana (Fig. $45 \mathrm{~B}, 8 \S 404,679$ ).

The following parts are not described elsewhere :-

Crista epitrochlearis.-This name may be applied to the ridge which extends proximad from the epitrochlen (Fig. 69).

$F^{\prime} m$. (foramen) medullare-The medullary or nutrient foramen (Fig. 70).-This opens upon the caudal aspect of the diaphysis, at about the junction of the middle and distal thirds of the bone. It points distad from the surface.

F3. (fossa) olecranalis-The olecranon fossa (Fig. 71).-This is a deep and irregular depression upon the dorsal aspect of the extromitas distalis. When the antebrachium is extended, the olecranon of the ulna is received by it. We have never observed a perforation of the bone at this point, as is sometimes the case with man. 

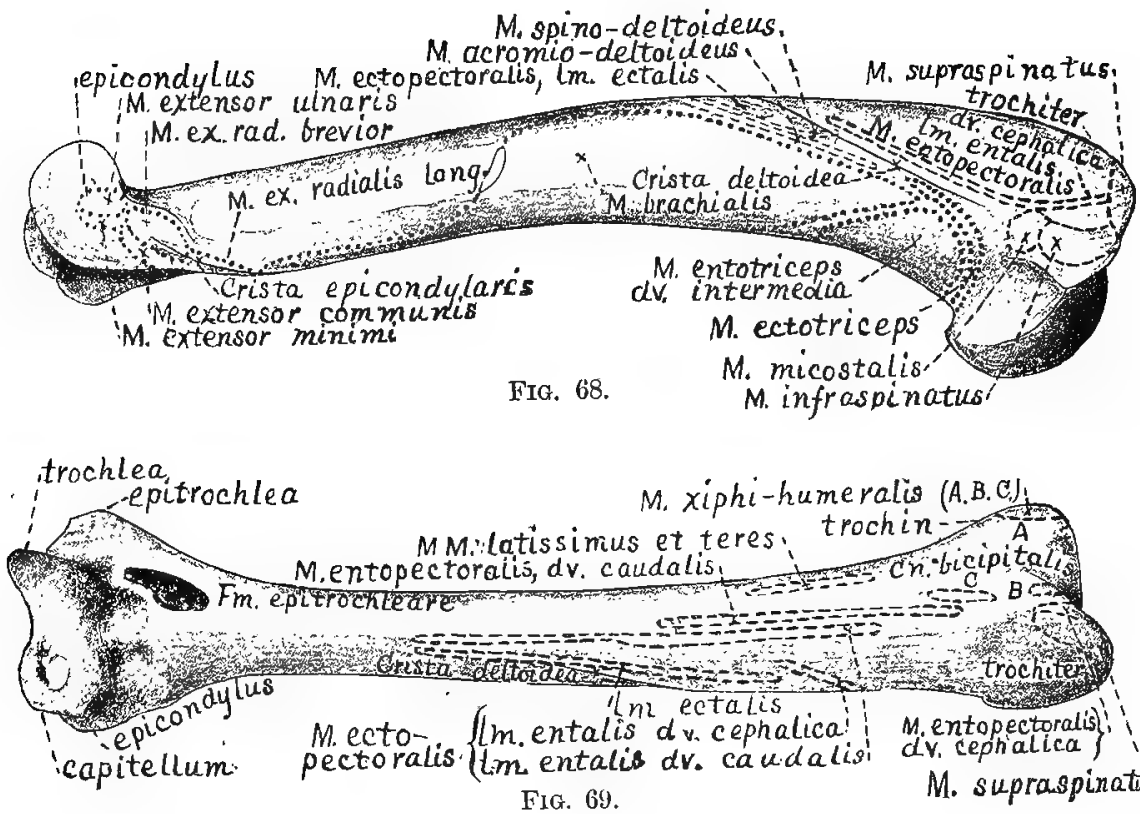

Fig. 69.

M.entopectoralis)! ¿iv. cephalica

M. supraspinatis
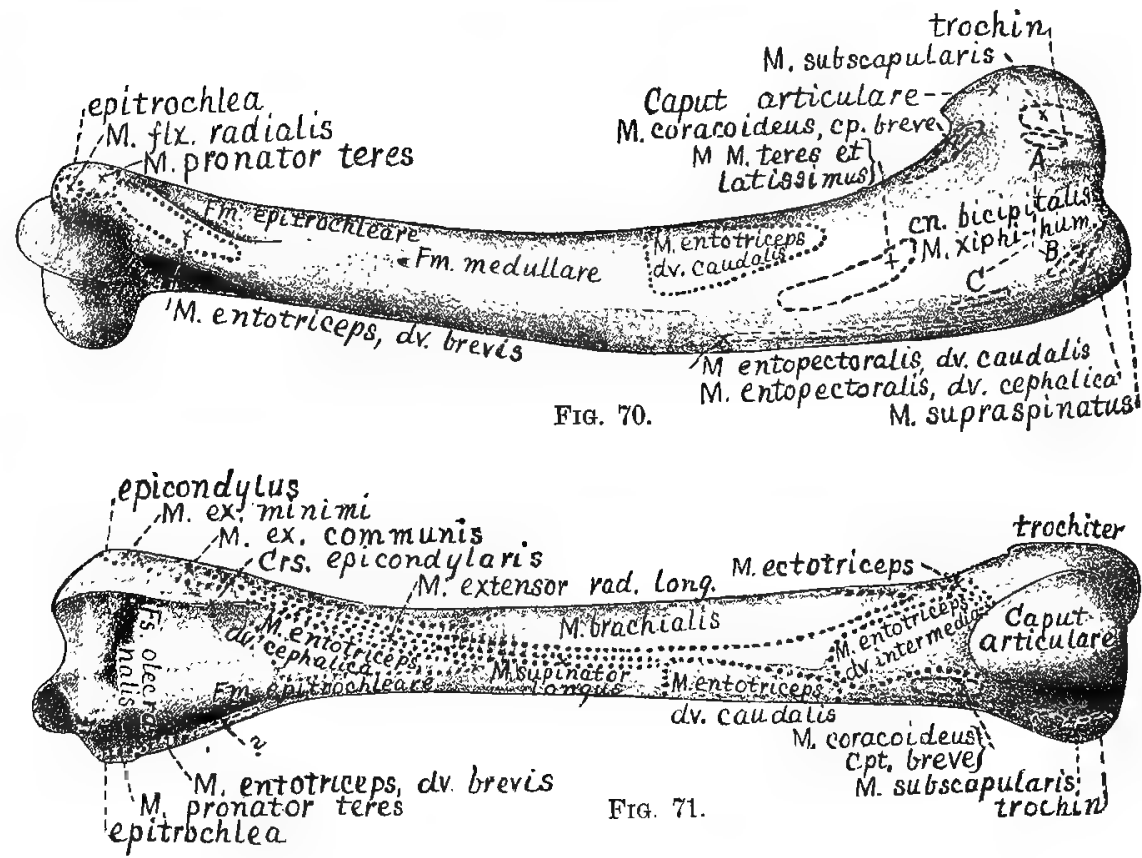

Fig. 68-71.-ThE Humerds. 
- \$ 633. Upon Figures 68-71 are represented the insertion areas or lines of the following muscles; the list includes all which are inserted upon the humerus :-

Acromio-deltoideus (68), $\$ 676$; coracoideus, caput breve (70), caput longum (71), $\$ 668$; cctopectoralis, lm. ectalis $(68,69), \$ 649$; ectopectoralis, lm. entalis, do. caudalis $(69), \S 653$; ectopectoralis, $I m$. entatis, dv. cephalien $(68,69)$, \$ 651 ; entopectoralis, dv. caudalis $(69,70)$, $\$ 656$; entopectoralis, dv. cephalica $(69,70), \S 658$; infraspinatus $(68)$, \& 678 ; micostalis (68), $\$ 679$; spino-deltoideus $(68), \S 674$; supraspinatus $(68,69), \S 675$; teres $(69,70), \S 680$; xiphi-humeralis $(69)$, $\$ 660$.

As stated in $\$ 670$, the insertion of the coracoideus, caput longum, is so variable that the area is here indicated on Fig. 71 by an interrogation point.

$\$ 634$. The origin areas or lines of the following 13 muscles are represented upon Fig. 68-71:-

Brachialis (68,71), § 692 ; entotriceps, dv. brevis $(70,71), \S 688$, dv. caudalis $(70,71)$, $\S 687$, dv. cephalica $(71), \S 689$, dv. intermedia $(68,71), \S 686$; extensor digitorum communis $(68,71), \S 697$; extensor minimi $(68,71)$, $\$ 698$; extensor radialis brevior $(68), \S 696$; ext. rad. longior $(68,71), \S 694$; extensor ulnaris $(68), \S 699$; flexor radialis $(70), \S 702$; pronator teres $(70,71), \$ 701$; supinator longus $(71), \$ 690$.

\section{LATISSIMUS.}

§635. Synonymy.-Latissimus dorsi, Q., A, 1, 189 ; G., A, 374; "grand dorsal," S.-D., A, II, 339 ; “grand dorsal," CL., A, 217, Fig. 90 ; great dorsal, Ch. (Fl.), A, 203 ; latissimus dorsi, Miv., B, 137.

Figures.-Eetal aspect $(66,67,74)$; ental aspect $(72,73,75)$; insertion area $(69,70)$; transection $(99,100)$.

Posture.-Latericumbent, the venter toward the dissector; a block transversely under the thorax just caudad of the elbows.

Exposure.-Most of the muscle has been exposed by the removal of the spino-trapezius and dermo-humeralis, and needs only to have its ectal surface cleared. If the caudal region of the body has not been removed, the corresponding part of the latissimus may be exposed by dividing the skin and the dermo-humeralis along a dorso-caudal line from the already exposed caudo-ventral border of the latissimus to the crista ilii ( $\$ 230$, Fig. 51) of the opposite side, and reflecting the flap so formed across the dorsimeson.

General Description.-A large triangular sheet, covering rather more than the dorso-cephalic half of the abdomen and thorax, excepting so much of the latter as is between the scapulæ. It arises at the dorsimeson between the pelvis and the 5th thoracic spine, and is inserted upon the humerus, forming part of the bicipital $\operatorname{arch}$ (Fig. 73).

Dissection.-Lift the caudo-ventral border where it crosses the 7th rib (which is also the 7th counting from the last), and trace it both ways for $2-3 \mathrm{~cm}$. Trace the cephalic border from. the vertebral 
border of the scapula along a dorso-ventral line from the 5th thoracic neural spine.

Transect the muscle along a line between the 7 th rib and the vertebral border of the scapula, beginning at the caudo-ventral border and alternately lifting and dividing the successive parts. Toward the meson, along a line extending ventro-caudad from the 10th, 11th or 12th thoracic spine to the lateral border of the vertebral muscles, about $3 \mathrm{~cm}$. from the meson, the muscular sheet gives place to a strong fascia.

In reflecting the brachial portion of the muscle, note the attachment of the dermo-humeralis to its ectal surface (Fig. 74), its close relations with the subjacent $M$. xiphi-humeralis, and the presence of a lymphatic gland near the dorso-caudal border of the latter. Turn the arm so as to bring the convexity of the elbow dorsad, and thus expose the space between the brachium and the scapula, and note that the ental surface of the latissimus is joined, near its cephalic border, by a thick muscle, the teres (Fig. 75), from the glenoid border of the scapula, and that, from the ectal surface, close to the attachment of the dermo-humeralis, there proceeds distad a muscle, the epitrochlearis (Fig. 75).

The details of the connection of the latissimus with the humerus and the bicipital arch are more easily examined after the dissection of the pectoralis group, and the removal of the arm from the body.

$\S 636$. Origin.-In two parts: (A) by muscular or short tendinous fibers from the sides of the tips of the thoracic spines from the 4 th or 5th to the 10th inclusive, and from the corresponding interspinous ligaments ; this part of the origin is wholly covered by the origin of the spino-trapezins; (B) from the dorsimeson, between the 10th thoracic spine and the sacrum, by a strong triangular tendon, the lateral angle of which corresponds nearly with the lateral border of the vertebral mass of muscles.

Insertion.-At the junction of the $2 d$ and $3 d$ fourths of the humerus, upon its ventral aspect, by a thick tendon which forms the caudal pillar of the bicipital arch.

The detailed description of the insertion must include that of the $M$. teres ( $\$ 680$, Fig. 75); but though the tendons of the two muscles are inseparably united, there are indications of the manner of their junction. The joint tendon, at a point midway between the humeral end and the point of separation of the two muscles, is wholly tendinous as to the distal fifth of the caudal surface which seems to 
form the direct continuation of the latissimus, while the remainder is chiefly muscular. The cephalic surface presents the opposite appearance, being muscular as to its proximal fiftb. In general, it is as if the shorter tendon of the teres were applied upon the longer tendon of the latissimus in such a way that the distal border of the latter shows upon the caudal surface of the joint tendon, while the proximal border of the former shows upon the cephalic surface.

The area of attachment (Fig. 71, 72) is about one seventh of the length of the entire humerus, and forms an elongated fossa upon the caudal surface, near the ventral border; its proximal end is opposite the distal end of the area of insertion of the short head of the coracoideus.

\section{CLAVO-DELTOIDEUS.}

$\$ 637$. Synonymy.-The clavinular portion of the human deltoideus, G., A, 444 ; Q.,

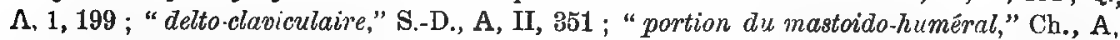
209 ; portion of the mastoido-humeral, Ch. (F1.), A, 197 ; portion of the cephalo-humeral, Miv., B, 147; Wood, 9, 101.

Figures.-Ectal aspect, clavicular end (66); ectal aspect of whole (7?, right side); ental aspect of antebrachial part (72, left side).

Posture.-Latericumbent, the venter toward the dissector. Secure the arm caudiducted so as to stretch the muscles upon the ventral aspect of the brachium and shoulder.

Exposure.-The proximal end of the muscle was exposed during the exposure of the clavo-trapezius. Connect the vertebro-presternal incision made in exposing the trapezii with the dorsal border of the antebrachium, at the junction of the proximal and middle thirds, by an incision along the ventral border of the brachium and passing caudad of the elbow. Reflect both edges of the skin for 2-3 cm. near the shoulder, and for 1-2 cm. near the elbow.

General Description.-Tæniate; along the ventral aspect of the brachium, from the clavicle to the ulna.

Dissection.-Draw the clavicular portions of the clavo-trapezius and clavo-mastoideus away from the body so as to expose the more or less distinct bands-hardly deserving the name of ligamentswhich pass from the ends of the clavicle to the shoulder and neck. In a subsequent dissection of the parts these connections may be studied in detail before division. Divide them and draw the same muscles cephalad so as to render tense the clavo-deltoideus, and indicate the general position and direction of its borders.

The caudal border begins at the sternal end of the clavicle, 
crosses obliquely the subjacent ectal lamina of the $M$. ectopectoralis (Fig. 72, right side), and, at the junction of the proximal and middle thirds of the brachium, is separated by only a slender line of connective tissue from the cephalic division of the pecto-antebrachialis.

The cephalic border is the direct continuation of the caudal border of the clavo-trapezius, but is attached quite firmly to the strong fascia covering the cephalic surface of the brachium. The muscle, with the clavicle attached, may now be dissected up as far as the ventral border of the antebrachium, but the distal half of the caudal border cannot usually be separated from the pecto-antebrachialis without cutting fibers. The examination of the insertion can be made more easily after the removal of some other muscles.

\$638. Origin.-As stated under the clavo-trapezius $(\$ 615)$, the clavo-deltoideus, viewed from its ectal surface, is apparently the direct continuation of that muscle; the two are really separated only by the transverse raphé, excepting that the ental layer of fibers of the clavo-deltoideus arises from the ventral border of the clavicle directly, or-near its scapular end-by short tendinous fibers.

Insertion.-At the ventral border of the antebrachium the cephalic border of this muscle is firmly connected with the general antebrachial fascia, and, at 8-10 $\mathrm{mm}$. from the ulna, the muscle joins the brachialis to be inserted with it, by a flat tendon, upon a rough and sometimes slightly depressed area on the caudal aspect of the ulna, just distad of the greater sigmoid notch, and about midway between the dorsal and ventral borders. See $\$ 693$.

Remark.-By its origin this muscle seems to be a member of the deltoid group of muscles, but its insertion associates it, functionally, with the flexors of the antebrachium. When the clavicle is wholly absent, as with the horse, etc., the MM. clavo-deltoideus, clarotrapezius and clavo-mastoideus seem to form a single muscle, the cephato-humeralis.

$\S 639$. Explanation of Fig. 72. - The pectoralis group of muscles, partly dissected, seen from the ventral aspect. The neck is toward the observer. The right and left of the figure correspond in position with the right and leit of the observer $(\$ 56)$.

Preparation.-The cat is dorsicumbent, resting upon the right side more than the left. The figure includes the thorax and caudal part of the neck, together with the arms to a little distad of the elbows. The arms are pulled away from the trunk so as to put the pectoral muscles upon the stretch. On the right side, the borders of the muscles have been defined by the removal of the fat and con- 
nective tissue, and in some cases the ectal layers have been slightly displaced. On the left the divisions of the ectopectoratis and pectoantebrachialis have been reflected, cut short or wholly removed; on this account the cephalic part of the mesal or interpectoral raphé is drawn dextrad out of line with the caudal part, giving a somewhat distorted appearance to the entire figure. The raphé itself is too sharply defined.

This figure fairly illustrates the crossing of the pectoral elements which is commented upon in $\$ 641$.

The figure represents the condition of things in the preparation from which it was taken ; but in some respects, especially as to the marked subdivision of the caudal division of the entopectoralis and the non-attachment of the xiphi-humeralis to the xiphisternum, it hardly indicates the usual arrangement.

Bones, etc.-Cartilago-The first costicartilage (Fig. 30, 49).-The costal end of this just appears on the left side.

Clavicula (\$ 422).-The position of the left clavicle is nearly iuverted with respect to its normal position, on account of the reflection of the clavicular end of the clavo-deltoideus. From its mesal and lateral ends are strips of fascia or thin ligaments passing respectively to the presternum and to tise muscles upon the scapula. On the right side the sternal end of the clavicle appears near the cephalic end of the mesal raphé.

Costa (Fig. 30).-Part of the first rib appears on the left side.

Epigastrium (\$ 228). -The name is written across this region, just caudad of the xiplisternum; the space was vacated by the removal of the $M M$. rectus, ectobliquus and other constituents of the abdominal parietes.

Trochin (Fig. 30, 46, $\$ 420$ ). -This, the "lesser" humeral tuberosity, appears on the left between the humeral ends of the caudal and cephalic divisions of the entopectoralis.

Xiphisternum (Fig. 49, $\$ 496$ ).-In some cases the xiphi-humeralis is attached to nearly the whole length of the narrower portion of this last sternal segment.

Muscles.-The following are not particularly described in this work; sterno-hyoideus, sterno-thyroideus, ectobizqus and rectus. The thoracic continuation of the last is shown in Fig. 73.

Biceps ( $\$ 691)$. -The distal part of this appears on both sides. On the left side its tendon (tendo bicipitis) may be seen just ventrad of the trochin.

Clavo-deltoideus (\$ 651). - The right is but slightly displaced; the left las been tran. sected and reflected.

Clavo-mastoideus (\$ 625) and clavo-traperius (\$ 615). -The edges of the clavicular ends of these muscles are seen on the right side of the neck.

Dermo-humeralis ( 829 ). - On the left side a fragment of this is seen connected with the latissimus.

Ectopectoralis, $l m$. ectalis ( $\$ 649$ ). -On the right this is partly hidden by the cephalic division of the pecto-antebrachialis and the clavo-deltoideus. On the left it has been transected very near the meson, and the humeral portion is reflected.

Ectopectoralis, $2 m$. entalis, dv. caudalis ( $\$ 653$ ). - Only part of this appears on the right. The humeral portion of the left has been reflected, and part of it removed so as to leave it shorter than the dv. cephalica.

Ectopectoralis, $l m$. entalis, $d v$. cephalica $(\$ 651)$.- On the right it is entirely hidden by the ectal lamina; the left has been treated like the caudal division, excepting that the reflected lumeral end is left longer. 
Entupertoralis, do. caudalis ( $\$ 656$ ). - The right humeral portion is hidden. The left was separable into two divisions.

Entopectoruls, ds. cephalica (\$ 658). - On the right little of this is visible. On the left it is almost wholly exposed.

Epitrochlearis (\$ 681). - The left is entire, passing from the latissimus to the elbow, where it is associated with the pecto-antebrachialis. Un the right side is seen the reflected distal half.

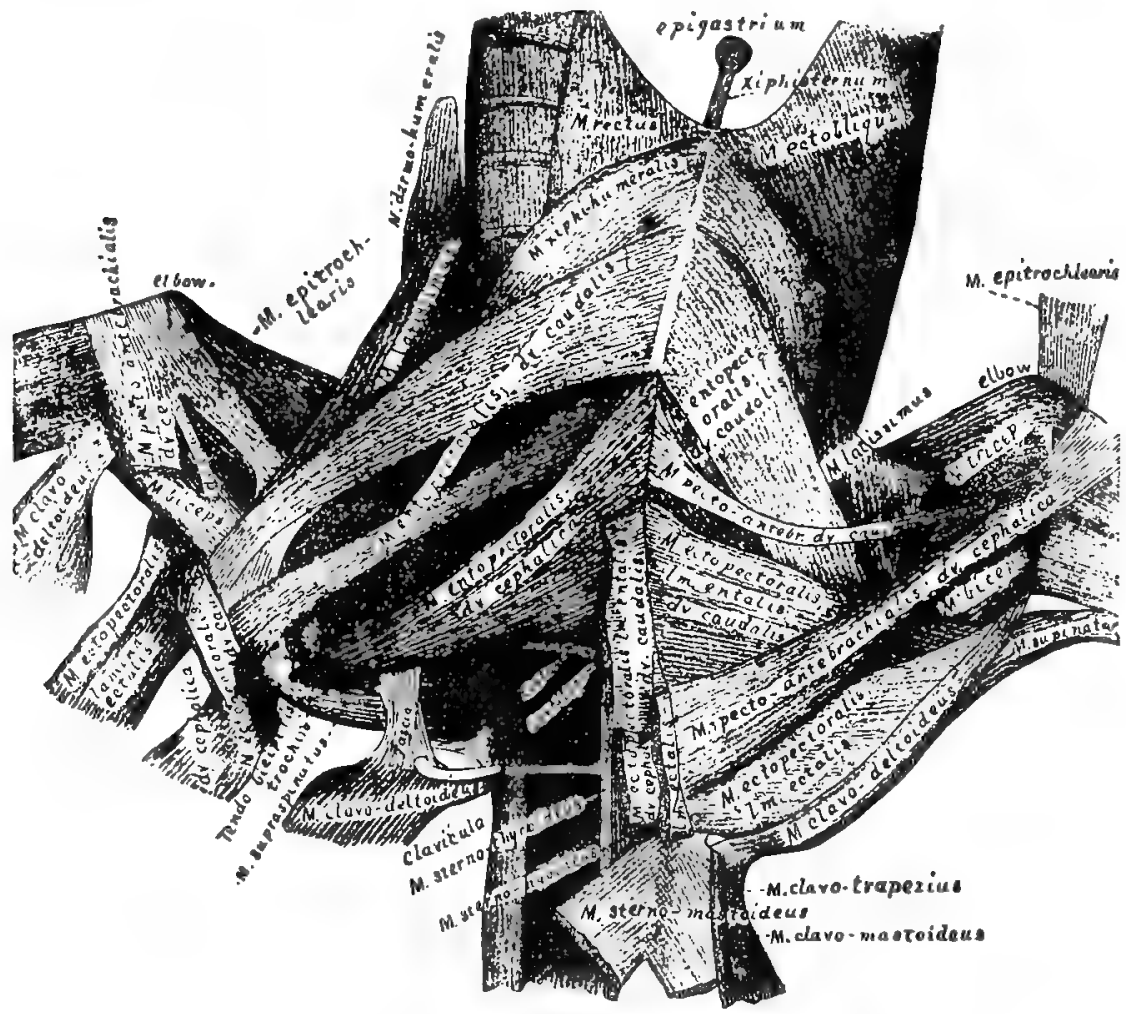

Fig. 72.-The Pectoralis Group of Mugcles, Partly Dissected.

Latissimus (\$ 635).-A fragment of the right is visible. The left has the appearance of passing ectad of the bicepss; in reality, however, most of it passes entad of the biceps, and what appears to be the continuation of the latissimus is the tendon of the xiphi-humeralis which passes entad of the caudal division of the entopectoralis.

Pecto-antebrachialis, do. cephalica (\$ 646), dv. caudulis (\$ 647). - These are shown eutire on the right, but on the left they have been removed excepting the distal ends.

Sierno-mastoideus (\$622).-The sternal ends of hoth are shown, including the mesal raphe formed by the interdigitation of their fibers.

Supinator longus (\$ 690). - Part of this appears on the right.

Supraspinatus (\$ 675). - This is more fully shown in Fig. 79, 74. 
Triceps (\$\$ 687-689).-The caudal or ectal aspect of the entotriceps appears on both sides, but the divisions are not defined.

Xiphi-huneralis ( $\$ 660)$. - On the right the sternal part is shown but not named. On the left, its course entad of the entopectoralis is indicated by the broken lines, and part of its humeral end is seen. Usually this muscle is more intimately connected with the xiphisternum.

\section{PECTORALIS GROUP.}

$\S 640$. General Remark.-The two pectoral muscles of man, ectopectoralis, "pectoralis major," and entopectoralis, "pectoralis minor," are represented in the cat by several muscles to which Straus-Durckheim and others have applied distinct names. Most of these divisions, however, may be recognized as parts of two masses, an ectal or superficial, arising nearer the cephalic end of the sternum, and extending laterad to the diaphysis of the humerus, and an ental or deep, arising from the caudal part of the sternum, and extending latero-cephalad to the head of the bone. The former, representing the ectopectoralis, tends to subdivide into superposed lamince; the latter, representing the entopectoralis, tends rather toward a division into parallel fasciculi. See §572, Humphrey, E, 110, and Wilder, 20, 306.

\$ 641. Crossing of the Pectoral Elements.-Excluding the $M$. pecto-antebrachialis, which is inserted upon the antebrachium, the pectoral mass may be roughly described as a series of four superposed laminæ crossing one another in such a way that the cephalie in origin is distal in insertion, while the caudal in origin is proximal in insertion.

The ectal lamina of the $M$. ectopectoralis (Fig. 72) arises from the præsternum and cephalad of it, and is inserted upon the middle third of the humerus. The ental lamina, as a whole, arises from the cephalic third of the sternum, and is inserted upont the proximal three fifths. The M. entopectoralis, as a whole, arises from the entire mesosternum, and is inserted upon the proximal third. Finally, the M. xiphi-humeralis arises from or near the xiphisternum, and is inserted upon the lhead and neck of the humerus.

It follows from this arrangement that the general direction of the fibers of the first portion is nearly transverse ; that, in the natural attitude of the arm, for a part of their course at least, the fibers of the last portion run nearly parallel with the meson; while the directions of the other two portions are intermediate.

A somewhat similar relation exists between the less distinctly separable regions of the human ectopectoralis as described by Gray (A, 400) and Quain (A, I, 193).

The insertion lines of the two Jaminæ of the ectopectoralis are nearly parallel, but almost meet at their distal ends (Fig. 69). If they were continuous, they might be described as a single line folded upon itself, and their tendons would be strictly comparable with the tendon of the human pectoralis major as described by Gray and Quain.

\$ 642. The Pcctoral Complexity.-In the cat there may be recognized eight or nine elements of the pectoral mass, more or less independent as to origin or insertion or both. In man, the $M$. entopectoralis $(P$. minur $)$ is distinct, and the $M$. ectopectoralis is more or less readily in different subjects separable into two or three portions, whose origins and insertions, however, are nearly or quite continuous.

It may be said, therefore, that the provision for separate and independent movement 
by means of the pectoral muscles is twice as great in the cat as in man. This will hardly surprise those who have watched a kitten at play, or a cat in any kind of vigorous action. It must be remembered, bowever, that this superior complexity of the muscles acting upon the brachium, aud thus upon the limb as a whole, does not confer peculiar powers upou the distal segment, and no one would regard the cat's manus as equal to that of man. In the quadruped, the specialization is proximal and the distal parts are relatively simple; with the bimanous biped, the muscles acting upon the arm as a whole are comparatively simple and in what may be regarded as a generalized condition, but the projection of the brachium from the thorax confers great freedom of movement, while the distal muscles are more distinct and independent than in the cat.

Those who are disturbed that any parts of a cat should be described as more complex than the corresponding human organs should compare the stomach and brain of man with the same parts of the pig, sheep and porpoise.

$\S 643$. Caution.-Excepting the muscles especially related to the vertebral column, there are probably none more difficult of dissection than the pectorales. This is due in part to the number and extent of the individual variations which have so far made it impossible to provide directions to meet all cases, but chiefly to the intrinsic complexity of superposition and attachment.

The student should proceed with great caution, follow the directions and descriptions as closely as possible, repeat the dissection upon the opposite side, and make careful notes and drawings of all peculiarities.

$\S 644$. Exposure.-As with the trapezins group, it is usually more convenient to expose all of the pectorales by lifting a single flap of skin.

Connect the antebrachial end of the incision made in exposing the clavo-deltoideus with the free border of skin left in exposing the dermo-humeralis and latissimus, or with the epigastrium (Fig. 72). Begin with the skin already raised from the ventral aspect of the brachium, and reflect the flap just circumscribed across the ventrimeson. 'To cut as nearly as possible in the direction of the fibers, the flap may be grasped at first by the angle near the shoulder, but later by its caudal margin. Great care must be taken to avoid injuring the subjacent muscles.

\section{PECTO-ANTEBRACHIALIS.}

§ 645. Synonymy.- "Pecto-antébrachial," S.-D., A, II, 352 ; "sterno-aponéurotique," Ch., A, 247 ; sterna-aponeuroticus, Cl. (Fl.), A, 232 ; part of " pectoralis, part 1," Miv., B, 145 ; not normally represented in man.

Figures.-Ectal aspect of both divisions ( ${ }^{1} 2$, right side; distal ends (72, left side).

General Description.-In two divisions, cephatic and caudal, from the median raphé at the prosternum and $3 d$ mesosterneber 
respectively, to the dorsal border of the antebrachium near the elbow.

Posture.-Dorsicumbent, the head toward the dissector ; a block under the shoulders so that the head and neck hang down.

$\S 646$. Dv. Cephalica.-Dissection.-The cephalic border has been indicated during the dissection of the clavo-deltoideus, to which it is attached excepting at the ends. Trace it for $2-3 \mathrm{~cm}$. both ways from the middle. Then feel upon the meson, about $3 \mathrm{~cm}$. from the tip of the præsternum, for the elevation corresponding with the first sternal node, or for the attachment thereto of the second costicartilages. Laterad from that point runs a white line, which marks the caudal margin of the muscle. Toward this line dissect up the muscle from its cephalic border, at about the middle of the length of the latter, and transect.

In reflecting the mesal part of the muscle, note its close attachment to the subjacent ectopectoralis, and that it joins its platetrope by a median raphé. The distal part of the muscle is much more easily separable from the subjacent muscle, but, about $1 \mathrm{~cm}$. ventrad of the level of the antebrachium, its caudal border is joined by the caudal division of the muscle. At this point the muscular fibers of both divisions are replaced by tendinous fibers.

The tendon thus formed seems to be continuous with the general antebrachial fascia, but, if this fascia be divided along a line corresponding with the cephalic border of the muscle, the tendon may be traced across the caudal surface of the antebrachium and found to terminate upon the dorsal aspect of the ulna. The examination of the details of the insertion may be deferred until after the dissection of the caudal division.

Origin.-From a median raphé common to it and its platetrope, and extending the whole length of the prasternum, excepting, sometimes, its caudal or cephalic 1-2 $\mathrm{mm}$.

Insertion.-By tendinous fibers along the distal third of the oblique caudal border of the subcutaneous surface near the proximal end of the dorsal aspect of the ulna. The cephalic border of the tendon is closely attached for part of its length to the caudal border of the clavo-deltoideus, and its caudal border is continuous with the fibers forming the tendon of the caudal division of the muscle.

\$ 64\%. Dv. Caudalis.-Posture and Exposure as with the cephalic division. The muscle is very slender and closely attached 
to the neighboring muscles, so that its isolation is not always easy. Sometimes it is absent altogether.

Dissection. - The distal part of the cephalic border has been indicated as united with the caudal border of the cephalic division. It is here $\mathbf{1 . 5 - 3} \mathrm{mm}$. wide, but widens gradually as it crosses the axilla and nears the meson.

At $3-5 \mathrm{~cm}$. from the meson, it leaves the border of the cephalic division, and becomes attached with equal closeness to the caudal border of the ental lamina of the ectopectoralis, which it accompanies, until it reaches the meson at the $3 d$ mesosterneber, about the middle of the length of the sternum, exclusive of the xiphisternum.

$\S 618$. Origin.-By very short tendinous fibers, from the meson of the $3 \mathrm{~d}$ or 4 th mesosterneber, just.caudad of the origin of the ental layer of the ectopectoralis. Sometimes the origin on one side is just caudad of that on the other. At the meson its width is 10-15 mm., but it narrows greatly toward the distal end. The caudal border of the distal end is connected with the epitrochlearis.

\section{ECTOPECTORALIS.}

§ 649. Synonymy.-The human pectoralis mrjor, G., A, 399 ; Q., A, II, 193 ; "large pectoral," S.-D., A, I, 342.

Exposure.-By the reflection of the MM. clavo-deltoideus and pecto-antebrachialis.

IAMINA ECTALIS.

Synonymy.-- Le premier chef du large pectoral," S.-D., A, I, 343 ; pectoralis, part 2, (in part), Miv., B, 146.

Figures.-Part of ectal aspect (72, right side); ental aspect of humeral end 172 , left side) ; insertion line $(68,69)$.

General Description.-Wide, tæniate, from the dorsimeson, at and cephalad of the prosternum, to the middle third of the ventral border of the humerus.

Dissection. - The caudal border extends almost directly laterad from the presterno-mesosternal node, where its mesal end underlies the mesal end of the caudal border of the cephalic division of the $M$. pecto-antebrachialis. Lift it with great care at about the middle.

The cephalic border lies nearly parallel with the caudal, at a distance of $2-3 \mathrm{~cm}$., and extends almost directly laterad from the point where the caudal end of the sterno-mastoideus (Fig. 72) passes entad of the pectoral mass. In well-injected specimens this border is indicated, at about the middle of its length, by the disappearauce, entad of it, of an artery which has emerged from the thorax and curved over the cephalic border of the pectoral mass. 
Lift the cephalic border at its middle and relax the parts so as to permit dissection entad of the muscle from one border to the other ; then transect.

In reflecting the mesal end of the muscle, cut an artery and nerve which emerge from the subjacent ental layer, and note that, near the meson, the cephalic border curves cephalad. The ental surface of the lateral part of the muscle is often so closely joined with the subjacent muscle that there is danger of cutting fibers. The tracer should be used in tearing the connective tissue until the bone is reached.

\$650. Origin.-From a median raphé common to it and its platetrope ; the caudal half or three fifths of the raphe is attached to the presternal keel, the remainder is continuous with the line of union of the caudal portion of the $M M$. sterno-mastoidei.

Insertion.-The middle of the length of the line of insertion corresponds closely with the middle of the length of the humerus, but the insertion includes rather more than a third of the length of the bone. Its distal end is almost in line with the middle of the distal end of the bone, but its proximal end is nearly midway between the ventral and cephalic aspects. The caudal border of the line of insertion is well defined, but the cephalic is not so clearly separable from the insertions of the spino-deltoideus and brachialis, and a strong fascia sometimes extends proximad from the border of the muscle toward the trochiter.

LaMLNa ENTALIS, DV. CEPHALICA.

§ 651. Synonymy.- " Le second chef au large pectoral, sa partie antérieure," S.-D., A, II, 343 ; pectoralis, part 5, "subclavicular part," Miv., B, 147.

Figures.-Sternal end (72, left side); humeral end, reflected (72, left side); insertion area $(68,69)$.

General Description.-Narrower and thicker than the ectal layer; 15-18 $\mathrm{mm}$. wide; from the prosternum and raphé to the proximalfourth of the cephalic side of the humerus.

Dissection. - The artery and nerve mentioned ( $\$ 649)$ as passing from the ental to the ectal layer of the ectopectoralis usually penetrate the former through a narrow interval about one third of the distance from the meson to the humerus. This interval usually marks the line of separation between the cephalic and caudal division of the ental lamina of the ectopectoralis. Mesad of the inte:val, the plane of separation is at a right angle with the surface of 
the muscle, but laterad of it the plane becomes oblique, with a dorso-cephalic direction which is more marked nearer the humerus. Transect by an ecto-ental incision opposite the artery.

In reflecting the sternal part, note the close union of the ental surface near the meson with the ectal surface of the sterno-mastoideus. In reflecting the humeral part, note that, excepting at the candal border, the muscular fibers cease along the ventral margin of the trochiter.

$\S 652$. Origin.-The caudal two thirds is from the lateral surface of the præsternum cephalad of the attachment of the first costicartilage; the cephalic third from the median raphé and from the ectal surface of the sterno-mastoideus.

Insertion.-Along a curved line upon the cephalic surface of the proximal fourth or fifth of the humerus (Fig. 68, 69). The line of insertion begins $3-4 \mathrm{~mm}$. proximad of the proximal end of the insertion of the ectal lamina, and sometimes very slightly ventrad of it. The distal two thirds of the line extend dorso-proximad to a point just ventrad of the slight elevation ( $T b c l$. micostale, see $M$. micostalis), distad of the Fs. trochiteriana (Fig. 45, B), where it usually turns slightly ventrad so that the proximal third forms an angle of 20-30 degrees with the rest. The line ceases about $3 \mathrm{~mm}$. from the proximal border of the trochiter at the edge of the insertion of the M. supraspinatus.

LAMTNA ENTALIS, DV. CAUDILIS.

§ 653. Synonymy.- "Le second chef du lurge pectoral, sa partie postérieure," S.-D., A, II, 313 ; pectoralis, part $\cong$ (in part), Miv., B, 146.

Figures. - Part of ectal aspect (71, right side); humeral end, reflected (72, left side); insertion line (69).

General Description. - The widest and longest portion of the ectopectoralis, excluding of course the perto-antebrachialis; from the cephalic part of the sternum to the second and third fifths of the ventral border of the humerus.

Dissection. - The cephalic border has been indicated during the dissection of the cephalic division, and the caudal border during the dissection of the caudal division of the pecto-antebrachialis. Lift both borders, but dissect up the middle of the muscle from the cephalic toward the candal border, using the tracer and the handle of the scalpel so as to aroid cutting the subjacent muscles; then transect, and reflect both ends until bone is reached. 
$\S 654$. Origin.-By fleshy fibers, from the lateral border of the presternal keel, and from the first mesosterneber and part of the second, thus filling the interval between the origin of the cephalic division and that of the pecto-antebrachialis, do. caudalis.

Insertion.-Along a line occupying, approximately, the second and third fifths of the ventral border of the humerus, thus ventrad of the lines of insertion of the cephalic division, and of the ectal lamina. The insertion is somewhat variable in detail, but a simple form is the following: The cephalic half continues fleshy to the bone, while the caudal half is inserted by a thin tendon $3-7 \mathrm{~mm}$. long. The proximal end of the line of insertion is nearly opposite, but $2-3 \mathrm{~mm}$. ventrad of, the distal end of the line of insertion of the cephalic division, and the distal end is close to the distal end of the line of insertion of the ectal lamina.

\section{ENTOPECTORALIS.}

$\S 655$. Remark.-The remainder of the pectoral mass forms at least three divisions which are sufficiently distinct in origin or insertion to warrant separate descriptions, but which, perhaps, are all parts of what may be regarded as a large representative of the "pectoratis minor" of man (\$572).

These muscular divisions are very variable in number, form, connection with each other and osseous attachment. In respect to size there are marked differences between individuals; in young or feeble cats, the masses may be not only thin, but more or less subdivided, while in adult or robust animals, they are sometimes almost continuons with each other.

Exposure.-So much as was not concealed by the $M$. ectopectoralis is covered by a dense layer of connective tissue which must be removed.

DV. CAUDALIS.

§ 656. Synonymy.-_"Le premier chef du grand pectorul," S.-D., A, II, 341 ; pectoralis, part 3 (in part), Miv., B, 147.

Figures. -The ectal aspect (172, both sides); humeral end, reflected (73); insertion line $(69,70)$.

General Description.-A thick band, from the 6th mesosterneber and sometimes the xiphisternum to the proximal half or two fifths of the ventral border of the humeral diaphysis.

Dissection.-The line of separation between the cephalic and caudal divisions is about midway of the width of the whole mass, at about $3 \mathrm{~cm}$. from the humerus; it coincides nearly with a line drawn from the surgical neck of the bone in the direction of the fibers of the muscle. The degree of separation varies greatly, and 
sometimes one or both of the divisions show signs of subdivision. In the specimen figured (Fig. 72), the cephalic division presents two well-marked subdivisions.

Near the humerus the interval between the two divisions is usually wide, but toward the sternum the cephalic border of the caudal overlaps the caudal border of the cephalic division, and sometimes their separation cannot be effected without cutting fibers. At the sternum, however, the overlapping sometimes hardly exists.

The caudal border has been exposed during the dissection of the latissimus and dermo-humeralis, whose humeral ends are connected with this muscle at the bicipital arch (Fig. 73); but the sternal half of this border is closely united with the cephalic border of the xiphi-humeralis, which it overlies in its humeral half. The xiphi. humeralis may be recognized by its loose and wide origin at the epigastrium. Carefully disengage the two muscles at their crossing, then transect the caudal division of the entopectoralis.

$\S 65 \%$. Origin.-By fleshy or short tendinous fibers from the border of the last 2 or 3 mesosternebræ, and sometimes from the cephalic part of the xiphisternum, and from the intervening nodes.

Insertion.-Variable and complex, and not easily described, excepting in connection with the other elements of the bicipital arch. At about $3 \mathrm{~cm}$. from its attachments to the ventral border of the humerus the caudal border is connected with the latissimus, and its ental aspect, along an oblique line passing proximo-cephalad, is sometimes united with the thin tendon of the xiphi-humeralis. For the sake of distinctness, this union may be severed close to the ental surface, some fasciæ passing from the caudal border of the tendon to the surface of the $M$. biceps may be removed, and the extent of the true insertion may be seen more distinctly.

Like that of the caudal division of the ental layer of the ectopectoralis, the insertion is partly muscular and partly tendinous. The line of attachment is about $3 \mathrm{~cm}$. long, and extends from the base of the trochiter along the ventral border of the humerus to a point near the junction of the second and middle fifths of the length of the whole bone, and opposite the junction of the muscular and tendinous parts of the insertion of the caudal division of the ental lamina of the ectopectoralis. The proximal third or two fifths of the insertion is fleshy, the rest is a thin tendon about $1 \mathrm{~cm}$. long. 
DV. OEPHALICA.

\& 658. Synonymy.- "Sterno.trochitérien," S.-D., A, II, 337 ; pectoralis, part 3 (in part), Miv., B, 147.

Figures.-Ectal aspect (72, left side); humeral end, reflected (73); insertion area (68, $69,70)$.

General Description.-A thick band, much widened at the sternum; from all the mesosternebra, excepting the first and sixth -or fifth and sixth - and from the intervening and terminal nodes, to the head of the humerus.

Exposure.-Both borders have been exposed, the cephalic by the reflection of the ectopectoralis, the caudal by the reflection of the caudal division of the entopectoralis.

Dissection.--The humeral end must be reflected with great care, and the preliminary examination should be made with the tracer rather than with the scalpel. Particular pains should be taken to avoid cutting or breaking a slender tendon which sometimes extends from the cephalic border, close to the humerus, to the coracoid process of the scapula (Fig. 73, Tn. $x$.).

The ectal fibers of the muscle cease at the supraspinatus, and seem to be inserted upon it, but the coracoid margin of the latter muscle may be dissected up for 1-5 mm.; there will be exposed a tendinous continuation of the entopectoralis, which, as to its cephalic half, cannot be separated farther from the tendon of the supraspinatus without cutting the tendinous fibers.

$\S 659$. Insertion.-At the border of the overlapping supraspinatus the fleshy part of the present muscle is replaced by a tendon which is attached to the caudal aspect of the trochiter, and along its ventral border ; this attachment is in line with the insertion of the caudal division, and terminates $1-2 \mathrm{~mm}$. from its proximal end. The caudal half or third of this tendon is thin; the rest is thick and fused with the ental surface of the tendon of the supraspinatus so that the respective areas of attachment can be ascertained only approximately. The latter muscle, however, is on the ectal side, and occupies the crest and cephalic aspect of the trochiter.

In addition to the tendon of direct insertion, a slender band sometimes extends from the cephalic border, just at the junction of the muscle and the tendon, and is attached to the border of the coracoid process between its tip and the prominent coracoid lip of the glenoid fossa. This tendon probably represents the coracoid 
insertion of the entire entopectoralis in some monkeys and-more commonly-in man. As to the frequency of the humeral insertion of the human $M$. entopectoralis, see Macalister, Proc. Roy. Irish Acad., X, 142.

\section{XIPHI-HOMERALIS.}

$\S 660$. Synonymy.- " Le second chef du grand pectorul," S.-D., A, II, 341 ; pectoralis, part 4, Miv, B, 147.

Figures.-Part of ectal aspect (72); humeral end, refected (73); insertion spots (69).

Exposure.-By the reflection of the other portions of the pectoral mass.

General Description.-The longest and most slender member of the pectoralis group ; from the median raphé at the epigastrium to the proximal end of the humerus. The length of the cephalic border is sometimes $20 \mathrm{~cm}$., while the width at the middle is only $4 \mathrm{~mm}$.

Dissection and Origin.-The caudal border was exposed during the dissection of the MM. latissimus and dermo-humeralis; the cephalic border during the dissection of the caudal division of the M. entopectoralis. Transect the muscle near the middle.

In reflecting the proximal part, note that, $1-2 \mathrm{~cm}$. from the meson, the loose connective tissue between it and the thoracic parietes is sometimes replaced by a firm tendinous attachment to the 8th costal cartilage and to the fascia covering the $M$. rectus; the muscular fibers cease at about the same point, and the thin, wide tendon is connected with its platetrope by a median raphé, the position of which, as regards the xiphisternum, is quite variable.

$\S 661$. Insertion.-The humeral connections are complex and variable. In passing the latissimus, it is usually connected, by tendinous fibers, with the ental surface of that muscle, then, indirectly, with the entopectoralis and the other elements of the bicipital arch. Just beyond this connection the narrow muscle is replaced by a tendon which usually widens as it nears the humerus. In the broad sheet so formed may usually be detected three more or less distinct bands with attachments as follows (Fig. 66, 71, 73): upon the bicipital border of the trochin, just cephalad of the insertion of the M. subscapularis ; (B) and (C) just caudad of the insertions of the cephalic and caudal divisions of the entopectoralis. As a whole, therefore, the tendon spans the bicipital groove. 
ARCUS BICIPITALIS-THE BICIPITAL ARCH (Fig. 73).

$\S 662$. This name is given to the tendinous arch through which passes the $M$. biceps (Fig. 73, 75). In man, normally, the ectopectoralis passes ectad of the biceps, while entad of it pass the tendons of the latissimus and teres. In the cat, as in the Mammals generally, there is a union of the ental with the ectal muscles so as to form a complete arch over the biceps. The ectal, or cephalic, pillar of the arch is formed by the caudal division of the entopectoralis; the ental, or caudal, pillar, by the teres and latissimus, while the xiphi-humeralis and dermo-humeralis are connected with one or the other pillar, with the muscles composing them, or with the convexity of the arch itself.

Explanation of Fig. 73.-The ental aspect of the muscles about the left shoulder, and the ectal aspect of the MM. serratus magnus and levator anguli scapuloe.

Preparation.-After the dissection and reflection or removal of the muscles of the trapezius and pectoralis groups, and of the other muscles already described as connecting the soma with the arm and shoulder girdle, the arm and scapula were turned dorsad.

Certain muscles (scaleni) not described herein have been wholly removed from the neck and cephalic part of the thorax, and of the M. ectobliquus (abdominis) there is left little more than the first six digitations. There are thus exposed the $M$. rectus with its wide, thin tendon, and parts of the costal cartilages $7-8$, with the intervening $M M$. intercostales.

Bones, etc.-Arcus bicipitalis, with its cephalic and caudal pillars (Clm. cephalica and caudalis)-The bicipital arch $(\S 662)$. - This is seen to embrace the M. biceps. There is considerable variety in the mutual relations of its constituents. In the preparation figured, the $M$. latissimus might be said to enter into the composition of both pillars, and the M. dermo-humeralis does not directly reach the arch at all.

Costa and cartilago (\$ $\$ 41,442$ ). - The first rib is exposed and the name is written thereon. The sternal ends of the eighth, ninth and tenth ribs are seen near the cut border of the $M$. ectobliquus. The first costicartilage affords insertion to the tendon of the $M$. rectus, and parts of the seventh to the tenth appear between the margins of the MM, rectus and ectobliquus; elsewhere their position is indicated by dotted lines.

Diapophyses cervicales-The transverse processes of the cervical vertebræ $(\S 481)$. These are numbered 1-7, but no name has been written near them. Note the gradual increase in the extent of their bifurcation toward the caudal end of the series.

Prc. (Processus) coracoideus-The coracoid process of the scapula (Fig. 43, 44, 45 A; $\S 389,400)$. 
Th. $x$. The tendinous slip from the border of the cephalic division of the $M$. ectopecto. ralis to the coracoid process $(\$ 659)$.

Trachea-windpipe (Fig. 89),-The transverse lines indicate merely the general appenrance of this tube, and not the number of its rings.

Xiphisternum (Fig. 49, 72, §426).

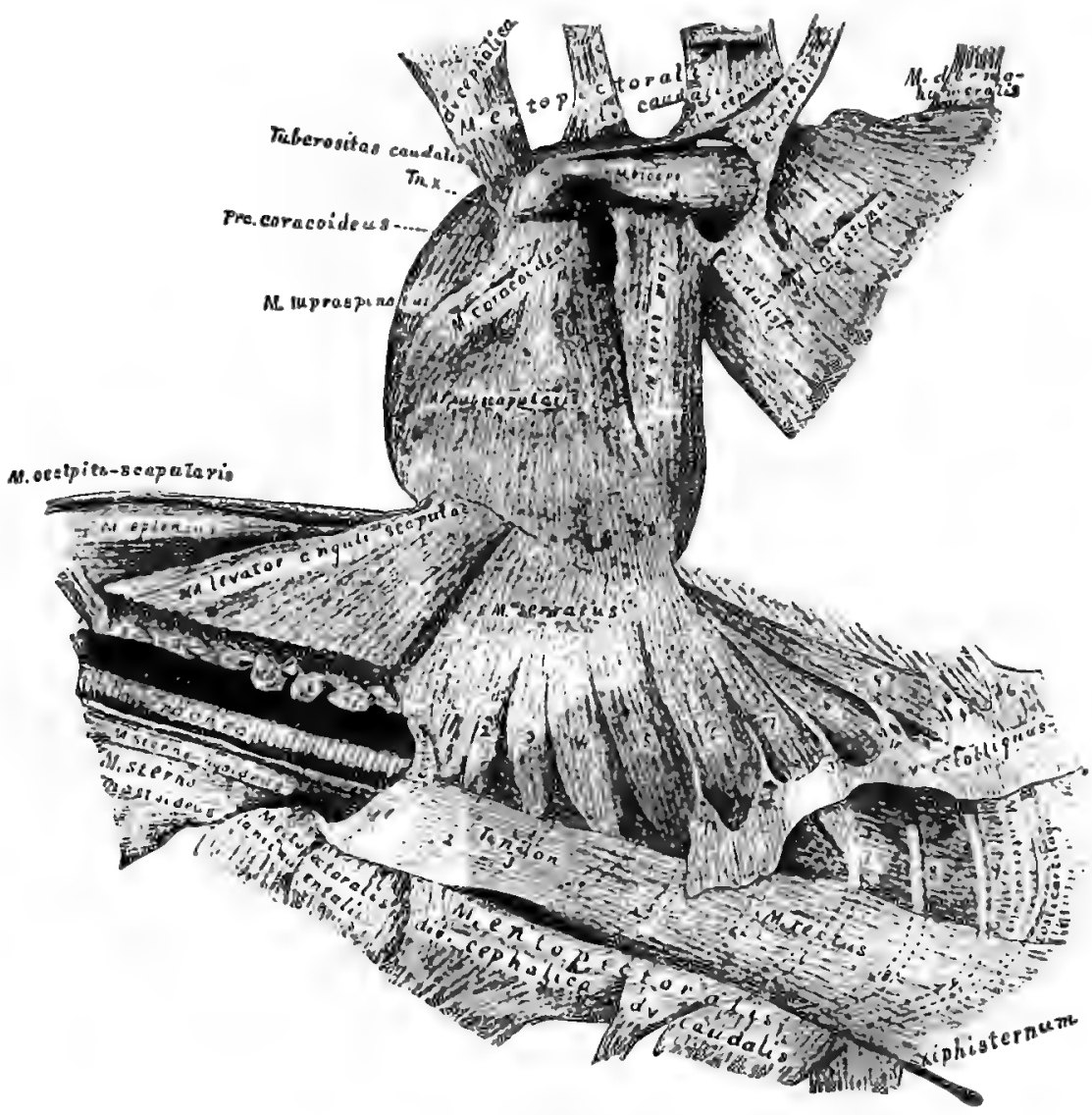

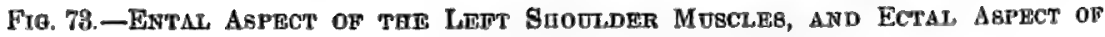
THE MM. SERRATUSALAGUS AND LEVATOR ANOULT SCAPULAR.

Muscles.-The following are not particularly described : the numbers placed after the natnes designate the other figures in which they appear :-

Splenius (67) ; sterno-thyroideus (72); rectus $(72)$; ectobliquus (72) ; intercostalis.

Biceps ( $\$ 691)$. - The proximal tendon is seen passing entad of the ligament which spans the Canalis bicipilalis (Fig. 46 and 45, B), and emerging agnin nearer the Pre. coraeoideus; it is not, however, inserted into it, but apon the Tuberculum bicipitale (Fig. 45, A).

Coracoideus (368).-Only the caput breve is seen, arising frow the coracoid process and passing to the neck of the humerus. The caput longum appears in Fig. 75. 
Dermo-humeralis $(\S 629)-A$ part of this is seen to pass to the ectal aspect of the $M$. latissimus.

Entopectoralis, dv. caudalis ( $\$ 656) .-A$ s in Fig. 72, which was taken from the same preparation, this division presents a well-marked subdivision.

Entopectoralis, dv. cephalica ( $\$ 658$ ).-Thicker than the caudal division, this is inserted chiefly upon the trochiter, but here, as in some other cases, a slender tendon (Th. $\left.x_{*}\right)$ passes to the coracoid process.

Latissimus ( $\$ 635$ ).-The ectal aspect was presented in Fig. 66 and 67 ; here are seen the ental surface and its connections with the arcus bicipitalis and the $M$. teres.

Levator anguli scapulas (\$666).-The general appearance of the muscle is fairly indicated, but the removal of the other soft parts from the line of origin has given the proximal end the look of having been cut off. The proximo-cephalic angle also extends somewhat too far cephalad. The interval between it and the serratus magnus was artificially produced. The two muscles are evidently parts of the same general muscular lamina, but there are sufficient practical reasons for treating of them separately.

Sterno-mastoideus (\$ 622). - This is better shown in Fig. 72.

Subscapularis (\$ 670).-Especially noteworthy are the appearances of its continuity with the adjacent muscles, serratus magnus, levator anguli scapula, teres and supraspinatus. Its tendon of insertion is crossed by the coracoideus.

Supraspinatus (\$ 675).-Between this and the humeral part of the subscapularis is the triangular interval mentioned in the dissection of the coracoideus (\$668).

Teres ( $\$ 680)$.-As in the other figures, the word major has been inadvertently added.

Xiphi-humeralis $(\$ 660$ ). - The humeral end is reflected like the two divisions of the entopectoralis.

Occipito-scapularis $(\S 617)$. - Its ventral margin is seen to thicken caudad.

\section{SERRATUS MAGNUS.}

\$ 664. Synonymy.-The human serratus magnus, G., A, 402, Q., A, I, 196 ; “grand dentelé" [thoracic portion], S.-D., A, II, 335 ; grand dentelé, Ch., A, 250 ; great serratus, Ch. (F1.), A, 236 ; serratus magnus, Miv., B, 145.

Figures.-Ectal aspect (73) ; scapular end (75); insertion area (43).

Posture.-Latericumbent; the venter toward the dissector; block transversely under the thorax just caudad of the elbows.

Exposure.-For the complete exposure of this muscle it is necessary to reflect all the muscles thus far enumerated, excepting the sterno-mastoideus; also to remove the larger part of the thoracic portion of the $M$. ectobliquus "external oblique muscle of the abdomen" (Fig. 73), and to remove or partly displace the thoracic portion of the $M$. rectus and some other muscles upon the cephalic region of the thorax.

Lift the elbow so that the brachium rests at a right angle with the side of the neck. Dissect out the fat and connective tissue thus exposed between the shoulder and scapula and the thorax, then divide and reflect the axillary vessels and nerves. This will permit the whole arm, with the scapula, to be turned dorsad so as to expose 
the lateral wall of the thorax. Sometimes there will be found a slender muscle extending from the muscles covering the ventral surface of the cervical vertebræ to the $M$. teres which lies along the glenoid border of the scapula.

General Description.-A thick, trapezoidal muscle, from all the ribs and cartilages, excepting the last 3 or 4, to the caudal three fifths of the vertebral border of the scapula. Its cephalie border is continuous with the caudal border of the levator anguli scapula, with which, indeed, it seems to form a single muscle. The independence of the two muscles in man results from the absence of so much of the lev. ang. scap. as-in the cat-arises from the last three cervical vertebræ.

Dissection.-Near the vertebral end of the first rib note the emergence of an artery and nerve. Extending caudad from this point note the slightly raised border of a long, flat muscle, one of the Scaleni ; divide this at the fourth rib, and reflect the two ends, to the 9th or 10th costal cartilage and to the cervical muscles respectively. Nearer the ventrimeson lies another, and wider, ribbonshaped muscle, the rectus thoracis, the thoracic continuation of the rectus abdominis. Reflect this muscle, together with the strong fascia between it and the ventrimeson, cephalad as far as the 1st rib, and caudad to the 9 th or 10 th.

Now turn the arm ventrad so as to expose the ental surface of the combined serratus magnus and levator anguli scapula. Dissect up, or divide, the rather dense fascia which extends from the dorso-caudal and dorso-cephalic borders upon the neck and thorax. Lift the arm from the thorax so as to put the whole muscle upon the stretch, and examine the ectal surface along a line extending from the point of emergence of the artery and nerve above mentioned-corresponding with the vertebral attachment of the first rib--to the vertebral border of the scapula at or near the junction of its coracoid and middle thirds, and opposite the vertebral end of the mesoscapula.

If, along this line, runs the principal branch of the artery, the division of the entire mass into a caudal part, the serratus magnus, and a cephalic part, the levator anguli scapuloe, can usually be made without cutting many fibers; but in some cases it may be better to leave the muscles connected. Note, in either case, that the $M$. serratus presents, in its thoracic half, divisions corresponding to the ribs, while the other muscle is continuous. 
Divide the connective tissue, sometimes quite firm, which unites these subdivisions, and then transect the muscle itself along a curved line about $3 \mathrm{~cm}$. from the scapula. For greater ease of examining the attachments of the subdivisions, continue the interval just caudad of the 4th rib to the cut edge of the muscle. In reflecting the two parts thus formed, note the passage of nerves and vessels to them from the intercostal spaces. The muscle is usually tender, and the tracer should be used rather than the knife in clearing away the connective tissue at the attachments of the subdivisions.

$\S 665$. Origin. $-A$ line drawn through all the origins describes about the fifth of a circle, extending from the middle of the 1st rib to the middle of the 9 th or 10 th. The 4 th subdivision extends almost directly dorsad, the first and last extend dorso-caudad and dorsocephalad respectively, while the intermediate ones vary in direction according to position.

The first subdivision arises from the 1st rib along nearly or quite the whole of its caudal border; toward the sternal end it is overlapped to some extent by the attachment of the scalenus above mentioned; the $2 \mathrm{~d}$, from the $2 \mathrm{~d}$ costal cartilage $1-2 \mathrm{~mm}$. from its union with the rib, and sometimes by a short tendon; the $3 d$ and 4th, from similar points upon their respective ribs or just at the point of union of the ribs and cartilages; the 5th, at the point of junction; the 6th, 7 th and 8 th, from their respective ribs, at gradually increasing distances from their junctions; the 9th, at about $2 \mathrm{~cm}$. from the junction. The lines of attachment of the first and the last coincide very nearly with the axis of the ribs; those of the next four are nearly at right angles, while those of the remaining three are oblique. A 10th subdivision, from the 10th rib, sometimes occurs. It should be carefully looked for.

Insertion.-The scapular attachment is continuous, but in two parts: (A) the caudal two to three fifths is by a short tendon along the ental edge of the vertebral border of the scapula; (B) the remainder is by fleshy fibers upon a triangular area near the vertebral border of the subscapular fossa, 10-15 $\mathrm{mm}$. long and 4-8 $\mathrm{mm}$. wide. The wider end of this attachment is opposite the vertebral end of the mesoscapula, and is continuous with the insertion of the levator anguli scapula. 


\section{LEVATOR ANGULI SCAPULE.}

§ 666. Synonymy.-The human levator anguli scapula, G., A, 376, Q., A, I, 192 ; "grand dentelè" [the cervical portion], S.-D., A, II, 335 ; angulaire de l'omoplat, Ch., A, 202 ; angrlur muscle of the scapula $\mathrm{Ch}$. (Fl.), A, 189 ; levator anguli scapuloe, Miv., B, 145.

Figures.-Ectal aspect (73); scapular end (75); insertion area (43).

Posture and Exposure.-These are the same as with the serratus magnus, excepting that the block may be under the neck, and the muscles (scaleni) covering the series of ventral tubercles of the diapophyses of the cervical vertebræ (Fig. 53) must be removed.

General Description.-Thick, trapezoidal, just cephalad of the $M$. serratus magnus, from the diapophyses of the $3 d-7$ th cervical vertebro to the subscapular surface of the scapula close to the vertebral border.

Dissection.-After the division of the serratus magnus, this forms the only union between the arm and the trunk. Note its compactness as compared with the muscle just named, although soinetimes the vertebral end shows signs of subdivision. Transect it at the middle.

$\$ 667$. Origin.-By fleshy fibers from the rounded ends of the dorsal tubercles of the transverse processes of all the cervical vertebræ excepting the atlas and axis, and from the ligaments between the tubercles. Entad of the attachment will be seen the shining tendons of the $M$. plagio-antobliquus cervicalis. The line of origin is about $6 \mathrm{~cm}$. long.

Insertion.-The scapular attachment is by fleshy fibers upon a triangular area about $15 \mathrm{~mm}$. long by $5-10 \mathrm{~mm}$. wide (Fig. 43). This area is continuous with the area of insertion of the serratus magnus, but the apex points in the opposite direction, that is, toward the coracoid border of the scapula. The ental surface sometimes receives the insertion of the occipito-scapularis $(\$ 617)$.

Remark.- The arm, with the scapula attached, has now been separated from the trunk, and may be dissected on a smaller tray. After reviewing the insertions of the muscles so far examined, the dissector may remove them to within $1-2 \mathrm{~cm}$. of their attachment.

\section{CORACOIDEUS.}

$\S 668$. Synonymy.-The human coraco-brachialis, G., A, 407, Q., A, I, 204 ; "corrco brachial," S.-D., A, II, 343; " coraco-brachicl," Ch., A, 268 ; coraco-humeralis Ch. (F1.), A, 254 ; coraco-brachiaiis, Miv., B, 148.

Figures.—Caput breve (73); caput longum (75) ; insertion areas (70, 71). 
Posture.-The brachium forms nearly a right angle with both the scapula and the antebrachium. Place the arm upon its cephalic surface, let the antebrachium and manus rest against the rim of the tray toward the dissector, and place a block flatwise under the scapula and shoulder so that the brachium forms an angle of about 45 degrees with the tray.

Exposure.-Remove the fascia, fat, connective tissue, vessels and nerves upon the caudal aspect of the shoulder and brachium. In doing this, watch very carefully for the slender tendon (of the caput longum, Fig. 75), which extends, in some cases, nearly the whole length of the brachium; use the tracer more than the knife, and remove nothing until sure that the tendon is not included.

General Description.-This muscle consists of two parts (caput longum and cp. breve), so distinct that, if they were larger, they probably would be regarded as two muscles (\$573). They arise from the coracoid process of the scapula and are inserted into the humerus near its proximal and distal ends. Both parts are so small as to be easily overlooked.

Dissection.-Feel for the coracoid process in the triangular interval at the head of the humerus between the distal ends of the subscapularis and supraspinatus (Fig 73, 75); the tip of the process is just at the border of the former muscle near the apex of the interval. Carefully lift the border of the subscapularis with the forceps, and use the tracer and scalpel to dissect between it and the slender coracoideus. The latter lies upon the capsule of the joint and sometimes adheres quite firmly to it.

Separate the connections, when they exist, with the tracer, and divide the muscle so as to follow the course of the shorter and more fleshy part (caput breve) from the coracoid process to the surgical neck of the humerus. The dissection of the longer and more slender caput longum should be done almost wholly with the tracer, and the delicate tendon should not be pulled in tracing it toward the distal end of the humerus.

$\$ 669$. Origin.-The common origin of the two heads from the tip of the coracoid process is by a tendon about $1 \mathrm{~mm}$. long.

Insertion. - The short head is inserted by fleshy fibers upon the caudal surface of the surgical neck of the humerus ; the length of the area of insertion is approximately equal to half the distance from its proximal end to the proximal end of the humerus, and the width equals half the length. The ventral margin of the area of its 
insertion is well defined, but the dorsal margin is in contact with the area of insertion of the proximal division of the internal head of the triceps, and sometimes fasciculi cross from one muscle to the other.

The long head is more often absent than present: it is not mentioned by Mivart, and its existence in the cat is denied by Meckel (A, VI, 281). Of the cases observed by us, no two were alike. The fleshy portion usually leaves the short head at about its middle, and is $2-3 \mathrm{~cm}$. long. Its tendinous continuation is sometimes filamentary and disappears among the intermuscular fascia; sometimes it is larger and divides, one portion joining the tendon of the epitrochlearis and the other inserting upon the humerus near the Fm. epitrochleare; more often this last is the only attachment, but the precise point varies so much that the area which was observed in one case is indicated on Fig. 71 by an interrogation point.

\section{SUBSCAPULARIS.}

\& 6r0. Synonymy.-The human subscapularis, G., A, 404, Q., A, I, 203 ; "sons-seapulaire," S.-D., A, II, 345 ; "sous-scapulaire," Ch., A, 266 ; subscapularis, Ch. (Fl.), A, 252 ; subscapularis, Miv., B, 148.

Figures.-Ental aspect $(73,75)$; origin area $(43)$; insertion area $(70,71)$.

Posture.-The same as for the coracoideus. The muscle is already fully exposed.

General Description.-Thick, subtriangular, from most of the subscapular fossa of the scapula to the trochin of the humerus.

Dissection.- In addition to the loose fascia previously removed, the free surface of the subscapularis is covered by a firmer fascia which, in places, adheres to the muscle. Complete the removal of connective tissue and fat from the triangular intervals near the glenoid ends of the glenoid and coracoid borders of the muscle, and between them and the supraspinatus and teres respectively. Note that the coracoid interval is twice the length of the other, and extends about two fifths of the length of the muscle.

Manipulate the muscle so as to indicate the direction of the fasciculi, and note that the central portion of the muscle is hidden near the glenoid end of the scapula by the converging glenoid and coracoid portions.

Transect the muscle to the bone by an incision connecting the two borders at the apices of the intervals above mentioned; bisect the vertebral end of the muscle and reflect the two sides, noting the 
extent and manner of connection between the muscle and the bone; then reflect the humeral end, noting its close attachment to the capsule of the shoulder joint.

$\$ 671$. Origin.-By fleshy fibers from the subscapular fossa, excepting: (A) the oblong area near the vertebral border which gives insertion to the levator anguli scapula and serratus magnus ; (B) an irregular quadrilateral area near the glenoid angle of the bone, the vertebral limit of which coincides nearly with the position of a vascular foramen about $1 \mathrm{~cm}$. from the lip of the glenoid fossa. In addition to this general fleshy origin from the periosteum lining the subscapular fossa, the muscle has at least two lines of tendinous attachment along the slight ridges which converge toward the glenoid angle.

Insertion.-By a strong flat tendon upon the dorsal border of the trochin of the humerus at the margin of the arthral surface.

\$ 672. Explanation of Fig. 74.-.The cephalic (outer) aspect of the left brachium and antebrachium, with the ectal muscles of the scapular region.

Preparation.-After examination of the MM. serratus magnus and levator anguli scapula, the arm with the scapula was detached from the trunk by the transection of those muscles.

The spino-deltoideus and acromio-deltoideus have been transected and reflected.

Bones, etc.-Acromion (Fig. 44, §392).-As seen in Fig. 67, the tip of this process coincides with the acromial margin of the M. acromio-deltoideus; but the muscle is here reflected so as to hide it, and the name has not been connected therewith by a dotted line.

Capitellum radii ( $\$ \$ 220,410)$. - This enlargement of the proximal end of the radius is shown but not named in Fig. 30. Its position here is nearly indicated by the beginning of the name.

Epicondylus (Fig. 30, 68, 69, 71, § 415).-The position is nearly indicated by the first letter of the name.

Olecranon (Fig. 30, § 220). - This proximal process of the ulna forms the angle of the elbow.

Trochiter (Fig. 30, 46, 68, § 405).-This has been exposed by the removal of the $M$. clavo-deltoideus (Fig. 66). By inadvertence it is marked ce.

Muscles.-The following have been sufficiently described in connection with the figures whose numbers are placed in parentheses :-

Dermo-humeralis $(66,67,72,73), \$ 629$; latissimus $(66,67,72,73)$, § 635 ; rhomboideus (67), $\$ 620$; suprcspinatus (67), $\$ 675$; teres, "teres major," (67), $\$ 680$.

Acromio-deltoideus (\$ 676) and spino-deltoideus ( $\$ 674)$. - These two muscles have been transected and reflected. The name of the former is written across the scapular end of both. 
Biceps ( $\$ 691)$. - After transection, the distal part of this was left in place; the proximal part is hidden, but appears in Fig. 75.

Brachialis ( $\$ 692$ ). - The somewhat thin ectal margin of this flexor of the antebrachium gives no adequate idea of its size and the extent of its origin area (Fig. 68).

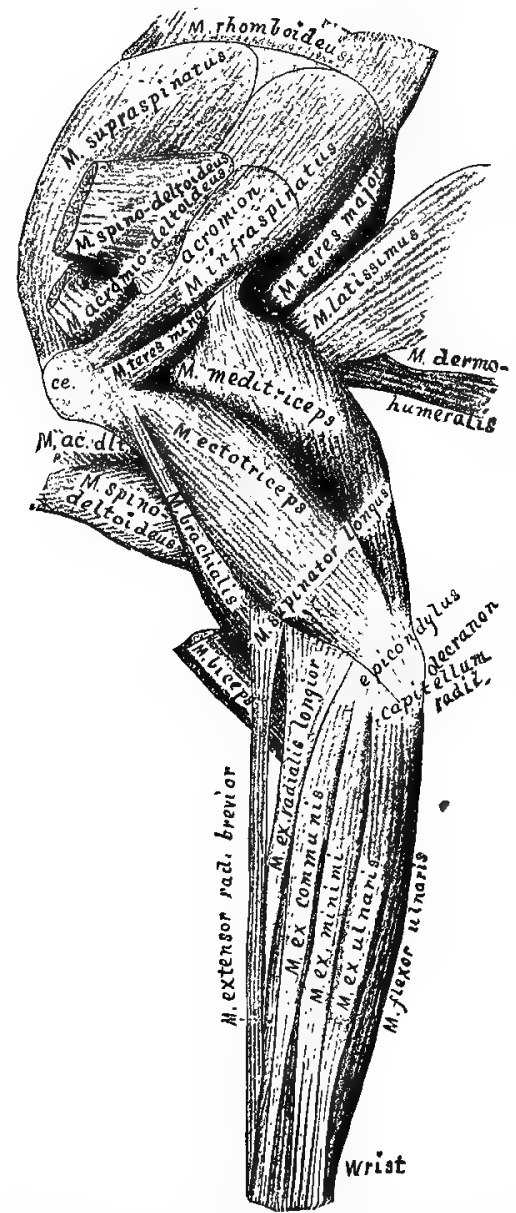

Fig. 74.- -The CePHaLic AsPeCT OF This Left ARM, with THe Ectal MusCLES OF THE SCAPULA.

Extensor digitorum communis (\$ 697).The origin areas of this and of the $e x$. minimi (§ 693) and ex. ulnaris $(\S 699$ ) are closely grouped on the epicondylus, and their bodies form a compact mass.

Extensor (carpi) radialis longior (\$694) and ex. radialis brevior ( $(696)$.- - The name is written upon the body of the former, but the latter is only partially visible, and its name is written along the side of the supinator longus.

Flexor ulnaris ( $\$$ 702). - The name is written along the side of the muscle.

Meditriceps-The "long, middle or scapular head of the triceps" (\$ 682).-The tendon of this strong muscle is comparatively small and is partly hidden by the micostalis (here called teres minor). The body of the muscle is also partly hidden by the ectotriceps.

Micostalis-"Teres minor" (\$ 679).-In the undissected arm this insignificant muscle is hidden by the acromio-deltoideus and spinodeltoideus.

Spino.deltoideus (\$ 674).-See acromio-deltoidens above.

Supinator longus $(\S 690)$ - - The $M$. of the name rests upon the proximal end of this muscle, which is seen to emerge between the $M M$. biceps and brachialis.

Teres minor-Micostalis, - See above.

\section{SUPRASPINATUS.}

\$ 673. Synonymy.-The human supra. spinatus, G., A, 405, Q., A, I, 200; “sนs-êpineur," S.-D , A, II, 336 ; "sus-êpineux, Ch., A, 265 ; supraspinatus, Ch. (F].), A, 251 ; supraspinatus, Miv., B, 148.

Figures.-Ectal aspect $(67,74)$; part of ental aspect $(73,75)$; origin area $(44)$; insertion area $(68,69,70)$.

Posture.-Similar to that for the dissection of the subscapularis, excepting that the arm should rest upon the caudal (inner) side, and the mesoscapula should be toward the observer.

Dissection.-Note that the ectal surface of the muscle is cov- 
ered by a firm fascia, which is closely attached along the border of the mesoscapula and the coracoid border of the scapula excepting where it was separated from the subscapularis. Divide these attachments, cutting from the glenoid angle of the scapula toward the vertebral border, then transect the muscle at its middle, and reflect both parts; the proximal part may be wholly removed.

Origin.-By fleshy fibers from the whole supraspinous fossa, and, by the ectal fascia, from the coracoid border of the scapula and mesoscapula.

Insertion. - The muscle passes over the capsule of the shoulder joint with but slight adhesions thereto, and ends in a short, thick tendon which is attached to the crest of the trochiter. Between the trochiter and the tip of the acromion process, the body of the muscle is closely attached to the border of the acromio-deltoideus. The distal $\mathrm{cm}$. of the other-the coracoid-border is connected with the insertion of the cephalic division of the entopectoralis, as described under that muscle $(\$ 658)$.

\section{SPINO-DELTOIDEUS.}

§ 674. Synonymy.-The spinal, or mesoscapular, part of the buman deltoideus, G., A, 404, Q., A, I, 199 ; " delto-spinal," S.-D., A, II, 338; part of “long abducteur du bras," Ch., A, 263: part of the long abductor of the arm, or scapular portion of the deltoid, Ch. (Fl.), A, 249 ; part of delloid, Miv., B, 147.

Figures.-Ectal aspect $(66,67)$; reflected (74); origin area (44) insertion area $(68)$.

Posture.-Let the arnı rest upon its caudal surface on the flat side of a block, with the elbow toward the dissector. The scapula may be permitted, at times, to hang over the end of the block, so as to render the muscle tense.

Exposure--Remove the skin upon the cephalic surface of the brachium to the junction of its middle and distal thirds. Remove the firm fascia covering the cephalic side of the shoulder.

General Description.-Thin, apparently subtriangular, but really trapezoital ; from the mesoscapula and metacromion to the deltoid ridge (Fig. 46, 68, 69), on the proximal half of the cephalic surface of the humerus.

Dissection.-The dorsal border forms a nearly direct line between the tuberosity of the mesoscapula and the humerus at the junction of the proximal and middle thirds. Lift it at its middle, where it crosses the angle formed between the muscles upon the 
glenoid border of the scapula and the dorsal border of the brachium. Here it is easily separated from the subjacent muscles, but nearer both ends it adheres very closely. Dissect the muscle up toward the tip of the metacromion. Lift the overlapping border of the acromio-deltoideus, which lies just ventrad of it near the humerus. At the side of the metacromion toward the tip of the acromion will be exposed the short, free, ventral border of the muscle. Connect the two borders and reflect the ends. In reflecting the scapular part, avoid cutting into the subjacent infraspinatus, some fibers of which take their origin from the ental surface of the spinodeltoideus. The humeral end passes entad of the acromio-deltoideus, most of the fibers of which are inserted upon its ectal surface.

$\S 675$. Origin.-By short tendinous fibers along the infraspinous border of the mesoscapula and metacromion, from the tuberosity of the former to the glenoid angle of the latter, and from a tendinous raphé between this muscle and the acromio-trapezius.

Insertion.-By a tendon 1-1.5 cm. long upon the deltoid ridge of the humerus; this extends obliquely distad along the proximal half of the shaft from the middle of the cephalic surface to the ventral border. The ectal surface of the tendon is wholly covered by the acromio-trapezius, and the line of insertion is nearly parallel with that of the insertion of the ectal lamina of the ectopectoralis.

\section{ACROMIO-DELTOIDEUS.}

§ 676. Synonymy. - The acromial, or intermediate, part of the human deltoideus, G., A, 401, Q., A, I, 139 ; "delto-acromial," S.-D., A, II, 338; part of the "long abducteur du bras," ('h., A, 263; part of the long abductor of the arm, or sccpular portion of the deltoid, Ch. (Fl.), A, 249 ; part of deltoid, Miv., B, 147 .

Figures, Posture and Exposure.-As with the M. spino-deltoideus.

General Description.-A short band, from the acromion to the deltoid ridge of the humerus.

Dissection.-The dorsal-and longer-border was lifted in order to expose the tendon of the spino-deltoideus. The shorter-or ventral-border may be traced from the tip of the acromion to a point near the proximal end of the line of insertion of the same muscle, and forms the dorsal limit of the subcutaneous area of the trochiter (marked $c e$ in Fig. 74).

$\S 677$. Origin.-By short tendinous fibers along the infraspinous 
border of the acromion, and the contiguous border of the metacromion to near the tip of the latter.

Insertion.-Most of the fibers seem to terminate upon the ectal surface of the tendon of the spino-deltoideus, but the ectal layers, especially at the borders of the muscle, are connected with the bone by a thin, tendinous sheet, which is attached along a shorter and straighter line than that of the insertion of the muscle just named and between it and the insertion of the ectal layer of the ectopectoralis. The proximal end of the line of insertion is $3-4 \mathrm{~mm}$. ventrad and distad of the proximal end of the line of insertion of the spinodeltoideus.

\section{INFRASPINATUS.}

§678. Synonymy.-The human infraspinatus, G., A, 405, Q., A, I, 200; "sous-épineux," S.-D., A, II, 344 ; "sous-épineux," Ch., A, 265 ; supraspinatus, Ch. (Fl.), A, 251 ; infraspinatus, Miv., B, 148.

Figures.-Ectal aspect $(67,74)$; origin area (44); insertion area (68).

Posture.-As for the $M$. supraspinatus.

Exposure.-By the reflection of the MM. spino-trapezius, levator clavicula, spino-deltoideus and acromio-deltoideus.

General Description.-From the infraspinous fossa to the Frossa trochiteriana of the humerus (Fig. 45, B).

Dissection.-The rounded mesoscapular border may be seen between the head of the humerus and the metacromion, where it is overarched by the acromion. The border is, for the most part, in close contact with the small micostalis, but close to the humerus is an interval filled by connective tissue. Follow this interval nearly to a point opposite the metacromion, and then divide the infraspinatus.

In reflecting the humeral part, note a synovial bursa between the tendon and the dorsal slope of the fossa of insertion. The scapular half separates readily from the micostalis (marked teres minor upon the figures), but from the teres, nearer the vertebral end of the scapula, it can be separated only by cutting fibers.

Origin.-By fleshy fibers from the entire supraspinous fossa, and by short, tendinous fibers from the raphé between it and the teres.

Insertion.-By a strong tendon into the ventral half of a depression (F's. trochiteriana, \$ 404) upon the cephalic aspect of the trochiter. The proximal end of the insertion is almost in contact with the attachment of the supraspinatus upon the crest of the trochiter. The 
insertion of the cephalic division of the ental lamina of the entopectoralis extends just dorsad from this depression, and the Tuberculum micostale is just distad of it.

\section{MICOSTALIS.}

$\$ 679$. Synonymy.-The human teres minor, G., A, 406, Q., A, I, 201 ; " micostal," S.-D., A, II, 345 ; "court abducteur du bras, ou petit rond," Ch., A, 265 ; short abductor of the arm, or teres minor, Ch. (Fl.), A, 250 ; teres minor, Miv., B, 148.

Figures.-Ectal aspect (74, where the muscle has its more common name teres minor); origin area $(43)$; insertion area $(68)$.

Posture. - As for the $M$. infraspinatus ( $\$ 678$ ).

Exposure.-By the reflection of the $M$. infraspinatus.

General Description.-Small, from part of the glenoid border of the scapula to the Tuberculum micostale (Fig. 68).

Dissection.-The mesoscapular border was exposed by the removal of the infraspinatus. The opposite border is nearly in line with the glenoid border of the scapula, and it is only necessary to remove some connective tissue between it and the oblique border of the ectotriceps, and to dissect up the muscle from the meditriceps, to which it adheres somewhat closely. In reflecting, note the close attachment of the ental surface to the capsule of the shoulder joint.

Origin.-By a sheet of tendinous fibers from the glenoid border of the scapula, beginning about one fifth of the length of the border from the glenoid fossa, and ending at its middle.

Insertion.-By a very short tendon upon the Tbcl. micostale on the cephalic aspect of the trochiter.

\section{TERES.}

\$ 680. Synonymy.-The human teres major, G., A, 408, Q., A, I, 202 ; "teres," S.-D., A, II, 339 ; " abducteur du bras, ou grand rond," Ch., A, 267 ; abductor of the arm, or teres major, Ch. (Fl.), A, 253 ; teres major, Miv., B, 148.

Figures.-Ectal aspect $(66,67,74)$; ental aspect $(75)$; origin area (43); insertion area $(69,70)$.

Posture.-The same as for the $M$. subscapularis.

Exposure.-If the bicipital arch has not been divided, it must be now ; the brachial artery and nerves accompanying it must be removed, and the body of the $M$. biceps pushed ventrad.

General Description.-A thick band; from the glenoid border of the scapula to the ventral surface of the humerus, a little distad 
of its head; associated at its origin with the subscapularis, and at its insertion with the latissimus.

Dissection.-Both borders of the muscle have been indicated during the examination of the subscapularis and latissimus. Connect them at the point of junction of the $M$. teres with the last named muscle. The scapular part of the muscle may be dissected from its attachment along the border of the scapula, but the humeral parttogether with the humeral part of the latissimus-can be reflected with ease.

Origin.-By fleshy fibers, from all but the glenoid sixth of a shallow groove along the glenoid border of the scapula; this groove intervenes between the true glenoid border and the marked ridge upon the subscapular surface which runs nearly parallel with it at a distance of $4-5 \mathrm{~mm}$. It arises also from the aponeurotic septum between it and the subscapularis.

Insertion. - This has been described in connection with the M. latissimus (\$635).

M. EPITROCHLEARIS.

$\S$ 681. Synonymy.-The dorso-epitrochlien of monkeys, and, by exception, of man, Q., A, I, 207; "triceps-interne," S.-D., A, II, 348; dorso-epitrochlear, Miv., A, 137.

Figures.-Ectal aspect (72, left side); distal end, reflected (72, right side); both ends (75).

Posture.-Let the arm rest upon its cephalic surface, with the olecranon and the gleno-vertebral angle of the scapula toward the dissector. The muscle was exposed during the dissection of the pecto-antebrachialis and clavo-deltoideus.

General Description.-A thin ribbon, from the vertebral border of the latissimus to the caudal border of the olecranon process of the ulna.

Dissection. - The ventral border was indicated in the dissection of the pecto-antebrachialis; the dorsal border may be seen if the latissimus is drawn toward the head of the humerus. Both borders should be freed from connective tissue and thin fascia; then the muscle may be divided at its middle.

Origin.-Variable and ill defined. By fleshy fibers from the ventral border of the latissimus, just opposite the oblique line of union of the latter muscle with the teres, and close to the place of reception of the dermo-humeralis; occasionally some of the fibers arise from the latter muscle. 
Insertion.-At the epitrochlea the fleshy fibers are replaced by a thin tendon which really forms part of the general antebrachial fascia, and is continuous with the tendons of the pecto-antebrachialis; so much of the fascia as belongs to this muscle is attached along the proximal 10-15 $\mathrm{mm}$. of the caudal border of the triangular subcutaneous area upon the dorsal aspect of the olecranon.

\section{MEDITRICEPS.}

\$ 682. Synonymy.-The human middle or scapular head of the triceps, G., A, 400, Q., A, I, 207; " biceps-moyen," S.-D., A, II, 348; "long extenseur et gros extensour de l'avant bras," Ch., A, 273 ; the long and large extensor of the forearm, Ch. (Fl.), A, 258; second part of the triceps, Miv., B, 149.

Figures.-Cephalic aspect $(66,67,74)$; caudal aspect $(72,75)$; origin area (43).

Posture.-At first the arm should rest upon its cephalic side, but the posture must be changed several times during the dissection.

Exposure.-By the reflection of the MM. Latissimus, teres and epitrochlearis.

General Description.-Prismatic, from the glenoid third of the glenoid border of the scapula to the olecranon.

Dissection.-At the middle of its length the ventral border of the muscle is indicated by the large nerve which lies between it and the $M$. entotriceps. Let the scapula be flexed upon the brachium so as to relax the muscle, and dissect from the caudal toward the cephalic border along its entire length, as far as the epitrochlea.

The cephalic surface is readily separable from the ectotriceps in its proximal fourth ; but for the rest of its length it is united with the dorsal border of that muscle by a strong fascia. In reflecting the distal part, note the close union of both borders of the muscle in its distal $5 \mathrm{~mm}$., and the presence of a synovial sack over the bifid tip of the olecranon.

Origin.-By a short tendon, the outline of the section of which is wedge-shaped, the base toward the glenoid end of the scapula and the apex toward the gleno-vertebral angle. The line of origin occupies nearly the glenoid third of the glenoid border, beginning about $1 \mathrm{~mm}$. from the glenoid fossa.

Insertion.-By a short tendon upon the rounded tuberosity which forms the dorsal angle of the olecranon; the ventral border of the tubercle is indicated by a slight transverse furrow.

$\S 683$. Explanation of Fig. 75.-The muscles on the caudal 
aspect of the brachium and antebrachium, and the ental aspect of the scapula.

Preparation.-The cephalic (outer) aspect of the same arm is shown in Fig. 75. The caudal aspect of the scapular region is also represented in Fig. 73, but the position of the whole limb is there reversed. The serratus magnus and levator anguli scapulce have been transected a little nearer the scapula than the rhomboideus, which appears ectad of them. The bicipital arch has been divided, and the biceps and epitrochlearis transected and reflected so as to expose the coracoideus and entotriceps. The muscles on the caudal and ventral aspect of the antebrachium have been merely freed from fat and fascia.

Bones, etc.-Antebrachium-the forearm; brachium-the upper arm or proximal segment of the arm.

Fm, (Foramen) epitrochleare (Fig, $46, \S 417$ ). - A part of its ventral orifice is covered by the humeral end of the $M$. entotriceps, dv. brevis.

Humerus (Fig. 45, 46, 68-71, $\S 407$ ).-Most of the caudal aspect of this bone is exposed ; the trochin appears at the proximal end.

Olecranon (Fig. 30, \$220).-The prominence of the elbow.

Prc. (Processus) coracoideus-The coracoid process of the scapula (Fig. 43, 44, 45, A, $\S 400$ ).-In the shaded space between the supraspinatus and subscapularis projects the tip of this process. Connected therewith are the origin tendon of the coracoideus and the tendinous slip from the insertion tendon of the entopectoralis, dv. cephatica.

Muscles.-The following have been sufficiently described elsewhere, and in the explanations of the figures whose numbers are placed in parentheses:-

Latissimus (67), § 635 ; subscapularis (73), §670; supraspinatus (67, 73), 8675 ; supinator longus (74), \& 690 .

Biceps ( $\$ 694)$.- The distal end is seen to pass to the ulna between the proximal ends of the two groups of muscles lying on the ventral aspect of the antebrachium: the flexores, including the pronator teres, and the extensores, including the supinator longus (see $\$ 695$ ). The tendon of origin passes entad of and through the Canalis bieipitalis to the Tbcl. bicipitate (Fig. 45, A) of the scapula, but in this figure it has the appearance of continuity with the slip from the entopectoralis to the Pre. coracoideus.

Brachialis (\$ 692).-Only a portion is seen between the MM. pronator and ectopectoralis.

Coracoideus (\$668).-The caput breve has been shown in Fig. 73, and appears here passing cephalad of (behind) the insertion tendon of the $M M$. teres and latissimus. 'I'he caput longum is seen to pass caudad of the same tendon, to become a very slender, threadlike tendon, and to become attached to the ventral margin of the $\mathrm{Fm}$. epitrochleare.

Ectopectoralis, lm. entalis, dv. caudalis (\$ 653). - The remnant of muscle so named evidently includes also part of the caudal division of the entopectoralis.

Entopectoralis ( $\$ 658$ ). - The name begins near the insertion tendon of the cephatic division.

Entotriceps, dv. brevis (\$ 688).-This is not only the shortest division of the entotriceps, but forms a very different angle with both the humerus and the olecranon.

Entotriceps, dv. caudalis (\$ 687); dv. cephalica (\$ 689) ; dv. intermedia (\$ 686).-The relative positions of these three divisions of the entotriceps are more clearly shown upon 
Fig. 71, where their origin areas are seen to be respectively intermediate, distal and proximal. It should be remembered that the names here applied to them are merely provisional.

Epitrochlearis (\$681). - The proximal end is in position; the distal tendon has been reflected; the tendons of the two divisions of the pecto-antebrachialis (\$8 645-647, Fig. 73), with which it is associated, have been removed.

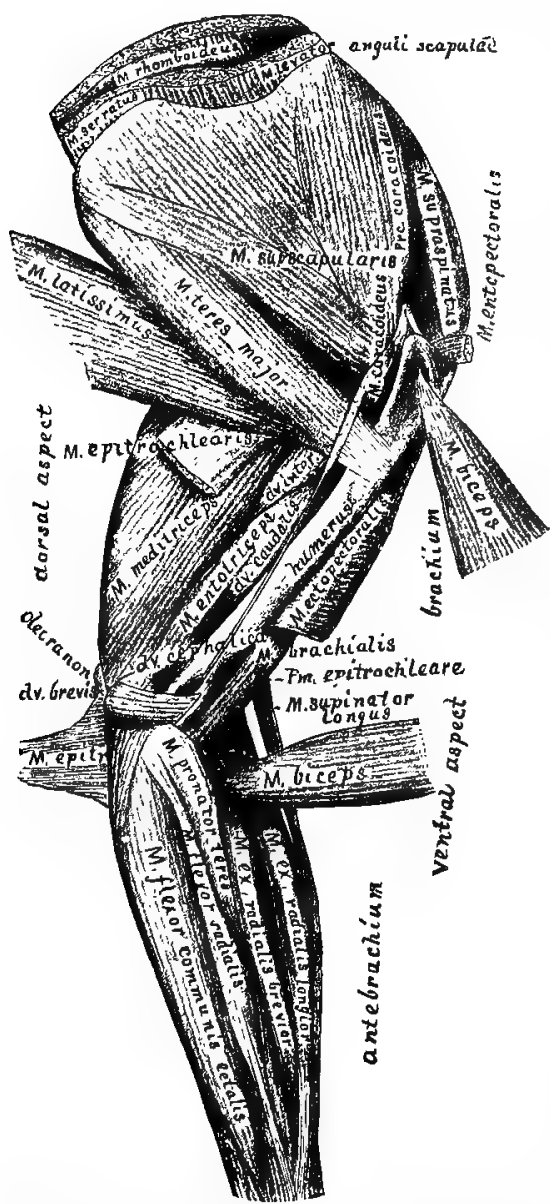

Fig. 75.-The MIUscles upon tie Ental ASPECT OF THE SCAPULA AND THE Caudal aspect of the Brachidu AND ANTEBRACHIUM.

of this muscle and the latissimus between the two heads of the coracoideus.
Extensor (carpi) radialis brevior $(\$ 696)$; ex. rad. longiur $(\$ 6 \& 4)$. - The former of these two associated muscles was but partially visible in Fig. 74.

Flexor (digitorum) communis ectalis.This represents the common flexor of the digits which, in Anthropotomy, is called $s u b$ limis, superficialis or perforatus. It is not particularly described herein.

Flexor (carpi) radialis (\$ 702).--The division of the insertion tendon described by Straus-Durckheim does not appear in this figure.

Latissimus (\$ 635).-After the division of the bicipital arch, the parts immediately concerned in its formation were removed. The figure shows the intimate association $f$ the distal ends of the latissimus and tcres, and the origin of the epitrochlearis from the ectal aspect of the former near its ventral margin.

Levator anguli scapulce (\$ 666). - As shown in Fig. 43 and 73 , the origins of this and of the serratus magnus are practically continuous.

Meditriceps ( $\$ 682$ ).-The cephalic aspect of the proximal end appears in Fig. 74.

Rhomboideus ( $\$ 620)$. - The scapular end of this muscle is seen to be coextensive with the scapular ends of the serratus magnus and levator anguli scapula, but, as shown upon Fig. 43 and 44, the insertion area is partly upon the ental and partly upon the ectal aspect of the vertebral margin of the bone.

Serratus magnus (\$ 664).-See lev. ang. scap., above.

Teres $(\$ 680)$. - As stated elsewhere, the word major is superfluous. The figure well shows the passage of the combined tendons 
M. ECTOTRICEPS.

\$ 684. Synonymy.-The external head of the human triceps, G., A, 409, Q., A, I, 207; "triceps externe," S.-D., A II, 347; " court extenseur de l'avant bras," Ch., A, 273 ; short extensor of the forearm, Ch. (Fl.), A, 259 ; first division of the triceps, Miv., B, 149.

Figures.-Ectal aspect $(66,67,74)$; origin area (71).

Posture.-The arm may rest upon the shoulder and wrist, the elbow looking upward, the dorsal aspect of the brachium toward the dissector, and the antebrachium leaning against a block.

Exposure.-The muscle is subcutaneous in its whole length, and was exposed in removing the skin from the cephalic aspect of the brachium.

General Description.-A flattened fusiform mass, from the proximal part of the deltoid ridge of the humerus to the cephalic aspect of the olecranon.

Dissection.-The dorsal border was cut from the cephalic border of the meditriceps, and the whole muscle is attached to the subjacent muscles only by connective tissue; an artery enters its ental surface a little proximad of the middle of the length, and must be divided. The ventral border is attached to the brachialis upon the cephalic side of the arm by a strong fascia, but at the middle of the muscle it is thinner than elsewhere, and may be cut first. Then transect the muscle and reflect it, dividing the fascia between its ventral border and the adjacent parts.

Origin.-By a thin tendon from the proximal part of the deltoid ridge and from the dorso-cephalic aspect of the neck of the humerus. The line of attachment is curved so as to pass ventrad of the tubercle for the insertion of the micostalis, and dorsad of the origin of the middle division of the entotriceps, some fibers of which seem to spring from the tendon of the ectotriceps. The line begins about $5 \mathrm{~mm}$. proximad of the proximal end of the line of insertion of the spino-deltoideus.

Insertion.-The proper tendon is $5 \mathrm{~mm}$. wide at its attachment to the cephalic border of the olecranon, but the distal half of the ventral border of the muscle is so firmly connected to the brachial and antebrachial fascia that it may be said to have a general insertion upon the region about the elbow.

\section{ENTOTRICEPS.}

$\S 685$. Remark.-The remaining extensors of the antebrachium are not easy to homologize with the parts of the human triceps, anconeus and subanconeus; the names 
here given to them are provisional and descriptive rather than designatory. They readily separate, and no special directions for dissection are required.

\section{INTELMEDIA.}

$\S 686$. Synonymy.-Straus-Durekheim (A, II, 350) calls this "le premier chef de l'anconé moyen"; Mivart describes it as the fourth division of the triceps (B, 149).

Figures. - In part (75); origin area $(68,71)$.

Origin.-By fieshy fibers from the dorsal surface of the surgical neck of the humerus, and from the proximal part of the shaft. Sometimes the attachment extends upon the arthral capsule. The length of the area of origin equals about one sixth of the length of the humerus. Its caudal border is encroached upon by the area of insertion of the $M$. coracoideus, caput breve, but the cephalic border is rounded, and embraced by the line of origin of the $M$. ectotriceps. The triangular apex of the area lies between the origins of the caudal division next to be described and the M. brachialis. The ventral border is indicated by the radial artery and nerve which lie between it and the dv. caudalis.

Insertion. - At the junction of the middle and distal thirds of the humerus the muscle ends in a slender, flat tendon which widens slightly as it nears the elbow, rests in the furrow at the proximal end of the bone, and is inserted into the oblique ridge which forms the dorsal limit of the furrow, thus slightly ventrad of the ridge of insertion of the meditriceps. Between the tendon and the floor of the furrow is a synovial capsule.

DV. CAUDALIS.

§ 687. Synonymy.-Called by S.-D. (A, II, 350) "le second chef de l"anconé moyen"; it is the third division of Mivart (B, 149).

Figures.-Caudal aspect (75); origin area $(70,71)$.

Origin.-By fleshy fibers from a subtriangular area, a little proximad of the middle of the dorso-caudal aspect of the humerus. The length of the area equals about one sixth of the lengtlı of the bone. Its apex points proximad, and is almost continuous with the apex of the origin area of the Dv. intermedia.

Along the candal border of the distal part of the muscle runs the ulnar nerve, and the radial artery and nerve cross the dorsal border at about the junction of the proximal and middle thirds.

Insertion.-By short, tendinous fibers upon the caudal border of the ventral aspect of the olecranon as far as the lip of the greater sigmoid notch (Fig. 30). 
DV. BREVIS.

\& 688. Synonymy.-The ancone interne of S.-D., A, II, 351 ; the fifth division of the triceps, Mivart, B, 149.

Figures.-Ental aspect (75); origin area $(70,71)$.

Origin.-By fleshy or short tendinous fibers from the ectal surface of the osseous bar which encloses the Foramen epitrochleare, as far as the origin area of the pronator teres upon the epitrochlea.

Insertion.-By fleshy fibers into the caudal border of the olecranon, just distad of the furrow for the insertion of the meditriceps.

DV. CEPHalica.

§ 689. Synonymy.-The ancone externe, S.-D., A, II, 350 ; the anconeus, Miv., B, 149. Figures.-Indistinetly (75); origin area (71).

Exposure.-The strong fascia upon the cephalic surface of the ellow must be removed.

Origin.-By fleshy fibers from the èlongated triangular surface upon the distal half of the dorsal surface of the humeral shaft. The cephalic limit of the area is indicated by the prominent ridge which extends obliquely distad from the middle of the dorsal surface to the epicondyle. The caudal border extends more nearly in the line of the axis of the bone.

Insertion.-By fleshy fibers upon the cephalic side of the olecranon, from the insertion of the middle division to a point opposite the distal lip of the greater sigmoid notch, which is just distad of the epicondyle. The insertion area is about $2 \mathrm{~cm}$. long.

The ental surface of the muscle is attached to the capsule of the elbow joint, but its thickness indicates that it serves some other purpose than that suggested by Straus-Durckheim, namely, to keep the capsule tense.

\section{SUPINATOR LONGUS.}

§ 690. Synonymy:-The human supinator longus, G., A, 415, Q., A, I, 215 ; "long supinateur," S.-D., A, II, 356 ; "long supinateur," Ch., A, 289 ; long supinator, Ch. (Fl.), A, 272 ; supinutor longus, Miv., A, 151.

Figures.-Cephalic aspect (74); caudal aspect (75); origin line (71).

Posture.-The arm may rest upon the caudal surface most of the time, but must be held in various positions at different stages of the dissection.

Exposure.-Divide the skin and the ectal fascia from the epicondyle to the wrist, and girdle the arm between the Eminentia 
hypothenaris (Fig. 105) and the base of the pollex. The girdling must be done with care, so as not to divide any of the tendons at the wrist. Reflect the skin and fascia first upon the caudal (ulnar) side of the antebrachium.

General Description.-A slender muscle from the cephalic side of the humerus to the distal end of the radius.

Dissection.-If the antebrachium is extended slightly, the free ventral border of the muscle will appear. The dorsal border is attached by connective tissue to the subjacent muscles, excepting close to the ventral border of the brachium, where a large nerve passes between it and the next muscle. Trace this border distad to near the end of the radius, noting that the connections with the subjacent muscles become firmer toward the wrist. Divide the muscle, and reflect the proximal end this sometimes adheres so closely to the cephalic surface of the brachialis that, for 5-7 mm. from the humerus, it cannot be separated without cutting fibers.

Origin.-By a very thin tendon, from the middle fifth of the dorsal border of the humerus; a third of the origin line lies between the origin areas of the brachialis and the cephalic division of the entotriceps; the rest of the line is a direct proximal continuation of the apex of the origin area of the last named muscle, and ceases at the distal end of the origin area of the caudal division.

Insertion.-By fleshy fibers upon the distal end of the radius, just proximad of the grooves for the tendons of the $M M$. extensores radiales and upon the adjacent ligaments.

\section{BICEPS.}

$\S 691$ Synonymy. -The human biceps brachialis, G., A, 408, or biceps flexor cubiti, Q., A, I, 205 ; "biceps," S.-D., A. II, 353 ; "long fléchisseur de l'avant bras ou biceps brachial," Ch., A, 271 ; long flexor of the foreurm or brachial biceps, Ch. (Fl.), A, 255 ; biceps, Miv., B, 148.

Figures.--Ventro-caudal aspect $(72,73)$ : cephalic aspect of distal end (74) ; reflected (75); origin point, tuberculum bieipitale (45, A, 43); insertion point, tuberositus bicipitalis (30).

Posture.-At first the arm should rest upon the cephalic side. The muscle was exposed by the division of the bicipital arch ( $\$ 662)$.

General Description.-A long, fusiform muscle extending the whole length of the ventral surface of the brachium, from the bicipital tubercle of the scapula to the bicipital tuberosity of the radius.

Dissection.-No preliminary dissection is needed beyond freeing the muscle from fascia and connective tissue. After transection, if 
the scapula is strongly flexed upon the brachium, the tendon of origin of the muscle may be seen to play in the bicipital groove. If now the capsule is opened, the tendon will be seen to be still covered by a special sheath, so that it does not lie free within the capsule. If it be desirable to expose the whole tendon, the dissector must divide the coracoid attachment of the entopectoralis and the expanded tendon of the xiphi-humeralis.

The distal end of the $M$. biceps must then be freed from general connective tissue, and special note taken of a rather firm tendinous band which connects the caudal side of the muscle with the fascia covering the $M$. pronator teres. The insertion cannot be seen until some of the antebrachial muscles are removed, but, by lifting the border of the muscle which arises from the epicondyle, it is possible to trace the tendon of the biceps toward a point upon the radius distad of the attachment of the clavo-deltoideus and brachialis.

Origin.-By a strong, rounded tendon, $1.5 \mathrm{~cm}$. long, from the prominent glenoid lip of the glenoid fossa of the scapula. (In man, a second tendon-that of the "short" or "coracoid" head-arises from the tip of the Prc. coracoideus.)

Insertion.-By a similar, though slightly shorter, tendon upon the bicipital tuberosity of the radius. An additional slight insertion is by the tendinous band above mentioned upon the ventral surface of the pronator teres.

\section{BRACHIALIS.}

§ 692. Synonymy.-The human brachialis anticus, G., A, 409, Q., A, 1, 206 ; " brachial," S.-D., A, II, 354 ; " court flechisseur de l'avant bras ou brachial anterieur," Ch., A, 272 ; short flexor of the forearm. Ch. (Fl.), A, 256 ; brachialis anticus, Miv., B, 148.

Figures.-Ectal aspect (74); ental aspect (75); origin area $(68,71)$; insertion area, indistinetly (30).

Posture.-With this and the remaining muscles the appropriate posture will readily suggest itself to the dissector.

Exposure.-By the reflection of the MM. biceps and supinator longus.

General Description.-From an irregular, long, v-shaped line upon the cephalic surface of the shaft of the humerus to the ulna near its proximal end.

Dissection.-Flex the brachium slightly upon the antebrachium so as to relax the muscle. At the border of the antebrachium push it slightly cephalad, and note that here it is attached to the hume- 
rus only by loose connective tissue, which may be torn with the tracer.

Note here the median nerve and brachial artery after their passage through the Foramen epitrochleare. Still keeping the brachium flexed, separate the cephalic side of the muscle from the series of antebrachial muscles arising from the cephalic side of the humerus. The muscle may be divided just proximad of its union with the clavo-deltoideus.

$\S 693$. Origin.-By fleshy fibers from an irregular, long, $\nabla$-shaped line extending almost the whole length of the cephalic surface of the shaft of the humerus. The apex of the $v$ is represented by a triangular area a little distad of the tubercle for insertion of the $M$. micostalis.

The dorsal and longer branch of the $v$ extends dorso-distad to near the middle of the length of the bone, thence distad to the crista epicondylaris, which it follows to opposite the proximal end of the Fm. epitrochleare.

The ventral branch runs ventro-distad parallel with the crista deltoidea, then distad to about the junction of the middle and distal thirds of the bone.

Each of these branches is $2-4 \mathrm{~mm}$. wide, and is really therefore a long and narrow area rather than a line. The triangular space between them does not give origin to fibers.

Insertion.-From this peculiar origin the fibers converge to form a flat tendon . $5-1 \mathrm{~cm}$. wide, which is closely attached by its ectal surface to the ental surface of the tendon of the $M$. clavo-deltoideus. The tendon of the brachialis is inserted upon the dorsal portion of the depressed rough area on the caudal aspect of the ulna just distad of the greater sig. moid notch and about midway between the dorsal and ventral margins of the bone.

This account of the $M$. brachialis is derived mainly from the illustrated Thesis of Homer Collins, B. S., a special student in the Anatomical Laboratory of Corvell University. The dotted lines upon Fig. 68, 71 approximately include the outline of the v-shaped line, but they should be double.

\section{EXTENSOR (CARPI) RADIALIS LONGIOR.}

§ 694. Synonymy.-The human muscle of the same name, G., A, 415, Q., A, I, 216; "premier radial," S.-D., A, II, 359 ; part of the "extenseur antérieur du métacarpe," Ch., A, 277 ; part of the anterior extensor of the metacarpus, Ch. (F1.), A, 262 ; extensor carpi radiaits longior, Miv., B, 151.

Figures.-Cephalic aspect (74); caudal aspect (75); origin area (71).

General Description.-From the epicondylar ridge of the humerus to the proximal end of the indical metacarpal. 
Dissection. - Just dorsad of the insertion of the supinator longus is the oblique border of the strong tendon of the $M$. extensor ossis metacurpi polliois. Fintad of this tendon may be seen another tendon evidently continuous with the muscle lying along the cephalic border of the antebrachinm. With the tracer, separate the tendon into two, an ectal and more slender, and an ental and thicker. The former may be traced proximad for two fifths of the length of the antebrachium, where it is continuous with its muscle, the extensor carpi (radialis) longior.

Divide the muscle at the middle; in reflecting the proximal end, note that it becomes thinner and wider, is wedged somewhat between two subjacent muscles, and, at the humerus; has a third or a fourth overlapped by the muscle arising just distad of it.

By pulling upon the teudon, and alternately flexing and extending the manus, it may be seen that the tendon passes across the carpus entad of the oblique tendon of the extensor metacarpi pollicis. With the arthrotome, cut the fascia at one side of the tendon upon the carpus, introduce the tracer, and thus indicate where more incisions may be made so as to expose the whole tendon as far as the proximal end of the indical metacarpal.

Note that, in its passage over the distal end of the radius, the tendon lies in a groove upon the dorso-cephalic side of the bone, separated by a triangular elevation from the groove for the tendon of the extensor metacarpi pollicis.

$\$ 695$. Remark. -By analogy with the less modified leg, the muscles of the arm which lie upon the dorsal aspect of the antebrachium and are inserted upon the carpus should be called flexors, and those upon the caudal aspect extensors. These and other considerations have been presented by the senjor author ( 1 and 4 ). In an ideal myological nomenclature, we believe the muscles should be named as above, but in the present practical work it seems best to retain the designations commonly accepted.

Origin.-By fleshy fibers from the epicondylar ridge of the humerus, between the origin area of the supinator longus and a point opposite the distal end of the Foramen epitrochleare. The larger part of the origin line lies between the slender distal prolongation of the origin area of the brachialis and the triangular origin area of the cephalic division of the entotriceps. The distal fourth or fifth is just ventrad of the origin line of the extensor communis (digitorum).

Insertion.-By a long tendon upon the dorsal border of the proximal end of the indical metacarpal.

Remark.-Like other tendons which pass over the wrist, this is held in place by ligamentous bands representing parts of the annular or armillary ligaments.

\section{EXTENSOR (CARPI) RADIALIS BREVIOR.}

§ 696. Synonymy.-The buman muscle of the same name, G., A, 416, Q., A, I, 216 ; " second radial," S.-D., A, II, 359 ; part of the " extenseur antêrieur du métacarpe," Ch., A, 277 ; part of the anterior extensor of the metacarpus, $\mathrm{Ch}$. (Fl.), A, 262 ; ext. carpi rad. brevior, Miv., B, 151.

Figures.-Cephalic aspect, in part (74); caudal aspect (75); origin area (68).

General Description.-From the epicondylar vidge of the humerus, just prozimad of the epicondylus, to the base of the medial metacarpal.

Dissection.-The thicker tendon mentioned as joined with that of the ex. rad. longior belongs to the present muscle. This tendon is shorter than the other, and the body proportionately longer, as well as thicker. Divide it $1 \mathrm{~cm}$. farther distad than in the case of the ex. rad. longior, and reflect both ends.

Origin.-By fleshy or short, tendinous fibers from the epicondylar crest, just distad of 
the origin of the ex. rad. longior. The origin area cannot be seen fully until after the removal of the ex. communis.

Insertion. -The tendon passes through the same groove as the tendon of the ex. rad. longior, and is inserted upon the dorsal side of the proximal end of the medial metucarpal.

\section{EXTENSOR (DIGITORUM) COMMUN1S.}

\$ 697. Synonymy.-The muscle has the same name in human anatomy, G., A, 417, Q., A, I, 216; extenssur commune des doigts, S.-D., A, II, 364 ; extenseur antêrieur des phalanges, Ch., A, 275 ; anterior extensor of the phalanges, $\mathrm{Ch}$. (Fl.), A, 263 ; ext. dig. communis, Miv., B, 151.

Figures.-Ectal aspect (74); origin area $(68,71)$.

Dissection.-The muscles remaining upon the cephalic and dorsal surfaces of the antebrachium are covered by a dense fascia which must be removed. Near the wrist note a wide tendon which passes ectad of the $\boldsymbol{M}$. abductor ossis metacurpi pollicis. Trace it to the corresponding muscle, and this to the humerus, and transect the muscle at its middle.

Origin.- -By fleshy and tendinous fibers from the epicondylar ridge. The origin area is about $9 \mathrm{~mm}$. long, and extends from the disto-cephalic angle of the origin area of the cephalic division of the entotriceps to the trochlea; it lies just dorsad of the origin area of the extensor rad. brevior and of the distal fifth of the ex. rad. longior.

Insertion.- The tendon lies in a groove upon the dorsal surface of the distal end of the radius, from which it may be disengaged by slitting up the ligament which converts the groove into a canal. It then divides into four tendons which may be traced to the dorsal aspect of the four ordinary digits.

\section{EXTENSOR MINIMI (DIGITI).}

§ 698. Synonymy,-The human muscle so called (G., A, 417, Q., A, I, 218), sends a tendon to on!y the minimus, while in the cat and dog what seems to be the same muscle supplies also the annularis and medius; (see Huxley, A, 418). S.-D., however (A, II, 368, 369), regards it as forming three separate muscles, one for each digit, excepting the pollex and index, which he terms E.r. prop. du verpus, paramese and micros, respectively. Extenseur laterale des phalanges, $\mathrm{Ch} ., \mathrm{A}, 279$; lateral extensor of the phalanges, $\mathrm{Ch}$. (Fl.), $\mathrm{A}, 264$; extensor minimi digiti, Miv., B, 151.

Figures.-Dorsal aspect (74); origin area $(68,71)$.

Origin.-From the ventral bordex of the epicondyle just distad of the origin of the extensor communis. The proximal part of the origin is by muscular fibers, and the distal by a short tendon At $2-3 \mathrm{~mm}$. from the origin the muscle divides into a slender ectal portion and a thicker ental part, which are in close contact, but may be separated without cutting fibers. The former becomes tendinous at the junction of the proximal and middle thirds of the antebrachium, and the latter at the junction of the distal and middle thirds.

Insertion.-The tendons join the corresponding tendons of the extensor communis.

\section{EXTENSOR (CARPI) ULNARIS.}

$\$ 699$. Synonymy.-Tlie human muscle of the same name, G., A, 417, Q., A, I, 218; cubital, S.-D., A, II, 360 : ex. carpi ulnaris, Miv.. B, 152.

Figures.-Dorsal aspect (74); origin area (68).

Origin.-In two parts : (A) by a short, broad tendon from the distal end of the epicondyle next to the origin of the extensor minimi; (B) by a smaller and rounded tendon from the proximal lip of the sigmoid notch of the ulna. 
Insertion.-The muscular fibers continue to within $1 \mathrm{~cm}$. of the wrist; the tendon, about $4 \mathrm{~mm}$. wide, passes over the distal end of the ulna, is connected with ligaments, and is inserted upon the tubercle at the caudal side of the proximal end of the minimal metacarpal.

\section{INDICATOR.}

$\$ 700$. Synonymy.-The extensor indicis or indicator, Q., A, I, 220; extensor indieis, G., A, 418 ; " indicator," Dunglison, A, 676 ; " indicateur," S.-D., A, II, 367 ; ext. indicis and ex. secundi internodii pollicis, Miv., B, 152.

Origin.-By fleshy fibers along the cephalic border of the ulna, from the lesser sigmoid notch to the junction of the distal and middle thirds of the bone, exclusive of the olecranon. The proximal third of the muscular portion resembles the ordinary antebrachial muscles, and is continuous with a slender tendon; the remaining fibers form a thin, loosely connected series of bundles extending distad at an angle of about 45 degrees with the bone to be attached to the tendon almost to the wrist.

Insertion.-At the wrist the tendon divides into two, both of which are connected with the indical tendon of the extensor communis. The more caudal of the two tendons sometimes receives a small tendinous slip from the medial tendon of the extensor minimi. In some cases the tendon divides into three, which are distributed respectively to the medius, the index and the second segment of the pollex.

\section{PRONATOR TERES.}

§ 701. Synonymy.-The human pronator radii teres, G., A, 411, Q., A, I, 209 ; "rond pronateur," S.-D., A, II, 357 ; pronator teres, Miv., B, 149.

Figures.-Caudal aspect (75); origin area (70, 71).

Origin.-By a short, strong tendon from the extremity of the epitrochlea just distad of the origin of the short division of the entotriceps.

Insertion.-By fleshy and short tendinous fibers for about $1.5 \mathrm{~cm}$. along the cephalic border of the radius at its middle.

\section{FLEXOR (CARPI) RADIALIS.}

\$ 702. Synonymy.-The human flexor carpi radialis, G., A, 411, Q., A, I, 210 ; "cercialis," S.-D., A, II, 362 ; flexor carpi radialis, Miv., B, 149.

Figures.-Caudal aspect (75) ; origin area (70).

Origin.-By fleshy and short tendinous fibers from the distal aspect of the epitrochlea.

Insertion.-The muscle becomes tendinous about $2 \mathrm{~cm}$. from the wrist, passes deeply entad of the surface, and, according to Straus-Durckheim, divides into two tendons which are attached to the indical and medial metacrrpals.

$\S 703$. Other Muscles.-In addition to the muscles described in the foregoing pages, the figures indicate more or less fully the position and connections of the following : MM. supra-cervico-cutaneus and cervico-auricularis (66); temporalis, masseter, digastricus and splenius (67); sterno-hyoideus, sterno-thyroideus, rectus and ectobliquus (abdominis) (72); intercostales, rectus, ectohliquus, splenins and sterno-hyoideus (73); flexor ulnaris and flearor digitorum communis ectalis (75).

The muscles just named, together with all others of the cat, are more or less fully described by Straus-Durckheim, and most of them are at least enumerated by Mivart. 
$\S$ 704. The Obvious Structure of Muscle.-A muscle is readily seen to be a collection of more or less elongated, reddish, fleshy bundles attached at each end more or less independently or by a common fibrous structure, the tendon. The bundles are easily separable into smaller bundles called fasciculi. A perimysium or sheath of connective tissue surrounds each muscle, and from this partitions extend between the fasciculi, furnishing each with a separate sheath.

\$ 705. Microscopic Structure.-Fibers.-Each fasciculus is composed of a variable number of cylindrical fibers.

Striation.-The fibers appear to be composed of alternate light and dark segments. A muscle which has been hardened, in alcohol for example, also presents an appearance of longitudinal striation, and if it be teased carefully, it may be divided into finer threads called fibrillox; each fibrilla shows the same alternating light and dark bands as the entire fiber.

Sarcolemma.-Surrounding each fiber is a delicate sheath of elastic tissue.

Nuclei or Muscle Corpuscles.-These are clear oval bodies found in the fibers. In Mammalian muscles they lie on the surface of the sarcolemma, but in Amphibia they are distributed throughout the substance of the fibers.

Length.-The average length of a fiber is nearly $3 \mathrm{~cm}$. They are of nearly uniform size throughout, but sometimes branched as in the torigue.

Connection with Tendons.-A. When continued in a direct line with a tendon, the fiber merges into the tendon somewhat abruptly, yet it can be distinguished from tendon only by the absence of cross striation in the latter.

B. When the fibers join the tendon at a more or less acute angle, they terminate in rounded ends which are received into corresponding depressions in the tendinous structures. Quain, A, II, 115. 


\section{CHAPTER VII.}

THE ABDOMINAL VISCERA, SALIVARY GLANDS, MOUTH, NECK AND THORAX.

LIST OF INSTRUMENTS AND MATERIAI-STOMACH-LIVER-PANCREAS- SMALL INTESTINE-LARGE INTESTINE-URINARY ORGANS-SALIVARX GLANDS-MOUTH-NECKTHORAX-TRACHEA - CESOPHAGUS-THYMUS-DIAPHRAGM.

\section{ABDOMINAL VISCERA.}

There is first given a general consideration of the parts, to enable the student to recognize them. This is followed by a more detailed description.

§ 706. It is advisable to employ at least two specimens for the abdomen, one for the viscera and the other for the blood vessels. If specimens cannot be obtained readily, the thoracic and abdominal viscera and vessels may be studied upon a single individual.

Preparation.-Just before the explanation of each figure there is given the method of preparing the part or organ for that particular figure. The directions for demonstration to be followed by the student are given in the text propor.

\$ 707. Names of Parts in Order of Examination.-(1) Peritoneum ; (2) Diaphragma, diaphragm ; (3) Hepar, liver, and cholecystis or gall bladder; (4) Stomachus, stomach; (5) Splen, spleen; (6) Omentum majus, great omentum; (7) Intestinum tenue, small intestine; (8) Urocystis, urinary bladder; (9) Intestinum amplum, large intestine; (10) Pancreas; (11) Mesenterium, mesentery ; (12) Ren, kidney; (13) Uterus (or vas deferens); (14) Ovarium, ovary.

Instruments and Material.-Coarse comb; 15 per cent. glycerin; injecting apparatus and material ( $\$ 336)$; scalpel; scissors; sponge; thread; towel ; tray ; tracer ; water.

\$ 708. Choice of Specimen.-Choose a young adult, lean cat. It may be fasting, but preferably the stomach should contain a moderate amount of solid food. 
$\S$ 709. Posture and Preparation.-Place the cat dorsicumbent; fasten the arms and legs laterad with cords tied to the loops on the edges of the tray (Fig. 76). Take the precautions for cleanliness ( $\$ 199)$. When the examination is made in warm weather or is to extend over several days, the arteries should be injected with alcohol as directed in $\S 284$. If the vascular system itself is to be

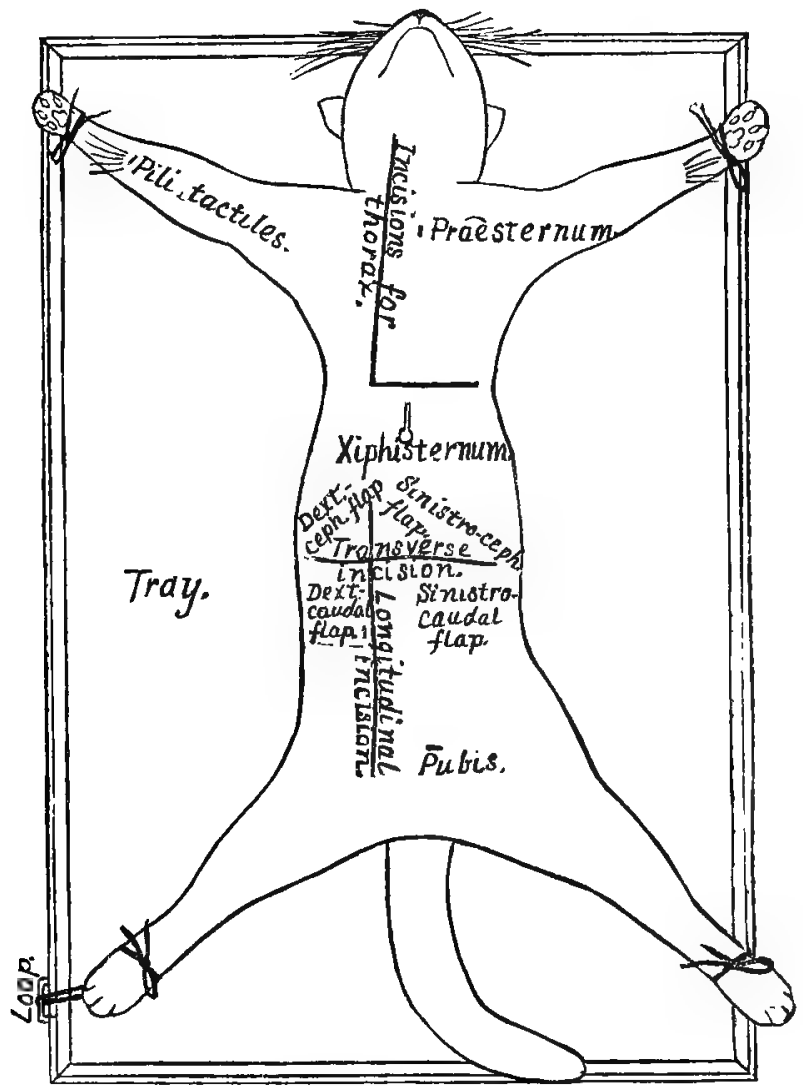

Fig. 76.-Lines of Incision for Exposing THE Thoracic and abdominal VISCERA ; $\times .25$.

studied in the same individual, both arteries and veins should be injected with plaster (\$ 352, 362).

If the thorax is not to be studied, open it (\$825), cut the pracava to allow the blood to escape, and inject caudad through the postcava and the aorta thoracica. If the vessels are to be studied on a separate specimen, the injection may be omitted.

Keep all exposed parts moist with the 15 per cent. glycerin ( $\$ 171$ ). 
\$ 710. Exposure--Determine the three following landmarks by pressing on the various parts of the abdomen where they are indicated (Fig. 76): (A) The ventrimeson by finding the xiphisternum (Fig. 30, 72, § 228); (B) the caudal margin of the 12th rib (Fig. 30, 72); (C) the pubis (Fig. 51, 76, § 228).

After having determined the three landmarks, the hair should be moistened and parted as directed above $(\$ \$ 354,599)$; then the incisions should be made along the lines indicated in Fig. 76. Make the incisions in the manner described for abdominal transection ( $\S 237$ ). Reflect the four flaps, being careful not to tear any of the thin membranes-urocystic and hepatic ligaments, etc.-attached along the meson.

Preparation and Exposure -Fig. 77.-The cat was fed a moderate amount of meat about an hour before it was killed with chloroform. After death it was placed dorsicumbent on the demonstration board, the head fastened with the pointed holder (see Fig. 77), and the limbs secured laterad with the straps.

After parting the hair (\$354), an incision was made from the angle of the mouth on the right, along the middle of the side of the neck, thorax and abdomen, then on the left side as far cephalad as the angle of the mandible. The mucous membrane and the thick muscles on the side of the face and neck were cut at the same level as the slrin, and the right mandibular ramus disjointed with the arthrotome. The hyoid bone (Fig. 30, \$ 224) was cut, and the left coronoid process (Fig. 61) broken with nippers; this made it possible to turn the mandible to the left so as to expose the pharynx and the floor and roof of the mouth.

The ribs and soft parts of the abdominal and thoracic wall were then cut with scalpel and nippers at the same level as the incision in the skin. The mediastinal septum and the diaphragm were cut with scissors close to the ventral wall, and the entire ventral wall of the body was removed.

In some specimens the ovary (ovarium) and kidney (ren) will not appear without displacement of the intestines, and in old animals the thymus may be absent (Fig. 77, Cp. thym.). The following parts will be exposed (Fig. 77):-

Description of Fig. 77.-Cardia, az.-Heart. Cholecystis, az. - Gall bladder. Cornu Uteri.-Left horn of the uterus. Costæ.Ribs (13). The cut ends are shown on each side of the thorax. Cp. thym., Corpus thymicum, az.-Thymus body or gland. Dia- 


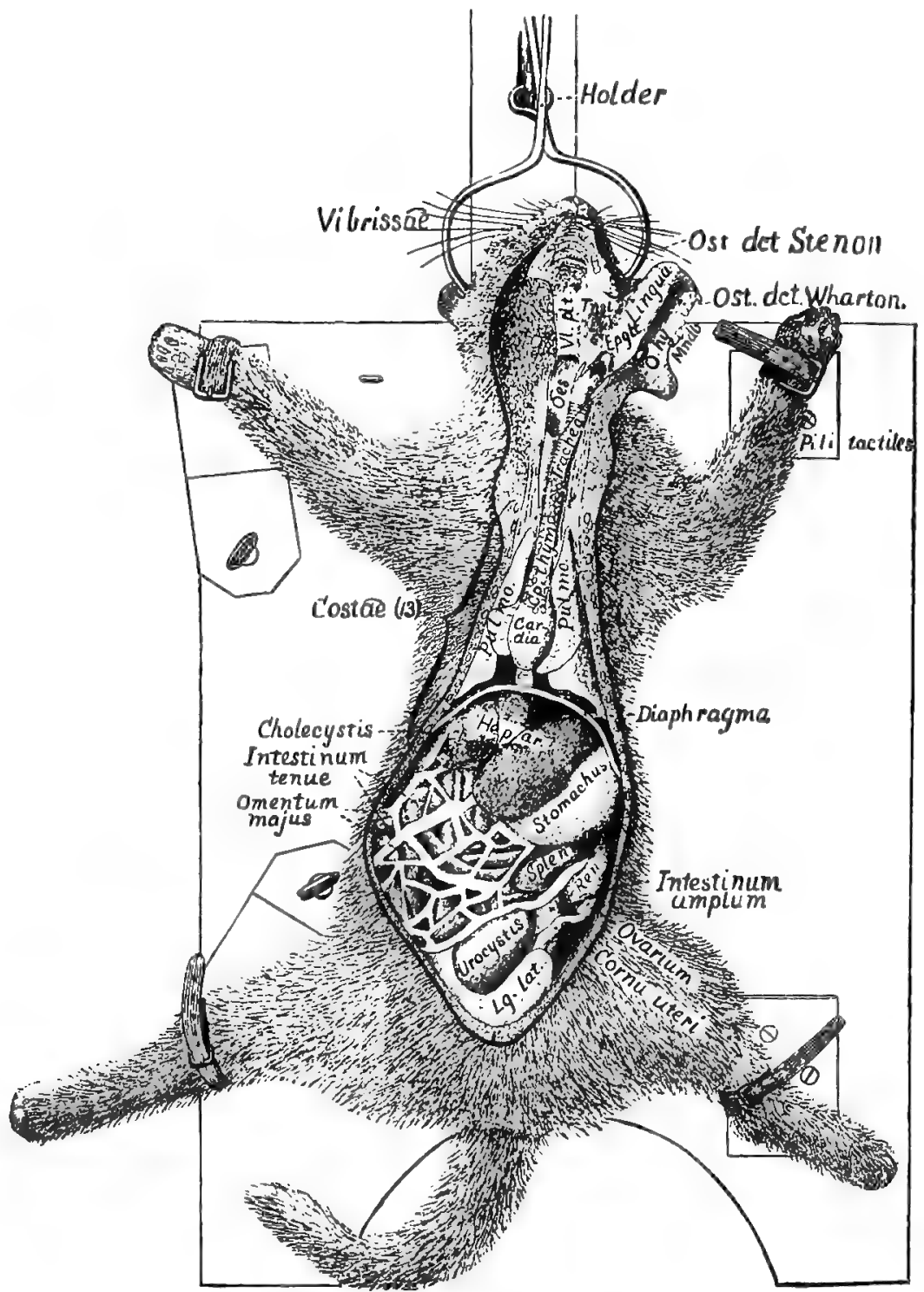

Fig. 7i.-General View of the Viscera; $\times .25$.

phragma, az.-Diaphragm, "midriff." Epglt., Epiglottis, az. Hepar, az.-Liver. Pointed Holder. Intestinum amplum, az.Large intestine. Intestinum Tenue, az.-Small intestine. Ig. lat., Ligamentum laterale.-Lateral ligament of the urocystis. 
Lingua, az.-Tongue. IMndb., Mandibula.-Mandible, lower jaw. Es., Esophagus, az.-Gullet. Omentum majus, az.-Epiploon, sreat omentum, caul. O. hy., Os hyoides.-Hyoid bone. Osì. det. Stenon., Ostium ductus Stenoniani.-Mouth or opening of the duct of Stenon, duct of the parotid gland. Ost. det. Wharton., Ostium ductus Whartoniani.-Mouth or opening of Wharton's duct, duct of the submaxillary gland. Ovarium.-Ovary. Pili tactiles.-Tactile hairs (see Fig. 105). Pulmo.-Lung. Ren.Left kidney. Splen, az.-The spleen. Stomachus, az.-The stomach. Tnsl., Tonsilla.-Left tonsil. (The tonsils are composed mostly of lymphoid tissue and are abundantly supplied with blood vessels and nerves. The function of the tonsils is not well understood; Quain, A, II, 335). Trachea, az.-Wind-pipe. Urocystis, az.-Urinary bladder. Vibrissæ.-Whiskers. Vl. plt., Velum palati, az.-This is the veil-like or pendulous part of the soft. palate ; its candal margin is free (Fig. 88).

$\S$ 711. Peritoneum ( $\$ 725)$. - This is the smooth shining membrane lining the abdominal cavity and covering the viscera. It may be separated from the muscular parietes over a small area by using the tracer.

§ 712. Diaphragma, az. (Fig. 77, § 734):-Grasp the free edge of the cephalic abdominal flaps (Fig. 76); draw them upward, and look toward the cephalic end of the abdominal cavity. The diaphragm will appear as a transverse muscular curtain separating the abdomen from the thorax.

§ 713. Hepar and Cholecystis, az.-Liver and gall bladder (Fig. 77, $\$ \$ 744,745$ ). - The liver is a deep red, multilobular organ occupying nearly the entire cephalic part of the thorax, but especially the dextral part.

The cholecystis is a reservoir for bile; it usually appears as a greenish sac in one of the lobes. If it does not appear, grasp the caudal margin of the liver and turn it slightly toward the thorax. The cholecyst will appear as a pear-shaped, greenish sac partly imbedded in the substance of the middle lobe.

$\S 714$. Stomachus, $\alpha z$. (Fig. 77, 79, $\S 735$ ).-This is a somewhat pear-shaped organ extending obliquely across the cephalic part of the abdominal cavity. Its larger, cardiac or cesophageal end (Fig. 79) is next the diaphragm and mainly in the left half of the cavity. The small or pyloric end is sharply curved. It is partly covered by 
the liver, and may be fully exposed by turning the edge of that organ cephalad.

The space occupied by the stomach depends largely on the amount of food it contains.

$\S$ 715. Splen, az.--Spleen (Fig. 103, § 737).-This is a deep red, usually single lobed organ, situated on the sinistro-caudal aspect of the stomach.

\$ 716. Omentum majus, epiploon, $\alpha z$._Great omentum (Fig. 77, \& 727).--This appears as a kind of transparent apron extending caudad from the stomach. It contains many strips of fat.

$\S 717$. Intestinum tenue, az.-Small intestine.-Very carefully turn the omentum over toward the thorax. The greatly coiled cylindrical small intestine will be exposed (Fig. 77, §738).

§ 718. Urocystis, az.-Urinary bladder (Fig. 77, 101, § 757).-In the caudal part of the abdomen will be seen a median sac, usually more or less filled with liquid. This is the urocystis, the receptacle of the urine.

§ 719. Intestinum amplum, az.-Large intestine (Fig. 77, §742). - Turn the coil of small intestine toward the left leg. The large intestine will be seen on the right side extending first cephalad from a blind extremity, the cæcum (Fig. 80), nearly to the stomach, then transversely across the cavity a little to the left of the meson, and finally somewhat obliquely caudad.

§ 720. Pancreas, $a z$. (Fig. 81, 103, \& 746).-Turn the large intestine to the left; the pancreas will appear as a pinkish, finely lobulated and elongated body within the great omentum near its dorsal attachment to the stomach. It extends from the spleen dextrad to the pylorus, and then for $5-10 \mathrm{~cm}$. along the small intestine (Fig. 83).

§ 721. Mesenterium, az.-Mesentery (Fig. 78, § 726).--Grasp the small intestine and lift it up. The mesentery is the translucent membrane supporting the intestine and serving to attach it to the body. It is a fold of peritoneum ( $\$ 726)$.

§ 722. Ren-Kidney (Fig. 101, § 761).-Turn the stomach and intestines to the right, and the left kidney, a dark red body, will appear resting on the ventral surface of the muscles of the back near the meson and but a short distance from the diaphragm.

§ 723. Uterus, az. (Fig. 77, § 759).-Turn the urocystis ventrocaudad, and if the animal is a female the uterus will be seen resting upon the rectum, and sending a prolongation-cornu or horncephalad on each side toward the kidney. 


\section{ABDOMINAL VISCERA, SPECLAL STUDY.}

\$ 724. Names of Parts in the Order of Examination.-Peritoneum ; Diaphragma, diaphragm; Stomachus, stomach; Splen, spleen; Intestinum tenue, small intestine (duodenum, jejunum, ileum); Intestinum amplum, large intestine (cæcum, colon, rectum); Hepar, liver ; Pancreas ; Ren, kidney; Urocystis, urinary bladder; Adrenal, suprarenal capsule ; Uterus, womb ; Ovarium, ovary ; Vas deferens, spermatic duct.

Instruments and material the same as for the general study.

Specimen.-The same specimen may be used, or it a different one is used, it should be prepared in the same way ( $\$ 709)$.

\section{PERITONEUM.}

$\S$ 725. Peritoneum.-The peritoneum is a serous sac lining the abdomen. It gives the ental aspect of the abdominal wall its smooth, glistening appearance. It way be separated easily from the abdominal wall over a small area with the tracer.

The peritoneal sac is closed in the male, but in the female the Fallopian tubes open into it, and hence it communicates, through these, with the exterior.

The mesenteries and the ligaments of the liver, urocystis and uterus are formed by duplicatures of the peritoneum.

All the organs of the abdomen are really outside of the sac. The apparent presence of some of the organs within it, and the way in which the mesenteries are formed, may be readily understood from the following diagram (Fig. 78).

This diagram represents an ideal transection of the cat's abdomen at the level of the kidneys. The kidneys (ren) are represented as projecting somewhat into the abdominal cavity, and covered only on their ventral surfuce by the peritoneum. The alimentary canal (Alt. canab), shown also in cross section, is represented as having moved far ventrad into the abdominal cavity, carrying with it a fold of peritoneum which forms the mesentery (\$726).

$\S 726$. The word mesentery is often used in a general way to indicate any of the duplicatures of peritoneum supporting the intestines; but strictly speaking, the term refers only to the duplicature of peritoneum supporting the portions of the small intestine known as jejunum and ileum. The proper term for the peritoneal duplicature of any other part of the intestine is formed by prefixing meso to the name of the part; thus, meso

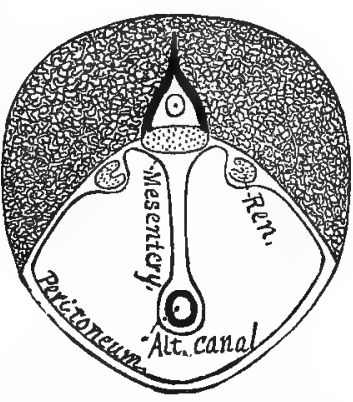

Fig. 78. -Dragram showing THE ReLations OF THE ABDOMINAL ORGANS AND The Peritoneum. duodenum, mesocolon, mesorectum, etc.

As shown in Fig. 78, the mesenteries and ligaments are double walled membranes. Demonstrate this by tearing away the mem- 
brane covering one side of a blood vessel. A similar membrane will remain on the opposite side.

$\S$ 727. Omentum majus, epiploon, $a z$.-Caul ( $\$ 716)$.-The epiploon is a double walled sac formed by a duplication of a double fold of peritoneum. The cavity of this sac is sometimes called the lesser peritoneal cavity. Demonstrate the sac-like character of the omentum by tearing it open and divaricating the two walls. Demonstrate that each wall of the sac is composed of two layers, as with the mesentery, by tearing away one layer with the tracer. This may be most easily and satisfactorily done over a blood vessel or a strip of fat.

It will be seen from the above that the omental sac as it lies collapsed on the intestines consists of four thicknesses of peritoneum.

\$ 728. Foramen Winslovii, $\alpha z$.-Foramen of Winslow.-This is the contracted mouth of the omental sac through which it communicates with the general peritoneal cavity. It is relatively larger than in man, and may be easily demonstrated by tearing open the sac near the pylorus, lifting the two walls slightly and divaricating them. There will be seen within the sac a small lobe of the liver (lobus Spigelii), and at the caudal margin of this lobe, sometimes partly filled by it, will be seen the foramen. It is about $2 \mathrm{~cm}$. in diameter, and is on the dorsal side of the ductus communis choledochus and Vena portce. The foramen may likewise be demonstrated by turning the duodenum to the left and finding the ductus choledochus and Vena portæ. This should be done in a perfectly fresh and uninjured specimen, and the omental sac inflated by blowing into the foramen with a bent glass tube or a large flexible blow-pipe.

$\$$ 729. The Obvious Structure of the peritoneum is like that of serous membranes generally-thin and transparent, smooth and glistening.

\$ 730. Microscopic Structure.-(A) Ectal layer of a single thickness of flattened nucleated cells. (B) Attached or ental layer of connective tissue containing elastic and white connective tissue fibers. Consult Fley, A; Quain, A, II, 197 ; Stricker, A, 569.

$\S$ 731. Glandulæ Mesentericæ, az.-Mesenteric glands.-The so called mesenteric glands belong to the lymphatic system. They are between the layers of mesentery, and are especially large near the cæcum, some of them being 1-2 cm. thick and $3 \mathrm{~cm}$. long. 
§ 732. Corpuscula Pacini, $a z$.-Pacinian corpuscles.-These are oval bodies about $2 \mathrm{~mm}$. long and $1 \mathrm{~mm}$. thick. They are between the layers of mesentery, and may be demonstrated, in lean cats, by lifting the mesentery and looking through it toward the light. They appear as translucent thickenings of the form and size mentioned above.

§ 733. Microscopic Structure.-(A) A connective tissue envelupe. (B) Many concentrically arranged layers of translucent connective tissue. (C) A semifluid, richly nucleated, central mass. (D) The termination of a single medullated nerve filer. (E) A capillary network of blood vessels. Stricker, A, 179 ; Quain, A, II, 150.

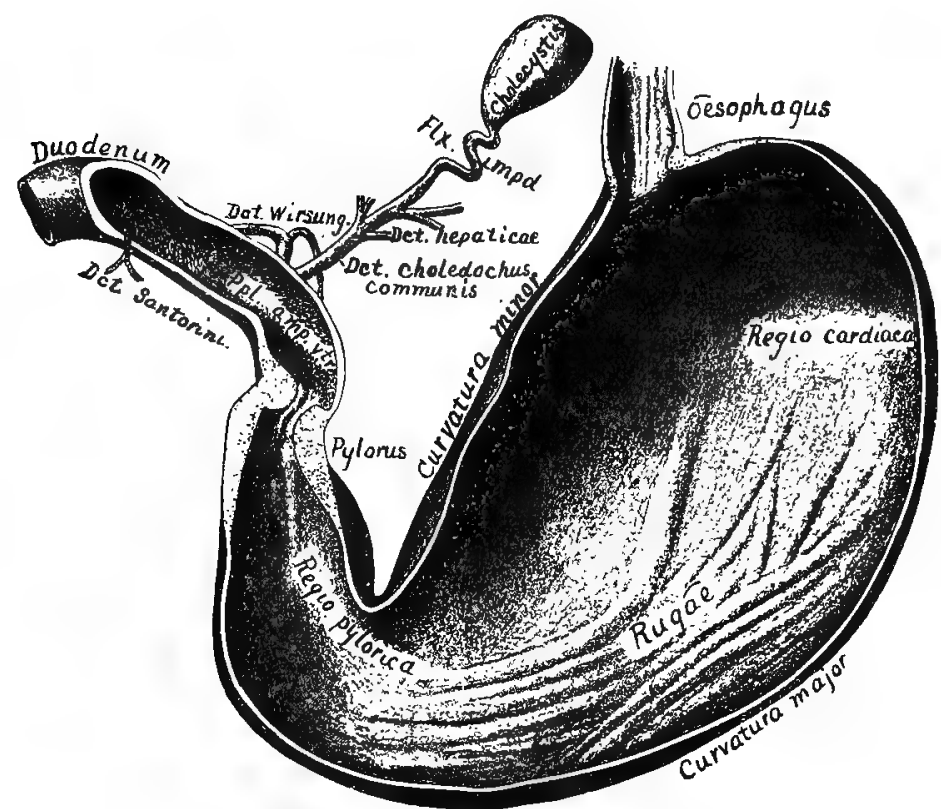

Fig. 79.-Stomach and Duodentm, Ventral View; $\times 1$.

§ 734. Diaphragma, $\alpha z .-D i a p h r a g m$ (Fig. 90, § 712).-Draw the stomach and liver somewhat caudad, and with a sharp scalpel perforate the diaphragm, provided the thorax was not opened to make the injection. This will allow the air to enter the thorax, and the stomach, etc., may be kept in view. The diaphragm is fully considered in $\$ 816$.

Preparation-Fig. 79. - The cat was fed a moderate amount of solid food about an hour before death. After death, the stomach 
was filled from the duodenum moderately with 95 per cent. alcohol. The omentum and mesentery were then carefully removed; the pancreatic and hepatic ducts isolated for a short distance; the cholecyst carefully separated from the liver; then the csophagus was separated from the diaphragm and ligatured about $2 \mathrm{~cm}$. cephalad of it. The duodenum was also ligatured, and then the whole was put into 95 per cent. alcohol for two days. 'The ventral portion was then removed, as shown, with a sharp scalpel, and the contents washed out.

Description of Fig. 79.-Cholecystis.-Gall bladder. Curvatura major.-The greater curvature of the stomach. Curvatura minor.-The lesser curvature of the stomach. Dct. (ductus) hepaticæ.- Hepatic ducts. Dct. (ductus) choledochus communis. Dct. Wirsung., Ductus Wirsungianus. - The pancreatic duct opening into the ampulla of Vater. Dct. (ductus) Santorini.-The pancreatic duct opening independently into the intestine. Duodenum.- $\$ 738$. Flx. impd., Flexuræ impedentes.-The impeding flexures of the cystic duct. Esophagus.-\$ 735. Ppl. amp. Vtr., Papillæ ampullæ Vateri. Pylorus. -8735 , C. Regio cardiaca. - The cardiac region, the region next the diaphragm, Regio pylorica.-The pyloric region. Rugæ.-Folds.

\$ 735. Stomachus, az.-Stomach (Fig. 79, § 714).-Demonstrate the following:-(A) The abdominal csophagus. Turn the left lobe of the liver cephalad, and the abdominal cesophagus will be seen emerging from the diaphragm and entering the cephalic or cardiac end of the stomach.

From the nearly cephalo-caudal direction of the dorsal part of the diaphragm, the dorsal side of the cardiac end of the stomach is applied closely to it, and hence there is an abdominal cesophagus only on the ventral side.

(B) The stomach as a whole is pear-shaped and curved upon itself. The curvatura major, or great curvature, faces sinistrocaudad, and the great omentum is attached to it. The curvatura minor, or lesser curvature, looks dextro-cephalad, and there is attached to it the lesser omentum.

The larger or cardiac end is next the diaphragm and receives the œsophagus. The pyloric or smaller end is curved sharply upon itself, and is partly concealed on its ventral side by the liver.

(C) Pylorus or pyloric valve (Fig. 79).- This is between the stomach and small intestine. It usually appears as an annular constriction, and is firm to the touch. The pylorus in the cat, as in man, is a ring-like fold of mucous membrane and a sphincter muscle formed by an increase in thickness of the general layer of circular muscular fibers of the alimentary canal. 
The general appearance may be demonstrated by making a longitudinal section of the pylorus and small intestine as shown in Fig. 79.

$\S$ 736. Obvious Structure of the Stomach.-With a scalpel, make a longitudinal incision in the stomach along its entire ventral surface, and wash out the contents. With scissors, cut out a piece of the stomach $2-3 \mathrm{~cm}$. square. Look at the cut edge with a tripod magnifier after observing it well with the naked eye. It will be seen to be composed of two very obvious coats, an ectal, firm muscular coat, covered by the thin peritoneum, and an ental, soft mucous coat. These are somewhat loosely connected together, and if the stomach is empty or but slightly filled, the mucous coat will be tbrown into folds or rugce, mostly longitudinal in direction, by the contraction of the muscular coat.

Microscopic Structure, commencing ectad :-(A) Peritoneal or serous coat. (B) Muscular (unstriped) coat of :-(1) Ectal longitudinal layer; (2) intermediate circuiar layer ; (3) ental oblique lajer. (C) Submucosa, loose connective tissue coat. (D) Muscularis mucosa, a thin layer of unstriped muscular fibers both circularly and longitudinally arranged. (E) Mucous coat, with peptic glands. See Stricker, A, 370 ; Quain, A, II, 350.

\$ 737. Splen, $a z$.—Spleen ( $\$ 715)$.- The relations, form and texture of the spleen should be carefully noted. It is one of the so called ductless glands, and its functions are not well understood.

§ 738. Intestinum tenue, $a z$. - Small intestine (Fig. 77, § 717). The small intestine is arbitrarily divided into three regions: The duodenum, the jejunum and the ileum.

(A) Duodenum. - That part of the small intestine along which the pancreas extends is called the duodenum. It is held rather firmly in position by a ligament from its caudal end. Into the duodenum empty the ductus choledochus communis (Fig. 81 and 82) and the two pancreatic ducts.

(B) Jejunum.-This is an ill-defined portion of the small intestine immediately following the duodenum. It is so called because in man it is often found empty after death.

(C) Ileum.-This is the caudal part of the small intestine, and is a continuation of the jejunum, as that is of the duodenum. It terminates in the large intestine, entering it obliquely. At its termination is the ileo-coecal valve, which allows the alimentary contents to pass from the small to the large intestine, but not easily in the opposite direction. The action of this valve may be demonstrated by cutting a slit in the small intestine $5-10 \mathrm{~cm}$. cephalad of its termination, and injecting water caudad. The water will pass readily into the large intestine.

Now cut a small slit in the large intestine and inject water toward the small intestine. It will pass with difficulty into the small intestine. 
Preparation-Fig. 80.-A cat which has been well fed five or six hours before death is best. The mesentery should be removed, and about $10 \mathrm{~cm}$. of the small and the same length of the large intestine should be left with the cæcum and ileo-cæcal valve. The

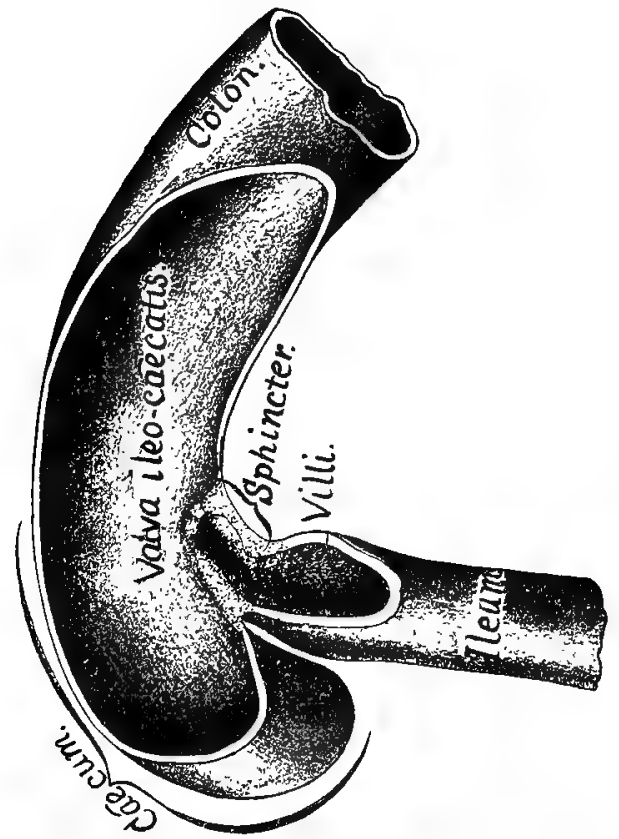

Frg. 80.-C TRAL VLEW ; $\times 1$. contents of both large and small intestine should be carefully removed and the large intestine ligatured. Then both should be moderately distended by injecting 95 per cent. alcohol into the small intestine. The latter should then be ligatured and the whole putinto 95 per cent. alcohol. After two to three days the walls may be removed with a sharp scalpel.

Cæcum, az.--See $\S 742$, A. Colon, az.-See $\S 742$, B. Ileum, az.-See § 738. Sphincter, $a z .-$ See $\S 739$. Villi-_See $\$ 740$, B. Valva ileo-cæcalis, az.-Ileo-cæcal valve. See $\S 789$.

\section{§ 739. Valva ileo-cæca-} lis.-The ileo-cæcal valve in the cat is quite unlike its homologue in man. In the cat it is a sphincter, composed of circular muscular fibers like the pylorus, and like the pyloric valve it is partly formed by an annular fold of mucous membrane, which in the ileo-cæcal valve projects into the large intestine (Fig. 80).

The thickest part of the sphincter is about $1 \mathrm{~cm}$. cephalad of the opening into the large intestine. The villi are not present caudad. of this point, hence about $1 \mathrm{~cm}$. of the mucous membrane of the small intestine of the cat is devoid of villi.

\$ 740. Obvious Structure.-(A) Cut out two or three square cm. of any part of the small intestine ; rinse it with water if necessary; it will be seen to be composed of two obvious coats like the stomach $(\S 736)$.

(B) Villi intestinorum.- Put a fresh piece of intestine in a watch glass of water or normal salt solution, and look at the mucous surface in profile. There will appear numerous slender finger-like processes with their free ends pointing toward the lumen of the 
intestine. These are the intestinal villi. They are only found in the small intestine, and are most abundant in its cephalic portion.

The small intestine of the cat has no valvulæ conniventes (Quain, A, 250 ; Gray, A, 779); but it is completely invested by peritoneum throughout its whole extent. In these two particulars it differs from the intestine of man.

\$ 741. Microscopic Structure--(A) Peritoneal or serous coat. (B) Muscular (unstriped) coat :-(1) Longitudinal layer ; (2) circular layer. (C) Submucosa, areolar or loose connective tissue coat. (D) Muscularis mucosæ of longitudinal and circular unstriped muscular fibers, some of which pass into the villi. (E) Mucous coat with villi and crypts of Lieberkühn, covered with columnar epithelium; Brunner's glands and Peyer's glands. Quain, A, II, 358 ; Stricker, A, 380.

§ 742. Intestinum amplum, az.-Large intestine (Fig. 77, § 719). -The large intestine is the part of the alimentary canal extending from the cæcum to the anus, the caudal opening of the canal. For convenience of description, the large intestine is divided into four parts, named in order:-Caccum, colon ascendens, colon transversum, colon descendens and rectum or terminal part.

Exposure.-Turn the small intestine toward the left leg.

(A) Crecum (Fig. 80).-This is the somewhat conical blind sac at the beginning of the large intestine. It lies on the right side and in about the middle of the abdominal cavity.

(B) Colon ascendens--Ascending colon.- This is the part of the large intestine which extends cephalad from the cæcum.

Respecting the use of the terms cephalad and caudad in designating the position or direction of parts of the alimentary canal, see $\$ 91$.

Topographically, the colon ascendens extends cephalad from the cæcum, but physiologically, that is, in respect to the passage of the contents, the entire colon is candad of the cæcum.

It is quite short, and reaches nearly to the pylorus. Its dorsal surface is in contact with the duodenum.

(C) Colon transversum-Transverse colon.-This is the continuation of the preceding. It extends transversely across the abdomen just caudad of the stomach.

(D) Colon descendens and rectum.--After extending nearly across the abdomen from right to left, the large intestine passes obliquely caudad, soon reaching the meson. It then extends caudad along the vertebral column to terminate at the anus. The last and straighter part is called the rectum.

\$ 743. Obious Structure of the Intestinum amplum.-If the large intestine is full of feces, press the contents of a small part of the colon transversum aside and cut out a piece 
1-2 cm. square. Rinse the mucous surface with water or normal salt solution. The structure will appear like that of the small intestine except that villi are absent.

Microscopic Structure of the Intestinum amplum.-(A) Peritoneal or serous cont. (B) Muscul.ur (unstriped) coat:--(1) Longitudinal layer ; (2) circular layer. (C) Submucosa, loose connective tissue coat. (D) Muscularis mucosæ, unstriped muscular fibers arranged both longitudinally and transversely. (E) Mucous coat, containing crypts of Lieberkühn and Peyer's glands.

The large intestine of the cat is completely invested by peritoneum, and is supported by a duplicature of the same; lience in these respects it differs somewhat from man. See Stricker, A, 391 ; Quain, A, II, 371.

\$ 744. Hepar, az.-Liver (Fig. 77, \$713).-Girasp the liver with the hand and draw it in various directions. It is deeply divided (lobed) and is supported in various parts by folds of peritoneum, the so called ligaments of the liver.

Lobi hepatici.--The lobes of the feline liver have not been satisfactorily homologized with those of the human liver, and the nomenclature is somewhat unsettled. (Owen, A, III, 485; Flower, 41). For convenience, the following provisional names may be used :-

(A) Lobus sinister.-This part of the liver is at the left of the suspensory ligament (the ligament parallel with the meson and serving to hold the liver against the diaphragm). This lobe is deeply divided, and its caudal or thin edge is on the ventral side of the pyloric region of the stomach (Fig. 79).

(B) Lobus cysticus-Cystic lobe.-This is at the right of the suspensory ligament, and occupies the right ventral part of the abdomen. It contains the cholecyst or gall bladder, and hence cannot be mistaken. It is in some cases deeply divided, and in others only slightly.

(C) Lobus dexter-Right lobe.-This is dorsad of the cystic lobe. It is usually deeply subdivided, and is in contact with the ventral aspect of the right kidney.

(D) Lobus Spigetii.--This is the smallest of the lobes of the liver. It is dorsad of the left lobe and dextro-cephalad of the lesser curvature of the stomach. It usually appears at the mouth of the foramen of Winslow, and may be fully exposed by tearing away the peritoneum $(\$ 728)$ at the lesser curvature of the stomach.

\$ 745. Cholecystis-Gall bladder. - This is a receptacle for bile, and as stated above is partly imbedded in the cystic lobe. It is a pear-shaped sac, and the larger end usually appears on the ventral surface of the cystic lobe (Fig. 7\%). To expose it fully, turn the free edge of the cystic lobe cephalad. 
§ 746. Ductus hepatici-Bile ducts.-Press on the cholecyst, and the contained bile will be forced into the various bile ducts:-

(1) Ductus cysticus. - This is the duct extending from the small end of the cholecystis to the ductus communis (3). It presents several loops, the so called impeding flexures, and serves to conduct the bile to or from the cholecyst (Fig. $79,81,82$ ).

(2) Ductus hepatici (Fig. 79).--These are the bile ducts proper. They convey the bile from the various lobes of the liver to the ductus communis (Fig. 79, 81). Press on the cholecystis again if necessary to make them evident. Isolate one by means of the tracer. Cut a V-shaped slit in it near its end and press all of the bile in the cholecyst out through the slit. Then insert a canula through this slit and inject toward the cholecyst with plaster $(\S 359)$. This will fill all the ducts as well as the cholecyst, and after the plaster has set they may be traced in the same way as arteries, ( $\$ 596[10])$.

(3) Ductus choledochus communis-The common bile duct.As the name implies, this receives all of the other ducts from the liver. It appears to be a continuation of the cystic duct. It reaches the duodenum about $3 \mathrm{~cm}$. from the pylorus, and enters it obliquely caudad. Within the walls of the duodenum, it empties into a small reservoir (ampulla of Vater), common to it and the duct of Wirsung (Fig. 84).

The character of the opening of the ductus choledochus and its relation to the ductus Wirsungianus are shown in Fig. 84.

577. Obvious Structure of the Liver (hepar).-(A) Lobi.-The liver is composed of several deep red lobes, which are smooth and shining on the surface from the presence of the peritoneal investment. This is very thin and is separated with difficulty, even over small areas.

(B) Lobuti-Lobules.--These are plainly visible as small areas about $1 \mathrm{~mm}$. in diam. eter, surrounded by rings of deeper color.

(C) Parenchyma.-If a piece is cut out, it may be readily crushed into a pasty mass, showing that the structure is pulpy and cellular rather than fibrous.

§ 748. Microscopic Structure-(A) Peritoneal or serotzs coat. (B) Fibrous coat (pro. jections of this accompany the vessels). (C) Liver substance; this consists of lobules composed of polyhedral cells arranged in a radiate manner around the center of the lobule.

Vessels of the Labule-(1) Intralobular vein or hepatic veinlet in the center of the lobule; (2) Interlobular or portal veinlet and the hepatic arteriole. These are between the lobules, and capillaries pass from them to the center of the lobule between the rows of hepatic cells. In addition to the above, are the beginvings of the hepatic ducts. See Stricker, A, 407 ; Qzzain, A, II, 386.

§ 749. Pancreas, az. (Fig. 81, § 720). -The cat's pancreas is greatly elongated, and so bent as to form two sides of a triangle. 
From the relations of the two parts, they are called respectively the gastro-splenic and the duodenal parts of the pancreas $(\mathrm{O}$ wen, $\mathbf{A}$, III, 495). The organ is wholly enveloped by peritoneum, thus differing from the human, which is covered by peritoneum only on its ventral surface.

In order to expose the pancreas fully, the ventral wall of the great omentum should be removed, and the large and small intestines should be drawn caudad.

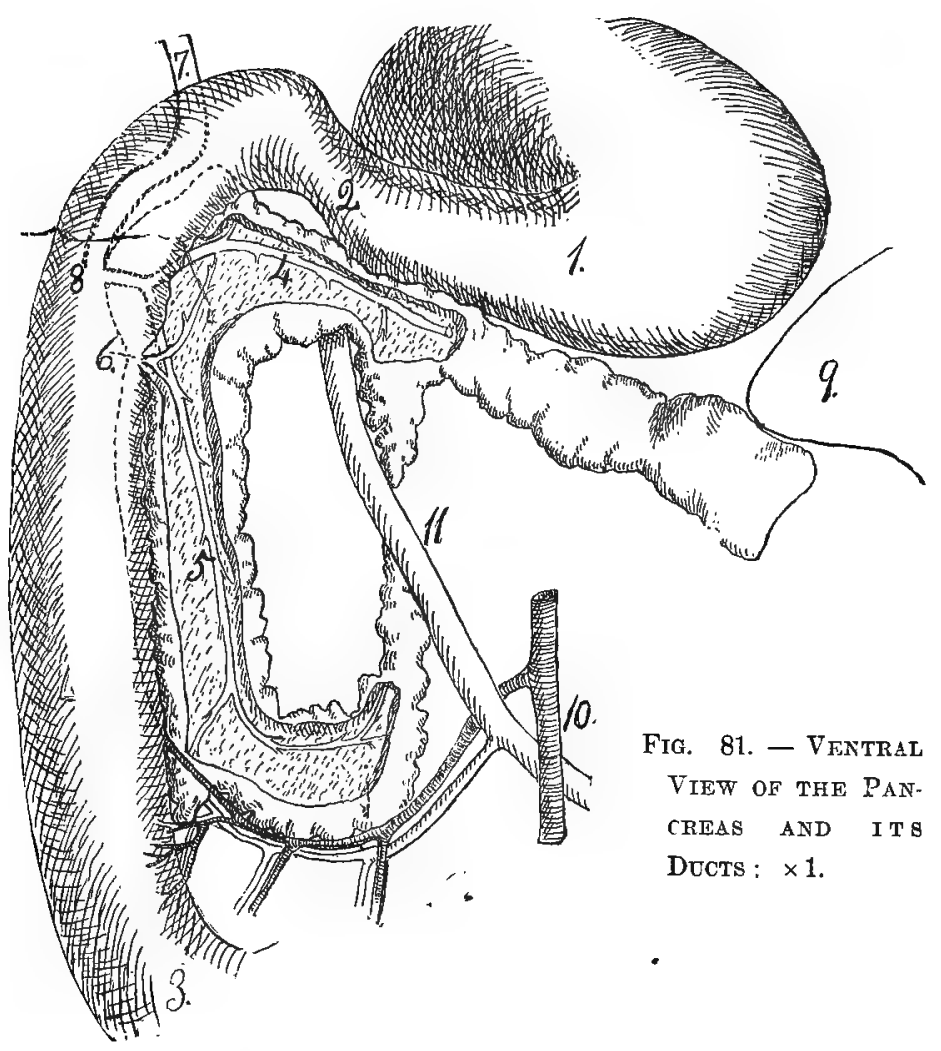

Like the human pancreas, that of the cat has two ducts-Ductus Wirsungianus and Ductus Santorini-which open separately into the intestine, although they anastomose in the substance of the pancreas (Fig. 81-83).

Preparation-Fig. 81.- The great omentım, the jejunum, ileum, 
colon and liver were removed the remaining parts shown in situ. The duct of Wirsung was afterward injected with Berlin blue, and both it and the duct of Santorini dissected out to show their branches and anastomoses.

Explanation of Fig. 8r.-1. Pyloric region of the stomach.

2. Pylorus. 2-3. The duodenum.

4. Gastro-splenic division of the pancreas, near the main branch of the duct of Wirsung.

5. The duodenal part of the pancreas and brancl of the duct of Wirsung.

6. Duodeuum at the point where the duct of Santorini pierces its walls. The dotted line shows the extent of the pancreas on the dorsal side of the intestine. The duct of Santorini is seen to anastomose with each division of the duct of Wirsung.

7. Ductus communis choledochus.

8. The point where the ductus choledochus and the duct of Wirsung enter the duodenum.

9. Tip of the spleen, somewhat displaced.

10. The superior mesenteric artery sonding the inferior pancreatico-duodenal branch to those parts.

11. Superior mesenteric vein receiving a corresponding branch.

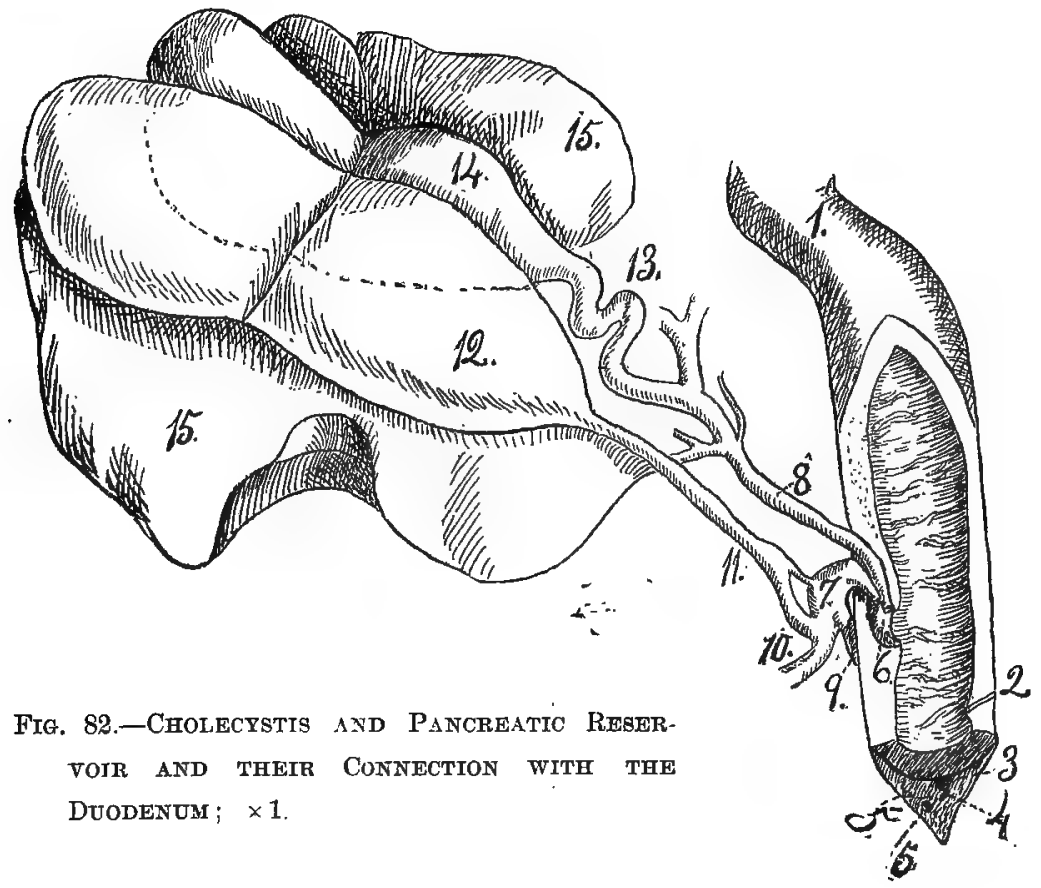

Preparation-Fig. 82.-The liver was turned to the right, bring- 
ing the concave side up ; the duodenum to the left, so that its right side looks directly upward; it was then sliced off to the ievel of the ampulla of Vater and the duct of Santorini.

Explanation of Fig. 82.-1. Pylorus.

2. The duct of Santorini passing obliquely through the duocienal walls.

3. Cut end of the inferior pancreatico-duodenal artery.

4. Same for the correspording vein.

5. The duodenal branch of the duct of Wirsung.

5. Cut end of the duodenal pancreas, showing triangular section, and the intestine partly enveloped by it.

6. The ampulla of Vater.

7. The duct of Wirsung, opening into the ampulla.

8. The ductus communis choledochus, also opening into the ampulla.

9. The duodenal branch of the duct of Wirsung.

10. The gastro-splenic branch displaced.

11. Duct from the pancreatic reservoir opening by a large branch into 10 , and by a small one into 7 .

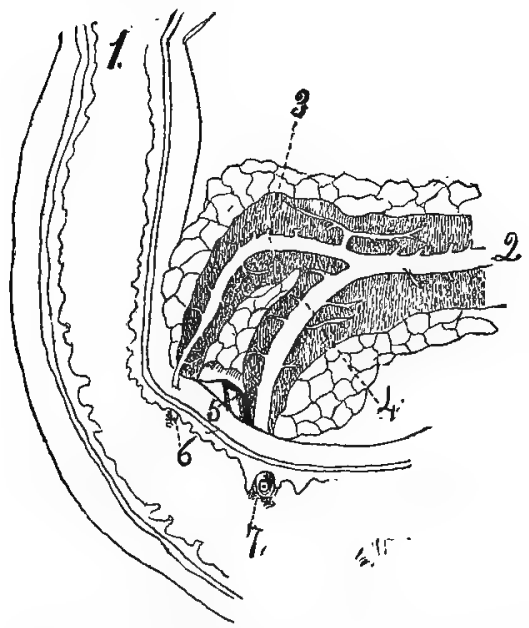

Fig. 83.-Ventral View of the Human Pancreatio Ducts. (After Bernard, 17). $\times .5$.

12. Pancreatic reservoir covering part of the cholecyst.

13. The "impeding flexures" in the cystic duct.

14. T'he cholecystis constricted in the middle, as is aiso the pancreatic reservoir, by a firm wide band possing over them.

15, 15. The cystic lobe of the liver.

Preparation--Fig. 83.--The ventral wall of the duodenum was partly removed to show the openings of the ducts, which had been exposed by dissection.

Explanation of Fig. 83.-1. Pylorus.

2 and 4. Duct of Wirsung.

3. Duct of Santorini anastomosing freely with the preceding, and opening into the intestine hetween the aperture of the ampulla of Vater and the pylorus.

5. Ductus communis choledochus.

6. Opening of the duct of Santorini at the summit of a papilla.

7. Openivg of the ampulla at the summit of a similar papilla. These openings are usually about $10-15 \mathrm{~mm}$. apart.

$\$ 750$. Ductus Wirsungianus-Duct of Wirsung, principal pancreatic duct.-This, in the cat; is usually the larger of the two pancreatic ducts, as in man. It opens into a small reservoir, am- 
pulla of Vater, within the walls of the duodenum. The ampulla appears as an oblique elevation on the ectal surface of the duodenum, and receives also the ductus communis choledochus (Fig. 84).

Preparation-Fig. 84.-The duodenum should be suspended in 95 per cent. alcohol for 2-3 days, or it may be hardened in Muller's fluid (see Frey, A). Then rather thick ireehand sections may be made with a razor or a very sharp scalpel. The sections may be studied to advantage with a tripod lens or with a ${ }_{4}^{8}$-in. objective and a compound microscope.

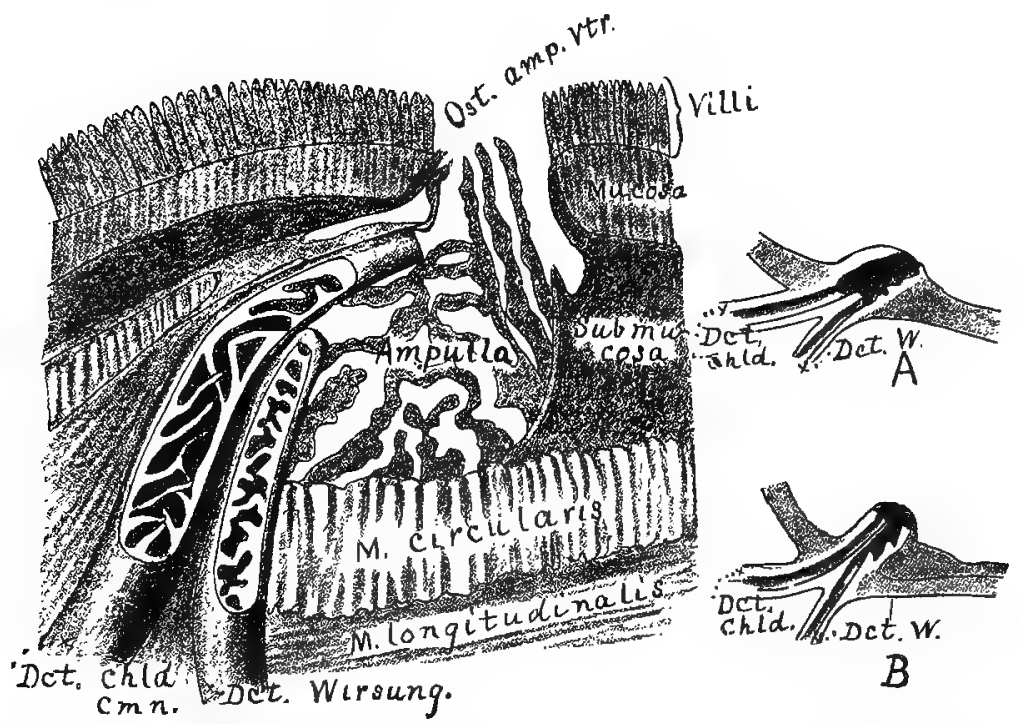

Frg. 81.-Longitudinat Section of the Ampulla of Vater, showing the Entratoe of the Ductus Choledocios and the Duct of Wirsung ; $\times 8.4$. A and B.Similar Sections of the ampdlda of Vater in Man. (A and B, after Claude Bernard, 17). $\times 1.75$.

Explanation of Fig. 84.-Ampulla, Ampulla of Vater.-The sac-like space in the wall of the duodenum into which open the ductus choledochus and the ductus Wirsungianus. The ampulla is not a free space, but is more or less filled by anastomosing processes springing from the walls. The ampulla opens into the duodenum through a single orifice on the summit of a slight papilla.

Det. chld. cmn., Ductus choledochus communis, $a z$.-The common bile duct.-It is seen to have its lumen partly filled with anastomosing processes which allow the bile to flow into the ampulla, but tend to prevent any regurgitation.

Dct. Wirsung., Ductus Wirsungianus, az-Duct of Wirsung.-The pancreatic duct emptying into the ampulla of Vater. In the cat it is usually much larger than the duct of Santorini (Fig. 81-83). 
Mucosa.-The mucous membrane of the small intestine.

M. circularis.-The circular layer of unstriped muscle.

M. longitudinalis. - The longitudinal layer of unstriped muscles.

Ost. amp. Vtr., Ostium ampullæ Vateri, az.-The opening or mouth of the ampulla of Vater.

Submucosa.- The lajer of connective tissue between the circular muscle and the muscularis mucnsæ.

Fig. 84, A and B.-Dct. chld., Ductus choledochus communis, az.-The common bile duct.

Dct. W., Ductus Wirsungianus.-The pancreatic duct opening into the ampulla of Vater. In A, both ducts open at the bottom of the ampulla. This is said by Bernard to be the normal condition. In $B$, the bile duct extends pearly to the orifice of the ampulla, as in the cat. This is not common in man, althongh normal in the cat.

\$ 751. Demonstration of the Duct of Wirsung, the terminal part of the Ductus choledochus, and the Ampulla of Vater.

Turn the duodenum to the left and trace the ductus choledochus to its point of entrance into the intestine. Now remove the peritoneal covering on the dorsal side of the pancreas just caudad of the termination of the ductus choledochus. Then tear away the substance of the pancreas very carefully, and the duct will be exposed. It looks like an uninjected blood vessel. Trace it for some distance from the intestine and it will be found to divide into two main branches, one for each part of the pancreas (Fig. 81-83). Open the duct about $1 \mathrm{~cm}$. from its point of entrance into the intestine, and pass a beaded bristle into it toward the intestine. With a sharp scalpel, slice away the intestine over the point of entrance of the ductus choledochus communis and the ductus Wirsungianus, making the slices parallel with the direction of the two ducts. Continue the cutting until the plaster in the ductus choledochus and the bristle in the ductus Wirsungianus are exposed. It will be seen that the two ducts penetrate the intestinal wall obliquely caudad, and open separately into the ampulla (Fig. 84). The ampulla then opens through a slight papilla into the lumen of the intestine.

Contrary to the common statement, the two ducts cannot be said to unite at all (Gage, 3,177 ).

In some respects it is better to employ a specimen whose hepatic ducts have not been injected with plaster to demonstrate the relation and termination of the two ducts. In this case a bristle should be put into each.

(B) Ductus Santorini-Lesser pancreatic duct (Fig. 83).-This, in the cat, is usually much smaller than the preceding, and hence its demonstration is more difficult. It opens on the left side of the 
intestine obliquely caudad of the preceding, and is demonstrated by tearing away first the peritoneum and then the pancreatic substance. The anastomosis of the two pancreatic ducts may be demonstrated by injecting the ductus Wirsungianus peripherad with Berlin blue.

\$ 752. Obvious Structure of the Pancreas.-It is composed of a great many small lobules arranged like a bunch of grapes, the ducts representing the stems of the grapes. Such glands are said to be racemose.

\$ 753. The Microscopic Structure as well us the obvious structure is in all essential particulars like that of the salivary glands (\$§ 788, 789). See Stricker, A, 295; Quain, A, II, 396.

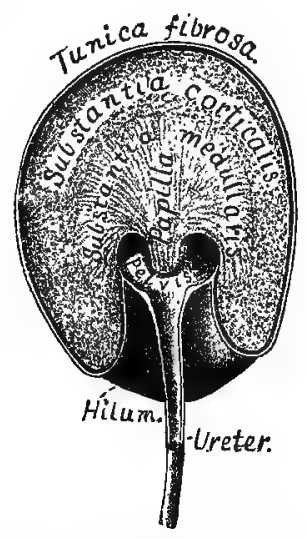

Fig. 85.-Dorso-ventral Section OF THE Right KIDNEY, Caddal VIEW ; $\times 1.1$.

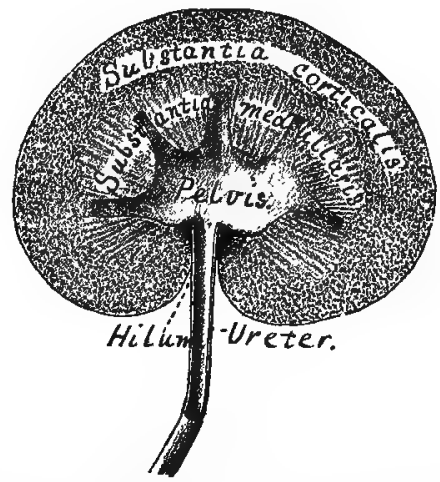

Fig. 86.-Longitudinal Dextro-stnistral SECTION OF THE RIGHT KIDNEY, VEN. TRAL VIEW ; $\times 1.1$.

Preparation-Fig. 85, 86.-The ureter and pelvis were inflated by injecting 95 per cent. alcohol through the ureter toward the kidney. Then the ureter was ligatured and the kidney carefully removed and placed in 60 per cent. alcohol for two days, then in 95 per cent. alcohol for the same time. When well hardened it was sliced off to the level shown in the figures.

Explanation of Fig. 85, 86.-Hilum.-The concavity of the mesal border of the kidney. It is at this point that the ureter and the blood ressels enter.

Papilla.-The apex of the medullary portion. From its free end the urine exudes. There is but one papilla in the cat. In Fig 86, the medullary portion seems to end by several papillæ, but the pyramids forming these converge at a ligher level than here shown, and finally end as shown in Fig. 85.

Pelvis.-The somewhat dilated space into which the papilla opens.

Substantia medullaris.-The medullary or central part of the kidney

Tunica fibrosa (Fig. 85).-The thick fibrous sac enclosing the kidney. It is reflected into the hilum and upon the ureter. 
§ 754. Ren-Kidney (Fig. 77, 78, 85, 86, 101, 103, § 722).-Turn the stomach and the intestines to the right, and the left kidney will be exposed. Remove any fat that can be removed without displacing the kidney. Its lateral aspect is convex, while the mesal one presents a deep concavity, the so called hilum of the kidney.

The right kidney is somewhat farther cephalad than the left, thus differing from man. Only the ventral surface is covered with peritoneum (Fig. 78); but the entire kidney is surrounded by a special fibrous capsule or covering (Fig. 85).

Obvious Structure of the Kidney.-With a sharp scalpel, make a longitudinal dextro. sinistral section of the kidney, removiug the ventral three fifths. The appearance shown in Fig. 86 will appear.

The ureter (\$ 756) commences as a funnel-shaped opening from the hollow or pelvic part of the kidney (Fig. 85, 86).

The solid part of the kidney is evidently composed of two portions-the ectal, peripheral or cortical, and the ental, central or medullary portions. The cortical or peripheral pcrtion is granular and of a desp color, while the medullary portion is lighter in color, smooth, compact, and of more or less triangular outline. The apex projects into the pelvis of the kidney and is called the renal papilla. There are several in man, but only one in the cat

\$ 75.5. Microscopic Structure.-(A) Tubuli uriniferi, or urinary tubules-tubes lined with cells and forming the kidney substance proper (B) Blood vessels and lymphatics. (C) Nerves and connective tissue.

In the medullary part of the kidney, the blood vessels and urinary tubules are mostly straight in direction, while in the cortical portion they are looped or couvoluted; both vessels and tubules are branched. The arterics form the so called glomeruli or Malpighian corpuscles by a multiple knotting at their termination. See Siricker, A, 460 ; Quain, A, II, 400 .

$\S$ 756. Ureter.-Grasp the kidney with one hand and the urocyst (Fig. 101) with the other. Draw the former cephalad and the latter caudad. There will be seen, stretching between the kidney and the neck of the urocyst, a narrow tense band, more or less covered with fat. This band is the ureter, or duct conveying the urine from the kidney to the urocyst.

Sometimes one may inflate the urocyst by blowing into the renal end of the ureter, but usually the ureter is too greatly contracted. The tubular character of the latter may be easily demonstrated, however, by using a beaded bristle $(\$ 136)$ or by commencing at the kidney and slitting it with fine scissors.

\$ 757. Urocystis, vesica urinaria-Urinary bladder (Fig. 77, $101, \S 718)$. - This is the receptacle for the urine.

A. Ligamentum suspensorium.-Grasp the cephalic or larger free end of the bladder and draw it ventro-caudad. A thin mem- 
brane like the mesentery will be seen between it and the ventrimeson, the ligamentum suspensorium. It is formed of a duplicature of peritoneum, and in young animals there may be seen between its two layers the remnants of the hypogastric arteries and of the urachus (Gray, A 812 ; Quain, A, II, 800).

B. Ligamentum laterale.-In addition to the suspensory ligament, one having the same general appearance will be seen on each side.

C. Cervix urocystis-Neck of the urinary bladder-Draw the urocyst caudad and its fixed point will be seen to grow narrow. This narrow part is the neck, and its continuation to the exterior is called the urethra or excretory canal of the bladder. The ureters penetrate the urocyst on each side of the neck ; their course through the wall is quite oblique, as may be demonstrated by passing a bristle from the ureter into the bladder.

$\$$ 758. Obvious Structure of the Urocyst.-Cut a slit in the urinary bladder to allow the urine to escape, then cut out a piece about $2 \mathrm{~cm}$. square. Rinse it with water or normal salt solution. 'There may be demonstrated a structure somewhat comparable to that of the stomach:-(A) An ectal serous (peritoneal) coat. (B) An intermediate firm or musculur coat. (C) An ental soft or mucous coat. The muscular and mucous coats are, however, more closely united than in the stomach.

$\S$ 759. Microscupic Structure.-(A) Serous (peritoneal) coat. (B) Muscular (unstriped) coat:-11) Longitudiual layer ; (2) circular layer ; (3) longitudinal layer. The three layers are arranged somewhat in the form of a figure of - 8. (C) Submucosa of loose connective tissue. (D) Mucous coat covered with stratified epithelium. See Stricker, A, 487 ; Quain, A, II, 423 .

\$ 760. Adrenale-Capsula renalis, capsula suprarenalis.-Turn the stomach and intestines to the right. About $2 \mathrm{~cm}$. meso-cephalad of the kidney will appear a pinkish, oval body about $2 \mathrm{~cm}$. long and $1 \mathrm{~cm}$. wide. Its caudal end is usually in contact with the V. renalis and its ventral surface is crossed by the $\mathrm{V}$. adreno-lumbalis (Fig. 101, adrn.).

The right adrenal is in about the same position with respect to the right kidney ; but as the $V$. cava and part of the liver are on its ventral surface, it is not so easily demonstrated as the left.

Both adrenals are covered on their ventral surface by peritoneum. Neither of them is in contact with the kidney. In this respect they differ from their human homologues.

$\S 761$. Uterus--Womb ( $\$ 723$ ).--If the cat is a female, there will appear between the rectum and the urocyst a mesal organ, the uterus, having the same general color and appearance as the intes- 
tines. It is a hollow organ and at its cephalic end bifurcates, forming the so called cornua or horns of the uterus. The horns extend obliquely latero-cephalad nearly to the caudal extremity of the corresponding kidney (Fig. 77).

§ 762. Tuba Fallopiana-Fallopian tube, Oviductus.-Near the ends, the horns of the uterus become quite small and more or less convoluted. This small part of the horn is called the oviduct or Fallopian tube. It opens directly into the peritoneal cavity, thus putting the peritoneal cavity in communication with the exterior of the body. The end is somewhat funnel shaped and one edge is applied to the ovary. Quain, II, 470.

Make an incision in one of the cornua near the beginning of the Fallopian tube and pass a beaded bristle into the uterus, and then along the Fallopian tube throughout its whole extent. This will be facilitated by severing tile connections of the tube so that it may be straightened.

\$ 763. Ligamentum uteri.-Grasp a uterine cornu and lift it up. A broad membranous band will appear extending laterad from it. This is the ligamentum latum or broad ligament of the uterus and its horns. Like the mesentery and ligaments of the urocyst, it is simply a duplicature of peritoneum.

Ligamentum rotundum-Round ligament.-Look through the ligament toward the light; a thickening will appear in it extending from near the middle of the horn caudad to the abdominal wall ventrad of Poupart's ligament (Fig. 39). This is the round ligament, and may be traced through the abdominal wall. It terminates in the external organs of generation.

\$ 764. Obvious Structure.-(A) Serous (peritoneal) coat. (B) Muscular coat. (C) A soft mucous coat. These points may be easily demonstrated by cutting out a small piece of the uterus or one of its horns.

§ 765. Microscopic Structure.-(A) Serous (peritoneal) coat. (B) Muscular (unstriped) coat ; the fibers greatly interlace and are mixed with abundant connective tissue. (C) Mucous coat. See Stricker, A, 606 ; Quain, A, II, 464.

$\S$ 766. Ovarium-Ovary.-At the cephalic end of each Fallopian tube ( $\$ 762)$ may be seen the ovary, a yellowish oval body about $1 \mathrm{~cm}$. long and $.5 \mathrm{~cm}$. wide. It is supported by an extension of the broad ligament.

§ 767. Microscopic Structure.-(A) Modified peritoneal coat. (B) Ovarian stromaconnective tissue, blood vessels and nerves, Grafian follicles with the ova. See Stricker, A, 510 ; Quain, A, II, 4 \%2. 
$\S 768$. Vas deferens. - If the subject is a male, there will be seen on each side a white cord, the vas deferens or spermaduct (Fig. 101), looping around the ventral side of the ureter and A. hypogastrica, and then extending toward the urethra.

If the vas deferens is traced peripherad, away from the urethra, it will be seen to penetrate the abdominal wall laterad of the $A$. epigastrica and ventrad of Poupart's ligament.

In traversing the abdominal wall, it passes through the canatis inguinalis. It is accompanied by the spermatic artery and vein and a duplicature of peritoneum, and all together form the spermatic cord. The opening of the inguinal canal within the abdominal cavity is called the annulus abdominalis internus or the internal abdominal ring, while the one on the ectal surface of the abdominal wall is called the annulus abdominalis externus or external abdominal ring (Fig. 39).

From the external abdominal ring, the spermatic cord extends obliquely caudad and entad of the skin to the testis.

\section{THE SALIVARY GLANDS, MOUTH CAVITY, PHARYNX, NECK, THORAX AND DIAPHRAGM.}

\$ 769.-Names of Parts in the Order of Examination.-Glandula parotis-Ductus Stenonianus - Glandula submaxillaris-Ductus Whartonianus - Lingua - Pharynz - Tuba Eustachiana - Larynx - Trachea - Esophagus - Pleura - Thymus - Pulmo-Cardia_ Diaphragma.

The vessels and nerves of these parts are treated in Chap. VIII and IX.

$\$$ 770. Instruments and Matericul the same as for the abdomen with the addition of a saw (Fig. 21), nippers (Fig. 11), beaded bristles (\$ 136), and about 100 cc. of the Berlin blue solution.

Choice of Specimen the same as for the abdomen.

\section{SALIVARY GLANDS.}

§ 771. References.-Quain, A, II, 335; Gray, A, 757 ; Bernard, A, 504 ; Chauveau, A, 387 ; Leyh, A, 372 ; Owen, A, III, 396 ; Cuvier, A, III, 409; Hyrtl, A, 241 ; Gegenbaur (Lankester), A, 519 ; Milne-Edwards, A, VI, 220 ; Chauveau (Fleming), A; Gurlt, A, 361.

§ 772. Posture and Preparation.-The cat should be dorsicumbent, with a block crosswise under the neck, and the head rotated dextrad so that the side of the face looks upward; the mouth should be held open with a cork. The animal should be injected from the femoral artery, making the plaster somewhat thinner than usual (\$S. 345, 352). The femoral vein should be injected with blue plas- 
ter ( $\$ 362)$. If the abdomen is not to be used ( $\$ 234)$, it is easier to inject from the postcava and the aorta ( $\$ 363,365)$.

As plaster will not pass the valves in the veins, it is best for this preparation, as for Fig. 101, to inject the jugular vein with fine blue mass (see $\$ 1450$ ) instead of blue plaster. This is only necessary, however, for permanent preparations or for special demonstrations.

$\S$ 773. Salivary Glands. - The salivary glands are the organs, belonging to the digestive system, which secrete the saliva and pour it into the oral cavity through single or multiple ducts. They are situated in close proximity to the mouth and mostly just entad of the skin. There are 5 on each side :-Parotid, submaxillary, sublingual, molar, zygomatic.

$\$ 774$. Preparation of the Ducts of the Salivary Glands.These should be injected with Berlin blue (see $\S 1449$ ), or if that is not at hand, there may be used a sufficient quantity of chrome green or orange ground in 15 per cent. glycerin to give a decided color. As the process of injection is somewhat troublesome, the ducts may bo demonstrated by inserting into them beaded bristles.

$\S$ 775. Preparation of Wharton's Duct.-This opens on the summit of a prominent papilla situated in the floor of the mouth just cephalad of the frænum (Fig. 88). It usually lies on the floor of the mouth. Grasp it near its free end with the fine forceps, and enlarge the opening with the probe of the tracer; then insert a beaded bristle or the canula for injection (\$358).

Injection. - The canula need not be tied, but merely compressed with the fingers while injecting. Before commencing the injection, the canula should be filled with the injecting mass to avoid air. In making the injection, the pressure should be light and continued for but a short time lest the duct be ruptured. If the injection is successful and the tongue be turned to the opposite side, the duct may be seen in the floor of the mouth extending nearly parallel with the mandibular ramus as far caudad as the last tooth.

\& 776. Preparation of Stenon's Duct.-With coarse forceps or the fingers, grasp the dorsal lip near the angle of the mouth and draw it laterad so as to expose the mucous membrane opposite the last præmolar tooth (Fig. 61). Just cephalad of the angle of the mouth and opposite the most prominent cusp of the last præmolar will be seen a slight ridge on the mucous membrane. The cephalic end of this ridge is about $1 \mathrm{~cm}$. from the edge of the lip, and at the end will be seen a slight circular depression, which is the opening 
of the duct. Enlarge the opening with the probe of the tracer, and insert a beaded bristle or a canula for injection. Inject as directed for Wharton's duct $(\$ 775)$.

\$ 777. Exposure of the Salivary Glands and their Ducts.Divide the skin as follows:-(A) Along the mesal border of the mandibular ramus from the canine tooth directly caudad as far as the caudal end of the larynx. (B) From the caudal end of the incision (A) to a point opposite the meatus auditorius externus. (C) From the maxillary canine to the dorsal border of the ear. (D) Along the edge of the lips from the mandibular to the maxillary canine, leaving a narrow band of skin with the lip.

Exposure of the Glands, etc.-Commence at the angle next the larynx (ventro-caudal angle) and dissect the skin free. Use a sharp scalpel and dissect close to the skin. Then commence at the same point and dissect free the thin dermal muscle $(\$ 608)$ in the same manner that the skin was dissected. It is necessary to take the greatest care in removing the muscle in order to avoid injury to nerves and vessels. Remove the caudal part first, thus exposing the glands and larger vessels and nerves, which are more easily seen than their branches. Compare the appearances presented with those shown in Fig. 67. If the gland ducts were injected with fine mass, the glands will be of the same color as the mass used. The duct of the parotid (Fig. 87) will be very conspicuons and will serve as a kind of landmark. The same is true of the $V$. jugularis (Fig. $87,101)$.

In exposing and isolating nerves and vessels in this preparation, it is necessary to work with the greatest care. The sharp tracer cannot be used as safely as in most cases. Use the dull tracer, fine forceps and scissors, and remove fat and connective tissue piecemeal. So many branches of the nerves enter the dermal muscle that it is necessary to dissect it very carefully, so as not to remove at the same time the larger branches of the nerves.

Explanation of Fig. 87.-A. fac., A. facialis.-Facial artery; a branch of the carotid. Ductus Stenon. (Stenonianus).-Stenon's duct; duct of the parotid gland. Glandula parotis.-The parotid gland, the largest of the salivary glands. Gl. (Glandula) submaxillaris.-The submaxillary gland. Gl. m., Glandula molaris.Molar gland. Mandible.-Inferior maxilla, lower jaw. Mr. clv. (clavo-) trapezius (Fig. 66). M. sterno-mstd. (mastoideus) (Fig. 
72). IM. tmp., M. temporalis. IM. mstr., M. masseter (Fig. 67).Masseter muscle. N. aur. (auricularis) magnus, N. tmp. fac., $\mathbf{N}$. temporo-facialis.-Branches of the temporo-facial division of the facial nerve (vii). N. crv. facial., N. cervico-facialis.--Cervicofacial division of the facial nerve (vii). N. tmp. aur., N. temporoauricularis.-Branches of the temporo-auricular division of the

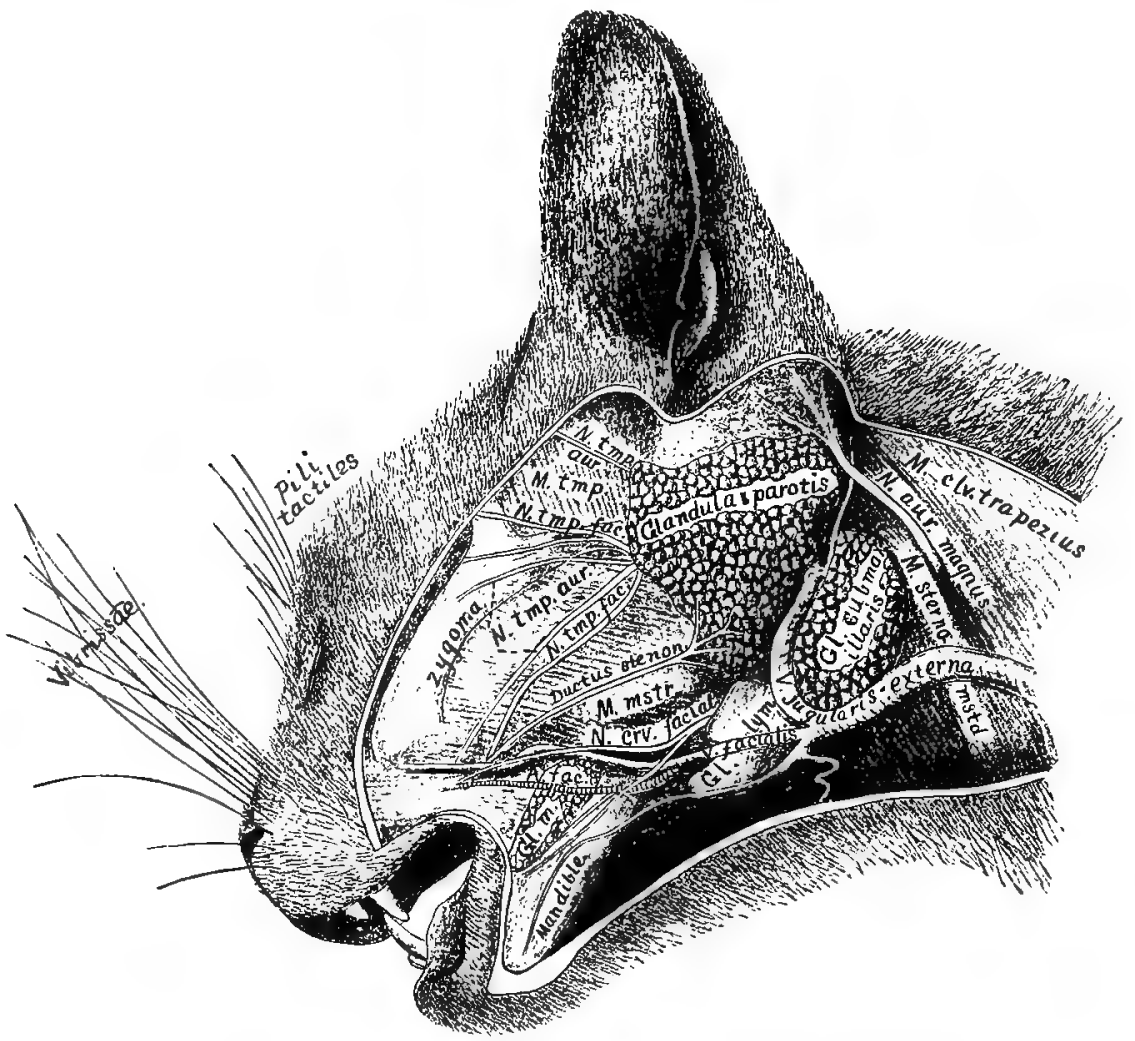

Fig, 87.-Salivary Glands 0:" the Lefit Side; $\times 1$.

trigeminus nerve ( $\nabla)$. Pili tactiles.-Tactile hairs. There are usually 5-8 of these. They are supplied by branches of the trigeminus nerve (v) and are supposed to be tactile hairs. Vibrissw.Whiskers. These stiff hairs are likewise supplied by branches of the irigeminus nerve and are also supposed to be tactile organs. $\boldsymbol{\nabla}$. facialis - Facial vein. A branch of the external jugular vein. V. jugularis externa.-The external jugular vein. It almost inva- 
riably lies between the parotid and submaxillary and separates the latter from the lymphatic immediately cephalad of it. Zygoma, arcus zygomaticus.-The zygomatic arch (Fig. 56).

For the manner of preparation, see $\$ \$ 772,777$.

$\S$ 778. Nerves.-Cervico-facial division of the facialis (vii) (Fig. 87).- This emerges from the ental surface of the lymphatic gland (Fig. 87, Gl. lym.), crosses the $\mathrm{V}$. facialis and divides into the two main divisions, one of which extends along the nandible, the other dorso-cephalad toward the angle of the mouth.

Temporo-facial division of the facialis (vii) (Fig 87, N. tmp. fac.).-About $1 \mathrm{~cm}$. dorsad of the parotid duct there emerges another branch which extends dorso-cephalad and spreads out in many branches over the side of the head and face.

Temporo-auricular division of the trigeminus (branch of the $3 \mathrm{~d}$ or inferior division of the $V$ nerve).- It has the same general dircction as the temporo-facial branch of the facialis and anastomoses Ireely with it. Its fine branches spread out over the head and face as do those of the temporo-facial.

At the dorso-cephalic angle of the parotid emerges another branch of the temporo-auricular (Fig. 87, N. tmp. aur.). It extends almost directly dorsad.

Auricularis magnus (Fig. 87).-This large spinal nerve emerges from between the $M M$. clavo-trapezius and sterno-mastoideus. It then extends dorso-cephalad and spreads out over the caudal surface of the external ear.

\section{GLANDUL丑 SALIVARI压.}

\& 779. Glandula parotis-Parotid gland-The position and relations of this gland are well shown in Fig. 87. It surrounds the ventral half of the external ear.

8 780. Ductus Stenonianus-Stenon's duct, duct of the parotid gland (Fig. 87).--It extends cephalad from the cephalic edge of the gland along the ectal surface of the masseter muscle, nearly directly toward the angle of the mouth. When near the edge of the lip it penetrates the cheek, passing entad of the facial vein (Fig. 87, V. facialis). It opens on the mucous surface of the cheek opposite the most prominent cusp of the last prænılar (Fig. 57).

Isolate the duct as directed for nerves and vessels (\$ 777). It may be easily traced if it has been injected or if a black bristle has 
been inserted. Near the gland it will be seen to divide into several branches.

§ 781. Glandula parotidea accessoria - Accessory parotid gland.-In about one subject in ten there may be found one or more small glandular masses connected with the parotid duct. These are sometimes in contact with the duct at some point of its course over the masseter, or they may be separated for a centimeter or more; if separated, a slender duct connects them with the main duct. Mivart, B, 173.

§ 782. Glandula submaxillaris-Submaxillary gland (Fig. 87). The submaxillary gland is ventrad and partly entad of the parotid. Its cephalic edge is also covered by the lymphatics. The lobulations of the submaxillary are coarser than those of the parotid ; and if uninjected it is of deeper color.

$\S$ 783. Ductus Whartonianus-Wharton's duct, duct of the submaxillary gland.-In order to expose this, the lymphatics and the vein should be removed. The duct appears at the cephalic border of the gland, and extends almost directly dorsad, passing between the MM. digastricus (Fig. 101) and masseter (67), until it reaches the floor of the mouth opposite the last tooth. In its passage between the muscles, its lateral surface is crossed by the gustatory branch of the $N$. trigeminus (v).

Exposure.Divide and rofect the M. digastricus (Fig. 101); then the duct may be traced to the floor of the mouth. Its passage along the floor of the mouth to the papilla may be more readily traced by removing part or all of the corresponding mandibular ramus.

\& 784. Glandula sublingualis-Sublingual gland.-The sublingual gland in the cat is quite small and so closely connected to the submaxillary that it appears as an accessory of it. It is elongated and extends cephalad from the submaxillary about $2 \mathrm{~cm}$. and parallel with Wharton's duct. Its duct, smaller than that of the submaxillary, extends parallel with it and opens upon the same papilla.

$\S$ 785. Glandula molaris-Molar gland.-This gland is small as compared with the parotid, but is of the same general appearance. It is situated near the angle of the mouth about $1 \mathrm{~cm}$. ventrad of Stenon's duct. It has several ducts which pass straight through the cheek and open on the mucous surface. This gland is considered by Cuvier (A, III, 424), Ward, (A, IV, 426), and Quain 
(A, II, 301), to be merely an aggregation of buccal glands; see $\$ 794$.

\$ 786. Glandula zygomatica-Zygomatic, subzygomatic or infraorbital gland.-This is a compact, somewhat elongated gland situated in the lateral part of the orbit. Its ventral end rests on the mucous membrane of the roof of the mouth just caudad of the last maxillary tooth (true molar, see Fig. 57, D. M.), and its duct opens at the same place.

$\S 787$. To demonstrate this gland, the mouth may be kept open by a cork between the teeth ; then the mucous membrane just caudad of the last maxillary tooth should be cut, and the gland will appear. Or the zygoma, the malar process of the maxilla and the masseter muscle may be removed to expose the lateral surface of the eyeball. The gland will be found at the ventro-lateral surface of the eye. To demonstrate its duct, carefully tear away the gland substance near its ventral end with a tracer.

$\$$ 788. Structure of Salivary Glands.-Tbe obvious structure of the salivary glands is that of the racemose type, that is, like a bunch of grapes, the ducts representing the stems and the lobules the fruit.

\$789. Microscopic Structure.-The ducts, except the very smallest, are lined with columnar epithelium; the smallest are lined with pavement epithelium. Their mole of termination "demands further investigation" (Stricker, A, 800). The lobules are composed of groups of spheroidal cells surrounded by a continuation of the connective tissue formiug the interlobular septa. Quain, A, II, 339.

CAVUM ORIS, MOUTH OR BUCCAL CAVITY, PHARYNX, az. (Fig. 77, 88).

References.-Quain, A, II, 300; Gray, A, 745 ; Cbauveau, A, 351 ; Chaureau (Fleming), A, 330 ; Leyh, A, 364: Owen, A, III, 383; ('uvier, A, III, 379; Hyrtl, A, 241 ; Gegenbaur (Lankester), A, 548 ; Milne-Edwards, A, VI, 11 ; Gurlt, A, 336.

$\S$ 790. The mouth cavity or cavum oris is the cephalic division of the alimentary canal. It is bounded cephalad by the lips and caudad by the velum palati and the cephalic opening of the pharynx. It contains the teeth, gums, alveolar margins, the jaws, the tongue and the tonsils, and into it open the dncts of the salivary and buccal glands.

§ 791. Pharynx, az.-See description of Fig. 88.

Exposure and Dissection-Fig. 88. - With a scalpel, divide the skin and soft parts upon both the dorsal and the ventral aspects of the head $1 \mathrm{~cm}$. sinistrad of the meson from the snout to a point opposite the $2 \mathrm{~d}$ or $3 \mathrm{~d}$ cervical vertebra. With the arthrotome, separate the mandibular rami at the symphysis menti, and with bone 
scissors, cut the left pier of the os hyoides (Fig. 30, § 224). Hemisect the tongue and the muscles along the ventral side, cutting about $1 \mathrm{~cm}$. to the left of the meson and entirely through to the buccal cavity. The trachea and cosophagus should be displaced to the right. Cover the hand with a towel and grasp the left side of the head, and divide the entire head with the saw, commencing at the snout a little sinistrad of the meson and making the strokes in the dorso-ventral rather than in the caudo-cephalic direction. With nippers, remove the left side of the vertebral arch of the atlas, axis and the skull as far as the meson.

$\$$ 792. If a permanent preparation is to be made, the remaining half of the mandible should be opened and a cork put between the teeth; the tongue should be drawn slightly cephalad and held in position by a jin pushed through its tip and into the floor of the mouth. The velum palati may be pinned out as in the figure. Finally. the esophagus and larynx should have one side cut away for a short distance and the tubular portion distended by filling the lumen with cotton; the opening of the Eustachian tube should also be tilled with cotton.

Begin the hardening in about 60 per cent. alcohol ( $\$ 286)$. After the head has thoroughly hardened, the brain may be sliced away to the meson, and the mesethmoideum and romer removed to show the turbinated bones and the passage from the prenaris to the postnaris. The surface, especially of the tongue, may be freed from mucous with a soft nail brush.

8 793. Obiious Structure of the Cavum oris or Mouth.-The free surface is usually quite firm and smooth, except in certain places, as the roof of the mouth, where there are many ridges and fine projections.

§ 794. Microscopic Structure.-The free surface is made up of stratified epithelium resting on a rather abundant submucous connective tissue, in which are small so called buccal glands of the racemose type ( 178 ), whose ducts open on the fiee surface.

\& 795. Obvious Structure of the Lingua or Tongue,-The free surface on the ventral side of the tongue is smooth and soft. On the dorsal side it is beset with numerous projections or papillae of various forms, named in tbe order of their abundance :-Filiform, odontoid, fungiform, circumvallate.

$\S 796$. Microscopic Structure.-Into all the papillæ extend loops of blood vessels. The odontoid variety are covered by a horny substance. In the walls of the circumvallate are imbedded the so called taste buds, flask-like in form and composed of modified epithelium. The principal mass of the tongue consists of stripod muscle and comnective tisgne. The muscles are arranged in an intricate net-work, the fibers sometimes branching near the inucous coat. The free or mucous coat consists of stratified epithelium with a small amount of subrucous connective tissue. Seo Stricker, A, 35: ; Quaiu, A, II, 327. 
Preparation-Fig. 88-See p. 303, exposure and dissectionFig. 88.

Explanation of Fig. 88.-Atlas, as.-The first cervical vertebra.

Axis, as.-The second cervical vertebra; its odontoid process (Fig. 62) is shown projecting cephalad into the ring of the atlns.

Callosum, Corpus callosum, ac.-The great commisenre of the hemispheres. (For this and the other parts of the brain, see Fig. 117.)

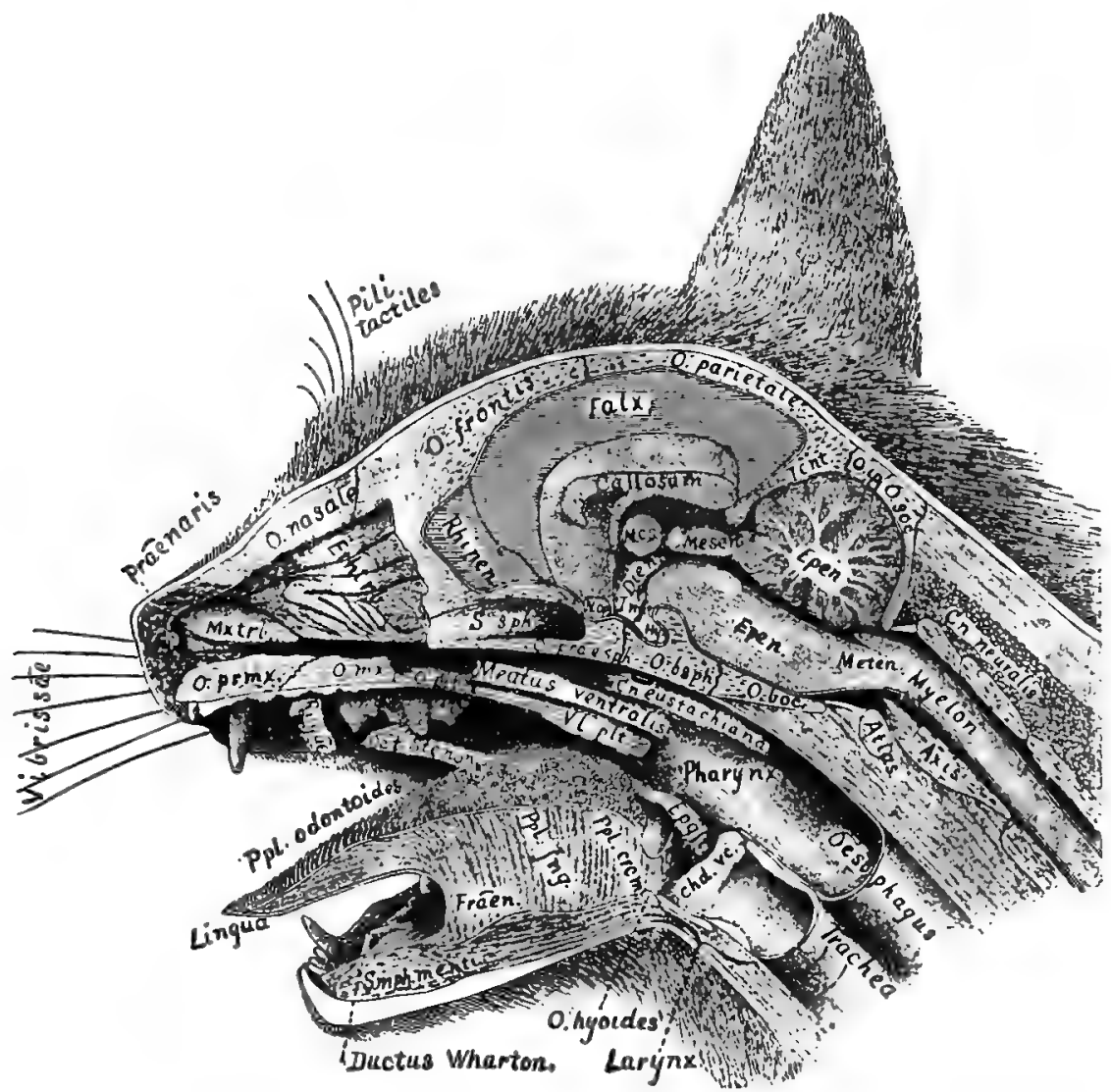

Frg. 88.-Hemregction of TEE Head; $\times 1$. Compare with Fig. 59 and 117 and with Plate II, Fig. 4.

Cn. (Canalis) Eustachiana.-The canal connecting the middle ear or tympanum with the pharynx. Its crescentic opening into the pharynx is shown in this figure.

Cn. (Canalis) neuralis, as.-Neural or vertebral canal. The neural canal is represented by the deeply shaded space in the vertebre in which the myelon rests. (The name is put upon the muecles dorsad of the roof of the arch of the atlas and axis. Through an inadvertence no dotted line is drawn from it to the canal) 
Chd. vc., Chorda vocalis.-Vocal cord.

Dien., Diencephalon.-The abbreviation is written in the space between the two thalami known as the diacelia or " $3 \mathrm{~d}$ ventricle."

Dct. Stenon., Ductus Stenonianus-Stenon's duct, Duct of the parotid gland (Fig. $87, \S 780$ ). - Its opening is hidden by the prominent cusp of the last præmolar, but a bristle is represented as coming from it.

Ductus Whartonianus-Wharton's duct, Duet of the submaxillary gland (§ 783).The prominent papilla at the summit of which the duct opens is shown.

Dura-Dura mater.-The dura is represented in this figure as a broad white line just entad of the cranium and then prolonged into the neural canal as a sheath for the myelon. It takes the place of the periosteum in the skull, but the neural canal is lined by a special periosteum, so that the dura in the neural canal belongs exclusively to the myelon.

Epen., Epencephalon, Cerebellum.-See Fig. 11\%.

Epglt., Epiglottis.-The triangular flap which aids in the closure of the glottis during deglutition.

Ethtrb., Ethmoturbinale-The ethmoturbinal bone (Fig. 59, $\$ 550$ ).

Falx, Falx cerebri, az. - The fold of dura separating the olfactory lobes and part of the mesal surface of the hemispheres of the right and left sides. In this figure it is shaded witb horizontal lines.

Fræn., Frænum linguæ.--The more or less plate-like cephalic part of the attachment of the tongue to the floor of the mouth.

Hy., Hypophysis cerebri, az.-Pituitary body.-See Chap. X.

Inf., Infundibulum, az.-The ventral prolongation of the diaccelia into the bypophysis.

Larynx, az.-The epecialized cephalic part of the trachea, containing the vocal cords.

Lingua, $a z$-Tongue.

Meatus ventralis.-The ventral and more direct passage from the prenaris through the nasal chamber to the Postnaris or opening into the pharynx.

Mcs., Medicommissura.-Middle commissure (Fig. 117).

Mesen., Mesencephalon.-Optic lobes.

Meten., Metencephalon, az.-Medulla oblongata.

Mxtrb., Maxilloturbinale.-Maxilloturbinal bone (Fig. 59).

Myelon, az.-Spinal cord (Fig. 104).

N. op., N. opticus.-Optic nerve.

Es., Esophagus, az.-Giullet ( $\$ 801$ ).

O. boc., O. basioccipitale, $a z$. (Fig. 59).

O. bsph., O. basisphenoideum, az. (Fig. 59).

O. præsph., O. præsphenoideum. $\alpha z$. (Fig. 59).

O. hyoides (Fig. 30, \& 224).

O. plt., O. palatinum (Fig. 59).

O. mx., O. maxillare (Fig. 59).

O. pmx., O. præmaxillare (Fig. 56).

O. soc., O. supraoccipitale, $\alpha z$. (Fig. 59 ).

O. ip., O. interparietale, $a z$. (Fig. 59).

O. parietale (Fig. 56).

O. frontis (Fig. 56).

O. nasale (Fig. 56, 59).

Papillæ filiformes-Filiform papillæ (\$ 795).-These are the fine projections from the dorsal surface of the tongue. Caudally they become broad and ligulate (1-3 mm. long), while in the middle of the cephalic part of the dorsal surface they are modified into the form next described (Stricker, A, 356). 
Ppl. (Papillæ) odontoides (Milne-Edwards, A, VI, 104).-These are the horny recurved papillæ on the dorsal aspect of the cat's tongue with which it rasps the surface of bones. For the other papillæ, see Quain, II, 325.

Ppl. fng., Papillæ fungiformes-Fungiform papillæ.-These are the bead-like projections on the dorsal surface of the tongue. They are especially abundant near the middle of its length.

Ppl. crcm., Papillæ circumvallatæ-Circumvallate papillæ.-These are few in number and are situated on the dorsal surface of the tongue in the locality indicated in Fig. 88. They appear like large fungiform papillæ with a trench and a raised border around them.

Pharynx, az.-The general cavity into which open the mouth, postnares, Eustachian tube, cesophagus and trachea.

Pili tactiles-Tactile hairs (Fig. 87).

Prænares-Nostrils.-The opening of the nasal clamber at the snout.

Rhinen., Rhinencephalon.-Olfactory lobes.

Rugæ.-The transverse wrinkles or folds in the roo: of the mouth. There are five or six of these, and they are covered with short, stiff papillæ.

S. șph., Sinus sphenoideum.--Sphenoidal sinus (Fig. 59).

Symph. (Symphysis) menti, az.-The amphiarthrodial articulation between the two sides of the mandible.

Tnt., Tentorium cerebelli.-The letters are on the bony tentorium (see Fig. 59), but this is seen to be lined on its ental surface by the dura. The dura is as easily separable from the bony tentorium as from the other parts of the skull, and the bony tentorium itself seems to be a part of the Os parietale, not an ossification of the membranous tentoriusa as stated by Flower, A, 99 ; see Cuvier, A, III, Article 10.

Trachea, az.-Windpipe.-The tube connecting the lungs with the pharynx (Fig. 77).

V1. plt., Velum palati, $a z$.-The pendulons or caudal portion of the soft palate. It is here represented as lifted from the tongue so that it extends nearly caudad instead of curving ventrad as in nature.

Vibrissæ-Whiskers.-See Fig. 87, 88. In Fig. 88 their free ends are cut off.

\$ 797. Collum, az.-Neck. - This is the constricted portion of the trunk cephalad of the first rib, $i . e_{\text {. }}$, between the thorax and head (Fig. 6).

\$ 798. Exposure.-Make an incision along the neck about $2 \mathrm{~cm}$. to the right of the ventrimeson as far caudad as the first rib; then a transverse incision just cephalad of the first rib to a point $2 \mathrm{~cm}$. to the left of the ventrimeson; dissect off the skin and the muscles covering the trachea (Fig. 76).

\$ 799. Trachea, az.-Windpipe (Fig. 77).-The trachea cannot be mistaken, as it is the first tube uncovered by the removal of the skin and muscles on the ventral aspect of the neck.

Cut out a segment of the trachea $3-4 \mathrm{~cm}$. long. It will be seen to be a cylindrical tube stiffened by rings of cartilage which do not meet on the dorsal side, excepting when the trachea is con- 
tracted. When uncontracted, the dorsal side is fleshy, something like the cesophagus.

800. Mieroscopic Structure of the Trachea.-(A) Its ectal layer is of rather dense connective tissue. In it are imbedded the incomplete rings of cartilage. (B) Entad of the connective tissue layer and extending around about the dorsal third of the circumference of the trachea is a layer of unstriped muscle. This is partly attached to the ectal surface of the rings of cartilage, and by its contraction causes the rings to meet or even overlap. (C) Entad of (A) and (B) is a layer mostly composed of elastic connective tissue. (D) Mucons membrane. This lines the Iumen of the trachea. It is composed of three layers of cells, the free layer of which is ciliated. (E) Imbedded in the wall of the trachea are many racemose glands whose ducts open on the free surface of the mucous membrane. See Stricker, A, 435 ; Quain, A, II, 266.

$\S 801$. Esophagus, az.-Gullet (Fig. 77, 109).-The œesophagus is the fleshy tube connecting the pharynx and stomach.

$\S$ 802. Demonstration.--Draw the trachea somewhat dextrad, and there will be seen the œsophagus, an entirely fleshy tube, dorsad and slightly to the left of the trachea. When empty it does not retain a cylindrical form like the trachea, but collapses.

$\S$ 803. Structure of the OEsophagus.-(A) The ectal layer is composed of striated muscular fibers arranged as in the intestine (\$ 741), with an admixture of unstriated muscle increasing caudad. (B) The intermediate layer is of connective tissue and forms the submucosa. It contains many small racemose glands. (C) The ental or mucous layer is composed of stratified epithelium. See Stricker, A, 361 ; Quain, A, II, 344.

\section{THORAX.}

$\S 804$. The thorax or chest is the part of the trunk between the diaphragm and first rib. Its cavity contains the lungs ( $\S 809$ ), the heart and great vessels (Fig. 91), and part of the trachea and œsophagus ( $\$ 8799,801$; see also Chap. VIII).

$\S 805$. Exposure.-Determine the position of the following landmarks : prcesternum (\$ 228); xiphisternum (\$ 228); first rib (Fig. $30,72)$; ventrimeson - the line between the xiphisternum and præsternum.

Incisions.-A. Make a longitudinal incision ( $\$ 599$ ) through the skin and muscles $3 \mathrm{~cm}$. to the right of the ventrimeson from the first rib to a point opposite the base of the xiphisternum. Taking care not to injure the vessels and nerves in the axillary region, reflect the skin for $2-3 \mathrm{~cm}$. on the right of the incision.

B. Make a transverse incision from the caudal end of the longitudinal incision to a point 4-5 $\mathrm{cm}$. to the left of the ventrimeson. 
Reflect the skin across the ventrimeson as far as the transverse incision extends. Scrape or dissect the muscles from the ectal surface of the ribs and cartilages near their union; see Fig. 50.

C. With the arthrotome or strong scissors, cut through the thoracic wall on both sides, just mesad of the junction of the cartilages and ribs.

$\S 806$. Pleura, Septum mediastinale.-Grasp the cut edges of the right costicartilages and turn the sternum partly to the left side. The ental surface of the thoracic wall is covered by a smooth glistening membrane, the pleura, a serous membrane like the peritoneum ( 725 ); like the latter, it may be detached over a small space by means of the tracer.

At the meson will be seen a transparent curtain containing: blood vessels and more or less fat. This is the mediastinum, mediastinal septum or septum thoracis (Fig. 7, 99, 100). It divides the thorax into a right and left half. Each half of the thorax is lined by a separate serous sac, and the meeting of these on the meson produces the mediastinal septum. The thorax thus differs markedly from the abdomen, where there is but one serous sac (\$725), but the thoracic organs, like those of the abdomen, are all properly ectad of or outside the serous membrane. Some of the organs, as the heart, are between the two walls of the septum, while others, as the lungs, are apparently within the sacs, as the alimentary canal is within the peritoneal sac (see Fig. 78).

$\S 807$. After the mediastinal septum has been examined, make, with bone scissors, a transverse incision from the right to the left between the incisions in the thoracic wall, cutting the soft parts in the intercostal space between the 9th and 10th ribs. Then cut the mediastinum near its attachment to the sternum to a point opposite the 4th costal cartilage, avoiding injury to the blood vessels. Finally, turn the sternum cephalad and secure it in this position with a string or a pin.

With the nippers, cut the ribs, excepting the first, on each side along a line about $3 \mathrm{~cm}$. from their tubercula, and with scissors or a scalpel, cut the soft parts and remove the freed portion of the thoracic wall.

$\S 808$. Thymus gland.-If the cat is young, there will be seen an elongated pinkish body (Fig. 77) extending along the ventral side of the trachea and great vessels from the heart to a point somewhat cephalad of the first rib. This is one of the so called ductless 
glands; it is also a temporary organ and may be entirely absent in old animals. Its function is not well understood.

References to the Lungs.-Quain, A, II, 269; Gray, A, 827; Chauveau, A, 493; Chauveau (Fleming), A, 466 ; Leyh, A, 444 ; Owen, A, III, 572 ; Cuvier, A, VII, 19 ; Hyrt1, A, 306 ; Gegenbaur, A, 572 ; Milne-Edwards, A, II, 334 ; Williams (T.), A, V, 258.

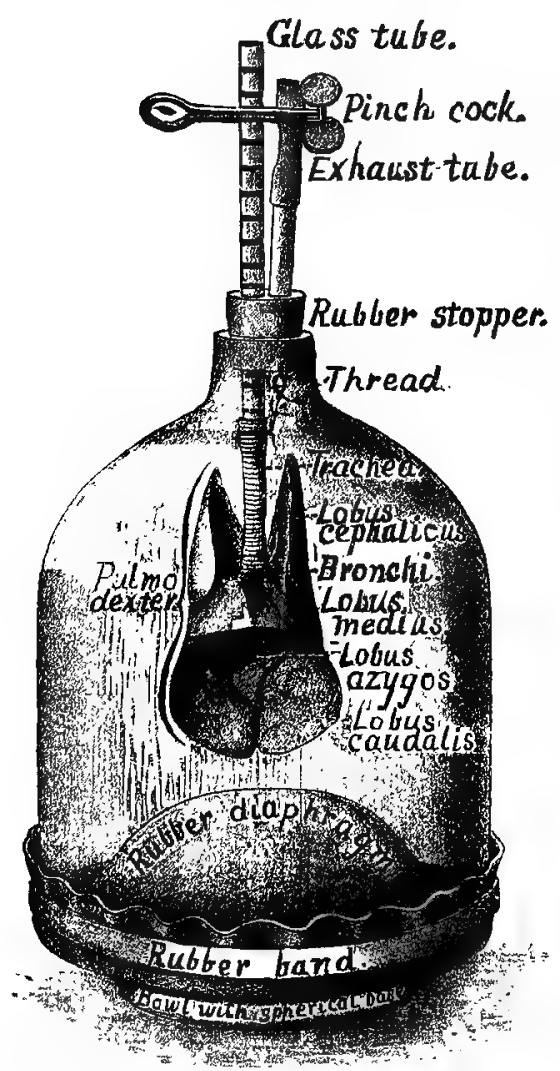

Fig. 89.-Lungs and Trachea; $\times$.2. (The apparatus is arranged as for an experiment; see Wilder, 23.)
§ 809. Pulmo - Lung (Fig. $77,89,99,100)$. - On each side of the thorax, extending from the diaphragm to the first rib, will be seen a sponge-like mass. Each of these masses is a lung; and the two are the essential organs of respiration. Insert a glass tube or a flexible blow-pipe into the trachea and inflate the lung. The trachea must be pressed closely against the tube to prevent the escape of air.

$\S 810$. Lobes.-Each half of the inflated lung will be seen to consist of several divisions, the so called lobes. There are three on each side-cephalic, intermediate or middle, and caudal (Fig. 89).

Azygous Lobe. - The right lung, in addition to the three lobes mentioned in the preceding paragraph, has a small one, the azygous lobe, projecting into a kind of pocket formed of pleura, dorso-caudad of the apex of the heart and between it and the diaphragm (Fig. 77, 103, C. 1., az.). The free edge of this pocket is bounded by the postcava. This lobe is sometimes deeply divided into two, the caudal of which is the larger.

\$ 811. Air cells.-Hold an edge of the inflated lung between the eye and the light, and note that it is divided into spaces about $1 \mathrm{~mm}$. in diameter. These are the air cells. See Structure, $\$ \$ 813,814$. 
§ 812. Bronchi and Bronchioli.-The trachea should be followed from the neck into the thorax, cutting or tearing away any blood vessels or nerves that cover its ventral suriace, and also the thymus (Fig. 77, \& 808). Near the intermediate lobe of the lung, the trachea divides into the two bronchial tubes, one of which goes to each lung. Tear away the substance of the lung sufficiently to follow one bronchus. It will be seen to continually divide and so form the bronchioli.

5813. Obvious Structure of a Lung.-On entering the lung the bronchus divides like a tree into branches (bronchioli). Near their termination the bronchioli dilate somewhat, thus forming the so called infundibula or ultimate lobules. From the wall of the infundibulum project sac-like recesses singly or in groups. Each recess is called an alveolus, and may be considered as the blind ampuiliform termination of the smallest division of an air tube. The alveoli may be readily seen by looking at the edge of an inflated lung as directed above (\$ 811).

\$ 814. Microscopic Structure.-This is very complicated, its main features being as follows:-(A) The air tubes are composed of an ectal dense, largely elastic, connective tissue layer, in which are found plates of cartilage. (B) Unstriated muscular layer. (C) $\Delta \mathrm{n}$ elastic connective tissue layer. (D) The mucous membrane with its ciliated epithelium.

In the smallest air tubes the cartilage disappears. In the larger ones are small racemose glands as in the trachea. As an air tube enters a lobule, its ciliated epithelium is supplanted by a stratum of cubical, nom-ciliated cells. The unstriped muscle also disappears, and finally the alveoli are lined with a single layer of pavement or scaly epithelium. See Stricker, A, 437 ; Quain, A, II, 273.

\section{DIAPHRAGMA, az-DIAPHRAGM.}

References.-Straus-Durckheim, A, II, 309 ; Quain, A, I, 308 ; Gray, A, 394 ; MilneEdwards, II, 406 ; Chauveau (Fleming), A, 245; Chauveau, A, 260 ; Gegenbaur (Lankester), A, 574; Hyrtl, A, 316 ; Cuvier, A, VII, 198 ; Owen, A, III, 1.

\$ 815. The Diaphragm (Fig. 77, 90) is a musculo-tendinous curtain completely separating the thoracic and abdominal cavities. Many structures transverse it, but they are joined to their respective apertures in such a manner that the partition is absolutely air-tight, and yet no hindrance is put upon the free movement of the diaphragm in respiration.

The tendinous part (Fig. 90) is near the middle. Its form is somewhat crescent shaped, the horns of the crescent pointing dorsad. From the tendon radiate the muscular fibers. Those of the dorso-mesal third converge and form two thickened masses called the crura or pillars of the diaphragm.

The diaphragm is attached to the xiphisternum, to the last five ribs, and somewhat loosely to the thick muscles which lie ventrad of the vertebræ. The crura unite, forming a single dense tendon, 
which is attached to the centra of the $2 d, 3 d$ and 4 th lumbar vertebroe.

It will be seen from the above that the diaphragm is attached very obliquely to the body wall so that the dorsal part is caudad of the ventral. The central part is strongly arched into the thoracic cavity.

\$ 816. Posture and Preparation.-The cat should be placed in a dorsicumbent posture, the abdomen opened as directed for the study. of the viscera ( $\$ 237,710$ ), and injected from the abdominal aorta and postcava $(\$ 363,365)$. The abdominal wall should be cut along a line about $2 \mathrm{~cm}$. caudad of the diaphragm. The liver and stomach should be drawn somewhat caudad; the suspensory ligament, the postcava and the asophagus cut, the two last named about $2 \mathrm{~cm}$. from the diaphragm. Care should be taken not to cut the diaphragm in any of the operations.

The kidneys (Fig. 101) should be removed, and the aorta about $2 \mathrm{~cm}$. candad of the origin of the superior mesentery artery. The vertebral column should be disarticulated between the 4th and 5th lumbar vertebræ (Fig. 30). The superior mesenteric artery and the coliac artery and the splanchnic nerve (Fig. 103, 107, A. c., N. splnch.), should be carefully isolated with the tracer, fine forceps and fine scissors. It is also desirable to isolate the vagus (gastric nerves) on the csophagus (see Fig. 103, 107, N. g. d. N. g. v.). The ribs should be cut near their tubercula, and then the ventral border of the diaphragm drawn strongly cephalad and held in position with large pins. Lastly, the fat and connective tissue should be removed from the muscles so as to show the direction of the diaphragmatic fibers and their interdigitations with those of the $M$. transversalis.

Preparation - Fig. 90.--The thoracic duct (Fig. 103), the abdominal aorta and postcava (Fig. 101) were injected. The cat was then transected, following a line about $1 \mathrm{~cm}$. caudad of the tip of the xiphisternum and going between the 4th and 5th lumbar vertebræ. The ventral border of the diaphragm was drawn strongly cephalad and cotton placed on the pleural side to make it nearly level.

Explanation of Fig. 9o.-Aorta, $a z$.

A. phrn., A. phrenica.-The phrenic or diaphragmatic artery.

A. adrn. Imb., A. adreno-lumbalis.-The adreno-lumbar artery, a small artery arising from the aorta and supplying the adrenal body and the cephalic part of the lumbar region.

C., A. cœliaca, az.-The cœliac artery or coliac axis. 
Crus dphrg., Crus diaphragmatica. - The left crus or pillar of the displuragm.

Dct. thr., Ductus thoracicus, az-The left thuracic duet , Fig, 103.

Gng. (Ganglion) semilunare. - The semilunar ganglion-dauglion of the solar plesus of nerves (Fig. 107).

M., A. mesenterica superior, az. (Fig. 103, 107).

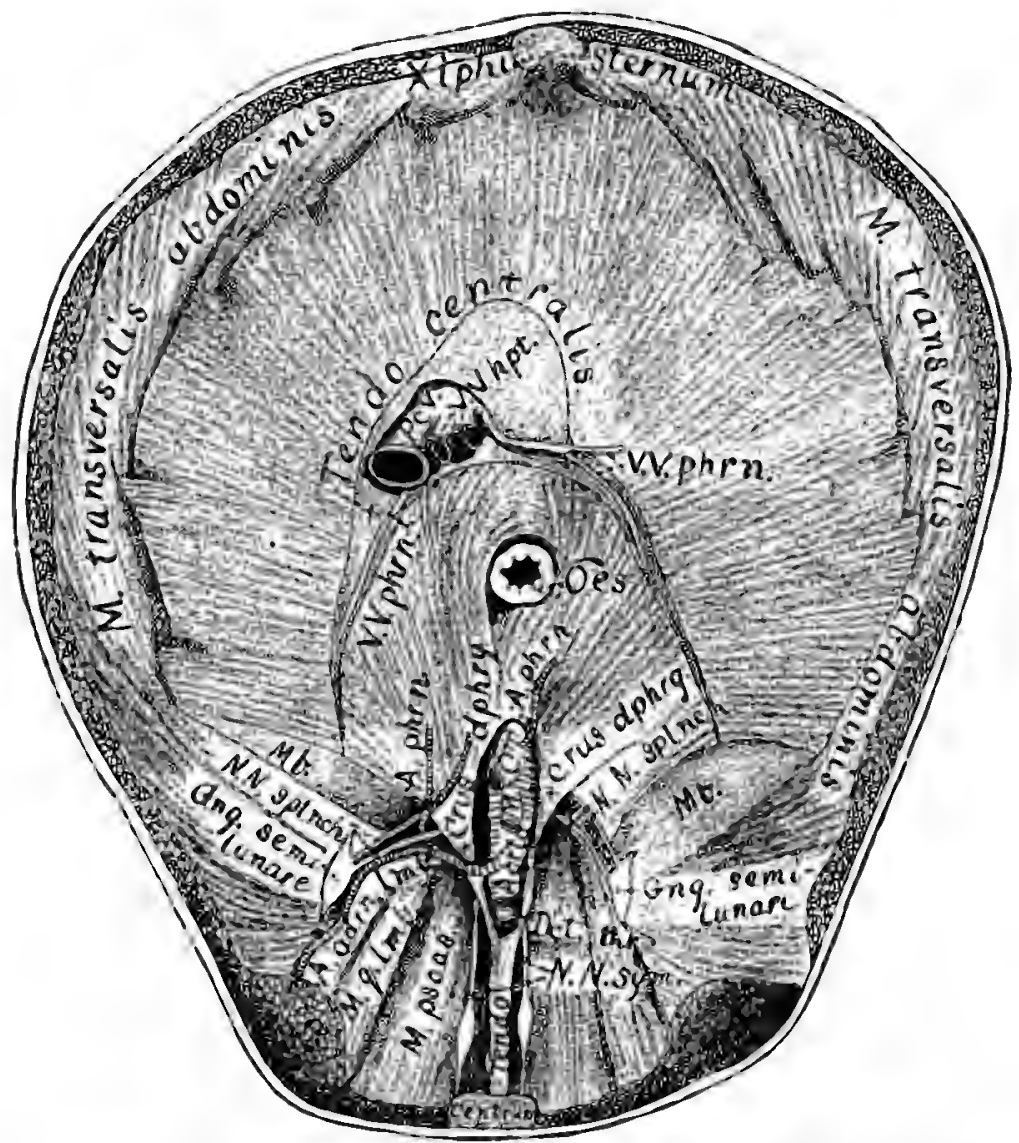

Fig. 90.-Cacdal Vtew of the Diapiragm with TBE structcres that thavek8e IT ; $\times .75$.

Mbrn., Membrana. - The somewhat crescent-ahaped membranes which. with thr museular portion of the diaphragm. complete it dorkad It ia said by Atraus llurekherm. A. II. 3t0. that in place of thege membranes. thepe are often muscular mllars-lateral pillars of the diaphragm-joining the dispophyses of the 24 lumbar vertebre.

M. transversalis-abdominis.

M. q. lumb., M. quadratus lumborum.

M. psoas. (The human psoas magnus.) 
NN. sym., NN. sympathici.-The sympathic nerves of the two sides continued into the aldomen.

NN. splnch., NN. splanchnici. -The two splanchnic nerves of each side joining the corresponding semilunar ganglion (Fig. 10\%, Gng. smln.),

N. gastricus-Gastric nerve.-There are two of these, one on the dorsal and one on the ventral side of the esophagus (Fig. 107, N. gstr. dor., N. gstr. vnt.). They are the continuations of the vagus nerves into the abdomen.

Es., Esophagus, az.-This is somewhat loosely attached to the diaphragm, and hence can move longitudinally quite freely (Fig. 107, CEs.).

Pcr., Postcava, az.-The great rein returning blood to the heart from the caudal half of the body (Fig. 101).

Tendo centralis, $a z .-$ The central tendon of the diaphragm.

Tendo, az.-The common tendon of the two crura of the diaphragm. It is very strong and firmly attached to the $2 \mathrm{~d}, 3 \mathrm{~d}$ and 4 th lumbar vertebræ.

VV. phrn., Venæ phrenicæ.-The phrenic veins. The large trunks follow mostly the two horns of the tendo centralis.

VV. hepat., Venæ hepaticæ, az.-'Two hepatic veius from the right lobe of the liver. They unite just as they enter the vena cava (Fig. 101).

Xiphisternum, $a z$. 


\section{CHAPTER VIII.}

THE VASCULAR SYSTEM.

GENERAL CONSIDERATIONS - HEART-ARTERIES-CAPILLARIES-VEINS - LXMPHATIC8THORACIC DUCTS.

\$ 817. General Considerations.--In the cat, as in man and the higher animals generally, the tissues are supplied with blood, and blood and lymph are removed from the tissues, by means of a series of closed tubes or vessels. These tubes all communicate more or less directly with one another, but-excepting the lymphatic stomata-present no obvious openings into any other parts. This closed series of tubes is known as the vascular system.

The vascular system as a whole consists of two main divisions,the blood vascular system and the lymph vascular system or lymphatic system.

$\S 818$. The blood vascular system is that by which the blood is (A) conveyed to the tissues in general for their nourishment and returned therefrom; (B) conveyed to the lungs for its own improvement and returned therefrom (Fig. 92). Those parts which are concerned in the transfer to and from the lungs constitute the pulmonic division of the blood vascular system, and the remainder constitute the general or systemic division. While in process of transfer, the blood is said to perform either the pulmonic or the systemic circulation.

\$ 819. Subdivisions of the Blood Vascular System.-There are four parts, continuous with each other, but more or less distinctly differentiated: (A) A central receiving and distributing organ, the heart (cardia); (B) tubes extending from the heart throughout the lungs and the organs generally, the arteries; these divide and diminish in size like the branches of a tree, and gradually merge into (C) the capillaries, the most minute vessels, which in turn unite and gradually merge into (D) the veins, which unite and increase in size as they diminish in number and finally terminate in the heart (Fig. 92).

The arteries are said to continually divide and decrease in size because the current therein is from the larger vessels toward the smaller. The veins are said to unite and increase in size because the blood current is from the smaller to the larger branches. Again, the arteries are said to extend from the heart peripherad, that is, in the direction of their blood current, while the veins are ald to extend toward the heart or centrad, as the current is toward the central organ. The veins differ from the arteries by having thinner walls and by the presence of valves in many (Fig. 102, B, C). 
\$ 820. The Lymph Vascular System.-This is that part of the general vascular system which collects the lymph from the tissues and the chyle from the alimentary canal and conveys them to the great veins; it is thus an auxiliary of the venous system (Bernard, A, 250). From the office of collecting both lymph and chyle, it is commonly considered as forming two divisions: (A) The lymphatics proper ; (B) the lacteals or chyle vessels.

\$ 821. The lymphatic system is somewhat comparable to the venous, since its vessels begin as capillaries at the periphery and extend toward the center. Like the veins also, the - lymphatic vessels contain numerous valves which prevent a reversal of the current, that is, a flow from the center tcward the periphery.

The lymphatic vessels differ from the veing in the following particulars: (A) They have thinner walls; (B) they do not so markedly increase in size as the veins, although they anastomose more frequently; (C) at various points along their course there are enlargements, the so called lymphatic glands, through which the lymph passes; (D) in. stead of joining the central organ of the vascular system directly as do the blood vessels, the lymphatic trunks open into veins (Fig. 103); (E) the lymphatic system differs from the venous also in having no direct communication througl capillaries with anything like an arterial system; (F) the lymphaties are found to communicate with serous cavities through minute orifices, the stomata (Quain, A, II, 188 ; Stricker, A, 222).

\section{CARDIA-THE HEART.}

\$ 822. References.-Quain, A, II, 242 ; Gray, A, 801 ; Cuvier, A, VI, 272 ; Gegeubaur (Lankester), A, 583 ; HyrtI, A, 300 ; Bernard, A, 274 ; Milne-Edwards, A, III, 473; Owen, A, III, 516 ; Chauveau, A, 529; Chauveau (Fleming), A, 499 ; Gurlt, A, 574; StrausDurckheim, B, II, 181 ; Foster and Langley, A, 91 ; Smith, E. N., A, Pl. 47, 48, 49, 55, 56 ; Bourgery and Jacob, A, Pl. 9, bis, PI. 13 ; Rolleston, B, 25-34 ; McAlpine, B, I, Pl. 23; Krause, A, 178, Fig. 13 ; Turner, A, 899 ; Sabatier, A ; Flower, A ; Pettigrew, 64; Pettigrew, A ; Parchappe, A ; Mojsisovics, A, 54 ; Leyh, A, 559; Mivart, B, 199-206.

Remnrk.-Most of the above refer to the human heart more especially, but the heart of the horse is chiefly described by Leyh, Chauveau and Gurlt, and that of the rabbit by Krause, McAlpine and Foster and Langley. Methods of preparation are given by Hyrtl, Straus-Durckbeim, Mojsisovics and Pettigrew. The only descriptions and figures purporting to refer to the heart of the cat are given by Mivart; unfortunately, however, in Fig. 102, the relative thickness of the right and lett ventricles is made the reverse of what it should be (a probable oversight which, although readily corrected by the anatomist, is sure to confuse the beginner); while the usefulness of the text is diminished both by the general uncertainty as to how much refers directly to the cat ( $\$ 127)$, and by the presence of an absolute misstatement upon pp. 201, 214 respecting the number of pulmonary veins.

$\S 823$. Before dissecting the heart, or even removing it from the body, the student will do well to familiarize himself with its general features and location as shown in Fig. 77 , $91,101$.

If he has been following the present work in order, he will have at least one cephalic region of a cat preserved in alcobol, and may run the risk of injuring the heart thereof in removing it before gaining any detailed knowledge of it. He must bear in mind, however, that all the cavities of such a heart will be collapsed and that the auricles especially will look very unlike the preparation shown in Fig. 91. Such a heart may serve for the examination of some parts, but eventually he should have at least two specimens well distended and hardened by alcohol, and if possible another filled witl plaster.

824. Preparation-Fig. 91.-The postcava and abdominal aorta. 
were injected with red and blue plaster respectively. After the plaster had hardened, a small hole was made in the left ventricle and a long canula inserted and pushed through the auriculo-ventricular opening into the left auriole. Red plaster was then injected so as to fill the pulmonary veins. Finally, the pericardium was removed together with the fat and connective tissue covering the cardiac vessels.

$\S 825$. Description.-This, the dorsal or " posterior" aspect of the heart, is less familiar to most persons and less frequently represented. It is, however, much more comprehensive and instructive than the ventral aspect, and is given in the following works : Quain, $\mathbf{A}$, II, Fig. 166 ; Smith, E. N., A, P1. 55 ; McAlpine, B, Pl. XXIII, Fig. 3 (rabbit) ; Gegenbaur (Lankester), A, Fig. 336 (pig).

The present figure fairly represents the size of a sornewhat large heart, and its form when injected. As stated, however, in $\S 829$, it is probable that the less thickness of the lateral wall of the right ventricle bas permitted it to yield to the force of the injection so that the right side is unduly convex.

I'he word ventriculi is written across the apex of the ventricular portion, and the abbreviation $\nabla$. crd. is just below the furrow between that and the auricular portion.

For the sake of showing certain parts more distinctly, the figure represents the organ as if rotated slightly upon its longer axis so as to expose more of the right than of the left side. The line of demarcation between the riglut and left auricles (Aur.. dxt., Aur. sin.) coincides nearly with a line connecting the $V$. of $V$. crd. with the left border of the procaza. The ductus arteriosus (\$ 867 ) is not shown.

\$ 820. General Description of the Heart.The heart is a hollow, quadrilocular (four chambered) muscular organ situated in the thorax. It is the anatomical and physiological center of the vascular system, simultaneously receiving

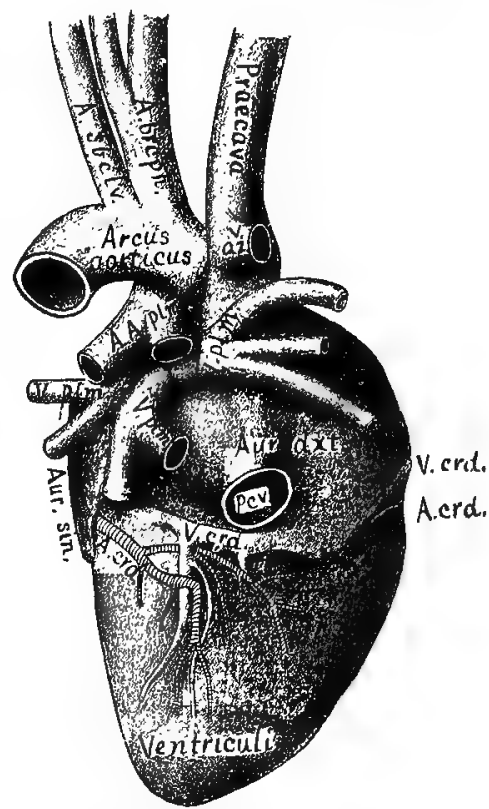

Fig. 91.-The Dorsal Aspect of the heart with tite Central PokTIONS OF THE LARger VeSSELS; from a Maltese cat ; $\times 1$. blood from the lungs and from all other parts of the body, and distributing it thoreto.

\$ 827. Location.-As seen in Fig. 77, 99, 101, the heart is on the meson, but extends a little farther to the left than to the right. In Fig. 101 it appears at a considerable distance from the diaphragm, but of the latter only the dorsal portion remains, and its cephalic convexity is really very closs to or in contact with the heart.

\& 828. Pericardium.-The heart proper is enveloped in a fibro-membraneous sac, the pericardium, which is attached about the roots of the great vessels, but elsewhere is unconnacted with the heart, which thus moves freely within it. The pe:icardium is best studied 
by girdling it at about the middle of the length of the organ and reflecting the attrehed end over the great vessels so as to display the line of attachment. Its relations to both the beart and the diaphragm will be mentioned in the descriptions of Fig. 99, 100.

\& 829. Form.-When moderately distended, the heart is approximately oval in shape, one end obtuse and the other pointed.

As seen in Fig. 92, however, this oval is not regular, one side being more curved than the other. This is due to the greater yielding of the thinner wall of the right ventricle to the pressure of the injection. As a whole also, the organ is slightly flattened in what will be found to be the dorso-ventral direction; this may be due to the greater resistance offered by the septum ventriculare to the pressure of the injection.

§ 830. Normal Position.-Naturally, the longer axis of the heart is oblique with respect to the meson. The smaller end points caudo-sinistro-ventrad. For purposes of description, however, it will be more convenient to assume that the two ends of the organ point respectively cephalad and caudad.

The obliquity in position of the cat's heart is probably less than that of man (as remarked by Owen, A, III, 525), but it is nevertheless decided. In frozen section No. 11 of the series from which Fig. 99 and 100 were made, the apex of the heart is at least $15 \mathrm{~mm}$. sinistrad of the meson.

\$ 831. Size.-Fig. 91 represents the natural size of the preparation from which it was made, but the heart was from a large cat, and was somewhat distended with plaster by injection. In round numbers, the heart of an average adult cat measures about $5 \mathrm{~cm}$. from the apex to the attachment of the procava and about $3 \mathrm{~cm}$. across the greatest width of the ventricular portion. We have not yet ascertained the average weight of the organ, but it is very considerable in comparison with the size and weight of the animal.

8 832. Designation of the Regions. -As shown in Fig. 92, 93, the cephalic and caudal ends of the heart differ not only in shape, but in the thickness of the muscular walls. Since the two cavities enclosed by the thinner cephalic walls are known as auricles, the cephalic or basal region or end of the organ is spoken of as auricular; in like manner, the other end or region is called ventricular from the name of the two cavities enclosed by the thicker walls.

As seen in Fig. 95, 96, there is a partition between the auricles and another between the ventricles. These septa divide the organ into two sides or portions like the sides of a double house. Since the planes of the septa are approximately dorso-ventral, it is customary to speak of the two sides as right and left. The other two surfaces and regions are then respectively dorsal and ventral.

$\$$ 833. Recognition of the Regions.-The cephalic and caudal regions are readily distinguished both from the greater size of the former, from the more compressible nature of the walls, and from the presence of the vessels which enter or leave the organ. In the natural attitude of man, the base of the heart is uppermost, but this somewhat puzzling feature does not appear when the animal is regarded as in the normal position of a vertebrate $(\S 35)$.

The ventral aspect of the heart is comparatively regular, the two auricular appendices (Fig. 95) projecting slightly at the sides of the two great arteries, aorta and $A$. pulmonalis. The dorsal aspect, on the contrary, is quite irregular in the auricular portion on account of the branching of the two arteries just named, and the entry of veins into the auricles (Fig. 91).

The right and left sides are of course determined by the determination of the ends and the other two sides. In addition, the wall of the right ventricle is decidedly thinner than that of the left, and that region is therefore the more compressible. 
\$ 834. Removal of the Heart.-For obvious reasons, a cat's heart intended for careful examination should be taken from a large adult. In most cases the same cat may be employed for the removal of the brain and for the separate study of the abdominal viscera (Chap. VII). Unless the vessels are to be filled with plaster, it is better not to bleed the animal, since the presence of the blood in the large veins near the heart facilitates their recognition.

Instruments and Materials.-Arthrotome ; tracer ; medium scalpel; coarse scissors ; bone scissors ; nippers ; coarse and fine forceps; 6 pieces of linen thread about $20 \mathrm{~cm}$. long; 2 pieces of thick muslin or flannel or chamois leather, each about $15 \times 7 \mathrm{~cm}$; basin of water and towel; large tray, with cords for securing the legs of the cat.

Landmarks.-Præsternum, xiphisternum and epigastrium ( $\$ 228$, Fig. 30, 49, 72, 76) ; clavicle (\$230, Fig. 30, 72). Observe also the general location of the thoracic viscera in Fig. 77 and 101 and the operations for their exposure ( $\$ 810,805$ ).

$\S 835$. Exposure.-Divide the skin ( $\$ 599)$ as directed for the exposure of the thoracic viscera $(\$ 805)$, but-unless the lungs are to be employed for study or experiment-begin the longitudinal incision (Fig. 76) dextrad of the prosternum instead of the larynx. It is also more convenient to make a second transverse incision from the cephalic end of the longitudinal one to or beyond the corresponding point upon the left side.

The triangular or quadrangular area of skin so indicated is to be raised as directed in $\S 600$ and reflected sinistrad. Divide the pectoral muscles (Fig. 72) on the right by a longitudinal incision parallel with the edge of the skin, taking care not to cut upon the clavicle. Grasp the pectoral mass and dissect it up from the proper thoracic wall to the meson. Repeat the operation upon the other side and then remove both masses from their attachments to the sternum. Divide each $M$. rectus thoracis (Fig. 72, 73) at the caudal transverse cut edge of skin and dissect them up cephalad.

Throngh the MIM. intercostales on each side near the middle of the exposed area, push the conjoined tips of the coarse forceps, and forcibly separate the blades for about $2 \mathrm{~mm}$. This will permit air to enter the thorax, and the lungs will collapse so as to be in less danger of injury during the subsequent operation.

With the arthrotome or bone scissors, divide the exposed costicartilages close to their junctions with the ribs. Cut across the sternum just cephalad of the xiphisternum, sever the septum mediastinale and other adhesions to the ental surface of the sternum, and reflect it cephalad with the costicartilages or remove it altogether. 
Push the lungs on both sides toward the dorsal part of the cavity and with the bone scissors, or nippers and bone scissors, remove the sternal ends of all the exposed ribs for $2-3 \mathrm{~cm}$., so as to facilitate access to the viscera.

\$ 836. Removal.-Lift the heart slightly by the pericardium and sever its pleural attachments to the diaphragm as far dorsad as the postcava (Fig. 101). Put two ligatures upon the postcara, the caudal one about $2 \mathrm{~cm}$. from the diaphragm if the abdominal vessels are to be injected. In passing the ligatures about the vessel with the fine forceps, avoid injuring the azygous lobe of the left lung. This ligature should not be tied very hard.

Double ligature (Fig. 41) the pracava near its bifurcation, and divide this and the postcava between the two ligatures.

If the lungs are to be studied or experimented upon, place the cloth or chamois over the cut edges of the thoracic parietes so as to protect the lungs from laceration by the sharp ends of the ribs.

Grasp the apex of the heart and draw it ventro-cephalad. This will expose the caudal and azygous lobes of the lungs (see Fig. 89), the thoracic aorta and osophagus. Insert the fingers dorsad of the lungs, lift them and sever their attachments as far cephalad as the central end of the azygous vein (Fig. 101). Double ligature this vein just centrad of the point of junction of the cephalic branch. Employ the tracer in isolating the vein so as not to injure other parts. Then divide between the ligatures.

Cut the aorta opposite the head of the 8th rib, and note that, usually, some blood remains therein. Grasp its central part and draw it ventro-cephalad, cutting the intercostal arteries and other connections as far as the origin of the A. subclavia (Fig. 101, 102). Then grasp the heart and lungs together, draw them ventrad and divide the trachea and the vessels cephalad of the heart at the desired points, and the organs may be removed from the thorax.

\$ 837. Separation of the Heart from the Lungs.-On the dorsal aspect, at the bifurcation of the trachea, is a mass of connective tissue and fat enveloping a dark glandular body. Remove these with the tracer and fingers so as to open a way ventro-cephalad to the ventral side of the bifurcation. With the tracer, expose the bronchus on each side, and note that, on its ventral side, the lung is connected with the heart by a group of vessels, the $A A$. et $V V$. pulmonales. Ligate very firmly the roots of the lungs and divide them 
peripherad of the ligatures, and thus complete the separation of the heart from the lungs.

$\$$ 838. Preservation of the Heart in Alcohol.-For the study of the cardiac cavities, the heart should not only be hardened in alcoliol, but distended therewith.

Instruments and Materials. - Coarse scissors; coarse forceps ; sharp tracer or syringotome ; four threads for ligatures, or two threads and two small compressors; syringe with canula adapted to the aorta and postcava (a rubber bulb syringe is most convenient in some respects); small jar or glass box ; 95 per cent. alcohol, about 250 cc. ; cotton; small pins; thread.

At the the time of first preparing a heart by the injection of strong alcohol into the cavities, we were unaware that it was recommended by Hyrtl and Mojsisovics (A, 58). The former ascribes $(\mathrm{A}, 305)$ the original idea to $\mathrm{Wm}$. Hunter.

\$39. Removal of the Pericardium. - In most cases this should be partly removed. Pinch it up at a point about one third of the distance from the base of the ventricles to the apex and make a transverse incision. Continue this incision around the heart so as to remove the apical two thirds of the pericardium. It may be preserved for reference.

$\$ 840$. Removal of the Blood.-The little blood that may remain in the left side of the heart can be easily expelled through the aorta. The right cavities usually contain considerable blood. Remove the ligatures upon the postcava by carefully pushing entad of it the point of the sharp tracer or the syringotome. If it has been tied very tightly, it may be necessary to cut off the end of the vessel.

Introduce the nozzle of the syringe into the postcava and inject water carefully; then manipulate the right auricle and ventricle at the same time so as to expel the blood through the postcava.

\$ 841. Tying the Vessels. -Tie firmly the A. brachio-cephatica and the $A$. subclavia sinistra at about $1 \mathrm{~cm}$. from their respective origins from the arcus aorticus (Fig. 102). The other vessels should have been tied in removing the heart, and the aorta and postcava are left open for the injection.

$\$ 842$. Injection of Alcohol. - A. Into the left side.-Insert the canula into the aorta so that the ligature about its tip may be just centrad of the emergence of the first intercostal artery. Tie it in place, and prepare a second ligature for tying it after the injection.

Naturally, the progress of the alcohol is checked by the sernilunar and bicuspid valves, but it may be caused to pass them by holding the heart with the apex up, and manipulating the base of the aorta and the base of the left ventricle. When the auricle and 
the $V V$. pulmonales are fully distended, apply the ligature firmly and remove the canula.

B. Into the right side. -In like manner inject through the postcava, but no special manipulation is needed for the liquid to fill the ventricle and the $A$. pulmonalis.

\$ 843. Hardening.-The heart may be carefully laid in strong alcohol upon cotton, with the base uppermost, or it may be suspended by a thread passed about a small pin pushed transversely through the extreme apex so as not to penetrate the ventricles. After two days' insertion it will be fit for section or dissection.

Injection with plaster.-See explanation of Fig. 91.

§ 844. Table of the Princrpal Parti and Fratures of the Heart (Cardia).

\begin{tabular}{|c|c|}
\hline $\begin{array}{l}\text { Hemicardia Sinistra (Left on } \\
\text { Steteino Portion?. }\end{array}$ & \\
\hline Special Names. & General Names. \\
\hline
\end{tabular}

Auricula sinistra.

Ventriculus sinister.

Sinus ; appendix...........

Base ; apex...............

Orificium aur. vnt. sin

VV. pulmonales (from the ? lungs)...............

Aorta (to the body), A A. cardiacæ $s$. coronariæ (to the heart from the base of the aorta)..............

Bicuspides 8.mi- S Septalis, ? trales....... \{ Lateralis.

Chd. tnd. sin

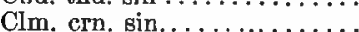

Semilunares aor- $\left\{\begin{array}{l}\text { Dorsalis, } \\ \text { Dextra, }\end{array}\right.$ tici ....... $\left.\begin{array}{l}\text { Dextra, } \\ \text { Sinistra. }\end{array}\right\}$

$\mathrm{Sn}, \nabla \mathrm{ls} \sin (8) \ldots \ldots \ldots \ldots$ hemicardia Dextra (Right or Pulmonic Portion).

Special Names.

A. Great Cavities (Loculi).

1. Reception.............. Auricula dextra.

2. Delivery.............. Ventriculus dexter.

B. Divisions of Great Cavities.

1 Of auricles........... \{ $\begin{gathered}\text { Sinus; } \\ \text { ovalis. }\end{gathered}$

2. Of ventricles. . ....... Base; apex; conus arteriosus.

C. Orifices and Vessels.

(1. Auriculo-ventricular or ? interlocular.......

f2. Vasa afferentia (enter-) ing the auricles)....

(3. Vasa efferentia (leav-) ing the ventricles)...

D. Valves (Valve) and the Parts associated therewich.

\{1. Auriculo-ventricular valves...........

2. Chordæ tendineæ

3. Columnæ carneæ.......

4. Arterial valves.........

5. Sinus Valsalva

Orificicum aur.-vnt. dxt.

VV. præcava et postcava (from the body): VV. cardiacæ \&. coronariæ (from the heart).

A. pulmonalis (to the lungs).

Tricuspides.... \{ $\begin{aligned} & \text { Eeptalis, } \\ & \text { Dorsalis, } \\ & \text { Ventralis. }\end{aligned}$

Chd, tnd. dxt.

C'lm, cru. dxt.

Semilunares Ventralis, $\left\{\begin{array}{l}\text { pulmonales.. } \\ \text { Dextra, } \\ \text { Sinistra }\end{array}\right.$ Sn. Vls. dxt. (3), Valva Thebesii.

E. Single Parts belonging equally to Both Sides.-1. Septum auriculare. 2. Septum ventriculare. 3. Ductus arteriosus. 
Fig. 92.-Diagrammatic representation of the heart, the great vessels, the pulmonic and systemic capillaries.

In this diagram, as in Fig. 91, the heart is seen from its dorsal (posterior) aspect; bence its right and left portions correspond in position with the right and left of the observer $(\$ 56)$.

Most of the parts are shown by outlines only, but the ventricular and auricular walls are shaded, and the lines representing the aorta and pulmonary artery are made a little heavier than those representing the vena cava and pulmonary vein.

The course of the blood is indicated by arrows, but the action of the parts will be considered in Part III. The difference between the valves upon the two sides will be explained in connection therewith.

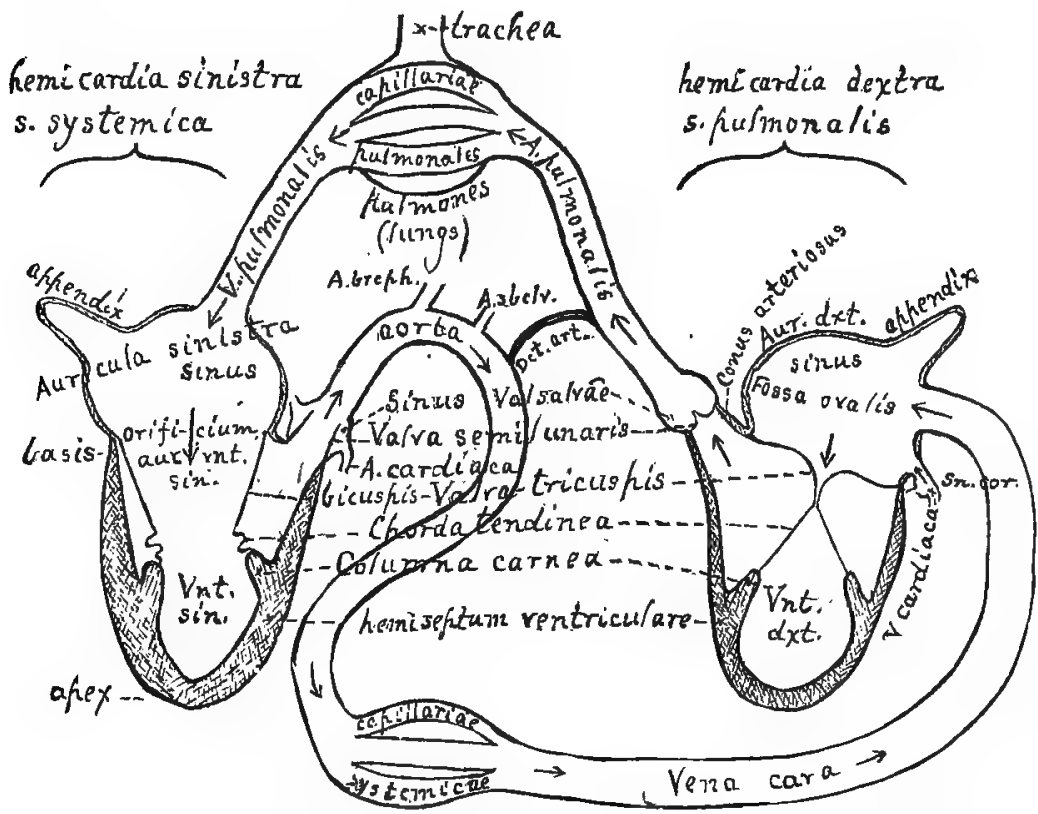

Fig. 92.-Diagram of the Cardiac Cavithes, eitc.; Dorgal agpect.

$\S 845$. The special objects of this diagram are:-

1. To represent the more essential parts of the heart in a single figure, to give their full technical names, and to indicate the relations of the cavities to each other and to the great vessels.

2. To illustrate the physiological fact that the mammalian heart, in the normal adult, really consists of two organs whose cavities have no direct communication whatever.

3. To show the many points of resemblance between the right and left portions.

4. To indicate the relations of the two portions of the heart, through their afferent and efferent vessels, with the lungs and the other organs of the body, and thus to justify the apparent paradox that whereas, anatomically, the heart may properly be described as a single and approximately mesal organ, situated between the two lateral masses of the lungs, physiologically, the right and left portions of the heart are separated, on the one hand by the lungs and on the other by the rest of the body. Anatomically, there are two lungs 
and the heart lies between them; physiologically, the lungs form a single organ which is interposed between the two hearts; Wilder, 1 .

$\S 846$. The diagram (Fig. 92) differs from an accurate representation of any actual section of the cat's heart in the following respects :-

1. The separation of tise right and left portions (hemicardice), involving the splitting of the septa auriculare et ventriculare and the elongation of the ductus arteriosus.

2. The non-crossing of the aurta and $A$. pulmonalis and the deflection of the former to the right instead of the left.

3. The representation of the cardiac (coronary) vessels by the beginnings of the two arteries and the ends of two of the veirs.

4. The representation of the systemic veins (postcava, procava and $V$. azygos) by a single vessel here named $V$. cava.

5. The representation of the several $V V$. pulmonales by a single vessel.

6. The representation of the two lungs by a single and simple sac.

7. The distance from one another upon the arcus corticus of the origins of the vessels supplying the head and arms, $A A$. brachio-cephalica et subclaria sinistra (A. breph., $A$. $s b c l v$.).

8. The representation of the branches and capillaries of the aorta and A. pulmonalis by only three divisions of each vessel.

9. The representation of the semilunar and auriculo-ventricular valves in each case by a pair of lines.

10. The omission of the fret-20ork upon the ental aspect of the walls, especially of the ventricles and the auricular appendices.

11. The positions and points of attachment of the appendices and of the veins which enter the aulicles have been assigned with a view to convenience of representation rather than absolute accuracy.

12. The omission of the ductus thoracicus, which might have been made to join the proximal portion of the Vena cava.

$\S 847$. The right and left portions (hemicardia) of the heart agrce with each other in the following respects :-

1. Their primary division into an auriche (anricula), which is relatively cephalic in position, irregular, rounded and thin walled; and a ventricle (ventriculus), which is caudal in position, regular, pointed and thick voalled.

2. The division of each auricle into a larger sinus and a smaller appendix.

3. The communication of each auricle with its ventricle by a slightly constricted orifice, guarded by valves of irregular shape.

4. The attachment of cusps of the free edges of the auriculo-ventricular valves to the ventricular walls by fibrous chorda tendinea springing from the apices of columna carnece.

5. The free communication of the auricles with veins ( $V V$. cava and $V V$. pulmonaria).

6. The exit from the ventricles of arteries (aorta and A. pulmonalis).

7. The guarding of each arterial orifice by three similar and regular semilunar valves.

8. The expansion of each artery opposite the valves to form three sinuses of Valsalva.

9. The aorta and A. pulmonalis are united by a fibrous band, the ductus arteriosus, the normally impervious remnant of a vessel which, in the fœtus, permitted the blood to pass from the latter vessel into the former peripherad of the origin of the vessels ( $A A$. brachio-cephalica et subclavia sinistra) which carried the purer maternal blood to the head and arms.

$\S 848$. The right and left portions (hemicardice) of the heart differ in the following resperts :- 
1. The afferent vassels ( $V V$. cavce) of the former come from the body in general, those of the latter ( $V V$. pulmonales) from the lungs. The efferent vessels of the former ( $A A$. pulmonales) go to the lungs, and those of the latter (aorta) to the body in general.

2. From two of the aortic sinuses of Valsalva arise the two $A A$. curdiace which are distributed to the substance of the heart itself.

3. Into the right auricle open the $\nabla V$. cardiace which come from the substance of the heart.

4. The septal wall of the right auricle presents an oval depression, fossa ovalis, the indication of a thin portion of the septum which, in the foetus, was absent, so as to permit the existence of the foramen ovale $(\$ 868$ ).

5. The lateral walls of the left ventricle are, upon the whole, from two to three times the thicker.

6. The right ventricle is prolonged as a conus arteriosus from which arises the $A$. pulmonalis.

7. The auriculo-ventricular valves on the right form three divisions, hence named $V V$. tricuspides, while those of the left form two, hence named bicuspides or mitral.

8. The foregoing are real and constant distinctions between the right and left hearts. In addition, in the diagram, on the left side, the semilunar valves are represented as closed, forming bags whose convexities bulge toward the ventricle as if presed upon by a column of liquid in the artery ; the tricuspid valves are also closed, as if by the pressure of a volume of liquid in the ventricle; they are restrained from being carried into the auricle by the chordo tendineos.

\$ 849. List of Abbreviations of Cardiac Names.-Ao., Aorta, \$ 851.-Arc. ao., Arcus aorticus, § 854.-A. brcph., Arteria brachiocephalica, § 855.-A. crd., Arteria cardiaca, § 856.-A. plm., Arteria pulmonalis, $\S 857 .-A$. sbclv., Arteria subclavia, $\S 858 .-A u r$. dxt., Auricula dextra, \$ 859.-Aur. sin., Auricula sinistra, \$ 860.Chd. tnd., Chordæ tendineæ, §864.-Clm. car., Columnæ carneæ, \$ 865.-Con. art., Conus arteriosus, \$866.-Dct. art., Ductus arteriosus, \& 867.-F's. ov., Fossa ovalis, § 868.-Orf. aur-vnt., Orificium auriculo-ventriculare, § 870.-Pcv., Postcava, § 871.-Prcv., Præcava, \& 872.-Spt. aur., Septum auriculare s. auricularum, \$ 873. -Spt. vnt., Septum ventriculare $s$. ventriculorum, $\S 874 .-$ Sn. aur. $d x t$., Sinus auriculæ dextræ, $\$ 875$.—Sn. aur. sin., Sinus auriculæ sinistræ, $\S 876$.-Sn. cor. s. crd., Sinus coronarius s. cardiacus, § 87\%.-Sn. Vals., Sinus Valsalvæ, § 878.-Tbcl. Low., Tuberculum Loweri, § 880.-Vlv. bic., Valva bicuspis, § 881.Vlv. slmr., Valva semilunaris, § 882.-Vlv. Thb., Valva Thebesii, \$ 883.- Vlv. trc., Valva tricuspis, § 884.-V. az., Vena azygos, $\S 885 .-V$. crd., Vena cardiaca $s$. coronaria, \& 887.-V. cv., Vena cava, §886.-V. plm., Vena pulmonalis, §888.-Vnt. dxt., Ventriculus dexter, § 889.-Vnt. sin., Ventriculus sinister, § 890 .

$\$ 850$. Descriptive List of the Parts of the Heart.- The beginner may find it advisable to read over this list with reference to 
the figures, before undertaking the dissection and detailed examination of the organ. Later, however, he should again consult it for the descriptive portions.

$\S 851$. Aorta (az.), Ao. (Fig. 91, 92, 93, 94, 95, 96, 99, 100, 101, 102, $103,107,108)$.- The large artery springing from the base of the left ventricle and giving off branches to all parts of the body. Its central portion (as shown in Fig. 91, 101, 102, 108) is strongly arched, and is thence named arcus aorticus. It then passes through the thorax into the abdomen, and the diaphragm therefore indicates the point of demarcation between the aorta thoracica and the aorta abdominalis. This vessel is sometimes, as by Stowell (1), called A. (Arteria) aorta, but the single word seems to be sufficient.

$\S 852$. Apex (ventriculi). - The smaller or caudal end of either ventricle, and of either the cavity or the fleshy wall thereof (Fig. 91, 92, 93, 98).

\$ 853. Appendix (auriculæ)-The ventral extension of either auricle (Fig. 91, 95, 96).--The other and larger division of the auricle is the sinus. The limits of the two divisions are not clearly defined, but as a whole the ental surface of the appendix presents more corrugations. Sometimes the appendix is called auricle, and the entire cavity is then known as atrium.

$\S 854$. Arcus aorticus (az.), arc. ao.-The arch of the aorta (Fig. 92, 101, 102, 108).-See aorta (\$ 851) and the explanation of Fig. 101, 102.

\$ 855. Arteria brachio-cephalica (az.), A. breph.-The brachiocephalic artery (Fig. 91, 92, 101, 102, 103, 107, 108).-See explanation of Fig. 102.

$\S 856$. Arteria cardiaca, A. crd.-A cardiac or coronary artery (Fig. 91, 92, 93, 94, 102).--There are two cardiac arteries, arising from the aortic sinuses of Valsalva and distributed to the substance of the heart. As seen in Fig. 102, they arise respectively from the right and left sinuses, and are distributed to the corresponding regions. The mouth of the right one is shown in Fig. 94. It is not covered by the valve when the latter is opened; see W. T. Sedgwick (1).

$\S 85 \%$. Arteria pulmonalis (az.), A. plm.-The pulmonary artery (Fig. 91, 92, 93, 95, 99, 100).-This springs from the conus arteriosus at the base of the right ventricle, passes sinistro-cephalad and ventrad of the aorta, and divides into two $(A . p l m$. dxt. and $A$. plm. sin.), which carry blood to the right and left lung respectively. 
§ 858. Arteria subclavia, $A$. sbclv. sin.-The subclavian artery (Fig. 91, 92, 101, 102, 108).-This arises from the arcus aorticus just peripherad of the A.brachio-cephatica. On Fig. 92 the abbreviation sin. is omitted.

§ 859. Auricula dextra, aur. dxt.-The right or pulmonary auricle (Fig. 91, 92, 93, 94, 95, 99, 100).-The cavity of the auricle is divisible into a larger sinus, dorsal in position and smoother walled, and a smaller appendix, more ventral in position and with corrugations and recesses upon the ental aspect of the wall. Into the right auricle venous blood is poured through the postcava, procava and the VV. cardiacce.

$\$ 860$. Auricula sinistra, aur. sin.-The left or pulmonary auricle (Fig. 91, 92, 93, 95, 96, 99, 100).-Like the right, the left auricle presents a sinus and an appendix. Purified blood is brought to it by the $V V$. pulmonales.

$\S 861$. Basis.-The base of either ventricle or of the entire ventricular portion of the heart.

$\$$ 862. Capillariæ pulmonales-The capillaries of the lungs.In Fig. 92, these are diagrammatically represented by three subdivisions of the single $A$. pulmonalis. In reality they are exceedingly numerous and minute.

\$ 863. Capillariæ systemicæ-The systemic capillaries.-The exceedingly numerous and minute subdivisions of the branches of the aorta are represented in Fig. 91 by three.

$\S$ 864. Chordæ tendineæ, chd.tnd.-The tendinous cords connected with the free borders of the auriculo-ventricular valves on both sides (Fig. 92, 93, 94 A).-These cords are very strong and inelastic. Their other ends are connected with the apices of the columna carnece, and they serve to prevent the free borders of the bicuspid and tricuspid valves from being forced back into the auricles at the time of the ventricular systole.

\$ 865. Columnæ carneæ, clm. car.-The fleshy columns of the ventricles (Fig. 91, 93, 94, 97, 98).-There are two large columns in the left ventricie and a variable number of smaller ones in the right. Consisting of muscle like the ventricular walls themselves, these columns are supposed to contract and thus keep the chordoe tendinece from becoming lax at the ventricular systole.

$\S 866$. Conus arteriosus $(d x t$.), con. art.-The arterial cone or bulb from which springs the $A$. pulmonalis (Fig. 91, 92, 94, 100).- 
This is a conical prolongation of the base of the right ventricle at its left corner, and is continued into the A. pulmonalis. In a heart of average size its length is about $1 \mathrm{~cm}$. Since there is no corresponding prolongation of the left ventricle, the semilunar valves of the aorta are upon a "lower" level than those of the A. pulmonalis, $i$. e., they are nearer the apex of the ventricle (Fig. 94). The ental surface of the conus is smooth.

$\S 867$. Ductus arteriosus (az.), dct. art. (Fig. 92).-By an oversight, this very significant remnant of an important fœtal structure is not represented upon any of the figures of actual preparations. It is slight and easily overlooked in even a somewhat careful examination of the parts. We haye never observed any depression corresponding with its attachment to the A. pulmonalis, and in the aorta the depression is usually very indistinct. The ductus begins at the cephalic side of the A. pulmonalis, just centrad of its bifurcation, and extends very obliquely along the slight interval between the artery and the aorta, to become attached to the latter a little peripherad of the origin of the $A$. subclavia sinistra, and somewhat at the ventral as well as caudal side of the vessel.

$\S 868$. Fossa ovalis $(\alpha z$.), Fs. ov.-The oval depression upon the right side of the septum auriculare (Fig. 91). - This is not distinctly represented upon any of the figures of actual preparations. It is most easily seen after the lateral wall of each auricle has been removed and the septum is held between the eye and the light. At about the middle of the septum there will appear a thinner area, bounded cephalad and ventrad by a roore or less distinct thickening. The thin portion is usually oval, and measures about $5 \times 2.5$ $\mathrm{mm}$. In the kitten before birth, the thin area is more or less completely absent, so that the two auricles communicate, and the blood from the postcava passes through the orifice, the Fm. ovale, into the left ventricle. Respecting the significance of the fossa ovalis and its appearance in the human heart, see the works cited and also Quain, A, II, 799-803, and Dalton, A, 699.

On the left side of the septum, the area corresponding with the position of the Fs. ovalis is sometimes quite smooth, but more often presents (as in Prep. 360, Museum of Cornell University) a crescentic elevation at its dorsal side.

\& 869. Hemicardia dextra-The right side or portion of the heart.-Since the entire organ is called heart or cardia, it is logically incorrect to speak of the two sides as the right heart and the 
left heart. The term hemicardia is analogous with the words hemisphere, hemipteron, etc. The left auricle and ventricle constitute the hemicardia sinistra.

Hemiseptum auriculare and hemiseptum ventriculare. - Not only ideally, as in Fig. 92, but actually may the interventricular septum be divided so that a portion remains as the mesal wall of either ventricle. Strictly speaking, each of these parts is not a septum, but a hemiseptum, but practically the latter term need seldom be employed.

\$ 870. Orificium auriculo-ventriculare dextrum, orf. aur.-vnt. $d x t$.-The right auriculo-ventricular orifice (Fig. 92, 93, 96, 97, 99). -This is the slightly constricted communication between the right auricle and ventricle. It is guarded by the tricuspid valves.

The similar orifice between the left auricle and ventricle is guarded by the bicuspid valves.

$\S 871$. Postcava $(a z),. p c v$. - The posterior or caudal vena cava or V. cava inferior s. ascendens (Fig. 91, 92, 95, 101, § 955).-This large vein enters the right auricular sinus on its dorsal aspect near the ventricle. Respecting the name, see $\$ 886$.

\$ 872. Præcava $(a z$.$) , prcv.-The anterior or cephalic vena cava$ or $V$. cava superior $s$. descendens (Fig. 91, 92, 93, 95, 101, §919). -This opens into the right auricular sinns at its cephalic aspect, just dorsad of the arch of the $A$. pulmonalis.

$\S 873$. Septum auriculare $\left(a z_{.}\right)$, spt. aur.-The partition between the right and left auricles (Fig. 93, 95, 96, 99).-This is hardly thicker than the lateral auricular wall and is very thin at the foss $\alpha$ ovalis. In Fig. 93, what is named septum embraces also the mass of connective tissue between the aorta and the bifurcation of the septum proper as seen in Fig. 96. The septum is really between only those larger portions of the auricles known as the sinuses.

\& 874. Septum ventriculare (az.), spt. vnt.-The partition between the right and left ventricles (Fig. 93, 97, 98).-The septum is about as thick as the lateral wall of the left ventricle.

\& 875. Sinus (auriculæ dextræ), sn. aur. dxt.-The sinus or larger and more dorsal portion of the right auricle (Fig. 91-96).Its walls are smoother within than those of the appendix. Into it open the postcava, the pracava and the $\nabla$. cardiaca.

§ 876. Sinus (auriculæ sinistræ), sn. aur. sin.-The left auricular sinus (Fig. 91, 92, 93, 95, 96).-The larger and more dorsally 
placed portion of the left auricle. Entad its walls are smoother than those of the appendix, and into it open the VV.pulmonales.

$\S 877$. Sinus coronalis $s$. cardiacus, sn. cor. s. crd.-The sinus of the coronary vein.-This is not distinctly shown in any of the figures. Its position is indicated on Fig. 91 by the abbreviatiou V. $c r d$. Both the sinus and the semilunar valve at its opening into the auricle are readily found by examining the dorso-caudal angle of the auricle close to the septum. Into the sinus open not only the principal $V$. cardiaca, but one or two smaller ones.

\& 878. Sinus Valsalvæ, sn. Vals.-One of the six sinuses of Valsalva (Fig. 92, 94, 96).-There are three of these at the mouth of the aorta and that of the A. pulmonalis. Each sinus may be described as an enlargement of the base of the vessel occupying a little less than one third of its circumference. Each is partly covered by a semilunar valve ( $\$ 882)$, and is thus open peripherad but closed centrad or toward the ventricle. As in man, they may be designated as approximately dorsal, dextral and sinistral in the aorta, and ventrab, dextral and sinistral in the pulmonary artery. From the right and left aortic sinuses arise the two $A A$. cardiaca or "coronary" arteries.

$\S 879$. Trabecula tenuis.-This name is applied, provisionally, to a slender and apparently fibrous filament which, in the preparation from which Fig. 98 was taken, spans the right ventricle near its apex. Its septal end springs from an independent little muscular elevation; its lateral end is attached to the base of a columna carnea. In Fig. 98 it is represented much too large; it is really hardly thicker than a spider's thread. Can it be the insignificant representative of the "moderator band" of Ruminants (Rolleston, B, $25-35)$ ?

\$ 880. Tuberculum Loweri-The tubercle of Lower.-This and the Eustachian valve, both of which have been described in connection with the fossa ovalis of some Mammals, we have not yet determined the distinct presence of in the cat. According to Hyrtl (A, 290), the former rarely if ever appears in the human heart.

$\S 881$. Valva bicuspis, $v l v$. bic.-One of the two bicuspid or mitral valves (Fig. 92, 93, 96, 97, 99).-As in maa, one of these wide valves is at the left or lateral side of the auriculo-ventricular orifice, and the other is toward the septum, thus also overhanging the entrance to the aorta. 
\$ 882. Valva semilunaris, vlv. stmr.-One of the six semilunar or sigmoid valves (Fig. 92, 94, 96, 100).-The mouths or bases of the aorta and $A$. pulmonalis are guarded each by three of these valves. Their free borders are nearly even, and naturally look peripherad. Like the sinuses of Valsalva, which they partly cover, the three aortic valves are approximately dorsal, dextral and sinisistral respectively, while those of the pulmonary artery are approximately ventral, dextral and sinistral.

§ 883. Valva Thebesii ( $\alpha z$.), vlv. Thb.-The valve of Thebesius.This name has been applied to the semilunar valve at the entrance of the $V$. cardiaca through the sinus coronarius into the right auricle (Quain, A, II, 246). It is easily seen in the cat.

\$ 884. Valva tricuspis, vlv. trc.-One of the three tricuspid valves (Fig. 92, 93, 94, 96, 97, 99). - As in man, one of these right auriculo-ventricular valves is nearer the septum, while the other two are, approximately and relatively, dorsal and ventral. There is considerable variation in their form. Usually the free border is quite irregular, but in the preparation from which Fig. 94 was taken, the free border is even and the chordæ tendineæ are attached at the lateral edges.

$\S 885$. Vena azygos (az.), V. az.-The azygous vein (Fig. 91, 99, $101, \S 920)$.-This vein opens into the pracava about $1 \mathrm{~cm}$. peripherad of its junction with the right auricle.

$\S 886$. Vena cava (az.), V. cv.-The adjective cava is applied to either of the two great veins through which the impure blood is brought from the organs in general to the right auricle. It is more commonly employed for the longer and larger of the two, which traverses the abdomen and penetrates the diaphragm. By Owen (A) the two are designated as the postcaval and procaval veins. The vessels are so large, so important and so often mentioned, that we have ventured to omit the vena and to designate them as simply procava and postcava. In the diagram (Fig. 92) the single Vena cava represents both procava and postcava.

$\S 887$. Vena cardiaca, $V$. crd.--One of two or more cardiac or coronary veins (Fig. 91, 92).-The blood which has traversed the tissues of the heart itself is returned to the right auricle by one large and one or more smaller veins, all of which open into a small sinus (sn. coronalis), which has been described above ( $\$ 877)$.

\$ 888. Venæ pulmonales_-The pulmonary veins (Fig. 91, 92).- 
Near the left auricle these form three wholly distinct groups of two each, which from their position may be called dextral, sinistral and intermediate or dorsal.

Disregarding the smaller subdivisions, some of which are not represented in the figures, each group consists of two trunks which open into the auricle by a common sinus of varying depth.

The intermediate and the sinistral sinus are indistinctly seen in Fig. 95 at the ends of the lines drawn from the abbreviation $V V$., but the separate orifices of the veins do not appear. The dextral sinus could not be shown in the same figure, but its position is indicated approximately by the $s$ of the word septum.

When traced to the lungs, it is found that the dextral and sinistral groups come from the right and left lungs respectively, but that of the two large constituents of the intermediate, the one nearer the right comes from the lung of that side, and the other from the left.

In man, there are usually two pulmonary veins on each side, opening independently into the auricle.

$\$ 889$. Ventriculus dexter, vnt. dxt.-The right or pulmonary ventricle (Fig. 91, 92, 93, 94, 97, 98, 99).-This is not only, as its name implies, relatively dextral in position, but also, in the natural attitude of the organ, somewhat more ventral than the left ventricle. Its walls are markedly thinner than those of the left, and its cavity does not so nearly reach the apex of the organ. The ental surface of its walls presents numerous elevations and depressions. In addition to the columnce carnece and the trabecula tenuis, there are many muscular trabeculæ passing obliquely from one part of the wall to another, forming a sort of coarse network. The sinistrocephalic corner of the ventricle is devoid of reticulations, and is prolonged as the conus arteriosus ( $\$ 866$ ).

$\S 890$. Ventriculus sinister, vnt. sin.-The left or systemic ventricle (Fig. 91, 92, 93, 94, 97, 98, 99).-This is not only sinistral, but also, relatively, somewhat more dorsal in position in the natural attitude of the heart. Its lateral walls are 2-3 times as thick as those of the right ventricle, and its cavity reaches more nearly to the apex of the organ. In the contracted state of the heart, the left ventricle occupies the more space on account of the greater thickness of the walls (Fig. 93) ; but the thinner walls of the right yield more to the pressure of an injection, and its cavity generally appears more capacious (Fig. 91, 97, 98). It presents two large columnæ 
carneæ and some other slight irregularities, but not the reticulations which exist in the right ventricle. The aorta springs from the base of the ventricle close to the septum.

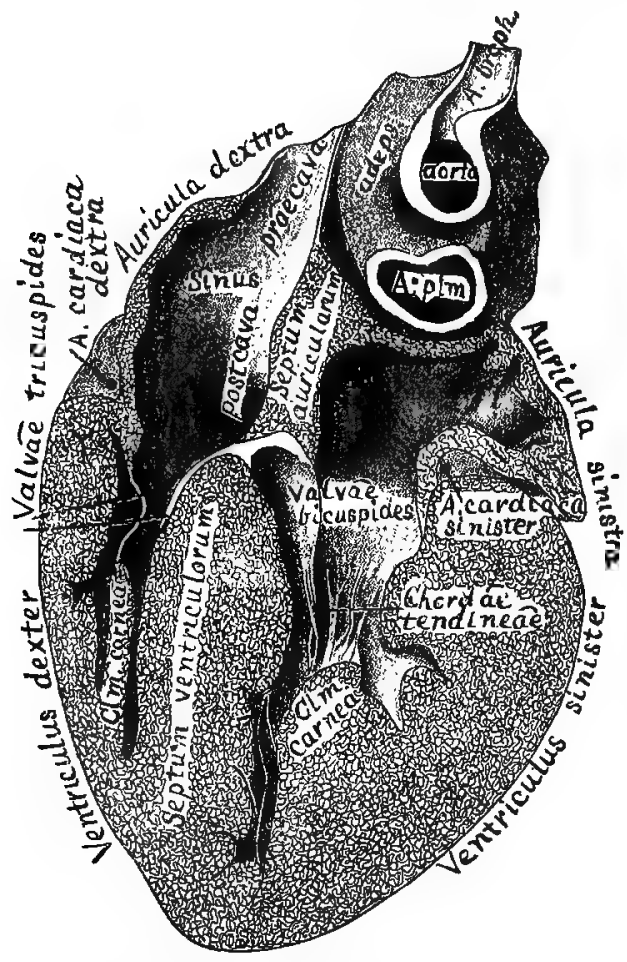

Fig. 93. -The Dorsal Part of the Heart, SEEN FroN The Ventral AsPeCT; $\times 1.5$.

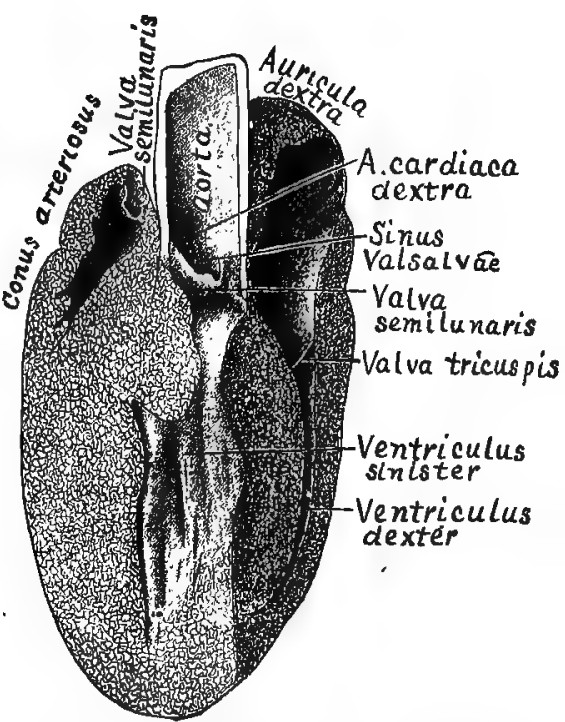

Fig. 94.-A Longitudinal Segment of the Heart, showing the Right Sindo of Valbalta and Cardiac artert; $\times 1.5$.

§ 891. Explanation of Fig. 93--Longitudinal, dextro-sinistral section of the entire heart, seen from the ventral surface.

Preparation.-This was the heart of a very large male (castrated) cat, a gift from Messrs. Melvin \& Badger of Boston, Mass. From the same cat were taken the scapula and humeri, ribs and vertebræ shown in Fig. 43-46, 50, 68-71. .

The heart was not injected or prepared in any special way, excepting hardening in alcohol upon cotton. Hence the cavities are small and the auricies corrugated. When hard, it was divided by a longitudinal dextro-sinistral incision with a large sharp scalpel. The dorsal part is here represented; the ventral part is a little the thicker.

The special object of the preparation is to exbibit the relative positions and oonnections of the four great cavities with the auriculo-ventricular valves. In addition, there are shown, (A) the entrance of the pracnva, which has been longitudinally divided; (B) the point of entrance or mouth of the postcura, as a dark spot at the beginning of the word ; (C) the aorta and pulmonary artery $(A, p l m$.$) ; both are transected, the former just at the$ 
place of origin of the A. brachio-cephalica; (D) the right and left cardiac arteries imbedded in the fat at the line of junction of the auricles and ventricles; (E) the part marked septum auricularum is not truly part of the septum, but the thick tissue ventrad of it as seen in Fig. 96.

§ 892. Fig. 94-Preparation.-This represents nearly the dextro-ventral fourth of the heart, but the two planes of longitudinal section are not quite at right angles with each other, and the preparation is so placed that both are foreshortened.

The special object is to show the dextral wall of the central part of the aorta, with the dextral sinus Valsalva and the mouth of the corresponding $A$. cardiaca. This latter is seen to be so high up that it would not be covered by the $V$. semilunaris even when fully extended $(\$ 856)$.

At the left are seen the cavity of the conus arteriosus, with a very small piece of the A. pulmonalis, and one of the semilunar valves, which is decidedly upon a higher (more cephalic) Jevel than those of the aorta ( $\$ 866$ ).

It will be understood that the communication between the right ventricle and the conus is behind the septum ventriculare.

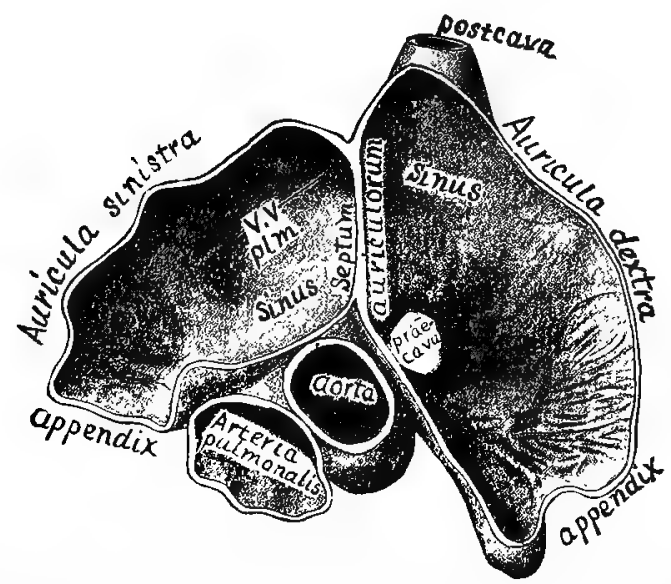

Fig. 95.-Transection of the Auricles, Caudal Aspect ; $\times 1.5$.

§ 893. Fig. 95-98-General Description and Mode of Preparation.-These four figures represent transections of the same heart through the auricles (Fig. 95, 96) and through the ventricles (Fig. 97, 98), and as viewed from the caudal (Fig. 95, 97) or the cephalio (Fig. 96, 98) aspect. The heart had been distended and hardened with alcolıol (\$ 842), and was then transected with the large scalpel at two levels.

In comparing the figures as representing continuous parts of the same organ, Fig. 97 is to be imagined inverted upon Fig. 98 , the inversion being from left to right; then Fig. 96 is to be placed upon Fig. 97 without inversion, and Fig. 95, inverted as before, upon Fig. 96. The entire heart will then, in the mind, be as if viewed from the base with the ventral aspect toward the observer, so that the right and left sides are inverted as compared with his.

\& 894. Fig. 95-The Interior of the Auricles from the Caudal Aspect.-The figure illustrates the extent of the septum auriculare, and the fact that the auricular sinuses appear upon the dorsal aspect, while on the ventral there are to be seen only the appen- 
dices projecting on either side of the anrta and pulmonary artery. Of these two vessels, the former is seen to be nearly in line with the auricular septum, while the latter, at this level, is ventro-sinistrad of it. The middle of the length of the septum auriculare should have been thinned to indicate the position of the fossa ovalis $(\$ 868$ ). In the left auricle the two ends of the line which is interrupted by the abbreviation $V V$. point respectively to the positions of the dextral and sinistral sinuses of the pulmonury veins $(\S 888)$; the third or intermediate sinus is overhung by the auricular septum, and its position is indicated approximately by the $s$ of the word septum. A little of the complete wall of the postcava remains, but the place of attachment of the precava is indicated only by an unshaded area.

\section{$\$$ 895. Fig. 96-The Auricles and Auriculo-ventricular Orifices seen from the}

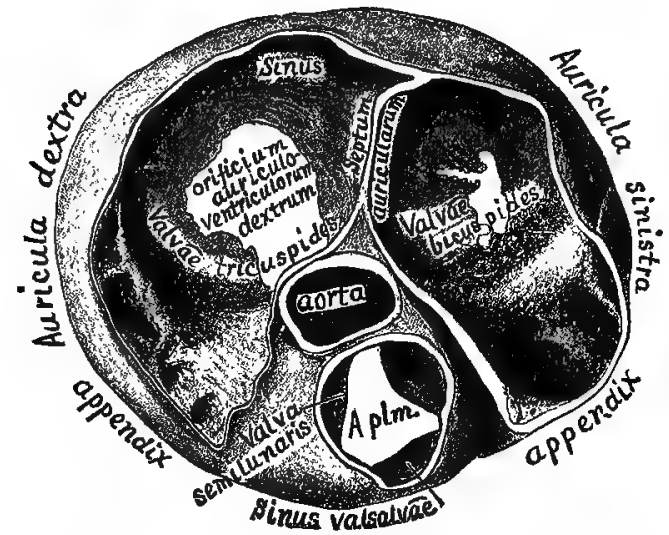

Fig. 96.-Transection of the auricles, Cephalic affect; $\times 1.5$.

Cephalic Aspect.-In addition to the features in common with Fig. 95, this figure illustrates the following points :-

(A) The relative positions of the three semilunar valves of the pulmonary artery, namely, ventral, dextral and sinistral.

(B) The fact that the aortic valves are upon a lower (more caudal) level than the pulmonary, the former, indeed, not distinctly appearing unless the preparation is so held as to admit the light directly into the vessel $(\$ 866)$.

The auriculo-ventricular valves are not especially well shown, but the form of the two unshaded areas indicatos, approximately, the fact that of the two bicuspides ( $\$ 881$ ), one is lateral and the other septal, while of the three tricuspides ( $\$ 884$ ), one is septal and the others respectively dorsal and ventral (more strictly dorso-lateral and ventro lateral).

In the right auricle, the word sinus designates the general cavity of the auricle aside from the appendix; but in the corner, and pointed at by the words sinus and septum, is the location of the small sinus of the cardiac veins ( $\$ 887$ ).

§ 896. Fig. 97.-The Ventricular Cavities, from the Cephalic Aspect.-In this and Fig. 98, the most striking features are (A) the greater thickness of the lateral wall of the left ventricle, in relation to its office of propelling the blood over all parts of the body, and $(B)$ the larger size of the cavity of the right. The difference of size is artificial, and due to the less resistance offered by the thinner wall to the pressure of the injected alcohol.

In the left ventricle is seen the cut end of one of the columnæ carneæ, still attached to 


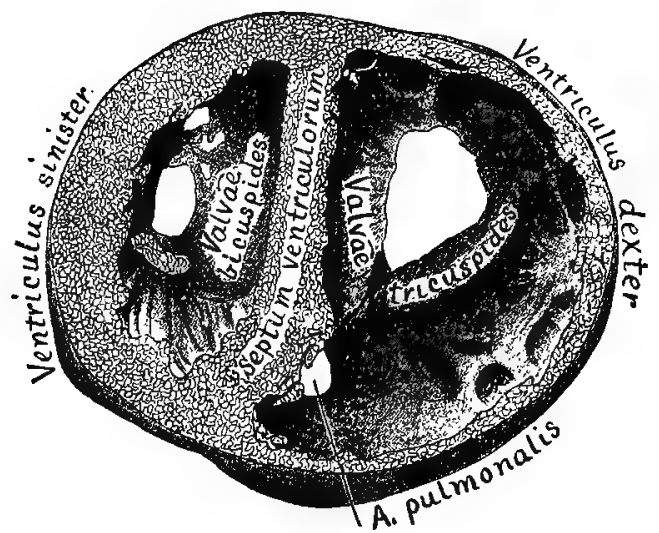

Frg. 97.-Transegron of the Venitricles, Caddal Aspeet ; $\times \mathbf{1 . 5}$.

the lateral bicuspid valve by its chordæ tendineæ. In the right, the surface adjacent to the place of exit of the pulmonary artery is seen to be smoother than in other parts $(\$ 860)$.

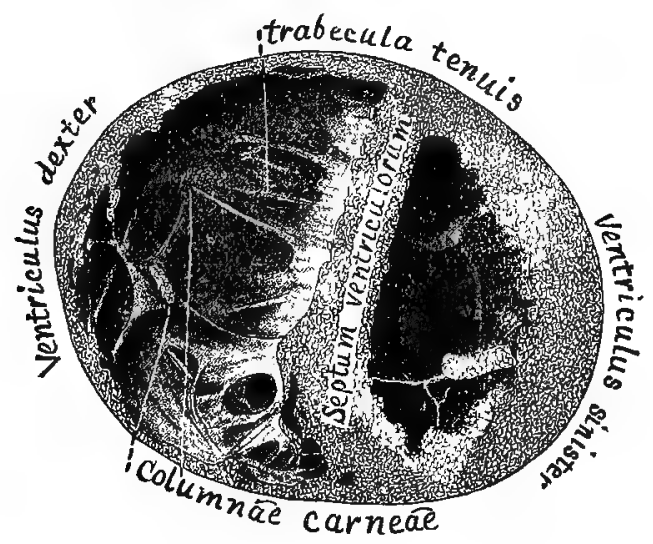

Fig. 98.-Transection of the Ventricles, Cephalio Aspect ; $\times 1.5$.

\& 897. Fig. 98-The Ental Aspect of the Apices of the two Ventricles.-In addition to points in common with Fig. 97, this figure well displays the very numerous reticulations and trabecula of the right ventricle, and especially the presence of a very delicate fibrous band, provisionally named trabecula tenuis, which connects the septum with the iteral wall ( 8879$)$.

\section{DISSECTION OF THE HEART.}

\& 898. After the careful inspection of the organ as a whole, and the study and comparison of the various sections represented in Fig. 93-98 (or at least of the figures if such preparations have not been made), the heart should be dissected as follows :- 
Instruments and Materials._-Sharp Charrière scalpel ; coarse or medium curved scissors; silver probe, syringotome or dull tracer; 15 per cent. glycerin ; a heart, the larger the better, with the pericardium removed. The dissection may be done upon a fresh specimen or upon one simply hardened in alcohol; it is easier, however, if the organ has been distended and hardened with alcohol ( $\$ 842)$.

\$ 899. Auricula dextra.-Pinch up with the forceps a bit of the lateral wall of the right auricle, for example, just dorsad of the appendix, and remove it with the scissors. Introduce the probe and ascertain the points of emergence of the postcava and pracava. With the scissors, remove the entire lateral wall, including that of the appendix, but leave the attachments of the cavæ.

Note (A) the smoothness of the ental surface of the sinus as compared with the fretwork in the appendix; $(\mathbf{B})$ close to the auriculoventricular furrow, just caudad of the postcava, the orifice of the sinus coronalis ( $\$ 877$ ), guarded by the valva Thebesii ( $\$ 883$ ).

$\$ 900$. Ventriculus dexter.-Pass the probe through the orificium auriculo-ventriculare into the right ventricle, noting that no obstruction is offered by the tricuspid valves. Ascertain by probing the limit of the ventricular cavity, and with the scalpel make a $\mathrm{V}$-shaped flap having its base at the base of the ventricle and including the entire lateral wall.

Turn this flap toward the auricle and note: (A) the two or three columne carnec; (B) the smaller trabecula, both fleshy and tendinous; (C) the chordæ tendineæ passing from the columns or directly from the ventricular wall to the borders of the three valvo tricuspides; (D) that two of these valves (dorsal and ventral) will be moved by the lifting of the flap; the third (septal) is applied closely to the septum, and its chordæ are very short, arising either from the septum directly or from very slight elevations.

$\S 901$. Conus arteriosus and A. pulmonalis.-Pass the probe sinistro-cephalad through the conus into the artery. Then introduce a scissors blade in the same direction and slit both up along the most prominent part of the convexity. On divaricating the sides, it will be found that the incision has either divided the ventral semilunar valve or gone between it and the dextral. Note $(\mathbf{A})$ the three valves; (B) the corresponding sinuses of Valsalva; (C) the bifurcation of the A.pulmonalis into right and left branches to the corresponding lungs. 
Ventriculus sinister.-It is easier to defer the examination of the left auricle until after the simpler ventricle has been opened.

With the scalpel, make a longitudinal incision through the lateral wall about midway of the width of the ventricle. This will permit a view of the cavity without injuring the massive columnæ carneæ which will generally appear one at either side. Then make a transverse incision at right angles with the basal end of the first, making sure that it is on the ventricular side of the furrow between the auricle and the ventricle. With the scalpel, remove the enclosed angles of the wall on either side so as to expose the cavity as much as desirable. Note that one of the wide bicuspid valves is applied against the septum so as to conceal the orifice of the aorta.

$\$ 902$. Aorta.--Pass the scissors blade behind the septal bicuspid valve into the aorta and slit it up. The incision will probably cut through one of the three semilunar valves. Note (A) the position of these valves, dorsal, dextral and sinistral; (B) the corresponding sinuses of Valsalva; (C) the orifices of the right and left cardiac arteries from the dextral and sinistral sinuses.

Farther peripherad, note (A) the origins of the brachio-cephalic and left subclavian arteries; (B) in some cases, at the opposite side of the aorta from those vessels, a slight depression indicating the place of attachment of the ductus arteriosus.

\$ 903. Auricula sinistra.-Returning to the left ventricle, pass the probe into the left auricle and ascertain the points of attachment of the pulmonary veins nearest the appendix. Then introduce a scissors blade and divide the parts from the ventricle just dorsad of the appendix, carefully avoiding the veins just mentioned.

Slit up the appendix to its tip. Then with the probe, seek out the orifices of the three groups of pulmonary veins as represented in Fig. 91. Cut along the auriculo-ventricular furrow so as to permit a better view of the cavity. Note the more or less marked crescentic fold which indicates the dorsal margin of the fossa ovalis. Hold the septum between the eye and the light and note its thinness at that place. Examine the right side of the septum for the foss $a$ and the left for the crescentic fold ( $\$ 868)$.

\section{FROZEN SECTIONS OF THE THORAX.}

$\S 904$. Figures 99 and 100 represent respectively the caudal and cephalic aspects of a frozen transection of the thorax, the thickness of the section being a trifle over $1 \mathrm{~cm}$. The manner of preparation has been described in $\$ 324$. 
These two figures may be compared with Fig. 101, 103, 107 and 109 in the present work, and with Plates IV and V of Dwight's " Frozen Sections of a Child " (B)

$\S 905$. Level of the Sections.- So far as we can judge by comparison with dissections and by the collation of these with the other sections of the same cat, the centrum of the 7 th thoracic vertebra appears in Fig. 99, and that of the 6th in Fig. 100, the intervertebral arthron being included in the thickness of the section. The section includes the bifurcation of the trachea, which appears as a mesal tube in Fig. 100, into the two bronchi which appear in Fig. 99 ; since plane surfaces only are shown, the ridge, at the place of bifurcation does not appear in Fig. 100. Between the oesophagus and the vertebra in Fig. 100 appear the transections of the $M M$. longus colli; but these terminate in the thickness of the section, and in Fig. 99 the Vena azygus is seen to be joined by the first intercostal vein.

$\$ 906$. The Heart.-From the fact that the heart was injected with plaster, while the lungs were not injected at all, the former occupies a disproportionally large space. The natural obliquity of the organ also interferes with the ready appreciation of the relations of the parts which appear in the two figures.

In Fig. 99, the right and left correspond with those of the observer, while they are reversed in Fig. 100. In the former, the vein presented includes a combination of the special features which have been observed in the longitudinal section (Fig. 93) and the transections (Fig. 96, 97). The non-injection of the left auricle accounts for the relatively larger size of the right in Fig. 99, and for the non-appearance of the left in Fig. 100.

In Fig. 100, not only are the right and left parts reversed in position with respect to the observer, but the appearances are less readily comparable with what are shown in the other figures of the heart. The right auricle is divided near the point of entrance of the pracava, but the non-injected left anricle just escaped; its cephalic end miglit fairly have been placed in the vacant area just sinistrad of (dextrad of on the figure) the word $A$. pulmonalis.

$\S 907$. Pleura ( $\$ 806$ ). - In both figures the lines representing the pleura are made disproportionally heary to facilitate their recognitiou.

The pleura is seen to form a continuous line upon the ental aspect of the thoracic parietes, to be reflected off at each side of the vertebra] centrum upon the adjacent structures, and then to follow the contour of the lung to the beart, where it forms the ectal lamina of the pericardium. At the ventrimeson it is reflected again upon the parietes. Hence, like the peritoneum (\$ 725), each pleura is a closed sac with continuous walls, and the viscera which appear to be within its cavity are really outside and in contact only with its ectal surface.

\& 908. Mediastinum.-The irregular space between the vertebra and the pericardium, and bounded on the sides by the undulating line of pleura as it, approaches the roots of the lungs, is the mediastinum. Within it are the asophagus and trachea, the aorta and other vessels and nerves.

$\$ 909$. Septum mediastinale.-At the ventrimeson the right and left pleural reflections are in contact, and form an apparently single membrane between the two cavities, which, as may be demonstrated very easily by experiment, are thus entirely disconnected. In Fig. 99, the mediastinal septum so formed is very short, but in Fig. 100, the pericardium is at some distance from the sternum and the septum is correspondingly extensive.

In man (Quain, A, II, Fig. 163; Dwight, B, Pl. IV), these two reflected layers of pleura do not meet at the level of the heart, and the small space between them is called the anterior mediastinum. For coinparison with man, therefore, the single mediastinum of the cat should be called dorsal, or posterior.

$\$ 910$. Pericardium. - Like the pleura, the pericardium is represented upon the figures by unnaturally heavy lines. In Fig. 99 , near the ventrimeson, it is seen to consist of two 


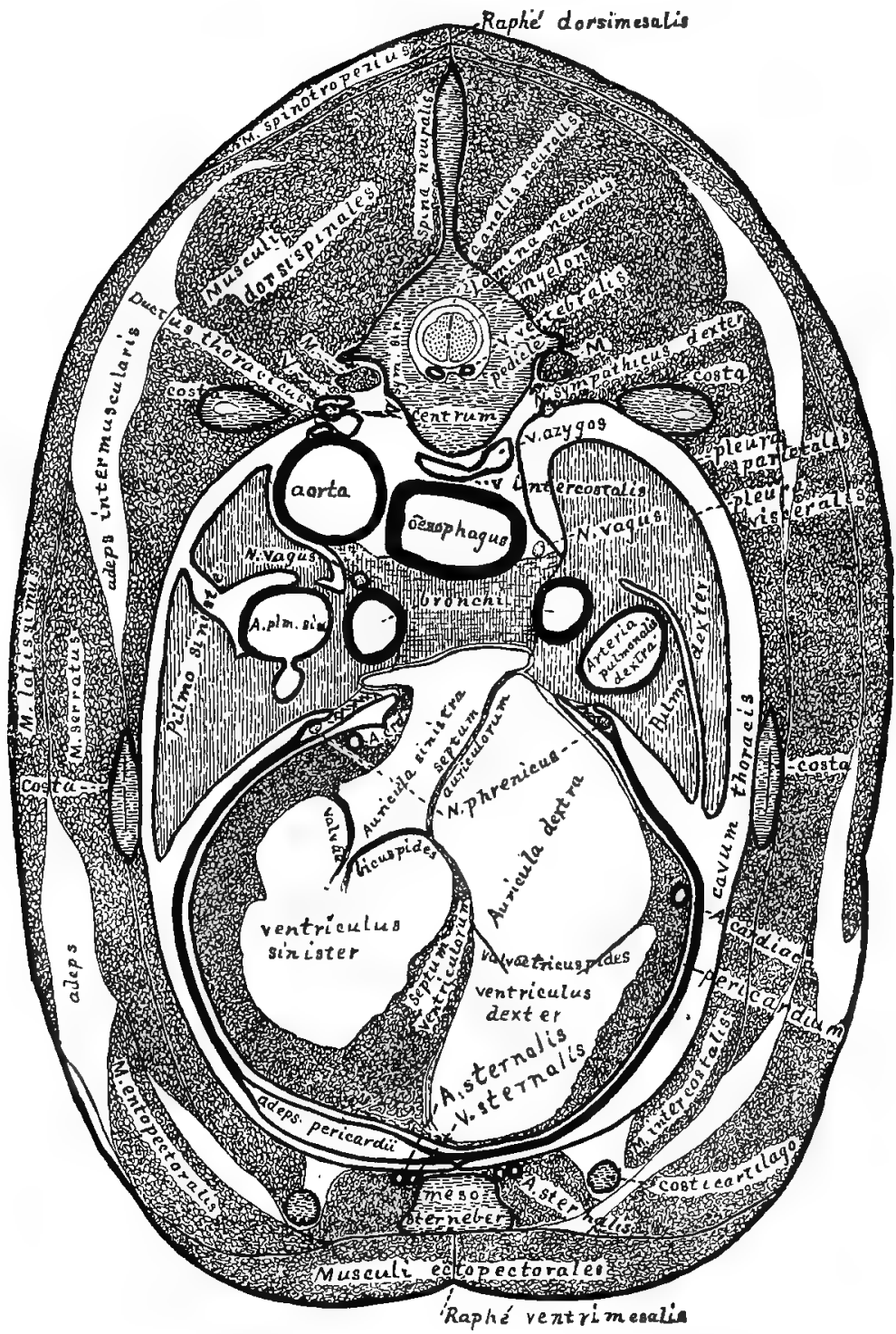

Fig. 99.-Frozen Trangection of the Thorax at the Heart, Caudal abpect; $\times 1.5$.

laminx embracing a deposit of fat (adeps pericardii). On both figures the ectal lamina may be traced to the root of the lung on either side, where it is reflected to form the risceral pleura of those organs, and to the ventrimeson, where it is reflected at the septum 


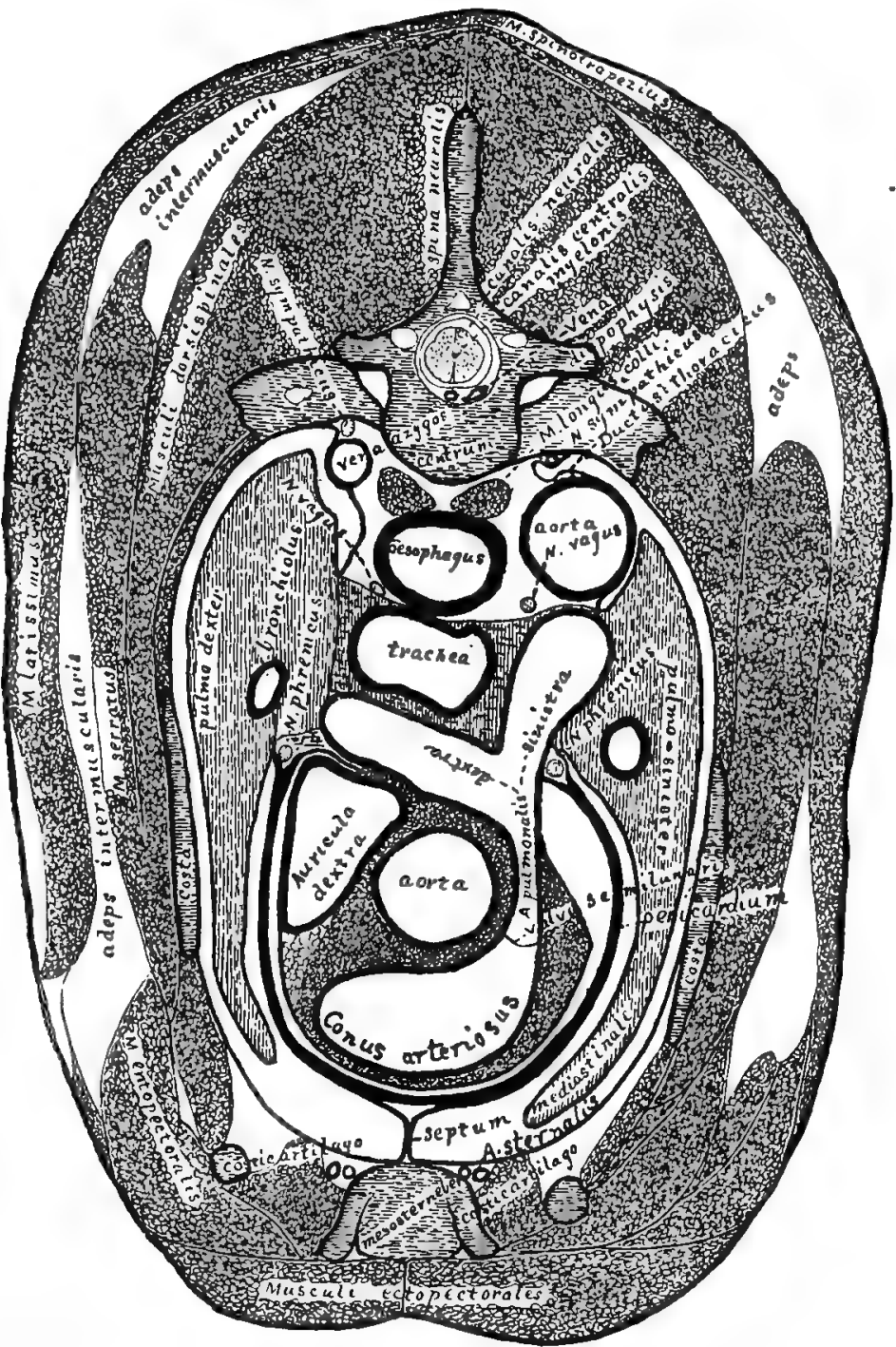

Fte. 100.-Trozen Traxsection of the Thorax, Cephalic Aspect; $\times 1.5$.

mediastinule to become continuous with the parietal pleura. The ental lamina is reflected to form the proper serous covering of the heart. At the dorsal points of junction of the two laminæe are the phrenic nerves.

The sternal arteries and veins appear dorso-laterad of the mesosterneber. The veins lie mesad of the arteries ( $\$ 021$ ).

$\S$ 011. Nerves, etc.-The sympathic trunic ( $N$. sympathicus) appear just ventrad of the heads of the ribs in Fig. 99, and in corresponding places in Fig. 100. 
The vagi ( $N$. vagus) lie ventro-laterad of the osophagus and dorsad of the roots of the lungs. The phrenics ( $N$. phrenicus) lie just ventrad of the roots of the lungs, and at the point of union of the ectal and ental laminæ of the pericardium. The recurrent laryngeal nerves do not appear at these levels.

The ductus thoracicus is represented by a circle just dorsad of the aorta; its walls are much thinner than here indicated, and are collapsed when the tube is empty. See Fig. 103 , where, however, at this level, the duct is double.

\section{BLOOD VESSELS OF THE TRUNK.}

\$ 912. Special Instruments and Material.-Arthrotome; scalpel; fine and coarse scissors and forceps; tracer; nippers; injecting apparatus and material (\$\$ 130, 336).

Arteries, References.-Quain, A, I, 343; Gray, A, 75; Cuvier, A, VI, 100 ; Gegenbaur (Lankester), A, 585 ; Hyrt], A, 643 ; Bernard, A, 244 ; Milne-Edwards, A, III. 511 ; Leyh, A, 566 ; Owen, A, III, 532 ; Chauveau, A, 545 ; Chauveau (Fleming), A, 515 ; Gurlt, A, 555; Straus-Durckheim, B, II, 183.

Veins, References.-Quain, A, I. 343 ; Gray, A, 564 ; Cuvier, A, VI, 226 ; Gegenbaur (Lankester), A, 589 ; Hyrtl, A, 713 : Bernard, A, 244 ; Milne-Edwards, A, III, 570 ; Leyh, A, 625 ; Owen, A, III, 549 ; Chanveau, A, 636 ; Chauveau (Fleming), A, 596 ; Gurlt, A, 656.

$\S 913$. Preparation.-Inject the right $V$.jugularis externa witl fine blue mass ( $\$ 386$ and 1450 ), and either the femoral artery or the abdominal aorta with thin plaster ( $\$ \$ 345,352,363)$.

See especially $\$ 596$ (10) for the method of dissecting vessels and nerves. Vessels when uninjected may be distinguished from nerves or bundles of connective tissue by cutting a slit in them and demonstrating their tubular character with the tracer.

\section{Table of the Systemic Veins of the Trunk, and the Portal Veins (Fig. 101, 103, 107).}

The Table of Veins is like that of the Arteries ( $\$ 916)$, inasmuch as the larger trunks are placed first. It should be remembered, however, that the larger trunks are formed by the smaller ones instead of dividing to give rise to the smaller ones, as with the arteries $(\$ 819)$.

§ 914. THORAX AND NECK.

VV. cardiacæ (Fig. 01, § 887).

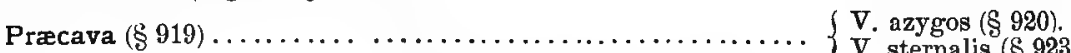

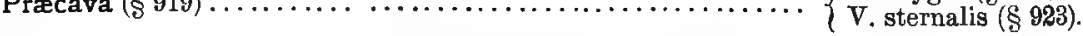

( V. vertebralis $(\$ 923)$

Vena brachio-cephalica (\$92:). I V. subclavia (\$924).

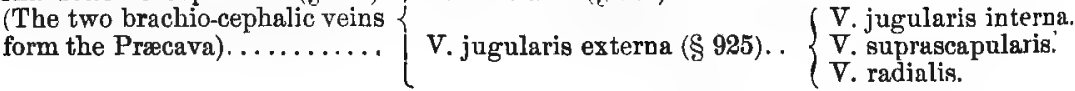




\section{§ 915. ABDOMEN.}

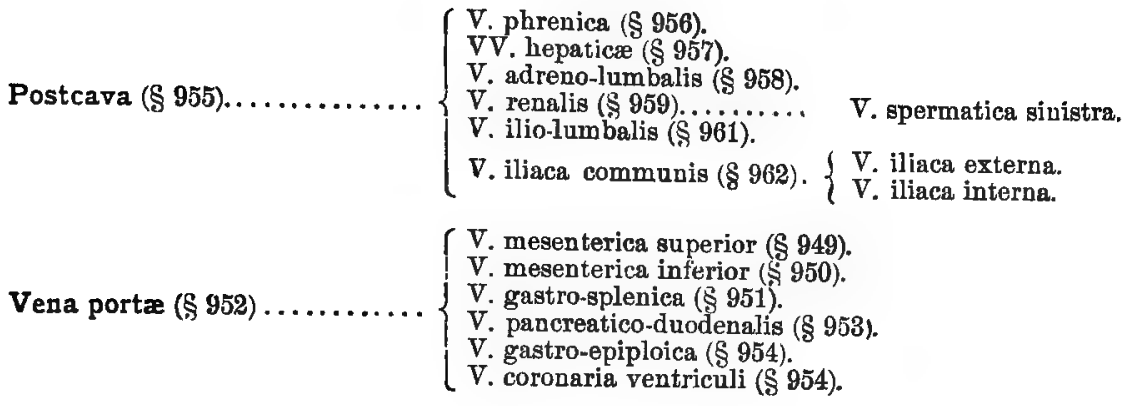

\section{Table of the Systemic Arteries of the Trunk and Arm (Fig. 101, 102, 105, 107).}

A brace placed after an artery includes the branches of that artery.

\section{§ 916. THORAX AND ARM.}

AA. cardiacæ $(\$ 856)$.

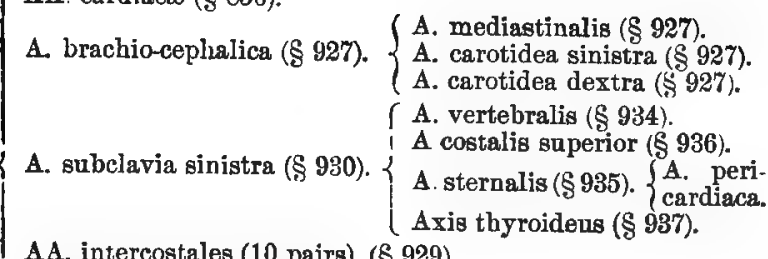

AA. intercostales (10 pairs), $(\$ 929)$.

Aorta thoracica (\$ 926). . \{ A. subclavia sinistra (\$ 980).

A. bronchialis (2), (\$930).

A. oesophagæa $(2-4),(\$ 931)$.

AA. lumbales (3 pairs), (\$932).

A. subclavia dextra ( $\$ 933$ ). (Continuation of the brachio-cephalica after the origin of the right carotid.)

A. axillaris ( $\$ 938$ ). (Continuation of either A. subclavia peripherad of the first rib.) .........

A. brachialis ( $\$ 943)$, (Continuation of the Axillaris peripherad of the origin of the common trunk of the circumflex and subscapular.)

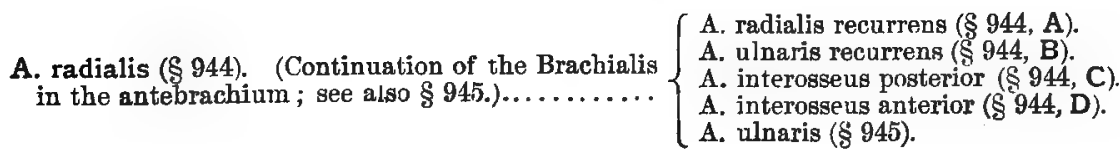

A. radialis (\$944). (Continuation of the Brachialis $\left\{\begin{array}{l}\text { A. radialis recurrens }(\$ 944, \mathrm{~A}) \text {. } \\ \text { A. ulnaris recurrens }(\$ 944, \mathrm{~B}) \text {. } \\ \text { A. interosseus posterior }(\S 944, \mathrm{C}) \text {. } \\ \text { A. interosseus anterior }(\S 944, \mathrm{D}) \text {. } \\ \text { A. unaris }(\S 945) \text {. }\end{array}\right.$

A. vertebralis (\$ 934).

A. costalis superior $(\$ 936)$.

A. sternalis (\$ 935).

Axis thyrojdeus (\$937).

A thoracica anterior ( $\$ 939)$.

A. thoracica longa $(\S 940)$.

A. circumfleza posterior ( $\$ 941$ ) et subscapularis (Fig. 102, ร 942).

A. circumflexa anterior ( $\$ 943, \mathrm{~A})$.

A. profunda superior ( $(943, \mathrm{~B}$ ).

A. nutriens $(\$ 945, \mathrm{C})$.

A. anastomotica magna ( $\S 943, D)$. 
§ 917. ABDOMEN.

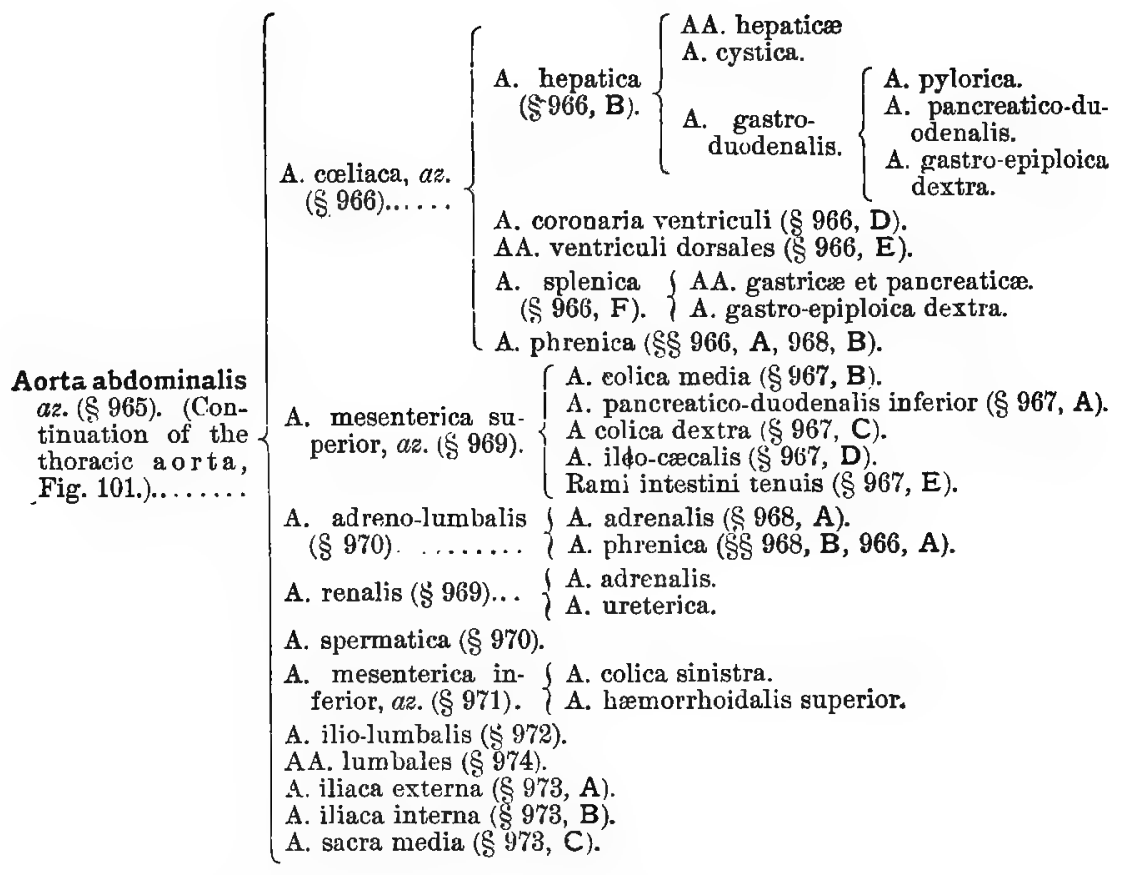

Preparation-Fig. 101.-A lean cat was killed with chloroform, and the femoral artery and vein were injected with red and blue plaster respectively ( $\$ 352-362$ ). The $V$. jugular is externa was then injected cephalad with blue gelatin (\$366). After an hour the ventral wall of the entire trunk was removed, together with all the viscera, except the heart, kidneys and urocystis, and enough of the diaphragm to expose the vessels. The liver was cut away within about $1 \mathrm{~cm}$. of the postcava. All the organs were then freed from fat and connective tissue with the fine forceps, tracer and scissors. It was necessary to proceed with great care, especially in separating the ureter, the spermatic vessels and the vessels into which the aorta divides.

The urocyst was turned caudad so as to expose its dorsal surface.

Fig. 101, $\mathbf{A}$ and $\mathbf{B}$, required no special preparation, as they are simply enlarged diagrams of the aorta and postcava with their branches. 


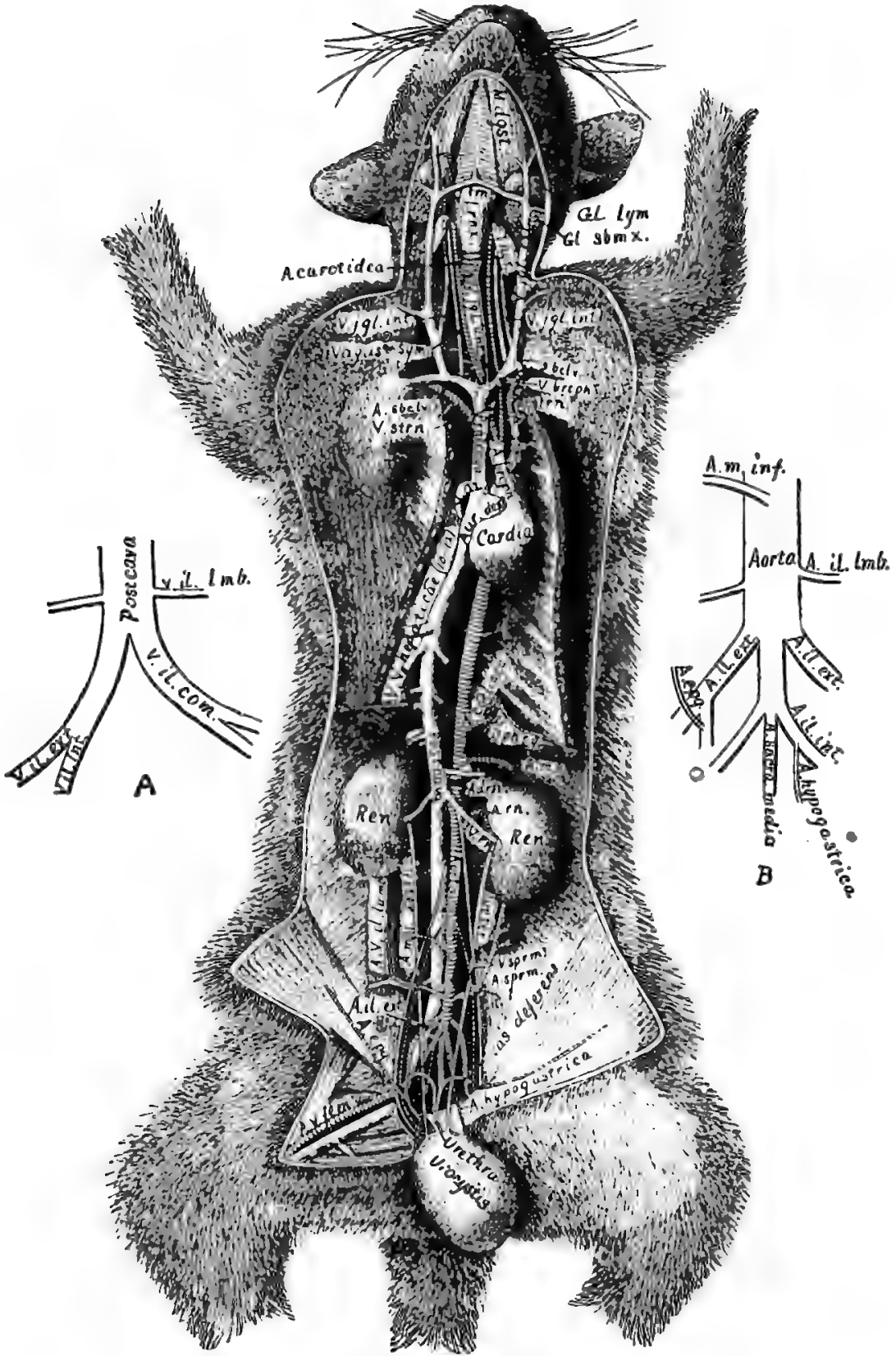

Fig. 101.-Gexerat View of the Blood Vegsels; $x$.8. A. Ohiorn of the Postcava. B. Termination of tere abdominal aorta. 
Description of Fig. I0r.-A. V. fem., A. V. femorales-Femoral artery and vein. A. hypogastrica-The hypogastric or superior vesical artery. A. epigastrica-The deep or inferior epigastric artery. A. il. ext., A. iliaca externa-The external iliac artery. A. V. il.-lumb., A. V. ilio-lumbales-The ilio-lumbar artery and vein. A. m. i., A. mesenterica inferior, $a z$.-The inferior mesenteric artery. A. sprm., A. spermatica-The spermatic artery. Aorta, $a$ z. A. renalis-The renal artery. A. m. s., A. mesenterica superior, $a z$. The superior mesenteric artery. A. cœliaca, $a z$-The cœliac artery or coeliac axis. A. brcph., A. brachio-cephalica, az.-The brachio-cephalic or innominate artery. A. subcl., A. subclavia-The subclavian artery. A. strn.; A. sternalis-The sternal or internal mammary artery. A. carotidea-The carotid artery. Adrn., Adrenale -The adrenal or suprarenal body. Au. dext., Auricula dextra-The right auricle of the heart. Cardia, az.-The heart. Dphrg., Diaphragma, az.-The diaphragm. Gl. sbmx., Glandula submaxillaris-The submaxillary salivary gland. Gl. lym., Glandula lymphatica-A lymplatic gland or pair of glands just cephalad of the submaxillary. Lrnx., Larynx, az. M. dgst., M. digastricus-Digastric muscle. Præcava, az.The superior or descending vena cava. Ren-The kidneys Thyr., Corpus thyroideum-The thyroid body; the two are connected in man. Urocystis, az.-The urinary bladder. Urethra, $a z$ - The canal leading from the urocystis to the exterior. Ureter -The canal conveying urine from the kidney to the bladder; see Fig. 85, 86. V. sprm., V. spermatica-The spermatic vein. V. rn., V. renalis. VV. hepaticæ (10-12), az.-Hepatic veins. V. az., V. azygos, az.-The azygous vein. V. strn., V. sternalis-The sternal or internal mammary vein. V. sbclv., V. subclavia-The subclavian vein. V. brcph., V. brachio-cephalica-The brachio-cephalic or innominate vein. V. jgl. int., V. jugularis interna-The internal jugular vein. V. jgl. ext., V. jugularis externa-The external jugular vein. V. jgl. ant., $V$. jugularis anterior. V. trns., V. transversa-A large vein connecting the two jugular veins across the meson. Vagus and Symp.-The vagus and sympathic nerves. Vas deferens-The canal conveying the semen from the testis.

Description of Fig. Ior, A, B.-A. sacra med., A. sacra media, az-The median sacral artery. A. il. ext., A. iliaca externa. A. il. int., A. iliaca interna. A. hypogastrica-The hypogastric or superior vesical artery. A. epg., A. epigastricaThe internal or inferior epigastric artery. Aorta, az. A. il.-lumb., A. ilio-lumbalis -The ilio-lumbar artery. Postcava, az.-The ascending or inferior vena cava. V. il. ext., V. iliaca externa-The external iliac vein. V. il. int., V. iliaca interna-The internal iliac vein. V. il.-lumb., V. ilio-lumbalis-The ilio-lumbar vein. V, il. com., V. iliaca communis.

Fig. 102-Preparation.-The abdominal aorta of an adult cat was injected with red plaster (\$352). After an hour the vessels were carefully isolated in the thorax and in the right arm to the middle of the antebrachium. This was done by removing muscles, connective and other tissues with the scalpel, tracer, scissors and fine forceps. The pericardium was then removed, and the proper cardiac arteries (AA. cardiacæ) were isolated for 1-2 cm. Finally, the auricles and part of the ventricles were cut away, commencing peripherad and following the aorta. After the aorta with its semilunar valves was isolated, the entire preparation was soaked in Wickersheimer's fluid $(\$ 300)$ for a day and then allowed to dry. 


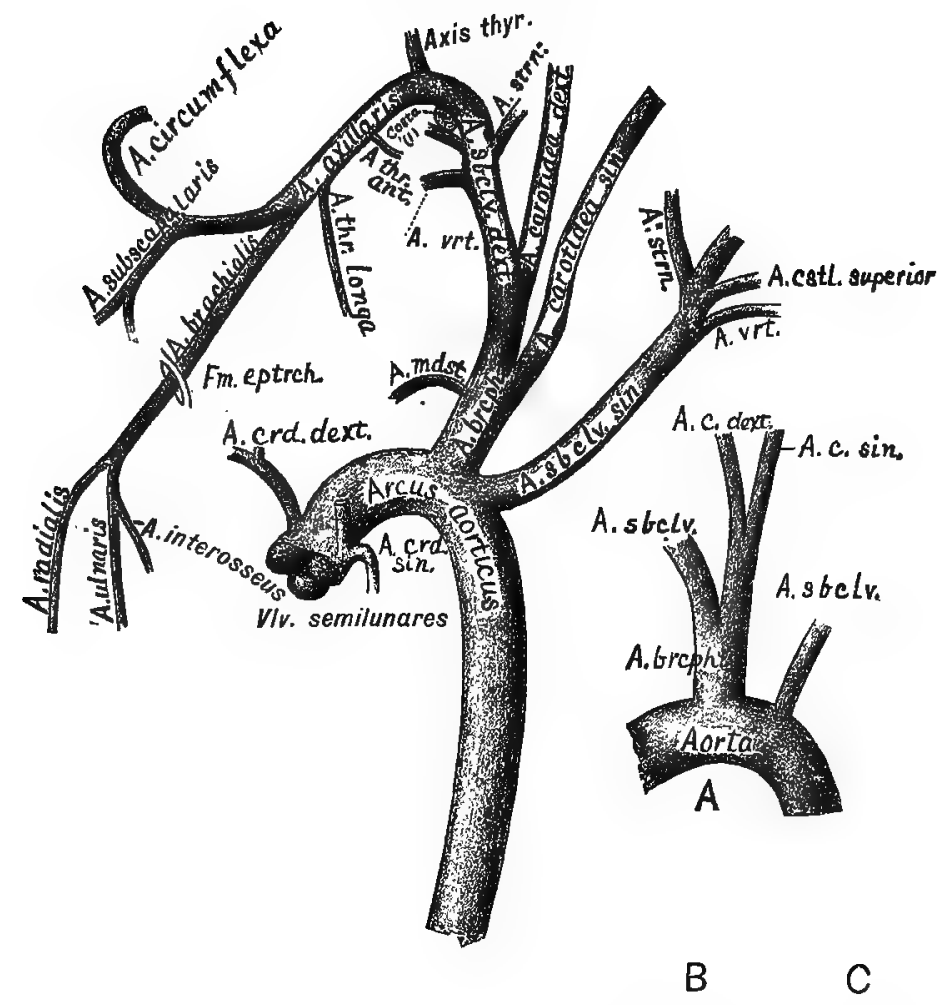

Fig. 102.-The Aorta, With ITs MaIN Branches in the Thorax and Right Arm; Ventrat VIew; $\times 1$. A. Origin of the Carotid ARTeries; $\times 1$. B, C. Vhins With VALves ; Quain, A, II, 174.

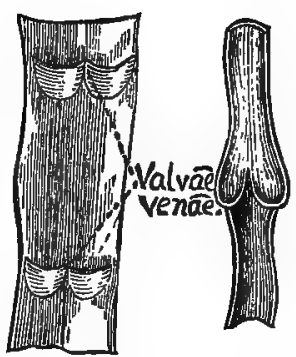

"The Wickersheimer's fluid prevents the brittleness that is so objectionable in plaster injected vessels that are dried.

B and C-Preparation.-B. A segment of the $V$. jugularis externa about $5 \mathrm{~cm}$. long was slit lengthwise and placed in water, and the valves floated up by blowing into the little pockets with a blow-pipe.

C. The external jugular or femoral vein was injected peripherad 
with plaster, and after the plaster was moderately hard, a hemisection of the vessel was made and the plaster removed.

Explanation of Fig. ro2.-Arcus aorticus, az.-The arch of the aorta. A. brcph., A. brachio-cephalica, $\alpha z$.-The brachio-cephaljc or innominate artery. A. crd. dext., A. cardiaca dextra-The right cardiac or coronary artery. A. crd. sin., A. cardiaca sinistra-The left cardjac or coronary artery. A. mdst., A. mediastinalis, az.-The mediastinal artery. A. sbclv. dext., A. subclavia dextra-The right subclavian artery, a continuation of the brachio-cephalica. A. carotidea sin. (sinistra)-The left carotid artery arising from the brachio-cephalica; see A. A. vertebralis-The vertebral artery. This arises from the subclavian. A. cstl. (costalis) superior-The superior costal artery. A. strn., A. sternalis-The sternal or internal mammary artery. A. axillaris-The axillary artery. A. thr. ant., A. thoracica anterior-The anterior thoracic artery. A. thr. (thoracica) longa-The long thoracic artery. A. subscapularis-The subscapular artery. A. circumflexa-The circumflex artery; a common trank arising from the axillary bifurcates to form this and the preceding artery; in man they arise separately from the axillary. A. brachialis-The brachial artery. A. radialis-'The radial artery. A. ulnaris-The ulnar artery. A. interos., A. interosseus-The interosseus artery. Costa (1)-The first rib in cross section. Fm. eptrch., Foramen epitrochleare. Vl. semilunares-The semilunar valves at the beginning of the aorta; they are closed.

Explanation of Fig. I02, A.-Aorta, az, A. brcph., A. brachio-cephalica, az.The brachio-cephalic artery. A. sbcl., AA. subclaviæ-The subclavian arteries. A. c. dext., A. carotidea dextra. A. c. sin., A. carotidea sinistra.

The carotids may arise separately from the brachio-cephalica as shown in Fig. 102, or less frequently they may arise by the bifurcation of a single trunk which is given off from the brachio-cephalica (Fig. 102, A). This common trunk is called by Owen (A, III, 535), the bicarotid, and by Milne-Edwards (A, III, 524), carotide primitive. It is stated by the latter that this is the normal condition in the cat, lion, dog, etc.

Fig. 102, B.-Vein slit lengthwise and spread out to show two pairs of valves.

Fig. I02, C.-Hemisection of a vein with closed valves.

In both $\mathbf{B}$ and $\mathbf{C}$ the central end is uppermost. $\mathbf{C}$ shows how the two valves prevent the flow of blood or injecting mass toward the periphery. Sometimes but a single valve exists, in which case the action is less efficient.

\$ 918. Posture and Exposure for the Thoracic Vessels.-Place the cat dorsicumbent, with the arms stretched laterad and fastened with cords to the loops in the edge of the tray (Fig. 76). If the dissection is to be long continued, the caudal half of the body may be removed $(\$ 234)$.

Commence on the right side, and continue the incision made in exposing the $V$. jugularis for injection (\$ 913) to a point opposite the base of the xiphisternum, carrying the incision $2 \mathrm{~cm}$. to the right of the ventrimeson; then, with the arthrotome, cut through the sterno-mastoid and pectoral muscles (Fig. 72), along a line about $2 \mathrm{~cm}$. to the right of the ventrimeson and parallel with it. Cut 
the thoracic wall on the right side at the junction of the ribs and their cartilages, and then across the meson (Fig. 76, § 825); do this with either the arthrotome or bone scissors. Then cut with nippers the right ribs about $2 \mathrm{~cm}$. from their heads. Turn the sternum to the left and the wall of the thorax to the right. This will expose the heart, right lung, præcava, the abdominal part of the postcava, the right carotid and sternal arteries, and the axillary vessels and nerves (Fig. 101, 105).

VEINS OF THE THORAX. (See Table, § 914.)

VV. cardiacæ $s$. coronariæ (see \& 887).

\$ 919. Præcava, $a z$., and its branches (Fig. 101).-The præcava is the prominent vessel extending from a point opposite the first rib to the cephalic part of the right auricle of the heart. It is noticeable just at the right of the meson.

$\S 920$ ! V. azygos, az. (Fig. 99, 101, 107, V. az.).-This enters the præcava just cephalad of the root of the right lung. Grasp the right lung and turn it toward the left, and with the tracer follow the V. azygos peripherad. At regular intervals branches from the intercostal spaces enter it. Those entering it near its termination are large, as they represent the trunk formed by the union of two or more intercostal veins. Opposite the 10th rib the vein becomes mesal in position, and is dorsad of the aorta (Fig. 107, V. az.). The $\mathrm{V}$. azygos can not be traced caudad of the diaphragm.

§ 921. V. sternalis (Fig. 101, V. strn., 99, 100)-A -A bout opposite the $3 \mathrm{~d}$ rib the sternal veins enter the præcava as a single trunk, but on the sternum there are two veins, one on each side just mesad. of the corresponding artery.

\$ 922. $\boldsymbol{\nabla}$. brachio-cephalica s. innominata (Fig. 101, 103, 107, V. brcph.).-If the thymus body is present, separate it from the præcava. About opposite the 1st rib the præcava will be seen to be formed by the union of two nearly equal branches, the $V V$. brachio-cephalica. Carefully isolate the right one.

\$ 923. $\mathbf{v}$. vertebralis.-Very near the 1st rib this large branch enters the dorsal side of the brachio-cephalica. It comes from the brain through the vertebrarterial canal in connection with the vertebral artery (Fig. 104, A. vrt.).

$\S$ 924. V. subclavia (Fig. 101).- Just beyond the entrance of , 
the $V$. vertebralis the $V$. brachio-cephalica is formed by the union of two nearly equal branches, the subclavian vein and the external jugular. The V. subclavia may be traced to the arm. Later, in studying the arteries of the arm, it will be found to follow them very closely.

$\S 925$. V. jugularis externa (Fig. 87, 101, 103, A).-This large vein comes from the head and face. In the neck it is covered only by the skin and cutaneous muscle. Opposite the cephalic part of the larynx there is a very large branch connecting the jugulars of the two sides (Fig. 101, V. trns.).

The external jugular receives the internal jugular (Fig. 101, V. jgl. int.), the combined trunk of the subcutaneous vein of the arm and the V. subscapularis. This trunk is shown in Fig. 101 just cephalad of the abbreviation $V . j g l$. int.

Veins of the Abdomen, see \$\$ 946-964.

ARTERIES OF THE THORAX AND ARM. (See Table, \$ 916.)

$\S$ 926. Aorta thoracica, az. (Fig. 91, 99, 100, 101, 102, 103, 107). --The aorta is the single great artery arising from the left ventricle of the heart. Through it and its branches, every part of the body is supplied with pure blood. To expose it, remove the pericardium from the ventral wall of the heart, also the præcava, the septum mediastinale, and the thymus body if that is present. The aorta curves sharply to the left (Fig. 102), thus making the arcus aorticus. The branches of the thoracic aorta are as follows, commencing centrad :-

\section{AA. cardiacæ $s$. coronariæ (see $\S 856$ ).}

\$ 927. A. brachio-cephalica $s$. innominata, $a z$. (Fig, 101, 102, 103, 107).- This arises from the convexity of the aortic arch and passes almost directly cephalad. Very near its origin it gives rise to the A.mediastinalis, which passes ventrad, then to the $A$. $c a$ rotidea sinistra (Fig. 101, 102, 102, A, 103, A, C, and 107), and the $A$. carotidea dextra. Sometimes the two carotids arise as a single trunk (Fig. 102, A). After the origin of the carotids, the A. brachio-cephalica is continued as the A. subclavia dextra (\$933).

\$ 928. A. subclavia sinistra (Fig. 101, A. sbclv., 102, 103, A. sbclv. sin., 107, A. s.). - For the branches of the A. subclavia, see §§ 934-945 and the Table ( $\$ 916)$. 
§ 929. AA. intercostales (10 pairs), (Fig. 103, AA. costales).-The intercostal arteries are given off from the dorsal side of the aorta. Each passes to an intercostal space and divides into three branches. One of these passes ventrad along the caudal margin of the cephalic of the two ribs between which it extends (Fig. 103); another goes to the deep muscles of the back, and the third enters the spinal canal through the intervertebral foramen ; see s 484 .

§ 930. A. bronchialis.-The arteries (2) to the lungs arise either from the aorta itself, about opposite the root of the lung, or from the 4 th intercostal arteries. They accompany the bronchi to the lungs.

8 931. AA. csophageaz (2-4), (Fig. 107, A. cs.).-These are all small branches and may sometimes arise from the intercostals instead of the aorta.

8 932. AA. lumbales (2-3 pairs).-On account of the great caudal projection of the diaphragm, 2-3 pairs of the lumbar arteries are given off by the thoracic aorta. Rarely also the $A$. coliaca arises in the thorax (\$966).

§ 933. A. subclavia dextra (Fig. 102, A. sbclv. dext.).-This is a continuation of the $A$. brachio-cephalica peripherad of the origin of the right carotid. Tear away carefully any connective tissue covering it as far peripherad as the ectal margin of the first rib. Note the large nerve covering its ventral face. This is the vagus (Fig. 107 and 108; the recurrent laryngeal branch may be seen winding: around to its dorsal side (Fig. 108, N. l. r.).

\section{Branches of the A. Subclatia.}

\$934. A. vertebralis.--This, the first branch, arises from the cephalic surface of the subclavian and passes dorso-cephalad to enter the vertebrarterial canal. It passes through this to the brain (Fig. 102, 103, A. vert., 104, A. vrt.). In Fig. 104, the dorsal wall of the vertebrarterial canal is removed and the artery shows throughout nearly its whole extent.

\$ 935. A. sternalis $s$. mammaria interna (Fig. 101, A. strn., 102, A. strn., 103, 107).-This arises from the ventral surface of the A. subclavia nearly opposite the origin of the $A$. vertebralis. It passes ventrad and reaches the sternum opposite the $2 \mathrm{~d}$ mesosterneber (Fig. 49). It sends two or three small branches cephalad, then extends caudad along the sternum, and, as stated above, is laterad of the sternal vein. Branches of this artery anastomose with the intercostal arteries (\$929), with the phrenic on the diaphragm, and with the epigastric on the abdomen (Fig. 101). The left sternal also usually supplies the $A$. pericardiaca to the pericardium.

$\S 936$. A. intercostalis superior (Fig. 102, A. cstl. superior).Just as the subclavian crosses the first rib, this artery arises from 
its dorsal surface. It extends only $2-3 \mathrm{~mm}$. before dividing into two trunks. One extends caudad on the ental wall of the thorax, supplies the 1st and $2 \mathrm{~d}$ intercostal spaces, and then passes between the 1 st and $2 \mathrm{~d}$ ribs to be distributed to the deep muscles of the back. It may be followed by tearing away the muscles with the tracer. Its final distribution can best be followed after the removal of the scapula. The other branch passes cephalad nearly parallel with the $A$. vertebralis, and reaches the dorsal side of the great nerve trunks of the brachial plexus (Fig. 107). It is distributed to the M. serratus magnus ( $\$ 664$ ). After the section of the brachial vessels and nerves and the lateriduction of the scapula, it may be very easily traced.

§ 937. Axis thyroideus (Fig. 105, A. spscp.)-Just laterad of the 1st rib, this trunk takes its origin. It passes laterad along the ental surface of the clavo-trapezius muscle, sending two or three branches cephalad, the most important one being to the longus colli muscle. The part extending laterad is called the $A$. suprascapularis. The $A$. suprascapularis supplies the clavicular end of the M. clavo-trapezius and the lateral surface of the supraspinatus. Its principal branch, however, accompanies the suprascapular nerve between the supraspinatus and subscapularis muscles and enters the suprascapular fossa about opposite the base of the acromion. In the supraspinous fossa it divides into two branches, one being distributed to the muscles in the supraspinous fossa, while the other passes through the incisura magna (Fig. 45) and is distributed to the structures in the infraspinous fossa (Fig. 43). The scalpel as well as the tracer should be used in tracing this vessel.

\$ 938. A. axillaris (Fig. 105, A. axl.). - This is the continuation of the A. subclcuia laterad of the 1st rib. It is very intimately associated with the axillary veins and nerves.

§ 939. A. thoracica anterior (Fig. 102 and 105, A. thr. ant.).This is the first branch furnished by the $A$. axillaris. It arises from the ventral surface of the axillary and passes with the nerve of the same name (Fig. 105) ventrad to the pectoral muscles. If the pectorals were divided at the proper level, this artery would remain with the arm.

\$ 940. A. thoracica longa (Fig. 102 and 105, A. thr. longa).It arises at about the same level as the preceding ( $\$ 939)$ and passes nearly caudad, sending several small branches to the pectorals, but 
is distributed principally to the ental surface of the $M$. latissimus ( $\S 635)$.

\$ 941. A. circumflexa (Fig. 102, 105, A. crem).-After the A. thoracica longa has been traced, cut through the pectoral mass and the $M$. epitrochlearis ( $\$ 681$ ) along the caudal edge of the $M$. biceps, that is, nearly parallel with the humerus. Turn the muscles aside, and there will be exposed the vessels and nerves of the brachium. A bout opposite the trochin (Fig. 45, B) the axillary artery will be found to divide into two slightly unequal parts (Fig. 102, 105). The larger of these, the brachial artery, continues along the arm; the other, the common trunk of the circumflex and subscapular arteries, proceeds toward the surgical neck of the humerus for about $1 \mathrm{~cm}$., when it divides into the branches just named.

The circumflex passes between the subscapularis and teres muscles in company with the circumflex nerve (Fig. 105) ; it winds dorsally around the surgical neck of the humerus and is distributed to the triceps muscles, and its terminal branches pass to the ental aspect of the $M$. clavo-deltoideus. Demonstrate these by separating the acromio- and clavo-deltoid muscles and raising the cephalic border of the latter. Branches of the circumflex artery and nerve will be seen ramifying on its ental aspect.

§ 942. A. subscapularis (Fig. 102, 105, A. sbscp.).-This arises in common with the circumflex, as stated above ( 894 ); it passes somewhat laterad and is distributed principally to the structures in the subscapular fossa (Fig. 44). Several branches are furnished, however, to other structures, viz., MM. latissimus, meditriceps and dermo-humeralis. This artery and its branches are best followed after section of the axillary vessels and nerves.

\$ 943. A. brachialis (Fig. 102, 105, A. br.).-This is the artery of the brachium. It is a direct continuation of the A. axillaris peripherad of the origin of the common trunk of the circumflex and subscapular (\$\$ 941-2). Isolate it with the tracer by tearing away connective tissue. The median nerve and brachial vein lie ectad of it and should be removed or turned aside. In its course along the caudal side of the arm, the brachial gives rise to several unnamed muscular branches and the following named branches:-

(A) A. circumflexa anterior (Fig. 105. It is the small vessel passing between $s$ and $p$ of "N. m. spiralis").--It passes to the caudal margin of the M. biceps, and then sends a branch proximad to the head of the humerus. 
(B) A. profunda superior (Fig. 105).-This arises opposite the musculo-spiral nerve (Fig. 105) and follows its course.

(C) A. nutriens.-In Fig. 105 it is the vessel passing between the $A$. and the $b r$. It is represented too large. It passes to the bone, and entering the nutrient foramen, is distributed to the shaft of the humerus.

(D) A. anastomotica magna (Fig. 105, between the abbreviations $h m r$. and trc., near the distal end of the humerus).- This artery passes to the convexity of the elbow.

Upon reaching the foramen epitrochlearp, the $A$. brachialis traverses it in company with the median nerve (Fig. 102, 105) and reaches the concavity of the elbow.

\$ 944. A. radialis (Fig. 102, 105).--The brachial artery distad of the concavity of the elbow is continued as the radial. It passes caudad of the tendon of the biceps muscle and entad of the pronator teres (Fig. 105, §701). About opposite the middle of the antebrachium the radial artery is covered only by skin and fascia. At this point the artery divides into two unequal branches; the smaller or ventral one follows the radial nerve, while the principal one inclines dorsad, and just distad of the styloid process of the radius it curves around to the dorsal side of the manus.

To expose the artery, divide the $M$. pronator teres about $2 \mathrm{~cm}$. from its origin and turn the two ends aside, and divide longitudinally the antebrachial fascia along its ventral aspect.

The radial artery gives rise to several muscular branches that are unnamed and to the following named branches:-

(A) A. radialis recurrens. - This arises in the concavity of the elbow on the caudal side of the tendon of the biceps (Fig. 105). It is distributed to the various structures in the concavity of the elbow.

(B) A. ulnaris recurrens (Fig. 105). - This is shown in the figure as arising from the radial opposite the point where it crosses the $M$. biceps. It extends proximad to the struetures at the convexity of the elbow. It is called unar recurrens on account of its distribution. See Quain, A, I, 408, and Gray, A, 520.

(C) A. interosseus posterior.-This artery arises from the ental aspect of the radial just distad of the tendon of the biceps. It extends nearly directly dorsad, and may be demonstrated by tearing away the nuuscles surrounding it.

(D) A. interosseus anterior (Fig. 105, A. interosseus).-This artery arises, either separately or as a common trunk with the ulnar, from the ental aspect of the radial about $3 \mathrm{~cm}$. from the $F \mathrm{~m}$. epitrochlecure. It passes distad along the antebrachium on the ventral side of the interosseus ligament. It may easily be followed by turning the radial artery slightly aside and then separating the muscles with the tracer.

§ 945. A. ulnaris (Fig. 102 and 105). - In man the ulnar artery is about equal in size to the radial, and the division of the brachial to form them is very near the elbow or even in the brachium (Quain, A, I, 403); but in the cat the ulnar is so small that it is sometimes not injected at all with plaster. In its course and distribution it cor- 
responds, however, with its hnman homologue. It arises from the radial at about the junction of the proximal and middle thirds of the antebrachium, and is covered at its origin by the $M$. pronator teres (\$701). It passes caudad and distad along the ental surface of the flexor muscles, and joins the ulnar nerve at about the point of its division into a dorsal and ventral branch. The artery divides at this point, and the two branches accompany the two branches of the ulnar nerve.

\section{ABDOMINAL BLOOD VESSELS.}

Posture.-Dorsicumbent, the limbs fastened laterad with cords (Fig. 76).

\$ 946. Preparation.-The arteries and veins should be injected with plaster as directed above (\$8 352-362), or the thorax may be opened and the thoracic aorta and postcava injected caudad; the latter is easier done if the thorax is to be studied on a different cat.

\$ 947. Exposure.-About half an hour after the injection is finished, npen the abdomen as directed for the exposure of the viscera $(\$ 710)$.

\section{Vena Porta. (See Table, §915.)}

The study of the portal vessels will be greatly facilitated by injecting them. To do this, turn the duodenum to the left, and extending along the mesoduodenum ( $\$ 729)$, near the edge of the duodenal part of the pancreas, will be seen a large vessel filled with blood ( $V$. mesenterica superior). Inject this vessel toward the liver, inserting the canula about opposite the caudal end of the pancreas. Employ uncolored plaster or that colored with chrome green or yellow. All the larger portal vesse]s will be filled except the peripheral part of the $V$. mesenterica superior, and that may be filled by reversing the direction of the canula

It will be necessary to change the position of the viscera often in tracing the portal vessels.

\& 948. V. mesenterica superior, az. (Fig. 103, 107, V. m. s.).This collects the blood from the small intestine. Trace it from the point where it was injected, first peripherad along the small intestine, and then centrad to a point opposite the pylorus.

§ 949. V. mesenterica inferior, az. (Fig. 107).-This usually empties into the preceding about opposite the ampulla of Vater (Fig. 84), or it may empty into the following, as in man. It comes from the large intestine.

\$ 950. V. gastro-splenica, az.-This joins the portal slightly nearer the liver than the preceding ( $\$ 950)$.- It comes from the spleen, from part of the stomach and from. the gastro- 
splenic part of the pancreas. Trace it to these parts. It may also receive the vessels mentioned in $\$ \S 949,950$.

$\S 951$. V. portæ, $a z$.-The trunk formed near the pylorus by the $V V$. mesenterica and the V. gastro-splenica is called the Vena portoe. It passes to the liver parallel with the ductus choledochus communis ( $\$ 746$ [3]), and receives the following branches when they do not empty into any of the preceding :-

\$ 952. V. pancreatico-duodenalis, $\alpha z$-Coming from the pancreas and the duodenum. This vessel empties into the $V$. porta on the dorso-dextral side of the pylorus.

$\S 953$. V. gastro-epiploica. $a z_{\text {. }}$-This comes from the pyloric part of the great currature of the stomach and the dorsal fold of the great omentum. It empties into the $V$. porta opposite the pylorus.

\$ 954. V. coronaria ventriculi, $a z,-A s$ the name indicates, this comes from the lesser curvature of the stomach. It empties into the $\bar{T}$. porte somewhat nearer the liver than the preceding. The three branches just described may all unite to form a single trunk before emptying into the $V$. portce.

\section{Systemic abduminal Venns. (See Table, $§ 915$ )}

$\S 955$. Postcava $s$. Vena cava inferior, az. (see Table, $\S 915$, Fig. 101, 103, Postcv., 107, Pcv.).--Turn the stomach and intestines to the left. The postcava, filled with blue plaster, will appear extending somewhat obliquely caudo-sinistrad along the meson. Just cephalad of the right kidney it penetrates the liver and is entirely surrounded by its substance. Opposite the last lumbar vertebra the vessel usually becomes entirely mesal in position and reaches the dorsal side of the aorta (Fig. 101). The vessel may divide opposite the kidneys into two nearly equal branches, which extend caudad, one on each side of the meson.

$\S 956$. V. phrenica (Fig. 90).-These pass mesad along the opposite halves of the diaphragm, and open into the postcava just as it penetrates the diaphragm. Draw the liver somewhat caudad, and the vein may be easily demonstrated with the tracer.

$\S 957$. VV. hepatica, az. (10-12), (Fig. 101).-These convey the blood supplied to the liver by the $V$. portec and the $A$. hepatica into the postcava. They may be demonstrated by tearing away the liver substance next the postcava. Their number is variable, as the lobes sometimes give off more than one branch each.

$\S 958$. V. adreno-lumbalis (Fig. 101, 108, 107).-This vein returns blood from the adrenal (\$760), from the $M$. psoas and the dorso-lateral parietes of the abdomen opposite the kidney. It. opens into the postcava just candad of the liver, or it may empty into the $\boldsymbol{V}$. renalis (\$959). To demonstrate it, turn the stomach and intestines aside, and employ the tracer. The vein usually rests in a groove on the ventral side of the adrenal (Fig. 101).

\& 959. V. renalis (Fig. 101, V. rn, 103, V. rn., 107, V. rn.).-This extends in nearly a straight line from the kidney and empties into the 
side of the postcava. The left is slightly cephalad of the right, and both veins usually lie caudad of the corresponding arteries (Fig. 103). The left renal vein usually receives the $V$. spermatica and nearly always contains a pair of valves just centrad of the entrance of the $V$. spermatica. The right may also contain valves, but it rarely receives the $V$. spermatica dextra. Both often receive the $V$. adrenolumbalis. The V. renalis, like the A. renalis, may be double ( $\$ 969)$. Finally, when the postcava is divided sufficiently far cephalad ( 962 ), the $V$. renalis may empty into the corresponding division instead of into the postcaval trunk.

$\S 960$. V. spermatica (male), ovarii (female), (Fig. 101).-The left opens almost invariably into the left renal (\$959), while the right nearly always empties directly into the postcava. Both are guarded by a pair of valves about $5 \mathrm{~mm}$. from their mouth.

The $V$. spermatica returns blood from the testis, and hence passes nearly longitudinally cephalad through the inguinal ring $(\$ 768)$ to the point where it empties. It is very slender and must be traced with great care. The $V$. ovarii is much shorter, passes obliquely laterad from the ovary, and increases greatly in size during gestation.

$\S 961$. V. ilio-lumbalis (Fig. 101).-This enters the postcava at right angles nearly opposite the Crista $i$ iii (Fig. 51). It returns blood from the free or ventral surface of the muscles in this region.

Divisions of the Postcava.-Usually the postcava is a single median vessel until it reaches a point opposite the junction of the 6th and 7th lumbar vertebræ. Here it lies between the aorta and the spinal column; hence the aorta should be removed. But this part of the dissection should be deferred until the arteries have been studied ( $\$ 965)$.

§ 962. Branches:--V. iliaca communis (Fig. 101, A).-The postcava in the cat is formed by two equal trunks opposite the junction of the 6th and 7th lumbar vertebræ. Each trunk is one of the common iliac veins. Sometimes (once in about ten cases) the postcava divides into the common iliacs much farther cephalad, rarely even cephalad of the kidneys. In such cases the postcava is on the ventral or lateral aspect of the aorta, never on its dorsal side.

§ 963. V. iliaca externa (Fig. 101). -This large vessel comes from the leg, passing into the abdomen dorsad of Poupart's ligament (Fig. 39). It unites with the following ( $\$ 964$ ) to form the $V$. iliaca communis. The vein should be traced peripherad upon the meros. Just as it enters the abdomen there is a pair of valves that usually stop the plaster injection completely. The valves may be easily demonstrated by slitting the vein longitudinally and then blowing peripherad with the blow-pipe (Fig. 102, B, C, §130). This 
demonstration will be especially satisfactory if the vein is immersed in water.

§ 964. V. iliaca interna (Fig. 101, A).-This returns blood from the pelvis, the hip and the thigh, etc. The main trunk of this vein is formed by branches within the body cavity. It unites with the preceding to form the V. iliaca communis. The branches of this trunk are so well supplied with valves that they are rarely injected with plaster.

Aorta Abdommalis. (Fig. 101, 103, 107 ; see Table, $\$ 917$.

$\S 965$. Turn the stomach and intestines to the right, press upon the median line against the spinal column, and the injected aorta will be felt. It may be exposed by tearing away the peritoneum with a tracer. Commence at a point just caudad of the diaphragm, expose the aorta, and note the following branches and their anastomoses:-

\$ 966. A cœliaca - Coliac axis, $\alpha z$. (Fig. 101, 103, 107). As the aorta enters the abdomen-sometimes just before-there is given off a large trunk, the coeliac axis, which extends nearly ventrad. Tear away the peritoneum and connective tissue covering the vessel, and note the following branches and their distribution:-

(A) A. phrenica $s$. A. diaphragmatica.-This artery is sometimes given off from the cephalic side of the cœliac axis near its origin from the aorta. More frequently it arises from the A. adreno-lumbalis ( $\$ 968)$. It soon divides into two branches and is distributed to the diaphragm.

(B) A. hepatica, az.-Turn the liver cephalad and draw the stomach sinistro-caudad. Tear away with the tracer the peritoneum from the lesser curvature of the stomach, and the caliac axis will appear. A large branch-A. hepatica-will be seen arising from the cœliac axis, and extending dextro-ventrad toward the liver. Trace it by tearing away the peritoneum and connective tissue with the tracer. Nearly opposite the pylorus it divides into three trunks :-

(a) A. hepatich, continuing to the liver and sending branches to each of the lobes.

(b) A. cystica, to the cholecyst (Fig. 79).

(c). A. gastro-duodenalis.-This is on the dorsal side of the pylorus. It furnishes three named branches :-

(1) A. pylorica, extending from about opposite the pylorus aloug the lesser curvature of the stomach, and finally anastomosing with the A. coronaria ventriculi. The A. pylor$i c \alpha$ sometimes arises directly from the $A$. hepatica, as in man.

(2) A. gastro-epiploica dextra.-This, like the preceding and following, passes dorsad 
of the pylorus; then it extends along the greater curvature of the stomach in the ventral fuld of the great omentum near its attachment to the stomach. It anastomoses with a branch from the spleen (gastro-epiploica sinistra?)

(3) A, pancreatico-duodenalis superior.-This passes along the dorsal side of the pylorus, then to the left of the duodenum. It finally anastomoses with the pancreatico-duodenalis inferior (Fig. 81).

(D) A. coronaria ventriculi, az.-This branch arises from the coliac axis only slightly peripherad of the A. hepatica, and is distributed to the lesser curvature of the stomach. One of its larger branches anastomoses with the A. pylorica $(\$ 966,[1])$.

(E) AA. ventriculi dorsales (Chauveau, A, 559).-There are usually two of these. They pass directly to the dorso-sinistral part of the stomach. Their homology is doubtful.

(F) A. splenica, az.-This is the largest branch of the celiac axis, and seems to be a continuation of it. It passes to the spleen, but before reaching that organ divides into two nearly equal branches, one going to each extremity.

The spleen is sometimes double, and in such a case each part is supplied by one of the branches just mentioned.

Numerous small branches pass from the A. splenica to the pancreas and to the stomach. One branch passes dextrad in the ventral fold of the great omentum to anastomose with the A. gastro-epiploica dextra $(\S 966$, [?]). This may be the homologue of the A.gastroepiploica sinistra of man. It is small, and only rarely is the anastomosis shown with the ordinary plaster injection. In fresh specimens the artery will contain sufficient blood to enable one to trace it. It is of course easily filled with a fine injecting mass ( $\$ 1450)$

§ 967. A. mesenterica superior--Superior mesenteric artery, $a z$. (Fig. 101, 103, 107).-Turn the stomach and intestines to the right. The artery arises about $2 \mathrm{~cm}$. caudad of the A. cœliaca. Expose it fully by tearing away the peritoneum and nerves covering it. Use the tracer, and the scissors occasionally if necessary. Observe the following branches and their connections and distribution:-

(A) A. pancreatico-duodenalis inferior (Fig 81 [10]).-This passes obliquely cephalad to the duodenal pancreas and anastomoses with the $A$. pancreatico-duodenalis superior.

(B) A. colica media. - This branch is of considerable size. It passes to the large intestine and sends branches cephalad and caudad. The cephalic branch anastomoses with the $A$. colica dextra (C) or, if that is absent, with the A. ileo-colica. The caudal branch anastomoses with the A. colica sinistra ( $\$ 971, A)$.

(C) A. colica dextra.-This is often absent in the cat. When present it passes to the large intestine, and anastomoses with both (B) and (D).

(D) A. ileo-colica.-This is a large branch, extending directly to the cecum. It sends branches caudad to the large intestine, where they anastomose with (B) or (C), and others cephalad to the ileum, where they anastomose with the Rami intestini tenuis.

(E) Rami intestini tenuis.-These are the branches into which the $A$. mesentericu superior finally breaks up, and, as the name indicates, they are distributed to the small intestine.

§ 968. A. adreno-lumbalis (Fig. 101, 103, 107).-This artery arises from the side of the aorta, just caudad of the origin of the A. mesenterica superior ( $\$ 967$ ). It passes directly laterad, giving off the following branches: (A) the A. adrenalis to the adrenal, and $(B)$ the $A$. phrenica to the diaphragm. The artery rests on the free or ventral surface 
of the M. psoas ; it is, however, partly covered by the adrenal and the ceptralic end of the kidney. It will be necessary to remove the fat and connective tissue with great care from the adrenal and phrenic arteries, and it may be desirable to use the forceps and scissors to remove tough connective tissue and nerves. As was stated above, the A. phrenica may be supplied by the coliac axis $(\$ 966)$. The left A. adreno-lumbalis much more often supplies an A. phrenica than the right. The lelt and its branches are more easily demonstrated than the right.

$\S 969$. A. renalis (Fig. 101, 103, 107).--The renal artery arises from the side of the aorta like the preceding, and passes nearly laterad to enter the hilum of the kidney. It usually gives from its ventral suriace a small branch to the adrenal, and often one to the ureter. The renal artery is covered on its ventral surface by the $V$. renalis; hence, to obtain a good view of the artery, the vein must be removed or drawn aside. Employ the tracer, working carefully in order that the branches named above may not be broken.

The $A$. renalis usually divides into two nearly equal branches about $1 \mathrm{~cm}$. from the kidney, and one branch goes to each side of the renal pelvis ; it is sometimes double (Fig. 103).

$\S 9 \%$. A. spermatica (male), ovarii (female), (Fig. 101).-This arises from the ventral surface of the aorta $1-2 \mathrm{~cm}$. caudad of the $A$. renalis. It passes somewhat obliquely caudad, supplying the ovary in the female and forming part of the spermatic cord in the male.

Expose it by turning the intestines to the right; then very carefully tear away the peritoneum covering the aorta. As the artery is only about $1 \mathrm{~mm}$. in diameter, great care is necessary in the dissection ; probably it will be necessary to employ the forceps and scissors as well as the tracer. In the female the artery is much convoluted; see $\$ 960$.

$\S 971$. A. mesenterica inferior, $a z$. (Fig. 101, 103, 107).-The artery arises from the ventral surface of the aorta about opposite the iliac crest (Fig. 51). It passes nearly ventrad.

Turn the large intestine to the right, and with the tracer follow it. Near the large intestine it divides into two branches:-

(A) A. colica sinistra, which extends cephalad along the large intestine and anastomoses with the $A$. colica media $(\$ 967, \mathbf{B})$.

(B) A. hæmorrhoidalis superior. This passes caudad along the large intestine and anastomoses with the hæmorrhoidalis media from the $A$. iliaca interna.

\$ 972. A. ilio-lumbalis (Fig. 101).-This artery arises from the side of the aorta slightly caudad of the origin of the preceding $(\$ 971$ ), and passes laterad over the ventral wall of the $M$. psoas, and to other muscles over and near the Ilium. It is comparable with the A. adreno-lumbalis ( $\$ 968$ ). To demonstrate it, turn the large intestine to the right, and tear away the peritoneum with a tracer. As it is the only artery passing laterad in this region, it cannot be mistaken. 
\$ 973. Divisions of the Aorta abdominalis (Fig. 101, B).-Exposure and Dissection.-Draw the urocystis (Fig. 101) caudo-ventrad; press the contents of the rectum cephalad; doubly ligate and cut it opposite the neck of the urocystis. Cut the mesocolon $(\S 726)$ and mesentery near their attachment to the intestine as far cephalad as the duodenum; then cut the small intestine at the caudal end of the duodenum, and throw the intestines away. If the remaining part of the rectum is washed out with a solution of ferric sulphate (copperas) by introducing the canula of the syringe into the anus, the unpleasant odor will be avoided. If the urocystis contains urine, it should be pressed out through the urethra, or a slit may be cut in it.

In demonstrating the ressels, employ the forceps and tracer mostly; but use the scissors when it is necessary to remove any tough masses.

(A) A. iliaca externa (Fig. 101, B).-This passes obliquely caudad, penetrates the abdominal wall dorsad of Poupart's ligament, and reaches the cephalic side of the meros where it is called the femoral artery. Just before the A. iliaca externa leaves the abdomen, it gives off a large branch from its mesal aspect. This branch supplies the A. epigastrica (Fig. 101), then penetrates the abdomen near the symphysis pubis, and sends branches to the skin in the pubic region, but is mainly distributed to the muscles on the cephalic and ventral aspects of the proximal end of the meros. The human homologue of this artery has not yet been satisfactorily determined.

(B) A. iliaca interna (Fig. 101, B).--This arises from the aorta about $1 \mathrm{~cm}$. caudad of the origin of the preceding. It passes obliquely caudad within the pelvis and dorsad of the pubis. A short distance from its origin the A.vesicalis superior (Fig. 101) is given off from its ventral surface. This small vessel is the remnant of the $A$. hypogastrica of the fotus. It occasionally arises from the aorta.

The A. iliaca interna supplies most of the pelvic viscera entirely and furnishes part of the blood supply to the innominate and meral regions.

(C) A. sacra media, $a$ a. (Fig. 101, B).-The aorta is continued to the tail by this small mesal artery, which passes through the arches formed by the chevron bones ( $\$ 465)$.

It will be seen by comparing the figure of the cat with that of man (Quain, A, I, 281, Gray, A, 324), that in the cat there is no common iliac as in man, but each iliac is a branch of the aorta. 
$\S$ 974. AA. lumbales (6 pairs).-The lumbar arteries arjse from the dorsal side of the anrta opposite the intervertebral fibro-cartilages. 'They extend directly dorsad for a short distance, and then turn laterad to be distributed to the ental or attached surface of the muscles on the ventral and lateral aspects of the spinal column. A small branch usually enters the intervertebral foramen to supply the myelon.

$\S 975$. Demonstration of the AA. lumbales.-Turn the stomach to the right, cut the left renal vessels and remove the left kidney and the left half of the diaphragm. Draw the aorta dextrad. Commence opposite the last rib, and with the forceps and tracer clear away the fat and connective tissue on the dorsal and sinistral aspects of the aorta. From the great caudal projection of the dorsal part of the diaphragm, the 1st, $2 \mathrm{~d}$, and sometimes $3 \mathrm{~d}$, pairs of AA. lumbales are given off within the thorax; hence the necessity of removing the diaphragm. The last pair is given off by the aorta just caudad of the origin of the A. iliaca externa. Tear and cut away carefully the muscles and connective tissue, and trace the $2 \mathrm{~d}$ or $3 \mathrm{~d} A$. lumbalis and its distribution as described above (\$ 974).

\section{STRUCTURE OF THE HEART AND BLOOD VESSELS.}

$\S 976$. Obvious Structure of the Heart.-(A) The heart is covered by a serous membrane-the ental (inner) layer of the pericardium ( $\$ 910$ ).

(B) Its main substance is composed of muscle, apparently but not really like the ordinary voluntary muscles. Compare $\$ \S 704$ and 705 .

The arrangement of muscular fasciculi in the auricles seems to be comparatively simple, namely, in two layers, of which the ectal bave a circular and the ental a longitudinal direction (Stricker, A, 183). But in the ventricular roalls the arrangement is very complex (Quain, A, I, Fig. 179). According to Pettigrew (A, 194), there are no less than seven more or less distinct layers, the fibers of which vary in direction from nearly circular to nearly longitudina], with several degrees of obliquity. The author just named recommends (p. 193, note 2) that for the study of the layers, the cavities should be filled witl bran and the organ then boiled for from 4-10 hours, according to size, and kept in alcohol for a fortnight before dissecting.

(C) The cavities (Fig. 92) are lined by a firm serous membrane-the endocardium.

(D) Valves and chorda tendinece (\$\$ 864, 881-884).

$\$ 97 \%$. Microscopic Structure.-(A) The serous covering and lining of the heart has the structure belonging to serous membranes generally ( $\$ 730$ ).

(B) "The muscular fibers of the heart differ remarkably from those of involuntary muscular organs in general, inasmuch as they present transverse stria. The striæ, however, are less strongly marked and less regular, and the fibers are smaller in diameter than in the voluntary muscles (\$ 705). They differ also from these in being made up of quadrangular cells joined end to end, and often presenting a branched or forked appearance near one extremity. Each cell has commonly a single clear oval nucleus situated near the center ; occasionally two nuclei are seen. The cell substance is faintly striated longitudinally as well as transversely; it presents no indication of an investing membrane or sarcolemma. As stated by Stricker, however (A, 182) the 'cells of muscles, like all other 
naked cells, must present a peripheral investment;' that is, like all cells which form part of a complex, compact structure, and do not simply float free in a liquid as do the leucocytes in the blood. The muscular fibers of the heart freely divide and anastomose, the junction with neighboring fibers being effected by the medium of the cell-offsets above noticed." Stricker, A, 179 ; Quain, A, II, 119, 240, 261.

\$ 978. Arteriz.-Their obvious structure is that of smooth-bored, thick-walled tubes which retajn their form when cut. They are elastic and flexible.

$\$$ 979. Microscopic Structure.-They are composed of three well-defined coats:-(A) Ectal or outside coat, tunica adventitia, of rather loose elastic and white connective tissue, with a general longitudinal direction. (B) Intermediate or middle coat, composed of elastic and white connective tissue and circularly arranged muscular fibers. (C) Ental or inner coat, composed of elastic tissue covered by endothelium on its free surface.

$\$$ 980. Muscular and Elastic Types of Avteries.-Ranvier and others have divided the arteries into the two classes just named, from the preponderance of elastic tissue or of muscular fibers in the middle cont. The larger arteries, like the carotids, the axillary and the aorta, contain very little muscular tissue, and hence they belong to the elastic type. The arteries of the limbs and the smaller arteries generally contain a large amount of muscular tissue in their middle coat, and hence represent the muscular type.

\$ 981. Venz.-The obvious structure of these is the same as that of the arteries, but the walls are thin, so that they collapse when cut.

\$ 982. Microscopic Structure.-There are three coats as with the arteries. The white connective tissue is more abundant in the middle coat. Smooth muscle is often present in the ectal coat of some large veins, like the Vena cava, while it is entirely wanting in others, as in most of those of the brain and pia mater; Quain, A, II, 173.

$\$ 983$. Capillaries.- “ The wall of the capillaries proper is formed entirely of a simple epithelioid layer, composed of flattened lanceolate cells joined edge to edge, and continuous with the corresponding layer which lines the arteries and veins." Quain, A, II, 177.

\$ 984. The structure of the lymphatic vessels very closely resembles that of the blood vessels. Quain, A, II, 186.

\section{THORACIC DUCTS AND LYMPHATIC VESSELS.}

References.-Quain, A, I, 504; Gray, A, 589 ; Cuvier, A, VI, 60 ; Gegenbaur (Lankester), A, 599 ; Hyrtl, A, 750 ; Bernard, A, 253; Leyh, A, 656 ; Owen, A, III, 511; Milne-Edwards, A, IV, 503 ; Chauveau, A, 675; Chanveau (Fleming), A, 634; Gurlt, A, 681 .

\section{Instruments and materials the same as for the blood vessels} (\$912).

§ 985. Specimen, Preparation, Posture and Dissection.-Employ a young, but especially a lean cat. Two hours before death feed it 200 cc. of milk mixed with a beaten egg. Kill with chloroform (\$189). Inject the right $V$. jugularis externa with fine mass as for the blood vessels (\$\& 366 and 1450), and either the femoral artery or the aorta just caudad of the origin of the A. mesenterica inferior (Fig. 39, 101, §352). In case the venous system is not filled with fine mass, the cephalic part of the præcava should be closed 
by a ligature or compressor (Fig. 13), and the dissection may commence fifteen minutes after the injection with plaster ; or if injected with fine mass, after an hour open the abdomen as for the abdominal viscera $(\$ 710)$, and the thorax as for the blood vessels ( $\$ 918$ ), except that the left side should be cut as well as the right, and the longitudinal incisions should be carried caudad till they reach the free edge of the abdominal flaps. The diaphragm should be cut next the ventral wall, and the ventral wall removed with great care so as to avoid wounding the veins or arteries. With nippers (Fig. 11), carefully cut the left ribs, except the first, about $2 \mathrm{~cm}$. from their heads. Turn the free edge of the thoracic wall to the left.

\$ 986. Vasa chylifera, Lacteals.-Turn the omentum majus cephalad and lift the intestines. Look at the mesentery, and the lacteal vessels will be seen as whitish or yellowish lines extending dorsad from the intestine and nearly parallel with the blood vessels. Draw the large intestine caudad, and there will be seen a very large lacteal trunk extending dorso-cephalad from the large mesenteric gland ( $\$ 831,992, \mathbf{B})$.

Dorsad of the crecum this vessel crosses obliquely the superior mesenteric vein (Fig. 103, V. m. s.), and extends dorsad nearly parallel with the superior mesenteric artery (Fig. 103, A. m. s.). Attempt to force the contained chyle toward the periphery, and the beaded appearance shown in Fig. 103 will result. This is due to the valves, which are similar in form and function to those in the veins (Fig. 102, B. c., § 963).

$\S$ 987. Receptaculum chyli, $a z$. (Fig. 103).-This is a fusiform enlargement at the caudal end of the left thoracic duct, into which empty the lacteals or vasa chylifera from the alimentary canal, and the lymphatics from the caudal half of the body (\$992).

Exposure.-Cut the peritoneum along the abdominal wall from the caudal end of the kidney to the diaphragm. Reflect the peritoneum and kidney mesad, and the receptaculum will appear as a fusiform yellowish sac on the dorso-sinistral side of the aorta, extending from a point about opposite the hilum of the kidney to the hiatus aorticus in the diaphragm (Fig. 90).

$\S 988$. Ductus thoracicus sinister (Fig. 103).-The left thoracic duct is the common trunk which receives the lymphatics of the entire caudal half of the body, including those of the alimentary canal-the lacteals or vasa chylifera-and those of the left side of the head, face, neck and thorax. It pours its contents-lymph or, 
during digestion, mixed lymph and chyle-into the V. jugularis externa.

The duct almost invariably divides into two or more trunks near the middle of its course. After extending for a few centimeters as a double or triple duct, it may unite and then divide again before emptying into the vein (Fig. 103). See Colin, A, article Thoracic Duct, for variations in the domestic animals.

\$ 989. Dissection.-Slit the diaphragm ventrad from the hiatus aorticus (Fig. 90), and turn the two crura or pillars of the diaphragm aside as shown in Fig. 103, Crus dphrg. Turn the heart and lungs to the right, and, if the cat is lean, the thoracic duct will be seen on the dorso-sinistral side of the aorta, as a continuation cephalad of the receptaculum (Fig. 103). Cephalad of the heart it rests on the ventral aspect of the $A$. subclavia sinistra. It finally crosses the $V$. brachio-cephalica sinistra and, receiving the vasa lymphatica from the head and neck, empties into the $V$. jugubaris externa near the angle of union of that vessel with the $V$. subclavia. As a rule, however, the thoracic duct opens into the vein in two places, as shown in Fig. 103. Both branches of the divided thoracic duct may receive a lymphatic vessel from the head and neck. In isolating the duct, it is necessary to proceed with great care. The tracer must be employed sparingly. The pleura and connective tissue are most safely removed piecemeal with scissors and fine forceps, as directed for exposing the nerves and salivary glands of the face $(\S 777)$. It is especially difficult to isolate the duct in its cephalic third, as it is usually double or triple, and each part therefore correspondingly small (Fig. 103, A).

The first rib must now be carefully cut and removed. The largest of the vasa lymphatica in the neck rests on the longus colli muscle entad of and parallel with the carotid artery.

\$ 990. Ductus thoracicus dexter.-The right thoracic duct or great lymphatic vein is the large lymphatic trunk into which empty the vasa lymphatica of the right side, of the thorax, of the head and of the neck. It opens into the right $V$. jugularis externa in the same way as the left empties into the left $V$.jugularis externa.

\$ 991. Exposure and Dissection.-Employ the same specimen that was used for the left duct, make an incision on the right along the neck, and expose the $V$. jugularis externa to its junction with the V. subclavia. Do this very carefully. 'The vasa chylifera will be seen dorsad of the aorta, and entering the veins as described after 


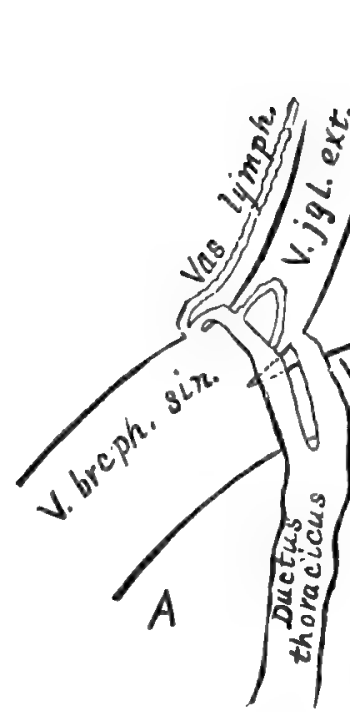

sinistra

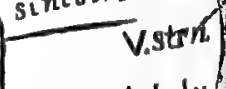

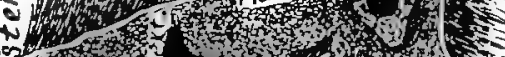

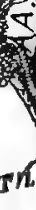

A.suclvi.

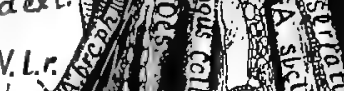

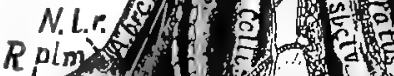
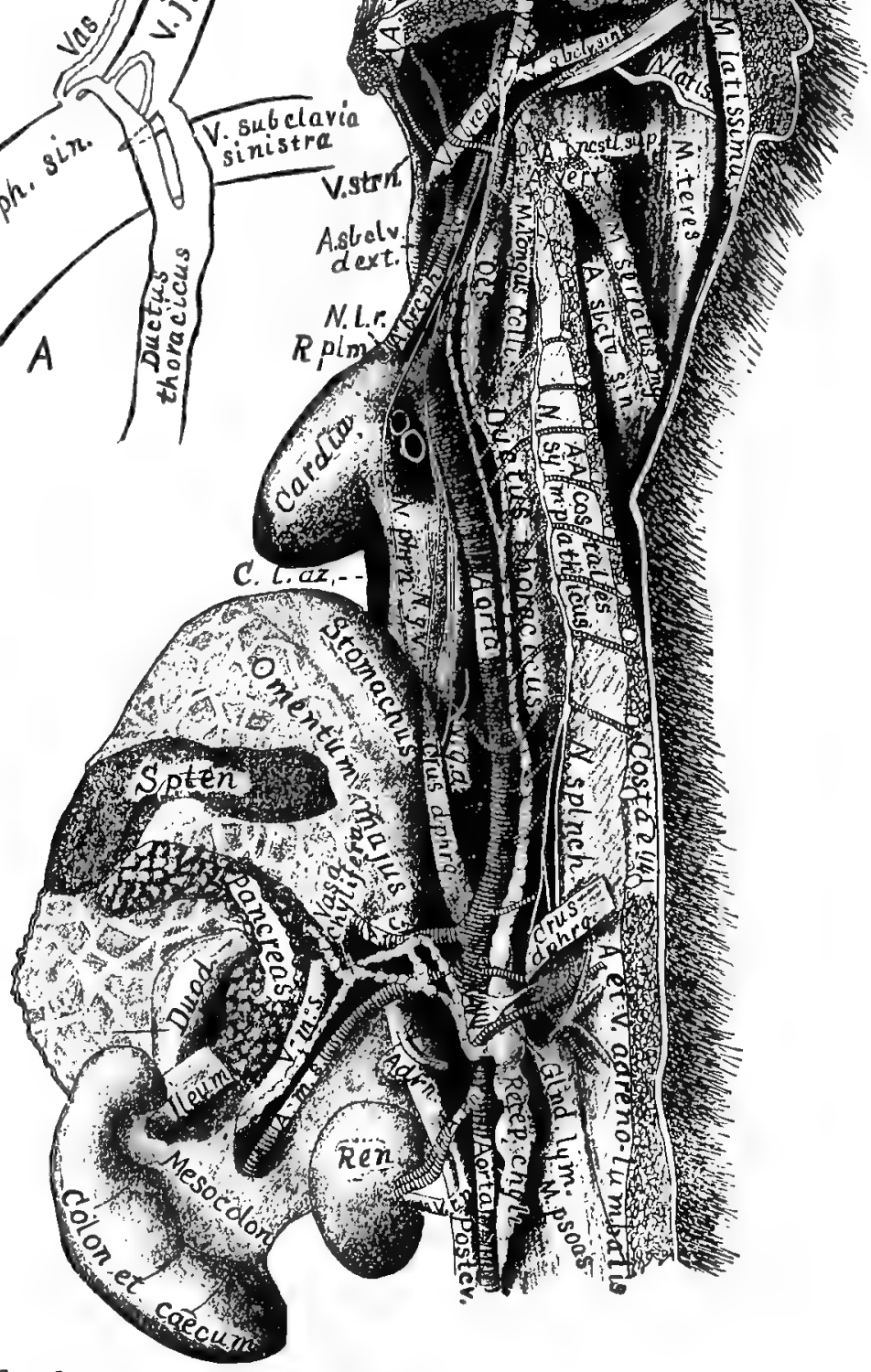

Fig. 103.-Tae Left Thomacic Duct, Sintstral View; $x .8$. A. Termination of The Thoracjc Dect; $\times 3$. 
uniting with the duct from the thorax. The lymph in these vessels will be pale, and hence they must be looked for with care. They may usually be recognized from their characteristic moniliform appearance (Fig. 103); they may be injected $(\S 992, \mathbf{A})$.

\section{LYMPHATIC VESSELS.}

The lymphatics, like the veins, contain many valves, making it impossible to inject from a large trunk peripherad, as with the arteries. The vessels may be filled, however, by the puncture method of Ludwig, which consists simply in thrusting a sharp pointed canula iuto the tissues and forcing Berlin blue through the canula with a syringe.

\$ 992. Lymphatics of the Arms and Legs.-To inject these prepare a glass canula (Fig. 32), leaving the point sharp. Fill the syringe with Berlin blue and connect it by means of a rubber tube to the canula. Then with the tracer perforate the skin covering the pad in the middle of the hand or foot of a cat just killed (see \$ 189194), and insert the canula. Push it into the tissues slightly and force the piston slowly down; at the same time compress the foot and press upon the limb in such a way as to facilitate the flow of the mass centrad. It is well to insert the canula into all the pads on the ventral surface of the manus or pes. It requires some time to fill the vessels well. In the cat the larger trunks follow the veins. If the leg is pressed as directed and the injection long enough continued (15-30 minutes), the thoracic duct may be filled.

\$ 992, A. Lymphatics of the Neck and Face.-To inject these employ a similar or the same specimen, and insert the canula in the naked place at the end of the snout, as directed for the par of the foot. Press on the nose and face. In this way the lymphatic vessels on the face and in the neck and the lymphatic glands in Fig. 87 (Gl. lym.) are very éasily injected.

$\S 992$, B. Injection of Lymphatic Glands.--The lymphatic glands upon an injected vessel are injected, since the vessels enter them. One may, however, inject the glands directly and so fill the efferent vessels. This is very easily done by inserting the canula at the peripheral edge of the gland and injecting centrad. The gland will first become very blue and then the mass will appear in the efferent vessels. Those at the side of the face (Fig. 87) and the mesenteric glands (\$ 731) are large and favorable for this operation.

If it is desired to make permanent preparations of the injected lymphaties, the blue should be mixed with half its volume of the blue gelatin mass ( $\$ 1450$, Frey, A).

For the structure of the lymphatic vessels, see $\S 984$

Preparation-Fig. 103. - A cat was fed as described above (\$985). Then the arteries, but not the veins, were injected, and the abdomen and thorax were opened as there described. The position of the large lacteal trunk crossing the $V$. mesenterica superior was then found as described, and a $\mathrm{V}$-shaped incision made in it with 
scissors (Fig. 40) at the point where it crosses the vein. A canula was inserted, but not tied. The injecting mass was thin and colored with chrome yellow $(\$ 344, \mathbf{E})$. A very gentle pressure was exerted in forcing down the piston of the syringe. The canula was lightly compressed with the fingers where it was inserted in the vessel. The receptaculum and duct were slightly compressed, the fingers being moved at the same time cephalad, to facilitate the movement of the injecting mass. When the duct seemed well filled, the canula was removed. After the injection of the duct, the most caudal part of the postcava was opened to allow the blood to escape, and then loosely ligatured centrad of the incision. Finally, the veins were injected with blue plaster $(\$ 342)$ from the axillary vein. After an hour the vasa chylifer , receptaculum and duct were carefully isolated as described above $(\$ 989)$. The specimen was preserved as directed (\$286).

Explanation of Fig. I03.-Adrn., Corpus adrenale-The adrenal or suprarena] body. Aorta. A. rn., A. renalis-The renal artery. A. m. s., A. mesenterica superior-The superior mesenteric artery. The lacteal vessel which was injected extends nearly parallel with this. A. c., A. cœliaca-The coliac artery or axis. A. adreno-lumbalisThe adreno-lumbar artery. AA. costales-The costal or intercostal arteries. A. brcph., A. brachio-cephalica-The brachio-cephalic or innominate artery. A. sbcl., sin., A. subclavia sinistra-The left subclavian artery. A. sbclv. dext., A. subclavia dextraThe right subelavian artery. A. vert., A. vertebralis-The vertebral artery. A. incstl. sup., A. intercostalis superior-The superior intercostal artery. AA. sternales-The sternal or internal mammary arteries. Cardia-The heart. C. 1. az., Cavum lobi azygi ( 8 810, Fig. 89). Colon et cæcum. Costæ (14)-Ribs. In this cat there were fourteen ribs. Crus dphrg. (diaphragmaticum)-One of the pillars of the diaphragm. Ductus thoracicus sinistra-The left thoracic duct. Duod., Duodenum. Glnd. lym., Glandula lymphatica-One of the lymphatic glands. Several small branches connect it with the injected lacteals. Humerus-See Fig. 46. Ileum-The part of the small intestine nest the cæcum. Mesocolon-The duplicature of peritoneum belonging to the colon. M. psoas. M. longus colli. M. serrat, M. serratus magnus $(\S 664)$. M. teres $(\S 680)$. M. latissimus, M. latissimus dorsi $(\S 635)$. N. splnch., N. splanchnicus-The trio splanchnic nerves are shown here (see Fig. 107). N. g. d., N. gastricus dorsalis-The dorsal gastric nerve. N. g. v., N. gastricus ventralis-The ventral gastric nerve. N. phrn., N. phrenicus-The left phrenic nerve. N. sympathicus-The left sympathic or sympathetic nerse. Omentum majus-Epiploon (\$ 727). Es., Esophagus. Postcv., Postcava.-The inferior or ascending vena cava. Pancreas. Recep. chyli, Receptaculum chyli. V.m. s., V. mesenterica superior-The superior mesenteric vein. V. sbclv. sin., V. subclavia sinistra-The left subclavian vein. V. jgl. ext., V. jugularis externa-The external jugular vein.

Fig. 103, A.-Termination of the left thoracic duct.

Vena subclavia sinistra-The left subclavian vein. V. brcph. sin., V. brachiocephalica sinistra. V. jgl. ext., V. jugularis externa-The external jugular vein. Vas lymph. (lymphaticum)-One of the lymphatic trunks from the head. 


\section{CHAPTER IX.}

\section{NEUROLOGY-THE STUDY OF THE NERVOUS SYSTEM.}

GENERAL CONSIDERATIONS-THE MYELON (BPINAL CORD) AND ITS NERVEB-THE BRACHIAL PLEXTS-THE VAGUS NERVE-THE BYMPATHIC SYSTEM-THE RELATIONS OF THE SYMPATHIC AND MYELENCEPHALIC (CEREBRO-SPINAL) STETEMS-STRUCTURE OF NERVOUS MATTER.

§ 993. General Considerations. - Nerves. - Throughout the body, distributed to all organs and membranes, there are white cords which are neither hollow like the vessels nor inextensible like the tendons, but composed of a greater or less number of fibers of a peculiar structure ( $\S 1048)$. These cords are called nerves; the larger ones are also distinguished as trunks, the smaller as branches, the yet smaller as twigs, and the final subdivisions as fibers or terminal filaments.

\$ 994: Ganglia. - The peripheral ends of the nerves are distributed to the various tissues constituting the muscies, bones, viscera, membranes, etc. Their central ends, however, are sooner or later traceable to collections of cells $(\$ 1048, \mathrm{~B})$, with which they are more or less closely and directly connected. Such collections of cells, whether or not intermingled with fibers, are called ganglia, or said to constitute ganglionic tissue.

\$ 995. Alba and Cinerea.-In the ganglia the gray protoplasm of the nerve cells imparts to the mass a more or less decided gray color. Hence the ganglionic tissue is commonly spoken of as the gray matter, or more technically the (substantia) cineren.

But although the central (ental) part of each nerve fiber is a band of gray protoplasm, it is in most cases so completely covered by a white substance (myeline, medullary sheath or voite substance of Schwann), that the prevailing color of the fibrous nervous tissue is white, and it is commonly known as the white substance, or more technically the (substantia) alba.

\$ 996. Primary Divisions.-The nerves and ganglia may be conveniently considered as forming two great divisions which are tolerably distinct in location and functions, but are nevertheless anatomically connected and physiologically associated : they are the sympathic (sympathetic) and the myelencephatic (cerebro-spinal) nervous systems. 
The latter is more directly connected with the skin and with the skeletal muscles which are commonly voluntary.

The former is chiefly distributed to the viscera and to the vascular system, and is regarded by some as merely a dependency of the other.

\section{997. Centrel and Peripheral Portions--Provisionally adopting the arrangement} above indicated as at least convenient, each of these two great divisions of the nervous system consists of a central and a peripheral portion. 'I he central portion of the sympathic is a double chuin of gang ia placed along the ventral aspect of the columna vertebralis, and thus within the ventral or visceral somatic cavity. Two pairs of these ganglia are shown in Fig. 109, that of the left side displaced dorsad. The peripheral part comprises the branches from these gauglia, with plexuses situated among or upon the viscera and vessels, and numerous small ganglia in the walls of the intestine and elsewhere. The details of the arrangement of this system will be given in connection with Fig. 107 and 109, where also will be pointed out the existence of rami communicantes between the sympathic and the cerebro-spinal systems.

The peripheral part of the cerebro-spinal system consists of nerves which occasionally form plexuses (Fig. 104, 105), and present ganglia at points to be presently indicated.

The central part of the cerebro-spinal system is lodged within the canalis neuralis 8 . cerebro-spinalis-the cavities of the cranium (\$ 491) and vertebral column (\$ $\$ 79$ ). It is a continuous mass of alba and cinerea. The cranial portion is the encephaton or brain, and the spinal portion is the myelon or spinal cord. The dorsal aspect of the brain and of part of the myelon is shown in Fig. 104.

\& 998. The Myelona' Alba and Cinerea.-The cinerea of the myelon presents the form of a column deeply fluted on four sides, dorsal, ventral and lateral. On a transection, the appearance is approximately that of a letter $H$, the ends of the two uprights being curved luterad Hence these areas are commonly known as the cornua or horns, two dorsal and two ventral.

The interspaces are occupied by the $a b b$. Since, however, the myelon is nearly divided into lateral halves by the fissure:3, dorsal and ventral, what would otherwise be single dorsal and ventral columns of alba are double; hence on each side there is a lateral column and a dorsal and ventral one. These features are indicated upon Fig. 109 and 112. The general arrangement of alba and cinerea in the brain will be described in connection with the structure of that organ in Chap. X.

$\S 999$. Motor and Sensory Nerves.-By means of experiments, it has been ascertained that certain nerves mainly or exclusively transmit sensory impressions, made upon the parts to which their peripheral ends are distributed, townrd the myelencephalon, that is, centrad or centripetally; while others convey motor impulses to muscles in the opposite direction, peripherad or centrifugally.

\$1000. Motor and Sensory Roots.-A nerve, especially a large trunk, usually contains come of both sets of fibers; but near the junction of the trunks with the myelon, each trunk divides into two roots, which are attached to the myelon upon its dorso-lateral and ventro-lateral aspects respectively. Anatomically, these roots are dorsal and ventral (an. terior and posterior), but physiologicall; , they differ as do certain of the cranial nerves: the dorsal ones transmit impressions centrad, and the ventral ones transmit motor impulses peripherad. Hence they are commonly designated as the motor and sensory roots of the spinal nerves. 
$\$$ 1001. The Cranial Nerves.-As will be shown in Chap. XI, there are reasons for regarding snme at least of the nerves which arise from the brain as representing the motor and sensory roots of ordinary myelonal nerves.

\& 1002. Functions of the Alba-So far as known, the nerves and other parts consisting wholly of the white or fibrous nervous tissue are simply capable of transmitting impressions which are made upon them ; their office is one of cunduction only.

$\S 1003$. Functions of the Cinerea.-Different portions of the gray or ganglionic nervous tissue have been found to act in one or more of the following ways :-.-

(A) As Trophic Centers.-The ganglia upon the dorsal roots of the myelonal nerve trunks (Fig. 109) seem to preside in some way over the uutrition of those roots, and are therefore said to have a trophic action. The same may be the case with the ganglia constituting the central portion of the sympathic system.

(B) As Centers of Automatic Action.-The ganglia in the substance of the heart, and perhaps in some otber localities, appear to possess the power of bringing about the action of the muscular fibers with which they are connected independently of other parts.

(C) As Centers of Inhibition - Certain portions of the cinerea seem to be able, either automatically or otherwise, to interfere with, check or inlibit the activity of other parts of the cinerea.

(D) As Agents of the Will.-Portions of the cortex cercbri appear to be immediately under the influence of volition, which is unable to directly actuate other parts of the brain or of the boty.

(E) As a Medium of Conduction. - Acting as the agent of the Will, the cortical cinerea must be capable of transmitting impressions and impulses to and from the rest of the body. Moreover, there are reasons for believing that the myelonal cinerea does, or at least may, take a share in the transmission of impressions and impulses between the brain and the body.

(F) As a Center of Reflex Action.-Many actions, especially those of the viscera and vesgels, are brought about iv an indirect way. An impression made at the peripheral end of a sensory nerve is transmitted througly the nerve and through the dorsal or sensory root to the cinerea of the myelon. Here it is apparently transformed into a motor impulse which leaves by one or more ventral roots and causes an action of the appropriate muscles or vessels or viscera. This kind of action, which may be very complex, is called reflex, and the cinerea is said to operate as a center for the reception and interpretation of the message and the determination of the response to be made.

$\$$ 1004. Analogies of the Nervous and Vuscular System:-(A) Somewhat remotely, the twofold division of the former into the sympathic and the cerebro-spinal may be.compared with the divieion of the latter into the lymph and the blood vascular systems.

(B) Confining the comparison to the better known and more extensive divisions, each presents a central and a peripheral portion. In each case the central organs are relatively astive and the peripheral passive.

(C) The vessels carry the blood, containing. the elements of nourishment and the products of waste, together with beat. The nerves transmit impressions and impulses, by means of which the different organs are connected and made capable of mutual and harmonious action. Both vessels and nerves are thus channels of communication; but they differ as do rivers, canals, roads and railways from the telegraph and the telephone. The relations established are of commerce on the one hand and intelligence on the other.

(D) Again, as the vessels are of two kinds, the arteries which carry blood from the heart and the veins which return it thereto, so there are two sets of nerves, motor and sensory, which transmit impulses in one direction and impressions in the other. The direc- 
tion in the arteries and the motor nerves is centrifuga ${ }^{7}$, while it is centripetal in the sensory nerves and the veins. [For this reason, upon colored diagrams, it is at least convenient to represent the motor nerves by red, the color of most arterial blood, and the sensory nerves by blue, the color of the blood in most of the veins].

(E) Like the two kinds of vessels, the motor nerves divide and subdivide so as to form smaller and smaller branches, while the sensory nerves unite and reunite to form larger and larger trunks.

§ 1005. Differences between the Nervous and Vascular Systems.-Here, however, the strict analogy ceases. The connection of the two sets of nerves at the myelon is not well understood, but it is certainly less simple than that of the great venous and arteria! trunks at the heart, separated as they are only by the lungs.

Again, while the capillaries establish a complete continuity of the peripheral ends of the arteries and veins, no such constant connection has been shown to exist between the motor and sensory nerves, which commonly terminate independently either in muscle or at sensitive surfaces; but see Beale, A, 240.

The subdivision of the vessels is like that of a large stream into several smaller, all being part of one. But as a rule the nerve fibers maintain their independence throughout their course from the myelon to the termination, and the larger nerves are made up by the association of many fibers, and not by their actual union into one large fiber.

Arteries and veins differ not only in that the former usually contain purer blood and the latter that which is less pure (the pulmonary vessels forming exceptions to the rule); nor even in that the current in the one is always toward the heart and in the other away from it. The real distinction is in their structure, the arteries containing more elastic and muscular tissue, by virtue of which they are more perfectly elastic and contractile; their walls also are relatively thicker, so that they remain open when empty instead of collapsing like the veins. But no such distinctions have been ascertained between the motor and sensory nerves, and the proof that a sensory nerve may transmit an impression in the direction opposite to the usual one goes far to indicate that their properties as well as their structure are identical, and that the äfference in their functions depends upon the connections of their central end with the dorsal and ventral regions of the myelon and of their peripheral ends with muscles or with sensitive parts.

\section{THE MYELENCEPHALIC OR CEREBRO-SPINAL NERVOUS SYSTEM.}

\& 1006: The IMyelon.-This, called also chorda spinalis or spinal cord, is the longer and more slender portion of the myelencephalon or central part of the cerebro-spinal axis, and is lodged within the canalis neuratis of the columna vertebralis (\$479).

References to the Myelon.-Quain, A, I, 568, and II, 489; Gray, A, 602; Hyrtl, A, 463; Gegenbaur (Lankester), A, 512 ; Chauveau, A. 709 ; Chauvean (Fleming), A, 666 and 747 ; Gurlt, A, 715 ; Owen, A, III, 73 ; Milne.Edwards, A, XI, 257 ; Leyl, A, 504.

As briefly stated in $\$ \S 997,998$, the myelon is a continuous mass of alba and cinerea, and is functionally a gigantic motor and sensory nerve, an elongated ganglion and a center of reflex actions. It may be conveniently divided into regions corresponding with those of the vertebral column, cervical, thoracic, lumbar, sacral and caudal; but the anatomical distinctions between them consist chiefly in the increase or decrease of the cinerea 
or of the alba. Corresponding with the origins of the large nerves fortning the brachial and sacral plexuses, and supplying respectively the arms and the legs, the myelon presents enlargements which, from their location, are known as the cervical and lumbar enlargements. The cephalic end differs from the rest in several respects, which will be indicated in Chap. X; it is commonly regarded as a division of the brain under the name of medulla or metencephalon.

The dorsal aspect of the cervical and of part of the thoracic myelon is represented in Fig. 104 ; transections of it are given in Fig. 99, 100, 109 and 112.

s 100\%. The Myelonal or spinal Nerves.-These arise from the myelon or spinal cord (Fig. 104) and, except in the lumbar region, pass almost directly laterad througlı the intervertebral foramina, and are distributed to the tissues. If a section of the body be made at the proper level, as in Fig. 109, it will be seen that the nerves are in pairs, and that each nerve trunk arises by two roots - the dorsal (posterior) root arising from the dorso-latera] aspect of the myelon, and the ventral (anterior) root from the ventro-lateral aspect. These extend toward the intervertebral foramen, piercing the dura on their way. In the foramen they unite to form the nerve trunk, but just as they unite there appears on the dorsal (posterior) root a swelling-the ganglion of the dorsal root ( $\$ 999)$.

'The nerve trunk soon divides into two primary divisions-the dorsal or posterior pri. mary and the ventral or anterior primary division (Fig. 109). The dorsal primary division supplies the parts dorsad of the spinal column, while the ventral supplies the parts ventrad of the spinal column, including the limbs. For the most part, the ventral are much larger than the corresponding dorsal divisions; the suboccipital and the great occipital nerves are, lowever, exceptions (see explanation of Fig. 104). The myelonal nerves are sometimes designated by the names of the groups of vertebre through whose intervertebral foramina they emerge-cervical, thoracic, lumbar, sacral and coccygeal or caudal (\$463).

Preparation-Fig. 104. - The muscles covering the spinal column were first carefully removed on the left to the level of the vertebral laminæ. The neural arch was removed with nippers, entirely on the leit, partly on the right. This exposed the myelon, the nerves, and-on the left-the vertebrarterial canal with its contained vertebral artery. The left nerves were then isolated by commencing at their origin and tracing the ventral primary division peripherad, removing the.muscles and connective tissue with the tracer, forceps and scissors. The MM. rhomboideus and serratus (magnus) were cut and the scapula strongly lateriducted, to expose the brachial plexus. The parts of this plexus were then carefully isolated; (compare with the ventral view, Fig. 106). On the right, the dorsal primary divisions of the nerve trunks were isolated as described for the left (see also $\S 1008$ ). They are as a rule smaller than the ventral, and hence require more care for their isolation. The myelonal dura was entirely removed from the left side with the fine forceps and scissors, but only to the level of emergence of the nerves on the right. Finally, the skull was nipped away from the dorsal and lateral aspects of the brain, comniencing at the foramen magnum (Fig. 55); see Chap. $\mathrm{X}$.

In preserving this specimen, the rectum was cleared and the abdomen and thorax filled with 95 per cent. alcohol ( $\$ 282)$; then it was suspended in a jar of alcohol (\$286) by a cord tied to the tail and legs. Cotton was placed in the metacolia or 4th ventricle, to raise the cerebellum, and between the hemispheres, to divaricate them sufficiently to show the callosum.

Explanation of Fig. I04.-Cerebellum, az.-Epencephalon; Chapter X. Costæ (7) -The first seven ribs. Dura-Dura mater of the myelon. Fm. alt., Foramen atlantale The atlantal foramen in the cephalo-dorsal margin of the atlas; through it pass the $A$. vertebralis and the $N$. suboccipitalis or 1st cervical nerve. Ganglion-Ganglion on the 


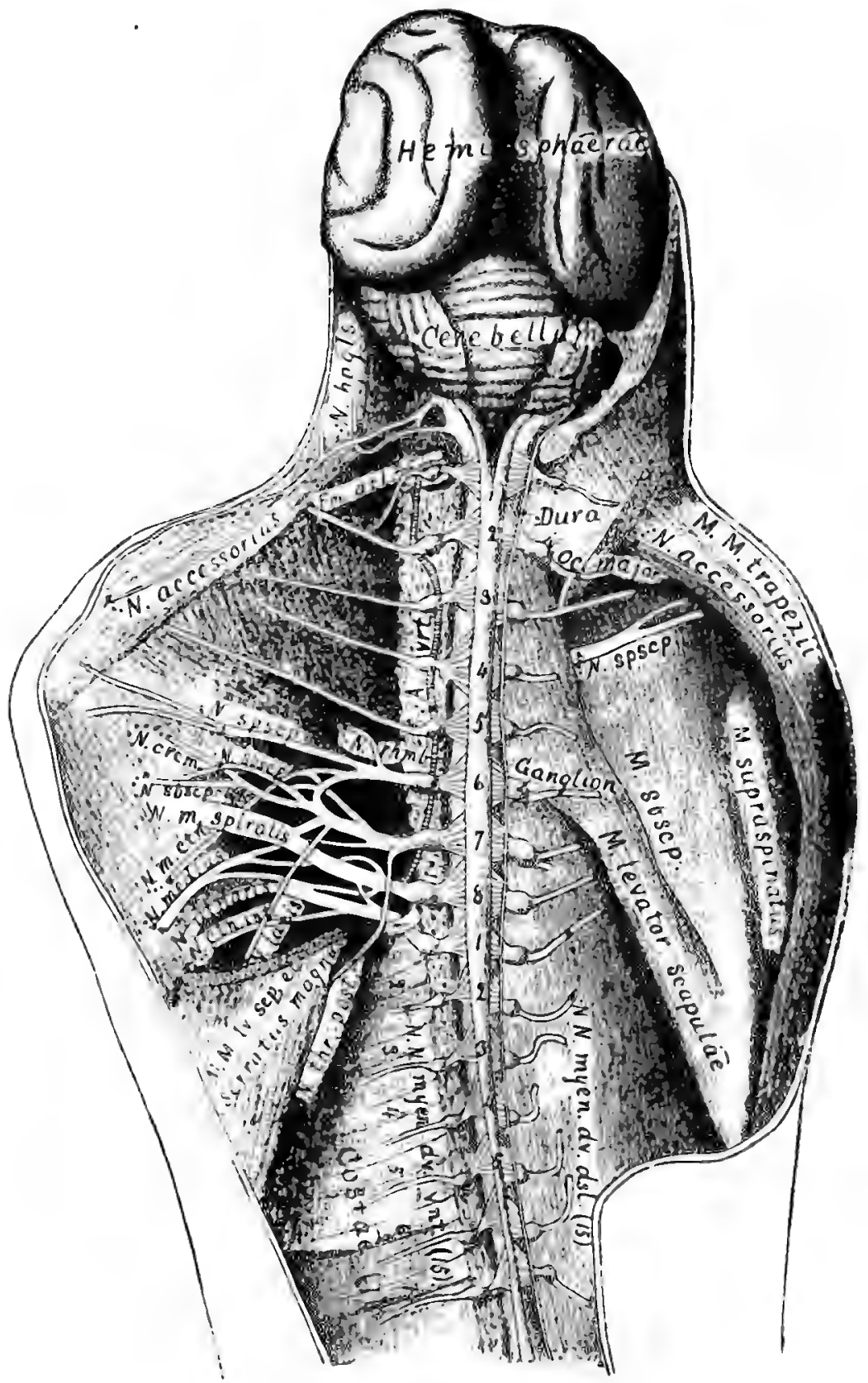

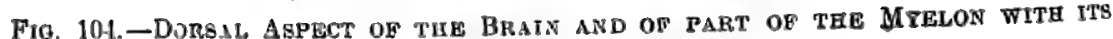
NenVES; $\times .75$. 
dorsal (posterior) root of the 6 th left cervical nerve; ganglia are present on all as shown in this figure; see also Fig. 109. Hemisphæræ-Cerebral hemispheres, prosencephalon ; see Chap. X. . MM. (lv. scp.) levator anguli scapulæ et serratus (magnus) (\$ 664). M. sbscp., M. subscapularis-The light streak between this and the preceding nuscle is the glenoid border of the scapula (Fig. 44). M. supraspinatus-See $\$ 673$. MM. trapezii-Trapezius muscles ; see Fig. 66. N. accessorius-The spinal accessory nerves (xi) ; this nerve passes along the ental surface of the trapezius muscle; see also Chap. $\mathbf{X}$. N. crcm., N. circumflexus (§ 1024). N. ctn. int., N. cutaneus internus $(\S 1021)$. N. hpgls., N. hypoglossus-The 12 th pair of cranial nervea ; Chap. X. N. latis., N. latissimus-Nerve of the $M$. Latissimus dorsi (\$1023). N. medius s. medianus $(\S 1025)$. N. m. spiralis, N. musculo-spiralis (\$1026). N. m. ctn., N. musculo-cutaneus (\$ 1022). NN. myen., dv. dsl., NN. myelencephalici, divisio dorsalis (15)-Dorsal (posterior) primary division of the first 15 myelencephalic or cerebro-spinal nerves. NN. myen., dv. vnt. (15), NN. myelencephalici, divisio ventralis-The ventral (anterior) primary division of the first 15 myelencephaiic or cerebro-spinal nerves. N. oc. (occipitalis) majorThis forms the dorsal part of the $2 d$ cervical nerve; it receives a large anastomotic branch from the $3 \mathrm{~d}$ cervical nerve; this nerve is distributed to the dorsal and caudal regions of the head; in the 1st and $2 \mathrm{~d}$ cervical nerves the dorsal primary divisions are as large or larger than the ventral (\$1007). N. rhmb., N. m. rhomboidei-Nerves of the romboideus muscle. N. sbscp., N. subscapularis ( $\$ 942$ and 1023). N. spscp., N. suprascapularis-A large nerve from the brachial plexus; it sends a branch to the clavieular end of the clavo-trapezius, but is mainly distributed to the scapular muscles; it is in company with the suprascapular artery; see $\S \S 937,1020$, and Fig. 105. N. suboccipitalis-The 1 st myelonal nerve ; its name is not written, but it traverses the $F m$. atl. with the $A$. vertebralis. N. thr. post., N. thoracicus posterior-The external respiratory of Bell (\$1029); see $\$ 1019$ for the internal respiratory or phrenicus. N. ulnaris (\$1028).

\section{$\$ 1008$. General Directions for Dissecting the Nervous Sys-} tem.-The precautions mentioned in $\$ 596$ (10) should be carefully observed, and especial care taken that the part under examination should be in a good light. Employ a tripod magnifier (Fig. 36) whenever necessary to determine the course or relations of small branches. Change the position of the cat as often as is necessary to get the given part in the best light or to make it more accessible.

Whenever a part is dissected, it should be kept moist by recovering it with skin or sheet rubber, or by placing upon it a tuft of cotton wet with 15 per cent. glycerin (\$170).

In fresh animals, nerves may be distinguished from connective tissue or empty vessels by their glistening and wavy or crimped appearance when looked at closely. An uninjected ressel may always be distinguished by cutting a slit in it and demonstrating its tubular character with the tracer.

$\S 1009$. Instruments and Material.-Fifteen per cent. glycerin; cotton; towels or cloths; pins; tracer ; fine, coarse and bone acissors: nippers; scalpels; arthrotome : injecting apparatus and material (Chapter IV); skeleton, and a natural skeleton of the $\operatorname{arm}(\S 252)$. 
Choice of Specimen.-A lean cat with milk teeth is preferable: young, because the bones are softer and more easily cut; lean, because the tracing of nerves is difficult even when unobscured by fat. The usual precautions for cleanliness should be observed ( $\$ 199)$, and if it be desired to demonstrate the vertebral artery as in Fig. 104, the arteries should be injected from the abdominal aorta or the femoral artery ( $\$ 8545,363$ ). The cat should be deprived of blood by opening the $V$. femoralis near Poupart's ligannent, centrad of the last pair of valves ( $\$ 62)$, or the postcava may be opened.

Posture.-Place the cat ventricumbent with a block between the arms and under the neck, so that the structures on the dorsal part of the body may be somewhat tense. The legs should be tied to the tray, so that the subject will not move too much.

$\S 1010$. Exposure and Dissection.-Part the hair (\$354) along a line $1 \mathrm{~cm}$. sinistrad of the dorsimeson, commencing at the base of the skull and ending opposite the gleno-vertebral angle of the scapula. Cut the skin along this line and reflect it for $2-3 \mathrm{~cm}$. on the left and just beyond the dorsimeson on the right. In reflecting the skin on the right, it should be torn from the body rather than cut, so that the nerves passing into the skin may be more easily detected. During the dissection the parts should be kept covered with skin as much as possible and moistened occasionally with 15 per cent. glycerin. In case it takes more than one day to complete the study, the preparation should be moistened with 15 per cent. glycerin, covered with skin and then with a damp towel, and kept in a cool place. As the dissection of a given part is completed, there should be laid upon it a tuft of cotton moistened with 15 per cent. glycerin.

\section{\& 1011. Demonstration of the Ventral Primary Division of} Nerves of the Left Side (Fig. 104).-After the skin is reflected on the left side, commence in the 7th intervertebral space and cut the muscles from the left half of the neural arch $(\$ 478)$, and with the tracer carefully isolate the nerve as it emerges through the intervertebral foramen ( $\$ 484)$. Then with the nippers remove the left half of the neural arch, and the origin of the nerve from the myelon will be seen. Continue to expose the nerves in this way until the $2 d$ cervical or great occipital nerve is reached. This does not emerge through a special intervertebral foramen, but between the arches of the atlas and axis ; hence the ganglion is imbedded in the muscles. It is most safely isolated by tracing it from the surface toward its origin. This nerve is easily seen on the caudal part of the head and through the clavo-trapezius muscle if the cat is lean. One might also begin with the $N$. auricularis magnus (Fig. 87) the ventral 
primary division of the $2 \mathrm{~d}$ cervical. Use the scalpel and tracer to remove the muscles. After the ganglion is exposed, the neural arch may be removed as usual with nippers.

The removal of the 1st or suboccipital also requires care, as it traverses the atlantal foramen. The muscles must be carefully removed from the dorsal side of the atlas, commencing caudad. The ganglion of this nerve is either in the muscles immediately covering the Fm. atlantale ( $\$ 474$ ) or just within the mouth of the foramen ; hence the muscles must be very carefully removed until the nerve is uncovered. It may then be traced peripherad as far as desired, and the dorsal part of the atlas removed with nippers.

If it be desired to follow the brachial plexus as shown in Fig. 104, the rhomboideus and trapezius muscles must be removed (Fig. 66,67 ), and also part of the serratus magnus (Fig. 73). Then the scapula should be strongly lateriducted. This will expose the brachial plexus and its anastomoses, and the final trunks may be made out by carefully dissecting with the tracer, fine fórceps and fine scissors. The 1st thoracic will be seen to pass entad of the 1st rib on its way to join the brachial plexus.

The distribution of the $\mathrm{N}$. accessorius (xi) may be easily found, as it extends along the ental surface of the $M$. trapezius, reaching this point soon after its emergence from the Fm. jugulare in company with the $N N$. vagus et glossopharyngeus; see $\$ 562$.

\$ 1012. Demorstration of the Dorsal (Posterior) Primary Division of the Nerves on the Right Side (Fig. 104). - In removing the skin from the right side, it should be torn rather than cut : then the branches of the dorsal primary division going to the skin will be seen as white cords which penetrate the muscles in the dorsal region and pass to the skin. These branches should be followed through the muscles, using tracer, scissors, scalpel and forceps as is necessary. Trace the dorsal division until it joins the ventral division (Fig. 109). After the nerves are isolated, the right side of the neural arch should be removed to the level of the intervertebral foramina. After the myelonal nerves are isolated and the myelon uncovered, some cotton wet with 15 per cent. glycerin should be placed upon the myelon and damp towels over the whole dorsal region, to protect the parts from injury or drying.

$\S 1013$. Exposure of the Brain.-The 12th, 11th, 10th, 9th and 7th cranial nerves (P1. I, II), on the left side should be isolated. In doing this, have at hand a skull and Fig. 56, 57, 59 and 107, also the Table in $\S 562$. If the structures are carefully removed opposite the points of exit of the various nerves, they may be easily found and traced as far peripherad as desired. After the nerves are isolated, the roof of the skull should be removed with nippers. In doing 


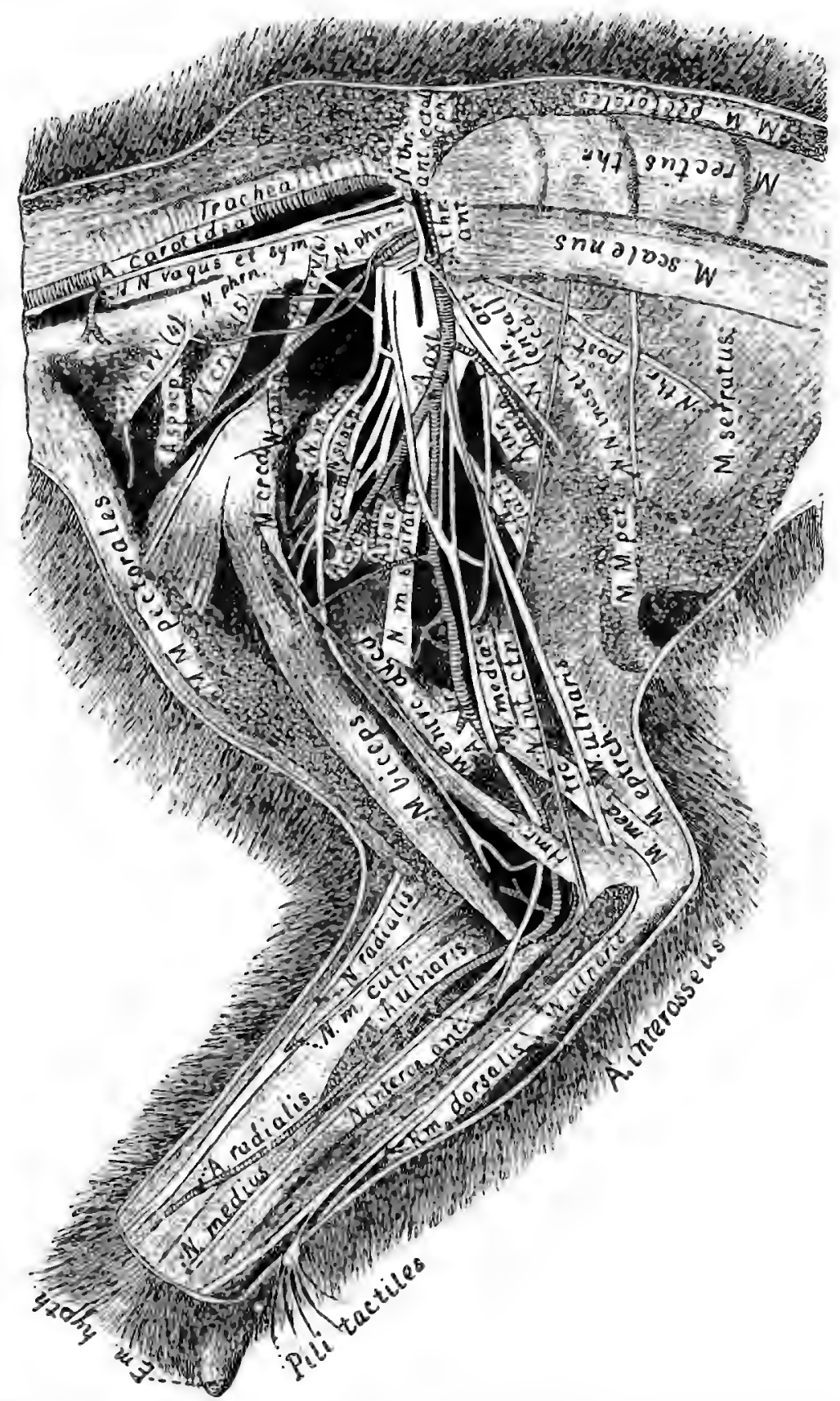

Fio 105.-Ventral Aspect of the Riget Brachial Plexts and ite Nerves: $x .9$ 
this, ventriduct the head and insert one blade of the nippers into the foramen magnum (Fig. 56, 57, 59), and remove the 0 . supraoccipitale piecemeal. Remove also the parietal and frontal bones in the same way until the dorsal aspect of the brain is entirely uncovered. The bony tentorium (Fig. 59, 88) will remain, but its dorsal border will be free. To remove it, separate slightly the hemispheres and cerebellum, insert the nippers and break the tentorium on both sides. It may then be removed with the coarse forceps.

$\$ 1014$. Removal of the Dura.-After the hard parts are removed from the central nervous system, the dura, a tough membranous sac enclosing it, should be partly removed by grasping the membrane at some point where it is relaxed and cutting away a piece at a time. Grasp the cut edge of the dura with the forceps and lift it away from the underlying nervous matter so that the scissors may be inserted. Remove the dura, proceeding with extreme caution, especially around the nerve roots. Sometimes it will be necessary to employ fine scissors and fine forceps and the tripod magnifier, especially in cutting the dura from the roots of the cranial nerves. The fan-like appearance of some of the nerve roots on their emergence may be well seen with the magnifier.

\section{THE BRACHIAL PLEXUS AND THE PRINCIPAL NERVES OF THE RIGHT} ARM AND SCAPULAR REGION.

References.-Quain, A, 1, 582; Gray, A, 671; Hyrt], A, 543; Gegenbaur (Lankester), A, 514; Chauveau, A, 800 ; Chauveau (Fleming), A, 754; Gurlt, A, 749 ; Owen, A, III, 170, 176 ; Milne-Edwards, A, XI, 239 ; Leyl, A, 537.

Instruments and material, see $\$ 1009$.

$\$ 1015$. The Brachial Plexus is the network of nerves from which the scapular region and the arm are supplied. It is formed by the intimate convection of the ventral (anterior) primary divisions ( $\S 1007$ ) of the 6 th, 7 th and 8 th cervical and the 1st thoracic nerves.

Fig. 105 and the description are given to illustrate the relations and distribution of spinal or myelonal werves in a well defined region.

For the study of this subject, the student should have before him a natural skeleton of the arm, inciuding the scapula (\$ 252).

§ 1016. Specimen, Posture and Preparation.-The same specimen may be employed as for Fig. 104, but it is better to use a different one. It should, however, be of the same character, viz., young and lean.

Preparation of Fig. r05.-The cat was injected from the abdominal aorta with thin plaster ( $\$ \S 345,363$ ). Then the skin was removed from the axillary and pectoral regions; and from the caudal aspect of the arm to the wrist. The pectoral, clavo-mastoid, clarotrapezius, epitrochloaris and pronator teres muscles (\$8 680,681) were then cut and turned 
aside. The veins of the arm were wholly removed, the arteries and nerves carefully traced with fine scissors, forceps and tracer. The animal was transected ( $\$ 234$ ) and the cephalic half preserved in alcohol ( 286$)$.

Explanation of Fig. I05.-A. carotidea-The right carotid artery (\$927). A. spscp., A. suprascapularis-The suprascapular artery ; the main branch of this artery accompanies the $N$. suprascapularis, but in this figure only the smaller branch of the artery to the trapezius muscle is shown; see $\$ 937$. A. crcm., A. circumflexa-The circumflex artery (\$941). A. sbsc., A. subscapularis-The subscapular artery (\$942). A. ax1., A. axillaris-The axillary artery (\$ 938). A. thor. (thoracica) longa-The long thoracic artery ( 940 ). A. thor. ant., A. thoracica anterior ( 9939 ). A. br., A. brachialis-The brachial artery ( $\$ 943)$. A. ulnaris-The ulnar artery ( $\$ 945)$. A. interosseus-The interosseous artery $(\S 944, D)$. A. radialis-The radial artery $(\S 946)$. Eminentia hypothenaris -The hypothenar eminence. Fm. eptrch., Foramen epitrochleare; see Fig. 46. MM. pectorales-The cut end of the pectoral muscles $(\$ 640)$. M. crcd, M. coracoideus ( $\$ 668)$. M. biceps $(\$ 691)$. M. entrc., dv. cd., M. entotriceps, divisio caudalis ( $\$ 68 \tilde{)}$ ). M. medtrc., M. meditriceps ( $\$ 683$ ). M. eptrch., M. epitrochlearis ( $\$ 681$ ). M. serratus (magnus), ( 864$)$. M. scalenus. M. rectus thor. (thoracicus). N. vagus et sym. (sympathicus), (Fig. 107). N. crv. (4), N. cervicalis (4). N. crv. (5), N. cervicalis (5). N. crv. (6), N. cervicalis (6). N. spscp., N. suprascapularis-The suprascapular' nerve $(1020)$. N. phrn., N. phrenicus-The phrenic, diaphragmatic or internal respiratory nerve ( $\$ 1019$ ); see $\S 1029$ for the external respiratory. N. thr. ant. (ectal \&. cph.), N. thoracicus anterior (ectalis \&. cephalicus)-The anterior (ectal or ceplajic) division of the thoracic nerve $(\$ 1018)$. N. m. ctn., N. musculo-cutaneus-The musculo-cutaneous or the external cutaneous nerve 1022$)$. N. sbscp., N. subscapularis-The subscapular nerve $(\$ 1023)$. N. crcm., N. circumflexus-The circumflex nerve $(\$ 1024) . N$, m. spiralis, N. musculo-spiralis-The musculo-spiral nerve $(\$ 1026)$. N. medius 8 . medianus-The median nerve ( $\$ 1025)$. N. int. ctn., N. cutaneus internus-The internal cutaneous nerve $(\$ 1021)$. N. latis., N. m. latissimi-Nerve of the latissimus or the long subscapular nerve $(\$ 1023)$. N. thr. ant. (ental. s. cd.), N. thoracicus anterior (entalis 8 . caudalis). - The anterior (ental or caudal) thoracic nerve $(\$ 1018)$. N. thr. post., N. thoracicus posterior-The postorior thoracic or the external respiratory of Bell ( $\$ 1018,1029$ ). N. radialis-The radiul nerve, a branch of the musculo-spiral (\$ 1026). N. interos. ant., N. interosseus anterior-The anterior interosseous nerve; the posterior interosseus is a branch of the musculo-spiral (\$s 1025, 1026). Pili tactiles-Tactile hairs ; to these extend a branch of the N. ulnaris ( $\$ 1028)$. Ramus dorsalis-The dorsal branch of the ulnar nerve (§ 1029). Trachea-The windpipe (§ 799).

Preparation of Fig. I06.-This is the same as for Fig. 105, excelt that all of the soft parts were removed so as to show the exit of the nerves from the intervertebral foramina.

Explanation of Fig. 106.-A. brachialis-The brachial artery. N. suprascapularis -The suprascapular nerve. N. crv. (6), N. cervicalis-The 6th cervical nerve. N. subscapularis-The subscapular nerve. N. crv. (7), N. cervicalis-The 7 th cervical nerve. N. musculo-cutaneus-The musculo cutaneous or exterual cutaneous nerve. $\mathrm{N}$. crcm., N. circumflexus-The circumflex nerve. N. thr. ant. (ectal. s. cph.), N. thoracicus anterior (ectalis 8 . cephalicus)-The anterior (ectal or cephalic) division of the thoracic nerve. N. medius 8. medianus-The median nerve. N. musculo-spiralis-The name is not written, but it is the large blank one arising from the 8th cervical ; see Fig. 104 and $105, \S 1026$. N. crv. (8), N. cervicalis-The Sth cervical nerve. N. ulnaris-The ulnar nerve. N. thr. (1), N. thoracicus 8 . dorsalis-The 1st thoracic or dorsal nerve. N. cutaneus internus-The internal cutaneous nerve. N. latis., N. m. latissimi-The 


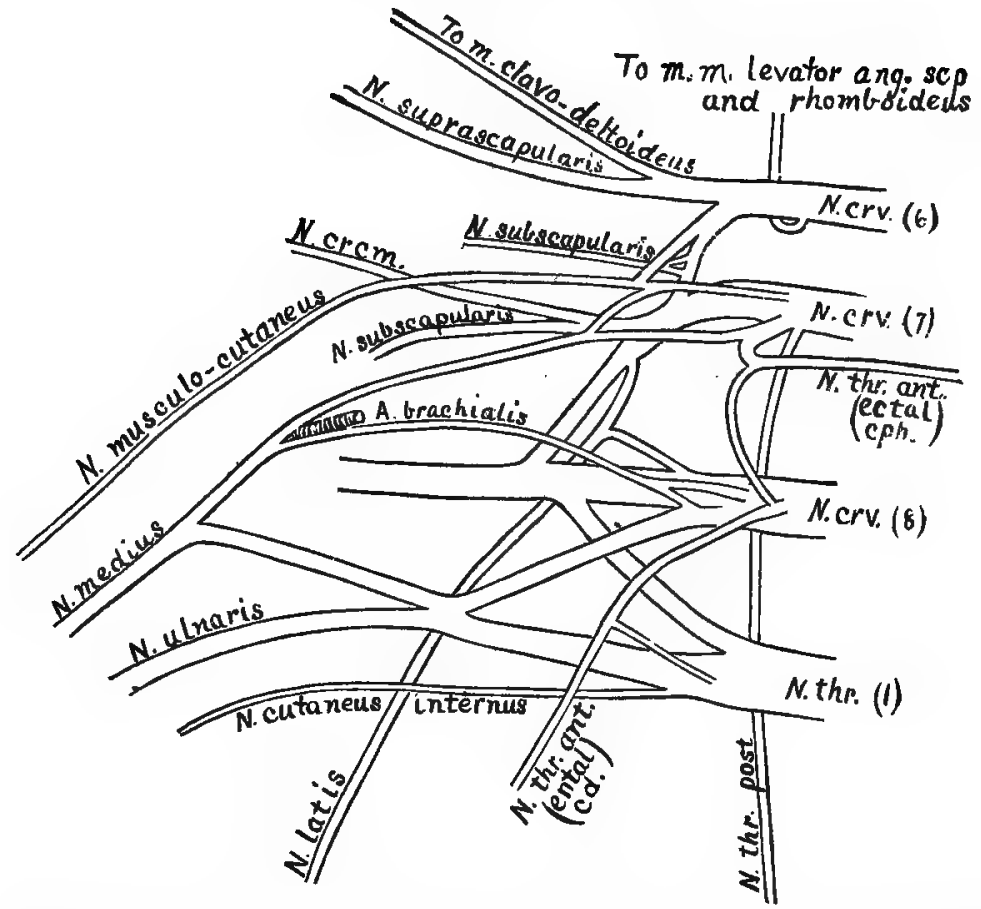

Fig. 106.-Diagram of the Right Brachial Plexds, Ventral View; $\times$ about 2.

nerve of the M. Zutissimus dorsi or the long subscapular nerve (\$1033). N. thr. ant. (ental. cd.), N. thoracicus anterior (entalis 8. caudalis)-The anterior thoracic nerve, ental or caudal division. N. thr. post., N. thoracicus posterior-The posterior thoracic nerve or the external respiratory of Bell. To M. levator ang. scp. and rhomboideusThe nerve to the levator anguli scapuloe and rhomboideus muscles. To M. clavo-deltoideus-The nerve to the clavo-deltoideus muscle.

Dissection of the Brachial Plexus.-The cat should be dorsicumbent and the arms secured laterad with cords as in Fig. 76. A block should be placed flatwise under the shoulders, so that the head may be slightly dorsiducted; later in the dissection, the arm may be supported by a block. The arteries should be injected with thin plaster $(\$ 345,352,363)$, from either the abdominal aorta or the femoral artery. If the cat is to be used only for this preparation, it should be transected $(\$ 234)$.

$\$ 1017$. Exposure.-Make a longitudinal incision through the skin $2 \mathrm{~cm}$. dextrad of the ventrimeson, commencing at about the middle of the neck and ending opposite the base of the xiphisternum. 
Then make a transverse incision $2-3 \mathrm{~cm}$. long, from the beginning of the longitudinal incision, dextrad. Then, commencing at a point opposite the 1st rib, cut through the skin along the caudal (upper in present posture) side of the arm to the elbow. After the nerves are studied in the brachium, the longitudinal incision on the caudal side of the arm will be continued to the pad in the palm of the hand.

Reflect the skin just across the ventrimeson by grasping the cut edge and tearing it from the structures which it covers. Grasp the corner of the cephalic flap and tear the skin from the arm and shoulder, exposing as much as is shown in Fig. 105. Reflect the skin from the dorsal side of the arm in the same way, because, if done in this manner, any nerves entering it can be readily seen, whereas if the scalpel is used freely, they will be cut with the connective tissue.

After the skin is reflected, make an incision through the muscles $2 \mathrm{~cm}$. from the ventrimeson, commencing and ending as for the skin. It is necessary to proceed with extreme caution, to avoid cutting the vessels and nerves entad of the muscles. In the neck the cut should divide only the muscles covering the carotid artery, the vagus and sympathic nerves (Fig. 101). It is best to uncover these structures at the beginning of the incision and keep them in view. In cutting the pectoral nuscles, one should proceed in the same manner. When the muscles are divided, the mesal edge should be grasped by the hand and turned mesad, taking care not to break any of the nerves entering them. The lateral ends of the muscles should likewise be turned laterad with the same caution. Finally, separate carefully the $M M$. epitrochlearis and biceps to the elbow, cutting, however, just deep enough to expose the internal cutaneous and median nerves (Fig. 105). The above incisions will expose the brachial plexus and the principal branches arising therefrom.

Commencing as near the meson as possible, with tracer, fine forceps and fine scissors, remove fat and connective tissue from the vessels and nerves. Fat is moderately tender, and both it and connective tissue are more easily torn than either blood vessels or nerves; the nerves toomay be recognized as directed above $(\S 1008)$; the arteries, being filled, will serve as landmarks. The axillary vein should be removed, great care being used so that no nerves are cut in the operation.

$\S 1018$. N. thoracicus anterior (Fig. 105, 106 ; Quain, A, I, 584; Gray, A, 672).-The anterior thoracic nerve passes ventrad from the 
brachial plexus to the pectoral region. It is divided into two parts, or rather there are two nerves - an ectal or cephalic division, accompanying the anterior thoracic artery between the muscles ( $\$ 939$ ), and an ental or caudal division, accompanying the long thoracic artery ( $\$ .940)$, and distributed to the pectoral and also somewhat to the latissimus muscle. The two are connected near their origin by an anastomosing branch, as shown in Fig. 105 and 106.

Dissection.-If the section was properly made through the pectoral muscles, the ectal or cephalic division will be turned mesad and the ental division laterad. Both are easily found, and their relations and distribution can be determined by using the tracer and fine forceps.

\$1019. N. phrenicus s. diaphragmaticus (Fig. 103, 105, 107; Quain, A, I, 578; Gray, A, 669).-This nerve is formed by two branches, one from the 5th and one from the 6th cervical nerve. It also occasionally receives a branch from the 4 th cervical, as in man. The latter condition is shown in Fig. 105, while the more frequent one is shown in Fig. 107. These branches unite near the 1st rib, and the phrenic then passes into the thorax on the mesal side of the $A$. sternalis (Fig. 107, 109). The right nerve rests on the lateral aspect of the præ- and postcavæ on its way to the diaphragm, to which it is distributed. The course of the left is well shown in Fig. 103 and 107, N.phrn.

Dissection-The branches forming the phrenic nerve are quite small, hence it is best to trace the nerve centrad from the point where it enters the thorax; the branches may then be isolated one by one. When this is done, the costicartilages and the thoracic walls may be cut and the two edges divaricated. The nerve will appear very clearly as a white cord passing along the lateral aspect of the præ- and postcavæ to the diaphragm.

$\$ 1020$. N. suprascapularis (Fig. 104, 105, 106 ; Quain, A, I, 583 ; Gray, A, 672).- The suprascapular nerve arises from the 6th cervical. It passes directly laterad for a short distance and then divides into two branches, one going to the ental surface of the $M$. clavodeltoideus, the other to the ectal surface of the scapula. These two branches accompany the branches of the suprascapular artery $(\S 937)$.

Dissection. - This nerve is easily traced. It follows closely the corresponding branches of the suprascapular artery, and may be traced as directed for that vessel ( $\$ 937$ ). 
$\S 1021$. N. cutaneus internus (Fig. 104, 105, 106 ; Quain, A, I, 585; Gray, A, 675). - The internal cutaneous nerve arises wholly from the 1st thoracic. It becomes subcutaneous at about the distal third of the brachium, emerging from between the epitrochlearis and biceps muscles about opposite the point where the $N$. radialis emerges from between the biceps, clavo-deltoideus and ectotriceps (Fig. 74) ; hence it is liable to be destroyed in removing the skin or in the exposure $(\$ 1017)$ unless care is taken. It gradually curves from the dorso-caudal to the ventral aspect of the arm, and is distributed mostly to the skin of the brachium and antebrachium on the caudal and ventral aspects.

Dissection.-It is best to isolate the 1st thoracic near its origin to find the origin of the internal cutaneous nerve; it may then be traced peripherad, or it may be found on the ventral side of the arm, where it becomes subcutaneous, and traced in both directions. The presence of the nerve of $W$ risberg has not been satisfactorily determined in the cat.

$\S$ 1022. N. musculo-cutaneus's. cutaneus externus (Fig. 104, 105, 106 ; Quain, A, I, 587; Gray, A, 674).-The musculo-cutaneous nerve arises from the ventral surface of the 6 th and 7 th cervical nerves. It is also closely connected with the $N$. medius (Fig. 106). It passes almost directly toward the shoulder joint, and when near the trochin gives several filaments to the coracoideus and biceps muscles. It passes entad of the long head of the $M$. coracoideus, and continues distad along the brachium, resting on the caudal aspect of the biceps. Opposite the Frn. epitrochleare, a small branch is given off which anastomoses with the $N$. medius through the foramen. In the concavity of the elbow it passes entad of the $M$. biceps to its cephalic aspect. On the antebrachium it is subcutaneous and extends along the cephalic border of the arm parallel with the $N$. radialis (Fig. 105). It is distributed to both skin and muscles, as the name indicates.

Dissection.-Commence near the shoulder joint and trace it first centrad to determine its origin and communications, then peripherad along its whole course. It will be necessary to employ a tripod or other magnifier in tracing the anastomosis with the median at the elbow. On the antebrachium the nerve is very near the surface, hence the skin and fascia should be removed only by degrees and while keeping the nerve in sight.

$\S$ 1023. NN. subscapulares (Fig. 104, 105, 106; Quain, A, I, 
584 ; Gray, A, 673).-There are two of these nerves, or, if the nerve of the latissimus be counted, three (Fig. 105, 106). 'They all arise from the dorsal side of the brachial plexus, as shown in Fig. 104 and 106. The most cephalic one goes to the $M$. subscapularis, and the intermediate one mostly to the teres. The long subscapular or nerve of the latissimus passes caudo-laterad to the $M$. latissimus in connection with a branch of the subscapular artery $(\$ 942)$.

Dissection.-The position of the nerves is indicated on the figures referred to. They may be isolated with the tracer; this should be done very carefully on account of their position with reference to the other nerves.

$\S$ 1024. N. circumflexus (Fig. 104, 105, 106 ; Quain, A, I, 584 ; Gray, A, 673).-The circumtlex nerve arises from a sort of intermediate branch joining the 6th, 7 th and 8 th cervical nerves. For a short distance it is in the closest relation with the cephalic of the subscapular nerves. It passes nearly laterad and follows the circumflex artery as it winds around the dorsal side of the proximal end of the humerus to terminate finally in the $M$. clavo-deltoideus ; see $\$ 941$.

Dissection. - Commence at the point where the nerve is crossed by the musculo-cutaneous, and trace it first to its origin, and then to the point where it disappears in company with the circumflex artery. The termination may then be seen by lifting the cephalic edge of the clavo-deltoideus just distad of the trochiter. Its entire course may be traced from the periphery by cutting away the muscles.

$\S 1025$. N. medius $s$. medianus (Fig. 104, 105, 106 ; Quain, A, I, 590; Gray, A, 675).-The median nerve in the cat is formed by three branches, shown in Fig. 106, the brachial artery passing between the cephalic and intermediate. It follows the direction of the artery, lying ectad of it in most of its course and traversing with it the $F \mathrm{~m}$. epitrochleare, where it receives an anastomosing branch from the $N$. musculo-cutaneus (\$1022). In the concavity of the elbow the nerve passes entad of the $M$. pronator teres and follows the general course of the bones of the antebrachium to the wrist. It furnishes branches to the muscles on the ventral aspect of the antebrachium, and especially the $N$. interosseus anterior, which accompanies the artery of the same name $(\$ 944, \mathbf{D})$. Finally, it is distributed to the structures on the palmar aspect of the manus.

Dissection.-Commence at about the midale of the brachium 
and trace the nerve to its origin, as shown in Fig. 105 and 106. Then it may be followed peripherad, but great care should be taken not to injure the $N$. cutaneus internus or break the anastomosing filament from the $N$. musculo-cutaneus ( $\$ 1022)$. In the concavity of the elbow the $M$. pronator teres should be divided and the nerve followed in the antebrachium as described for the radial artery (\$944).

$\S$ 1026. N. musculo-spiralis (Fig. 104, 105, 106; Quain, A, I, 592; Gray, A, 679). - The musculo-spiral nerve is formed largely from the 8th cervical nerve, although it receives branches from the 1st cervical and the 1st thoracic; it is the largest offset from the brachial plexus. It winds obliquely around the humerus, commencing at about its middle, being accompanied by the superior profunda artery $(\S 945, \mathbf{B})$. After reaching the cephalic side of the brachium, the nerve divides into two parts, which are known as the radial and the posterior interosseus nerves.

N. radialis.-This is the smaller of the two branches into which the musculo-spiralis divides. It becomes subcutaneous as it emerges from between the MM. ectotriceps, brachialis and clavo-deltoideus (Fig. 74), near the distal end of the brachium. It then follows the course of the radius, remaining subcutaneous through the whole length of the antebrachium. This nerve is closely associated with the musculo-cutaneous. The single large superficial vein of the arm also follows its course. It is partly cutaneous in its distribution.

$\S 1027$. N. interosseus posterior.-This is the larger of the two branches into which the musculo-spiralis divides. It passes along the dorsal side of the antebrachium to the wrist. This nerve is almost wholly muscular in its distribution.

Dissection. - The skin should be torn from the dorsal and cephalic sides of the brachium, then the muscles carefully cut along the course of the nerve. The $N$. radialis will have been exposed in the study of the musculo-cutaneus. The $N$. interosseus may be followed by cutting and tearing the muscles very cautiously with a tracer.

§ 1028. N. ulnaris (Fig. 104, 105, 106; Quain, A, I, 588; Gray, A, 677).-The ulnar nerve arises from the 8th cervical and the 1 st thoracic. It passes along the brachium parallel with the brachial artery as far as the elbow, and then turns to the dorsal side of the elbow. It passes along the dorso-caudal border of the antebra- 
chium to a point somewhat distad of its middle. Here it divides into two divisions-a dorsal, which winds around to the dorsal side of the manus, a ventral, which extends along the ventral surface to the palm. Near the distal third of the antebrachium the ventral division sends a considerable branch to the group of tactile hairs near the hypothenar eminence (Fig. 105). If the skin is torn from the antebraohium at this point, the roots of the hairs will be seen to be similar to those of the vibrissæ (Fig. 87, 88). In distributiou the ulnar is partly cutaneous, but chiefly muscular.

Dissection.-The dissection of the ulnar nerve is very simple, and needs no special directions.

$\S 1029$. N. thoracious posterior-The external respiratory nerve of Bell (Fig. 104, 105, 106 ; Quain, A, I, 576 ; Gray, A, 671).-The long thoracic or external respiratory nerve (so called because the phrenic was called internal respiratory) arises wholly from the 7th cervical near its roots. It passes nearly caudad to be distributed to the serratus magnus muscle. It may be seen readily as shown in Fig. 105.

Dissection.-The rhomboideus and trapezius muscles should be divided and the vertebral border of the scapula turned laterad. The nerve may then be easily traced from its distribution to its origin.

NervUs VAGUS, s. PAR VAGUM, s. N. PNEUMOGaSTRICUS, 10th PAIR. (Fig. 101, 105, 107; Pl. II, Fig. 3, N. v. [X]).

References.-Quain, A, I, 557 ; Gray, A, 660 ; Hyrt1, A, 521; Gegenbaur (Lankester), A, 518; Chaureau, A, 772 ; Chauveau (Fleming), A, 728; Gurlt, A, 737 ; Owen, A, III, 159 ; Milne-Edwards, A, II, 340 ; Leyh, A, 526 ; Dalton, A, Fig. 482 ; Stowell, 1.

$\S 1030$. The vagus or pneumogastric nerve $(\mathrm{x})$ arises apparently from the side of the medulla, passes laterad and traverses the $\mathrm{Fm}$. jugulare in connection with the glossopharyngeus and accessorius. Just as it emerges from the skull or just within the foramen, it has a gangtion, the ganglion of the root, and about $1-2 \mathrm{~cm}$. farther peripherad it presents a second ganglion, the ganglion of the trunk. The nerve passes along the neck in company with the carotid artery, and from the ganglion of the trunk to a point near the 1 st rib is so closely connected with the sympathic nerve that the two appear like one (Fig. 107). The vagus enters the thorax mesad of the A. sternalis, extends caudad along the ventral aspect of the $A$. subclavia sinistra ( $\$ 930)$, crosses the arcus aorticus, and while crossing it gives off a long branch, the $N$. laryngeus recurrens, which winds around the arch and extends cephalad along the trachea to the larynx (Fig. 107). The vagus then passes caudad along the dorsal side of the root of the lung. Slightly caudad of the root of the lung it divides into two parts; one, extending along the ventral side of the cesophagus, soon unites with a branch from the right vagus (Stowell, 1), and the combined trunk, the ventral gastric nerve, then passes along the œsophagus, penetrates the diaphragm and 
is distributed to the ventral aspect of the stomach; the other branch of the vagus winds to the dorsal side of the cesophagus, where it is joined by a small branch from the right vagus. 'l'be union of the two forms the dorsal gastric nerve, which penetrates the diapliragm on the dorsal side of the cesophagus, and is distributed to the dorsal aspect of the stomach and especially to its greater curvature (Fig. 79).

The vagus supplies branches to the larynx. trichea, heart, lungs, asophagus and stomach. Throughout its entire extent it is closely related to certain spinal nerves and to the sympathicus.

The origin, course and distribution of the right vagus is nearly the same as that of the left, except, that the recurrent laryngeal is given off opposite the A. subclavia dextra (Fig. 108), and winds around to its dorsal side to reach the trachea.

The account of the vagus is introduced partly in order to illustrate the origin, course, distribution and relations of a peculiar cranial nerve, but especially on account of its importance from an experimental standpoint.

\section{DISSECTION OF THE VAGUS NERVE.}

\$ 1031. Specimen and Preparation. -The specimen should be young and lean (\$ 1008). The vascular system should be filled from either the femoral or abdominal vessels ( $\$ \$ 352$, 363). The same speciren is to be used for the sympathic ( $\$ 1042)$.

\$1032. Exposure and Dissection.-A block should be placed under the cat's neck so that the head may be slightly dorsiducted. After parting the hair $(\$ 354)$, make an incision from the left external auditory meatus to a point opposite the tip of the xiphisternum, going about $2 \mathrm{~cm}$. to the left of the ventrimeson. The skin should be reflected on both sides of the incision for $2-3 \mathrm{~cm}$. and held aside with pins. Find the position of the larynx and trachea (Fig. 88) by pressing on the ventrimeson.

Make a longitudinal incision through the sterno-mastoid muscles just laterad of the trachea, commencing near the 1st rib and cutting cephalad to the bulla (Fig. 57). It is necessary to proceed with caution, however, in order not to injure the vessels and nerves on the ental side of the muscle. Turn the cut edges of the muscle aside and fasten them with pins. This will expose three structures lying parallel to the trachea:--the carotid artery, which, being injected, will be very apparent; at the same level as the carotid, but laterad of it, the combined vagus and sympathic nerves; and finally between the two, but somewhat ventrad, the internal jugular vein (Fig. 101).

Commence near the middle of the neck and follow the nerve cephalad, using the tracer, fine forceps and scissors. Near the bulla (Fig. 57 and 107, B), the submaxillary and lymphatic glands (Fig. 87) and the thyroid body (Fig. 101) must be removed. At the ganglion of the root the vagus may be easily separated from the 
sympathic (Fig. 107). - From the ganglion of the root the N. laryngeus superior (Fig. 107, N. l. sup.) may be traced entad of the carotid to the larynx.

$\S 1033$. N. hypoglossus (Fig. 107, N. hpgls.).-A little cephalad of the superior laryngeal nerve this will be seen crossing the ectal surface of the carotid and extending to the tongue.

$\S$ 1034. N. glossopharyngeus (Fig. 107, N. gph., Plate II, Fig. 3, N. gph. [ix]).-Divide the M. digastricus (Fig. 101) near its middle and reflect the two ends. This will expose the bulla, and passing along its caudo-mesal aspect will be seen the small glossopharyngeal nerve. From its exit at the Fm. jugulare (Fig. 57, \$ 562), it passes entad of the carotid on its way to the tongue.

$\S 1035$. N. accessorius.-The spinal accessory may also be seen piercing the sterno-mastoid muscle and passing latero-caudad from the Fm. jugulare to be distributed to the ental surface of the clavotrapezius muscle (Fig. 104, 107, N. ac.).

$\S 1036$. The path of the vagus through the foramen jugulare and its relations with other nerves, also the ganglion of the root (Stowell, $\boldsymbol{1}$ ), may be made out by nipping away the skull upon the dorso-lateral side of the foramen.

After the parts just described have been determined, the nerve should be followed caudad. Near the 1st rib the sympathic inclines laterad (Fig. 107). The vagus passes into the thorax entad of the V. brachio-cephalica (Fig. 101, 109).

$\S 1037$. Exposure of the Left Vagus in the Thorax.-Divide the pectoral and other muscles, and costicartilages, about $1 \mathrm{~cm}$. from the meson, and turn the sternum dextrad, securing it with chain hooks $(\$ 140)$ or strings. Then cut the left ribs with the nippers about $2 \mathrm{~cm}$. from their heads, and either remove this part of the thoracic wall entirely or pin it down so that the thoracic cavity shall be readily accessible.

Cut the left brachio-cephalic vein and turn it mesad as shown in Fig. 107. The sternal artery ( $\$ 935)$ may be cut near the sternum and pinned laterad. Then the vagus may be followed with the tracer, fine forceps and fine scissors. Note the phrenic nerve (\$1019), which crosses the vagus just cephalad of the sternal artery. The phrenic may be easily traced along the thorax ventrad of the root of the lung to the diaphragm; by pulling upon it near where it crosses the vagus, and turning the lungs to the left. 
In tracing the vagus, be very careful to preserve all the branches either given off or received by it. The main nerve is so large that there is little danger of injuring it, but its branches are often very small. It is a great help to pull the nerve in various directions with the fingers or forceps; then the presence of branches or anastomoses may be detected by the tense lines extending from the main nerve. There are several of these branches which leave or join the nerve on its way from the 1st rib to the aorta. One or two of these come from the thyroid ganglion, two or three from the vertebral ganglion. When near the arch of the aorta, the ventral side of the vagus is crossed by the lesser cardiac nerve on its way to the cardiac plexus from the vertebral ganglion (Fig. 107, $N$. crd.).

$\$ 1038$. N. laryngeus recurrens $s$. inferior (Fig. 107, 108).Just before crossing the ventral side of the aorta, the left vagus gives off a large branch, which winds around to the dorsal side of the aorta and follows the trachea cephalad to the larynx. Its origin may be readily determined; but to trace it on the trachea, the vessels should be carefully removed in order to expose the trachea. This should be deferred, however, until the vagus is traced to the stomach, or it should be traced upon another specimen.

$\S 1039$. The vagus gives many branches to the heart and lungs near the point where the nerve crosses the arch of the aorta. Their branches are usually rather small, but their course may be made out by pulling on the main nerve.

In tracing the vagus caudad of the arch of the aorta, the lung should be turned mesad, so that the nerve may be seen as it passes along the dorsal side of its root (Fig. 107). In following the nerve in the remainder of its course, it is especially desirable to draw it tense, for in this way is most surely and easily determined the presence of branches. Just caudad of the root of the lung, the nerve will be found to divide, one part passing along the dorsal aspect of the œsophagus, and the other remaining on its ventral surface.

$\S 1040$. Follow the ventral branch (N. gastricus ventralis, Fig. 103, 107). About half way between the root of the lung and the diaphragm, there will be seen a branch joining it from the right. This can be seen easily by pulling the nerve cephalad and to the left. This branch is the ventral division of the right vagus. The combined trunks now pass along the diaphragm and penetrate it with the cesophagus. The diaphragm may be cut away, and then 
if the nerve trunk be pulled, the fan-like expansion of the nerve on the ventral side of the stomach may be seen.

$\S 1041$. N. gastricus dorsalis (Fig. 103, 107). - Now follow the dorsal division of the vagus. It winds around to the dorsal side of the œsophagus, and when within about $2 \mathrm{~cm}$. of the diaphragm, a large branch may be seen to join it. This is the dorsal division of the right vagus. Follow the combined trunk to the diaphragm, then cut the diaphragm so as to expose the csophagus; do this carefully, so as not to injure the nerve. Now pull upon the dorsal gastric nerve, and its fan-like expansion on the great curvature of the stomach will be seen, and also a tense line passing dorso-caudad to the semilunar ganglion of the solar plexus (Fig. 107, Ramus cm., Gng. smln.). The left semilunar ganglion is at the left of the A. mesenterica superior. It is a pinkish white body not difficult to distinguish.

Preparation of Fig. I07.-The arteries and veins were injected from the abdominal aorta and postcava ( $\$ 8363,365)$; the skin was then removed as shown in the figure, and the ribs cut within $2-3 \mathrm{~cm}$. of their heads ; the costicartilages were cut at about the same distance from the sternum, and then the thoracic and abdominal walls were removed. The arm was amputated near the middle of the brachium; the sterno- and clavo-mastoid muscles were also removed. The sternum and heart were turned to the right, together with the brachio-cephalic vein. The left lung was cut off at its root and removed. The vesseis and nerves were then isolated as directed above ( $(1008)$.

Explanation of Fig. 107.-In the description of this figure, under the general heads, which are arranged alphabetically, the special parts are named commencing at the caudal extremity.

A. c., A. cœliaca $(\$ 966)$. A. m. s., A. mesenterica superior (\$967). A. œs., A. œsophagea-One of the cesophageal arteries. Aorta (\$965). A. V. cost., A. V. costales-The intercostal arteries and veins (Fig. 103). A. brcph., A. brachio-cephalica (Fig. 101, 102, 103). A. s., A. subclavia (\$ 933). A. axillaris (Fig. 105, § 938). A. sternalis (\$935). A. carotidea-The left carotid artery; the name is written on the trachea just mesad of the artery (Fig. 101, \& 927). A. thyr., Axis thyroideus ( $\$ 937$ ). A. 1., A. lingualis-The lingual artery, a branch of the carotid. A. fac., A. facialisThe facial artery, a branch of the carotid (Fig. 87). Adrn., Corpus adrenale-The adrenal or suprarenal body ( $(760)$. Cardia-Heart (\$ 822). Costæ (13)-Ribs. Costæ sternales (9). Dphrg., Diaphragma, (Fig. 90, \& 815). D. Stenon., Ductus Stenonianus-Duct of the parotid gland (Fig. 87, \& 780). Gng. smnl., Ganglion semilunareThe semilunar ganglion, the largest ganglion of the solar plexus ( $\$ 1044)$. Gng. vert., Ganglion vertebrale-The caudal cervical ganglion of the sympathic (\$ 1042). Gng. thyr., Ganglion thyroideum-The thyroid or middle cervical sympathic ganglion (\$ 1043). Gng. crv. sym., Ganglion cervicale superius sympathici-The superior cervical ganglion of the sympathic ( 81043 ). Gng. inf. (vagus), Ganglion inferius vagi -The inferior ganglion of the vagus, called also the ganglion of the trunk. G1. (Glandula) parotis-The parotid gland (Fig. 87, $\$ \S 778$, 779). Humerus (Fig. 46, $\S 407$ ). M. serratus ing. (magnus), (Fig. 73, \& 664). M. teres (Fig. 75, 8680 ). M. latis., M. latis- 


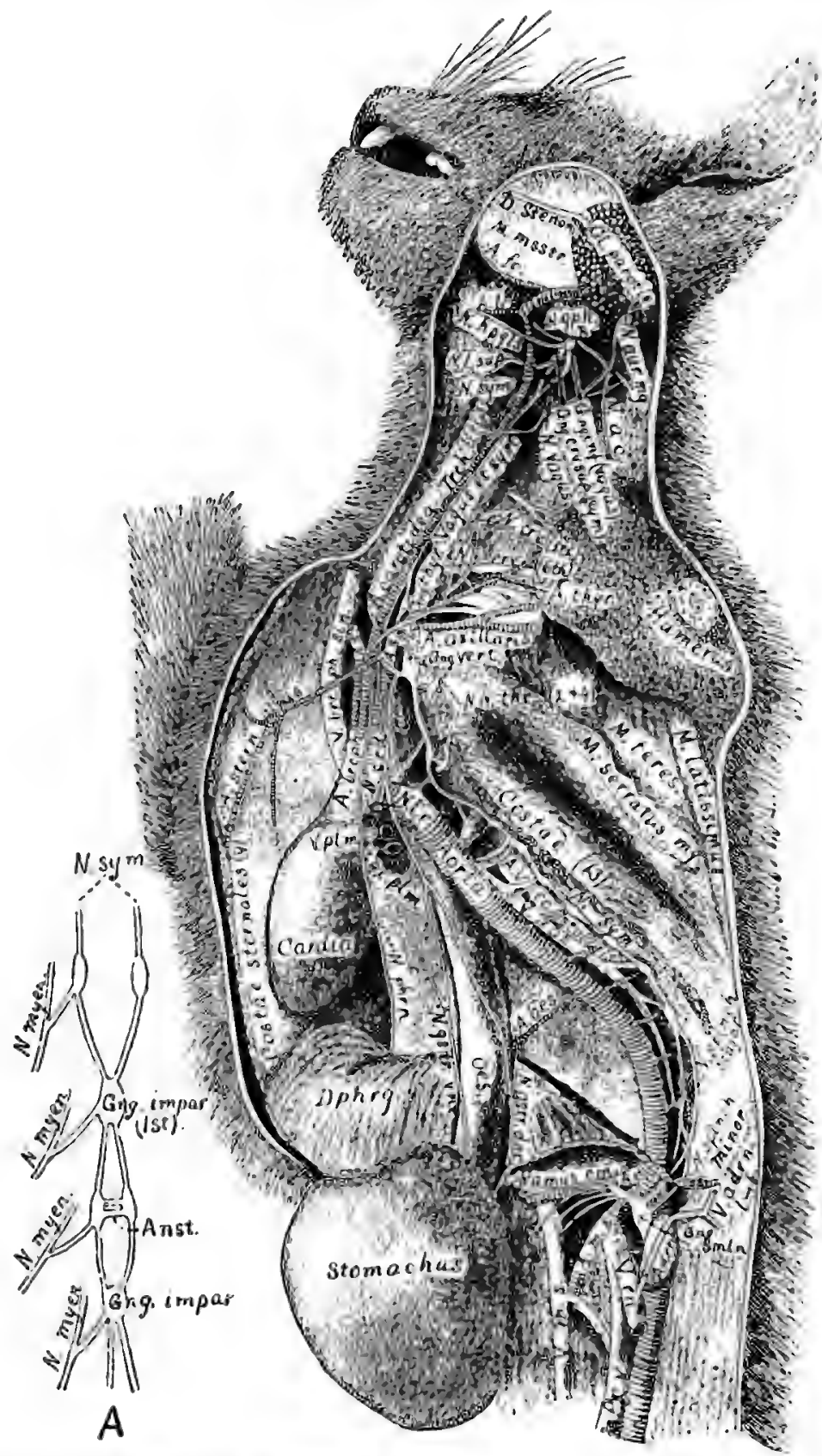

Fio. 107.-The Vagtb and simpatule Nerves; $x$. A. Diagram of tue Sacral

Part of The SxMpateic Nerve; $\times$ about 3. 
simus (Fig. 67, $\$$ 635). M. msstr., M. massetericus. N. splnch. minor, N. splanchnicus minor (Fig. 103, $\S$ 1044). N. splnch. major, N. splanchnicus major, (Fig. 108, $\$ 1044$ )-Both splanchnic nerves are seen to join the semilunar ganglion (gng. smln.), N. gstr. vnt., N. gastricus ventralis-The ventral gastric nerve; it is formed partly by the right and partly by the left vagus, as is also the following (see Fig. 103, $\$ 1040$ ). N. gstr. dor., N. gastricus dorsalis-The dorsal gastric nerve (\$1011); a large branch (ramus $\mathrm{cm}$.) is seen to unite this with the semiluuar ganglion $(g n g . s m l n$.$) . N. phrn., N.$ phrenicus (Fig. 105, N. phrr.). N. sym., N. sympathicus-The sympathic nerve in the thorax ( $\$ 1043)$. N. 1. r., N. laryngeus recurrens-The recurrent largngeal of the left side winding around the aorta (see Fig. 108 and $\$ 1038$ ). N. crd., N. cardiacus-The lesser cardiac nerve from the sympathic (Fig. 109). NN. thr. (2 and 4), NN. thoracici (5 et 6)-The 5th and 6th thoracic nerves; each receives a branch from the sympathic (Fig. 109, § 1012)。 N. crv. (6th), N. cervicalis (6th). N. crv. (5th), N. cervicalis (5th) -The phrenic nerve is seen to arise from this and the preceding (see also Fig, 105, 81019 ). N. vagus $(x)$, (Fig. 103, $\$ \$ 562,1030$ ) - The vagus and sympathic appear as a single trunk in the neck, but are easily separated, especially at their ganglia by the bulla $(B$.) and near the 1st rib. N. sym., N. sympathicus 8 . N. sympatheticus-The great sympathic or sympathetic nerve with its ganglia on the left side (Fig. 103, $\S 1042$ ). N. 1. sup., N. laryngeus superior ( $\S 1038$ ). N. hpgls., N. hypoglossus (Fig. 104, $\$ \S 562,1033$, Plate II, Chapter X). N. ac., N. accessorius (spinalis xi), (Fig. 104, Plate II, Chapter X, $\$ 8562,1035$ ). N. gph., N. glossopharyngeus, ix (Plate II, $\$ \$ 562,1034$, Chapter $X$ ). N. aur. mg., N. auricularis magnus (Fig. 87). CEs., CEsophagus (Fig. 109, § 801). Pcv., Postcava (Fig. 101, § 955). Ramus cm., N. ramus communicans-The nerve putting into communication the gastric branch of the vagus and the semilunar ganglion. Rx. plm., Radix pulmonalis-The root of the left lung. Stomachus (Fig. 79, \& 735). Trch., Trachea (Fig. 88, § 799). V. rn., V. renalis (Fig. 101, §959). V. m. s., V. mesenterica superior ( $\$ 949$ )-The branch entering the $V$. m. s. as it crosses the $A$. m. s. is the $V$. mesenterica inferior (\$ 95U). V. adrn.-lumb., V. adreno-lumbalis (Fig. 103, § 958). V. az., V. azygos (Fig. 91, 107, \& 220 ). V. plm., V. pulmonalis-One of the pulmonary veins just before entering the left auricle (Fig. 91). V. brcph. sin., V. brachio-cephalica sinistra (\$922).

Fig. 107, A.-The sympathic of the two sides in the sacral and part of the coccygeal regions, showing the fusion or close connection of the ganglia and their connection with the myelencephalic nerves; $\times$ about 3 .

Preparation of Fig. I07, A.-The ventral half of the pelvic girdle $(\$ 455)$ was cut away with nippers, and then all the pelvic viscera were removed. The sympathic nerves were found resting on the lumbar vertebræ by divaricating the psoas muscles. The nerves were carefully traced to the sacral and coccygeal region, and their anastomoses with the spinal nerves and the fusion of the ganglia were exposed by removing muscle, connective tissue and the middle sacral artery with fine forceps, scissors and tracer, using also the tripod magnifier whenever the branches became so small as to be in danger of injury from not being distinctly seen.

Explanation of Fig. 107, A.-Anst.-Anastomosis of the two ganglia. Gng. (ganglion) impar-A single ganglion formed by the fusion of those of the two sides; in the one just cephalad the ganglia are not completely fused, but connected by anastomosing fibers. Gng. (ganglion) impar (Ist)-The 1st fused ganglion of the sympathic; it is opposite the $2 \alpha$ sacral vertebra $(\S 458)$. N. myen., N. myelencephalicus-Four myelencephalic or cerebro-spinal nerves with their anastomosing branches from the sympathic. N. sym., N. sympathicus-The nerves of the two sides approaching each other on the sacrum. 
Preparation of Fig. 108.-The vagus and sympathic, together with the arteries, were isolated as directed above ( $\$ 1008)$. The left cardiac nerves from the thyroid and vertebral ganglia frequently pass ventrad of the vagus, as shown in Fig. 107, and those of the right side often pass to the heart along the ventral side of the blood vessels instead of as here shown; see Quain, A, I, 632.

The figure is meant to illustrate the origin and course of the recurrent laryngeal nerve on the two sides-(A) representing the right side, with the recurrent winding around the A. subclavia dextra, and (B) the left side, with the recurrent nerve winding around the arch of the aorta.
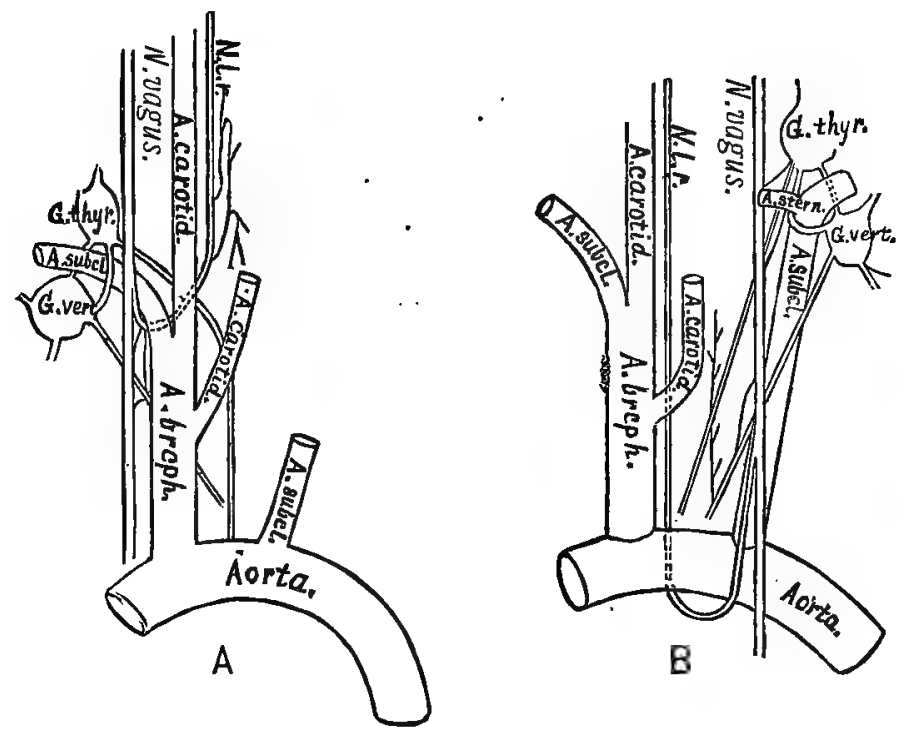

Fig. 108.-The Recurrent Laryngeal Nerves, Ventral View, (Stowell, 1); $x$ about 1 .

Explanation of Fig. I08.-The abbreviations are the same in the two figures.

Aorta, $a z$. (\$ 926). A. brcph., A. brachio-cephalica, $a z .(\$ 943)$. A. subcl., A. subclavia ( $\$ 933$ ). A. carotid., A. carotidea. A. stern., A. sternalis s. mammaria interna. G. vert., Ganglion vertebrale (\$ 1043). G. thyr., Ganglion thyroideum (: 1043) N. vagus $(\$ 1030)$. N. 1. r, N. laryngeus recurrens $(\$ 1038)$.

\section{NERVUS SYMPATHICUS s. SYMPATHETICUS. (Fig. 90, 101, $103,105,107,108,109)$.}

References.-Quain, A, I, 696 ; Gray, A, 696; Hyrtl, A, 572 ; Gegenbaur, A, 522 : Chauveau, A, 328; Chauveau (Fleming), A, 781; Gurlt, A, 767, 774; Owen, A, III, 181; Milne-Edwards, A, II, 331 ; Leyh, A, 550.

\$ 1042. The sympathic nervous system as a whole comprises a central system consisting of a double chain of ganglia extending alons the ventral side of the spinal column. The two chains also extend into the head and into the tail. The ganglia are connected by 
intervening nerves and give rise to nervous branches which are distributed to the viscera and blood vessels. Besides the chain of ganglia, the sympathic nerves may have ganglia developed upon them at almost any point in their course. The various parts of the sympathic are intimately associated with each other and also with the cerebro-spinal nervous system. So close is this connection that some authors consider the sympatlic as belonging to the peripheral part of the cerebro-spinal system (Quain, A, I, 519).

There are nominally as many pairs of sympathic ganglia as vertebræ, except in the cervical region and rarely also in other regions. From each ganglion passes a branch to the corresponding spinal nerve (Fig. 107, 109).

\$1043. Specimen, Preparation and Dissection. - The same specimen may be employed as for the vagus, and it should be prepared in precisely the same manner $(\S 1030)$. The exposure is also the same, and the method of dissecting the nerves $(\$ 1031)$.

Nearly dorsad of the ganglion of the root of the vagus is the similar, fusiform, superior cervical ganglion of the sympathic. It may be carefully isolated; then, by carefully tearing the sheath of the combined vagus and sympathic, the two may be separated in the neck. About $1 \mathrm{~cm}$. from the 1st rib the sympathic inclines laterad, and very near it there appears in most subjects a small ganglion, the thyroid or middle cervical ganglion (Fig. 107, Gng. thyr., Fig. 108). Pull the nerve, and it will be found to divide into two parts, one of which passes dorsad and the other ventrad of the A. subclavia (Fig. 109). Cut the axillary artery and turn it mesad. The two nerves 'may then be followed to their termination in the ganglion vertebrale or the inferior cervical ganglion just caudad of the 1st rib (Fig. 107, Gng. vert., 108, 109). Pull upon the nerve and make out the branches passing from this ganglion. They are shown in Fig. 107, 108 and 109. After these branches are traced, tear away the pleura and follow the sympathic chain along the thorax. Note that there is a ganglion for each vertebra, and that each ganglion communicates with the corresponding spinal nerve (Fig. 107).

§ 1044. NN. splanchnici.-The sympathic ganglion about opposite the last rib is somewhat larger than those preceding it, and there is given off a branch, the $N$. splanchnicus major, which passes caudad and slightly ventrad and penetrates the diaphragm. The diaphragm should be cut, the abdomen well opened and the viscera turned to the right; then by pulling upon this nerve it will be seen to move the semilunar ganglion, showing that it joins that body. Now follow the sympathic from the origin of the great splanchnic. It becomes more nearly mesal in position, and when near the dia- 
phragm gives off another branch, the $\mathcal{N}$. splanchnicus minor. This also penetrates the diaphragm and joins the semilunar ganglion (Fig. 90, 107).

$\S 1045$. Ganglion semilunare (Fig. 90, 107).-The semilunar ganglion belongs to the so called solar plexus of nerves, a network of nerves formed partly from the vagus ( $\$ 1041$ ), but mostly from the sympathic. The ganglion is a slightly pinkish body situated laterad of the origin of the superior mesenteric and caliac arteries, as shown in Fig. 10\%, and very near the adrenal body. It is quite tough, and when grasped and pulled the nerves may be seen spreading out in all directions like the rays of the sun.

$\S 1046$. The abdominal sympathic.-In order to follow the sympathic in the abdomen, the blood vessels should be removed. The nervous cords of the two sides closely approach each other, so that both may be traced at once.

The caudal part of the sympathic is partly shown in Fig. 107, A, and directions for tracing it are given under the preparation of this figure.

Preparation of Fig. 109.-The arteries were injected with red and the veins with blue plaster ( $\$ 5362,363)$. The sternum was removed, and the thyroid and vertebral gang]ia of the sympathic and their branches isolated as directed above ( $\$ 1008)$. The thyroid ganglion of the right side was displaced somewhat dextrad, the vertebral ganglion of the left side drawn donsad, and the muscles removed from the vertebra and the pleura and connective tissue from the other parts. The œesophagus was slightly distended with cotton and the two roots of the myelonal nerves slightly separated and held apart with cotton. The preparation was bardened and preserved in alcohol ( $\$ 286)$.

Explanation of Fig. Iog.-A. sbclv. sin., A. subclavia sinistra. A. sbclv. dext., A. subclavia dextra-The right subclavian is shown in two places as if it were double; the artery is not double, but is shown in its natural position and also displaced in order to more clearly indicate the ring of the sympathic (ansce s. annulus Vieussenii) surrounding it; see Fig. 108 and Foster, A, Fig. 37. A. c., A. carotidea ( $\$ 900)$. A. sternalis-On the left the name is written on the artery. A. thyr., A. vertebralis-By an inadvertence the wrong abbreviation was used. The artery is shown on the right dorsad of the origin of the $A$. sternalis.

Alba-The white substance of the myelon. It is dotted to indicate the cut ends of nerve fibers $(\$ 1048)$.

Cn. centralis-The central canal of the myelon; see Chapter $X$, Fig. 112, A.

Centrum-The centrum or body of the $2 \mathrm{~d}$ thoracic vertebra (\$462).

Cinerea-The gray or ganglionic substance of the myelon $(\S 104 \%)$. It is left white in the figure, and appears something like a letter $H$ in the interior of the myelon (\$ $\$ 998)$.

F. dms., Fissura dorsimesalis-The dorsimesal (posterior) fissure of the myelon.

F. vms., Fissura ventrimesalis-The ventrimesal fissure of the myelon. It is directly opposite the dorsal fissure, and the two divide the myelon nearly in half (\$ 998). 
Gng. thyr., Ganglion thyroideum-The thyroid or middle cervical ganglion of the sympathic (Fig. 107, 108, § 1043).

Gng. vrt., Ganglion vertebrale-The vertebral or inferior cervical ganglion of the sympathic (Fig. 107, 108, § 1043).

Myelon-The myelon or spinal cord is the part of the central nervous system situated within the neural canal (Fig. 104, \& 479).

MM. Ig. colli-The longus colli muscles.

N. thr. (Ist)-The first thoracic or dorsal nerve. The abbreviation is written on the ventral primary division. Centrad of the abbreviation, the dorsal primary division is seen

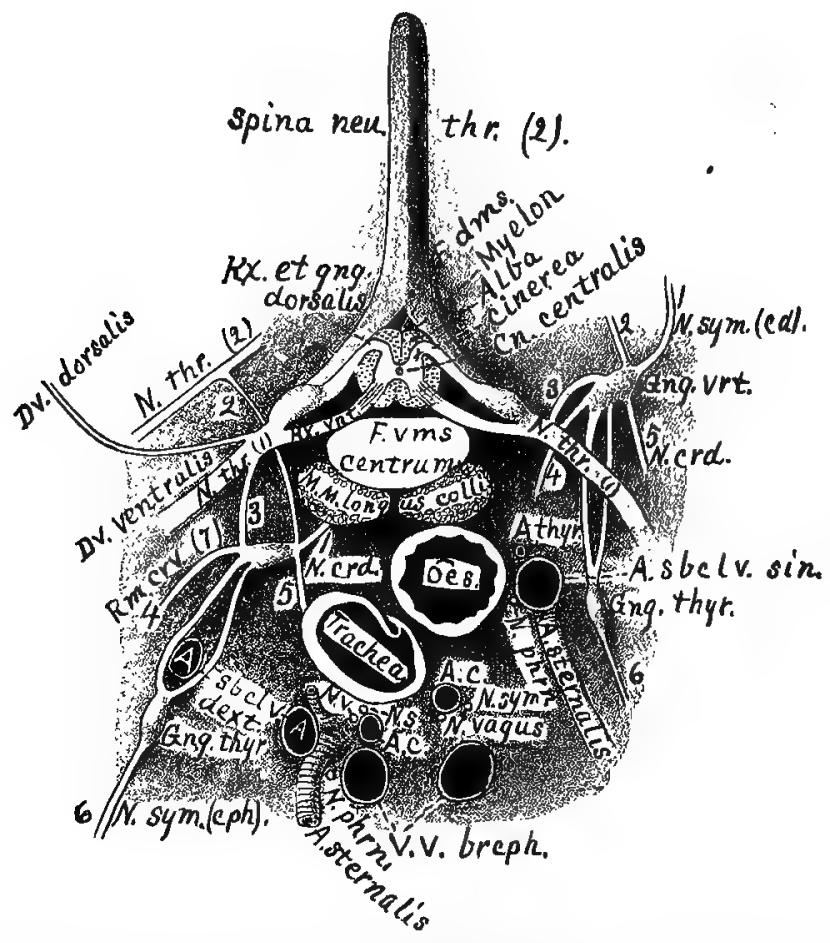

Fig. 109.-Trangection of the Thorax, showing the Origin of a Patr of Spinal

Nerves and the Relations of The Sympathic and Cerebro-spinal Systems;

Cephatio View; $\times 1.5$.

to join the ventral, and the trunk is formed by the uniou of the doral and ventral nerve roots from the myelon $(\$ 1007)$.

N. thr. (2d), N. thoracicus (2d)-The second thoracic nerve, showing the ramus communicans connecting it with the vertebral ganglion.

N. sym. (cd.) I, N. sympathicus (caudalis)-The sympathic nerve extending caudad of the vertebral ganglion in the thorax (Fig. 107, $\$ 1042$ ).

Ramus communicans 2-The communicating branch between the vertebral ganglion of the sympathic and the $2 \mathrm{~d}$ thoracic myelonal nerve. 
Ramus communicans 3-The branch or ramus connecting the vertebral ganglion of the sympathic with the 1st thoracic myelonal nerve.

Rm, crv. 4 (7), Ramus communicans cervicalis ( 7 th)-The branch connecting the 7 th cervical nerve with the vertebral ganglion.

N. crd. 5, Nervus cardiacus minor-The lesser cardiac nerve from the vertebral ganglion. The heart receives several filaments from the vertebral and thyroid ganglia, but only this large one is shown.

N. sym. (cph.) 6, N. sympathicus (cephalicus)-The sympathic nerve extending cephalad of the thyroid ganglion along the neck. It is somewhat displaced to the right; see Fig. 107.

N. sym., N. sympathicus-The cut end of the sympatbic nerves of the two sides, showing their relations to other parts. That of the left side $(N . s y m$.) is on the left of the corresponding carotid artery, while the one on the right $(N . s$.$) is dorsad of the right$ carotid and very close to the vagus.

N. v., N. vagus-The cut ends of the vagus or pneumogastric nerves, showing their relations.

N. phrn., N. phrenicus-The cut ends of the phrenic nerves, showing their relations to the other structures.

Es., Esophagus (Fig. 103, 107, § 798)-The cesophagus is seen to be somewhat to the left of the meson and dorso-sinistrad of the trachea. It is represented as somewhat contracted, hence the wavy outline of the mucous membrane.

$\mathbf{R x}$. et Gng. dorsalis, Radix et Ganglion dorsalis-The dorsal (posterior) root of the 1st pair of myelonal nerves with its ganglion. (The adjective dorsalis belongs only to radix, bence the feminine form.)

Rx. vnt., Radix ventralis-The ventral (anterior) root of the 1st pair of myelonal nerves (§ 1007).

Spina neu. thr. (2), Spina neuralis thoracica (2)-'The $2 \mathrm{~d}$ neural thoracic spine.

Trachea-The trachea is represented as slightly contracted so that the cartilaginous rings overlap somewhat.

VV. brcph., VV. brachio-cephalicæ-The cut ends of the two brachio-cephalic veins (Fig. 101, § 922).

\$1047. Obvious Structure of Nervous Tissue.--Nervous tissue is obviously of two kinds:-(A) Alba (white matter). This js found in the central nervous system, where it has a dense, lusterless white appearance, but principally in the nerves ( $\$ 995)$, wbere it is white and glistening; the nerve also has a wavy or crimped appearance when relaxed. The nerves themselves have something of the same obvious structure as striated muscle $(\$ 704)$, that is, a more or less cylindrical fasciculus, surrounded by a slicath (epineurnm), and this mass in turn being composed of smaller bundles (funiculi) each funiculus having its special sheath (perineurium, neurilemma).

(B) Gray matter. 'The gray nervous matter is soft, so that it is easily crushed in the hand ; in color jt is of a delicate gray or reddish brown, and is collected in masses ( $\$ 994$ ), the largest being in the brain and myelon ( $\$ 997)$.

$\S 1048$. Microscopic Structure of Nervous Matter.-(A) White matter (alba). Each funiculus, like the fasciculus of a striated muscle, is composed of a variable number of cylindrical fibers which are of two kinds:-(1) Medullated, composed of three parts, viz., a primitive sheath somewhat comparable to the sarcolemma of the muscle fiber ( $\$ 705)$; it has a wavy outline and presents nuclei: Myeline or vohite substance of Schoonn, this is a white fatty substance just within the primitive sheath; it is interrupted at regular intervals 
(1-2 mm.), forming the nodes of Ranvier: Axis band, band axis, axis cylinder; this forms the central part of the nerve fiber; it is continuous, subcylindrical, grayish, rather tena. cious, finely fibrillated and with straight outline. The axis cylinder is the essential part of the nerve, and is the only part found at its origin and termination. Medullated nerves neither give nor receive anastomoses except very near their termination. In cross section, a bundle of nerve fibers appears like a bunch of lead pencils, the band axis corresponding to the lead. (2) Non-medullated fibers. These are of a pale gray appearance, owing to the absence of the myeline. They possess a nucleated sheath and an axis cylinder like the medullated fibers, but differ from them in the absence of the medullary sheath and from the fact that they anastomose frequently throughout their entire course; Ranvier, A. 776.

(B) Gray matter. Gray matter is composed of an interlacing network of nerve fibers, peculiar connective tissue (neuroglia) and nerve cells, the latter being the characteristic feature. The nerve cells of the brain and mselon apparently possess no proper sheath, and present two forms, viz., (1) cells containing a nucleus and nucleolus, surrounded by. the gray or reddish brown protoplasm which gives off one or more processes, giving rise to the so called multipolar cells, one or more of the poles being continued as the band axis of a nerve fiber; (2) cells much smaller than the preceding, composed apparently of only a nucleus and nucleolus. The nerve cells of the ganglia possess a proper sheath, which is merely an expansion of the primitive sheath of a nerve fiber. Typically, these cells are pyriform and possess a pole at each end which is continued into a nerve fiber. Stricker, A, 116 ; Quain, A, II, 125. 


\title{
CHAPTER X.
}

\author{
ENCEPHALON-THE BRAIN.
}

GENERAL CONSIDERATIONS-NAMES OF THE PRINCIPAL PARTS OF THE AMPHIBIAN BRAIN

-DIAGRAMS OF AN IDEAL STMPLE BRATN-EXAMINATION OF THE BRAINS OF THE FROG ANDD MENOBRANCHUS-REMOVAL OF THE CAT'S BRAIN-MACROSCOPIC VOCABULARY-DESCRIPTION OF FIGURES AND PLATES-DISSECTION OF THE BRATNSYNONYMS AND REFERENCES-FISEURES AND GYRI.

\$1049. General Considerations.-Definition.-Excluding Amphioxus, whose brain is peculiar and apparently incomplete, the vertebrate encephalon may be defined as the enlarged, differentiated and incompletely segmented cephalic portion of the myelencephaton (cerebro-spinal axis), contained within the cranium.

The junction of the brain proper with the myelon proper ( $\$ 1006$, Fig. 104) is formed by the part commonly called medulin (oblongata), but metencephalon by Quain (A, II, 755), and in the present work. By its obvious characters and real structure the medulla is only a modified continuation of the myelon (Stricker, A, 758-764, Spitzka, 1, 46), and Wyman describes it $(\mathbf{3 4}, \mathbf{7})$ as a division thereof; most writers, however, regard it as a part of the brain, and this is practic $r l y$ the more convenient way.

$\S 1050$. Importance and Difficulty of the Study of the Brain.-An acquaintance with the obvious features, the intimate structure and the functions of the brain is desired by not only the physician and the practical veterinarian, but also by the systematic zoologist, the comparative anatomist, the physiologist and the psychologist.

The nature and extent of the obstacles to the acquisition of this knowledge are most fully appreciated by those who have advanced the farthest in its pursuit; some idea of them may be gained from any recent compendium by an original investigator in either of the branches of inquiry above indicated (Quain, A, II ; Stricker, A ; Ferrier, A).

Deferring for the present any considerations of the histology and functions of the mammalian brain, its mere topography is far from easy to understand.

$\S 1051$. Methods of Studying the Brain.-The brain is commonly figured and described as a fibrous and cellular mass penetrated here and there by inconsiderable cavities. Little attention is paid to the membranes which line these cavities and invest the entire organ. Stress is laid upon its complex structure and remark- 
able functions. Finally, the human brain is usually presented, or at least employed as a standard for comparison.

From personal experience and from the uniform testimony of our students, we have been led to the following conclusions:-

(A) The arrangements of the solid parts of the brain are more readily perceived and more easily remembered after the relations of the cavities are fully understood.

(B) An adequate idea of the circumscription of the cavities involves a distinct recognition of their lining and of the investment of the whole brain.

(C) The general plan of the organ is most readily appreciated if we disregard altogether its organic composition and its direct subservience to mental operations, and view it primarily as we might any artificial structure, like a house or a piece of furniture of homogeneous material.

(D) Even if, as is commonly the case, the human brain is the ultimate object of inquiry, the brain of some Amphibian should be examined first.

$\S 1052$. So far as we know, the first three of these propositions have not been distinctly enunciated heretofore.

The advantage of studying first the cavities of the brain seems to arise from the fact that while the walls are subject to great modificatiouts as to form, thickness, histological composition and connections, the cavities can present differences of only size, shape and degree of circumscription; their connections are invariable, and of course no structure is predicable of them. Hence fewer considerations are presented to the mind, a matter of no small importance to the beginner.

$\$ 1053$. To the physiologist the membranous envelopes are of interest only as concerned in the vascular supply of the proper nervous tissue, and for most anatomical purposes they are better removed. With them, however, are apt to come away some atrophied parts of the parietes together with the lining of the cavities, so as to leave the latter open at certain points. Hence many figures and descriptjons indicate or imply that there are free communications between the cavities and the outside of the brain.

With possibly a single exception ( $\$ 1082$ ), this is not the casé, in adults at least, and indeed the existence of such communications would be out of keeping with what is known of the mode of development of the organ. Hence any clear and adequate conception of the relations of the cavities involves the distinct recognition of the presence of the membranes and of their general arrangement.

$\S 1054$. As to the third proposition, the comparative anatomist and the systematic zoologist especially desire the identification of the various regions of the brain, and the determination of suitable names and terms for description, Even where the histology and functions of the organ are the ultimate objects of its study, the student must first become familiar with the order of succession of the parts, their constant topographical relations and the connections of their cavities, and with the names of them all.

Now this may be done not only as well, but in our opinion more easily, if all other 
features are for the time ignored, and the brain is viewed simply as a series of cavities with parietes of varying thickness, more or less distinctly divisible into walls, floor and roof.

$\S 1055$. The fourth proposition is in accordance with the following general aphorism :-

"In all departments of investigation, it is right to commence with the study of that which is common, simple and regular, and thence to proceed to inquire respecting that which is [complex], unusual and irregular."-Bucknill and Tuke, A.

The specific idea is admirably expressed in the following passage from a paper in which it is practically carried out:-

"With man and the other mammals, the cerebrum and cerebellum so far transcend all the other organs of the encephalon, that the parts which in a morphological point of view are of equal value have been frequently overlooked, as forming either integral parts or primary subdivisions.

"In frogs ... while no one part takes an excessive development, there is at the same time no one of the fundamental ones either wholly deficient or so far reduced as to deprive the general plan of any of its more important features. The brain is so far reduced in the relative proportion of its different parts, and so far stripped of the 'accessory organs of perfection,' as to enable the student to obtain with ease a clear conception of the geveral plan, a conception always so difficult to acquire when studying the brain of mammals or of man."-Wyman, 34, 6.

\$ 1056. The advantages presented by the frog's brain may be categorically stated as follows:-

(1) The various parts, while far from equal in size, differ much less than in the higher Vertebrates.

(2) No part, is completely hidden by another.

(3) All lie in the same plane, the organ not presenting the perplexing " cranial flexure" (Quain. A, II, 733) of most of the higher Vertebrates.

(4) The cavities are relatively large.

(5) The parietes vary little in thickness.

(6) While all the primary components of the brain are present, there are but few special additions or modifications to distract attention from the general plan.

Yet the frog's brain is by no means an ideally perfect type of the vertebrate brain, or wholly adapted for study, for the following reasons :-

(1) It is undesirably small. Hence the student should select for this purpose the very largest individuals, if possible of the bullfrog, Rana Catesbiana (pipiens of some writers).

(2) The tissue is very soft. Hence great care is needed, and the organ should usually be hardened.

(3) The cerebellum is disproportionally small.

(4) The cavity of the optici presents a projection of the wall which renders a section of the region somewhat juzzling.

(5) The passages ( porta or foramina of Monro) between the mesal and the lateral cavities are undesirably small.

(6) The cephalic divisions, the Lobi olfactorii, which are separate in all other Vertebrates, are, in the frog and toad and other anourous Amphibia, not only in contact upon the meson, but there united by somewliat firm connective tissue, constituting a feature which has seriously misled some anatomists, including even Wymun $(\mathbf{3 4}, \mathbf{8}, \mathbf{9})$.

(7) The plexuses are nearly or wholly absent.

In respect to the last four objections, the brain of Menobranchus is preferable to that of the frog. But the animal is less easily obtained, the cerebellum is even smaller, and the optici are so slightly differentiated from the parts caudad and cephalad as to render somewhat difficult the recognition of their limits. 
Nevertheless, the brain of Menobranchus is well worthy of examination upon the following grounds:-

(1) The slight differentiation of the regions is an interesting reminder of the presumed condition of all brains at an early stage of development.

(2) The size of the cavities and communications and the thinness of the parietes permit the effects of inflation with air to be at once apparent.

(3) The Lobi olfactorii are disconnected, as in all Vertebrates excepting the Anura.

(4) The aula is large and well defined.

$\S 1057$. The method of viewing the general constitution of the brain which was suggested by Wyman and is herein adopted may be called the comparative anatomy way. There is another, the embryological way, which is theoretically more satisfactory and complete, but practically not well adapted to beginners. It would be well, however, for the some.what advanced student to obtain a collection of fotal pigs, kittens, etc, of different ages, and carefully expose their brains so as to observe the gradual increase of the hemispheres and cerebellum, the formation of the gyri and the progressive thickening of the walls in the greater part of their extent.

$\S$ 1058. Partial Vocabulary.-The following List includes only the names of the principal parts of the Amphibian brain. A more complete macroscopic vocabulary of the organ will be given later in this chapter.

Names of the Principal Parts of the Amphibian Brain, with their more Common Synonyms.-Aula-Ventricle of the " unpaired cerebral rudiment." Aulatela-Atrophied or membranous roof of aula. Auliplexus-Plexus choroideus aulæ. Cerebrum-Hemisphæræ, larger portion of prosencephalon. Cerebellum-Dorsal portion of epencephalon. Chiasma-Commissura optica, chiasma nervorum opticorum. Conarium-Corpus pineale, pineal gland, epiphysis. Crus cerebri-Floor of mesocœlia. Diacœlia-Ventriculus tertius. Diencephalon-Deutencephalon, thalamencephalon. Diaplexus-Plexus choroideus ventriculi tertii. Diatela-Atrophied or membranous roof of third ventricle. Endyma-Ependyma, lining of the coliæ. Epicœlia-Ventriculus cerebelli, cephalic part of ventriculus quartus. Epencephalon-Hind brain. Hemisphæra -Hemicerebrum. Hypophysis-Corpus pituitarium. Lobus olfactorius-Lateral half of rhinencephalon. Mesencephalon-Lobi optici and crura. Mesocœlia-Ventriculus loborum opticorum, aquæductus Sylvii, iter a tertio ad ventriculum quartum. IMetacolia-Caudal portion of ventriculus quartus. Metatela-Atrophied or membranous roof of ventriculus quartus. Metencephalon -Medulla. Myelon-Chorda spinalis. Opticus-Lobus opticus. Pia-Pia mater. Porta-Foramen Monroi. Portiplexus-Plexus choroideus foraminis Monroi. Postcommissura-Commissura posterior. Præcommissura-Commissura anterior. Procœlia-Ven- 
triculus lateralis. Proplexus-Plexus ventriculi lateralis. Prosencephalon-Cerebrum, hemisphæræ. Pseudo-commissura-Connective tissue between lobi olfactorii in Anura. Rhinencephalon-Lobi olfactorii. Rhinocœlia-Ventriculus olfactorius. Terma-Lamina terminalis, lamina cinerea. Tuber cinereum. Thalamus-Thalamus nervi optici, wall of diaccelia. Valvula-Valve of Vieussens.

\$1059. List of some of the Technical Names of the Parts most frequently mentioned, with the terms (in black letter) which are Preferred to them.-Aquæductus Sylvij-Mesocœlia. Chorda spinalis-Myelon. Commissura anterior-Præcommissura. Commissura posterior-Postcommissura. Corpus pineale-Conarium. Corpus pituitariumHypophysis. Ependyma-Endyma. Foramen Monroi-Porta. Iter a tertio ad ventriculum quartum-Mesocclia. Lamina terminalis s. cinerea-Terma. Lobus opticus -Opticus. Medulla oblongata-Metencephalon. Pedunculus cerebri-Crus cerebri. Plexus choroidens ventriculi tertii-Diaplexus. Plexus choroidens ventriculi lateralisProplexus. Ventriculus lateralis-Procœlia. Ventricle of the " unpaired cerebral rudiment," mesal part of ventriculus lobi communis-Aula. Ventriculus quartus-Metacolia. Ventriculus tertius-Diacclia.

Comparntive Brevity of the Terms here adopted.-In the above list there are 19 new names composed of 20 words and about 150 letters. The corresponding old names comprise 40 words and about 300 letters. Since the parts specified are very frequently mentioned in treating of the macroscopic anatomy of the brain, it is evident that a substantial saving is effected by the employment of the shorter terms.

$\S 1060$. The Encephalic Segments.-All brains present more or less marked constrictions with intervening enlargements; the caudal region also is single or mesal, while the cephalic is double or in two lateral parts. Hence the brain may also be defined as an incompletely segmented tube of nervous tissue, bifurcated at one end.

In each of these segments there is one organ or pair of organs constituting its principal or characteristic portion, but there are always some other parts of greater or less importance.

$\$ 1061$. Names of the Encephatic Segments. - That region of the brain which includes the (lobi) optici, which is easily recognized in most adults and is very prominent in the embryo, has been almost uniformly designated by the technical term mesencephaton, or by its vernacular equivalents mittelhirn or midbrain.

With regard to all the other segments, however, there has been such diversity of usage that the student is apt to be confused in comparing the statements of different writers. In the following Table are given the principal synonyms of the names of the encephalic segments herein adopted, which, as may be seen by comparing the second and seventh columns, are almost identical with those which are given in the Human Anatomy of Quain (A). 


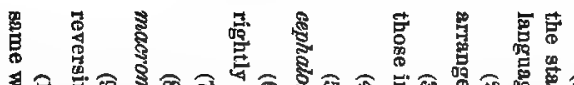

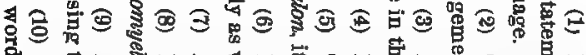

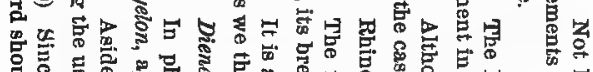

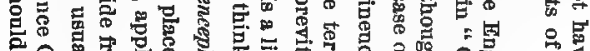

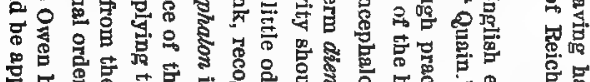

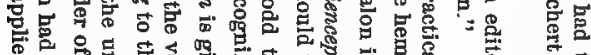

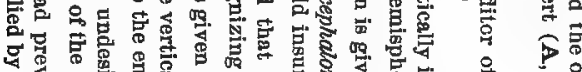

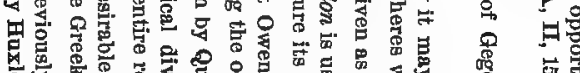

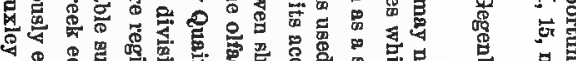
s.

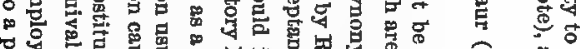

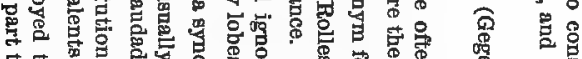

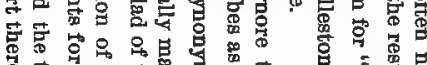

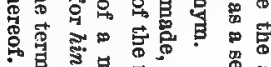

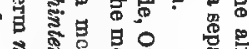
告 保 s s. 鬲高

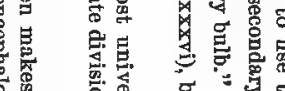

$$
\text { ह }
$$

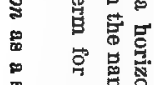

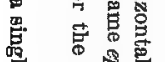

\%

突

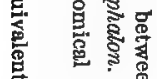

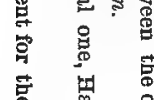

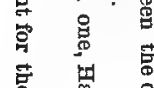

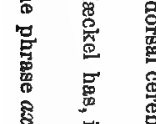

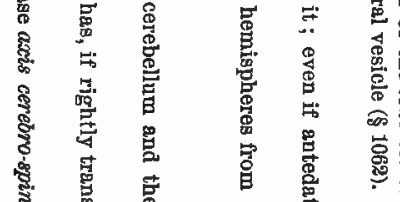

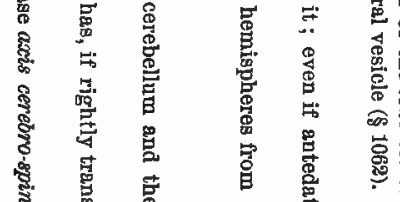

है

$$
\text { i }
$$

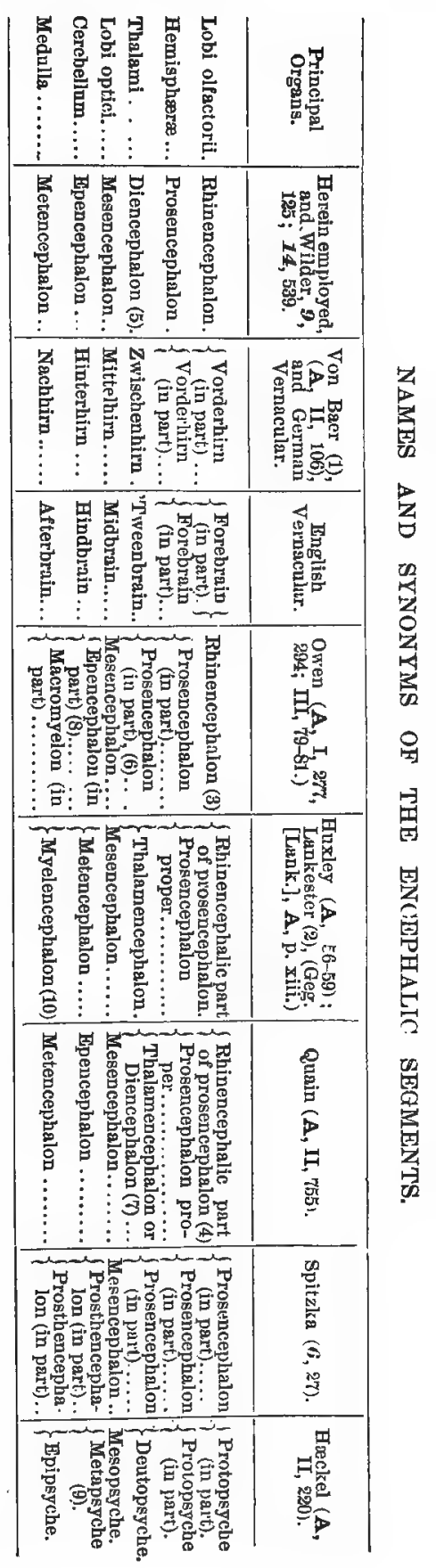


$\S$ 1062. Unequal Morphical Value of the Segments.-So far as appears in the second column of the Table, the six segments are equal primary divisions.

In one sense this is certainly not the case. According to most accounts (Reichert, $A$, II, 11 ; Mihalkovies, A, 21 ; Spitzka, 6, 27; Huxley, A, 56; Quain, A, II, 750), the embryonic brain consists of three primary vesicles; of these the middle is developed into the mesencephalon, while each of the others is again divided into secondary vesicles, from which the other five regions are formed. Balfour, lowever (A, II, 345), admits only twoo primary vesicles.

Aside from the verbal distinctions indicated in the Table, most of the differences are due to the unequal estimates placed by writers upon the sereral segments from an embryological point of view.

Anatomically, there seems to be no objection to the arrangement here adopted, for while the cerebellum and hemispheres preponderate in the higher animals, the optic lobes are larger in some "fishes," the olfactory lubes are enormous in some sharks and placed at a considerable distance from the prosencephalon, and in a skate (Torpedo) the largest part of the brain is the medulla.

For practical purposes, then, the six segments may be regarded as coördinate divisions.

$\S 1063$. Advantages of Using the Segmental Names. - There are tbree advantages in the use of terms designating the encephalic segments :-

(1) They indicate the segmental constitution of the brain.

(2) Each designates a general region which may consist of several more or less distinct parts. Misenceplaalon, for example, includes not only the optic lobes, but the crura cerebri, etc.

(3) They are single words capable of inflection,

\$.1061. Names of the Cavitier.-On account of the peculiar condition of things in man and the higher Mammals, certain portions of the general cavity have been recognized as such, while others have been called passiges or ignored altogether. In modern times the larger cavities have been usually called ventricles, from the Latin ventriculus.

The incongruity of the anthropotomical designations of the encephalic cavities has been pointed out by Owen (A, I, 294, note), and the senior author (9, 125, 14, 539).

The canalis centralis expands into a cavity which, although the first of the series, is called the fourth ventricle. The more or less distinct cavities corresponding to the cerebullum and the optici are not called ventricles at all, and the second is known by either of the following phrases: aquaductus Sylcii and iter a tertio ad ventriculum quartum. The diencephalic cavity is the third ventricle. The two "lateral "ventricles are rarely men. tioned as the first and second, but since the numbers must be understood in order to account for the third and fourth, the student desires, in vain, to know which is the first and which the second. In point of fact, if the enumeration is begun at the cephalic end of the series, the lateral ventricles are the third and fourth, since, in most air-breathing Vertebrates, there are well-developed ventricles in the Lobi olfactorii. Finally, a "fifth ventricle" is mentioned, which is not only at a great distance from the fourth, but has no normal connection with the other ventricles, and is, in fact, no part of the series.

It is hardly possible to imagine less appropriate and consistent appellations for a series of essentially similar cavities.

Ventriculus is objectionable because of its length, because its use is apparently established in connection with the cardiac cavities, and because, as a Latin word, it is not readily combined with Greek prefixes. No one of these objections is fatal, but combined they may be regarded as warranting the use of another word if such can be found. 


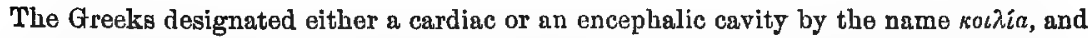
the senior author has proposed $(\$, 1 \% 5,14,540)$ to substitute it for ventriculus, and to des. ignate the several encephalic cavities by terms formed by its combination with the characteristic prefixes of the encephalic segments. This gives us rlinoccelia, procelitu, diacolia, mesocelia, epiccolia and metaccelca. These terms are capable of inflection, and the longest of them is no longer than the Latin ventriculus, which requires a prefix or qualifying word. Finally, when the student has once leurned the order and significance of the names of the encephalic segments, he has only to aequire a single term and apply thereto the characteristic prefixes with which he is already familiar.

$\S 1065$. Aula and Porta. -These names were proposed by the senior author $(5,9,14$, 540) upon the following grounds :-

(1) To substitute brief single words for the phrases: "ventriculus communis," "ventriculus lubi communis," cavity of the "cerebral rudiment," unpaired hemisphere vesicle or "secondary forebrain," mesal part of the "common ventricular cavity," foramen Monroi, etc.

(2) Because the phrase most commonly employed, foramen Monroi, is used to designate at least three different cavitice or orifices: (A) The cavity by which either proccelia communicates with the mesal series of cœliæ; (B) the two lateral orifices together with the intervening space; (C) the mesal (cephalic) orifice of the diacœlia. We have been unable to ascertain by whom the phrase was first employed, and the description by Monro secundus (A, 12 16), in whose honor it was applied, is somewhat vague (Wilder, 3).

(3) In order to indicate our opinion of the desirability of recognizing the aula as morphologically an important element of the colian series.

$\S$ 1066. Telee and Plexuses.-The atroplied or membranous roofs of certain coeliæ are called tela vasculosa or tela choroidea, superior, inferiur, etc, and the vascular plexuses formed by them are designated as plexus choroideus ventriculi tertii, ete. If once the general names for the encephalic segments and cœliæ are adopted, we bave only to employ the characteristic prefixes and gain the single and definite names metutela, diatela, aulatela, metaplexus, diaplexus, auliplexus, portiplexus and proplexus.

$\S 1067$. Commissurce. - Of the bands of fibers, or aggregations of cells and fibers, by which the parts of the brain-especially corresponding parts upou the two sides-are connected, some are called commissures, while others have received special uames. These latter-callosum, fornix, pons and chiasma-are retained, but the other three-as proposed by the senior author $(9,126,14,538)$-are here simplified by prefixing to the word commissuru the syllabies proe, post and medi.

§ 1068. Tabular Vien of the Encephalic Segments and their parts in the Amphibian Brain.-The accompanying Table contains the names of the principal parts of the brains of the frog and Menobrauchus arranged according to the segments which they constitute (Fig. 110-112). Attention is called to the recurrence of the prefixes charaterizing the segments in the names of the corresponding ccelice, telce and plexuses (\$1066). A somewhat similar table is given by Mihalkovics $(A, 48)$, including also the names of the parts of the mammalian brain; see also Quain (A, II, 755).

The abbreviation $\alpha z$. indicates that the part is azygous or unpaired; the rest are lateral and paired. 

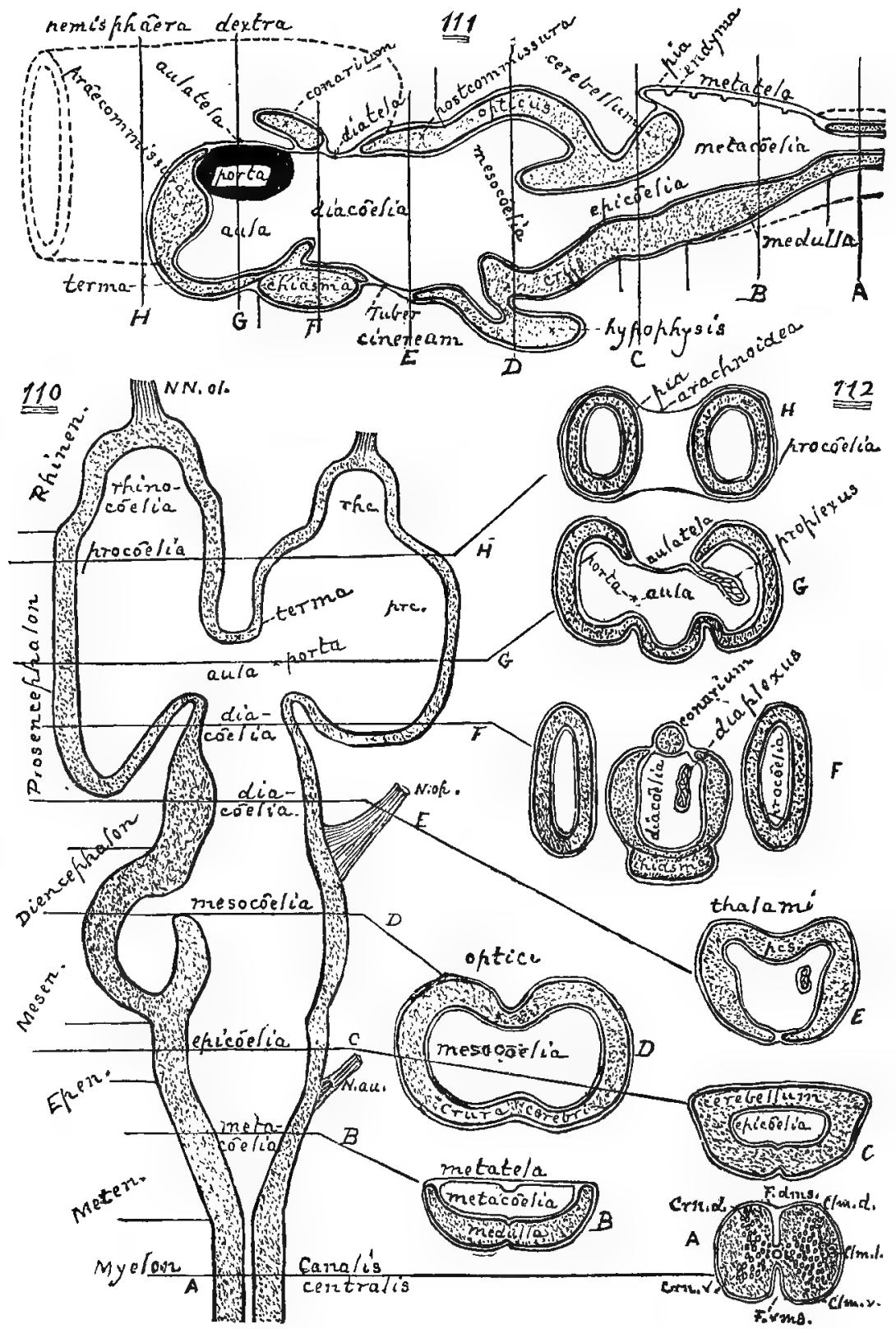

Fig. 110-112.-Schematic Diagrams of a Simple Brain. 


\section{§ 1099. TABULAR ARRANGEMENT OF THE NAMES OF THE PRINCIPAL} PARTS OF THE AMPHIBIAN BRAIN.

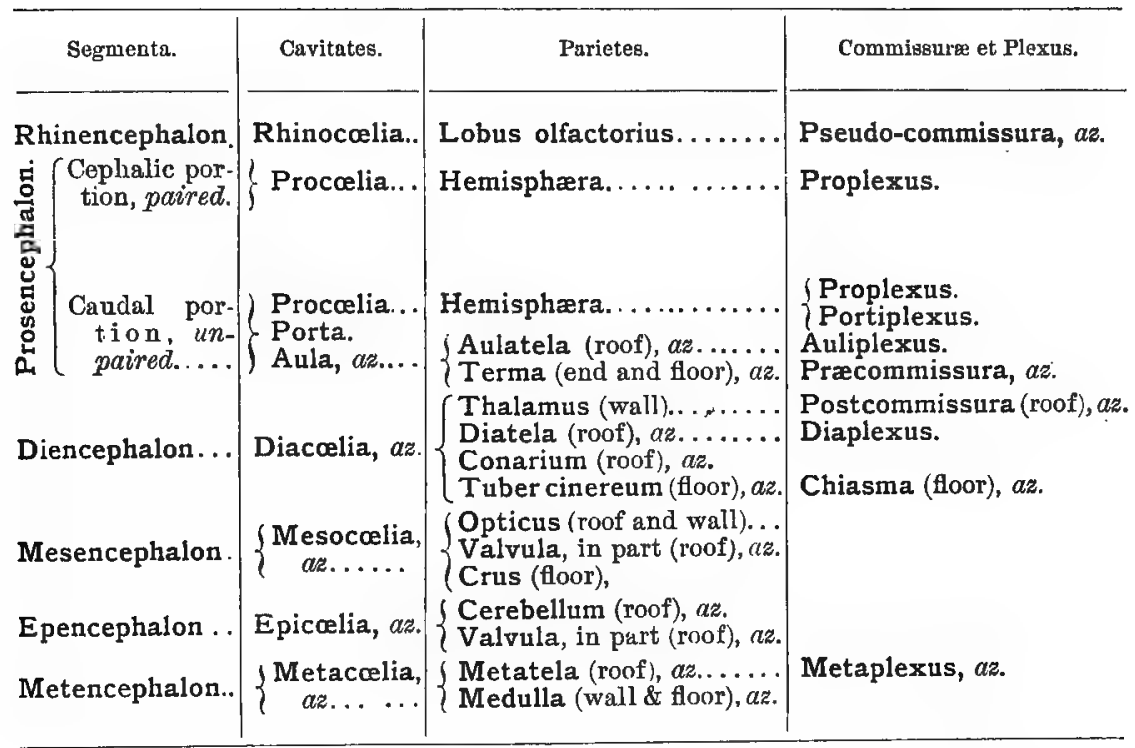

\$1070. An Ideal Simple Brain.-In accordance with the general plan of this work and the propositions given above $(\$ 1051)$, the examination of the actual brains of the frog and cat may be advantageously prefaced by the careful study of the preceding diagrams (Fig. 110-112), which present to the eye certain essential and fundamental facts.

Fig. 110-112. Schematic Diagrams of an Ideal Simple Brain.-Fig. 110-Longitudinal dextro-sinistral section, showing the relations of the cavities, the sequence of the encephalic segments and the relations of the cceliæ.

Fig. 111-Mesal aspect of the right half after hemisection, showing the contour and constitution of the colian floors and roofs.

Fig. 112-Transection of several segments, showing the colian parietes.

$\$ 1071$. Comments upon the Diagrams of the Brain.-Aside from the prominence given to the aula, these diagrams, so far as they are correct, convey no information or ideas not already the common property of neurologists; they are intended merely as visual aids to the student in the somewhat oncrous task of learning the sequence of parts and associating the names therewith.

They do not accurately represent the actual condition of things in any known brain at any stage of development. They correspond most closely with the brains of the frog and Menobranchus, but differ from the former in the disjunction of the Lobi olfactorii, from 
the latter in the greater differentiation of the segments, and from both in the subglobular form of the right hemisphere.

All that is shown, however, might really exist in a brain without contravention of our general knowledge of the structure and development of the organ ; hence, even if considerable modification of detail should be required, such diagrams would still be useful as an elementary introduction to the study of the brain.

At the outset the stident will do well to regard the diagrams as purely geometrical figures or as representing hollow masses of wood or iron. The shading is conventional, and intended to be uniform excepting with the three nerves of special sense in Fig. 110. No attempt is made to indicate the difference between the true nervous parts and those (conarium and hypophysis) which may consist of very different material, between the fibrous and cellular portions of the nervous tissue, or between the longitudinal and transverse fibers of the former.

The names, however, are of course those which apply to the various parts of the real brain. They are usually written in full upon or near the parts, but in Fig. 112, A, and a few other cases, abbreviations are employed upon one or both sides of the symmetrical figures.

The idea of diagrams like Fig. 110,111, was derived from the very clear and suggestive views of the typical brain given by Huxley (A, Fig. 19, 20); the only diacrams known to us comparable with those in Fig. 112 were published loy the senior author (2\%, Pl. III), but the membranes were not included. The transections of the frog's brain given by Stieda (22, Taf. XVIII) are of actual sections and likewise omit the membranes.

§ 1072. Fig. 110-Horizontal Section.-This affords the most complete view of all of the encephalic cavitjes (coelix) and of their relations to each other and to the segments of the organ.

The cœliæ form two series, mesal and lateral. The mesal cœliæ are direct continuations of the canalis centralis of the myelon, and open through the portæ into the lateral coliæ on either side.

The walls of the cœliæ are made continuous and of nearly uniform thickness ; in general the two sides are similar, but those of the mesocoelia are made unlike so as to indicate one of the most marked distinctions between the brains of the frog and Menobranchus, which will be specified later. The left of the two lateral masses has approximately the form of the hemisplere in the adult Amphibian; the right has what may be regarded as the primitive form of the hemisphere in the embryo in all Vertebrates ; compare Balfour, A, II, Fig. 25\%.

The pairs of ditted lines at the left of the figure indicate the limits of the encephalic segments, the names of which are written between the ends of the lines. Of these names, only one, prosencephalon, is given in fall; to the abbreviations of the others, meten., epen., etc., the word crphaton is to be added in each case. On the right, the short straight lines are likewise intersegmentrl) like those in Owen ( $\mathrm{A}, \mathrm{III}, \mathrm{Fig} .46)$; but the longer lines (A-H), which pass to the similarly lettered parts of Fig. 112, are persegmental; that is, they pass through the several segments instead of between them.

Three special sense nerves are represented. The auditorius $(N . a u$. $)$ arises from the metencephalon; the opticus $(N . o p$.) from the mesencephalon and diencephalon, and the numerous olfactorii ( $N N$. ol.) from the rhinencephalon.

The only portion of the wall here particularly specified is the terma (lamina terninalis), forming the cephalic boundary of the aula, the last of the mesal series of cavities.

$\$ 1073$. Fig. 111-Mesal aspect of the right half of an ideal simple brain, exclusive of the rhinencephalon and the cephalic portion of the prosencephalon.-Of course only the 
mesal colic appear in this figure, but the extent of the right procolia is indicated at the cephalic end of the right hemisphæra.

The porta is represented by the dark spot.

The order of succession of the ccliæ and of the segments is seen to be the same as in Fig. 110.

As in Fig. 110, the shorter transverse lines are intersegmental, and the longer ones (A-H) persegmental. In most cases the latter correspond closely with those of the persegmental lines in Fig. 110, but the line $\mathrm{H}$ is placed farther caudad, there being, in the frog and Menobranchus, no considerable difference between the parts of the hemi. spheres.

The several coeliæ are named as in Fig. 110, but in place of the names of the encephalic segments are given the names of their principal parts ( $\$ 1069$ ).

The floor of the mesocalic is formed by the parts called crura cerebri in the higher Vertebrates, and its sides and roof by the optici. Between the optici and the cerebellum proper, and perhaps belonging in part to both segments, is a thin and incurved portion of the roof, the valvula (Vieussenii).

The roof of the diacelia is variously constituted. Its caudal portion consists of norvous tissue, which in the frog, according to Wyman $(\mathbf{3 4}, 11)$, presents commissural fibers, the postcommissura (Stieda, 22, 17 ; Ecker, B, Abt. II, 10). The cephalic part consists mostly of the membranes, but presents the thickening commonly known as conarium or epiphysis or pineal gland (Wyman, 34,11), which, however, may be ouly an indication of the place of attachment of the true conarium (\$1084). The depressed floor presents a diverticulum, the infundibulum, leading to the hypophysis or pituitary body. Ventrad of the cephalic portion of the floor is a transverse band of fibers, the chiasma of the optic nerves.

The aula forms the last or most cephalic of the mesal series of cavities. Most of its floor and part of its cephalic boundary is formed by the terma, of which the pracommissura ( $p$ rcs.) is really a thickening and differentiation.

In this figure, instead of the unbroken lateral walls of the cclir, there are seen the roofs and floors of the meral series, irregular in contour and variously constituted in different parts. The proper nervous tissue is atrophied in several places, and the colian parieties consist chiefly of the two mcmbranes, the enveloping pia and the lining endyma $(\S 1080)$.

Each of these membranes is represented by a narrow black line, while in this, as in Fig. 110, the surface of the nervous tissue is represented by a heavy line.

The metatela, or roof of the metacclia, consists chiefly of the two membranes. The transverse ridges upon its ventral aspect in the frog are indicated by the undulations of the ental line. The roofs of the aula and of the ceplatic part of the diaccelia are also membranous (aulatela and dialela). In the frog and Menobranchus, although not in the higher Vertebrates, the diaccelian floor is devoid of nervous tissue along the meson. but no special name is given thereto.

$\$ 1074$. Fig. 112.-Transections of an ideal simple brain at several points.

The points of transection ase indicated by the lines connected with Fig. 110 and by the letters $\mathbf{A}-\mathbf{H}$. Of course the continuity of the coeile cannot appear in these sections, but they combine the distinctive features of the other two in exhibiting at one view the peculiarities of the sides and of the roof and floor.

(A) Transection of the Myelon.-More accurate representations are given of this in Fig. 99, 100, 109, but this indicates the existence of the canalis centralis, which expands to form the cceliæ, and the peculiar form of the deeply fluted column of cinerea, which 
is here made black ; the shaded portions represent the lateral, dorsal and ventral columns of alba.

The abbreviations signify as follows:-Clm. $d$., Columna dorsalis_- posterior white" column. Clm.l., Columna lateralis_-"lateral white" column. Clm.v., Columna ventralis- " anterior white" column. Ir. $d m s$., Fissura dorsimesalis-" posterior" fissure.

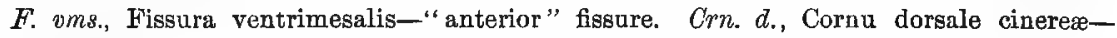
"posterior horn of gray matter." Crn.v., Cornu ventrale cinereæ- " anterior horn of gray matter."

(B) Metencephalon.-The ventrimesal fissure is nearly obliterated, but the sides of the dorsimesal are widely separated, and the central canal opened into connection with the space, metacœlia, so formed. The roof of the metacœlia is the metatela, composed of the pia and endyma, und the mesal ridge is indicated by the undulation of the latter.

(C) Epencephalon.-The floor and sides of the epicolia are similar to those of the metacoelia in the frog and Menobranchus, but in Mammals the former is reinforced by the pons, and the latter presents three sets of fbers, the pedunculi of the cerebellum. This latter is here represented in its essential character as a bridge over the epiccolic (Ecker, B, Abt. II, 8), without the mesal furrow which indicates the junction of the optici and thalami of the two sides.

(D) Mesencephalon.-Aside from its greater width, the chief diffcrence between this region and the epenceplalon is the existence of the distinct dorsimesal furrov, whence the name corpus bigeminum. In the higher Vertebrates, the floor of the mesocelia is more or less differentiated as the crura cerebri. Iu Menobranchus the mesocelia is large and simple; in most Mammals it is narrowed by the approximately uniform thickening of all the walls, and may be reduced to a mere passage. In the frog the cavity is very irregular, and no attempt is here made to indicate its form, excepting on the left of Fig. 110; hence the difference from Fig. 16 and 17 of Stieda (22).

(E) Diencephalon.-This transection is through the caudal portion of the segment. The thalami constitute the sides of the diacolia, and their roof presents the special band of fibers known as the postcommissura (pcs.). At the ventrimeson the proper nervous floor is absent, but the two membranes are unbroken. The slightly protuberant floor answers to the more distinct tuber cinereum of the higher Vertebrates; compare Stieda (22, Fig. 18). In the right of the diaccelia is represented a section of the free part of the right diaplexus of Menobranchus (\$ 1097), which does not exist in the frog.

(F) Diencephalon.-This is through the cephalic portion of the segment. The two lateral figures represent the eandal ends of the hemisphare, which project candad considerably beyond their points of attachment. In the diencephalic portion of the figure, the sides are the thinner cephalic portions of the thalami, and the floor is reinforced by the chiasma ; the roof is membranous at the sides, but thickened at the meson to form the conarium or its continuation (\$ 1084).

(G) Prosencephalon-Aula and Porta.-The two procœliæ are seen to communicate with a mesal cavity, the aula, through the two portæ. The floor of the aula is here formed by the term $\alpha$, but the roof (aulatela) consists of only the membranes. Compare Stieda (23, Fig. 21), where, however, the membranes are omitted, and the caudal border of the precommissura is included, so as to separate the dorsal part of the aula proper from the cephalo-ventral portion.

(H) Prosencephalon-Hemisphcerce.-This figure may represent the transection of almost any portion of the prosencephalon or rhinencephalon cephalad of the terma; compare Stieda ('20. Fig. 22). The hemispheres are usually in contact, but are united organically only in Mammals (by the callosum). Their cavities (proccitics) communicate with 
each other only through the porte and aula. In addition to the line representing the pia in direct contact with the niasses, the arachnoided (arch.) is represented by the line bridging the interval between them.

$\S 1075$. Relations of the Cœliæ.-These are most clearly indicated in Fig 110, representing a horizontal section of the typical brain. The coliæ form two series, caudal and cephalic. The former are mesal or azygous, the latter are lateral or paired. The arrangement may be roughly compared to a two-tined fork, the handle representing the mesal series and the prongs the two lateral extensions.

A more accurate analogy is with the apartments of a house. A narrow passage (the canatis centralis of the mýelon) opens into a wider apartment, or rather a suite of apart. ments but slightly distinguished from each other. From the farther (cephalic) end (aula) a passage (porta) upon either side opens into a wing or lateral extension, each containing two apartments, the second of which is closed at the farther end.

$\S 1076$. Comparison of the Brain with a House.-Let us inagine that a house consisting of a series of apartments in the order represented in Fig. 110 is completely enveloped by a continuous layer of tarred paper, and that its rooms are lined throughout with wall paper, the ceilings and floors being covered with the same.

Now it is conceivable that (1) the proper wooden wall of any apartment might be so reduced in thickness at any point as to hardly merit the name; (2) it might be omitted altogether along a given line, leaving only the two layers of paper ; (3) a fold of the cntal or lining paper might hang within the apartment; (4) between the two layers of the fold might be interposed a fold of the ectal or covering paper ; (5) instead of a complete fold of the ectal paper there might be supported in the fold of the ental some looped strings or fringes connected primarily with the ectal layer.

It is also evident that (1) while the fold of ental paper is really projected into the apartment, (2) the fold of ectal paper, or the strings or fringes of that paper, are covered by the ental paper, and are therefore not really within the apartment; (3) any force applied from within or without will be likely to rupture the wall along the line of interruption of the proper wooden wall, corresponding with the line of reflection of the ental paper therefrom to form the fold.

\$ 1077. Arachnoidea.-After the removal of the craninm and the dura which lines it, the brain of the frog, cat, man, and presumably of all Vertebrates, is found to be covered by two membranes. Of these, the ectal is the more delicate, and is known as the arachnoid. It was formerly described as presenting two layers, a visceral next to the brain and a parietal lining the dura; according to Quain (A, II, 573), there is insufficient evidence of the existence of the latter, and it is not represented herein.

The arachnoid passes from lobe to lobe and from fold to fold across intervening spaces or fissures, or dips but slightly therein.

\$ 1078. Pia.-This is in direct contact with the brain, follows closely the contour of the lobes and folds, is pigmented in the frog and some other animals, and supports bloud vessels which send branches into the substauce of the brain.

The pia is represented in all parts of Fig. 111 and 112; the arachnoid only in Fig. 112, $\mathrm{H}$.

$\S$ 1079. Endyma.-As intimated by Todd (A, 634), Duval (2, 164), Wyman (34, 15), Balfour (A, II, 364), and Quain (A, II, 540), and confirmed by our observations, all parts of the true cavities of the vertebrate brain are lined by a smooth epithelium called ependyma or endyma, the shorter name being preferable. This is akin to a serous mem. brane, and secretes a watery liquid which may (as in hydrocephalus) be produced in large amount. 
\$1080. Telce. - The pia bears to the brain which it covers the relation which the tarred paper does to the house, while the paper covering the interior walls is represented in the brain by the endyma.

Where the proper nervous substance of the cclian parietes is atrophied so that the pia and the endyma are nearly or quite in contact, the resultant is called a tela.

With the cat, and probably with other animals, the telæ sometimes seem to be not altogether devoid of nervous structure; indeed, it would seem quite possible that between a true membranous tela and a thin nervous lamina like the cephalic part of the vaivula there may be at least one intermediate condition.

When the pia is removed, the telæ, being connected tilerewith, are apt to be torn off; but the consequent exposure of the carities is no more proof of the presence of a natural orifice than is the fontanelle of a child, after the removal of the scalp and the dura, evidence that the cranial cavity is naturally in free communication with the outside of the head.

\& 1081. Plexuses.-Notwithstanding the more or less elaborate accounts in works upon Descriptive Anatomy, and some recent efforts to elucidate their mode of development, the precise structure and arrangement of the plexuses is far from well ascertained. Indeed, it is probable that a plexus may be formed in two or more ways in different species or in different parts of the same brain.

As we understand the matter, a ccelian plexus is formed in one of two ways :-

(A) Certain vessels of the pia are protruded entad of the proper nervous parietes so as apparently to enter the cœliæ.

(B) Certain parts of the pia containing vessels are carried as folds entad of the parietes so as apparently to enter the coliæ (Fig. 121).

In either case the endyma along the line of interruption of the proper nervous wall is reflected upon the intruded pia or vessels and covers them completely; lence, while the thinness of the epithelium permits osmosis to occur practically as if the vessels were free, a yet from a morphological point of view they are not free, but are excluded from the cavity just as the kidney or the intestine is excluded from the abdomen by the visceral layer of peritoneum (Fig. 78); in fact, the cases are strictly comparable.

$\S$ 1082. The alleged "Foramen of Magendie."-Magendie described (A), under the name "orifice des cavités encephaliques," an opening which he believed to exist in the metatela near the caudal end of the metacclia. I cuschka figured it (A, 'Taf. III, Fig. 1). but no other representation is known to us, although its existence is generally admitted (as in Quain, A, II, 513 ; Mihalkovies, A, 59).

Todd, however $(A, 641)$, believes such an orifice to be artificially produced; the senior author (14,543,555) could not find it in the cat, and its natural presence is emphatically denied by Duval $(\boldsymbol{1}, 33)$. See, however, Westbrook (I).

\$ 1083. Complete Circumscription of the Cavities.-First in 1876 (Wilder, 4;9, 136), and frequently since, we have made upon the cat's brain experiments (by inflation with air and by the injection of alcohol, water and plaster), which failed to indicate the presence of any natural communication (as by the rima or "fissure of $\mathrm{Bi}$ chat" or "great transverse fissure") between the coliæ and the exterior.

Upon a point of general arrangement like this there is every presumption in favor of uniformity throughout the vertebrate series; hence we may fairly regard the coelice (excepting at an early stage 
of development) as completely circumscribed, usually by nervous tissue, always by membranes.

This idea is more or less distinctly enunciated, upon various grounds and respecting various animals, by the following writers : Foster and Langley (A, 224); Balfour (A, 364); Todd (A, 634) ; Mihalkovics (A, 115); Duval (2, 33) ; Quain (A, II, 546); Hadlich (1); Löwe. (A) : Mivart (B, 266); and probably others.

\$ 1084. The Conarial Tube.-The above statement respecting the complete circumscription of the coeliæe excluded the earlier embryonic stages on account of the views of Götte (A). According to this observer, as briefly stated by Balfour (A, 856), the conarium is the remnant of the canal by which, as is commonly believed for Vertebrates in general, the cavity of the embryo myelencephalon communicates with the ectal surface of the bead. According to Stieda, as stated by Balfour (A, 357), a part of the conarial tract persists upon the outside of the cranium with some Amphibia, and the corresponding orifice of the cranium is identified as the parietal foramen of some fossil Reptiles by Owen (1). The entire "conario-hypophysial tract," as it is termed by Owen, has great morphological interest, but for our present purposes it seems best to omit any detailed account of the various views, and to refer only to the generally accepted opinion as to the primitive origin of the hypophysis from the alimentary canal. (Owen, 1 ; Balfour, A, II, 3.58 ; Quain, A, II, 735 ; Foster and Balfour, A, 91 ; Parker and Bettany, A, 10; Mihalkovics, A, 83).

\section{STUDY OF 'THE AMPHIBIAN BRAIN.}

$\S 1085$. Obtaining the Animals.-Directions for procuring frogs and Menobranchi and caring for them will be given in the Appendix. Large examples are to be preferred for the study of the brain," and they should be obtained alive or freshly killed.

$\S 1086$. Killing.-Place the animal in a jar or covered vessel of water, and pour off any water in excess of what is needed to simply submerge it.

Pour in a little chloroform, not more than 5 cc.; it will sink to the bottom as oily looking drops. The movements of the animal will usually diffuse it more or less, and the vessel may be shaken if necessary. Death will ensue in 10-30 minutes.

If ether is used, it will float upon the top of the water, the vessel must be shaken, a longer time is required, and the animal is more likely to revive unless the subsequent operations are done without delay.

If no anæsthetic is at hand, decapitation may be performed with the bone scissors by cutting caudad from each angle of the mouth to the caudal margin of the brachium in the frog and the caudal gill in Menobrauchus, and then cutting transversely so as to separate the cranium and maxilla, with the first two or three vertebræ, from the mandible and the rest of the body. This should be first 
practiced upon dead animals, and should be done very rapidly and by only three strokes of the scissors.

If only the cephalic portion of the brain is wanted for some special purpose, the animal may be pithed as directed in the Appendix; the brain should be exposed at once so as to prevent the clotting of blood about it.

If a toad is used, it should be put into a small jar or under an "open-top" bell jar, and a sponge saturated with chloroform suspended by a string near the top. In handling the toad, it is well to protect the fingers with gloves or a cloth, and care should be taken not to get the acrid dermal secretion into the eyes.

$\S 1087$. Injection.-For the special study of the telos and plexuses, the vessels should be injected. This is most conveniently done with cold flowing blue material from the bulbus arteriosus ; see Appendix.

$\S 1088$. Exposure of the Frog's Brain-Instruments and $M a-$ terials.-Small tray with waste paper; bit of cloth ; arthrotome; coarse forceps; pointed nippers; bone scissors, not too dull; wide mouthed vial containing at least 25 cc. of $62-67$ per cent. alcohol ; (to 10 cc. of water add 15 or 20 cc. of 95 per cent. alcohol; see $\S 273$ ); refer to some figure of the brain ( $\$ 1093$ ).

Fix the head by introducing the tip of the left index into the mouth and applying the pollex upon the snout. With the scissors or arthrotome, divide the skin between the cephalic angles of the eyes.

With the forceps, grasp the caudal cut edge of skin, and with the scissors, cut the skin along a line extending approximately caudad and just mesad of the eye and the ear (membrana tympani) on each side as far as a point opposite the caudal border of the brachium. Connect the caudal ends of the two incisions so as to remove the flap.

Grasp the left dorsal eyelid, and with the scissors cut mesad of the eyeball, gradually everting it and cutting the muscles until the round, white $N$. opticus is divided. Then cut more boldly and remove the entire ball.

From the orbit push a scissors blade through the mucosa into the mouth, and cut cephalad through the snout. Then cut caudad in the same way, along the same line or a little farther laterad, as far as the skin was removed. Finally, cut from, the angle of the mouth so as to remove the bony projection containing the left tympanum or middle ear.

With the nippers, tear up the muscles dorsad and laterad of the caudal part of the cranium and the first two or three vertebræ.

Cautiously nip off the projecting border of the cranium at the 
orbit until the left Lobus olfactorins or the hemisphere is exposed. Continue to remove that side of the cranium and the roof in very small pieces and with great care. The widest part of the brain (mesencephalon, optici) lies opposite where the tympanum was removed, and is liable to injury unless the adjoining cartilaginous capsule of the internal ear is removed very cautiously.

Caudad of the mesencephalon the brain is narrower and merges into the myelon, which must be exposed by the removal of the neural arches; it will be necessary to cut away part of a thin cartilaginous plate upon the shoulder, the suprascapula (\$383).

Pass a scissors blade through the pharynx to the dextral angle of the mouth and cut obliquely, so as to separate the cranium and two or three vertebræ from the rest of the body.

\& 1089. Exposure of the Brain of Menobranchus.-Instruments and materials ( $\$ 1088)$. With the arthrotome, cut the skin upon a transverse line just cephalad of the eyes. With the scissors, cut caudad along a line just mesad of the eye on each side to a point opposite the cauda! gill. Raise and remove the flap so outlined, noting that the skin adheres more closely than in the frog, and that between it and the cranium there are considerable muscles.

With the arthrotome scrape the muscles from the bone, beginning at the cephalic end of the exposed area. Alternately ventridnct and dorsiduct the head so as to indicate the position of the occipito-atlantal arthron. With the arthrotome, carefully pick up the membrane between the atlantal neural arch and the cranium so as to expose the metencephalon (medulla).

With the nippers remove the neural arches of the first two or three vertebræ, taking care not to wound the myelon. Then remove the occiput in like manner, inserting the nipper blade but a very little way. The larger part of the cranial roof is very thin and may often be lifted in slivers upon the point of the arthrotome, but with large individuals the nippers may be needed. Special pains should be taken not to disturb the metatela, a pigmented and vascular curtain just cephalad of the occiput, which sometimes adheres to the skull or is caught by the point of the instrument.

When the dorsal aspect of the brain is exposed, with the scissors cut away the left side of the head along the line of incision of the skin, and then cut across the vertebral column and other parts obliquely from the caudal end of the incision to the dextral angle of the mouth. 
\$ 1090. Preservation.--Place the preparation in the $62-67$ per cent. alcohol for at least two days. Then transfer it to 95 per cent. alcohol, where it should remain if a permanent preparation is to be made. For most purposes it is best to leave the brain supported by part of the cranium. If the base of the brain is to be specially studied, the base of the skull must be more completely removed; it is thin, but so closely in contact with the brain that much care is required.

§ 1091. Labeling.-To the vial containing the brain should be affixed a $\operatorname{tag}$ (§ 162) benring the name of the genus and, if possible, of the species, and stating the sex and locality of the animal, the date of the preparation, and the name of the preparator.

The genus will be either Menobranchus (sometimes called Necturus), Rana (frog), or $B u f o$ (toad). Probabiy there is only a single species of Menobrancbus, lateralis, but the doubt upon this point renders the noting of the locality essential. Of the toad there is but one species, lentiginosus (Americana), common in the Northern United States. There are several species of frogs, the largest being the pipiens of some authors, but the Cateshianc according to Jordan's " Manual " (A, 188).

If any generalizations are to be based upon the specific characters of the brain, and there is doubt respecting the determination, the entire body should be kept for reference, and labeled like the brain.

With the female Menobranchus the vent is a simple longitudinal slit, but with the male it presents numerous papillæ, especially in the spring. If in doubt, open the abdomen and note that the testes are elongated solid organs, while the ovaries are thin walled sacs, one on each side, with ova of two or more sizes, the largest in the spring being yellow and nearly as large as small peas. With frogs and toads the testes are small oval bodies, while the ovaries are voluminous laminæ with dark colored eggs.

$\S 1092$. General Inspection of the Amphibian Brain.-In what follows, including the dissection of the brain, no attempt is made to give exhaustive descriptions or directions.

Notwithstanding the numerous figures and descriptions of the frog's brain, none of its parts are known as they should be, and that of Menobranchus can hardly be said to be known at all excepting in the most general way. But our ignorance of many details of their anatomy need not prevent our use of them for the sake of illustrating certain features of brain construction which are comparatively obvious with them, but obscured with the brains of the bigher Vertebrates.

Since the frog is the more common and likely to be used more frequently, the directions apply primarily to it, while the peculiarities of Menobranchus are separately mentioned.

\$ 1093. Frogs' brains are figured and more or less fully described in the following works and papers. So far as we can judge, the figures are original in only the first seven : Wyman, 34 ; Ecker, B ; Gegenbaur (Lankester), A, Fig. 283; Leuret et Gratiolet, A, Pl. II ; Laurencet, A, Pl. II ; Spurzheim, A, Pl. III ; Bourgery and Jacob, A, VIII, Pl. 22, Fig. 4; Mivart, A, Fig. 74; Huxley, A, Fig. 59 ; Cyon, A, Taf. 24 ; McAlpine, A, Pl. 21 ; Burdon-Sanderson, A, Pl. 109 ; Packard, A, Fig. 373.

Figures of the brain of Menobranchus or other urodelous Amphibia are given in the following ; the accompanying descriptions are usually very meager: Mayer, A ; Laurencet, 
A. Pl. II ; Wyman, 34, P1. I ; Duges, 4, Pl. 10 ; Bourgery and Jacob, A, VIII, Pl. 22, Fig. 7 ; Owen (Linnean Transactions, XVIII, Pl. 27).

\$1094. Instruments and Materials.-Tripod magnifier; tine forceps ; beaded bristles ; small dish of 62-67 per cent. alcohol or 15 per cent. glycerin in which the brain may be dipped, or a vial of the same with a quill duster for applying the liquid.

Keep the brain wet with the alcohol or glycerin.

Remove the white masses of calcareous crystals at the side of the caudal region.

Compare the several regions with Fig. 110, or with figures in other works $(\$ 1093)$.

$\S 1095$. Note the lateral expansion of the myelon to form the metencephalon (medulla), abrupt in the frog, very gradual in Menobranchus.

Note the pigmented metatela which forms the dorsal surface of the metencephalon.

If the metatela is in place, its cephalic border more or less completely covers the cerebellum. Grasp its sinistro-cephalic angle with the forceps and invert it gently until it lies dextrad of the metencephalon. Note its thickness as compared with the myelonal pia, and the symmetrical corrugation of the ental surface. The cavity thus exposed is the metacolia (ventriculus quartus).

In Menobranchus the metaccelia is muoh elongated, and the caudal part of the metatela is very thin.

Cautiously remove the arachnoid from the dorsal aspect of the entire brain, excepting just between the divergent caudal ends of the hemispheres; it is little if at all pigmented, and bridges the interhemispheral fissure as shown in Fig. 112, $\mathbf{H}$.

The widest region is the mesencephalon (optici), the pia of which is thickly pigmented. Note the marked mesal furrow between the two lateral convexities, and that the longer axes of the latter diverge cephalad.

In Menobranchus the mesencephalon is but little wider than the adjoining segments, the mesal furrow is very shallow, and the lateral masses hardly merit the name of lobes.

Between the optici and the metacclia is a narrow transverse band, unpigmented and with its caudal margin dorsiverted, and separated from the optici by a somewhat deep furrow. This is the cerebellum, the dorsal part of the epencephalon. 
In Menobranchus the cerebellum is very narrow, its caudal margin is not dorsiverted, there is no furrow between it and the optici, and it is distinguishable from them mainly by the absence of pigment.

The diencephalon is decidedly narrower than the parts cephalad and caudad of it and is partly covered by their projections, and the mesal furrow is less deep than between the optici. In Menobranchus its caudal limit is not easy to assign.

Upon the cephalic part of the diencephalon rests the conarium (or its continuation, $\S 1084$ ) ; this body is not round, as almost universally shown, but oval, and anteverted so as to rest also upon the roof of the aula.

If the cephalic (really dorsal) end of the conarium is cautiously elevated, there will be exposed a delicate membrane, the aulatela, or roof of the aula.

The hemispheres, the largest portion of the prosencephalon, constitute the widest part of the brain with Menobranchus, and the longest in both it and the frog. In the latter they are compressed, in the former depressed. As already indicated, their caudal ends flare laterad so as to partly embrace the diencephalon and nearly reach the optici in the frog. Their mesal surfaces are in contact, but may be separated in the cephalic part by blowing between them or by a beaded bristle.

At the cephalic end of each hemisphere is attached the corresponding Lobus olfactorius, the lateral half of the rhinencephalon. The Nervi olfactorii may be traced thence to the nasal capsules. The lobes are separate in Menobranchus as in most Vertebrates, but in the frog and other Anura they are united by connective tissue constituting a pseudo-commissura.

$\S$ 1096. Dissection of the Amphibian Brain.-The arachnoidea and the metatela have been removed as directed in $\$ 1095$.

Instruments and Material.-Tripod magnifier; fine forceps; beaded bristles; very sharp scalpel, preferably small ; fine scissors; syringotome or tracer ; flexible blowpipe ( $(138)$; alcohol or glycerin as directed in $\$ 1094$.

Note at the bottom of the metacœlia a mesal furrow, which is continuous caudad with the canalis centralis of the myelon.

With the flexible blowpipe, blow cephalad under the cerebellum, and note that all the parts of the brain are inflated, showing the existence of a continuous series of cavities, as represented in Fig. 110. 
With the scalpel previously dipped in alcohol; or with the fine scissors, cut off obliquely the latero-caudal angle of the left hemisphere. This exposes the corresponding procœlia, and blowing into it inflates all the other parts. With the scissors, remove the entire latero-dorsal wall of the hemisphere, noting its extension, rhinoccelia, into the base of the Lobus olfactorius.

Blow gently upon the mesal wall of the hemisphere at about its middle, and note the presence of an orifice through which air passes into the other cœliæ ; this is the left porta or "foramen of Monro." Note the continuity of the hemisphere wall at all other points.

Wyman mentions $(34,15)$, but does not figure, another opening from the procolia dorsad of the thalamus; this, as he suggests, would probably correspond with the rima or " fissure of Bichat." It could not appear until after the removal of the pia, and we have not satisfied ourselves of its existence.

Pass the beaded bristle through the porta toward the opposite hemisphere, and note that it enters the other procœlia, as indicated by the protrusion of its wall at some point.

Just cephalad of the porta is an elliptical raised surface which is thought by some (Wyman, 34, 15) to represent the striatum of the higher Vertebrates, but there is doubt upon this point.

In Menobranchus the porta is partly filled by a plexus which extends cephalad in the procolia; this is the proplexus, which may be snipped off with the scissors, but never pulled upon. The porta is much longer than in the frog, but there is no thickening of the mesal wall of the hemisphere Jike the supposed striatum of the frog.

With the scalpel, remove the lateral prominence of the left opticus, and note that a somewhat expanded lateral portion of the mesocclia communicates by a constricted orifice with the mesal portion and so with the corresponding expansion in the right opticus. Then remove the dorsal wall of the entire mesocœlia with the scalpel and scissors, and note the marked folding, the valvula, by which the cerebellum is continuous therewith. Pass a bristle ventrad of the cerebellum into the mesocœlia, and then sinistro-cephalad through the diacclia so as to emerge in the left procolia.

Slice off the caudal part of the diacœlian roof, including the part known as postcommissura. Note that the walls (thalami) are quite thick and nearly in contact, but that the interval is a nearly simple vertical fissure and not a wide and partly divided cavity like the mesocoelia. Pass a bristle cando-ventrad so as to enter the hypophysis. 
By placing the bead of the bristle, or the tip of the tracer or syringotome, in the cephalic part of the diacclia, note that the roof, diatela, is partly membranous and partly formed by the thickening known as the conarium $(\$ 1084)$.

Then slice away the thalami to a still lower level so as to expose the diacclian floor. Note that it is depressed, and bounded caudad and cephalad by transverse ridges; also that there are no plexuses therein.

Slice off both hemispheres to the level of the conarium. Then remove another slice, including the conarium and the aulatela upon which it rests. This will expose a wedge-shaped cavity, the aula, which is bounded as follows: caudad, by the ridge mentioned above as the cephalic boundary of the diacclia; laterad, by the portce whose length nearly coincides with its own; cephalad, by the terma, a lamina of nervous tissue uniting the mesal walls of the hemispheres at the cephalic end of the portæ; (the terma is more easily seen if the left $\mathcal{L}$.ol. and cephalic end of the hemisphere are gently pushed away from the right) ; dorsad, by the aulatela, the atrophied continuation of the terma.

If the mesal wall of the left hemisphere be removed, the extent and form of the right porta may be seen.

$\S 1097$. With Menobranchus the conditions are so different as to require special directions :-

Introduce a scissors blade just caudad of the cerebellum on either side, and cut cephalad to the caudal end of the hemisphere; do the same on the otlier side, and turn the flap so formed cephalad upon the hemispheres. This exposes the epi-, meso- and diacclia. Note that the lateral walls are thicker than the roof, and that the ental surface of the roof presents a slight transverse ridge which indicates the division between the diencephalon and the mesencephalon.

The tloor of the mesocolia is nearly level, and its cephalic margin overhangs the passage-the canal of the infundibulum-leading to the hypophysis.

In the diacœlia lie side by side a pair of string-like plexuses-the diaplexuses-which are attached to the aulatela or diatela and extend the whole length of the cavity. They should be drawn to the sides or cut off, but not pulled upon at all. The floor of the diacclia is then seen to be irregular, sloping from each side to a mesal furrow. 
The cephalic end of the diacœlia is decidedly narrowed, and the walls are thin.

$\S 1098$. Remove the basis cranii so as to expose the basis encephali as far as the aula. Observe the sub-cordate hypophysis underlying the mesencephalon, but attached by its base to the slight intumescence-the tuber cinereum-forming the floor of the diencephalon.

In case more than one brain is examined, the second should be transected with a very sharp scalpel, and drawings made to show the form of the cœliæ at different points. A third brain may be divided upon the meson, and a fourth opened from the ventral side. With all of these the metatela should be left in place. Still others may be prepared to show special points. When many brains are available, each should be devoted to a given section or dissection, all other parts being untouched, so that the special features may be more easily recognized.

\section{REMOVAL OF THE BRAIN OF THE CAT.}

$\S 1099$. The method first described is to be preferred when the brain is wanted entire, and especially when the length of the nerve roots is an object; see Wilder, 11.

Instruments and Materials.-Medium scalpel, Charrière scalpel; arthrotome; tracer; curved scissors; bone ecissors ; forceps; nippers, medium or large and small; large tray for the cat ; small tray, or a folded cloth, for the head; block; small towel, or piece of muslin, for aiding the grasp of the head; waste paper; basin and towel; dish of 7 per cent. brine, about $6 \mathrm{~cm}$. deep and 20 wide. containing some well soaked cotton; bowl of normal salt solution (15 grams of salt to $2000 \mathrm{cc}$. of water), sufficient to cover the head after its separation from the body); bowl for catching the blood; glass box ( $\$ 307)$; wide mouthed jar or covered dish of $62-67$ per cent. alcohol, with some well souked cotton at the bottom; a cat's skull ; figure of the basis cranii (Fig. 5\%); figure of the busis encephali (Fig. 115, Pl. II, Fig. 3) ; Table of the cranial foramina (\$ 562).

$\$ 1100$. Killing the Cat.-The cat may be drowned, but is more conveniently anæsthetized ( $\$ 192)$. Kill the fleas as directed in $\S 193$. As soon as respiration ceases, suspend it by the head over a pail or the sink, and expose and divide the femoral artery and vein (Fig. 39) centrad of the valves in the vein ( $\$ 362)$; even if little blood escapes, the amount in the brain will be reduced. If it be desired to ascertain the weight of the entire animal, the blood should be caught in the bowl and weighed.

For injection with alcohol, see $\$ \$ 284,285$.

For injection of the plexuses, see $\$ 1126$.

If the more delicate internal parts or the microscopic structure are to be studied, the remaining operations for the procurement of the brain should be performed within 24 hours. But if the specimen is desired only for the fissures or the coarser anatomy, removal may be deferred for a week, provided the head be kept in a cool place. It should not, however, be allowed to freeze.

\$ 1101. Decapitation.-From the dextral angle of the mouth divide the skin along a line extending nearly caudad for $6-8 \mathrm{~cm}$. If 
the skin is to be mounted, this should be the only incision, and the skin must be dissected from the mandible as well as from the rest of the head. But if, as is more often the case, the skin is not to be preserved, while the vessels and nerves of the neck are to be examined, make a corresponding incision from the angle of the mouth upou the opposite side.

In all subsequent operations, unless otherwise stated, both sides are to be treated alike.

Dissect the skin from the maxilla as far as the ventral margin of the orbit and cut the nasal cartilages. Dissect the skin from the nasal and frontal regions, including the dorsal and ventral lids but leaving the third lid, Membrana nictitans, attached to the ball. Remove the skin from the rest of the head, dividing the meatus auditorius close to the head. The parotid gland (Fig. 87) will be removed with the ear, but the submaxillary, of a darker color, will remain with the head. Reflect the skin from the cervical muscles for about $2 \mathrm{~cm}$. caudad of the crista lambdoidalis.

With the arthrotome, dissect the origin of the $M$. massetericus from the zygoma, noting that its cephalic and caudal borders are strengthened by tendinous bands which must be cut $(596,19)$. Push a nipper blade between the eyeball and the cephalic root of the zygoma, and nip the latter as close as possible to the maxilla. Then nip the caudal root at the angle between the transverse and longitudinal parts of the zygoma, just laterad of the Fossa glenoidalis; remove the zygoma with the bone scissors.

Grasp the lateral aspect of the eyeball with the forceps, and rotate it mesad so as to expose its attachments, by the muscles and $N$. opticus, to the bottom of the orbit; cut the attachments with scissors, leaving the Mb. nictitans connected with the ball. If the eyes are to be studied or preserved, mark them right and left by numbers or tags; the proper position is always indicated by the Mb. nictitans; see Chap. XI.

Slightly ventriduct the mandible and move it from side to side so as to indicate the position of the Arthron temporo-mandibulare. Usually the capsule has been opened already in nipping the caudal root of the zygoma. If not, it is to be cut while on the stretch by inserting the arthrotome, and cutting until separation is complete on that side.

Dissect the $M$. temporalis from its cranial origin, and then from its insertion upon the Processus coronoideus of the mandible. 
Bring the mandible to a right angle with the rest of the head, and divide the soft parts at the angles of the mouth if any remain. In doing this, insert the arthrotome sidewise so that it may pass between the Prc. coronoideus and the projection left after the removal of the cephalic root of the zygoma.

Feel for the caudal border of the hard nalate and for the tips of the Ossa pterygoidea (Fig. 57); at a point midway between push a scissors blade entad of the soft palate, and divide it; then divide the mucosa forming the dorsal wall of the postnares, and dissect it up as far as the atlas.

The mandible is now attached to the rest of the head by some muscular fasciculi and by the slender piers of the hyoid arch (Fig. $30, \S 224)$. These last join the skull at the lateral side of the bullæ, where they are to be divided with the arthrotome; if it be desired to examine the mode of their attachment, they may be cut with the bone scissors at a little distance from the attachment.

Ventriduct the tip of the mandible still farther, and dissect off the muscles, rectus anticus capitis ( $\$ 628$ ), that are inserted between the bullæ; near the caudal ends of the mesal borders of the builæ emerge several nerves, which should be divided with the scissors or a sharp scalpel at about $1 \mathrm{~cm}$. from the skull. By continuing the removal of the muscles across the Arth. atloöido-occipitale this is exposed. Put the mombranes upon the stretch, and divide them with a Charrière scalpel along the cephalic border of the atlas. This exposes the myeron, which is to be divided in the same way. The remaining ligaments and the cervical muscles may be cut with the arthrotome, and the skull proper is then separated from the rest of the body. Place the skull in the normal salt solution, and wash the hands and the instruments which have been used.

\$ 1102. Exposure of the Brain.-In the later stages of the operation there is considerable risk of injuring the brain by the unintentional pressure of the nippers.

In whatever way the bone is grasped, when force is applied, the tendency is to approxi mate the cutting edges as nearly as possible, and thus to bring their planes into right angles with the surface of the bone. This of course crowds the convexity of the ental blade against the brain, and may crush it seriously. It may occur either from the turning of the nippers in the hand, or more frequently from the escape of the skull from the grasp of the other hand. The accidents may usually be aroided by keeping the danger in mind, by having the right hand dry, and aiding the grasp of the more or less slippery skull by a small towel or bit of conse muslin; this last is also desirable during some stages of the operation as a protection of the hand itself from abrasion. 
In using the nippers, another precaution is to be observed. If the bit of bone to be removed is attached only to bone, it may be either cut or broken or twisted off ; but if it adheres to the dura or other soft parts, only cutting should be employed; it is safer to use the bone scissors after nipping the bone.

When any bit of the skull is broken off, lift it very carefully, and examine its ental aspect for nerve attachments.

During the exposure of the brain, the head should be frequently dipped into the $n$. s. s. If obliged to suspend the operation for more than an hour, wrap the head in a cloth wet with the $n$. s. s., and set in a cool place.

Trim off the soft parts, including the meatus auditorius. Nip off the caudal root of the zygoma, including the Fs. mandibularis. Insert a nipper blade into the meatus auditorius, and remove the bulla in fragments. With the scissors, cut away the membranes attached to the margin of the Fm. magnum.

Nip off the occipital condyles, with the intervening area of the basioccipital for $2-3 \mathrm{~mm}$. from the foramen. Insert a nipper blade between the dura and the bone 5-6 $\mathrm{mm}$. from the meson and in line with the mesal border of the cephalic part of the bulla, and nip out the basioccipital as far as the middle of the length of the bulla.

At or near the angle left after the removal of the condyle and the basioccipital, the $N$. hypoglossus enters the Fm. condylare, and passes cephalad to emerge on the ventral aspect of the skull by the $F m$. jugulare. If the series of roots do not appear, carefully remove a little more bone until they do. If the nerve roots are to be specially studied, endeavor to nip off the bone surrounding the Fm. condylare, so as to save the trunk. On emerging upon the ventral aspect of the skull, the $N$. hypoglossus will be found to lie very near the Fm. jugulare, and to be more or less intimately attached to the NN. glossopharyngeus, vagus and accessorius, which penetrate the bone by that foramen. In attempting to separate the $N$. $h y p$., great care must be used to avoid any traction upon the roots, which readily pull out of the medulla.

of the other three nerves, the accessorius is the most caudal, and the most readily distinguished, but at this stage it is as well to leave them together, simply endeavoring to remove the bone surrounding the foramen, and to save the trunks pretty long, at least upon one side. Upon the other, it will save time to cut the roots just entad of the skull, and the same may be done on one side with the remaining nerves, or with all upon both sides in case the brain is not to be employed for the study of the ectal nerve origins.

The dorsal wali of the bulla is hard, but readily crumbles be- 
tween the nippers. It may be removed in small pieces, so as to save the $N N$. facialis and auditorius which enter the Meatus auditorius internus, and the little Lobulus appendicularis of the cerebellum which is lodged in a slight fossa (Fs. ap.) just dorsad of the meatus (Fig. 59, M. a. $i$.).

Since no nerves are transmitted by the mesal region of the basis cranii, it may be removed with comparative freedom as far cəphalad as the sella (Fig. 59), where some care is needed to avoid injuring the hypophysis (Fig. 88, Hy.).

The skull may now be held more securely by the facial region, especially if a towel is employed. In removing the bone at each side of the meson, and just cephalad of the bullæ, great care is required to disengage the nerves which emerge by the Fm.Fm. ovale, rotundum and lacerum anterius. These nerves, the $N N$. oculomotorius, trochlearis and abducens, with the ophthalmic, superior maxillary and inferior maxillary divisions of the $N$. trigeminus, penetrate the bone more or less obliquely, and are closely surrounded by dense connective tissue.

The entire maxilla is now to be removed by first nipping the interorbital region just cephalad of the fronto-maxillary suture, and then, with the bone scissors, cutting toward this point from just caudad of the cephalic root of the zygoma. The scissors should be kept as far cephalad as possible, so that the Lobi olfactorii may not be injured. This plan serves equally well for some dogs, but with the larger breeds, which have prominent olfactory lobes, the interorbital region should be nipped at about the middle of the length of the nasal bones.

$\S 1103$. Remove the mesal wall of the orbit and the turbinated bones, using care not to crush the very soft Lobi olfactorii. The olfactory nerves should be divided, a few at a time, with the scissors or the tip of the scalpel, and all pulling and twisting of the parts must be avoided.

The large and white Nervi optici will have been seen in the orbit, and should be divided near the bone. In removing the plate (Fig. 60) upon which the chiasma rests, use care not to pull upon it, lest the terma or other delicate parts should be torn.

During the remaining steps of the operation, the head must be held by the parietal regions, and with great care, so as to avoid pressure of the tips of the fingers upon the brain. The bone also must now be cut by the nippers rather than twisted or broken. 
Nip off the supraoccipitale, including the dura, as far as the Crista lambdoidalis. Then nip off the crista itself in its whole extent until the attached border of the bony tentorium is seen to be free from the parietalia. Then divide the ventral ends of the tentorium as follows:-

Hold the head with the ventral side down, support the caudal divisions of the brain with a disengaged finger, and with tracer and scissors separate the cephalic surface of the cerebellum from the tentorium.

Introduce a nipper blade between it and the hemisphere on either side, in such a way that the greater convexity is toward the hemisphere rather than the cerebellum; the cut is to be made at the level of the Sutura squamosa; the width of the tentorinm at this point is about $8 \mathrm{~mm}$., and the nipper blade should not be introduced to a greater depth than that, for fear of injuring the optici. In closing the blades, the head should be held very firmly so that no rotation may occur. The detached tentorium may be extracted by the forceps, or by the nippers used as forceps, any adhesions being carefully separated with the tracer or scissors.

Hold the head over the $\boldsymbol{\gamma}$ per cent. brine, with the ventral side down, and nip out, piecemeal, a triangular piece of the calva. The mesal adhesions of the dura may be divided with the scissors, but elsewhere the dura is to be left upon the hemispheres. As the hemispheres begin to fall, hold the head so that they are supported by the brine, and then snip all remaining adhesions until the entire brain is free and floats in the liquid.

$\S$ 1104. Removal of the Dura.-Saturate some cotton with the brine, and place it under the brain, so that about one third of the organ projects above the surface. Avoid handling and lifting the brain; move it by shifting the cotton or by grasping the dorsal portions of the dura. Remove the dorsal and lateral parts of the dura by grasping the free borders left by cutting along the dorsimeson, and cutting out piece by piece with the scissors. Then grasp the falx just dorso-caudad of the Lobi ol., at the straight transverse fissure- $F$. cruciata ; introduce the scissors about $5 \mathrm{~mm}$., and cut the falx. Gently draw the cephalic portion cephalo-ventrad between the Lobi ol., and remove it. Draw the caudal portion caudad, and carefully cut all its attachments.

Turn the brain upon its dorsal surface, and remove the ventral portions of the dura with great care and in small pieces. Especial 
pains are needed in connection with the hypophysis and the nerves, and all pulling must be avoided. On one side, at least, it is well to leave the dura still attached to the nerves and the great Gasserian ganglion upon the $N$. trigeminus (Fig. 115; Plate II, Fig. 3, Gn. G.), to be more completely removed at the time of the removal of the pia.

\& 1105. Transfer to the Alcohol.-Place a large spoon or watch glass at the side of the brain, and pull the cotton which supports it, so as to roll it into the glass, resting upon its dorsum. Let the brain slide from the glass into the alcohol so as to rest upon the cotton therein, still with the ventral side up.

Set the bowl with the alcohol in a cool place, and change the position of the brain at intervals of 5-10 hours during the first three days, by pulling the cotton in various directions. At the end of about three days, transfer the brain to 95 per cent. alcohol, where it may remain indefinitely. For a few days, however, it should rest upon cotton, and its position be occasionally changed. For the management of alcohol employed in the preservation of brains, see \$§ 286, 296.

$\S 1106$. Weighing the Brain.-If this is to be done, handling of the brain may be avoided as follows: Place the bowl of alcohol into which the brain is to be put upon the scales, and pour in alcohol of the same strength until it balances an even number of grams, e. g., 400,410 or 420 . While the brain is in the spoon or watch glass, pour over it some of the same alcohol, and then let the latter drain off as much as possible by tilting the glass and supporting the brain with the fingers or a bit of cotton. Then transfer to the bowl of alcohol as above directed, and the increase in weight will represent, with approximate accuracy, the weight of the brain.

$\S 1107$. Removal of the Pia.-This is most easily accomplished at the time of the removal of the brain to the stronger alcohol. At any subsequent period the pia is apt to be more firmly adherent. If the brain has been allowed to dry at all during its removal from the skull, the pia comes off with great difficulty, or parts of the cortex are torn off with it.

$\$ 1108$. Instruments and Materials.-Coarse and fine forceps; medium or fine scissors; wetting bottle of 15 per cent. glycerin; cotton thoroughly wet with water, and so molded as to form a sort of shallow cup in which the brain may be grasped, or on which it may rest without danger of rolling off.

Place the brain upon the cotton, and wet it with the glycerin. 
Then let it rest upon its ventral side, and grasp it in the cotton, firmly yet gently. Grasp with the forceps the fold of pia which occupies any one of the tissures, especially at the point of forking or junction with another fissure, and pull along the line of the fissure. Usually the fold of pia will come out easily, and with it will be removed some of the pia covering the free surface of the gyri between it and the adjoining fissures.

Proceed thus until the pia has been removed from the dorsal and lateral aspects of the hemispheres. Avoid pulling across the line of the fissures. The larger forceps are easier to work with, and less apt to puncture the brain ; but the fine forceps are sometimes required for the removal of the pia from the bottom of a deep fissure. The caudal surface of the hemispheres may be reached by slightly ventriducting the cerebellum. The mesal pia can be removed only at the margins of the hemispheres.

On one side, preferably that on which the $N$. opticus was cut shorter, raise the mass of nerves formed by the divisions of the $N N$. trigeminus and abducens by its lateral border, and cut with the scissors the $N$. oculomotorius which holds the mesal border close to the brain. This will permit the mass to be turned caudad so as to expose the course of the slender $N$. trochlearis which emerges from between the hemispheres and the cerebellum. It also permits the removal of the pia from the region just laterad of the hypophysis. Grasp the pia on the ventrimeson just caudad of the Lobi ol., and pull caudad so as to remove it as far as the chiasma, taking care not to tear the delicate terma just dorsad of the chiasma. Then remove the pia from the olfactory tracts.

In removing the pia from the metencephalon, the position of the nerve roots should be constantly kept in mind, the tripod (Fig. 26) should be frequently used, and traction should beavoided. It is very difficult to preserve the delicate funiculi of the $N$. hypoglossus, for their connection with the pia seems to be closer than with the brain. Sometimes it may be necessary to submerge the brain in water or alcohol, so as to float the roots out and render them more apparent. In some cases it is safer to cut carefully about the point of passage of the root through the pia, leaving a bit of the membrane attached thereto.

As suggested on a previous page, it is often as well to leave the roots longer on one side than the other, but the choice may be determined mainly by the degree of success in the various operations which have been described. 
\$ 1109. Other Methods of Removing the Brain.-The following methods are simpler and more expeditious than the precoding, but less satisfactory in some respects.

(A) By Nipping away the Calva (Vault of the Cranium).-This method is especially adapted for the rapid exposure of the dorsal and lateral aspects of the hemispheres or the cerebellum. When, for example, the brain of a recent or alcoholic specimen of some rare animal is believed to be so soft that extraction by the usual method is not feasible, a drawing or photograph can be taken so as to represent at least the form, and the arrangement of the fissures.

Instruments and Materials.-These are the same as for the method already described ( $\$ 1099)$.

Decapitation.-This is to be performed as directed in $\$ 1101$, and the eyes, zygomata and temporal muscles removed.

\$1110.-Exposure.-With the large or medium nippers cautiously penetrate the dorso-lateral aspect of the cranium, but without piercing the dura. Pass the tracer between the bone and the dura at all sides, keeping the point away from the brain; then remove so much of the bone as is separated. When the orifice is sufficiently enlarged, employ the probe or the handle of a scalpel in place of the tracer. If the adhesions along the meson are very firm, remore the bone on both sides, and divide them with the arthrotome or scalpel.

In exposing the cephalic part of the hemispheres, the large frontal sinuses (Fig. 56, 59, 60, §524) will be opened; note the density of the ectal layer (tabula externa) of the cranium, and the elliptical orifices of the infundibula.

If the hemispheres only are to be exposed, or if it is desirable to preserve the facial region, the dura may be divided with the scissors along the margins of the bone, and the falx (Fig. 88) carefully cut near the cephalic and caudal ends of the hemispheres, so as to permit the removal of the dorsal dura. The lateral aspects of the hemispheres may be exposed so far as desired, the olfactory crura (Pl. I, Fig. 2) may be divided, and the hemispheres together with the parts covered by them removed by holding the head so that its frontal region tends to fall out, and successively dividing the $N N$. optici, the infundibulum and the other nerves as they appear at the base of the brain, and lastly the crura cerebri.

$\S 1111$. Commonly, however, the removal of the dura should be postponed, and the olfactory lobes should be carefully exposed by the removal of the thin bones which surround them; this is of course facilitated by removing the entire maxillary region as directed in $\S 1102$. 
The caudal regions of the hemispheres rest upon the bony tentorium (Fig. 59, 88, $\S 552$ ); note that the dura is continued upon its surface and closely attached, but quite independent of it.

Remove the supraoccipitale ( $\$ 1013)$, leaving the dura upon the cerebellum if possible, and then the Crista lambdoidalis and the parts of the parietalia between the latter and the attachment of the tentorium. The latter may then be nipped at each side as directed in $\S 1103$, and the central part removed. The tentorial dura commonly adheres so closely that it must be removed likewise by snipping it along the attached border of the tentorium. In removing it, care must be taken not to injure or tear away the conarium (Pl. II, Fig. 4, cn.). The entire dura so far as exposed may now be lifted as directed above.

Remove more of the right side of the skull so as to expose the Lobulus hypocampa (Pl. I, Fig. 2, Ll. $h m p$.); the perioticum (Fig. 59, pro.) should be cracked with the nippers and removed in small fragments.

$\S 1112$. Removal.-Hold the head so that the right side of the brain tends to fall out of place. The dextral nerves are to be divided with the scissors or small scalpel, beginning with the most caudal. If the hypophysis is to remain attached to the brain, the division of the optic and trigeminus nerves should be deferred until after it is dislodged or the bone is nipped from about it. Finally, the sinistral nerves may be divided and the brain allowed to fall into the brine.

8 1113. With some modifications, the method just described is well adarted to the removal of the brain from young human subjects. If the skull cannot be cut wit ti.e nippers or bone scissors, it may be trephined in various places and the intervening areas broken away. The dura should be retained as long as possib'e, and the entire head should be floated in strong brine so that the brain way not bear its own weight at all. If the head is still attuched to the body, the latter may be laid upon a box as high as the dish so that the head may hang over the side of the latter.

8 1114. (B) By Hemisection of the Head with a Saw.-.The advantages of this method are :- (1) It is comparatively expeditious; (2) the skull may be preserved ; (i) the brain may be hardened in situ, safely transported if desired and removed at a future time.

The two following objections are more apparent than real :-

(1) The skull is " mutilated."-On the contrary, for nearly all purposes of study, to halve a skull is to double its value. Even if it is to be mounted with the skeleton or within the skin, the two halves may be readily conjoined.

(2) The brain is injured by the saw. - If the hemisection is accurately mesal, the only parts really destroyed are the conarium and crista; all other parts which cross the meson are recognizable, and the mesal aspects of the hemispheres are often untouched. If it be 
especially desirable to preserve the mesal surface of one hemisphere and the parts upon the meson, the plane of section may be $1-2 \mathrm{~mm}$. laterad (usually sinistrad) of the meson. The lateral incisor will be destroyed unless previously removed; but the rest of the skull will be unharmed, and its meson will be available for study.

\$1115. Instruments and Materials. - The same as for the method first described ( $\$ 1099$ ), with the exception of the nippers, and with the addition of the small back saw (\$152) and the macrotome (Appendix).

§1116. Decapitation.-Remove the skin as directed in $\$ 1101$; if the skin is to be mounted, divide it only from the dextral angle of the mouth.

With the arthrotome divide the $M$. temporalis along the dorsal margin of the zygoma and the caudal margin of the orbital process of the 0 . malare, and dissect up the muscle for 1-2 $\mathrm{cm}$.

This will expose the end of the Prc. coronoideus of the mandible; cut about it so as to free it from muscular and tendinous attachments.

Separate the masseter (Fig. 67) from the ventral border of the zygoma, cutting to the Prc. coronoideus. At a point nearly midway between the meatus aud. and the orbital process of the malar is situated the Arthron temporo-mandibulare; determine its exact position by pressing with the finger while the mandible is moved in various directions. Open the arthron as directed in $\S 1101$.

Ventriduct the mandible and cut any remaining attachments; then proceed as directed in $\S 1101$.

\$ 1117. Mesal Hemisection.-With the arthrotome cut between the two mesal incisor teeth and between the nasal bones; also upon the meson at the Crista lambdoidalis and the caudal border of the o. basioccipitale.

Adjust the slide so that when the head is forcibly pressed against it the cuts just made coincide with the slit and groove of the macrotome. Rest the head with the canine teeth in the rebate, hold the saw obliquely so that it runs in the slit and cuts through the facial region. Then hold the saw more nearly horizontal; finally, place the skull flat upon the board and complete the hemisection with long, steady strokes.

§ 1118. Lateral Hemisection.-If it is desirable that the meson should be absolutely uninjured, remove a mesal incisor, and make the initial guiding cuts through the periosteum about $1 \mathrm{~mm}$. laterad of the meson, so that the saw may pass through the vacant socket 
and through corresponding points upon the basi- and supraoccipital bones.

$\$ 1119$. As already indicated ( $\$ 1114$ ), the brain may be hardened while in the cranium. Each half should be loosened a little as presently directed, and 95 per cent. alcohol injected between the dura and the brain at two or three points. The half heads may then be placed in 62-67 per cent. alcohol for two days and then transferred to 95 per cent.

Usually, however, the brain should be removed ( $\$ 1121$ ), and the dish of alcohol should have a flat bottom, or a piece of flat glass should be placed in it, so that the hemiencephala may rest upon their mesa. The operation will be facilitated by reference to a hemicranium or to a figure of its ental aspect.

$\S 1120$. Weighing.-(A) Direct method:-As soon as the hemiencephala are in the normal saline solution, lift them out by means of a slip (\$95), upon which their mesa should rest, let the liquid drain off, and place them, still upon the slip, upon the weighing pan. Upon the other pan place a similar slip which has been dipped in the same liquid.

(B) Indirect method:- Just before removing the brain, and after snipping off the falx, weigh the two halves of the head. Weigh the hemicrania immediately after the removal, and the difference will represent the weight of the brain.

$\$ 1121$. Removal.-Wash the mesa with a gentle stream of water, or by movement to and fro in a dish of water. If the falx (Fig. 88) is upon this side, grasp the edge with the forceps, and snip it along its attachment to the skull. Note the position of the tentorium, of the fossa olfactoria, and of the foramina upon the prepared skull; also, upon a figure, the ectal origins of the cranial nerves.

Hold the skull with the venter upward, and gently push the medulla mesad with the tips of the scissors. Cut the $N$. hypoglossus close to the dura. Successively cut the $N N$. accessorius, vagus and glossopharyngeus as they enter the $F m$. jugulare. Tilt the skull so as to permit the slight dislodgement of the meten. and epen. and divide the $N N$. auditorius and facialis; this will allow the small Lobus appendicularis to leave its fossa. Cut the $N$. abducens and the $N$. trigeminus, with the tracer and scissors dislodge the hypophysis, and cut the $N$. opticus. Lastly, divide the $N N$. trochlearis and oculomotorius. 
Turn the cephalic end upward, and very carefully dislodge the Lobus olfactorius. When this is done, the cephalic end of the hemisphere will be easily freed from the dura. Hold the skull with its venter down over the normal salt solution, and let the brain roll into it.

\$1122. Hardening.-For a few hours the hemiencephala should rest upon their mesa; then a little bed of absorbent cotton should be made for each, so that it may rest with the meson uppermost and yet not be distorted.

After two days transfer to 95 per cent. alcohol, changing the position as above.

$\$ 1123$. The Zinc Chlorid Process.-The preservation of brains by means of a solution of chlorid of zinc is mentioned or more or less fully desaribed by Gratiolet (A, II), Bischoff $(12,11)$, Giacomini ( $\boldsymbol{l}$ [abstract in Jour. of Anat. and Phyø., XIII]), Rolleston (1) and Osler $(\boldsymbol{1}, \boldsymbol{Z})$.

Our own experience is not yet sufficiently extensive to enable us to form an opinion respecting its merits, and we here give (slightly modified verbally) the condensed directions which accompanied the admirable preparations of the human brain exhibited by Osler at the meeting of the Am. Assoc. Adv. Sei., 1879, und substantially reproduced in his second paper $(\boldsymbol{Z})$.

“(1) Immerse in a zinc chlorid [saturated] solution ("Burnett's' will answer) Turn two or three times a day. On the second day remove the pia. Let it remain until it no longer sinks (8-10 days).

"(2) Immerse in alcohol of commerce [95 per cent.] for 10-12 days, turning often to prevent distortion.

"( $(3)$ Immerse in good glycerin to which has been added 1 per cent, of carbolic acid. Let it remain until it sinks to the level of the liquid.

"(4) Set aside for several days until the surface is dry. Then cover with several layers of gum-elastic varnish."

\$ 1124. Injection of the Coelix.-(A) With alcohol.-When the coliæ and plexuses are to be studied, it is an advantage if the former have been first filled with strong alcohol. This is most readily done after the entire brain has been placed in the weaker alcohol (\$ 1105).

Gently detach the hypophysis with the forceps, or leave it attached by a part of the infundibulum. Then fill a small syringe with 95 per cent. alcohol, apply the outlet (without a canula) to the Fm. infundibuli, and inject slowly until the $L l$. hypocampa (Pl. II, Fig. 3) is seen to swell slightly. Repeat the operation several times at intervals of 1-3 hours.

$\S 1125$. (B) With plaster.-This is for the sake of obtaining a cast of some part of the coliæ. It is best done while the brain still rests in the calva (\$1103). See description of Fig. 119. This metbod was employed by us before Welcker's method of injecting roax was known to us ( 1 [abstract in Jour. Anat. and Phys., XIII, 283]).

After the injection the brain should remain wholly undisturbed for at least half an hour. The parts about the $F m$. infundibuli, constituting the floor of the diacolia, may then be gently cut away with scissors so as not to break the cast. It is probable that a fracture 
will occur at the portæ, but the parts may be reunited with glue and monnted upon glass under a watch glass so that the separated parts are contiguous.

$\$$ 1126. Injection of the Plexuses. - The larger arteries of the brain are filled when the cephalic region of the body is injected with plaster ( $\$ \$ 352-367$ ).

For the special study of the plexuses, gelatin or Berlin blue may be injected from the aorta as follows :-

Expose the thoracic vessels as directed in $\S 918$, and note their positions and connections as shown in Fig. 101, 102.

Ligate the aorta near its base, the $A$. subclavia sinistra near the aorta, and the $A$. subclavia dextra near its junction with the $A$. carotidea. Open the aorta peripherad of the place of origin of the $A$. subclavia sinistra, expel any blood, and inject cephalad.

\section{A MACROSCOPIC VOCABULARY OF THE BRAIN.}

\section{§ 1127. AbBreviations of the General Names.}

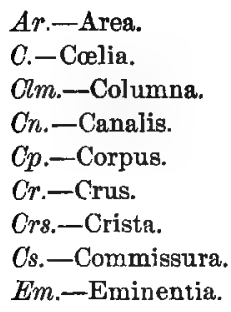

$F$.-Fissura.
$F m$. -Foramen.
$F n$. -Funiculus.
$F s$. -Fossa.
Inc.-Incisura.
L.-Lobus.
Ll.-Lobulus.
N.-Nervus.
Pt.-Portio.

F.-Fissura.

Tm.-Foramen.

$F^{\prime} n$.-Funiculus.

Fr. -Fossa.

Inc.-Incisura

L.-Lobus.

N.-Nervus.

Pt.-Portio.

\section{1128. List of aberevitations of the Spectal Names.}

In this list are given only the names adopted in this work ; the synonyms are given in the descriptions which occur later in the alphabetical order of the names.

Those marked $a z$. are mesal or azygous ; the rest are in pairs.

a.-Aula, $\alpha z$.

$a b n$-Albicans, (Corpus).

alb.-Alba, (Substantia).

apx.-Auliplexus.

$a r b$. vt. - Arbor vitæ, $a z$.

arch.-A Arachnoidea.

$A r$. cr.-Area cruralis, $a z$.

$A r$. icr.-Area intercruralis, $a z$.

Ar. el.-Area elliptica.

Ar. ov.-Area ovalis.

Ar. ppn.-Area postpontilis, $a z$.

Ar. prch.-Area præchiasmatica, $a z$.

Ar. spt.-Area septalis.

$c \alpha$-Carina, $\alpha z$.

$c b$.-Cerebrum, $a z$.

$c b l$-Cerebellum, $a z$.

$c d$. s. -Cauda (Corporis) striati.

cel. m. -Cella media.

ch.-Chiasma, az.

cin.--Cinerea, (Substantia). .

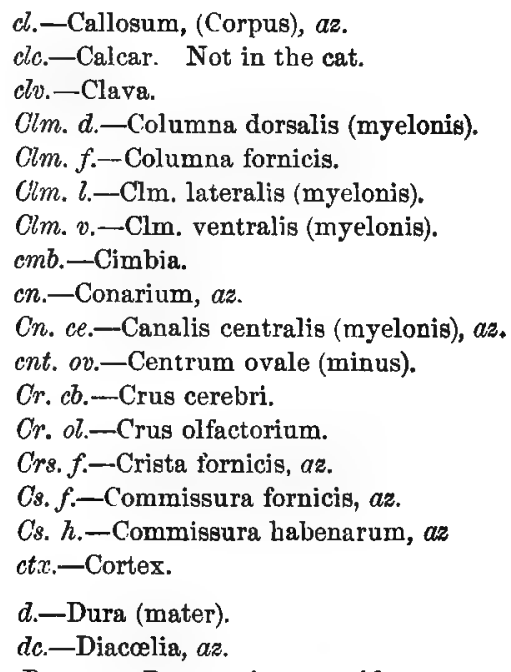

cl.-Callosum, (Corpus), az.

cle.-Calcar. Not in the cat.

clv.-Clava.

Clm. d.-Columna dorsalis (myelonis).

Clm. f.-Columna fornicis.

Clm. l.-Clm. lateralis (myelonis).

Clm. v. $-\mathrm{Clm}$. ventralis (myelonis).

cmb.-Cimbia.

$c n$.-Conarium, $a z$.

Cn. ce.-Canalis centralis (myelonis), $a z$.

cnt. ov.-Centrum ovale (minus).

Cr. $c b$. - Crus cerebri.

$C r$. ol.-Crus olfactorium.

Crs. f.-Crista fornicis, $a z$.

Cs. f-Commissura fornicis, $a z$.

Cs. h.-Commissura habenarum, $a z$

ct.x-Cortex.

d.-Dura (mater).

$d c .-D i a c c l i a, a z$.

Dec. py.-Decussatio pyramidum. 
den.-Diencephalon, $a z$.

dlt.-Delta (fornicis), az.

apx.-Diaplexus.

dul.-Diatela, $a z$.

Em. au.-Eminentia auditoria.

end.-Endyma.

epe.-Epicoelia, az.

epen.-Epencephalon, $a \approx$.

F. $a$. - Fissura anterior.

F. an.-Fissura ansata.

$F^{\prime}$. $c f$. - Fissura confinis.

F. cl.-Fissura callosalis.

Fr. cor.-Fissura coronalis.

F. cr.-Fissura cruciata.

$F$. $d g$.-Fissura diagonalis.

F. $d l$. - Fissura dorsilateralis (myelonis).

F. $d m s$.-Fissura dorsimesalis (myelonis), $a z$.

F. $f m_{1}$-Fissura fimbriæ.

F. fl.-Fissura falcialis.

F. hmp.-Fissura hypocampæ.

$F$. in.-Fissura intermedia.

F. l. -Fissura lateralis.

$F$. In. -Fissura lunata.

$F$. $m l$. -Fissura medilateralis.

Fr. $m r$. - Fissura marginalis.

F. ol.-Fissura olfactoria.

$F$. $p$.-Fissura postica.

F. per.-Fissura postcruciata.

F. pmr.-Fissura postmarginalis.

F. $p r d$.-Fissura postradicalis.

F. prh.-Fissura postrhinalis.

F. prrd.-Fissura præradicalis.

F. $p s$. Fissura postsylviana.

F. $r h$.- Fissura rhinalis.

F. 8.-Fissura Sylviana.

F. ofl. - Fissura subfalcialis.

$F$ ' 'sp.-Fissura splenialis.

$F$. so.-Fissura superorbitalis.

$F$. ss. -Fissura supersylviana.

$F$. vl.-Fissura ventrilateralis (myelonis).

F. vms.-Fissura ventrimesalis (myelonis), ag:

f.-Fornix, $a z$.

flc.-Flocculus.

Fm. ce.-Foramen cæcum, $a z$.

$F m$. cn.-Foramen conarii, $a z$.

Fm. inf.-Foramen infundibuli, $a z$.

fmb. - Fimbria.

\author{
ficl.-Fasciola. \\ g.-Genu, $a z$. \\ h.-Habena. \\ hem.-Hemisphæra. \\ $h m p$.-Нуросатра. \\ $h m s p t$.-Hemiseptum (cerebri). \\ $h p h$.-Hypophysis, $a z$.
}

Inc. hmp.-Incisura hypocampæ.

inf.-Infundibulum, az.

inop. - Interopticus. Not in the cat.

ins.-Insula.

it.-Iter, $a z$.

L. . -Lobus lateralis (cerebelli).

$L$. ol.-Lobus olfactorius.

L. tmp.-Lobus temporalis.

$L l$. ap.-Lobulus appendicularis (cerebelli).

$L l$. $h m p$.-Lobulus hypocampæ.

lm. alb. - Limes alba.

lm. cin.-Limes cinerea.

lq. c.-Liquor coeliarum.

$l y$.-Lyra, $a z$.

mes.-Medicommissura, $a z$.

meu.-Medicornu.

$m p d$-Medipeduncnlus, $a z$.

$m s c$-Mesoccelia, $a z$.

msen.-Mesencephalon, az.

mtc.-Metacœlia, az.

mten.-Metencephalon, az.

mtpx.-Metaplexus.

mttl.-Metatela, $a z$.

$m y$.-Myelon, $a z$.

N. $a b d .-$ Nervus abducens (vi).

N. ac--Nervus accessorius (xi).

N. $a u .-$ Nervus auditorius (viii).

$N$. $f$.-Nervus facialis (vii).

N. $g p h$.-Nervus glossopharyngeus (ix).

$N$. $h g$.-Nervus hypoglossus (xii).

N. ocm.-Nervus oculomotorius (iii).

N. ol.-Nervus olfactorius (i).

N. op.-Nervus opticus (ii).

$N$. tr.-Nervus trochlearis (iv).

N. trg.-Nervus trigeminus (v).

N. v. -Nervus vagus ( $\mathrm{x}$ ).

ob.-Obex.

olv.-Oliva.

op.-Optictrs, (Lobis). 
p.-Porta.

pi.-Pia (mater).

pcs.-Postcommissura, $a z$.

pcu.-Postcornu. Not in the cat.

pgn.-Postgeniculatum, (Corpus).

po, ol,-Pero (olfactorius).

pop.-Postopticus, (Lobus).

$p n$.-Pons (Varolii), $a z$.

ppd.-Postpedunculus.

ppf.-Postperforatus, (Locus), az.

$p p x$.-Portiplexus.

prc.-Procœlia.

pres.-Præcommissura, $a z$.

preu.-Præcornu.

pren.-Prosenceplalon, az.

prgn.-Prægeniculatum.

prpd.-Præpedunculus.

prpf.-Præperforatus, (Locus).

prpx.-Proplexus.

ps. ol.-Pes (olfactorius).

Pt. d.-Portio depressa (præperforati).

Pt. den.-Portio diencephalica (cruris cerebri).

Pt. msen-Portio mesencephalica (cruris cerebri).

Pt. p.-Portio prominens (præperforati).

py.-Pyramis.

psc. -Pseudocœlia, az.

$q$-Quadrans.

$r$-Rima.

R. a.-Recessus aulæ, az.

$R$. op.-Recessus opticus.
R. prpn.-Recessus præpoutilis, $a z$.

$R g$. a.-Regio aulica, $a z$.

rhc.-Rhinocolia.

rhen.-Rhinencephalon, $a z$.

rm.-Rostrum, az.

rp.-Ripa.

rst.-Restiforme, (Corpus).

$R x$. in.-Radix intermedia.

Rx. l.-Radix lateralis.

Rx. mt.-Radix motoria.

$R x$. ms. -Radix mesalis.

$R x$. $s n .-$ Radix sensoria.

8.-Striatum, (Corpus).

Sl. $h .-$ Sulcus habenæ.

Sl. ic. l.-Sulcus intercruralis lateralis.

S. ic. ms.-Sulcus intercruralis mesalis, az

Sl. li.-Sulcus limitans.

Sl. trd.-Sulcus triradiatus, az.

sp.-Splenium, $a z$.

Spt. lu.-Septum lucidum (cerebri), $a z$.

str. lng.-Stria longitudinalis (callosi).

t.-Terma, $a z$.

Tbcl. Rol.-Tuberculum Rolandii.

T. cin-Tuber cinereum, az.

th. -Thalamus.

Tr. op.-Tractus opticus.

Tr. prh.-Tractus postrhinalis.

Tr. $r$ h.-Tractus rhinalis.

tz.-Trapezium.

$v l .-$ Velum (interpositum), $a z$.

vm.-Vermis, az.

vv.-Valvula, $a z$.

$\S 1129$. Most of the above names are those in common use, with the omission of superfluous elements like corpus, and the genitives of the names of more comprehensive parts. Most of the apparently new names will be found to be old acquaintances under such thin disguises as translation, transposition, abridgement, and the substitution of prefixes for qualifying words. In a few cases the old names are wholly discarded for briefer new ones (e. g., cimbia for Tractus transversus pedunculi). Most of the new names, however, refer to parts apparently unobserved hitherto (e. g., crista, carina, delta), or to parts which -although probably observed-seem not to have been regarded as needing a special designation (e. g., aula, quadrans, pero).

For the names of the segments, cœliæ, telæ and plexuses, see $\$ 1063$.

So much of each name as immediately follows the abbreviation is regarded as a sufficient designation of the part under ordinary circumstances; sometimes it may be necessary to add the words in parenthesis.

The names are tabulated according to segments in $\$ 1138$. 


\section{THE ENCEPHALIC SEGMENTS IN THE CAT.}

$\S 1130$. The recognition of the several segments is less easy than with the frog in some respects on account of certain features of the mammalian encephalon.

\$1131. Differences between the Brains of the Cat and the Frog.--In a general way these have been indicated already $(\$ \$ 1055$, 1056); they may be more definitely stated as follows:-

(1) In the cat the coeliæ are irregular in form and relatively reduced in extent (Fig. 113; Pl. II, Fig. 4).

(2) The parietes are relatively thicker, and more differentiated as to both contour and strueture (Fig. 113 ; Pl. II, Fig. 4; P1. III, Fig. 13).

(3) With several segments the dorsal portion (roof) is much more extensive than the ventral (floor), (Fig. 117; Pl. II, Fig. 4).

(4) The longitudinal axis of the entire brain presents a decided flexure, the convexity of which is dorsal and coincides in position nearly with the mesencephalon (Fig. 88).

(5) The dorsal portions of two segments (epencephalon and prosencephalon) are so greatly enlarged as to cover all the others excepting parts of the metencephalon and rhinencephalon, the extremes of the series (Fig. 88, 104, 117; Pl. I, Fig. 1, 2).

(6) In addjtion to the Amphibjan commissures (chiasma, postcommissura, proecommissura), there are in the cat's brain more or less distinct fibrous fasciculi constituting other commissures-longitudinal (Crura cerebri, Pl. II, Fig. 3, Pl. III, Fig. 11; Crura olfactoria, Pl. II, Fig. 3, 4 ; postpedunculi, præpedunculi) ; transverse (pons, Fig. 117 ; P1. II, Fig. 3 , pn. ; decussatio pyramidum, medipedunculi, Commissura fornicis, Pl. IV, Fig. 14, cs.f.); and oblique (fornix, Pl. IV, Fig. 14,f.).

(7) Between the apposed surfaces of the thalami is established a connection, the medicommissura (Fig. 122; Pl. II. Fig. 4, mes.), which occupies a large portion of the diacclia.

(8) The apposed surfaces of the hemispheres are connected along a slightly curved line by a thick band of fibers, the callosum (Fig. 104, 115 ; Pl. III, Fig. 13, PI. IV, Fig. 15, cl.), the largest and presumably the most important of the commissures.

(9) The dorsal aspect of the mesencephalon presents a transverse furrow, distinguishing a cephalic pair of lobes, the optici proper, from a caudal pair, the postoptici (Fig. 114; Pl. III, Fig. 7); hence the bigeminum of the frog becomes the quadrigeminum of the cat.

(10) The lateral aspect of the diencephalon presents at least two elevations, postgeniculatum and prægeniculatum (Pl. III, Fig. 7, 9, p!/n., prgn.).

(11) Of the diacclian roof, the membranous part (diatela, Pl. III, Fig. 10) is relatively more extensive, while the nervous part (postcommissura, Pl. II, Fig. 4, pcs.) is correspondingly diminished; concomitantly the conarium is attached near the caudal end of the diencephalon and is retroverted so as to rest upon the mesencephalon. 
(12) Each lateral half of the rhinencephalon consists of a Lobus olfactorius connected with the prosencephalon by a distinct Crus olfactorium (Pl. II, Fig. 3, 4, Cr. ol.).

(13) Each procolia may be subdivided, somewhat vaguely, into a central cella media (Pl. IV, Fig. 15, cll. m.), and two curved prolongations, the pracornu (Pl. II, Fig. 121, Pl. IV, Fig. 15, 16, prou.), and the medicornu (Pl. IV, Fig. 15, 17, 18, mou.)

(14) Into each cornu projects a decided eminence. That of the præcornu is the striatum (Pl. IV, Fig. 15, 16, s.), a thickening of the parietes. That of the medicornu is the hypocampa (P1. IV, Fig. 15, 16, hmp.), which is chiefly an involution or folding of the parietes"(Fig. 121).

(15) In addition to the atrophied portions of the coelian parietes noted in the frog, the proper nervous substance of the hemisphere is abrogated along a line from the porta to near the tip of the medicornu, constituting the rima or " fissure of Bichat" (Fig. 121; Pl. IV, Fig. 14, 17, R.). Along this line enters the larger portion of the proplexus.

(16) As in Menobranchus, there are metaplexus, diaplexus and proplexus, the latter two being continuous through the intermediation of the auliplexus and portiplexus.

(17) The surface of the cerebellum is convoluted, presenting numerous thin folds (laminœ) with intervening fissures (Fig. 104, 117 ; Pl. I, Fig. 1, 2).

(18) The surface of the hemispheres is also convoluted, presenting a limited number of fissures arranged according to a somewhat definite pattern (Fig. 124, 125 ; PI. I, Fig. 1, 2). Most of the fissures involve only a certain depth of the mass, but the $F$. hypocampce, " hippocampal fissure," represents an involution of the entire thickness of the parietes (Fig. 121, 125 ; P1. IV, Fig. 15, 17, F. hmp.).

(19) With the cerebellum and hemispheres, most of the cinerea is located near or at the surface, constituting a cortex (Pl. II, Fig. 4, PI. IV, Fig. 14, 15). The extent of the cortex is of course increased by the convolutions.

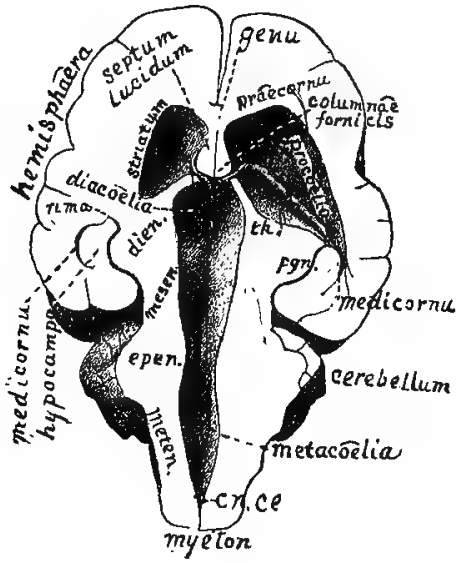

Fig. 113.-Ventral Exposure of THE Coelia; $\times 1$.

Fig. 113.-Ventral exposure of the coliæ; preparation No. 458, Museum of Cornell University. Compare with Fig. 110.

Objects.-To show (A) the general succession of the coelim; (B) the relations of the portæ to the aula and procœliæ; (C) the thickness of the parietes and concomitant reduction of the coliæ; (D) the undulations of the colian roofs.

Preparation.-From the ventral aspect of a well hardened brain successive slices were removed with the large scalpel until the porte and Canalis centralis were exposed. With the Charrière scalpel there were excavated successively so much of the various segments as to expose the entire series of coliæ, excepting the rhinocoliæ, the rhinencephalon having been removed.

On the right (left of the figure) the convexity of the striatum was cut off. On the other side enough more of the hemisphere and thalamus was removed to expose the proplexus and thus throw the præcornu into direct communication with the medicornu.

Explanation.-Most of the names are written in full, and the parts mentioned are described elsewhere.

Cn. ce.-Canalis centralis myelonis. 
Dien., epen., mesen., meten, are abbreviations of diencephalon, etc. So much of the preparation as is not included therein belongs to the prosencephalon.

Pgn.-Postgeniculatum. Th.-Thalamus.

The sides of all the colliø are beveled oft so as to expose their roofs more clearly. The widest portion is the metacclia, whose proper roof (metatela) is so thin that the laminæ of the overhanging cerebellum show through it .

The epicclia presents two very different portions-a caudal, which is short but wide and high, reaching up into the cerebellum '(Pl. II, Fig. 4), and a cephalic, Jonger but narrower and lower, excepting at its cephalic end, where its roof, the valvula, rises to join the postoptici.

The succeeding contracted portion represents the mesoccelia; the next mesal cavity is the diaccelia, the roof of which presents the two parallel diaplexuses, diverging in the aula to connect through the portæ with the proplexuses.

On the right (left of the figure) the crescentic line representing the transection of the medicornu should reach the end of the line indicating the boundary between the hemisphere and the postgeniculatum; see Fig. 121.

The membranes and the relative areas of alba and cinerea are not shown.

Fig. 114.-Dorsal aspect of the mesencephalon, exposed by the separation of the cerebellum and hemispheres; from Prep. No. 390, M. C. U.

Objects.-To expose (A) the dorsal aspect of the mesencephalon, the cephalic aspect of the cerebellum and the caudal aspect of the hemispheres; (B) the origin of the NN. trochleares from the cephalic part of the valvula (vv.); (C) the caudal position and retroversion of the conarium.

Preparation.-The fresh brain was carefully held and the caudal portions of the hemispheres gently pushed cephalad, the attachments to the mesencephalon being divided with the tracer, until the conarium was exposed; then the cerebellum was in like manner tilted caudad, care being had not to tear the delicate valvula.

By the above operations the natural encephalic flexure was so exaggerated as to bring the ventral aspects of the crura olfactoria and the metencephalon

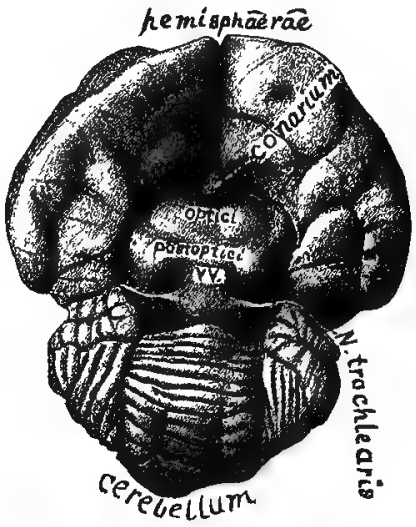

Frg. 114.-Dorgal Aspect of THE Mesencephalon, With SOMe AdJacent PaRTs; $\times 1$. into contact. The brain was secured in this condition upon a bed of wet cotton by pushing cotton against it, and covered with 95 per cent. alcohol.

Explanation.-The names are written in full, excepting vv. for valvula. The furrow between the optici is not sufficiently distinct, and the word postoptici obscures the fact that the elevations so named are separated by a somewhat wide and flat valley rather than by a narrow depression..

In the preparation, the infundibuliform mesocolian orifice is visible through the transparent valvuja. Compare Pl. III, Fig. 7.

Fig. 115.-The callosum after removal of the dorsal portions of the cerebellum and hemispheres ; from Prep. No. 540, M. C. U.

Objects.-To show (A) the fact of the connection of the hemispheres by the callosum; 
(B) the cephalo-caudal extent of the latter; (C) the manner of cleavage of the hemispheres; (D) the irregular lamination of the cerebellum.

Preparation.-The hemispheres and Lobi olfactorii of a hardened brain were sliced to near the level of the callosum and in a plane coinciding with the larger part of its course.

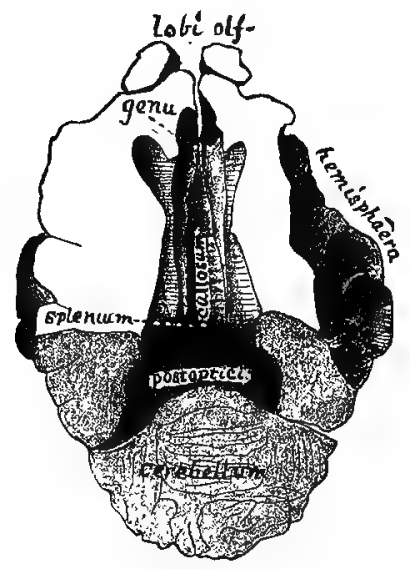

Fig. 115.-DoRsal ASPECT OF the Callosum after ParTIAL Removal OF THE HEMISPHERES ; $\times 1$.

The slight portions overhanging the borders of the callosum so as to constitute the FF. callosales (Fig. 116, 122 ; Pl. III, Fig. 13) were then pushed dorso-laterad with the tracer; the torn surfaces so left are represented by the striated areas. On the right, a part of the hemisphere was torn off so as to indicate the wavelike arrangement of the fasciculi.

An oblique section was then made from opposite the splenium through the caudal portion of the hemispheres and the cerebellum, so as to expose the postoptici.

Explanation.-The name is written lengthwise of the callosum ; perhaps the line at each side represents a stria longitudinalis. The curvature ventrad at the genu and splenium is better shown in section (Fig. 116; P1. II, Fig. 14). The postoptici do not appear distinctly, and no attempt was made to indicate the areas of cerebral alba and cinerea, or of the pes, pero or cœlia in the rhinencephalon.

$\S 1132$. Preliminary Examination of the Basis Encephali.-As seen in Fig. 116, 118, and Pl. II, Fig. 3, the base of the brain presents numerous parts distinguished by their elevation, their subdivision, their color or the direction of their fibers. Including the twelve cranial nerves, about fifty different parts, more or less comprehensive, are named upon Fig, 116.

The cranial nerves are treated of in the next chapter. Of the remaining parts, the beginner is advised to study first only those which have been observed in the Amphibian brain, or which serve either as topographical landmarks or as aids in recognizing the limits of the encephalic segments. Later, it will be desirable to identify each feature by the aid of the figures (Fig. 118 ; Pl. II, Fig. 3 ; Pl. III, Fig. 11) and the detailed descriptions.

Fig. 116.-Diagram of the base of the brain, including the nerve roots.

Explanation.-The names are written in full. As suggested above, the following parts are to be noted first :-

Myelon, expanding into the metencephalon.

Cerebellum, greatly enlarged and convoluted.

Hemisphæræ, still more enlarged and likewise convoluted.

Lobi olfactorii, elongated and in apposition, but not organically united.

Chiasma, Tuber cinereum, infundibulum, hypophysis, Crura cerebri (one of which is designated on Fig. 116 by the word co'us) and pons.

Of the parts just named, all but the last have been noted upon the mesal aspect of the Amphibian brain, and are mentioned in the Table ( $\$ 1069)$.

The pons is a wide and thict band of transverse fibers connecting the lateral lobes of the cerebellum across the ventrimeson. It is likewise a prominent landmark, serving, with the chiasma, to divide the entire basis encephali conveniently into three general regions-caudal, Area postpontilis ; cephalic, Area præchiasmatica ; and intermediate, Area cruralis. 


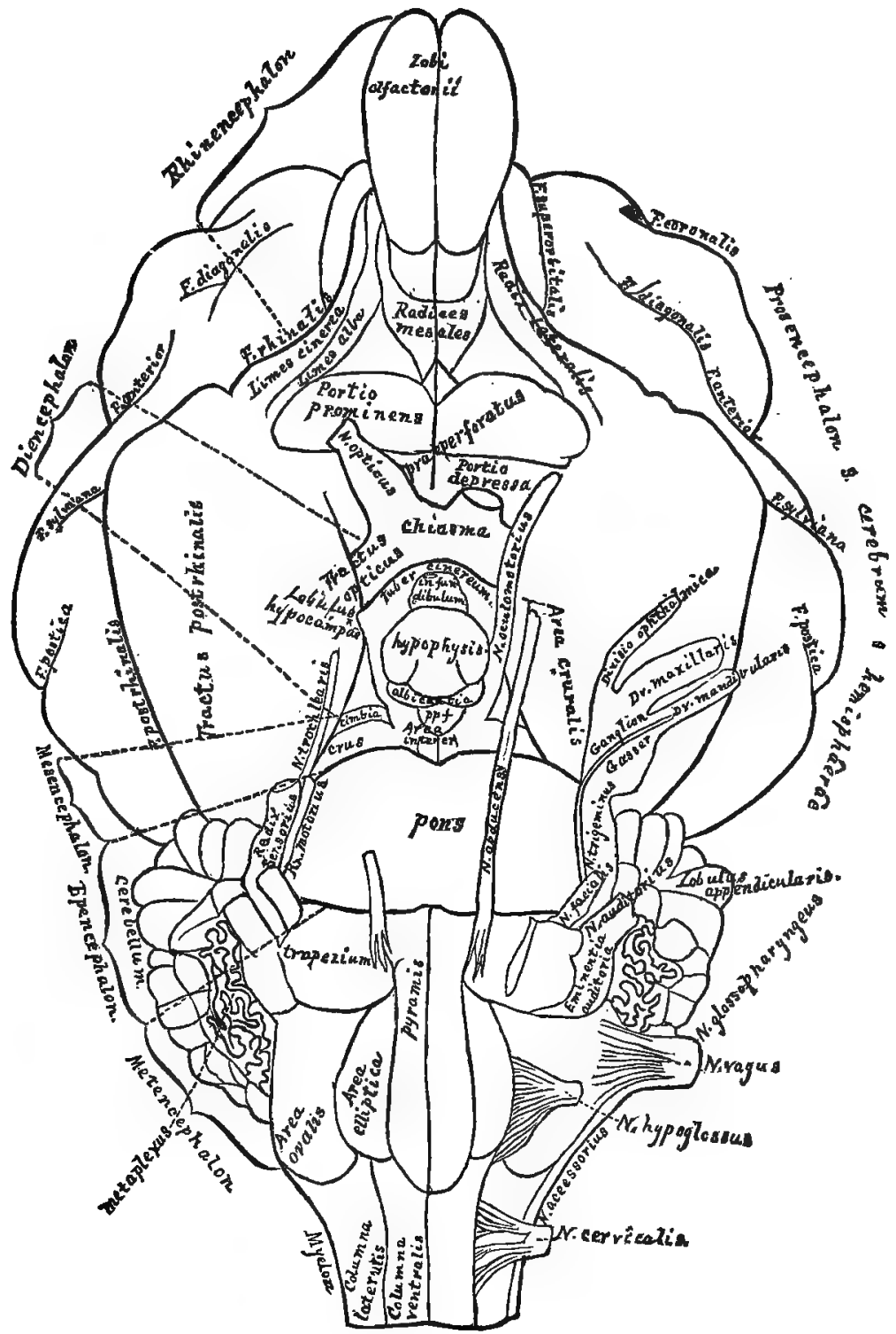

Fit. 116.-Diagram of the Basis Encephati ; $\times 3$.

It is to be observed that, notwithstanding the preponderance of the cerebellum and the hemispheres, all the encephalic segments appear upon the ventral aspect, most distinctly at and near the meson. On the left (right of the figure) the names of the prosencephalon are written along its expanded dorsal portion, but upon the ventrimeson it may be 
regarded as extending only from the middle of the chiasma to the diamond-shaped area just caudad of the rhinencephalic Radices mesales.

The extent of the metencephalon coincides nearly with that of the pyramis.

$\S 1133$. Hemisection of the Brain.-The encephalic segments are more easily recognized and examined upon a hemiencephalon than upon the entire organ because (1) all the segments and most of the cœliæ are then exposed; (2) the parts can be more readily bent upon each other or dissected apart.

Instruments and Materials.-A brain, symmetrical and well-hardened; distorted specimens may serve for dissections, but for the hemisection select the most perfect. Razor or large scalpel, with very keen, smooth edge; if the scalpel is used, it should be especially sharp at the heel, which is usually neglected. A small dish or vial of alcohol, in which the knife may be dipped. A piece of sheet cork, or of soft wood free from knots, at least $6 \times 4 \mathrm{~cm}$, and preferably $16 \times 8$. Remove from the brain any pins which may cross the meson.

$\S 1134$. Upon a preparation or figure (Fig. 115, 116; Pl. II, Fig. 4), note the position of the callosum and the angle which it forms with the dorsal and ventral surfaces of the brain.

Place the brain upon its basis, with its long axis coincident with the length of the cork or the grain of the wood, and the cephalic end of the brain to the left.

Dip the scalpel in alcohol, and grasp it bow-fashion. Introduce the tip of the blade between the hemispheres at their highest part, and gently push it ventro-cephalad until the point reaches the cork a little in advance of the Lobi olfactorii, and the back of the bladeif a scalpel is used-is about on a level with the dorsal margins of the hemispheres. The blade then forms an angle of about 30 degrees with the cork, and its heel is a little cephalad of the cerebellum.

Grasp the brain gently but firmly as follows:-The pollex should be on the left, against the lateral surface of the cerebellum and the caudal part of the hemisphere; on the right the tips of the index, medius and annularis press respectively upon the lateral aspect of the cerebellum and the temporal and frontal regions of the hemispheres. The minimus may be raised out of the way. The pressure upon the two sides of the brain must be equal and uniform.

Draw the scalpel slowly caudad, keeping the convexity near the tip constantly upon the cork, and the entire instrument at the same angle until it emerges through the cerebellum and the medulla.

At the beginning of the hemisection, the edge of the scalpel was firmly held by the closely approximated hemispheres, and its edge rested on the callosum, so that the latter was probably divided accurately on the meson. But a very slight initial deflec- 
tion may have brought the blade out 1-2 $\mathrm{mm}$. laterad of the ventrimeson.

Ascertain the extent of the departure by apposing the two halves of the brain and noting the position of the cut with respect to the ventrimeson. If the cut and the meson coincide, the surfaces of the two sides of the brain will be practically identical; but according to the degree of the separation there will be found a difference which is sometimes so great as to be puzzling to the beginner, especially since in these cases neither surface resembles that shown in the figure.

After becoming somewhat familiar with the organ, it may be well to make purposely a hemisection about $1 \mathrm{~mm}$. laterad of the meson, so that the commissures and other mesal structures may be dissected out in relief; but at the outset it is better to secure a view of the mesal surface itself.

Select that half of the brain to which part of the other half is attached, and hold it with the meson up and the base toward you; grasp the large scalpel bow-fashion, and apply the heel at the ventrimeson, upon the chiasma if it be the right half, or at the ventrimesal ("anterior median") fissure of the metencephalon if the left; cut away from you with a long steady sweep, taking care not to cut upon the other side of the meson. Certain parts of the surface may require subsequent special treatment in order that the surfaces may be fairly exposed.

$\S 1135$. Aside from detailed comparison of the surface with the figures, the best test of the mesal surface is the exposure of the series of mesal cœliæ, extending, as in the Amphibian brain (Fig. 111 ), in uninterrupted continuity from the ventro-caudal angle of the cerebellum to a point dorsad of the chiasma. Just ventrad of the cerebellum the lateral extent of the cavity, epicclia, is considerable, but at other points it is no more than 1-2 mm., so as to appear on the mesal surface like a shallow depression. The most obscure portion of the cavity is the cephalic part of the epicolia, where the lateral extent is considerable, while its vertical diameter is very slight, and the roof, valvula, is quite thin.

Fig. 117.-Mesal aspect of the left hemiencepbalon; a diagram enlarged from Pl. II, Fig. 4.

Explanation.-Upon this diagram are named nearly all the parts and surfaces which are exposed by a mesal hemisection.

To avoid turning the volume in referring to it, the figure has been divided. Unfor- 
tunately, it is reversed as compared with Fig. 111 and Pl. II, Fig. 4. The cephalic part of the valvula is represented much too thick.

\$ 1136. Preliminary Inspection of the Mesal Aspect. - Before entering into details, the student should compare the mesal series of coliæ in Fig. 116 with the same in Fig. 111, and recognize the following parts of their parietes by name, even although their appearance may be quite unlike : cerebellum, valvula, opticus, conarium, postcommissura, præcommissura, chiasma, Tuber cinereum.

The lateral coliæ, procelia and rhinoccelia, do not appear, and the porta itself is not visible, but its position coincides nearly with that of the little eminence called crista, just ceplualad of the medicommissure.

\$ 113\%. Surfaces exposed by Hemisection of the Brain.-Four different kinds of surface appear upon the mesal aspect of the hemiencephalon, and they should be recognized and distinguished before the details are examined :-

(1) Dorsad of the callosum the hemisphere is natural or free and covered by pia.

(2) The several cceliæ are lined by endyma, which likewise form a natural surface.

(3) The commissures and other parts which extend

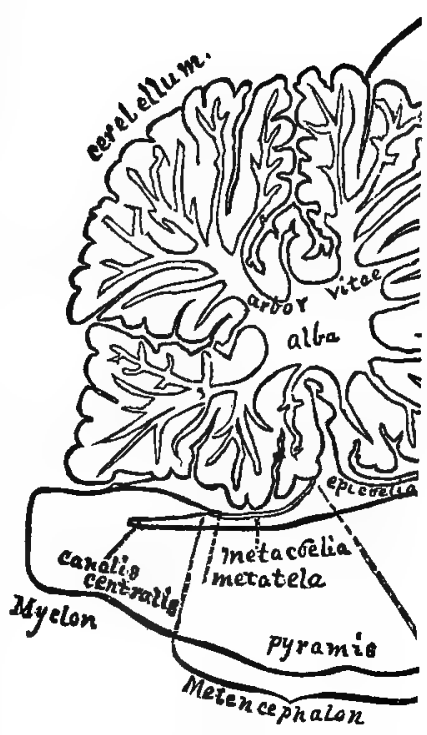
across the meson have been divided and the resulting surfaces are cut or artificial. With the callosum, præcommissura and postcommissura, chiasma, ental portion of cerebellum, etc., the parts are white in color and fibrous in texture $(a l b a)$, but the medicommissure, terma, ectal layer of cerebellum, etc., are cellular and formed by cinerea.

(4) Caudo-ventrad of the cephalic part of the callosum is a triangular area which is naturally attached by connective tissue to the corresponding surface of the other hemiencephalon. This is the Area septalis. In man the surfaces are free, forming the lateral boundaries of the pseudo-ceitia.

\$ 1138. Segmental Arrangement of the Names of the Principal Parts of the Cat's Brain.-The 'Table upon pages 48 and 49 is intended to enable the student to learn more readily the large number of names according to their respective segments, and to see at a glance the relations which the parts bear to each other.

In each case, excepting perhaps the caudal portion of the prosencephalon, the part first mentioned constitutes the principal mass of the segment.

The indenting of a name signifies that the part is a subdivision of the part named above; for example, the striatum is a division of the hemisphere, and itself includes the cauda striati.

In most cases the apposition of names in different columns signifies that the parts are in some way correlated; $e . g$., under epencephalon, the arbor vita is a surface exposed upon bemisection of the cerebellum, and the pons is the great cerebellar commissure. Where the parts are not correlated, the vertical line between the columns is reinforced by a short vertical line; e. g., between carina and ripa.

In the column headed superficies are also included the names of elevations, depressions, recesses, sulci, and even cavities. 


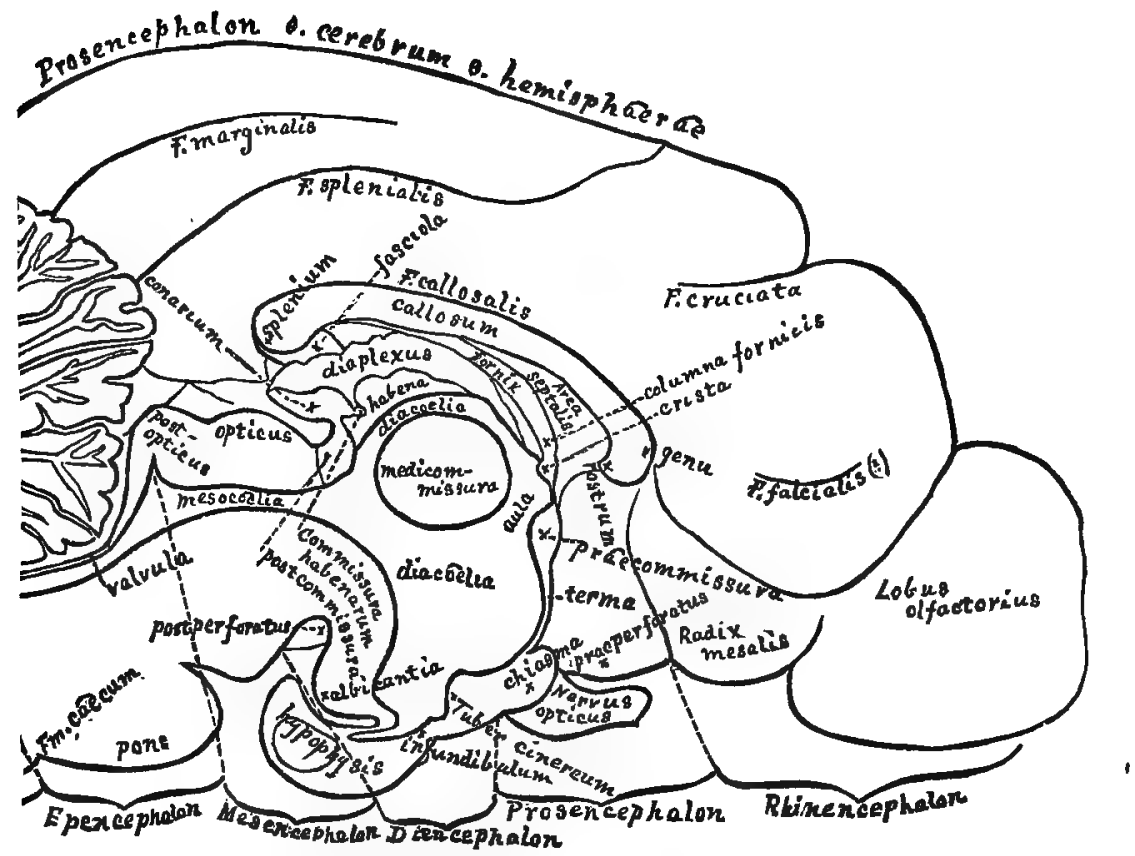

Fig. 117.-Diagram of the Megal aspect of the Left Hemiencephaton; $\times 3$. The caudal part of the figure is upon the opposite page.

The fornix is difficult to classify. In some respects it and the fimbria seem to be commissural.

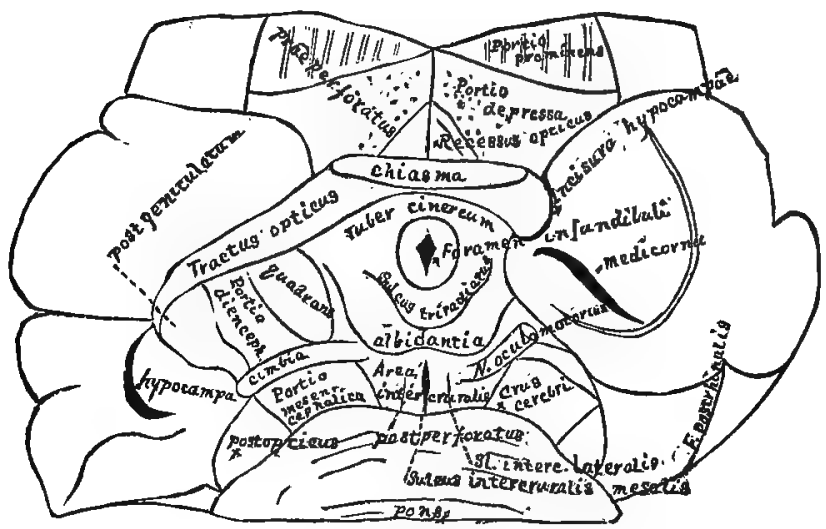

Fig. 118.-Diagram of the Area cruralis; $\times 2$. T'his figure is enlarged and reversed from P1. III, Fig. 11, and the description of that figure may be consulted for the details and for the mode of preparation. 


\section{TABULAR ARRANGEMENT OF THE PARTS OF THE CAT'S BRAIN.}

This Table differs from that upon page 409 mainly in that the names of the encephalic segments, and of the ccliæ, telæ and plexuses thereof, are placed together above the names of the particular parts, surfaces and commissures. The synonyms of the names are given in $\$ 1180$ et seq.

RHINENCEPHALON.

RHINOCGLIA.

\begin{tabular}{|c|c|c|}
\hline Partes. & Superficies. & \\
\hline $\begin{array}{l}\text { Lobus olfactorius. } \\
\text { Pero (olfactorius). } \\
\text { Pes (olfactorius). } \\
\text { Crus olfactorium. } \\
\text { Radix mesalis. } \\
\text { Radix intermedia. } \\
\text { (not in cat ?) } \\
\text { Radix lateralis. }\end{array}$ & 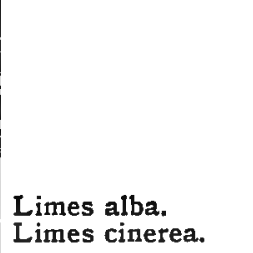 & . \\
\hline
\end{tabular}

PROSENCEPHALON (Cephalic Portion, Paired).

Procolia - Propuexus.

\begin{tabular}{|c|c|c|}
\hline Partes. & Superficies, etc. & Commiseuræ. \\
\hline Hemisphæra. & $\begin{array}{l}\text { Centrum ovale. } \\
\text { Centrum ovale majus, } a z \text {. }\end{array}$ & $\begin{array}{l}\text { Callosum, az. } \\
\text { Splenium, } a z . \\
\text { Genu, az. } \\
\text { Rostrum, } a z .\end{array}$ \\
\hline Septum, $a z$ & $\begin{array}{l}\text { Area septalis. } \\
\text { Pseudo-cœlia (not in cat). }\end{array}$ & \\
\hline $\begin{array}{l}\text { Striatum. } \\
\text { Cauda striati. } \\
\text { Insula. }\end{array}$ & Præcornu. & Præcommissura, $a z$. \\
\hline $\begin{array}{l}\text { Hypocampa. } \\
\text { Lobulus hypocampx. } \\
\text { Fasciola. }\end{array}$ & Medicornu. & $\left.\begin{array}{l}\text { Fornix, } \\
\text { Fimbria. }\end{array}\right\} ?$ \\
\hline Calcar (not in cat). & $\begin{array}{l}\text { Postcornu (not in cat). } \\
\text { Lyra, az. } \\
\text { Præeperforatus. } \\
\text { Portio depressa. } \\
\text { Portio prominens. } \\
\text { Fissuræ cerebrales. }\end{array}$ & \\
\hline
\end{tabular}

PROSENCEPHALON (Caudal Portion, Unpaired).

adla, Porta, Procoglia-Aulatela-Aultplexus, Portiplexus, Proplexds.

\begin{tabular}{|c|c|c|}
\hline Partes. & Superficies, etc. & Commissurx. \\
\hline $\begin{array}{l}\text { Terma, az. } \\
\text { Fornix, } a z \text {. (See } \$ 1457 \text {.) } \\
\text { Columna fornicis. } \\
\text { Carina, } a z \text {. }\end{array}$ & Ripa. & Commissura fornicis, az. \\
\hline $\begin{array}{l}\text { Crista, } a z . \\
\text { Fimbria. } \\
\text { Tænia. }\end{array}$ & $\left\{\begin{array}{l}\text { Delta, } a z . \\
\text { Aula, } a z . \\
\text { Recessus aulæ, } \alpha z . \\
\text { Recessus opticus. } \\
\text { Rima. } \\
\text { Sulcus limitans. }\end{array}\right.$ & \\
\hline
\end{tabular}




\section{DIENCEPHALON.}

Diacolia - Diatela - Diaplexug.

\begin{tabular}{|c|c|c|}
\hline Partes. & Superficies, etc. & Commisenrse. \\
\hline $\begin{array}{l}\text { Thalamus. } \\
\text { Pulvinar (not in cat). } \\
\text { Habena. } \\
\text { Conarium, az. } \\
\text { Prægeniculatum. } \\
\text { Postgeniculatum. } \\
\text { Crus cerebri (portio dien- } \\
\text { cephalica). } \\
\text { Tuber cinereum, az. } \\
\text { Infundibulum, az. } \\
\text { Hypophysis, az. } \\
\text { Albicans. }\end{array}$ & $\begin{array}{l}\text { Sulcus habena. } \\
\text { Foramen conarii, az. } \\
\text { Tractus opticus. } \\
\text { Quadrans. . } \\
\text { Foramen infundibuli, az. } \\
\text { Sulcus triradiatus, ar. } \\
\text { Postperforatus, az. }\end{array}$ & $\begin{array}{l}\text { Medicommissura, az. } \\
\text { Postcommissura, } a z \text {. } \\
\text { Commissura habenarum, } a z \\
\text { Chiasma, } a z .\end{array}$ \\
\hline
\end{tabular}

MESENCEPHALON.

Mesocoglia.

\begin{tabular}{|c|c|}
\hline Partes. & Superficies, etc. \\
\hline $\begin{array}{l}\text { Opticus. } \\
\text { Postopticus. } \\
\text { Crus cerebri (portio mesen- } \\
\text { cephalica). } \\
\text { Cimbia. }\end{array}$ & $\begin{array}{l}\text { Sulcus intercruralis later- } \\
\text { alis. } \\
\text { S1. ic. mesalis, az. }\end{array}$ \\
\hline
\end{tabular}

EPENCEPHALON.

EPICGELA.

\begin{tabular}{|c|c|c|}
\hline Partea. & Superficies. & Commiseuræ. \\
\hline $\begin{array}{l}\text { Cerebellum, } a z \text {. } \\
\text { Lobus lateralis. } \\
\text { Lobulus appendicularis. } \\
\text { Vermis, } a z \text {. } \\
\text { Valvula, } a z .\end{array}$ & $\begin{array}{l}\text { Arbor vitz, az. } \\
\text { Foramen cæcum, az. } \\
\text { Recessus præpontilis, az. }\end{array}$ & $\begin{array}{l}\text { Pons, } a z \text {. } \\
\text { Medipedunculus. } \\
\text { Postpedunculus. } \\
\text { Prepedunculus. }\end{array}$ \\
\hline
\end{tabular}

METENCEPHALON.

Metaccelta - Metateida - Metaplexds

\begin{tabular}{l|l|l}
\hline \multicolumn{1}{c|}{ Partes. } & \multicolumn{1}{c}{ Superfleies, etc. } & \multicolumn{1}{c}{ Commisarre. } \\
\hline $\begin{array}{l}\text { Medulla, } a z . \\
\text { Pyramis. }\end{array}$ & Area postpontilis, $a z$. & Decussatio pyramiđum, $a z$. \\
Oliva. & Area elliptica. & \\
Tuberculum Rolando. & Area ovalis. & \\
Restiforme. & & \\
Trapezium. & \\
Eminentia auditoria. & & \\
Clava. & & \\
\hline
\end{tabular}


$\S 1139$. Examination of the Encephalic Segments.-Instruments and Materials.-A hemiencephalon, well hardened; the condition of its lateral aspect may be disregarded. Large and Charrière scalpels, both very sharp. Syringotome or tracer. Fine scissors. Blowpipe. Coarse and fine forceps. Beaded bristles. Small dish or wide-mouthed vial of alcohol, into which the brain and the scalpels may be dipped.

In dissection of the brain, the scalpels should be very sharp, and dipped in alcohol just before each incision.

Rhinencephalon (examined first on account of its liability to injury during the study of the other parts). - Ventriduct the Lobus olfactorius so as to expose the dorsal aspect of the Crus ol.; also the Fissura ol. (Pl. III, Fig. 5, $F$. ol.) on the ventro-cephalic surface of the hemisphere in which rests the dorsal border of the crus.

At the junction of the crus with the lobus, note the slight prominence of the softer ectal layer, the pero, from which directly arise the olfactory nerves (Pl. II, Fig. 3, $N N$. ol.). Remove the pero with the forceps and tracer, and note that there remains a thin smooth lamina, the pes ol., which is partly fibrous and forms the immediate continuation of the crus.

The rhinocœlia will be more easily seen upon the dissection of another specimen.

$\S 1140$. Metencephalon.-Of this segment, one part, the pyramis, has been noted. Laterad of the cephalic part of the pyramis is a quadrangular area, the trapezium (Pl. II, Fig. 3, tz.) ; laterad of its caudal and longer portion are two elevations, here called from their outlines, Area elliptica and Area ovalis.

The dorsal aspect of the metencephalon is overhung by the cerebellum. Tilt the latter cautiously latero-cephalad, using the tracer to disengage from its ventral aspect the delicate metatela, the roof of the metacœlia (Pl. III, Fig. 12, mttl.). Even if, in so doing, the metatela is torn from its connection with the border of the metacœlia (so as to produce a "Foramen of Magendie," \$ 1082), it may usually be traced continuous with the ventral surface of the cerebellum just caudad of the epicœlia.

Now tilt the cerebellum meso-cephalad and note the mass of membrane and blood-vessels forming the metaplexus lateralis (Pl. II, Fig. 4 ; Pl. III, Fig. 12, mtpx.).

Upon removal of the metatela and metaplexus, there will be 
seen a transverse band (Tractus auditorius) passing from the Eminentia auditoria across the wall of the metacœlia.

\& 1141. Epencephalon.-Tilt the cerebellum caudo-laterad, so as to expose the limits of the epicclia. Note the distinction between its caudal portion, which extends dorsad into the cerebellum, and the rest, which has for its roof only the thin valvula. Separate the valvula from the cerebellum, noting the continuity of the two at the caudal end of the former.

With the forceps and tracer tear and push off the lateral part of the cerebellum so as to expose the medipedunculus, the continuation of the pons into it. In like manner, remove the caudal part of the cerebellum, and note a less distinct fasciculus, the postpedunculus, passing from the Tractus lateralis of the metencephalon to the cerebellum just mesad of the medipedunculus. The præpedunculus is exposed by the removal of the cephalic part of the cerebellum ; it forms the lateral wall of the cephalic part of the epicelia, and is continuous with the base of the postopticus.

Push the tracer entad of the medipedunculus, and then divide it with the scissors. Grasp the ventral piece and tear off the pons as far as the meson. Note that the pons concealed the continuity of the pyramis with the Crus cerebri.

$\S 1142$. Mesencephalon.-Note the slenderness of the mesocœlia, whence the names iter and aquaductus Sylvii.

Ventriduct the meten. and epen. so as to leave a space between them and the hemisphere. Remore the caudal end of the latter by a dorso-ventral incision corresponding with the convexity of the splenium. On the cut surfaces, note the darker color of the ectal cinerea, the cortex, as compared with the ental alba. There will be exposed a cavity, the medicornu, and a rounded elevation, the hypocampa. These may be disregarded for the present.

With the pollex tear away the Lobulus hypocampæ (P1. I, Fig. $3, L l . h m p$.) and so much of the hemisphere as readily comes off with it; then cut off as much more as may be necessary to expose the Tractus opticus ( $T r$. op.).

The parts thus exposed will be found covered by pia, which forms a distinct fold in the $F$ ? hypocampa (Fig. 121).

This covering of the mesencephalon and part of the diencephalor is called the velum (interpositum). Remove it with the forceps, beginning at the postopticus. Note the slender $N$. trochlearis 
passing laterad and then ventrad just caudad of the postopticus from its ectal origin on the cephalic part of the valvula.

Observe the contrast in form and position of the opticus and postopticus. The latter is farther from the meson and more sharply defined; the opticus presents a more gradual slope to the meson. Extending laterad from the lateral slope of the opticus, note the cimbia, the ventral part of which crosses the crus in the Area cruralis (Fig. 116, 118; Pl. II, Fig. 3, and Pl. III, Fig. 11, cmb.). The cimbia seems to indicate the boundary between the mesencephalic and diencephalic portions of the crus.

§ 1143. Diencephalon.-Note the great dorso-ventral extent of the diaccelia, the presence of the medicommissura (mcs.) in its dorsal part, and the absence of any distinct boundary between the diacœlia and the aula.

The thalami constitute the lateral parietes of the diacclia, and its ventral and cephalic boundaries are sufficiently obvious, but the dorsal require specification.

The most caudal part of the roof is formed by the postcommissura (pcs.), which intervenes between the opticus and the conarium $(c n$.$) .$

Between the medicommissure and the fornix the most prominent part, especially with an injected preparation, is a longitudinal plexus, the diaplexus.

Lift the diaplexus with the tracer at about the middle of its length and note that it is attached throughout by one edge, and that it partly covers a ridge upon the mesal aspect of the thalamus. This, the habena $(h$.$) , is widest near its caudal end, which joins its$ platetrope by a slender band, the Commissura habenarum $\left(C s . h_{\text {. }}\right.$, just dorsad of the conarium.

The habena becomes narrower and less prominent cephalad and disappears at the cephalic convexity of the thalamus, corresponding with the dorsal limit of the porta.

Grasp the diaplexus with the fine forceps and gently pull upon it. Note that it readily separates from the other parts excepting at the ends. The ends are connected with somewhat large vessels, but the precise arrangement is not clear to us. Recall the relations of the diaplexus in Menobranchus ( $\$ 1097$ ), where it is free excepting at the cephalic end.

Remove the diaplexus by carefully cutting the caudal and cephalic connections, and note that it was attached to the ventral or 
ental aspect of a delicate membranous roof of the diacœlia, the diatela (Fig. 122).

By slightly pushing the hemisphere away from the thalamus, it will be seen that the diatela springs from the dorsal margin of the habena, along a slight furrow, the sulous habenæ (Sl. $h$.), and curves dorso-mesad to meet its other half from the opposite side.

$\$ 1144$. On the lateral aspect, between the cimbia and the tractus opticus, note a decided elevation, the postgeniculatum (Fig. 18; Pl. III, Fig. 7, 9, pgn.). Ventrad of it is a ridge of the crus, partly embracing a depressed area, the quadrans (Fig. 18; Pl. III, Fig. $11, q$.$) .$

Ventriduct the parts still more, and trace the tractus opticus into an expanded elevation just cephalad of the postgeniculatum. This, the prægeniculatum ( $\mathrm{Pl}$. III, Fig. 7, 9, prgn.), is practically continuous with the thalamus, the principal part of the diencephalon, which has been seen above as the lateral wall of the diacolia. Note the absence of any distinct caudal protrusion of the thalamus, such as forms the human pulvinar. At the meson, in the depression between the thalamus and the opticus, note the half of the conarium, more or less enveloped by the velum, and inclined caudad from its attachment so as to rest upon the opticus.

By an incision beginning just dorsad of the callosum and extending laterad and very slightly dorsad, remove the dorsal portion of the hemisphere. The features of the cut surface may be disregarded here; the object is to permit what is left to be raised more easily. On lifting this portion, note that its ventral surface presents slight fissures and striæ, trending latero-caudad. This is the formix (Pl. IV, Fig. 14, $f$.), including the lyra $\left(l y_{*}\right)$, the limits of which have not been determined. Note that, for a short distance from the splenium, the plane of the fornix coincides with that of the callosum, but that it curves ventrad so as to become nearly vertical at its cephalic end. The general shape of the fornix is triangular ; its larger portion is the body, and the narrower cephalic end is the columna. The band which forms its lateral border is the fimbria, which, as will be seen later, is continued nearly to the tip of the Ll. hypocampa.

Between the fornix and the diencephalon is a fold of pia, the velum. When freed from the velum, note the difference between the dorsal surface of the thalamus and the mesal surface already 
examined ( $\$ 1043)$; the latter is entocolian and covered by endyma; the former is wholly ectocœlian (Fig. 122).

But, along the free border of the fimbria, the velum, or vessels thereof, passes into the procolia, covered by the endyma, whose attachment may be noted along the entire length of the fimbria (Fig. 121). This solution of the continuity of the nervous tissue along the border of the fimbria is the rima ( $\mathrm{Pl}$. IV, Fig. 14, r.), and the intruded border of the velum is the proplexus (Pl. IV, Fig. 15, ppx.).

$\$$ 1145. Aula and Porta.-Cut off the larger part of the fornix with the corresponding portion of the callosum, gently push the formix from the thalamus, and note that, at about the junction of the body with the columna, the adhesion of the velum to both fornix and thalamus ceases suddenly, and that the surfaces of both, ventrad of the adhesions, are covered by the smooth endyma which is characteristic of the colian cavity.

So much of the interval between the fornix and thalamus as lies ventrad of the lines of adhesion is the porta. Its ventral limit is formed by the continuity of the two masses (Fig. 123).

The adhesions coincide with the lines of reflection upon the intruded velum of the endyma from the columna and thalamus. The slight space mesad of the porta is the aula, and the irregular cavity laterad of the porta is the procolia, which will be examined later.

So much of the brain as has not been specified appertains to the prosencephalon.

$\$ 1146$. Demonstration of the Procœlia, Rhinocœlia and Porta ("Lateral and olfactory ventricles and Foramen of Monro").-The procclia and porta have been incidentally exposed during the examination of the encephalic segments. The following directions deal particularly with their parietes and with the continuity of the procclia with the rhinocœlia.

Instruments and Materials.-The same as for the examination of the segments $(\$ 1139)$. The syringotome is preferable to the tracer.

For the most satisfactory study of the cœliæ and the plexuses, the arteries should have been injected with gelatin $(\$ 1126)$ and alcohol thrown into the Foramen infundibuli (\$1124). The following figures should be consulted before and during the dissection : 117, 119-123 ; Pl. II, Fig. 4; Pl. III, Fig. 13 ; Pl. IV. Fig. 14, 15, 16, 19.

\$ 1147. Opening the Cella Media of the Procelia.-Apply the edge of the large scalpel along a line $2 \mathrm{~mm}$. dorsad of the main part 
of the callosum, and make a slight incision. Then dip the scalpel in alcohol, and cut horizontally along that line so as to remove the dorsal part of the hemisphere.

On the surfaces so exposed note the ental alba (centrum ovale) and the ectal cinerea (cortex); also the undulations of the line of their junction, according to the depth of the fissures between the gyri. Slice the removed dorsal piece in various directions so as to show the continuity of the cinerea.

If the surface of the alba of the ventral part is uniform in color and continuous, remove successive slices, not more than $.5 \mathrm{~mm}$. thick, until, about $5 \mathrm{~mm}$. from the meson, there appears a group of dark points ; the removal of another very thin slice will then expose the summit of an arched cavity, the cella media of the proccelia, and a slightly undulating convex surface, the cephalic part of which is the fornix and the caudal the hypocampa ("hippocampus major").

Opening the Medicornu and Exposure of the Hypocampa.Push the syringotome very cautiously latero-caudad between the hypocampa and the cut edge which overhangs it, and then, with the scalpel, remove a wedge-shaped slice so as to expose more of the hypocampa. Repeat the operation, bearing in mind that the direction of the hypocampa and of the cavity - the medicornu-into which it projects, is successively caudad, laterad, ventrad, cephalad and mesad; see Fig. 119.

The anthropotomical terms indicative of these directions are backward, outwoard, downward, forward and inward, the initial letters of which form the mnemonic word bodfi.

During this exposure of the hypocampa, there is danger that some part of its surface will be sliced off, and the syringotome should be used as an explorer before each incision. When near the tip of the Ll. hypocampce, be especially careful not to cut too deeply; the tip of the cornu is here separated from the ectal surface by a very thin lamina.

When the entire length of the hypocampa is exposed, pass the convexity of the tracer along its caudal border, and then slice off the overhanging portions of the caudal wall of the medicornu. Note that the width of the medicornu varies somewhat, but that there is no sign of the caudal prolongation which, in man, the monkeys, seals and some cetacea, forms a postcornu. Note also two slight oblique ridges which cross the hypocampa in opposite directions, 
and the existence of something like the terminal expansion which, in the human brain, is called the "pes hypocampa."

\$ 1148. Demonstration that the Hypocampa is only a Modified Portion of the Proccelian Parietes.-Carefully pass the tracer between the opticus and the caudal border of the hemisphere, and rotate it so that the point may penetrate the hypocampa and appear in the medicornu. Withdraw the instrument without disturbing any connections ; see Fig. 121 and Pl. IV, Fig. 18, 19.

$\S 1149$. Opening the Procornu and Exposure of the Striatum.From the roof of the procoelia, cephalad of the orifice first exposed, remove a thin wedge-shaped slice, and thus more completely expose the fornix, which is seen to be continued along the cephalic border of the hypocampa as a flat band, the fimbria.

Cephalad of the fornix is a marked elevation, the striatum; that part of the procelia into which it projects is the præcornu. Between the striatum and the fornix and fimbria is a depression, the Sulcus limitans, into which projects a plexus, the proplexus. The sulcus and the plexus may be traced along the cephalic border of the fimbria to near the tip of the medicornu ; their relations will be seen better at a later stage of the dissection.

Note that the cella media does not quite reach the meson, on account of the continuity of the fornix and the hypocampa with the callosum. As will be seen later, the only place where the procoelia does reach the meson is at the bottom or mesal end of the Sulcus limitans, where the porta communicates with the aula and thus with the mesal series of cœliæ.

Exposure of the Mesal Aspect of the Striatum.-Along a line passing dorso-ventrad about $2 \mathrm{~mm}$. cephalad of the chiasma, make an incision $1 \mathrm{~mm}$. deep at the venter, its dorsal end reaching the mesal border of the striatum, as already exposed.

From the mesal aspect of the olfactory lobe and cephalic part of the hemisphere remove a slice about $1 \mathrm{~mm}$. thick, and then, with the small scalpel, cut successively thin wedge-shaped slices so as to expose the mesal aspect of the striatum and the cavity, the pratcornu, into which it projects. Note the somewhat sharply defined ridge which separates the mesal from the dorsal surface of the striatum, and the greater extent of the former.

$\S 1150$. Opening the Rhinocoelia.-The ventro-cephalic angle of the præcornu presents a slight funnel-shaped prolongation, which may be traced cephalad into the Crus olfactorium, and to within 
about $2 \mathrm{~mm}$. of the end of the Lobus. This canal is the reduced representative of the rhinocœlia, which is quite large in most lower vertebrates and in many mammals, but nearly or quite obliterated in man. It is most easily traced by means of a beaded bristle. If the bristle is gently moved to and fro, enough of the coloring matter of the bead will adhere to the sides of the canal to make its recognition more easy as it is exposed, either by removing very thin slices or by following it up with the tracer. The diameter of the canal is about $.5 \mathrm{~mm}$., but it is usually expanded a little at its extremity.

\$ 1151. Demonstration of the Porta.-Recall the position of the aula upon the meson. Hold the brain in alcohol or water so that only the surface cephalad of the dorso-ventral incision projects above the surface; then blow toward the aula from between the striatum and the mesal wall of the præcornu. The escape of bubbles of air will demonstrate the connection, through the porta, of the aula with the procœlia; see Fig. 120 and Pl. IV, Fig. 16.

\$1152. Exposure of the Proccommissura.-Remove the cephalic end of the brain by an incision at about the middle of the striatum, and note, on the cut surface, the alternation of alba and cinerea on account of which the name was applied. Remove other slices, cutting a little obliquely, latero-cephalad, and note the increasing distinctness of an oblong white area, the oblique section of a fibrous fasciculus-the præcommissura-which unites the striata and Lobi olfactorii across the meson, and which has been observed already in the examination of the mesal surface; see also Pl. IV, Fig. 14.

Fig. 119.-Plaster casts of the medicornua, inverted.

Fig. 120.-Plaster casts of the diaccelia, aula, right porta, and part of the right procœlia of the sheep, inverted.

Fig. 121.-Diagram of a transection of the left medicornu.

Fig. 122.-Diagram of a transection of the diacœlia.

Fig. 123.-Dingram of a transection of the porta.

$\S$ 1153. Fig. 119.-Plaster casts of the medicornua, inverted ; $\times 1.5$. Compare with Pl. IV, Fig. 15.

Objects.-To show (1) the shape and extreme curvature of these parts of the procœliæ; (2) that they are completely circumseribed, and do not open by a " fissure of Bichat" upon the surface of the hemisphere.

Preparation.-The plaster was injected through the Foramen infundibuli while the brain was supported by the calva (\$ 1125). After resting undisturbed for an hour, the brain substance was carefully torn and cut sufficiently to extricate the casts.

Explanation.-The ventral ends are thinner and somewhat expanded, excepting the extremities, which are decidedly contracted. This contracted finger-like portion is wholly enclosed by true nervous parietes; all the rest is bounded along the concave (cephalic) 


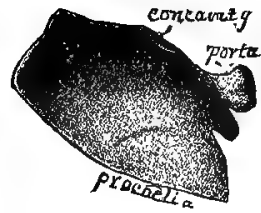

FIG. 120.

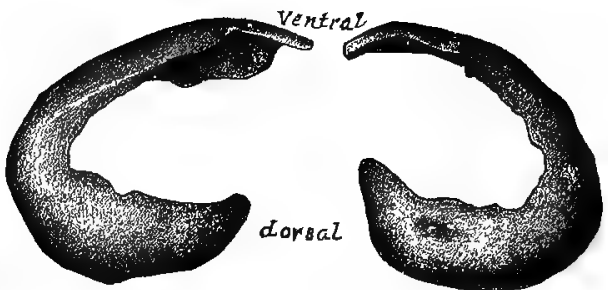

FIG. 119.

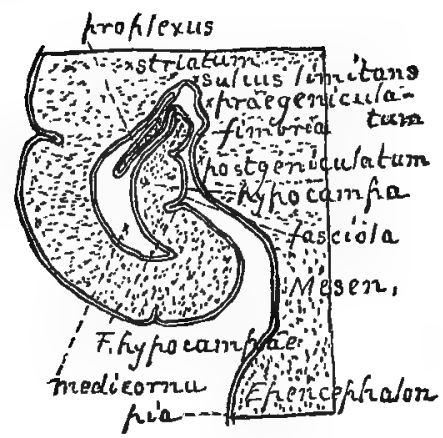

FIg. 121.

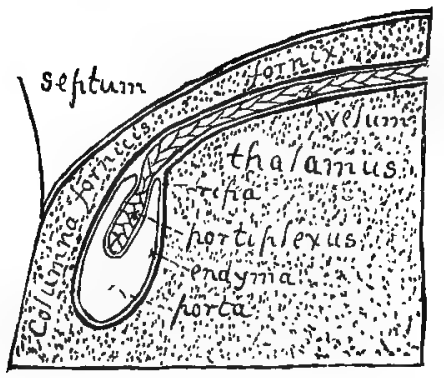

FIT. 123.

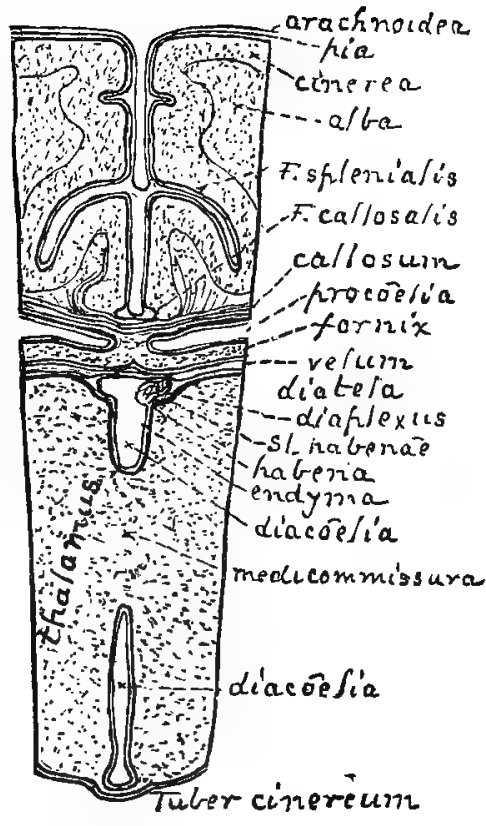

FIG. 122.

Fig. 119-123.-Casts of The Collie and Dragrams indicating the Manner of THeIR Circumscription. 
border by the rima, where the continuity of the endyma reflected upon the proplexus, and its adhesion along the fimbria and in the Sulcus limitans prevented the escape of the injected material.

\$ 1154. Fig. 120.-Plaster casts of the diacclia (dorsal portion), aula, and right porta and præcornu of the sheep, inverted ; $\times 1.5$. Compare with Fig. 113, 122 ; Pl. III, Fig. 7, 10 ; Pl. IV, Fig. 16, 18, 19.

Objects.-To show that (1) the porta permits the passage of injection mass from the aula into the procolia; (2) the aula, porta and diaccelia are completely circumscribed; (3) the porta is a passage of some length, and strongly compressed.

Preparation.-The plaster was injected through the Foramen infundibuli (\$ 1125). Notwithstanding all possible caution in disengaging the casts, a fracture occurred just between the porta and the aula. In mounting, a slight interval was left between the parts. The left porta and procolia are not included in the figure. The ridge on the ventral aspect of the cast of the diacolia corresponds with the dorsal contour of the medicommissure. The cast of the precornu is quite thin, and its lateral aspect presents a sharp longitudinal depression corresponding with the ridge upon the striatum (Pl. IV, Fig. 16).

Qualification.-The three following figures (121-123) are diagrammatic representations of our present understanding of the relations of the plexuses to the membranous parietes of the porta, diacoelia and medicornu.

It is probable that microscopic sections will be needed in order to elucidate certain points, especially the question whether the proplexus and portiplexus are formed by the intrusion of the entire pia or only of vessels therefrom (\$1081). We have thought best to represent the former view provisionally.

Admitting that in respect to this detail we may be in error, we wish to insist upon the more essential point, viz., that these cavities are really and completely circumscribed by the continuity of the endyma reflected from the borders of the nervous parietes upon the plexuses.

\$ 1155. Fig. 121.-Diagram of a transection of the left medicornu. Compare with Fig. 113, 119 ; Pl. III, Fig. 11; Pl. IV, Fig. 14, 15 ; also with Duval (2, Pl. I, Fig. 2).

Object.-To show that the medicornu is completely circumscribed, although the proper nervous parietes are absent from the porta to near its tip, constituting the rima.

Explanation.--The figure is based upon Fig. 113, but the cornu is disproportionally enlarged, as are also the distances between the hemisphere and the mesencepha] on and diencephalon. The membranes and the proplexus are also introduced.

It should be remembered that this represents a transection of the length of the medi. cornu, although, from the fact that the plane of section is horizontal with respect to the brain as a whole, the cavity closely resembles a longitudinal exposure of the postcornu of man and the monkeys, which does not exist in the cat.

The ectal surfaces of the hemisphere and of the epencephalon, mesencephalon, postgeniculatum and prægeniculatum are covered by pia. The cornu is lined by endyma.

In the frog and presumably in the embryo of the cat, the entire length of the medi. cornu has complete nervous parietes, as has the tip of the cornu in the cat and man. The endyma and the pia would then be wholly separated.

But the abrogation of the nervous continuity between the border of the fimbria and the Sulcus limitans permits the vessels of the pia, or perhaps a fold of the pia itself, to 
intrude apparently into the cornu. In so doing, however, the endyma is carried before so as to encompass the fold or the vessels, as an abdominal viscus is surrounded by the peritoneum. Hence, also, the cornu is really completely enclosed by the endyma, even where the nervous parietes are absent.

The general name striatum is given to the thickened part of the hemisphere, but the cauda striati and tonia are not represented.

§ 1156. Fig. 122.-Transection of the brain through the diacclia. Compare with Fig. 120; Pl. III, Fig. 7, 10, 13 ; Pl. IV, Fig. 15.

Objects.-To show (1) that the diacclia has a roof (diatela) independent of the velum and fornix ; (2) that the dorsal surface of the thalamus is ectocolian; (3) that the arach. noidea does not reach the bottom of the interhemispheral fissure.

Explanation.-The diaccelia is seen to be divided into a larger ventral and a smaller dorsal portion by the large medicommissura. The walls (lateral parietes) of both portions are the thalami.

The dorsal surface of the thalamus is convex and covered by pia. The mesal is nearly flat and covered by endyma. The rounded angle between these two surfaces presents a ridge, the habena, and a furrow, the Sulcus habenæ.

The endyma leaves the thalamus along the sulcus (Pl. IIf, Fig. 7), and is reflected first dorsad and then mesad so as to. roof in the diacolia completely, as indicated in Pl. III, Fig 10, and by the definite form of the plaster cast shown in Fig. 120.

In the frog and in the embryo mammal, the only parts dorsad of the diacolia are this endyma and the pia and arachnoid which envelope the entire brain, together constituting a membranous diatela. But in the adult mammal, the hemispheres not only project dorsad of the diencephalon, but unite along the meson so as to form the callosum and the mesal part of the fornix (lyra) ; hence these parts are dorsad of the diatela, and the latter is often ignored on account of its tenuity.

The relation of the diaplexus to the diatela is not altogether clear. In the figure only one is represented. There is no evidence that it is formed by the intrusion of the pia along the Sulcus habenæ, and more probably it is primarily prolonged from the aula, as in Menobranchus, but acquires a somewhat distinct adhesion to the diatela.

$\$ 1157$. Fig. 123.-Diagram of a transection of the porta (foramen of Monro). Compare Fig. 113, 120, and Pl. III, Fig. 18, 19.

Object.-To show that the porta is completely circumseribed, notwithstanding the absence of the proper nervous parietes at the dorsal end where it adjoins the rima.

Description.-The plane of section is dorso-ventral and longitudinal, practically parallel with the meson. Regarding the porta as a short passage with an approximately transverse direction, it is here transected.

The larger mass is the dorso cephalic portion of the thalamus. Cephaiad and dorsad of it is the fornix with its Columna, these being continuous with the corresponding half of the septum lucidum.

The dorsal surface of the thalamus is convex and covered by pia (velum), but the cephalic surface is concave and covered by endyma. In man and in some cats, the boundary between the two surfaces is indicated by a more or less distinct tubercle. In all cases there is a point of reflection of the endyma cephalad toward the fornix.

The corresponding surfaces of the fornix are likewise pial and endymal ; there is no elevation of the surface to mark the boundary between the two, but nearly opposite the point of reflection of the endyma from the thalamus it is reflected also from the fornix.

So much of the interval between the thalamus and the fornix as lies ventrad of these two points of reflection is the porta. Primitively, there is reason to believe, the endyma 
continued directly across, as the lining of the original nervous roof. The abrogation of the nervous portion of that roof permitted the intrusion of the pia (or of its vessels) to form the portiplexus, upon which the endyma is reflected.

The boundaries of the porta are, then, as follows: caudal, the thalamus ; cephalic, the fornix ; ventral, the continuity of the two; dorsal, the endyma, reflected upon the portiplexus.

\section{EXPLANATION OF THE PLATES.}

$\S 1158$. The four lithographic plates of the brain illustrated the senior author's paper (1t) in the Proceedings of the Philosophical Society of Philadelphia, by courtesy of which they are included in the present work. With slight alterations, the following explanations are the same as given in that paper, but quotation marks are used only where specially needed.

As with the other figures of the brain, all of the preparations from which the figures were drawn are in the Museum of Cornell University, and are accessible for examination to those who may desire to verify the figures or the descriptions.

In most cases, each figure is based upon more than one preparation. Encephalotomists need not be reminded of the difficulty of obtaining a preparation which shows many points of strncture equally well. Since the present account is only general, and does not aim to indicate individual peculiarities, or those of sex, breed or age, most of the figures may be regarded as representing what may be called an average cat's brain. It is obvious that a very large number of specimens would need to be carefully compared in order to confer upon any generalization respecting sex, etc., a trustworthy character.

"Most of the figures are twice the diameter of the preparations, and, with the exception of Fig. 1 and 2, it would have been better to make the enlargement four or five diameters. Aside, however, from the greater expense which this would have involved, such a degree of enlargement would have rendered it not only possible but necessary to show certain details of structure upon which my information is, at present, imperfect.

"All of the figures have been drawn from my own preparations by Miss G. D. Clements, B. S., at the time a student in the Natural History Course in Cornell University.

"Artists and anatomists who have undertaken to represent the details of encephalic structure understand the difficulties of the task, and will admit that the omissions and inaccuracies to which attention is called in the descriptions are both few and unimportant compared with the general thoroughness of the work. Indeed, for all the deficiencies, I hold myself much more responsible than the artist, by whom some of the figures were drawn at least four times, twice upon stone."

The abbreviations are explained in $\$ \$ 1127,1128$; synonyms, references and brief descriptions are given in the latter part of this chapter ( $\S 1181-1333$ ).

\section{PLATE I.}

\$1159. Fig. I.-The dorsal aspect of the brain; $\times 2$.

"The general form and some of the fissures are drawn from Prep's 288 and 289, the bisected brain of a white and Maltese $q$; but the fissures of the right hemisphere are derived from several different preparations. 
"The Lobi olfactorii ( $L . o l$.) are made somewhat too prominent; there is considerable difference among cats in this respect, although much less than among dogs.

"The general features of the cerebellum $(c b l$.) are well shown. The Lobi laterales $(L . l$.) have only a fair proportion to the median lobe or vermis $(v m$.), instead of the preponderance which they have in the human brain The lateral contortion which characterizes the caudal aspect of the vermis in adult cats (as shown in my paper, 11, 221, Pl. I, Fig. 1 and 2) does not affect the dorsal part.

"As already stated, the fissures of the hemispheres are differently represented upon the two sides. The combination of the two kinds of fissural arrangement in a single figure serves to illustrate the extent of the lateral variation and compensation to which attention was called by me in $1873(\mathbf{1}, 282)$."

$\$ 1160$. Fig. 2. - The sinistral aspect of the brain. From Prep. 288; $\times 2$. Compare with Fig. 124.

The Lobus olfactorius $(L . o l$.$) is made somewhat too prominent. The curved line$ upon its lateral surface indicates, approximately, the boundary of the more cephalic portion of the pero or ectal layer, whence arise the Nervi olfactorii. These nerves are not shown.

The features of the Crus olfactorium indicated by $l m$. cin. and $l m$. alb. are more fully shown upon Fig. 3.

The Nervus opticus $(N . o p$.) projects from the ventral margin of the figure, and the Fissura Sylviana $(F . S$.) is seen dorso-caudad of it.

The ventral end of this fissure, as is always the case in the cat, joins the fissure which forms the dorso-lateral boundary of the Tractus olfactorius ( $T r, o l$.$) , and the cephalic and$ caudal divisions of that fissure are called respectively rhinalis and postrhinalis ( $F F$. $r$. and $p r h$. .). So much of the hemisphere as lies caudad of the $F$. Sylviana forms the Lobus temporalis $(L \cdot t m p$.$) , the ventral extremity of which is the Lobulus hypocampæ ( L l$. hmp.).

The cerebellum $(c b l$.$) presents the narrow median lobe or vermis (vm.), and the Lobus$ lateralis $\left(L . l_{\text {. }}\right)$. Near the ventro-cephalic angle of the latter, two or three of the laminx of the second tier project as the Lobulus appendicularis $(L l . a p$.), which is seen better in Fig. 3.

The metaplexus shown in Fig. 3 (mtpx.) has been removed so as to expose the prominent Eminentia auditoria ( $E m . a u$.), whence springs the N. auditorius $(N . a u$.).

Just ventrad of the eminence is the trapezium ( $t z$.), and cephalad of this is the pons $(p n$.).

Between the pons and the hemisphere appears a part of the Crus cerebri $(C r . c b$ ), and cephalad of this is the slender $\mathrm{N}$. trochlearis $(N, t r$.$) , which, by inadvertence, seems to$ emerge from the $F$. postrhinalis instead of from between the cerebellum and the hemisphere.

The N. trigeminus $(N . t r g$.) has been cut short, in order the more clearly to show that it usually emerges just caudad of the pons, and not through it as in man.

The remaining nerve origins are indicated only by dots. Those of the NN. glossopharyngeus, vagus and accessorius ( $N N . g p h$, v. and $a c$.) form a series. At the side of the myelon, near the dorsal and ventral borders, are seen the origins of the first cervical nerve (N. $c v .1)$.

In this figure the fissures are accurately represented as they are in the preparation, excepting that the small F. lunata $(F, l /$.$) has been added from Prep's 519$ and 520. The small $F$. intermedia might well have been inserted between the dorsal ends of the FF. anterior and postica ( $F F$. $a$. and $p$.). 


\section{PLATE II.}

\section{$\$ 1161$. Fig. 3. - The basis encephali, or ventral aspect of the brain; $\times 2$.} Compare with Fig. 116.

The proportions and general features are from the brain of an adult $q$, Maltese and white, Prep's 288, 289. Some details of the Area præchiasmatica (the region ceplralad of the chiasma) are from 461 and 527 ; of the Ar. postpontilis (the region caudad of the pons) from 358, 454 and 491 ; and of the intermediate Ar. cruralis from 422,506 and 527.

Most of the nerves and cerebral fissures are lettered on the right side, and most of the other parts on the left. Some of the left nerves are cut short, and the left $\mathbf{N}$. trochlearis is not shown at all.

The Lobi olfactorii ( $L l . o l_{\text {. }}$ ) are made too long, and the hypophysis ( $h p h$.) is too short.

Attention is called to the following points, chiefly in comparison with the human brain :-

The absence of a distinct Radix intermedia ( $R x$. in.) of the Crus olfactorium, corresponding with the so called "middle root of the olfactory nerve" in man. The part so designated upon the plate is appurently only an area, comparatively undifferentiated, between the more or less fibrous tracts forming the Radix mesalis and Rx. lateralis.

The turning of the Rx. mesalis (Rx. ms.), ("internal root"), over the margin of the brain so as to appear upon the meson.

The distinction of the Rx. lateralis ( $R x . l$.), (" external root "), into a lateral gray and a mesal white tract, the Limes cinerea ( $L m$. cin.) and the Lm. alba.

The great extent of the (Locus) praperforatus ( $p r p f$. ), and its division into a cephalic more prominent portion (Pt. p.) and a caudal depressed portion (Pt.d.). Both portions are "perforated," but the degree of furrowing of the Pt. prominens varies considerably. These furrows exist in some other Carnivora.

The width of the hypophysis ( $h p h$.) and the crenation of its caudal border, indicating the existence of an ental subspherical mass, which is covered by an ectal layer, the thinness of which, in the caudal region, permits the contour of the former to be seen.

The slight degree of separation of the albicantia ( $a b n$.$) , which are here nearly con-$ cealed by the hypophysis, but more fully shown in Fig. 12.

The distinctness of the cimbia ( $c m b$.), which is better seen in Fig. 11.

The slight extent of the true postperforatus ( $p p f$.) ; the only part which is really "perforated" is a small area just caudad of the albicantia, and partly hidden by them.

The less caudo-cephalic extension of the pons (pn.); this exposes more of the Area intercruralis ( $\mathcal{A} r$. ic.) than in man, and uncovers the trapezium (tz), which, in man, is wholly concealed. Connected also with this feature of the pons is the fact that the $N$. abducens $(N, a b d$.) passes directly cephalad from its origin a little caudad of the pons, whereas in man it is forced to curve around the caudal border. Finally, the N. trigeminus $(\boldsymbol{N} \cdot \operatorname{trg}$.$) , instead of emerging through the pons near the cephalic border as in man,$ emerges close to its caudal border or clears it completely ; see Chap. XI.

The greater extent of the Ar. cruralis, which may be ascribed both to the less extent of the pons and the less degree of flexure of the whole brain at the mesencephalic region.

The greater width of the Tractus postrhinalis (Tr.prh.), which includes the surface of the Lobulus hypocampa $(L l . h m p$.). In man, indeed, this part is hardly visible on account of the prominence of the convolutions laterad of the $F$. postrhinalis.

The apparent origin of the $N$. oculomotorius $(N$. ocm. ) laterad of the meson and just caudad of the cimbia $(\mathrm{cmb}$.). 
The apnearance of a division of the ectal layers of the pontile fibers into three groups, cephalic, caudal and intermediate, the latter partly overlapped by the other two.

The appearance of a faint band crossing the trapezium a little obliquely between the origins of the NN, abducens $(N . a b d$.$) and facialis \left(N . f^{\prime}\right)$. The distinctness of this band varies.

The origin of the $\mathbf{N}$. hypoglossus $(N . h g$.) at the lateral border of the Area elliptica (Ar. el.), which is thought by some (Am. Jour. of Neurology, etc., I, 102) to be the surface of the oliva or "olivary body" of man. The determination of this point involves some comparisons and sections which we have not yet made.

The close association of the roots of the $N N$. glossopharyngeus ( $N$. gph.), vagus ( $N . v$.) and accessorius ( $N$. ae.). See Stowell (I) and Chap. XI.

The marked prominence of the ventro-lateral region of the metencephalic continuation of the Columna lateralis myelonis (Clm. l.), forming an elevation to which I have applied the provisional name Area ovalis $(A r .0 v$,), but which is thought by some (Am. Jour. of Neurology, etc., I, 102) to represent the Tuberculum Rolandii.

The absence of any superficial decussation of the pyramides (py.). Hence, the $F$. ventrimesalis ( $F$. vms.), or "anterior fissure," is uninterrupted. The $F$. ventrilateralis $\left(F . v l_{.}\right)$is deflected at the caudal end of the Area elliptica.

$\$ 1162$. Fig. 4.-The mesal surface of the right hemiencephalon; $\times 2$. Compare Fig. 114.

The general features are from the same brain as Fig. 3, but some are derived from Prep's 290, 304 and 454.

The surfaces shown in this figure are of four kinds, as follows (\$ 1137) :-

(1) The natural surfaces which are covered by pia. These are the mesal aspects of the hemisphere (hem.) and the Lobus olfactorius ( $L$. ol..).

(2) The natural mesal surface (Ar. spt.) of the right half of the septum lucidum, which, in the cat, is in contact with its platetrope, or separated therefrom only by a thin layer of connective tissue. We bave never observed an interval corresponding to the pseudocolia or "fifth ventricle" of man.

(3) The natural endymal surfaces of the true coelice or "ventricles." Of course the procœliæ ("lateral ventricles") do not appear.

(4) The cut surfaces of the commissures and other parts which cross the meson or lie upon it. In the cerebellum ( $c b l$.), the relative areas of the ental alba and the ectal cinerea, forming the arbor vita (arb.), are indicated by the shading; with less definiteness, the alba is shown in the callosum (cl.), the fornix (f.), the pracommissura (pres.), the postcommissura (pcs.), the commissura habenarum (cs. h.), and the chiasma.(ch.). The section of the medicommissura (mcs.) should appear as if composed, at least chiefly, of cinerea, but no attempt has been made to indicate the nature of the cut surfaces of the Crista fornicis (Crs. f.), the terma (t.), the hypophysis (hph.), the infundibulum (inf.), the conarium (cn.), the optici and postoptici (op. and pop.), the valvula (vv.), the Crura cerebri (Cr. cb.), the metatela (mttl.), or the rest of the epencephalon and metencephalon (mten.). The extent of the transverse fibers of the pons $(p n$.) should have been represented, at least approximately.

So much of the cephalic boundary of the aula (a.) as intervenes between the pracom. missura (pres.) and the crista (Crs. f.) is very thin, and is too indistinctly shown in the figure: Neither bere nor at any other point is there any such interruption of the wall as would form a communication between the true caliæ and the pseudocolia or the ectal surface of the brain. It is probable that the presence of such a communication as is 


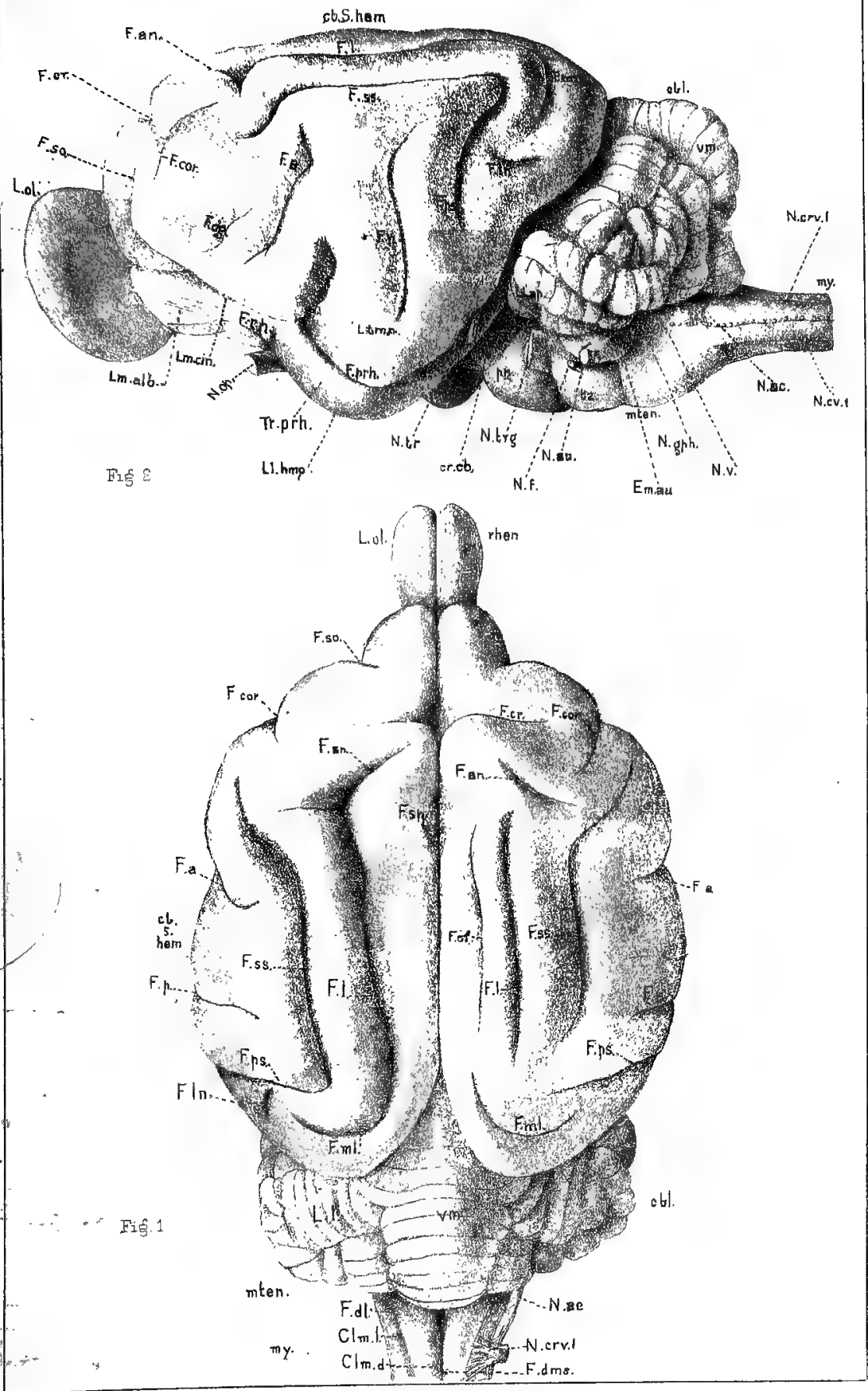





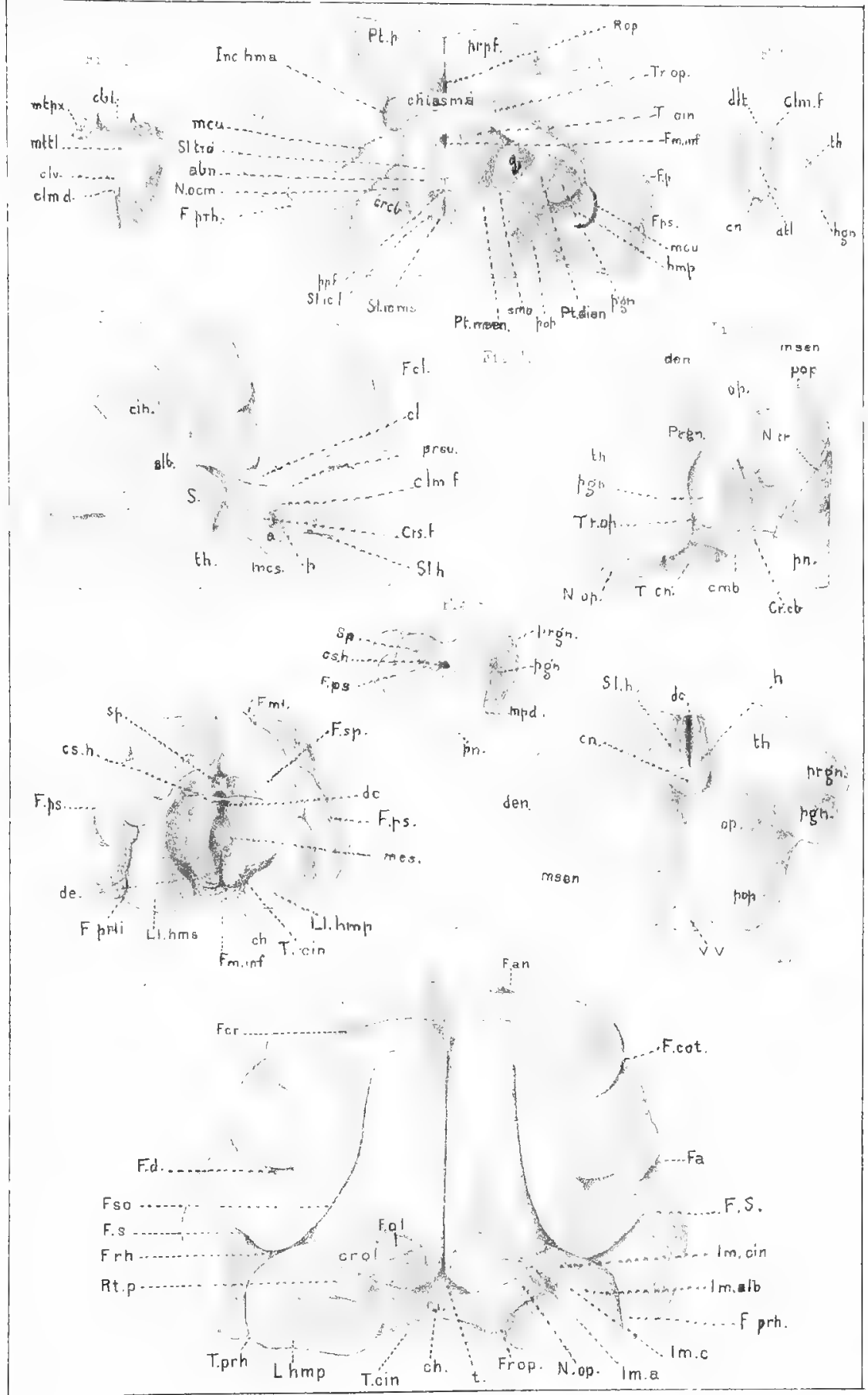




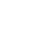

, 


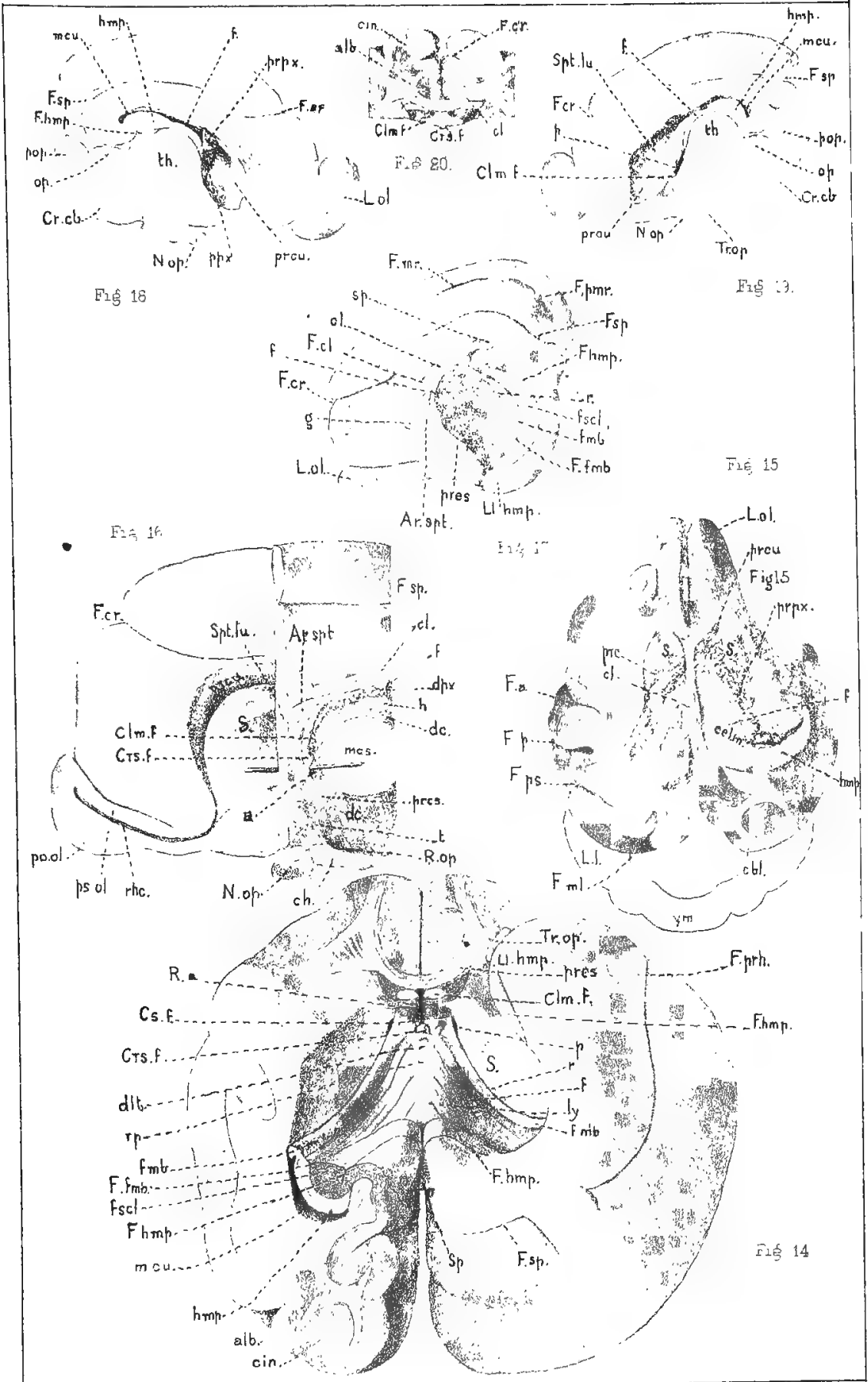


ascribed to the human embryo and to some animals in Quain (A, II, 543) is due to the artificial rupture of the natural connections; see p. 536 of the same work.

Attention is called to the following points, chiefly in comparison with the human brain :-

The appearance of the Rx. mesalis ( $R x . m s$.) on the meson, and the presence of two shallow fissures, postradicalis and preradicalis (FF. prd. and prrd.) between it and the adjoining surfaces of the hemisphere.

The large size of the commissures, especially the medicommissura, which nearly fills the dorsal part of the diacœlia $(d c$.).

The non-appearance of the porta when the meson is viewed squarely; it is doubtful whether the human "foramen of Monro" is really visible from the meson.

The less extent of the callosum, especially of its rastrum $\left(r m_{*}\right)$. In some human brains the rostrum does not extend so far as is usually represented.

The darker spot on the section of the hypophysis represents the space occupied by the ental mass, which has been removed.

The relations of the pia are not indicated at all, and are not well understood, especially between the cerebellum and the metencephalon and mesencephalon.

\section{PLATE III.}

With the exception of Fig. 13, all the figures upon this plate represent the natural surfaces of regions which are more or less completely concealed by other parts in the undissected brain.

\section{$\S$ 1163. Fig. 5.-The cephalic aspect of the prosencephalon. From Prep. $294 ; \times 2$.}

The hardened brain was transected at the $f$. postica, so that the preparation includes only the cephalic two thirds of the prosencephalon.

The drawing represents the preparation tilted up so as to expose the ventral aspect foreshortened.

As compared with Fig. 6, this might well have been made of the natural size. A less regularly symmetrical brain would have been more instructive. One of the Crura olfactoria should have been divided at a little greater distance from the prosencephalon.

So far as appears in the figure, the fissures are remarkably alike upon the two sides; the left F. ansata ( $F$. an.), however, only the meso-cephalic end of which appears in the figure, presents the somewhat unusual but very suggestive condition of entire independence of the lateralis (which is invisible) and the coronalis ( $F$. cor.). On the right side it is joined by the former fissure.

The right F. Sylviana $(F . S$.) is shorter than the left, and presents a slight terminal bifurcation which is not shown.

In consequence of the removal of the Lobi olfactorii and the tilting of the whole preparation, so much of the $F$. rhinalis $(F . r h$.) as lies cephalad of its union with the superorbitalis ( $F . s o$.) is practically obliterated, and the remainder of it is so foreshortened as to appear as an insignificant intermediate portion of an extensive u-shaped fissure formed by the FF. Sylviana ( $F, S$.$) and superorbitalis (F .80$.$) . "The appearances thus presented$ are suggestive in view of the idea of Meynert $(1,12)$, which I also entertained at one time (11,225), that the F. superorbitalis represents the "anterior branch" of the human F. Sylviana, and that the intervening region corresponds to the "operculum." "

A slight preponderance of the left hemisphere just caudad of the $\mathbf{F}$. Sylviana is some- 
what exaggerated in the figure, and the Crura olfactoria $(C r . o l$.$) should differ less in form$ and in their distance from the meson.

The FF. olfactoria $(F, o l$ ) appear as little more than shallow furrows.

On account of the foreshortening of the ventral aspect, the line of separation of the Portio prominens (Pt.p.) and the Pt. depressa (Pt. d.) is indistinct. T'he hypocampal lobule ( $L l . h m p$.) on each side has been flattened by pressure, and is so represented.

$\$ 1164$. Fig. 6.-The caudal aspect of the prosencephalon, with part of the dien. cephalon. From Prep. 292, an adult $q ; \times 1$.

The diencephalon has been transected so as to leave a concave surface which, at the meson, is close to the caudal border of the medicommissura (nces.), but rises at the sides so as to coincide nearly with the caudal surface of the prosencephalon.

The postcommissura has been removed, and the slender transverse band (Cs. $h$.) just ventrad of the splenium ( $s p$.) is the Commissura habenarum. Had the postcommissura been left, the intervening space would be a foramen, Fm. conarii.

The shallow depression of the ventricaudal surface of each hemisphere just laterad of the splenium represents the area of contact of the opticus.

The cerebral fissures are markedly unsymmetrical, and thus in contrast with those of Fig. 5. The right postrhinalis $(F \cdot p r h$.) is the longer, and the right postsylviana ( $F . p 8$. joins the supersylviana, although the place of union does not appear in the figure. On the contrary, by reason of the perspective, it seems to be joined by the F. medilateralis (F. $m l_{0}$ ).

The hypocampal lobules ( $L l$. hypocampa) have their proper rounded form in this preparation.

Part of the diacclia ( $d c$.) appears dorsad of the medicommissura, and part on its ventral side (Fig. 122). In man, the commissure is smaller and the colia correspondingly more extensive. On account of the removal of the hypophysis and infundibulum, the diaccelia opens freely at the Fm. infundibuli ( $F m$. inf.).

$\S 1165$. Fig. 7.-The dorsal aspect of the diencephalon and mesencephalon. From Prep's 397 and 494, adult $q$, 423, a nearly adult $\&$, and 506 ; $\times 2$.

The principal features of this figure were drawn from Prep. 506. The preparation was made by lifting the caudal ends of the hemispheres and gradually separating them, with the callosum, fornix and velum, from the subjacent parts. The epencephalon and metencephalon were then removed by a transection just caudad of the postoptici.

The valvula (vv.) is drawn as it appeared in Prep. 494, after inflation by blowing air from the diacœlia through the mesocœlia or iter.

The Commissura habenarum $(C s . h$.) is really more distinct in Prep. 397 than appears in the figure. The habenæ $(h$.$) , their sulci (S l . h$.) and the lines of reflection of the endyma are taken from Prep. 422, and their distinctness is not exaggerated in the figure; see Fig. 122.

The complete roof of the diacolia, the diatela (dtl.), is shown in Fig. 10.

As compared with the homologous parts in man, the feline postoptici (pop.) and geniculata (pgn. and prgn.) are larger, while the thalami proper (th.) seem to be only the mesal continuations of the progeniculata (prgn.), and to lack altogether the pulvinar or "posterior tubercle" of man.

$\S 1166$. Fig. 8.-The caudal aspect of the mesencephalon, with parts of the adjoining regions. From Prep. $506 ; \times 1$.

The plane of transection coincides nearly with the caudal surface of the postoptici $(p o p)$, and has divided the pons ( $p n$.$) a little caudad of its middle. The valvula (vv.) was$ 
torn from this preparation, and the line of its attachment is not distinctly shown. Something of its position may be judged from Fig. 7. The caudal orifice of the mesocclia (mse.) is shown here as a nearly regular circular spot; in reality, it presents a slight mesal extension at both the dorsal and ventral sides. Indeed, when carefully examined, the so called "aquæductus Sylvii" is far from being a perfectly simple and uniform tube : its form in man is indicated in Reichert's Fig. 31 (A, Taf. 4). Among the lower mammals it is usually larger, and with the lower vertebrates it often has the proportions of a true colia, with lateral extensions.

The cimbia ( $\mathrm{cmb}$.) is partly seen on the right. The geniculata ( $p g n$. and prgn.) do not project as far as they should. The optici are wholly hidden from view by the prominent postoptici ( pop.).

$\S 1167$. Fig. 9.-The sinistral aspect of the mesencephalon and diencephalon. From Prep's 491 and $506 ; \times 2$.

The only cut surface shown in this figure is that caused by the oblique transection between the diencephalon and the prosencephalon; the plane of section followed the cephalic border of the Tractus opticus ( $T r . o p$. ), and corresponds with the Bulcus limitans between the thalamus and the striatum.

Crossing the crus $(O r . c b$.), just caudad of the postgeniculatum ( $p g n$.$) , is seen the$ cimbia ( $\mathrm{cmb}$.).

"Upon this figure should appear the Lemniscus superior and $L$. inferior, and the postbrachium and probrachium, provided they exist in the cat as distinct parts visible at the surface. I have not been able to satisfy myself respecting their exact position and limits in the human brain, and refrain from expressing any opinion concerning them."

$\S 1168$, Fig. I0.-The dorsal aspect of the diencephalon, including the diatela. From Prep. 301, a half grown $\delta ; \times 1$.

The object of this figure is to show the existence of a distinct roof of the diacnelia independent of the fornix and velum, which have been removed. This diatela $(d t l$.) presents the appeurance of something more than the lining endyma, but its structure has not, so fax as we know, been examined. The darker triangular area at the cephalic end of the diatela corresponds with the delta fornicis (dlt. $f$.).

\$1169. Fig. II.-The Area cruralis, with part of the pons and of the Ar. præchiasmatica. From Prep's 506, 425 (nearly adult + ) and $461(\hat{\delta}) ; \times 1.5$.

This figure, reversed, is reproduced in outline in Fig. 118. The general relations of the medicornu are also indicated in Fig. 113, and the relations of the cornu, rima and proplexus in Fig. 121.

The Lobi temporales have been divided at different levels on the two sides. From the right, only the extremity, or $L l$. hypocampa, has been removed, and the section of the medicornu (meu.), which is here cut very obliquely, is a slightly curved space completely circumscribed by a nerrous wall. Neither in the cat, nor-contrary to the common belief and the explicit statement in Quain (A, II, 542, 544)_-in man, dues the rima or "great transverse fissure" extend to the tip of the medicornu.

Where the $L l$. hypocampa rests against the Tractus opticus (Tr. op.), there is usually a deep notch which may be called the Incisura hypocampa (Inc. hmp.).

On the left side, the liemisphere was dissected off so as to leave two cut surfaces. One of these surfaces is plane and nearly horizontal, and lies at about the level of the dorsal end of the postgeniculatum (pgn.). The other is convex, and extends from the cephalic border of the former obliquely to the ventral surface of the brain ; it corresponds closely with the cephalic border of the Tractus opticus. 
The left medicornu is cut at about the middle, and at nearly a right angle with its course ; hence its lumen presents its characteristic crescentic section, the ental boundary being the convex surface of the hypocampa ( $h \mathrm{mp}$.).

The cephalic margin of the medicornu is here seen to reach the surface of the hemisphere close to the Tractus opticus, and this narrow line of interruption of the true nervous wall of the cornu constitutes the rima. The scale upon which this figure was drawn did not permit the relations of the pin, the velum and the proplexus to be shown, and the undulations of the ectal surface, corresponding with the $F F$. hypocampo and fimbrice, and the fasciola and fimbria are hidden by the projecting postgeniculatum.

Most of the cephalic portion of the brain has been removed, but the Portio depressa (Pt.d.) of the præperforatus is seen, with part of the Pt. prominens (Pt. p.). The removal of most of the chiasma (ch.) exposes the form and extent of the Recessus opticus (R. op.).

The pons has been transected obliquely, and its caudal portion removed, together with the rest of the epen. and the metencephalon.

The left crus $(C r . c b$.) is seen in its whole length, excepting a small part concealed by the slightly projecting cephalic border of the pons. The well marked cimbia ( $\mathrm{cmb}$.) may perhaps be regarded as the boundary between the diencephalic portion of the crus ( $P t$. dien.) and the mesencephalic portion (Pt. msen.), which more directly supports the optici and postoptici ; in man, this part seems to be more nearly concealed by the pons.

The right $\mathrm{N}$. oculomotorius $(N . \mathrm{ocm}$.) is seen to emerge from the crus just caudad of the mesal end of the cimbia and just laterad of the Sulcus intercruralis lateralis ( $S$. ic. l.). A marked longitudinal ridge of the crural fibers separates from the postgeniculatum (pgn.) a depressed area, the quadrans $(q$.$) .$

The albicantia $(a b n$.) are more closely united than in man, but large, white and perfectly distinguishable. The shallow furrow between them, together with the u shaped furrow which forms their cephalic boundary, is the Sulcus triradiatus (Sl.trd.).

The hypophysis has been removed so as to expose the Tuber cinereum (T. cin.) and the thin raised margin of the $\mathrm{Fm}$. infundibuli ( $F m$. inf.).

Just caudad of the albicantia, and partly overhung by them, is a small triangular depressed space with distinet perforations; this seems to be the true postperforatus (ppf.).

The entire Area intercruralis may be more completely exposed by removing the cerebellum and dorsiducting the "medulla," as in Prep. 425.

$\$ 1170$. Fig. 12.-The dorsal aspect of the metencephalon. From Prep. 397 (adult \%), 464 and $491 ; \times 1$.

"The metatela, like the diatela, seems to consist of more substantial tissue than simply pia and endyma, but I am not aware that its microscopic structure has been ascertained. I am in doubt respecting the precise limits and attachments of the metatela and metaplexuses." No "foramen of Magendie " was seen.

\$ 1171. Fig: I3.-Part of an oblique transection of the prosencephalon and diencephalon to show the form and position of the Crista. From Prep. $441 ; \times 2$.

The brain was traneected obliquely at an angle of about 45 degrees with the general longitudinal axts. The plane of section passed from a point nearly dorsad of the genu, through the aula, the medicornu and the albicantia. The figure includes only a part of the caudal aspect of the slice.

The dorsal borders of the hemispheres are divaricated slightly, and the callosum (cl.) is seen crossing the interval ; the slight notch on each side just dorsad of the callosum is the F. callosalis ( $F . c l$.). 
The striata (s. s.) are seen in section just ventrad of the lateral expansion of the callosum, while the lower part of the figure is occupied by the thalami (th.), united by the medicommissura (mos.). Between each thalamus and the corresponding striatum is a groove, the Sulcus limitans ( $\$ 7$. li.).

The Columnze fornicis $(C l m, f$.$) are divided nearly at a right angle with their course,$ and at a point just dorsad of the crista (Crs. f.), which is particularly well shown in this preparation. The open space between the fornix and the thalami is the aula (a.), and at the sides are the portz $(p$.$) leading into the proccelice. All the membranes and plexuses$ have been removed.

\section{PLATE IV.}

Unlike those of Plate III, all of the figures upon this plate represent cut surfaces, although some natural surfaces are shown also.

\$1172. Fig. I4.-A ventro-caudal view of the fornix, with the adjacent parts. From Prep's 507, 433 and 396 (adult of); $\times 2$.

The preparations were made while the brain was fresh, so as to permit more flattening of the hemispheres, and consequent exposure of the fornix.

After the removal of the rhinen-, meten-, epen- and mesencephalon, the thalami and geniculata were excavated piecemeal, so as not to injure or displace the fornix. The cut surface (s.) at each side of the fornix $(f$.$) is the plane of division of the diencephalon frcm$ the striatum.

The cephalic end of the prosencephalon was then sliced down to the level of the procommissura (prcs.), which is seen to send a distinct fasciculus toward the $L$. olfactorins on each side. Then the right hemisphere was sliced obliquely from near the meson dorsolaterad so as to cut the medicornu (mcu.) and hypocampa ( $h m p$.) at about the middle of their length. On the left side, the $L$. temporalis was allowed to fall somewhat by its own weight so as to expose the fornix more fully.

The velum and all the plexuses were removed so as to display the peculiar markings of the fornix and its mesal area, which is supposed to represent the lyra (ly.).

The portæ ( $p$.$) appear both shorter and narrower than they really are, on account of$ the obliquity of their planes to the line of vision. The v-shaped line called ripa (rp.), which connects the two portæ, separates the delta (dlt.) or entocœlian part of the fornix from the remaining surface, which is wholly outside of the colian cavity. The delta forms the roof of the aula, the cephalic continuation of the diacolia between the two portæ, and the ripa is the line of reflection of the endyma upon the two auliplexuses; the removal of these plexuses causes the rupture of the endyma along the ripa.

At each side, the ripa curves dorsad somewhat sharply so as to reach the dorsal end of the porta; at this point, and dorso-caudad for the entire length of the rima $(r$.), the endyma is simply reflected from the contiguous surfaces of the fimbria ( $f m b$.) and the corresponding border of the striatum. Hence the rima is virtually closed, and thus wholly distinct from the porta.

On the meson, between the portæ, is seen the crista (Crs. f.), which is unusually rounded in this preparation. The carina, which sometimes appears as a slight mesal ridge extending dorso-caudad from the crista, does not appear in this preparation. The Recessus aulæ $(R . a$.) is the cleft between the two Columna fornicis ( $C l m \cdot f$.) whose cut ends are seen just caudad of the pracommissura. The shading on the candal aspect of the columnæ indicates, but rather too distinctly, a slightly depressed area, of which the dorsal part, close to the crista, sometimes presents the appearance of a transverse band, for which the senior author has suggested the name Commissura fornicis (Cs. $f$.). 
“ After a prolonged examination of many preparations, I am unable to define accurately the limits of the fornix proper and the lyra (ly.). A comparison of the accounts given in standard works with the appearances presented by the limited materials at my disposal leads me to doubt whether the relative extent of the two parts in the human brain is well determined."

The fasciola ( $f s c l$.) is thick, and no part of it presents the denticulations from which its more ventral portion, in man, is called "fascia dentata." The peculiar curve of the hypocampa, medicornu and fasciola is well indicated by the fact that the $F$. hypocampa (F. $h m p$.), which corresponds nearly with them, in direction, is visible in this preparation only at its two ends, near the splenium (sp.), and near the tip of the L1. hypocampæ ( $L l$. $h m p$.). Between the fimbria and the fasciola is a depressed line which may be called the Fissura fimbriz $(F, f m b$.$) .$

$\S 1173$. Fig. I5.-The dorsal aspect of the procclia. From Prep. $465 ; \times 1$.

The special object of this preparation is to show that, in the cat, no part of the thalamus appears in the procalia. The cerebelium (cbl.) is shown only in outline.

Both hemispheres were sliced from the dorsum to the level of the intermediate part of the callosum $(c l)$. This laid open both procoeliæ in some degree. The central part of each procolia is called cella media $(c e l . m$.). The right medicornu was then opened to the tip, which, however, cannot be seen from the dorsal side.

The floor of the procelia is seen to be formed by the striatum (s.), the fornix $(f$ ) and the hypocampa ( $(\mathrm{mp}$.). The proplexuses have been turned in opposite directions for the sake of showing the absence of any interval between the fornix and hypocampa-or the fimbria which forms the border of the latter-and the striatum, such as would permit the appearance of the thalamus in the procolia. See Fig. 121.

$\S 1174$. Fig. I6.-The rhinocœlia and procœlia. From Prep's 425 and $493 ; \times 2$.

This figure shows the continuity of the proccelia with the rhinoccelia and its communica. tion through the porta with the aula and diaccitia. See $\$ 1151$.

The right half of the brain was transected through the caudal part of the medicommissura (mes.). A slice was then cut from the mesal aspect so as to include the genu. This exposed the præcornu (prcu.) with the mesal aspect of the striatum (s.), the rhinocœlia $(r h c$.), and the relative extent of the pes ( $p s$.$) , and the pero (po.) of the Lobus olfacto-$ rius. A bristle was then passed through the porta from the præcornu into the aula ( $a$.). Just ventrad of the bristle are the præcommissura (prcs.) and the terma $(t$.). The latter is traced distinctly to the chiasma ( $c h$.), so that the cephalic wall of the coelian cavity is complete. The deeper shadow just dorsad of the chiasma indicates the position of the Recessus opticus ( $R$. op.).

Just dorsad of the bristle, the crista (Crs. f.) is seen divided upon the meson, and continuous with the Columna fornicis $(C l m . f$.). The indentation between the crista and the præcommissura corresponds with the Recessus aulæ $(\boldsymbol{R} . a$.$) . The triangular Area sep-$ talis (Ar. spt.), between the fornix and the callosum, is the mesal surface of the right half of the Septum lucidum (Spt. lu.) and is in contact with its platetrope in the undissected brain. The thickness of the hemisepta in the car renders the adjective lucidum wholly inapplicable.

$\$ 1175$. Fig. $17 .-$ The mesal aspect of the right hemisphere, with the Lobus olfactorius. From Prep's 296 and $401 ; \times 1$.

The caudal divisions of a hemiencephalon were removed, and the thalamus carefully excavated so as to leave undisturbed the fornix $(f$.$) and the fimbria ( f m b$.). In this respect, this figure may be compared with the left half of Fig. 14. 
The special object of this figure is to show the hypocampal fissure $(F$. $h m p$.) in its whole length at once. So great is the curvature of the parts that this is possible only in a single position of the preparation in which the meson is foreshortened. In general, this figure may be compared with those given by Flower (13) of the rabbit and sheep.

The dorsal end of the $F$ ? hypocampce is seen to turn sharply around the splenium ( $8 p$.), so as to become continuous with the callosalis $(F$. cl. $)$. The fasciola $(f s c l$.$) is wide and$ devoid of denticulatious, but is crossed obliquely by a shallow furrow. In this position of the preparation, the F. fimbria ( $F$. $f m b$.) appears to be continuous with a short line passing cephalad to a point ventrad of the callosum; in reality, however, this latter line is only one of the markings of the ventral surface of the fornix. and the $F$. fimbrice, like the callosalis, turns sharply dorso-caudad to terminate just cephalad of the splenium (Fig. 125).

$\S 1176$. Fig. 18. - The right procœlia seen from the right or ectal side. From Prep, $495 ; \times 1$.

The right half of the brain was removed in successive slices until what remained was about $3 \mathrm{nmm}$. thick. The remainder of the striatum was then everted from the præcornu (preu.). The proplexus (prpx.) is slightly displaced, but the porta is hidden by the portiplexus $(p p x$.$) . The medicornu (meu.) and the hypocampa (hmp.) are shown in sec-$ tion, and the other parts will be readily recognized. The relative heights of the opticus (op.) and the postopticus ( $p o p$.), at a little distance from the meson, are well displayed. The short curved line at the cephalo-ventral end of the proccelia represents the beginning of the passage to the rhinocoelia (Fig. 16).

$\S 1177$. Fig. 19.-The left præcornu and porta exposed from the left or ectal side. From Prep. $495 ; \times 1$.

This figure represents the other side of the same brain from which Fig. 18 was drawn. The preparation was made in the same way, but in addition the proplexus and portiplexus were carefully snipped off so as to expose the porta.

The porta $(p$.) is seen to open between the Columna fornicis $(C l m . f$.) and the cephalic end of the thalamus $(t h$.). The orifice would appear larger if the preparation had been so placed as to leave its plane parallel with the picture-plane.

The membranes could not be shown well on so small a scale (see Fig. 123). In this and in the previous figure, the fornix is seen to be continuous with the hemiseptum (Spt. lu.) which forms part of the mesal wall of the precornu.

$\S 1178$. Fig. 20.-Transection of the fornix with the crista. From Prep. $508 ; \times 1$.

The object of this figure is to show the decided elevation formed by the crista (Crs. f.). Only enough of the rest of the section is included to locate the crista.

\$ 1179. Other Figures of the Cat's Brain.-Since most published figures of the cat's brain illustrate the fissures rather than the structure, they will be mentioned in connection with Fig. 124, 125, later in this chapter.

\section{SYNONYMS AND REFERENCES.}

$\S 1180$. The principal parts of the Amphibian brain are enumerated in $\S 1058$ and tabulated according to their segments in $\S 1069$.

Most of the parts of the Mammalian brain which are visible to the unaided eye are named in $\S 1128$ in the alphabetical order of their abbreviations, and in $\S 1138$ they are tabulated according to 
their segments. Synonyms of about twenty of the principal parts are given in $\$ \$ 1058,1059$.

In the following pages ( $\$ \S 1181-1333$ ) the parts of the mammalian brain are enumerated in the alphabetical order of the technical names herein employed. Unless otherwise mentioned, these names are the same as adopted by the senior author $(\boldsymbol{9}, \mathbf{1} \boldsymbol{\mathbb { A }})$; the apparently new terms really differ but little from those in common use $(\$ 1129)$.

Following the abbreviation of each name are references to figures and sections in the present work and to the works on Human Anatomy by Gray and Quain. The principal technical and English vernacular synonyms are then given, with in most cases a brief description or commentary upon the part itself or its designation.

As has been stated already, our information is far from satisfactory, and all parts of the cat's brain need monographic treatment.

$\S$ 1181. Alba, (Substantia), alb.-\$§ 995, 1047 ; Fig. 112, 116; P1. III, Fig. 13 ; P1. IV, Fig. 14. Gray, A, 60; Quain, A, II, 558.

Syn.-Substantia alba, white matter, white or fibrous nervous tissue.

The alba constitutes the columns of the myelon, most of the ental portions of the cerebrum and cerebellum, and so much of the entire brain as is not formed by the cinerea, which see, $\$ 1204$.

§ 1182. Albicans, (Corpus), abn.-Fig. 115, 116 ; P1. II, Fig. 3, 4; Pl. III, Fig. 11 ; §1161. Gray, A, 621; Quain, A, II, 535.

Byn.-Corpus candicans, corpus mammillare, bulbus fornicis.

The albicantia are a pair of white elevations a little cephalad of the pons and overhang ing the postperforatus. They are distinct, but less so than in man. For their relation to the Columnæ fornicis, see $\$ 1207$.

§ 1183. Arachnoidea, (Membrana), arch.-Fig 112, H ; § 1077. Gray, A, 608 ; Quain, A, II, 519 ; Westbrook, 1,348 .

Syn.-Arachnoideus, meninx media, tunica serosa.

Like the pia, this membrane needs thorough examination in the cat.

\$ 1184. Arbor vitæ, arb. vt.-Fig. 88, 117; Pl. II, Fig. 4. Gray, A, 634; Quain, A, II, 519.

The ectal foliation of the cerebellum gives to the surface of a dorso-ventral section a tree-like appearance, to which the above name is applied.

\$1 1185. Aula (az.), a.-Fig. 110-112; Pl. II, Fig. 4 ; Pl. III, Fig. 13 ; Pl. IV, Fig. 16 ; $\S \S 1065,1145 . \quad$ Meynert (Stricker, A, Fig. 253); Balfour, A, II, Fig. 257.

Syn.--" Le vestibule des ventricules latéraux "-Milne-Edwards, A, XI, 305.

Cavity of the prosencephalon primitivum--Mihallkovics, A, 30.

Cavity of the "secundäres vorderhimbläschen"-Mihalkovics, A, Pl. 1, Fig. 1.

Cavity of the "unpaaren grosshirnblase "-Löwe, A, 93. Fig. 11, 12, " es."

Mesal portion of the cavity of the "lobus communis"-Huxley, $\boldsymbol{1}, 00$.

Mesal portion of "Furamen commune anterius"-Todd, A, 676; Quain, A, II, 544, and other writers, according to Bell, B, Pl. X.

"Iter ad tertium ventriculum"-Monro, $A, 9$. 
"Rima ad infundibuli, s. vulva"-Various authors, according to Dunglison, A, 906.

Mesal portion of the foramen of Monro-Balfour, A, II, 257 .

Mesal portion of the "ventriculus communis"-Stieda, $6,180$.

Mesal portion of the " common ventricular cavity"-Spitzka, $6,31$.

The aula is the most cephalic part of what is commonly known as the third ventricle. Its best defined portion lies between the two portæ, and is bounded cephalad by the fornix and caudad by the medicommissura. Ventrad it reaches the chiasma so as to include the Recessus optici; dorsad it is bounded by the triangular area of the fornix, called delta. The torm of the cavity is therefore peculiar and irregular.

The Name.-The origin of the name and the reasons for its use are briefly stated in $\$ 1065$. Much remains to be done, especially in Comparative Anatomy and Embryology, before the limits of this cavity can be well defined.

$\$ 1186$. Auliplexus, apx.-Fig. $113 ; \S 1066$.

The aulic portion of the "plexus choroideus ventriculi tertii" or diuplexus.

This portion of the plexus is so slight that it would hardly need a separate designation but for the possibility that in the cat, as in Menobranchus, the larger diaplexus may be only an extension of the more primitive auliplexus.

$\S 1187$. Area cruralis (az.), Ar. cr.-Fig. 116, 118 ; Pl. II, Fig. 3 ; Pl. III, Fig. 11.

A convenient name for the ill-defined and non-homogeneous area of the basis encephali bounded by lines projected laterad from the pons and chiasma. See Area intercrurulis (ร 1189).

§1188. Area elliptica, Ar. el.-Fig. $116 ;$ Pl. II, Fig. $3 ; \$ 1140$.

According to the Am. Jour. of Neurology, etc. (I, 102), this is the surface of the oliv", notwithstanding the funiculi of the $N$. hypoglossus emerge laterad of it instead of mesnd as in man.

§ 1189. Area intercrutalis (az.), Ar. icr.-Fig. 116, 118 ; Pl. II, Fig. 3 ; Pl. III, Fig. $11 ; \$ 1133$.

Syn.-Interpeduncular space ; Area intercruralis (manuscript)-Spitzka, $\%, 165$.

If the diverging fibrous tracts sometimes called pedunculi cerebri are to be called crura, then the space bounded by them and by the pons and chiasma, should be intercrural rather than interpeduncular.

§1190. Area ovalis, Ar. ov.-Fig. 116 ; Pl. II, Fig. 3 ; 1140.

The surface of an elevation of the ventro-lateral aspect of the metencephalon, laterad of the Area elliptica.

According to the Am. Jour. of Neurology, etc. (I, T02), this corresponds with the Tubercle of Rolando, "tubercolo cinereo."

$\S 1191$. Area postpontilis (az.), Ar. ppn.-Fig. 116; P1. II, Fig. 3 ; \& 1133.

The ventral aspect of the metencephalon. It includes the Area elliptica, the Ar. cvalis, the pyramis and trapezium, and the ectal origins of several nerves.

$\$ 1192$. Area prachiasmatica (az.), Ar. prch.-Fig. 116; Pl. II, Fig. 3.

The ventral aspect of the basis encephali cephalad of the chiasma.

§ 1193. Ar. septalis, Ar. spt.-Fig. 117; Pl. II, Fig. 4; Pl. IV, Fig. 16.

The mesal surface of either half of the Septum lucidum; see pseudocceliu, $\S 1297$.

Septal area-Flower, 13,634. The name is ascribed to Huxley.

\$ 1194. Calcar (avis), cle.-Gray, A, 625; Quain, A, II, 542.

This is the brief synonym of hippocampus minor, ergot and unciform eminence. It designates a projection into the postcornu of man and monkeys, and has not been observed in the cat, where the postcornu is not normally developed. 
8 1195. Callosum (az.), cl.-Fig. 88, 104, 115, 117, 122, 125; Pl. II, Fig 4 ; P1. III, Fig. 13; Pl. IV, Fig. 15-17, 20. Gray, A, 623; Quain, A, II, 537.

Syn.-Corpus callosum, commissura magna, trabs cerebri.

A broad band of nerve fibers connecting the mesal surfaces of the hemispheres along a line dorsad of the fornix and curved ventrad at each end. The cephalic curvature is the genu and the caudal the splenium.

In nearly its caudal half, the callosum is in contact with the fornix at the meson and for 1-2 mm. laterad (F'ig. 122, § 1149). With care the callosum may be dissected up from the fornix, and at the splenium the two are then seen to be continuous. In fact, a simple way of describing their relations is the following :-

The two hemispheres, originally separate, become united along two lines represented by the callosum and the fornix. The former is approximately straight, excepting at its ends, the cephalic corresponding with the genu and rostrum. The latter forms the segment of a circle, but the caudal end turns dorsad to become continuous with the caudal end of the callosal line which is curved ventrad at the splenium.

The fibers constituting the larger part of the callosum pass dorsad of the procolia and thus constitute its roof; the fibers of the fornix pass ventrad of the procolia and thus enter into the composition of its floor; the fibers of the splenium are intermediate, and are partly continued into the hypocampa and partly into the general caudal part of the hemisphere. These relations are indicated in Pl. IV, Fig. 19, 20.

Notwithstanding the fact that the callosum exists only in mammals, is larger, as a rule, in the higher members of the group, and presumptively has great physiological importance, there are recorded several cases of its more or less complete absence from man, once without serious lack of mental or physical power (Malinverni [Henry, 1]); and the senior author has reported (13) a case of its complete absence in a cat which is not known to have been peculiar during life.

$\S$ 1196. Canalis centralis (myelonis), (az.), Cn. ce.-Fig. 99, 100, 109, 113, 117 ; Pl. II, Fig. 4. Gray, A, 68; Quain, A, II, 500.

The central canal of the "cord."

The mesal canal of the myelon, which expands cephalad and is opened up into the dorsimesal fissure so as to form the metacolia. In the cat this canal persists through life, but in man it is said to be commonly obliterated in the adult, excepting at the cephalic end.

\& 1197. Carina (az.), ca.-This is a mesal ridge upon the ventro-caudal aspect of the fornix, extending from the crista the entire length of the delta. It varies in distinctness and does not appear upon any of the preparations here figured, but is very well marked in Prep. 530, M. C. U.

$\S$ 1198. Cauda striati, $c d$. s. - This is not distinctly shown in the figures and has not been accurately observed by us.

The slender tail-like prolongation of the striatum.

Former writers who mentioned this portion of the striatum (Todd, Gratiolet, Hirschfeld, and Cuvier, B, III, 51) gave it no special name. Recently it has been described by Dalton $(1,12)$ under the name of surcingle. Having vainly requested him to substitute for this vernacular term some equivalent technical one, the senior author reluctantly proposed $(\%, 134)$ the name here employed, which is approved by Spitzka, $\%, 165$.

II, 540 .

$\S$ 1199. Cella media, cel. m.--Pl. IV, Fig. 15; § 1147. Gray, A, 625; Quain, A,

The central or intermediate portion of the procolia ; its limits are not defined.

$\$ 1200$. Cerebellum (az.), $c b l .-F i g .88,104,113-117$; Pl. I, Fig. 1, 2 ; Pl. II, Fig. 3, 4; Pl. IV, Fig. 15; \& 1074 (C). Gray, A, 632 ; Quain, A, II, 515.

Syn.-Cerebrum parvum. 
Next to the cerebrum, the largest portion of the brain. A single foliated mass, forming with the valvula the dorsal part of the epencephalon and constituting the roof of part of the epicolia.

$\S 1201$. Cerebrum ( $a z.), c b .-F i g .88,104,113-117,124,125$; PI. I, Fig. 1, 2 ; Pl. II Fig. 3, 4; Pl. III, Fig. 5, 6, 13 ; Pl. IV, Fig. 14-20. Gray, A, 615; Quain, A, II, 522.

Syn.-Prosencephalon, hemisphæræ.

The largest portion of the brain, forming two convoluted lobes between the Lobi olfactorii and the thalami; the former are partly, the latter wholly, covered by them. The striata and hypocampos are thickenings of certain parts.

The thalami and optici are not properly " internal parts of the cerebrum."

\$ 1202. Chiasma (az), ch.-Fig. 116-118; Pl. II, Fig. 3, 4; Pl. III, Fig. 5, 11; Pl. IV, Fig. 16. Gray, A, 621, 639; Quain, A, II, 536.

Syn.-Chiasma nervorum opticorum, optic commissure.

The subcylindrical $\mathrm{x}$-shaped mass at the base of the brain formed by the union and decussation of the two optic tracts; from it the $N N$. optici pass to the eyes.

Remark - We are not aware that special observations have determined the extent of the crossing or decussation of the fibers at the chiasma in the cat; the precise arrangement seems not to have been determined for man (Meynert [Stricker, A, 688]; Ferrier, A, 72; Wadsworth, 1, 528).

§ 1203. Cimbia, cmb.-Fig. 116, 118; P1. II, Fig. 3 ; Pl. III, Fig. 9, 11 ; § 1142.

Syn.-Tractus transversus pedunculi-Gudden, as quoted by Meynert (Stricker, A, 737).

A fibrous band crossing the Orus cerebri just cephalad of the ectal origin of the $N$. oculomotorius. It may be traced from between the opticus and the postgeniculatum to pear the ventrimeson, where it suddenly enters the crus.

The senior author has suggested $(14,554)$ that the cimbia may be regarded as indicating the line of junction between the mesencephalic and diencephalic portions of the crus. The name was proposed as a brief substitute for Gudden's descriptive term ; it signifies in architecture a band or filiet about a column.

$\$ 1204$. Cinerea, (Substantia), cin.-Pl. III, Fig. 13; Pl. IV, Fig. 14, 15, $20 ; \S 995$. Gray, A, 622 ; Quain, A. II, 558 ; Meynert (Stricker, A, 651).

Byn.-Gray matter, ganglionic or cellular nervous tissue, vesicular neurine.

The myelonal cinerea has been mentioned in $\$ 998$. The encephalic cinerea is arranged by Meynert (Stricker, A, 651) in four categories : cortex cerebri; busal ganglia [striata and thalami] ; centrat tuberar gray [lining the coeliæ] ; cerebellar cinerea. The central tubular gray is the subject of a paper by Spitzka (1).

§ 1205. Clava, clv.-Pl. III, Fig. 12. Gray, A, 612 ; Quain, A, II, 505.

Syn.-Processus clavatus, funiculus gracilis, pyramis posterior.

The slender fibrous band forming the margin of the metacclia. It is the cephalic contivuation of the slender "posterior median column" of the myelon.

The name is used in accordance with the remark of Spitzka $(\boldsymbol{y}, 165)$. We have not encountered it elsewhere.

$\$ 1206$. Columna dorsalis (myelonis), Clm. d.-Fig. 112 ; Pl. I, Fig. 1; Pl. I, Fig. 12. Gray, A, 605 ; Quain, A, II, 494.

Syn.-Columna posterior, the " posterior white column of the cord."

Excepting in Fig. 112, no distinction is indicated between the larger Clm. dorsalis and the smaller and more mesal "posterior median column," which is commonly regarded as merely a part thereof, and is continued as the clava.

$\$$ 1207. Columna fornicis, Clm. f.-Fig. 113, 117, 123 ; Pl. II, Fig. 4 ; Pl. III, Fig. 13 ; Pl. IV, Fig. 14, 16, 20 ; \$1145. Gray, A, 628; Quain, A, II, 543. 
Syn.--Crus fornicis anterius, anterior pillar of the fornix.

The fibrous fasciculus which forms the cephalic boundary of the porta, extends ventrad to the albicans and dorso-caudad as the lateral half of the body of the fornix. The columna is a differentiated part of the embryonic terma and is continuous with the hemiseptum.

The two columnæ are separated by the Recessus aula and conjoined by the Commissura fornicis.

\$ 1208. Columna lateralis (myelonis), Olm. l.-Fig. 104, 112 ; Pl. I, Fig. 1, 2 ; Pl. II, Fig. 3. Gray, A, 605; Quain, A, II, 494.

The lateral white column of the "cord."

$\S 1209$. Columna ventralis (myelonis), $C l m$. v.-Fig. $112 ; 116 ;$ Pl. II, Fig. 3 Gray, A, 605 ; Quain, A, II, 494.

The " anterior white column of the cord."

\$ 1210. Commissura fornicis (az.), Cs. f.-Pl. IV, Fig. 14.

This name was provisionally applied by the senior author $(14,543)$ to what appears in some preparations to be a transverse band just ventrad of the crista fornicis.

$\S$ 1211. Commissura habenarum (az.), Cs. h.-Fig. 117 ; PI. II, Fig. 4 ; Pl. III, Fig. $6 ; \S 1143$.

A narrow band of apparently nervous tissue connecting the caudal ends of the habenæ and constituting the cephalic boundary of the Foramen conarii.

$\S 1212$. Conarium (az.), cn.-Fig. 111, 112, 114, 117 ; Pl. II, Fig. 4 ; P1. III, Fig. 7, 10 : §̧ 1084, 1143. Gray, A, 630; Quain, A, II, 549.

Syn.-Glandula pinealis, epiphysis cerebri.

A subglobular mass forming a part of the roof of the diacolia just cephalad of the postcommissura. There is no good evidence of its true nervous structure, and its functions are unknown.

§ 1213. Cortex, ctx.-Fig. 123; Pl. II, Fig. 4; Pl. III, Fig. 13 ; Pl. IV, Fig. 15, 20 ; \& 1147. Gray, A, 623; Quain, A, II, 521, 559 .

The ectal cinerea of the cerebrum or cerebellum, more commonly of the former. It consists of several more or less distinct layers, whose structure and relations are figured and described by Meynert (Stricker, A, 660) and Bevan Lewis $(1,88)$.

$\S$ 1214. Crista fornicis (az.), Crs. f.--Fig. 117 ; Pl. II, Fig. 4 ; Pl. III, Fig. 13 ; Pl. IV, Fig. 14, 16, 20.

A hemispherical or semioval elevation of the caudal surface of the fornix just dorsad of the Recessus aulæ, between the portæe and opposite the cephalic convexity of the medicommissura. It is continued dorso-caudad as the carina.

The crista seems not to have been observed prior to the senior author's paper ( 7 ). He has observed it in the brains of a sheep and human subject, but has not looked further for it. Probably it will be fourtd in most if not all mammals. Its histological composition, function and morphological significance are unknown. We can only surmise that it may mark the dorsal limit of the primitive terma. 9,11 .

$\S 1215$. Crus cerebri, $C r$. $c b .-F i g .111,112,116-118$; Pl. II, Fig. 3,4 ; Pl. III, Fig.

Syn.-Pedunculus cerebri, caudex cerebri, crus anterius medullæ oblongatæ.

The fibrous mass forming with its fellow the support of the mesencephalon and dien. cephaus, and extending from the pons to the chiasma.

$\S$ 1216. Crus olfactorium, $C r$. ol.-Fig. 116, 117, 124, 125; Pl. II, Fig. 3,4 ; Pl. III, Fig. 5 ; \& 1139. Gray, A, 620, 637; Quain, A, II, 566.

Syn.-Crus rhinencephali, Owen, A, I, 298; olfactory nerve or tract, Quain, A, II, 566 ; Gray, A, 038. 
The contracted portion of the brain between the prosencephalon and the Lobus olfactorius. Its ventral surface is continuous caudad with the Tractus rhinalis.

$\$ 1217$. Delta (fornicis), ( $\alpha z$.$) , dlt.-Fig. 120$; P1. IV, Fig. 14.

The triangular entocalian area of the ventro-caudal surface of the fornix, constituting the roof of the aula. Its base coincides with a line between the portæ, and its two other sides are ripa, lines of reflection of the endyma upon the intruded auliplexus. It does not appear to have been observed prior to the senior author's paper (9).

§ 1218. Diacœlia $\left(a z_{0}\right), d c_{.}-F i g .110-113,117,120,122$; Pl. II, Fig. 4; Pl. III, Fig. 7; Pl. IV, Fig. 16; $\$ 1143$. Gray, A, 629 ; Quain, A, II, 546.

Syn.-Ventriculus tertius, third ventricie, middle ventricle, mediventricle.

The irregular mesal cavity between the thalami, bounded dorsad by the diatela, postcommissura and conarium; ventrad by the diencephalic portion of the Crura cerebri, the albicantia, Tuber cinereum and terma (reinforced by the chiasma), continuous cephalad with the aula and caudad with the mesocoelia. Most of its dorsal portion is occupied by the medicommissura. The diacolia represents the cavity of the primitive "anterior cerebral vesicle."

The reasons for adopting this and the other names for the encephalic cavities are stated in $\$ 1064$ and the papers there referred to.

$\S$ 1219. Diaplexus, $d p x$-Fig. 112, 113, 117, 122; P1. II, Fig. 4; Pl. IV, Fig. 16 ; \$1143. Gray, A, 628; Quain, A, II, 545.

Syn.-Plexus choroideus ventriculi tertii, plexus choroideus medius.

The string-like vascular plexus extending the entire length of the diaccelia on each side; it is slightly attached to the diatsla and bas firmer connections by vessels at its ends, which are not clear to us. It is contiuuous cephalad with the auliplexus. For the name, see $\$ 1066$.

§ 1220. Diatela (nz.), $d t l$. Fig. 111-113, 117, 122 ; Pl. III, Fig. 10. Reichert, A, 155.

The membranous or atrophied nervous roof of the diacelia. Its exact composition has not been ascertained, but it seems to consist of something more substantial than endyma. Judging from current statements respecting the roof of the "third ventricle," this delicate tela is usually torn off with the fornix, and no notice is taken of the ragged lines of its separation along the Sulcus habence on each side. In the Museum of Cornell University, however, there are preparations of the cat and rabbit which show the diatela after the removal of the fornix and velum, while the ripas or lines of reflection are apparent upon many others, as in that shown in Pl. III, Fig. 7.

§ 1221. Diencephalon (az.), den.-Fig. 88, 110-113, 116-118, 122 ; P1. II, Fig. 3, 4; Pl. III, Fig. 6, 7, 9-11; Pl. IV, Fig. 16, 18, 19 ; 1061 . Gray, A, 111; Quain, A, II, 755.

Syn.-Deutencephalon, thalamencephalon, interbrain, 'tweenbrain.

The encephalic segment between the mesencephalon and the prosencephalon. Its cavity is the diacoelia ("third ventricle"), and its chief constituents are the thalami.

§ 1222. Dura (mater), d.-Fig. 88: § 1104. Gray, A, 606; Quain, A, II, 569.

The firm membrane which lines the cranial cavity, is reflected upon the osseous tentcrium (\$552), and is produced between the hemispheres on the falx cerebri; it is an entocranial periosteum.

Notwithstanding its feminine form, dura is frequently employed without the substantive mater.

§ 1223. Eminentia auditoria, Em. au.-Pl. I, Fig. 2; Pl. II. Fig. 3; § 1140.

The name was suggested by the senior author $(14,536)$ for the distinct elevation just laterad of the trapezium, whence springs the $N$. auditorius. It is continuous mesad with a Tractus auditorizes, which does not appear in the figures. 
II, 540 .

§ 1224. Endyma, end.-Fig. 111, 112, 121-123; § 1079. Gray, A, 630; Quain, A,

Syn.-Ependyma, pia mater interior.

The epithelial lining of the cœliæ, which is reflected upon the plexuses.

§ 1225. Epencephalon (az.), epen.-Fig. 88, 104, 110-114, 117; Pl. I, Fig. 1, 2; Pl. II, Fig. 3, 4; Pl. III, Fig. 12 ; Pl. IV, Fig. 15. Gray, A, 111; Quain, A, II, 755.

The encephalic segment including the cerebellum, pons, pedunculi, valvula, and the corresponding part of the "medulla."

While it is convenient to recognize the segment, its precise limits are difficult to assign, and Spitzlia $(\boldsymbol{7}, 165)$ inclines to abandon the segmental name altogether. If the caudal boundary coincides with the margin of the pons, the trapezium of man will be included therewith, while that of the cat remains in the metencephalon.

$\$ 1226$, Epicœlia (az.), epc.-Fig. 110-113, 117; P1. II, Fig. 4; §1141. Gray, A, 685 ; Quain, A, II, 512.

Syn.-Ventriculus quartus or fourth ventricle, the cephalic part; ventriculus cerebelli.

The cavity of the epencephalon, covered by the cerebellum and valvula. II, 545.

§ 1227. Fasciola, fscl.-Fig. 121; Pl. IV, Fig. 14, 17. Gray, A, 627; Quain, A,

Syn.-Fascia dentata, fasciola cinerea.

The somewhat thickened margin of cinerea along the fimbria. In man the ventral portion has commonly been called fascia dentata; in the cat, however, there is no denticulation, and the name fascia is certainly misleading; hence the senior author proposed $(\boldsymbol{9}, 135)$ to employ fasciola for the whole.

§ 1228. Fimbria, fmb.-Fig. 121; Pl. IV, Fig. 14, 17. Gray. A, 627; Quain, A, II, $542,544$.

Syn.-Corpus fimbriatum, tænia bippocampi.

The strip of alba forming the border of the hypocampa and one of the boundaries of the rima.

§ 1229. Fissura ansata, $F$. an.-This and the other cerebral fissures (including all the fissures named in $\$ 1129$ excepting the four appertaining to the myelon) are briefly discussed in the last part of this chapter. See especially the Table of Synonyms $(\S 1342)$.

$\S 1230$. Fissura dorsilateralis (myelonis), F. dl.-Fig. 112 ; Pl. I, Fig. 1. Gray, A, 605 ; Quain, A, II, 493.

$\S 1231$. Fissura dorsimesalis (az.), F. $d m s .-F i g .112$; Pl. I, Fig. 1 ; P1. III, Fig. 12. Gray, A, 605 ; Quain, A, II, 493.

Syn.-The "posterior fissure of the cord."

$\S 1232$. Fissura ventrilateralis, $F$. vl.-Fig. 112, 116 ; Pl. II, Fig. 3. Gray, A, 605 ; Quain, A, II, 493.

$\S 1233$. Fissura ventrimesalis (az.), $F$. vms.-Fig. 99, 100, 109, 112 ; P1. II, Fig. 3. Gray, A, 605 ; Quain, A, II, 493.

The "anterior fissure of the cord."

§ 1234. Flocculus, fic.-Gray, A, 634; Quain, A, II, 518.

We are not sure that this is represented in the cat, although it has been homologized by some with the Lobulus appendicularis. II, 504.

$\S 1235$. Foramen cæcum (az.), Fm. ce.-Pl. II, Fig. 3, 4. Gray, A, 610; Quain, A,

Syn.-Fossa cæca-Spitzka, 3,6. 
The name here employed was used by Vicq d'Azyr (A, PI. XVIII, “48”), and is given in Dunglison; it should, we think, be retained, notwithstanding the somewhat unusual use of foramen.

§ 1236. Foramen conarii ( $\alpha z)$, Fm. cn.-Pl. III, Fig. 6.

The interval between the postcommissura and the Commissura habenarum.

\$ 1237. Foramen infundibuli (az.), Fm. inf.-Fig. 118; PI. III, Fig. 11.

The orifice left after removal of the hypophysis and infundibulum.

$\therefore \quad \$ 1238$. Fornix (az.), f.-Fig. 117, 122 ; Pl. II, Fig. 4 ; Pl. III, Fig. 13 ; Pl. IV, Fig. 14-20 ; $\$ \$ 1144,1172$, Gray, A, 627 ; Quain, A, II, 543.

Byn.-Camara, testudo cerebri.

A subtriangular fibrous sheet, forming successively the cephalic boundary of the aula and porta, the roof of the aula, and part of the floor of the procœliæ. (See $\$ 1457$.

The form, constitution, direction and relations of the fornix are exceedingly difficult to describe, and indeed are not fully understood. The fornix proper includes the following parts : columna, commissura, crista, carina, delta and lyra. The fimbria are its caudo-lateral prolongations to the tips of the hypocampal lobules, while the fibrous fasciculi constituting the columnæ are described as beginning in the thalami, passing ventrad to form a figure-of-eight turn in the albicantia, then passing dorsad just caudad of the præcommissura to form the lateral halves of the "body" of the fornix, which again are continued into the fimbrix. Between the thicker lateral parts of the body of the fornix is the thin and illdefined surface known as lyra.

The caudal portion of the fornix is in contact with the ventral aspect of the callosum at and for a short distance laterad of the meson, and the two are continuous at the splenium. See callosum ( $\$ 1195)$.

The feline fornix is proportionally much wider than the human, and may be torn into several bands on each side, as indicated by the $\nabla$-shaped lines in Pl. IV, Fig. 14.

§ 1239. Genu (az.), g.-Fig. 117; P1. II, Fig. 4 ; P1. IV, Fig. 17. Gray, A, 623; Qnain, A, II, 538 .

The knee-like cephalic curvature of the callosum, ending in the rostrum.

$\S 1240$. Habena, h.-Fig. 117, 122 ; PI. II, Fig. 4 ; Pl. IV, Fig. 16 ; $§ 1143$. Gray, A, 630 ; Quain, A, II, 549.

Syn.-Habenula, pedunculus conarii.

The ridge along the dorso-mesal aspect of the thalamus; it may be said to be the dorsal limit of the mesal surface, as the Sulcus habenæ is the mesal limit of the dorsal surface.

The habena terminates on the cephalic slope of the thalamus as a more or less distinct tubercle which marks the dorsal limit of the porta; it joins its platetrope by the Cs. habenarum, forming the cephalic boundary of the Fm. conarii.

$\S 1241$. Hemiseptum (cerebri), hmspt.-Fig. 117; Pl. IV, Fig. 16.

This is the lateral half of the septum (lucidum), which see ( $\$ 1315)$; its mesal surface, the Area septalis, is joined with its platetrope by connective tissue $(\$ 1137,4)$.

\& 1242. Hemisphæra, hem.-Fig. 104, 110-118, 124, 125 ; Pl. I, Fig. 1, 2 ; Pl. II, Fig. 3, 4 ; P1. III, Fig. 5, 6, 11, 13 ; Pl. IV, Fig. 14-20.

Byn.-Ganglion hemisphæricum, hemicerebrum, lobus prosencephalicus.

The lateral half of the largest portion of the brain, united with its platetrope by the fornix, callosum and præeommissura. Its cavity is the procolia, and the striatum, hypocampa, and (in man and monkeys) calcar are thickenings or involutions of the parietes. The surface is convoluted, presenting fissuræ and gyri. The cinerea is mostly near the surface, forming the cortex cerebri. See cerebrum, $\$ 1201$. 
§ 1243. Hypocampa, hmp.-Fig. 122; Pl. III, Fig. 11; Pl. IV, Fig. 14, 15, 18, 19; § 1147. Gray, A, 627; Quain, A, II, 541.

Sym.- Hippocampus major, cornu Ammonis.

A thickening and involution of the parietes of the medicornu, forming its floor.

The convexity constituting the hypocampa corresponds with a well-marked cctal fissure, the $F$. hypocamper. The ectal surface also presents the fasciola, fimbria and $F$. fimbria.

The grounds for preferring the name employed by Vicq d'Azyr (A, 61, Pl. VII, Fig, 1, 3 ; Pl. VIII, Fig, 2), and ascribed by him to Arantius and Varolius, are briefly stated by the senior author $(9,125 ; 14,541)$.

\& 1244. Hypophysis $(a z)$, hph.-Fig. 111, 112, 116, 117; Pl. II, Fig. 3, 4 ; § 1084. Gray, A, 621 ; Quain, A, II, 535; Balfour, A, II, 358.

Syn.-Corpus pituitarium, pituitary gland.

A subcordate mass attached to the Tuber cinereum by a tube, the infundibulum. It does not appear to consist of true nervons tissue, and its functions are wholly unknown; but it is constant throughout the vertebrate series, excepting Amphioxus; see Owen, 1 .

\$ 1245. Incisura hypocampæ, Inc. hmp.-Fig. 118 ; Pl. IV, Fig. 11.

A more or less distinet crenation of the mesal border of the hypocampal lobule, where it abuts against the Tractus opticus.

§ 1246. Infundibulum (az.), inf.-Fig. 116, 117; Pl. II, Fig. 3. 4. Gray, A, 621; Quain, A. II, 535.

The short thin-walled tube by which the hypophysis is connected with the Tuber cinereum.

It has several antiquated synonyms, but the name here given is almost universally employed.

§ 1247. Insula, ins, 一Gray, A, 616; Quain, A, II, 525.

Syn-Insula Reilii, Gyri operti, Lobulus Fissuræ Sylvii, Lobulus Corporis striati.

In man and monkeys, and perhaps some other mammals, the corter cerebri opposite the striatum is elevated and more or less convoluted. By the subgrowth of the neighboring regions, it becomes nearly or quite concealed, whence the name Gyri operti. The insula has not been identified in the cat; see striatum, $\S 1318$.

$\S 1248$. Interopticus, inop.-The interoptic lobe of some Reptiles (Spitzka, $\mathbf{4}, \mathbf{5}, \boldsymbol{1 1}$ ); we have not observed it in the cat.

\$ 1249. Iter (az.), it.-See mesocolia (\$ 1263). The entire name is iter a tertio ad quartum ventriculum, but as the only other iter (iter ad infundibulum) is rarely used, the senior author has suggested $(0,135)$ the single word as a convenient designation of the contracted mesoccelia of mammals.

§ 1250. Limes alba, lm. celb.-Fig. 116 ; Pl. I, Fig. 2 ; Pl. II, Fig. 3.

The Radix lateralis of the olfactory lobe presents two distinct tracts or paths for which, from their color, the senior author has proposed $(0,135 ; 14,537)$ the names limes alba and limes cinerea. The latter is laterad of the fornix.

$\S 1251$. Limes cinerea, $l m$. cin.-See limes alba (\$ 1250).

$\S 1252$. Liquor cœliarum, $l q$. c.-The liquor ventriculorum cerebri. The collian surfaces are always moistened by a serous liquid, secreted presumably by the plexuses. We have never observed an abnormal increase of this liquid in cats, but hydrocephalue has been recorded of several domesticated animals as well as of man.

$\$ 1253$. Lobulus appendicularis (cerebelli). Ll. ap._Fig. $116:$ Pl. I. Fig. 2 ; PI. II, Fig. 3 .

The name has been applied to the more or less distinct projection consisting of two or 
three folia or lamine of the lateral lowe of the cerebellum. It rests in the Fossn appen. dicularis of the periotic bone (Fig. 59, $F_{s} . a p$.). It is larger in dogs (śee Wilder, 11, 217, Fig. 1), and very large and long in the bear and seal. The name flucculus lias sometimes been applied to it, but its homology with that part of the human cerebellum is not clear.

\$ 1254. Lobulus hypocampæ, $L l$. hmp.-Fig. 116 ; Pl. I, Fig. 1 ; Pl. II, Fig. 3 ; Pl. III, Fig. 6 ; Pl. IV, Fig. 14, 17.

Syn.-Alveus (?), subiculum (?), protuberantia natiformis.

The senior author had suggested $(9,135)$ for this the single name monticulus, but withdrew it $(14,537)$ on the representation of Spitzka $(\boldsymbol{\gamma}, 165)$ that the name had been applied to a part of the cerebellum.

$\S 1255$. Lobus lateralis (cerebelli), L. l.-Fig. 116 ; Pl. I, Fig. 1, 2 ; Pl. II, Fig. 3 ; Pl. IV, Fig. 15. Gray, A, 634; Quain, A, II, 517.

Sy/l.-The lateral lobe of the cerebellum. This and the mesal lobe or vermis are not well defined from each otler. The Lobuins appendicularis is an appendage of the $\mathrm{L}$. lateralis.

$\S 1256$. Lobus olfactorius, L. ol.-Fig. 116, 117 ; Pl. I, Fig. 1, 2; Pl. II, Fig. 3, 4 ; Pl. IV, Fig. 15-19. Gray, A, 636 ; Quain, A, II, 506.

Syn.-Bulbus olfactorius, olfactory lobe, olfactory nerve.

The enlarged extremity of each half of the rhinencephalon which gives off the olfactory nerves. In man, it and the crus are so small as to have been called olfactory nerve. It contains, however, a distinct rhinocolia; see pero and pes.

§ 125\%. Lobus temporalis, L. tmp,_Pl. I, Fig. 2 ; Pl. II, Fig. 3 ; Pl. III, Fig. 5 ; P1. IV, Fig. 14. Gray, A, 616; Quain, A, II, 530.

That portion of the hemisphere which is caudad of the Sylvian fissure. Its dorsal limit is not defined. Its ventral end is the Lobulus hypocampa, and the surface ventromesad of the $F$. postrhinalis is the Tractus postrhinalis.

\$ 1258. Lyra (az.), ly.-Fig. 122 ; Pl. IV, Fig. 14. Gray, A, 628 ; Quain, A, II, 544.

Syn.-Psalterium, corpus psalloides, lamina medullaris triangularis cerebri, spatium trigonum.

This name is applied to part of the ventral surface of the fornix. It is not well defined.

\$ 1259. Medicommissura (az.), mes.-Fig. 117, 122 ; Pl. II, Fig. 4 ; Pl. III, Fig. 6, 13 ; Pl. IV, Fig. 16 ; § 1143. Gray, A, 630; Quain, A, II, 546.

Byn.-Commissura media, commissura mollis, the middle or soft commissure.

The junction of the two thalami in the dorsal part of the diaccelia. It seems to consist of cells rather than fibers.

\$ 1260. Medicornu, meu.-Fig. 113, 118, 119, 121 ; P1. III, Fig. 11 ; Pl. IV, Fig. 14, 15 ; \$ 1147. Gray, A, 626 ; Quain, A, II, 541.

Syn - Cornu medium, cornu descendens, cornu inferius, digital cavity.

The strongly curved extension of the cella media of the procolia to the tip of the $L$. temporalis; its floor is formed by the hyrocampa.

$\$ 1261$. Medipedunculus (cerebelli), mpd.-\$1141. Gray, A, 655; Quain, A, II, 516.

Syn.-Pedunculus medius, crus medium, processus e cerebello ad pontem, brachium pontis.

The subcylindrical fibrous mass connecting the pons with the cerebellum. It is overhung and concealed by the $L$. lateralis. It was called pontibrachium by the senior author $(9,136)$ under a misapprehension.

$\S$ 1262. Mesencephalon (az.), msen.-Fig. 110-114, 116-118; § 1061. Gray, A, 111 ; Quain, A, II, 755.

Syn.-Midbrain. It embraces the optici, postoptici and crura. 
§ 1263. Mesocœlia (az.), msc.-Fig. 110-11s, 117; Pl. II, Fig. 4; \& 1055. Gray, A, 630 ; Quain, A, II, 552 .

Syn.-Aquæductus Sylvii, iter a tertio ad quartum ventriculum, ventriculus opticus, ventriculus mesencephali.

The mesencephalic cavity, enclosed by the crura cerebri, the optici and postoptici, and opening cephalad into the diacolia and caudad into the epicoelia.

§ 1264. Metacœlia (az.), mtc.-Fig. 113, 117 ; Pl. II, Fig. 4 ; § 1065 . Gray, A, 635 ; Quain, A, II, 512.

Syn.-Ventriculus quartus, caudal portion. As has been admitted (\$ 1225), it is diffcult, perhaps impossible, to define aceurately the limits of the metacclia and epiccelia.

§ 1265. Metaplexus, mtpx-Fig. 116 ; Pl. II, Fig. 3. Luschka, A, Pl. III ; Gray, A, 636 ; Quain, A, II, 513.

Syn.-Plexus choroideus ventriculi quarti, plexus choroideus inferior.

We have not yet satisfied ourselves respecting the nature and connections of this plexus, and have provisionally designated the prominent plexus between the dorsal border of the medulla and the cerebellum as the metaplexus lateralis.

$\S$ 1266. Metencephalon ( $a z)$, mten.-Fig. 110-113, 116; P1. I, Fig. 1, 2 ; P1. II, Fig. 3, 4; PI. III, Fig. 12. Gray, A, 111; Quain, A, II, 755.

Syn.-Medulla, as far as the pons. See $\$ \S 1061,1225$.

\$ 1267. Metatela (az.), mttl.-Fig. 111, 112, 116; Pl. II, Fig. 4 ; Pl. III, Fig. 12. Luschlia, A, PI. III.

Syn.-Tela choroidea inferior.

Notwithstanding this atrophied roof of the metacœlia is so obvious with Amphibia as to have been once mistaken for the cerebellum, it is usually ignored in the dissection of the mammalian brain, probably because of its tenuity and its liability to be torn off with the cerebellum.

As has been stated in several places, there is much to be learned respecting the structure of the metatela, the arrangement of the metaplexus and the "Foramen of Magendie." The probability of the existence of the latter is increased by the experiments of Westbrook (1), which should be repeated upon the cat; detailed descriptions and enlarged figures are required $(\$ 1082)$.

§ 1268. Myelon (az.), my.-Fig. 88, 104, 109-113, 116 ; Pl. I, Fig. 1, 2 ; Pl. II, Fig̣. 3, 4. Gray, A, 604; Quain, A, II, 489.

Syn.-Medulla spinalis, spinal cord, spinal marrow, chorda spinalis.

It is described in $\$ 1006$ and in connection with the figures above named.

$\S 1269$. Nervus abducens, $N$. $a b$. - This and the other cranial nerves (i-xii) are treated of in the next chapter. The ectal origins are shown in Fig. 116 and in Pl. II, Fig. 3.

$\$ 1270$. Obex, ob.-We have not yet identified this in the cat. It is mentioned by Spitzka (3, 18), and also, we think, by Meynert (Stricker, A).

$\$ 1271$. Oliva, olv.-According to the Am. Jour. of Neurology, etc. (I, 102), the elevation called Area elliptica (Fig. 116 ; Pl. II, Fig. 3, Ar. el.) represents the oliva or Corpus olivarium, notwithstanding the funiculi of the $N$. hypoglossus emerge laterad of it.

\$ 1272. Opticus (Lobus), op.-Fig. 110-112, 114, 116, 117; Pl. II, Fig. 4; Pl. III, Fig. 7, 8, 9, 12 ; Pl. IV, Fig. 18, 19 ; §1142. Gray, A, 681; Quain, A, II, 551.

Syn.-Corpus bigeminum anterius, natis cerebri, one of the corpora quadrigemina, cephalic lobe of the mesencephalon.

As seen in Pl. III, Fig. 7, 8, 9, the opticus is more regularly convex and less elevated than the postopticus. 

261).

§ 1273. Pero (olfactorius), po.-Pl. IV, Fig. 16; § 1139. Meynert (Stricker, A, Fig.

This name was proposed by the senior author $(9,135)$ for the softer ectal layer of the Labus olfactorius from which the olfactory nerves arise. 'The word signifies a kind of boot made of raw hide, and seems more appropriate than the term Bulbus olfactorius used by Meynert (Stricker, A, 671).

$\S$ 1274. Pes (olfactorius), ps. ol.-Pl. IV, Fig. 16; § 1139. Meynert (Stricker, A, Fig. 261).

This name was proposed by the senior author $(9,136 ; 14,538)$ for the ental and fibrous portion of the Lobus olfactorius. It is in harmony with the term crus, already in use, and with pero, which was proposed at the same time, and less apt to be misunderstood than the term Lobus ol. used in this restricted sense by Meynert (Stricker, A, 671). II, $\mathbf{5} 71$.

$\S$ 1275. Pia (mater), pi.-Fig. 111, 112, 121-123; § 1078. Gray, A, 609 ; Quain, A,

Syn.-Meninx vasculosa, membrana vasculosa, membrana tenuis.

The immediate envelope of the myelencephalon, dipping into the fissures and supporting the vessels. Its relations to the telæ and plexuses are not fully understood.

$\S$ 1276. Pons (Varolii), (az.), pn.-Fig. 116, 118; Pl. II, Fig. 3, 4 ; Pl. III, Fig. 9, 11. Gray, A, 610 ; Quain, A, II, 511, 756.

Syn-Pons cerebelli, tuber annulare, protuberantia basilaris.

The bridge-like mass upon the basis encephali connecting the two sides of the cerebellum. It forms a prominent landmark of the mammalian brain, and is not present with the lower vertebrates.

§ 1277. Porta, p.-Fig. 110-113, 120, 123; Pl. III, Fig. 13; P1. IV, Fig. 14, 16, 18, 19 ; \$1065. Gray, A, 630; Quain, A, II, 544.

Syn.-Foramen Monroi, lateral orifice of the y-shaped Foramen Monroi.

This more or less constricted communication between the aula and the procclia is described in connection with the figures above named and in $\$ \S 1096,1145,1151$. 'The reasons for adopting the single word in place of the compound term have been given by the senior author (3).

$\S 1278$. Portio depressa (præperforati), $P t$. d.-The caudal and depressed portion of the (Locus) præperforatus, which see $(\S 1293)$.

$\S 1279$. Portio diencephalica (Cruris cerebri), Pt. den.-Fig. 116, 118; Pl. III, Fig. 9 , $11 ; \S 1142$.

In the cat the Crus cerebri is traversed by the cimbia, and its ventral surface is separated thereby into a caudal or mesencephalic portion and a cephalic or diencephalic. The latter presents a longitudinal ridge, mesad of which is the quadrans, while the postgenicnlatum lies just laterad of it.

\$1280. Portio mesencephalica (Cruris cerebri), Pt. msen.-Fig. 116, 118; P1. II, Fig. 3 ; P1. III. Fig. 11 ; § 1142.

This is the part of the crus which is visible upon the undissected brain between the pons and the hemisphere. See Crus cerebri (\$1215), cimbia (\$ 1203), and Portio diencephalica (\$1279).

$\S$ 1281. Portio prominens (præperforati), Pt. p.-The cephalic, elevated and usually furrowed portion of the (Locus) præperforatus, which see ( $\$ 1293)$.

\$ 1282. Portiplexus, $p p x-$ Fig. $113,123$.

This name was proposed lyy the senjor author $(9,136)$ for that small portion of the plexus which bangs in the porta. 
$\S$ 1283. Postcommissura (az.), pes.-Fig. 111, 112, 117; Pl. II, Fig. 4; P1. III; $\S 1143$. Gray, A, 630; Quain, A, II, 546.

Byn.-Commissura posterior.

Composed of transverse fibers forming the caudal part of the roof of the diacœlia, and thus joining the dorsal portions of the thalami.

§ 1284. Postcornu, pcu.-Gray, A, 625; Quain, A, II, 542.

Syn.-Cornu posterius ventriculi lateralis, cavitas digitata.

This candal prolongation of the procolia is normally present only in man, monkeys, seals and cetacea. The hydrocephalous brain of a dog reported by the senior author (23) presented a distinct postcornu, so that perhaps it may be regarded as normally rudimentary or undeveloped rather than totally absent in the ordinary mammals.

§ 1285. Postgeniculatum, pgn.-Fig. 118; Pl. III, Fig. 7-11; § 1144. Gray, A, 631; Quain, A, II, 552.

Syn.-Corpus geniculatum internum.

The elevation at the side of the diencephalon, between the Tractus opticus and the cimbia. It is proportionally much larger in the cat than in man.

$\S$ 1286. Postopticus, pop.-Fig. 114 ; Pl. II, Fig. 2 ; Pl. III, Fig. $7-9$; Pl. IV, Fig. 18, 19 ; § 1142. Gray, A, 631; Quain, A, II, 551.

Syn.-Testis cerebri, corpus bigeminum posterius, one of the corpora quadrigemina, caudal lobe of the mesencephalon.

The marked elevation just cepha?ad of the cerebellum and valvula.

$\S 1287$. Postpedunculus, $p p d .-\$ 1141$; Gray, A, 635 ; Quain, A, II, 516.

Syn.-Processus e cerebello ad medullam oblongatam ; restibrachium (Spitzka, 7,165$).$

The fibrous fasciculus passing from the dorsal aspect of the metencephalon dorsocephalad to the cerebellum, mesad of the medipedunculus.

$\S$ 1288. Prægeniculatum (Corpus), prgn.-Pl. III, Fig. 7-9; $\$ 1144 . \quad$ Gray, A, 631; Quain, A, II, 552.

Syn.-Corpus geniculatum externum.

There seem to be no distinct lines of demarcation between the Tractus opticus, the prægeniculatum and the thalamus.

$\S$ 1289. Postperforatus (Locus), (az.), ppf.-Fig. 116, 118 ; Pl. II, Fig. 3 ; Pl. III, Fig. 11. Gray, A, 621; Quain, A, II, 535 .

Syn.-Locus perforatus posticus, pons Tarini.

A small triangular area overhung by the albicantia, and presenting a few pores for the transmission of vessels. It is apparently smaller than in man.

$\S 1290$. Præcommissura (az.), prcs.-Fig. 111, 112, 117 ; Pl. II, Fig. 4 ; Pl. IV, Fig. 14, 16, 17 ; $\$ \$ 1067,1152$. Gray, A, 630; Quain, A, II, 546.

Syn.-Commissura anterior cerebri.

At the meson this commissure is a subcylindrical fibrous fasciculus just cephalad of the Columnæ fornicis. It expands laterad and reaches the striata and Crura olfactoria, which it thus connects across the meson. In man, according to Meynert (Stricker, A, 680), the connection of the olfactory Jobes with the commissure is slight.

$\S 1291$. Præcornu, preu.-Fig. 113, $120 ;$ Pl. IV, Fig. 15, 16, 18, $19 ; \S 1149$. Gray, A, 625 ; Quain, A, II, 541.

Syn.-Cornu anterius ventriculi lateralis.

The cephalic portion of the procelia. It is quite high, but compressed, the striatum projecting into it. 'The ventro-cephalic end narrows suddenly to become continuous with the rhinoccelia. 


\section{\$ 1292. Præpedunculus, prpd.-\$1141. Gray, A, 635: Quain, A. II. 576.}

Syn.-Pedunculus cerebelli superior, processus $\Theta$ cerebello ad testim, crus cerebelli superius, testibrachium.

The præpedunculi extend from the cerebellum to the base of the postoptici, and form the walls of the cephalic and longer part of the epicœlia.

$\S 1293$. Præperforatus (Locus), prpf.-Fig. 116, 118; Pl. II, Fig. 3 ; Pl. III, Fig. 11. Gray, A, 621 ; Quain, A, II, 536.

Syn.-Locus perforatus anterior s. anticus, anterior perforated space.

The irregular area just cephalad of the chiasma. In man it is comparatively small, but in the cat it is larger and presents two quite distinct portions, a cephalic which is more or less elevated and often distinctly marked by longitudinal furrows and ridges, and a caudal which is smaller, depressed, and smooth excepting for the vascular perforations which characterize the entire area. The two divisinns are hence named Portio prominens and Portio depressa. Between the depressed portions is the slight gray elevation of the terma, forming part of the floor of the Recessus opticus.

§ 1294. Procœlia, prc.-Fig. 110-113; Pl. IV, Fig. 15, 16, 18, 19 ; § 1064. Gray, A, 624 ; Quain, A, II, 539.

Syn.-Ventriculus lateralis, ventriculus tricornis, lativentriculus, first or second ventricle.

The lateral cavity of the prosencephalon, communicating through the porta with the aula and thus with its platetrope, and with the mesal series of coliæ. Ceplualad it opens into the rhinoccelia.

$\S 1295$. Proplexus, prpx.-Fig. 118, 121 ; PI. IV, Fig. 15, 18 ; $\$ \S 1066,1149$. Gray, A, 627; Quain, A, II, 545.

Syn.-Plexus choroideus ventriculi lateralis.

The larger part of this plexus of the procolia is formed by the intrusion of the velum or of vessels thereof between the fimbria and the Sulcus limitans so as to appear, still covered by endyma, in the medicornu. Its length thus coincides with that of the rima.

A smaller portion of the proplexus projects laterad into the præcornu, as shown in $\mathrm{Pl}$. IV, Fig. 18.

Respecting the continuity of the endyma upon the proplexus, see Todd (A, III, 704); Balfonr (A, II, 364, Fig. 260, 261) ; Mivart (B, 267); Gray (A, 627); Quain (A, II, 546).

\$ 1296. Prosencephalon (az.), pren.-Fig. 110-118; P]. I, Fig. 1, 2 ; Pl. II, Fig. 3, 4; Pl. III, Fig. 5, 6, 11, 13 ; Pl. IV, Fig. 14-20; $\$ \$ 1$ 1061, 1138, 1145. Gray, A, 111; Quain, A, II, $\%$ ริ9.

Syn.-Cerebrum, hemisphæræ, forebrain.

The cavities of the prosencephalon are the aula, the porte and the procolice.

§ 1297. Pseudocœlia (az.), psc.—\$§ 1064, 1137 (4), $1162(2)$. Gray, A, 627; Quain, A, II, 543.

Byn.-Ventriculus quintus, ventriculus septi pellucidi, ventriculus Sylvii, incisura septi, sinus septi pellucidi, fifth ventricle.

In man the mesal surfaces of the hemispheres between the callosum and fornix are separated by an interval, the "fifth ventricle." In the cat the two hemisepta are in contact, and the pseudocœlia"does not exist.

§ 1298. Pyramis, py.-Fig. 116; P]. II, Fig. 3,4 ; § 1140. Gray, A, 612 ; Quain, A, II, 504.

Syn.-Corpus pyramidale, prepyramid, ventripyramid, anterior pyramid.

At the side of the meson, just caudad of the pons and extending a little caudad of the 
Area ovalis. The pyramids are commonly regarded as continuations of the lateral rather than of the ventral columns of the myelon.

The use of the short term pyramis was suggested by Spitzka $(\boldsymbol{\gamma}, 165)$.

The "decussation of the pyramids" does not appear at the surface, but may be demonstrated by divaricating their caudal portions.

§ 1299. Quadrans, q.-Fig. 118 ; Pl. III, Fig. 9, 11 ; \$ 1144.

This name was proposed by the senior anthor $(9,136 ; 14,544)$ for an area of the ventral aspect of the Crus cerebri which is approximately the fourth of a circle. It is not always distinctly marked.

§ 1300. Radix intermedia (Cruris olfactorii), Rx. in.-Gray, A, 638; Quain, A, II, $53 \%$.

Syn--Radix media, the middle root of the olfactory tract. As stated in $\$ 1161$, it is not apparently differentiated in the cat.

This and the two following technical terms for the olfactory "roots" were proposed by the senior author $(9,136 ; \mathbf{1 4}, 538)$ as less apt to be misunderstood than the current vernacular words.

$\S 1301$. Radix 1ateralis (Cruris olfactorii), Rx. l.-Fig. 116; Pl. II, Fig. 3 ; 1161. Gray, A, 638 ; Quain, A, II, 536.

Syn.-Radix externa, external root of the olfactory tract.

See Limes alba, $\S 1250$.

§ 1302. Radix mesalis (Cruris olfactorii), Rx. ms.-Fig. 116; Pl. II, Fig. 3,4 ; 1161. Gray, A, 637; Quain, A, II, 537.

Syn.-Radix interna, inner or internal root of the olfactory tract.

In man this root is wholly, ventral in position, but in the cat it passes obliquely mesad and is continuous with the region between the postradical and præradicai fissures.

\$ 1303. Radix motoria (Nervi trigemini), Rx. mt. - The smaller motor root of the $N$. trigeminus; see Chap. XI and Pl. II, Fig. 3.

$\$ 1304$. Radix sensoria (Nervi trigemini), Rx. sn. - The larger and sensory root of the N. trigeminus ; see Chap. XI and Pl. II, Fig. $\hat{\jmath}$.

$\S 1305$. Recessus aulæ (az.), R. a.-Fig. 117; Pl. II, Fig. 4 ; Pl. IV, Fig. 14; § 1172.

The slight space between the Columnæ fornicis just ventrad of the Crista and dotsad of the præcommissura. Its cephalic wall is exceedingly thin and has not received a special name, but is evidently a part of the original terma.

\& 1306. Recessus opticus (az.), R. op.-Fig. 117, 118.; Pl. II, Fig. 4; Pl. III, Fig. 11.

As best seen in Pl. III, Fig. 11, there is a distinct recess just dorsad of the chiasma on each side. It seems to correspond with what Mihalkovics $(A, 79)$ calls by the name above given, Together they constitute the ventro-cephalic part of the aula.

$\S 1307$. Recessus præpontilis (az.), R. prpn.-Fig. 116, 117, 118; Pl. II, Fig. 3, 4 ; Pl. III, Fig. 11.

This name was proposed by the senior author $(9,136 ; 14,538)$ for the mesal pit formed by the overhanging of the cephalic border of the pons ; it is sometimes quite deep.

$\$ 1308$. Regio aulica $(a z),. R g$. a. - This name was proposed by the senior author $(9,136 ; 14,538)$ for the complex region about the aula. Within a radius of $1 \mathrm{~cm}$. from the Crista fornicis occur a large number of parts the structure and relations of which are inadequately known and far from easy to elucidate.

$\S 1309$. Restiforme (Corpus), rst.-Gray, A, 611; Quain, A, II, 505.

Syn.-Crus e cerebello ad medullam.

This part of the metencephalon is not distinctly shown upon any of the figures, and we 
have not accurately compared it with the corresponding part in man. As indicated by the synonym, the restiforme is sometimes regarded as identical with the postpedunculus, but even if they contain the same fibers, the latter should probably be defined as the continuation of the former to the cerebellum (Quain, A, $\mathrm{II}, 505$ ).

Between the restiforme and the Area ovalis is a smooth rounded elevation which we have not been able to identify. Nearly opposite the cephalic end of the Area elliptica it ceases, apparently covered in by the union of the parts at its sides.

$\S 1310$. Rhinencephalon (az.), rhen.-Fig. 110-112, 116, 117 ; Pl. I, Fig. 1, 2 ; Pl. II, Fig. 3. 4; Pl. IV, Fig. 15-19; § 1061 . Quain, A, II, 755.

This name seems to have been employed first by Owen (A, I, 283) as a convenient designation of the olfactory lubes and their crura. The observations of Milnes Marshall (3), as presented by Balfour (A, II, 382), require considerable modification of the views here adopted respecting the constitution of the encephalic segments. The term rhinencephala is used by Balfour (A, II, 366); $\$ 1150$.

$\S$ 1311. Rhinocclia, rhc.-Fig. 110-112; Pl. IV, Fig. 16; § 1150. Gray, A, 115; Meynert (Stricker, A, Fig. 261).

Syn.-Ventriculus lobi olfactorii, ventriculus rhinencephalicus, ventriculus olfactorius.

The cavity of the Lobus olfactorius, communicating with the ventro-cephalic part of the præcornu. Though slender in the cat, it is perfectly distinct, but is either obliterated in the adult human subject or so small as to have escaped notice. The human olfactory lobes are rarely obtained in a fit condition for accurate observation.

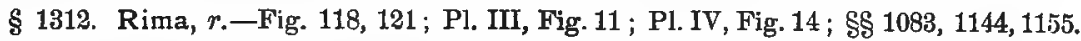
Gray, A, 627 : Quain, A, II, 544.

Syn.-Rima transversa cerebri magna, fissura transversa magna, fissura Bichatii.

The line of atrophy or abrogation of the proper nervous parietes of the procolia from the dorsal end of the porta to near the tip of the medicornu. Along this line there enters either the margin of the velum, which is a fold of pia, or vessels therefrom, to constitute the proplexus. Since, however, the endyma is continued from the margins of the rima upon the intruded pia or vessels, these latter can be said to enter the procollia only in the sense in which an abdominal viscus enters the peritoneal cavity.

of the two margins of the rima, one is certainly formed by the fimbria, which is continuous, through the hypocampa, with the caudal and thus with the dorsal part of the hemisphere. Concerning the other margin we are in doubt. In man it is probably the Tania (semicircularis), but this part has not yet been identified in the cat, and we have not personally examined the Cauda striati. We content ourselves, therefore, with the general statement that the fimbria lies in the Suleus limitans and that the proccelian endyma is reflected upon the proplexus from the fimbria and from the striatum or such other parts as may form the cephalic slope of the Sulcus. At about $5 \mathrm{~mm}$. from the tip of the cornu the rima ceases, and the cornu is completely encompassed by nervous substance.

The Name.-The terms above enumerated are not, strictly speaking, synonyms. They were all applied under a misapprehension still commonly entertained that there is a lack of all the colian parietes along a line extending between the tips of the medicornua, so that the medicornua and diacolia were in direct communication both with each other and with the ectal surface of the brain. With human brains in the condition in which they frequently are obtained, removed without sufficient care and roughly handled, such a solution of continuity may easily be demonstrated; but it is certainly artificial, and the names applied to it need not be retained in the same sense. To avoid, however, the introduction of a new term, the senior author proposed $(9,136 ; 14,541)$ the single short word rima 
to signify so much of the "rima transversa cerebri magna" as has been indicated above. When used for the interval between the chorda vocales, the compound term rima glottidis is commonly employed.

§ 1313. Ripa, rp.-Pl. III, Fig. 7; Pl. IV, Fig. 14.

This name was proposed by the senior author for the line formed by the rupture of the endyma along the lines of its reflection from entocolian surfaces. It is a ragged edge of endyma, sometimes quite distinct, as in Pl. III, Fig. 7. The ripa may be traced along the Sulci habenarum after removal of the diatela, along the margins of the delta, along both borders of the rima and on the thalamus and fornix at the nearly opposite points whence the endyma is reflected upon the portiplexus. The surfaces separated by the ripa are always unlike, the one being entocclian and the other ectocolian.

§ 1314. Rostrum (az.), »m.-Fig. 117; Pl. II, Fig. 4; Pl. IV, Fig. 17. Gray, A, 623; Quain, A, II, 538.

The tip of the genu of the callosum. It is shorter in the cat than in man, and sometimes less extensive in man than is commonly figured.

\$1315. Septum lucidum (cerebri), (az.), Spt.lu.-Fig. 113.

Syn.-Septum pellucidum, speculum, mediustinun s. diaphragma ventriculorum lateralium.

This consists of two lateral halves, the hemisepta. Fach hemiseptum is so much of the mesal wall of the procelia as is intercepted by the callosum and the fornix when the two apposed surfaces of the hemispheres are united. In man, although a space, the pseudocolia, remains between them or is formed by absorption, the compound septum so constituted is so thin as to have received the name lucidum. In the cat and in most other mammals, the adjective is wholly inapplicable. See hemiseptum (\$ 1241) and Area septalis (\$ 1203).

$\S 1316$. Splenium (az), sp.-Fig. 114, 115, 117 ; Pl. II, Fig. 4; Pl. III, Fig. 6 ; Pl. IV, Fig. 14, 17 ; §§ 1131, 1195, 1238.

The rounded caudal border of the callosum. When the caudal portions of the liemispheres are separated, the splenium appears as a thick white band. Its fibers pass laterad into the caudal portions of the hemispheres. Its ventral surface is continnous with the lyra.

The term is in common use, but we have not found it in the works of Gray or Quain.

$\S$ 1317. Stria longitudinalis (callosi), Str. lny.-Fig. 115 (?). Gray, A, 624 ; Quain, A, II, 537 .

Syn.-Stria Lancisi, nervus Lancisi.

The human callosum is described as presenting several more or less distinct longitudinal lines. We have not satisfactorily observel them upon the fresh brain of eitber man or the cat, but presume they are represented by the longitudinal striation vaguely shown in Fig. 115.

$\S$ 1318. Striatum (Corpus), s.-Fig. 113 ; Pl. III, Fig. 13 ; Pl. IV, Fig. 15, 16 ; $§ 1131$ (14), 1149. Gray, A, 625 ; Quain, A, II, 547, 564.

Syr.-Nucleus caudatus, eminentia lenticularis, ganglion cerebri anterius, apex cruris medullæ oblongatæ.

As indicated by the above synonymy, the corpus striatum of the older anatomists included the entocœlian ("intraventricular") portion, which appears in the procolia (PI. IV, Fig. 16), and the ectocclian ("extraventricular") portion, which is commonly described as imbedded in the wall of the hemisphere. The former is specified as Nucleus caudatus and the latter as Nucleus lenticularis. In man, between the two is a mass of 
alba called corona radiata, and between the Nucleus lenticularis and the insula, which lies ectad of it, is a thin lamina of cinerea, the claustrum.

The presence and arrangement of these divisions in the cat have not yet been determined by us, and we have therefore preferred to use the comprehensive term striatum. The student will avoid some confusion if he bears in mind that all of them are portions of the thickened procoelian parietes.

Spitzka has commented $(7,166)$ apon the misleading use of the term Nucleus in this connection; we suggest that the caudate and lenticular portions of the striatum be known as (Corpus) caudatum and (Corpus) lenticulare.

\section{$\S$ 1319. Sulcus habenæ, Sl. $h$.-Fig. $122 ;$ Pl. III, Fig. 7 ; $\S \S 1143,1156,1165$.}

This name was proposed by the senior author $(9,136 ; 14,538,544)$ for the more or less distinct furrow along the dorso-mesal angle of the thalamus just dorsad of the habena. It coincides nearly with the line of reflection of the diacclian endyma toward the opposite side.

$\S 1320$. Sulcus intercruralis lateralis, Sl. ic. l.-Fig. 118; Pl. III, Fig. 11.

The Area intercruralis of the cat presents some features which may not exist in man or may have escaped notice. They are most distinctly visible when the cerebellum is removed and the "medulla" is dorsiducted as in Fig. 118 and P1. III, Fig. 11.

Caudad of the small (Locus) postperforatus there is a mesal fissure, the Sulcus intercruralis mesalis, and on each side a Sulcus intercruralis lateralis. Between them, of course, are two ridges.

$\S 1321$. Sulcus intercruralis mesalis, sl. ic. ms.-See $\S 1320$.

\$ 1322. Sulcus limitans, Sl. li.-Fig. 121 ; Pl. III, Fig. 13 : $\$ \$ 1149,1155$.

This name was proposed provisionally by the senior author $(\boldsymbol{y}, 137 ; 14,538)$ for the "depression between the thalamus and the striatum" (Gray, A, 625; Quain, A, II, 549), which is obvious and usually mentioned, but has apparently not been named. So long as both the bodies above mentioned are regarded as parts of the procelian floor, this furrow might not require special designation any more than the furrow between the fornix and the hypocampa. But, in the eat at least, " this furrow is the line of separation between the entoccelian surface of the striatum and the ectocelian surface of the thalamus. A shorter term is, however, desirable."

$\S 1323$. Sulcus triradiatus (az.), Sl. trd-Fig. 118 ; Pl. III, Fig. 11; $\$ 1169$.

This name was proposed by the senior author $(14,554)$ for the three-pointed shallow depression which demarcates the albicantia from each other and from the Tuler cinereum. It is much deeper in the human brain.

8 1324. Terma (az), t.-Fig. 110-112, 117 ; Pl. II, Fig. 4; Pl. IV, Fig. 16. Gray, A, 620 ; Quain, A, II, 536.

Syn.-Lamina terminalis 8. cinerea.

The thin lamina between the præcommissura and the chisma and crista, and forming the cephalic boundary of the caudal portion of the aula.

The name was proposed by the senior author $(9,137: 14,541)$ as a brief and significant substitute for the compound terms commonly employed. It is the termination of the mesal series of ccliæ, and therefore has considerable morphological significance ; but it is so delicate as to be sometimes overlooked, and is usually ruptured in the extraction of the human brain.

\$ 1325. Thalamus, th-Fig. 110-113, 117, 122, 123; P1. II, Fig. 4; Pl. III, Fig. 6, 7, 9, 10, 13; Pl. IV, Fig. 16, 18, 19 ; §§ 1143, 1144, 1156, 1157. Gray, A, 629; Quain, A, II, 535 . 
Syn.-Thalamus opticus, thalamus nervi optici, ganglion cerebri posticum, eminentia magna cerebri.

The thalami form the walls (lateral parietes) of the diacclia, and are connected by the medicommissura.

Most of the important features of the thalami are described in the sections referred to ; there is one, however, which may properly be insisted upon here, inasmuch as it is not commonly recognized; viz., its complete exclusion from the procolia in the cat.

In most works upon Descriptive Anatomy, the thalami are mentioned as appearing in the "lateral ventricles," and in Gray' (A, Fig. 364) and Quain (A, II, Fig. 383), the surfaces of the striata aud thalami are apparently similar and continuous. Now the fornix (including the hypocampe and fimbriæ) of man is relatively narrower than that of the cat, and it is quite possible that in the adult, even without the rupture of the membranes at the rima, a part of the dorsal aspect of the thalamus may appear in the procœlia; if so, however, that part of the surface must be covered by the proccelian endyma, and the line of reflection (ripa) upon the proplexus should be represented. Upon these points our materials for observation do not enable us to speak more fully.

But in the cat and dog, we are prepared to state emphatically (\$1083), that the margins of the rima are in close apposition, excepting for the intruded tements of the proplexus, and that in these animals and in other mammals examined by us, no portion of the thalamus appears in the procolia or enters into the formation of its floor; any statements to the contrary should not be accepted without detailed descriptions and figures.

§ 1326. Tractus opticus, Tr. op.-Fig. 116, 118; Pl. II, Fig. 3; PI. III, Fig. 9, 11; 1144. Gray, A, 638; Quain, A, II, 533.

The subcylindrical fibrous band which arises from the diencephalon, and perhaps from the mesencephalon, and meets its platetrope cephalad of the Tuber cinereum to form the chiasma. The larger part of the tract seems to be a direct continuation of the prægeniculatum, but we have not traced the fibers in detail. See Chap. XI.

§ 1327. Tractus postrhinalis, Tr. prh.-Fig. 116 ; Pl. I, Fig. 2 ; Pl. II, Fig. 3.

This and the following name were proposed by the senior author for the caudal and cephalic portions of the ventral aspect of the prosencephalon and rhinencephalon, bounded Jatero-dorsad by the $F$. postrhinatis and $F$. rhinalis respectively. They are of course continuous with each other.

In the adult human brain these' tracts are relatively so small and so obscured by the overhanging convolutions of the hemisphere proper that they appear not to have been defined.

Tractus rhinalis, Tr. $r h .-$ See $\$ 1327$.

§ 1328. Trapezium, tz.-Fig. 116 ; P1. I, Fig. 2 ; Pl. II, Fig. 3 ; §§ 1140, 1161, 1276. Quain, A, II, 511.

Syn.-Corpus trapezoides-Meynert (Stricker, A, 726) ; Huxley, A, 64.

The quadrangular, slightly convex portion of the Area postpontilis in the angle formed by the caudal margin of the pons and the lateral margin of the pyramis. It is sometimes crossed by a faint cephalo-caudal band, as indicated on the right (left of the figure) side of Pl. II, Fig. 2. This band seems to be continuous with the Area oralis, and there is sometimes (as in Prep. 407, M. C. U.) an equally distinct band close to the pyramis and apparently continuous with the Area elliptica.

Laterad, the trapezium is continuous with the Eminentia auditoria; the N. abducens arises between it and the pyramis, and the $\mathrm{N}$. facialis between the pons and its laterocephalic angle.

In man this area is covered by the greater caudal extension of the pons. 
$\S 1329$. Tuber cinereum (az.), T. cin.-Fig. 111, 112, 116-118, 122 ; Pl. II, Fig. 3, 4; Pl. III, Fig. 5, 9, 11 ; §1074 (E). Gray, A, 621; Quain, A, II, 535.

The gray eminence at the cephalic part of the Area cruralis just caudad of the chiasma. To it is attached the hypophysis by the infundibulum which covers the mesal Foramen infundibuli. The Tuber cinereum is really continuous with the terma, but the chiasma forms an ectal interruption.

\$1330. Tuberculum Rolando, Tbcl. Rol.-\$1190. Gray, A, 613; Quain, A, II, 510.

According to the American Jour. of Neurology, etc. $(I, 102)$, the elevation herein named Area ovalis is homologous with the Tuberculum Kolando or Thbercolo cinereo.

$\S$ 1331. Valvula (az.), vv.-Fig. 111-114, 117; Pl. II, Fig. 4 ; Pl. III, Fig. 7 ; §§ 1141, 1165. Gray, A, 631 ; 'Quain, A, II, 552.

Syn. - Valvula Vieussenii, vv. cerebelli, vv. Willisiana, vv. magna cerebri, velum interjectum cerebelli, velum medullare anticum.

The delicate and transparent roof of the longer and cephalic portion of the epicolia. Cephalad it is continuous with the postoptici, and candad with the cerebellum just $t_{3}$ cepphalad of the highest part of the epicoelia. Near its cephalic end arise the NN. trochlearga. .

We have not ascertained whether the valvula is covered by a fold of pia ; apparently there is between it and the overhanging cerebellum only a jittle connective tissue. Neither do we know the precise constitution of the delicate substance of the valvula; it is, so thin that it might well be included with the other telæ as the epitela.

$\S 1332$. Velum (interpositum), (az.), vl.-Fig. 122 ; \$ 1142, 1144; 1156. Gray, A;628; Quain, A, II, 545.

As has been admitted elsewhere, our knowledge of the velum is incomplete, especially in respect to its relation with the diatela. As commonly described, and as appears to be the case in the cat, it is the fold of pia between the dorsal aspect of the mesencephalon and diencephalon and the ventral aspect of the superincumbent fornix, a part of the prosencephalon. Theoretically, and doubtless actually in the embryo mammal, the two layers of this fold are simply continuous at the line of junction of the prosencephalon with the diencephalon; but the growth of the former and its recumbency upon the latter brings them into contact, and perlaps their distinction is altogether lost.

Primarily, too, the diencephalic layer must have been in contact with the primitive roof of the diacoelia and entered into the formation of the diatela: but the close approximation and, perhaps, fusion of the two layers in the adult renders it possible to remove them together, and the diatela which remains must consist, therefore, of merely the endyma with the atrophied nervous substance of the roof.

The relations of the velum to the proplexus are referred to in $\$ 1295$.

$\$ 1333$. Vermis (cerebelli), (az.), vm.-Fig. 114 ; PI. I, Fig. 1, 2; Pl. IV, Fig. 15; $\$ 1200$. Huxley, A, 64 .

Syn.-Lobus medius cerebelli.

The name vermis seems to be used in anthropotomy in" a restricted sense for a portion of the median lobe of the cerebellum, but Huxley designates by it the entire lobe.

In the adult cat the vermis is markedly contorted, although regular and symmetrical in the new-born kitten (Wilder, 11, Pl. I, Fig. 2).

$\S 1334$. Other Figures of the Brain of the Cat or other Felidx.-The following list, arranged alphabetically according to the names of the authors, includes all the works and papers known to us to contain figures of feline brains. Additions and corrections will be thankfully received. The figures represent the cat's brain unless otherwise specified. 
(1) Bell, T.: A, Fig. 201 ; the dorsal and dextral aspects of the brain of "the lion." The lateral aspect represents a brain so distorted as to be unrecognizable; the dorsal aspect is evidently of a cat's brain, and, although very imperfect, is copied by Owen, A, III, Fig. 83 .

(2) Bourgery et Jacob : A, VIII, Pl. 16; Fig. 1, ventral aspect of myelon and its nerves and of the caudal portion of the brain; Fig. 2, dorsal aspect of the myelencephalon. In Fig. 1, some features are obviously incorrect. Both are apparently original.

(3) Cuvier (Audouin, ete.) : B, II, PI. III, Fig. 6 ; dorsal aspect of the brain of the lion, reduced. "Tirée de l'atlas de M. Leuret" [Leuret et Gratiolet, A].

(4) Dareste : 13, P1. 2; Fig. 3, lateral aspect of right bemisphere; Fig. 4, dorsal aspect of hemispheres. Diagrams, not wholly correct, of the fissures. Arparently original.

(5) Ferrier: A, Fig, 34, 35; dorsal and lateral aspects, with the "motor areas" marked upon the latter. The figures are original and correct; respecting the enumeration of the external convolutions, see $\$ 1364$.

(6) Flower : 6, Pl. 27; Fig. 12, the mesal aspect of the right hemisphere. Correctly represents the mesal fissures excepting part of the hypocampal; original.

(7) Gall and Spurzheim: A; according to Owen $(35,135)$, the cat's brain is figured in this work.

(8) Gegenbaur (Lankester): A ; Fig. 286, C, represents a partially dissected cat's brain ; the figure is evidently original, but vague in some respects.

(9) Gervais, P. . 146 ; there are several figures of feline brains, but the work is not at present accessible to us, and they cannot be specified.

(10) Hammond, G. M. : 1, Fig. 1; a transection of the deft hemisphere through the striatum. This original figure is intended to display certain histological features.

(11) Hitzig: A, Fig. 7; the lateral aspect; a fissural diagram.

(12) Jones, T. R.: A, Fig. 411; the ventral aspect of the lion's brain. Reduced nearly one half, and reversed, from Tiedemann, A, Pl. III, Fig. 4 ; unacknowledged.

(13) Leuret (Leuret et Gratiolet, A, Pl. V): dorsal and lateral aspects of the brain of a lion and lateral aspect of that of a panther. Fig. 1-3, dorsal, mesal and lateral aspects of the cat's brain. All are obviously origrinal and very correct, excepting some of the struc. tural features of Fig. 2, especially the relations of the fornix and callosum.

(14) Lussana e Lemoigne: A ; Fig. 123-125, the mesal aspect of the left hemisphere, with two schernatic diagrams ; Fig. 126-131, the dorsal, ventral and lateral aspects of the hemispheres, actual and schematic; Fig. 132-135, the ventral, mesal, dorsal and lateral aspects of the hemispheres of a leopard.

(15) Marshall, J. : A, Fig. 2 ; dorsal aspect ; poor.

(16) Meynert, T. : 1 . Fig. 8, 17, dorsal and lateral aspects of the hemispheres of a lion ; Fig. 16, lateral aspect of the hemisphere of a wild-cat ; Fig. 23, the lateral aspect of a lion's hemisphere. The figures are original, clear and correct.

(17) Mivart: B, Fig. 125, 126, 129 : the lateral, dorsal and mesal aspects. These figures are unacknowledged, but evidently copied from Leuret, Pl. V, Fig. 1-3 ; the inaccurate representation of the pseudocolia is exaggerated.

Fig. 127 is an apparently original representation of a preparation made by tilting the cerebellum caudad and the hemispheres cephalad, as in Fig. 114 of this work, so as to expose the optici, thalami, etc. A very erroneous impression is given by the exposure of the striata and the introduction of a tubular and wholly imaginary pseudocelia.

Fig. 128, the ventral aspect of what purports to be the brain of a cat; aside, however, from the general outline and the exposure of the trapezium, it might be the brain of a monkey, and some of its features have never been observed in the cat. 
(18) Owen : 25, Pl. 20; Fig. 1-3, dorsal, lateral and mesal aspect of the brain of the Cheetal Felis jubata, shaded; Fig. 4-6, the same of the cat, outline. The former are somewhat vague, especially as to the Sylvian fissure; the latter are clear, and correct excepting the non-extension of the $F$. postrhinalis in Fig. 5, and the indication upon Fig. 6 of an improbable fissure near the caudal end of the hemisphere.

(19) Owen : A, III ; Fig. 83, dorsal aspect of eat's brain. Unacknowledged, but evidently copied from the very poor figure of Bell (A).

(20) Owen : A, III ; Fig. 86, the mesal aspect of the right hemisphere; apparently original. A good outline diagram, excepting the presence of the line marked (13), purporting to represent the $F$. lambdoidalis, and apparently the same as shown in Fig. 3 and 6 . Whether or not such a fissure exists in the Cheetah, or whether, if present in any feline brain, it is the homologue of the "lambdoidal " or " occipito-parietal "fissure, need not be discussed here; but among the many (over 200) cat's brains examined by us, none have presented any fissure in that region. Probably the line was accidentally introduced or may represent a vascular furrow (\$ 1341).

(21) Owen: A, III; Fig. 91, the lateral aspect; an outline diagram, apparently reduced and slightly changed from the same author' Fig. 5 (25, Pl. 20). The superorbitalis, however, has disappeared, and the diagonalis is made, incorrectly, to join the rhinalis.

(22) Pansch : 1, Taf. XIV, XV ; Fig. 23-38, dorsal, lateral and mesal aspects of the hemispheres of adult, new-born and fœtal cats. These are excellent original diagrams of the fissures.

(23) Serres : A, P1. XIV ; Fig. 264, 265, the lateral and dorsal aspects of what purports to be the brain of a lion, but, as remarked by Leuret (Leuret et Gratiolet, A, Pl. 5, p. 10), is really that of a cat.

(24) Spurzheim : A, Pl. IV ; Fig. 5, dorsal aspect of a somewhat distorted brain.

(25) Stowell, T. B. : $\boldsymbol{I}$; Fig. 1, 2, ventral and lateral aspects of the cat's brain, with special reference to the ectal origins of the cranial nerves; the fissures and other parts are shown diagrammatically; Fig. 3-12, ectal nerve origins and distribution of the vagus.

(26) Tiedemann, F. : A, Tab. III ; Fig. 3-5, dorsal, ventral and mesal aspects of a lion's brain ; original and mainly accurate ; Fig. 6, the brain "Felis nondum adulti, quod cerebro Leonis persimile est." Unless the author had positive knowledge as to the immaturity of this brain, it must be regarded as that of an adult domestic cat.

(27) Wilder : 11 ; PI. I, Fig. 1, 2, portions of the cerebellum, showing the vermis and the Lobulus appendicularis; Pl. III, Fig. 15,17, dorsal and lateral aspects of the hemispheres, diagrams of the fissures; P1. IV, Fig. 18, 19, lateral aspect, young Asiatic and African lions, fissural diagrams.

(28) Wilder : $\boldsymbol{8}$; Fig. 1, 2, fissural diagrams of the lateral and mesal aspects.

(29) Wilder : 14 ; Pl. 1-3, Fig.1-5, the dorsal, lateral, ventral, mesal, cephalic and caudal aspects of the entire brain or of the hemispheres; Pl. 3, 4, Fig. 7-20, sections and dissections illustrating the gross anatomy.

\section{THE CEREBRUM AND ITS FISSURES.}

$\S 1335$. Form of the Cerebrum.-As compared with that of most dogs, the cat's cerebrum is remarkable for its width. The average width is $34-37 \mathrm{~mm}$. With four well preserved adult brains, taking the width at 100 as the standard, the lengths were respectively $93,97,100$ and 103 , while the heights were $75,67,71$ and 72. In round numbers, then, the lateral and longitudinal dimensions of 
the combined hemispheres are approximately equal, and the height is about three fourths as much.

As compared with most dogs, also, the region cephalad of the cruciate fissure is very short.

\section{THE CEREBRAL FISSURES.}

§ 1336. References.--The principal works and papers in which the cerebral fissures are treated of are named in connection with the synonymy of the $F$. eruciata ( $\$ 1361$ ).

\$1337. As was remarked in $\S 1131(17,18)$, the brain of the cat differs from that of the Amphibia in that the hemispheres and cerebelium are not only larger in proportion, but convoluted-the surface presenting depressions (fissur $\boldsymbol{\text { s }}$ ) and intervening folds (gyri).

The cerebellar fissures are numerous and apparently irregular; and we are not aware that their arrangement has been studied in detail.

The cerebral fissures are comparatively few (about 30 ) and simple, so that it is not difficult to delineate or describe them as they appear upon a given brain.

§ 1338. The fissures should be studied before the gyri.-Notwithstanding the probability that the fissures are only the results of the outgrowth constituting the gyri, and the fact that experiments are made upon the exposed surfaces of the latter, the study of cerebral topography should begin with the fissures, and they should be identified and named before the gyri are described and designated. As remarked by the senior author (11, 219), the "sides of a fissure are usually near together and parallel, so that the fissure may be described as a single line of certain direction; but the opposite borders of any one gyrus are rarely parallel throughout their whole extent. Indeed, it would be as hard to designate gyri without first identifying fissures as to describe the countries of Europe without mentioning its rivers."
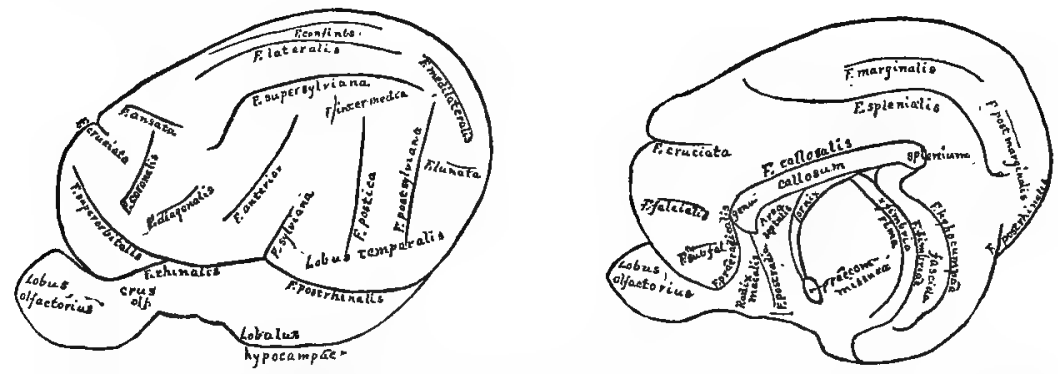

Fig. 124, 125.-Diagrams of the Lateral and Mesal aspects of the HemiSPHERES, SHOWING THE FIsSURES; $\times 1.5$.

Fig. 124,-Diagram of the lateral aspect of the left hemisphere, showing the fissures; $\times 1.5$.

Fig. 125.-Diagram of the mesal aspect of the right hemisphere, showing the fissures. The diencephalon and the segments caudad of it have been removed as in PI. IV, Fig. 17, so as to expose the $F$. hypocampa and the adjacent parts ; $\times \mathbf{1 . 5}$.

On both the figures too little distinction is indicated between the constant and the inconstant fissures. Upon the Table (p. 496) the former are printed in heavier faced type, and they are enumerated in $\S 1366$. 
$\S 1339$. Study of the Fissures.-The student should make outline drawings of one or more hemispheres, especially of the lateral aspect. If possible, both hemispheres of the same brain should be drawn and compared with respect to the amount and character of lateral variation and compensation (Wilder, 11, 232). The sex should always be noted, and the age, known or estimated, stated upon the drawing.

The drawings of fissures should be in outline only, and most attention should be given to the union or independence of fissures which approach each other.

$\$ 1340$. Sometimes it is difficult to distinguish between a true fissure and a depression in which was lodged a superficial vessel. Such vaseular trenches, however, have usually more abrupt and sharply defined edges than the fissures (Wijder, 11, 221).

The mesal aspect of the hemisphere is largely a plane surface; but the lateral and other aspects present difficulties in respect to perspective which are common to convex surfaces. Where a hemisphere is very peculiar, the drawings should be based upon photographs. The method of drawing fissures described by the senior author in 1873 (11, 219), in which the perspective was ignored, is no longer recommended by him.

$\$ 1341$. Indicating the Relative Depth of Fissures.-As appears in Fig. 122, and in P1. III, Fig. 13, and PI. IV, Fig. 15, the fissures vary considerably in deptl. They also vary in different parts of their course, being usually deepest near the middle of their length. Where a fissure is wholly superficial, it may be represented by a shading; but otherwise a line must be employed, the width of which may indicate the depth of the fissure in different parts of its extent and as compared with other fissures. The depth may be ascer tained by carefully " sounding" with a graduated, thin, smooth and rounded rule, or by sections of the brain after the location, extent and connection of the fissures have been indicated by lines of uniform width.

$\S 1342$. Table of the Cerebral Fissures.-The accompanying Table (p. 496) includes an alphabetical list of the feline cerebral fissures, with the abbreviations employed in this work, and the principal synonyms.

The constant fissures are printed in black letter.

The synonymy is limited to writers who have made special additions to the technical nomenclature, and excludes those who have employed phrases or vernacular names, or who have used the names of other writers in purely physiological papers.

$\$ 1343$. Sources of the Names.-The following brief statement respecting the names is quoted from the senior author's paper $(8,50)$ :-

"Owen's 'postsylvian' should not be displaced by Krueg's 'suprasylvii posterior,' nor his 'murginal' by 'suprasplenialis.' Likewise, Flower's 'supraorbital' has priority cver my ' presylvian,' which Krueg has adopted [and is free from the implication of a doubtful homology].

"On the other hand, Krueg's 'anterior' and 'postica' are so much more usable than previous names as to be worthy of acceptance, especially as they may be regarded as abbreviations of the phrases by which the fissures in question were designated by Owen and myself. 'Splenialis' is to be preferred to supercallosal or calloso-marginalis, so long as the human homologue of the fissure is uncertain. If marginalis is to be retained, postmarginalis will be better than ' postsplenialis.'

"I am particularly gratified to find that Krueg admits as fissural integers the ansatre and the diagonalis ; the former I had intended to call transversa and the latter intermedia, but Krueg's names must be retained. We agree also in regarding Owen's 'medilateral' as composed of two fissures, which Krueg terms medilateral and confinis. I had intended to leave Owen's name attached to the fissure which is really mesad of the lateralis and to eall the [caudal] curved division lunata; the name lunata has now been given to what otherwise would have been sublunata. I have applied the name intermedia to a fissure which Krueg mentions but does not name." 


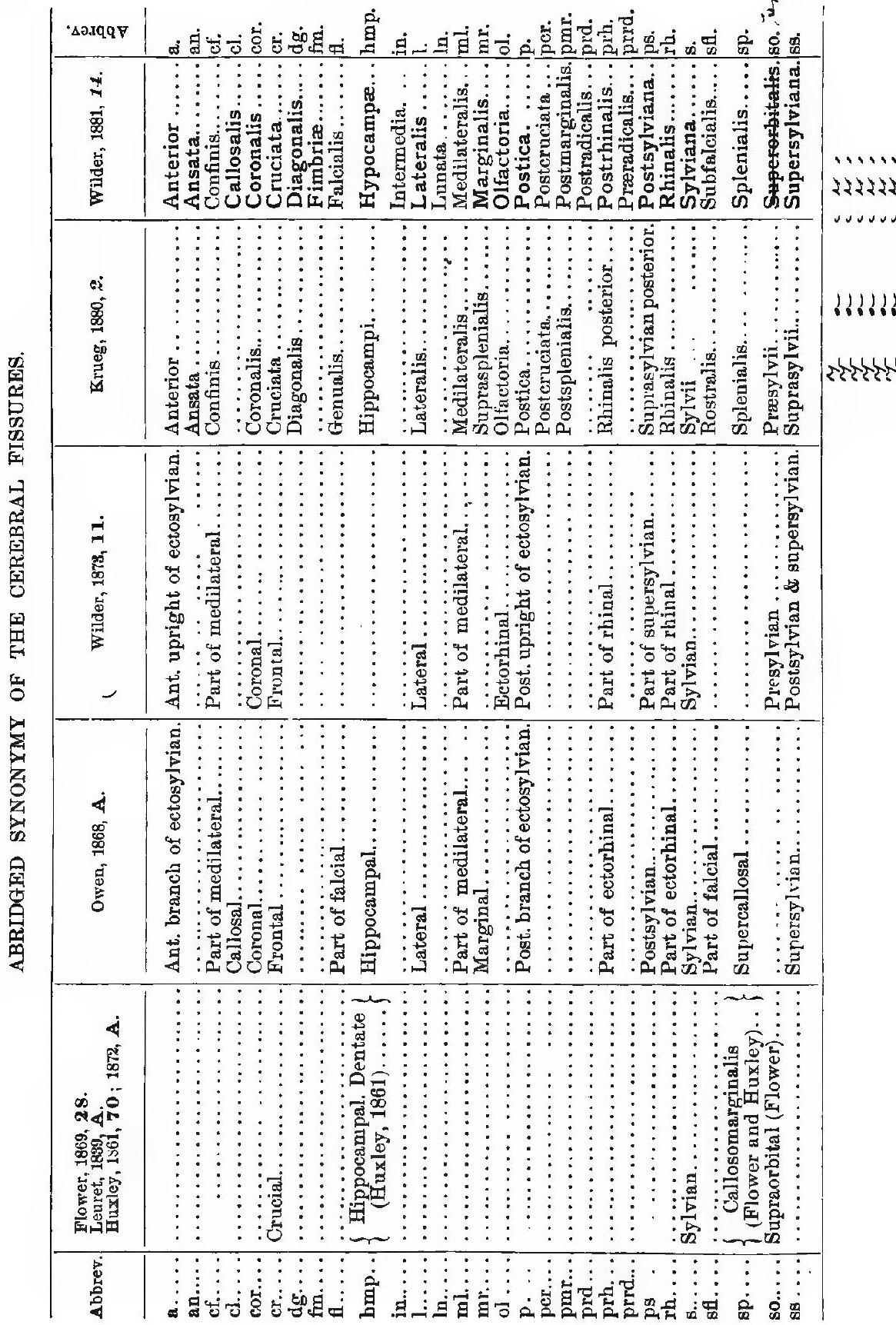




\section{\$ 1344. Problems Connected with the Cerebral Fissures.-} The study of the fissures of a single brain is comparatively uninteresting and unprofitable unless three general questions are considered :-

(1) What relations do the fissures bear to the ental structures?

(2) What is the fissural pattern in the cat?

(3) How do the fissures of the cat compare with those of man and other mammals ?

$\$ 1345$. Formation of Fissures.-At birth the cat's hemispheres present fewer and shallower fissures than in the adult. Presumably they were entirely smooth at an earlier period, as is the case in all other mammals which have been examined.

The hypocampal fissure represents an involution of the entire thickness of the parietes, the hypocampa ( $\$ 1243$ ) being the reverse elevation. The callosal fissure, and perhaps some of the others already enumerated (\$1343), are formed in some peculiar way.

So far as the other and ordinary fissures are concerned, although sometimes described as depressions, it is probable that they are to be regarded as lines of less elevation as compared with the intervening folds. More extended and accurate observations are needed upon this matter. For the formation of the cruciate fissure, see $\$ 1359$.

\& 1346. Structural Relations of Certain Fissures.-So far as appears from sections of the cat's brain at any period after birth, only eight of the cerebral fissures have any intimate or constant relation to structural features, viz., the callosal, fimbrial, hypocampal, olfactoria, postradical, proradical, rhinalis aud postrhinalis. These have all been mentioned in connection with the parts with which they appear to be correlated.

$\S 1347$. In man two other fissures, which do not exist in the cat, are related to ental structures : the calcarine to the calcar $(\$ 1194)$ and the collateral to the Eminentia collateralis at the place of departure of the postcornu from the medicornu.

A few of the fissures will now be mentioned separately.

$\S 1348$ F. callosalis, $F$. $c l$, the callosal fissure.-Fig. 117, 122, 125 ; Pl. II, Fig. 3 ; Pl. III, Fig. 18; Pl. IV, Fig. 17, 20.

This coincides with the dorsal border of the callnsum, curves about the splenium to join the hypocampal (Fig. 125) and about the genu to be continuous with the F. proeradicalis when the latter is distinct.

§ 1349. F. fimbriæ, F. fmb., the fimbrial fissure-Fig. 121, 125 ; Pl. IV, Fig. 14, 17; $\S 1172$.

A distinct and apparently constant depressed line between the fasciola and the fimbria, thus coinciding with the margin of the cinerea. It is not a true cortical fissure, and perhaps should not be enumerated with the rest.

§ 1350. F. hypocampæ, F. hmp., hypocampal fissure-Fig. 121, 125; Pl. IV, Fig. 14, $17 ; \$ 1172$.

Syn.-F. hippocampi, bippocampal fissure.

This is not the deepest of the cerebral fissures, but it is one of the longest, and is perhaps the most constant of all among the Mammalia, being present when the hemispheres are otherwise smooth or indented only by the rhinalis and postrhinalis.

It extends from near the tip of the Lobulus hypocampce to the splenium, where it is continuous with the $F$. callosalis. In the larger part of its course it presents a decided caudal convexity, forming nearly the half of a circle and coinciding in general with the medicornu and with the hypocampa, of which it is the depression in reverse. Near the 
splenium, however, it presents a short and quite sharp cephalic convexity. The cinerea cephalad of the $F$. hypocampo constitutes the fasciola.

$\S$ 1351. F. Sylviana, $F . S$, the Sylvian fissure.-Fig. 124; P1. I, Fig. 2 ; P1. II, Fig. 3 ; Pl. III, Fig. 5 ; $\$ 1163$.

Byn.-Fissura Sylvii ; the "posterior" or longer branch of the human F. Sylvii.

By common consent, this short and distinct fissure of the cat and dog is called by the name originally applied to the much more extensive and complex human fissure. To discuss the homology would occupy undue space. The chief questions are (1) as to the representation of the insula ( $\$ 1247$ ), which in man is concealed between the dorso-cephalic and the ventro-caudal lips of the fissure; and $(2)$ as to the correspondence of the relatively extensive region between the $F$. Sylviana and the $F$. superorbitalis with the human operculum ; Meynert, 1, Fig. 16; Wilder, 11, 225, and 14, 551.

$\S 1352$. The statement $(11,223)$ of the senior author as to the presence of the Sylvian fissure in all brains which are fissured at all (quoted in the last two editions of Dalton's Physiology, A, 413) referred originally only to the lateral fissures, and may not be correct with even that qualification, for the rhinalis and postrhinalis seem to occur in some mam. mals when the Sylvian is either absent or very indistinct.

\& 1353. Fissura ansata, F. an.-Fig. 24 : Pl. I, Fig. 1, 2.

This fissure is peculiar and presents some difficulties. Most commonly it seems to form simply a conjunction between the lateralis and the coronalis, with a branch pointing meso-cephalad. Less frequently is it independent of the coronal, as in Pl. I, Fig. 2, and the left side of Fig. 1; most rarely is it wholly isolated as a simple diagonal fissure, as in Fig. 21 and the right of Pl. I, Fig. 1. Nevertheless, Krueg and the senior author came independently to the conclusion that this is the primitive condition of the fissure and the one to be represented upon a diagram.

$\S 1354$. Fissura anterior, $F$. a.-Fig. 24 ; Pl. I, Fig. 1, 2; Pl. III, Fig. 5.

The disconnection of the dorsal end of this fissure from that of the postica, so that the keystone of the first arch is absent, constitutes a constant distinction between all the Felidæ and the feral Canidæ and most domestic dogs. The two fissures sometimes approach quite closely and even overlap, but we have never observed a junction. On the other hand, while the two form a continuous fissure in all feral Canidæ and most domesticated dogs, in the latter the arch is sometimes broken, giving this region of the brain a feline aspect (Wilder, 11, 229, and Fig. 13, 16). In tbis as in other respects, the dog displays more variability than the cat.

$\S$ 1355. Fissura cruciata, F. cr.-Fig. 117, 124, 125 ; Pl. I, Fig. 1, 2 ; Pl. II, Fig. 4 ; Pl. III, Fig. 5; Pl. IV, Fig. 16-19.

$\$$ 1356. Constant and Peculiar Characters.-Indents the dorso-mesal margin of the hemisphere near its cephalic end, so as to appear upon both the dorsal and mesal aspects. Length of the dorsal and mesal portions approximately equal. Dorsal portion at a right angle with the meson. Line formed by the dorsal portions of the two fissures about one half the length of the line representing the $F$. interhemispheralis, with which it forms a Roman cross. Lateral end simple and independent.

$\S 1357$. Variable Characters.-Caudal end of mesal part of fissure usually dorsad of the junction of the cephalic and middle thirds of the callosum, and about two fifths of the distance from the callosum to the dorsal margin of the hemisphere, thus about midway between the callosum and the cephalic end of the $F^{\prime}$. spleninlis. Rarely are these two fissures continuous.

$\S 1358$. The dorsal part of this fissure is a marked feature of the dorsal and cephalic aspects of the hemisphere, on account of its straightness, simplicity and independence, and 
its relation to the $F$. interhemispheralis. Its symmetry is also remarkable; very rarely is the mesal end on one side farther caudad than on the other.

The mesal portion is less uniform. The caudal half often tends slightly dorsad and the end is sometimes forked. Rarely does the caudal end join the $F$. splenialis. According to Krueg ( $\because, 620)$, the union was observed once by Guillot (A, Fig. 172); Krueg himself has seen it only twice $(2,620)$, and Pansch $(1,21$, Fig. 27) three times out of fourteen. Out of about 400 hemispheres of adult cats dissected by us or our students or preserved in the Museum of Cornell University and in the Museum of Comparative Zoology, it was noticed in only 4 .

In view of Broca's idea (1) that the cruciata and splenialis are morplologically parts of a single fissural integer, their relations should be carefully examined; note should be taken as to whether a junction is effected by means of branches or by a deflection of one or both of the fissures themselves; whether the combined fissure is shallower at the place of junction, and whether the junction exists npon both hemispheres.

This fissure is also interesting on account of the various opinions which have been expressed as to its human homologue (see $\S 13 \% 0$ ), and because several well-marked "motor centers" have been found about its dorsal portion; apparently none have been discovered upon the mesal surface of the hemisphere.

§ 1359. Formation. - As intimated by the senior anthor $(11,226)$, and more distinctly shown by Pansch (1, Fig. 32) and Krueg ( $\because$, Taf. XXXIV), the $F$. crucicta really begins upon the mesal aspect of the hemisphere, as a shallow depression which gradually approaches the margin, indents it, and finally extends laterad for a distance equal to or greater than its mesal part. Upon a series of kitten's brains, from a week before birth to a week after, the formation of this fissure is beautifully illustrated.

$\S 1360$. The Name.-Owen's "frontal" is descriptively significant, but it implies a not yet proven homology with one of the human frontal fissures, and is antedated by Leuret's "crucial." As to the technical form, there seems little to choose between crucialis and cruciata. Personally we prefer the former, but Krueg has employed the latter, and his name is here adopted.

$\$ 1361$. Synonymy. - The following synonymy is chronological, and intended to include all the works and papers in which the cruciate fissure is mentioned. It forms part of an unpublished paper by the senior author which is mentioned in 8,49 , and 14,524 . II, 157 .

Cuvier (1805); "en avant, un sillon court qui la traverse en croix ;" Carnivora; C,

Owen (1833): " a transverse anfractuosity-the transverse anfractuosity-the first transverse fissure ;" cat, cheetah ; 35, 133, 134 ; P1. XX, " $1 . "$ 122.

Owen (1835); "the anterior transver'se anfractuosity ;" Cercoleptes (kinkajou) ; $\mathbf{5 3}$,

Leuret (1839) ; "sillon crucial ;" cat and the Carnivora generally ; Leuret et Gratiolet, A. I, 379, etc., Pl. V, Fig. 3, opposite " $a . "$

Cuvier, Dumeril, etc. (1845); "sillon crucial ;" Felidæ and most other Carnivora ; Cuvier, B, III, 93.

Dareste (1855); "sillon crucial ;" cat and several other Carnivora ; 13,110, Pl. II, Fig. 1, 2, 4, 8, “f."

Owen (1868); "the frontal fissure;" cat and the "Gyrencephala " generally ; A, III; 116-136, Fig. 91, etc., "14."

Flower (1869) ; "erucial sulcus, crucial fissure;" Proteles cristata and the Carnivora generally; $\boldsymbol{2} \boldsymbol{8}, 479,482$, Fig. 1, 2,4 , “c." 
Flower (1870) ; "crucial sulcus ;" Ailurus fulgens ; 35, 755, Fig. 1, 2, 8, "c."

Lussana e Lemoigne (1871) ; "scissura crociata;" cat and many other mammals ; A, I, 142, Fig. 127, 191.

Fritsch und Hitzig (1870): "frontal fissure ;" $\operatorname{dog} ; \mathbf{1}, 312$.

Gervais (1870); cephalic part of "sillon crucial;" most Carnivora ; 146, 105.

Huxley (1870) ; "crucial sulcus-anterior end of the calloso-marginalis ;" $\operatorname{dog} ; \mathrm{A}, 420$.

Hitzig (1873) ; "frontal fissure;" $\mathrm{dog} ;$ 4, 434, Fig. 1, 2, 3, "14."

Wilder (1873) ; "frontal fissure ;" cat and the Carnivora generally ; 1 1, 225, Fig. 15, 17 , etc., " $f . "$

Hitzig (1874) ; "sulcus cruciatus-frontal fissure-fissura frontalis;" cat and dog ; A, 13, 46, 96, 129, Fig. 1, 7, "14" or " 8. c."

Garner (1876); "transverse or crucial sulcus ;" $1,153$.

Ferrier (1876) ; "crucial sulcus ;" cat, dog ; A, 145-154, Fig. 32-35, "b."

Meynert (1877) ; “ der Leuret'schen querfurche-der vordere aufsteigende ast der randfurche;" wild-cat, etc. ; 1, 12, Fig. 17, etc., "cm.-call. m."

Huguenin (Duval et Keller), (1878) ; “sulcus cruciatus ; " dog, fox, etc. ; A, 55, 56, Fig. 40, 41, " $8 c . "$

Broca (1878); 1 ; (this reference has been mislaid, and the paper is not now accessible to us).

Foster and Langley (1878) ; "crucial fissure ;" dog ; A, 219.

Krueg (1878); " sulcus oruciatus;" $\operatorname{dog}$ and Carnivora generally ; 1, 335, 345, Taf. XXI, "cr."

Horsley (1879); "fissura cruciata ;" Carnivora; 1, 277.

Clevenger (1879); "carnivoral crucial sulcus;" Carnivora ; 1, 7.

Pansch (1879); "das vordere ende der mediale hauptfurche;" cat and Carnivora generally ; 1, 21, etc., Fig. 26, 32, 43, etc., " $m$. hf." "cr."

Krueg (1880); " fissurce cruciata ;" most Carnivora, 2, 610, Taf. XXXIV, XXXVII,

Wilder (1880); " fissura cruciata ;" 8,50 , Fig. 1, 2.

Mivart (1881) ; "crucial sulcus ;" B, 259 and 261, Fig. 125 and 126, "c."

Wilder (1881); " fissura cruciata ;" cat ; 14, 534 ; P1. I, Fig. 1, 2 ; P1. II, Fig. 4 ; Pl. II, Fig. 5; Pl. IV, Fig. 16-19, " $F$. or."

$\$ 1362$. Designation of the Gyri.-The Sigmoid Gyrus.-This name has been used, especially by English writers, to designate the approximately s-shaped fold which curves about the lateral end of the cruciate fissure (P1. I, Fig. 1). Its surface includes several quite constant and well-marked "motor areas" (Ferrier, A, Fig. 32 ; Dalton, A, Fig. 113, 114).

§ 1363. The External or Arched Gyri.-The four arched gyri which are so regularly arranged in the fox and wolf were enumerated by Leuret (Leuret et Gratiolet, A, I, 374; Atlas, 9), beginning with that which immediately borders the Sylvian fissure, and ending with that which forms the margin of the hemisphere.

Since the fissure is constant and the margin variable, this would seem to be a natural arrangement, and it has been adopted by Huguenin and some others. As appears, however; from the accompanying Table, several writers have modified it or reversed the order altogether. Such transpositions are troublesome enough for skilled anatomists, and can hardly fail to perplex the beginner. 


\section{Table showing Fodr Methods of Endmerating the "Arched GrRi."}

\begin{tabular}{|c|c|c|c|c|}
\hline LOCATION. & $\begin{array}{l}\text { Leuret } \\
\text { (Leuret et } \\
\text { Gratiolet), } \\
\text { A, } 380 .\end{array}$ & $\begin{array}{l}\text { Ferrier, A, } \\
\text { 145. }\end{array}$ & $\begin{array}{c}\text { Flower, 28, } \\
479 .\end{array}$ & $\begin{array}{l}\text { Mivart, B, } \\
259 .\end{array}$ \\
\hline Next the mesal border of the hemisphere...... & Fourth... & First.... & Third.... & Superior. \\
\hline 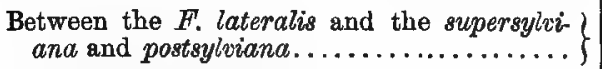 & Third.... & Second... & Second... & Middle. \\
\hline 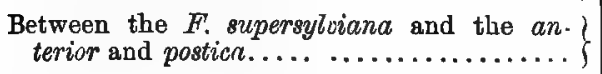 & Second. & Third... & & \\
\hline 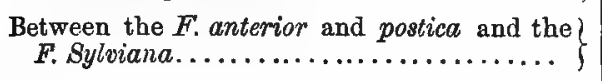 & First .. & Fourth.. & First. . . & Inferior. \\
\hline
\end{tabular}

\$ 1364. The Angular Gyrus.-This name is applied to a fold of the hemisphere in man and monkeys. By a few writers it has been given also to the caudal portion of what Leuret called the third arched convolution. As indicated below ( $\$ 1369$ ), we do not think the homologies of the fissures or folds are sufficiently well determined to warrant the application of this name to the Carnivora.

$\S 1365$. The Fissural Pattern.--'Whe fissures of the cat, dog and fox can be homologized in most respects, yet they differ to such an extent as to be readily distinguishable. If we could accurately determine the arrangement of fissures which is common to all domestic cats and peculiar to them, we might be able to define the fissural pattern of the species.

In view of the inadequacy of our knowledge, we have thought best to confine the discussion of the fissural pattern to a brief statement of what appear to be the constant and the inconstant fissural characters of Felis domestica.

\$ 1366. Constant Characters.-(1) Presence of the following nineteen fissures : anterior, ansata, callosatis, coronalis, cruciata, diagonalis, fimbrio, hypocampa, lateralis, marginalis, olfactmia, postica, postrhinalis, postsylviana, rhinalis, Sylviana, splenialis, superorbitalis, supersylviana.

(2) Fissura Sylviana rather short, forming not more than one third nor less than oue eighth of an imaginary line coinciding therewith and extending from its ventral end to the dorsal margin of the hemisphere.

(3) Nine fissures are so placed with reference to the Sylvian as to form three irregular arches dorsad of the Sylvian, corresponding with the more regular arched fissures of the fox and wolf ; the first consists of the postica and the anterior, the diagonalis often being continuous with the latter; the second, of the postsylviana and supersylviana; the third, of the lateralis, with the medilateralis when present, the ansata and coronalis.

(4) Absence of a fissure ( $F$. ectolateralis of Canidæ) between the caudal portions of the lateralis and the supersylvian.

(5) Disconnection of the dorsal ends of the anterior and postica.

(6) Independence of the $F$. olfactoria.

(7) Independence of the dorsal ends of the anterior, postica, superorbitalis and Sylviana; of the ventral ends of the hypocampa, coronalis and medilateralis; of the caudal ends of the splenialis and postradicalis (when present); of the lateral end of the cruciata; of the mesal end of the ansata. 
(8) Continuity of the rhinalis with the postrhinalis; of the Sylvian with the point of their junction; of the superorbital with the rhinalis; of the callosal with the hypocampal, and with the praradicalis when present.

\$ 1367. Variable Characters.-(1) More or less frequent presence of the following ten inconstant fissures: confinis, falcialis, intermedia, lunata, medilateralis, postcruciata, postmarginulis, postradicalis, proradicalis, subfalcialis.

(2) Frequent union of the a/sata with the coronalis and lateralis; of the lateralis with the ansuta and meditateralis; of the diagonalis with the anterior; of the supersylvian and postica; of the marginalis with the postmarginalis.

(3) Occasional unions of the medilateralis and confinis; of the cruciata and the splenialis.

(4) Very rare unions of the postica and the rhinalis; of the anterior with the supersylvian.

$\S 1368$. Homology of the Human and Feline Fissures.-The determination of the identity of the human fissures with those of the other Mammalia has Jong been desired from the standpoint of Comparative Morphology and Systematic Zoology. Referring in 1868 to his Lectures on the Brain in 1842, Owen says (A, III, 116): "The main object which I had in view was the determination of the homologous and superadded convolutions in the more complex prosencephalon of man."

Since the discovery in 1870 by Fritsch and Hitzig (1) of the electrical excitability of certain areas of the cerebral cortex in the dog and cat, and the confirmation of this upon monkeys by Ferrier (A, 138), there have been likewise physiological and psychological reasons for the determination of these fissural homologies, and at this time probably few biological events would be more generally welcomed than the presentation of incontrovertible evidence as to the human homologue of the carnivoral Fissura cruciata, or the representative of the human centralis (Fissure of Rolando) in the cat and dog.

$\S 1369$. The following are sufficient general examples of the difficulties which surround the subject and of the differences of opinion among high authorities :-

Gratiolet wrote $(\mathrm{A}, 10)$ :-

"We need only compare the brain of an ape with that of a carnivore or a ruminant to see that the convolutious present very dissimilar general arrangements in the several orders of Mammalia. These differences are so great that it would be imprudent to establish correspouding subdivisions and to investigate their homologies. In fact, this question has as yet no basis of certainty, and we think that for the present it should not be undertaken."

Owen says ( $\mathbf{2 5}$ ) that the same names apply to the fissures of the Aye-aye and the cat, while the very next paper in the volume of the Zoological Transactions contains the admission of Flower (6) that, as between the Lemurs and the Carnivora, the "nomenclature utterly fails."

$\S 1370$. Special examples of the diversity of opinion are furnished by the two fissures already named, the cruciata or "frontal" of the cat and dog and the centralis of man.

The centralis is homologized with the superorbitnlis by Duval and Keller (A, 57, note), and apparently by Broca; Hitzig (A, 136, 137, Fig. 10, 11) makes it equivalent to the ansata together with a part of the sippersylviana, a view which derives some support from the occasional interruption of the human centralis; it is the homologue of the coronalis in the opinion of Owen (A, III, 130), Meynert (1), and Pansch $(1,47)$. In an earlier paper, however, Pansch regarded the centralis as homologous with the oruciata, and this is the opinion of Ferrier and Clevenger.

The cruciate fissure of the Carnivora is said by Ferrier (A, 199) to be experimentally the equivalent of the centralis, and Clevenger $(\mathcal{Z}, 14)$ states that the two fissures are " his- 
tologically as well as physiologically analogous," although in a previous paper $(\boldsymbol{I}, 24)$ he had declared that " anatomically, the crucial and Rolandic are not capable of comparison ;" Lussana and Lemoigne (A, Fig. 75) make the cruciata equivalent to the calloso-marginalis of man, while Duval and Keller (Huguenin, A, 57, note) consider it as "l'analogue du sillon perpendiculaire externe ou sillon occipital de l'homme;" Broca $(1,407)$ is sure that the cruciata is not the representative of the centralis, and its existence with the Primates is denied altogether by Hitzig (A, 430) and Meynert $(1,659$, note).

After having followed up all the clues at our disposal, and spent upon this single matter more time than we supposed would be required for the elucidation of the gross anatomy of the entire brain, we are forced to admit our inability to satisfy ourselves completely with respect to the homology of the carnivoral fissures with those of man, excepting of course the hypocampal and callosal, which have never presented any difficulty on account of their relation to structural features; as to the existence of a "lambdoidal " or " occipito-parietal " fissure in the cat, see $\$ 1834$ (20).

So long, indeed, as any donbt exists with regard to the correspondence of the fissures of the cat, seal and raccoon, and of man, Macacus and Lemur, it is hardly to be expected that the homology between the members of the two groups should be altogether clear.

$\$ 1371$. The following lines of inquiry seem likely to be most productive of results :-

(1) Numerous and careful preparations and drawings should be made of the brains of all Carnivora and monkeys, especially of the young. The same should be done for peculiar foetal and adult human brains.

(2) Between the ordinary Carnivora and the monkeys are two groups whose brains should be studied with especial care : the seals have a rudimentary postcornu and occipital lobe, and these parts are said to be well developed in the Lemurs, which have affinities with both the Carnivora and the Primates.

Respecting the brains of the lower Vertebrates, see Appendix, $§ 1455$. 


\section{CHAPTER XI.}

THE CRANIAL NERVES AND ORGANS OF SENSE.

GENERAL CONSTDERATIONS-CLASSIFICATION OF THE CRANLAL NERVES-TABLE OF THE CRANIAL NERVES-SEIN-TONGDE-NOSE-EYE AND ITS APPENDAGES-EAR.

$\S 1372$. The Cranial Nerves-General Considerations.-As briefly described in $\$ \S 996,1007$, and shown in Fig. 104, 109, most of the nerves of the trunk and limbs are connected with the myelon by two sets of roots which respectively emerge from its dorso-lateral and ventro-lateral aspects opposite the dorsal and ventral cornua of the cinerea. The dorsal roots bear each a ganglion, and they are sensory, while the ventral have no ganglion (Fig. 109) and are motor.

Each dorsal root is joined by a corresponding ventral root, and the trunk so formed has both motor and sensory functions.

The roots and trunks vary in size and in the number of their rootlets and branches; they vary also to some extent in their relations with the sympathic nerves and the viscera. Upon the whole, however, they form a series all the members of which are readily and quite closely comparable.

$\S 1373$. But the cranial nerves (which either arise from the encephalon or eventually escape through cranial foramina) present no such simplicity in any animals, and in man and most mammals their irregularities in origin and distribution are so great that the older anatomists seem not to have attempted any comparison with the myelonal type. Their functions likewise were imperfectly known, and they were therefore numbered in order, beginning at the cephalic end of the brain, and their names referred mainly to their anatomical connections. 
§ 1374. TABLE OF THE SYNONYMS OF THE CRANIAL NERVES.

\begin{tabular}{|c|c|c|c|}
\hline sơmmering. & Technical names berein adopted. & Synonyms. & Willis. \\
\hline 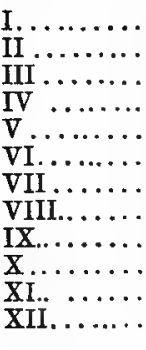 & 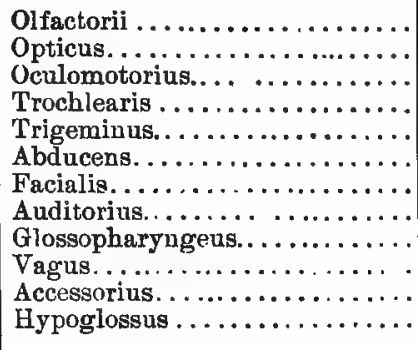 & 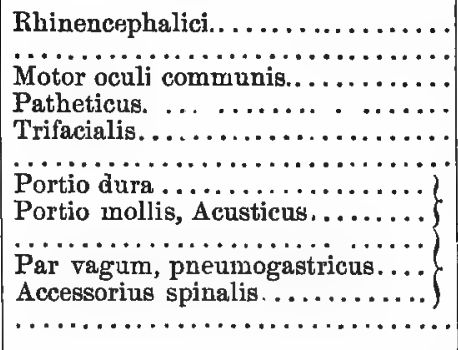 & $\begin{array}{l}\text { I. } \\
\text { II. } \\
\text { III. } \\
\text { IV. } \\
\text { V. } \\
\text { VI. } \\
\text { VII. }\end{array}$ \\
\hline
\end{tabular}

\$1375. Designation of the Cranial Nerves by Numbers. Among the older anatomists (as may be seen from Vicq d'Azyr, A, "Explication," 48-50), the cranial nerves were variously enumerated. At the present day only two methods are commonly employed, those of Sömmering and Willis. As indicated upon the accompanying Table, the difference between these two concerns only half of the twelve. The 7th and 8th of Sömmering constitute the Portio dura and the Portio mollis of Willis's 7th; the 9th, 10th and 11th of Sömmering are included in the 8th of Willis, and the 12 th of the former represents the 9 th of the latter.

Fortunately, the nerve most often concerned in medicine and surgery is the 5th, the seat of toothache and most other forms of facial neuralgia. Upon the whole, it would be better to abandon the use of the numbers altogether and employ only the technical names here given, with, perhaps, the substitution of the shorter word acusticus for auditorius. Nevertheless, in the Descriptions ( $\$ 1380-1391$ ) and in the Table (p. 520), the numerical order is followed for convenience of reference.

$\S$ 1376. Arrangement of the Cranial Nerves.-These nerves have been variously classified in accordance with physiological or morphological facts and theories.

The following Table exhibits the provisional physiological arrangement which was outlined by Wyman $(\mathbf{3 4}, 40)$ and has been elaborated by Dalton (A, 447). 
§ 1377. PROVISIONAL PHYSIOLOGICAL ARRANGEMENT OF THE CRANIAL NERVES. (Slightly altered from Dalton, A, 447.)

A. Nerves of Special Senge, lacking Graneral Sensibixity.
I. Olfactorii.
2. Opticus.
3. Anditorius.
B. Motor and Sensory Nerfes comparable with Mrelonal Nerves.

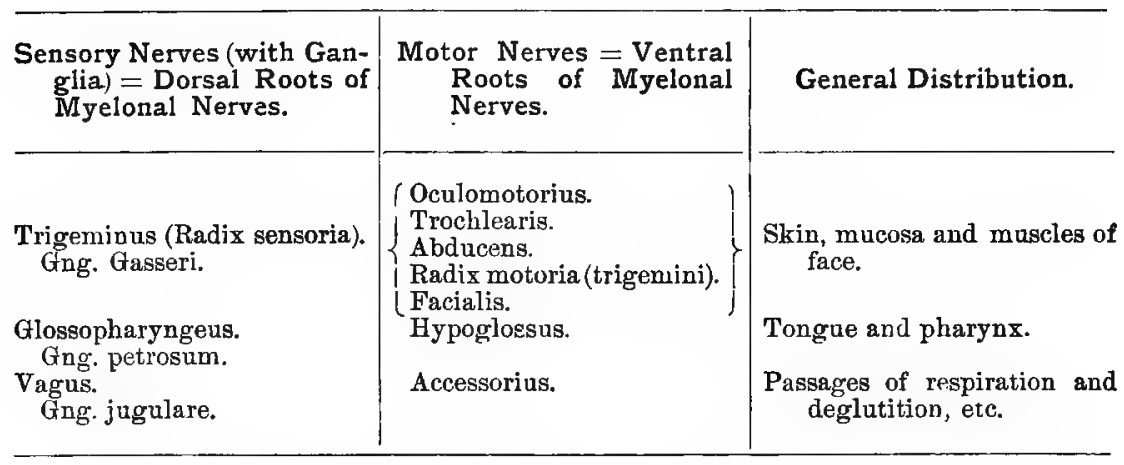

$\S 1378$. Dalton recognizes two great divisions, the nerves of special sense and ordinary motor and sensory nerves. Since some of the latter also possess special sensibility, the former may perhaps be characterized as nerves of special sense which lack general sensibility and have no ganglia.

The other and larger division includes nerves which are anatomically and physiologically distinguishable into two groups, corresponding respectively to the dorsal and ventral roots of myelonal nerves. Three of these bear each a ganglion, like that upon the dorsal root of a myelonal nerve, and are, at their origin, exclusively sensitive. The others apparently lack ganglia and are functionally motor.

But while the foregoing Arrangement is at least convenient and serves to impress upon the mind of the student the probability that, as the "medulla" is a modification of the myelon, so some at least of the cranial nerves are modifications of the myelonal type, yet sight should not be lost of certain ascertained or probable facts of Embryology and Comparative Anatomy which are not in full accord therewith.

These facts and considerations are admitted by Wyman and Dalton, and others are presented in the compendiums of Huxley, A, 66-71, 
Gegenbaur (Lankester), A, 515-522, and Balfour, A, II, 374-383, and in the papers there cited. See also A. M. Marshall (4).

$\S 1379$. In the following brief descriptions of the cranial nerves, only the ectal or superficial or apparent origins are given. Their ental (real or deep) origins in man are presented briefly in Gray and Quain, and more fully in Meynert (Stricker, A, 727-751), and in special papers.

$\S 1380$. (I) Nervi olfactorii, $N$. ol., the olfactory nerves.-Fig. $110 ; \S 1160$. Gray, A, 620 ; Quain, A, I, 526.

The true olfactory nerves of the cat and man are soft fibrous fasciculi which pass from the surface of the pero through the olfactory foramina of the cribriform plate of the ethmoid bone (Fig. 60,88), to be distributed to the nasal mucosa ( $\$ 1398$, membrana Schneideriana).

On uccount of the small size of the Lobus olfactorius in man, it was formerly regarded as a nerve, and is still often so called. As shown both by development and by comparison with the lower animals, it is really a protrusion or lobe of the brain.

$\S 1381$. (II) Nervus opticus, $N$. op., the optic nerve.-Fig. 110, 116, 117; P1. I, Fig. 2 ; Pl. II, Fig. 3, 4 ; Pl. III, Fig. 5 ; Pl. IV, Fig. 16, 18, 19. Gray, A, 628 ; Quain, A, I, 527.

Each optic nerve is a cylindrical white cord springing from the side of the chiasma ( $\$ 1202$ ) and passing through the optic foramen (Fig. 57, $F m$. op.) to the eyeball.

The optic nerves are formed by protrusions of the primitive diencephalon, and are hence, like the olfactory lobes, regarded by Gegenbaur (Lankester), A, 515, as prolonga. tions of the brain.

The cavity is obliterated, and in man the fibers constituting the nerve have been traced not only to the thalami and geniculata, but also to the optici. We lave not traced them carefully in the cat.

$\$ 1382$. (III) Nervus oculomotorius, $N$. ocm., the oculomotor nerve.-Fig. 116; PI. II, Fig. 3. Gray, A, 640 ; Quain, A, I, 528.

The trunk of this nerve is cylindrical and about $1 \mathrm{~mm}$. in diameter. It arises, slightly flattened, from the Area intercruralis, about $2 \mathrm{~mm}$. from the meson and just caudad of the cimbia ( $\$ 1203$ ), is closely associated with the ophthalmic division of the N. trigeminus, and emerges therewith by the Fm. lacerum anterius to be distributed to all the muscles of the eyeball which are not supplied by the trochlearis and abducens; it goes also to the levator palpebræ dorsalis muscle.

\& 1383. (IV) Nervus trochlearis, $N$.tr. the trochlear or patheticus nerve.-Fig. 116 ; Pl. I, Fig. 2 ; Pl. II, Fig. 3 ; Pl. III, Fig. $9 . \quad$ Gray, A, 641 ; Quain, A, I, 519.

This, the smallest of the cranial nerves, arises from the valvula by three fasciculi. The trunk is involved in the pia and easily torn away therewith.

It passes laterad and then ventro-cephalad between the cerebellum and the hemisphere, associates itself with the ophthalmic division of the trigeminus, and emerges therewith by the Fm. lacerum anterius to supply the M. trochlearis ("obliquus superior"). According to the Thesis of C. E. Manierre, this nerve enters the ocular aspect of the muscle in the cat, while in man it enters the orbital or ectal aspect.

$\$$ 1384. (V) Nervus trigeminus, $N$. trg., the trigeminal or trifacial nerve,-Fig. 116; P1. II, Fig. 3. Gray, A, 647 ; Quain, A, I, 532.

This is the largest of the cranial nerves and peculiar in several respects. Althongh 
commonly described as a single nerve, it really consists of two, the larger being sensory (Radix sensoria) and the smaller motor (Radix motoria). It thus conforms to a myelonal nerve, excepting that the ectal origins of the two roots are closely associated.

The sensory root is a large, slightly fattened band which lies across the pons just where it is contracted to form the medipedunculus. When lifted from the pons, it is found to have its ectal origin either just caudad of it or from its surface close to the caudal border.

In the senior author's paper $(\boldsymbol{1 4}, 548)$, the nerve is simply said to arise caudad of the pons. This statement was questioned in the Am. Jour. of Neurology, etc. (I, 103); a reëxamination of several preparations shows the existence of the variation above indicated, and a qualification has been published (Wilder, $2 \mathbf{2 4}$ ).

In man the ectal origin is through the pons nearer the cephalic than the caudal margin. In part, at least, the difference is due to the greater caudal extension of the human pons $(\$ 1161)$.

At the cephalic border of the pons the root presents a large flattened ganglion (Gng. Gasseri) and then separates into three divisions - the ophthalmic, maxillary and mandibular. Of these, the first is the most mesal in position and the smallest, but with it are associated the oculomotorius, trochlearis and abducens, all emerging together from the $\mathbf{F m}$. lacerum anterius.

The maxillary division is intermediate and escapes by the $\mathrm{Fm}$. rotundum. The mandibular division is lateral, is joined by the Radix motoria and emerges by the Fm. ovale.

The distribution is stated upon the Table $(\$ 1392)$, and corresponds in the main with the three regions of the face; some filaments also supply the dura.

The motor root (Radix motoria) is much smaller than the sensory and not easily recognized. Upon a fresh brain it may be seen as a narrow, Jight band crossing the sensory root and the ganglion from the mesal side of the former, to attach itself to the mandibular division. Upon an alcoholic brain, a dull tracer may be used to isolate it in the middle of its course and follow it to its origin mesad aud slightly cephalad of that of the sensory root. We have not ascertained whether, as in man, it is separated therefrom by a few fibers of the pons or of the trapezium.

In man the motor root is distributed to the muscles concerned in mastication (Dalton, A, 464).

$\S 1385$. Nervus abducens, $N$. abd., the abducens nerve.-Fig. 116 ; PI. II, Fig. 3. Gray, A, 641 ; Quain, A, I, 519

A ribbon-shaped nerve, arising by three or four funiculi from the trapeziun just laterad of the pyramis. The attachment is very feeble and the nerve is apt to be torn off with the pia. It associates itself with the ophthalmic division of the trigeminus and emerges therewith by the $\mathrm{Fm}$. lacerum anterius to be distributed to the MM. choanoidei and the rectus lateralis (" externus").

$\S 1386$. (VII) Nervus facialis, $N$. $f$., the facial nerve or " portio dura."-Fig. 116 : Pl. II, Fig. 3. Gray, A, 642 ; Quain, A, I, 548.

In some respects this is the motor counterpart of the trigeminus, being distributed to most of the muscles of the face and head, excepting the muscles of mastication. It is small as compared with the trigeminus, just caudad of which it arises from the laterocephalic angle of the trapezium. It traverses the ental auditory foramen, the Aquæductus Fallopii and the stylo-mastoid foramen ( $\$ 506)$.

$\S 1387$. (VIII) Nervus auditorius, $N$. au., the auditory or acoustic nerve or portio mollis.-Fig. 110, 116 ; Pl. II, Fig. 3. Gray. A, 639 ; Quain, A, I, 548.

This is commonly grouped with the olfactory and optic nerves as a nerve of special 
sense; but according to Gegenbaur (Lankester), A, 515, it is developed like an ordinary cranial nerve.

It springs from the cephalic part of the Eminentia auditoria, traverses the ental auditory foramen, and is distributed to the sensory organs of the labyrinth or "internal " ear.

$\$ 1388$. (IX) Nervus glossopharyngeus, $N$. gph., the glossopharyngeal nerve.-Fig. 116 ; P1. II, Fig. 3. Gray, A, 658; Quain, A, I, 554 ; Stowell, 1.

The origins of this nerve and of the vagus and accessorius are associated so as to form a series extending caudad from the Eminentia auditoria for a considerable distance along the lateral aspect of the cervical myelon. We have not examined these origins in detail, and the reader is referred to the figures and descriptions of Stowell. As has been stated elsewhere, the points of origin cannot be defined accurately until the ectal features of the "medulla" are more satisfactorily understood.

The nerve has a ganglion (Gng. petrosum), emerges through the Fm. jugulare, and is distributed, in man, to the base of the tongue, the soft palate and the pharynx.

$\S 1389$. (X) Nervus vagus, $N$. v, the vagus or pneumogastric nerve.-Fig. 116 ; P1. II, Fig. 3. Gray, A, 660 ; Quain, A, I. 556 ; Stowell, 1.

This nerve, remarkable alike for its distribution, its accessions from other (motor) sources, and its numerons and peculiar functions, arises just caudad of the glossopharyngeus by several funiculi, which, according to Stowell, form two series, dorsal and ventral. As stated under the glossopharyngeal, we have not fully examined the origin, and the student is referred to the figures and descriptions of Stowell.

The nerve presents a ganglion (Gng. jugulare, ganglion of the root), in the proximal end of the foramen of exit, and about $15 \mathrm{~mm}$. peripherad of the foramen another, the Gug. inferius or the ganglion of the trunk (Fig. 107; Stowell, 1).

Relations of the Ganglia.-The Gng. jugularis is connected by anastomotic filaments with the N. facialis (VII), glossopharyngeus (IX), accessorius (XI) and sympathicus. The ganglion of the root is connected with the $\mathrm{N}$. glossopharyngeus (IX), accessoriug (XI), hypoglossus (XII), and sympathicus; (Stowell, I).

Besides the connections just named, the vagus furnishes the following branches: NN. pharyngeus, laryngeus superior, laryngeus recurrens, rami cardiaci, rami pulmonares, gastricus dorsalis and gastricus ventralis (Fig. 103, 107; $\$ \$ 1040,1041$ ). It also gives many anastomotic filaments to the sympathicus ( $\$ 1041$; Stowell, 1 ).

$\$ 1390$. (XI) Nervus accessorius, $N$. $\alpha c$., the accessory or " spinal accessory " nerve.Fig. 116 ; Pl. II, Fig. 3. Gray, A, 686 ; Quain, A, I, 564; Stowell, 1.

This nerve has a peculiar and extensive origin by funiculi scattered along the lateral aspect of the metencephalon and cervical myelon, from just caudad of the origin of the vagus to a point opposite the 6 th or 7 th cervical nerve.

The trunk enters the cranium by the Fm. magnum, and then associates itself with the vagus and glossopharyngeus to emerge through the Fm. jugulare. In man it is distributed to the trapezius and sterno-mastoid muscles, and also, by the fibers which join the vagus, to the heart.

$\$ 1391$. (XII) Nervus hypoglossus, N. hpg., the hypoglossal nerve.-Fig. 116 ; Pl. II, Fig. 3. Gray, A, 646 ; Quain, A, I, 565.

Arises by several (10-15) funiculi from the ventral aspect of the metencephalon just laterad of the caudal half of the Area elliptica (oliva?). (The difficulty in the homology of this area is referred to in $\$ \$ 1161,1188$.)

The funiculi are very slightly attached and apt to be torn off with the pia. The trunk escapes through the Fm. condylare, and is distributed, in man, chiefly to the muscles of the tongue.

This nerve has much the aspect of the ventral root of a myelonal nerve. 
获要

के

范

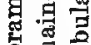

㝬

品

붕

案然

욥 원

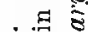

安苋

进

压 $>$ S

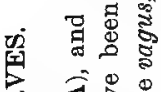

ड芒

명 के

业总总总

㻤

过

ᄃㄷㅇ 密

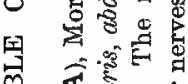

य

F 즁

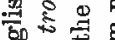

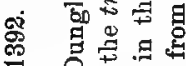

$\cos$ 岁它责 응 穹晨

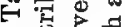
总焉 몽 峜

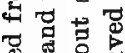
题 哓 密 . 穼 空灵 曲 농 E 雨瓷 路융 近些能

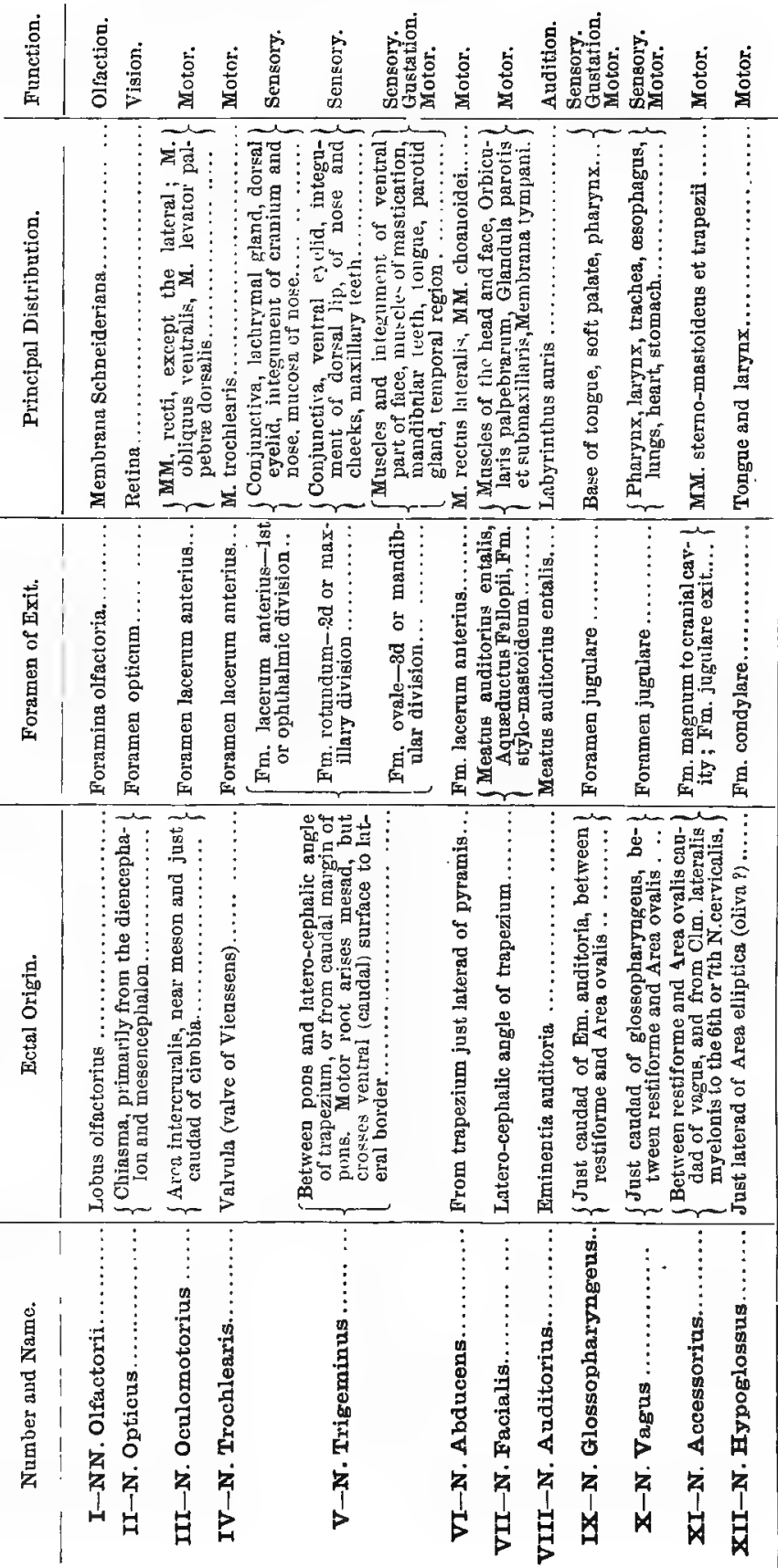


\& 1393. The demonstration of the cranial nerves requires much care and skill on account of the great number crowded into small space, and also because they pass through various bony canals and foramina or are concealed by bony processes. The beginner can hardly hope to demonstrate all satisfactorily on one specimen. It is especially necessary to employ a young lean animal, in order that fat may not obscure the nerves and that the connective tissue may not be so tough as to render the tracing of fine nerves impossible. The general directions for dissecting nerves given in $\$ \S 1008,1037$, should be faithfully followed. Some of the nerves may be traced on one side and some on the other. Whenever a saw is employed, it is best to protect underlying parts if possible by a cloth. In case a vessel or nerve is inadvertently severed, the two ends may be slightly lapped and tied with a thread. The nippers employed should be sharp and narrow pointed (\$146). In using the nippers, remove very small pieces, and use the tracer often to make sure that all branches are pushed aside. Have at hand for constant reference a prepared skull, the figures of the skull (Fig. 56-62), and the Tables of foramina $(\$ 562)$ and nerves $(\$ 1392)$.

\section{THE ORGANS OF SENSE.}

$\$ 1394$. The organs of sense are the specialized parts of animals which, being acted upon by objects in the external world, are capable of transmitting the impressions so received to the central nervous system by means of nervous connections. Huxley, $\boldsymbol{5}$.

The organs are five, corresponding to the five senses.

(A) Cutis and mucosa.-The skin, and mucous membranes near the exterior (Dalton, A, 510). These are the organs of Touch (taction, tactile sensibility).

(B) Iingua (tongue), Soft Palate and Fauces.-These are the organs of Toste (gustation, gustatory sensibility).

(C) Nasus, nose.-Its mucous membrane, especially that of the maxillo-turbinals, forms the organ of Smell (olfaction, olfactory sensibility).

(D) Oculus, eye.-The organ of Vision (sight or visual sensibility).

(E) Auris, ear.-The organ of Hearing (audition or auditory sensibility).

(F) General Sensibility.-In addition to the special sensibilities 
just named, requiring a special apparatus, there is the "so called general sensibility of the body. 'This is manifested whenever an ordinary sensory nerve is stimulated in any part of its course; when slight, it is called feeling; when more intense, pain.

\section{CUTIS-THE SKIN.}

References.-Gray, A, 83 ; Quain, A, II, 211; Dalton, A, 510; Foster and Langley, A, 165 ; Foster, A, 589 ; Leyh, A, 350; Stricker, A, 792 ; Chauveau, A, 841 ; Chauveau (Fleming), A, 792 ; Owen, A, III, 186, 610 ; Gurlt, A, 815 ; Milne-Edwards, A, XI, 411; Bernstein, A, 10 ; Flint, A, 381, 751.

\$1395. Cutis, skin.-The skin or integument forms the covering of the entire body. It is elastic and flexible, tough and dense, hence well adapted as a protecting envelope. It is continuous with the mucous membranes at the various natural orifices.

$\S 1396$. The skin is composed of two layers, ectal and ental.

Ectal Layer, Epidermis, Cuticle.-Composed of nucleated cells, those nearest the surface are flat; the deeper ones are spherical or columnar and form the Rete mucosum, which contains the coloring matter of the skin.

The function of the epidermis is almost wholly protective, and it is devoid of sensibility.

Ental Layer, Derma, Corium, Cutis vera (true skin).-The true skin is composed of elastic and white connective tissue, plain muscles, blood vessels, lymphatics and nerves. The principal function of the Cutis vera is tactile sensibility or the determination of the presence and character of objects which come in cuntact with the epidermis.

Into it are implanted the appendages of the skin-hair and claws, sweat and sebaceous glands.

The sweat glands serve to get rid of some of the waste products of the body, while the sebaceous glands pour ont upon the surface an oily substance which keeps it soft and pliable.

The hair and claws serve as protectors of the body. The hair covers the entire surface of the body except at the tip of the snout, the pads of the hands and feet and the hypothenar eminences. It protects the surface and assists more or less in touch, since a nervous filament is connected with the implanted end of each hair. In addition to the ordinary hairs forming the protective covering of the body, there are specialized hairs which, from their connections, are undoubtedly tactile organs. These are situated, in the cat, on the dorsal lip (Vibrissa, Fig. 87, 88), in the eyebrows (Fig. 87, 88), and on the antebrachium (Fig. 76, 105, pili tactiles). They differ from common hairs in being longer and stiffer, but especially in being implanted very deeply and in having a larger nervous and vascular supply.

For the structure and presence of tactile hairs in various animals, see Owen, A, III, 187 ; Curtis, L., 1, 166; Schoebl, 10 ; Ranvier, A, 913 ; Milne-Edwards, A, XI, 424 . For the structure of the skin of the dog, see Stirling, 1, 465 .

\section{LINGUA-THE TONGUE.}

References.-Gray, A, 707; Quain, A, II, 325; Dalton, A, 463, 513; Foster and Langley, A, 176 ; Foster, A, 586 ; Leyh, A, 349 ; Stricker, A, 352 ; Chauveau, A, 355, 
859 ; Chauveau (Fleming), A, 344, 813; Owen, A, III, 190 ; Gurlt, A, 344, 814 ; MilneEdwards, A, XI, 437 ; Bernstein, A, 295 ; Flint, A, 759.

$\S 1397$. Lingua, tongue.-The tongue is the principal organ of taste or gustation. It is a very movable muscular organ covered with mucosa and situated in the mouth. It is also possessed of a high degree of tactile sensibility.

By its muscular structure it takes part in the processes of mastication and deglutition and in speech. To it are distributed three nerves, the trigeminus to the tip, the glossopharyngens to the base and the hypoglossus to the muscles. The glossopharyngeus is also distributed to the mucosa of the soft palate and the pillars of the fauces; bence their mucosa possesses a certain amount of gustatory sensibility. The parts upon the tongue supposed to be the special seats of gustatory sensibility are the fungiforn and circumvallate papillob. These are briefly described in connection with Fig. 88, p. 306.

For supposed gustatory structures in the epiglottis of the dog and cat, see Schofield, 1,475 .

Demonstration.-The muscular structure of the tongue may be made out by such a section as that shown in Fig. 88. The papillæ may be seen on such a section, but better on a tongue which has been removed with the mandible. The nervous supply of the tongue may be determined by following the general directions for the dissection of nerves; see also Fig. 107, $\S 1008$, and for the vagus and sympathic $(\S 1037)$.

\section{NASUS-THE NOSE.}

References.-Gray, A, 710 ; Quain, A, II, 664; Dalton, A, 517 ; Foster and Langley, A, 176 ; Foster, A, 584 ; Hyrtl, A, 385 ; Leyh, A, 349 ; Stricker, A, 792 ; Chauveau, A, 466, 862 ; Chauveau (Fleming), A, 439, 815 ; Owen, A, III, 204 ; Gurlt, A, 814 ; MilneEdwards, A, XI, 453 ; Bernstein, A, 285 ; Fint, A, 754.

$\$ 1398$. Nasus, nose.-In Fig. 69 and 88 are shown longitudinal sections of the nasal passages. It will be seen, especially in Fig. 88, that there is a tolerably direct passage from the pronaris to the postnaris through the so called meatus ventralis (inferior). Dorsad of the meatus ventralis are the turbinated bones which are most intricately convoluted.

The membrana Schneideriana is the mucosa of the nasal fossæ. Through the comparatively minute spaces formed by the scrolls of the turbinated bones, the air may pass from the præ- to the postnaris, but its movement is much slower than when passing through the meatus ventralis. The forms of the turbinated bones and the passages through them may be well seen by transecting a cat's head just cephalad of the mesal capthi of tixe eyes ( $\S 1400$ ). Through the lamina cribrosa (Fig. 60) pass the olfactory nerves to be distributed to the mucosa upon the ethmo-turbinals; this mucosa is the seat of the olfactory sensibility proper, that is, of the appreciation and distinction of perfumes and odors. The mucosa of the maxillo-turbinals and meatus ventralis is supplied by nervous filaments of the nasal branch of the 1st or opththalmic division of the trigeminus and possesses sensibility more like that of the skin. It takes cognizance of the pungent vapors of such substances as anmonia.

The nervous supply of the nose may be determined by following the general directions for the dissection of nerves $(\$ 1008)$. 


\section{OCULUS-THE EYE AND ITS APPENDAGES.}

General References.-Gray, A, 713 ; Quain, A, II, 583 ; Dalton, A, 519; Foster and Langley, A, 178, 244 ; Hyrtl, A, 391 ; Leyh, A, 323 ; Straus-Durckheim, A, 201 ; Stricker, A, 802 ; Morrell, A, 260 : Chauveau, A, 862 ; Chauveau (Fleming), A, 817 ; Owen, A, III, 246 ; Foster, A, 510 ; Gurlt, A, 775 ; Milne-Edwards, A, XII, 94 ; Bernstein, A, 48 ; Flint, A ; Le Conte, A.

Instruments and Material.-Scalpel; arthrotome; fine and coarse scissors; fine and coarse forceps ; tracer ; flexible blow-pipe ; nippers; tripod lens; beaded bristles ; skull prepared as in Fig. 56.

$\S 1399$. The eye is the organ of the sense of sight $(\S 1394, \mathbf{D})$. It is situated in the orbital fossa (Fig. 56), which in the cat is incomplete.

In connection with the eye proper are certain appendages which protect, lubricate and move the eyeball.

\section{APPENDAGES OF THE EYE.}

$\S 1400$. Palpebræ, eyelids. -The eyelids are modified folds of skin which protect the cephalic surface of the eye. There are two for each eye, which from their position are called respectively the dorsal (upper) and ventral (lower) lid. Their ectal surface is covered with hair, but there are no eyelashes ; their ental surface, that next the eyeball, is lined with a smooth mucous membrane, the conjunctiva (Fig. 126, enjet.).

The two points where the eyelids meet are called the canthi or angles of the lids; a mesal (inner) or nasal and a lateral (outer) or temporal canthus.

Dissection of the Lids.-Make an incision through the skin from the angle of the mouth to a point opposite the base of the ear, and then transversely to the dorsimeson. Then make an incision from the lateral canthus directly caudad to the transverse incision. Reflect the ventral eyelid and the skin nearly to the mesal canthus, noting that the smooth mucous membrane (conjunctiva) lining the lid is continuous with the covering of the eyeball (Fig. 126).

$\S 1401$. Meibomian Glands.-Grasp the free edges of the lids and evert them. On the mucous membrane there will be seen many parallel, broad, yellowish lines, extending from the edge of the eyelid for about $2 \mathrm{~mm}$. (Fig. 126). These are the Meibomian glands. They secrete a sebaceous substance which is poured ont upon the edges of the lids through minute orifices; these may be seen at the top of slight elevations by employing a tripod magnifier. 
§ 1402. Membrana nictitans, third eyelid (Fig. 126). - The third eyelid is a fibrous, crescent-shaped organ situated at the mesal canthus between the eyelids proper and the eyeball. Both of its surfaces are covered by a continuation of the conjunctiva. Its cephalic edge is free and dark bordered, its caudal edge is flexibly attached to the eyeball (Fig. 126).

The office of the membrana is to keep the eye free from dust. The generalization made by Chaureau, $\mathbf{A}$, is, "That it is most developed in animals that are unable to use their cephalic limbs for removing foreign particles from the eye."

Demonstration.-The movement of the membrana depends upon that of the eyeball, not upon the action of special muscles. There are, however, a few striated muscular fibers in the band of connective tissue passing from the rectus ventralis to the membrana as shown in Fig. 126. If the membrana is not visible, press upon the cornea so as to force the eyeball farther into the orbital fossa, and it will appear. It may be made to entirely cover the cornea. To cause it to disappear, cut the masseter muscle and force a scalpel handle into the orbital fossa so as to push the eyeball cephalad.

$\S 1403$. Lachrymal Apparatus.-This consists of the lachrymal or tear glands and their ducts. The glands are, in general structure, like the salivary glands (\$8 788, 789). They are situated near the lateral canthi of the lids.

Demonstration.-(A) Glandula lachrymalis, lachrymal gland proper.-Nip the orbital process of the frontal and malar bones, cut the soft parts connecting the malar process, and turn the end dorsad. The lachrymal gland will cling to the reflected part. It appears like the molar gland ( $\$ 785)$, and is so formed as to mold itself to the eyeball upon which it naturally rests.

(B) Glandula Harderi.-Harder's gland, situated on the convex or ectal surface of the membrana nictitans, extends from near its attached border over about one third its width (Fig. 126). It is very apparent after the removal of the eyeball $(\$ 1415)$.

It is found only in animals possessing the third eyelid (MilneEdwards, A, XII, 121).

Lachrymal Canal.-On the free edge of each eyelid, about 3 $\mathrm{mm}$. from the nasal canthus, is the opening of a lachrymal canal. These canals collect the tears and convey them to the lachrymal duct.

Demonstration.-The openings of the lachrymal canals may 
be seen easily by drawing the eyelids well apart and looking with a magnifier at their free edges near the mesal canthus. Insert a beaded bristle into each canal.

Lachrymal Duct.-This is formed by the union of the two canals and extends to the nasal cavity. Its beginning is somewhat dilated and is called the lachrymal sac.

Demonstration.-Slightly expand the prænaris and push the beaded bristles mentioned above until they appear at the nasal opening of the lachrymal duct. This opening is just ventrad of the cartilaginous prolongation of the maxillo-turbinal bone (at a point ventrad of the $\mathrm{M}$ of the abbreviation "Mxtrb." in Fig. 88). The opening is quite large and will readily receive the probe of the tracer. If it is desirable to trace the lachrymal duct throughout its course, a bristle should be put into it. Then the head should be hemisected, and the duct traced with nippers and arthrotome, commencing at its nasal termination.

\section{MUSCLES OF THE EYE.}

The cat's eye possesses 12 muscles, ten belonging to the eyeball (4 recti, 4 choanoid, the trochlearis and the obliquus ventralis), and 2 to the lids-orbicularis palpebrarum $(\$ 1404)$ and levator palpebrae dorsalis ( $\$ 1409)$. Besides the special muscles of the eyelids, the muscles of the face assist and modify their movements.

$\S 1404$. MI. orbicularis palpebrarum (Fig. 126, M. orb. plpbr). -This is the circular muscle surrounding both lids and serving to close them. To demonstrate it, cut either of the lids transversely. Just entad of the skin will be seen the cut ends of a thin layer of pale, striated muscular fibers. The fibers are plentifully mixed with elastic and white connective tissue.

For the M. levator palpebræ dorsalis, see $\$ 1409$.

$\S 1405$. Exposure of the Muscles of the Eyeball.-These as well as the levator palpebra are within the orbital fossa. To expose them, cut with nippers the two ends of the zygoma (Fig. 56). Grasp the orbital process with the forceps and lateriduct it. At the same time sever the soft parts close to the bone with an arthrotome, so that the zygoma can be removed. Remove the temporal and masseter muscles by grasping their cephalic edge and severing the attachments. In doing this, be very careful not to include any of the fibrous capsule of the eye. After these muscles are removed, nip away half of the mandible; then remove the part of the maxilla 
containing the molar and the last or largest præmolar tooth. This will expose the lateral aspect of the eyeball and a muscle (pterygoid) extending obliquely from the mandible to the floor of the orbit. There is also brought clearly into view the superior maxillary artery and the superior maxillary division of the 5th nerve. Both extend along the floor of the orbit laterad of the eyeball to the infraorbital foramen (Fig. 50, Fm. inf. or.), from which point they are called infraorbital artery and nerve. They should be carefully removed. Two of the muscles of the eyeball will appear, 1v. rectus ventralis, IM. rectus lateralis. Raise the eyeball somewhat with a scalpel handle and cut the pterygoid muscle at its origin in the floor of the orbit. Note that the edges of the recti muscles slightly overlap near their origin, but separate like the sepals of a flower as they extend cephalad toward the eyeball. Separate them, and trace first the Mr. rectus lateralis to its attachment on the eyeball, removing the loose fibrous substance with scissors. Do not injure the tendon of the ventral oblique (see $\$ 1406$ ).

Pass a scalpel handle entad of the free edge of the muscle, raise it and free it from the underlying tissue with a tracer. When free, raise the muscle by the scalpel handle and make it tense. It will be seen to terminate in a broad ribbon-like tendon which is inserted into the sclerotic at the caudal margin of the white zone of the eyeball (Fig. 126, Z. a.).

$\S 1406$. IM. obliquus ventralis (inferior), the ventral or inferior oblique muscle.-In clearing away the fibrous tissue from the ball to expose the lateral rectus, this muscle also will be exposed. It appears as a circular band overlapping the ventral rectus. Separate the body of the muscle from the other tissues, and lift it up with the scalpel handle as for the lateral rectus, and it will be seen to insert itself by a broad tendon along the edge and cephalad of the tendon of the lateral rectus. The tendons of these two muscles form a right angle. Raise the eyeball with a scalpel handle and trace the ventral oblique to its origin from the orbital surface of the maxilla just laterad of the Os lachrymale (just laterad of the " $h$ " of the abbreviation "O. lch." in Fig. 56).

$\S 1407$. M. rectus ventralis (inferior), (Fig. 126, M. r. vntr.).This appears on the ventral side of the eyeball. Draw it out so as to show the attachment; then dissect the body of this muscle as directed for the lateral rectus, and raise it in order to note its inser- 
tion at the caudal margin of the white zone as with the lateral rectus (Fig. 126).

\$ 1408. M. rectus dorsalis (superior), (Fig. 126, M. r. drsl.).Cut for about $1 \mathrm{~cm}$., close to the bone, the fibrous band holding the eyeball to the postorbital process of the frontal. Draw the eyeball cephalad, and the dorsal rectus will appear on the dorsal side of the eyeball. Dissect it as described for the others $(\S 1405)$, and note that its insertion is at the same level on the eyeball, as shown in Fig. 126.

A considerable band passes from the ventral rectus to the Membrana nictitans. This is composed mostly of connective tissue, but with the microscope a small number of muscular fibers may be found in it. It is thus a retractor of the Membrana nictitans.

$\S 1409$. M. levator palpebræ dorsalis (superioris).-This very thin, slender muscle may be seen by grasping some of the fibrous substance near the cornea and drawing the ball cephalo-ventrad. It is on the ectal surface of the dorsal rectus for the first fourth of its length, then it inclines mesad. Isolate it with the greatest care. About opposite the point of insertion it spreads out into a broad tendon, which is attached to the dorsal lid (Fig. 126). Grasp the edge of the dorsal lid and raise it from the ball; then pull upon the muscle, and the traction can be seen on the ental surface of the lid.

$\S 1410$. M. rectus mesalis (internus). - Divide the ventral oblique and sever the fibrous connection of the eyeball with the orbital fossa on the ventral side to a point about opposite the opening of the lachrymal canal on the dorsal lid; then evert the Membrana nictitans and draw the eyeball laterad. This will expose the mesal rectus. Its attachment to the eyeball should be determined as described in $\$ 1405$.

$\S$ 1411. IM. trochlearis $s$. obliquus dorsalis (superior).-This muscle will appear mesad of the rectus mesalis. Draw the eyeball caudad and laterad and isolate the muscle from its origin toward its insertion. When about opposite the middle of the eyeball, it merges into a slender tendon which extends to a point a little caudad and entad of the mesal canthus, where it passes through a fibro-cartilaginous ring. This ring is held somewhat loosely to the bony orbit, nearly directly opposite the origin of the ventral oblique, by a strong fibrous band about $4 \mathrm{~mm}$. long extending directly dorsad, and a slender one about $25 \mathrm{~mm}$. long attached to 
the postorbital process of the frontal (Fig. 56, Prc. po.). To demonstrate these bands, draw the eyeball laterad and pull upon the fibro-cartilaginous pulley. The tense lines show the direction of the bands and serve as guides in isolating them. Continue to draw the ball laterad and isolate the tendon of the M. trochlearis after it passes the pulley. Draw it taut and it will be seen to pass directly laterad toward the eyeball. It passes entad of the M. levator palpebræ and then expands into a thin sheet which is inserted into the eyeball at right angles to the insertion of the dorsal rectus, as the ventral oblique is inserted into the ball at right angles to the lateral rectus $(\$ 1406)$.

$\S 1412$. IMM. choanoidei, $s$. IM. choanoideus, s. MMM. recti minores, $s$. MIM. recti posteriores, $s$. MI. suspensor oculi.-These are four straight muscles like the recti proper, but smaller. They may be demonstrated by separating the recti muscles. They will be seen to alternate with the recti as they extend along the eyeball to their insertion, which is by a broad, thin tendon near the middle of the eyeball. This is true of all but the ventral (inferior) one, whose tendon, like those of the recti, is inserted into the edge of the white zone of the sclerotic (Fig. 126).

$\S 1413$. Origin of the MIuscles of the Eye.-All of the muscles of the eye described above, except the orbicularis palpebrarum $(\$ 1404)$ and the obliquus ventralis $(\$ 1406)$, arise in a circle surrounding the optic nerve at its exit from the skull, thus forming for it a muscular sheath.

The levator palpebrce dorsalis arises near the sutura frontoorbito-sphenoidea, dorsad of the origin of the dorsal rectus, which arises very near the foramen opticum.

The trochlearis is in like manner ectad of the mesal rectus.

The lateral rectus passes between the tendon of the ventral rectus and the combined tendon of the choanoid muscles to be inserted into the septum between the optic and anterior lacerated foramina.

The ventral rectus arises from the lateral and ventral aspects of the foramen lacerum anterius, and just ectad of its origin is the common tendon of the four choanoid muscles (Fig. 126).

In determining the origin of the muscles, the connections of the eyeball should be so far separated from the socket that one may work in any part of the orbital fossa without difficulty.

$\S 1414$. Action of the Muscles of the Eye.-With the recti this is probably as in man, viz, that they move the eye in the direction of the four cardinal points according to 
their attachment. The oblique muscles are so differently attached from those in man that the action could hardly be the same (Quain, A, I, 277). It would seem from the anatomical arrangement that the ventral oblique would simply rotate the eyeball laterad and the trochlearis would rotate it mesad.

$\S 1415$. The nervous supply is given in the Table of cranial nerves (\$ 1392), distribution of the $3 \mathrm{~d}, 4$ th, 6 th and 7 th nerves. The nerves may be made out on a fresh or alcoholic specimen by following the general directions for dissecting nerves in $\$ \S 1008,1037$.

\section{GLOBUS OCULI-EYEBALL.}

$\S 1416$. How to Obtain the Eyeball.-The one exposed in dissecting the muscles may be severed from the head by cutting the muscles near their middle and the optic nerve about $1 \mathrm{~cm}$. from the ball; or a fresh eye may be exposed as described for studying the muscles ( $\$ 1405)$ and then severed as just described. In case it is undesirable to injure the skull, or the eye of an ox or sheep is to be obtained, grasp the lids successively, and turning the concavity of the curved scissors toward the eyeball, sever the connection with the lids. Grasp the membrana nictitans and draw the eyeball cephalad. Keeping the concavity of the scissors toward the ball, cut its fibrous and muscular connections with the orbit; cut also the optic nerve about $1 \mathrm{~cm}$. from the ball.

After the eyeball is removed, carefully free it from all tissue except the membrana nictitans and the lateral and dorsal recti muscles. These should be left to enable one to determine the aspect of the eye.

$\S 1417$. Note the following:-(A) In form, the eyeball of the cat is spheroidal and somewhat pointed cephalad (Fig. 126). (B) The cephalic third (cornea, $\$ 1421$ ) is transparent and continuous with (C) the sclerotica ( $\$ 1421)$, which forms the rest of the ectal wall of the eyeball. (D) $N$. opticus, at the caudal part of the eyeball enters the large cylindrical optic nerve.

$\S 1418$. Iris et Pupilla (Fig. 126).-Upon looking into the cornea there will be seen a golder-yellow circular curtain, the iris. This curtain is not complete, but in the middle is an opening, the pupil. The form of the pupil in the cat is circular when fully dilated, as in a cat killed with chloroform. When partly contracted it is elliptical, but when fully contracted it is a dorso-ventral slit. These various forms are readily seen in a living cat's eye by transferring the cat from a dim into a brilliant light.

$\$ 1419$. Images Formed by the Eye.-If the eye is perfectly fresh, so that the cornea is transparent, rub some strong glycerin on 
the caudal part of the sclerotic to make it transparent; hold the eye with the cornea toward a well-lighted window or a lamp tlame. The image of the window or flame will be seen on the caudal aspect of the eyeball; the image is real, and hence inverted like that formed by a photographer's camera. Raise or lower the eyeball, and the image will be seen to move in the opposite direction. If the eye of a large animal is used for this experiment, a piece must be removed from the caudal part of the sclerotic on account of its opacity.

$\S 1420$. Tunicæ oculi-Coats of the eye (Fig. 126).-For the study of the remaining parts of the eye, a fresh one may be used, but one hardened in alcohol is desirable, as such a one retains its form and the various parts are less easily torn and displaced. To harden an eye, cut a slit in the sclerotic at one side and place the eye on absorbent cotton in 62 per cent. alcohol for a day; then remove to 95 per cent. for two days or more.

Dissection. - With forceps and scissors make an incision from about the middle of the cornea to near the optic nerve, taking care to cut only through the wall. Connect the same two points by another incision in such a way as to remove a segment containing one fourth or one fifth of the entire wall of the globe. In this segment the different tunics may be studied.

$\$ 1421$. Sclerotica et Cornea (Fig. 126).-Together these form the ectal covering or framework of the eyeball.

The sclerotic covers the caudal three fourths of the eyeball and becomes thickened before merging into the cornea. This thickening has the appearance of a white band around the eyeball, and for convenience may be called the Zona alba (Fig. 126, Z. a.) or white zone. At the caudal margin of this zone are inserted the recti muscles. Its width indicates the length of the plica ciliares (\$1422).

In the ental wall of the sclerotic are many pigment cells (Zamina fusca), giving it a dark appearance; and on the line where it merges into the cornea these pigment cells extend through to the ectal wall of the sclerotic. The cornea completes the framework of the eyeball cephalad. It is transparent and intermediate in thickness between that of the white zone and the rest of the sclerotic.

\$ 1422. Choroidea (Fig. 126). - The choroid coat of the eye is just entad of the sclerotic. It is a vascular coat, but contains also much pigment, hence its dark appearance. With a tracer separate the choroid and sclerotic as shown in Fig. 126. The choroid does 
not extend cephalad of the iris. Opposite the white zone it is folded into plaits (Plicæ ciliares, Fig. 126). There are about seventy of these plaits or folds.

$\S 1423$. Iris.-The iris, as stated above, is the circular perforated curtain caudad of the cornea. Its caudal surface is black, its cephalic a golden yellow, which gives color to the eye. It is attached at its circumference to the choroidea, the cornea and the sclerotic.

§ 1424. Retina (Fig. 126).-The retina is the ental coat or tunic of the eye. It is an expansion of the optic nerve to which are added nerve cells and various other parts (see Quain, A, II, 605). It is the sensitive part of the organ of sight. It may be separated from the choroid as shown in Fig. 126 by using a scalpel handle. Note that it is of nearly uniform thickness until it reaches the margin of the ciliary folds, Ora serrata (Fig. 126). Its extension upon the folds becomes thin and is called the Pars citiaris retina. The entrance of the optic nerve appears as a round white spot, macula lutea (blind spot).

$\S 1425$. Tapetum (Fig. 126).-In the eye of the cat, as in many other animals, the retina does not contain pigment over its whole extent, but is devoid of it in its dorso-mesal part. Here the choroid is brilliantly colored, forming the so called Tapetum. The color is metallic golden-blue green. In this part of the choroid is a deposit of mineral salts of calcium which assists in giving the luminous appearance to the cat's eye in a dim light (Milne-Edwards, XII).

$\S 1426$. Humor aqueus.-The aqueous humor is a clear watery fluid which fills the space between the cornea and the iris and lens and also the small space between the iris and the plicæ ciliares (Fig. 126). These spaces are called, from their position, Camera aquosa cephalica (anterior), cephalic or anterior aqueous chamber (Fig. 126, C. aq.), and Camera aquosa caudalis (posterior), caudal or posterior aqueous chamber (Fig. 126, C. a.).

$\S 1427$. The corpus vitreum or vitreous humor is a transparent jelly-like substance occupying the greater space of the eyeball. It is bounded cephalad by the lens and ciliary processes and at all other points by the walls of the eyeball.

§ 1428. Lens (lens crystallina)-Crystalline lens (Fig. 126). The lens is the double convex transparent body situated between the aqueous chambers and the vitreous body. Its cephalic convexity 
is greater than its caudal. In the fresh eye it is perfectly transparent, and its ectal part is soft, while its ental part is firmer. In an alcoholic eye it is hard and mostly opaque.

$\S 1429$. Capsula lentis (Fig. 126, Cpsl.).--This is the sac surrounding the lens. Grasp the cut edges of the white zone, and attempt to spread the eyeball out flat. There will be seen a tense line passing from the plicæ ciliares to the edge of the lens. At the same time look at the lens, and there will be seen enveloping it a thin transparent membrane, which is the capsule. Blow with a blowpipe (Fig. 19) against the lens where the tense line is attached, and the air will get between the lens and its capsule, thus making the latter very evident.

§ 1430. Zonula Zinnii, s. Ligamentum suspensorium (Fig. 126, Z. Z.). -This is the fibrous connection of the lens capsule with the plicæ ciliares. By pulling upon the lens capsule and the sclerotic, this suspensory ligament will be seen attaching itself to the Ciliary plica (Fig. 126).

Preparation of Fig. I26.-A cat's head was removed and frozen solid. The skin was cut with a scalpel along the dorsal surface of the head to indicate the direction of the section. Then the section was made with a fine tooth back-saw (Fig, 21). The débris was removed by carefully scraping with a scalpel, and the outlines were obtained immediately by means of a photographer's camera. The relative position, size, insertion and origin of the muscles were obtained by subsequent careful dissection on several specimens. The form of the eyeball and the relations of the parts were verified on six eyes by carefully removing and freezing them and then making sections with a watch-spring saw.

The plice ciliares (P. c.) are about 70 in number, but in order that they might be shown distinctly only a few of them were drawn. The ciliary muscle in the cat has not been sat. isfactorily worked out, hence its limits and size have not been clearly indicated in the figure. On the ventral side the retina and choroidea are shown as separated from the sclerotica and from each other. Finally, the muscles of the head closely related to the $\mathbf{M}$. orbicularis palpebrarum have not been indicated.

Description of Fig. 126.-Camera aquosa posterior (C. a.).-The posterior (caudal) aqueous chamber. It is situated between the Zonula Zinnii and the iris.

Camera aquosa anterior (C. aq.).-The anterior (cephalic) aqueous chamber. It is bounded by the lens, iris and cornea.

Canalis Schlemmii ( $\mathrm{Cn}$. Shlm.). - The canal of Schlemm in the cat is double and often triple. It is a venous sinus.

Capsula (Cps1.).-Capsule of the lens. This is an elastic sac completely inclosing the lens. To it is attached the suspensory ligament or zone of Zinn.

Choroidea, s. Tunica vasculosa. - This is the dark brown membrane composed chiefly of blood vessels and lying between the sclerotica and retina. It extends cephalad to the iris. Opposite the zona alba of the sclerotica the choroidea is plaited or folded, forming the plices ciliares or ciliary processes.

Conjunctiva (Cnjct.).-The conjunctiva is the mucosa lining the lids, covering both 


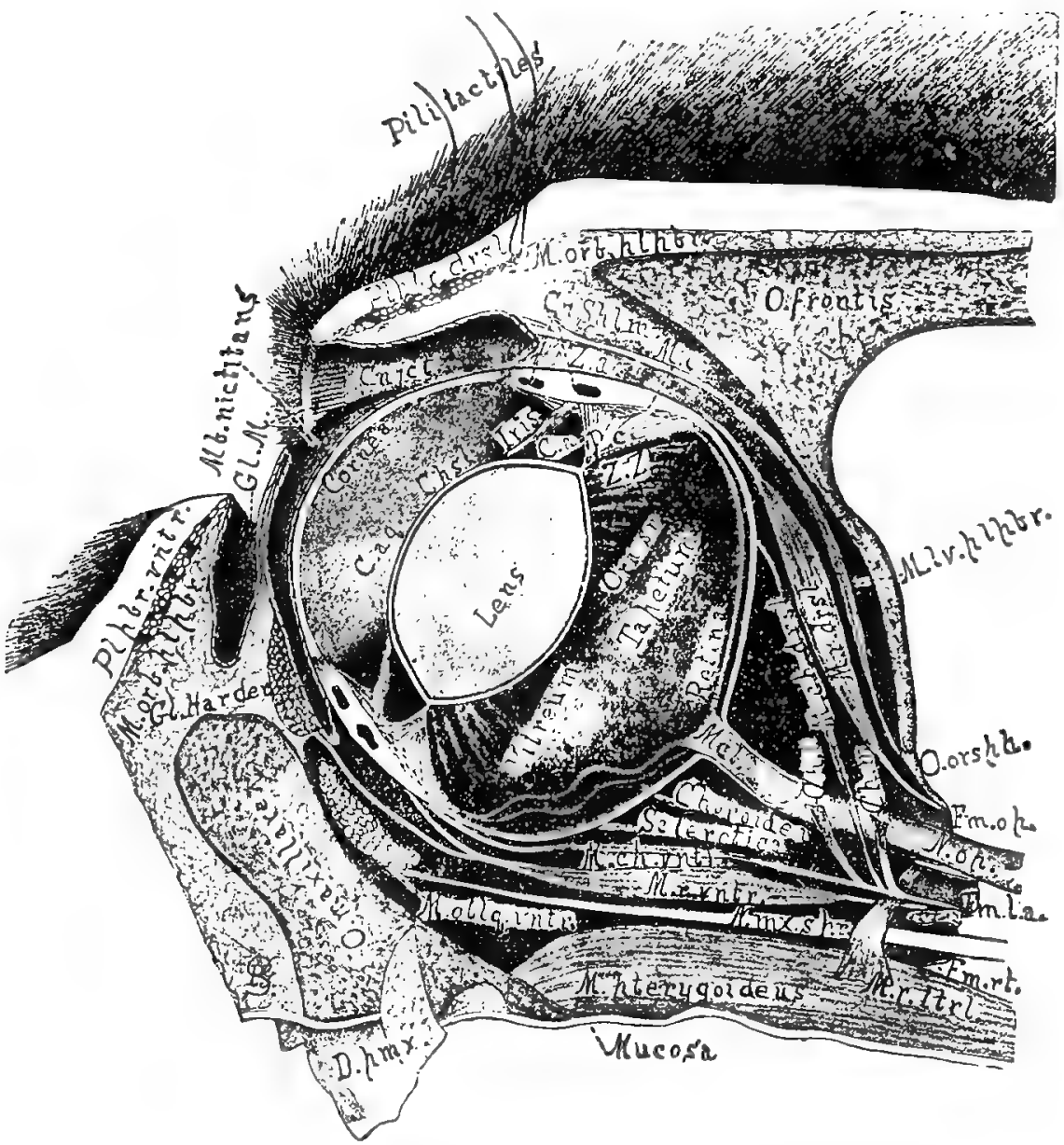

Fig. 126. -Dorbo-ventrat (Vertical) Section of the Cat's Laet Eye, in ait 0. $\times$ about 2.5.

surfaces of the membrana nictitans and the cephalic surface of the eyeball. It is indicated by oblique cross lines.

Cornea.-The transparent cephalic part of the eyeball. It is a continuation of the sclerotica. Their junction is indicated by a dar's band.

Foramen opticum (Fm. op.).-Foramen for the optie nerve (Fig, 57 , § 562).

Foramen lacerum anterius $(F \mathrm{~m}$. 1. a.) g. Fissura sphenoidalis.-A great many structures traverse this foramen ( $\$ 562$ ), and from its lateral wall arise the ventral rectus and the choanoid muscles.

Foramen rotundum (Fm. rt.), (\$ 562).

Glandula Meibomiana (Gl. M.).-The Meibomian glands (\$ 1401) pour out a seba. ceous substance on the edge of the lids. 
Glandula Harderi (G1. Harder).-A small lachrymal gland pouring out a lubricator on the ental or concave surface of the $M b$. nictitans (\$1403, B).

Iris.-This is the contractile diaphragm just cephalad of the lens. Its cut edges are shown in the figure, and it is represented as dilated to a circle $(\$ 1418)$. Its muscles are of the unstriped variety, yet they act rapidly and appear as if almost voluntary in the cat.

Lens.-The lens in the cat is double convex as in man, but unlike the human lens, that of the cat is the more convex cephalad ( $\$ 14 \% 8$ ).

Macula lutea (Mcl.).-This is the white or blind spot of the eye. It is the end of the optic nerve as it enters the eyeball.

Membrana (Mb.) nictitans. - This is the internal or third eyelid. It bas a black free border (\$1402).

Mucosa.-Mucous membrane of the roof of the mouth.

M. ciliaris (M. c.).-The ciliary muscle. This is attached to the sclerotica and choroidea. By its contraction and relaxation the lens is made more or less convex, and hence accommodates the eye for near or distant objects. The limits of this muscle bave not been satisfactorily determined in the cat.

M. levator palpebræ dorsalis (M. Iv. plpbr.).-The elevator of the dorsal (superior, upper) lid is a slender muscle. It must be greatly aided by the ectal muscles of the head.

M. rectus dorsalis (superior), (M. r. drsl.).-The dorsal straight muscle of the eye. It is cut longitudinally, hence its tendon is seen on edge.

M. rectus lateralis (externus), (M. r. 1trl.).-The muscle is cut and reflected ventrad to show its origin from the bony septum between the Fm. op. and Fm. I. a., and also that it is between the tendon of the ventral rectus and the common tendon of the choanoid muscles (\$ 1405).

M. rectus ventralis (inferior), (M. r. vntr.).-The ventral rectus has been divided longitudinally and is seen on edge. Its origin is from the lateral wall of the Fm. I. a.

M. choanoideus mesalis (M. ch. m.).-The tendon of this muscle crosses the optic nerve as shown.

M. choanoideus dorsalis (M. ch. drsl.).-This muscle and its tendon are seen on edge.

M. choanoideus lateralis (M. ch. 1.).-The tendon of this muscle is seen in its width.

M. choanoideus ventralis (M. ch. vntr.).-The ventral of the choanoid muscles. The common tendon of the choanoids seems to be a continuation of this as it goes to its origin from the $\mathrm{Fm}$. 1. a. (\$1413).

M. obliquus ventralis (inferior), (M. oblq. vntr.).-The cut end of the ventral oblique muscle (§ 1406).

M. orbicularis palpebrarum (M. orb. plpbr.).-The cut ends of this circular muscle are shown in each lid. The ectal muscles of the head mingle with this, but they are onitted from the figure.

M. pterygoideus.-This corresponds to the external pterygoid of man. It passes from the O. palatinum to the mesal side of the mandible (Straus-Durckheim, A, II, 217).

$\mathrm{N}$. maxillaris superior (N. mx. spr.). - The second division of the trigeminus nerve. Its distribution is given in the Table (\$ 1392).

N. opticus (N. op.).-The optic nerve is seen traversing the optic foramen on its way to the eyeball. It is surrounded by dura which is continuous with the sclerotica.

Ora serrata (Ora sr.).-The retina at the beginning of the ciliary plicæ becomes thin, and, as in man it is somewhat indented, it is called ora scrrata. The serrated appearance is not marked in the cat. The retina is very thin from the ora to the tips of the ciliary processes, where it ceases. This part of the retina is called the pars ciliaris retinæ (\$1424).

O. frontis. -The frontal bone ( $\$ 516)$.

O. orbito-sphenoideum ( $O$ orsph.), (\$515). 
Palpebra dorsalis.-The dorsal (superior or upper) eyelid. The doreal lid does not contain a tarsal cartilage as in man (Quain, A, II, 584), and there are no eyelashes (cilia), $(\S 1400)$.

Palpebra ventralis (Plpbr. vntr.).-The ventral (inferior) or lower lid, like the dorsal, possesses no lashes $(\$ 1400)$.

Pili tactiles.-Tactile hairs. The bulb of one is shown to indicate its great size and deep implantation (\$1396).

Plicæ ciliares (P. c.) s. processus ciliares, ciliary plicæ or processes.-They are foldings of the choroidea. The abbreviation is written on one shown in its full extent. It is approximately triangular in outline, and to its tip is attached the zonula of Zinn, and into it pass many strong bands forming part of the suspensory ligament. The folds bifurcate caudad and gradually merge into the general surface of the choroid (\$ 1422).

Retina.-The nervous tunic of the eye. It is the ental of the three coats. In the figure its cut edge is crossed by lines, and on the ventral side it is drawn away from the choroid and toward the center of the eye ( $\$ 1424)$.

Sclerotica.-This with the cornea forms the ectal coat of the eye. In the cat it is very thin except opposite the iris and ciliary plicæ. Here it is thickened and has been termed, provisionally, Zona alba ( $(1421)$.

Tapetum. -The brilliantly colored part of the ental surface of the choroidea (\$ 1425).

Vitreum.-The vitreous body or vitreous humor. It fills the entire space caudad of the lens and zonula of Zinn. It is clear and somewhat jelly-like. The canal of Petit is merely an interval between the vitreum and the zonula. As it is not supposed to exist during life, and certainly did not appear in the sections of the frozen eye, it has been omitted.

Zona alba ( $Z$. a.).-This is a thickening of the sclerotica giving firmness to the eyeball. Into its caudal edge are attached the recti muscles (\$§ 1407, 1408).

Zonula Zinnii (Z. Z.) s. Ligamentum suspensorium lentis.-The suspensory ligament in the cat is attached to the lens capsule at the edge of the lens and passes not only to the summits of the ciliary processes, but in to their substance as shown in the figure. Moreover, there is an especially strong part of the ligament opposite the summit of each ciliary process.

\section{AURIS-ORGANUM AUDITUS-EAR. (Fig. 127, 128.)}

General References.-Gray, A, 729 ; Quain, A, II, 626 ; Dalton, A, 554; Flint, A ; Foster and Langley, A, 201 ; Foster, A, 574; Hyrt1, A, 413 ; Leyh, A, 338; Stricker, A, 950; Chauveau, A, 880; Chauveau (Fleming), A, 846 ; Owen, A, III, 235 ; Gurlt, A, 79, 799 ; Milne-Edwards, A; XII, 1 ; Bernstein, A, 164.

Instruments and Material. - Watch spring saw; nippers; dissecting instruments (\$ 181) ; tripod magnifier ; 15 per cent. glycerin ; cat's bead ; macerated skull prepared as in Fig. 57.

$\S 1431$. Auris, ear.-This, the organ of hearing in the cat as in the higher animals generally, is composed of three parts, two of which (labyrinth and tympanum) are completely encased in bone.

(A) Auris ectalis (externa), ectal or external ear.-This includes the external ear, commonly so called, which is partly cartilaginous, and the meatus auditorius ectalis (externus), a partly bony canal 
leading to the membrana tympani. Both are lined with skin, which extends also over the membrana tympani.

(B) Tympanum $s$. Auris media (Fig. 127). - The middle ear is an elliptical cavity in the bone, lined with mucous membrane and containing the bones of the ear and their muscles and the chorda tympani nerve. Into it opens the Eustachian canal (Fig. 58, 88), which puts it into communication with the pharynx.

(C) Labyrinthus $s$. Auris entalis (interna), (Fig. 127).-The ental (internal) ear or labyrinth is the sentient portion of the ear. It consists of three parts, a common cavity (vestibulum), from which extend the other two-in one direction the canales semicirculares, in the other the cochlea. In the living body the cavity of the internal ear is closed and lined with a thin periosteal membrane, but in the macerated skull the fenestra rotunda and fenestra ovalis put it into communication with the tympanum.

Dissection.-Remove the head of a young cat and wash away the blood.

\$ 1432. Auris ectalis.-The external ear. The outer prominent part turns its concave surface latero-cephalad. Note its flexibility and elasticity, also the little pocket on its lateral border (Fig. 87). Spread the edges apart. Note the numerous ridges and winding ways, and that it is only partially covered by conspicuous hairs. At its latero-ectal aspect is the opening into the Meatus auditorius ectalis, the walls of which are firm and, near its termination, bony.

Remove the mandible, os hyoides, tongue and larynx, also the skin from one side, including the external ear. Then isolate the facial (7th) nerve as it emerges from the Fm. stylo-mastoideum (Fig. 57, Fm. stm.). Partially isolate also the Eustachian canal. The opening of the canal will be exposed as shown in Fig. 88, by slitting the soft palate lengthwise and turning the flaps aside. From this point the canal extends cando-laterad to the bony Eustachian tube (Fig. 58, Cn. Eu.). Remove from around the bulla (Fig. 57) everything except the Eustachian canal and the facial nerve. Cut the cartilaginous part of the meatus where it joins the bony part, but do not allow the instrument to enter the latter.

\$ 1433. IMembrana tympani (Fig. 127). - Place the head in a good light and look into the bony ectal meatus. A nearly transparent membrane will be seen, the Membrana tympani. It separates the ectal ear from the tympanum. Note the white rod (handle of the malleus) extending across its dorsal third. 
$\S$ 1434. Tympanum $s$. Auris media.-With nippers remove the wall of the mesal chamber of the tympanic bulla (Fig. 58), and note the delicate membrane lining it, also the bony septum (Fig. 58, Spt. tym.) separating this chamber from the tympanum proper except at a point directly ventrad of the fenestra rotunda, where it is notched, thus forming a free communication between the two chambers of the bulla (Fig. 58). Carefully remove the septum with nippers and use the scissors for cutting its lining membrane, so that nothing may be removed by inadvertence. Holding the head in the hand, allow the light to fall upon the tympanum from various directions. Note the attachment of the membrana tympani to a ring of bone terminating the ectal bony meatus, and that the membrane is conical in form, the apex projecting into the tympanum.

\$ 1435. Canalis Eustachiana (Fig. 58, 88). - This, as stated above (Fig. 88), is the canal connecting the pharynx and tympanum. Insert a beaded bristle into the pharyngeal opening (Fig. 88) and it will appear at the tympanic opening in the cephalic part of the tympanum, just dorsad of a projecting shelf of bone.

Ossicula auditus, Bones of the Ear (Fig. 127).--There are three of these, malleus, incus and stapes, extending in a chain from the membrana tympani to the fenestra ovalis.

$\S$ 1436. Malleus, hammer (Fig. 127).-The malleus is the first of the chain of ear bones. Its handle stretches partly across the membrana tympani. With the tracer move the handle; it will sway but little. Note that the neck and head of the malleus form an angle with the handle, and that attached to a small cylindrical process of bone arising from the mesal aspect of the neck, is the short tendon of the tensor tympani muscle (the $M$. Eustachianus of Straus-Durckheim, A, II, 200). This muscle is nearly spherical and occupies a concavity slightly cephalad of the fenestra ovalis. The fossa may be seen on a prepared skull. With scissors cut the tendon of the muscle and then the dorsal part of the bony ring supporting the membrana tympani, and carefully remove the malleus adhering to the membrana. Note the rounded head of the malleus and also the long flat process (processus gracilis) arising from the lateral aspect of the neck.

\$ 1437. Incus, anvil (Fig. 127).-This, the second of the chain of bones, resembles a molar tooth with two divergent fangs rather than an anvil (Quain, A, II, 631). To expose it, remove the cephalic part of the tympanum and the tensor tympani muscle. Examine 
under a strong light with a magnifier. Note the larger part with its concavity for articulation with the malleus and, extending ventromesad, a slender process which articulates with the stapes. Continue to use the magnifier, and with a tracer sever the connection with the stapes; then, by moving the incus slightly, it will be seen to occupy the caudal part of an elongated fossa caudo-laterad of the one occupied by the tensor tympani muscle. The shorter process (fang) is held in position by a ligament. The cephalic part of the fossa is occupied by the head of the malleus. Carefully nip away the lateral wall of the tympanum and fully expose the longer process of the incus and its ligament.

\& 1438. Stapes, stirrup (Fig. 127). - This is the third and last of the chain of bones. (The so called Os lenticulare between the stapes and incus belongs properly to the incus.) The narrow part of the stapes, termed the head, articulates with the longer process of the incus, and its broader part or base is inserted into the foramen ovale. With the tracer or forceps move the bone slightly from side to side, and there will be seen passing caudo-laterad the tendon of the MI. stapedius. Remove the lateral wall of the tympanum, the mastoid process, etc., around the Fm. stm. (Fig. 57), and the muscle will be seen to originate near the aquæductus Fallopii $(\S 506)$ entad of the 7th nerve. On the prepared skull the space occupied by the stapedius may be seen to be separated from the fossa occupied by the incus by a septum of bone. After the muscle is well made out, grasp its tendon and pull gently. The stapes will be drawn out of the fenestra ovalis. Note the small bony process on the caudal side to which is attached the stapedius muscle.

\section{J.ABYRINTHUS 8. AURIS ENTALIS (INTERNA). (Fig. 127.)}

§ 1439. Fenestra rotunda s. Fenestra cochlece; Fenestra ovalis s. Fenestra vestibuli.-These two gateways to the labyrinth have already been exposed. Note the membrane covering the fenestra cochleæ. The fenestra vestibuli was closed by the base of the stapes and its connecting soft parts. These foramina open respectively on the summit and side of the cylindrical cochlear eminence.

$\S 1440$. Cochlea.-The cochlea is situated mainly in the cylindrical elevation at the caudo-lateral aspect of which is found the Fenestra rotunda $s$. cochleæ. It consists obviously of the tapering canals (scalæ) separated by a lamina of bone (lamina spiralis, Fig. 128). These scalæ are coiled about a central piece (modiolus), some- 
thing like the shell of a snail. The large end of the canal is visible through the membrane covering the Fenestra rotunda, and, since it faces into the tympanic cavity, is called scala tympani. The other canal opens into the vestibule and is called the Scala vestibuli.

To demonstrate the parts of the cochlea, rest the head on the occiput, and with a watch-spring saw make a section across the cochlear eminence so that the Fenestra vestibuli is divided in half. If a watch-spring saw is not at hand, one may employ the nippers. Remove the fragments made by the saw or the nippers by blowing with the blowpipe. This will expose the vestibule, a cross section of the cochlea and the opening of the scala vestibuli. The appearance shown in Fig. 127 will be seen, except that the membranous part of the septum may be torn. The direction of the cochlea beyond the vestibule is nearly in a line, connecting the centers of the foramen jugulare et ovale (Fig 57), and if the ventral wall of the cochlear eminence be removed along such a line, the cochlea will be exposed and a clear view obtained both of the Lamina spi. ralis and the Modiolus or center piece around which the coils are made.

To remove the ventral wall of the cochlear eminence, press a blunt-pointed scalpel or arthrotome against the wall of the scala tympani and pry carefully. Usually it will come off without the least difficulty. The exposed cochlea will look like a cork-screw. It should be remembered that in addition to the lamina spiralis which forms a partition between the two scalæ, there will appear a complete wall of bone separating the different whorls.

In the center of the modiolus is a cavity or canal, and the lamina spiralis is perforated by many small holes, giving the appearance of a sieve, and under the tripod it is seen that through these the branches of the auditory nerve pass, to be distributed to the sentient part of the cochlea.

$\S 1441$. Canales semicirculares (Fig. 127).-There are three of these, each forming about two thirds of a circle, in the periotic bone. They are related somewhat as are related the three dimensions of a cube, and open into the vestibule in pairs. From their position, they are named as follows in man, and the terminology has been retained for the cat: external, superior and posterior. The external one (horizontal) is nearly in a dextro-sinistral plane and surrounds a small fossa nearly caudad of the fenestra ovalis. The superior one is in a dorso-ventral (vertical) transverse plane. It is 
in the ridge forming the caudal boundary of the Fossa appendicularis (Fig. 59, Fs. ap.).

The posterior semicircular canal is in a dorso-ventral (vertical) longitudinal plane, just laterad of the Fm. jgl. (Fig. 56). As stated above, the canals open into the vestibule in pairs. The opening of one end of the posterior and superior canals may be seen by looking into the vestibule. The two other openings are situated near the edge of the fenestra ovalis, one laterad and one mesad of the opening just described. The mesal one is the common opening for the posterior and external canals, while the lateral one is for the superior and external canals. These three openings are situated in a line connecting the middle of the Fm. jugulare and of the fenestra ovalis (Fig. 57, Fm. j., Ft. ov.).

To trace these canals, remove the perioticum from the rest of the skull, and, commencing at the central or common opening of the posterior and superior canals, with the nippers and arthrotome carefully remove the bony walls of the canal. To demonstrate all the canals and their openings, one should take a skull, cleaned preferably by maceration ( $\$ 250$ ), and after separating the perioticum from the rest of the skull, remove the wall from the middle of the length of the various canals ( $\$ 1441$ ), to expose them; then insert fine bristles in both directions. In this way the three openings of the canals may be found, and the ends of two bristles will be found projecting from each opening.

$\S 1442$. Aquæductus cochleæ.-This is a canal through the perioticum which transmits a vein from the scala tympani. One opening of the canal is near the fenestra rotunda and the other is just caudad of the Meatus auditorius internus (entalis). It may be readily demonstrated by inserting a bristle into the scala vestibular opening. It is mentioned so that it may not be mistaken for the opening of a semicircular canal.

For a sketch of the development of the eye and ear in the pig, see Hunt, D., 1 ; for the external ear passages, 2 ; and for the development of the middle ear, 3 .

Figure 127 was suggested by the diagram of the human ear given in Huxley and Youmans, A, 195, Fig. 82. It is meant to represent the three parts of the ear in their relative order. The first division or auris ectalis is removed, except the bony and a small part of the cartilaginous meatus.

The bones of the ear were placed as nearly as possible in their proper position and outlined with a camera lucida.

Explanation of Fig. 127.-Aquæductus cochleæ (Aq. chl.).-A passage through the petrosum for a vein from the scala tympani ( $\$ 1442$ ).

Canalis Eustachiana ( $\mathrm{Cn}$. Eu.).-The Eustachian canal opening into the cavity of the tympanum or middle ear (§ 1435).

Canalis semicircularis posterior (P.).-This canal is represented in its whole length and its opening at one end with the external and at the other with the superior canal. 


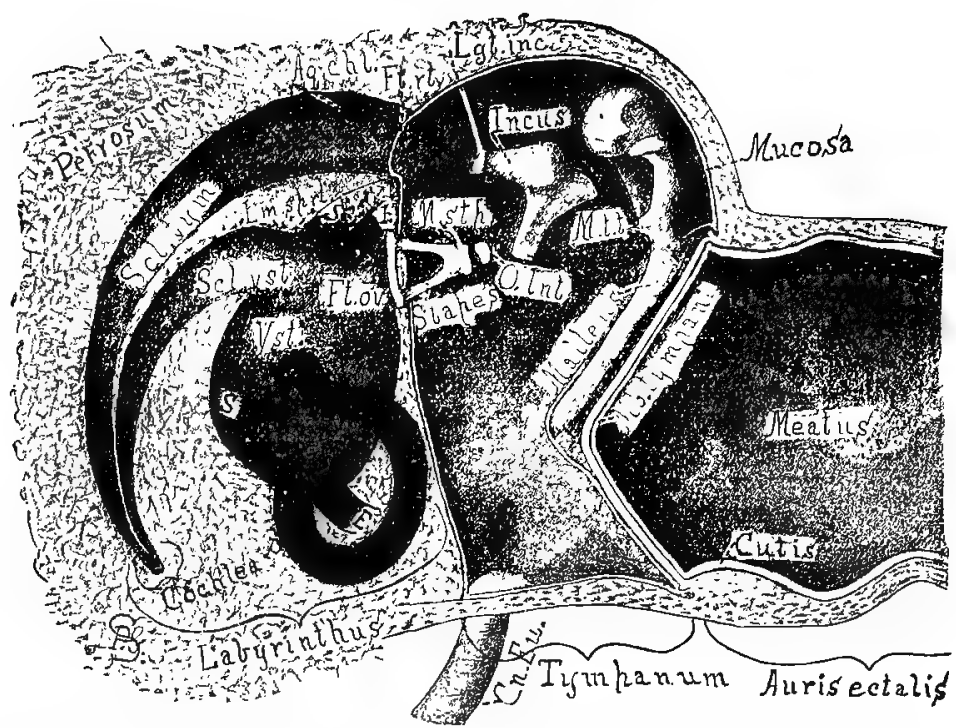

Fig. 127.-Diagram of the Cat's Right Ear, Ventral View ; x about 5.

Canalis semicircularis superior (S.).-Only the beginnings of this are shown. One of them commences in common with the posterior and one with the external canal (\$1441).

Canalis semicircularis externa (E.). Only the beginnings of this canal are shown, one of them opening with the superior and one with the posterior canal ( $\S 1441$ ).

Cochlea.-This is represented as unrolled and the ventral surface removed so as to expose the scalæ and the lamina spiralis (\$1440).

Cutis, the skin.-It is continuous over the meatus and becomes very thin as it extends over the membrana tympani to form its ectal layer.

Fenestra rotunda (Ft. rt.) s. Fenestra cochlea.-In life this is inclosed by a membrane. It leads into the scala tympani of the cochlea.

Fenestra ovalis (Ft. ov.) s. Fenestra vestibuli.-This is the passage from the tympanum to the vestibule. In life it is covered by the base of the stapes with its connecting soft parts.

Incus. -The middle bone of the ear. It is also called anvil and dens molaris ( $\$ 1437$ ).

Labyrinthus.-This is the third and last or sentient part of the ear. It is often called internal ear.

Lamina spiralis ( $\mathrm{Lm}$. spr.).-This is a plate of bone arising from the modiolus or column of the cocblea, and with its membranous continuation divides the cavity of the cochlea into the two scalæ (Fig. 128).

Ligamentum incudis (Lgt. inc.).-The ligament holding one of the processes of the incus to the wall of the tympanum.

Malleus.-The first of the small bones of the ear. It is by far the largest of the three. It gains its name from its resemblance to a bammer. Its handle stretches partly across the Mb. tympani.

Meatus.-The name is written in the meatus ectalis or passage from the exterior to the membrana tympani ( $\$ 1432)$. 
Membrana (Mb.) tympani.-The membrane is somewhat funnel-shaped as shown. It is composed of three layers, the skin or cutis (see cutis), a fibrous central or intermediate part and a continuation of the tympanic mucosa. The latter covers the handle of the malleus and helps to bind it to the tympanum, and is only partly shown.

M. stapedius (M. stp.).-The name is connected with the bony process of the stapes to which the muscle is attached (\$1438).

M. tensor tympani (M. t. t.).-There is here shown the bony process of the malleus to which the tendon of this muscle is attached (\$ 1436 ).

O. lenticulare (O. lnt.).-The small, nearly cylindrical bone between the stapes and incus ( $\$ 1438)$.

Petrosum s. perioticum.-The dense bone containing the parts of the labyrinth ( $\$ 510$ ).

Scala vestibuli (Scl. vst.).--The chamber of the cochlea opening into the vestibule. It is divided into two chambers by the membrane of Reissner (Fig. 128).

Scala tympani (Scl. tym.).-This chamber of the cochlea is separated from the tympanum only by membrane.

Stapes.-The last of the small bones of the ear. Its oval base fits into the fenestra ovalis. Near its small end is a bony process, to which is attached the stapedius muscle.

Tympanum.-This is the second or middle chamber of the ear ( $\$ 1434)$.

Vestibulum (Vst.).-The vestibule is the common chamber of the labyrinth : from it extend the semicircular canals and the scala vestibuli of the cochlea (\$1431. C).

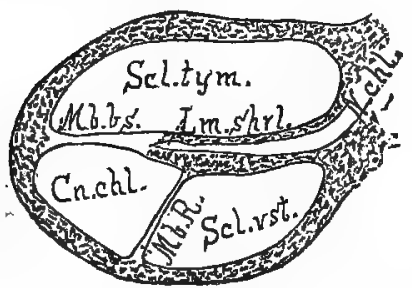

Fig. 128.-Transection of A CoIl OF THE COCHLEA, (From Quain, after Henle.)

Explanation of Fig. 128.-Canalis cochleæ (Cn. chl.).-A division partitioned off from the scala vestibuli by the membrane of Reissner. It is separated from the seala tympani by the membrana basilaris (Mb. bs.).

Lamina spiralis ( $\mathrm{Lm}$. sprl.). - This is the bony partition between the two scalæ. The partition is completed by the membrana basilaris (Mb. bs.). It arises from the modiolus and through it passes the cochlear nerve (N. chl.).

Membrana basilaris (Mb. bs.) s. lamina spiralis membranacea.-This with the osseous spiral lamina completes the separation between the scalæ.

The organ of Corti is on the side of this membrane toward the canalis cochlex; no attempt is made to indicate it here.

Membrana Reissneri (Mb. R.), s. lamina denticulata, s. limbus laminos spiralis.-This membrane divides the scala vestibuli into two parts the canalis cochlea and the scald vestibuli proper. 


\section{A P P E N D I X.}

TERMS OF DESCRIPTION ( $(1443)$-NIPPERS ( $\$ 1444)$-DROPPING-BOTTLE OILER ( $\$ 1445$, Fig. 129)-LIQUID GELATIN (\$ 1446)-OBTAINING ALCOHOL FREE OF TAX (\$ 1447)DRYING JARS QUICKLY WITHOUT HEAT (\$ 1448)-SOLUBLE BERLIN BLOE ( 1449$)$ BLDE GELATIN FOR INJECTIONS ( $(1450)$-OBTAINING FROGS AND MENOBRANCHI (\$§ 1451, 1452)-PITHING FROGS (\$ 1453, Fig. 130)-MACROTOME (\$ 1454)-BRAINS OF LOWER VERTEBRATES $(\$ 1455)$-USE OF MICA $(\S 1456)$.

$\S 1443(\S 23)$. Terms of Description.-In a notice of the senior author's paper (14) upon the brain of the cat, the Medical Record (New York) for May 13, 1882, has the following:-

"We feel disposed to especially endorse his recommendation for the more general adoption of such terms as cephatic and caudal, dorsal and ventral, in place of superior, inferior, posterior and anterior. The latter are often rendered ambiguous and confusing to the student on account of the natural difference in posture between man and other mammals, and the fact that the human cadaver is usually examined in a supine position."

The American Journal of Neurology and Psychiatry (I, Feb., 1882), in a notice of the same paper (pp. 101-104), and in other notices and editorials (pp. 46,50,55), employs the terms cephalic, ectal, ental, ventral, ventrad, dorsal, caudad, cephalad, caudocephalad.

The same terms are consistently employed by Prof. T. B. Stowell in his admirable paper (1) on the Vagus nerve in the cat, and by our colleague, Prof. J. Henry Comstock, in his Report for 1880 (A) as U. S. Entomologist, and in his Guide to Practical Work in Entomology (B); in the latter, Prof. Comstock has made valuable suggestions respecting the whole subject of toponomy, especially with regard to the use of cephatic and caudal.

$\S 1444(\$ 146)$. Nippers.-Besides the ordinary surgical bone forceps, there are the dental "wedge-cutters," which closely resemble the nippers, but are highly polished and provided with a spring for separating the handles; they cost about $\$ 3.25$. 
$\$ 1445$ ( $\$ 147)$. Dropping-bottle Oiler.-A bottle of the kind shown in Fig. 129, used largely in mieroscopic work, is found to be a very convenient oiler. The glass tube is prepared as directed for glass canulæ (\$340), except that its large end is slightly flared while hot by pressing it upon some blunt, pointed object. The bulb is a pure rubber nipple.

$\$ 1446$ (\$ $251, \mathrm{~A})$. Liquid Gelatin. - The liquid gelatin referred to is prepared as follows: 75 grams of the best translucent glue is put into a clean towel and crushed with a hammer. It is then placed in a fruit jar and $100 \mathrm{cc}$. of com. mercial acetic acid poured over it. After standing three days or more in a warm place, there should be added $100 \mathrm{cc}$. of water and $100 \mathrm{cc}$. of 95 per cent. alcohol. This preparation will remain liquid at the ordinary temperature of a sitting room $\left(20^{\circ} \mathrm{C}\right.$.). It should be of such a consistency that when spread upon ordinary note paper it will dry on the surface without penetrating the paper. If too thick, it may be thinned by adding the liquids in the proportion given above, or it may be thickened by adding glue. This liquid glue or gelatin may be used like common mucilage. Both are sometimes improved by mixing them. The brush used must be mounted in quill or something that will not rust. A quill duster, with the addition of a wooden handle, answers very well.

$\S 1447$ ( $\$ 264)$. Obtaining Alcohol Free of Tax.-(A) The original bill granted the privilege only to incorporated or chartered institutions ; later provisions apply to all educational institutions.
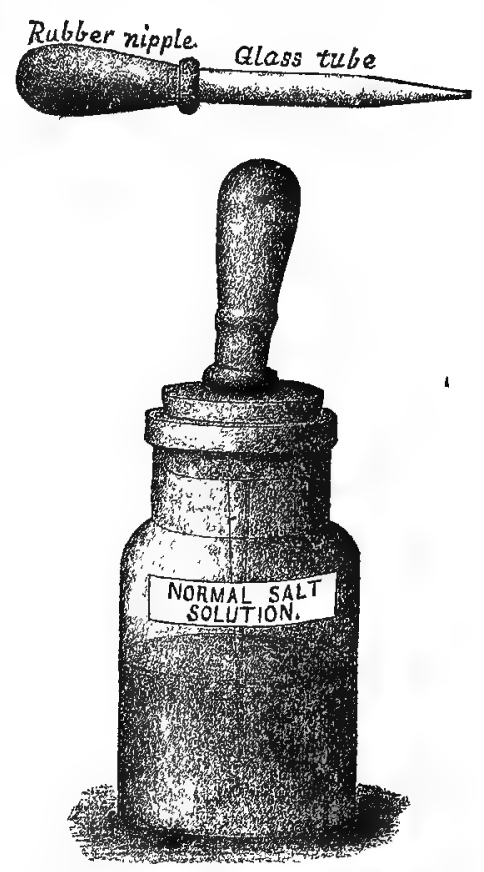

Fig. 129.-Dropping-BotTle OILER, WITH Dropping TUBe ReMOVED; $\times .5$.

(B) In the application and the bond, the first name of each person named must be written in full or an unmistakable abbreviation must be given.

(C) The bond must be executed for a sum equal to double the amount of tax due upon the alcohol withdrawn.

$\S 1448$ ( $\$ 338$, A). To Dry Jars Quickly without Heat.-Clean them properly, rinse them with rain (or distilled) water, and let this drain off. Then rinse with strong alcohol and finally with ether. The evaporation of the latter will leave the surface perfectly dry. Recommended by F. Lenggenhager in the Druggists' and Chemists' Cireular.

\& 1449 (\$ 336). Soluble Berlin Blue.-This form of Berlin blue is used largely in making fine injections and many experiments. Directions for preparing it are given in nearly all the modern works on Microscopy, and, being so widely used, it has become an article of commerce, and may be had of most dealers in microscopic materials. It should be obtained in the solid form and a saturated solution prepared with distilled or rain water. Such a solution may be used for a cold-flowing injecting mass (\$ 1087), or it may be mixed with glue as directed immediately below.

\$ 1450. Blue Gelatin for Injections.-To prepare this, soak fine glue in clean cold water until it becomes soft; then transfer it to a metal dish and heat over a water bath 
till the swollen glue melts. Add to this about an equal volume of the saturated Berlin blue ( $\$ 1449$ ) solution beuted to about 80 or 90 Centigrade. Mix thoroughly, and if any dirt is present, filter tirrough fine flannel. T'he solution may be thinned with water. It must of course be warmed when it is used, and the animal into which it is to ke injected should be warmed to at least $30^{\circ} \mathrm{C}$. The material soon becomes moldy in warm weather, so only about the amount needed should be made at a given time.

Injections with colored glue are made precisely as directed in § 359 . After the injection is finished, allow cold water to flow upon the animal or set it away in a cool place.

$\S 1451$ ( $\S \S 1085,1091$ ). Obtaining Frogs and Menobranchi.-Notwitlestanding the abundance of frogs in certain lncalities, they are not always obtainable when wanted for anatomical and physiological purposes, and laboratories should keep a supply on hand.

Boys sometimes capture them, but are apt to injure them. Large frogs may sometimes be had from restaurants or from large dealers in fish lise E. G. Blackford, of Fultom Market, New York city. Frogs abound in the marshes near Montezuma, N. Y, and may be had at a moderate price from Mr. Fennimore Helmer of that I.lace.

Frogs may be transported long distances in a box of wet grass if a few airholes are made. They should be kept in a spring, or in a dark covered tank or vessel through which water flows, or in which it is frequently changed. The cover is needed both to prevent their escape and to retard their vital changes. In spite of care, few survive more than three months; the larger ones often die within as many weeks.

§ 1452. Menobranchi abonnd in the lakes of central New York, but are not easily taken with the net on account of the depth of the water, and the supply from that source is

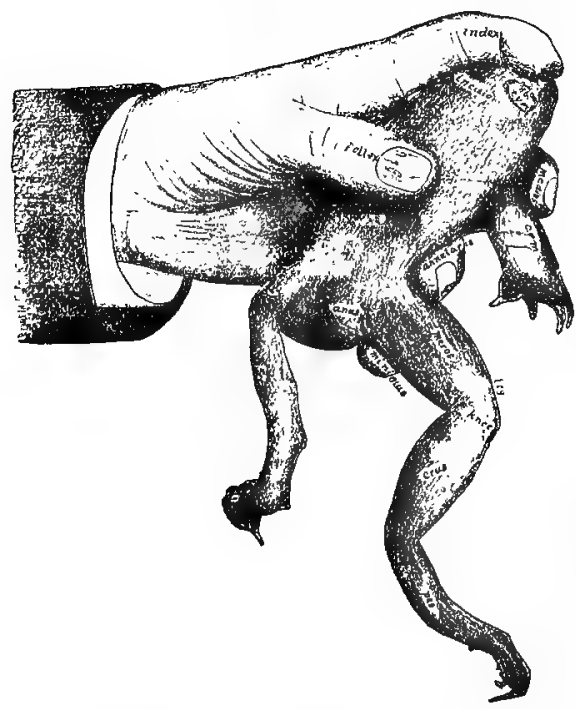

Fig. 130.-Method of Holding a Frog for Pithing. therefore variable. We have usually been able to obtain them in considerable numbers at short notice from Mr. Russel Dee, of Harmar, Ohio. Menobrancli cannot live long out of water, and the water should be changed often. For transportation, not more than three should be put in a pail of water, and they should be shipped very promptly. They may be kept easily in aquaria, especially if a shelter is provided under which they can screen themselves from the light.

Alcoholic examples of the above named animals, as well as of many other forms, domestic and foreign, may be had from the Natural History Establishment of Prof. H. A. Ward, at Rochester, N. Y.

81453 (\$ 1086). Pithing FrogsThis is an expeditious and approximately painless way of killing frogs for anatomical and experimental purposes. The frog is to be grasped as shown in Fig. 130-the pollex upon the middle of the back, the medius, annularis and minimus across the belly so that their tips are related to the right arm and leg as represented, and the index upon the head. In this way the most active and powerful frog may be held securely, but the grasp may be aided by a bit of 
cloth. When first seized, the caudal end of the frog should be held downward or away from the operator, so that the urine may not be discharged upon the clothes.

To pith, ventriduct the head with the index, and pass the tip of the right index from between the eyes caudad until a distinct depression is felt at the meson, upon a line corresponding nearly with the caudal margins of the membranæ tympanorum. With a sharp-pointed Charrière scalpel, divicle the skin transversely for about $3 \mathrm{~mm}$, and then plunge the scalpel deeper so as to divide the "medulla." Respiratory movements cease, and the frog is supposed to be dead and incapable of feeling.

If the cephalic portions of the brain are wanted, the head should be cut off and the brain exposed without delay ( $\$ 1087$ ) ; or reflex movements of the limbs should be prevented by breaking up the myelon with the probe or a piece of wire introduced from the incision into the neural canal.

$\S 1454$ (§ 1115). Macrotome.-This name is applied to a simple apparatus upon which sections of the head or other parts may be made with a saw. It is, in effect, a kind of "miter-box," and is made as follows:-

A block of hard wood (ash, oak, etc.), $6 \mathrm{~cm}$. thick and about $18 \mathrm{~cm}$. long, has a rebate ("rabbet") cut out along one of its long corners to a depth of $12 \mathrm{~mm}$., and is then accurately divided at about the middle of its length.

The pieces are then to be screwed securely across a perfectly level board, about $36 \times 18$ $\mathrm{cm}$., at about $10 \mathrm{~cm}$. from one end. The pieces are to be separated by only the thickness of the blade of the small back saw (§ 152) used in making the sections, and the rebate is to be above, toward the shorter portion of the board.

At the right of the division between the blocks (or at the left if the operator is lefthanded) is to be placed a block about $4 \mathrm{~cm}$. thick; it is to be adjustable by means of a thumb-screw passing through a slot. In $\S 1117$, this block is called the slide; in some cases it may be dispensed with.

The macrotome may be used not only for hemisection of the head, but,also for making frozen sections of limbs and other parts. The saw should be kept sharp, and clean but nct oily.

$\S 1455$ ( $\$ 1371$ ). The Brains of the Lower Vertebrates. -The brains of Amphibia and of the air-breathing Vertebrates (Reptiles, Birds and Mammals) are readily comparable with that of the frog or Menobranchus. But those of the "fishes" (sharks, skates, lamprey-eels, Ceratodus, gar-pikes and Teleosts) have not yet been homologized satisfactorily, and the beginner is not advised to undertake their examination; some idea of the difficulties may be gained from the special papers by various writers upon the brains of the several groups and from a brief summary by the senior author (26).

$\S 1456$ ( $\$ 318$ ). The Use of Mica for Mounting Alcoholic Specimens.-Prof. Leslie A. Lee has kindly called our attention to the use of slips of mica for the mounting in alcohol of preparations which require some support. It can readily be eut into the desired shape, and holes can be drilled through which may be passed the threads for supporting the specimen.

$\$ 1457$ ( $\$ 1128,1138,1238$ ). The Fornix.-According to an editorial in the Am. Jour. of Nearology and Psychiatry (I, 402), there are two fornices, right and left, one for each hemisphere, as there are two fimbria, etc. This commends itself to us upon some grounds, but the commonly accepted view should not be hastily abandoned. 


\section{B I BL I O GR A P H Y.}

In the following Bibliography, the names of the authors are arranged alphabetically. The books of an author are designated by heavy letters, and the papers by heavy Arabic figures. If the papers are recorded in the Royal Society's Catalogue, the corresponding numbers are herein given; otherwise they are numbered with heavy italicized figures (\$ 3).

The more common book sizes are indicated by the initials: F. (folio), Q. (quarto), O. (octavo), D. (duodecimo).

The names of periodicals are abbreviated as in the Royal Society's Catalogue.

The Arabic figures in ordinary type at the end of a title indicate the pages in the present work where the book or paper is referred to.

General references to authors are given in the Index.

Agassiz, L. : A.-Contributions to the natural history of the United States. "On Morphology and Nomenclature." III. Chap. 2, Sect. 4...14.

Alcock, B. : A.-Article, "The fifth pair of nerves." Todd's Cyclopædia of Anatomy and Physiology, II, pp. 268-316. (This paper should have been referred to on p. 507.)

American Journal of Neurology and Psychiatry... .464, 482, 491, 508, 534.

Argyll, Duke of : 1.-A fourth state of matter. "Nature," June 24,1880; 168 . Repr, in "Science," July 17, $1880 \ldots .12$.

Baker, R. : A.-Cursus osteologicus. Appendix on the preparation of bones. D. London, $1699 . . .111$.

Baley, T. : A.-A pocket book for chemists, chemical manufacturers, etc. $32 \mathrm{mo}, \mathrm{pp}$. 421. London and New York, 1878...112, 136.

Balfour, F. M.: A.-A treatise on comparative embryology. Royal O., 2 vols., 429 fig. London, $1881 \ldots .8,29,32,37,41,406,410,413,416,472,473,485,487,507$.

Barclay, John : A.-A new anatomical nomenclature, relating to the terms which are expressive of position and aspect in the animal system. O., pp. 182, 5 plates. Edinburgh, $1803 \ldots .12,21,25$.

Barnard, W. S.: 1.-Observations on the membral musculation of Simia satyrus (orang), and the comparative myology of man and the apes. From the Proc. Amer. Assoc., 1875....193, 196.

Beale, L. S. : A.-How to work with the microscope. O., pp. 518, 100 plates, 5th ed. London, 1880...197, 372.

Bell, Sir Chas. : B.-The anatomy of the brain explained in a series of engravings. Q., pp. vii, 87, 12 plates. London, $1802 \ldots . .472$.

Bell, T. : A.-Article Carnivora. Todd's Cyclopædia of Anat. and Phys. (q. v.), I, p. $480 \ldots 492$. 
Bernard, Cl. : A.-Leçons de physiologie operatoire. 0., pp. 614, 116 fig. Paris, $1879 \ldots .80,144,148,192,199,200,297,316,342,363$.

$290,291$.

17.-Memoir sur le pancreas. Supplément aux Comptes Rendus (1856)...

Bernstein, J. A. : A.-The tive senses of man. D. pp, 301, 91 fig. New York, 1876 -...512-514, 526.

Bichat, X.: A. - Traité d'anatomie descriptive. 5 vols., D., 2517 pages. Paris, 1801-1803. Vol. III terminé et publié par Buisson; IV par Buisson; $V$ par Roux.... $53,166$.

Bigelow, M. T. : A.-Punctuation, and other typographical matters, for the use of printers, authors, teachers and scholars. 16mo, pp. 112. Boston, $1881 \ldots .2$.

Birdsall, W. R. : 1. -The embryogeny of the sympathetic nervous system. Archives of Medicine, April, 1879. (This paper should have been referred to on p. 394.)

Bischoff, T. L. W. : 12.-Die Grosshirnwindungen des Menschen mit Berücksichtigung ilhrer Anordnung bei den Affen. München Akad. Abhandl. X. 1870, pp. $389-498 \ldots . .435$.

Bourgery et Jacob: A.-Traité complet de l'anatomie de l'homme. Paris, 1844. (Vol. 3, neurology; Vol. 8, embryology, including comparative neurology)....316, 418, 419, 492 .

Bowditch, H. P.: 1.-The collection of data at autopsies. Boston Med. and Surg. Jour., 1880, CIII, p. 148....136.

Broca, P.: 1.-Anatomie comparée des circonvolutions cérébrales; le grand lobe limbique et le scissure limbique dans la serie des 'mammiferes. Rev. d'Anthrop. Paris, 1878, pp. 385-498....499, 500, 503.

Brown, T. : A.-The taxidermist's manual. 21 ed., D., pp. 149, 6 plates. London and Edinburgh, $1870 \ldots 111$.

Bucknill and Tuke : A.-A manual of psychological medicine. 0. London, 1858.... 402.

Champneys, F.: 1. - On the muscles and nerves of a chimpanzee (Troglodytes niger) and Cynoeephalus anubis. Jour. Anat. and Phys, VI, 1872, pp. 176-211....193.

Chauveau: A.-Traité d'anatomie comparée des animaux domestiques. 2me edition, 0., pp. 992, 368 fig. Paris, 1871....152, 157, 161-163, 166, 167, 169, 174, 193, 194, 206, 212 , 214, 215, 217, 219, 221, 223, 225, 229, 231, 236, 247, 250, 254-258, 260, 263, 265-270, $297,303,310,311,316,342,359,363,372,379,387,394,512-514,526$.

Chauveau (Fleming) : A.-2d edition, translated and edited by G. Fleming. The comparative anatomy of the domesticated animals. O., pp. 957, 450 fig. New York, 1873.... $152,157,161-163,167,174,192,206,212,214,215,217,219$, 221, 223, 225, 229, 231, 236, $247,250,252,254-258,260,265-270,297,303,310,311,316,342,363,372,379,387,394$, 512-514, 526.

Clark, W. F. : A.--A manual of the practice of surgery. Revised and edited by an American surgeon. O. New York, $1879 \ldots 86$.

Cleland, John : A.-A directory for the dissection of the human body. D., pp. 175. London, 1876....70, 192.

4.-On a peculiar ligament connecting the opposite ribs in certain vertebrata. Edinb. Roy. Soc. Proc., IV, 1858, pp. 61-63 ....166.

- 5 . - On the structure, action and morphological relations of the ligamentum conjugale costarum. Edinb. New Phil. Jour., IX. 1859, pp. 258-266...166.

7.-On the serial homologies of the articular surfaces of the mammalian axis, atlas and occipital bone. Edinb. Phys Soc. Proc., II, 1859-1862, pp. 221-226; Nat. Hist. Review, 1861, pp. 151-154 ...169. 
Cleland, John: 13.-On ribs and transverse processes, with a special theory of the vertebrate skeleton. Brit. Assoc. Rep., 1861 (Pt. 2), pp. 164-165....169.

- 1.-A remarkable double monstrosity of the head. Jour. Anat. and Phys., XIII, 164-172 ; 2 plates. $1879 \ldots .22,23$.

Clevenger, S. V. : 1.-Cerebral anatomy simplified by comparative anatomy studies. Chicago Med. Jour. and Examiner, Nov., 1880, pp. 9, 12 fig....53, 500, 503.

2.-Comparative neurology. Am. Naturalist, Jan. and Feb., $1881 \ldots .502$.

3.-Plan of the Cerebro-spinal nervous system. Read before Amer. Assoc.

1880. Jour. of Nerv. and Mental Disease, Oct., 1880. Pp. 40, 26 fig. (See p. 506.)

Colin, G. : A.-Traité de physiologie comparée des animaux, etc. $2 d$ ed., $0 ., 2$ vols., 206 fig. Paris, 1871-1873....365.

Comstock, J. H. : A.-Report of the Entomologist of the U. S. Department of Agriculture for 1880 , and in later reports....534.

B -A laboratory guide in Entomology, Part I. 1882 ...534.

Coues, E.: 36.-On the myology of the Ornithorhynchus. (1870.) Essex Institute Comm. VI, 1871, pp. 127-173....192.

47. - Osteology and myology of Didelphys Virginiana. Boston Soc. Nat. Hist. Mem. II, 1873, pp. 41-156...57, 192, 193, 206.

- 1.-Antero-posterior symmetry, with special reference to the muscles of the limbs. Medical Record, June-Nov,, $1870 \ldots . .22,92,192,194$.

2.-The nature of the human temporal bone. American Journal of Otology, IV, Jan., 1882. (This paper should have been referred to on p. 174.)

Crooke, H. : A.-Mikrokosmographia, a deseription of the body of man. London, $1681 \ldots 197$.

Cunningham, D. J. : 1.-The relation of nerve supply to muscle homology. Jour. Anat. and Plyss., Oct., 1881, XVI, pp. 1-9...196.

Curtis, L.: l.-On tactile hairs. Amer. Jour. Mic. and Pop. Sci., IV, 1879, pp. 166-172...512.

Cutter, G. R. : A.-A dictionary of the German terms used in medicine. D., pp. 304. N. Y., $1879 . . .16$.

Cuvier, Georges : A.-Leçons d’anatomie comparée, recueillies et publiées par Duméril, seconde édition, corrigée et augmentée. 8 volumes. D. Paris. [Tome troisième, contenant le système nerveux et les organes des sens, revu par MM. F. G. Cuvier et Laurillard.] Puris, 1836-1816....169, 173, 192, 194, 297, 302, 303, 307, 310, 311, 316, 342, 363.

B.-Le règne animal, publ. par Audouin, Blanchard, Deshayes, Milne-Edwards, Valenciennes, etc. 11 vols. de texte et 11 vols. d'Atlas, renf. 993 plchs. coloriées. Paris, 1849 et suiv. .. 174, 474, 492, 499.

C.-Leçons d'anatomie comparée, recueillies et publiées sous les yeux de l'auteur par MM. Duméril et Duvernoy. O., 5 vols. Paris, 1800-1805. -499.

Cyon : A.-Methodik der physiologischen Experimente und Vivisectionen. Rnyal O., pp. 566, Atlas of 54 plates. Giessen, $1876 \ldots 418$.

Dalton, J. C. : A.-A treatise on human physiology, for students and practitioners of medicine. 7th edition, O., pp. 722, 252 fig. Philadelphia, 1882 ...328, 38\%, 498, 505, $506,508,511-514,526$.

1.-Cerebral anatomy, with special reference to the corpus striatum. Annals of the Anatomical and Surgical Society, II, pp. 1-18, 6 fig. Jan., 1880. Reprinted in "Brain"....474.

Dana, J. D. : 123.-The classification of animals based on the principle of cephalization. Am. Jour. Sci., XXXVI, 1863, pp. 315-321....56.

Dareste, C. : 13.-Troisième mémoire sur les circonvolutions du cerveau chez les 
mammifères. Ann. Sci. Nat., III, 185̄ (Zool.), pp. 65-111. Paris, Comptes Rendus, XL, 1855 , pp. 683-686...492, 499 .

Darling and Ranney: A.-The essentials of anatomy. 0., pp. 629, with figures. New York, $1880 \ldots 173$.

Dugès, A. : 4.-Note sur une nouvelle espéce d'azolotl ; le Siredon Dumérilii (1870). Ann. Sci. Nat., XV, 1872 (Zool.), Art. 17... .419.

Dunglison, R. : A.-Medical Lexicon. A dictionary of medical science, etc. Edited by R. J. Dunglison. Royal O., pp. 1131. Philan, 1874....3, 12, 22, 271, 473, 510.

Duval, M. : 1.-De la non-existence des trous de Monro. Progrés Méd. No. 25, p. 483, et No. 26, p. 503....413-415.

2.-La corne d'ammon (morphologie et embryologie). Archives de Neurologie, II, 161-173, Sept., 1881; III, 1-35, Jan., 1882; Pl. 1-4. ...459.

3.-Des plexus choroides et des trous de Monro. Comm. à la Soc. de Biologie, June 14, 1879. (This paper should have been referred to on p. 460.)

- 4.-Developpement de la région lenticulo-optique dans le cerveau humain. Comm. la Soc. Biologie, June 21, 1879.

Duval et Keller : A.-See Huguenin : A.

Duvernoy, G. L.: 100.-Des caractères anatomique des grandes singes pseudo-anthropomorphes. Paris, Mus. Hist. Nat. Archiv., VIII, 1855-1856, pp. 1-248. . . 193.

Dwight, Thos. : A.-The anatomy of the head, with 6 lithographic plates representing frozen sections of the head. Boston, pp. viii, 136...131, 136.

136,339 .

B. -Frozen sections of a child. Q., pp. 66, 15 plates. New York, 1881....131,

- 1.-On the preservation of anatomical specimens. Publications of the Mass. Med. Soc., $1870 \ldots 136$.

Ecker, Alex. : B.-The cerebral convolutions of man, represented according to original observations, especially upon their development in the foetus; intended for the use of physicians. Transl. by R. T. Edes. O., pp. 87, 6 fig. New York, $1873, \ldots .411,412,418$ :

Egleston, T. A. : A.-Tables of weights, measures, coins, etc., of the United States and England, with their equivalents in the French decimal system. 16mo, pp. 60. New York, $1871 \ldots \%$.

Eliot, C. W.: 1.-The new education. Atlantic Monthly, March and Feb., Vol. XXIII, 1869, pp. 203, 358....17.

Ferrier, D. : A.-The funetions of the brain. $0 .$, pp. 323, 68 fig. New York, 1876.... $400,475,492,500-502$.

Flint, A. : A.-A text-book of human physiology for practitioners and students of medicine. Large O., pp. 978, 3 plates, 313 fig. New York, 1876...512-514, 526.

Flower, W. H. : A.-An introduction to the nsteology of the mammalia. D., pp. 337, 126 fig. London, 1870...94, 152, 153, 157, 160-164, 167, 169-172, 174, 179, 184, 185, 188, 307,316 .

6.-On the brain of the Javan Loris (Stenops javanicus, Illig.). 1862. Zool. Soc. Trans., V, 1866, pp. 103-111...492, 502.

8. - On the optic lobes of the brain of the Echidna. Zool. Soc. Proc., 1864, pp. 18-20, 2 fig. (This should have been referred to in $\$ 1131$ [8].)

13.-On the commissures of the cerebral hemispheres of the Marsupialia and Monotremata as compared with those of Placental mammals. Phil. Trans., CLV, 1865, 633-651, 4 plates. Abs. in Roy. Soc. Proc., 1865, 71-74 . 471, 473.

26. - On the value of the characters of the base of the cranium in the classific)tion of the order Carnivora, and on the systematic position of Bassaris and other disputed forms. Zool. Soc. Proc., 1869, pp. $4-37 \ldots 185$. 
Flower, W. H. : 28.-On the anatomy of the Proteles cristatus, Sparrman. Zool. Soc. Proc, 1869, pp. 474-496...499.
35.-On the anatomy of the Allurus fulgens, Fr. Cuv. Zool. Soc. Proc., 1870, pp. $752-769 \ldots .500$.

36. - On the composition of the carpus in the dog. Brit. Assoc. Rep., XLI, 1871 (sect.), pp. 138 ; Journal of Anat. and Phys., VI, 1872, pp. 62-64 ...41. (This paper should have been referred to on p. 161.)

41.-On the arrangement and nomenclature of the lobes of the liver in Mammalia. Brit Assoc. Rep., XLII, 1872 (sect.), pp. 150-151; Nature, VI, 1872, pp. 346-365 ....286.

Ford, Corydon L. : A table of the cranial nerves. ...510.

Forel, A.: 1.-Untersuchungen über die Hauben-region und ihre oberen Verkniupfungen im Gehirne des Menschen und einiger Säugethiere. Archiv für Psychiatrie. Berlin, 187\%. (This and the following paper should have been referred to in $\$ 1325$.)

- 2.-Beiträge zur Kenntniss des Thalamus opticus und der ihn umgebenden Gebilde bei den Säugethieren. Sitzungsberichte der Kaiserlichen Akademie der Wissenschaften ; LXVI Band, 1 Heft, Juni 1872 ; S. 25-58, Tafeln 1, 2 ; 18 i2.

Foster, M. : A.-A text-book of physiology. 3d edition, D., pp. 804, 60 fig. New York, $1880 \ldots . .396$.

Foster, M., and Balfour, F. M. : A.-The elements of embryology. Pp. 272, 71 fig. $16 \mathrm{mo}$, Part I, $1874 \ldots . . .415$.

Foster and Langley : A.-A course of elementary instruction in practical physiology. 3d edition, D., pp. 276. London, 1878...7, 57, 316, 415, 500.

Frey, H. : A.-The microscope and microscopical technology. Translated and edited by G. R. Cutter. O., 2d ed., pp. 624, 385 fig. N. Y., 1880 . . .280, 291, 298, 363, 367.

Fritsch und Hitzig : 1.-Ueber die elektrische Erregbarkeit des Grosshirns. Arch., f. Anat., Phys. und Wissen. Med., Leipzig, 1870, S. 300-332 . . 500, 502.

Gadow, Hans : 3.-Observations in comparative myology. Jour. Anat. and Phys., XVI, pp. 493-514, July, 1882. (This paper was received too late for use on p. 196.)

Gage, S. H. : 1.-Plaster of Paris as an injecting mass. American Naturalist, Nov., 1878, pp. 717-724 ...57, 139, 148.

- 3.-The ampulla of Vater and the pancreatic ducts in the domestic cat, Felis domestica. Amer. Quarterly Microsc. Jour., Jan., 1879, pp. 123-131, and April, pp. 169$180 \ldots 5^{r}, 292$.

- 5. - The inter-articular ligament of the head of the ribs in the cat. Amer. Assoc. Proc., 1879, pp. 421-424,...166.

the Amer. Assoc., 1879.

Gall et Spurzheim: A.--Recherches sur le system nerveux en genéral, et celui du cerveau en particulier. Q. Paris, $1809 \ldots 492$.

Galton, J. C.: 1.-Note on the epitrochleo-anconeus or anconeus sextus (Gruber). Jour. Anat. and Phys., IX, 169-175, PI. I. ...193.

Ganser, S. : 1.-Vergleichend-anatomische Studien über das Gehirn des Maulwurfs. Morphologisches Jabrbuch, VII, 591-725, 5 taf. (Unfortunately this important paper wan received too late for reference in the body of the work.)

Garner, R. : 1.-On the Brain of the Canidæ. Brit. Assoc. Rep., 1876, pp. 152-153 ...500.

Gegenbaur, C. : A.-Grundriss der vergleichenden Anatomie. Leipzig, 1874. O., pp. $660,320 \mathrm{fg} . . .22,42,174,310,394$. 
Gegenbaur, C. : B.-Untersuchungen zur vergleichenden natom.e er Wirbelthiere. Q., pp. 308, 15 plates. $1864-65 . . .161$.

C. - Untersuchungen zur vergleichenden Anatomie der Wirbelthiere. Zweites Heft. 1, Schultergürtel der Wirbelthiere. 2, Brustfösse der Fische. Q., pp. 176, 9 plates ... 162 .

$141 . . .14$

59.-Ueber das Archipterygium (1871), Jenaische Zeitschr. VII, 1873, pp. 130-

(Lankester): A.-The elements of comparative anatomy. Translated from the German by F. J. Bell. Edited by E. R. Lankester. O., pp. 45, 35 fig. London, 1878 $\ldots .162,297$, 303, 311, 316-317, 342, 363, 372. 379, 387, 405, 418, 492, 506, 507, 509.

Gervais, P. : 146.-Memoir sur les formes cérébrales properes anx carnivores vivants et fossiles suivi de remarques sur la classification de ces animaux. Paris Mus Hist. Nat. Nouv. Archives, VI, 1870, pp. 103-162....492, 500.

Giacomini, C. : 1.-Nuovo processo per la conservazione del cervello. Communicazione alla reale accademia di medicina di Torino, $1878 \ldots . .435$.

Gill, T. : 1.-Arrangement of the families of fishes. .Smithsonian Miscellaneous Collections No. 274. 1872...58.

Goodsir, J. : A.-Anatomical memoirs, edited by Wm. Turner. 2 vols., 0., pp. 993, 14 plates, 26 fig. Edinburgh, 1868....12, 197.

Götte : A.-Die Entwickelungsgeschichte der Unke (Bombinator igneus). Leipzig, $1875 . . .415$.

Gratiolet: A.-Memoire sur les plis cerebraux de l'homme et des primates. Q.,pp. 104, with folio atlas of 13 plates. 1854. (The volumes bear no date, but the one above given is contained in an advertisement of the work upon a fly-leaf of Lauret et Gratiolet, A.) . . . 435, 502.

Gray, H.: A.-Human anatomy, descriptive and surgjcal. New American, from the 8th enlarged English edition, to which is added, Landmarks, medical and surgical, by L. Holden. Royal O., pp. 983, 522 fig. Philadel phia, 1878....94, 143, 154, 157, 161-163, $166,167,169,173,212,214,215,219,221,223,229,231,235,238,247,250,252,254-258,260$, $263,265-271,285,295,297,303,310-311,316,342,354,361,363,372,879,382-387,394$, $472-491,507-509,512-514,526$.

Giinther, A. C. L. G. : A.-An introduction to the study of fishes. 0., pp. 720, 320 fig. Edinburgh, $1880 \ldots 115,124,136$.

Guillot, N. : A.-Essai sur le cerveau. Q Paris, 1829...4499.

Gurlt, E. F. ; A.-Handbuch der vergleichenden Anatomie der Haus-säugethiere. 5th edition, O., pp. 903,170 fig. Berlin, 1873...206, 297, 303, 316, 342, 363, 372, 379, 387, $394,512-514,526$.

Hadlich : 1.-Ueter die bei gewissen Schädeldifformitatäten yorkommende Gehirnmissbildung mit Verwachsung der Grosshirnhemisphären. Arch. für Psychiatr., Berl, 1879, X, pp. 97-114, 1 plate....24, 415.

Haeckel, E. : A.-The evolution of man: A popular exposition of the principal points of human ontogeny and phyllogeny. Translated from the German. 2 vols., D., pp. 971, 330 fig., 15 plates. New York, $1879 \ldots .14,405$.

Hammond, G. M. : 1.-A new cortical centre. Med. Record, Mar. 19, 1881, 309, 310, 1 fig....492.

Harrison: A.-The Dublin dissector, or system of practical anatomy. 2 vols., D., pp. 870, 160 fig. Dublin and London....141, 148.

Haughton, S. : 115. - Notes on animal mechanics. No. IV. On the muscular anatomy of the lion. Roy. Irish Acad. Proc., IX, pp. 85-93, a fig., June 13, 1864... 194. 
Heath, C. : A.-Practical anatomy : A manual of dissections. 2d ed., O., pp. 572, 247 fig. Edited by Keen. Philadelphia, 1870...111, 148, 192, 202, 203.

Henry, A. : 1.-Malinverni on a case of absence of the corpus callosum without disturbance of intelligence (Abstract). London Medical Record (No. 135), May 27, 1874, pp. 319-320 .. 474.

Hitzig, E. : A.-تUntersuchungen über das Gelirns. O. Berlin, 1874. . .492, 503.

- 4.-Untersuchungen zur Physiologie des Gehirns. Arch. für Anat., Phys. und Wissen. Med., 1873, pp. 397-435...500.

Hodges : A.-A dissector's guide. 1st ed....192, 203.

Holmes, O. W. : 1.-Letter to B. G. Wilder on Anatomical Nomenclature. Science, No. 48 , May 28, $1881 \ldots . .11$.

- 3.-The rectus sternalis muscle. A description and historical sketch. Boston Med. and Surg. Jour, Nov. 19, 1857, 321-324, 1 fg. (Paper read and specimen shown by Dr. R. M. Hodges, at the meeting of the Bost. Soc. for Med. Improvement, Oct. 12, 185\%.) (This should have been referred to in connection with Fig. 73.)

Holmes, T.: A.-A system of surgery, theoretical and practical, in treatises by various authors. 4 vols., pp. 3715. London, 1860-1864.. \& 86.

Holtzapfel: A.-Turning and mechanical manipulation. 4 vols., many figures (incomplete)....79.

Horsley, V. : 1.-The fissures of the cerebral hemispheres in the Ungulata. Nature, $\mathrm{XIX}$, , 1879, pp. 276-278. Notice of Krueg, 1 . . .500.

Huguenin, G. : A.-Anatomie des centres nerveux. Traduit par Keller (Th.), annoté par Duval (M.). O., pp. 368, 149 fig. Paris, 1879...500, 502, 503.

Humphrey, G. M. : A.-A treatise on the buman skeleton (including the joints). 0 , pp. 620, 60 plates. Cam uridge and London, 1858.....152, 156, 157, 160-164, 167, 169, 173.

E.-Observations in myology. O., pp. 192, 9 plates, 48 fig. Cambridge and London, 1872...28, 192, 194, 195, 235.

- 10. - On some points in the anatomy of the chimpanzee, and the consideration of the term "quadrumanous" as applied to that animal. Cambridge Phil. Soc. Proc., I, 1866-67, pp. 49-50; Jour. Anat. and Phys., I, 1867, pp. 254-268...193.

Hunt, D. : 1.-A comparative sketch of the early development of the ear and eye in the pig, together with a new account of the development of the meatus externus, drum and Eustachian tube. Transactions of the International Otological Congress, 1876. . .531. 2.-On the development of the external ear passages. American Journal of the Medical Sciences, Jan., 1877....531. Oct., $1877 \ldots 531$.

3.-The development of the middle ear. Boston Medical and Surgical Journal,

Hutchinson: A.-Article Thorax, Todd's Cyc. Anat. and Phys. (q. v.), Vol. IV, pp. 1016-1087....164.

Huxley, T. H. : A.-Manual of the comparative anatomy of vertebrated animals. 0 ., pp. 431, 110 fg. New York, 1873. (The paging differs slightly from that of the English edition.)...24, 41, 193-194, 223, 270, 405-406, 410, 418, 496, 500, 506.

B.-Article Amphibia. The Encyclopædia Britannica, 9th edition. I, pp. 750777,26 fig. $1875 \ldots 18,174,185$.

C.-The crayfish, an introduction to the study of zoology. The international scientific series, Vol. XXVIII, O., pp. 371, 82 illustrations. New York, 1880...12, 16.

44.-On the theory of the skull. Roy. Soc. Proc., IX, 1857-59, pp. 381-457, 10 fig. [Repr. in Ann. Nat. Hist., III, 414-439.] . . .174.

70.-On the brain of Ateles paniscus. Zool. Soc. Proc., 1861, pp. 247-260, 1 plate, 2 fig....14, 496 . 
Huxley, T. H. : 108.-On the classification of the Dinosauria, with observations on the Dinosauria of the Trias. Geol. Soc. Jour., XXVI, pp. 32-50. 1870...18.

- 1.-Contributions to morphology. Ichthyopsida, No. 1. On Ceratodus Forsteri, with observations on the classification of fishes. Zool. Soc. Proc., Jan. 4, 1876, pp. 24-59, 11 fig.....472.

2.-The evolution and classification of mammals. Zool. Soc. Proc., 1880. Reported in Nature, Jan. 6 and 13,1881....22.

3.-On the cranial and dental characters of the Canida, with figures. Zool. Soc. Proc., London, 1880, Vol. II, pp. 238-288....58, 174.

- 4.-On the limbs of vertebrates. A lecture before the Royal College of Surgeons, Feb. 6, 1864. Abstract in Med. Times and Gazette, Feb. 20, 1864...26, 37.

- 5. - On sensation and the unity of structure of sensiferous organs. Nineteenth Century, V, 1879, pp. 597-611. Popular Science Monthly, XV, 1879....511.

Huxley and Youmans: A.-The elements of physiology and hygiene. O., pp. 420, many figures. $1868 . \ldots 531$.

Hyrt1, J. : A.-Handbuch der praktischen Zergliederungskunst als Anleitung zu den Sectionsübungen und zur Ausarbeitung anatomischer Präparate. 0., pp. 762. Wien, $1860 \ldots .79,136,141,148,192,199,297,303,310,311,316,321,330,342,363,372,379$, $387,394,513-514,526$.

- B. - Onomatologia anatomica. Geschichte und Kritik der anatomischen Sprache der Gegenwart, mit besonderer Berïcksichtigung ihrer Barbarismen, Widersinnigkeiten, 'Tropen, und grammatikalischen Fehler. O., pp. 626. Wien, 1880...157. This book should bave been referred to on p. 14 also.

Jones, T. R. : A.-General outline of the organization of the animal kingdom. London, $1861 \ldots . . .492$.

Jordan, D. S. : A.-Manual of the vertebrates of the Northern United States, exclusive of marine species. O., pp. 342. Chicago, 1876...418.

Joulin : 1.-Anatomie et physiologie comparée du bassin de mammifères. Archives gén. de Med., III, 1864, pp. 29-46...167.

Keen, W. W.: 1.-On chloral hydrate as a preservative. Phila. Medical Times, March 21, 1874...125, 136.

- 2.-The anatomical, pathological and surgical uses of chloral. Am. Jour. Med. Sci., July, 1875, pp . 76-86 ...125, 136.

Krause, W.: A.-Die anatomie des Kaninchens in topographischer und operativer Rücksicht. O., pp. 271, 50 fig, Leipzig, 1868....57, 192-193, 206, 316.

Krueg, J. : 1.-Ueber die Furchung der Grosshirnrinde der Ungulaten. Zeits. für Wissent. Zool., XXXI, pp. 297-345. 1878...500.

2.-Ueber die Furchen auf der Grosshirninde der zonoplacentalen Säugethiere. Zeitschrift für Wissen. Zool., XXXIII, 4 Heft, $1880 \ldots 496,499,500$.

Laurencet : A.-Anatomie du cerveau dans les quatre classes d'animaux vertébrés. D., pp. 164, 13 plates. Paris, $1825 \ldots . \ldots 18$.

Lee, R. : A.-Memoirs of Baron Cuvier. O., pp. 351. London, 1833....193.

Le Conte, J. : A.-Sight : An exposition of the principle of monocular and binocular vision. International scientific series. D.. pp. 275, 132 fig. New York, 1881...514.

Lewis, B. : 1.-On the comparative structure of the cortex cerebri. "Brain," April, 1878, pp. 79-96, 4 plates....476.

Leuret et Gratiolet : A.-Anatomie comparée du systeme nerveux considere dans ses rapports avec l'intelligence. 2 vols., $0 ., 1284 \mathrm{pp}$, with folio atlas of 32 plates. Paris, 1839, 185\%. (Vol: I is hy Leuret, Vol. II by Gratiolet; as stated in a note appended to the explanation of Pl. xvii, Pl. i-rxii were drawn under the direction of Leuret; but the 
explanations of xvii-xxii were prepared by Gratiolet according to Leuret's method; the remaining plates were prepared under the direction of Gratiolet.)...418, 492, 496, 499, 501.

Leyh, F. A. : A. -Handbuch der Anatomie der Hausthiere mit besonderer Berücksichtigung des Pferdes. 2d ed., O., pp. 724, 224 fig. Stuttgart, 1859... 152, 157, 161-163, 166-167, 169, 174, 206, 297, 303, 310, 316, 342, 363, 372, 379, 387, 394, 512-514, 526.

Littré et Robin : A.-Dictionaire de médicine, de chirurgie, de pharmacie, de l'art vétérinaire et des sciences qui s'y rapportent. 14th ed., 2 vols., O., pp. 1896, 532 fig. Paris, 1877 ....3.

Löwe, L.: A.--Beiträge zur Anatomie und zur Entwickelungsgeschichte des Nervensystems der Säugethiere und des Menschen. Folio, pp. viii-126 18 taf. Berlin, 1880. Auch mit dem Titel : I, Bd. die Morphogenesis des centralen Nervensystems....415, 472.

Luschka, H. : A.-Die Adergeflechte des menschlichen Gehirnes. Q., pp. 174, 4 plates. Berlin, 1855....414, 482.

Lussana e Lemoigne: A.-Fisiologia dei centri nerrosi encefalici. 0., 2 vols., many figures. Padova, $1871 \ldots 492,500,503$.

Macalister, A. : 9.-Contributions toward the formation of a correct system of muscular homologies. Ann. Mag. Nat. Hist., I, 1868, pp. 318-322 ....192.

- 12.-On the homologies of the flexor muscles of the vertebrate limb. Journal of Anat. and Phys., II, 1866, pp. 283-289...192.

19. - Further notes on muscular anomalies in human anatomy and their bear. ing upon homotypical myology. (1867.) Irish Acad. Proc., X, 1870, pp. 121-164...192.

21.-On some points in the myology of the chimpanzee and other of the primates. Ann. Mag. Nat. Hist., VII, 1871, pp. 341-351....193.

41.-The muscular anatomy of the gorilla. (1873.) Irish Acad. Proc., I, 1873-74, pp. 501-506...193.

- 1.-British Association Report, 1877, p. 91....58.

2.-Proceedings of the Royal Irish Academy, X, p. 142....244.

Maclise, J. : A.-Surgical Anatomy. Folio, pp. 156, 68 plates. Philadelphia, 1866. ....169.

pp. 298-300....14.

Magendie : A.-Recherches sur le liquide céphalo-rachidien. Paris, 1842....414.

M'Alpine, D. and A. N.: A.-Biological atlas : A guide to the practical study of animals and plants. Q., 423 colored figures, with accompanying text, containing glossary, classification, etc. Edinluurgh and London, 1880 ...418.

M'Alpine, D. : B.-Zoological atlas, with practical directions and explanatory text. F., 231 colored figures and diagrams. London and Edinburgh, $1881 \ldots .316,317$.

Markoe, T. M. : 1.-Remarks upon fractures of the epicondyles. Proc. N. Y. Surgical Soc., March 9, 1880. In N. Y. Med. Record, July 10, $1880 \ldots 160$.

Marsh, O. C. : 1.-The limbs of Sauranodon, with notice of a new species. Am. Jour. of Science, Feb., 1880, pp. 169-171, 1 fig....14, 41, 42.

Marshall, J.: A.-Ontlines of physiology. O. Phila., 1868 .. 492.

Marshall, A. M.: 3.-The morphology of the rertebrate olfactory organ. Quart. Jour. Mic. Science, XIX, $1879 \ldots 487$.

4.-The segmental value of the cranial nerves. Jour. Anat. and Pliys., XVI, pp. 305-354, 10 plates, April, 1882. (Through an oversight, this paper was not received in time for use in preparation of Chap. XI.)

Mayer, A. : A.-Analekten für vergleichende Anatomie. Q. $1835 \ldots 418$.

Mayer, A. F. J. C.: 1.-Blase für den Saft des Pankreas. Meckel, Deutches Ar- 
chiv, I, 1815, S. 297-298. (A reference to this paper should have been made in connec. tion with Fig. 82, p. 289.)

47. - Ueber ein neuentdektes Band, Jochband der Rippen (Ligamentum costarum conjugale) Müller, Archiv, 1834, S. 275-277....166.

Mayer, A. M. : 1.-Eulogy upon Joseph Henry. Science, Sept. 11, 1880 ...52.

Meckel, J. F. : A.-Traité général d'anatomie comparée. Translated from the German. O., 6 vols. Paris, 1828....192, 194, 252.

Medical Record (New York) : Aug. 20, 1881...88. Apr., 1880...124.

Meynert, T.: 1. - Die Windungen der convexen Oberflïche des Vorderhirns bei Menschen, Affen, und Raubthieren. Archiv für Psychiatrie und Nervenkrankheiten. Band VII, Heft 2, S. 32, 23 fig. $1877 \ldots . .465,492,498,500,502,503$.

The brain of Mammals. This article is referred to as a part of Stricker (A).

Mihalkovics, V. von : A.-Entwickelungsgeschichte des Gehirns, nach Untersuchun. gen an höheren Wirbelthieren und dem Menschen. Q., pp. 105, 7 plates. Leipzig, 1877. ....405-407, 414-415, 472, 486 .

Milne-Edwards, H. : A.-Leçons sur la physiologie et l'anatomie de l'homme et des animaux. 14 vols., $O$. Paris, 1857-80 . 19, 168, 169, 174, 297, 308, 307, 310-311, 316, $342,348,363,372,379,387,394,472,512-515,523,526$.

Minot, C. S. : 1.-Note on whether man is the highest mammal. Amer. Assoc. Proc., 1881, pp. 240-242...56.

Mivart, St. George : A.-The common frog. Nature series, London, 1874. D., pp. 158, $88 \mathrm{fig} \ldots 22,21,94,236,252,267,418$.

B.-The cat : its history, anatomy, psychology and origin. An introduction to the study of backboned animals, especially mammalia. O., 200 illustrations. London and New York, 1880...22, 24, 27, 41-42, 56-57, 152, 159, 161-163, 184, 194, 212, 214-215, $217,219,221,223,225,229,231,238,239-241,243-244,247,250,254-260,263-267,268-271$, $302,316,415,418,492,500,501$.

3.-Contributions toward a more complete knowledge of the axial skeleton in the primates. Zool. Soc. Proe., 1865, pp. 545-592....169.

4.-On some points in the anatomy of the Echidna hystrix. Linn. Soc. Trans., XXV, 1866, pp. 379-404....192.

8. - On the appendicular skeleton of the primates. (1866.) Phil. Trans., CLVII, 1867, pp. 299-430 : Roy. Soc. Proc., XV, 1867, pp. 320-321 ; Ann. Mag. Nat. Hist., XIX, 1867, p. 219....167.

19.-Contributions toward a more complete knowledge of the skeleton of the primates. Part I. The appendicular skeleton of Simia. Zool. Soc. Trans., VI, 1869, pp. $175-225 \ldots . .167$.

24.-On the vertebrate skeleton. (1870.) Linn. Soc. Trans., XXVII, 1871, pp. 369-392....169.

1.-The genesis of limbs. Nature, XVIII, 1878, pp. 282, 309, 331...22, 24, 26, 27. 2.-Tails. Nature, XX, 1879, pp. 508, 537...25.

Mojsisovics, E. von Mojsvar: A.-Leitfaden bei zoologisch-zootomischen Präparirübungen für Studirende. O., pp. 232,110 fig. Leipzig, 1879...79, 136, 148, 192, $316,321$.

Monro, Alexander, secundus : A.-Observations on the structure and functions of the nervous system. Illustrated with tables. F., pp. 176, 47 plates. Edinburgl,; 1788.... 407, 472 .

Morrell, G. H. : A.-Supplement to the anatomy of the mammalia. Containing dissections of sheep's heart and brain, rat, sheep's head, and ox's eye. 0. , pp. 269 . London, $1872 . . .57,174,510,514$. 
Morse, E. S. : 18.--On the tarsus and carpus of birds. N. Y. Annals, Lyceum, X, pp. 22, 2 plates, $8 \mathrm{fg} . \ldots 17,42$.

1.- - On the identity of the ascending process of the astragalus in birds with the intermedium. Anniv. Mems. of the Boston Soc. Nat. Hist. Q., pp. 10, 1 plate....42.

Newton, E. T. : A.-The typical parts in the skeleton of the cat, duck and codfish, being a catalogue with comparative descriptions arranged in a tabular form. 0,1 folio plate, pp. 28. London, $1875 \ldots . . .57$.

B.-An introduction to animal physiology. with directions for practical work. 5th ed., D., pp. 186, 98 fig. London, $1878 \ldots . .67$.

Osler, W. : 1.-Giacomini's method of preserving brains with chlorid of zinc. Journal of Anat. and Phys., XIV, 1879, p. 144 ..435.

- 2 - On Giacomini's method of preserving the brain. N. Y. Med. Rec., 1880, XVII, p. $315 \ldots 435$.

Owen, R. : A.-Comparative anatomy and physiology of vertebrates. O., 3 vols., pp. 2155, 1471 fig. London, 1861, 1868. . . 12, 14, 18-19, 23-24, 94, 152, 157, 169, 173, 194, 286, $288,297,303,310-311,316,318,331,342,348,363,372,379,387,394,405-406,410,487$, $493,496,499,502,512-514$.

35.-On the anatomy of the Cheetah (Felis jubata Schr.). Zool. Soc. Proc., I, 1835, pp. 129-136...492, 493, 499, 502.

53.- Notes on the anatomy of the Kinkajou (Cercoleptes convolvulus, Ill.). Zool. Soc. Proc., III, 1835, pp. 119-124...499.

101.- On a new species of the genus Lepidosiren [Protopterus]. Linn. Soc. Trans, XVIII, 1841, pp. 327-362, 4 plates. . . 419 .

166.-Report on the archetype and homologies of the vertebrate skeleton. Brit. Assoc. Rep., 1846, pp. 169-340; Froriep, Notizen II, 1847, col. 148-152 ; Silliman Jour., IV, 1847, pp. 123-130...14.

- 231. - Recherches sur l'archétype et les homologies du squelette vertébré. Paris, Comptes Rendus, XXVII, 1853, pp. 389-394...169.

- 1.-On the homology of the conario-hypophysial tract or the so called pineal and pituitary glands. Jour. Linn. Soc. Zool., XVI, No. 90, pp. 131-149; British Assoc., 1881 ; reported in Nature, Sept. $1881 . . .415,480$.

Packard, A. S., Jr. : A.-Zoology for high schools and colleges. 2d ed., 0., pp. 718, 545 fig. New York, $1880 \ldots 418$.

- 1. - Memoir of Henry James Clark, 1826-1873. Read before the National Academy of Sciences, April 23, $1874 \ldots 52$.

and Cope, E. D., editors : 1.-Laws of nomenclature. Aner. Naturalist, March, 1881, editorial, pp. 219-220...13.

Pansch, A.: 1.-Beitrüge zur Morphologie des Grosshirns der Säugethiere. Morphologisches Jahrbuch, $\nabla, 1879 \ldots 493,499-500,502$.

Parchappe: A.-Du cour, de sa structure et de ses mouvements. Paris, 1844 . 0., avec atlas in $\mathrm{Q} . . .316$.

Parker, W. K. : A.-A monograph on the shoulder girdle and sternum in the Vertebrata. Folio, pp. 337, 30 plates. Roy. Soc. Publ., London, 1868. . 152-153, 155, 162.

- 25.-On the structure and development of the skull in the pig (Sus scrofa). Roy. Soc. Proc., XXI, 1873, pp. 402-407; Monthly Microsc. Jour., X, 1873, pp. 140-144. ....174.

Parker and Bettany : A.-The morphology of the skull. O., pp. 368, 85 fig. Lon. don, $1877 \ldots .14,94,174,415$.

Pascoe, F. P. : A.-Zoological classification. 2d ed., 16mo. London, 1880...8. 
Pettigrew, J. B. : A.-The physiology of the circulation in plants, the lower animals, and in man. $0 .$, pp. 329, 151 fig. London, 1874. . .316, 36\%.

2.-On the arrangement of the muscular fibers in the ventricles of the vertebrate heart, with physiological remarks. (1863.) Phil. Trans., 1864, pp. 445-500...316.

Pye-Smith, H. : 1.-Suggestions on some points of anatomical nomenclature. Jour. Anat. and Phys., XII, pp. 151-175, Oct., 1877....14.

Quain : A.-Elements of anatomy. 8th edition. Edited by W. Sharpey, Allen Thompson and E. A. Schäfer. 2 vols., 0, pp. 1530, 1000 fig. London and New York, 1877... $22,94,143,152,154,157,160-163,166-167,169,172-174,180,184,188,192,206,212$, $214-215,219,221,223,229,231,235,238,247,250,252,254-260,263,265-272,280-281$, 283, 285-287, 293-297, 302, 303-304, 307-308, 310-311, 316-317, 328, 331, 339, 342, 347, 354, 361, 362-363, 372, 379, 382-387, 394-395, 399-400, 402, 404-406, 413-415, 467, 472-491. $507-509,512-514,520,522,526,528$.

Ranvier, L. : A.-Traité techuique d'histologie. O., pp. 976, 324 fig. Paris, 18751882 (incomplete)... . 399, 512.

Reichert, C. B. : A.-Der Bau des menschlichen Gehirns. Zweite Abtheilung. 0., pp. 192, 9 plates and 10 fig. Leipzig, 1861...405-406, 467.

Roberts, M. J. : 1.-Museums as educational adjuncts to medical colleges. American Veterinary Review, June, July and Aug., 1882. (This paper should have been referred to on p. 136.)

Robinson, F. E. : A.-A table of the crania] nerves....510.

Rolleston, George ; A.-Forms of animal life ; being outlines of zoological classification based upon anatomical investigation and illustrated by descriptions of specimens and figures. $O$, pp. 268, 12 plates. Oxford, $1870 \ldots 2,57,405$.

B.-The Harveian oration for 1873. D., pp. 90, 4 fig. London, 1878. . . 22, 316, 330.

13.-On the homologies of certain muscles connected with the shoulder-joint. (1868.) Linn. Soc. Trans., XXVI, 1870, pp. 609-630....192.

- 1.- Note on the preservation of encephala by zine chlorid. Jour. Anat. and Phys., XIII, part 2, pp. 232-233, Jan., 1879....435.

Royal Society's Catalogue of Scientific Papers: Compiled and published by the Royal Society of London. Q., 1800-1873, 8 vols....3.

Sabatier, A. : A.-Etudes sur le cour et la circulation centrales dans la serie des vertéḅrés. Montpelier, 1873....316.

Sanderson, J. B. (editor) : A.-Hand-book for the physiological laboratory. O., text, pp. 585 ; atlas, 123 plates. Phila., 1873. Reprint of the English edition with slightly different paging. A single volume edition has been published since. . .418.

Sanders : 1.-Contributions to the anatomy of the central nervous system in vertebrate animals. London. Pp. 44, 8 plates....24.

Schöbl, J. : 10.-Ueber die Nervendigung an den Tasthaaren der Säugethiere so wie über die feinere Structur derselben. Prag, Abhandl, V, 1872. Archiv mikrosk, Anat, IX, 1873, pp. 197-219...512.

Schofield : 1.-Taste goblets in the dog and cat. Jour. Anat. and Phys., April, 1876, p. 475,1 plate. ...513.

Sedgwick, W. T. : 1.-The functions of the semilunar valves of the aorta. N. Y. Med. Ree, May 28, 1881....326.

Serres, E. R. A. : A.-Anatomie comparée du cerveau dans les quatre classes des animaux vertébrés appliquées à la physiologie et à la pathologie du system nerveux. 2 vols., o. Paris, 1824-27... 493. 
Smith, E. N .: A.-The descriptive atlas of anatomy : A representation of the anatomy of the human body. Q., 92 plates, with Index. Phila., 1880 .. 316-317.

Spitzka, E. C. : 1.-The central tubular grey. Jour. of Nerv. and Mental Disease, April, 1880, p. 40...22, 400, 475 .

- 2.-Notes on the anatomy of the encephalon, especially the larger ganglia. "Science," II, 14, Jan. 15, 1881. (This should have been referred to in $\$ 1325$.)

- 3.-The peduncular tracts of the Anthropoid apes. Jour. of Nerv. and Mental Disease, p. 27, July, 1879...478, 482.

4.-The higher ganglia of the mid and hind brain. Jour. of Nerv. and Mental Disease, July, $1880 \ldots . .480$.

- 5.-The brain of the Iguana. Ibidem. Reprinted in N. Y. Med. Record, 1880. $\ldots 480$.

6.-The architecture and mechanism of the brain. Preliminary considerations. Jour. of Nerv, and Mental Disease, 1878...405-406, 473, 478.

- \%.-Letter on the nomenclature of the brail. "Science," April 9, 1881, pp. $165-166 \ldots .11,22,473-475,478,481,484-486,489$.

11.-Some new features of the corpora quadrigemina. Medical Record, Mar. 13,1881 , pp. $282-284 \ldots . .480$.

14.-Organolingy of the island of Reil. Proc. of N. Y. Neurological Soc., Jan. 6, 1879 ; reported in N. Y. Med. Record, May 25, 1879... (This should have been referred to in $\$ 1247$.)

Spurzheim, J. G. : A.-The anatomy of the brain. $2 d$ Amer. ed. 1886. O., pp. 244, 18 plates .. 418, 493 .

Stieda, L. : 22.-Studien über das centrale Nerven-system der Wirbelthiere. Zeits. wissen. Zool., 1870, S. 273-456, Taf. 17-20...410-412, 473.

Stirling, Wm. : 1.-Contributions to the anatomy of the cutis of the dog. 2 plates. Jour. Anat. and Phys., Vol X, 1876, pp. 465-474. ‥512.

Stowell, T. B. : 1.-The vagus nerve in the domestic cat (Felis domestica).-Amer. Phil. Soc. Proc., XX, pp. 123-138, 4 plates. Read July 15, 1881; publ. July, 1882 . . .326, $387,389,394,464,493,509,534$.

Straus-Durckheim, Hercule: A-Anatomie descriptive et comparative du chat, type des mammifères en general, et des carnivores en particular. 2 vols., O., pp. 1020, with folio atlas of 25 plates. Paris, $1845 \ldots .14,87,90,94,152,157,159-163,167-169,172-173$, $182,185,189,193-194,207-208,212,214-217$, 219, 221, 223, 225-226, 229, 231, 235-236, 238-241, 243-244, 247, 250, 252, 254-260, 268-271, 311, 313, 514, 525, 528.

B.-Traité pratique et thenretique d'anatomie comparative, comprenant l'art de dissequer les animaux de toutes les classes et les moyens de conserver les piéces anatomiqucs. 2 vols., D., pp. 870, 4 plates. Paris, 1842...79, 111, 148, 192, 316, 342.

Stricker : A.-A manual of histology. O., pp. 1106, $431 \mathrm{fig}$. Translated from the German. New York, 1872....280, 281, 283, 28̄̄-287, 293-296, 303, 304, 306, 308, 311, 316, 362-363, 399-400, 472, 475-476, 482-484, 487, 490, 507, 512-514, 526.

Tait, P. G. : 1.-On the formula of evolution. Nature, Nov, 25, 1880. [Repr. in "Science," Dec. 31, No. 26.]...12.

Thacher, J. K. : 1.-The mesial and paired fins of fishes. Trans. of the Conn. Acad. of Sciences....23, 26.

Tiedemann, F. : A.-The anatomy of the foetal brain, with a comparative exposition of its structure in animals. D. Edinburgh, 1826 ...492-493.

Todd, R. E.: A.-Cyclopædia of anatomy and plysiology. 5 vols., 0. London, 1835-1859...413-415, 472 . 
Treasury of Natural History : By Maunder; revised by T. S. Cobbold. 6th ed. D., pp. 798, 900 fig. New York, 1872....111.

Turner, W. : A.-Article Anatomy. Encyc. Brit., 9th ed., Vol. I, 1875, pp. 799-908. ....23, 316 .

Turner, H. N. : 4.-Observations on the distinctions between the cervical and dorsal vertebræ in the class Mammalia. Zool. Soc. Proc., XV, 1847, pp. 110-114; Froriep, Notizen VIII, 1848, Col. 177-180...174.

United States Dispensatory, 13th ed....112, 136.

Vicq d'Azyr: A.-Traité d'anatomie et de physiologie, avec des planches coloriées représentant au naturel les divers organes de l'homme et des animaux. Folio. Paris, $1786 \ldots . .14,479-480,505$.

Von Baer: A.-Entwickelungsgeschichte der Thiere. 1828-37...405.

Wadsworth, O. F.: 1.-Report on the decussation in the chiasma. Bost. Med. and Surg. Jour., May 6, 1875, pp. 528-529... .475.

Ward : A.-Article Saliva in Todd's Cyclopædia of anatomy and physiology, IV, pp. 415-433....302.

Watts : A.-A dictionary of chemistry and the allied branches of the sciences. $0 ., 8$ vols., many figures. $1866-81 \ldots . .112,136$.

Welcker, H. : 1.-Zwei Hülfśsmittel bei Demonstration des Gehirns und des Herzens. Archiv für pathologische Anatomie, LXXIV, 1878, pp. 500-504, 1 plate ; Jour. Anat. and Phys. (wax casts of the ventricles of the brain), XIV, 1879, p. 144....435. (Unfortunately this very suggestive paper was known to us only by the Abstract until after p. 435 was in type.)

Westbrook, B. F: : 1. - Some points in the anatomy of the encephalon. Annals of the Anat. and Surg. Soc. of Brooklyn, N. Y., Vol. II, 1880, pp. 387-854....414, 482.

Wilder, B. G.: 1.-Contributions to the comparative myology of the chimpanze日 (Troglodytes niger). Boston Jour. Nat. Hist, VI, pp. 354-384, 1861....192-193, 269.

4.-On morphology and teleology, especially in the limbs of mammalia. (1863.) Boston Soc. Nat. Hist., Mem. I, 1866-69, pp. 46-80...192, 269.

- 5. - On the morphological value and relations of the human hand. Amer. Jour. Sci., XLIV, 1867, pp. 44-48....92.

- 10.-Intermembral homologies, the correspondence of the anterior and posterior limbs of vertebrates. Boston Proc. Nat. Hist. Soc., XIV, pp. 154, 5 figures.... 19, $37,40,42,92,167,169,192,194$.

11. - The outer cerebral fissures of mammalia, especially the carnivora, and the limits of their homologies. Amer. Assoc. Proc., XXII, 1873, pp. 214-233, 4 plates.... $57,462,465,481,491,493-496,498-500$.

12.-Cerebral variation in domestic dogs and its bearing upon scientific phrenology. Amer. Assoc. Proc., XXII, 1873, pp. 234-249....21, 56.

13. - Lateral asymmetry in the brains of a double human monster. Amer. Assoc. Proc., XXIL, 1873, pp. 214-234, ...21.

17.-The need of a uniform position for anatomical figures. Amer. Assoc. Proc., XXII, 1873, p. 274....31, 37.

18. - Lateral position of the vent in Amphioxus and in the larva of Rana pipiens (catesbiana). Amer. Assac. Proc., XXII, 1873, pp. 275-300, 10 fig...21.

- 19.-On the composition of the carpus in dogs. Amer. Assoc. Proc., XXII, 1873, pp. 301-302, 3 fig....161.

20. -The pectoral muscles of mammalia. Amer. Assoc. Proc., XXII, 1875, pp. $305-307 . . .18,192-195,235$.

21.-Variation in the pectoral muscles of domestic dogs. Amer. Assoc. Proc., XXII, 1873 , p. $308 \ldots . .56,193$. 
Wilder, B. G.: 1.-Should comparative anatomy be included in a medical course? New York Medical Journal, Oct., 1877 . . . 324.

- 2 .-The anatomical uses of the cat. N. Y. Med. Jour., Oct., 1879, p. 16. ...53, $56-58$.

3.-The foramina of Monro: some questions of anatomical history. Boston Med. Surg. Jour., CIII, Aug. 12, 1880, 2 pages....18, 57, 488.

4.- Notes on the anatomy of the cat's brain. Read at the meeting of the Amer. Assoc., 1879 ...57, 414.

- 5.- On the foramina of Monro in man and the domestic cat. Read at the meetiag of the Amer. Assoc., 1880. Partly reported in the Boston Daily Advertiser, Iug. 30,1880 , and in the N. Y. Med. Record, Sept. 18, 1880 ...57, 407 .

- 6.-A partial revision of the nomenclature of the brain. (Same as No. 5.)...57.

- 7 .-On the crista fornicis, a part of the mammalian brain apparently unob. served hitherto. (Same as No. 5.)....57, 476 .

8.-The cerebral fissures of the domestic cat (Felis domestica). "Science," I, No. 5, July 31, 1880, pp. 49-51, 2 figures. . .57, 493, 495, 499-500.

9.-A partial revision of anatomical nomenclature, with especial reference to that of the brain. "Science," II, No. 38, pp. 122-126; No. 39, pp. 133, 138 ; Mar. 19 and $26,1881 \ldots .11-13,18,25,27,37,57,405-407,414,472,474,477,480-481,483,486-487,489$.

10.-The two kinds of vivisection, sentisection and callisection. Medical Record, Aug. 21, 1880, p. $219 . \quad$ Reprinted in "Nature," Sept. 30, 1880, and in "Science," Oct. 23 , p. $210 \ldots 80$.

- 11.-How to obtain the brain of the cat. "Science," II, No. 41, April 9, 1881, pp. $158-161 \ldots .18,67,428$.

12.-Criticism of Spitzka's "Notes on the anatomy of the encephalon, etc." "Science," No. 31, p. 48, Jan. 29, 1881. (Embodies a statement as to the dorsal limits of the diaccelia.)....57.

13.-On the brain of a cat lacking the corpus callosum. Presented at the meeting of the Amer. Assoc. Adv. Sci., 1879, but not yet published....21, 57, 474.

14.-The brain of the cat (Felis domestica). A preliminary account of the gross anatomy. Amer. Phil. Soc. Proc., XIX, pp. 524-562, 4 plates. Read July 15, 1881; publ. Dec., 1881...57-58, 405-406, 414, 461, 472, 474-477, 480-481, 483, 486-487, 489, $493,496,498-500,508,584$.

15.-On a mesal cusp of the deciduous mandibular canine of the domestic cat (Felis domestica). Amer. Assoc. Proc., 1881, p. 242 ...189.

- 17.-On a method of collecting and arranging information. Boston Proc. Nat. Hist. Soc., May 15, 1867, p. 242...52.

- 21.-Frozen sections of the cat preserved in alcohol. Read at the Amer. Assoc., Aug., 1879....131.

22.-On the brains of Amia, Lepidosteus, Acipenser and Polyodon. Amer.

Assoc. Proc., XXIV, 1874, pp. 168-185̄, 2 plates. . . 410.

23.-An apparatus to show the action of the diaphragm in respiration. Boston

Soc. Nat. Hist. Proc., XX, 1870, p. 50....310.

24.-Note on the ectal (" apparent") origin of the $\mathrm{N}$. trigeminus in the cat. Amer. Jour. Neurology and Psychiatry, I. ...508.

_- 26. - On the brains of some fish-like vertebrates. Amer. Assoc. Proc., 1876, pp. 3, 5 fig....587.

Williams, H. S. : A.-The bones, ligaments and muscles of the domestic cat. $\quad 0 ., p p$. 86, with atlas of 12 folio plates. Copies, reduced one third, of the outline plates in StrausDurckheim's A. The text is an explanatory index. New York, 1875...14. 
Williams, H. S. : 1.-Comparison of the muscles of the chelonian and human shoul. der girdles. (1872.) Conn. Acad. Trans., II, 1871-73, pp. 301-308. ...154.

Williams, T. : A.-Article Respiration in Todd's Cyclopædia of anatomy (q. v.). ...310.

Witt, G. : A.-Compendium of osteology ; appendix on preparation of bones. Folio. London, $1833 . .111$.

Wood, J.: 7.-On human muscular variations and their relation to comparative Anatomy. Jour. Anat. and Phys., I, 1867, pp. 44-59...194.

- 9.-On a group of varieties of muscles of the neck, shoulder and chest, with their transitional forms and homologies in the Mammalia. (1869.) Phil. Trans., CLX, 1870, pp. 83-116 ; Roy. Soc. Proc, XVIII, 1870, pp. 1-3...215, 217, 223, 231.

Wyman, Jeffries : 34.-Anatomy of the nervous system of Rana pipiens. Smithsonian Contributions to Knowledge, V, Art. 4, 1855 . Q, pp. 51, 2 plates....2, 400, 402, $411,413,418-419,421,505$.

47.-Dissection of a black chimpanzee (Troglodytes niger). Boston Proc. Nat. Hist. Soc., V, 1854-56, pp. 274-275. . . 193.

- 75.-On symmetry and homology in limbs. (1867.) Boston Nat. Hist. Soc. Proc., XI, 1868, pp. 246-278. . .92.

76.-Observations on crania. Boston Nat. Hist. Soc. Proc., XI, 1868, pp. 440 462... 174, 191.

Young, A. H. : 1.-Myology of Viverra civetta. Jour. Anat. and Phys., XIV, pp. 166-177, Jan., $1880 \ldots 194$.

In addition to our obligations to many of our laboratory students for useful hints upon various points, we feel especially indebted to those named below, who have at our suggestion investigated special parts of the cat's anatomy for their graduating Theses :-

Collins, Homer, class of '82: The brachial flexor muscles in man, lion and cat.

Curtice, F. C., class of '81: A study of the tracheal region of the cat.

Dounce, G. A., class of ' 79 : Comparative myology of the shoulder of man and the cat.

Hoag, W. I., class of '81: On the masseter and temporal muscles in the cat.

Manierre, C. E., class of ' 80 : The fourth cranial nerve in man and the cat.

Schenck, H. D., class of ' 82 : The distribution of the nerves to the museles of the eye in the cat.

Smith, Theobald, class of ' 81 . The peritoneum and its principal blood vessels in the cat.

Young, J. H. W., class of '79 : Comparative myology of the arm in man and the cat. 


\section{N D E X.}

In the following Index, technical words and words used in a technical sonse are accented with the principal accent. In this, Webster's Dictionary has been followed whenever possible. There is also herein given the etymology of the technical terms not found in the last edition of Webster's Unabridged Dictionary.

\section{Abbreviations for Parts of the Brain, $436-438$.}

Abdo'men, a division of the trunk, 36.

ABDOM'INAL and thoracic viscera, lines of incision for exposing, 274 .

blood vessels, 355 .

transection, how to do, 98-101.

viscera, 273-297.

Abdu'cens nerve, origin and distribution, 508 .

Absorbent cotton, 62 .

ACCESSORY or spinal accessory nerve, origin and distribution, 509 . parotid glands, 302.

Accuracy, limits to in description, 27 .

Acous'tic or auditory nerve, origin and distribution, 508

Actions of muscles, 202 .

Action of the muscles of the eye, 5019.

Ad'ipocere, 105.

Adjective and adverbial forms, 27.

Adrena'le, 295.

ADVERBIAL and adjective forms, 27. ending, 27.

Air cells of lungs, 310 .

$\triangle L^{\prime} B A$, functions of, 371 .

structure of, $398-399$.

white nervous matter, $369,472$.

Al'bicans, 472 .

$\mathrm{AL}^{\prime} \mathrm{COHOL}$, a perfect preservative, 113.

absolute, 112 .

admission of into abdomen, 118.

amount required for different purposes, 121.

anatomical uses of, 112-124.

as a deodorizer, 83 .

care of, 113.

changing the percentage of, 115-116.

coagulation of tissues by, 117 .

cost of, 113.

crystalline deposits in, 123.

deterioration of, 122.
AL'COHOL, determination of percentage of, 114.

economics of, 117 .

filtration of for clearing, 122.

filtration of for deodorization and decoloration, 123 .

how to obtain free of tax for scientific purposes, 113, 535 .

inflammability of, 114.

injection of into arteries, 119.

injection of into hollow viscera, 119.

injection of into lungs, 119.

injec on of into thorax and abdomen, 118.

its importance and general character, 112.

measures accessory to immersion in, 117-119.

methyl, or wood spirit, 124.

percentage of required for brains, etc., 120.

percentage of required for ligaments, etc., 121.

percentage of required for muscular organs, 120.

precautions against fire from, 114.

purification of, 122-123.

reduction of with water, 116 .

removing from hair, 131 .

settling of for clearing, 122.

special characters of, 113.

specific gravities of various grades, 112.

strengthening of, 123 .

use of appropriate grades of, 117.

vapor as a preservative, 123.

varieties of, 112 .

Alcoholom'eter (alcoömeter), 114.

Alcoöm'eter (alcoholometer), 114.

Alisphe'noid bone (Latin, ala, a wing, and

Greek, $\sigma \phi \eta \nu$, a wedge, eidos form), 181.

$\mathrm{Al}^{\prime}$ veolar margin of the jaw, 184. 
Al'veus, lobulus bypocampæ, 481.

American Metric Bureau, 5.

Amphibia, 8.

Amphibian brain, table of parts, 409.

Amphiox'us lanceola'tus, 10 .

AMPULLA OF VA'TER, 292.

figure of, 291.

Anæsthet'ic box and figure of, 81 .

Analogies and differences of vascular and nervous systems, $371-372$.

Anapoph'ysis (avá, on, and $\phi v e \iota v$, to grow or be produced), 172 .

ANATOM'ICAL instruments and material, 59.

Pbilistinism, forms of, 204.

ngles of the eyelids, 514 .

Angular gy'rus, 501.

An'gulus mandibula'ris, 188.

Animal charcoal, 83.

Animal charcoal for filtration of alcohol, 123.

An'nulus abdomina'lis exter'nus and inter' nus, 297.

ANTERIOR fissure of the spinal cord, 478. perforated space, 485 . pillar of the for' nix, 476 .

AOR'TA, 388.

abdomina'lis, 358.

abdomina'lis, injection of, 147.

and its principal thoracic branches, figure of, 347 .

thorac'ica, 350 .

Apex of heart, 326.

APHORISMS for dissectors, 197-198. general, 52-54.

respecting nomenclature, 12.

Aponeuro'sis, an undesirable name, 196.

Append'ages of the skin, 512.

Appendic'ular parts of the body, 25.

$\triangle{ }^{A}$ ZADDUC'TUS coch'leæ, 531 . Syl'vii, 482 .

$A^{\prime}$ 'queous humor and aqueous chambers of the eye, 522.

Arachnoi'dea, 413, 472.

$\Delta \mathrm{r}^{\prime}$ bor vi'tæo, 472 .

Arched $\mathrm{gy}^{\prime} \mathrm{ri}, 500$.

$\mathrm{AR}^{\prime} \mathrm{CU}$ S bicipita'lis, 245. neura'lis, 172.

A $^{\prime}$ REA crura'lis, 473. crura' lis, diagram of, $44 \%$.

ellip'tica, 473 .

intercrura'lis, 478.

ova'lis, 473 .

postponti'lis, 473 .

præcbiasma'tica, 473.

septa'lis of brain, 473.

Areas and lines of muscular attachment, 195.

Areom'eter, 114.

ARM, cephalic or anterior limb, 29, 30.

muscles of, witb figures, 225-271.

Arrangement of cranial nerves, 506 .

ARTE'RIA adre'no-lumba'lis, 359.

anastomo' tica mag'na, 354 .

axilla' ris, 352.
ARTE'RIA brachia'lis, 358.

bra'chio-cephal'ica s. innomina'ta, 350.

bronchia'lis, 351 .

cardi'aca, 326.

carotidæ' $^{\prime}$, figures of, $345,347,392$.

carotidæ'a, injection of, 148.

circumflex'a, 353.

circumflex' $\mathrm{a}$ anterior, 359 .

coeli'aca, 358.

co'lica dex'tra, 359.

co'lica me'dia, 359.

co'lica sinis'tra, 360.

corona'ria ventric' uli, 359.

cyst $^{\prime}$ ica, 358 .

epigas' trica, 361.

femora'lis, injection of, 142.

gas't ro duodena'lis, 358.

gas'tro-epiplo'ica dex'tra, 358.

hæmorrhoida' ${ }^{\prime}$ lis supe'rior, 360 .

hepat'ica, 358.

hypogas' trica, 361 .

il' eo-co'lica, 359.

il'eo-lumba'lis, 260 .

ili'aca exter'na, 361 .

ili'aca inter'na, 361 .

intercosta' ${ }^{\prime}$ lis, 351 .

interccsta' lis supe'rior, 351.

in teros'seus ante'rior, 354 .

interos'seus poste'rior, 354 .

lumba'lis, 351,362 .

mesenter'ica infe'rior, 360.

mesenter'ica supe'rior, 359.

nu'triens, 354 .

œesophagæ'a, 351 .

ova'rii, 360 .

pancreat'ico-duodena'lis, 359.

pancreat'ico-duodena'lis inferior, 359.

pericardi'aca, 351.

phren'ica, 358.

profun'da supe'rior, 354.

pulmona'lis, 326,337 .

pylor'ica, 358.

radia'lis, 354 .

radia'lis recur'rens, 354 .

rena'lis, 360 .

sa'cra me'dia, 361 .

spermat'ica, 360 .

splen'ica, 359.

sterna'lis \&. mamma'ria inter'na, $\mathbf{3 5 1}$.

subcla'via, 327 .

subcla'via, branches of, 351 .

subcla'via dex'tra, 351 .

subcla'via sinis'tra, 350.

subscapula'ris, 353.

suprascapula'ris, 352.

thorac'ica ante'rior, 352.

thorac'ica lon'ga, 352.

ulna'ris, 354.

ulna'ris recur rens, 354.

ventric'uli dorsa'lis, 30 9.

vertebra'lis, 351 .

vesica' ${ }^{\prime}$ lis supe'rior, 361.

Ar'teries, distinction from reins, 315 . 
AR'TERIES of the thorax and arm, 350 . of the trunk and arm, 34:3-344. structure of, 363 . to inject, 142.

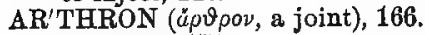
capitel'li, 171 . costicartilag'inis, 166.

il'io-sacra'le, 167.

mesosterne ${ }^{\prime}$ bri, 166. of ribs, 165.

tuber'culi, 1\%1.

AR'THROTOME ( $\dot{\rho} \rho \vartheta \nu \nu$, a joint, and $\tau \varepsilon \dot{\mu} \mu \nu \varepsilon \iota \nu$, to cut), 62 .

how to use, 201.

figure of, 66 .

Articula'tions of bones of skull, 176.

Aspects of a vertebrate, figures of, 30 .

Astrag'alus, 41.

Atlan'tal fora'men, 172.

At'las, 171.

Attachment lines and areas, 195.

Audi'tion, hearing, 511, 526 .

AU'DITORY apparatus, 526.

or acous'tic nerve. origin and distribution, 508 .

AU'LA (Latin, a court-yard), 472. and por'ta, 407, 454 .

in frog, 422 .

Auliplex' us (Latin, aula, a court-yard, and plexus, a twining or braiding), 473 .

Au'ricles of heart, section, 333-335, Fig. 93-96.

AURIC'ULA dex'tra, 327. sinis'tra, 327, 338.

Auricula' ${ }^{\prime}$ is mag' nus nerve, 301.

$\mathrm{AU}^{\prime} \mathrm{RIS}$ (Latin, the ear), 511, 526. bones of, 528 .

enta'lis, labyrinth' us, 529.

me'dia, 528.

Automat'ic nervous centers, 371.

Ax'ial part of body (soma), 25.

AX'IS, 171.

band or cylinder of nerve, 399 . coeli'aca, 358.

thyroi'deus, 352.

$A Z^{\prime}$ YGOUS and paired, meaning of, 32 .

lobe of the lungs, 310 .

Ball of the eye, globus oculi, 520.

Band axis of nerve, 399.

Barnard, F. A. P., 5.

BASE of brain, figure showing segments and nerve roots, 443.

of the heart, 327.

Basilar membrane of the $\operatorname{coch}^{\prime}$ lea, 593.

Basioccip'ital bone (Latin, basis, the base, and occiput, the back of the head), 181.

Basisphe'noid bone ( $\beta$ arıs, a base or pedestal, $\sigma \phi \dot{\eta} \nu$, a wedge, and $\varepsilon l \delta_{o \zeta}$, form), 181.

Batrachians, Amphibia, 8.

Beaded bristles, 63 .

Belly or body of a muscle, 195.
Benzine, 81.

Berge, J. and H., 59.

Bicip'ital arch, 245.

Bicus' pid valves, 329-330.

Bigem ${ }^{\prime}$ inum (Latin, double, twofold), 482.

Blackford, G., 475 .

Bleaching bones, 110.

Bleeding as preliminary to preservation, 117 .

Blind spot of retina, 522.

Blocks for dissecting, 64 .

Blood-vascular system, 315.

Blood-vascular system, subdivisions of, 315 .

BLOOD VESSELS, figure of general, 345.

in femoral region, figure of, 142 .

isolating and drying, 346.

metbod of connecting when severed, 511.

of the trunk, 342 .

of the trunk, figure of, 345 .

structure of, 362.

Blow-pipe, care of, 74,

figure of, 66 .

flexible, 64 .

Blue for injections, 139, 535 .

BODY cavities, dor'sal and ven'tral, 23.

figure of principal divisions, 38 .

its primary divisions, 29 .

of a vertebra, 172 .

or belly of a muscle, 195 .

planes of, 33

planes, figure of, 34.

table of principal divisions, 39 .

Bone nippers, 62, 67.

Bone, structure of, 191.

BONES, bleaching of, 110 .

cleaning by the liquid soap process, 106.

freeing them from grease, 110 .

maceration in water, 105.

of limbs, 40 .

of the ear, 528 .

of young animals, preparation of, 109.

preparation of, 103.

removing soft parts from alcoholic specimens, 105.

soft parts removed by ants or Dermestes, 105.

Borders of muscles, designation of, 197.

Bottle brush, figure of, 129.

Box, glass, figure of, 126 .

BRA'CHIAL plex'us, 378-387.

plex' us, figures and diagrams of, 374 , 378,381 .

Bra'chium pon'tis, medipedunculus, 481.

BRAIN, abbreviations of names, $436-438$. and its fissures, 494 .

and myelon, figure of dorsal view, 374 .

cavities completely closed, 414.

ce'liæ or cavities, 406.

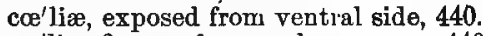

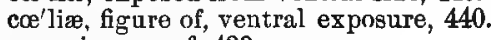
commissures of, 439 .

comparison to a house, 413.

definition of, 400 . 
BRAIN, exposure of, 377, 425 .

exposure by removing calva, 431.

figure of the base, showing segments, and nerve roots, 443.

figure of the mesal aspect, 446-447.

figure showing the relations of the cavities, 408.

figure of a transection through the diacolia, 458.

four plates of, with explanation, 461-471.

general considerations, 400.

hardening in alcohol, 429.

hardening with zinc chlorid, 435.

hemisection of, 444 .

ideal, simple, 408,409 .

ideal, simple, diagrams of, 408 .

importance and diffleulty of its study, 400.

injection of coliæ, 435 .

its form, 493.

killing animal for, 423.

macroscopic vocabulary of, $436-438$.

mesal aspect, showing segments, etc., 446-447.

methods of study, 400 .

names of parts, $403-404$.

names, synonyms and references, alphabetically considered, 471-491.

of Amphibia, dissection of, 420 .

of Amphibia, exposure of, $37 \%$.

of Amphibia, general inspection, 418.

of Amphibia, labeling, 418 .

of Amphibia, preservation, 418 .

of Amphibia, special methods, 423 .

of cat, removal, 423 .

of cat, segmental arrangement of names, 446.

of cat and frog, differences of, 439 .

of felines, figures by various authors, with comments, 492-493.

of frog, advantages for study, 402 .

of frog, exposure, 416.

of Menobranchus, description and dissection, $42 \%$.

of Menobranchus, exposure, 417 .

of young human subject, method of removal, 432.

plexuses, injection of, 436 .

removal after hemisection of the head, 432-433.

segments, 404-406.

segments, examination of, 450 .

segments in the cat, 439 .

showing dorsal aspect of mesencephaIon, etc., 441.

study for comparative anatomist and systematic zoologist, 401.

supporting with brine while removing, 428.

Table of parts in Amphibia, 409.

transferring to alcohol, 429 .

ventricles of, cœliæ, 406.

weighing, 429 .
Brain, weight determined from capacity of the skull, 191.

Brains, etc., percentage of alcohol required for, 120 .

Breneman, A. A., 123.

Brevity of technical terms, importance of, 15.

Brine for supporting brain, 125, 428.

Bristles, beaded, 63 .

Bron'chi, 311.

Bron'chial tubes, 311.

Bronchio'li, 311.

Brown, F. H., 8.

Bul'bus for'nicis, 472.

Bul'la tympan'ica, 185.

Cæ'cum and Ileo-creal Valve, 284-285.

Calca'neum, 41.

Cal'car (Latin, a epur) part of brain, 473.

CALLO'SUM (Latin, hard, firm), 439. corpus, of brain, 474 .

figure of its dorsal aspect, 442 .

Cam'era, fornix, 479.

CANA'LIS bicipita'lis, bicipital arch or groove, 158.

centra'lis, of myelon, 406, 474 .

cer'ebro-spina' lis, 370.

coch'leæ, 500 .

Eustachia'na, 185, 500.

Iachryma'lis, 179,500 .

neura'lis, 172, 370.

Schlem'mi, 500.

semicireula'ris, 500.

Canalic ${ }^{\prime}$ ulus (Latin, diminutive of canalis, a canal), 191.

Canalic'uli of bone, 191.

C'an'thi or angles of the eyelids, 514.

CAN'ULA, inserting and securing, figure of, 145 .

introduction into vessels, 144 .

of a syringe, character and preparation of', 138 .

CAPACITY of organs, determining, 135. of skull, determining, 191.

CAP'ILLARIES, general character of, 315 . structure of, 363.

CAPITEL'LUM (Latin, diminutive of caput, a head), 159.

$\cos ^{\prime}$ tæe, 166.

hu'meri, 159.

Cap'sula len'tis, 523.

$\mathrm{CA}^{\prime}$ PUT (Latin, a head), 159.

hu'meri articula're, 159.

Car'dia, heart, 316.

$\mathrm{CAR}^{\prime} \mathrm{DI} A \mathrm{C}$ arteries, 326. cavities, 323.

CARE of instruments, 73. of syringe, 137.

Cari'na for' nicis, 474.

Carmine solution for injection, 139.

Carot'id artery, injection of, 148.

CAR'PUS and tar'sus, 41. comparison with $\operatorname{man}, 41$. of lion and dog, figure of, 161 . 
Cat, advantages for study as compared with the dog, rat, rabbit, etc., 56 .

CATALOGUE, data for specimen, 46. slip box, figure of, 50 .

CA'TALOGUES, library, 46. of manufacturers, 59 .

$\mathrm{Cau}^{\prime} \mathrm{da}$ stria'ti, 474.

$\mathrm{Cau}^{\prime}$ dal and cephal'ic, use of, 23.

Cau'dal or tail vertebræ, 170.

Caul, omentum, 280.

CAVITIES of the body, 33 . of the brain, importance and advantage of for study, 401.

of the brain, communication with the exterinr, 401.

of the brain, completely closed, 414 .

of the brain, names of, 406,409 .

of the brain, their relation, 413 .

Ca'vum o'ris, 303.

Cel'la (Latin, a cell), me'dia, 474.

Cements for bones and teeth, 107.

Centigrade thermoneter, 3.

CEN'TRAL canal of myelon, $413,474$. tubular gray, 475 . use and significance of, 26 .

Cen'trum vertebra'le, 172.

Ceplual'ic and cau'dal, significance and use of, 23.

CEREBEL'LAR cine'rea, 475. fos'sa, 186.

CEREBEL'LUM, 474-475. lateral lobe of, 481 . of frog, 419.

CER'EBRAL fos'sa, 186. pedun'cles, 476.

Cer'ebro-spi'nal nervous system, 369, 372.

CER'EBRUM and its fissures, 493-503.

its form, 493 .

parvum, 474 . prosencephalon, 485 .

$\mathrm{CER}^{\prime}$ VICAL nerves, 373. vertebræ and determination of, 170.

Cer'vico-fa'cial division of the facial nerve, 301.

Cer'vix of a rib, 166.

Chain hooks, 65 .

Changes in terminology, objects and methods, 13.

Charcoal, animal, for filtering alcohol, etc., $83,123$.

Charrière scalpel, 69.

Chaussière, 15.

Chest, thorax, 308.

Chevron bones, 171.

Chias'ma ( $\chi$ iar/la, the mark of the letter $\chi$ ). $475,50 \%$.

Chloral hydrate as a preservative, 124.

Chloroforming animals, 80 .

Choice of origin and insertion of a muscle, 195.

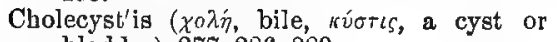
bladder), $277,286,289$.

Chor'da spermat'ica, 143.
Chor'da spina'lis, 372, 482.

Chor'dæ tendin'eæ, 327.

Cho'roid plex'us of lateral ventricles, 485.

Choroi'dea, 521.

Chyle vessels, 364 .

CIL'LARY muscle, 525.

plicæ or folds, 522 .

$\mathrm{Cim}^{\prime}$ bia, tractus transversus pedunculi, 475.

CINE'REA (Latin, of or like ashes), gray nervous matter, $369-370,475$.

functious of, 371 .

of the lorain, $4 \% 5$.

structure of, 398-399.

Clark, C. F., 64 .

Class Mammalia, 8.

Claseification of animals, Table of, 9.

Cla'va (Latin, a knotty branch), 475.

Clav'icle, clavic'ula, 56.

CLAVIC'ULA, clav'icle, 56.

determination of right and left, 150 .

of cat, figured, 162 .

Claw points, removal of, 102 .

Claws as appendages of the skin, 512 .

CLEANING bones, 103-111.

canula and syringe, 146.

Cleanliuess, precautions for, 81 .

Cleavage of muscles, 195.

Cleaves, Prof. E. C., vi, xxi.

Clements, Miss G. D., xxi, 461.

Clipping the hair, 204.

Coagulation of tissues by a]cohol, 117.

Coarse injections of blood vessels, $137-148$.

Coats of the eye, 521-522.

Coccyge'al nerves, 373.

COCH'LEA, 529.

canal of, 531 .

scalæ and spiral lamina of, 529.

transection of a coil of, 533 .

Codmun and Sluurtleff, 79.

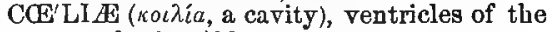
brain, 406.

relations of, 413 .

ventral exposure of, with figure, 440 .

Co'lum, trunk ravity, 37.

Col'lum, neck, 39, $30 \%$.

Co'lon, 285.

Colors for injections, 139.

COL[M' ${ }^{\prime} \mathrm{NA}$ dorsa'lis (myel'onis), 475.

for'nicis, 475 .

latera'lis (myelonis), 476.

poste'rior, 475 .

ventra'lis (myelonis), 476.

vertebra'lis, 169-173.

Colum'næ car'neæ, 327 .

Columns of alba in the myelon, 370.

Combination of words, 28.

COMMISSU'RA ante'rior, præcommissura, 484.

for'nicis, 476.

habena'rum, 476.

mag'na, callosum, 474.

me'dia s. mol'lis: medicommissura, 481. 
Commissu'ra poste'rior, postcommissura, 484.

$\mathrm{Com}^{\prime}$ missures of the brain, 407, 439 .

Common ventricular cavity, aula, 472-473.

Compound words, connecting vowel in, 28.

Compress'or, figure of, 65.

Conarial tube, $\$ 15$.

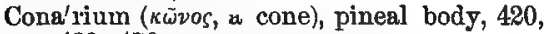
439. 476.

Conductive action of nerves, 371.

$\mathrm{CON}^{\prime}$ DYLUS mandibula'ris, 189. occipita'lis, 183.

Conjuncti'va, 514.

Connect, definition of term, 197.

Co'nus arterio'sus, 337 .

Copperas as a deodorizer, 83.

Co'rium, derma, cutis vera, true skin, 512.

Corks and rubber stoppers, 126.

Cor'nea, 521.

COR'NU Ammo'nis, hypocampa, 480. ante'rius ventric'uli latera'lis, præcornu, 484 .

me'dium s. descen'dens, medicornu, 481.

poste'rius, postcornu, 484.

Cor'onal suture, 183.

COR'PUS al'bicans, 472.

bigem'inum ante'rius, optici, 482.

bigem'inum poste'rius, postoptici, 484 .

callo'sum, 474.

can'dicans, albicans, 472.

fimbria'tum, fimbria, 478.

genicula'tum exter'num, 484.

genicula'tum inter'num, 484.

mammilla're, albicans, 472 .

pinea'le, conarium, $420,439,476$.

pituita'rium, hypophysis, 480 .

pyramida'le, pylamis, 485.

stria'tum, 488 .

trapezoi'des, trapezium, 490.

vit'reum, 522.

Corpus'cula Paci'ni. 281.

COR'TEX (Latin, a rind), 440.

cer'ebri, 440. 475 .

of the encephalon, 476 .

Cor'ti, position of the organ of, 533 .

Cos'ta, rib, 167.

Cos'tal car' tilage, costicartila'go, 167.

COTTON, absorbent, 62 .

filtration of alcohol through, 122.

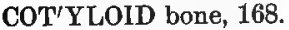

fos'sa, 168.

CRA'NIAL fos'sæ, 186.

NERVES, 371.

comparison with spinal, 504.

method of demonstration, 511.

numbering, 505 .

physiological arrangement, with Table, 506.

Table of origin, exit, distribution and functions, 510 .

Table of synonyms, 505 .

Cra'nium and face, 174.
Crib'riform plate of the ethmoid, 188.

CRIS'TA (Latin, a crest) deltoi'dea hu'meri, deltoid ridge, 159.

for'nicis, 476 .

il'ii, 167 .

lambdoida'lis, 179.

pectora'lis hi'meri, pectoral ridge, 159. tempora'lis, 179.

CRU'CIAL FISSURE, constant and variable characters, 498-499.

its homology and formation, 500-502.

$\mathrm{Cru}^{\prime}$ ral area, 473.

CRUS ante' rius medul'læ oblonga'tæ, 476.

cerebel'li supe'rius, præpedunculus, 485.

ce' rebri, 476.

e cerebel'lo ad medul'lam, postpedunculus, 486.

for'nicis ante'rius, 476 .

me'dium, medipedunculus, 481.

olfacto' rium 8 . rhinenceph'ali, 476 .

Crys'talline lens, 522.

Cuboi'des, 41.

Cu'neiforme, 41.

Curtis, Miss I. M., xxi.

Cusps of deciduous canines, $56,188$.

C'u'ticle, epidermis, 512.

CU'TIS, skin, 512.

ve'ra, derma, true skin, 512.

\section{DECAPITA'TION of a Cat for the} Brain, 423.

of a frog or Menobranchus, 415.

Decimal systems, 3 .

Dee, R, 536 .

Del'ta for'nicis, $47 \%$.

Del'toid ridge of the humerus, 159.

Deo'dorizers, 83.

Derivative words, formation of, 28.

Der'ma ( $\delta_{\varepsilon}^{\prime} \rho \mu \alpha$, the skin), cutis vera, true skin, 512.

DESIGNATION OF organs, organonomy, 14.

position and direction, toponomy, 20, 34.

the cranial nerves by numbers, 505 .

DETERMINATION OF muscular homologies, 196.

right and left, 149.

right and left with entire limbs, 151.

the capacity of the skull, 191.

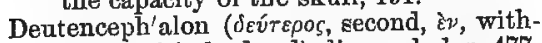
in, $\kappa \dot{\phi} \phi \lambda \dot{\eta}$, the head), diencephalon, 477 .

Dex'tral and sinis'tral, use of, 24 .

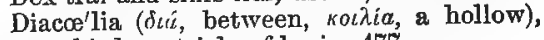

third ventricle of brain, $47 \%$.

Diagonals of solids, designation of, 35.

Diagrams of ideal, simple brain, 408 .

DIAPHRAG'MA, 277, 281, 311.

figure of calidal or peritoneal surface, 313.

partition between thorax and abdomen, 37. 
Diaphrag'ma, tendons of, 311 .

Diaph $^{\prime}$ ssis ( $\delta \iota \dot{\alpha}$, througb or between, and фúeıv, to grow or be produced), humeri, shaft of a bone, 158, 159.

Diaplex'us ( $\delta i \dot{\text {, }}$ through or between, and Latin plexus, a twisting or braiding), plexus of third ventricle, $422,47 \%$.

Diapoph'ysis of a vertebra, 172.

Diate'la ( $\delta c x$, through or between, and Latin tela, a web), 477 .

DIENCEPH'ALON ( $\delta u \dot{u}$, between, $\dot{\varepsilon} v$, within, and $\kappa \varepsilon \phi \pi \lambda \eta$, the head), $47 \%$.

examination, 452 .

in Amphibia, 420.

Differences of brain of cat and frog, 439 .

Direct lines, 34-35.

DIRECTION AND POSITION, designation of, 20,34 .

on direct lines, 35.

on oblique lines, 35 .

Disarticulating skulls, 109.

Display of alcoholic specimens, 129 .

DISSECTING gown, 65.

instruments, how to use, 199-201.

DISSECTION a fine art, 193.

of muscles, instruments and materials for, 198 .

of muscles, preparation of cat for, 198.

of the amphibian brain, 420 .

of the heart, 336 .

of the brain, $450-457$.

wounds, 85.

Dissections by beginners should not be published without careful examination, 193.

DISSECTORS, aphorisms for, 197-198. practical suggestions for, 201-203.

Dis'tal, significance and use of, 25 .

DIS'IINGUISHING the groups of vertebræ, 170.

vessels from nerves, 375 .

Dividers, duplicating, $6 \%$.

DIVISIONS of the body, figures of, 30 , 38.

of a muscle, 195 .

of the eensory nerves, 506 .

DOR'SAL and ven'tral, use of, 24 .

or thoracic vertebræ, and determination, 170.

or upper eyelid, 514.

primary division of myelonal nerves, 373.

primary division of nerves, demonstration, 377.

Double knot, 144.

Drawing materials, 67 .

Drills, 67.

Dropping-bottle oiler, figure of, 535 .

Drowning animals, 80 .

Drying to be avoided in dissection, 203.

DUCT of the parotid gland, 301 .

of the submaxillary gland, 302 .

Ducts of salivary glands, preparation, 298.
DUC'TUS arterio'sus, 328.

choled'ochus commu'nis, 287.

cyst'icus, 287.

hepat'jici, 287.

lachryma'lis, 516 .

Santorini, 292.

Stenonianus, 301.

thorac'icus dex'ter, 365.

thorac'icus in sections of thorax, 342.

thorac'icus sinis'ter, 364 .

thorac'icus sinis'ter, figure of, 366 .

Whartonianus, 302.

Wirsungianus, 290.

Duode'num, 283.

DU'RA (mater), 477.

removal from myelon and brain, 379 , 428.

EAR, Auditory Apparatus, 526-533.

auris, bones of, 528 .

diagram of, 532 .

ectal or external, 526 .

ental (internal), labyrinthus, 529.

middle, or tympanum, 529.

muscles of, 528,529 .

semicircular canals, tracing of, 530 .

Ear-pocket, 56.

EC'TAL ('́kтós, ontside, external), $2 \%, 36$.

and ental and their derivatives, 36.

and ental, significance and use, 27.

muscles of neck and shoulder, figure of, 211.

or external ear, 526 .

Elimination of slips, 47 .

Em'bryos, percentage of alcohol required for, 120.

Emery, 76.

EMINEN'TIA audito'ria, 477.

lenticula' ris, 488.

mag' na cer'ebri, 490.

ENCEPHAL'IC cavities, names of, 406 .

segments, 404-406.

segments, examination of, 450 .

segments in the cat, 439 .

segments, unequal value of, 406 .

ENCEPH' ${ }^{\prime}$ LON, definition, 400.

general considerations, 400 .

vocabulary of parts, $436-438$.

Endocar'dium, 362.

En'dyma ( $413,478$.

ENT'AL ('cotós, within, internal), 27, 36.

and ectal and their derivatives, 36 .

and ectal, significance and use, 27.

En'terotome, 70.

Enumeration of parts in series, 42.

EPENCEPH'ALON ( $\varepsilon \pi i$, upon, $\dot{k} r$, within,

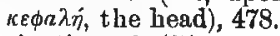

examination of, 451 .

Epen'dyma, endyma, 478 .

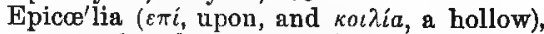
fourth ventricle, 478.

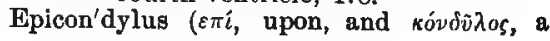


knob or head), external condyle of humerus, 160.

Epider'mis, cuticle, 512:

Epip'loon, 278, 280.

Epipodial'ia ( $\varepsilon \pi i$, upon, $\pi$ ov́s, a foot), 41.

EPITROCH'LEA ( $\varepsilon \pi i$, upon, and r $\rho \circ \chi \tilde{a} \lambda \hat{i} a$, a pulley), 158, 160 .

internal condyle of humerus, 160.

Epitroch'lear fora'men, 160.

Ergot, hippocainpus minor, calcar, 473 .

ERRORS of interpretation in dissection, 194.

of manjpulation in dissection, 193.

Ether, inflammability of, 114.

E'therizing animals, 80 .

EUSTACHIAN canal, 185, 528. valve, 330 .

Examination of brain segments, 450 .

Exhibition and storage of specimens, 125126.

EXPOSURE OF brain, 425.

frog's brain, 416.

heart, 319.

the salivary glands and their ducts, 299 .

EXTERNAL abdominal ring, 297.

auditory meatus, 184.

condyle of the humerus, 160 .

EYE, action of muscles of, 519 . definition and situation, 514 .

frozen sections of, 523 .

hardening in alcohol for study, 521.

images formed by, 520 .

muscles of, 516 .

nerves of, 520 .

oculus, and its appendages, 514 .

of cat, dorso-ventral section, 523 . tumics or coats of, 521 .

EYEBALL, form of, 520 . how to obtain of cat, ox or sheep, 520.

Eyelashes, absence of in cat, 514 .

EYELID, third, action of, 515 . palpebra, 514.

Face and Cranium, 39, 174.

Fa'cial nerve, origin and distribution, 508.

Fahrenheit's thermometer, 3.

Fallopian tube, relation to ovary and peritoneum, 296.

Falx (Latin, a sickle), removal of, 428 .

FAS'CIA, 196. denta'ta, 478.

Fascic' uli of muscles, 272.

Fasci'ola (Latin, a small bundle), cine'rea, 478.

Fau'ces, 513.

Feline brains, comments on figures of, 491.

Fellow of the opposite side, platetrope, lat. eral homologue, 32.

FEM'ORAL artery, injection of, 142. blood vessels, figure of, 142 . vessels, injection of, 142, 146.

FE'MUR, 40. determination of right and left, 151 .
FENES'TRA (Latin, a window), 184.

ova'lis, 184, 529 .

rotan' $\mathrm{da}, 184,529$.

Fibril'læe of muscle, 272.

Fi'bro-cartila' go-intervertebra'lis, 171.

FIB $^{\prime}$ ULA, 40.

determination of right and left, 151.

Fifth ventricle, pseudoccelia, 485.

Filter, earthenware, 123.

Fim'bria, 4 is.

Fim'brial fissure, 497.

Finder, tracer, 72 .

Fire, precautions against, 114.

Fishes, injection of, 141.

FISSU' RA ansa'ta, 478, 498.

ante' rior, $^{4} 498$.

Bichati, rima, $48 \%$.

callosa'lis, 497.

crucia'ta, its constant and variable characters, 498.

crucia'ta, its homology and formation, 499.

crucia'ta, the name, synonyms with authorities, 499.

dorsilatera'lis (myel'onis), 478.

dorsimesa ${ }^{l}$ lis (myel'onis), 478.

fim'briæ, 497 .

hy pocam' pæ, 497 .

Sylviana, 498.

Sylviana, its constancy as compared with the FF. rhinalis and postrhina]is, 498.

transver'sa mag'na, rima, 487.

ventrilatera'lis (myel'onis), 4 ri 8.

ventrimesa'lis, 478 .

FIS'SURES, cerebral, and gyri, study of, 494.

comparison with those of man and other mammals, 502 .

figure of the lateral and mesal aspects, 494.

formation of, 497 .

four problems connected therewith, 497 . homology with those of man, 502.

list of the constant ones in the cat, 495-496.

of the cerebellum, 494.

pattern in the cat, 501.

promising lines of inquiry, 503.

relation to ental structures, 497 .

relative depth, and method of determining and indicating on drawings, 495.

structural relations, with list, 497.

Table, with synonyms, 496.

FISSU'RAL PATTERN, constant characters, 501.

variable characters, 502 .

Fleas, killing with benzine, 64,80 .

FLEXIBLE blow-pipe, 64.

specimens, how to preserve, 121.

Floc' culus, of the cerebellum, 478 .

Folsom, 52. 
Foltz, 42.

FORA'MEN atlanta'le, 172.

$\cos ^{\prime}$ cum, 478.

cona'rii, 479 .

condyla're, 183.

epitrochlea're s. supracondyloi'deum, 160.

infundib' uli, 479 .

jugula're, 183.

lac ${ }^{\prime}$ erum $m e^{\prime}$ dium, 183.

Monroi, porta, 483.

neura' $]$ e, 172.

obturato' rium, 167.

of Magendie, 414.

of Monro, porta, 407,421 .

of Winslow, 280.

ova'le, of heart, 328.

palati'num poste'rius, $180,184$.

sphe'no-palati'num, 180.

sty'lo-mastoi'deum, 183.

verteisrarteria'le, 171, 173.

Foram'ina of skull, Table of, 190 .

FORCEPS, care of, 74 .

coarse and fine, 67.

figure of, 66 .

how to use, 201.

Form of the cerebrum, 493.

Formation of fissures, 497 .

Forms of muscles, 196.

For'nix (Lrtin, an arch), 479.

FOS'SA appendicula'ris, 180.

cæ'ca, 478.

cotyloi'dea, 168.

mandibula'ris, 184.

olecrana'lis hu'meri, 160.

orbita'lis, 180.

ova'lis, 328.

radia'lis hu'meri, 160 .

tempora'lis, 180.

thy ro-hya' lis, 184.

ulna'ris hu'meri, 160.

Fos'se of skull, 186.

Fourth ventricle, metacclia, 478.

Frazer, Persifor, 5.

Fresh specimens in alcohol should not touch sides of jar, 121 .

Frog and Menobranchus, killing for brain, 415.

FROG'S BRAIN, advantages for study, 402. exposure, 416.

From and of, use of with adverbs, 27.

FRON'TAL bone, 181. si'nus, 183.

FROZEN dissections, 132. sections, method of making and preserving, 131.

sections of the eye, 523 .

sectious of the thorax, 338-343.

FUNCTIONS of the alba, 371 . of the cinerea, 371 .

FUNIC'ULUS (Latin, diminutive of funis, a cord), grac'ilis, cla'va, 475 .

of a nerve, 398 .
Gaboriau, 53.

Gall bladder, 277, 286.

Gan' glia, 369.

GAN'GLION cer'ebri posti'cum, 490.

cervica' le supe' rius, 395.

hemispher'icum, 479.

infe'rius of vagus, 509.

jugula're of vagus, 509 .

petro'sum of glossopharyngeus, 509.

semiluna're, 391, 396.

Ganglion'ic nervous matter, cinerea, 475.

General sensibility, 512 .

Ge'nu (Latin, a knee). of the callosum, 479.

German technical terms, 16.

Giacomini's method of hardening the brain, 435.

Girdle, definition of term, 197.

GLANDS, sweat and sebaceous, 512. lachrymal, 515.

GLAN'DULA (Latin, a gland) Harderi, 515.

lachryma'lis, 515 .

Meibo'miana, 514 .

mesenter'ica, 280.

mola'ris, 302.

paro'tis, 301.

pinea'lis, conarium, 476.

sublingua' lis, 302 .

submaxilla'ris, 302.

zygomat'ica, 303 .

GLASS dishes and boxes for specimens, 126. jars, $127,128$. jars, cleaning, 128, 585 .

GLO'BUS OC'UI, eyeball, 520. form of, 520 .

how to obtain of cat, ox or sheep, 520 .

Glossopharynge'al nerve, origin, relations and distribution, 509 .

Glot'tis, gustatory structures in, 513 .

Glycerin, 15 per cent., bottle for, 72 .

Goodnow and Wightman, 59.

Gould, John Stanton, $7 \mathrm{P}$.

Grades of alcohol, use of appropriate, 117.

Gram, 4.

GRAY matter of the brain, 476 . nervous matter, cinerea, 475 .

Grease, removal from bones, 110 .

Groups, muscular, 194.

Gudden, 475.

Gul'let, cesophagus, 308.

Gusta'tion, taste, gustatory sensibility, 513.

GY'RI and fissures, study of, 494 .

angular, 501.

arched, 500 .

designation of, 500-501.

oper'ti, 480 .

Habe'na (Lrtin, a thong, that by which anything is held), 479.

Habeniula (Latin, a small thong), habena, 479.

HAIR, clipping the, 204 .

disposal of, 204 .

parting for incisions, 143,204 . 
Hair, parts from which absent, 512.

Hairs, tactile, 512.

Hamerton, Philip Gilbert, 52.

Harder's tear gland, 515 .

Haversian canals of bone, 191.

Head, figure of a hemisection, 305.

Hearing, audition, auditory sensibility, 511, 526.

HEART and blood vessels, structure of, 362. $\mathbf{a}^{\prime}$ pex, 326 .

appendix, auricular,326.

base of, 327.

cardia, 316-338.

cavities and parts, diagram of, 323 .

designation of regions, 318 .

difference between right and left, 324 .

dissection of, 336.

dorsal aspect, figure of, 317.

exposure of, 319 .

figures of sections, 333-336.

form, normal position, size, 318 .

general description, 317.

hardening in alcohol, 322.

injection with alcohol, 321.

iujection with plaster, 316 .

in sections of thorax, 339 .

location of, 317 .

physiologically double, 323 .

preservation in alcoliol, 321.

recognition of regions of, 318 .

removal of, 319.

removal of blood from, 321.

separation from lungs, 320 .

septum of, 329 .

structure of its muscles, 362.

Table of parts, 322.

transection of auricles, 334.

transection of ventricles, 336 .

ventricles of, 332 .

Helmer, F., 536.

HEMICAR'DIA dex'tra, 328. difference between right and left, 324.

Hemicer'ebrum, 479.

Hemisec'tion (i $\mu \iota$, half, and Latin, secare, to cut), of the brain, 444 .

Hemisep'tum (i ${ }^{\prime} / \mu t$, half, and Latin, septum, a partition), cer'ebri, 4 \% 9 .

Hemisphæ' ræ, 475, 479, 485 .

Hem'ispheres of frog's brain, 420.

He'par, liver, 277, 286.

Hepat'ic ducts, 287 .

Hi'lum of kidney, 294.

HIPPOCAM'PU'S major, hypocampa, 480. minor, calcar, 473 .

Holding scalpel, figures of ways of, 199, 200.

Hollow viscera, preparation of, 132 .

HOMOL'OGIES, intermembral, 42.

muscular, determination of, 196.

Homol'ogy of the cerebral fissures of cat and $\operatorname{man}, 502$.

Hone, coarse and fine, 76.

Honing and stropping instruments, description and figure of, 76 .
Horns or cornua of gray matter in the myelon, 370 .

Horns of the uterus. 296.

Human subjects, small, 55 .

HU'MERUS, 40, 157-16L.

determination of right and left, 150 .

figures of, $156,158,228$.

figures showing muscular attachments, 228.

general description, $15 \%$.

its anatomical head, 159 .

its anatomical neck not distinct in cat, 159.

its arthral or anatomical head, 159.

its diaphysis or shaft, 159.

of eat does not appear twisted, 158.

special mnemonics of, 152.

$\mathrm{Hu}^{\prime}$ mor a'queus and the aqueous chambers, 522.

Hybrid words, 28.

HYDROMI'ETER, British, 114

jar for, 115 .

specific gravity, 114.

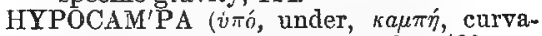
ture), hipposampus major, 480 .

dissection, exposure, etc., 455 .

Hypocam' pal fissure, 497.

Hypoglos'sal nerve, origin, relations and distribution, 509 .

HYPOPH'YSIS, derivation from the alimentary canal, 415 .

of Amphibia, 423.

pituitary body, 480 .

Ideal, Simple Brain, with Diagrams, 408-409.

Il'eo-cæ'cal valve, 284.

$\mathrm{II}^{\prime} \mathrm{eum}, 283$.

Il'iac crest, 167.

Il'io-pectine'al line and eminence, 169.

Il'io-sa'cral arthron, 167.

Il'ium, 168.

Images formed by the eye, 520 .

INCIs'IONS for injection, figures of, 143144.

for thorax and abdomen, 274, Fig. 76.

INCISU'RA hypocam'pæ, 480.

vertebra'lis, 173 .

Inconsistencies of nomenclature in this book, 19 .

In'cus (Latin, an anvil), 528.

India ink, 67 .

Inferior articulating process, 173.

Inflation of hollow viscera with air or alcohol, 133-134.

Inflection of technical words, 27.

Infraorb'ital gland, 303.

INFUNDIB' ULUM (Litin, a tunnel or funnel), of frontal sinus, 181.

of hypophysis, 480 .

In'guinal ring, 143, 297.

Inhib'itory centers, 371.

Injec'tion, choice of specimen for, 141. 
INJEC'TION, coarse, of blood vessels, 137148.

jncision in vessels for, 143 .

making an, 145.

masses, 139.

of aldominal aorta, 147 .

of brain cavities with alcohol or plaster, 435 .

of carotid artery, 148.

of femoral vein, 146 .

of femoral vessels, 142 .

of heart with alcohol, 321.

of plexuses of brain, 436 .

cf postcava, 147.

of $V$. jugularis externa, 147.

time of making, 141.

Innom'inate boue, 168 .

Innomina'tum, determination of right and left, 150.

Insertion and origin of muscles, 195.

Instrument cases, 74 .

IN'STRUMENTS and materials, anatomical, 59 .

and materials for abdominal transection, 99 .

and materials for the dissection of muscles, 198.

dissecting, how to use, 199.

polishing of, 75 .

sharpening of, 76 .

value of, 62 .

In'sula (Latin, an island), Reilii, 480.

Integers, muscular, 194.

Interartic'ular ligament of ribs, 165.

In'terbrain, diencephalon, 477.

Intercru'ral (Latin, inter, between, and crurulis, belonging to legs), or interpeduncular space or area of brain, 473 .

Intermediate, use of', 25.

Intermem'lural (Latin, inter, between, and mombrum, a member), homologies, 42.

INTERNAL auditory meatus, 181. condyle of humerus, epitrochlea, 160.

Interop'ticus (Latin, inter, between, and

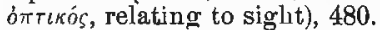

Interpari'etal (Latin, inter, between, and $p a$ rins, a wall) bone, 181.

Intestinas, removing to avoid odor, 84 .

INTESTI'NUM an'plum, 278, 285. te'nue, 278,283 .

Intrinsic toponomy, 23.

Introduction of canula into vessel, 144.

Iris and pupi], 520 .

Is'chium, 168.

$I^{\prime}$ ter (Latin, a way), aqueduct of Sylvius, 480.

JARS for Specimens, figures of, $12 \%$. and bottles, how to dry quickly, 535 . Jeju'num, 283.

$\mathrm{J} \mathbf{u}^{\prime}$ gular vein, injection of, 147 .

KID'NEY, Ren, 278, 294.

figures of sections, 293 .
KID'NEY, relation to peritoneum, 279.

relative position, 294 .

sections of, 293 .

structure, 294.

KILLING Amphibia, 415.

animals for dissection, 79.

cats for brain, 423 .

fleas with benzine, 80 .

Knots, surgeon's and square, figures of, 144.

Kölliker, 37.

Kreider, 8.

\section{LA'BELING Bones, 103.}

brains of Amphibia, 418.

Labyrinth'us (Latin and Areek, a labyrinth), ental or internal ear, $\mathbf{6 2 7}$.

LACH'RYMAL apparatus, 515.

bone, 181.

cunal, 179, 515.

duct, 516 .

sac, 516.

Lac'teals, 316, 364 .

Lacu'næ of bone, 191.

LAMBDOID ${ }^{\prime}$ AL crest, 179.

suture, 183.

Lamel'la ventra'lis, 172.

LAM'INA cine'rea, 489.

cribro'sa, and figure of, 188.

denticula'ta, 533 .

fus'ca of sclerotica, 521 .

neura'lis, 173.

spira'lis membrana'cea, 533.

spira'lis of cochlea, 529-530.

termina' ${ }^{\prime}$ lis, 489.

Lam'inæ, layers of muscles, 195.

Large intestine, $278,285$.

LA'TERAL homologue, platetrope, 32.

ligament of urinary bladder, 295.

use of, 24 .

ventricle, 485 .

white column of the spinal cord, 476 .

Lead chlorid, 83.

Leakage of alcohol, 125.

Lean animals better for dissection, 198 .

Lens of eye and its capsule, 522-523.

LIGAMENTS, etc., percentage of alcohol required for, 121.

of the liver, etc., formed of peritoneum, 279.

LIGAMEN'TUM incu'dis, 123.

interarticula're $\cos ^{\prime}$ tæ, 165.

latera'le of the urocystis, 295.

Poupartii, 143, Fig. 39.

suspenso'rium of lens, 523.

suspenso' rium of urocy'stis, 294.

u'teri, 296.

Ligatures, 144.

LIMBS, 25, 29.

bones of, 40 .

designation of direction and relative position, 35.

designation of regions, 25 .

normal position of, 37 . 
Limbs, segments of, 40.

Lim'bus (Latin, a hem), lamina spiralis, 532. LI'MES (Latin, a path), 480.

$\mathrm{al}^{\prime}$ ba (of rhinencephalon), 480 . cine'rea, 480 .

LIMITS of accuracy in description, 27, 49. of terminological change, 18.

LINES and areas of muscular attachment, 195.

of incision for exposing the thoracic and abdominal viscera, 274.

Lin'gua, tongue, 512.

Liquid soap, 106.

Liq'uor coelia'rum, liquid in the ventricles

Liter, 4. of the brain, 480 .

Liver, hepar, 277, 286.

LOBES of the liver, 286. of lungs, 310 .

LOB' ULUS appendicula'ris cerebel'li, 480. cor'poris stria'ti, 480 . hy pocam'pæ, 481.

LO'BU'S latera'lis (cerebelli), 481. olfacto'rius, in frog and other vertebrates, 420 .

olfacto'rius not a nerve, 507.

olfacto'rius, olfactory bulb, 481 .

op'ticus, 482.

prosencephal'icus, 479.

tempora'lis, 481 .

LO'CUS perfora'tus ante'rior, 485. perfora'tus pos'ticus, 484 .

LUM'BAR nerves, 372. vertebræ and determination of, 170 .

Luna're (Latin, Luna, the moon), 41.

LUNGS and lobes of, 310.

and tra'chea, figure of, 310.

protection of in removal, 320 .

separation from the heart, 320 .

Lymph vascular system, 315-316.

LYMPHAT'IC glands, 316, 365-368. glands, injection of, 367 . vesse]s, injection of, 367 .

LYMPHAT'ICS, 316.

comparison with veins, 316.

of the arms and legs, injection of, $36 \%$. of the neck and face, injection of, 367 . $\mathrm{Ly}^{\prime} \mathbf{r a}, 481$. structure of, 363 .

Macera'tion of Bones in Water, 105.

$\mathrm{Ma}^{\prime}$ cula (Latin, a spot), lutea of retina, 522.

Magnifier, tripod, figure of, 72.

$\mathrm{Ma}^{\prime}$ lar bone, 181 .

Malinverni, 474.

Mal'Ieus (Latin, a hammer), 528.

Malo'dorous parts, 84 .

Mamma'lia, class, 8.

Mammilla're, albicans, 472.

Mam'millary process, 173.

Man'dible, lower jaw, figures of, 188, 189.

MANDIB'ULAR condyle, 189.

division of the 5th nerve, 508,510.
Manuscript, steps in the preparation of, 51 .

Mar'go alveola' ris of the jaw, 184.

Matches, care of, 114.

Mate' rials and instruments, anatomical, 59

MAX'ILLARY bone, 181.

division of the 5th nerve, 508, 510 .

MEA'TUS (Latin, a passage), audito'rius ecta'lis (exter' nus), 184, 527 .

audito' rius enta'lis (inter ${ }^{\prime}$ nus), 181, 509. ventra'lis of nose, 306,518 .

Mediasti'num, 339.

Medicommissu'ra (Latin, medius, intermediate, and commissura, a joining together), 481 .

MEDICOR'NU (Latin, medius, intermediate, and cornu, a horn), 481.

cornu medium, 481.

opening, 455.

transection of, 458 .

Medipedun'culus (Latin, medius, intermediate, and pedunculus, a small foot), 481.

MEDUL'LA (oblongata), 400, 482.

in Amphibia, 419 .

spina'lis (myelon), 482.

Med'ullary sheath, 369.

Med'ullated nerves, 398 .

Meibo'mian glands, 514 .

Mem'bra, limbs, 29 .

MEMBRA'NA arachnoi'dea, 472.

basila'ris, of cochlea, 5833 .

nic'titans, action and use, 515.

Reissneri, 533.

Schneideriana of nose, 513.

tym'pani, 527, 533.

vasçulo'sa cer'ebri, 483

Me'ninx vasculo'sa ( $\mu \tilde{\eta} \nu \iota \gamma \xi$, a membrane, and Latin. vasculum, a little vessel), 483.

MENOBRANCHUS and frog, killing, 415.

brain of for study, 403 .

distinguishing male and female, 418.

exposure of brain, 417.

Mental symphysis, 189.

MES'AL (mĕz'al) ; ( $\mu \varepsilon^{\prime} \sigma o \varsigma$, middle), 24.

aspect of the brain, showing segments, etc., 446,447 .

incisions, objections to, 202.

or azygous organs, 43.

significance and use of, 24 .

MESENCEPH'ALON ( $\mu \dot{\varepsilon} \sigma 0$, , middle, $\varepsilon^{\prime}$, in, and $\kappa \varepsilon \phi a \lambda \dot{\eta}$, the head), 481 .

figure of doraal aspect, 441.

in frog and Menobranchus, 419.

Mesenter'ic glands, 280 .

Mesente' rium, mesentery, 278-279.

Mesocœ'lia ( $\mu \varepsilon \dot{\sigma} \sigma \circ$, middle, and $\kappa \circ \iota \lambda i \alpha$, cavity), 482.

Meso-co' 1 on, 279.

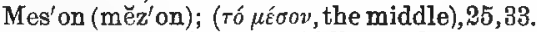

Mesopodial'ia ( $\mu \varepsilon \sigma o s$, middle, and Tor's, a foot), 41.

Mesorec'tum, 279. 
Mesostern'ebra ( $\mu \dot{\varepsilon} \sigma o s$, middle, and $\sigma \tau \dot{f} \rho \nu o \nu$, the sternum), $16 \%$.

Metace'lia ( fourth ventricle, $419,482$.

Metaplex'us ( $\mu \varepsilon \tau u ́$, after, and Latin, plexus, a twiving or braiding), 482 .

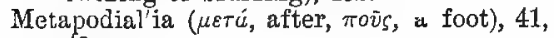
42.

Metapoph'ysis ( $\mu \varepsilon \tau$ ć, after, anó, from, and фve $i$, to grow), 173.

Metate'la ( $\mu$ ric $\dot{c}$, after, and Laíin tela, a web), 482.

Metal boxes and cans for specimens, 126.

METENCEPH'ALON ( $\mu \varepsilon \tau^{\prime} i$, after, $\dot{\varepsilon} \nu$, within, and $\kappa \varepsilon \phi a \lambda \dot{\lambda}$ the lead), 419,482 . examination of, 450 .

METER, 4 yard, figure of, 6 .

Methods, importance of, 53, 55.

Meth'yl alcohol, 124.

METRIC and inch measures, figure of, 6. and other measures compared and reduced, 7 . and other systems, comparison, 5 .

METRIC SYSTEM, 4. how to learn, 6 . in medicine, 8,15 .

Midbrain, mesencephalon, 481.

MIDDLE ear, tympanum, 527. or medi-ven'tricle, $47 \%$. plane (meson), 24,33 .

Mitral valves, 330.

Mod'erator band of heart, 330 .

Modi'olus (Latin, the nave of a wheel), of the cochlea, 529 .

Molar gland, 302.

MO'TOR and sensory nerves and roots, 370 , 504.

root of 5th nerve, 507-508.

Mouth cavity, 303.

MUSCLES, designation of the borders of, 197.

ectal of neck and shoulder, figure of, 211.

forms of, 196.

instruments and materials for the dissection of, 198.

method of description, 206.

names of, 206.

of arm and scapula, figures of, 254, 262.

of ear, 528,533 .

of eye, 516 .

of eye, action, 519 .

of eye, nerve supply, 520 .

of eye, origin, 519 .

of left shoulder, figure of, 246 .

of neck and shoulder, figure of second layer, 218.

origin and insertion of, 195 .

parts of, 195 .

pectoral, figure of, 234.

structure of 272 .

the study of, Chap. VI, 192-272.
Muscles, twisting of, $19 \%$.

MUS'CULAR fibers, 272.

groups, 194.

homologies, determination of, 196.

integers discussed, 194.

organs, percentage of alcohol required for, 120 .

subdivisions, 195 .

variations, 193.

MUS'CULCS, definition of, 194.

acro'mio-deltoi'deus, 256 .

acro'mio-trape'zius, 214.

bi'ceps, 266.

brachia'lis, 267 .

choanoi'deus, 519.

cilia'ris, 525.

cla'vo.deltoi'deus, 231.

cla'vo-mastci'deus, 223.

cla'vo-trape'zius, 215.

coracoi'deus, 250.

der'mo-humera'lis, 225

ec'to-pectora'lis, 235-241.

ec'to-tri'ceps, 263 .

en'to-pectora'lis, 241-244.

en'to-tri'ceps, 263-265.

epitrochlea'ris, 259.

exteu'sor (car' pi) radia'lis bre'vior, 269.

exten'sor (car'pi) radia'lis lon' gior, 268.

exten'sor (car'pi) ulna'ris, 270 .

exten'sor (di'giti) win'imi, 270.

exten'sor (digito'rum) commu'nis, 270.

flex' or (car'pi) radia'lis, 271 .

indica'tor, 271.

infraspina'tus, 257.

latis'simus, 229.

leva'tor an'guli scap'ulæ, 250.

leva'tor clavic'ulæ, 223.

leva'tor pal' pebræ, 518-519.

meditri'ceps, 260.

micosta'lis s. te' res mi'nor, 258.

obli'quus dorsa'lis (superior), 518-519.

obli'quus ventra'lis (inferior), $517,519$.

occip'ito-scapula'ris, 217.

orbicula'ris palpebra'rum, 516, 519.

pec'to-antebrachia'lis, 236.

platys'ma-myoi'des, 227.

prona'tor te'res, 271.

pterygoildeus, 525 .

rec'tus dorsa'lis (superior), 518-519.

rec'tus latera'lis (externus), 517, 519.

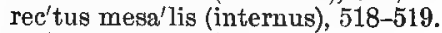

rec'tus mi'nor, 519 .

ree'tus poste'rior, 519.

rec'tus ventra'lis (inferior), 519.

rhomboi'deus, 219.

serra'tus mag'nus, 247.

spi'no-deltoi'deus, 255.

spi'no-trape'zius, $21 \%$.

stape'dius, 5?9, 533 .

ster'no-mastoi'deus, 221.

subscapula' lis, 252.

supina tor lon'gus, 265.

supraspina'tus, 254 . 
MUS'CULUS suspen'sor oc'uli, 519.

ten'sor tym ${ }^{\prime}$ pani, 528, 533 .

te'res, 258.

trochlea'ris (dorsal or superior oblique), 518-519.

xiph'i-humera'lis, 244.

Museum catalogue data, 46 .

Myelencephal'ic nervous system, 369, 37\%

My'elin ( $\mu v \varepsilon \% o ́ s$, marrow, fat), 369, 398.

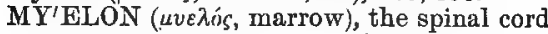
or spinal marrow, $19,372,482$.

in Amphibia, 419.

MYEL'ONAL alba and cinerea, 370.

nerves, 373.

MYOL'OGY, general considerations upon, 192-194.

technical terms of, 194-19\%.

the study of the muscles, Chap. VI, 192-272.

\section{NAMES and Abbreviations on the Fig-} ures, 20.

indicating relative position, 17 .

of brain, segmental arrangement of, 446. of muscles, sources, 208.

$\mathrm{Na}^{\prime}$ 'sal bone, 181 .

$\mathrm{Na}^{\prime}$ sus, nose, 513.

$\mathrm{Na}^{\prime}$ tis cer'ebri, opticus, 482.

NATURAL skeletons, flexible, 109.

skeletons, preparation of, 108.

Neck (part of soma), 307.

NERVE cells, 399.

fibers, anastomoses, 399 .

roots shown in figure of the base of the brain, 443.

supply of the muscles of the eye, 520 .

NERVES, comparison of cranial with myelonal, 504, 506.

connection with the limbs, 500.

cranial, general considerations, 371, 504 .

cranial, method of demonstration, 511.

cranial, numbering, 505.

cranial, physiological arrangement, with Table, 006.

cranial, Table of origin, exit and functions, 510.

cranial, Table of the synonyms, 505.

distinguishing from vessels, 375 .

figure of a transection of the' thorax, showing the relation of the cerebrospinal and sympathic, 397.

medullated, 398.

motor and sensory, 370 .

non-medullated, 399.

of the face, 301 .

subdivisions, 369 .

termination of, $37 \%$.

NERV'OUS and vascular systems, analogies and differences, 371-372.

matter, structure, $398,399$.

plexuses, 370

system, central and peripheral portions, 370.
NERV'OUS system, general directions for dissecting, $37 \%$.

system, primary divisions, 369 .

NER'VUS abdu'cens, origin and distribution, 482,508 .

accesso' rius (XI), 377, 389, 509.

audito'rius, origin and distribution, 508.

auricula'ris mag'nus, 301 .

circumflex' 'ts, 385.

cuta'neus inter'nus, 384 .

fạcia'lis and audito'rius, 427.

facia'lis, origin and distribution, 508 .

gas'tricus dorsa'lis, 391, 509 .

gas'tricus ventra'lis, $390,509$.

glos'so-pharynge'us (IX), 389, 509.

glos'so-pharynge'us, va'gus and accesso' rius, relations, 426,509

hypoglos'sus (XII), 389, 426, 509 .

interos'seus poste'rior, 386 .

Lancisi, 488.

larynge'us recur'rens, 388, 390, 394, 509.

larynge'us supe'rior, 500 .

maxilla' ris supe' rior, 508 .

me'dius, 385 .

mus'culo-cuta'neus s. cuta'neus exter' nus, 384.

mus'culo-spira'lis, 386 .

oc'ulo-moto'rius, 507.

oc'ulo-moto'rius, trochlea' ris and abdu' . cens, relations, 427 .

olfacto'rius of Amphibia, 420.

olfacto'rius, 507.

op'ticus, $50 \%$.

phren'icus s. diaphragmat'icus, 383.

pneu'mo-gas' tricus (vagus), 387, 509.

splanch'nicus, 395.

subscapula'ris, 384 .

suprascapula' ris, 383.

sympath'icus, sympatheticus, 394.

sympath'icus in the abdomen and tail, 396.

thorac'icus ante'rior, 382.

thorac'icus poste'rior, or external respiratory of Bell, 387 .

trigem'inus, and its three divisions (1st or ophthalmic, 2d or maxilla'ris, $3 \mathrm{~d}$ or mandibnla'ris), 508.

trigem'inus, its gauglion (Ging. Gasseri), 508.

trigem'inus, its motor root, origin and distribution, 507-508.

trigem'inus, sensory root, origin, 508.

trochlea'ris, 507.

ulna' ris, 386.

$\nabla a^{\prime}$ gus, dissection of, 388 .

va'gus, pneumogastricus, origin, distribution and relations, $387,388,509$ 510.

$\mathrm{NEU}^{\prime} \mathrm{RAL}$ and visceral cavities, 33.

arch, 172.

canal, 172.

fora'men, 172 .

lam'ina, 173. 
Neu'ral spine, 173.

Neurol'ogy, general considerations, 369, 398.

Nichols, William, 48.

Nictita'ting membrane, 515.

NIPPERS (bone), 62, 67, 534 . care of, 74 . cross-cutting, 68.

figures of, $62-63$.

NOMENCLA' URE, inconsistencies of in this book, 19.

or terminology, 10.

Nom'ina impudi'ca, objections to, 15.

Non-medullated nerves, 399.

NORMAL POSITION, 21.

of limbs, 37 .

Nose, nasus, 513.

NOTES, cost of material for, 50 .

distribution of, 48 .

elimination of, 47 .

proper, 47.

slip system of, 45 .

storage of, 48.

subdivision of, 48 .

$\mathrm{Nu}^{\prime}$ cleus cauda'tus, stria'tam, 488.

Numbering the cranial nerves, 505.

$\mathrm{O}^{\prime}$ bex (Latin, an obstacle), 482.

OBLIQUE lines, 35.

muscles of the eye, $517-518$.

Obtura'tor fora'men, 167 .

Occip'ital con'dyle, 183.

Oc'ulo-mo'tor nerve, 507.

OC'ULUS (Latin, the eye), eye and its appendages, 514 .

frozen sections of, 523 .

hardening in alcohol for study, 521 .

jmages formed by, 520 .

muscles of, 510 .

section, figure of, 523 .

tunics or coats of, 521 .

Odon'toid process of axis, 172.

Odors, perception by olfactory nerves, 513 .

Fs oph'agus, gullet, 308 .

Of and From, use of with adverbs, 27.

Oil-stone, coarse and fine, $7 \%$.

Oiler, 68, 535 .

Olfaction, olfactory sensibility, smell, 513 .

OLFAC'TORY foesa, 186.

lobe or bulb, 481,487 .

lobes, examination of, 450 .

nerves, distribution to the nose, 513 . nerve or tract, 476 .

Oli'va (Lriti", an olive), area elliptica, 482.

Omen'tum, 278. 280.

Ophthal'mic (1st) division of the trigeminus nerve, 508, 510.

OPTIC chiasma, 475.

nerve, origin, 507 .

tract, 490 .

Op'ticus (lo'bus), 482.

$O^{\prime}$ ra serra'ta (Latin, serrated border), 522.

Orb'ital fossa, 180 .

Orb'ito-sphe'noid bone, 181.

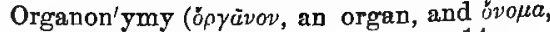
a name), designation of organs, 14.

ORGANS, determining volume and capacity of, 135-136.

of hearing, 526 .

of sense, 511-533.

Or'ganum (Latin and Areek, an organ or instrument) audi'tus, ear, and diagram of parts, 526 .

Orific'ium auric'ulo-ventricula're, 329.

ORIGIN and insertion of muscles, 195. more important for determination of muscular homologies, 196.

of the muscles of the eye, 519 .

of the slip system, 52 .

OS alisphenoi'deum, 181

basioccipita']e, 181 .

basisphenoi'deum, 181.

cotyloi'deum, 168.

ethmoturbina' Ie, 187.

fron'tis, 181.

innomina'tum, 168.

interparieta'le, 181.

juga'le, 181.

lachryma'le, 181.

lenticula're, 529 .

mala're, 181.

maxilla're, 181.

mesethmoi'deum, 187.

nasa'le, 181.

orbito-sphenoi'deum, 181.

palati'num, 181.

parieta'le, 181

periot'icum, 181.

pla'nuin, 181.

præmaxilla're, 182.

pu'bis, 168.

supraoccipita']e, 181.

tempora'le, 181.

vo'meris, 187.

zygomat ${ }^{\prime}$ icum, 181.

Ossic'ula audi'tus, bones of the ear, 528 .

Osteol'ogy, 149-191.

Ova'rium, ovary, 296.

Pacinian Corpuscles, 281.

PACKING alcoholic specimens, 129.

instruments for transportation, 75 .

Pagenstecher, 37.

Pail, waste, 81 .

PAIRED and azygous, meaning of, 33. or lateral organs, 43.

organs, 33.

PAL'ATE bone, 181. soft, 304, 307, 512 .

Pal'pebra (Latin, an eyelid) dorsalis, 514, 526.

Pan'creas and figures of, 278, 287, 288.

PANCREAT'IC DUCTS, 288, 290.

in man, and figure, 292.

lesser, 290.

principal, 290.

reservoir, 289-290. 
Papil'læ, fun'giform and circumval'late, $305,513$.

Paramas'toid (napu, by the side of, and

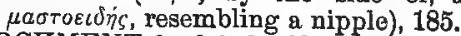

PARCHMENT for labels, 68. numbers, 68.

Pari'etal bone, 181.

Paroccipita'lis ( $\pi a p u ́$, by the side of, and Latin, occiput, the back of the head), proces'sus, 185.

Paroccip'ital process, 185.

Parot'id gland, 301.

Pars cilia' ris ret'inæ, 522.

Parting hair for incísions, 143.

PARTS in series, 42. of a muscle, 195.

Patel'la, 40.

PATTERN of the cerebral fissures, constant characters, 501. of the fissures, variable characters, 502.

PEC'TORAL muscles, figure of, 234. ridge of the humerus, 159 .

Pec'toralis group of muscles, general considerations, 235 .

Ped'icle of a vertebra, 173.

PEDUN'CULUS (Litin, a small foot) medius, medipedunculus, 481 .

cerebel'li inferior, postpeduneulus, 484. cerebel'li superior, præpedunculus, 485 . cer'ebri, crus cerebri, 476 . cona'ril, habena, 479.

Pel'vic bone, determination of right and left, 150.

$\mathrm{Pel}^{\prime}$ vis and figure of, 167,168 .

Pericar'dium, 317, 321, 339, 332.

Perimys'ium ( $\pi \varepsilon \rho i$, around, $\mu \dot{v} s$, a muscle), 272.

Perineu'rium ( $\pi \varepsilon p \hat{i}$, around, $\nu \varepsilon \hat{v} \rho o v$, a nerve), 398.

Perios'teum, attachment of museles to, 195.

Periot'ic ( $\pi \varepsilon \rho i$, around, and ovंs, the ear), 181 .

Periph'eral, use and significance, 26.

Peritone'al sac, 279.

PERITONE' UM, 277, 279.

and viscera, diagram showing relations of. 279 .

structure of, 280.

Permanganate of potash, 83 .

$\mathrm{Pe}^{\prime}$ ro (Latin, a rawhide boot) olfactorius, 483.

Pes olfacto'rius, 483.

Petit, canal of, in eye, 526.

Phalan' ges, 41 .

Phar'ynx, 307, Fig. 88.

Philistinism, anatomical, forms of, 204.

Philosophical Society, American, vi, 461.

PHYSIOLOG'ICAL and topographical position, 285.

sense, use of words in, 44.

PI'A MA'TER (Latin, pius, tender, mater, mother), 483 .

interior, 478.

removal from brain, 429 .
Pigeon hole case and figure of, 51.

Pi'li tac'tiles, 512.

Pineal body, conarium, 476.

Pisifor'me, 41.

Pithing a frog, figure of, 536 .

Pitu'itary body (hypophysis), 480.

Planes of the body, and figure of, 33,34

Plaster casts of medicornua, porta, etc., 458.

Plaster of Paris injection mass, 139, 140.

Plates of the brain, explanation, 461-471.

Plat'etrope ( $\pi$ rúros, breadtl, width, and $\tau \rho \dot{\varepsilon} \pi \varepsilon(\nu$, to turn). lateral homologue, fellow of the opposite side, 32 .

Pleu'ra, 44, 309, 339.

PLEX'US CHOROI'DEUS infe'rior, metaplexus, 482.

me'dius, diaplexus, 477 .

ventric' uli latera'lis, proplexus, 485.

ventrie'uli ter'tii, diaplexus, 473.

ventric' uli quar' ti, metaplexus, 482.

PLEX' USES and telæ, 370, $40 \%$.

not in procoelia of frog, 422 .

of brain, 414 .

of brain, injection of, 436 .

Pli'cæ ciliä'ris, 522.

Pneumogas'tric or vagus nerve, 509.

Poisoning dried preparations, 134.

Polishing instruments, 75 .

PONS cerebel'li, 488.

Tarini, 484.

(Varo'iii), 483.

POR'TA (Lutin, a door), foramen of Monro, $421,454,483$.

and $\mathrm{au}^{\prime} 1 \mathrm{a}, 407,454$.

demonstration, 454 .

existence demonstrated by plaster injection, figures, 458,459 .

PORTFOLIO for sheets, 51.

for slips, 48.

POR'TIO depres'sa (praeperfora'ti), 483. diencephal'ica (cru'ris cer'ebri), 483. mesencephal'ica (cru' ris cer'ebri), 483. prom'inens (praeperfora'ti), 483.

Portiplex' us (Latin, porta, a door, and plexus, a plaiting or braiding), 483 .

POSITION AND DIRECTION, designation of, $20,34,35$.

on the soma, 31 .

Position in a physiological sense, 44 .

POSTCA ${ }^{\prime} V A$, ve'na ca'va inferior, 329,331 , 356.

injection of, 147.

Postcommissu'ra (Latin, post, behind [caudad of], and commissura, a counection), 484.

Postcor'nu (Latin, post, and cornu, a horn), 484.

POS'TE'RIOR fissure of the spinal cord, 478. white column of the spinal cord, 475 .

Postgenicula'tum (Latin, post, and geniculum, a little knee), 484 .

Postna'ris (Latin, post, and naris, a nostril), 513. 


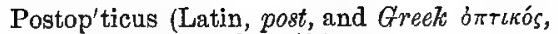
relating to sight), 484 .

Postpedun'culus (Latin, post, and pedunculus, a little foot), 484 .

Postperfora'tus (Latin, post, and perforare, to bore through), 484 .

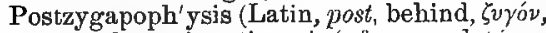
a yoke or junction, $\dot{a} \pi o$, from, and $\phi \dot{v} \varepsilon \nu$, to grow, 173 .

Potassium permanganas, 83 .

Pouparti, ligamentum, 143.

Practical suggestions for dissectors, 201-203.

Præca'va, for v. cava, superior, 329, 331, 449.

PRACOMMISSU ${ }^{\prime}$ RA (Latin, $p r c$, before [cephalad of], and commissura, a connection), 484 .

demonstration, $4 \overline{\%} \%$.

Præcor'nu (Latin, pree, and cornu, a horn), 454,484 .

Prægenicula'tum (Latin, pro, and genieulum, a little knee), 484.

Præna'ris (Latin, prce, and naris, a nostril), 513.

Præpedun'culus (Latin, pre, and pedunculus, a little foot), 485 .

Præperfora'tus (Latin, prce, and perforare, to bore through), 485.

Præzygpoph'ysis (Latin, pre, and $\zeta \bar{y} \gamma^{\prime} v$, a yoke or junction, $a \pi o ́$, from, and $\phi \dot{v} \varepsilon u$, to grow), 178.

PRECAUTIONS against dissection wounds, 85.

against fire, 114.

for cleanliness, confort, and health, 81 .

PREPARATION of bones, 103-111.

of ducts of salivary glands, 298.

of hollow viscera, 132 .

of natural skeletons, 108.

of skulls, 107.

provisional, of specimens, 121.

PRESERVATION of amphibian brains, 418. of brain, 429 .

of heart in alcohol, 321.

PRIMARY divisions of the body, 29 . divisions of spinal nerves, 372 .

PROCES'SUS clava'tus, clava, 475.

e cerebel'lo ad medul'lam oblonga' tam, postpedun'culus, 484.

e cerebel'lo ad pon'tem, medipedun'culus, 481.

e cerebel'lo ad tes'tim, præpedun'culus, 48 ?.

odontoi'deus, 172.

paroccipita'lis, 185.

PROCEE'LIA ( $\pi \rho n$, before [cephalad of], and

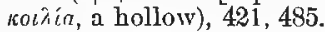

and rhinoce'lia, demonstration of, 454

Proof spirit, 115.

Proplex'us ( $\pi \rho n$, before, and Latin, plexus, a twining or plaiting), 454, 485 .

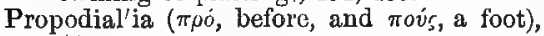
41.
PROSENCEPH'ALON ( $\pi \rho{ }^{\prime}$, before [cephalad of ], $\dot{\varepsilon} \nu$, witlin, and $\kappa \varepsilon \phi a \lambda \dot{n}$, the head), cerebrum, 475, 485.

of frog, 420 .

Protuberan'tia basila'ris, pons, 483.

Provisional preparations, 121.

Prox'imal, use and significance, 25.

Psalte'rjum (Latin, a hare), ly ra, 481.

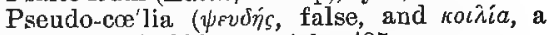
hollow), fifth ventricle, 485.

Pseudo-commissu'ra of olfactory lobes in frog and other Anoura, 420.

PU'BIC bone, 169. sym' plysis, 169.

Pul'mo, lung, 310.

Pul'monary artery, 326.

PUPIL and iris, 520 . form in the cat, 520 .

Putnam, J. Pickering, 5.

Pylo'rus, 282.

PYR A AlIS, anterior pyramid, 485. posterior, clava, 475 .

Quad'rans (Latin, a fourih part), 486.

Quadrigem'inum (Latin, four or four fold),

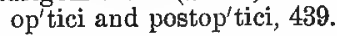

Ra'dius, 40 .

determination of right and left, 150 .

RA'DIX (Latin, a root), exter'na, external root of the olfactory tract, $\mathbf{4 8 6}$.

interme'dia (cruris olfactorii), 486.

inter'na, inner or internal root of the olfactory tract, 486 .

latera'lis (cruris olfactorii), 485 . mesa'lis (cruris olfactorii), 486. moto'ria (nervi trigemini), 486. senso'ria (nervi trigemini), 486 .

Ra'mi intesti'ni ten'uis, 359.

Ra'mus (Latin, a branch), mandibularis, 188.

Razor strop, 178.

Readers' and Writers' Economy Co., 46.

REASONS for selecting the cat, 55 .

for giving prominence to the viscera, 58.

for treating only part of the body, 57 .

Receptac' ulum cliy']i, 364.

RECES'SUS au'li, 486.

opticus, 486 .

præponti'lis, 586 .

Rec'tum, 285.

Rec'tus muscles of the eye, 517-519.

Recurrent laryngeal nerves, figure of, 394 .

Reduction of alcohol with water, 116.

REFERENCES, use of slips for, 47 .

to books and autbors, how made, 2-3.

to other publications, reasons for, 2 .

Re'flex nervous centre, 371.

Re' gio au'lica, 486.

Regions of the vertebral column, 170 .

Reissner's membrane, 533.

Relation of cavities of the brain, 413 . 
Relative positions in an animal, and names for, $17,35$.

REMOVAL of brain, 423.

of dura (mater), 428 .

of heart, 319 .

of pia (mater) from brain, 429 .

of skin for muscles, 205.

of the tail, 102 .

REN, kidney, 278, 294. section, 293.

Restifor'me (corpus), 486.

Restibra'chium, postpedun'culus, 484.

$\mathrm{Re}^{\prime}$ te muco'sum, 512 .

RET'INA, 522.

its ora serrata and pars ciliaris retinæ, 522.

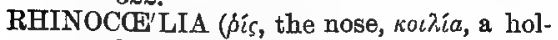
low), 454, 487.

and procoelia, demonstration of, 454. examination of, 454 .

RHINENCEPH'ALON ( $\beta$ is, the nose, $\varepsilon v$, within, $\kappa \varepsilon \phi a \lambda \eta$, the head), 450,487 . examination of, 450 . of Amphibia, 470.

RIB, costa, 167 . head, neck, etc., 166.

figure of a pair of, 166. joints of, 165 .

Right and left, determination of, 149, 151 .

Right heart, 328.

Ri'gor mor'tis, removal of, 205.

$\mathrm{Ri}^{\prime}$ ma, great transverse fissure, $48 \%$.

Ri'pa, 488.

Ros'trum, 488.

Rouge for polishing, 76 .

Royal Society's Catalogue, 3.

RÜBBER gloves, 68.

tubing, 68.

Rudd, W. N., 72.

Sa'crum, 169.

Sag'ittal suture, 183.

SAL'IVARY GLANDS, 297, 298. und ducts, exposure of, 299. figure of, 300 .

Sarcolem'ma, 2\%2.

Saw, care and figure of, 68,74 .

SCA'LA tym' pani, 530, 533 . vestib' uli, 580, 533 .

Scales, weighing, 68.

SCAL'PELS and cutting instruments, care of, 74 .

figures of, 66,69 .

figures of ways to hold, 199, 200.

how to hold, 199.

Scaphoi'des, 41.

SCAP'ULA, description of, 152. determination of right and left, 150. figures of, 154, 155 .

Schneiderian membrane of nose, 513.

SCISSORS, and figures of, 63,70 . care of and sharpening, 74, 79. how to use, 200 .
Sclerot'ica ( $\sigma \kappa \lambda \eta p o ́ s$, hard), 521.

Seba'ceous glands, 512.

Seeker, tracer, 72.

Segmen'tal arrangement of encephalic names, $409,446$.

SEG'MEN'TS of brain, 404.

of limbs, 40.

Semicir'cular canals, 530.

SEMILU'NAR valves and figure of, 331, 347.

valves, situation, 338 .

Sense organs, 511-533.

Sensibil'ity, general, 511.

SEN'SORY and motor nerves and roots, $370,372,505,506$.

nerves, two great divisions, 506 .

Separation of heart from lungs, 320 .

Sep'ta of beart, 329.

Sep'tal area of brain, 473 .

SEP'TUM auricula're, 329.

lu'cidum cer'ebri, 488.

mediastina'le, $309,339$.

thorac' is, 309 .

tympan'icum, 528.

ventricula're, 329 .

Series, enumeration of parts in, 42 .

Sero'sa of heart, 341 .

Sharpening instruments, 76 .

Sharpness, determination of, 78.

Shepard and Dudley's Catalogue, 59.

Sight, vision or visual sensibility, 511, 514 .

Simple, ideal brain, fgures of, 408.

Sinis'tral and dextral, use of, 24.

SI'NUS corona' lis, 330.

fronta'lis, 183 .

of cardiac vein, 330 .

of heart, 329 .

of Valsalva, situation of, 330,338 . sphenoida'lis, 187.

Skel'eton seen from left, figure of, 88 .

Skel'etons, natural, preparation of, 108.

SKIN, cutis, appendages of, 512.

coloring matter, 512.

cutting the, 204.

removal for muscles, 205.

structure of, 512 .

skinning, three methods of, 205.

SKULL, 173.

articulations of bones, 176 .

base, figure of, 182 .

cleaning of, 107 .

disarticulating, 109.

dorsal aspect, figure of, 180 .

figure of hemisected, 186.

Table of bones, 174.

Table of its foramina, 190.

SLIP box, 50.

portfolins, 48.

system of notes, $45-52$.

SLIPS, accumulation and elimination of, 47.

use of, 51 .

Small intestine, $278,283$. 
Smell, olfactory sensibility, olfaction, 511, 513.

Snowden, Catalogue of, 59.

Soft palate, 513 .

SO'MA ( $\sigma \tilde{\omega} \mu \alpha$, the body), and its divisions, 36.

axial part of body, 15, 29.

Sömmering, 505.

Specific gravity hydrometer, 114 .

SPEC'IMENS, dishes and boxes for, 126127.

display of, 129.

for injection, 141.

jars, figures of, 127.

storage and exhibition of, 125-126.

transportation of, 129.

Spec'ulum, sep'tum luci'dum, 488 .

Spencer, 194.

Spermat'ic cord, 143, 297.

Sphenoid'al si'nus, 187.

Spigelian lobe of the liver, 286.

Spi'na neura'lis, 173.

SPI'NAL cord, myelon, 372, 482. nerves, 373.

Spi'ral lam'ina of cochlea, 529.

Spleen, splen, 278.

Sple' nium, 488.

Sponges, 70.

Sta'pes, stirrup, bone of the ear, 529.

Stenon's duct, and preparation of, 298, 301.

Sternum, figure of, 163.

STOM'ACH, preparation and drying, 132.

and duodenum, figure of, 281.

Stom'achus, stomach, 277, 28\%.

Stom'ata of lymphaties, 316.

STORAGE and exhibition of alcoholic speimens, 125.

of notes, 48 .

temporary, of specimens, 125.

STRI'A longitudina'lis, 488 .

Lancisi, 488.

STRIA'TUM (Latin, striare, to mark with grooves or channels), 488.

exposure, etc., 456 .

supposed representative in frog, 421.

Stropping instruments, 78.

Structural relations of certain fissures, 497 .

STRUCTURE OF the heart and vessels, 362-363.

large intestine, 285.

liver, 287.

mouth, 304.

lung, 311 .

muscle, $2 \% 2$.

nervous matter, 398-399.

cesophagus, 308.

ovary, 296.

Pacinian corpuscles, 281.

pancreas, 293.

peritoneum, 280.

salivary glands, 303 .

small intestine, 284.

stomach, 283.
STRUCTURE of the tongue, 304.

trachea, 308.

urocyst, 295.

uterus, 296.

Stubs's nippers, 68 .

Subdivisions, muscular, 195.

Sublin'gual gland, 302.

Submax'illary gland, 302.

SUL'CUS (Lutin, a furrow) habe'næ, 489.

intercrura'lis latera'lis, 489.

intercrura' lis mesa'lis, 489 .

lim'itans, 456, 489.

triradia'tus, 489.

Sulphate of iron as a deodorizer, 83.

Superior articulating process, 173.

Supracon'dyloid foramen, 160.

Supraoccip'ital (Latin, supra, above, and occiput, the back of the head) bone, 181.

Suprare'nal capsule, 295.

Sur'cingle (cauda striati of brain), 474 .

Surgeon's knot, 144, Fig. 41.

SUSPEN'SORY LIGAMENT of the lens, 523.

of the urinary bladder, 294.

SUTU'RA corona'lis, 183.

lambdoida'lis, 183.

sagitta'lis, 183 .

Sut'ures of the skul], 174, 176.

Sweat glands, 512.

Swift Manufacturing Co., 75.

Syl'vian fissure, its constancy, etc., 498, 501.

Symmetry, 43.

Sympathet'icus, sympath'icus, 369, 392.

SYMPA'TH'IC and vagus nerves, figure of, $366,392$.

nervous system, 369, 394.

SYMPATH'ICUS ( $\sigma \dot{u} v$, with, $\pi \dot{v} \vartheta \circ$, suffering), sympathet'icus, 369,394 .

in sections of thorax, 341 .

SYM'PHYSTS men'ti, 189.

pu'bis, 169.

Synonymy of muscles, Table of, 207.

SYR'INGES, use and care of, 137.

figures of, 138, 139.

Syrin' gotome, figure of, 66,71 .

TABLE illustrating the subdivision of notes, 49 .

of bones of skull, 174 .

of cranial nerves, origin, exit, distribution and function, 510 .

of fissures, with synonyms, 496 .

of foramina of the skull, 190.

of metric measures, 5 .

of names and synonyms of encephalic segments, 405 .

of parts of Amphibian brain, 409.

of parts of heart, 322 .

of physiological arrangement of the cranial nerves, 506.

of principal divisions of body, 39 .

of synonymy of muscles, $20 \%$.

of synonyms of cranial nerves, 505 . 
TABLE of systemic arteries of the trunk and arm, 343, 344 .

of thorac'ic veins, 342 .

showing three methods of enumerating the arched gyri, 501 .

TAC TILE hairs, 512.

sensibility of tongue, 513 .

sensibility, taction, touch, 511,512 .

Tac'tion, touch, tactile sensibility, 512.

Tæ'nia hippocam'pi, fim'bria, 478.

Tags for labels, 71 .

TAIL, relation to body, 25. removal of, 102.

Tailing to be avoided in dissection, 203.

Tape'tum ( $\tau i \pi \eta s$, a carpet), 522, 526.

TAR'SUS and car' pus, 41. comparison with man, 41.

Taste, gustation, gustatory sensibility, 511, 512 .

Tear glands, 515.

TECHNICAL TERMS compared with vernacular, 16, 17.

desirability of retaining, 15 .

necessity for, 16.

of myology, 194-197.

Teeth, fastening in the skull, 107.

TE'LA (Latin, a web), 414. and plexuses, 407.

choroi'dea infe'rior, metate'la, 482. of brain, 414 .

Teleol'ogy, 56 .

TEM'PORAL bone and fossa, 180, 181. crest, 179.

Tem' poro-auric' ular division of the trigem'inus nerve, 301.

Tem'poro-fa'cial division of the facialis nerve, 301.

Tenac'ulum, 71.

TEN'DON, 195, 272. of the diaphragm, 311.

Tensor tympani muscle, 528, 533.

TENTO'RIUM (Latin, something stretched ont, a tent), $30 \%$.

bony, of cat, $18 \%$.

bony, removal, 428 .

Ter'ma (т́́p $\mu a$, a limit, a terminus), lamina terminalis, $422,489$.

Terminolog'ical changes, limits of, 18, 130.

TERMINOL'OGY, general considerations, 11. or nomenclature, 10.

TERMS applying to whole body, 26. of position and direction, 20 . teehnical, of myology, 194-197.

Tes'tis, 297 .

Tes'tis cer'ebri, postopticus, 484.

Testu'do (Latin, a tortoise shell), 479.

Thalamenceph'alon ( $A \dot{a} \lambda a \mu \nu$, a bed, $b v$, within, $\kappa \varepsilon \phi a \lambda \eta \dot{n}$, the head), diencephalon, $47 \%$.

THAL'AMUS ner' vi op'tici, 490. op'ticus, $489,490$.

Thermometers, comparison and reduction of scales, 3-4.
THIRD eyelid, and action of, 515 . ventricle, and aula, 472.

THORAC'IC ducts, 363.

ducts, figure of the left, 366 .

nerves, 372 .

or dorsal vertebræ, and determination, 170.

transection, 102.

THO'RAX, 37, 308.

figures of a frozen transection, 341.

flgure of ideal transection, 43 .

frozen sections, 338-342.

transection showing relation of sympathic and spinal nerves, figure of, 397.

Thread and twine, linen, 61 .

Thy'mus body, 309.

THY'ROID axjs, 353.

body, 224.

or middle cervical ganglion, 395 .

Tib'ia, determination of right and left, 151.

TONGUE, lingua, 512.

nervous supply, 513.

papillæ of, 306, 307, 513.

tactile sensibility, 513 .

uses of in speech, etc., 513 .

Tools, carpenters', 71.

Topon'ymy ( designation of position and direction, $20,23$.

Torsion of humerus, not apparent in cat, 158.

Touch, taction, tactile sensibility, 512.

Towels, 71.

Trabec'ula (Latin, a small beam), of heart, 330.

TRACER, seeker, finder, 66, 71, 79. how to use, 201.

Tra'chea, windpipe, 307.

TRAC'TUS op'ticus, 490. postrhina lis, 490 .

rhina'lis, 490 .

transver'sus pedun'culi, cimbia, 475.

Tralles, alcoömeter of, 114.

Transect, definition of term, 197 .

TRANSEC'TION (Latin, trans, across, and secare, to cut), 98.

abdominal, how to make, 98-101.

ideal of body, 30 .

ideal of thorax, figure of, 43 .

thoracic, 102 ,

Transverse process of a vertebra, 172.

Trape'zium, 490.

Trape'zius group of muscles, 208.

Trays, and care of, 73,74 .

Treatment of dissection wounds, 86 .

Treman, King \& Co., 68.

Tricus' pid valves, 329, 331.

TRIFA'CIAL or trigeminal nerve, 507.

nerve. its sensory and motor roots. 507, 508.

Trigem'inus or trifacial nerve, its two roots, $507,508$. 
Tripod magnifier, 72 .

TROCH'IN ( $\tau \rho \circ \chi \chi^{\prime} s$, anything spherical or circular), 158, 160.

the caudal, lesser or inner humeral tuberos'ity, 160 .

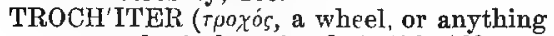
spherical or circular), 158, 160.

the cephalic, greater or outer, humeral tuberosity. 160.

Troch'lea, 160.

Trochlea'ris or patheticus nerve, 507 .

Troph'ic ( $\tau$ poøís, a feeder), centers, 371.

True skin, cutis vera, corium, derma, structure and function, 512.

Trunk and its divisions, 36 .

Tu'ba Fallopiana, 296.

T'U'BER annula're, pons, 483. cine'reum, 423, 491.

TUBER'CULUM (costæ), 167. Loweri, 330 .

Tuberos'ity of the ischium, 169.

TU'NIC A oc' ali, 521. vasculo'sa, 523 .

Tunnels, 72 .

Tur'binated bones, scrolls of, 187,513 .

Turner, H. W., 227 .

Turpentine, spirits of, 110, 114.

Tying vessels, 144, 146.

TYMIPAN'IC bulla, 185. membrane, $52 \%$.

$\mathrm{Tym}^{\prime}$ panum, middle ear, 527, 528.

UL'NA, 40. determination of right and left, 150 .

Unciform eminence, calcar, 473.

Uncifor'me (Latin, uncess, a hook, and forma, form), 41.

UPPER (dorsal) eyelid, 514. jawbone, 181.

Ore'ter, 294.

$\mathrm{U}^{\prime}$ rinary bladder, 278, 294.

U'rine, expelling, for cleanliness, 84 .

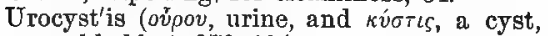
a bladder), 278, 294.

Use of dissecting instruments, 199.

$\mathrm{U}^{\prime}$ terine ligaments, 296.

U'TERUS, 278, 295.

horns or cornua of, 296.

VA'GUS (Latin, vagus, wandering), 388, $392,509$.

and sympathic nerves, figure of, 366 , 392.

in sections of thorax, 342 .

or pneumngastric nerve, origin, etc., 509.

VAL'VA bicus' pis, 330.

il'eo-cæer'lis, 284.

semiluna'ris, 331.

Thebesii, 331 ,

tricus' pis, 331 .

VALVES of veins, 347 .

in veins, figure of, 34 ?
Val'vula (Latin, diminutive of valva, a valve), valve of Vieussens, 491.

Variations of muscles, 193.

Vas def'erens, 297.

Va'sa chylif'era, 364

Vas'cular system, general considerations, 315 .

VEINS, character and distinction from ar. teries, 315 .

of the abdomen, Table, 343 .

of the thorax. 349 .

of the thorax and neck, Table, 342.

structure of, 363.

to be injected, 142 .

$V^{\prime}$ 'lum pala'ti, figure, 307.

VE'NA adre'no-lumba'lis, 356.

az'ygos, 331, 349.

bra'chio-cephal'ica, s. innomina'ta, 349. cardi'aca, 331.

ca'va, 331.

ca'va infe' rior, postcava, 356.

corona' ria ventric' uli, 356 .

femora'lis, injection of, 146 .

gas'tro-epiplo'ica, 356 .

gas'tro-splen'ica, 355.

hepat'icæ, 356.

ili'aca commu'nis, 357.

ili'aca exter'na, 357.

ili'aca inter'na, 358 .

il'io-lumba'lis, 357 .

jugula'ris exter'na, 350 .

jugula'ris exter'na, injection of, 147.

jugula'ris inter'na, 350 .

mesenter'ica infe'rior, 355.

mesenter'ica supe' rior, 355 .

ova'rii, 357.

pancreat'ico-duodena'lis, 356 .

phren'ica, 356 .

por'tæ, 355-356.

pulmona'riæ, 331.

rena'lis, 356 .

spermat'ica, 357.

sterna'lis, 349 .

subcla'via, 349 .

subscapula'ris, 350 .

vertebra'lis, 349 .

VEN'TRAL and dorsal, use of, 24.

primary division of nerves, demonstration, 376.

VEN'TRICLES of brain, cœliæ, 406, 440. of heart, relative thickness, 332 . of heart, transection, figures, 336 .

VENTRIC'ULUS (Latin, the belly, a cavity), commu'nis, aula, 472-473.

dex'ter of heart, $332,33 \%$.

latera'lis, procolia, 485 .

olfacto'rius, rhinoccelia, 487.

op'ticus, s. mesencephalici, 482.

quar'tns, metaccelia, 482.

quin'tus, pseudocolia, 485 .

sinis'ter of heart, 332,338 .

ter'tius, diacolia, 477 .

Veruacular terms compared with technical, 16,17 . 
VER/TEBR丑, 37.

cervicales, figure of, 172.

figure of, 173 .

VER'I'EBRAL column, regions of, 170. ganglion, 395.

notch, 173 . ring, 172.

Ver'tebrate, figure of various aspects, 30.

Vertebrarte' rial (Latin, vertebra, and arteria) foramen, 171, 173.

Vesi'ca urina'ria, urocystis, 294.

VESSELS, distinguishing from nerves, 375. how to connect when severed, 511 . of thorax and arm isolated, 347.

Vestib'ulum (of the ear), 527, 533.

Vial for brain or heart, figure of, 128.

Vibris'sæ (Latin, vibrare, to move quickly to and fro), 512.

Vil'li of intestine, 284.

VIS'CERA, abdominal, and peritoneum, diagram showing relations, 279 . general view of, 276 . preparation of, 132.

$\nabla$ is'ceral and 'neural cavities, 32.

Vision, sight or visual sensibility, 511, 514.

Visual sensibility, sight vision, 511, 514 .

Vit'reous humor, 522.

Vit'reum (Latin, of or like glass), 522, 526.

VOCABULARY, macroscopic of brain, 436. partial, of amphibian brain, 403.

Voli'tion, center of, 371 .

Volume of organs, determining, 136.

Vo'mer, 187.

Ward, Henry A., 536.
WASTE pail, 81.

papers, 73.

pit, 82 .

Water for the reduction of alcohol, choice of, 116.

Wax and tallow injection masses, 141.

Weighing pan, 69.

Weight of brain, determined from capacity of skull, 191 .

WETTING bottle, and mixture, 72, 73. bottle for 15 per cent. glycerin, figure of, 72 .

Wharton's duct, and preparation of, 298, 302.

Whetstone, coarse and fine, 76 .

Whitall, Tatum \& Co., Catalogue of, 59.

WHITE, nervous matter, 472.

sulustance of Schwann, 369, 398 .

zone of the eyeball, 521,526 .

Wickerscheimer's liquid, preparation and use, 124.

Wigglesworth, E., 8.

Wilkinson, A. J., Catalogue of, 59.

Willis, 505.

Windpipe, trachea, 307.

Womb, uterus, 295.

Wood spirit or methylic alcohol, 124.

Words, formation of compound and hybrid, 28.

Wounds, dissection, 85.

Wyman on anatomical use of cat, 56 ,

Zinc chlorid for hardening brain, 435.

Zona alba of the eye, 521, 526.

$\mathrm{ZO}^{\prime}$ nula Zinnii, 523.

Zoological classification, 8.

Zygomat'ic gland, 303. 








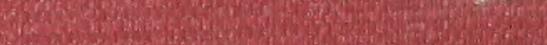

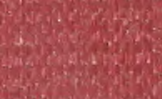

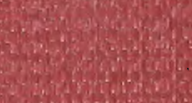
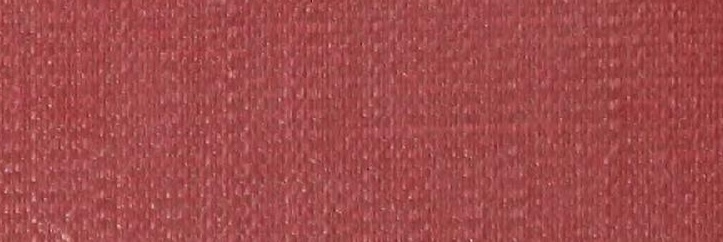

Hos
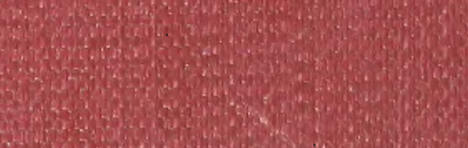

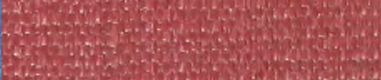

H.

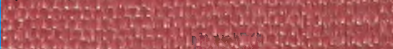

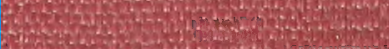

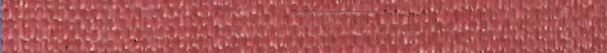

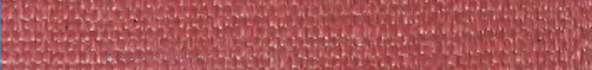
Whate

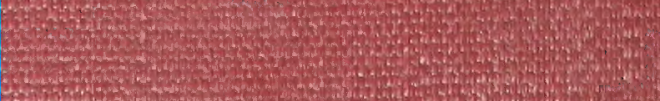
240.

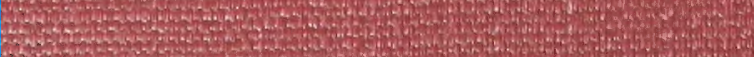
ment

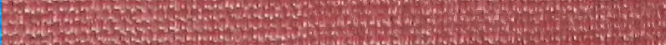

